


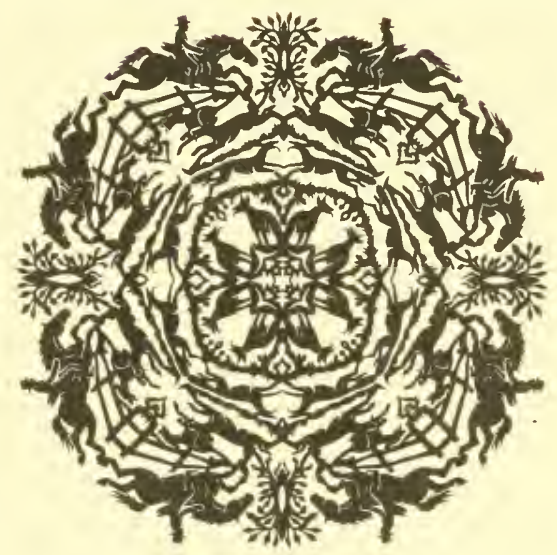

JOHN A.SEAVERNS 


THE PRINCIPLES AND PRACTICE OF VETERINARY MEDICINE. 

TUFTS UNIVERSITY LIBRARIES

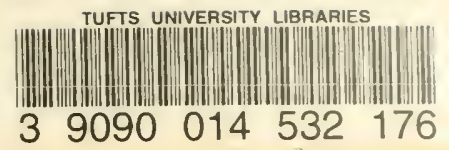




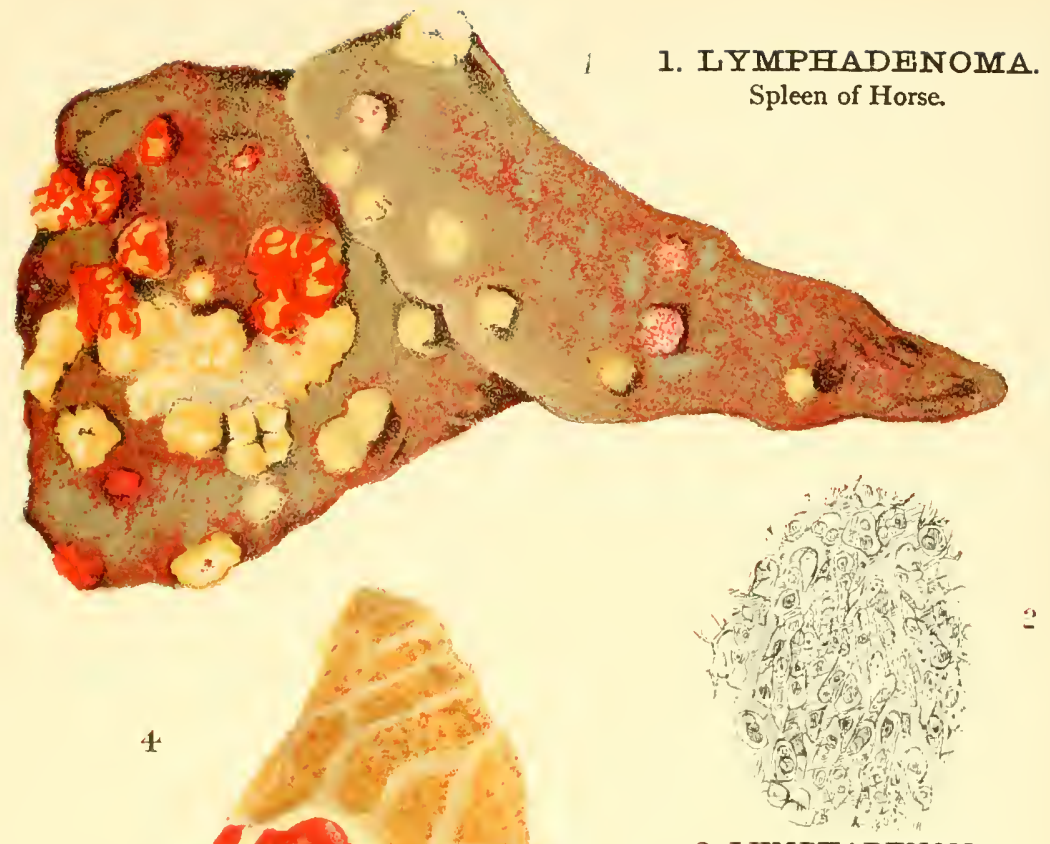

2. LYMPEADENOMA.

Microscopic view $\times 300$.

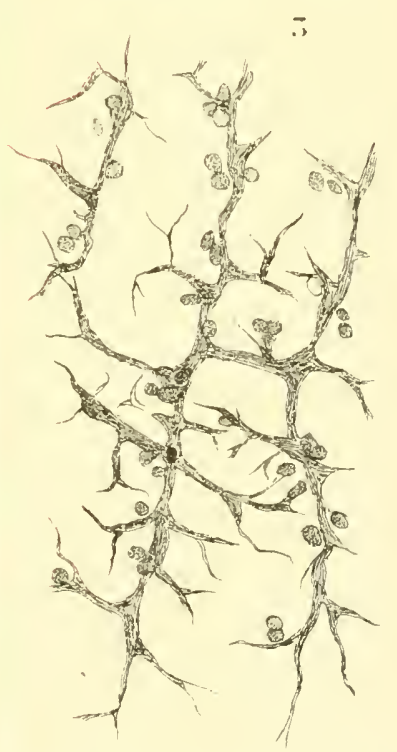

3. LYMPHADENOMA, showing Fibres. Some of the Cells pencilled out. $\times 300$. 
BRONCHO-PNEUMONIA, or so-called. Pleuro-Pneamonia.

American Contagious

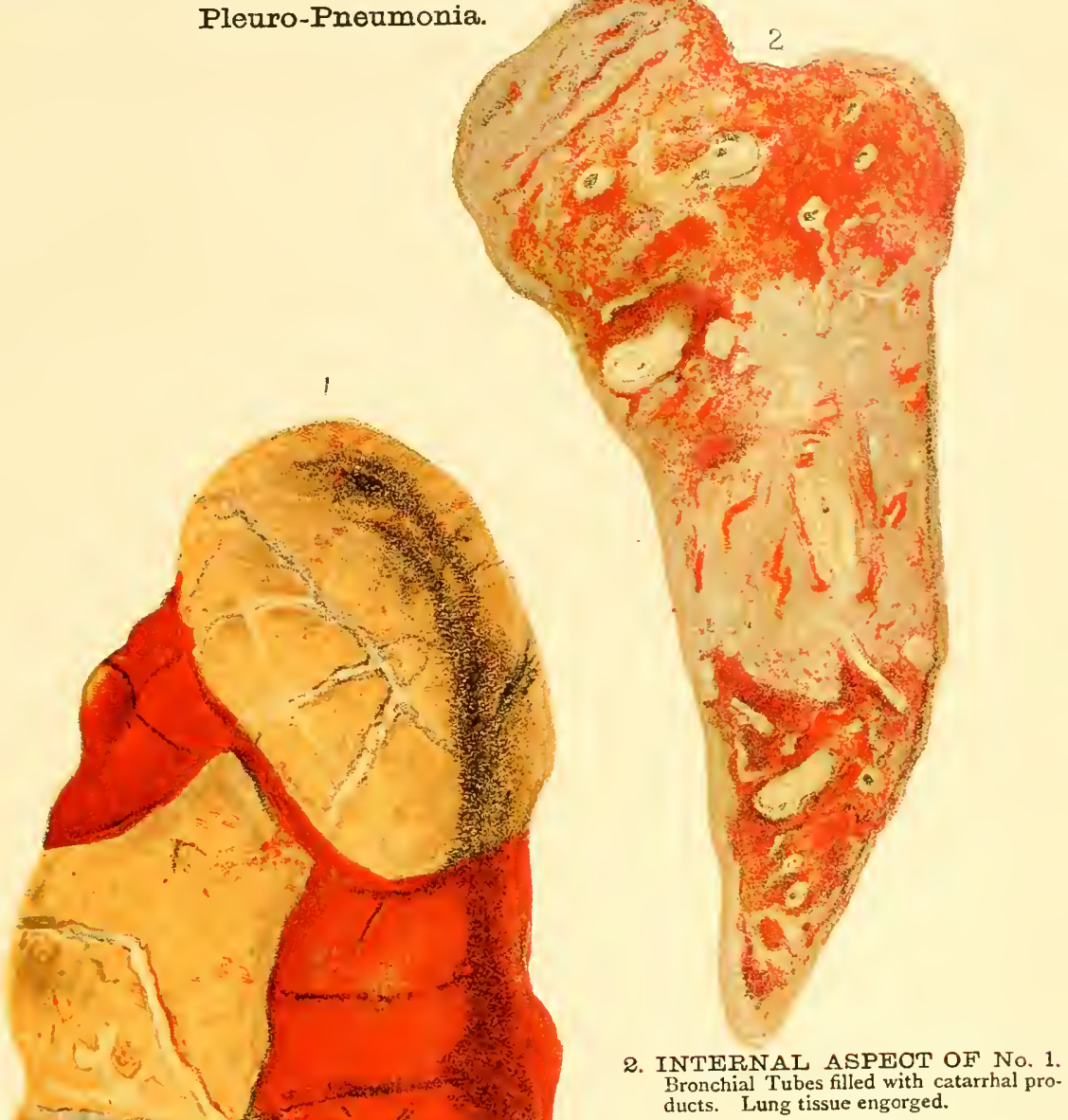





TUBERCOLAR PERICARDITIS.

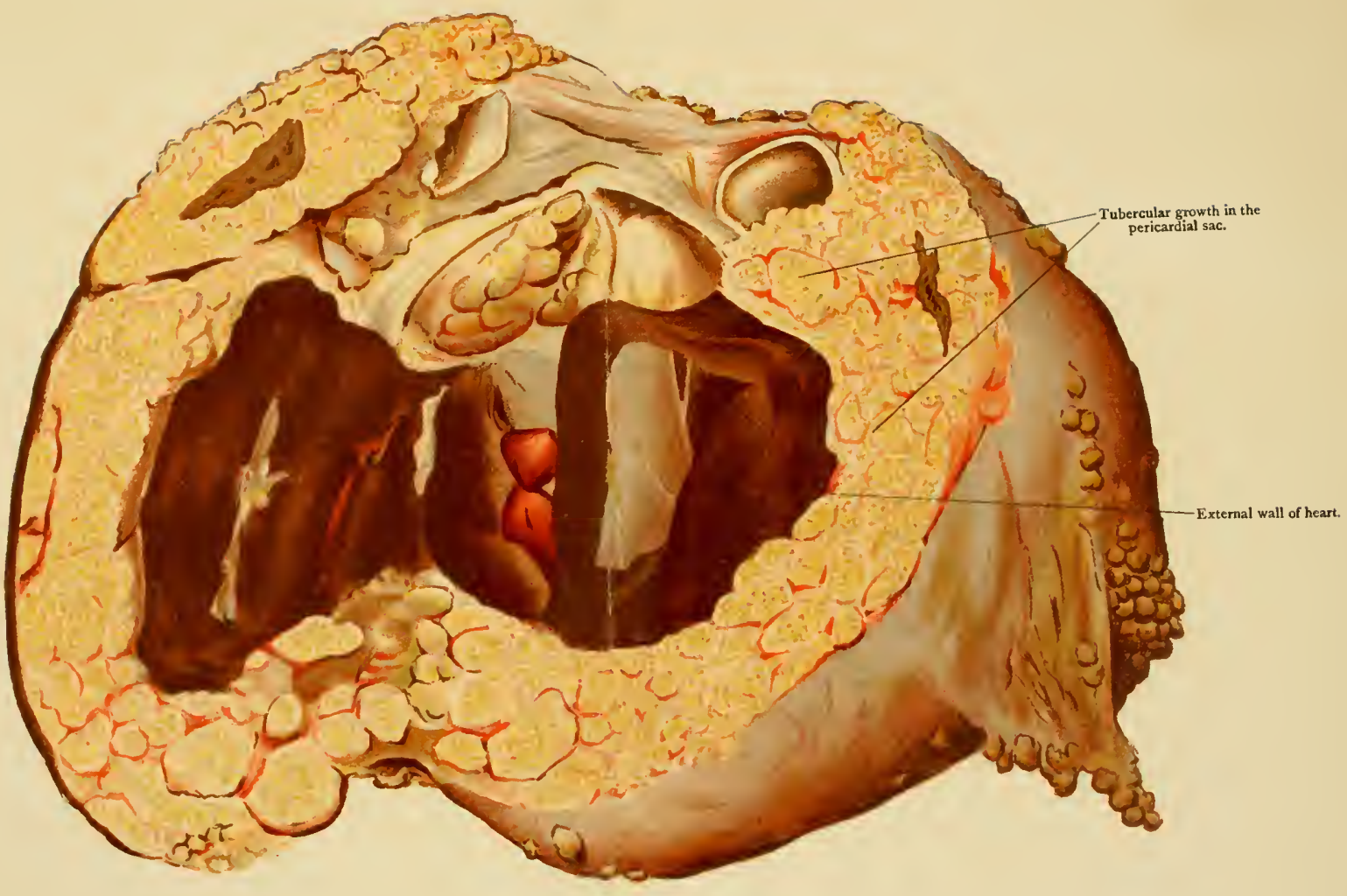

The Heart and Lungs were from a Cow, and together weighed $56 \mathrm{lbs}$. 

TUBEROULOSIS OF OUTSIDE OF LUNG. ("GRAPFS.")

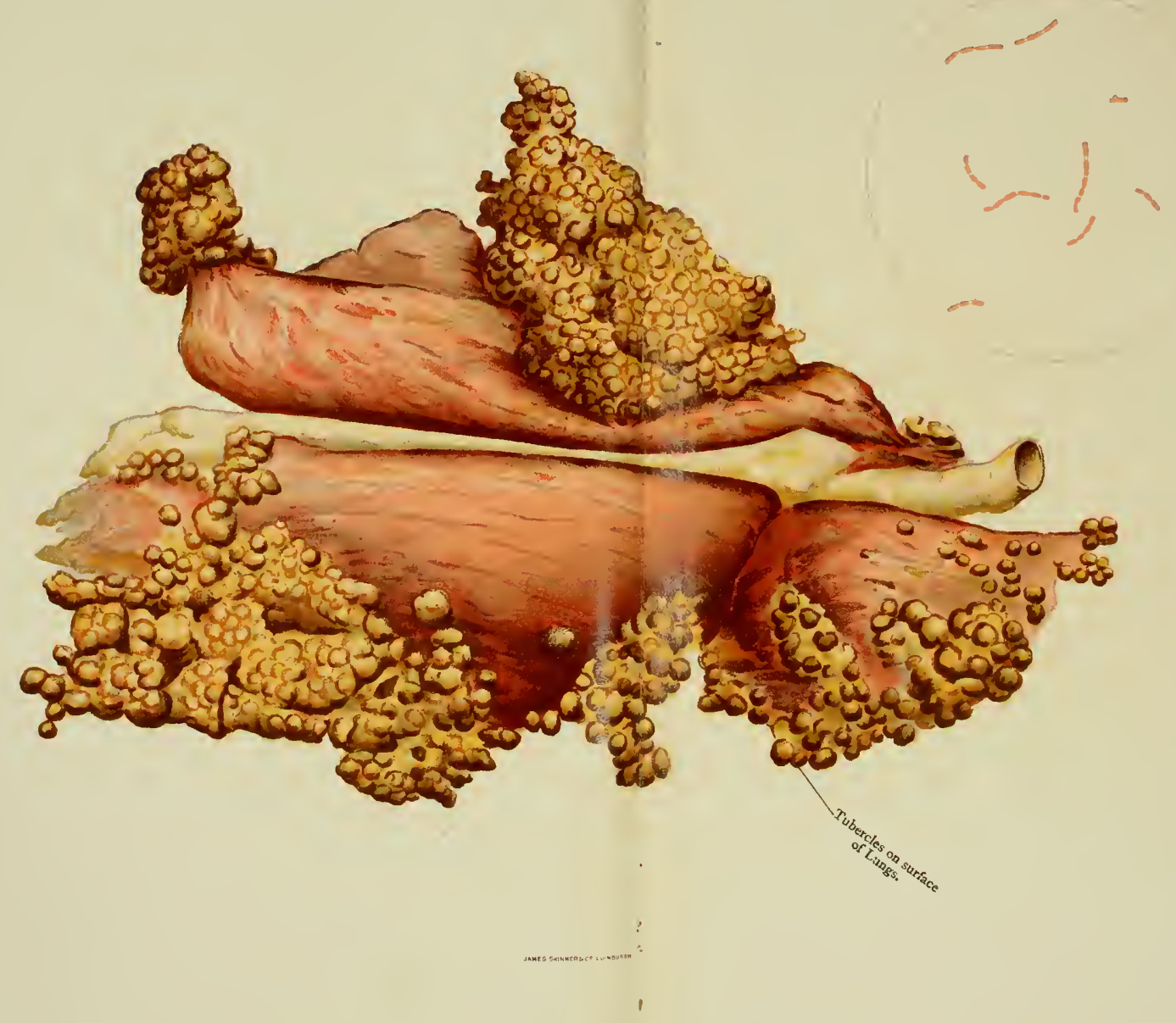



CLEAR VACCINE LYMPH.

Source-Typical Jennerian vesicle. Cover-Glass preparation.

a. Minute isolated spores of micrococci.

b. Larger forms of micrococci, showing com mencement of opacity.

Size-'s $\mu$ to $5 \mu$.

Koch's aniline methyl-violet stain.

$\times 1045$.

Buist.

OPAQUE VACCINE, LYMPH.

a. Diplococci.

b. Sarcina-like groups.

c. Chains or necklaces.

d. Irregular colonies.

e. Very large micrococci.

Koch's aniline methyl-violet. $\times 1045$. TROUP.

$:$

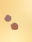

$\because$

$\because$

$\alpha$
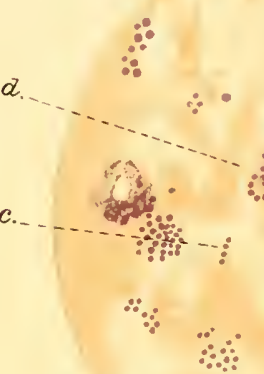

d........ :

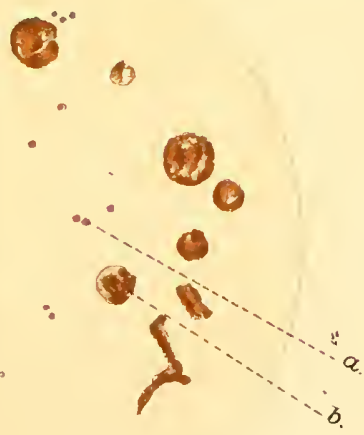

e.

Left Half.

CLEAR VARIOLOUS LYMPH.

Shows spores of micrococci.

. $2 \mu$ to $3 \mu$.

Gentian violet.

$$
\times 1045 .
$$

Buist.

\section{Right Half.}

OPAQUE VARIOLOUS LYMPH.

a. Large forms of micrococci and diplococci.

b. Torulæ variolæ (not always present).

$$
\text { Size }-{ }^{\circ} 5 \mu \text { to }{ }^{\circ} 8 \mu \text {. } \quad \times \text { ' } 1045 \text {. }
$$

Gentian violet and chysoidin.

Buist. 



\title{
PRINCIPLES AND PRACTICE
}

\author{
OF
}

\section{VETERINARY MEDICINE.}

BY

\section{WILLIAM WILLIAMS,}

F.R.C.V.S., F.R.S.E., ETC.

EX-PRESIDENT AND MEMBER OF COUNCIL OF THE ROYAL COLLEGE OF VETERINARY SURGEONS; PRINCIPAL, AND PROFESSOR OF VETERINARY MEDICINE AND SURGERY AT THE NEIV VETERINARY COLLEGE, EDINBURGH;

EX-EXAMINER IN VETERINARY HYGIENE FOR B.SC. (AGRICULTURE), EDINBURGH UNIVERSITY ; PROFESSOR OF VETERINARY SURGERY AND EXAMINER IN AGRICULTURE FOR THE HIGHLAND AND AGRICULTURAL SOCIETY OF SCOTLAND; FOREIGN CORRESPONDENT OF THE SOCIÉTÉ CENTRALE DE MÉDICIN VÉTERINAIRE; AND AUTHOR OF "THE PRINCIPLES AND PRACTICE OF VETERINARY SURGERY."

EIGITH EDITION.

REVISED BY THE AUTHOK, ASSISTED BY HIS SON. WV. OIVEN WILLIAMS, F.R.C V.S., F.R.P.S., F.R.S.E.

JOHN MENZIES \& CO., EDINBURGH AND GIASCGOW: BAILLIERE, TINIAIL, \& COX, IONDON. 
PRINTED FOR THE AUTHOR BY J. SKINNER \& COMPANY, AND PUBLISHED BY

JOHN MENZIES \& CO, EDINBURGH AND GLASGOW. london, . . . . . Ballier, Tindal, \& Cox. 


\section{PREFACE TO THE EIGH'TH EDITION.}

SInce the last edition of this work appeared, in 1893, many important discoveries have been made in Pathological and Biological Science, more particularly in the departments that treat of the evolution, the physiological or pathological effects, and all the various phenomena associated with the development and life-history of the lower forms of life known as Bacilli, Bacteria, and Microbes. The Author has therefore been compelled to completely recast the Bacteriological portions of his work.

In 1882 an attempt was made by certain parties to disparage and minimise the importance of a discovery which the Author clained to have made, namely, the organism of the Tick. since then the Anthor has continued assiduously his investigntions, with the result that his former conclusions have been abundantly confirmed. For further information on this point, he refers the reader to the chapter on what is popularly termed Louping-IIl, which he has ventured to term Ixodic Toximia. This chapter, along with that on Texas Fever (termed by the Author Ixodic Animia), which he had special opportunities of studying while in Jamaica in the summer of 1896, will, he trusts, be found interesting as being founded on the theory that certain saprophyte filaments, innocuous in themselves, may, when ingested with foods which they infest, becune pathogenic 
or disease-indueing to the higher animals, when they have passed throngh the blood of the lower forms of life, such as Ticks, and have been transmitted by these into the circulation of the higher animals. This view to some extent supports that of Buchner, who states that the ordinary Hay Bacillus-Bacillus subtilis-attains all the virulent properties of the Bacillus anthracis after it has been cultivated in animal media, meatextract, blood, \&c.

Since this work has gone to press, the Anthor has received further corroboration of the seemingly anomalous oceurrence of simple filaments as retrogressive stages in the development of an organism, apparently dependent upon a change in the nutrient medium, - a fact previously noticed in the course of his "Louping-Ill " investigations, 1882, which has led to a considerable amount of controversy.

These so-called "involution" stages, as exhibited by the Bacillus typhosus, will be found described and illustrated in a paper by Dr. Edward 1'. Carter in the Bulletin of the Johns Hophins Hospital for June of this year.

The thanks of the Author are specially due to his colleagne Dr. James Hunter, whose unwearied labou's and whose acute judgment and profound learning have contributed greatly to the fulness and accuracy of many of the illustrations, in themselves unique, in the present work.

W. W.

New Veterinary Coldege, Eminburgh, September 1897. 


\section{PREFACE TO THE SEVENTH EDITION.}

Iт is a source of much gratification to the Author that a new edition of this (the seventh) and the companion work, "The Principles and Practice of Veterinary Surgery" (the eighth) have again been called for. Both works have been carefully revised and bronght down to date, and while in the latter the changes required have not been so great, the advance in the science of Veterinary Medicine has been so important as to necessitate considerable alterations in portions of the text. It has been the Author's endeavour to watch closely, both by means of original research and by the study of the work of the most eminent Pathologists, all the developments which have taken place in that science, and professional men and students will find the results incorporated in this work.

The diseases of Domesticated Animals, and their preservation in a state of health, have long since become important factors in connection with the vital question of the food supply of the conntry, and the Members of the Veterinary Profession must continue to give their utmost attention to the advancement of Veterinary Science, as more than ever this question has become of national importance, especially since the depression in Agriculture, and the substitution therefor to a great extent of the rearing and feeding of Cattle, and also their importation in such large numbers from Canada, the United States, and other countries. 
viii PREFACE TO THE SEVENTII EIITION.

So far back as 1879 the differentiation of Pleuro-Pneumonia Contagiosa and Broncho-Pneumonia had been a disputed question between the Veterinary Officer's of the Privy Council and the Author, and this matter has recently assumed alarming proportions in consequence of the great and useless slaughter of Cattle involved muler the Privy Comncil Orders, and the consequent immense financial loss to Farmers and Cattle Importers, and also to the public in increased taxation, as well as the nation generally in the enhancerl priee of its food supply.

The Author desires to call the attention of his professional brethren to the Chapters on Pleuro-Pneumonia Contagiosa for the history of this question, and the Illustrations there given, as he consider's that the matters discussed therein are calculated to benefit the publie, and, if thoroughly understood, to mise the standard of Veterinary science.

The Author has to thank l'rofessors Hun'tek and W. O. Williars, and others, for the preparation of sections of various diseased structures throughout the work.

W. W.

New Veterinary College, Enmburgh, Jamur!! 1893. 


\section{CONTENT'S.}

CHAPTER

I. INTRODUCTORY, . . . . . . . 1.3

II. Pathologr, . $\quad$. $\quad$. $\quad$. $4-22$

III. The Morbid Phenomena-Simptoms añ Signs of Disfase

一SEMIOLOGY, . . . . . . . . . 23.25

IV. General Symptoys of Disease, . . . . 26-46

V. Morbin Elementary Pronucts-Complex Vital Processegs whose Phenomena constitute Disease, . . . 4 $7-51$

VI. Atrophy and Degenerations of Tissul: Atrophy, $52-56$

ViJ. Atrophy and Degenerations of Tissue: Degenerations, $57-70$

ViII. Changes in the Blood, . . . . . . 71-81

IX. Changes in the Blood: Anemia; Leukwaia ; Plethora, 82-8i

X. Changes in the Blood: Defective Excretion, . . 88-93

XI. Changes in the Blood: Hyperemia or Congestion; 1,ipemia, or Fatty Blood; Septic enia, . . . 94.112

XiI. Mlodes of Death, . . . . . . . 113.125

XIII. Classification of Diseases, . . . . . 126.128

XIV. Contagion and Contagious Diseases, . . . 129-136

XV. Contaglous Diseases : Cattle Plague, . . . $137-159$

XVII. Contagious Diseases : Pleuro-Pneumonia Contagiosa, . 160.187

XVIII. Contagious Diseases: Eozema Contagiosa, • . 188-195

Xix. Contagious Diseases: Infectious Abortion, 196-201

XX. Contagious Diseases: Variola Vaccine (Cow-Pox). 202-209

XXI. Contagious Diseases: Variola Ovine (Sheep-Pox), . 210-220

XXII. Contaglous Diseases: Rabies, . . . . $221-234$

XXIII. Contagious Diseases : Glanders and Farcy (Equina), . 235-254

XXIV. Contagious Diseases: Maladie du Coït, 255-268

XXV. Contagious Diseases: Canine Distemper, . . 269-276

XXVt. Contaglous Diseases: Axthrax, • • . . 277-309 
XXVII. Contagious Diseases: Hog Cholera-Swine FeverPneuno-Enteritis, . . . . . 310-322

XXViIt. Contagious Diseases : Tuberculosis, . . . 323-345

XXIX. Enzootic and Eprzootic Diseases : Influenza, 346-378

XXX. Enzootic and Epizootic Diseases: Malignant Catarrhal Fever of the Ox, . . . . . . 379-380

XXXI. Eruptive or Petechial Fevers : Purpura Hemorrhagica 一Scarlatina, . . . . . . . . 381.394

XXXII. Septic Blood Diseases, . . . . . 395-408

XXXiII. Blood Diseases: Axemil-Surra, . . . 409-415

XXXIV. Blood Diseases : Ixomic Andma-Texas Fever, eTc. . 416-437

XXXV. Sporanic Diseases : Rheumatism, . . . 438-443

XXXVI. Dietetic Diseases, . . . . . . 444-447

XXxvil. Dietetic Diseases: Drabetes Ixsipinus - Polyunia Hytruria - Azoturia - Oxaluria - Ren Water Anthma, Broken Wind; Lean Poisonixg; Palaffin Orl Poisoning; Rhododendron Poisoning: Yew Poisoning; Milk Sickness; Cake Poisonisg; LatimRIASIS ; LtPINOSIS, . . . . . 448-497

XXXVIII. Local Diseases : Diseases nf the Nervous Srstear, ～$\quad 498-502$

XXXtX. Lacal Diseases: Cephalic Diseases, . . . 503-506

XL. Local Diseases: Encephalitis, Inflamation of the Brain and Membranes; Cerebro-Meningitis, Phrenitis, Mad Staggers, Phrenzy, Coma, Sleepy Staggers, Stonach Staggers, AbDoninal Vertigo, etc.

$507-515$

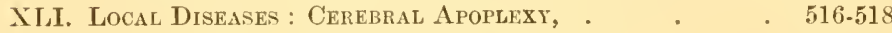

XLII. Local Diseases: Apoplexy from Congestion, . . 519-530

Xliit. Local Diseases : Adventitious Substances in the Brain and Cranial Cavity-causing, generally, Symptoms of Connitions variously termed Sleepy Staggers,

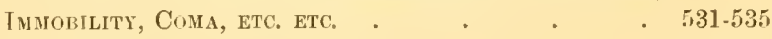

Xliv. Local Diseases: Diseases of the Spinal Corn and its Membranes, . . . . . . . $536-540$

XIV. Local Diseases: Paralysis, • . . . $541-573$

Xlvi, Local Diseases: Diseases of the Respiratory Organs, 574-594

XliviI. Local Diseases: Diseases of the Respiratory Organs: Catarrh and Laringitis, . . . . . 595-598

XI,ViII. Local Diseases: Diseases of the Respiratory Organs: Croup-Cynanche Trachealis ; Diphtheria, 599-604 
XliX. Local Diseases: Diseases of the Respiratory Organs:

InFLaMHation of the Respiratory Organs-Bron-

CHITIS, . . . . . . 605-620

I. Local Diseashes : Diseases of the Respiratory Organs:

Chronic Emphysema of the Lungs, . . . 621-623

LI. Local Diseases: Diseases of the Respiratory Organs:

Congestion of the Lungs-Pulionary Apoplexy-

Mechanical Engorgement, . . . . 624-628

LII. Local Diseases: Diseases of the Respiratory Organs:

Inilamiation of the Lungs-Lobar (Sporadic)

Pneumonia, . . . . . . 629-64

LiII. Local Diseases : Diseases of the Respiratori Organs :

Pleurist-Pleuritis, . . . . . 645-653

LIV. Local Diseases: Diseases of the Heart and its Mem-

BRANES, . . . . . . 651-669

LV. Local Diseases: Affections of the Diaphragi, a 670-671

LVi. Local Diseases : Diseases of the Digestive Organs, . 672.673

LVII. Local Diseases : Diseases of the Stomach, • • 674-688

LViII. Local Diseases: Diseases of the Bowels: Constipation:

Colic : Impaction of the Colon : HeMorrhoids oR

Piles : Imperforate AnUs, . . . . . 689.698

LIX. Local Diseases: Diseases of the Bowels: Inflaminatory

Diseases OF the INTestines-Enteritis, . . 699-703

LX. Local Diseases : Diseases of the Bowels : Intussuscep-

TION AND Volvolus, . . . . . 704-706

LXI. Local Diseases: Diseases of the Bowels: Intestinal

Concretions, . . . . . . . $707 \cdot 710$

LXII. Local Diseases: Diseases of the Bowels: Diarkhea, 711.712

LXIII. Local Diseases : Diseases of the Bowels: SuperpulgaTION,

LXIV. Local Diseases: Diseases of the Bowels: Dysentery, $718-719$

LXV. Local Diseases: Diseases of the Bowels: Ascites, or

Dropsy of the AbDomen, . . . . $720-721$

LXVI. Local Diseases : Diseases of the Liver, 722-738

LXVII. Local Diseases : Diseases of the Spleen and Pancreas, 739-741

LXVII1. Local Diseaseis : Diseases of the Kibneys, · · 742-74t 
LXIX. Lucal Diseases: Inflamimation of the KinnetsNephritis,

LXX. Local Diseases: Crstitis, or Inflamation of the BLADDER,

LXXI. Local Diseases: Hrsteria,

LXXII. Local Diseases: Diseases concurient with or immediately succeeding Parturition, . . . $759-762$

LXXiti. Parasitic Diseases, _ . . . . . . 763-798

LXXIV. Parasitic Diseases: Diseases caused by Nimatoda or Round-Worms, . . . . . . . 799-820

LXXV. Parasitic Diseases: Trematoda-Tot in Sheep, _ \$21-826

LXXVi. Parasitic Diseases : Cestoda, . . . . $827-842$

LXYViI. Bacteria, . . . . . . . . 843-844 


\section{LIST OF ILLUS'TRATIONS.}

\section{Plates.}

PAfF

Comparative View of Pleuro-Pneumonia and Broncho-Pneumonia, or so-called American Contagious Pleuro-Pneumonia ; also Lymphadenoma of Spleen of Horse, (Frontispicce.)

Tubercular Pericarditis, . . . . . (Do.)

Tuberculosis of Outside of Lung, . . . . . . (Do.)

Bacillus Tuberculosis, . . . . . (Do.)

Micrococcus of Variola Vaccinæe in Clear and Opaque Vaccine

Lymph, .

I. Cattle Plague: Mouth and Palate of a Bullock, showing excoriated Ulcers and Aphthous Deposit, . . .

II. Cattle Plague: Fourth Stomach, Pyloris, and Commencement of Duodenum of a Cow, showing Congestion of Aphthous Deposit,

III. Cattle Plague : Small Intestine of a Cow, showing Aphthons Patches, Mucous Membrane entirely congested, verging towards Gangrene, . . . . . .

Cattle Plague: Gall Bladder of a Cuw, showing Aplithous Patches,

V. Cattle Plagu : : Heart of Ox, showing Petechial Spots, . 151

VI. Foot and Mouth Disease: Excoriatious on Tongue, as desuribed on page 189 ,

VI ${ }^{1}$. Pleuro-Pneumonia Contagiosa : Broncho-Pneumonia : Bacterium of Broncho-Pneumonia, . . . . 177

VI.1. Glanders affecting Nostrils of Horse, . . . . 237

VIB. Farcy Buds in various stages, $\quad . \quad$. $\quad . \quad 239$

VIc. Swine Plague : Bacillus Luteus, . . . . . 315

VII. VIII. Plates shwwing Life-History and Development of Cestoda, 837.838 IX.-X.-XI. Do. do. do. of Nematoda, 839-841

XII. Do. do. do. of Trematoda.

Diptera, ${ }^{7}$ and Trachearia, . . . . . 842

XIII. Plate showing Life Hist ory and Development of Bacteria, . $\$ 11$

Woui ENgRavings, \&c.

Sphynographic Illustrations of various kinds of Pulse, . . 38

1. Fatty Degeneration of Muscular Tissue, . . . 58

$\therefore$. Fatty Infiltration of Heart, . . . . . 65

2.1. Blood Corpuscles in Lipæmia, . . . . . 103

3-4. Transverse Section of Broncho-Vascular System, . . . 168

5. Section of Lung in Pleuro-Pneumonia, . . . . 170

6. Microscopic Appearance of 'do. . . . . . 170 
6A. Pure Pneumonia as seen in Pleuro-Pneumonia, . 179

6B. Broncho-Pnenmonia as seen in "Corn-Stalk" Disease, . . ' 180

7. Glanders Bacilli, . . . . . . . 238

8. Ulceration of Dental Surface of Lips, . . . . . 251

9. Ulceration of T'ip of Tongue, . . . . . . . 251

10. Ulceration of Skin of Lips and Cheeks, . . . . 252

11. Transparent Rods of Bacillus Anthracis, . . . . 280

12. Spore-bearing Filaments of do. . . . . . 281

13. Spores of do. . . . . . $\quad 281$

14. Anthrax Bacilli and Blood Corpuscles, . . . . 282

I4A. Blood Corpuseles and Bacilli of Anthrax,. . . . 292

14B. Portion of Cæcum of Pig affected with Swine Plague, . . 317

15. Tubercle Bacillus, . . . . . . . . 326

16. Tuberenlar Nodule, . . . . . . . . 326

17-18. Tuberculosis Bovis, . . . . . . 344

19. Congestive Patch on Murous Membrane of Stomach in Influenza, . 357

20. Microbes in Epizootic Cellulitis, mostly in pairs (Diplococci), $\quad 360$

21. Do. do. in groups, . . 361

22. Monarls in Rat's Blood in Surra, . . . . . 412

23. Micro-Parasites in Ixorlic Anæmia, seen as minute points, . 419

24-25. Organism in Blood of Ox in Ixodic Anæmia in various stages, 436

26. Crystals of Nitrate of Urea, . . . . . . 454

27 . Crystals of Oxalate of Lime, . . . . . . . . 458

28. Constituents of Urine in Red Water, . . . . 464

29. The Sheep Tick (magnified), . . . . . 555

30-31. Mieroscopic Views of Gelatinous MLatter from Spinal Canal of Sheep, containing Bacillum Chorex Ovis, . . . . . 558-559

32. Microscopic View of Bacillum Chorea Ovis, showing Cultivation from Spinal Fluid, . $\quad . \quad$. $\quad . \quad 5661$

33. Do. do. showing Cultivation from Bloukl, . 563

34-35. Do. do, showing Cultivations from Tieks, $564-565$

35 A-35K. Photo-Nicrographs of Organism of Louping-Ill, . . 573

36. Small Bronchus in Acute Bronchitis, . . . 608

37. Portion of Lung showing Bronchitis in earliest stages, . . 611

38. Pleural aspect of Pulmonary Lobe in do. . . . 613

39. Section of portion of Lung in do. . . . 614

40. Bronchus (medium-sized) in Acute Bronchitis, . . . 615

41. Section through Air Vesicles in Acute Catarrhal Pneumonia, . 617

12. Cirrhosis of the Liver, . . . . . . 730

43. Lymphadenoma of Spleen, . . . . . . 740

44. Strongylus Filaria, embryonic stages, in Alveoli of Lungs, . $\quad 809$

45. Strongylus Contortus, . . . . . . 809

46. Do. Bursa of Male, . . . . 809

47. Do. Genital Papillæe of Female, . . 809

18-49. Microscopic Appearances of Strongylus Tetracanthus, . . 812

50. Appearance of Mucous Membrane of Colon of Iceland Pony, natural size, $\quad . \quad . \quad . \quad . \quad . \quad 813$ 
THE PRINCIPLES AND PRACTICE OF VETERINARY MEDICINE. 



\section{PRINCIPLES AND PRACTICE}

\section{or \\ VETERINARY MEDICINE.}

\section{CHAPTER I.}

I N TROD U C TORY.

TIIE external diseases incidental to the domesticated animals laving been discussed in my work on Veterinary Surgery, I purpose in the present volume to enter into a consideration of the more purely medical or internal ailments from which our patients are liable to suffer.

I have advisedly made a distinction between Veterinary Medicine and Veterinary Surgery, not only for the reason that the subjects, thus divided, are more easily dealt with, but because conclusions which may be considered almost hypothetical and speculative in medicine, are replaced in surgery by the tangible and demonstrable.

Medicine is studied as a science and as an art: as a science, when it inquires into all the circumstances under which diseases become developed, the condition of their existence, and into their nature and causes; as an art, when it is directed towards the recognition, the prevention, and cure of diseases. In fact it is the art of understanding the nature of diseases, so far as to appreciate their causes, to prevent their occurrence when possible, and to promote their cure or to relicve them when they occur.(Biglow. Aitkex.)

In order that the student be enabled to comprehend Medicine as a science, and its application as an art, it is necessary he 
should understand PATHoLOGY, which, in its full and proper meaning, implies a knowledge of diseased processes, abnormal conditions, and morbid structures, as well as what precedes them and what results from them. For this purpose a knowledge of many collateral branches of science is essential, more particularly a knowledge of Pirsiologr; and no one ean be a physiologist without being an anatomist and a chemist. By Physiology is meant that science which treats of the conditions, phenomena, and laws of life whilst the animal body is in a state of health. Without a knowlectge of the laws of health, it is impossible to grasp and comprehend the laws of disease, for it may be truly said that the latter are but perversions of the former, and are natural, or physiological, under the operation of existing circumstances and canses.

In addition to Pathology and Physiology, Medicine comprehends Therareutics, or the science which explains the actions of remedies upon the animal body, the means by which disease may be naturally overcome, and a return to health assisted and promoted; and Hrgiene or Prophylaxis, which treats of the sanitary condition, food, and surroundings whereby disease may be prevented, and all other methods by which health may be preserved.

Disease may also be studied CLINICALLY: that is to say, disease may be studied as it presents itself in each particular case to the attention of the observer. The term clinic can scarcely, with propriety, be applied to any method by which diseases of the lower animals are studied, as it means "a patient who keeps his bed," but for the want of a better, and as it is now a generie term, I am constrained to retain it.

Before proceeding further with our object, it is necessary that I endeavour to give a definition of disease, and this I can only do by following the rules alrealy liud down by our latest pathologists. A definition of disease can only be arrived at by comparing it with the standard of health, and health, says Williams, "consists in a natural and proper condition ared proportion in the functions and structures of the several parts of which the body is composed;" but no fixed rule can be applied to this, for what is health in one may be disease in another, and there are degrees or gradations of health which cannot be said to be due to disorder or disease. For example, one animal may fatten and maintain the most robust health upon the same 
quantity of food that would keep another thin and poor. "In plain words, health does not signify any fixed and immutable conditions of the body, nor does health necessarily imply the integrity of all the bodily organs: it is not incompatible with great and permanent alterations, nor even with the loss of parts, that are not vital. Our comprehension of health being thus indefinite, our idea of disease must be indefinite also; and the best definition that can be given of it is, that it is a deviation from the state of healtl, consisting generally in a change in the properties or structure of any tissue or organ, which renders such tissue or organ inadequate to the performance of its healthy actions and functions."-(IVATSON.)

It must not, however, be supposed that disease, as exhibited by an unnatural or morbid condition, and by phenomena which are seemingly abnormal, is unnatural in itself; for in reality disease may be looked upon as the natural expression of a combination of conditions, the essential and proper consequences of some cause or influence which has acted, or is acting, upon the animal body. To adduce a familiar example, let us suppose a blister is applied to the skin: the inflammation of the skin which is thus produced is certainly unnatural, and may with propriety be called disease ; but if we look further into the matter, we can easily understand that the inflammation, vesication, or even ulceration so induced are the natural results of the action of the irritant,-in fact, the proper and healthy reaction of a healthy organism to the irritation of the blister. The absence of this reaction-the skin remaining healthy-under such conditions wonld indeed be unnatural, unlooked for, and incomprehensible. This may be taken as a type of eause and effect in all diseases: it is not the inflamed condition of the slin, nor even the constitutional disturbance which may be caused by a severe blister, but the presence of the blister that is really unnatural. This illustration, homely as it may seem, is important, as all real advancement in prevention and treatment is based upon a due appreciation of the canses of disease; for in the past, more particularly in Veterinary Medicine, the aim and purpose of practice has been directed to the treatment of symptoms-to deal with effect, often ignoring the canse. Hence the many systems, founded on erroneous bases, by which diseases have been combated, have been productive of much harm. 


\section{H A P TER II.}

\section{PATHOLOGY.}

Patiology, or more properly, when applied to the lower animals, Zoo-Pathology, is derived from the Greek words $\Pi a^{\prime} \theta$ os, a disease, and $\Lambda$ óyos, a discourse-the doctrine of disease; and is divided into General and Special Pathology.

General Patiology includes-1st. Etiology, or a knowledge of the causes of disease : $2 d$. Shinology or Symptomatology, or a knowledge of the morbid phenomena or symptoms by which disease is manifested: $3 d$. PAtirogeny, which deals with the seats or localities of disease: $4 t h$. Nosology-its division and classification: 5th. Diagrosis-the methods by which it is detected-its distinction: $6 t h$. Progrosis-its probable results; and Morbid Aratomy, including Histology-the method by which the morbid alterations of structure and the elementary constituents of diseased products are discovered.

Etiology.-The eauses of disease, or, in other words, the circumstances which precede it, and to which its occurrence is clue, are arranged under three heads, namely-the predisposing, the exciting, and the proximate.

The term proximate is used to represent the pathological condition or essential bodily change on which the symptoms of disease depend; in fact, the proximate cause has been stated to be the disease itself, and for this reason some writers have expunged the term, and have been content to arrange the causes of disease under the heads predisposing and exciting. Later writers, however, have revived the term, and I)r. Bristowe gives the following illustration: "A woman who has been frequently exposed to the contagion of scarlet fever without taking the disease, becomes at the period of childbirth again exposed, and now suffers from a virulent attack. Here, parturition (which, 
as we know, renders women peculiarly susceptible of the contagious fevers) is the predisposing cause, the scarlatinal contagium is the exciting cause, and the inflammatory processes going on in the skin, tonsils, and elsewhere, the proximate cause of most of the symptoms which the patient manifests. But the exciting cause of the scarlet fever is obviously the proximate cause of that disease, and the proximate causes of its several secondary phenomena are just as obviously their exciting causes." The distinction between the exciting and the proximate causes is thus purely artificial; whereas the differences between the predisposing and exciting causes are generally well marked; but the co-operation of both of these kinds of causes is, however, generally necessary to produce disease.

Predisposing causes of disease differ from a predisposition to disease. The first may be certain influences operating upon the animal body from without, such as heat, cold, vitiated atmosphere, inordinate work, the quality of the food, poisons, \&c.; whilst a predisposition to disease is always intrinsic, existing within the animal body, and is very frequently found to arise from some hereditary taint. For example, horses of certain breeds become roarers, or otherwise defective in their wind, from no appreciable cause. Animals thus affected are said to have a hereditary predisposition to these infirmities. In such instances, the predisposition may be truly said to be the predisposing influence which has given rise to the disease of which roaring or other defect in the respiratory function is the symptom; but it cannot be maintained that a violent inflammation of the larynx or of the lungs, when succeeded by roaring, constitutes a predisposition, although they are most certainly the predisposing, and in some instances the exciting causes of the same pathological condition.

Predisposing causes of disease commonly consist of various circumstances which influence the functions or structures of the body in an unfavourable manner, yet short of actual disease; or, in other words, those general, non-specific conditions which by their influence so alter the health of the system, or the condition of parts of it, as to render them specially suitable for the development of certain diseases, provided that an animal so predisposed be subjected to the influence of the excitants of such diseases.

The exciting causes of disease are those circumstances and 
agents which, operating on the body, especially when predisposed, may excite disease; amongst them are included those specific causes, or elements of disense, which stamp their individuality on the morbid processes which ensue in the animal body when the germs of such diseases happen to become implanted therein (as in glanders, rabies, rinderpest, scabies, $\& c$.$) , and are divided into the cognizable and non-cognizable.$ The first includes all the physical and other agents of whose existence we can take cognizance, independently of their operation in producing disease; but these so-called non-cognizable causes are now found to consist of certain organisms, the majority of which have been demonstrated to be living matters having specific effects.

The predisposing and exciting causes of disease, when existing within the system, are called intrinsic, cndopathic, or internal; but when they arise without the system, they are denominated extriusic, exopathic, or external causes of disease.

Predisposing causcs of discuses. - The most important and generally recognised predisposing causes of disease are-

(1.) The infucence of age.-This is not so striling in the lower animals as in man, but still it plays an important part. In the dog, for example, the period of dentition render's the animal liable to fits of convulsion, paralysis, disturbances of the digestive process, with vomition, irregularity of the frecal discharges, weakness, and even inflammation of the eyes, and attendant unthriftiness. Hickets is also a disease which is only seen during the early period of life, and is witnessed in all our domesticated animals, but more particularly in the dog.

Canine distemper may be manifested during any period of life: as a great rule, it is only seen during the first few weeks or months of the animal's existence. Again, the strangles of the horse is generally a disease of adolescence.

It is also well known that the invasion of parasites is much more common during the earlier period of an animal's life; thus we find the Conurus cerebralis developed in the brains of sheep and cattle during the first months of life. The Strongylus filaria of the lamb, and its analogue, the Strongylus micrurus of the calf, as a general rule induce disease in these animals in early life. The Trichonema arcuati, witnessed by me in Icelandic ponies, and referred to in the latter part of this work, are rarely found in horses above two years old. 
The bones are also much more liable to disease during youth than middle age ; for example, osteo-porosis is rarely witnessed in an animal which has passed its fifth year. I say rarely witnessed, for I have seen some horses affected with this disease which had passed the age of seven years; but even here I was induced to think that the morbid changes had protably originated during the earlier years of the affected animals' lives.

Again, we find that disease of the facial bones is generally seen in young horses, and is doubtless closely connected with the process of dentition. Nor must we forget to mention that dentition is not always free from attendant ill consequences; in some cases the erowns of the temporary molars are not shed, but become entangled in the newly cut permanent ones, causing difficulty in mastication, indigestion, and unthriftiness, more particularly in horned eattle; whilst in the horse, at about the agge of four years, a true dental congh results from irritation induced by the cutting of the sixth molar tooth.

It becomes a matter of speculation whether those causes of lameness which are so commonly met with in young horses are results of predispositions or not. When it is taken into consideration that the bones of the young are more vascular or succulent, contain less earthy matter than those which have arrived at full maturity, and that these young bones are subjected to inordinate work, concussion, and alteration of incidence by erroneous shoeing, we must conclude that there is not always a predisposition to disease, but that disease is induced by subjecting the animal to work which its physiological condition and strength are unable to withstand. At the same time, many animals develop diseases of the bones and joints without the aid of exciting causes, which leads us to conclude that in them there is at this period of life a predisposition to disease.

Both foals, calves, and lambs are very early-indeed often within the first few days of their existence-subject to disense of their articulations, very frequently without any apparent cause. Here we may safely conclude that great vaseularity of the extremities of the bones constitutes a predisposing eause of disease.

During the middle period of life, animals as a rule have few special tendencies to disease. Such affeetions as navicular arthritis, and those conditions of the respiratory track which give rise to roaring, whistling, \&c., are more frequently witnessed during the 
midule period; lut as the predispositions to these affections are supposed to be hereditary, they will receive further consideration.

The cow is perhaps an exception to that exemption from diseases occurring in middle life, for in this animal we find that when its powers are fully developed, liability to that fatal disease, parturient apoplexy, is much enhanced.

At the approach of old age, and during its continuance, diseases arising from tumours, degeneration of organs and tissues, manifest themselves; the heart, liver, \&c. undergo fatty metamorphosis; the circulation becomes feeble; digestion impaired; the bloodvessels, particularly those of the brain, undergo a calcareous change; the joints become stiff; the bones brittle, and the cutaneous surface liable to be infected by various parasites. In the dog, the crystalline lens undergoes a retrograde change, and soft cataracts are commonly seen; whilst deafness and dropsy are not rare amongst dogs of adranced age.

In the ox tribe, again, we find that age influences the seat of disease: for example, in young animals, charbonous disease manifests itself externally, whilst in those of mature age its lesions are generally witnessed in the internal organs.

Sex.-The difference in the organisation of the male and female. In addition to those variations in diseases necessarily arising from the peculiarities of the generative organs of the two sexes, as affections of the testicles, \&c. in the one, and of the ovaries or uterus, as well as hysteria, in the other, we find that sex has to a limited extent an influence at least in one disease, which occurs in both sexes; thus roaring is much more frequently met with in horses and geldings than in mares.

Peculiarities of breed and conformation.-In connection with breed and conformation, we often witness predispositions to certain forms of disease; as, for example, canker and chronic grease, common enough in heavy-legged cart-horses, are but rarely seen in the better bred ones. Roaring is very often associated with certain conformation of the neck. Large horses with long necks, particularly if fine or small at the throat, are much more predisposed to roaring than those with shorter necks; smaller horses are more rarely affected, and ponies very seldom indeed become roarers. Round-chested horses are more liable to become broken-winded.

There are also certain forms of lameness which conformation 
and size have much to do with. Ossification of the lateral cartilages is rarely witnessed in the better bred, but is very common in the cart-horse. Navicular disease, so rife amongst better bred horses, is a very rare cause of lameness in the carthorse; and, not to adduce too many examples, high-bred, nervous animals are more liable to nervous diseases than those of a lower breed.

The effects of colour in predisposing to disease are very curious, as shown in the frequency of melanoid sarcomma, and in the intractability of tumours which do not contain pigment in grey or white horses; and the liability to cutaneous eruptions upon the white parts of the body only has caused one discase to be called "white face and foot disease."-(Crusta labialis, see Veterinary Surgery.)

Tribe or species.-The influence of species in favouring or resisting certain forms of disease is really remarkable. Thus the horse, whilst liable to glanders, resists diseases to which cattle and sheep are peculiarly liable, such as rinderpest, pleuro-pneumonia, eczema epizootica. Rabies, again, is primarily a purely canine disease. Charbonous disease is transmissible to man and to all the domesticated animals, with the exception of the domestic fowls and birds generally; but here it has been found-and probably this discovery explains why some contagia affect certain animals only-that resistance to charbonous contagion is due, not to conformation, but to temperature; for when the temperature of the fowl has been lowered by removing its feathers and keeping it in water, it has become affected with the disease.

T'emperament, which consists in excess of or defect in some function or set of functions, certainly predisposes to particular diseases.

The sanguine temperament, implying an activity of the circulatory and blood-producing organs, tends to diseases of an inflammatory character. This temperament is best exhibited in lighly bred horses, whereas in lower bred animals the lymphatic temperament seems to predominate. In this temperament there is a deficiency of arterial tonicity, a want of nervous power, the circulation is languid, the capillaries and lymphatics, particularly in the extremities and depending parts of the body, are often congested and the limbs odematous, which conditions disappear for a time with exercise. Inflanmatory diseases 
run a less favourable course in animals with this temperament, there being a tendency to the effusion of serum, to deliquescence of organs, and to gangrene, and not to the exudation of plastic lymph, as in animals in which tonicity is not defective.

Cattle and sheep are generally considered to possess a lymphatic temperament. This supposition is to my mind hypothetical, for, with the exception of liability to become more rapidly anemic, and infested by parasites when improperly fed, and to suffer from the dropsies associated with the mal-condition so induced, it is found that these animals do not exhibit the same tendency to odematous swellings and other signs of debilitated circulatory powers as the horse, and in the ox particularly lymph is abundantly exuded in some inflammations.

The nervous temperament is manifested by agitation and excitement, and although it is called nervous, it does not follow that an animal possessing it has extra nervous power. Indeed, the contrary is often the ease, for an animal of this kind generally becomes exhausted in a very short time. The term seems to imply a want of proportion of some of the functions or properties of the nervous system.

Nervous animals are easily frightened, and a sudden fright may be followed by serious illness, such as violent purgings, loss of appetite, tremblings and excitement, which may continue for several hours or even days, and, what is more serious, sudden death from rupture of the heart. I have seen one sudden death in the horse from this cause-of one that was quite well at the time. In another, not of death, but of serious illness. I have it recorded that an old hunter, every time it was shod, was seized with purging, loss of appetite, tremblings, snorting with fear, and other symptoms of excitement, which always continued for three days.

Previous discases are also predisposing causes of new ones. Thus we find many horses recovering or recovered from an epizootic become the subject of rheumatism in a joint, bursa, or tendon. Catarrhal fever is often succeeded by purpura. Chorea succeeds distemper in the dog. Lymphangitis predisposes to succeeding attacks of the same affection; and if under this head we classify artificial diseases, as induced by the operation of medicine, purgative, or other evacuant, we can understand how, during the prevalence of the epizootic influence, epizootic 
disease is easily excited in an animal which has been physicked a short time previously. Even a surgical operation will, during the prevalence of an epizootic disease, cause its development in au animal previously liealthy.

Present discase, and conditions bordering on discase.-Diseases of the heart, causing engorgement of the veins, often lead to congestion of the lungs and liver, dropsy of the cavities and areolar tissue ; excessive evacuations, as in diarrhœa and diabetes, predispose to other diseases, as glanders or tubercle; feeble digestive and assimilative powers, to anæmia and dropsies; inflammations, particularly in horned cattle, to caseous tumours, in parts other than those originally inflamed.

Hereditary tendency.-Many diseases, such as curbs, spavin, ringbones, navicular disease, chorea or stringhalt, run in certain breeds of horses; tubercle and scrofula in the best breeds of horned cattle.

Dr. Fleming states that French authors are unanimous in asserting that the disease termed "roaring" in the equine species, and which now generally affects horses in Normandy, was unknown there until the arrival of Danish stallions.

The influence of climate in overcoming hereditary predisposition is well shown in India, where horses the progeny of roarers are as a rule exempt from this infirmity.

Diathcsis, idiosyncrasy, or aptitude.-Diathesis is a term used to describe a particular tendency to certain forms of disease, such as the rheumatic, tubercular, and scrofulous. In animals of this constitution, the particular disease to which they are predisposed, or to which they have a tendency, is caused by different exciting circumstances, and serious diseases are induced by trivial causes, although such animals may present no external signs of idiosyncrasy.

Exopathic predisposing causcs. - Whilst the endopathic causes of disease are generally beyond the influence of our preventive power, those existing external to the animal are to a very great extent subject to our control, and, by a careful study of them, we discover that many diseases are preventible. As a rule, however, extrinsic causes are generally excitants of disease, and it would be a needless repetition if I were to describe them here. I shall therefore pass on to-

The cxciting causes of discase.--These again present themselves 
to us under two heads-namely, the intrinsic or endopathic, and the extrinsic or exopathic. The endopathic exciting causes are(1.) Mechanical, which include strictures of orifices and tubes, by contraction of their coats, pressure from without, or impacted concretions-as stricture, intus-susception, intestinal or urinary calculi; presence of parasites in the ducts of the liver, in the bronchial tubes and cerebral hemispheres; the pressure of tumours on the brain and other parts of the body; hernix; obstructions in the larynx or cesophagus; nasal polypi, \&c.; and (2.) Chemical causcs, which include all retained secretions of excretory organs-such as urea and the other products of the metamorphosis of the nitrogenous constituents of the food and body-occurring as a result of disease of the kidneys, or when nitrogenous products are too abundantly present in the blood, as in azoturia. Again, when from some impediment to the respiratory function carbonic acid is retained, the animal may die from delirinm and coma. If the liver fails to perform its function, jaundice follows, succeeded by anæmia, dropsy, and death.

Chemical and mechanical causes are also external or exopathic, and include all poisonous sulustances, mineral and vegetable-as acids, caustic alkalies, opium, strychnia, aconite, lead, \&c. \&c., and all forms of external violence.

Disease Germs.-Contagia, morbid poisons, and specific disease, virus are now included under exopathic causes of disease, although the evidence is not very clear upon all points, but they are generally propagated by infection, contagion, and jnoculation (see Origin of Contagious and Infectious Diseases). There is yet some evidence of their endopathic origin.

Exopathic predisposing and exciting causes are those due to the influence of climate, food and water, ventilation and drainage, locality, geological formation, weather and seasons, malaria, germs, work, and the want of it.

Of the influence of climate, the diseases named kumree and bursatee, common in the East, afford well-known examples.

Food and water:-The solid and liquid ingesta are fertile sources of disease-1st, by being insufficient or ill-proportioned in quality; $2 d$, by being deficient or excessive in quantity.

Unsuitability of food, either in consequence of a deficiency, over-abundance, or improper combination of nutritive constituents, is a very frequent cause of disease. This has been 
well shown in the experiments of Majendie and others. Dogs, geese, donkeys, and other animals, when fed entirely on sugar, gum, starch, oil, or butter, died with symptoms of starvation, almost as soon as if they had been kept without food. Even bread, when too fine, is insufficient nourishment. A dog fed on pure white bread lived only fifty days, whereas another fed with the coarsest brown bread was well nourished, and seemed capable of living for an indefinite period. Again, according to the researches of a Commission of the French Institute, animals fed on pure fibrin or albumen died of starvation, almost as soon as if not fed at all.

Experiments have proved that in order to support health and strength it is essential that, in addition to water, food contain at least three classes of constituents, namely-(1st.) Nitrogenous, to nourish muscular and other albuminoid tissues: (2d.) Hydrocarbons and carbo-hydrates, which undergo combustion in the body, and assist in the maintenance of animal heat and in the assimilation of the nitrogenous compounds; and (3d.) Salines, to supply materials for the building up of the solid structures of the body, maintaining them in health, and assisting in the processes of assimilation and elimination, conveying new materials into the system and removing old ones out of it. If these various constituents are deficient, absent, or present in undue quantities, hoalth cannot be maintained, and common experience has taught that all animals are kept in the best health when fed on a mixed diet And as types of all proper food we have two examples. (1st.) Milk offer's us the best example, as it contains caseinnitrogenous, oil and sugar-hydrocarbons, water and salts. Hence young animals thrive best, and are maintained in health by the food which nature has provided for them. (2d.) Grass may also be adduced as a food containing exclusively all the in rredients required for the support of animal life.

As examples of the bad effects of food good in itself, but illproportioned in quality, we may adduce those diseases, namely, fatty degeneration of the liver and anæmia, so commonly met with in sheep too exclusively fed on turnips. During good seasons, when the turnips are firm, well-grown, and healthy, they tend to overload the system with saccharine matter, and to induce a degenerative change in the liver, which renders it of a palish yellow colour, friable, and incapable of performing its functions. This condition may be associated with fatness: indeed sheep 
so affected begin to die when almost fit for the butcher, and the best plan, when they thus begin to fill off, is to make the remainder of the flock so fed into mutton as soon as possible.

During wet seasons, turnips, although apparently fully grown, may contain but little nourishment, but are loaded with watery particles. Animals then have to consume large quantities to maintain life, and in consequence the digestive powers become weakened; debility, anæmia, and death are the results.

Now if the stockowner bear in mind that the fodder with which he is supplying his animals does not contain the essential constituents of sound food, he will avoid his losses by supplementing turnips with cake, corn, and particularly with some long food, as it must be borne in mind that all ruminants thrive best upon food requiring to be remasticated. The horse also, though not a ruminating animal, does not thrive except on food, some of which at least is coarse, and requiring much mastication ; and the bad effects of feeding the horse on a diet easily swallowed are seen when it is fed on cooked food or on bran mashes exclusively. I have seen cases of fatal impaction and of rupture of the stomach caused by feeding on bran alone; and my experience points to numbers of instances where severe indigestion, with colicky pains, and foetor of the breath, have been induced when coarse food has been withlheld from horses suffering perhaps from a sore throat or other clisease.

But whilst hay or straw, which may be called the coarser articles of diet, are necessary, alone they are insufficient to maintain an animal in robust health, as the indigestibility of the quantities necessarily ingested becomes a source of disease, indigestion, broken wind, languor and debility, or lead to such a condition of the system as to predispose it to succumb to the influences of epizootics.

In addition to being insufficient or ill-proportioned, food may be bad in quality, as in rainy seasons, where the vegetation is too watery in its nature, its nutritive constituents washed out as it were; or damaged by mould, or other causes in operation and extending over districts and even comntries. What is more common after a bad harvest, hay or corn, than to see scores of horses affected with the disease termed diabetes insipidis, induced by some as yet unknown agent developed in the food by the operation of wet, heating, and fermentation, or all of these operations combined. 
Again, it is found that wheat and rye are affected with ergot, and oats with a fungus growth, eminently fatal in its action on the animal body; and that all kinds of forage by becoming rusty acquire unliealthy properties, infected with cryptogamic plants belonging to Uredo or Puccinia species, or mouldy-blue-mould -when attackel by the Mucor mucedo.-(See FlenING's Sanitary Science and Police on this point.)

Of the bad effects of grass in rainy seasons, when it is loaded with watery particles, numerous instances of tympanitis, diarrhoea, and dysentery of a fatal nature, particularly among sheep, are witnessed during wet years.

But if the season be too dry, forage becomes hard, innutritions, and indigestible from want of moisture as one of its constituents; causing constipation, impactions, with their attendant conditions of the body, unthriftiness and debility, leading on to anæmia, and even death. In young cattle particularly, this condition of the pastures, at first giving rise to indigestion, induces, if long continued, a mal-condition of the osseous system, whereloy the bones become fragile or brittle, with stiffness of the joints and liability to spontaneous fractures. In some parts of Scotland a similar condition of the skeleton is brought about by feeding young cattle on turnips grown on land highly dressed with the phosphates, and urinary calculi are not uncommon in animals highly fed on cakes and other artificial food.

Food may be excessive or deficient in quantity. The more common effects of food partaken of in excessive quantities are colies, enteritis, impactions, and ruptures. As a rule, we find that animals partake of food in quantities sufficient to satisfy appetite and maintain health; but there are exceptions to this, and we find some, partieularly horses, habitually greedy in their desire for food, eating voraciously, hurriedly, and masticating imperfectly. Others again are voracious from accidental long fasting, and the evil consequences of this kind of feeding are a soturce of great loss to the horseowner, and of great anxiety to the veterinary surgeon, as most of the fatal cases of disease of the digestive organs arise from this cause. To avoid such loss, a little forethought would go a long way. If a horse be habitually greedy, it should be made to take the edge off its appetite by an allowance of hay before its corn, then be fed sparingly on the latter, and the quantity which is generally 
given at once ought to be divided into two or three parts and given at intervals; if this be not done, colic, rupture, enteritis, or laminitis may ensue. If a horse seem voracious from long fasting, a similar course would often avert an attack of perhaps a fatal disease; and at all times, if it be deemed expedient, owing to a damaged condition of the grain, to give cooked food -an unnatural kind of food for horses, cattle, and sheep-it ought to be given in small quantities, and often.-(See Diseases of the Digestive Organs, also effects of Potatoes.)

Food defective in quantity or nourishment causes debility, wasting, œdematous legs, susceptibility to the attacks of parasites, anæmia, dropsy, and even death.

Chusat found that in animals gradually starved to death the temperature of the body progressively declined, and unless maintained artificially, the animals scemed to die of cold. All the textures, even the bones, sustained great loss of weight, but those of the nervous system less than any others.

Sudden change of food, even if it be of good quality, is often a cause of disease. For example, what is more common than a sudden outbreak of disease amongst cattle, particularly young ones, hardly kept in the winter, when first turned into rich pastures. The same applies to sheep, and I have often witnessed a fatal form of enteric discase amongst sheep-in one instance extending to cattle and horses-when depastured during early summer upon land lately drained and limed. So great was the fatality, that the farm seemed unfit for grazing purposes, for, after liming and draining had been completed, the more benefit the land seemed to derive from the cultivation and the richer the pasture, the greater the mortality.

The influence of water.-Much prejudice exists, particularly among horsemen, as to the use of water. In consequence of this we find that hunters and racehorses are severely punished before they are called upon to hunt or race, by having but a very limited and insufficient allowance of water prior to the performance of their task. What harm a sufficient supply of water, partaken of several hours before the hunt or race, can effect, is beyond my comprehension; indeed I have found that a hunter properly fed and watered on a hunting morning has been enabled, provided always that it is otherwise in condition, 
to do its work with greater ease, and to last longer than animals deprived of so necessary an element as water.

Water containing organic impurities, or too hard from the over-abundance of earthy salts-that is to say, water containing more than twelve degrees of hardness-is apt to induce disease.

Burton, in First Footsteps in Africa, says, at Zeyla, a large Somal town on the East Coast of Africa, all the pits within the walls supply brackish or bitter water, fit only for external use, and that is the reason why vegetables are unlinown, and why a horse, mule, or even a dog, is not to be found in the place.

Organic impurities, either suspended or in solution, and more particularly during the hotter months of summer, are apt to induce diarrhœa, anthracoid and putrid diseases, which may prove fatal. Animals certainly seen to become habituated to impure water, and sometimes prefer it to that which is clean and wholesome, but even these are not exempt from its evil consequences; and indeed the very depravity which leads them to prefer what is so unnatural may be looked upon as of itself a diseased condition. It may, however, be stated that there seems to be a resistance, particularly in cattle, to the ill effects of water contaminated with vegetable products, whereas, if these be of animal origin-human ordure, decomposed blood, milk, \&c.- disease is almost certain to follow. Continual drinking of very warm water is said to induce disease.

But, as a rule, however, pure water may with benefit and advantage be allowed in such quantities as the animal seems to require, taking its condition into consideration at the time. If it be heated or exhausted by work, water must be supplied in moderate and repeated quantities, and not too cold, until its thirst is assuaged, or enteritis, diseases of the skin, or inflammation of the feet, may result. With this exception, I repeat that water ought at all times to be freely but judiciously allowed.

Water in the form of dew or hoar frost is believed to be a cause of disease. It is very possible, if a very hungry animal were to eat dew-covered grasses ravenously and quickly, that digestive disturbances might arise; but I fail to see how food, masticated and retained in the mouth until its temperature is elevated almost as high as that of the body, can he inducive of disease. Dr. Fleming tells us (Sanitary Science and Police) 
that honey-dew has been supposed to cause aphthous and other affections. "Intelligent agriculturists in Saxony have remarked this dew as a cause of epizootics, and the shepherds take the precaution of leaving their crook on the grass in driving home their flocks, and examining it in the morning before driving them out again. If they observe the dew which has gathered on the crook to be only water, the flocks may then be allowed to pasture; but if it resembles oil or honey, then they must remain until the dew has evaporated."

Geological formation and locality.-Mr. Robertson, Kelso, in his little work Hints to Stockowners, says-" The substrata on which soils rest, and to which in part they owe their formation, is always an important element in determining their character, and one which remains undisturbed in its permanence, notwithstanding cultivation, and the improvements of modern scientific agriculture. From an acquaintance with the nature of this formation, we can in many cases predict what will be the character of the stock bred and reared on such lands, as also the diseases to which they are more particularly liable, or from which they are exempt. For example, it is a fact well known to the majority of our sheep-breeders, that on certain soils, chiefly those resting on the igneous rocks, sheep are liable to suffer from a form of abdominal consumption, known as pining, and that no system of treatment is so efficacious as their removal to soils resting on the sandstone formation. There are also diseases of particular structures of the animal body, as the bones and nervous system-enzootic - that is, confined to circumscribed districts of country, which seem to owe their origin to the redundancy or absence in the soil, and materials grown thereon, of certain organic or inorganic materials. These diseases are always difficult of prevention, when only methods of cultivation, or systems of folding the animals on these lands, are adopted. They would require to have access to those situations known to be dangerous only at particular periods, and to have what food is given them from such soils supplemented by others of a very different nature."

In several parts of Scotland, it is well known that stock suffer to an enormous extent in certain pastures during spring, or until the appearance of the white clover. It is difficult to account for this, unless by the supposition that, owing to the geological 
formation of such districts, the grasses, no matter how abundant they might be in quantity, are defective in some constituents, and calculated rather to induce disease than support life, until such grasses have attained a certain age, coincident with the period of the blossoming of the white clover.

In soils deficient in certain mineral constituents, the plants grown thereon will also be deficient in those, if not artificially supplied; as, for example, as stated by Mr. Fleming, there is no phosphate of lime in the soil of the alluvial tracts on the banks of the Phine. What is necessary for the growths of plants is derived from the atmosphere, which supplies no less than 400 grammes to each hectare, according to Burrell, and which is deposited by the rain. In dry years, however, this supply fails, and the plants are then deficient in this most essential ingredient. The consequence is, that the creatures which consume these plants suffer more or less, and this is now recognised as one of the causes of that special affection of the bones of animals in those regions which has received the name of Osteoclasty.

It is well known in Scotland that certain nervous diseases, more particularly that known as "louping-ill" in lambs, are only witnessed in certain localities,-very often the breadth of a river being sufficient to separate the unhealthy from the healthy ground. Many sheep-farmers and shepherds have long believed, what is now demonstrated to be the case, that "loupingill" is only seen upon land infested with ticks, and that ticks are the cause of the disease; while it is possible to have land infested with ticks, and yet have the sheep free from loupingill, as all ticks do not seem to contain disease germs, and it is clearly proved that louping-ill is not seen where ticks are at.sent.-(See "Louping-Ill.")

The influence of marshes and undrained lands in predisposing to and exciting disease is well known, and need but be merely referred to here. Such diseases as rheumatism, dysentery, and typhoid diseases in animals, and intermittent fevers in man, are traced to the influences-malarial-of such lands. It is now demonstrated that anthrax diseases are due to a specific germ, the Bacillus anthracis, and that they cannot be developed except by the introduction of the germs into the animal body. The subject will be discussed hereafter: at the present, I have only to state that moist lands have a great influence on the development of anthrax, and that since this country has been to a 
great extent drained, at least one form of anthrax, namely, gloss-anthrax or blain, has disappeared. Again, rot in sheep, as well as other parasitic diseases, prevail to a much greater extent on damp than on dry soils, and the influence of a wet season, even on dry and well-drained soils, induces the development of many diseases, amongst which the grouse disease may be cited.

Imperfect ventilation, to which may be added defective drainage, is a fertile cause of disease ; indeed it may be stated that defective ventilation is a source of greater loss to owners of horses than all other causes of disease combined, particularly in large towns. A deficiency of oxygen, excess of carbonic acid, and a scanty supply of fresh air, stint the vital processes, and the gradual accumulation of the ammoniacal products of the decomposition of excrementitious matters by which animals are surrounded, are almost certain excitants to diseases, particularly those of the respiratory organs; and when disease is once excited by such causes, a recovery becomes almost an impossibility until the sufferer is removed from their influence. It will be pointed out hereafter that in the treatment of lung inflammation, pure air is an absolute necessity; and if we bear in mind that inpure air is often a cause of this inflammation, can we wonder at the fatality of diseases when animals suffering from such a disease are kept under the very influences which have induced its development? Let the owners of thorough-bred stock, who periodically suffer serious losses, reflect that, no matter how skilful the treatment of such animals might be, it is futile so long as the operation of causes is not taken into account.

In large horse establishments scarcely a fresh animal arrives which is not shortly laid prostrate, not so much by change of locality and food, as by the direct influence of a contaminated atmosphere. After a time, however, even the horse may become habituated to some extent, and present all the signs of health; but if an epizootic influence at any time prevail, an animal so circumstanced is always the first and the most seriously to suffer.

With regard to the drainage of stables, I am of opinion that within the buildings they should be on the surface, and that the grating leading to a necessary underground drain should be some fect upon the outside of the stable walls. Where drainage is 
bad, disease is apt to assume a pyæmic character, and an ordinary inflammation may lead to the formation of multiple abscesses, purulent infiltrations, or even gangrene.

Work-over-exertion-by its debilitating effects, tends to induce disease: for example, we have congestion and apoplexy of the lungs, as well as laminitis, myositis, and spasm of the diapbragm, from severe exertion, especially when a horse is not in condition; and from the more continued over-work, as seen in cart-horses, stiffness of the joints and back, unthriftiness, a tendency to and actual development of diabetes, and its sequelæ, farcy and glanders.

Exercise is beneficial to all animals, but when in degree or continuance it exceeds what the strength can bear or rest can recruit, the animal functions are exhausted and lose their balance, muscular tone is impaired, nervous excitability takes the place of strength, the circulation fails, congestions ensue, the blood is not properly purified, and the various organs are on the brink of disease. Hence it is that animals which do not actually become diseased from over-exertion are more prone to succumb to the influence of any epizootic that might prevail at the time.

Want of exercise is a frequent cause of disease; the muscular system, and with it the circulation of the blood, is the first to suffer, the movements become sluggish, sweats break out upon the least exertion, there is want of condition, in horse phrase, swelled legs, grease; the respiration being but little exercised, the task of decarbonizing the blood falls upon the liver, hence the accumulation of fat and the occurrence of derangements of that organ.

Heat and cold are very prevalent causes, and act as follows:Heat relaxes the whole system; under its influence the muscles, and with them the heart and arteries, lose power and tone, perspiration becomes profuse, the internal organs especially are too much stimulated by blood which has lost more than usual of its water, and less of its hydrocarbon. You will often find that upon the sudden accession of hot weather horses suffer greatly from congestive diseases. In May and June 1867 this was the case in Bradford. The weather had been cold, but very suddenly it became hot, and immediately numerous cases of congestive hepatitis occurred; in other seasons we had to contend 
with congestive pneumonia. Cold again acts as a sedative and debilitant if long continued or severe; it weakens the circulation, especially that of the surface of the body, causes internal congestions, and directly lowers all the vital energies; and the most fatal cases of pleurisy and pneumonia are observed to prevail during and towards tine close of severe winters. A temporary application of cold to the healthy animal is followed by a favourable and invigorating reaction, but when long continued its effects are most injurious. 


\section{CHA P TER III.}

\section{PATHOLOGY-continued.}

\section{THE MORBID PHENOMENA-SYMPTOMS AND SIGNS OF DISEASE-SEMIOLOGY.}

"For my own part" (says Watson), "if I were called to define a symptom, I should say, ' Every thing or circumstance happening in the body of a sick person, and capable of being perceived by himself or others, which can be made to assist our judgment concerning the seat or nature of his disease, its probable course and termination, or its proper treatment.' Every such thing or circumstance is a symptom."

Symptoms are signs of disease, but it is only by mental effort and experience that the practitioner is able to convert symptoms into signs. The idea associated with symptom is much more vague than that which is connected with sign. Some writers have endeavoured to restrict the word symptom to the phenomena depending on vital properties, whilst those phenomena of disease which are more directly physical are by them called signs. Again, some restrict the term symptom to the phenomena manifested by present disease only; but this is contrary to the custom by which we speak of precursory and consecutive symptoms. Symptoms of disease are obvious to all persons alike: for example, the manifestation of pain, the symptoms in an animal suffering from enteritis, are plainly seen by all the surrounding atterdants, but it is only the skilled and experienced veterinarian who can detect the expression, the condition of the pulse, \&c., the signs by which this malady is diagnosed or distinguished from a mere colic. "Symptoms may be considered as resembling so many words. When taken separately, or when put together at random, the words have no force or signifi- 
cation. Arrange them in due order, reduce them into a sentence, and they convey a meaning. The sentence is a sign or expression of something which is thus revealed. Symptoms become signs when their import can be interpreted."-(Sir Thos. Watson.)

According to the arrangement of Reynolds, symptoms are to be considered as parts of a disease, for he says- "So long as disease was regarded as some material put into, added to, or engrafted upon the body, the words symptom or sign described the means by which we might recognise the presence of such a material; but when we define disease as being the sum of changes in function and structure presented by the living being, the words symptom and sign describe only those parts of the disease which are appreciable to the observer's senses. We call a disease by a particular name, which may express its primary or most important fact, but we cannot separate this one fact from others, as exhibited by the symptoms, but must regard them as integral parts of the malady. Thus the different phenomena of pneumonia, the cough, lung-sounds, respirations, \&c., are as much parts of the disease as are the structural changes in the lung. We cannot imagine the existence of symptoms without disease, and vice vcrsa." I must, however, differ from the abovenamed writer, as I am strongly convinced that when symptoms are thus arranged they are apt to be therapeutically looked upon with too much significance, and thus indnce the practitioner to pursue a course of treatment more calculated to modify them than to remove their causes.

Symptoms are local or general, according to whether they are confined to the diseased part, or affect, more or less, the whole system; idiopathic, when directly proceeling from the disease; sympathetic or secondary, when arising from those produced by the primary disease, or from secondary disorder; premonitory or precursory, when they precede the full development of disease, generally resulting from the first operation of its cause, such as signs precursory to an inflammation; commomorative, when developed in the previous history of the disease. Again, symptoms and signs are further divided into diagnostic, prognostic, and therapoutic, when they are specially applicable to the distinction, the determination of the event, and the suggestion of the treatment of the disease; into objective, when they present themselves to the scrutiny of the practitioner; and these 
are again divided into the active or dynamical, when they are only discoverable by motion or manipulation, and the passive or statical, when they are obvious without such action; positive, when they consist of phenomena actually present, and negative, when they consist in the absence of phenomena. Diagnostic symptoms are those symptoms which point out the distinction of one disease from another, and the art of diagnosis is that by which the practitioner is enabled to arrange symptoms into signs of disease. A symptom or a set of symptoms which are peculiar to any particular disease, are said to furnish pathognomonic signs of such disease, and are called pathognomonic or pathognostic. A simple symptom is rarely pathognomonic, but two or three conjointly often are so. For example, a discharge is seen to issue from a liorse's nose. This is a symptom. It may, however, be due to a catarrhal inflammation, to disease of the teeth, or of the bones of the face; to an affection of the lungs, to a collection of pus in the guttural ponches or facial sinuses, or to glanders. The discharge is so far a sign, that it indicates the formation of pus. If we discover, on examination, that the discharge is associated with ulceration of the Schneiderian membrane, with enlargement of the submaxillary lymphatic glands, and more especially if the discharge itself is of a glutinous consistence and starchy appearance, we conclude that it is lue to glanders. Taken collectively, these symptoms constitute a diagnostic sign of glanders, and are said to form the pathognomonic symptoms.

The interpretation of symptoms requires the closest observation, and in many instances repeated examinations of the patient; for the veterinarian is never assisted by what are termed in human medical practice the subjcctive signs of disease, namely, the sensations felt and described by the patient himself. In veterinary practice all signs and symptoms are objective, and each sign or group of signs has to be duly considered, compared one with another, and each with all; so that a close observation is essentially necessary before a due and proper conclusion can be arrived at and an opinion pronounced. It is by comparing at intervals the various modifications and alterations which occur in the signs and symptoms of disease, that we are enabled to prognosticate the nature of its termination, or, in other words, to malie our prognosis. 


\section{CHAPTER IV.}

\section{PATHOLOGY-continucd.}

\section{GENERAL SYMPTOMS OF DISEASE.}

\section{THE VISIBLE MUCOUS MEMBRANES.}

Tire general appearance of the visible mucous membranes is of great assistance to the veterinarian in the diagnosis of disease. The natural colour of the Schneiderian membrane and conjunctiva is a palish red, or carnation ; any appreciable deviation from this is indicative of some disorder. Increased vascularity and heightened colour-floridity-are indicative generally of disturbance and over-excitement of the circulatory system. It does not follow of necessity that disease is present, as the same condition of the membranes is brought about by severe exercise; in fact, any cause of excitement may produce increased redness and vascularity of the visible mucous membranes. This condition is not usually apparent in the tongue and mouth, for the epithelium, more particularly on the tongue, is sufficiently thick to obscure any increased vascularity.

The appearance of the mouth, however, is occasionally of great importance, redness of it being indicative of an irritable and congested conclition of the digestive organs generally ; vesication, of sporadic or epizootic eczema; a peculiar yellowish or salmon colour, with desquamation of its epithelium, more particularly in the neighbourhood of the incisor teeth and within the lips, of rinderpest; whilst sudden pallor of the mouth and tongue, with coldness, is symptomatic of approaching death from hæmorrhage. The appearance of petechial spots on the visible mucous membrane denotes a depraved condition of the blood, as in purpura. Yellowness of these membranes inticates disease of the liver; livility, a carbonized or non-oxygenated condition of the 
llood, as in bronchitis and pulmonary congestion; a slate-coloured appearance, a condition of the blood due to the poison of glanders, or a state of the system predisposing to that disease; rusty colour, some forms of epizootic diseases; pink, or pink-eye, epizootic cellulitis; pallidity, anæmia and general debility, or, if occurring suddenly, hæmorrhage. Mere fulness of the, capillaries of these membranes, with increased redness, is not always dependent on an increased circulation, for in many diseases, where the powers of the circulatory organs are much diminished, the colour and vascularity may be greatly augmented. In several cases, where death was approaching from a degenerate conditiou of the structure of the heart, I have noticed that the visible membranes became very greatly congested, and their colour increased; but the shading of the colour has been different to that seen in inflammatory diseases; the circulation in the vessels has been sluggish; the power of the heart-vis à tergo--has been insufficient to propel the blood through the minute capillaries; hence the congestion. The purplish line mentioned by some writers as being indicative, when not dependent upon an altered condition of the blood, of debility, has not been present in these cases; incleed the difference, except in the shading of the colour, from the appearance in many inflammatory diseases has been very slight; whilst the state of the heart itself has been recognised by violent palpitations, great irregularity of its action, and almost imperceptibility of the pulse at the jaw and arm. A foul appearance of the mouth and tongue, so valuable an aid to diagnosis in human medical practice, is rarely observable in the horse and ox, but is commonly seen in the dog. However, in some forms of dyspepsia, a slightly foul and soapy condition of the buccal membrane is seen even in the horse and ox, with an acid condition of the salivary secretions, which smell sour and even fœetid. Dryness of the mouth is often indicative of inflammatory diseases, more particularly those affecting the organs of digestion. A moist state of the mouth, from excessive secretion of saliva, is symptomatic of disease of the teeth, of catarrhal disease, tetanus, or of the presence of a foreign body, such as a pin or thorn, in some of the oral structures. 


\section{SYMPTOMS FURNISHED BY THE CONDITION OF THE SURFACE OF THE BODY AND EXTREMITIES.}

In a state of health, if the animal is dry and not exposed to extremes of temperature, the surface of the body and the extremities present a warm genial sensation to the touch of the observer, but the variations of temperature in disease are great and important. In severe inflammatory diseases, the legs, ears, and general surface of the body may be cold; the legs and ears presenting a sensation, when handled, of being what is termed "deathly cold." This symptom, if continuous, indicates grave diseases or important lesions; and if, in addition to the coldness of the legs and ears, there be perspiration over the body, or what is termed a cold sweat, the life of the animal is in great jeopardy.

In milder diseases, and those which run through their course slowly, such as many epizootics, peculiar alternations in the temperature of the surface and extremities are generally witnessed: thus one leg may be cold while the other three are warm, or three legs may be cold and one warm, one ear cold and the other warm, \&c. \&c. This eccentricity is often increased by the warm and the cold condition becoming more or less suddenly changed in situation; the leg which was warm in the morning may be found cold in the evening, the cold leg or legs being warm.

The signs furnished by these alternations are indicative of a depressed and erratic nervous force, and of a tendency to metastatic congestions.

A dry, scurfy appearance of the skin is often a symptom of indigestion, and of the presence of oxalic acid salts in the blood: and what is called "hide-bound," a condition where the skin seems to have lost its pliability and softness, is a symptom of a general state of mal-nutrition, arising from indigestion, improper food, worms in the intestinal canal, or a want of proper exercise, \&c. A staring coat often accompanies the above described conditions,-very often a staring coat is the only symptom of ill health. Whenever an animal is seen disposed to shiver, with staring of the coat, upon exposure to moderate cold, or whera this tendency exists in an animal whilst not so exposed, in all 
probability such an one is bordering on disease. A persistently staring coat, without any other symptom of disease, often indicates the incubative stage of glanders or farcy, and recurrent rigors are generally the fore-runners of strangles or other suppurative diseases. The premonitory stage of lymphangitis is marked by a fit of rigor or shivering, and in one case which came under my notice, acute glanders was ushered in by a continuous shivering fit of three days' duration.

THE PULSE.

The pulse is the beating of the arteries, and is usually felt at the jaw-the submaxillary artery, or on the inner side of the arm-the brachial artery. In the cow, whilst recumbent, the pulse may be very distinctly felt on the posterior part of the fore fetlock, and in the dog it is best felt at the femoral, on the inner surface of the thigh.

The pulsations felt by the finger are principally due to the fact that the artery expands during the contraction of the heart, and returns to its previous condition during the relaxation of that organ.

The arterial movements, as indicated by the sphygmograph, constituting the pulse, are described as follows by Dr. Burdon Sanderson:-

"1. At the moment that the heart begins to contract, a vibratory movement of the blood contained in the aorta is produced, in which the molecules of liquid are projected forwards in the axis of the vessel. A similar vibratory movement occurs the moment the ventricles cease to contract. These two movements differ only in this respect, that in the former the primary shock is directed towards the periphery, in the latter towards the heart.

" 2. Each of these movements is propagated in the direction of the stream at a rate of about ninety feet per minute,- the one expressing itself at the radial artery by a sudden expansion of the arterial tube, the other by a sudden collapse.

" 3 . The exact moment at which each movement occurs is indicated by the sphygmograph,- the former by a sudden vertical ascent of the lever, the latter by a descent. In either case the primary movement is followed by a succession of smaller move- 
ments in alternately opposite directions. By measuring the time which intervenes between the first and second vibrations, the exact duration of the systole of the heart may be ascertained, for the one occurs at the instant that the ventricle hardens in contraction, the other at the instant that the aortic valve becomes tense in closing.

" 4. Both movements manifest themselves with much greater distinctness in some cases than in others. In this difference it seems probable that the respective valves have much to do, and that the systolic vibration is produced by the sudden tightening of the mitral valve, just as the diastolic vibration is due to the sudden tightening of the aortic valve. The precise physical conditions on which the degree of vibration depends, have not as yet been ascertained, but observation points to the conclusion that the intensity of the systolic vibration is greatest when the arterial pressure is lowest at the close of the diastolic period.

"5. From the moment that the ventricles begin to contract, the fulness of the arterial system, and consequently the arterial pressure, rapidly increases. As, however, the arteries at first yield readily to the tide of blood, the tension does not attain its maximum until some time after the hardening of the ventricles in contraction. The duration of the interval between the one event and the other-that is, between the closure of the mitral valve and the moment of highest pressure in the radial artery-varies. It is longest when the arterial system is full; shortest when it is comparatively empty. Hence the measurement of this interval comes to be of considerable importance.

" 6 . From the moment that the artery attains its greatest distension, it begins to collapse; the form of that part of the sphygmographic tracing which corresponds to the period of relaxation is, as has been recently pointed out by Dr. Rivers, parabolic. In the normal pulse the parabolic form is not easily distinguished, the line of descent being nearly rectilinear; but in all those pulses in which the collapse is rapid, it is very obvious, and most of all in what has been called the monocrotons-single-form, which corresponds to the thready pulse of authors.

" 7 . In certain conditions of the circulation, the radial artery, immediately after the distension produced by the contraction of 
the left ventricle, suddenly collapses, and then as suddenly expands again, the second expansion being sometimes nearly equal in intensity to the first. This constitutes dicrotism." 1 - (Dr. Burdon Sanderson's Handbook of the Sphygmograph, to which the reader is referred for further information.)

I have already stated that the pulse is the beating of the arteries. In each pulsation as felt by the hand the arteries are slightly expanded, distended, and elongated by the wave of blood; sometimes they are laterally displaced, and then return to their original position, after which there is a short period of rest-the interval.

It seems probable that when the heart contracts of itself, it does so gradually and peristaltically, its constituent fibres being brought successively into action; and that, in so far as the movement is deprived of its automatic character by the influence of stimuli acting through the spinal cord, it becomes sudden and instantaneous.- See on this point Dr. Burdon SANderson, or Dr. Bell Pettigrew's Paper on the Physiology of the Circulation, in Lancet, 1872.) The influences concerned in the production of the pulse are those of the heart, the arteries, and the blood. The heart gives the impulse by which the expanding wave of blood is carried onward, after which the artery contracts in virtue of the elasticity of the yellow fibrous tissue which enters into the composition of its middle coat, or by its additional muscular force.

In health there is a nearly uniform relation between the frequency of the pulse and of the respiratory movements; the proportions being, as nearly as possible, one respiratory movement to three or four pulsations. Thus the pulse of the healthy horse beats about forty times per minute; the respirations are from twelve to fifteen in the same time. In the cow the same relationship does not exist, even in health, between the pulse and respiratory movements; for during rumination it may be observed that the pulse is seventy to eighty a minute, and the respirations not more than ten. Indeed the pulse of the cow in a state of confinement, in so far as regards the number of its beats, cannot be depended upon in the diagnosis of disease; the states of pregnancy and obesity, the effects of artificial food, and of the activity of the lactiferous glands, as well as the excitement caused by the act of rumination, generally produce such

I Double pulse. 
an impression upon the nervous system as to cause the action of the heart to be much increased, such increase being entirely consistent with a state of perfect health in an animal so circumstanced.

The relation between the pulse and respiratory movements, with the exception above mentioned, is generally maintained where the pulse and respirations are naturally accelerated, as after exercise; but in disease it generally ceases to exist. In many diseases accompanied by increased frequency of the pulse, the acceleration of the respiratory movements bears no comparison to the increased number of the pulsations, and in many other cases the respiration may be very rapid without a proportionate increase in the number of the pulsations; in some instances the pulse may actually fall below its normal standard, whilst the respirations are increased.

The natural pulse of the horse beats about forty times a minute, that of the cow about from forty to fifty, with the exceptions already mentioned, and the pulse of the dog ranges from eighty to one hundred, depending upon the size and breed of the animal, and the pulse of the sheep from seventy to cighty.

The frequency of the pulsations, and the regularity and irregularity of their succession, depends upon the heart, and the pulse at the jaw and arm, when it can be felt, always corresponds to the contraction (systole) of the ventricles, allowance being made for the very slight interval that must elapse before the wave of blood reaches these parts. To the heart's action must also be referred most of the healthy conditions as well as many of the peculiarities in disease; but the vital condition of the arterial system is not without its influence on the character of the pulse.

The arteries are gifted with a certain amount of vital contractility or tone, given to them by their muscular coat, upon which their healthy firmness depends; without this tone they become relaxed and flaccid, yielding readily to the force of the wave of blood, and but slowly recovering their calibre when the expanding wave has passed onwards. It is evident that, in this condition of the vessels, a portion of the impulse of the heart, which is expended in dilating the arteries, is not restored to the blood by the contraction of their coats; the impulse being thus gradually diminished as the arterial wave preceeds on its course, 
the pulse becomes weak, and is not in such instances to be considered a proper indication of the cardiac contraction. The contraction may be strong while the pulse is comparatively feeble. As a rule, however, except in valvular incompetency or muscular degeneration of the heart, there is a general correspondence between the cardiac and arterial pulsations, which renders the one a tolerably correct measure of the other. The quantity of blood also has undoubtedly some influence on the character of the pulse. When it is deficient, the pulse is likely to be smaller than natural. This is not an absolute rule, for in animals which may be considered to be in a plethoric condition the pulse is sometimes comparatively small. A modification of the quality of the blood affects the pulse, and this is most especially exemplified in cases of blood disease, such as hæmo-albumenuria and purpura hæmorrhagica, where it is frequently found that the pulse is " double," "fluttering," or " trembling."

The varieties of the pulse which are described by authors as being recognisable by the touch are-

(1.) As regards the number of pulsations within a given time, the pulse is said to be frequent or infrequent,-pulsus frcquens, pulsus rarus.

(2.) As regards the time which seems to be occupied by each beat, the pulse is said to be quick or slow,--pulsus celer, pulsus tardus.

(3.) As regards the dilatability of the artery in length and breadth, the pulse is said to be large or small,--pulsus magnus, pulsus parvus.

(4.) As regards the compressibility of the artery, the pulse is said to be hard or soft,-pulsus durus, pulsus mollis. ${ }^{1}$

\section{THE SYMPTOMS AFFORDED BY THE PULSE.}

The frequent pulse-pulsus frcquens.-Frequency of the pulse, or its number in a given time, varies slightly within the limits of health in animals of the same breed and similar conformation, and more so in animals of different breeds and temperaments. If the standard in the horse be set at forty beats per minute, it is not at all inconsistent with health to find it differ ten beats in two animals differing but slightly in breed and configuration. Thus, one may have a pulse of thirty-five and

1 Dr. Burdon Sanderson: Handbook of the Sphygmograph. 
the other a pulse of forty-five per minute, and both may be in a state of most perfect health; but a difference beyond this indicates some degree of excitement, or some amount of disease.

A frequent pulse-say a pulse above fifty-five per minute-if the horse is not in an excited state, from exercise or other accountable or natural cause, indicates a fevered condition, but not necessarily an increased vigour of the system; on the contrary, a very frequent pulse is generally associated with extreme prostration and debility. A certain quantity of blood is requisite to the existence of the several parts of the body; and when the heart is too feeble to act forcibly, it is compelled to excessive frequency of contraction to compensate for such want of strength. Excessive evacuations either of blood or of any of the secretions are generally attended with frequency of the pulse.

The old belief that "bleeding lowered the action of the heart and arteries," is disproved by the fact that all evacuations increase the number of the pulsations. I have repeatedly observed the effects of bleeding upon a healthy animal, and found that when from three to five quarts of blood have been withdrawn, the pulse has increased in frequency ten to twenty beats per minute; when the bleeding has been pushed beyond this, say to the extent of from seven to ten quarts, the pulsations have risen to extreme frequency - to 100,120 , or more per minute. As the pulse increases in frequency it becomes diminished in volume, supporting the conclusion that a pulse is small in proportion to its frequency.

The quich pulse-pulsus cclcr.-This term is made use of to denote that condition of the circulation in which the heart accomplishes its contraction almost instantaneously. The terms quick and slow have been objected to by Sir Thos Watson, as over-refinements, "simply because it was not certain what was meant by the terms by those writers who used them," and that it was very doubtful whether the quality of quickness or celerity of each pulsation could be truly appreciated by the finger. But the terms are now revived, for the qualities which they express are made manifest by the use of the sphygmograph, an instrument by which the movements of the artery are transferred to paper, in such a mauner as to render it possible to measure their extent and relative duration.-(See Dr. Burdon SANDERson's Handbook of the Sphygmograph.) 
Quickness of the pulse differs from frequency. Quickness refers to the time occupied by each beat of the pulse, whilst frequency has reference to the succession of the pulsations. A frequent pulse is that in which the number of beats is greater than usual in a given time; a quick pulse is that in which each beat occupies less than the usual time, though the whole number may not be much increased. Quickness of the pulse is not so difficult to appreciate by the touch in the larger domestic animals as it seems to be in man, and for the reason that the approach to frequency and quickness is normally much greater in man than in the horse and ox. The contrast, therefore, between abnormal quickness and the natural condition is more manifest in the latter than in the former.

Quickness of the pulse is due to the mode in which the ventricles contract, and always indicates irritation and little strength; the heart makes a quick, short contraction, which differs much from the somewhat prolonged, and more or less forcible, contraction of real energy and excitement. The jerking pulse is a modification of the quick pulse, and is characterised by a quick, forcible beat, followed by a sudden abrupt cessation. This pulse is found in cases where the nervous irritability is much increased, or it may be due to some deficiency in the aortic valves.

The slow pulse-pulsus tardus-or, as it is termed by Dr. Sanderson, the long pulse, is the reverse of the quick pulse, and indicates a prolonged or slow contraction of the cardiac ventricles. In inflammatory fever the pulse was formerly described as frequens magnus et celer, and was said to differ from that of plethora, which was described as magnus et tardus,-distinctions which are not now held to be competent.

An infrequent pulse--pulsus rarus.-This kind of pulse is often associated with slowness, and indicates clisease of the brain (compression), a disordered condition of the digestive apparatus, and is often indicative of that condition of the system manifested by excessive urination-polyuria. In degenerative disease of the heart the pulse is often more infrequent than the cardiac contractions, because the impulse of the heart is too feeble to be transmitted to the arteries.

The mutual relation of the pulsations may refer to their succession, their comparative duration, and their strength. In 
all these respects the pulse is often irregular, and the source of the irregularity is to be found in a deranged or debilitated action of the heart. An intermitting pulse is that in which a pulsation is occasionally omitted. This omission may be regular, occurring at the end of a certain number of beats, or it may be irregularly intermittent, and along with intermission of its beats there may be irregularity in its tone, volume, \&c. For example, several beats may occur in rapid succession; these may be followed by others at long intervals, or some beats may be strong, others weak, some quick, others prolonged; and often several run on successively of one character, to be followed by more or less of another character. Occasionally all these irregularities, or most of them, may occur in the same subject. They are indicative of important functional or nervous disorder, or of organic disease of the heart. An intermitting pulse may often be observed in horses presenting all the appearances of perfect health; indeed, an intermitting pulse is very commonly met with in the cart-horses of this city; and, reasoning from the frequency of indigestion, and the nature of their food, one is forced to the conclusion that this irregularity of the heart's action is due to some disorder of the digestive apparatus. When these animals are attacked by any disease, the irregularity of the pulse frequently disappears for a time, or until the animal is restored to its former condition of apparent health.

The volume of the pulse may be greater than usual, in which case it is said to be large-pulsus magnus, or it may be less than usual, when it said to be small-pulsus parvus. Largeness or fulness of the pulse may depend upon general plethora, or a prolonged and forcible contraction of the ventricles; to a certain extent on relaxation of the arterial coats, and on obstruction of the capillaries, without diminished power of the heart. It may be associated with strength or with feebleness of the pulsations. It is important not to confound a full feeble pulse with a full strong one. The feeble pulse is known by the weakness of the impulse, and by the artery yielding to the pressure of the finger. In pulmonary congestion of a severe character the artery is full, and the pulsations feeble. This has been called the "oppressed pulse of pneumonia" by veterinarians, and depends upon excessive congestion of the pulmonary vessels. A pulse of this character is improved in tone by a moderate abstraction of blood. 
The fecble pulse, if associated with softness, the artery yielding readily to the finger, indicates general or cardiac debility. It is sometimes so weak as to give one the idea that the artery is filled with air, hence it has been called the gaseous pulse, and is not unfrequent in an anæmic condition of the body.

The small pulse-pulsus parvus-may result from anæmia; from congestion of some important organ, as the lungs ; from feeble contraction of the heart; or from great tonicity of the arterial coats.

The hard pulse-pulsus durus-hardness of the pulse, is that property by which the artery resists compression, and results from contraction of the muscular coat of the arterial walls. Hardness of the pulse is often associated with smallness-pulsus durus et parvus; it is then termed corded, wiry, or thready, and this condition is often met with in the earlier stages of inflammatory disease, particularly during the rigor-in endocarditis, and in dangerous inflammations of serous membranes-and, experimentally, the thready pulse has been produced by division of the pneumo-gastric nerves.

Dr. Sanderson describes this pulse as follows:- "When the pulse is small and hard, as during the rigor at the outset of acute diseases, and in certain dangerous forms of carditis, the expansion of the radial artery is sudden and of short duration; the suddenness of the movement not depending upon the rapidity with which the arteries empty themselves by the capillaries, but on the violence with which the heart itself contracts. In this form of pulse there is no second beat. The explanation is clear: the difference between the arterial and venous pressure is so considerable, and the range of variation in the peripheral arteries so limited, that no perceptible diastolic retardation takes place in the capillaries, and consequently no second expansion."

The hard pulse is seldom associated with largeness; the nearest approach to the hard full pulse-pulsus magnus et durnsis the strong full pulse of laminitis. It may, however, depend upon hypertrophy of the left ventricle of the heart, and be present indepenclently of any inflammatory disease. When the pulse is very hard, the lateral displacement of the submaxillary artery is very apparent; it is then called by veterinarians a hard rolling pulse. 


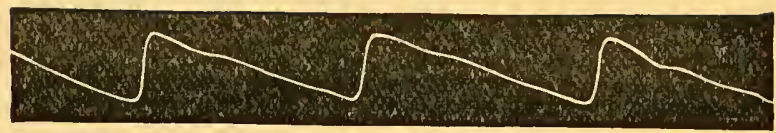

Firm and long pulse of health.

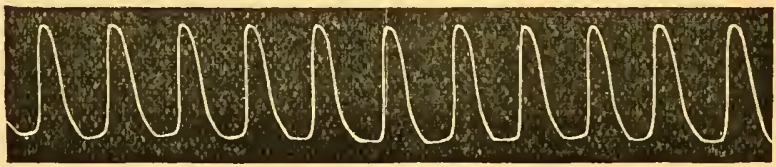

Hard pulse.

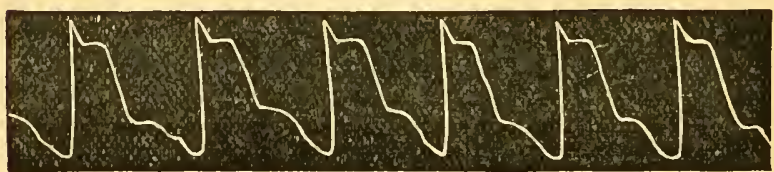

Hard and long pulse of hypertrophy of left ventricle.

The soft pulse.-Of this kind of pulse several varieties are described. In the small soft pulse, towards the close of progressive exhaustive diseases, the impulse of the heart is slight, the tension of the arteries climinished, and the volume of blood small. In some cases of anæmia, however, or of slow action of the heart, or of great relaxation of the arterial coats, or of these conditions combined, there may be a soft full yet feeble pulse. It is readily known by the feebleness of the impulse, and by the artery yielding readily to pressure.

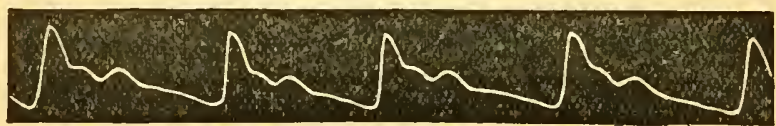

Soft natural pulse.

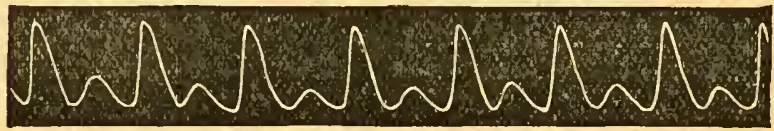

Soft frequent pulse of mild fever.

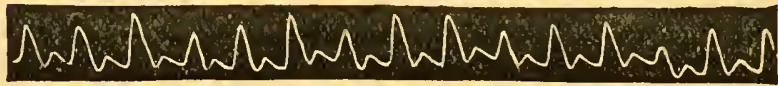

Soft frequent pulse of irritative fever. 
The double pulse.-This pulse is occasionally present in hæmo-albuminuria (red water) in cows, and in purpura in the horse. It denotes, according to Dr. Sanderson, that condition of the circulation in which arterial pressure is diminished, while the venous is increased, and that the capillary current, instead of being constant in its rate of movement, is markedly accelerated during the diastole, and retarded during the diastolic interval. In the undulatory double pulse of typhus, says Dr. Sanderson, the intensity and suddenness of the second expansion appears nearly as great as the first; the explanation being, that in this condition the contractions of the heart are extremely feeble, the arterial pressure being so low that the progressive movement of the blood ceases entirely in the capillaries during the diastolic interval.

The strength of the pulse depends chiefly upon the force with which the ventricles contract; in some measure upon the tonicity of the arteries, and upon the volume of the blood. In order to have a strong pulse there must be vigour of impulse, steady resistance to pressure, and a certain degree of fulness. There may be a sharp, forcible beat; but if this is not sustained by a certain amount of subsequent pressure, it indicates irritation rather than energy. A very contracted pulse, however sharp the impulse, cannot be called a strong one. A strong pulse is considered a sign of a sthenic or vigorous state of the system. But it is not invariably so, for hypertrophy of the heart will give strength to the pulse, even though the general powers of the animal be weak.

Weakness of the pulse.-In order to have a weak pulse it is essential that the impulse be slight, the tonicity of the arteries diminished, and the volume small. In some cases, however, it will be found, if there be a slow action of the heart, or great relaxation of the arterial coats, that there will be a full, and at the same time a feeble pulse. It is important not to confound this with strength.

The venous pulse-Pulsation of the veins is often seen in the jugulars of the ox, especially during rumination, and seems to be quite compatible with health. If the head or neck of the horse be extended, there will generally be an appearance of a jugular pulse. This, however, arises from the beating of the carotids, which is made visible by the parts being tensely 
drawn and pressed together. When a true venous pulse exists independent of heart disease, it is due to the veins filling when the ventricles of the heart contract, and the sudden closing of the auriculo-ventricular valves. It is supposed that there is no actual reflux of blood, but a sudden arrestment of its flow intu the right ventricle.

In some forms of heart disease the jugular pulse becomes a diagnostic symptom.-(See Diseases of the Heart.)

\section{SYMPTOMS CONNECTED WITH THE RESPIRATORY FUNCTIONS.}

The respiratory movements may be quickened, difficult or laborious, wheezing, roaring, stertorous, spasmodic or convulsive, irregular, slow, thoracic or abdominal; and be attended with such signs as sneezing, coughing, sighing, or yawning. The breath may be hot, as in pneumonia, fever, \&c., and cool or cold, as in cases of great prostration; fotid, as in gangrene and abscesses of the lungs, or in nasal gleet arising from diseased bones or teeth. The matters discharged from the air passages are also signs of great value, as blood, pus, mucus, or a mixture of all of them.

(a.) Quickened breathing.-Rapidity of the respiratory movements, although present in many diseases, is easily produced by auy cause which accelerates the circulation of the blood, and is generally proportioned to the rapidity of tine circulation, and the corresponding need of change in the blood. Thus, exercise, which accelerates the circulation and changes of the blood, also augments the respiratory movements.

(b.) Difficulty of respiration (dyspnœa) is a prominent symptom of disease, and may depend upon various causes. In inflammation of the lungs, or pleurisy, there are many circumstances in operation which impede the respiratory movements-as pain, or exudation into the substance of the lungs or cavity of the chest, which mechanically resists the entrance of air into the lungs. Diseases of the larynx or trachea, by diminishing their calibre, are generally associated with extreme dyspnœa, and with sounds, such as roaring or whistling, which furnish pathognomonic signs of the seat of the disease.

Difficulty of breathing, when not accompanied by certain sounds detectable by auscultation, if not sufficiently loud to be 
heard at a slight distance from the animal, is not, however, to be regarded as symptomatic of disease of the respiratory organs only, for in many acute diseases the breathing not only becomes quickened, but laboured and difficult to an extreme degree.

Difficult breathing must always be regarded as a symptom of importance, not ouly as being diagnostic of certain diseases, but as indicating grave alterations in the condition of the blood or in the functions of the nervous system. Diseases are also seriously aggravated, and a tendency to a fatal termination, owing to the non-oxidation of the blood, is augmented by difficult breathing.

(c.) Stertorous breathing (Snoring).-This arises from a relaxed state of the velum palati, and is a symptom of brain disease. So long as the respiratory movements are strong and frequent, it does not indicate immediate danger, but when the respiratory powers are impaired, the movements slow-that of inspiration delayed, and then performed with a sudden noise and a jerking effort, and the susceptibility to outward impressions diminished - stertorous breathing is a symptom of the near approach of death. Snoring, so common an accomplishment in the human being during sleep, is but rarely so in the lower animals.

(d.) Abdominal breathing, or respiratory movements performed with the ribs fixed as much as possible, owing to pain or mechanical obstruction in the chest, is a symptom of pleurisy, hydro-thorax, and pleurodynia.

(e.) Thoracic breathing is that in which the abdominal muscles are prevented from participating to their natural extent in the performance of the respiratory movements. This condition is dependent upon abdominal tumours of great magnitude, ascites, tympanitis, or acute abdominal-peritoneal-pain.

(f.) Irregular breathing is that condition where there is a want of harmonious correspondence between the inspiratory and expiratory movements, and is seen in the disease which is commonly known as "broken wind." The inspiratory movement in this affection is performed quickly and with a jerky effort, whilst the expiratory is performed slowly and with a double action, more particularly of the abdominal muscles. Irregular breathing often becomes spasmodic or convulsive during the paroxysms of the disease.-(See Broken Wind.)

Of the phenomena and sounds which attend the respiratory 
functions besides those already mentioned, the act of coughing and the varieties of cough demand our careful attention.

A cough is a violent spasmodic action, and the sound is produced by a sudden and forcible expiration, preceded by a firm closure of the glottis; and is for the most part an involuntary effort to remove some source of irritation from the lungs, trachea, or larynx.

The varieties of cough which are met with in veterinary practice are denominated moist, dry, short, hacking, violent, spasmodic, the broken-winded cough, and the cough of the roarer.

The moist cough attends cattarrh, bronchitis, and other diseases where the secretion of the mucous follicles of the air passages is increased.

The dry cough indicates a non-secretory condition of the air passages, and is usually present during the early stages of catarrhal affections. In pleurisy the dry cough remains throughout the disease; it is also painful, short, and hacking, from the pain which the forcible expiratory movement causes the animal to feel. The dry cough has several modifications from that of broken wind, where it is a miserable apology for a cough, to the loud paroxysmal cough, caused by the irritation of teething, and which may be properly named the dental cough, and the hollow, sepulchral cough of the roarer. Cough does not always arise from direct irritation of the air passages, lungs, or pleura, for we often find an animal coughs when suffering from indigestion, worms in the intestinal canal, diseases of the liver, \&c. These sympathetic coughs are always characterised by dryness.

\section{THE SYMPTOMS AFFORDED BY THE SECRETIONS.}

The secretions may be either diminished, increased, or perverted. In the early stages of inflammatory disease of a secretory organ, it secretory function becomes diminished. Thus, in the first stages of pleurisy the surfaces of the serous membrane are dry and rub against each other, producing the "friction sound;" but as the disease advances some of the natural fluid secretion of the membrane, along with a quantity of inflammatory products in a fluid state, is thrown out, and the surface, which was dry, becomes unnaturally moist. The same changes occur when a mucous membrane or a gland is inflamed. In the febrile state 
there is occasionally a diminution of all the secretions, but this can only continue for a short time, as some particular organ, the bowels, kidneys, or skin, is excited to increased action by the presence of effete materials in the blood, and its secretion thus increased, while those of the rest are diminished. General increase of the secretions is scarcely possible, and much more frequently the increase, like the diminution, is confined to one or more secretory organs. Excessive and exhaustive secretions are termed colliquative.

Perversion of the secretory functions is characterised by an alteration in the character of the secreted materials. The products of secretion are sometimes wholly changed in character from those of the same organ in health, becoming excessively irritant, and yielding evidence to chemical re-agents of the presence of new constituents. In some instances, again, one secretory organ will perform the function of another, as when biliary matters are excreted by the kidneys when the liver ceases to act.

\section{SYMPTOMS BELONGING TO THE CALORIFIC FUNCTIONS.}

The animal body is so constituted as to be able to bear great extremes of heat and cold, and be yet in itself never greatly above or below the mean standard of health-never greatly above, and never much below, until vitality has ceased, or has been so far reduced as to have surrendered the body to the operation of surrounding influences.

The mean temperature of the body in those internal parts which are most easily accessible-as the mouth and rectummay be estimated at from $99^{\circ}$ to $102^{\circ} \mathrm{F}$. In thirty-four healthy horses, all under the same conditions, the temperature was found to be under $101^{\circ}$ in ten; $102.2^{\circ}$ in one; and $101.2^{\circ}$ to $101 . \mathrm{S}^{\circ}$ in twenty-one. In young animals the temperature is commonly about $102^{\circ}$, but in very old ones $I$ have seen it as low as $96^{\circ}$. The external parts of the body become lowered in temperature the farther they are removed from the centre, and are liable to much variation from the state of the surrounding atmosphere.

The method of ascertaining the temperature of the body in the lower animals is by introducing a properly registered thermometer into the recturn. The practice of thus measuring the changes of 
the temperature of the animal body has now made progress in veterinary medicine, and is considered of great importance as an aid to diagnosis and prognosis. It has been advocated by clinical teachers since 1754, when Antonius de Haen, of the Hospital of Vienna, impressed his pupils with the necessity of attending to the temperature of the body in disease, as measured by a thermometer, instead of being judged by the hand. He showed that even in the cold stage of ague, with the teeth chattering and the body shivering, the temperature of the blood is rapidly rising, although the pallid skin may be cooler than usual, its supply of blood being diminished by the contraction of its vessels.

The production of animal heat is due to certain chemical and vital changes, which are continually taking place in the body; and consist in the absorption of oxygen by the pulmonary capillaries, the combination of that oxygen with the carbon and hydrogen derived from the disintegration of animal tissues, and from certain elements of the food which have not been converted into tissue. This combination of oxygen, or oxidation, not only takes place in the blood, which may be looked upon as a fluid tissue, but in the tissue cells of all parts of the body, the animal heat being thus maintained by the natural changes which are essential to a healthy condition.

The oxidation and natural decay of the tissues, the process by which they are rendered fluid and fitted for absorption, has been termed the secondary digestion, and is thus described by Bennett:- "Growth having been effected, it is necessary that the particles "of the tissues which have fulfilled their function and are worn ont should be removed to give place to new ones. This constitutes the so-called secondary digestion, - that is, in the same way that a piece of food, say flesh, is broken down, rendered molecular and fluid, and is absorbed into the blood to add bulk to the frame, so is our own living flesh constantly breaking down, rendered molecular and fluid, and absorbed into the blood, to be finally thrown out of the system. Thus the blood receives matter from two sources, the primary and secondary digestions; and is continually giving off matter in two directions, one to build up the tissues and form the secretions, the other to produce the excretions." - (BENneTT's Clinieal Lectures.)

In many diseases this secondary digestion or metamorphosis of tissues, which is mainly due to their oxidation, is abnormally 
increased, and the animal heat unnaturally elevated, and if continued for some time, indicates the existence of the febrile condition. The tissues are rapidly consumed, the blood becomes charged with the effete products resulting from the metamorphosis; the appetite being at the same time impaired, the animal becomes more or less rapidly emaciated, and if the abnormal process be not arrested, naturally or otherwise, finally dies.

The determination of abnormal heat in disease has a very great importance, and to detect it practitioners have bee: in the habit of placing their hand to the skin, and their fingers within the mouth of the patient; but the method of measuring the heat by the thermometer holds a highly important position, not only in cases of illness where the symptoms are apparent, but more particularly in the incubative stages of such diseases as glanders, pleuro-pneumonia-bovina, rinderpest, and other contagious or infectious ailments. Here the early recognition of disease plays an important part in preventing the development and propagation of such maladies, and depends much upon the use of a delicate thermometer. When an outbreak of such diseases as pleuro-pneumonia-bovina or glanders occurs, it is very possible that out of a large herd of cattle or stud of horses but one or two may present any appreciable signs of disease, all the rest remaining apparently healthy; but although they may not present any signs of disease, it does not follow that they are not tainted with the malady in its incubative stage. It must not, however, be supposed that elevation of temperature is diagnostic of any particular disease; it merely indicates the febrile condition, which of itself may be due to a great variety of causes, external and internal, specific and non-specific. Some practitioners are so expert as to diagnose even the incubative stages of pleuro-pneumonia, by placing their fingers in the mouth of the animal, and, from the elevated temperature so felt, be able at once to give an opinion as to the probabilities of the animal being affected, provided always that the disease has already attacked others in the herd. If it be possible to do this by the mere touch, how much more certain and satisfactory will it be, by means of a delicate instrument, to measure the degrees of temperature, and give an opinion based upon conclusions derived from an exact admeasurement.

In glanders and pleuro-pneumonia, the two contagious diseases 
which the veterinarian is most likely to be asked to pronounce an opinion upon, now that rinderpest is so safely guarded, the examination of all the animals which have been herded or stalled with those labouring under this disease should be systematically made, and all those presenting an elevation of temperature of from two to three degrees should at once be isolated, and looked upon with suspicion, until time proves whether they are suffering from the malady or not. Nothing can be lost by this, but much may be gained, the most important gain being the destruction of the centres of contagion, and the preservation of the remaining stud or herd; for when an animal is kept mixed with others until the disease be more fully developed, it remains there as a generator of contagious material, and thus propagates the disease over the whole number.

The best thermometer for the purpose is a straight instrument with a registered index. Such an instrument can be removed and read when convenient, for the index, being fixed in the stem, will denote the height to which the column of mercury has been raised. I find it desirable to keep the instrument in the rectum for about three minutes, and to make two observations, and if the two observations correspond, to remain satisfied with its correctness. The pocket thermometer invented by Professor Brown is very convenient and handy, and is specially recommended to the veterinarian.

Whilst elevation of temperature is generally associated with rapid waste of tissue, it is found that rapid emaciation occurs without a corresponding elevation of temperature, as in polyuria, and that the temperature may be elevated without much increased tissue waste. These phenomena are due to the condition of the "heat centre," which, when active, prevents elevation, but loses its inhibitory power when its condition is depressed. 


\section{CHAPTER V.}

\section{PATHOLOGY-continued.}

\section{MORBID ELEMENTAPY PRODUCTS-COMPLEX VITAL PROCESSES WHOSE PHENOMENA CONSTITUTE DISEASE.}

THE effects of disease, when they can be rendered obvious by dissections, by the application of various instruments, such as the microscope, and of chemical re-agents, are found to consist for the most part of-

1. Transformations in the elementary textures of the body generally, and altered conditions of the fluids.

2. The presence of new formations foreign to the normal condition of an organ or system of organs.

3. Change in the position or form of some of the organs or parts of organs.

4. Deposits in and around the elementary parts of tissues, or changes of a degenerative or retrograde kind in them. (See Aitken's Science and Practice of Medicine.)

Anatomical investigations of morbid parts, conducted with the aid of the microscope and other instruments of research, show that the material of which their substance is made up is of very various structure, sometimes combined in forms of one kind throughout, and sometimes varied by the development and combination of many elenientary forms, more or less solid, soft, or fluid.

An analysis of the morbid material, carried as far as scientific means at present enable us, shows that the elementary conditions in which morbid products are found may be described as follows:-

1. Fluid matter and hyaline substance, more or less soft. 
2. Simple elementary forms of the nature of deposits, sometimes of a mineral or inorganic character; c.g. (a.) amorphous granules; (b.) crystalline structures in a granular state.

3. Simple but organised products capable of growth,--simple cells, compound corpuscles, fibres.

The various appearances and conditions which these simple forms may assume in disease, as well as the functional states with which they are frequently associated, lead to a further enumeration and classification of morbid elementary products, as well as of more complex disease processes, as below.

MORBID ELEMENTARY PRODUCTS.

I. Exudations more or less soft, semi-fluid or fluid, and formed of-

(a.) Germinal plastic and formed material, which has sometimes been called blastema, coagulable lymph, false membrane, or fibrin, as seen adhering to free surfaces.

(b.) Aqueous matter, as seen in the morbid state termed dropsy, and œdema of parts.

(c.) Gaseous exudations, as seen in the various forms of pneumatosis.

II. Exudations more or less consolidated, and consisting of-

(a.) Molecular or granular material, from $\frac{x}{80} \bar{t}$ th of a line to an immeasurably sniall size, and consisting chiefly of the simple forms of-

(1.) Fatty molecules of granules.

(2.) Forms of an organic kind capable of growth, and invariably taking origin from a pre-existing structure.

(3.) Deposits of an inorganic kind, generally calcareons salts.

(4.) Pigment granules.

(b.) Coagulable compounds, resisting the action of most reagents, such as are seen in the elements of tubercle, scrofula, oleo-albuminous formations.

(c.) Exudutions of a transitional nature, organised, capable of 
growth, which may become vascular, growing from preexisting structures, and composed of-

(1.) Consolidated cellular material, passing to

(2.) A fibrilloid arrangement of the molecular or granular. particles composing connective substance, and a subsequent formation of fibres in it or from it.

(3.) The formation of pyoid cells, and of fibro-plastic or connective tissue cells, passing into fusiform cells and fibres as the material becomes consoliclated.

(4.) The formation or exudation of fluid matter holding pus, or other more compound cells.

III. Growths and exudations of a more or less specific lind.

(a.) Lymph of small-pox and cow-pox.

(b.) Matter of glanders, malignant pustule, and of the plague.

(c.) Fluids of infecting chancre, and of some forms of secondary syphilitic lesions.

(d.) Material of tubercle and scrofula.

(e.) Material of cancer.

$(f$.$) Growth of Peyer's glands during typhoid fever.$

(g.) The growth of Peyer's glands in cases of cholera.

(h.) Melanotic or pigmentary germs.

IV. Matcrial of a complex kind.

(a.) Media of repair and reproduction of injured or lost parts-substance of granulations and cicatrices.

(b.) Hypertrophy of parts.

(c.) Tumours, $\left\{\begin{array}{l}\text { Innocent. } \\ \text { Malignant. }\end{array}\right.$

(d.) Concretions.

V. Parasitic formations.

VI. Organic germs_-such as Bacilli and Micrococci.

COMPLEX VITAL PROCESSES WHOSE PIIENOMENA, MORE OR LESS COMBINED, CONSTITUTE DISEASE.

(1.) Fever-Pyrexia.

(5.) Depression.

(2.) Inflammation.

(6.) Atrophy.

(3.) Irritation.

(7.) Degeneration.

(4.) Congestion. 
Such a classification as the above is merely intended to bring before the student at a glance the variety of morbid material which is concerned in the expression of many of these phenomena seen in the comrse of disease, the distinctions made being mainly based on structural analysis and microscopic examinations.

It was formerly a much debated question whether diseases had their principal seat in the fluids or solids of the body. At the present time the purely solidist, as well as the purely humoral principles, may be said to lave died a natural death, and we can only wonder how men could have advocated exclusively one view or the other, and refused to allow that both solids and fluids had their share in the production of morbid phenomena. That many diseases are caused by the presence of a morbid material in the blood, which of itself is perhaps the first to be modified, there can be no doubt; at the same tine it cannot be denierl that the tissue plays a most important part in the various pathological processes which constitute disease. In pleuropneumonia contagiosa, for example, the blood is undoubtedly the recipient of the contagium; but it would be erroneous to suppose that the operation of the morbid material was confined to the blood alone, and that the exudation found on the pleura and in the parenchyma of the lungs was a mere precipitation of a product formed within the blood-vessels. In reality, the poison, though taken by the blood to all parts of the animal frame, is limited in its effects to the tissues of the lungs and pleura, and, like a medicine which has special affinities, passes harmlessly over all the other organs and structures of the body. In such an instance the blood contains the cause of the disease, whilst the solids of the parts affected are the seats of the diseased processes. So it may truly be said that both fluids and solids are alike the seat of the disease, that they are mutually dependent one upon the other, that the solids are almost of necessity liable to be affected by the fluids, and the fluids by the solids.

In some diseases it is found impossible, with the aid of the most exact instruments, to detect any structural change, or the presence of any of the morbid products aheady enumerated. Such diseases are termed functional, whilst those in which an alteration of the part can be detected are called structural or organic diseases.

Functional derangement is undoubtedly due to an alteration 
in the nutrition of the part, and is a condition which cannot exist for any length of time without the occurrence of an organic or structural change. The most familiar functional derangements are the conditions of increased sensitiveness, irritability, and contractility of muscular tissue, and tonicity, which is a modification of ccntractility,-termed passive, in opposition to active contractility, by Mr. Bowman. Of excessive contractility we have examples in spasmodic colic and in chorea, where the contractions are sudden, and alternate with sudden relaxations. Contractions of this kind are termed clonic spasms; and of excessive tonicity, or tonic spasms, we have examples in the arteries, when the pulse indicates a hard, unyielding condition of the arterial walls; in cramp of some of the voluntary muscles, and, in an extreme degree, in tetanus.

Opposed to the above conditions we have loss of sensibility, anæsthesia, and paralysis. 


\section{CHAPTER VI.}

\section{PATHOLOGY-continued.}

\section{ATROPHY AND DEGENERATIONS OF TISSUE.}

ATROPIIY.

Atrophy implies a wasting of a part, arising from a diminution in the size or a decrease in the number of the tissue elements of which that part is composed. Atrophy of the normal structure of a part may take place without any apparent diminution, but may be coincident even with an increase in size. Thus, the fibres of a muscle may be atrophied, whilst its whole bulk is increased by an interstitial exudation, which, pressing upon the true sarcous elements, canses their wasting and absorption. Again, a glandular organ may be apparently enlarged, whilst its true gland structures are considerably lessened.

Atrophy is divided into simple and numerical atrophy. Simple atrophy is applied to the diminution in the size of the tissue elements, and numerical atrophy to the process by which their number is diminished. The two varieties are often associated. Simple atrophy may, however, exist independently, but the numerical never occurs without the simple. Atrophy is not always a morbid process, as, for example, in the disappearance of the thymus gland, which is large in the foetus, and is removed shortly after the birth of the animal.

Simple atrophy is well shown in ordinary emaciation, in which fat disappears from the subcutaneous adipose tissue. Here there is no destruction of the fat cells, but simply an absorption of some of their contents, by which their size becomes more or less diminished. The sime process may take place in the cells of glandular organs, and so produce a diminution in the size of 
the whole organ. Muscular tissue also wastes in the same way, by a decrease in the size of the primitive fasciculi, as exemplified in the voluntary muscles and the heart.

Numerical atrophy resembles gangrene in some respects, for death of the part is common to both; but they differ in the following points. In gangrene, tracts of tissue are affected. It results from some sudden arrest of nutrition, and a gangrenous mass remains at the termination of the process, in such a condition that the structure can be usually recognised; whereas in necrobiosis ${ }^{1}$ the change is a molecular one, with gradual exhaustion of the vital power, a molecular disintegration and destruction of elements, so that at the termination of the process all that remains is a glandular débris, in which no trace of the natural structure of the part can be discovered. This form of atrophy is of much greater importance than the simple; for in the simple the elements of the part continue to exist, and repair can be effected withont any new formation; whilst in the numerical, restitution is only possible by the production of new elements.

Inactivity of a part, obstruction of its blood-vessels, failure of its own vital energy, continued pressure upon its surface, and the process of inflammation, are all well-known causes of atrophy. A muscle, if unused, becomes small and pale, and its tissue degenerates. This atrophy of muscnlar tissue from diminished activity is often seen in the horse. For example, a horse is chronically lame in a foot. In consequence of the pain felt by the animal, the limb is kept in a state of quietude and repose; the animal moves it as little as possible. The muscles, thus kept inactive, diminish in size, and the whole limb seems to shrink. If the cause of the lameness be removed, and the limb regain its former activity, the wasted muscles are restored to their natural condition and strength. Paralysis also leads to shrinking of a limb; and it is recorded that injury to a trunk of the sympathetic nerve will prodnce the same condition without impairment of motion or sensibility. Atrophy and softening may result from the ingestion of improper food. Majendie kept animals upon food containing no nitrogen and incapable of supplying sufficient nourishment; and one curious result was

I The term necrobiosis is applied by Virchow to the destruction of histological elements, which occurs both in atrophy and degeneratıu. 
atrophy and soitening of the cornea, which melted down and disappeared. Again, the stomach may be softened and its mucous membrane dissolved by the action of the gastric juice. This occurs in the dead body only, although it has been mistaken for the effects of disease. Atrophy may also be caused by the action of medicinal substances, such as mercury, iodine, bromine, and the alkalies. Iodine and mercury are said to exercise an influence on the lymphatic system, and bromine upon the generative organs. Bone is sometimes atrophied both in the horse and in the ox. This atrophy of bone is attended by a diminution in its weight, but the size of the bones in the specimens which I possess does not seem to be altered. The compact tissue is thin, the cancelli have mostly disappeared, the medullary canal, filled with a lardaceous looking fat, extends from one extremity of the bone to the other, and the periostial surface of the bone presents a more or less cribriform appearance.

The various structures of which an organ is composed may suffer from atropliy. Some, however, do so more than others. The fibrous tissue generally increases in amount. In glands, the secretory cells are the first to suffer. They become small, and often granular, from the presence of oil globules. In muscles, the fasciculi become smaller, and lose their transverse striæ, and finally the whole contents of the sarcolemma may be absorbed, and leave nothing but fibrous tissue, which may or may not be transformed into fat.

\section{DEGENERATIONS OF TISSUE.}

The wellbeing of every part depends upon the nutritive processes being actively performed by its cells, and upon a due supply of properly constituted plasma-formative material, germinal matter-protoplasm (Huxley), bioplasm (Beale)being conveyed to it by its vessels. The formative processnutrition-is performed by the cell, which is the seat of nutrition and function, each individual cell being in itself an independent organism, endowed with all the properties of active life. Various opinions are held as to the constitution of cells. Some writers maintain that a cell is composed of a cell wall, enclosing a cavity containing a nucleus and fluid contents (Schwann, Remak, Virchow, \&c.); others state that the existence of a celi 
wall is in many cases non-evident, more particularly in embryonic cells, in those of many rapidly growing new formations, blood corpuscles, pus, and mucus; that a cell is a small mass of matter enclosing a nucleus, and that the existence of a cell wall is due to a degenerative process going on in the outer layers of the mass. Thus Beale says, "A cell of epithelium consists of bioplasm or bioplasmatic matter, surrounded by formed non-lixing matter, which was once in the bioplasmic state. In the same way an oval yeast particle consists of the bioplasm, with an envelope of formed material, which has resulted from changes occurring when particles upon the surface of the bioplasm died."

Brücke, Stricker, and others consider that the existence of a nucleus is not essential to the constitution of a cell; and they base their conclusions upon the fact that in some of the lowest forms of life cells occur which show no trace of nuclei. Notwithstanding this conclusion-that a cell is an elementary organism capable of exhibiting all the phenomena of life-the nucleus is exceedingly constant in all cells, and presents fewer varieties in form and size than the cells themselves; is spherical or oval in shape, and often contains one or more minute round or angular bodies-the nucleoli; offers greater resistance to chemical reagents than the other constituents of the cell, and in disease often remains after these have been destroyed. It seems to be structurally homogeneous or slightly granular, is more deeply stained by carmine-a property peculiar to formative material -and is in some cases invested by a limiting membrane.

The cell contents have the power of spontaneous movement, and these, as well as the alterations in form characteristic of young cells, are due to the germinal living matter, which is probably the sole seat of the nutritive and formative power of the cell. This germinal matter, or bioplasm, differs in volume and consistence in different cells, and in the same cell at different times. It is capable of imbibing and giving up fluids, and of undergoing corresponding alterations in volume.

It is now generally accepted that every cell originates from a pre-existing cell, that tissue is formed by the natural formation and growth of cells, and that the deviations from this process constitute the basis of every pathological change.

The multiplication of cells may take place-1st. By simple division; $2 d$. By budding-gemmation; and $3 d$. By the forma- 
tion of new cells within the parent cell-endogenous growth. The multiplication by simple division is the most frequent method. The cell divides and forms two cells, each of which divides again and forms two more, and so on. In nucleated cells the nucleus generally divides first.

In multiplication by budding, a small portion of the germinal matter projects from the cell, and becomes detached by constriction at its base, and forms a new cell.

Endogenous multiplication occurs in cells with an indurated outer layer-dense cell wall_as in some varieties of epithelium. The germinal matter divides within the membrane, and forms a number of new cells. We have thus a parent cell enclosing a brood of young cells, of necessity smaller than the parent cell, which are subsequently liberated by solution of the parent cell wall, or escape by their own inherent power of movement.

Nutrition may then be said to be dependent on two things:1st. Upon a due supply of plasma; and $2 d$. Upon the appropriation and elaboration of the plasma by the cellular elements having vital properties, and possessing within themselves the powers of growth and reproduction.

When nutrition becomes absolutely arrested, death of the part where such arrestment occurs is the consequence. When the arrest of nutrition is general, the whole body dies; when it is local, gangrene or necrosis is the result. These two latter conditions having been already described in my work on Veterinary Surgery, I will pass on to those morbid conditions which are dependent on impairment of nutrition, namely, Atrophy and Degenerations. 


\section{CHAPTER VII. \\ PATHOLOGY-continued.}

\section{ATROPHY AND DEGENERATIONS OF TISSUE-continued.}

\section{DEGENERATIONS.}

Atropily and Degeneration resemble one another, in so far that, in both, nutrition is impaired and function interfered with. In atrophy the part may be said to be altered in quantity, the waste of the tissue being in excess of the formation of new material. In degeneration, however, the structure of the part is altered in quality, a new formation being present in the tissues, impairing their vitality and interfering with their functional activity.

The degenerations are divided into two classes, namely, the Metamorphoses and the Infltrations.

\section{THE METAMORPHOSES.}

These consist of an alteration in the albuminoid structures, whereby they are changed into new materials, with disappearance of the elements of the tissue proper, softening of the intercellular substance, and loss of function. The metamorphoses are the fatty, calcareous, mucoid, colloid, pigmentary, and amyloid degenerations.

\section{FATTY DEGENERATION.}

This is the most common of the degenerations, and consists in the transformation of the alburninoid constituents into fat, or rather in the replacement of the healthy tissues of a part by drops or molecules of an oily nature, which appear as 
minute granules within the cells of the tissues. A muscular fibre thus affected shows that the sarcous elements, the real

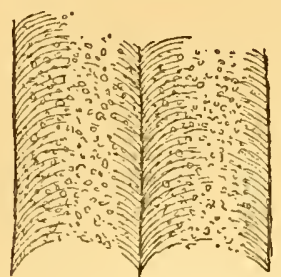

FIG. 1.-Fatty degeneration of muscular tissue. External matter replaced by oil particles. contractile tissue, within the sarcolemma, are replaced by shining oil particles, so that the contractile power of such fibre is destroyed. These oil granules, when examined microscopically, present a sharp contour, a dark colour, and strongly refract light. They are soluble in cther; and as they increase, they, by coalescence, sometimes form distinct drops of fat, but usually they retain their granular form. The cells, however, which contain them increase in size and become globular in shape, the nucleus and cell wall are destroyed, and the cell transformed into a mass of fat. The destruction of the cell is an essential element in fatty degeneration, and distinguishes it from fatty infiltration, where the cells, within which the fat accumnlates, still remaiu.

This pathological process is very similar to some of the physiological ones. Thus, in the formation of milk, the cells lining the lobuli of the mammary gland multiply abundantly, and are converted into fat; then they break up, and constitute the creamy particles (corpuscles) of the milk. The corpuscles thus formed are pushed forward in the ducts by the formation of new cells, which in their turn undergo the degenerative process, and in this manner a continuous formation and destruction of cells take place.

Fatty degeneration is clearly a kind of atrophy, but is not identical with the simple form, for it may be seen, in degeneration of the heart more particularly, that the muscular fibres may be atrophied with loss of their transverse striæ, and yet contain not a single drop of oil.

When much tissue is affected, the change can be readily distinguished with the naked eye by the yellowish white appearance, and by a diminution in consistence and elasticity.

The parts most commonly affected by the fatty metamorphosis in the horse are the laryngeal muscles-producing the disease which is so well known by the term "roaring," - the voluntary muscles, particularly those of the shoulder, the coats of the 
arteries, the walls of the heart, and the cartilaginous basis of bones.

Causes of fatty degeneration.-All influences which interfere with the nutrition of a part may cause this change. Indeed tatty degeneration may be looked upon as an advanced stage of atrophy, and depending upon the same causes-namely, diminished supply of blood, inflammation, decreased functional activity, and diminished nervous influence. Rapid growth is also a cause of degeneration of a part. Thus a rapidly growing tumour is liable to softening and degeneration, as seen in the softening of the central portions of cancer, \&c.; and lastly, old age, by impairing the vital activity, may cause degenerations and softening of various parts of the economy.

The formation of the fat depends upon changes in the tissue itself, and not upon the deposit of it from without; that is to say, upon a conversion of the albuminous constituents of the tissue into fat, in a manner similar to that process which occurs after death, when from spontaneous decomposition a peculiar kind of fat, named adipocere, is formed out of the albuminoid tissues.

The fatty metamorphosis may be looked upon as a salutary change when it occurs in new formations, in thrombi, and in inflammatory exudates. Indeed it is only through the occurrence of this change that they are rendered liquid and capable of absorption.

Various changes may take place in the fatty degenerated material. Sometimes it gradually dries up into a yellow substance resembling cheese. This process is termed easeation, and appears to be owing to a natural dryness of the tissue, and is most frequent in parts which are not very vascular, or in those where the vessels are occluded. This process of caseation is most frequently seen to take place in new formations, composed of closely crowded cellular elements, such as tubercle, growths in the lymphatic glands, \&c.; and in them the material, after gradually drying up, may become enveloped in a capsule of fibrous tissue, and remain inert in the midst of surrounding structures. These cheesy matters are often met with, particularly in horned cattle, in the lungs, pleura, and peritoncum, and are apt to cause considerable confusion, as it has been the custom to look upon all cheesy matter as tubercular. To conclude that these cheesy masses 
are always tubercular is erroneous. In themselves, they merely indicate that the growth has undergone a fatty metamorphosis, but they do not furnish any evidence of any one particular form of growth. As a result of pleuro-pneumonia, they merely indicate that the exudate has undergone this form of metamorphosis, not that there has been a tubercular growth. At a subsequent period caseous material may be either calcified, or may become softened, absorbed, tainting the blood-mass, or give rise to the formation of tubercule-like tumours.

\section{CALCAREOUS DEGENERATION.}

Calearcous degeneration or calcification is an advanced stage of caseation, and is chiefly found to take place in masses of caseous material which are enclosed, and isolated from the influence of the external air, and it is a sure evidence that the growth, whatever may be its nature, has been existent for some length of time. Rokitansky considers the earthy materials not so much as new deposits, but as precipitations of salts, principally consisting of the phosphate of lime and magnesia, and carbonate of lime, from their natural combinations with pre-existent animal matters.

Calcareous degeneration does not always depend upon a previously fatty degenerate state of a part, although it often results from such. It may occur under two opposite conditions, namely, when there is an absolute increase of earthy matters in the blood, and the excess deposited in the tissues; and when there is no such increase, the deposition of earthy salts which then takes place being consequent upon some alteration in the tissue itself. The former condition is oftener met with in man than in the lower animals, and results from extensive caries and other degenerative bone diseases, where the lime salts are removed from the bones into the blood, and deposited in other tissues.

In such cases, the calcification affects many organs simul. taneously, and it is not an uncommon thing to find the lungs, kidneys, stomach, intestines, liver, and even the dura mater, infiltrated with lime salts. Again, it is found in man that chronic disease of the kidneys leads to calcification of several organs. This is due to the fact that the lime salts, which ought to have been excreted by the hidneys, accumulate in the blood 
and are deposited in those portions of the tissues which immediately surround the blood-vessels.

Calcification is, however, a process most commonly dependent on local causes--depending not upon any undne qrantity of lime salts in the blood, but upon a change in the part itself, whereby the salts which are normally held in solution in the blood are deposited in the tissues. This process is often characterised as an ossification, and not without some reason, as the so-called bone cells or lacuna are often found in the calcareous substance; they are, however, imperfectly and irregularly developed. I have one specimen in my possession, presented by Mr. Robertson of Kelso, where very well developed lacunæ are found to exist in the calcareous mass. The specimen consists of a bony or calcareous tube surrounding a piece of wood which had been lodged in the inguinal region of a horse for some years. It is a most perfect specimen of the ossification of an exudate, having no connection with a bone.

Calcareous degeneration seems generally to take place in parts whose vitality has been lowered by previous morbid changes, and in degenerating new formations. A part which has become calcified undergoes no further change, but remains as an inert mass: on this account it must be looked upon as a salutary termination of a diseased process. It is thus the most favourable termination to all degenerative diseases, as exemplified in the calcification of many new formations, inflammatory products, tubercle, and atheroma of the arteries.

Rindfleisch states that the cause of the deposition of the salts in the substance of atrophied tissue is partly owing to the stagnation of the nutritive fluids in the part, owing to which the free carbonic acid, which holds the salts in solution, escapes, in consequence of which they are precipitated; and partly to the non-assimilation of these fluids by the degenerated tissue. The saline matters are seen at first as fine molecules, scattered irregularly through the intercellular substance, and are characterised, when viewed by transmitted light, by their opacity, dark colour, and irregularity of outline. They are solnble in dilute mineral acids, after which the original structure of the part, if not destroyed by previous change, may again be recognised.

Softening "consists in the liquefaction of the caseous substance, probably owing to some chemical change in its constituents. It 
commonly occurs in parts which come in contact with the air." -(Grees.) The caseous mass is converted into a thin puriform fluid containing curd-like matter, composed of animal débris, fat, and cholesterine crystals, which, if not absorbed or discharged, may ultimately dry and become calcified.

\section{TIIE MUCOID AND COLLOID DEGENERATIONS}

Are conditions which are rarely met with in the lower animals. The reader is therefore referred to works upon human pathology, more particularly to Green's Pathology and Morbid Anatomy.

\section{PIGMENTARY DEGENERATION.}

In this process pigment takes the place of the tissue elements, in the same manner as fat or earthy salts in the fatty and calcareous degeneration.

I have witnessed this form of deposit in the lungs of horses. It is, however, of little importance, and I shall merely refer the reader to authorities upon the subject, amongst whom may be mentioned Aitken, Bennett, Virchow, and Green.

\section{AMYLOID DEGENERATION.}

This morbid process consists in the transformation of the tissues into a peculiar homogeneous substance, having a relation in some respects to the chemical characters of cellulose or starch.

This degeneration has been long known by medical men under a variety of names, such as the lardaceous, waxy, bacon-like, and albuminoid degeneration. I have met with several cases which presented the physical appearance of this form of disease both in the liver and in the kidneys; but I have only satisfied myself in one instance of the character of the morbid product. For an account of the method by which this disease has been investigated, I take the liberty of making the following quotation from Aitken, who, after mentioning a number of names by which the disease has been known, proceeds to say that "Chemistry and micro-chemical investigation have modified the views regarding the nature of the disease, and now and then have led to modifications in the nomenclature. Under this kind of inquisitive 
investigation it has been described-(1.) By Virchow under the name of 'animal amyloid,' he believing, from the behaviour of the transformed substance with iodine and sulphuric acid, that the substance must be classified with the vegetable carbo-hydrates -cellulose and starch: (2.) Meckel retains the name of 'lardaceous' or 'cholesterine disease,' believing that the essential character of the degeneration consists in the development of a peculiar fatty or lardaceous matter of the nature of cholesterine: (3.) The more extended and definite examinations by Friedreich and Kekule have shown that the substance of the purest amyloid degeneration more closely resembles the albuminous principles than any other substance we know of; and (4.) Schmidt has arrived at the same conclusion. The question, therefore, is not yet definitely settled as to the exact nature of the substance into which the tissues are transformed in the socalled amyloid degeneration; but the weight of evidence points to its being albumen in some form." The conclusions that the material is allied to albumen are confirmed more recently by Kiihne, who, by submitting the amyloid organs to a process of artificial digestion, has completely succeeded in isolating the new material, and has thus been able to determine its albuminous nature. Starch-like bodies (the corpora amylacea) have been discovered in various parts of the body, more especially in the nervous system, the ependyma of the ventricles, the white substance of the brain, the choroid plexus, the optic nerve and retina, and the spinal cord of the aged; and larger varieties of the same bodies in the prostate gland of almost every adult, accumulating here sometimes to such an extent as to form large concretions.

The characteristic feature of amyloid is its reaction with iodine, iodine and sulphuric acid, and methylaniline violet. A solution of iodine applied to the amyloid organ causes the affected portions to change to a deep reddish brown colour, which is not, however, permanent, but gradually passes off, and the part regains its former appearance. If the application of iodine is followed by the cautious addition of sulphuric acid, a blackish blue or violet tint is produced. Great nicety of manipulation is, however, necessary to obtain the reaction of the sulphuric acid.

General characters of tissues which have undergone the amyloid dcgeneration.-The cut surface of an organ so affected has a 
semi-transparent appearance. It feels like a piece of soft wax, or of lard and wax combined.-(WILks.) It euts into portions of the most regular shape, with sharp angles and smooth surfaces, and the thimnest possible slices may be removed by a sharp knife for microseopical examination. They are abnormally translucent. Water and alcolıol, acids and alkalies, do not produce any change upon the transformed parts, which may be kept for a length of time withont decomposition. The organs affected are increased in volume, in solidity, and in weight, absolute and specific. Anæmia is predominant; but the colour of the blood, or of tissue, shines through the semi-transparent morbid substance.

Amyloid degeneration is generally widely diffused, so much so, that a constitutional state of ill-health seems associated with its production; and in cases preceded by a local disease, such as caries of a bone, the degeneration may be found in the adjacent lymphatic glands only.-(Billroth.) This is the earliest appearance of the degeneration yet recognised.-(AITKEx.)

The anyloid substance almost invariably affects the capillaries and small arteries. Their coats become thickened by the deposit, and at last pellucid, transparent, and hyaline. The deposit then extends into the surrounding tissues, and invades both its cells and intercellular substance. The cells become filled with the amyloid material; they gradually increase in size, become round and regular in shape, their nuelei disappear, and they are finally converted into homogeneous bodies, having a translucent or glistening appearance. The effect of this change in the nutrition is to impair or even destroy the function of the affected organ :-1st. By obstructing its eirculation, by diminishing the calibre of small arteries; and $2 d$. By the pressure of the new material upon its proper substance, whereby secondary atrophic changes are induced. It is important to remember that this form of degeneration is secondary to some serious constitutional disease. It is supposed that it is due to some change in the blood, and that the deposit is in reality an infiltration of an albuminoid or fibrinous substance from the blood into the tissnes, which there becomes consolidated. In the human being it is found to succeed chronic suppurative diseases, and the way in which the tissnes are affected is as follows:The ehange almost invariably commences in the small nutrient blood-vessels, and extends from them to the surrounding parts, 
and the general characters of the affection, sereral organs being simultaneously involved, point to some alteration in the composition of the blood as the cause of the change. In the instance where I succeeded in tracing the nature of the deposit, the horse liad been lately bought, suffering at the time from what appeared to the purchaser to be the remains of a cold, sore throat, or strangles. When seen by me, five weeks after purchase, the animal was much emaciated, and presented symptoms of disease of the liver, and was evidently in a dying condition. The post mortem revealed the liver much enlarged, weighing over twenty pounds, and of a glistening, palish yellow colour throughont, the new material being stained by the biliary matters; the kilneys were also enlarged, very solid, pale, and translucent in appearance; and when cut, the surfaces were smooth, bloodless, and glistening. The iocline test was most conclusive. It is important to bear in mind that amyloid disease is essentially chronic in its character, and when deposited to any extent, is a sure sign that the animal has been in ill health for a period extending over many weeks, or even montlis.

\section{FATTY INFILTRATION.}

This essentially diflers from fatty degeneration. In degeneration, the fat is derived from a change of the protoplasm itself, leading ultimately to the destruction of the cells of the part. In infiltration, the fat is deposited between, as well as within the cells, where it displaces and obscures the nuclei and protoplasm, but does not destroy them. In degeneration, the fat occurs in a granular form within the cell contents. In infiltration, the fat is deposited as distinct drops, which may gradually increase and coalesce, so that the cells, as in the figure, may be completely

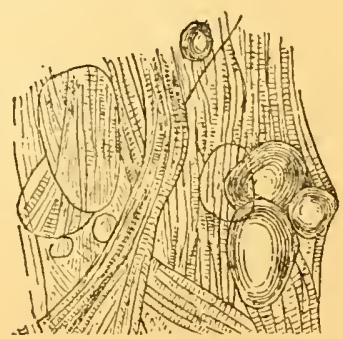

FIG. 2.-Fibuty infiltration of heart. Large fat cells intermixed with muscular tissue. filled and distended with it. The functions of the cells are but little impaired by the accumulation, and are restored to their natural condition by the removal of the fat. 
Fatty infiltration is due to an increase of oleaginous materials in the blood, which arises from high feeling and want of exercise. It occurs as a physiological process in the fattening of animals, where the connective tissne becomes filled with fat.

In the human being it has been observed that this form of infiltration is occasionally owing to the presence of fat in the blood, arising from its absorption from some particular tissue and 'its deposition in another: for example, the general emaciation of pulmonary phthisis is sometimes associated with fatty infiltration of the liver. "Why the deposition should take place in certain tissnes, and the fat be removed from some and deposited in others, is not known."-(GreEN.) Fatty infiltration, more particularly of the liver, has occurred in my experience involving legal points of some consequence. In two cases which more particularly occur to me, the animals died within a few days after being purchased. In one case the liver weighed thirty-five pounds, was of an opaque yellowish white colour, its surface smooth, the edges round and thick, doughy to the touch, pitted on pressure, and when cut into, the knife was coated with. oil, which oozed from the cut surfaces pretty freely. In the second case, death occurred twelve days after purchase: the liver was not so large as in the former instance, the deposition occuring more as isolated patches than as a general infiltration. In both instances the purchasers succeeded in obtaining a return of the purchase-money. Fatty infiltration is caused by high feeding, more particularly by food containing an excess of the hydrocarbons-such as the oilcakes-and is apt to occur in animais quickly prepared and made fat for sale. It also frequently occurs in very highly fed cart-horses employed for slow work, and often causes death by the liver enlarging to such an extent as to burst its capsule.

Why the liver should suffer from the accumulation of fat, is explained by the fact that when animals are fed on hydrocarbonaceous food, an excess of fat in the blood is the consequence, such fat being, before it is finally disposed of, deposited in the hepatic cells, more particularly in those cells in close contact with the capillaries of the vena porta, at the circumference of the hepatic lobules. This temporary infiltration occurs after every meal, and the fat passes from the cells at the 
circumference to those situated more within the liver, then into the blood-vessels, and is finally disposed of, either by being deposited in other tissues, or destroyed in the maintenance of the animal heat.

There is thus a temporary accumulation of fat in the liver, which is gradually removed, and the vitality of the hepatic cells is not impaired. By bearing these facts in mind, we can the more readily understand that when an animal is constantly fed on such a diet, the accumulation may be too great to be disposed of or consumed, and that it remains permanently within the hepatic cells, or so long as the animal continues to be so fed and treated, interfering to some extent with the secretory powers of the organ, and rendering it unfit for ordinary work. A liver subjected to the stimulating influences of such food is prone to attacks of congestion, which are occasionally so great as to cause a rupture of Glisson's capsule, and bring on death from hæmorrhage. It is well to remember that when a horse is excessively fat, the liver may be in this condition; that there is a possibility of death occurring from congestion or rupture; and that the probabilities of recovery from diseases which may have no immediate connection with the liver itself are much lessened. If, however, the animal be carefully brought, into condition, regularly but moderately worked or exercised, and fed on food containing no excess of the hydrocarbons, but rich in nitrogenous materials-such as oats, beans, and hay-the liver cells will be again restored to their normal condition by the absorption of the contained fat.

As a veterinary legal question the matter is of some importance, as horses often die if not cautiously and carefully treated when loaded with fat.

It is well known that a practice prevails in many parts of the country of fattening horses rapidly prior to showing them for sale, by giving them an immoderate quantity of highly carbonaceous or even oleaginous food. To the eye, such animals present all the signs of good health. They are, however, totally unfit for work, and too well does the experienced veterinarian know, that when they are seized by an ordinary attack of disease-such as a cold, influenza, \&c.- that the ehances of recovery are more remote than they otherwise would be, by the liver and other internal organs being loaded with fat. The ten- 
dency to produce fat rather than muscle prevails to a very great extent in this country, encouraged and fostered by our agricultural societies, and by purchasers who buy to "please the eye." The question presents itself-Who is responsible when a horse dies in a few days after purchase, either directly or indirectly from this cause? The buyer or the seller?

Fatty infiltration must be carefully separated from fatty degeneration. Degeneration of normal tissues is at all times a morbid process indicative of chronic disease. Infiltration, on the other hand, is a physiological process depending on wellknown causes, and furnishes no evidence of a chronically diseased condition of the organ affected. As an example of the importance of the separation of the two conditions, we will suppose a case analogous to what often happens, of a horse in poor condition-thin-being purchased from the brceder and rapidly fed for sale. Such an one puts on fat quickly, and thrives as well as its purchaser can desire. We will say that it is sold and put to work, and that it dies in the course of a week or two after the second sale. Altogether it has not been out of the hands of the breeder more than two months. Now, the last purchaser naturally looks for restitution to the person from whom he bought it, who, in his turn, may make a claim on the breedcr. In many cases the matter is amicably settled by the three interested parties dividing the loss amongst them. Is this just? Decidedly not; and in all such instances the man who sold the horse before the fattening process had been commenced should not be made to suffer, the enlargement of the liver being merely due to an accumulation of fat, and not to any disease: and if one individual is to suffer, let the man who resorted to the forcing system of producing fat be made responsible; or let the loss be divided between him and the man who bought what pleased his eye, and who must have known, or ought to have known, that an animal loaded with fat was unfit for useful purposes. But should an examination reveal that the condition of the liver was due to degeneration, the case is entirely different, for beyond a doubt the disease must have been present prior to the first purchase, and the breeder ought then to be responsible for the whole loss.

Degeneration generally occurs in the aged, and may be associated with general emaciation, and with degeneration of other 
organs. Infiltration occurs in both the young and old, and is associated with a general obese condition of the whole system.

\section{NUTRITION INCREASED.}

Opposed to the conditions of atrophy and the degenerations, is that of increased nutritive activity, or hypertrophy. In hypertrophy there is an absolute increase of the normal tissues of a part, resulting from an increase in its functional activity.

Hypertrophy, although sometimes depending on disease, is of itself a compensating process, whereby injury from damage to an important organ may be avoided; as, for example, in the thickened condition of the heart in disease of the valves, and in the same condition of that organ when not associated with any disease, but arising from the animal being repeatedly forced to severe trials of strength and endurance, in racing, hunting, \&c. In the first instance the hypertrophy of the cardiac walls arises from the necessity of a more powerful contraction for the purpose of overcoming valvular obstruction, and sending a due supply of blood to the various organs of the body, and in the other case of contracting powerfully to attain the same end, during repeated and severe exercise.

As a physiological process, hypertrophy of muscular tissue is produced by the process of training, where the muscles of the whole body are enlarged, strengthened, and made capable of performing extraordinary feats of strength and endurance. Who can fail to see in a well-trained racehorse the muscles standing, as it were, prominently one from another about the quarters and thighs, "hard as iron" to the touch, and giving the animal an appearance of being thin and poor. The appearance of thinness is the result of the great prominence of each individual muscle, of increase in their volume and weight, and an extraordinary lealthy tonicity.

"The cause of real hypertropliy seems to be always the increased exertion of the organ; more than usual effort is demanded of it, and according to the law of the circulation, which we have noticed, more blood flows to the part than usual. This, if the organ be in a healthy state, not only supplies its waste, but furnishes material for increase and development. The heart in various diseased states of its valves, the urinary bladder in stric- 
ture of the urethra, the remaining kidney when one is destroyed, the muscles, and even the solid bones themselves, when long and actively exercised, afford excellent examples of true hypertrophy."-(C. Havdfield JoNes.)

Whilst increased nutritive activity may lead to hypertrophy, or an increase of normal tissue only, it may, on the other hand, lead to the production of new materials in a part, to which the narne of new formations-tumours-are given. These have been, to some extent, described in my work on Veterinary Surgery. 


\section{CHAPTER VIII.}

\section{PA T H O L O G Y-continued.}

\section{CHANGES IN THE BLOOD.}

Healthy BLOOD, when it can be seen flowing in the vessels of a living part, appears as a colourless fluid-the liquor sanguiniscontaining minute solid particles-the blood globules-of which the greater part are red, and the remainder colourless or white.

Analyses of blood show that it is composed of, in 1000 parts-

Water,

Red corpuscles,

Albumen,

Saline matters, .

Extractive matters

Fibrin, $784^{\circ}$

$131^{\circ}$

$70^{\circ}$

6.03

$6 \cdot 77$

$2 \cdot 2$

$\overline{1000 \cdot 00}$

The above constituents being proportioned as follows:-

Water,

Albumen,

Fibrin,

Red corpuscle

Fatty matters,

Inorganic salts,

$\begin{aligned} & \text { Phide and phosphate of iron, } \\ & \text { Oxis }\end{aligned}$
Extractive matter, with salivary matter, urea,
biliary colouring matter, gases, and accidental
substances, .

$\left.\begin{array}{l}\begin{array}{l}\text { Oxide and phosphate of iron, } \\ \text { Oxias }\end{array} \\ \text { xtractive matter, with salivary matter, urea, } \\ \text { biliary colouring matter, gases, and accidental } \\ \text { substances, . }\end{array}\right\}$ substances, .

$$
\begin{aligned}
& \left\{\begin{array}{l}
\text { Globulin, } \\
\text { Hrmatin, }
\end{array}\right. \\
& \text { Cholesterine, } \quad 0.08 \\
& \text { Cerebrine, . . } 0.4 \\
& \text { Seroline, . . } 0.02
\end{aligned}
$$

784

70

\section{$2 \cdot 2$}

$123 \cdot 5$

$7 \cdot 5$

$1 \cdot 3$

$3 \cdot 6$

0.56

0.2

0.84

$0.2 \mathrm{~S}$

0.25

0.5 
These several constituents are subject to some variations consistent with health. Thus the water varies in quantity according to the period since food has been eaten, the amount of bodily exereise, the state of the atmosphere, and all other events that affect the ingestion or the excretion of fluids. According to these conditions, it may vary from 700 to 800 parts in 1000 . On the whole, however, a degree of uniformity is maintained, because nearly all those things which tend to lower the proportion of water in the blood, such as active exercise, or the addition of saline and other solid matter, excite thirst; while, on the other hand, the addition of an excess of water to the blood is quickly followed by its more copious excretion in sweat and urine.

Dr. Zimmerman has shown that the quantity of water in the llood is increased during its abstraction from an artery or vein. In bleeding dogs he found that the last drawn portion contained 12 or 13 parts more of water in 1000 than that first drawn. This experiment goes to prove that the fluid of the tissues must be rapidly absorbed during the operation.

The water in the economy is required for many important purposes. (1.) The proper viscidity of the blood, and the degree of its adhesion to the blood-vessels, is due to the presence of water in proper proportion. (2.) It determines to a great extent the volume of the blood. (3.) Water is the general solvent of the other materials of the liquor sanguinis. (4.) The activity of absorption by the blood-vessels depends upon the due fluidity of the materials to be absorbed, for it is well known that no fluids quickly penetrate the vessels but such as are of lesser density than the blood.

If the presence of water in excess continues beyond a short period, it is a certain symptom that the other constituents are defective in quantity, and that the condition termed anæmia is present.

Defieiency of water in the blood is witnessed in super-purgation, diarrhoea, \&e. The deficiency, however, is generally of a temporary character, for nature prompts the animal to overcome it by drinking freely. The water aiso becomes deficient when an animal sweats profusely from any canse, and when prevented from allaying his thirst for a considerable period, as when hunting or going long journeys. A great mistake is made 
by those in charge of horses who have a belief that it is a bad thing to allow a horse to drink while out on a journey or in the liunting-field. Small and repeated drinks are always beneficial, and prevent much suffering.

The albumen of the blood may vary, consistently with health, from 60 to 70 parts in 1000 . Its amount, according to Andral and Gavarret, is notably increased in various diseases; but this excess does not appear to be characteristic of any. In acute rheumatism an increase was found varying from 4 to 24 ; in pneumonia the highest increase was about 12 ; in pleurisy the extraordinary amount of 34 in excess was once observed; and several other instances are mentioned of a lower degree. Excess of albumen in the blood is witnessed in the horse in a disease which the late Mr. Haycock termed hysteria.-(See Azoturic or Uramic Paralysis.) Very probably the albumen in this disease is not only excessive in quantity but deteriorated in quality.

The albumen is defective in cases of albuminuria, in the "rot" in sheep, and in dropsies, whether dependent on organic disease of the heart, or on the presence of entozoa-distomatain the liver. It is a remarkable fact, recorded by Andral, Gavarret, and Delafond, that in sheep affected with anæmia, with deficiency of the red globules, but without dropsy or entozoa, there was no deficiency in the albumen. Dr. Willianus conclucles from this, that cachexia and anæmia attended by dropsy owe this concomitant to a want of albumen in the blood; and he seems to be of opinion that a deficiency of the albumen, by destroying the spissitude of the blood, and allowing it to transude through the coats of the vessels, is a chief feature in the dropsical diathesis.

The fibrin of the blood.- When fresh drawn blood is left to itself, it very soon coagulates or becomes solid, and the substance thus becoming spontaneously solid is the fibrin, which, when separated from the blood, is a tough, elastic, tolerably firm, whitish-grey looking material, insoluble in water. Under the microscope the fibrin appears as a homogeneo-granular matter, with a more or less marked tendency to fibrillate. The white globules are usually seen imbedded in the mass, but they do not appear to modify the character of healthy fibrin. This coagulation is due to the formation of fibrin, a material which does not exist in the blood circulating in healthy vessels, but is formed in the blood and lymph when removed from the borly. At first 
the crassamentum or clot is soft, consisting of a few fibres on the surface. These soon extend throughout the mass, which is now called cruor. In the horse coagulation takes place but slowly, and the red corpuscles consequently sink; the upper surface, being thus deprived of their colour, assumes a strawcoloured appearance, and is called the buffy coat. Although the whole mass solidifies, the fibrin is only present in quantity of about 3 in 1000 .

MM. Andral and Gavarret record that in the domestic animals the fibrin diminishes before but increases after parturition. In the human female, however, it seems that the fibrin is decreased during the first six months of pregnancy-the average being 23 ; whilst in the last three months it is so increased as to average 4. Fibrin is more abundantly formed in arterial than in venous blood; and it is mentioned by Schmidt that the blood of the portal vein contains only one-third of the ordinary amount of fibrin found in the blood of the jugular vein.

An excess of the fitrin-liyperplasma or hyperinosis-exists in all true inflammations. In acute rheumatism it is generally very high; in some cases, according to MM. Andral and Gavarret, as high as 12 parts in the 1000 . In some exhansting diseases, and even in anremia, the fibrin is found occasionally increased in quantity. In rapid emaciation, more especially when accompanied by total loss of appetite, the quantity of fibrin is very materially increased. This is explained by the fact that fibrin results from the metamorphosis of the albumen of the blood and of the tissnes. An increase of fibrin is found in meagre, half-starved horses, amounting to 7 or 8 above the healthy mean. In one case, where no food was given for four days, it was found increased from 5 to 9 . As the fibrin is increased by all inflammatory diseases, such increase can also be artificially induced by the application of external irritants, such as blisters, setons, or the actual or potential canteries. This fact is of great importance therapeutically, and it should be borne in mind that the application of the so-called counter-irritants in extensive inflammatory diseases, tends to increase a condition of the blood which is already present to an abnormal extent.

Another very important circumstance connected with the increase of fibrin in the blood is pointed ont by Andral, namely, that the effect of bleeding was not to diminish it. In M. Andral's experiments the red globules became diminished in number by 
each abstraction of blood, whilst the fibrin often rose, sometimes considerably. The following examples are very conclusive :-

First Second Third Fourth Fifth Sixth Bleeding. Bleeding. Bleeding. Bleeding. Bleeding. Bleeding.

\begin{tabular}{|c|c|c|c|c|c|c|}
\hline Acute Rheun & $\mathrm{sm}, 6 \cdot 1$ & $7 \cdot 2$ & $7 \cdot 8$ & $10 \cdot 2$ & $9 \cdot 0$ & $7 \cdot 0$ \\
\hline Pneumonia, & . $7 \cdot 1$ & $8 \cdot 2$ & $9 \cdot 0$ & $10 \cdot 0$ & - & 一 \\
\hline Peritonitis, & - $3 \cdot S$ & $4 \cdot 7$ & $6 \cdot 1$ & & - & \\
\hline Pleurisy, . & . $\quad 3 \cdot 9$ & $5 \cdot 8$ & - & 一 & - & \\
\hline
\end{tabular}

MM. Andral and Gavarret found that the fibrin production was increased in tubercular disease. In the crude state of the tubercles, the mean is about 4 , when softening has commenced it is about 4.5 , and when cavities have formed it is from 5 to $5 \cdot 5$, the red globules decreasing from the first.

Deficieney of fibrin-hypoplasma, hypinosis, or defective coagulating power-is observed in varions morbid states, in disease, and in conditions bordering upon it, and is indicated by imperfect coagulation of the blood when drawn. It is deficient in all conditions where the blood is imperfectly arterialized; and this is in accordance with the physiological law that arterial blood is more fibrinous than venous. Thus, in death from asphyxia, the blood often remains semi-fluid, of a dark, tarry appearance, and coagulates only after being exposed to the air and absorbing oxygen. Various poisons seem to have the power of preventing the coagulation of the blood. Among these are hydrocyanic acid, carbonic acil, sulphuretted and carburetted hydrogen, and ammonia. Andral states that if a concentrated solution of carbonate of soda be injected into a vein, the animal presents the symptoms of blood disease, and that the blood is found fluid in che vessels. The same result may be produced by arsenious acid, digitalis, and oxalic acid. Other substances, although analogous to the carbonate of soda, seem to have no effect. Thus, the nitrate of potash does not always impede the coagulation of the blood; and Dr. Haudfield Jones states that he has observed that liquor potasse, although given for several days, and until the animal's health was interfered with, did not at all interfere with the coagulation of its blood; and he infers from these observations that it is not the alkalies, as such, nor the neutral salts, as such, which produce the effects usually ascribed to them upon the blood, but certain substances of 
particular qualities. In low fevers, influenzas of a typhoid character, particularly when animals so affected are stabled in ill-ventilated louses, the blood becomes remarkably fluid, and gravitates, even in cases that recover, to the depending parts of the body. Thus we find that in the so-called purpura hæmorrhagica the extremities and inferior portions of the abdomen, breast, and face become the seats of extensive swellings, caused by an accumulation of a preternaturally fluid blood in the vessels, and even in the tissues, whilst the appearance of petechire, vibices, and the occurrence of hæmorrhages are due to the same cause. The partial suffocation to which animals are sometimes exposed when confined in buildings accidentally or otherwise burnt, produce a remarkable degree of fluidity in the blood. I have often witnessed this in horses and cattle which had been early removed from burning buildings,-in fact, when they had not been exposed to any fire, but to the effects of the smoke of smouldering straw, wood, \&c. At the time, the symptoms of distress have not always been noticeable, and little has been thought of the matter. In the course of time, varying from a few hours to three days, the animals have presented symptoms of great respiratory disturbance, with hrmorrhage from the vessels of the lungs, the blood presenting a dark olive appearance, and coagulating but very imperfectly, even when exposed to the influence of the air. In such cases, it is quite fair to presume that the coagulating powers of the blood have been destroyed by the action of the products of combustion contained in the smoke, and that it-the blood-gradually accumulates in the vessels of the lungs until they have become congested, even to rupture of their walls. In apoplexy of the spleen and in "septicæmia" the same hypinotic condition of the blood is seen, and is probably the cause of the congestions and extravasations which characterise those diseases. Although it may justifiably be said that imperfect coagulation, as well as the abnormal fluidity of the blood, depends upon the absence of fibrin, modern research has proved that filurin, as such, does not exist in the blood, but is formed by the cumbination of two substances -or two modifications of the same substance-namely, globulin ${ }^{1}$ and fibrinogen; the former of which existing in the serum of the blood, and in some tissues of the body; whilst the latter, so

1 Fibrino-plastic.-(Sснитт.) 
far as it is known at present, exists only in the plasma of the blood, of the lymph, and of the chyle, and in fluids derived from them.

"Coagulation of the blood" (says Professor Huxley) "is an altogether physico-chemical process, dependent upon the properties of certain of the constituents of the plasma, apart from the vitality of that fluid. This is proved by the fact that if blood-plasma be prevented from coagulating by cold and greatly diluted, a current of carbonic acid passed through it will throw down a white powdery substance. If the white sulustance be dissolved in a weak solution of common salt, or in an extremely weak solution of potash or soda, it after a while coagulates, and yields a clot of pure fibrin. It would be absurd to suppose that a substance which has been precipitated from its solution, and re-dissolved, still remains alive.

"There are reasons for believing that this white substance consists of two constituents of very similar composition, which exist separately in living blood, and the union of which is the cause of the act of coagulation. These reasons may be briefly stated thus:-The pericardium and other serous cavities in the body contain a clear fluid, which has exuded from the blood-vessels, and contains the elements of the blood without the blood corpuscles. This fluid sometimes coagulates spontaneously, as the blood-plasma would do, but very often shows no disposition to spontaneous coagulation. When this is the case, it may nevertheless be made to coagulate, and yield a true fibrinous clot, by adding to it a little serum of blood.

"Now, if serum of blood be largely diluted with water, and a current of carbonic acid gas passed through it, a white powdery substance will be thrown down; this, re-dissolved in a dilute saline, or extremely dilute alkaline solution, will, when added to the pericurdial fluid, produce even as good a clot as that obtained with the original serum.

"This white substance is called globulin. It exists not only in serum, but also in smaller quantities in connective tissue, in the cornea, in the humours of the eye, and in some other fluids of the body.

"It possesses the same general chemical properties as the albuminous substance which enters so largely into the red corpuscles, and hence at present bears the same name. But 
when treated with chemical re-agents, even with such as do not produce any appreciable effect on its chemical composition, it very speedily loses its peculiar power of causing serous fluids to coagulate. For instance, this power is destroyed by an excess of alliali, or by the presence of acids.

"Hence, though there is great reason to believe that the florino-plastic globulin (as it has been called) which exists in serum does really come from the red corpuscles, the globulin, which is obtained in large quantities from these bodies by the use of powerful re-agents, has no coagulating effect at all on pericardial or other serous fluids.

" Though globulin is so susceptible of change when in solution, it may be dried at a low temperature, and kept in the form of powder for many months without losing its coagulating power.

"Thus globulin, added, under proper conditions, to serous effusion, is a coagulator of that effusion, giving rise to the development of fibrin in it."

The latest theories of coagulation slightly modify the above. Thus Schmidt says that the fibrin is formed by the coming together of two proteid substances which occur dissolved in the plasma, viz.-(1) fibrinogen, i.e., the substance which yields the chief mass of the fibrin; and (2) fibrino-plastic substance, or fibrino-plastin. The latter terms are now rarely used, having been replaced by either of the following-serum-globulin or paraglobulin. In order to determine the coagulation a ferment seems necessary, and this is supplied by (3) the fibrin ferment.

Both fibrinogen and fibrino-plastin belong to the group of proteids called globulins. They are insoluble in pure water, but are soluble in dilute saline solutions (common salt, 1 per cent.), phosphates of the alkaline earths, and calcium sulphate. They are not distinguished from each other by any marked chemical characters, but differ in the following particulars: Fibrino-plastin or paraglobulin-serum-globulin-is only appreciably precipitated when a solution of sodium salt of at least 20 per cent. has been added, whereas fibriuogen is easily precipitated by the solution of salt (sodium chloride), when it only reaches 16 per cent.

Again, the very light granular precipitate of paraglobulin is more easily dissolved than that of fibrinogen, and its solution only begins to coagulate at $133^{\circ} \mathrm{F}$., whereas that of fibrinogen 
readily coagulates at $105^{\circ} \mathrm{F}$. The precipitate of paraglobulin forms a granular powder, whilst that of fibrinogen adheres to the sides of the vessel as a sticky deposit.

The theory of Schmidt now seems to be superseded by that of Hammersten, and his researches have led him to conclude that fibrino-plastin is quite unnecessary for coagulation, and that the fibrin is formed from one body,-viz., fibrinogen, which is present in plasma when it has been acted upon by the fibrin ferment, which, however, has not as yet been obtained in the pure state.

Neither Hammersten nor Schmidt assert that this body is of the nature of a ferment, but they use the term for convenience. It is quite certain that fibrin may be formed, when no fibrinoplastin is present, by the addition of calcic sulphate or casein rrepared in a certain way; but as yet the views of specialists on this subject are by no means settled. Halliburton argues that the ferment supposed to be the determining agent in coagulation is really itself a proteid-in fact cell globulin; but as several experiments of others seem to show that the salt extract of fibrin can be very completely freed from all proteid matter without any loss of fermentive activity, this ferment can scarcely be a globulin. On the other hand, it might be assumed that the coagulative power is really due to the calcium sulphate (GreEs), which salt is well known to favour clotting even in very dilute solutions. But finally, as against this latter supposition, remains the fact that in solutions of the fibrin ferment which have been most carefully freed from everything giving proteid reactions, and also carefully freed from saline matters by dialysis, the power of inducing coagulation only becomes lost after heating to about $154^{\circ} \mathrm{F}$, a condition that conld by no means influence calcium sulphate, even if traces of it were present. Buchanan in 1831, Mantegazza in 1871, and Schmidt a year later, had all suspected that the white cells were the source of a ferment connected with coagulation.

Among several recent methods of separating the ferment, perhaps the best is the prolonged action of strong alcoholfifteen times the volume of the serum used-for two weeks or more. Subsequent filtering, drying and powdering the precipitate, and dissolving it in distilled water to twice the original volume of the serum, gives an extract with which coagulating experiments can be made. 
In addition to those above named, Halliburton and Rauschenbach consider that plasma entirely free from white cells cannot be made to coagulate in the usual way, but information on the fibrin ferment is much less accurately determined than is the case with almost any other known inorganic ensyme.

\section{FERMENT CONTAINED IN THE SECRETIONS OF ANIMALS AND PLANTS.}

The following appears to be all that is really known:-(1.) Temperature is of much account, action stops after $132 \frac{2}{5}^{\circ} \mathrm{F}$. lias been reached: 2 . The amount of coagulative change is not affected by the quantity of the ferment, only the rate, as with all known ferments; when the quantity is small the clotting is slow: (3.) The ferment is precipitated along with gelatinous matter found in its solutions; 4. It is most probably not used up, and a very small quantity indeed seems to be sufficient.

It seems, however, to be the general opinion that the ferment is perhaps derived from the disintegration of the white corpuscles when blood is shed, or within the economy in certain conditions, such as septic fevers, diseases of the blood-vessels, foreign bodies introduced into them, pleuro-pneumonia contagiosa, the microbe of which seeming to determine coagulation of the blood within the vessels, and of the exudate in the surrounding structures of the lungs, i.e., parenchyma cells, alveoli, and bronchi ; and it is also found that an injection of ichor into the blood causes the destruction of an enormous number of corpuscles, resulting in spontaneous coagulation within the circulation, after which the coagulability of the blood is diminished. From the foregoing we can understand how in metritis and other septic diseases where thrombi are abundantly formed, the blood itself may coagulate more feebly than natural.

Whilst fibrin is absent in healthy blood, it must be admitted that the materials from which its factors are derived must be contained in their proper conditions and proportions. But it is equally evident that circulating blood contains no ferment so long as both the fluid and the walls of the vessels are in a normal condition.

Coagulation of the blood varies in different animals, and is 
modified by disease. The blood of the horse, for example, coagulates more slowly than the blood of other animals and of man; hence what is termed the buffy coat is observable in coagulated healthy horse's blood, the buffy appearance on the top of the clot being ascribable generally to the fact that slow coagulation gives time to the blood globules, which are of higher specific gravity than the liquor sanguinis, to sink towards the bottom of the vessel before being incarcerated in the meshes of the coagulating fibrin. To prove this, fill two vials with the blood of a healthy horse, and to the blood in one add a small quantity of the chlorate of potash, and leave the other to coagulate naturally. In the course of a very short time the blood to which the chlorate has been added will be found firmly coagulated, and with but a slight or without any buffy coat; whilst the blood to which no addition has been made remains fluid for a much longer time, the globules falling to the lower parts, leaving the upper portion of the clot a straw colour.

During the first stage of coagulation, which is termed that of gelatinisation, the clot appears as a semi-solid mass. In the course of some time, however, it divides into serum and clot (crassamentum), the serum being pressed out by the fibrin. This shows that the fibrin possesses the power of contraction; and to the presence or absence of this power the further changes in the clot are due. The clot may be defective in soliditygelatinous-or it may present an excess not only of fibrin, but of contracting properties. The shrunk, contracted, or cupped appearance of the clot indicates increased contractility and a sthenic state of the system generally; whereas the gelatinous clot is indicative of an aplastic condition of the blood and an asthenic state of the system. 


\section{CHAPTER IX.}

\section{PATHOLOGY-continucl.}

\section{CHANGES IN THE BLOOD-continued.}

\section{ANEMIA, IIYDRAMIA, OLIGAMIIA.}

Truese terms are applied to that condition of the system in which there is a deficiency and poverty of the blood, a state brought about by loss of blood (hrmorrhage), profuse discharges of any of the natural fluids of the body, as those of diarrhoea or diabetes, in which it seems as if the blood globules were melted down to supply the profuse discharge; insufficient food, deprivation of fresh air, exhausting diseases, and the action of some deleterious agents, as witnessed in horses kept in ill-ventilated stables, or suffering from the influence of the glanders poison.

The symptoms of anæmia are paleness of the visible mucous membranes, with often an unhealthy or slate-coloured appearance of that of the nasal cavity. In some instances a coppery appearance is witnessed; this is undoubtedly due to an altered condition of the globules, and a disordered state of the powers by which they are formed; the conjunctiva is pale, the month cool, and the tongue generally unnaturally soft. Young, growing animals, insufficiently fed and improperly cared for, exposed to the vicissitudes of the weather during the winter months, are reduced to an anæmic condition, manifested by emaciation, debility, and often a depraved appetite. When thus reduced, great eare is to be taken that such are not suddenly turned into rich pastures, for it seems that the process of the formation and elaboration of the blood, and the organs concerned in it, are in such a state of disorder from long disuse, that the alimentary matters contained in luxuriant grass or nourishing food are transformed into an imperfect blood-plasma,- that is to 
say, into blood abundant in quantity, but defective in quality, plasticity: and consistence, incapable of nourishing the tissues, and which ultimately becomes of itself diseased. From this causenamely, the unwise and indiscriminate method in which young, rapidly growing, and imperfectly fed cattle are turned into rich pasturage in the spring, or put upon cake in the wintergastric, blood, nervous, and other important diseases are cansed, and the life of valuable stock destroyed. In horses I have repeatedly witnessed the anæmic condition associated with such extreme debility of the muscular system as to simulate spinal paralysis, and in some cases paralysis, from the pressure of serous fluid on the spinal cord, has been actually present; but in the horse, as in horned cattle, active signs of disease are not manifested until there has been a sudden change to a nourishing rliet. Sheep fecl on food containing an excess of moisture, and during long-continued wet weather, become anremic and dropsical. During the winter of 1872-3 this condition was exceedingly prevalent owing to the inferiority of the grasses, the moist condition of the turnips, and the humidity of the atmosphere; for in many instances no organic disease or parasitic invasion were present.

The anrmic condition is announced by abnormal sounds in various parts of the vascular system. These are called venous murmurs, and are ascribed to the thin, watery blood rumning with great rapidity in the ill-filled vessels, and are heard at the breast, at the base of the jugulars more particularly, and are, according to Dr. Williams, of the nature of ripples, " the natural inequalities of the surface over which the current of blood passes being sufficient to occasion, in its dilute and diminished condition, vibrations and sonorous gushes, which would not occur in a fluid of greater density.

The nutrition of the various structures of the body being dependent upon a healthy condition of the circulating fluid, it naturally follows that wasting, emaciation, and debility are the results of anæmia. In none of the anæmic states, unless it be intensified by an excessive discharge, as in diarrhœa, is rapid emaciation so prominent a symptom as in that caused by the presence of the glanders poison.

The pulse in anæmia is feeble, thready, often jerky and irregular, and any sudden excitement produces a degree of palpitation of the heart, leading one to suppose that the organ is 
hypertrophied, so loud, sharp, and knocking is the character of its contraction.

The contractile power of the muscles is generally much impaired, fatigue being soon induced; the bowels are often constipated, the constipation being due to a want of tone in the muscular coat.

Though the muscular system manifests great loss of power and tone, the nervous system seems to be exalted, excitement being induced by very trivial causes, but this seeming exaltation is in reality due to loss of strength and power. "The nervous system in the anæmic condition may be likened to a spring which originally was of a certain strength, requiring a certain impressing, and reacting with a corresponding force, but having become much weakened, is bent by much less force, and reacts also with much less. Mobility and debility may be said briefly to be the chief characteristics of the nervous actions in the anæmiated."-(Dr. HANDField Jones.)

The digestive powers are feeble and imperfect, and colicky pains are induced by trivial causes; tympanitis is frequent, and results from the defective power of the muscular coat of the alimentary canal. The secretion of the gastric juice is also impaired, and results from the gastric follicles being insufficiently supplied with nutritive materials. This constitutes a further cause for the continuance and aggravation of the anæmic condition, and acts as a bar to healthy digestion, though the food supply be liberal and good, for healthy chyle cannot be formed to renovate the blood if the primary digestion be impaired. Most probably the accession of disease, when nutritious food is supplied to half-starved animals, is due more particularly to this cause.

Anasarcous swellings of the limbs is a common symptom of anæmia in the horse, but is rarely witnessed in horned cattle. In sheep, as already stated, dropsy of the cavities is frequently witnessed. Under the term "spontaneous anasarca," Mr. Brown of Melton-Mowbray has described the anæmic condition, with dropsy of the cavities, in the horse. Mr. Brown says the disease attacks one and two year old colts, grazing during the winter season in wet, poor moorland, and is very prevalent in Lincolnshire; the disorder being manifested by swelling of the legs, sheath, belly, and lips, and finally the eyelids, the breath and 
excretions exhaling a peculiar odour: The animal turns sluggish and depressed, loathes its food, and seldom lies down; the respiration bccoming accelerated and the pulse feeble. Loss of flesh, prostration of strength, short and difficult respiration, frequent and indistinct pulse, and finally diarrhoe, which, if once established, carries off the animal in despite of all remedies. The post mortem examination reveals yellowness and flaccidity of the muscular system, effusion into the areolar tissue, thickening of the pericardium and pleura, with effusion into their cavities, often so great as almost to cause collapse of the lungs and arrest of the heart's action; effusion into the omentum, mesentery, and peritoneum.-(See Veterinarian, 1832.)

The causes of anæmia being generally apparent, I need scarcely state that they must be removed, as a primary step in its successful treatment; and for this purpose, it was, or is, the custom in Lincolnshire, to take up young colts for the month of August, and feed them during that time exclusively on dry provender. When anæmia is due to the operation of a specific virus, as that of glanders, or when at any time its cause cannot be discovered, the employment of any mere remedial measures is of very doubtful utility. Again, the anæmic condition, which can be ascribed to well-known and preventible causes, may have existed so long, or operated so strongly on a constitution perhaps naturally weak, as to act as a determining cause of some cachectic condition such as tuberculosis, or to disease of the osseous system; or may have so impaired the action of the heart-the fibres of which have become organically altered, degenerated-as to prove fatal, notwithstanding all rational treatment. The treatment calculated to overcome anæmia consists in allowing nutritious diet, carefully selected, and of easy digestion: for horses—oats, beans, crushed linseed, the latter in small quantities, and good hay; for cattle and sheep-an allowance of oilcake in addition to their ordinary fodder, which should be of good quality, and sufficient but not over-abundant in quantity; in addition to which the salts of iron are to be given in small and repeated doses. If the digestion be weak and the appetite bad, the iron is to be withheld, the vegetable tonics and the mineral acids given. Should the appetite be depraved, or if there be an unnaturally acid condition of the juices of the stomach, manifested by a tendency on the part of the animal to lick the walls and grind its teeth, with sourness of the mouth and fœtor of the fxces, the alkalies are to be com- 
bined with the vegetable bitters. For the constipation, which is due to debility of the muscular walls, nux vomica is very useful, mashes being now and then allowed. To the food above prescribed, it may be necessary in aggravated cases to allow the animal to have a quantity of milk twice a day-say one gallon of skimmed milk night and morning, mixed with oatmeal or porridge; this it will usually become very fond of in a day or two. The system of forcing food, by horning it, upon animals is not to be recommended; for what an animal does not eat spontaneously is not digested, but disorders the digestive system, and destroys any little appetite that may possibly remain.

Horses, during the spring and autumn, when casting their coats, suffer more or less from anæmia and debility, and are incapable of performing the same amount of work as at other seasons of the year. A little rest and gentle treatment at this period often prevent the accession of disease. Again, horses whose coats are very long, if kept in stables of even ordinary warmth, suffer from anæmia, with anasarca, and even dropsy of the serous cavities, which will increase notwithstanding the most powerful tonics and the most nutritious food, until their heavy coats are removed by clipping. I have seen the lives of many valuable horses saved by timely clipping.

In all anæmic cases the secretory organs-the kidneys, skin, \&c.- - are very apt to become torpid ; the urine is scantily secreted, the skin dry and harsh, the animal hide-bound, as it is termed. These complications must not be overlooked, or the blood will become loaded with effete materials. To stimulate the kidneys the spirits of nitrous ether may be given occasionally, and to excite the skin nothing is to be compared to good grooming, particularly damp wisping the whole surface of the body. Warmth, comfort, gentle exercise, and pure air all play an important part in the treatment, and should by no means be neglected.

The changes and alterations in the several constituents of the anæmic blood are briefly as follows:-(1.) The red corpuscles are remarkably diminished; they have been known in the human being to sink as low as 27; they also appear to contain less hrmatin, and are somewhat paler than those of healthy blood. (2.) The amount of white corpuscles does not appear to be altered. (3.) The fibrin, or the constituents by the interaction of which the fibrin is formed, is never found below the normal standard, 
in some cases its quantity is notably increased, particularly if inflammation of any organ is present. (4.) The solids of the serum are not found much altered. (5.) The quantity of water is more or less increased in proportion to the diminution of the globules.

\section{LEUKAMIA OR LEUCOCYTHEMIA}

Is a condition of the blood characterised by a large increase in the number of the white corpuscles, associated with a new formation of lymphatic tissue (lymphadenoma) in the lymphatic glands, spleen, and other organs; but the association of leukæmia with lymphadenoma is not constant.

An increase of white corpuscles is also witnessed in some cases of farcy and glanders; but the true nature of leukæmia seems to depend more upon an inability in the white and lymph corpuscles to form red blood globules, than upon any actual growth of lymphatic tissue.

POLY EMIA, PLETHORA.

The possibility of there being at any time an increaser quantity of blood in the animal body-plethora-is denied by some observers. The condition, however, is recognised by veterinarians as one of not infrequent occurrence, more especially in young fast-thriving animals, when fed upon very highly nutritious food. The occurrence of a general plethoric condition is also recognised by Sir Thomas Watson, who says"Perhaps it may not be so obvious that the whole quantity of blood throughout the body is sometimes in excess.

Full living and a sedentary life are causes likely to occasion general plethora, and they do occasion it. The full diet, so long as the digestive powers are perfect, provicles more chyle, conducts into the blood a larger quantity of its proper pabulum.

Their entire vascular system is preternaturally distended. If you open a vein you find that they [persons in a plethoric condition] bear a copious abstraction of blood without fainting, and are even refreshed by it; and the blood drawn separates into a large and firm mass of coagulum, with but little serum. Keeping to the nomenclature we have already employed, we might say that there is here hypertrophy of the blood."

The treatment of these cases is sufficiently obvious-abstraction of blood, purgatives, a restricted diet, and increased exercise. 


\section{CHAPTER X. \\ PATHOLOGY-(continued). \\ CHANGES IN THE BLOOD-(continued).}

DEFECTIVE EXCRETION.

Sir Thomas Watson, in his graphic language, says that "the animal fluids are-the blood, the fluids that enter the blood, and the fluids that proceed from the blood.

"The fluids that enter the blood are of two kinds-

" 1 . Those by which it is renewed and enriched.

"2. Those which enter it in order that they may be conveyed out of the body."

A defective condition of the fluids which enter the blood to renew and enrich it -namely, the clyyle-produces the condition already described-anæmia. A modified or altered condition of the chyle- that is to say, chyle imperfectly developed, containing abnormal constituents, products of imperfect digestion or elaboration, or directly derived from the ingesta-is a frequent source of alteration in the blood and of disease.

The fluids which enter the blood for the purpose of being conveyed out of it are the products of the metamorphosis of the chyle and tissue, accidental materials contained in the food, products of imperfect digestion, superabundant nutritive elements unappropriated by the tissue cells, and undergoing degradation within the blond-vessels, or in organs such as the liver. For the removal of these effete materials the organs of excretion are provided. When the organs of excretion are disordered or diseased, it follows that such produsts accumulate in the blood, contaminate and render it unfit for the proper nourish. ment of the body. 
The most remarkable and perhaps the most important change in the blood from defective excretion is that produced by arrested or deficient secretion of urine. In some experiments performed by MM. Prevost and Dumas, who removed the kidneys of dogs and cats, on the third day after the operation vomiting came on, with diarrhœa of a copious brown liquid containing ammonia, from transformation of the urea, eliminated from the intestinal mucous membrane; fever, with heat sometimes as high as $110^{\circ}$, sometimes as low as $92^{\circ}$; small and very frequent pulse, laboured breathing, and death, from the fifth to the winth day.

The post mortem appearances were, effusion of serum in the brain, much mucus in the bronchial tubes, bilious fluid and frces in the intestines, an appearance of inflammation of the liver, great contraction of the urinary bladder, the blood more watery than usual, and containing urea; five ounces of blood of a dog yielding twenty grains of urea, and two ounces of cat's blood ten grains.

Defective secretion of urine, resulting from disease of the kidneys, induces symptoms very similar to the above, differing in degree and intensity according to the extent of the kidney disease. Thus, in acute nephritis, or in rapid degeneration of the kidneys, such an amount of urea and other effete urinary matters may collect in the blood as to cause convulsions, delirium, suffocative discharge of mucus from the bronchi, diarrhœa, and serous effusion into the cavities and areolar tissue. In subacute eases dropsy gradually ensues, the animal finally becoming cachectic. All these effects may be traced to excrementitious materials retained in the blood, especially urea, which if in great amount acts as a narcotic poison, in smaller, as an irritant, inducing inflammations in various membranes; in the horse, more especially in the membranes of the spinal cord, and the neurilemma of the great nerves given off from the lumbosacral plexus.

TRANSFORMATION OF CHYLE AND OF THE TISSUES.

The blood is kept in its normal or healthy condition by being supplied with new elements derivable from the chyle, and by having the products of the metamorphosis of the tissue and all 
its effete constituents removed by the various excretory organs. When any of these conditions are absent, or when, as sometimes happens, the waste of tissue is so rapid as to preclude the elimination of all its products, a mal-condition of the blood-mass is induced, which may lead to grave local complications and diseases of important organs.

Some chemists hold the opinion that urea more especially is derivable from waste of tissue only, and not from any change or metamorphosis in the chyle or blood. I shall, however, endeavour to prove that an uræmic condition of the blood may be caused by chyle imperfect in quality or excessive in quantity; the imperfection of the quality being the result of disordered digestion, or of the ingestion of food unfitted for healthy chylification; and excessive in quantity from the animal being fecl upon aliment too rich for its requirements. In order to understand this, however, the student must be made still further acquainted with the process of mutrition, and this may be divided into-(1.) The introduction into the stomach and alimentary canal of the food to be digested: (2.) The formation of the chyle and blood, and the changes which the latter undergoes in the lungs: (3.) The passage of nutritive plasma from the blood to be transformed into tissues: (4.) The metamorphosis and re-absorption of the tissues: (5.) The excretion of these effete materials from the body by the various excretory organs.

I shall, however, confine myself, very briefly, first, to the digestion of the food, and the formation of the chyle; and second, to the changes and transformation of the chyle and blood in the vessels.

All kinds of food are resolvable into carbon, hydrogen, oxygen, nitrogen, and mineral constituents; and food to maintain the animal body in a state of health must contain all these various substances in due and proper proportions, and in quantities depending upon its necessities and wants under the circumstances in which it is placed, the amount of work it is compelled to perform, the amount of air it breathes, and upon various peculiarities affecting its powers of digestion, whether the animal be carnivorous, omnivorous, or herbivorous.

It is immaterial to what natural order the animal may belong, the proximate chemical principles required for its nourishment are the nitrogenous-albuminous, non-nitrogenous-starch, gum, 
sugar, oil, and the mineral salts, more particularly the phosphate of lime and the chloride of sodium, with iron, potash, \&c.; and it has been proved that all nutritive food must contain the three principles, and that the absence of any one of them induces starvation and death. It is also proved that the chemical constitution of plants and animals is nearly identical, and hence food derived from plants contains the substances of which the animal body is made, and by which it is nourished. Sonie animals are carnivorous, and must be maintained on flesh. In others life is supported by plants and grain. The digestive organs differ somewhat in these; but the digestion-that is to say, the ultimate end of the digestive process -is the same in all, namely, the formation of a whitish emulsion, the chyle, which is absorbed from the intestinal canal, and conveyed into the general circulation, there to be transmitted to all parts of the body, giving to each materials which it may at the time stand in need of for its developnent, growth, and vitality. In all animals water is also necessary, not only as a diluent, but as forming a component part of the biood and tissues.

It must be understood that mere nitrogenous, non-nitrogenous, and saline materials of food will not serve the purposes of healthy nutrition; they must be blended and united to form the kind of food natural to each class of animal, and capable of being finally converted into albumen, fat, and salts, as all these exist in every tissue; the fibrous and muscular organs containing much albumen, the glands and areolar tissue fat, and the bones mineral matters.

The quantity of food required is greatly influenced by the weather. If cold and condensed, the air contains more oxygen, which, rapidly uniting with the tissues for the purpose of maintaining the normal standard of animal heat, the body demands more nourishment to prevent emaciation. Again, work or severe bodily exercise causes waste of tissue, and at the same time, if not too severe, stimulates the appetite, and improves the powers of digestion. The demand for more food, the increaserl appetite, and the exalted digestive powers being necessary to the wellbeing of the animal, as compensating agents, by which more food is partaken of and digested, in order to make up the waste of tissue induced by the bodily exercise, a more or less even balance is thus kept up between the secondary digestion, 
or destructive process, and the primary digestion, or compensating process.

It is very true that a healthy animal will retain its appetite and be capable of partaking of food and digesting it for a considerable time without exercise, and, under certain circumstances, which sometimes seem inexplicable, remain in health. Generally, however, the excessive amount of nutriment is stored up amongst the fatty tissues in various organs; and the storage of fat may so far be looked upon as an act of excretion, bearing out the proposition of Treviranus, that " each single part of the body, in respect to its nutrition, stands to the whole body in the relation of an excreted substance," or, in other words, every part and tissue of the body, by taking from the blood the peculiar substance of which it is made, or which it can accommodate and store for future use, acts as an excretory organ, inasmuch as it removes from the blood that which, if retained, would be injurious to the rest of the body.

In many cases, however, this act of accommodation, as it may be termed, is insufficient; and in animals fed upon rich food, particularly nitrogenous food, the blood-mass becomes so impure, from the presence of effete materials, that the whole body is poisoned. For example-and this not at all uncommon-a horse is kept in the stable for some days, perhaps three or four days, and fed upon its usual liberal allowance of food, consisting of hay, corn, and water. When put to its work it usually commences its labour with the greatest spirit and animation, every nerve and muscle being, as it were, in the highest state of functional perfection; but before it has proceeded more than a few miles on the journey, very often before it has gone a mile, it suddenly staggers and falls paralysed. Sometimes it may be able to rise and walk slowly to its stable; sometimes it never rises again. Shortly after the attack it is observed to pass large quantities of a dark coffee-coloured urine, so dark, indeed, as to lead many observers to suppose that it is blood. Careful observations have, however, enabled me to determine that such urine contains no blood, and little or no albumen, but that the darkness of its colour depends upon an excessive quantity of colouring matter, urea, and probably other products of the degradation of the nutrient materials which had accumulated in the blood-mass during the period the animal was at rest. 
It has been maintained by some writers that urea is a product formed in the kidneys, and by others that it is an oxidized albuminous material, due to the degradation of muscular tissue, brought about by muscular exertion only, and merely excreted by the kidneys.

Without a doubt urea is an oxidized or degenerated condition of albumen, but the occurrence of the immense quantity of it in conditions of the animal body opposed to that of tissue change, I think, disposes of the idea that urea is due only to metamorphosis of tissue, and supports the theory that it is formed in the blood itself, or in the liver, as advocated by Meissner.

The rules for the proper treatment of disease depending on blood loaded with effete materials are-1st. To clear the intestinal canal of the materials with which it may be loaded, and from which additional matter may be absorbed and conveyed to the blood: $2 d$. To stimulate the powers of the excretory organs, kidneys, and skin: and $3 d$. To attend to the comfort of the patient, and particularly to supply it with abundance of water, which not only allays the thirst, but dilutes the blood and stimulates excretion.

\section{MELLITEMIA.}

The sugar in the blood is increased in certain diseased conditions-diabetes mellitus and in some cases of azoturia in the horse. It is then partly excreted by the kidneys. True diabetes mellitus is but rarely witnessed in horses, and perhaps never in cattle and sheep, but is not uncommon in dogs; and to replace the loss of sugar thus given there is an increased metabolism of the nitrogenous structures, and consequently the amount of urea is increased. There is loss of flesh, the glands atrophy, and the crystalline lens becomes opaque, and there being an increased amount of sugar in the humors of the eye, the water of the lens is extracted (Osmosis). 


\section{CHA P T ER XI.}

\section{P A T H O L O G Y-continued.}

\section{CIIANGES IN THE BLOOD-continued.}

\section{HYPER TEMIA OR CONGESTION.}

THE liability of the blood to be variously altered and modified having been described in the preceding chapter, it now becomes necessary to enter into a consideration of certain changes which may ocenr in its circulation, arising from an alteration in the condition and properties of the blood-vessels, and causing an interference with its natural flow.

By hyperemia is meant an excess of blood in the vessels of a part. It is of two kinds, namcly, active or arterial, and mechanical or venous.

Active hyperæmia is an excess of blood in the arteries, with, most commonly, an increased rapidity of the flow, and arises from increased blood pressure and from diminished arterial resistance.

Hyperemia from increased blood pressure occurs from interruption of the main current of blood in any particular part, owing to which increased pressure is thrown upon the collateral vessels, which thus become dilated, the amount of blogd in them being increased, and its flow accelerated. This kind of congestion is seen after the main current of a part has been obstructed from any cause, and is known as collateral hypercemia.

Congestion of the vessels of one part from an obstruction to the flow of blood in another, is also well exemplified in congestion of the internal organs from contraction of the superficial capillaries, so often produced by exposure to cold.

In some instances, the intropulsive operation of cold is so great that the internal capillaries become loaded with blood to such 
an extent that their walls give way, and the blood is extravasated into the surrounding tissue. In this manner apoplexy of the lungs is brought about, and so great is the extravasation in some rare instances, that the blood has found its way into the bronchi and trachea, and has been finally expelled through the nostrils. The local constringing effect of cold is best witnessed upon the human body, more especially in persons of weak circulation, in whom, very often after washing or bathing in cold water, the fingers are quite bloodless, pale, and numb. If we reason upon this circumstance, and bring such reasoning to bear upon our own subject, can we wonder that exposure to external cold is followed by internal congestions.

In many cases, however, nature provides a guard to the influence of cold upon the skin, by furnishing animals with coats of hair or wool suitable for their several necessities, and it is only in animals which are casually exposed to such cold that we witness its effect in causing internal congestions.

Congestion from diminished resistance arises from atony of the wall of the vessels. This atony, loss of power, or relaxation of the ressels, is generally due to paralysis of their muscular walls, and most commonly occurs in vessels least provided with muscular fibres, that is, in the capillaries and veins of organs easily distended, and which are composed of much areolar tissue. This paralysis of the muscular walls may be due to an influence operating upon the vaso-motor nerres, or to irritation of a sensory nerve. Pressure upon the great sympathetic nerve in the neck causes active congestion of the head and neck, and most probably the congestion of the brain, redness of the conjunctive, suffusion of tears, and other signs of fulness of the vessels of the facio-cranial region, with the coma and delirium which are sometimes associated with gastric or intestinal disease, are due to pressure upon the cardiac branches of the great sympathetic or ganglionic nerve. It is very true that some emotions are attended with hyperæmia; and it may be said that the meningeal or cerebral congestion of parturient apoplexy is due to some sympathetic influence which the stomach has upon the brain. The question need not be discussed here, as it will again come under consideration; and let it suffice us for the present to know that disorder of the stomach is capable of producing congestion of the brain. 
In hemiplegia, a disease rarely witnessed in the horse, but to which horned cattle are liable, congestion of the vessels of the paralysed side is a prominent symptom.

Atony of the arterial walls may be due to degeneration of their muscular and internal coats. It is most commonly the cause of the various congestions seen in old animals.

Mechanical congestion occurs chiefly in the veins, and is produced by anything which interferes with the flow of the blood through them, such as obstruction of the vein leading from a part, arising from pressure upon its walls, or the pressure of a thrombus or clot within its canal. Thus congestion may be induced by the pressure of a tight bandage; and again, congestion of the portal vein is caused by cirrhosis or other disease of the liver, which causes pressure upon its venous branches. Disease of the valves of the heart produces fulness of both the pulmonary and systemic veins. When the incompetency is limited to the mitral valve, the congestion is mostly limited to the lungs, while insufficiency of the tricuspid causes congestion of the systemic veins. The character of congestion beginning in the veins is that the veins as well as the capillaries are distended, giving the part an arborescent appearance, which has a much more permanent character than congestion beginning at the arteries. Arterial congestion, especially that of an active type, very often disappears shortly after death by the post mortem contraction of the vessels.

In addition to mechanical obstruction to the flow of blood, there are various and important causes of venous congestions, such as diminished cardiac power, gravitation, an altered condition of the blood, and defective secretions.

1. Diminished cardiac powcr.-The contractile power of the heart becomes diminished in many diseases, and death may result, not so much from the original disease from which the animal has been suffering, as from congestion of some vital organ, more especially the lungs or the brain, arising from the inability of the heart to propel the blood through the vessels of such organ. The example most familiar to the veterinarian of this form of congestion is acute congestion of the lungs caused by over-exertion. Many instances of this occur in the hunting-field during severe runs; horses out of condition, and unfit for very prolonged and severe galloping, falling down and rapidly sinking 
from this cause. This form of congestion becomos aggravated by the blood remaining unchanged, for it is found that when the blood is not properly changed in the lungs, its passage through them is partially impeded, that it accumulates in them, in the right side of the heart, and in the veins generally.

2. Gravitation.-The veins of the extremities and other depending parts of the body are congested from this cause. It indicates an atonic condition of the system generally, with diminished cardiac force; and is commonly associated with all the exhausting diseases of the horse, more especially if the animal is not exercised. Congestion from gravitation is also caused in many healthy horses by want of exercise. Indeed, a due amount of exercise, when in a condition to take it, seems to be of greater importance to the horse than to horned cattle. The cow or ox will remain in the stall for weeks, or even months, tied in one position and receiving no exercise, but the limbs never swell, and there are seldom any signs of venous congestions; not so with the great majority of horses. Now and then, however, a horse is met with whose limbs never swell from want of exercise, but as a rule horses when not exercised suffer from swellings of those depending parts of the body farthest removed from the centre of the circulation. Other horses habitually suffer from swelled legs, no matter how carefully and properly they are fed and worked. The debility produced by the process of "casting the coat" causes congestion of the vessels of the depending parts.

A very remarkable example of the effects of gravitation in producing congestion used to be frequently witnessed in the days when bleeding was so commonly performed, and when inflammation of the jugular vein was more frequently met with than now.

The loss of a jugular from the formation of a thrombus within it, and consequent obliteration of its canal, did not seem to interfere with the cervical circulation until the animal was turned out to grass; but immediately this was done, the animal's head would commence to swell to a most alarming extent, proving that, although the collateral circulation was sufficient, so long as the horse was fed from the rack or manger, it was quite insufficient when the head was depressed whilst grazing.

3. Altcred conditions of the blood.-Many congestive diseases, 
more especially those of horned cattle and sheep, are caused by an altered condition of the blood. How this determines a congestion of an organ or a system of organs it is difficult to understand; but such diseases as apoplexy of the spleen, quarter-ill, and other anthracoid diseases manifested by extensive congestions and extravasations, are all due primarily to blood alterations, the lesions of organs being secondary to and depending upon the blood disease. A thin watery state of the blood, as well as the presence of some specific blood poisons, and the operation of malarious influences, have also in many cases a tendency to cause congestions of organs.

4. Defeetive sceretion, either from an organ or a secretory surface, is a cause of congestion of such an organ or surface; and it is found that the means which increase the secretion will often remove the congestion. It must, however, be borne in mind that diminished secretion is usually the result of the congestion, and that the means which have the power of exciting the secretion of such a part very often, in virtue of their irritating effects upon it, increase the congestion, or, what is perhaps of greater moment, transform a mere congested condition into that of inflammation.

Many writers seem to confound congestion with inflammation, and we continually see slight inflammations described as congestions. It appears to me that a distinction should be made between the two conditions, and that a congestion should bo defined to be a fulness of the vessels of a part or parts arising from various causes, without the occurrence of any primary important perversion of the nutrition of that part; and that inflammation is a perverted condition of the nutritive function of a part, accompanied in the majority of cases by a congested condition of its vessels; such congestion, however, not being necessary to the inflammatory process, for it is found that inflammation involves the non-vascular as well as the vascular structures of the animal body.

The results of mechanical congestion are transudation of serum, hæmorrhage, thrombosis, and gangrene; and of active lyyperæmia, increased redness, elevation of temperature, and a sense of throbbing, succeeded, if the congestion continues, by enlargement of the small arteries and thickening of their walls; function heing in some cases exalted, more especially if the 
congestion be in a nervous centre, whilst in otler instances function becomes diminished. Thus a long-continued congestion of a secretory organ arrests, or very considerably diminishes, the function of that organ.

The congestion or fulness of the vessels is somewhat relieved by transudation of serum into the tissues, or into cavities, constituting œdema, anasarca, and dropsical effusion. For this reason the limbs swell when their vessels are congested from debility or want of exercise ; and the peritoneal cavity becomes filled with fluid, constituting ascites, when the portal veins are in any way obstructed. The transuded serum generally differs from that of the blood, in containing less of the solid blood constituents and more water; it has also a lower specific gravity. If exposed to the air it will sometimes coagulate, forming rather a gelatinous mass than a firm fibrinous clot.

When the obstruction is very great the serum partakes more of the nature of the blood plasma; and it is now ascertained that in some instances the blood globules may escape through the walls of the capillaries without rupture. This fact was dis. covered by Cohnheim, who, by applying a ligature to the femoral vein of the frog, observed, when the congestion of the vessels became very great, that the red corpuscles passed through the capillary walls, and in so doing assumed an hour-glass shape, or became constricted in their middle. This fact explains to some extent how, in some cases of purpura, transudation of blood takes place into the tissues without rupture of the vessels.

Congestion from obstruction producing gangrene is seen in strangulated hernia, and in invagination of a portion of the intestines. Gangrene from obstruction is always of the moist kind, whereas that from arterial incompetency is of the dry kind. In the one the blood supply is scarcely or not at all interfered with, but the return of that blood is arrested, stasis is produced, the blood thus imprisoned transudes into the tissues, and, rapidly dying and decomposing, adds to the rapidity of the death and decomposition of adjoining structures. In the other, or gangrene from want of blood, the part turns pale and dry, shrinks, becomes mummified, or crumbles into fragments.

The occurrence of thrombosis, or the formation of clots in the congested vessels, results from the blood coagulating within them during life, and may arise in congestion of any part, and 
from any of the causes already enumerated. When caused by direct pressure, the coagulation commences at the point where the obstructing cause comes into contact with the vessel, and extends as far as the collateral branches; when from debility, diminished cardiac power, or gravitation, the coagulation commences first behind the flaps of the valves, and appears to be owing to the force of the current being insufficient to completely open the valves. The blood thus allowed to lodge and stagnate coagulates and forms a thrombus or clot, which may finally obstruct the whole venous channel as far as the next large collateral branch.

Treatment of congestion.-The most important means for the removal of congestion are those which tend to remove its cause; and if the causes already enumerated are borne in mind, the practitioner will at least have some guide to the proper line of treatment. When the congestion is due to the influence of cold upon the surface, clearly the first step is to restore the circulation of the skin by warmth, friction, and by the administration of remedies, such as the spirits of nitrous ether, which are known to promote diaphoresis. If the congestion be due to diminished cardiac power, or debility of the vessels-and this is often induced by want of exercise-the lost power and tone must be restored by good food, pure air, regular and moderate exercise, and tonics. If the debility of the vessels be very great and obstinate, the effects of ergotine may be tried. This remedy acts by producing contraction of the blood-vessels; it may be very conveniently used hypodermically. The effects of bandaging, in supporting the weakened vessels of congested limbs, should not be lost sight of, nor its application neglected. In many instances a watery condition of the blood is associated with debility of the circulatory apparatus : in such cases tonic remedies combined with diuretics act most favourably.

In severe internal congestions, where not only the organ chiefly affected is filled with blood, but the right side of the heart and the great veius also, the necessity of affording mechanical relief becomes a serious question, and very often an urgent necessity. It is very true that severe internal congestions, more especially those of the lungs and brain, are generally associated with greatly diminished cardiac power, feeble pulse, and nervous prostration; and if we were to admit what is so commonly in- 
sisted upon, that the course of treatment is to be guided by the condition of the pulse, then the withdrawal of blood would be contra-indicated in every instance. But practitioners who have thought over the matter know full well that a moderate bleeding is followed by a diminished acceleration of the pulse, and improvement in its tone, and that many congestions are immediately relieved by such procedure. The explanation is not difficult. In cerebral congestions the diminished cardiac power and feebleness of the pulsations are caused by the pressure of the congested vessels upon the brain substance: in many instances this pressure is increased by the transudation of serum from such congested vessels. By diminishing the brain pressure by the abstraction of blood, the cause of the debility is at once removed. The heart is now able to beat, fully and strongly, and thus a small, feeble, or even thready pulse is changed into a full, soft pulsation. The variety of opinions held as to the possibility of causing an alteration of the fluids within the cranium by bleeding or otherwise, need not be discussed at present. My own experience leads me to the conclusion that a comatose condition produced by cerebral or cerebro-meningeal congestion is often removed, the pulse being at the same time improved in tone, by a moderate bleeding. Pulmonary congestion and the feebleness of the heart's actionthe "oppressed action" as it has been termed-are aggravated by the right side of the heart and the great veins being choked with blood. In this instance, it is true, the withdrawal of blood does not relieve by removing the primary cause-that is, the congestion of the pulmonary capillaries-but, a most important complication, it diminishes the supply of blood to the already overloaded heart, and through it to the already congested vessels. Bearing this in mind, the rapidity with which the withdrawal of a large stream of blood produces an impression will be understood; indeed, it can most truly be stated that the relief afforded does not depend so much upon the quantity withdrawn, as upon the rapidity of the withdrawal. For example, six or eight quarts of blood might be abstracted in a small or trickling stream, and afford no relief, but, on the contrary, do much harm, by causing debility or exhaustion; but a smaller quantity, if withdrawn rapidly, may be followed by a marked remission of all the distressing symptoms. In the one case, the blood supply t 
the heart within a given time has scarcely been interfered with, while, in the other, the quantity returned by the veins has been most sensibly diminished, time has been afforded to the congested vessels to become to some degree emptied, and regain their lost tone, and for the circulation generally to be restored to its equilibrium. In these congestions the administration of diffusible stimulants after the bleeding will still further restore the lost power of the propulsive force and of the debilitated walls of the vessels ; and, in virtue of their exalting action, will diminish the internal congestion, by causing an increased circulation of the vessels of the slin and excretory organs.

\section{LIPAMIA, OR FATTY BLOOD.}

In February 1882 I was consulted by a gentleman living in the West Highlands regarding a fatal disease which liad appeared amongst his ewes, mostly among gimmers.

The history of the outbreak was very meagre, and was from the farm manager, who stated-

"The appearance when first attacked, or rather when we notice them, is that they do not follow the flock, and, when walking, propping their legs as appearing to keep them from falling, which they often do, always going down head first.

"Their droppings not at all natural, and mixed with a slimy mixture of blood. They live from three to four days after we detect them ill.

"Their food for the last three months, turnips carted out on grass land, with an allowance of lialf a pound of Indian corn and cotton cake mixed per day.

"I may add that they were turned into the turnip fields to pick up broken and small turnips, but were only there about three weeks, during which time they had full turnips, and looked the very picture of health.

"Mostly all the dead ewes when opened have shown a great deficiency of blood."

The proprietor in a short note informed me that "the only treatment that has been attempted is giving a close of castor oil, and that some of those showing symptoms of disease improved since the giving of cotton cake was discontinued." 
These letters were accompanied by the carcase of a sheep of the Shropshire Down breed in an advanced state of decomposition.

On a post mortem examination being made the whole body was found to be loaded with fat; that of the abdominal parietes being several inches in thickness; the sublumbar region had many pounds of fat in it, and here it was found that the interior of the fatty masses had undergone crystallisation; well-formed crystals of stearine and margarine being found in the midst of a dry kind of clébris.

The structures of the liver and of the heart were not only thoroughly infiltrated with oil globules, but had undergone fatty degeneration to an advanced degree; the lungs were congested, and the large pulmonary vessels filled with thrombi ; the cardiac ventricles were quite full of blood, showing that death had been induced by resthenia and pulmonary congestion.

Microscopically examined, the blood (which was pale in colour when seen with the naked eye) from the heart, pulmonary vessels, and from other parts, was found to be loaded with oil globules, as represented in the drawing, which was made on the spot.

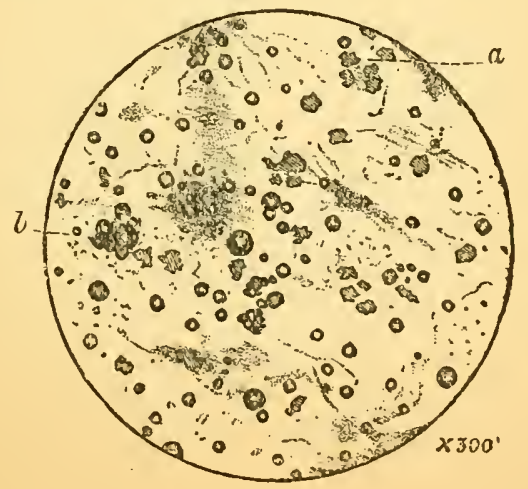

FIG. 2A. - (a) Blood corpuscles altered in shape. (b) Oil globules, isolated and in groups, scattered about the field.

It will be seen that the oil globules vary in size, some being smaller, some much larger than the red blood corpuscles. It was unfortunately, however, impossible to prepare microscopic slides 
of the blood and tissue owing to the advancing lecomposition; but notwithstanding this, the fat gave the usual reactions with perosmic acid and other staining materials.

I am not aware that this disease has ever been observed by any veterinary writer, and it was only in 1874 that Professor Kussmaul first drew attention to it in the Deutsches Archiv für Klinischc, as causing a remarkable mode of death in diabetes mellitus of the human being; the fatal symptoms consisting in a peculiar kind of dyspnœa, which after a time was accompanied by and ended in coma.

In 1879, the late Professor Sanders, and D. J. Hamilton, M.B., Edinburgh, investigated two cases after death from diabetes, and found a condition of the blood similar to that described above.

Death from lipæmia has also been observed in cats. which have suffered from exhaustive diseases, accompanied by great emaciation, but no cases have hitherto been recorded in which this condition of the blood has accompanied obesity of the body generally.

Fatty embolism of the pulmonary artery is a cause of death in some cases of compound fracture of the long bones in man, and is accounted for by the fact that the medulla of the osseous canal gains entrance into the ruptured blood-vessels, and is thus mixed up with the general circulation, becoming arrested in its passage through the pulmonary vessels, and there forming a block to the pulmonary circulation, which finally effectually stops the aëration of the blood, and causes death by apnœa.

On comparing the fat condition of the body of this sheep to the emaciation of diabetic subjects, one cannot help being struck with the contrast between the two extremes. It is certainly difficult to answer the question, how is the fat produced in the blood of diabetic patients? But in this sheep the conclusion seems self-evident: that we had an animal which (along with others) had been fed up to such a point that it could no longer either assimilate, utilise, or excrete the lighly nutritious matters contained in its food.

The Shropshire Down breed of sheep are noted for their fattening properties, and in this case we had an example of an animal having been fed beyond the point of perfection. The winter of $1881-2$ was a remarkably open one; grass was plentiful 
throughout the season, and, in addition to an abundance of grass, these sheep had a full allowance of turnips, besides corn and cotton cake. This rich food, the quantity of which, however, does not strike one as being outrageously great, given in addition to the turnips and grass to animals whose fattening qualities is so well known, was evidently too much. After feeding upon it, the sheep would have little or no inclination to roam about; indeed, grass being abundant, there would be no necessity for them to travel far in search of food; fat was consequently so rapidly formed that all the tissues of the body become loaded with it, and finally the blood, where it caused an obstruction to the circulation, and thus induced death.

\section{SEPTICEMIA-PROGRESSIVE GANGRENE.}

Definition.-A condition produced by the absorption or introduction of putrescent matter into the system, characterised by great prostration, metastatic lameness, colicky pains, with purging, the freces being fœtid, fœtor of the breath, and rapid emaciation in the horse. In horned cattle and sheep it is generally seen after parturition, and manifested by great prostration, collapse, fluttering pulse, expulsion of a coffee coloured fluid from the uterus, violent straining, purging, and great restlessness; also known as braxy, affecting sheep in their first year (hoggs), and occurring in the late autumn and early winter months, especially when the weather is cold, and the grass covered with hoar frost. It is also very destructive to lambs, and to calves a few clays old.

These varying forms of septicæmia will be found described under various heads in the context of this work, as well as in the Prineiples and Practice of Veterinary Surgery.

The artificial induction of Septic mula has been the subject of various experiments-first of all by M. Davaine, and afterwards by M. Bouley, Director of the Alfort Veterinary School. The experiments were as follows:- Six watch-glasses were placed on a table. Into the first 100 drops of water were placed, and to these was added one drop of blood from a septicæmic rabbit; the whole was stirred so as to produce a solution of $\frac{1}{100}$. One drop of this was placed in the second glass containing 100 drops of 
water, and a dilution of $\frac{1}{10,000}$ produced. In the third glass, a

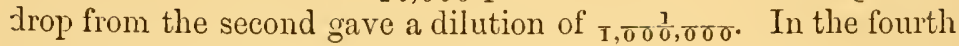
glass a drop of third dilution added to the 100 drops of water

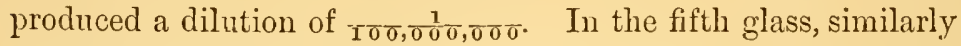

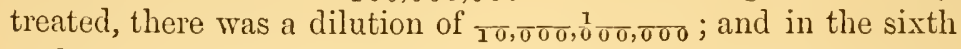
a drop of the dilution gave a trillionth $\left(\overline{1}, \overline{0} \overline{00}, \frac{1}{000, \overline{000}, 000}\right)$. Matters being so disposed, four rabbits were inoculated respectively with the first, second, third, and fourth dilutions, a horse with the second, and a guinea-pig with the first. Next day all the rabbits were dead, the guinea-pig fell ill, but recovered, and the horse sustained no harm. M. Bouley communicated an account to the Académie de Medicine of numerous experiments he had made confirming the above, and stated that dogs can also be killed by the septicæmic virus derived from the rabbit, and that such virus derived from the horse possesses much less virulence than that furnished by the rabbit.

I have repeatedly experimented on birds and rabbits with the undiluted blood of pyæmic-septicæmic (Vogel)—rabbits and birds, and have always induced death, either by a rapid corruption of the whole blood-mass, from which the animals have died in a few hours, or, resisting the first effects of the morbid inoculated material, from pyæmia with abscesses in various internal organs.

Selecting mice as being specially adapted for experiments on infective diseases, Koch has made some remarkable discoveries pointing to the conclusion that death may be induced by (a) a soluble poison, "Sepsin ;" by (b) a true septicemia induced by the multiplication of the bacteria introduced by the inoculation; and by $(c)$ a progressive destruction of tissue (gangrene), commencing at the point of inoculation and spreading rapidly to adjacent parts.

Blood or meat infusion, which have putrefied for a short time, act more injuriously than when putrefaction has extended over a longer period, and five drops of such blood, if injected under the skin of the back of a mouse, kills it within a short time. There are marked symptoms of illness immediately after the injection. The mouse becomes restless, runs about constantly, but showing great weakness and uncertainty in all its movements; it refuses food, the respiration becomes irregular and slow, and death takes place in from four to eight hours. 
In such a case the greater part of the injected fluid is found in the subcutaneous cellular tissue of the back in much the same condition as before it was injected. It contains bacteria of the most diverse forms, irregularly mixed together, and as numerous as when examined before injection. No inflammation can be observed in the neighbourhood of the place of injection. The internal organs are also unaltered. If blood from the right auricle be introduced into another mouse, no effect is produced. Bacteria cannot be found in any of the internal organs, nor in the blood of the heart.

Koch therefore concludes that an infective disease has not been produced as the result of the inoculation, but that the death of the animal is due to a soluble poison-Sepsin-which has been shown by the researches of Bergmann, Panum, and others to exist in putrid blood. This supposition is confirmed by the fact that when less fluid is injected the symptoms of poisoning which follow are less marked, and are quite absent when one or at most two drops have been injected. After the use of such small quantities mice often remain quite well, but a third of them, on an average, become ill after the lapse of abont twenty-four hours. The less amount of putrid fluid injected, the fewer mice become affected, but less than one drop is sometimes sufficient; thus, of twelve inoculated with one-twentieth to one-tenth of a drop each, only one was successfully infected.

The first symptoms of the success of the inoculation with this minute quantity is an increased secretion from the conjunctiva. The eyes appear dull, and a whitish mucus collects between the lids, and finally glues them together. At the same time lassitude sets in, the animal moves little and languidly; as a rule it sits quite still, with its back much bent and its extremities closely drawn up. It then ceases to eat; its respirations become slower; weakness gradually increases, and death comes on almost imperceptibly. Convulsions never precede it (they always do so in anthrax). After death the animal still remains in the sitting posture with its back strongly bent; whilst a mouse which dies from authrax is always lying on its back or sicle, with its stiffened limbs fully extended. Thus, by the position of the body after death, a fatal result produced by the inoculation of putrefying blood is at once distinguished from that occasioned by inoculation with the material of anthrax. 
On post mortem examination there is found at the place of injection or inoculation slight œdema of the subcutaneous cellular tissue. This, however, is often absent, and the internal organs, with the exception of a considerable swelling of the spleen, appear quite unaltered. One-tenth of a drop of the fluid of the subcutaneous cedema, or of blood from the heart of such animal, inoculated into another mouse, is sufficient to cause exactly the same symptoms, and death in about fifty hours. From this second animal a third may be injected, and so on through as many successive animals as one pleases.

Koch says that the certainty with which the infective material can be carried from one mouse to another is greater than in anthrax, as in the latter the material for inoculation must be taken from the spleen, because the blood of mice affected with anthrax oftell contains very few bacilli; but in septicrmia, produced by putrefying blood, it is a matter of indifference from which organ the material for inoculation is taken, and even the smallest quantity will produce an effect with certainty.

The blood of unice which became ill after intravenous injcetion of one to ten drops of putrefying blood was found to contain, as a rule, different varieties of bacteria in small numbers, micrococci, and large and small bacilli. If, however, the animals died after inoculation with putrefying or septicrmic blood, small bacilli alone appeared. This result was invariable, and the bacilli were in large numbers.

These bacilli are often attached to each other in septicæmic blood in pairs, either in straight lines or forming an obtuse angle. Chains of three or four bacilli also occur, but they are rare. They show at first sight a great resemblance to small needle-like crystals, but that they are undoubtedly vegetable bodies is evident, for when septicæmic blood is kept in an incubation apparatus, these grow in a manner similar to the bacilli of anthrax, not forming long threads like the latter, but dense masses, which consist of isolated bacilli sometimes containing. spores. They are extremely difficult to see, and it is necessary to stain thein with methylaniline violet or blue. The best way to do this is to dry the fluid containing them on a glass slide, stain with methylaniline, and mount the specimen in Canada balsam.

The relation of these bacilli to white corpuscles of the blood is peculiar. They penetrate into these and multiply in their 
interior, and it is often found that there is hardly a single white corpuscle in the interior of which bacilli cannot be seen.

Disease induced by the inoculation of the blood of septicæmic mice cannot so successfully be induced in so many different animals as with that of anthrax. Thus Koch failed to cause it by inoculating rabbits, and even field mice resisted the inoculations.

Occurring along with the septicæmic bacillus, Koch has found in mice, after the introduction of putrefying blood, a micrococcus in the neighbourhood of the place of injection. This organism is of rapid growth, and forms very regular chains. As a rule, when an animal dies of septicemia after about two days, none of the numerous forms of bacteria which were injected with the putrid blood can be discovered, except the septicrmic bacilli, or it may be a few residual specimens growing with difficulty. It must therefore be supposed that none of the other bacteria injected at the same time find in the body of the living mouse a suitable soil, and that they therefore die more or less quickly. Koch's attention was therefore arrested when he found in some cases, growing at and about the inoculated spot, micrococci in unusual abundance and of a constantly characteristic form. They were not present in the blood, and by inoculation with the blood the septicæmic bacilli alone were transmitted. In order to test whether they could be inoculated, it was necessary that the material should be taken from the place of injection. Inoculations carried out in this way were successful in producing both forms of disease, and the virulence of the serum from the subcutaneous cellular tissue containing these micrococci was just as marked as that of the septicæmic blood. When the point of a knife which had been well cleaned was merely brought in contact with the subcutaneous tissue at a spot about one centimetre and a half from the place of injection or inoculation, and when with this knife another animal was immediately inoculated, the inoculation was successful on evcry occasion. Septicæmia was of course always produced at the same time, because the serum used contained also septicæmic bacilli. The influence of these micrococci on animal tissues, and their mode of spreading, can be best traced on the ear of a mouse, and it is specially instructive to compare an ear on which only septicæmic bacilli have been inoculated with one 
into which both the bacilli and the chain-like micrococci have been introduced. In the former the cellular tissue is full of red corpuscles and lymph cells, so that the bacilli can often be recognised only with great difficulty among the numerous cell nuclei. The other ear presents totally different appearances. Spreading from the place of inoculation, one can see extremely delicate and regular micrococcus chains, here pressed together so as to form thick masses, traceable ahmost to the base of the ear, all the tissues of the part occupied by them being remarkably altered. As far as the micrococci extend, neither red blood globules nor nuclei of lymph or of connective tissue cells can be seen. Even the extremely resistant cartilage cells, and the plasma cells so richly present in the mouse's ear, and which are likewise characterised by great resisting power, are pale and scarcely recognisable. All the constituents of the tissue look as if they had been treated with caustic potash; they are dead, they have become gangrenous. Under these circumstances the bacteria develop all the more vigorously; and the micrococci penetrate in numbers into the damaged blood and lymphatic vessels, and here and there they fill them so completely that the vessels appear as if injected. Among these the septicæmic bacilli, no longer obscured by nuclei, are seen very distinctly in small groups, which are at times very dense, and can be traced up to the root of the ear, and indeed beyond it, having at the same time increased enormously in the blood, and ultimately cansing the death of the animals. The micrococci, on the other hand, and the destructive process associated with them, have only extended during the same time (about fifty hours) as far as the vicinity of the root of the ear, where their limit is sharply defined. The appearances presented by the ear on a post mortem examination lead to the conclusion that the action of these micrococci in causing gangrene is somewhat as follows:-Introduced by inoculation into living animal tissues, they multiply, and as a part of their vegetative process they excrete soluble substances, which get into the surrounding tissues by diffusion. When greatly concentrated, as in the neighbourhood of the micrococci, this product of the organisms has such a deleterious action on the tissue cells that they perish and finally completely disappear. At a 
greater distance from the micrococci the poison becomes more diluted and acts less deleteriously, only producing inflammation and accumulation of leucocytes. Thus it happens that the micrococci are always found in the gangrenous tissue, and that in extending they are preceded by a wall of nuclei, which constantly melts down on the side directed towards them, while on the opposite side it is as constantly renewed by deposition of fresh lencocytes.

These observations refer to inoculations with fluid containing both micrococci and bacteria, and it might have been supposed that the septicxmic bacteria were necessary forerumers of the micrococci, and must to a certain extent prepare the way for them.

Koch attempted by various means to separate them from each other, but for a long time with no avail. But chance led him to the proper method. A field mouse, which he had already found out possessed an immunity from septicæmia, was inoculated with bacteria and chain-like micrococci. The experiment was made in the expectation that neither parasite would develop: This expectation, however, was not fulfilled, for, thongh the rods, as usual, underwent no development, the rnicrococci increased and spread in exactly the same manner as had been the case with the domestic mouse.

Beginning at the point of inoculation in the root of the tail, the gangrene spread onwards along the back, passing deeply among the dorsal muscles, and downward on both sides to the abdominal wall. The animal died three days after inoculation. The parts affected with the gangrene were partially denuded of epidermis and hair, and contained chain-like micrococci in extraordinary numbers. The same micrococei were also found on the surface of the abdominal organs, although there was no visible peritonitis. The blood and the interior of the organs were, on the other hand, quite free from them. From this animal other field mice; and from these again domestic mice in various successive series, were subsequently injected, and with always the like result-namely, that only chain-like micrococci, and in their train progressive gangrene, were obtained.

The development of bacteria even in a suitable soil can be checked by certain influences, physical, biological, and chemical, and of these none are more potent than temperature. No fully 
developed bacteria have as yet been found to survive after long exposure to $60^{\circ} \mathrm{C}$. when in the moist state, and but few resist a temperature $50^{\circ} \mathrm{C} .=112^{\circ} \mathrm{F}$.

The spores of bacteria, however, can resist a much higher temperature than that which destroys the adult organism, and all agree that the boiling-point of water is the lowest which can be relied upon to destroy spores; but in the dried state they are much less readily destroyed, requiring three hour's' exposure to a temperature of $140^{\circ} \mathrm{C} .=284^{\circ} \mathrm{F}$.

Again, intense motion and sunlight checks bacterial life, but electricity seems to have no effect upon them.

of the chemical influences which destroy or retard bacterial life the most powerful is corrosive sublimate, its action being instantaneous even in a dilution of one part to five thousand. Next to the bichloride are placed bromine, iodine, and chlorine; but carbolic acid is not so reliable, as nothing less than a three per cent. solution destroys spores, and requiring at least seven days, and a five per cent. solution requiring from twenty-four to fortyeight hours. When dissolved in oil, Koch says it is harmless both to spores and developed bacteria. When immersed in it in the dried state and dissolved in alcohol, it is also inert.

The only other agents which can be depended upon to destroy microbic life in less than twenty-four hours are permanganate of potash in fire per cent., and osmic acid one per cent. solution. A five per cent. solution of chloride of iron destroys within six days, hut neither sulphate of copper nor chloride of zinc seem to have any effect. 


\title{
CHAPTER XII.
}

\section{PA TH O L O G Y-continued.}

\author{
MODES OF DEATH.
}

Following the example of Dr. Williams, Sir Thomas Watson, and other writers upon medicine, I purpose very briefly to consider the chief varieties of the modes of death.

An inquiry into this subject, says Watson, "is not one of merely curious interest, but has a direct bearing upon the proper treatment of clisease. It will teach us what we have to guard against, what we must strive to avert in different cases."

A continuance of "life is inseparably connected with the continued circulation of the blood. So long as the circulation goes on, life, organic life at least, remains. When the blood no longer circulates, life is presently extinct; and our investigation of the different modes of dying resolves itself into an investigation of the different ways in which the circulation of the blood may be brought permanently to a stand.

"Observe the ample provision that is made in the construction of the body for carrying on and maintaining this essential function. First, there is an extensive hydraulic apparatus distributed throughout the frame, consisting of the heart and other blood-vessels. Next, there is a large pneumatic machine, forming a considerable part of the whole body, composed of the lungs and the case in which they are lodged. Lastly, the power by which this machine is to be worked and regulated is vested in the nervous system. Each of these systems must continue in action, or the circulation will stop, and life will cease. The functions they respectively perform are consequently called vital functions; and their main organs -the heart, the lungs, the brain-are denominated vital organs. 
The functions of any one of the three being arrested, the functions of the other two are also speedily extinguished."(Watson's Principles and Practice of Physic.)

Death, it is observed, occurs as follows:-

1. Death beginning at the heart-syneope--asthenia.

2

3 . "

$4 . "$

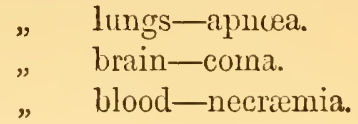

DEATH BEGINNING AT THE IIEART.

Death beginning at the heart may oceur in two ways:-1st. Suddenly, or by syncope; and $2 d$. By a gradual cessation of the heart's action. Syncope may oecur in two ways:-1st. By the heart losing its irritability, so that it eeases to contractasthenia; and $2 d$. By tonic spasm, in which it remains rigidly contraeted. Death from tonic spasm of the heart is rare amongst the lower animals. I have, however, seen one instance, where a valuable horse died from this cause in eonsequence of a sudden fright.

In both these cases denth is instantaneous. In the first case, each ehamber of the heart is found after death to be filled with its proper kind of blood, upon which it has been unable to contract. Sir Benjamin Brodie, in some experiments recorded in the Philosophical Transactions for 1811 and 1812, found that when death was oecasioned by the upas antiar, that the heart was full after leath, with purple blood in its rigltt and scarlet blood in its left cavities. This proves that the heart has ceased to contract upon the contained blood. In the second case, the heart appears very small and hard, the ventricles, partieularly the left, firmly eontracted and containing no blood, and the heart substance very firm. 'This state of the heart was supposed to depend upon concentrie lypertrophy, but its true nature was pointed out by Cruveilhier and Dr. G. Budd. Should any doubt remain as to its true nature, if the heart be kept for a few days, it will, if the eondition be that of spasm, lose this tonicity, and may easily be restored to its regular dimension by a little manipulation. Both conditions of the heart-namely, loss of irritability and tonie spasm-may arise from similar causes. Shock, for example, may produce the one or the other 
condition; and wounds of the heart are sometimes followed by paralysis, sometimes by rigid contraction of that organ. In sudden death from drinking very cold water when the body is heated, the heart has been found contracted.

In the slower modes of death beginning at the heart, it is found that the same conditions exist after death. In death from hæmorrhage-anæmia-it is found that the heart is firmly contracted, if the animal be examined shortly afterwards; the circulation failing, not because the heart has lost its power of contraction, but because blood does not arrive in sufficient quantities in its cavities. That the heart does not lose its power of contraction in such cases is proved by the transfusion of blood from an animal into the veins of a human being or other animal apparently dead from hrmorrhage, where it has been found that animation may thus be restored. "It is quite clear," says Watson, "that this introduction of fresh blood could be of no avail in a case where the heart was unable to act upon the blood which had already reached it."

Approaching death from hæmorrhage is manifested by great and rapidly increasing pallor of the mucous membranes, coldness of the mouth, surface of the body, and extremities, cold sweats, a dazed or dim appearance of the eyes, convulsive struggles-if the animal is down, at first strong but rapidly becoming feeble, if standing, the animal will balance its body upon the extremities; the pulse feeble, irregular-sometimes slow, sometimes fast-cold breath, convulsive breathing, dilatation of the pupils, and very often just before death the animal gives a loud scream or delirious neigh.

In another form of gradual death beginning at the heart, it is found that the heart has gradually lost its power of contractility. This is the most common mode by which deatl occurs, and is the termination of many exhausting diseases, especially those which destroy the strength without directly interfering with the more vital functions, such as influenza, diarrhœe, diabetes, fevers, as well as inanition from want of food, or other causes which reduce the powcrs of the whole body.

The two conditions of the heart found after death beginning at that organ may each be brought about in two ways:-1st. Suddenly, as in instantaneous death from shock or great violence; and $2 d$. When death, although it may be said to occur suddenly, 
presents some symptoms of its approach, however brief those symptoms may be, as from hæmorrhage, the operation of some poisons, as aconite, hydrocyanic acid, upas antiar, tobacco; from lightning, electricity, and various animal poisons, as suake-bites; the operation of debilitating influences, as well as diseases which tend to weaken and paralyze muscular force, and to produce asthenia.

In death from gradual asthenia the pulse becomes very feeble, frequent, and often irregular; the respirations feeble, sometimes sighing; the muscular debility extreme. Pallor of the membranes comes on gradually, or in some cases they may present an unhealthy reddened or brownish-red appearance from the blood remaining in the veins, the extremities become cold, and œdematous.

In various wasting diseases, such as pleuro-pneumonia-bovina, diarrhœa, polyuria, or any disease which more gradually exhausts the power's of life, death is cansed by anæmia and asthenia. The blood gradually diminishes in quantity and deteriorates in quality, therefore the supply to the substance of the heart hecomes diminished, and in a condition unsuited to nourish the heart properly. The power of the heart, as well as of the muscles generally, is gradually lesseued, and at leugth ceases altogether; and when death is so produced the heart is not contracted and empty as in death from anæmia, nor so full and engorged as in death from asthenia. This form of death is called by Watson death by inanition, and its typical form is that occurring from starvation.

\section{DEATII BEGINNING AT THE LUNGS.}

By this it is meant that the function of breathing is the first to fail, improperly termed death from asphyxia-literally pulselessness-more correctly termed death by apnoca, privation of breath, or suffocation.

This form of death is caused by diseases of the lungs or bronchial tubes, pleurisy terminating in hydrothorax, where air is prevented from entering the lungs by the pressure of the pleuritic fluid, mucus, pus, or blood, filling the tubes and air cells; by laryngitis, spasm of the glottis, or when tumours press upon the larynx or trachea; by œdema of the glottis; diseases of the 
heart affecting the quantity of blood in the lungs and great thoracic vessels; strangulation; drowning; pressure upon the diaphragm, forcing it forward and preventing admission of air into the lungs, as in severe tympanitis of the stomach and bowels, or from any circumstance which may paralyze the diaphragm-fracture or dislocation of the upper cervical vertebrae, and pithing, for example-or obstruct the passage of air through the nostrils, larynx, trachea, or bronchial tubes.

Sudden deatl from apnœa is not often witnessed as the result of disease, but is generally brought about by accident or design, accidentally when the upper cervical vertebræ are fractured or displaced; in cases of parturient apoplexy, when fluids gain entrance into the lungs, and from impaction of foreign bodies, or polypi in the larynx; and designedly, when an animal is pithed. Death is also thus produced by the entrance of air into the veins.-(See Prineiples and Practice of Veterinary Surgery.)

Death from apncea takes place most commonly from the supply of air being gradually cut off by morljid changes in the respiratory organs, and is often accompanied by asthenia and coma; but generally the symptoms belonging to apnœa are plainly predominant. In death, when the passage of air into the lungs is arrested suddenly and completely, it has been observed that the muscles of respiration exhibit strong and violent contractions: that the efforts to breathe are very great, struggling but ineffectual, and very distressing. This extreme distress, however, soon passes away, and is succeeded by vertigo, stupor, loss of consciousness, and convulsions, till at length all efforts cease, except a few irregular twitchings of the limbs; the muscles then relax, the sphincters yield, but even then the movements of the heart and the pulse continue for a short time after all signs of life are gone. The other signs of this method of death are congestion and lividity of the visible mucous membranes, a full, staring eye, protrusion of the nose, dilatation of the nostrils, and sometimes flapping of them. When it arises from inflammation or spasm of the muscles of the larynx, tumours, abscesses, or obstruction in the trachea, there will be a loud, roaring, inspiratory sound. In the slower forms of apnœa, from diseases of the lungs, air tubes, or hydrothorax, where the interruption is less complete, the efforts less violent, the congestion of the membranes less marked, there may be little 
perceptible protrusion of the nose; indeed, in bronchitis, stupor is present to such a degree that the animal generally hangs its head. The functions of the body together gradually failing, the symptoms of suffocation are less decided.

The pathology of this mode of dying is now pretty well understood. Bichat made several experiments which went to prove that maërated blood not only reached the left side of the heart, but was sent to all the arteries of the body.

His experiments were as follows:-A ligature was applied to the trachea of a living animal; a small opening was then made in one of the carotid arteries. Presently the slender stream of blood which issued began to lose its arterial tint, and to assume the dark colour of venous blood; but, contrary to what had been previonsly supposed-namely, that the circulation would be immediately arrested in the lungs, the quiescence of the lungs, consequent upon the cessation of the alternate movements of the thorax, forming a mechanical impediment to the transit of blood through them (Haller), or that the mnaërated blood passecl through the lungs and entered the left auricle and ventricle, but went no farther (Dr. Godwin)-Bichat discovered that the blood continued to flow from the opened carotid, and that its aftlux upon the brain was marked by convulsions and insensibility. This led lim to believe that the blood underwent no obstruction in its passage through the lungs, but that, remaining unpurified and venous, it acted as a poison upon every jart to which it was carried by the arteries-first upon the nervous system, and ultimately (passing through the coronary arteries) upon the muscular substance of the heart itself. "There are, however," says Watson, "two well-known facts lpon which this theory is inexplicable-the comparative emptiness of the left chambers of the heart, and the restoration of the suspended functions by the timely performance of artificial respiration. The air conld never reach and revivify or depurate the venous blood stagnating in the capillaries of the heart." Sir James Kay Shuttleworth, in his work on Asphyxia, and later, Mr. Erichsen, in the Edinturgh Medical and Surgical Joumal, have endeavoured to prove that stagnation of the blood conmences in the pulmonary capillaries, that the stagnation is due to its non-arterialization, and that the movements of the left side of the heart are brought to an end principally by the deficient supply of blood from the lungs. 
Venous blood (say these observers), circulating through the arteries, has no directly poisonous operation, but is capable, though much less effectually than arterial blood, of supporting in some degree the irritability of the muscles; and it appears that the primary and main cause of the failure of the circulation seems to be the difficulty with which the non-arterialized blood passes through the capillaries of the lungs, and partly the imperfect stimulus afforded by the venous blood to the walls of the heart, as well as its incapability to support the functions of the brain and nervous system. The appearance of engorgement of the pulmonary arteries and venous system generally, with distension of the right side and comparative emptiness of the left side of the heart, seen after death, are more constantly visible in the slower forms of apnoea than after sudden suffocation; for after sudden death blood seldom coagulates, and if the animal is not shortly examined, the engorgement is apt to disappear by gravitation.

The conclusions of Sir James Kay Shuttleworth and of Mr. Erichsen, although correct enough, fall rather short of a full explanation; for it has been ascertained by many observers that when an animal is strangled by a tight ligature on the trachea, and examined immediately after death, the lung tissue is always found void of blood, whilst the pulmonary artery, up to its smallest branches, the great veins and the leart, are greatly engorged. Dr. George Johnson, in explaining this, says that some opposing power is brought into play, more than equal to the propelling power of the lieart, and that this opposition is due to the firm contraction of the muscular walls of the ultimate branches of the pulmonary artery. He calls this contraction the stop-cock action of the ultimate pulmonary arteries. Dr. Jolnson proved by experiments of his own that this condition exists whether the ligature be applied after or before a full iuspiration; whether the lungs were at the time full or comparatively empty of air. "These," says Watson, "are the plain and unquestionable facts of the case. They show that some opposing power must have been called into play more than equal to the propelling power of the right ventricle of the heart. Now, such a power-and it is the only conceivable one-actually exists at the very place where the venous current meets with its curb, and it consists in the firm contraction of those muscular 
fibres of the minute arteries, the function (f which is to regulate the blood supply in accordance with the varying requirements of the part. This function again is determined by those unsleeping sentinels the (vasa-motor) nerves. Were it allowable, for the sake of illustration, to impersonate the vital forces concerned in this marvellons adaptation, we might liken the process to the intelligent stopping of the traffic on an obstructed line of railway by a backward telegram."

In order to test the correctness of Dr. George Johnson's experiments, I have repeatedly examined strangled dogs, and found that the heart will continue to beat for more than three minutes after the respiratory efforts have ceased, and that the lungs and great vessels are always as he has described them, namely, the lung substances pale and empty, the pulmonary artery and right side of the heart, with the carre, immensely engorged, and the pulmonary veins and left side of the heart containing a small quantity of venous blood. From these experiments it appears very conclusive that death by apncea occurs in two ways, and that two distinct pathological conditions of the lungs are observable after death:-1st. When death is sudden, as from suffocation by strangulation, drowning, or any cause whereby the supply of air is suddenly cut off, the stop-cock action of the ultimate pulmonary arteries prevents the blood from flowing in its ordinary quantity to the pulmonary capillaries, veins, and left side of heart, and that the lungs are pale when examined immediately after death: $2 d$. When death more slowly occurs, the blood accumulates in all the vessels of the lungs, which after death appear engorged, congested, and black. This is the condition of the lungs seen when an animal is galloped to death, in pulmonary apoplexy, and congestion; and in death from some acute diseases, where towards the close of the disease the heart becomes too enfeebled to propel the blood through the intricacies of the pulmonary system, or when the blood itself becomes so altered as to be incapable of proper arterialization.

To prevent death by apnœa, it is obvious that the respiratory function must be restored where it is defective, that all impediments to the passage of pure air into the lungs must be removed. The subject of treatment will, however, be considered in another place, and I may merely state here that nothing has been - found 
so effective and so useful in balancing the circulation and in restoring the passage of blood through the lungs as the application of heat to the whole body, frictions, stimulating applications to the extremities, warm clothing, and bandages, in addition to abundance of pure air for the animal to breathe, and that bloodletting frequently affords apparent relief.

\section{DEATH BEGINNING AT THE BRAIN.}

Death by coma, or beginning at the brain, is inuch less commonly witnessed in the lower animals than death by apnœe or by asthenia. Such diseases, however, as parturient and idiopathic apoplexy, cerebral meningitis, and other allied affections, which act upon and destroy the functions of the brain, canse death in this way. Death by coma is also induced by certain narcotic poisons, such as opium, as well as by inordinate quantities of effete materials in the blood, more especially urea and carbonic acid; by fractures of the cranial walls, the pressure of tumours, abscesses, serum or extravasated bloorl, and by coagula in the cerebral arteries, obstructing the flow of blood, and causing anæmia of the cerebral mass.

The symptoms of coma are stupor, insensibility, suspension of voluntary motion, which come on sometimes suddenly, as in apoplexy and injuries of the head, whilst in other cases they supervene gradually. The breathing becomes slow, irregular, stertorous; the instinctive motion of breathing still continues, but all voluntary attention to the act is lost. The feeling of the want of air is still sufficiently strong and powerful to excite, through the medium of the pneumo-gastric and branches of the fifth nerves, the reflex power of the medulla oblongata to sustain the involuntary movements of the thorax; but at length this fails also, the chest ceases to expand, the blood is no longer aërated, and thenceforward precisely the same internal changes occur as in death by apnœa.-(WATsos.)

Coma ultimately destroys life in the same way as apnœa, with this difference, that in death by apnoea the aerration of the blood and the functions of the lungs cease first-the circulation of the non-arterialized blood destroying the functions of the brain; whilst in coma the functions of the brain cease first, and in consequence of the loss of nervous power, the movements of 
the chest and the aëration of the blood cease also. The post mortcm appearances of death by coma and of that by apnœa are the same, except, indeed, in those cases where the cause of the coma remains, when it will be present in addition to those of apnoea. Reasoning upon the conclusion that the circulation ceases consequent upon the cessation of the act of respiration, arising from suspension of the nervous power, Sir Benjamin Brodie was led to think that by continuing respiration artificially in animals labouring under nareotic poisons, the circulation of the blood might be kept up until the effect of the poison upon the brain had passed off. The correetness of the supposition he soon verified by experiment. He inserted some woorara into a wound which he had made in a young eat: this after a short time entirely destroyed the respiratory movements, and the animal appeared to be dead, but the heart could still be felt beating. The lungs were then artificially inflated about forty times a minute. The heart continued to beat regularly. When artificial respiration had been kept up for forty minutes, the pupils of the cat's eyes were observed to contract and dilate upon the increase and diminution of light, but the animal remained perfectly motionless and insensible. At the end of an hour and forty minutes there were slight involuntary contractions of the muscles, and every now and then there was an effort to breathe. At the end of another hour the animal, for the first time, showed some signs of sensibility when roused, and macle spontaneous efforts to breathe, twenty-two times in a minute. The artificial breathing was, therefore, now discontinued. It lay as in a state of profound sleep for forty minutes longer, when it suddenly awoke and began to walk about. It must be clearly understood that artificial respiration can be beneficial only where there is a suspension of the nervous functions, as in cases of poisoning, and not where there is clestruction of them by disease, injury, or the long-continued action of poisons.

Professor Sewell experimented upon the horse with the woorara poison, and found that by artificial respiration life could be maintained until the action of the poison had passed away, and that afterwards the animal regained consciousness and recovered from its effects. 


\section{DEATII BEGINNING WITH THE BLOOD.}

Necrcemia, or death beginning with the blood.-In rinderpest, splenic apoplexy, black-quarter or quarter-ill, braxy, purpura limorrhagica, and scarlatina, death may be said to be due to the death and decomposition of the blood, the action of the heart ceasing because the blood is no longer capable of affording the necessary stimulus.

At an early stage of these diseases, when they occur in their worst form, the blood exhibits changes in its composition, manifested by petechire and vibices on the skin and mucous membranes, extravasations into the subcutaneous and muscular tissues, and by congestion of, and hæmorrhages into, the internal organs.

The blood is ftuid, of a dark colour, and possesses pathogenic properties, as manifested in its deleterious operations on other animals and on man.-(See Malignant Pustule.) It decomposes rapidly, and wherever a spot of extravasation-ecchymosis-may be found, there, almost simultaneously with the extravasation, will the presence of a gas, resulting from the decomposition of the blood, be detected, whilst a peculiarly putrid odour is exhaled from the surface of the body, and from the excretions.

"The blood," says Dr. Williams, "the natural source of life to the whole body, is itself dead, and spreads death instead of life. Almost simultaneously the heart loses its power; the pulse becomes weak, frequent, and unsteady; the vessels lose their tone, especially the capillaries of the most vascular organs, and congestions occur to a great amount; the brain becomes inactive, and stupor ensues ; the medulla is torpid, and the powers of respiration and excretion are imperfect; voluntary motion is almost suspended; secretions fail ; molecular nutrition ceases; and, at a rate much more early than in other modes of death, molecular death follows close on somatic death-that is, structures die, and begin to run into decomposition as soon as the pulse has ceased ; nay, a partial change of this kind may even precede the death of the whole body (Somatic Death-Dr. Pritcriard); and the fotid aphthous patches in the throat, the offensive colliquative diarrhoea of persons in the last stage of various fatal diseases, parts running into gangrene, as in the carbuncle plague, the sphacelous throat of malignant scarlatina, and the sloughy sores of the worst forms of typhus, and in the large 
intestines in dysentery, and the putrid odour exhaled even before death by the bodies of those who are the victims of similar pestilential diseases-are so many proofs of the early triumphs of dead over vital chemistry."-(WILLIams' Principles of Medicine.)

This mode of death is not recognised by some writers, but the truth of the above quotation will, I have no doubt, strike many who have witnessed the course and progress of the blood diseases of the domesticated animals ; the extensive congestions and extravasations of black, tar-like blood, and its rapid decomposition in splenic apoplexy and quarter-ill; the gangrenous throat, and sphacelated patches of skin in purpura hæmorrhagica and scarlatina; the putrid odour of the secretions and the aphthous patches of rinderpest, and the very general advent of an exhaustive diarrhea in those cases which do not succumb to the shock of the disease; the peculiar odour exhaled, as well as the rapid decomposition of the whole body after death;--indeed it may truly be said that decomposition of the blood has set in prior to death in many instances, for if the blood be immediately examined under a high microscopic power, bacteria may be found in great abundance.

The symptoms of the approach of death by necremia are generally as follows:-A dusky livid colour of the visible mucous membranes; slight exanthematous patches on the skin, petechial spots-in rinderpest these were thought to be small-pox pustules-or extensive extravasations and the production of a foetid gas subcutaneonsly; oozing of a thin sanguineons fluid from the nostrils or intestinal canal, or of blood in a semi-coagulated condition, extreme prostration of strength; the pulse accelerated, feeble, soft, sometimes presenting a double beat; twitchings of the limbs; dilatations of the pupils, the eye often fixed and staring, but sometimes with the lids closed; unequal and frequent respirations; cold perspiration, and a gradual fall of the animal temperature. The horse will generally stand until the last, and will only fall from prostration; it will then struggle ineffectually to rise. The cow and sheep, however, will lie down in the earlier stages of disease, and scarcely ever struggle to rise.

The prognosis in all diseases which tend to terminate in necræmia is seldom or never favourable, but it is generally considered expedient to endeavour to avert the tendency to a fatal termination; and there are certain considerations which may be 
useful guides to the practitioner to effect this enıl-namely, 1 st. That such diseases tend to cause congestions of some organ or organs, as the lungs, spleen, stomach, and bowels, and consequently means should be taken to prevent as much as possible the occurrence of such congestions by removal of the patient from the contamination of foul air or bad drainage, and the avoidance of gastric or intestinal irritants. $2 d$. That the blood to a varying extent loses its plasticity, and is apt to exucle through the walls of the vessels into the looser and softer tissues, and there undergoing rapid decomposition, causes gangrene of the tissue with which it is in contact, as in blackquarter, \&c.

Means must therefore be taken to overcome this condition of the blood-mass by the administration of the chlorate of potash, which has a remarkable effect in restoring both colour and plasticity to the altered blood; oil of turpentine, which is not only a most valuable antiseptic, but is also a styptic, and in virtue of its diuretic properties assists the kidneys in the removal of effete materials. Quinine, the mineral acids, and the salts of iron will be useful at a later stage, should convalescence be established. Ammonia and its salts are to be avoided, as they tend to decrease the blood's coagulability. Egrs, milk, and, as some recommend, beef tea, may, however, be administered in the earlier stages of the maladies, and continued until there be a return of appetite. 


\section{CHAPTER XIII.}

\section{PATIIOLOGY-continued.}

\section{CLASSIFICATION OF DISEASES.}

A correct classification of diseases, at once simple and suggrestive, is a subject of profound importance, essential to the existence of veterinary science, and necessary for its teaching; and were the reterinarians of Europe to assemble in a great congress, and there agree upon a system by which diseases might be named, defived, enumerated, and classified, they would indeed attain a great object. At the present time, however, the science of nosology is very unsatisfactory as applied to the diseases of the lomesticated animals, and incomplete even in the science of lnmman medicine.

In a work of this kind it is necessary that I should refer to the efforts of physicians to arrive at a satisfactory system of nosology, and by reading books on melicine it will be found that diseases have been classified in different ways. In the system of Sauvages diseases were divided into ten classes-vitia, febres, phlegmasioe, spasmi, anhelationes, debilitates, dolores, vesanice, fluxus, cachexia. Linnæus, Vogel, and Sagar's classifications were also of this kind, and Cullen's method was an unnatural simplification of it, by which all diseases were arranged in four classes-myrexice, neuroses, cachexia, and locales. This system of classification is based upon the symptoms, and regards them as the essences of diseases; hence it is exceedingly unsatisfactory, and has been abandoner.

Believing that nosology should be founded upon correct pathology, Pinel divided diseases into five kinds, namely, fevers, inflammations, hocmorrhages, neuroses, and organic affections. This classification is necessarily imperfect from the state of pathological science in Pincl's days, but it approaches very 
nearly the standard of a pathological nosology; was further developed by Bichat, and adopted by Dr. Craigie in 1836 .

The results of modern investigations into disease prove that many ailments hitherto supposed to be merely functional are in reality accompanied by organic alteration of tissue; and it is not unreasonable to suppose that many of the so-called funetional diseases will be found to depend upon an alteration of structure, and even where no organic change can be detected in eases where the existence of disease cammot be doubted, we may attribute our failure to the imperfection of onr means and instruments of investigation, or our modes of using them.

Dr. Aitken, to whose works the reader is referred for further information, observes that "a perfectly philosophical or natural system of classification aims at having the details of its plan to agree in every respect with all the facts as they exist in nature, and to be, as it were, a 'translation of the thoughts of the Creator into the language of man.' To effect this end, arrangements, as they naturally exist, require to be traced out, not devised. The tracks in which such a pursuit must be followed up, and in which our knowledge is as yet deficient, may be shortly indicated under the following heads, namely:-

"(1.) The affinities or alliances of diseases with each other. (2.) The morbid anatomy of diseased parts. (3.) The communication, propagation, inoculation, generation, development, cause, and spontaneous natural termination of diseases. (4.) The connection of the phenomena recognised during life with the facts of morbid anatomy. (5.) The geographical distribution of diseases. (6.) The succession of diseases, as far as they can be traced throngh past ages; the peculiarities they have exhibited at different periods in the world's history, or withim comparatively recent cyeles of years.

"But the time has not yet come for a classification on a basis so comprehensive, simply beeanse the material does not exist; and attempts to make so-called natural systems of arrangement must end in disappointment, on aecount of the uncertain and fluctuating data on which they must be based."

The arrangement of animal diseases, and their division into classes, groups, and orders, is a very difficult matter. They might certainly be arranged according to their pathology, to their causes, to the tissue or the systems of the animal body 
affected, or anatomically, according to the parts which they invade. The simplest plan, however, seems to me to be that adopted by Reynolds, namely, the division of diseases into two great groups.

The first group includes those diseases in which the whole organism appears primarily and prominently deranged, and the second group those diseases in which special organs or systems of organs are in like manner affected. The first group is divided into two classes:-A, those in which the disease appears to be developed by causes operating from without; and $\mathrm{B}$, those in which it depends upon change within the body. In the first class we have those ciseases which are caused by atmospheric influences, contagion, and other external eauses; and in the second, hereditary diseases, rheunatism, rickets, and other obscure bone diseases.

The second group, or that in which special organs are diseased, is again subdivided into many classes, consisting of diseases of systems of organs, such as-A, diseases of the nervous system; $\mathrm{B}$, diseases of the digestive system and its appendages; $\mathrm{C}$, discases of the circulatory system; $D$, diseases of the respiratory system; $\mathrm{E}$, diseases of the urinary system; $\mathrm{F}$, diseases of the reproductive system; $\mathrm{G}$, diseases of the locomotive system; and $\mathrm{H}$, diseases of the cutaneous system. 


\section{CHAPTER XIV.}

\section{CONTAGION AND CONTAGIOUS DISEASES.}

The origin of contagious diseases has been, and is yet to some extent, a matter of controversy. It is, however, generally accepted that they are caused by specific pathogenic microorganisms, having the power of inducing specific morbid lesions when introduced into the animal body.

Many writers assert that a contagious disease can only arise from the introduction of a specific microbe existing in the fluids and solids of a diseased animal into the body of another, and Koch laid down the following postulates, to which it was supposed there could be no exceptions; but further observations have proved that there are many exceptions to his conclusions, and an explanation is thus given to the belief in a spontaneous origin of a contagious disease. Koch's postulates are as follows :-

1. The micro-organism must be found in the blood or diseased tissue of men or animals suffering from or dead of the disease.

2. These micro-organisms must be isolated from the blood, lymph, or tissues, and cultivated in suitable media outside the animal body. To obtain these cultivations pure they must be carried on throngh successive generations of the organism.

3. A pure cultivation thus obtained must, when introduced into the body of a healthy animal, produce the discase in question.

4. Lastly, in the inoculated animal the same micro-organism must again be found.

Although coming from such a high authority, these postulates must not be accepted in their entirety, for we have many microbic diseases where this form of origin cannot possibly have an 
existence. For example, septicæmia originates from the accidental introduction into the circulation of surrounding external micro-organisms, naturally existing in the air, or within the body itself. Again, tetanus, now recognised as a microbic disease, is induced by an organism located in the ground. These examples might be multiplied, but will be referred to when dealing with microbic diseases specially. It may, however, be mentioned that these so-called non-pathogenic microbes are classified under the term saprogenic or putrefactive bacteria, and acknowledged that they play an important part in the economy of nature.

Some of these multiply in dead organic matter, others proliferate accidentally within the body, their natural habitat being the external surroundings; but many of them can lead a double existence, suproplyytes growing on dead organic matter, and parasites which lead their existence in a living host.

Parasites which exist in and on living matter only are termed obligatory parasites; those that lead a double existence facultative parasites; and in addition to these there is another class, called non-contagious facultative parasites, which live in their normal condition in the external media, and only incidentally develop within the living organism, where, not meeting with favourable conditions for their vitality, their power becomes exhausted, and the disease which they induce is not transmitted from one animal to another,- such as metritis, omphalitis, arthritis of young animals, tetanus, \&c.; and other diseases described under their various heads are microbic but noncontagious.

In accordance with the above statement, pathogenic microbes are arranged under these heads-

I. Contagions obligatory parasites.

II. Contagions facultative parasites.

1II. Non-contagious facultative parasites.

Of the first it may be stated that their virulence is sometimes diminished, sometimes increased, but never wholly destroyed, whilst passing through the living organism, as in rabies, where the virulence of the yet undiscovered microbe is diminished whilst passing through the body of the monkey and increased in that of the rabbit. Some of them perish immediately on leaving their host, immediate transmission of the virus being 
essential, as in rabies, pleuro-pneumonia, and other diseases where inoculation or cohabitation is necessary for the transmission of the disease.

Other contagious obligatory parasites, more resistant, are preserved for varying periods of time outside of the animal body, without, however, multiplying, and conveyed to healthy animals by various means, such as water, soil, clothing, \&c.contact of a healthy with a diseased animal not being necessary.

Contagious facultative parasites are those which, as already stated, live and multiply not only in the bodies of animals, but also in external media,-dead organic matter, water, soil, and even food. Some of these lose their virulence whilst passing throngh the animal body, and the disease which they induce more or less quickly dies out. We can in this way account for the subsidence, or, as is sometimes the case, the total disappearance of certain epizootics, - such as epizootic catarrhal fever, epizootic cellulitis, \&c.-and for the sudden reappearance of these diseases. Whilst the majority of these parasites seem to have their virulence gradually attenuated whilst passing through the animal body, they still retain the power of transmitting their pathological effects, when conveyed by a diseased or newly recovered animal from one part of the country to another; hence it may be stated that a disease miasmatic in its origin may become contagious.

It often follows that after a severe and widely distributed outbreak of some epizootic the disease disappears for a long period of time; may it not be concluded from this fact that the germs have a resting stage, and that they are only called into activity by some telluric or atmospheric conditions, the nature of which has as yet escaped detection? Cognisable atmospheric changes have but little effect in calling the dormant virulence into activity, for we witness outbreaks of epizootic diseases in all linds of weather. Long-continued wet or damp weather has sometimes an appreciable effect, but there are so many exceptions to this that it cannot be said to be a determining condition.

It is already hinted that germs can be held in the air, in water, in the soil-particularly in the superficial layers-foods, stables, byres, sheep pens, vehicles, and utensils, as well as in the various secretions and excretions of animals suffering from 
their speeific effects; hence the importance of the destruction or disinfection of these carriers of contagion.

\section{CLASSIFICATION OF MICROBES.}

There are various systems of elassification,-namely, of Muller, 1773, who established two genera-monas and vibrios-and grouped them with the Infusoria, but a seientific elassifieation was not made until Elirenberg in 1838 described four genera, namely :-

I. Bacterium filamenti-straight, rigid.

II. Vibrio- " - snake-like, flexible.

III. Spirillum $" \quad$-spiral, rigid.

IV. Spirochate " - spiral, flexible.

J)ugardin in $18+1$ united spirillum and spirochate, and classed them thus:-

J. Bacterium flamenti-rigid, vacillating.

II. Vibrio- " -

III. Spirillum \# -spiral, rotatory.

And up to 1853 bacteria were considered as Infusoria; but Robin then pointed out their affinity to Leptothrix, and Davaine in 1859 demonstrated that they were vegetables and allied to the Algre, and his conelusions are now universally adopted.

The classification of Cohn is now generally accepted, and he considers that the bacteria are a distinet group belonging to the Algre, and divisible into forr tribes, including six genera.

I. Sphrrobacteria-globules (Mieroeoecii).

II. Microbacteria-short rods (Baeteria).

If the length of a rod be less than twice its breacth, it is considered a bacterium, if longer, a bacillus.

III. Desmobacteria-long rod (Bacillus and Vibrio).

IV. Spirobaeteria—spirals (Spiroehæte and Spirillum).

Colm noted, notwithstanding the plaeing of them with the Algæ, that the absence of chlorophyll connected the bacteria to fungi; and Nageli subsequently adopted this view, and termed them sehizomycetes (cleft or fission fungi).

A word or two may now be said about the action of microbes; 
some of them excite fermentation, others putrefaction. The fermentative ones have the power of peptonising and liquefying culture media containing gelatine, and in many instances of producing a poisonous alkaloid-ptomaine-which causes septic poisoning, a condition distinct from septic infection. The effect of septic poisoning depends upon the dose, a small dose producing only mild and transient effects, whilst a large one may induce a fatal result; whereas in septic infection a small dose may induce severe disease and death, because the poison introduced is a living or ganism capable of propagation and numerical increase.

The researches of Brierger have enabled him to isolate various ptomaines from dead bodies, putrid meat, fish, and cheesecalaverin, putrescin, saprin, peptotoxin, and many others, varying in their toxic properties.

Associated with or independent of the production of the roisonous alkaloids, a poisonous albumose is secreted in culture media, and in addition the following substances are evolved:-

(a.) Gases.-Carbonic acid, hydrogen, light carburetted hydrogen, sulphuretted hydrogen, and ammonia.

(b.) Nitrates, water, and sulphur.

(c.) Volatile substances (trimethylamin, alcohol, formic acid, acetic acid, propionic acid, butyric acid.

(d.) Fixed acids,-lactic, malic, succinic, oxalic, and tartaric.

(e.) Taurin, leucin, alanin.

(f.) Tyrosin, phenol and kresol (aromatic bodies).

(g.) Carbo-hydrates, albumoses, peptone, hydrolytic ferments.

(h.) Colouring matters.

When germs gain entrance into the animal economy, a kind of battle royal takes place between them and a defensive army of phagocytes, consisting mostly of white corpuscles, aided by the connective tissue corpuscles, the endothelial cells of the capillary. vessels, soft epithelial and the cells of muscular fibres. These endeavour to arrest the invasion of the microbes by attempting to destroy them. When they fail in this the disease is developed, when successful in their efforts the disease is not developed; when the virulent germs are numerous and strong, they, in a susceptible subject, paralyse and overcome the phagocytes; but when the virus is attenuated, or when the subject is non-susceptible or immune, the 
leucocytes accumulate around the point of entrance, take up the germs into their substance and digest them; in fact, they kill and eat their enemies.

Again, some germs are antagonistic to others, and it is now well known that the microbes of putrefaction are destructive to those of anthrax; but whilst anthracic blood and tissue are thus rendered impotent, it does not follow that they are rendered innocuons, for inoculations with such blood have proved that death may result, not from anthrax, but from septicæmia; and again, the ptomaines of pathogenic germs in many instances, when properly prepared, attenuated, and introduced by inoculation into the bodies of healthy animals, have the power of giving protection or immunity to such animals against the specific effects of the microbes themselves. A knowledge of this fact, first elaborated by Pasteur, has revolutionised modern medicine, and is likely to confer even greater blessings both upon man and the lower animals.

Many microbes that require a free supply of oxygen are called aërobic, whilst others seem to be destroyed or fail to grow when oxygen is present; these are called anaërobic fungi. Some, however, can live and grow with or without oxygen. Microbes are consequently divided into three groups by Liborius :-

I. Ouligatory anaërobes, which flourish in the absence of free oxygen-c.g., Bacillus butyricus, and of malignant œdema.

II. Facultative anaërobes.-These grow in free oxygen, but continue to live and multiply, but more feebly, in the absence of it-e.g., Koch's comma bacillus.

III. Obligatory aërobes, which do not grow in the absence of oxygen-e.g., Bacillus anthracis and Bacillus subtilis.

Pleomorphism.--Lister in 1873 (Quarterly Journal of Microscopic Science) indicated that several micro-organisms in their life-cycle exhibit successively the forms of cocci, bacteria, bacilli, and streptothrix. This view is now generally adopted; and Lankester, also in 1873, observed a series of form-phases in the case of a peach-coloured bacterium, which led him to suppose that the natural species of these plants were within the proper limit protean, and that the existence of true species of bacteria must be characterised not by the simple form-features used by 
Cohn, but by the ensemble of their morphological and physiological properties, as exhibited in their life history (Crookshank).

Again, Chauvin has pointed out that the Bacillus pyogenicus, by modifying the basis on which it grows, may assume successively the character of a coccus, a long thread, or a spirillum. This theory of pleomorphisin is generally but not universally accepted.

It is also stated by some observers that bacteria are not constant in their properties, and the experiments of Nrgeli, Davaine, Buchner, and Wernich seem to indicate that both the morphological and physiological characters of microbes are mutable, that changes in the nutrient medium may have effect on their form and size, on their mode of multiplication, and on their physiological and fermentative properties.

Nregeli says that a given bacillus does not invariably produce bacilli of the same structure, and does not always pass through the same developmental stages. A bacterium which under given conditions gives rise to a definite fermentation may lose this property when cultivated under other conditions, and Buchner states that the hay bacillus can be transformed into the Bacillus anthracis. He says if hay bacilli are injected into the blood of animals they do not give rise to anthrax. If, however, they are bred for several generations in meat extract, and then in the arterial blood of the rabbit, they acquire noxious properties, and give rise to anthrax in mice after two to nine days' incubation; and conversely, if anthrax bacilli are properly cultivated, they can be transformed into bacilli whose properties are identical with those of hay bacilli. Without entering into the question of the mutability both of form and physiological properties of micro-bacteria, rejected by many observers, it is satisfactory to know that the change in their power of virulence brought about by cultivation and attenuation, which has already done so much for the prevention and even cure of microbic diseases, will yet do more in assisting in the suppression of many others.

Pcriod of Incubation.-The period of latency, or that intervening between the reception of a disease-inducing microbe into the economy and the manifestation of the effect, varies very considerably. Some of the most virulent produce their deleterious effects in a very short time, while others have a prolonged period of incubation. For example, splenic fever and death may be induced 
in a few hours after inoculation, whilst the deleterious effects of the pleuro-pneumonia contagium, or that of rabies, may not be developed for weeks or even months after introduction into the body. Some contagious poisons again seem to affect one class of animals: thus pleuro-pneumonia is peculiar to horned cattle; epizootic eczema originates in cattle and sheep, but is capable of transmission by direct contact to many other animals, and even to man ; glander's affects the horse and ass, but is communicable to man, dogs, sheep, goats, rabbits, and mice by inoculation; and rabies, originating in the dog, is capable of transmission to the majority of animals and to man by inoculation. These peculiarities in the action of morbid poisons are mysterious and unaccountable. We must, however, accept them as facts that investigation may some day throw light upon.

Contagious diseases, whether induced by obligatory or by facultative parasites, may assume an enzootic, epizootic, or even panzootic character, the great feature of which is a tendency to spread rapilly, attacking large numbers of animals in a short space of time, destroying many and incapacitating a large majority.

The term epizootic is derived from the Greek, $E \pi \iota$, upon, and $\Sigma \hat{\omega} o \nu$, an animal. Diseases of this order are said to arise from enzootic influences-from $E \nu$, in, and $\Sigma \hat{\omega} o \nu$, an animal. Enzootic influences are those peculiar to certain districts, and result from conditions or agencies peculiar to a locality, which favour the development of various miasmatic diseases, such influences becoming epizootic, or affecting the many, from causes as yet unknown. In this order of diseases may be included the catarrhal fever or influenza which prevails more or less at all times amongst the horses of large cities and certain localities. Such diseases may be said to dwell in certain localities, having, however, the tendency to spread rapidly. They are then said to be epizootic, or even panzootic. 


\section{II A P TE R X V.}

CONTAGIOUS DISEASES.

\section{CATTLE PLAGUE.}

Synonyms.-Rinderpest (G.); Typhus boum contagiosus ${ }^{1}$ (L.) ; Fièvre pestilenticlle du gros bétail ( $\mathrm{F}$.)

Definition.-A specific, malignant, and contagious fever, said by Semner to be due to a streptococcus which liquefies gelatine, and which induced the disease in a calf inoculated with the cultivation. The calf died in seven days. The cocci were said to lose their virulence by successive cultivation, and a protective vaccine was thus obtained. Other observers have described them as spore-bearing bacilli, transformed into micrococci, isolated or in chains, and as a short bacillus with rounded ends, which may also present the appearance of a micrococcus or leptothrix. Inoculations with cultures induced the disease. It is indigenous to the Asiatic steppes of Russia, also Hindostan, Persia, China, Burmah, Cochin-China, Thibet, Ceylon, \&c., never occurring in this country but as the result of direct or indirect communication between imported cattle (which have been exposed to the contagium) and those of our own shores. It is unknown in America and Australia. The disease has a period of incubation varying from four to eight days, at the end of which time its local manifestations are generally developed. It runs a definite course, and usually terminates fatally; but where recovery takes place the animal is rendered unsusceptible to another attack.

It is essentially a disease of the bovine family (the ox, auroch, and zebu), but it may be communicated to the sheep, goat, deer, camel, giraffe, antelope, gazelle, and even the peccary.

1 The word contagiosus was introduced into medicine by Vegetius, and is now recognised as a generic term. 
It is said that an accidental inoculation of the human skin with the juices of an animal which had died of rinderpest has caused the formation of a pustule similar to that of variola vaccinr.-(See Appendix to the Third Report of the Commissioners appointed to inquire into the origin, nature, \&c. of the Cattle Plague, page 79.)

\section{PATHOLOGY AND SYMPTOMS.}

The specific contagrium absorbed into the blood gives rise to primary fever. This fever, as indicated by a rise in the temperature, precedes all other symptoms, and occurs in a period ranging from thirty-six to forty-eight hours after an animal has been inoculated. The elevation of temperature varies to some extent. As an average of the normal temperature of the healthy ox during different periods of the day, $101^{\circ}$ may be accepted. During rumination it may be as high as $103^{\circ}$, and may then fall to $100^{\circ}$, or even lower. Taking $101^{\circ}$ as the standard average, it has been found that the temperature may rise $2^{\circ}$, or even $3^{\circ}$, in from thirty-six to forty-eight hours after inoculation, namely, to $103^{\circ}$ or $104^{\circ}$; this elevation of temperature occurring when the animal appears to be otherwise perfectly well. From this we learn that the true period of incubation-latency-is very short indeed, and that although there are no symptoms visible to the ordinary observer, the disease has actual existence.

Two days after the perceptible rise of temperature has begun, an eruption on, and a peculiar appearance and condition of the mucous membrane of the mouth is seen, having some resemblance at first sight to the appearance of that found in the footand-mouth disease. In some rare instances, however, this characteristic symptom has been absent. I have seen it in every case of the plague but one which has fallen under my own observation, and usually veterinary surgeons have been able to diagnose the disease by its presence. Almost simultaneonsly with this appearance of the mouth, the mucous membrane of the vagina of the cow becomes peculiarly altered. One or other of these signs is rarely absent; so that when they are taken in connection with the elevation of the temperature, the diagnosis of the disease can be made with certainty. 
On the day following the eruption in the mouth, or about seventy-two hours after the first elevation of temperature, the animal may be observed to be a little ill, to have less appetite than usual, and to ruminate irregularly. Even at this tine, however, the pulse may be unaltered. On the following day, the fourth from the first rise of temperature, the animal, for the first time, shows marked symptoms of illness; and this period, which may be one hundred and ten hours after the real commencement, is usually considered by superficial observers as the beginning of the disease. The seriousness of the oversight is obvious, on account of the great importance of the earliest possible separation, isolation, or more particularly the slaughter of the diseased animal, and of all cattle with which it may have been in contact.

After the fourth day is over, the constitution is thoroughly invaded. Then ensue the urgent symptoms-the drooping head, hanging ears, distressed look, rigors and twitchings of the superficial muscles, failing pulse, oppressed breathing, fotid breath, and the discharge from the eyes, nose, and mouth.

During the sixth day there occurs a great diminution of the contractile force of the heart and voluntary muscles, the pulse becomes feeble and thready, the respiratory movements are modified, and the animal sometimes shows such weakness in the limbs that it has been thought that some special affection of the spinal nerves must exist. The temperature now rapidly falls, and signs of a great diminution in the normal chemical changes in the body present themselves.

Death usually occurs on the seventh day from the first perceptible elevation of temperature.

Although this is given as the typical course of the disease, there are great deviations from it, as some animals live a longer, many a much shorter time, and the severity and sequence of the symptoms vary considerably.

The disease is induced by a microbe, which causes the formation of a ptomaine, for, coincident with the first elevation of temperature, and, of course, long before there is the least outward appearance of ill-health, the blood of an animal which has taken the disease contains an agent which can produce the plague in another animal. In other words, the earliest fact which can be made out after infection is, that the blood contains 
the poison of the disease, so that serum obtained from it will give the disease by inoculation. This fact was ascertained by Dr. Sanderson, and is a most important discovery, and, according to the Commissioners, "is pregnant with consequences in medical doctrine;" for though (they say) the existence of a similar fact has long been suspected in several human diseases, it has never been proved in any. When the Commissioners made this assertion they were evidently ignorant of the fact that Coleman had demonstrated the presence of the glanders poison in the blood, and had induced glanders by transfusion.

The morbid poison is also contained in the discharges from the month, eyes, intestinal canal, \&c. of an animal ill from the cattle plague, and a small quantity of the mucus from the nose, eyes, or mouth, when placed in the blood of a healthy animal, increases so fast, that in less than forty-eight hours, perhaps in a shorter time, the whole mass of blood, weighing many pounds, is infected, and a very small particle of that blood contains enough poison to give the disease to another animal. This at once accounts for the rapid spread of the cattle plague. The microbe is multiplied to a large amount in a very short space of time. How sooll after it is put into the blood the animal becomes capable of giving the disease by natural infection to other animals is not cletermined, but in all probability long before it is, as it were, impregnated with the poison; for it stands to reason that all parts of the body, out of which secretions are poured, must contain more or less morbific material when that material is contained in the whole mass of blood.

It has not been determined to what length of time the blood and textures retain the power of propagating the disease. Professor Jessen of Dorpat, however, says that the mucous discharges, carefully protected, occasionally retain their power of causing the disease by inoculation for no less than eleven months.

The virus, which is both volatile and fixed, can be diffused and the disease communicated by the air for a distance of about five hundred yards; but beyond this distance it remains inoperative. It is also conveyed by flies, which, after resting on a sick animal, or its offal, fly about and alight on healthy animals; and by the offal of animals dead of the disease; by hay, bedding, dung, 
vessels, men, dogs, conveyances; in fact, by any material contaminated by a diseased animal. The contagium is destroyed by dry air, by a temperature of about $140^{\circ} \mathrm{F}$., by chlorine, carbolic acid, \&c., and, it is stated, by putrefaction. Its virulence is greatest at the outset of an outbreak.

The fever, as shown by the elevation of the temperature, begins when the poison has infected the whole mass of blood, or within from about forty to sixty hours after its first entrance into the system. Coincident with the elevation of temperature, the chemical changes in the body are augmented, and, according to Dr. Marcet, one of the products of degradation of tissue--the urea-is largely increased. Soon afterwards the blood is otherwise altered, the amount of fibrin largely increased, the amount of water lessened, and the physical condition of the albumen alterer ; and, according to Ir. Beale, the proportion of soluble substances is also largely increased.

About the third or fourth day local congestions are to be seen on the skin and visible mucous membranes, varying in intensity and in size. Stagnation takes place in the small capillaries of many parts of the body, the blood in which becomes coagulated. A great increase of granular matter is found to occur both within and withont the vessels of the congested parts. The capillaries become greatly enlarged, and the spaces between them lessened or obliterated. At the same time considerable nutritive alterations take place in the mucous membrane and skin, leading to very rapid and imperfect growth of many of the cellular elements, and followed by rapid disintegration and detachment in the form of discharges. As that portion of the mucous membrane which is most essential for digestion is most affected, the appetite soon fails, rumination ceases, and large accumulations of undigested food are met with in the first stomach. In many cases the villi of the small intestines are so destroyeil, that even if food were taken it would scarcely be absorbed in sufficient quantity to maintain life, and hence the rapid exhaustion, failure of the heart's action, depression of the animal heat, and general sinking of the powers. In some cases, when the process is more superficial, the membrane rapidly recovers its healthy condition; and it is curious to find that one affected part may be healing while another is just beginuing to suffer. 
When, as sometimes happens, the mucous membrane most affected by the congestion is that of the bronchi, the phenomena are not less severe; indeed, the disease is sometimes even more rapidly fatal. A slight cough is soon followed by accelerated breathing, which rapidly increases; and, according to Drs. Sanderson and Bristowe, the difficulty in breathing becomes so great, that some of the pulmonary vesicles are broken, and emphysema, not only of the lungs, but of the subcutaneous tissues of the neck and back, is thus induced. I cannot, however, endorse this, being of opinion that both the pulmonary and subcutaneous emphysemæ are due to the formation of gases in the areolæ of the connective tissue.

Dr. Beale, after microscopically examining the discharges from the mucous membranes with the highest magnifying power, namely, the $\frac{1}{50}$ th of an inch objective glass, arrived at the conclusion that the abundant granular matter which he thus discovered consisted of living particles-germinal matter - that they constituted the morbid poison, and that the various local congestions were due to the abundance of these particles, blocking up the minute capillaries. Although not at the time generally accepted, this conclusion of Beale's has proved fairly correct.

With reference to the true nature of the cattle plague, and its identity with or resemblance to various human maladies, many theories have been advanced by writers upon the disease. Some have considered it to be the precise counterpart of typhoid or enteric fever, and this view has been held by the German pathologists for a considerable period, and was undoubtedly the view held by most veterinary surgeons who had the opportunity of witnessing it in this country. Dr. Murchison, however, successfully combats this view, and very lucidly points out its error. He says: "Human enteric fever is characterised by definite and easily recognised anatomical lesions; and for my own part I have been unable to discover any analogy whatever with them in those of rinderpest. The alterations in Peyer's patches in the latter disease are clearly the result of the general inflammation of the mucous membrane, and tend to obliterate the glands, instead of rendering them more prominent. Their resemblance to the lesions of enteric fever is much less than that of the condition of the corresponding glands in cases of cholera, small- 
pox, scarlatina, pyæmia, and other blood diseases. It is probable that the diminution in the size of Peyer's patches in rinderpest, as compared with the glands in a healthy ox, may be due in part to the length of time that has elapsed since digestion has been arrested, as it is well known that these glands are larger and more developed during the digestive process than during fasting. But, whatever be the cause, nothing can be greater than the contrast between the appearance of these glands on the tenth day of rinderpest and on the corresponding day of human enteric fever. In the former case the glands have almost, if not entirely, disappeared; in the latter they are enormously prominent, owing to abnormal deposit in and around the glandules." Dr. Murchison quotes the writings of several observers, who agree that the intestinal glands are not enlarged; on the contrary, that they are usually diminished in size, often covered over with a layer of discoloured and softened mucous membrane." "Peyer's glands," says Professor Simonds, in his Report on the Cattle Plague, published in the Royal Agricultural Socicty's Journal, 1857, " are not invariably diseased; but like other follicular openings of the digestive canal, they are often covered with layers of lymph, beneath which ulceration is occasionally observed, but more frequently the surface is healthy, although tinged with blood." After further comparing cattle plague with typhus, influenza, dysentery, erysipelas, scarlatina, and variola, Dr. Murchison concludes that cattle plague has no resemblance to typhoid fever, typhus, scarlatina, erysipelas, influenza, or dysentery, but that it resembles small-pox.

The promulgation of this thenry during the prevalence of the plague in 1866 led to the belief that vaccination would prove a preventive, and immediately cattle all over the country were vaccinated, very much to the benefit of the vaccinators but not of the cattle, for it proved a total failure; except, indeed, that it confirmed the view that rinderpest was not variola, but a disease having some resemblance to cholera, scarlatina, and to diphtheria, at the same time having characteristics of its own which separate it from all other diseases, and prove it to be an incurable and highly contagious malady, very properly denominated "the cattle plague."

Mr. John Gamgee, in his work on the Cattle Plague, very 
successfully controverts the conclusions of Dr. Murchison, and if the reader is desirous of further information he may consult that work with great advantage.

In addition to the general symptoms of cattle plague which are embraced in the foregoing observations, the more particularly visible signs are worthy of notice; and these are slivering, muscular twitchings, restlessness, often a husky congh, yawning, sometimes great dulness, with drooping of the ears, sometimes excitement approaching to delirium, appetite at first capricious, but soon becoming entirely lost, suspension of rumination, and secretion of milk arrested. The latter symptom was one of great significance amongst cattle owners during the prevalence of the plagne, the arrestation being much more sudden and complete than in any other disease. The shiverings and museular twitchings are not always observable in the earlier stages of the disease, but often seem to usher in the second stage, when the symptoms present a more aggravated eharacter. The animal now incessantly grinds its teeth, arehes the back, and draws its legs together, moans, and otherwise shows signs of much uneasiness. The eyes, mouth, and nose are at first dry, hot, and red; the legs and ears are generally cold. At first the bowels are constipated, but this condition is succeeded by violent purging, and the dry condition of the month, nose, and eyes is followed by a discharge of a glairy watery character, soon assmming an opaque or turbid nature, which is very typical of the disease. 'The respiratory movements are generally lut not always accelerated; the inspiratory movement is quickened, whilst the expiratory is rather prolonged, and accompanied by a low moan.

The colour of the visible mucons membranes becomes peculiarly altered. In some cases they present a characteristic salmon-red colour over their whole extent, with deep crimson-red patehes interspersed here and there.

Dr. Sanderson, in his Report to the Commissioners, and Professor Gamgee, in his book on the Cattle Plague, quote the observations of Jessen and other Continental veterinarians. Jessen says- "The appearances observed by me on the mucous membrane of the mouth, both in the natural and inoculated disease, are as follows:- ' In some cases, small round nodules, seldom larger than a millet seed, are observed, which are still covered with epithelium, through which a yellowish or yellowish- 



\section{PLATE $I$.}

\section{CATTLE PLAGUE.}

\section{MOUTH AND PALATE OF A BULLOCK, SHOWING EXCORIATED ULCERS AND APHTHOUS DEPOSIT.}

(By permission of J. H. B. HALt.eN, Esq., M.R.C.V.S., F.R.C.S.E., F.R.S.E., Inspecting Veterinary Surgeon H.M. Indian Army, General Supcrintendent of Horse-Breding Operations in India.)

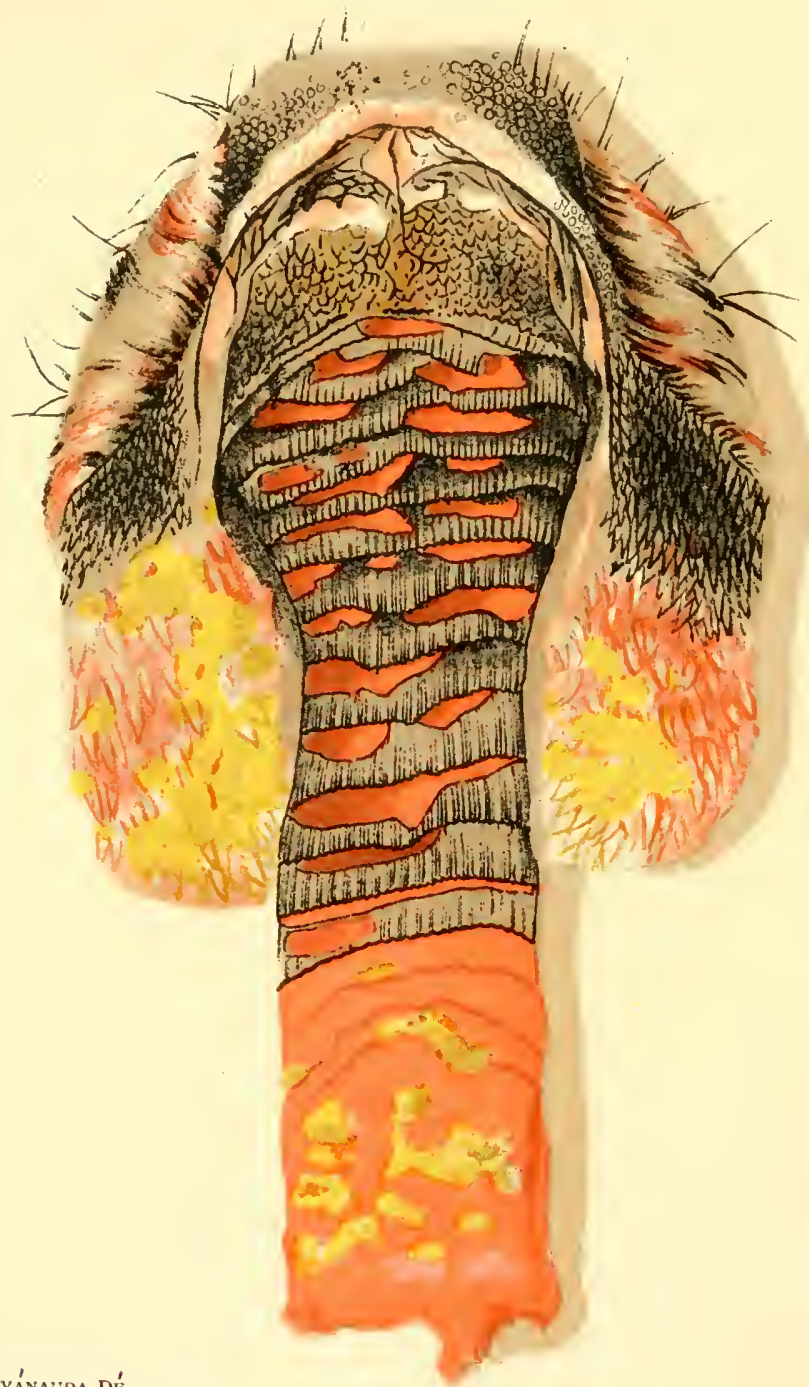


grey material can be distinguished. A f'ew hours later (sometimes not till twenty-four hours) the epithelium gives way, and the contents become visible; hence results a superficial lesion, which, after the removal of the material lying upon it, is scarcely recognisable; it heals in a few days, leaving no cicatrix. In other cases these nodules become confluent, and then give rise to an excavated ulcer of considerable extent, with irregular margins, which, however, usually heals quickly, leaving no cicatrix. In another form of the affection the epithelium is raised in the form of small vesicles, which contain either a elear or slightly turbid fluid, and leave behind shallow, round excavations, with smooth edges.'"

Dr. Brauell of Dorpat, who has made very extensive observations on the anatomy and microscopy of the disease, says that the buccal mucous membrane is usually reddened in patches of greater or less extent; these patches being in some places merely deprived of epithelium, in others covered with layers, varying in size from that of a lentil to that of a fourpenny piece. These layers are of a yellow or greyish colour, and of irregular form; they project from one to two lines above the surface of the recklened mucous membrane, and are so soft that they can be readily stripped off with the tip of the finger. ${ }^{x}$ The mucous membrane beneath is more or less reddened by congestion of the capillaries and hremorrhage, and in some cases broken down in its texture; usually, however, it is entire. Similar alterations are found at the margins of the lips and nose. In addition to these changes, roundish depressions about as large as a hemp seed, their bases covered with a yellowish material, were observed in the mucous membrane of the lips, and sometimes along with them greyish nodules, which might on superficial examination be easily mistaken for vesicles or pustules. On pressing these, a yellowish, semi-fluid mass could be squeezed out, leaving behind one of the depressions or pits above described. These were the appearances seen in animals suffering from the disease naturally cuntracted. Those seen in inoculated animals did not materially differ. On the third or fourth day after inoculation there was redness of the gum around the incisor teeth, and, more rarely, injection of the mucous membrane of the lower lip. At these spots the membrane was covered with a yellowish-white material, which could easily be stripped off, 
leaving small roundish pits. About the same period of the disease, nodules similar to those above described were observed on the lower lip.

"As the disease advances the animal becomes exceedingly restless, lying down and rising again, and otherwise denoting abdominal and colicky pains. Whilst lying down, the head is generally turned upon the upper flank. The voluntary muscles are, in most cases, affected with clonic spasm, and constant twitchings of them occur, more particularly about the neck, shoulders, and hind quarters. Diarrhoea sets in, and the animal becomes in some cases very thirsty; in others, there is loathing both of food and drink. The intestinal discharges, at first black, become of a pale greenish-brown colour, and are very fœtid; and as these are voided they canse much straining (tenesmus) by irritating the rectum. The urine is rather scanty, dark coloured, and sometimes albuminous." The pulse now becomes much accelerated and very feeble-as high as 120 to 140 per minute; the surface of the body deathly cold; the general weakness increases rapidly, the animal standing with difficulty, and lying most of the time; the cough is weak and soft; the subcutaneous areolar tissue becomes, but not invariably, emphysematus; the buccal membrane and gums are covered with masses of bran-like epithelial scales; the angles of the mouth ulcerated; the vagina of the cow and the preputial orifice of the male present a dark, deep redness. The animal is drowsy and unconscious; the breathing is performed with a spasmodic jerk; the alæ of the nostrils spasmodically open and shut; and as death approaches, the mucous membranes acquire a leaden hue; whilst dark coloured spots of erosion and ecchymosis stud their surfaces. Tympanitis sets in, and the discharges from the bowels are involuntarily excreted, whilst muscular twitchings denote the approach of death.

Some cases of cattle plague recover, and in these cases it is observed that the skin over the neck, withers, \&c. becomes covered with a yellowish sebaceous secretion, but there are no vesicles or pustules. In some of the fatal cases it is said that there is an eruption: the disease in this respect must, however, differ in different localities.

I have seen recovery take place where subcutancous emphysema was present over the greater part of the body. 
Now and then a relapse takes place after signs of improvement have become established, and in some instances animals apparently quite convalescent commence to purge, and to sink from intestinal and other complications.

Mr. Gamgee says that the disease is periodical in its manifestations: "Improvement in the morning; exacerbations at night; a distinct subdivision of an attack into stages, and from the date of the crisis either sudden aggravation or gradual abatement of alarming symptoms." This periodicity may exist to some extent; it is, however, so slight as to escape ordinary observation. Indeed, in many instances I have observed that the symptoms were quite as bad in the morning as in the evening.

The mortality in Great Britain is always very great. Amongst Russian cattle mild cases of the disease are not of uncommon occurrence, the animals passing through the disease presenting but slight symptoms of it. But even in Russia from 80 to 90 por cent. is reckoned the usual mortality, and under the most favourable circumstances 53 and 56 per cent. have been witnessed.

Although the disease is so highly contagious, it is found that some cattle resist its influence, remaining healthy whilst surrounded with the plague; but it is also no less remarkable that an animal thus exposed to the contagium will, whilst resisting the malady itself, convey the disease to other cattle, the morbid material being lodged about its body.

The post mortem appearances of cattle plague vary in different stages.

In the first stage there is congestion of the mucous membranes of the mouth, larynx, pharynx, and particularly of the fourth stomach near its pyloric end, the small intestines are marked with streaks and patches of red, and the follicles are uniformly reddened.

The surface of the mucous membrane is covered with a viscid, tenacious, and bloody secretion; is denucled of its epithelium, whilst the submucous tissue is charged with a turbid semi-fluid exudate. The condition of the first two stomachs calls for no special remarks. Sometimes the rumen presents patches of congestion on its mucous surface, approaching in tint the colour of port wine, and in a very small number of cases sloughing of the membrane has been observed.

The condition of the third stomach has been supposed to givo 
origin to the disease; hence the Germans have called it Löserdürre-impaction of the third stomach. The late Professor Dick for a long time held out that the disease was neither more nor less than this impaction of the omasum, that it was consequently a non-contagious disease, and that if by the timely administration of purgatives the stomach could be unloaded, the animal would be restored to a healthy condition. And Dr. Bristowe, in his report to the Commissioners, says, "The contents of the omasum are almost invariably preternaturally dry and caked, and as they are at the same time moulded accurately to the highly papillary surface of the folds of this stomach, it is probably due to the concurrence of these two conditions that the epithelial covering of the folds (whien, as often occurs, its normal attachments are loosened) becomes so frequently in this disease removed with the food." Now, I am of opinion that the majority of observers have fallen into some degree of error as to the abnormal state of the contents of this viscus; and have concluded that, because the food is found dry and moulded to the leaves, the condition is unnatural, whereas in reality (as may be confirmed any day by calling at a slaughter-house where healthy cattle are slaughtered) the condition of the contents of the omasum is almost invariably firm, the viscus having the appearance of' being impacted.

In some instances the leaves of the omasum are quite healthy; sometimes they are slightly reddened, the vessels which radiate from their attached border being more or less injected, and sloughing may occur in patches.

The fourth or true digestive stomach-the abomasum.-The contents of this stomach are nearly always fluid, and sometimes mixed with blood, and the specific lesions of the disease are intensely marked in this organ. Its mncous membrane ${ }^{1}$ is not only intensely red and covered with adhesive mucus, but is studded with numerous superficial erosions, like those which are so common in the ordinary catarrhal inflammation of the human stomach.-(Murchison.) The mucous membrane is easily removed from the submucous tissues, and the gastric glands are filled with granular epithelium and with blood. "In addition to the general redness," says Dr. Murchison, "which is most intense in the pyloric region, the mucous membrane at this part often presents circular or irregular patelies of a claret colour, 1 see Plate II. 


\section{PLATE $\|$.}

\section{CATILE PLAGUE.}

FOURTH STOMACH, PYLORUS, AND COMMENCEMENT OF DUODENUM OF A COW, SHOWING CONGESTION AND APHTHOUS DEPOSIT.

(by permission of J. H. B. HAlleN, Esq., M.R.C.V.S., F.R.C S.E., F.R.S.E., Inspecting Veterinary Surgeon H.M. Indian Army, General Supcrintendent of Horse-Breeding Operations in India.)

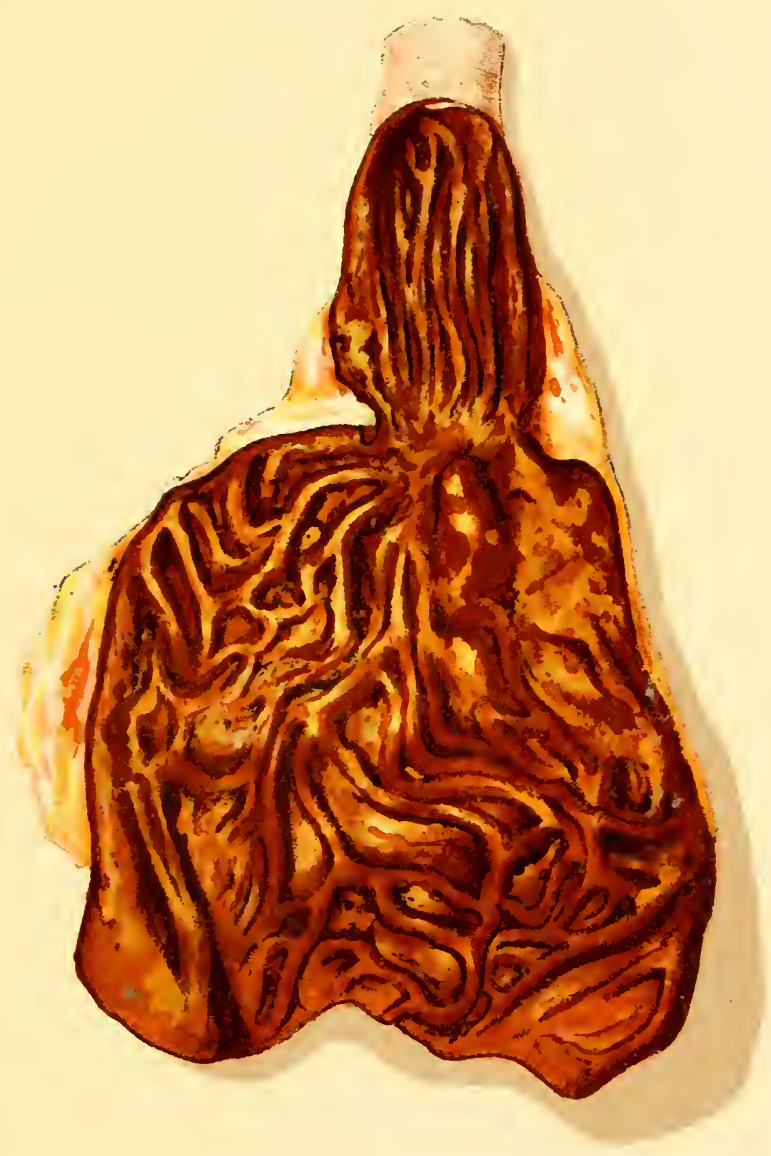






\section{CATTLE PLAGUE.}

SMALL INTESTINE OF A COW, SHOWING APHTHOUS PATCHES. MUCOUS MEMBRANE INTENSELY CONGESTED, VERGING TOWARDS GANGRENE.

(By permission of J. H. B. HALlEN, Esq., M.R.C.V.S., F.R.C S.E., F.R.S.E., Inspecting Veterinary Surgeon H.M. Indian Army, General Superintendent of Horse-Breeding Operations in India.)

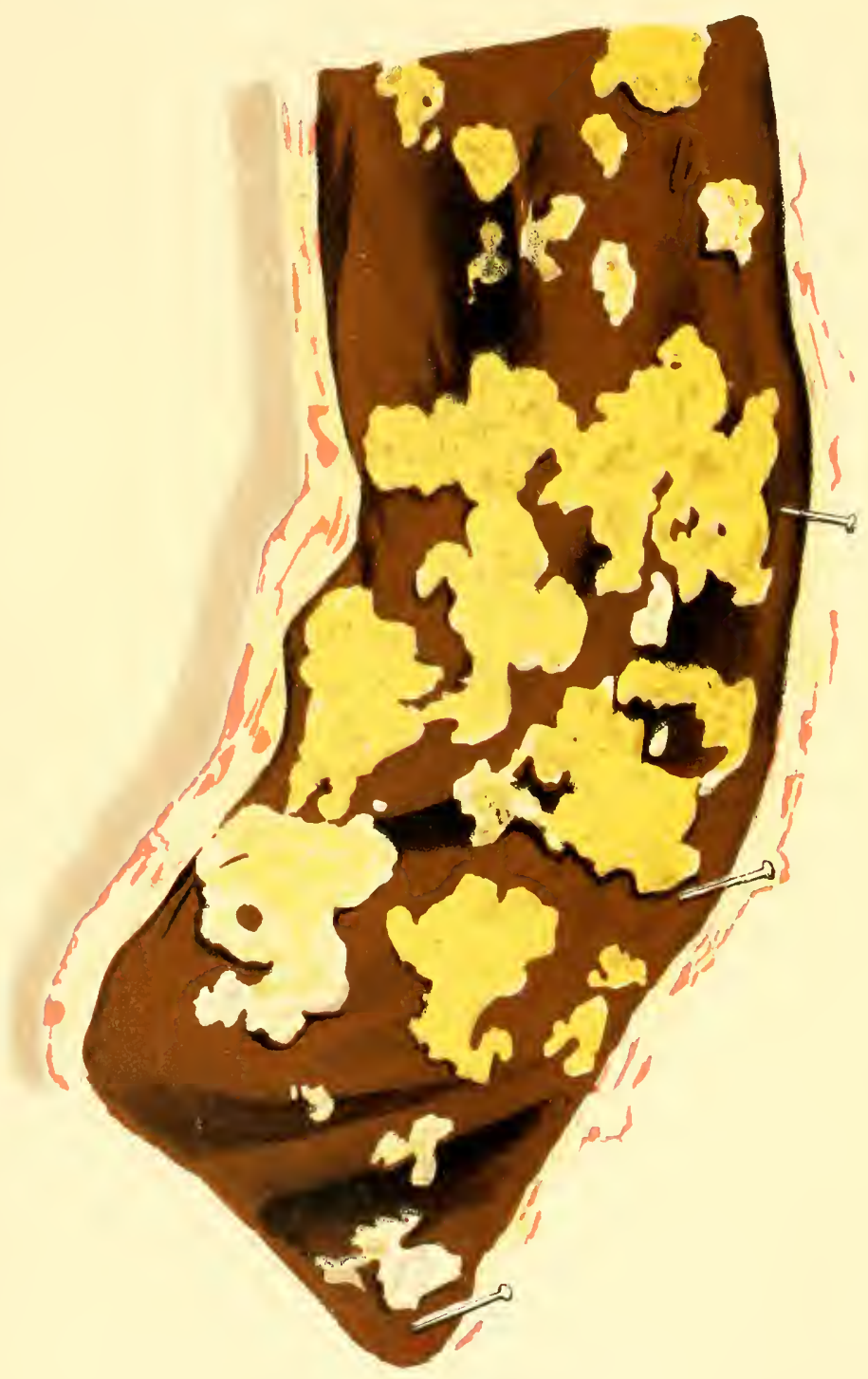


varying in size from a mere speck to a crown piece. This appearance is due to the extreme vascular injection of the parts in question, and sometimes to actual ecchymosis. The colour may be uniform over the patch; but at other times it is limited to its circumference, forming a coloured rim, with a central greyish-yellow portion. Occasionally these patches may be seen surrounded by a distinct fissure, and in rare cases the membrane corresponding to the patch separates as a slough, which may be found more or less extensively adherent. On separation of the sloughs, deep, excavated ulcers, penetrating the mucous membrane, and even the entire muscular coat, may remain."

With regard to the mucous membrane of the small intestines, it is generally more or less inflamed throughout. Sometimes, however, it has been found almost free from disease. When it exists, however, the inflammation is most intense at the iliocæcal opening, and about the middle of the crecum. The serous surface of the bowels is of a bluish aspect, dotted with spots of ecchymosis, and softened. The discolorations vary much in colour, some spots being scarlet or rose-red, whilst others are of the deepest purple. True ulceration of the bowels is rare, though the mucous membrane is easily removed, and croupous exudates are now and then found in the canal. ${ }^{1}$

The large intestines, according to Professor Simonds (see Report on the Cattle Plague, Journal of Royal Agricultural Society, 1857), show marks of the disease even to a greater extent than the small ones. The observations of Professor Simonds were made on the Continent: in this country, the large intestines rarely manifested such signs of inflammation as the small. As already stated, Dr. Murchison has very carefully compared the lesions of the intestinal glands with those occurring in the typhoid fever of man, and has arrived at the conclusion that there is no resemblance. In the healthy ox the glands of Peyer are very often found enlarged and hardened, and when these enlarged glands have been discovered in cattle dead of the plague, microscopic examinations have determined that their contents were chiefly composed of granular masses with cholesterine; products more indicative of a chronic than of an acute alteration.

The lining membrane of the respiratory track invariably presents signs of congestion, and is covered in patches with a soft membranous (croupous) exudation. In the majority of cases 
the rim of the glottis is considerably swollen, and the tonsils much enlarged. Emphysema of the lungs is very commonly met with, and is a condition which causes great distress to the animal prior to its death. The remarks of Dr. Sanderson upon this point are very interesting. He says, "During the first stages of the disease respiration is performed in a perfectly natural manner, but about the fiftl day irregularities begin to be observable. From time to time the rhythmical movements of the chest are interrupted, and expiration is accompanied by an audible moan. On the sixth day (that is, in fatal cases, the day before death) the breathing usually assumes a character which is so remarkable that if once observed it cannot be forgotten. The chest dilates suddenly, but apparently with considerable effort, in consequence of, as I believe, the umnatural permanent expansion of the lungs due to obstructed expiration. This inspiratory movement is immediately followed by closure of the glottis, the expiratory muscles being at the same time thrown into violent action, much in the same way as they are in the act of rumination. The closure of the glottis is always attended with a sound (such as might be produced by the sudden closure of a soft leather valve) so loud that it can be heard at a considerable distance. This sound coincides with the resisted expulsive effort, which is often so strong as to throw the whole body of the animal into oscillation. The chink of the glottis is continuously closed for one and a half to two and a half seconds, the chest remaining expanded and motionless. At the end of this period the air shut up in the thoracic cavity is expelled with a peculiar grunting noise, which is quite as characteristic as the valvular sound already described. It is immediately followed by a renewed inspiratory effort." Dr. Sanderson says that this peculiarity of movement is the cause of the interlobular emphysema. He says, "The pause, instead of occurring between each expiration and the succeeding inspiration - that is, when the breathing apparatus is in a state of relaxation-occurs when the chest is expanded, or, more accurately speaking, in the middle of the expiratory act; for, inasmuch as the expulsion of air from the chest commences immediately after the chest is filled, a considerable quantity escapes before the glottis has had time to close. At the moment that closure takes place the air confined in the chest is strongly compressed by the action of the 


\section{PLATE IV.}

\section{CATTLE PLAGUE.}

GALL BLADDER OF A COW, SHOWING APHTHOUS PATCHES.

(By permission of J. H. B. HALleN, Esq., M.R.C.V.S., F.R.C S.E., F.R.S.E., Inspecting Veterinary Surgeon H.M. Indian Army, General Superintendent of Horse-Brecding operations in India.)

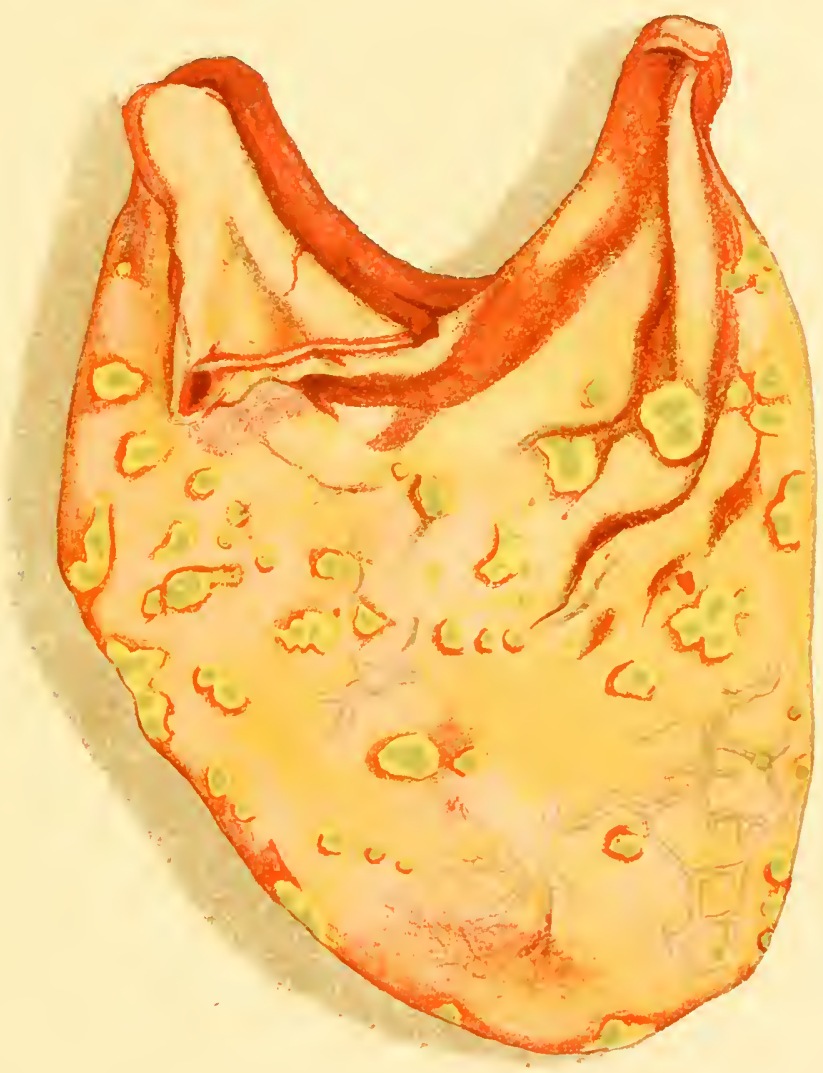






\section{PLATE V.}

\section{CATTLE PLAGUE.}

HEART OF OX, SHOWING PETECHIAL SPOTS.

(From a Model in Museum of New Veterinary College.)

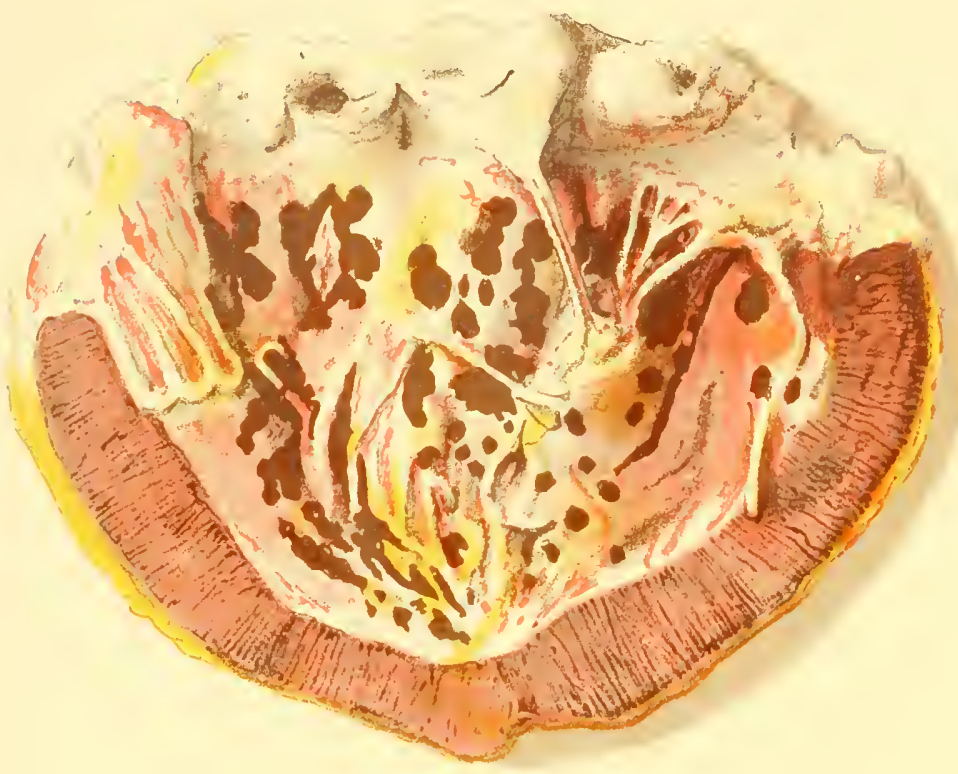

PLATE VI.

FOOT AND MOUTH DISEASF.

EXCORIATIONS ON TONGUE. 
expiratory muscles, and in this way gives rise to the interlobular emphysema, which is so commonly observed in cattle plague." I feel compelled to differ from Dr. Sanderson on this point; my own observations leading me to the conclusion that the emphysematous condition of the lungs is due to the generation of gases in the interlobular lung tissue, and that the peculiarity of the breathing results from, and is not the cause of, the empliysema.

The other organs of the body do not present many post mortem signs which may be called peculiar to the disease. The heart is often covered, both externally and internally, with petechial spots.-(See Plate V.) The liver is sometimes pale, sometimes dark; the gall bladder is usually full of bile, and sometimes covered with aphthous patches.-(See Plate IV.) The spleen is usually healthy, a circumstance of great interest, says Professor Gamgee, when taken in connection with the condition of this organ in the typhus and typhoid fevers of man.

The lining membrane of the vagina and uterus, like the mucous membranes of other parts of the body, are deeply tinged with various degrees of redness and purple, and their epithelium is in a softened and semi-detached condition.

The skins of animals suffering from cattle plague present a variety of appearances, such as crusts, eruptions, pustules, and elevations. In inoculated animals dermic alterations are said to be pretty constant, but, as already indicated, they were absent in those cases which fell under my notice whilst the disease was raging in Yorkshire. They were, however, carefully looked for in every post morten examination which I made. Dr. Sanderson says that "they may be described generally as consisting in the first place of incrustation of material exuded in a soft or semi-solid state from the glandular follicles of the skin; and, secondly, of pathological changes in the superficial structures of the skin, which, without taking anything for granted as to their nature, may be designated by the term eruption. Subcutaneous emphysema was not infrequent as an ante as well as a post mortem condition. 


\section{SHORT HISTORY OF THE CATTLE PLAGUE IN BRITAIN.}

In $1348-9$ a plague or murrain broke out amongst the eattle in England. It appeared just after the black death had destroyed thousands of human beings, and seemed to be similar to the cattle plague. Dr. Fleming, in his work on the History of Animal Plagues, gives much interesting information upon the subject. The importance of the "stamping out"system seemed to be comprehended in those days. The diseased cattle were slaughtered, and infected herds were as much as possible separated from those which were sound, while the herdsmen who attended the former were not allowed to come in contact with the latter. In 1480 another outbreak of disease (murrain) took place, and committed great devastation. There are, however, no accurate accounts of the symptoms, \&c. of these diseases. It is therefore impossible for any one to state that they were identical with cattle plague; but there is every reason to believe that the outbreak occurring in 1715, which renewed its attack in 1745 , and remained here till 1757 , was the same as the plague of 1865 .

The plague of 1745 was brought to this country by two white calves imported from Holland by a farmer living near Poplar. Another account states that it was brought here by a tanner, who bought a parcel of distempered hides at Zealand. It appears these hides had been forbidden to be sold there, and were ordered to be destroyed. Both accounts state that the disease broke out near London, and that it continued to ravage the herds for twelve years, and was only suppressed by vigorous measures being taken by local and other authorities.

It is curious to note that the local anthorities of those days were very similar to those of the present time. In the First Report of the Cattle Plague Commissioners, 1865, it is stated that in 1747 various orders were issued stopping local fairs, and empowering local authorities to do so when they found it expedient.

"The plague, in consequence of these orders, was extinguished where the local authorities acted with vigour, but lingered in other places, from whence it spreacl after a time as rapidly as ever." When the history of the present epizootics-pleuropneumonia and the foot-and-mouth disease-comes to be written, 
it can very truly be said that some of the local anthorities tried to suppress these diseases, and would have succeeded, had it not been for the laxity of other local authorities.

The origin of the outbreak of 1865 is, according to Professors Simonds, Gamgee, and others, as follows:- "Early in 1865 an Englishman, James Burchell, was engaged in the Russian province of Esthonia collecting together a cargo of "cattle for the English trade. He was in partnership with Mr. John Hönck and a Mr. Baker. The Esthonian Agricultural Society had undertaken to supply Burchell with 800 oxen and 800 sheep. The Society collected 640 oxen at Revel, out of which 400 had to be chosen. 46 of these animals had travelled from St. Petersburg or its neighbourhood, in four horse waggons, but the remainder were Esthonian oxen. The steamer 'Tonning' left London on the 9 th or 10th of May, and arrived at Revel on the 16th or 17th. She left Revel with 331 oxen and 330 sheep selected by Mr. Burchell."

Professor Gamgee says- "I have taken considerable pains to investigate the documents relating to these transactions; and of the oxen chosen by Mr. Burchell, three were sold sick to a butcher named Siehbert in Revel, one died, and a fifth was ill after the steamer had left Esthonia.

"According to Burchell 13 of the 46 oxen from the interior of Russia were amongst those shipped for England. Mr. John Hönck says that all the cattle placed on board the 'Tonning' were Esthonian. The St. Petersburg cattle were, however, mixed with the Esthonian cattle at Revel; and although they might not be seriously ill, it is a well-known fact that Russian cattle infect others when they themselves are not suffering, or are very slightly attacked. The plague was in the vicinity of St. Petersburg at the end of 1864, and probably later.

"The oxen purchased by Mr. Burchell were to have been landed in London, but they were entered in the manifest for Lowestoft, 'so as to avoid the cloctors.' I have seen a letter in which this sentence occurs. After all, the steamer made for Hull. The cattle were inspected at Hull; but it appears that, owing to the number being so very great, the inspection was not a very rigid one-600 animals having been inspected in three hours and a half by two inspectors.

"On the arrival of the cattle at Hull, Mr. Hönck 'put them in 
the hands of a Mr. Coulson,' who sold 106 of them to go to Derby or Leeds. A Mr. Hickman took 40 to sell at Manchester.

"The cargo arrived on the 29th of May, and the first 146 animals were disposed of on the 30th. The remaining 175 came to London, and they were sold on the Thursday following, ' after they had arrived in London on the Monday night.' The cattle for London were placed in Mr. Hönck's lairs in York Poad, adjoining the Cattle Market. They were then sold for slaughtering, with the exception of 20, which Mr. Baker sent on the following day to Gosport. The 155 animals were disposed of entirely to metropolitan butchers. The sheep were sold at Hull for killing purposes. The steamer 'Tonning' was disinfected by the free use of chloriue of lime, and after certain repairs were done to her engine, she started for Tonning. In a leading article in the Times, published on the 15th August, it is said that as far back as the 12th June animals affected with the steppe murrain were seen in the Metropolitan Market. As these were not the Rinssian cattle, they must have been animals which caught the disease in or near the market at the begimning of June. On the 19th of June, Mr. Carvell of Lambeth Walk purchased two cows, and one of these on the 24 th showed signs of sickness."-(GAMGEe on the Cattle Plague.)

In the end of July the disease appeared in Aberdeenshire. It was brought there by four calves which were sent by train to Huntly from the south.

By the beginning of November the plague had been conveyed over the length and breadth of the land, embracing thirty counties in England, seventeen in Scotland, and one in Wales.

The following summary for the week ending December 30 th will show how it had spread by the end of the year:-

Total number of farms, sheds, or other places in which the disease had appeared :-England, 7,443 ; Scotland, 2,065; Wales, 245.-Total, 9,753.

Total number of cattle on farms, in sheds, or other places where the disease had been officially reported to exist:-England, 110,647; Scotland, 44,527; Wales, 4,536.-Total, 159,710.

Number of animals slaughtered healthy:-England, 10,636; Scotland, 6,578; Wales, 152.-Total, 17,366. 
Total number of diseased animals reported since the commencement of the disease-

\begin{tabular}{|r|r|r|r|r|r|}
\hline & Attacked. & Killed. & Died. & Reeovered. & Remaining. \\
\hline England, . . . & 48,964 & 11,142 & 27,177 & 3,655 & 6,990 \\
Wales, . . . . & 2,257 & 93 & 1,565 & 218 & 411 \\
Scotland, . . . & 22,298 & 2,696 & 12,749 & 3,172 & 3,681 \\
\hline Total, . . & 73,549 & 13,931 & 41,491 & 7,045 & 11,082 \\
\hline
\end{tabular}

The disease continued to spread, and to commit great havoc, until an Order in Council was issued, making it compulsory to slaughter and bury all animals affected with the plague, as well as those which had been in contact with them.

The beneficial effects of this order were soon made apparent, the number of animals attacked gradually falling off until the disease was finally stamped out. It is true that every now and then outbreaks occurred at various places, without any trace of contagion or infection being discovered, leading people to think that the plague was a sporadic, non-contagious disease; and even to this day some believe that it was never imported here at all, and but for the most determined action of the Government, supported by the opinions of veterinary surgeons, all legislative restrictions upon the cattle traffic would have been removed.

Had the restrictions upon cattle traffic been removed, we should again have felt the effects of another visitation of the plague in 1872, for in July and August of that year the disease was brought to Deptford, Hull, and Leith, and but for the prompt action of the authorities, the affected animals would have conveyed the disease all over the country. As it was, the plague did appear at Patrington on the 10th, and at Yapham on the 19th of August. The origin of the outbreak was as follows:A vessel, the "Joseph Soames," arrived in Hull on the 25th July, from Cronstadt, with fifty-eight oxen on board. Two, it is stated, had died on the voyage, and in consequence of information the cattle were examined by Professors Simonds and Brown, who found eighteen of them infected with the plague. On the 
following day (the 27th) all the animals were slaughtered, and their carcases were placed on lighters and sunk. The hatches of these lighters were improperly fastened, and the carcases floated again, and were washed ashore on different parts of the coast. This washing ashore of the infected carcases caused much alarm, and it was feared the contagion would thus be conveyed. However, no evil results followed.

The origin of the disease at Patrington and Yapham is as follows:-_" The ship with the diseased cattle on board was lying for two days in the Humber Dock, close to the 'Forin,' and not five hundred yards from the cattle market, and, in spite of all that could be done, there was an open communication between the vessel and the shore.

"For nearly a fortnight cattle affected with the plague had stood on the ship's-the 'Joseph Soames'-deck, and it is surely no exaggeration to say that the amount of contagium in the excretions which had accumulated was sufficient to infect every head of cattle in the kingdom.

"Is it necessary, then, to question the probability of some of the virus being carried into the Hull market only the day after the carcases of the Russian oxen were sent to sea? The precise manner of the conveyance of the poison has not been, and certainly will not he ascertained. No one, in short, saw one of the men who had been engaged about the diseased cattle rub his hand or coat against an animal in the market or on its way thereto. But when a centre of infection has been established, it is not usual to cavil much about the passible means of communication between that centre and a place a few hundred yards off.

" The next link in the chain of facts is the purchase of two animals, a heifer and a steer, at the auction sale in Hull market on July 29th, by a butcher at Patrington. These came from farms in the neighbourhood of Hull, where no disease had existed. They were taken by railway on the day of purchase to Patrington, and placed in a field adjoining one belonging to $\mathrm{Mr}$. Sanderson, a miller, whose stock-two cows and two calveswere grazing in his meadow.

"Twelve days after reaching the field at Patrington, the heifer in the butcher's field was found to be ill, and was slaughtered on August 12th. A difficulty occurs here, which we hasten to 
meet. Twelve days is a long time for the incubation of cattle plague; but we have to observe, for the information of our nonprofessional readers, that the date of the discovery of cattle plague is quite distinct from the real date of its outbreak in a herd."-(Veterinarian, October 1872.)

By the activity of the authorities the outbreak was confined to three infected districts, namely, Bridlington, Patrington, and Pocklington, to which places the disease was clearly traced by the writer of the article in the Veterinarian.

I think the origin of this outbreak is most clearly traced, and that the difficulty of the long incubative period (twelve days) is no difficulty at all, for in all probability the heifer had been more or less ailing for several days before the illness was discovered. I think it right to state that I have dwelt longer upon the history of this outbreak than perhaps was necessary, in consequence of Dr. Parkin in his work on Epidemiology having thrown discredit upon the source of origin, and stated most dogmatically that the disease was of spontaneous origin, and due to something to be mentioned in a book " to be shortly issued."

\section{TREATIENT OF THE CATTLE PLAGUE.}

The cattle plague may be classified as one of those diseases in which all methods of medical and hygienic treatment have hitherto proved unsuccessful; and, judging from the nature of the malady, always will prove unsuccessful.

The disease, when induced by inoculation, has proved of a less severe nature, and the per-centage of recoveries has been greater in comparison than in that induced naturally. It has therefore been supposed that if inoculation were generally adopted (the disease never occurring a second time in the same animal), that the great mortality might be to some extent avoided. Professor Gamgee, however, concludes his exhaustive observations on this point as follows :-

"First. Inoculation induces, as a rule, a mild form of disease amongst steppe cattle. In cattle of other breeds the loss has sometimes been slight, but is usually as severe as that arising from natural rinderpest.

"Seconcl. The inoculated disease is communicable by reinoculation and cohabitation. My experience would indicate 
that the animals affected by simple cohabitation with inoculated cattle suffer more than those inoculated, and die in considerable numbers.

" Third. The results of inoculation are seriously aggravated by cold, wet, and exposure. The most successful cases have been those attended to indoors.

"Fourth. In my opinion inoculation never can be resorted to with success, even in Russia, as a means of exterminating rinderpest.

"Fifth. Means adopted for the cultivation or modification of the virus have proved unsatisfactory, and camnot be relied on.

"Sixth. Any liquid from the body of a sick animal serves to communicate the disease by inoculation.

"Seventh. The period of incubation is usually from four to five days after inoculation, and the invasion of the disease is indicated, first, by elevation of temperature, and secondly, by distinct white epithelial eruptions on the inner side of the lower lip, below the corner incisor, and on the inside of the nose.

"Eighth. The cutancous eruption not constant in natural rinderpest is usually seen in inoculated animals.

"Ninth. Sheep can be inoculated from cattle, and then again cattle from sheep, without modifying the virulence of the virus.

"Tenth. I have not witnessed any modification in the inoculation with different secretions. I have tried blood, milk, abdominal fluid, contents of the intestines, frothy mucus from the windpipe, saliva, and tears.

"Eleventh. I have reason to believe that glycerine modifies and then destroys the virus, as in the case of pleuro-pnemonia. On this point further experiments are required, but I feel tolerably certain that mixing glycerine with the virus of rinderpest cannot be relied upon to obtain a mild form of the discase.

"Twelfth. All animals that escape after inoculation without indicating symptoms, such as elevation of animal heat and eruption in the mouth, are not protected from further attacks.

"Thirteenth. The produce of animals which have had rinderpest is as susceptible to attacks as any other.

"Fourtecnth. Age does not materially affect the results of inoculation."-(GAMGeE on the Cattle Plague.)

Seeing then that the cattle plague is beyond the power of all medical and hygienic remedies, and that the inoculated disease 
commits fearful havoc amongst stock, we can only trust that the Government of this country will ever remain uninfluenced by those who would wish that cattle were admitted from all parts of the world without any restrictions whatever, and carefully guard our coast from another visitation of this dreadful malady; but should it again have access to our shores, there is only one method of dealing with it, and that is to stamp it out, by destroying all affected with it, as well as those which have been in contact with them.

In conclusion, I feel in duty bound to pay my tribute of respect to Professor John Gamgee for the earnest endeavours he made, as far back as 1857, to rouse up the Government to the fact that this country would be visited by the cattle plague if measures were not taken to prevent its entrance. I am aware that Professor Gamgee received much abuse, but he was a true prophet, and if he has received no other reward, has the satisfaction of knowing that he has performed an important public service.

RINDELPEST IN SHEEP.

It was at one time affirmed that cattle only were subject to the plague, "the cattlc tribe bcing alone its victims."-(Simonds" Report to Agricultural Society, 1857.) Experience has, however, proved that sheep, though less susceptible, are still capable of being affected with the plague.

The symptoms are the same as in the ox, and any one familiar with the disease in the latter animal will at once be able to recognise it in the sheep. The incubative stage is more variable than in cattle; the disease induced by inoculation appears in from five to eight days; naturally caused by cohabitation or contact, in from five to twenty days. Sheep kept in fields with cattle suffering from the plague remain a long time unaffected with the disease, many escaping it altogether; but if kept in closed sheds they are almost certain to become affected in a very short time.-(Professors Varnell and Pritchard, Report to Commissioners, 1866.)

Experiments in Inoculation.-The upshot of all the experiments has been that the operations were brought to a close, as it was found that the mortality was almost as great among the inoculated ones as among those that took the disease naturally. The Russian Government thereupon closed the establishments at Orenberg and Kerson. During the outbreak in France in 1871 a further series of inoculations was tried with similar results. In one experiment seven cattle were inoculated, and seven died, but the recent experiments conducted by Koch in South Africa seem to prove that the inoculation of 10ce. of bile from an animal that has died from an acute attack, say a week after the initial symptoms (rise of temperature, \&c.) have appeared, gives immunity, but six days must elapse after the inoculation before protection is afforded. After the lapse of six days animals so protected could not be made to take the disease by the inoculation of virulent rinderpest blood, nor from exposure to contagion 


\section{CHA P'TER XVII.}

\section{CONTAGIOUS DISEASES-continued.}

\section{PLEURO-PNEUMONIA CONTAGIOSA.}

Definition.-A contagions febrile disease, peculiar to horned cattle, supposed to have originated in Central, and has been conveyed to all parts of Continental Europe, to Britain, Africa, America, Anstralia, India, and New Zealand. It is dne to a contagium, which gains access to the system by the lungs, and which after an incubative period of from two to three weeks to as many months, induces complications in the form of extensive exudations within the substance of the lungs, and upon the surfaces of the pleura, finally resulting in consolidation of some portions of the lungs, occlusion of the tubes, embolism of the vessels, and generally adhesion of the pleural surfaces. In some cases there is extensive and rapid destruction of lung tissue, with death from suffocation; but most commonly the disease is of a lingering character, symptoms of great prostration manifesting themselves, with blood poisoning from absorption of the degraded pulmonary exudates, and death from marasmus and apnoea.

Synonyms.-Lung disease, pleura, new disease, new delight (Yorkshire), pulmonary murrain, epizootic pleuro-pnenmonia, \&e.; called by the Germans lungen scuche and peri-pneumonia exudativa contagiosa; by the French maladie de poitrine du gros bétail and péripneumonie contagieuse.

PATIIOLOGY AND SYMPTOMS.

There is much variety in the manifestations of the disease In some instances, more especially during its first outbreak in a 
district, it runs a rapid course, destroying life in the course of a few days, the lungs after death presenting the appearance of a congestive inflammatory change, with embolism and hæmorrhagic infarction. In other cases-and these are the most numerousthe onset, course, and termination of the disease occupy a period of from two to eight weeks, or even longer, the animal becoming much emaciated, and finally succumbing to an exhausting diarrhœa; imperfect aëration of its blood; hydrothorax; the depressing influence of degenerated animal matters absorbed into the blood, and anæmia.

Premonitory symptoms of the Disease.-As in cattle plague, the commencement of the disease is often not observable. Thus attention is only called in many instances to an animal for the first time appearing unwell, but in which an examination brings to light the fact that changes of structure have taken place, to such an extent as to convince the observer that disease has been gradually increasing for a lengthened period. The thermometer is not even a true guide by which we are able to discover the end of the incubative and the beginning of the active stage, as later investigations have proved that extensive pulmonary changes may exist without elevation of temperature, and that the fever is probably concomitant with the invasion of the pleural surfaces. For example, the introduction of the disease into Australia from this country proves that the first manifestation of illness is not at all to be depended upon as a guide to its true commencement; for the carriers of the contagion-bulls for breeding purposes-were three months on the voyage, the disease only breaking out after they were landed; but it is of great importance that thermometric observations should be made during the prevalence of pleuropneumonia, in order that owners of stock might be warned in time, and that measures be taken to isolate or otherwise dispose of all animals in which the temperature is found to be rising. In a suspecter herd, all animals showing a temperature above $102^{\circ}$ should be carefully watched. If the heat rises above this, there can be little doubt that disease is at work. When the disease is established the temperature may rise to $105^{\circ}, 106^{\circ}$, and rarely to $107^{\circ} \mathrm{F}$. In 134 cases, 61 of which were recorded by Mr. Elphick, V.S., Newcastle, and the remainder by myself, only in 8 did the temperature reach $107^{\circ}$. 
The palpable or obvious symptoms are slight rigors or shiverings, the hair merely standing the wrong way, loss of appetite to some extent, secretion of milk diminished; in some cases the animal "knuckles over" at one hind fetlock, usually the right one; an occasional cough is heard, which is dry and hard in character, not the painful cough of pleurisy, as one would suppose, nor the moist hoarse cough of inflammation of the bronchial mucous membrane; rumination becomes irregular, and although there is some loss of appetite, the animal seems fuller than its fellows, which are healthy and eating vigorously. The bowels are rather constipated, and the urine is scanty and high coloured.

The pulse of cattle, as I have already stated, is not, more especially with regard to its number, a good guide to the practitioner in this or many other diseases; however, as it advances, the pulse becomes accelerated and of a feeble character-sometimes a large soft pulse, other times a small wiry one. These insidious symptoms may continue for several days, the most careful examination of the chest denoting nothing unusual except a tenderness upon pressure applied to the intercostal spaces of one or both sides, and pressure upon the back causing the animal to wince and perhaps give a slight groan. Some cases in an infected herd will at this stage begin to give obvious signs of recovery, and in a few days seem well again, the morbid material having evidently been expelled from the body without cansing any extensive pulmonary change. In all cases, however, some amount of irritation and alteration of the lung tissue has been induced, as a cough remains for some time longer. These cases, however, are a source of great mischief, as they become the media through which the virus continues to be conveyed to the cattle with which they are herded, for a considerable period after their apparent recovery. Should recovery not take place, the signs of general disturbance gradually, sometimes rapidly, increase; the cough becomes more persistent, the mucous membranes, except that of the nose, are generally pale, the respiratory movements increased in frequency, more abdominal and shallow; when the animal stands, the elbows are turned out, the nose extended, back arched, and the hind limbs drawn under the body and knuckling over at the fetlocks; when recumbent, the animal throws the weight of its body upon the sternum, and, 
owing to the anatomical conformation of this bone and its articulation with the true ribs, the chest is thus expanded.

The breathing becomes painful, and is often accompanied by a moan or grunt, emitted during each expiration, and the nostrils are dilated; but even these signs are not constant, many animals with extensively consolidated lungs being but slightly distressed in the breathing when left quiet; but accelerated respirations are easily induced by percussion applied to the sides, or by compelling the animal to move. A discharge sometimes issues from the eyes and nose, which is at first colourless, but aften becomes purulent and yellowish, but is seldom profuse. The extremities, as well as the horns and ears, vary much in temperature. Sometimes all are cold; often, however, one ear and one horn may be cold whilst the others are hot, and so on with the extremities. The surface of the body becomes harsh and dry, the skin appearing tightly bound to the subcutaneous structures, and there is rapid loss of flesh. Constipation of the bowels continues in many instances for a long period ; in others it is succeeded at an early stage by a diarrhœea, which, if not too persistent, seems to have a salutary effect; but if it assume the colliquative character, the animal dies in from two to three weeks from the first visible manifestation of the symptoms.

The physical signs upon percussion are tenderness and some amount of dulness, the dulness increasing in proportion to the exudation and consolidation. If at the outset of the disease it can be determined that both lungs are inflamed, the prognosis in all cases is unfavourable; but if, on the contrary, it can be demonstrated that but one ling is affected (the right lung, according to my experience, being more prone to suffer, but showing a greater tendency to recovery), there are some hopes that the case may recover, for very frequently the morbid action is confined to the side primarily attacked.

Auscultation will detect friction and other sounds, which indicate that the disease is not confined to the pleural surfaces; thus we have crepitations, both large and sinall, indicative of inflammation of the lung connective tissue, with rhonchus and sibilus, denoting bronchial disease. It is seldom, indeed, but that some sound or other is detectable in all parts, except the lower portions, of the chest; the consolidation of the lung, extensive though it may le, being insufficient to mask or hide the abnormal sounds em:tted by the lung tissue, pleura, or bronchial tubes. 
When the disease is confined to one lung, the respiratory murmur in the healthy lung is louder than natural, owing to its having to admit more air than when both are in a state of health. This must not be confounded with a diseased condition; and in order not to make a mistake, percussion must be applied,- - the healthy side will be resonant, the diseased one dull. I have seen some cases where the diseased and consolidated lung has enlarged to such an extent as to push the ribs immediately covering it outwards to some extent, the animal appearing rounder and larger on that side in consequence; and some of these cases have afterwards thriven and become fit for the butcher.

Now and then it is found that some portion of the lung becomes gangrenous, and is coughed up; these cases are, however, very rare. When gangrene occurs, the discharge from the nose is sanious and fotid, and a fœetid diarrhœe soon carries off the suffering beast. Abscesses in the lungs are an occasional consequence. An animal apparently recovers from the disease ; but after a time begins to lose flesh, and sinks from exhaustion, the post mortem revealing caseons tumours or a large abscess or abscesses in the lungs. In some instances of very extensive consolidation, the sounds detectable by auscultation and those emitted by percussion are very trivial. In such it is found that the alteration of structures is most extensive in the central portion of the lungs. One symptom is very diagnostic of this condition-namely, much coughing when the animal attempts to swallow; this is caused by the exudate pressing upon the œsophagus within the chest, and retarding the action of deglutition. When the exudate presses upon the large blood-vessels, there may be turgescence of the jugulars and the venous pulse.

\section{POST MORTEM APPEARANCES.}

On opening the chest of an animal which has been slaughtered early in the disease, the visible changes are dulness of the affected pleural surfaces; the substance of the lungs, particucularly of the large lobes, is red, congested, and more or less consolidated; the redness and congestion being often in patches, each patch being generally surrounded by whitish bands of thickened interlobular tissue, giving them a marbled appearance. 
In other instances the redness is diffused over a more or less extensive surface, and is due to staining of the interlobular bands by extravasated blood, one or several affected lobules forming one patch, whilst the lung tissue for some distance may be more or less healthy, until another patch of more or less consolidated tissue is arrived at. The groups of consolidated lobules vary much in colour, some showing the varying tints of recent inflammation-blood brown, or dark brown redness; whilst others present signs that the exudation is not only consolidated, but is undergoing a change of colour. We have thus the red and a yellowish grey hepatization, the red denoting the early, the other the more advanced stages of the inflammation; but the true grey hepatization is seldom or never seen now-a-days, as cattle are slaughtered before the exudate has undergone this change.

In some animals from an affected herd it has been noticed that the first change is a dilatation of the blood-ressels, and the lung assumes a scarlet colour. There is no exudation into the interlobular connective tissue, but the lung is increased in weight, and sinks farther in water than healthy lung.

The true grey hepatization seems to indicate a drying up of the inflammatory products, the formation of new connective tissue in the lung, and the disappearance of the alveolar spaces; but the apparent grey or marbling hepatization seen in the early stages of pleuro is due to a distended state of the interlobular lymph vessels surrounding the infarction in the lobule.

The peculiarities in the character of the inflammation of pleuro-pneumonia are its tendency to invade small groups of lobules, more especially those composing the iarge lobes, or even a single lobule, and to spread not only by diffusion, but also by the invasion of non-contiguous lobules. In this respect it simulates to some, extent the pulmonary inflammation induced by the glanders poison, and is characteristic of a local change depending upon a cause existent in the blood; and we may conclude that the true nature of the inflammation differs from that of an ordinary one; that it is secondary to a general infected condition; that the exudates do not tend to become highly organised, but rather to become gangrenous, or 
degrade into lower forms of matter-i.e., caseous or calcareousand rarely to change to cicatricial tissue, and to remain latent in the part for an indefinite period; and that it may commeneo primarily in the parenchyma of the lungs, and that finally the tubular portion and vessels of the lungs become obliterated, not only by the pressure of exudates external to them, but by histological elements and clots within them. In consequence of these changes, the diseased lung becomes very heavy, sometimes attaining the weight of twenty, thirty, or even fifty pounds, sinks in water, is resistant, solid, non-crepitant, and on sectiou presents the marbled appearance already mentioned.

In those rapid cases of death which occur after the first introduction of the disease into a locality, the changes in the lung tissue are those of an acute character; there is great redness or blackness of the parenchyma-hæmorrhagic infarction -which is at same time loaded with much serosity, and is very friable, soft, and easily broken by the finger; but in the examples which most frequently fall under the pathologist's notice the cadaveric lesions are-1st. Extensive deposits of yellowish, friable layers of false membrane upon the surface of the pleura and upon the pericardium. These false membranes exist upon one or both sides, as the ease may be. Portions of the lungs are found adherent to the sides and to the diaphragm, but in many cases the bands of lymph are of a friable nature, the adhesions very imperfeet, and the opposing surfaces easily separated, while there is, in old-standing cases, generally much fluid in the cavity of the thorax, in which flakes of fibrin are seen floating. The effused fluid is of a yellowish colour, contains much albumen, and if exposed to the atmosphere will often coagulate into a gelatinous elot. There is much variety in the quantity of the fluid. In some cases the pleural cavity and pericardium will contain several gallons, whilst in others the quantity may only measure a few ounces.

The pulmonary pleura, in addition to the bands of lymph, which form the adhesions already alluded to, is invested by a firm layer of lymph, which may be stripped off; leaving the lung rough, mottled, and having papillæ-like eminences upon its surface. These bands of lymph, as well as the flakes found floating in the serum, are composed of fibrin. When examined 
microscopically, cellular elements, partaking of the nature of pus cells and white blood globules, are seen imbedded in the fibrin, which is sometimes granular, but often filamentous. The turbidity of the serum is caused by these globules and some amount of fatty matter.

The tissue connecting the pleura to the thoracic walls is but slightly involved, and when removed after slaughter, the underlying structures seem healthy, but a careful examination of the part will show the ribs to be bare, stripped of their pleural covering, and to have an unnaturally clean whitish appearance, and the intercostal flesh, in advanced cases, unhealthy, moist, or even dropsical. The pericardium is also covered with flakes of lymph, and greatly thickened by exudative materiais.

It has been considered that the primary seat of the disease is situated in the pleura; that the inflammation is of a rapidly spreading character, causing the formation of what have been termed by Rokitansky "the croupy exudates," both upon the surface of the serous membrane and in the substance of the lungs. As a rule the inflammation in pleuro-pneumonia contagiosa is more pronounced in the parenchyma than upon the pleural surface; but this is liable to exceptions, for in some cases the pleural disease is more marked than the parenchymatous. Some writers think that the disease may begin in the bronchi and air cells, and that the irritation extends from the tubes and cells into the parenchyma and pleura. Their conclusions are based upon the fact that inflammatory products are generally found, in the advanced stages of the disease, in the air vesicles, blood-vessels, and bronchi, as well as in the other portions of the inflamed part.

Dr. Yeo, in an elaborate report published in the Veterinary fournal and other periodicals, concludes that the disease originates in the bronchial tubes, and that the surrounding tissue is in a state of chronic inflammation for a lengthened period before any symptoms are manifested. He says-"I am convinced that the lung disease usually exists for months without being suspected, and invariably the beast is first thought to be sick only when the affection has spread to the pleura, and caused intense inflammation of that membrane with its accompanying well-marked symptoms." 
These very confident conclusions of Dr. Yeo, if allowed to go unchallenged, are calculated to lead to much misapprehension, and even to actions at law. They prove to me that he has had but little or no opportunity of studying the disease in the living animal, and that he bases his conclusions upon what he has observed in the laboratory.

Now, if pleuro-pneumonia were a species of bronchitis, or, as he puts it, if the virus acted primarily on the bronchial mucous membrane, one would naturally conclude that the bronchial walls would in the earliest stages show evidence of discase; but such is not the case, and only occasionally do we meet with congestive patches along their course in those parts of the lungs already involved in the disease. As the disease advances, however, the bronchi, as shown by Dr. Yeo in the woodeut,
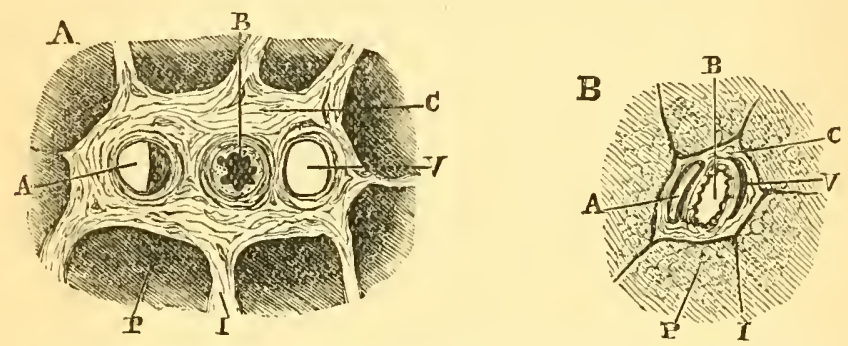

Figs. 3 and 4.-Transverse section of Broncho-vascular System, contrasting the healthy with the diseased state.

A. State of advanced disease. A. Artery, partially occluded by a thrombus. B. Bronchus, contracted and plugged. V. Vein. C. Common broncho-vascular sheath, thickened by exudation. I. Interlobular tissue. P. Lobular parenchyma.

B. A corresponding broncho-vascular system in health. ${ }^{1}-$ (YEo.)

1 The cut was kindly sent me by Dr. Fleming.

become occluded, their walls invaded by the inflammation, and they, as well as the blood-vessels, alveoli, and air cells, are filled with a solid exudate, differing, however, from that of bronchitis, where the occluding material is composed of more or less fluid catarrhal products; and one most important condition is absent, namely, the blood-vessels of the inflamed lobules are not occluded by coagula as in pleuro-pneumonia contagiosa. In some instances, rarely met with since early slaughter has been made compulsory, the bronchial tubes of a non-invaded 
lung territory are found occluded, and the lung tissue surrounding them collapsed by the gravitation or entrance into them of the degraded, softened products of the pneumonia, which, having escaped from the seat of formation, have been forced by the inspiratory act into the healthy tubes. Here we certainly find the bronchi filled with diseased products, and the pleural surface presenting little or no signs of disease; but an examination of the pneumonic products will at once prove that they consist of degraded fibrinous exudates, and that the parenchymatous disease is antecedent to the occlusion of the bronchi. In the second place, Dr. Yeo concludes that, in the common run of cases where pleurisy is associated with extensive disease of the lung, the latter gives the impression that it is of much older standing than the pleural affection. He says, "The pleurisy is commonly acute; while in the lung we usually have evidence of such chronic change as would require a very long time for their development."

If Dr. Yeo had had clinical experience amongst cattle he would have seen that these conclusions are quite opposed to facts. Many instances have occurred in my experience where animals, apparently healthy, which had been slanghtered in consequence of having been in contact with others affected with pleuro, have presented, post mortem, round patches of consolidation in one or more parts of their lungs, always associated with pleural exudation, and sometimes even adhesion of the opposing pleural surfaces; indeed, in some instances the most pronounced evidences of the presence of the disease have been patches of pleural exudation with but little or no corresponding invasion of the lung parenchyma.

Then, as to the chronicity of pleuro-pneumonia, the statements of Dr. Yeo are against all clinical facts, and consequently must not go unchallenged. A reference to the accompanying woodcut (fig. 5), and a short history of the case from which it was obtained, will illustrate this point. The large dark patches reveal the condition of dark red consolidation, and the smaller ones the commencement of that process, which is evidently that of hæmorrhage into the alveoli ; the dark patches are surrounded by œdema of the tissue contiguous to the points of extreme congestion; the interstitial tracts are much distended, and if 
carefully cut, a quantity of straw-coloured lympl, only found

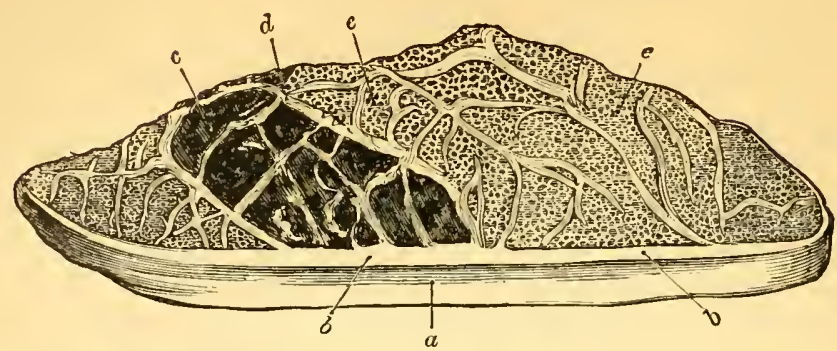

Fig. 5.-(a) Pleural surface presenting a smooth opaque appearance. (b) Subpleural exudation. (c) Red consolidation. (d) Thickened interlobular tissue. $(e, e)$ Hæmorrhagic spots, due to extravasation into alveoli.

in recently invaded lung territory, flows from the cut surface. This may be collected and used for inoculation purposes. At a

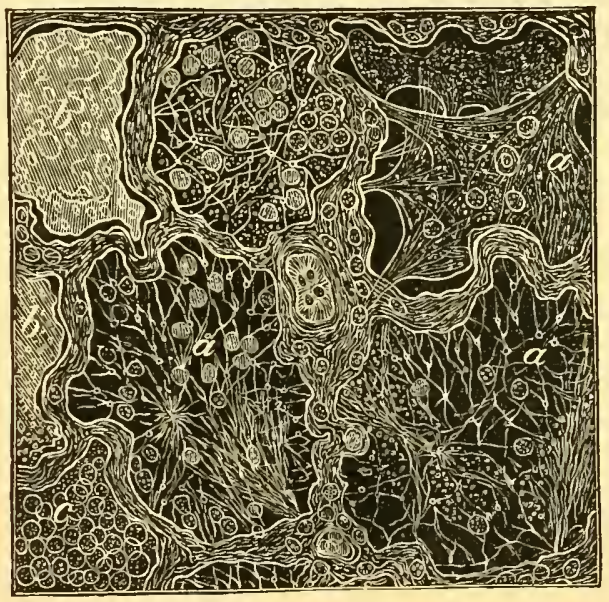

FIG. 6.- $(a, a)$ Exudation in air vesicles, composed of a fibrinous network, with leucocytes lying in it. $(b, b)$ The same caseating. (e) Air vesicle filled with leucocytes only. (480 diam.)

In the centre is seen a blood-vessel filled with a fibrinous plug, and around it numerous leucocytes in the substance of the wall of air vesicle.

later stage this is more gelatinous in consistence. The pleural 
surface is much thickened, the exudate being both within and upon the serous membrane. Microscopically examinel, as shown in fig. 6 , the diseased products have every appearance of being of recent origin, the secondary or degenerative changes $(b, b)$ which occur very early in pleuro where the blood supply is cut off by plugging of the blood-vessels, having only commenced. The history of the animal from which the specimen was obtained was clearly traced. It had been brought from a healthy herd six weeks prior to slaughter, throve and milked well up to the two days prior to slaughter, when it presented slight signs of illness, became rapidly worse, and when examined clear evidence was obtained that both lungs were gravely invaded. I may state that, along with four others, all of which succumbed, the animal had been brought into a byre where the disease had previously prevailed, but which had been declared free under the Act of 1868. Taking into consideration the clinical fact, which has forced itself upon me upon many occasions, that it generally takes from a month to six weeks for the disease to develop in a healthy animal introduced into an infected byre, and the condition of the diseased products in the above case, one cannot help concluding that pleuro-pneumonia is often a very acute disease, and that it may rapidly involve large tracts of lung tissue.

In other instances, however, the invasion is confined to small areas; the disease may then assume a mild type, and the animal recover; whilst in others the disease becomes chronic, the animal dying, not so much from destruction of lung structure, but from a mal-condition of the whole system, arising from absorption of the degraded products of the inflammation.

The cause of pleuro-pneumonia, in this country at least, is undoubtedly contagion and infection, and these only. My experience of the disease enables me to state that no mismanagement with regard to feeding, housing, or the general treatment of stock, will induce'an outbreak of pleuro-pneumonia contagiosa.

I do not say, with some writers, that it nowhere originates spontaneously; but I do say that it never originates spontaneously in this country. 


\section{PREVENTION OF PLEURO-PNEUMONIA.}

When the disease breaks out in a herd, the affected animals are to be isolated, or, if "in condition," slaughtered for beef, experience having proved that such beef is good and wholesome if the animal is killed early in the disease. If the animal be ont of condition, slanghter and burial are the safest, and, in the end, the most economical ; indeed, it may be distinctly laid down that the slanghter of the whole herd for human food is the cheapest in the end, the hides, hoofs, \&c. being carefully kept from coming in contact with any healthy cattle. Some recommend the destruction of hides, hoofs, \&c. I think this a wasteful destruction of property, and fail to see how the disease can be propagated by them; unless, indeed, they are actually brought into contact with healthy cattle-not a very likely occurrence.

Disinfection.-All sheds, cow-houses, or other premises which have contained cattle affected by the lung disease should be thoroughly cleansed and disinfected. The best and cheapest way of doing this is-1st. To burn sulphur in the buildings ; $2 d$. To whitewash the walls, stalls, roofs, and every nook and crcvice within the building with lime-wash containing carbolic acid (crude), in the proportion of one pint to each bucketful of whitewash.

Inoculation of Plcuro-Pneumonia.-Inoculation was attempted by Dieterichs with no satisfactory results. The experiments were repeated by Vix, who obtained results in the form of pneumonia, which was due in all probability to pyæmia. Nothing conclusive, however, was obtained until Dr. Willens of Hasselt, Belgium, instituted a series of experiments, the result of which led him to arrive at the following conclusions :-

"1. Pleuro-pneumonia is not contagious by inoculation of the blood or other matters taken from diseased animals and placed on healthy ones.

" 2 . That the blood and the serons and frothy liquid squeezed from the lungs of a diseased animal in the first stage of pleuropneumonia are the most suitable matters for inoculation.

" 3 . The inoculation of the virus takes from ten days to a month before it manifests itself by symptoms.

"4. The matter employed for the inoculation has, in general, 
no effect upon an animal previously inoculated or having had the disease.

" 5 . The inoculated animal braves the epizootic influences with impunity, and fattens better and more rapidly than those in the same atmosphere with it that have not been inoculated.

" 6 . The inoculation should be performed with prudence and circumspection, upon lean aninals in preference; and towards the tenth day after the operation a saline purge may be given, and repeated if necessary.

“7. By inoculating pleuro-pneumonia a new disease is produced; the affection of the lungs, with all its peculiar characters, is localised in some part on the exterior, but whether it occasions apparent morbid manifestations or not, the inoculated animal is preserved from pleuro-pneumonia.

"8. The virus is of a specific nature; it does not always act as a virus. The bovine race alone is affected by its inoculation, since other animals of different races, inoculated in the same manner, and with the same liquid, experience no ill effects."

Method of inoculation.-The virus is to be selected from the interlobular tissue of a lung in the first stage of a mild attack. The lung will then be found distended with a yellow semi-fluid exudate. All parts of the lung which present any appearance of dark red colour, and are consolidated, or which have the appearance of being gangrenous, should at all times be avoided. The selected portion of lung, after having been cut along the lymph spaces to allow the fluid to ooze out, is to be placed whilst warm in a strainer, over a clean stoneware or glass bowl, covered over by flannel or cloths, to keep in the warmth and to prevent dust, \&c. gaining access. In a short time a quantity of clear yellow fluid will be obtained, which, if not required for immediate use, is to be enclosed in glass tubes, about four inches long, threeeighths of an inch in diameter, and, when filled, hermetically closed by a blow-pipe flame.

The tip of the tail is the spot which should be selected for inoculation, and a single drop of the virus is sufficient. It is better to inoculate on the upper than on the lower surface of the tip of the tail, removing the hair, and then slightly scarifying the skin, the scarification being quite superficial. The point of 
the tail is selected in preference to any other part, because it can be easily amputated if gangrene occur.

In the most favourable instances, a slight heat and swelling occurs round the inoculated spot in a period varying from a week to two months; generally, however, the eruption manifests itself from the ninth to the sixteenth day, accompanied by slight rigors, loss of appetite, and slightly diminished secretion of milk. When the operation has been properly performed, and the virus carefully selected, the effects are generally as above described; but when the virus is putrid or badly selected, or, as sometimes happens, some peculiarity exists in the inoculated animal, the primary swelling is excessive, the tip of the tail becomes gangrenous, the animal suffers from a high state of fever; secondary deposits occur at the root of the tail, around the anus, and in the abdominal glands, and death occurs in a few days after the inoculation.

The conclusions arrived at by the Belgian Commission with regard to inoculation are as follows:-

"1. The inoculation of the liquid extracted from the lungs of an animal affected with pleuro-pneumonia does not transmit to healthy animals of the same species the same disease-at all events so far as its seat is concerned.

" 2 . The appreciable phenomena which follow the inoculation are those of local inflammation, which is circumscribed and slight on a certain number of the animals inoculated; extensive and diffuse, with general reaction proportioned to the local disease, and complicated by gangrenous accidents, on another number of the inoculated animals, so that even death may result.

" 3 . The inoculation of the liquid from the lungs of an animal affected with pleuro-pneumonia exerts a preservative influence, and invests the economy of the larger number of animals subjected to its influence with an immunity which protects them from the contagion of this malady during a period which has yet to be determined."-(GAMGEE.)

The losses sustained during the experiments of the Commission amounted to 11.11 per cent.; the number of animals on which the operation was benignant was 61.11 per cent.; the proportion in which there was gangrene and loss of a portion of the tail was $27 \cdot 77$ per cent. ; in twenty-one subjects the inflammation 
was very severe, and complicated by gangrenous phenomena, causing the death of six; and lastly, the recoveries amounted to 88.88 per cent.

Following the method of Willens, Professor John Gamgee introduced preventive inoculation into Edinburgh about 1857, but the casualties were very numerous, many animals dying from blood-poisoning induced by the operation; in fact the operation was condemned as being worse than the disease. In London it was condemned by Professor Simonds and others, and to the present time it finds no favour there.

Inoculation as reintroduced by Mr. Rutherford, V.S., Edinburgh, has been attended with great success.

Dr. Burdon Sanderson and other experimentalists recommend the injection of the lymph into the venous system by means of a small syringe, selecting the superficial aural vein for that purpose. This method of inoculating, while inducing no local results, is said to be quite as effective as that of inoculation of the tip of the tail.

Further investigations on the cultivation of virus, as recommended by Pasteur with those of anthrax and fowl cholera, may reveal the fact that an attenuated virus is quite as effective as that removed from the pneumonic lung, and that successful inoculation by direct injection into a vein may be performed without inducing any local ill effects.

Intravenous injection has also been investigated by Professors Thiernesse and Degive, who, having found that inoculation with the lymph of pleuro-pneumonia in the cellular tissue of the dewlap generally caused death, performed a series of experiments, and concluded-

1st. That the injection of pleuro-pneumonic virus info the veins is not at all dangerous, if care is taken that not a single drop of this liquid falls into the cellular tissue.

$2 d$. That this infusion possesses the same properties as caudal inoculations, - that is to say, that it invests the animal body with a real immunity.

Professors Thiernesse and Degive inject the virus into the jugular, and recommend certain precautions to prevent all contact between the virulent fluid and the cellular tissue. If, however, the steel cannula be plunged into a vein, and then 
the syringe adapted carefully, and care taken that all the fluid is injected into the vein before withdrawing, first the syringe, then the cannula, there will be little or no danger of this occurring.

By the Contagious Iiseases (Animals) Act, 1878, it is enacted that all cattle suffering from pleuro-pneumonia are to be immediately slaughtered; and by the Pleuro-Pneumonia Slaughter Order of 1888, all cattle being or having been in the same field, shed, or other place, or in the same herd, or otherwise in contact with cattle affected with pleuro-pneumonia, are to be slaughtered within ten days after the fact of their having been so in contact has been ascertained, or within such further period as the Privy Council may in any case direct. All cattle which have been certified by an inspector of the Privy Council to lave been in any way exposed to the infection of pleuropneumonia are also to be slaughtered within such period as the Privy Council may direct.

THE PATHOGENIC MICROBE OF PLEURO-PNEUMONIA.

At the meeting of the National Veterinary Medical Association held in Edinburgh in 1886, I stated that pleuro-pneumonia was due to an organism. This announcement was received with derision by certain members present. At that time, after several year's' work, I had found two organisms, a bacillus and a micrococcus, and was of opinion that the micrococcus was the pathogenic microbe. The question has been since studied by various Continental observers. Lustig found that there are generally four organisms in the exudates of pleuro. These four, however, may be classified into two groups, as seen under the microscope:-1st. A bacillus which rapidly liquefies gelatine in cultivation tnbes: $2 d$. Micrococci, which are proved by cultivation to be of three linds-(a.) a micrococcus whose colonies are white, like boiled white of egg; (b.) a micrococcus the cultivations from which assume a golden yellow colour; and (c.) a micrococcus whose cultures on gelatine resemble drops of wax. These four organisms were studied by Lustig in 1885, and by Cornil and Babes in 1886, but the results which the latter obtained were not very precise; and they concluded that their 


work, and that of all investigators who had preceded them, would have to be repeated.

Arloing and others have also found four different organisms: -1st. A bacillus called by Arloing Pneumobacillus liquefaciens bovis ; $2 d$. Three micrococci-(a.) a non-liquefying coccus whose colonies resemble drops of wax-Pneumococcus guttc-cerei; (b.) a micrococcus the white colonies of which spread in a thin layer, and as this grows older it becomes wrinkled and foldedPneumococcus lichenoides; and (c.) a micrococcus whose colonies, elongated or round, assume a beautiful orange tint-Pneumococcus flavescens. Arloing is now of opinion that the Pneumonia bacillus liquefaciens is the pathogenic microbe of pleuropneumonia ; for, by means of subcutaneous inoculations of cattle with pure cultivations of the microbes, it was found that the bacillus produced the greatest effect, and when injected in larger doses pulmonary effects were induced closely resembling those of pleuro-pneumonia. Arloing has repeated his experiments, and says-" I have reproduced in the bovine with pure cultures of the pneumobacillus taken between the second and tenth generations the typical alterations produced under the skin or in the chest by the virus of the peripneumonia contagosia. I then peremptorily say - (first) that the virulent agent of contagious peripneumonia is an ordinary microbe; (second) that this microbe is the pneumobacillus liquefaciens bovis."(Compte Rendus d'Académie des Sciences). Further experiments are, however, desirable.

BRONCHO OR CATARRHAL PNEUMONIA, CORN-STALK DISEASE, TRANSIT PNEUMONIA.

Pleuro-pneumonia has been confounded with another disease -broncho-pneumonia, called by American veterinarians the "corn-stalk disease," and transit pneumonia by the Calladians, described by Billings as interstitial pneumonia, following a septicæmia induced by eating decomposing corn-stalks (Indian corn-stalks)-by the veterinary officers of the Board of Agriculture and others, and I feel warranted in relating the history of that disease as seen in this country.

Early in 1879 pleuro-pneumonia was reputed to have been found amongst American cattle landed at Liverpool, and the 
shippers there, in order to satisfy themselves of the correctness of the diagnosis of the veterinary advisers of the Privy Council, and who now hold the same position on the Board of Agriculture, requested Professors $M^{\prime}$ Call, Walley, and myself to visit Liverpool and examine the cattle, and watch the post-mortem results; and here commenced the dispute, as, after having seen a few diseased lungs, I maintained that the malady was not pleuro-pneumonia, but a catarrhal or broncho-pneumonia, combined in a few cases with pleurisy, but in others the pleurisy was absent. Professors M'Call and Walley agreed with the advisers of the Privy Council, and I found myself in a magnificent minority. During the spring and summer of that year several condemned lungs were sent to me from Liverpool, and after further investigation I found no reason to cliange my opinion.

The matter remained in abeyance so far as the profession was concerned until April 1891. It is very true we now and then heard that pleuro had been detected amongst American cattle landed in this country, but we had no means of verifying or contradicting these reports; but some two years or so ago the American Govermment sent inspector's to the various ports where American cattle are landed, for the purpose of investigating and reporting upon the cattle slaughtered; and, wonderful to relate, we heard no more of American pleuro, except one notable report from Dundee, until a lung of an ox was found at Deptford in April 1891, and said by Professors Brown and Duguid and Mr. Cope, and confirmed by Professors Walley and M'Fadyean, to have pleuro-pneumonia contargiose, and in further support of the correctness of the diagnosis Mr. Chaplin, the Minister of Agriculture, said it was pleuro, for he had seen the lungs. But Dr. Wray, the American veterinary inspector at Deptford, disputed this opinion, and on 18th April brought a portion of the lungs to me.

I, along with my son, examined this portion of lung, and we both came to the conclusion that it was not pleuro-pneumonia. I was again in the minority, and nothing more would have been heard of the matter, at least so far as I am concerned, had not a remarkable coincidence occurred in Paris during the winter of last year, when it appears that MM. Redon, Godbille, and Blier, well-known veterinarians and sanitary inspectors at $\mathrm{La}$ Villetethe Parisian cattle market-where thousands of American cattle 
were exposed for sale. On three several accasions these veterinarians affirmed that some of the animals were affecterl with an unusual disease of the respiratory organs. In November 1890 , in a lot of more than 400 cattle direct from Illinois and Indiana, one died, and two were very ill, the symptoms leading to the suspicion that they were affecterl with contagious pleuro-pneumonia.

"The sick animals were killed, and the lungs examined by Godbille, who found no pleurisy, but such a suspicious appearance that, considering the importance of the matter, the two sets of lings were sent to Alfort, to be submitted to M. Nocard, one of the most illustrious of veterinarians and pathologists, and he reported that at the first glance a section of the hepatised tissue presented the appearance of a recent lesion of pleuropneumonia contagiosa. The tissue was dense, compact, friable, the colour varied from bright red and deep brown to almost

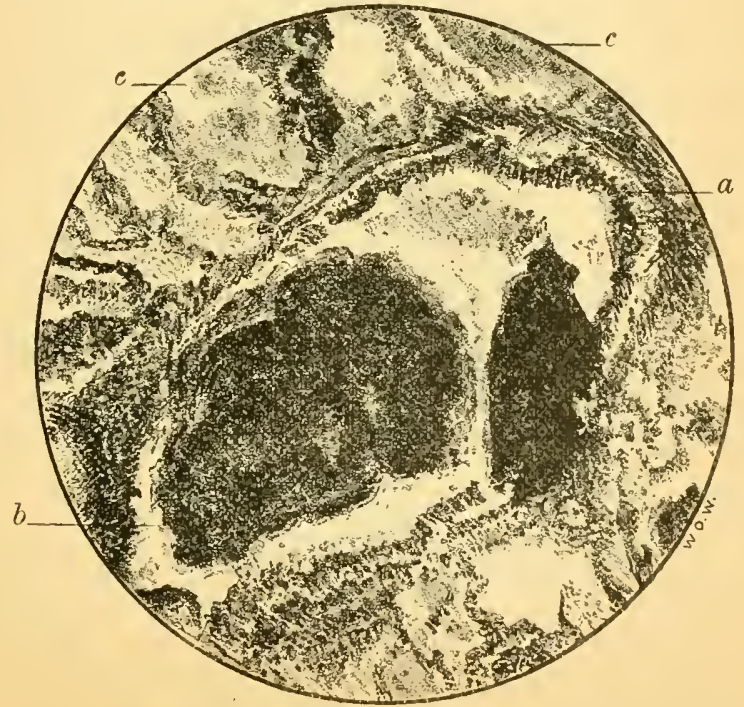

Photo-Micrograph by W. Forgan.

$\times 200$.

Fra. 6A.-Pure Pneumonia as seen in Pleuro-Pneumonia.

a. Healthy epithelium of bronchus.

b. Fibrinous plug.

c. Air vesicles containing croupous-fibrinous-exudate.

black, whilst the lobules were isolated from one another by thick septa, infiltrated with a considerable quantity of yellow limpid fluid, \&c."-See Veterinary Journal, October 1891. 
These appearances were startling, and if the investigator had been a less eminent man than he is, he might have made his conclusions from these naked eye appearances, and said it was contagious pleuro; but there were great international and commercial considerations in question, as well as the scientific bearing of the subject; and Nocard did what our own Government officials ought to have done, if not in 1879, at least in 1891. He made further investigations, and arrived at the conclusion that he had other than pleuro to deal with. $\mathrm{He}$ found, in the first place, that the infiltration of the connective tissue was less abundant, the serum less albuminous; that the tissue of the lobule in the thickened girdle of connective tissue had not the uniformity of tint and consistence that characterise pleuro-pneumonia lesion; that it was harder, more manifestly

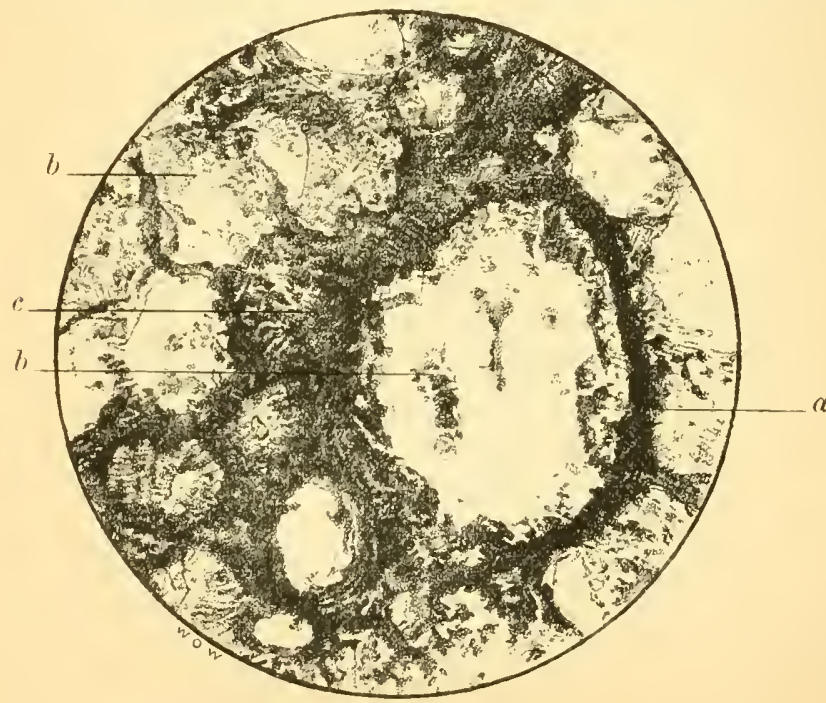

Photo-Micrograph by IV. Forgan.

$\times 200$.

Fig. 6B.-Broncho-Pneumonia as seen in "Corn-Stalk" Disease, from American ox slaughtered at Deptford 14th April 1891, and referred to in text.

a. Bronchial walls, epithelium desquamating and shed.

$b$. Catarrhal products in tubes and vesicles.

c. Increased fibrons tissue-fibrosis.

hepatised in the centre than at its periphery-the invasion being from the bronchial, and not from the perilobular tissue. "In pleuro" 
it is the opposite, notwithstanding what Drs. Yeo and Woodhead have stated, and which statements have been partly accepted by Professor M'Fadyean.

There was another important differential sign. Pressure caused a notable quantity of thick, viscid, light yellow pusanalogous to that observed in certain forms of verminous broncho-pneumonia-to issue from the bronchioles (this condition was very manifest in the Liverpool cattle in 1879); but a microscopic examination of this fluid and of the ling-pulp did not lead to the detection of ova, embryos, or worms of any kind. The bronchial mucous membrane was inflamed, thickened, corrugated, and more or less denuded of its epithelium, while the submucous connective tissue was infiltrated with yellow serum, and considerably thickened in places; but the bloodvessels contained no thrombi.

This muco-pus from the bronchioles was found to contain an abundance of short, oval, mobile bacteria, which appeared to be the only microbes present. The organisms were also found, as if in a state of pure culture, in the hepatised tissue, and more especially in the limpid serosity that distended the perilobular lymphatic sac. This single character alone sufficed to affirm that the lesion was not of a pleuro-pneumonic kind; for it is well known that the lung serum in that disease is very poor in figured elements, and that when it is collected pure from the infiltrated septa it does not usually contain any microbes.

In this instance the microbe, which existed in great numbers, belonged to the large class of ovoid bacteria of which the rounded poles fix aniline colouring matters more strongly than the centres. It is well known, as pointed out by Nocard, that the fowl cholera, the duck cholera, the septicæmia of rabbits and ferrets, the game plagne, the swine plague, \&c., all have analogous microbes, and which can only be distinguished from each other by the collective biological characters, and more especially by the effects of their inoculation on different kinds of animals.

Experimental inoculations were now made by M. Nocard, when he found that mice, rabbits, guinea-pigs, and pigeons, when inoculated subcutaneously with two or three drops of the serum or the culture, succumbed in less than forty-eight hours, without cedema at the point of inoculation, with intense congestion of all the viscera, but without any definite localisation. 
Sheep and calves inoculated subcutaneously, or in the trachea, with a cubic centimetre of culture, of serosity, or of virulent pus, did not die; they suffered, however, from intense fever, and remained prostrate, without appetite, for some days, but soon regained their normal condition.

Intra-pulmonary inoculation was more effective, as a calf of eight months and a ram of two years, inoculated in the right lung with five drops of peritoneal pus from a guinea-pig, died in less than forty-eight hours with fibrinous pleurisy and exudative broncho-pneumonia, analogous to that observed in American cattle, the lesions being very rich in bacteria. Nocard concludes that the disease does not resemble any known in France, and considers it is a malady special to American cattle, and is inclined to think that it is the affection known in the Western States as the "corru-stall disease," described by Gamgee, and more recentily by Billings, of whose observations there is an account by Bowhill in the Veterinary Journal for February 1892. And the question arose as to whether the importation of this microbic broncho-pneumonia would prove a source of danger to France. M. Nocard was able, however, from his close observation of facts, and the experimental study of the conditions of contagion, to state that the malady had only very feeble contagious properties. In three very large importations in which the disease was detected, notwithstanding the considerable number of animals in each, and their long and close contact with each other, the affection did not spread, and the cases remained isolated ones. From all these facts, his opinion was there was no urgent danger, or any necessity for special measures.

Dr. Fleming, in a leader upon M. Nocard's observations (see Veterinary Journal, October 1891), says :- "The question that now arises on this side of the Channel is one of some moment, not only from an economical, but also from a pathological point of view. Many cargoes of American cattle have been condemned because it was found that one or two in each lot were affected with what was supposed to be the specific lung plague. United States veterinary surgeons have strenuously denied the existence of that malady among cattle, and Professor Williams on two occasions, when the matter has been referred to him, has concluded that lung plague was not present, but the lesions were those of catarrhal or broncho pneumonia; and now Nocard finds 
what we are almost forced to conclude is the same disorder, examines and experiments with it, as a scientist of his position and responsibility should do, and-mirabile dictu_arrives at the same conclusions as Williams, and even designs it by the same name.'

Notwithstanding this, the North of England Veterinary Medical Association passed a formal resolution-" That the best methods of recognising pleuro were by the naked eye and hands, and that when these methods failed, little reliable assistance could be obtained from the examination of microscopic sections; that is to say, as far as determining it to be absolutely a case of pleuro-pneumonia was concerned."(Veterinary Record, 17th December).

At a meeting in Liverpool on 16th December 1891 a description was given by M'Fadyean of the post mortem appearance of pleuro, and a careful perusal of that description will at once convince any impartial reader that he labours very hard to prove the catarrhal origin of that disease. In one passage it was stated by M'Fadyean that- " The alveolar contents are by no means always fibrinous in character. In many lobules the change is distinctly a catarrhal one, air vesicles being filled with round nucleated cells, apparently the progeny of the swollen and proliferating epithelium. This change may affect the lobular bronchi also. Sometimes the catarrhal proliferation is associated with a peculiar transformation of the alveolar epithelium, that having become distinctly columnar in character. The fact that catarrhal changes are common in contagious pleuro-pneumonia has been strongly insisted on by several writers upon the subject, notably by Dr. Gerald Yeo and Dr. Woodhead."-(Veterinary Periodicals.)

With regard to the conclusions of Yeo, I have already demonstrated their fallacy; and with reference to those of Woodhead, published in the Journal of Comparative Pathology, \&c., 1888, I wish to draw attention to a review of that article by M. Delaforge, sanitary veterinary surgeon for the Department of the Seine-Recueil de Méd. Vétérinaire for 15th May 1889, page 324. He says:- "In an analysis of "Studies on the Pathological Anatomy of Pleuro-Pneumonia,' by Sims Woodhead, which appeared in the Recueil for 15th March, I read as follows: "The pathological process consists in an irritation of the mucous membrane of the smaller brouchi, followed by shedding of the epi- 
thelium of the pulmonary vesicles and a catarrhal proliferation of their elements. The virus then penetrates from these vesicles and the smaller bronchi into the neighbouring lymphatics (peribronchial lymphatics), but not into those of the bronchi," \&c. From this it appears that this author's opinion is that the initial start of the malady is in the bronchioles, and from these to the lymphatics or lymphatic spaces-the perilobular networks. This idea is radically false. Sims Woodhead mistakes the epiphenomena for the primordial phenomena-such as clinical observation shows very distinct. Every practitioner who cares to take the trouble to inform himself otherwise than through his imagination will readily perceive that at the periphery of the hepatised mass there are almost constantly met with, in an extent of $5,10,15$, and 20 centimetres, connective tissue bands, with their ramifications distended by the exudate of pleuropneumonia, without any alteration, any change even in colour, in the pulmonary lobules, which are surrounded by these advanced-guard projections. When two such projections run parallel at a short distance from each other, we can clearly see, from their point of emergence from the hepatisation, the graduated effects of the compression they exercise on the imprisoned strip of lung. The congestion and density of the lobules is then marked in decreasing proportion from the base to the terminal divergence of the two infiltrated bands (or spaces); while, on the contrary, the lobules outside them and touching them remain absolutely healthy. The clinical facts, which are very common, I repeat, prove distinctly that the evolution of contagious pleuro-pneumonia takes place from the connective tissue septa (interlobular spaces) towards the pulmonary vesicles, and not-as Sims Woodhead asserts-from the bronchioles towards these septa. The obstruction of the vesicles, the catarrhal and ephithelial transformations, the blood stasis, the pulmonary hepatisation, in a word, are evidently not the initial essence here, but rather physical corollary or epiphenomena. It is in this course, by this order in succession of the pleuro-pneumonia lesions, that in a first case, for instance, the practitioner can most certainly affirm the contagious nature of the malady; for whatever may be said and written to the contrary, there is observed in the bovine species a non-specific pneumonia and pleurisy, which may or may not be coincident, and the altera- 
tions in which are physically similar to those of contagious pleuro-pneumonia. I have had cases, the circumstances attending the origin of which, as well as the consecutive circumstances, left no doubt in my mind with regard to this. And apart from inoculation, which is not always practicable, I did not know until then of anything that had been published that informs us how we can differentiate between what is specific and what is not. Nevertheless, this means is, if not always constant, at least so in the majority of cases. To find it, it is only necessary to make an attentive and minute examination of all the periphery of the diseased parts ; if only for a few centimetres, we find an interlobular exndation advancing into the soft rosy tissue, we may be certain that we have before ns the contagious pleuro-pneumonia. It is less with the view of rectifying the above error than of establishing this indication that I request the insertion of this short note."

Further, in order to prove the catarrhal theory, $\mathbf{M}^{\prime}$ Fadyean says that the piece of lung brought to me by Dr. Wray might have had broncho as well as pleuro-pneumonia. I do not deny the possibility of the two conditions being sometimes-but, as a matter of fact, very rarely-co-existent; but it is a remarkable thing that the very many specimens of American and Canadian lungs that I have seen since the commencement of 1879 should all have been catarrhal, and the many hundreds of pleuroed ones that I have seen in this country should have the catarrhal conditions absent, and since then I have received a portion of another lung from Dr. Wray, in which the lesions are identical with those found in the 1891 lung. Portions of this lung were sent to M. Nocard, who kindly examined them, and I now append his opinion :-

"Alfort, 29th December 1891.

"Mr Drar Colleagde-You will exeuse my not having replied sooner, but I did not wish to limit myself only to an examination of the sections you sent me; for I was desirous of thoronghly studying the piece of lung you also forwarded. This study I have now made comparatively, with pieces I myself collected at the commencement of this year or end of 1890 from American cattle, the history of which I brought before the Société Centrale Vétérinaire in July last under the title of 'Infectious Broncho-Pneumonia.'

"I have made a great number of sections of your specimens and of my own, and have treated them by the same methuls of 
staining, which I have varied as much as possible. I have examined them with much care, and I believe I am now in a position to conclude:-1. The lesion which you have submitted to me is certainly not that of pleuro-pneumonia; 2. It is a bacteridian broncho-pneumonia, which, in all probability, is of the same nature as that I have already described. On the latter point I am not so positive as on the first, for although the distribution is very analogous, and the microbes have the same form and the same dimensions, and react in the same way to the different staining methods, you know as well as I do that we cannot, on these alone, affirm the identity of two microbes. In order to do this we must cultivate your bacteria, and then stndy them comparatively with mine, to see how they behave in the different culture media, and observe their pathogenic action on various animals. By doing this I shall doubtless be able to affirm or deny that we are dealing with one and the same organism. However this may be, I . again repeat that it appears probable to me that you and I have studied one and the same disease.

E. NOCARD."

A statement having been made that the microbe found was an ordinary putrefactive one, I wrote to Dr. Wray, and I append his reply, with the simple observation that when the lung arrived in Edinburgh it was free from putrefaction:-

\section{"No. 39 Belvedere Road, Upper Norwoon, LoNDon, S.E., Sth January 1892.}

"W. Williass, F.R.C.V.S., \&c., Principal,

New Veterinary College, Edinburgh, Scotland.

"My Dear Professor-In reply to yours of the 6th instant, the section of lung that I showed you on 18th April 1891 was taken from the lungs of a bullock that was slaughtered at Deptford on the afterrioon of 14th April 1891. The lungs from the said bullock were sent to the Royal Veterinary College the same afternoon. The next morning, 15th April, I inspected the lungs in company with Professor Dnguid, and Doctors Coghill and Shaw, the section I took you being cut off immediately after the inspection, Professor Duguid cutting off an adjoining section at the same time. The section I had was placed in a tin box, and there kept nutil I arrived home the same evening, was then taken out of the box and wrapped in a linen cloth saturated with a solution of bichloride of mercury [1 to 1500], and placed on ice, where it remained until 10 P.x. of the 16th, when it was taken unt of the cloth, re-saturated with the solution, then packed in a clean box, having been removed from the box twice previous to your seeing it on the morning of the 18th. I took the utmost care 
to get the section to you in as fresh a state as possible. When you saw the section I remember distinctly that it was quite fresh and free from any signs of putrefaction. If I can be of any assistance to you in this case, please command me. With many happy returns of the past holiday season to you and yours.-Very respectfully,

$$
\text { "W. H. Wray." }
$$

Now, in conclusion, I have simply to say that the American disease is not pleuro, but that it agrees in every respect with what Gamgee, Billings, and Nocard have described as bronchopneumonia, and that it is identical-but the specimens show a more chronically diseased condition - with what I discovered in both States and Canadian cattle landed in Liverpool in 1879.

One other observation. I trust that this controversy will lead to good results; that veterinary surgeons will in future have greater faith in microscopic investigations, as by such methods it has been proved that pleuro-pneumonia is a disease commencing in the perilobular tissue, and that catarrhal or broncho pneumonia proceeds from the tubes and air cells, from which pus can be pressed out, and that its best result is seen to be a desquamation of the epithelium of the inflamed tubes; that in pleuro fibrin is exuded in abundance into the connective tissue, air vesicles, bronchioles, and blood-vessels, where it coagulates into permanent clots; that pus is seldom or never found, and that in broncho-pneumonia more or less purulent matter is found, and the remarkable exudation of fibrin into the vessels, bronchi, and pulmonary tissue is notable by its absence.

In October 1892 pleuro-pneumonia was reported to have been detected in Canadian cattle bought at Dundee. This outbreak has given rise to another difference of opinion, and up to the date of going to press the veterinary officers of the Board of Agriculture maintain the correctness of their opinion. The coloured drawing, page 176, is an exact reproduction of the miscroscopic appearance of a portion of the lung in dispute. 


\section{CHA PTER XVIII.}

CONTAGIOUS DISEASES-continued.

\section{ECZEMA CONTAGIOSA.}

Synonyms.-Eczema epizootica; epizootic aphtha; aplithous fever; foot-and-mouth disease; murrain; epidemic.

Definition.-A highly contagious and infectious febrile disease, associated with a vesicular eruption in the mouth, between the pedal digits, and around the coronets. In some cases the mouth only is affected, in others the feet may be the seat of the eruption, the membrane of the mouth remaining free. In milch cows it sometimes happens that a vesicular ermption occurs on the mammary gland, and within the lactiferous ducts: when such occurs, the milk, contaminated by the vesicular discharge, is rendered unfit for use, either as food for the human being or for the lower animals, as it may induce a vesicular eruption in the mouth, larynx, pharynx, and intestinal canal.

\section{PATHOLOGY AND SYMPTOMS.}

Unlike rinderpest and pleuro-pneumonia, which are almost confined to the ruminantia, contagious eczema affects cattle, sheep, pigs, dogs, poultry, and even human beings are subject to the influence of its virus. It is amongst horned cattle that we find the ravages of this disease to be most extensive, they seemingly being most susceptible to the influence of the contagious poison; sheep and goats, however, suffer very severely, and dogs, pigs, and poultry often die from it.

After a period of incubation, varying from twenty-four hours to three or four days, the invasion and progi'ess of the disease are characterised by an elevation of temperature of from two to four 
degrees; by the formation of vesicles, varying in size from that of a sixpence to that of half-a-crown, on the tongue, inside the lips, roof of the mouth, and sometimes on the udder; whilst smaller blisters make their appearance between the digits, and around the coronets and heels, and there is a discharge from the eyes and nose. The animal presents signs of uneasiness in the mouth, by constant movement of the lips, champing of the teeth, a flow of saliva from the mouth, and difficulty in masticating its food; the affection of the feet being indicated by some degree of lameness in the foot or feet affected. In a short time the epithelial and cutaneous structures enclosing the vesicles are separated and thrown off in more or less rounderl patches leaving raw surfaces (see Plate VI., p. 182), which are, however, speedily re-covered by epithelium. In some instances there is an entire separation of the hoofs from the feet.

The symptoms are undoubtedly due to the presence of a contagious micrococeus in the animal economy, and are manifestations of the elimination or excretion of a virus, which evidently has some special affinity to the mucous and cutaneous structures of the several parts of the body which it invades. Eliminated by the feet and by the mouth, pastures very soon become impregnated with it, and hence we find that, owing to the great facility by which it is thus spread over a wide district of country, it appears to be the most infectious of maladies. Beyond question the virus of eczema is conveyed from field to field, from parish to parish, from county to county, by small game, vermin, and dogs ; and looking to this fact, we can account for the sometimes mysterious outbreak of the disease in places and districts into which no fresh cattle have been admitted. This frequently unaccountable appearance of the foot-and-mouth disease has led to the belief that it is of spontaneous origin; some believing that, although originating spontaneously, it is propagated by contagion; others that it originates in all cases independently of contagion, and that it is due to climatic or atmospheric causes, exposure, and the debilitating effects of long journeys by land or sea. It is very true that increased susceptibility to the disease is induced by any debilitating cause, but neither age, condition, management, climate, temperature, nor any common cause of disease seems to exert any modifying influence. No extremity of privation, nor the continued action of ordinary causes, is 
capable of inducing it; and one reason for the indifference which has been shown in respect to its ravages is to be found in the belief in its spontaneous origin, an idea which arises out of the observations of its frequently unaccountable appearance in isolated places.-(Professor Brown on Mouth and Foot Complaint.)

It was at one time believed that an animal which had once suffered from eczema contagiosa was guarded against another attack. This, however, is a fallacy; and it is now generally believed that an animal is only once attacked during a season. As a general rule this may be said to be the case, and consequently a herd which has gone through the disease early in the seasonlet that be the spring or autumn, the periods when, in consequence of the increased movements of cattle from place to place, the disease is most rife-is considered more valuable to the purchaser, and worth more money in the market. This rule is, however, liable to many exceptions, and it is by no means impossible for the same animal to suffer from two, three, or even four attacks during a period of a few months.

The microbe of this disease has been described by Nosotti, who says that in the pure liquid of the vesicle a micrococcus, easily stained with the aniline dyes, is constantly found, and which he has successfully cultivated and inoculated. It is both volatile and fixed, and can be transmitted directly and indirectly to healthy animals. Klein describes the microbes as diplococci or streptococci, which sometimes form little chains with a score of sections; if grown in coagulated serum or gelatine, they produce thin membranes marked with fine points formed of very delicate globules, and, by mixing these cultures with food, he has produced the disease in sheep, but subcutaneous inoculations have failed to have any effect. The blood corpuscles are very often stellate or star-like in form, and rapidly change to their natural circular shape; there is, however, no speciality in this as regards the blood of cattle, as the same thing occurs in splenic apoplexy, rinderpest, and even when an animal is apparently in a healthy condition. Professor Brown attaches some importance to the presence of vibriones, bacteria, and monads, which are found in the discharge from the eyes, saliva, and the contents of the vesicles. The milk also contains these bodies, and upon this head Professor Brown is very clear and distinct. He says"Milk from cows affected with foot-and-mouth disease acts energetically upon young animals to which it is given warm. 
Calves occasionally die quite suddenly after sucking cows affected with the malady; and fatal effects have followed the administration of the milk to young pigs. It has been alleged that there is not any direct evidence of injurious consequences arising from the consumption of the milk by human beings; but it must be remembered that the fluid is very rarely taken immediately from the cow, and still more rarely in an undiluted form, and it may be not altogether an unfortunate circumstance that the decrease of the secretion during the prevalence of the disease necessitates a large admixture of water in order to keep up the quantity which is required for daily consumption.

"In the early stage of the disease the milk presents few abnormal characteristics, the specific gravity falls to 1024, and the milk corpuscles are always found to be arranged in clusters.

"Minute moving specks are also seen under the quarter-inch objective, and those under the $\frac{1}{25}$ th are resolved into bacteria and spherical bodies.

"When the disease is fully developed, about the third day from the first appearance of vesicles, the milk invariably contains morbid products of a very pronounced character, viz., large granular masses of a brownish yellow colour, numerous pus-like bodies, bacteria, vibriones, moving spherical bodies, and a few milk corpuscles. It is particularly worthy of remank that these morbid elements were found in specimens of milk which in their physical character presented no appreciable peculiarity.

"In some specimens which were viewed with the micrometer eye-piece the milk corpuscles varied in size from $\frac{1}{2000}$ th to $\frac{1}{10,000}$ th of an inch in diameter, and the granular masses from $\frac{1}{500}$ th to $\frac{1}{1000}$ th of an inch. Milk from animals affected with cattle plague, and also with pleuro-pneumonia, was also found to contain an abundant quantity of the granular masses and puslike bodies; and in cases of cattle plague similar elements weredistinguished in the curdy exudation which existed in the mucous membrane of the mouth, pharynx, trachea, and bronchial tubes.

"Examples of milk taken from animals in different stages of foot-and-mouth disease afforded very interesting results. At the commencement the specific gravity fell to 1024-5, and continued to range between the two numbers until the animal was convalescent, when it rose to 1026-7, which standard was not exceeded for two months after recovery. 
“ The granular masses and pus corpuscles decreased in number as the affection subsided; but in all the specimens examined after the animals had recovered, they were found scattered here and there among the milk corpuscles, and even in specimens which were examined a month after recovery they were detected. The granular masses were not found in milli from the same animals two months after recovery; but even in these specimens a few pus-like corpuscles were present.

" Two examples of milk taken from cows on the fourth day of the disease were found to be highly charged with granular masses; the milk, however, was remarkably rich in quality, having a specific gravity of 1034 , and yielding a large proportion of crean. Diminution of the quantity of milk is invariably observed during the progress of any febrile disease, and in footand-mouth complaint the loss is sometimes considerable.

"Cows, when suffering from the worst form of disease, lose nearly all their milk; but when the attack is mild in character, the decrease will not be more than one-third of the usual yield. The average loss in a large dairy while the disease is going through the sheds will vary from one-third to two-thirds, according to the number of severe cases. As all the milk obtained is mixed, the worst milk will be to some extent modified by the addition of that which is less highly charged with morbid elements, and the whole is further diluted by the addition of water, which, judging from some specimens obtained from an establishment where the disease was known to exist among the cows, is sometimes added to the extent of 40 per cent. Boiling the milk has been recommended for the purpose of preventing or lessening its injurious action; but, as a matter of fact, it may be stated that boiling does not alter the appearance of the morbid elements, nor does it arrest the movements of bacteria "in the fluid."

In ordinary cases of eczema the symptoms are not very severe, and provided the feet are not seriously implicated, the animals recover in about a week from the first manifestation of the febrile symptoms. In many instances, particularly if the weather he cold and the cattle exposed, a hoarse tracheal cough is present, with much discharge from the trachea and bronchial tubes, whilst in aggravated cases the mucous membrane of the intestinal canal is very seriously involved, and when vesicles appear 
at the anus, $\mathrm{it}$ is fair to presume that vesication exists along the whole alimentary track; and post mortem examinations of such reveal the presence of much inflammation of the intestinal mucous membrane, patches of denudation here and there, more especially in the fourth stomach, giving the disease very much of the characteristic appearance found in cattle plague, to which disease it is to some extent allied.

It is when the feet are much affected that the loss to the stock-owner becomes great, for not only does the animal lose condition from the disease, but also from inability to roam about its pasture in search of food. Such cases should at once be comfortably housed, provided with proper aliment, and have the feet properly treated.

In sheep, goats, and pigs, the feet are the principal parts affected, the vesicles occurring between the digits and around the coronets. ${ }^{1}$

During the summer of 1872 eczema contagiosa assumed a very malignant character, especially in some herds of Iceland cattle brought to Leith and Edinburgh, the ordinary symptoms being complicated with swelling of the legs and other parts of the borly, the swelling when opened discharging a very fotid fluid. In all there was great prostration of strength, and many cases terminated fatally.

The loss from foot-and-mouth disease to the dairyman, from the diminished secretion of milk, and to the owner of cattle nearly fit for the butcher, is very great. I do not think $\mathfrak{E} \pm$ a head too lhigh an average; but when it is confined to lean or store cattle, provicled the attack is mild, the loss of condition is quite ephemeral, and can scarcely be accounted a loss at all, for such animals appear to make flesh much faster after than prior to an attack of the disease.

As sequele to eczema contagiosa, the following alterations of structure have been observed, namely, swelling of the joints, open joints, with sloughing of interdigital substance, gangrene of a whole foot, marasmus, and disease of the bones.

1 For distinguishing character of foot-and-mouth disease and foot-rot in sheep, see Principles and Praetice of Vetcrinary Surgery. 


\section{TREATMENT OF ECZEMA CONTAGIOSA.}

Mild cases require little medical treatment, foot-and-mouth disease being one of that kind which, rmming through a definite course, terminates in recovery. Whilst the mouth is very sore, all that the animal requires is a plentiful supply of cold water: if the fever be high, the water may contain an onnce of nitrate of potash dissolved in each bucketful. As a rule, however, even this is unnecessary. The food should be soft and easy of mastication-bran mashes, pulped roots, or grass. The feet must be looked to, and if suppuration be detected, with separation of some parts of the horn, the detached horn is to be removed, and the exposed sensitive surfaces dressed with a mild astringent, such as a solution of acetate of lead, containing a small modicum of carbolic acid. What I usnally prescribe is an ounce of the concentrated solution of pure carbolic acid-one part acid to sixteen hot water-to twelve of the white lotion. Pledgets of tow dipped in this lotion are to be bound to the foot by a bandage. This simple expedient, so generally neglected, will often prevent a lameness of many weeks' or even months' duration ; for suppuration, once established to any great extent in the sensitive structures of the foot, causes violent pain, increases the inflammation, and induces the process of slonghing. Some writers recommend a variety of remedies both to the mouth and feet. My experience leads me to the conclusion that, except carefully washing the feet, the less medical interference the better.

Now and then cases will be marked by extreme prostration of strength, entire loss of appetite, inability to stand from weakness as well as from the lameness; such ought to be more actively treated, and stimulants, such as whisky, brandy, or spirits of nitrous ether, administered internally, and the feet washed repeatedly with cold water, and kept cool with wet cloths fastened around them. In all instances, bleeding, purging, and other depleting measures are most injurious. The bowels, perhaps constipated at first, become loose as the disease advances; but this condition is not to be interfered with, for the diarrhoea may be looked upon as one result of the vis medicatrix naturce, by which the morbid material is expelled from the system. Extensive sloughing of the structures of the feet is best treated by more powerful antiseptics-solutions of chloride of zinc, or 
strong solutions of carbolic acid, say one part of crude acid to six or eight of oil.

Sheep lose flesh very rapidly whilst suffering from the footand-mouth disease. They suffer more in the feet than cattle, and consequently require more careful vigilance. It is a good plan to cause the affected flock to walk through a shallow trough, containing the above-named weak astringent and antiseptic solution, once or twice a day. And finally, it is my opinion that both cattle and sheep orght to be washed before they are allowed to be driven on a public road or exposed in a market, if they have recentiy recovered from this disease. It may be difficult to do this with large herds of young cattle, and its compulsion would be considered a liardship, but of two evils it is incomparably the lesser. 


\section{CHAPTER XIX. \\ CONTAGIOUS DISEASES-continuel}

\section{INFECTIOUS ABORTION.}

Abortion in cows is often seen as an epizootic, both in this and other countries, and its pathology has been profoundly studied in France and Switzerland, more particnlarly by Nocard, and its elinical aspect by Strebel, Galtier, de Poncius, and Org; ly Lavat, who has witnessed it in sheep; by Biot, who eonsiders it as an infectious inflammation of the serous lining of the uterus; and by Herr G. Sand, Copenhagen, and Professor Bang. In this country, $11^{\prime}$ Fadyean, Woodheal, and Aitken endeavoured to throw light upon its pathology. Their experiments were repetitions of those of Nocard, crudely performed, and leal to no fresh conclusions; but a very valuable paper was read upon the sulject by Mr. Harry Olver, F.R.C.V.S., Tanworth, at the meeting of the National Veterinary Association at Birmingham, 1895 .

Mr. Olver, who seems to have had great practical experience, rightly describes the disease under two heads, namely, noncontagious and contagious abortion, and states that abortion occurs at from the third to the seventh or eighth month. $\mathrm{He}$ also states that sporadic abortion is due to many eanses, such as cold, wet, fright, injuries, food, water (when drunk icy eold); but he traverses the opinion of C. Stephenson and others, that stagnant water contaminated by sewage has any appreciable effect. ${ }^{1}$

Contagious abortion rarely occurs in the mare, ewe, or goat, and still more rarely as an epizootic in this country, but cases are recorded of undoubted outbrealss in America, France, Australia, \&c.

1 See Transactions of National Veterinary Asscciation, 18.5. 
The disease occurs at all periods of gestation after the third month, and in the same animal at a later stage in each succeeding pregnancy, until at length the foetus is carried its full term, if the mother has not become sterile. The calf is generally dead at birth, but in some instances it is born alive; it is, however, generally in bad health, emits a peculiar lowing, and after the third clay is attacked with diarrhoea, which rapidly terminates fatally.

Abortion succeeding to fright or injury may become infectious ; hence arose the supposition that it was due to sympathy, and thus spread from cow to cow in a byre. It is true that there is often great excitement amongst the cows in a byre during and after abortion by one of them, and that there is also often a peculiar odour emitted by both mother and offspring. It has also been attributed to ergotism, monldy food, cold, contusions, unwholesome drinks, \&c. All these may be predisposing causes, but it is now fairly demonstrated by Nocard and others that the virus exists in the discharge from the mother, and in the uterine fluids, and that by the introduction of these fluids from an aborted cow into the vagina of healthy ones abortion will be induced in from nine to twenty days.-(Brauer.) Lehnert thus produced it in twelve to twenty days, and Trincherra obtained a purulent vaginal catarrh and abortion in from nine to nineteen days. Inoculation matter obtained by scraping the surface of the chorion of an aborted cow produced a like result. The transmission from cow to cow is most probably by immediate contact of the material with the genitals of cows in the same byre, the discharges being conveyed by the groop or drain which is immediately behind the cows in all byres.

It can easily be understood how the virus thus conveyed contaminates the tails and hind extremities of the cows when recumbent, and how easily natural inoculation is thus effected by the movements of the tainted tails, more especially if the drain leads from the aborted to the healthy ones.

Nocard states that abortion takes its origin in diverse germs met with in the uterus of aborted cows, but which are never found in healthy ones. These germs are also present in the amniotic fluid in the alimentary canal of aborted calves, as well as in the medulla oblongata of those which during life give utterance to the peculiar lowing sound already referred to. 
Nocard states-"The autopsy of diseased animals does not reveal any alteration of the thoracic or abdominal viscera, with the exception of the uterus. The peritoneal serous membrane is normal, incision of the uterus reveals the presence between the mucous membrane and the chorion of a fibrinous mucopurulent matter, which is more or less abundant and often acid, in which the microscope shows epithelial cells, leucocytes, a large number of isolated micrococei, which are generated or associated in short chains of three, four, or five sections, and a few short bacilli, which are isolated or associated in pairs; in the cotyledonous liquid the bacilli are predominant; in the product obtained by scraping the uterine mucous membrane both micro-organisms exist in almost equal numbers. The amniotic fluid also contains them. In the aborted foutus the intestinal mucous membrane is the seat of abundant epithelial desquamation; its tissue seems infiltrated with various microbes, which also exist in abundance in the contents of the intestines. These micro-organisms, which are contained in the digestive tube, give an explanation of the diarrhcea by which the calves which have been aborted in an advanced state of gestation are affected in two or three days after birth. Micrococei identical with those found in the amniotic fluid are also found in the medulla oblongata in those ealves which continually bellow during the days preceding death." Nocard arrives at the following conclusions:-

1. In aborted cows, even in primipara, there exists in the uterus, between the mucous and the fotal membranes, especially in the cotyledonous crypts, several micro-organisms which cannot be found in newly calved cows, which belong to a locality where abortion does not exist.

2. These micro-organisms do not seem to exercise any noxious action upon the uterine mucous membranes of the mother, either during the period of gestation, which must be interrupted suddenly, or even after abortion.

3. Repeated abortion in the same subject can easily be explained, if we admit the pathological influence of the nicrobe, by the persistency of the latter in the uterine cavity till the time when it is able to exercise its action on a new foetus or on its envelopes. 
4. Also cases of sterility which are consecutive to abortion can be explained by the acid reaction of the uterine mucus, in which the microbes thrive, the spermatozoa not being able to preserve their vital properties except in alkaline elements.

5. Epizootic abortion seems indeed to be a microbic disease of the fotus and its envelopes, but one which does not affect the mother.-(REvBET, 1889.)

This form of abortion, while prevalent in certain districts, is unknown in others, but it may be conveyed from one district to another by the introduction of cattle from an infected area. Upon this point a mass of evidence is adduced by Sand, ${ }^{1}$ Royal Teterinary College, Copenhagen, who hints at the conclusion that abortion, independently of its isolated sporadic occurrence in widely separated districts, occurs in an epizootic or enzootic but non-contagious form from accidental causes, such as musty food, bad or cold water, insanitary surroundings, stall feeding, and in-and-in breeding.

In addition to the evidence as to its spread by the introduction of affected cows, proof is brought forward establishing the fact that the disease is also introduced by using bulls from an infected herd, and instances are recorded where bulls have been known to suffer from severe preputial inflammation after having served diseased cows. It is also stated that the vaginal mucous membrane is reddened, permeated throughout with cordlike swellings, and showing numerous small reddish-yellow or reddish-brown nodules. There are also small eruptions about the root of the tail, anus, and vulva. These symptoms, however, are not common, but there is often a chronic purulent discharge from the vulva.

As already stated, a first abortion occurs earlier than later ones, often at the third or fourth month, whilst the period is gradually extended in later ones, and the explanation seems to be that the microbes gradually lose their virulence, until finally the animal has immunity. This fact is very important, and contradicts the generally adopted method of eradicating the clisease, namely, that of disposing of aborted cattle and replacing them with fresh ones, which in their turn become affected and maintain the continuance of the disease in a herd; whereas, if aborted cattle are kept, only those proving sterile being disposed

I See Infectious Abortion, by Jno. A. IV. Dollar,-Veterinarian, June 1893. 
of, they become immune, and the disease dies out in from two to four years, as it gradually loses its virulence, appears at a later period of gestation, and attacks fewer animals.

The best and cheapest method of disinfection is to thoroughly cleanse the byres by washing and scrubbing, and then brush them over with a hot 1 per cent. solution of chloride of lime. Everything should be thoroughly cleansed twice a year, the stalls, walls, and floors once a week, and the drains daily rinsed with the chloride of lime solution. Animals showing signs of abortion should be at once isolated and provided with separate attendants.

When the fotal membranes are retained more than twelve hours they should, as far as possible, be removed by hand, and, together with the dead fotus, destroyed by fire or buried in quicklime. The uterus should be washed out with 1 per cent. creoline, or 5 per cent. lysol solution, and this should be continued weekly or oftener as along as vaginal discharges continue (usually two or three months after calving), and the cows not put to bull until their entixe discontinuance. This both prevents the spread of the disease, and allows the majority of the animals to become pregnant.

In the apparently healthy cows and calves the vagina should be carefully washed out before they are allowed to enter the cow-shed, and afterwards the vulva, tail, and surrounding parts washed daily with the same solution, and the sheaths of infected bulls should be similarly washed out.

Or Nocard's recommendations may be substituted. They are as follows :-

(1.) The floors, \&c. are scraped, thoroughly cleansed, and sprinkled with a solution of sulphate of copper.

(2.) Each week from the date of conception the vaginæ sliould be thoroughly injected by means of a large syringe with the following tepid solution :-

Distilled water,

36 per cent. alcohol and glycerine, aa. 3 ounces, $=$ iii.

Bichloride mercury, $2 \frac{1}{2}$ drams, . . 3ii.ss.

The vulva, anus, and surrounding parts of all the pregnant cows should be carefully sponged with the same solution.

After removal of the foetal membranes of an aborted cow, the 
uterus should be irrigated, by means of a long tube introduced to the bottom of its cavity, with eight or ten quarts of the solution (tepid), but containing only half of the sublimate.

Aborted fotuses and fotal membranes should be destroyed by fire or boiling water.

In the cow abortion generally takes place from the third to the seventh month; in the mare from the fourth to the ninth; and the act will be indicated by redness of the vaginal mucous membrane, on which pimply eruptions are often seen; there is a discharge from the vulva of a reddish fluid, and a diminished secretion of milk, which becomes thick, simulating colostrum. These signs may continue for three days, when the fotus is easily expelled, leaving no other symptom than an increased vaginal discharge.

It lias been stated by some writers that the administration of half-ounce doses of crude carbolic acid prevents the spreal of infectious abortion. 


\section{CHA P TER XX.}

\section{CONTAGIOUS DISEASES-continued.}

\section{VARIOLA VACCINA (COW-POX).}

\section{Synonyms.-Vaccine (F.); Kuhpocken (G.)}

Definition.-A contagious, febrile, and eruptive disease, known in most parts of the globe, resulting from the presence of a specific fixed virus, which is reproduced and multiplied in the animal body luring the course of the malady. After a period of latency of from six to nine days, the contaginm causes the development of an eruption on the surface of the mammary gland, which eruption passes throngh the stages of pimple, vesicle, pustule, and scab. The disease runs a definite and mild course, and destroys the susceptibility of the affected animal to another attack; but in hot countries it sometimes assumes a diffuse and severe character.

\section{PATHOLOGY AND SYMPTOMS.}

Cow-pox has been described by high medical authorities as a malignant disease which destroyed cattle almost as extensively as small-pox did the human race. This view of the malignancy of cow-pox is evidently the result of a pathological error, which led observers to confuse the cow-pox with cattle plague.

Jenner believed in the identity of cow-pox and small-pox, and that both had a common origin in the "grease" of the horse. Jenner did not, however, perform any inoculations of cattle with the lymph of human small-pox. The first successful experiment of inoculating the cow with human small-pox was performed at the Berlin Veterinary College in 1801; the efforts previous to that period having been unsatisfactory. In 1807 Gassner of 
Giinsburgh inoculated eleven cows with small-pox matter, the result of which was the production upon one of them of vesicles having all the character of vaccine vesicles, and from which a stock of genuine lymph was obtained. In 1830 Dr. Sonderland of Barmen infected cows with the disease by enveloping them in blankets taken from the bed of a patient who had died of smallpox. Various other experiments have been made to this end by Mr. Ceely of Aylesbury, Mr. Badcock of Brighton, and by Mrr. Macpherson in India. Mr. Ceely, in the tenth volume of the Transactions of the Provincial Medical and Surgical Association, gires a most interesting history of the communication of the disease to cattle from man without inoculation. "At the village of Oakley, about sixteen miles from the town of Aylesbury, small-pox had been epidemic from June to October 1840. Two cottages, in which three persons resided during their illness, were situated on each side of a long narrow meadow, comprising scarcely two acres of pasture land. One of these three patients, though thickly covered with pustules of small-pox, was not confined to her bed after the full development of the eruption, but frequently crossed the meadow to visit the other patients, a woman and a child, the former of whom was in great danger from the confluent malignant form of the disease, and died. According to custom she was buried the same evening; but the intercourse between the cottages across the meadow was still continued. On the day following the death, the wearing apparel of the deceased, the bed-clothes and bedding of both patients, were exposed for purification on the herges bounding the meadow, the chaff of the child's bed was thrown into the ditch, and the flock of the deceased woman's bed was strewed about the grass over the meadow, where it was exposed and turned every night and for several hours during the day. This purification of the clothes continued for eleven days. At that time eight milch cows and two young heifers were turned into the meadow to graze. They entered it every morning for this purpose, and were driven from it every afternoon. Whenever the cows quitted the meadow the infected articles were again exposed on the hedges; and the flock of the bed was spread ont on the grass and repeatedly turned. These things remained till the morning, when the cows were readmitted, and the contaminated articles were supposed to be withdrawn, It appears, 
however, that the removal of the infected articles was not always accomplished so punctually as had been enjoined, so that, on one occasion at least, the cows were seen in the midst of them, and licking up the flock of the bed which lay on the grass. These cows were in perfect health when first put out to graze in this meadow; but in twelve or fourteen days five (ont of the eight) milch cows appenred to have heat and tenderness of the teats. The teats became swollen, and sinall, liard pimples could be distinctly felt upon them as if imbedded in the skin. These pimples daily increased in magnitude and tenderness, and in a week or ten days rose into blisters-vesicles-passing into brown or blackish scabs. When the teats were in this conclition and very tender, constitutional symptoms of ill health became developed. Sudden loss of milk, drivelling of saliva from the mouth, frequent inflation and retraction of the cheeks, staring of the coat, tucking up of the flanks, sticking up of the back," and rapid loss of flesh, were the appearances observed. By the middle of the third week the pustules were matmre, "and the srusts and loose cuticle began to be detached." One interesting fact was brought out in the course of this outbreak, namely, that heifers may be affected with variola as well as milking cows, an occurrence which up to this period ras supposed to be impossible. The reason for this is obvious, heifers before calving, bulls, and oxen not being subjected to the same chances of accidental inoculation as milking cows.

The interesting papers on this subject by Mr. Ceely, published in the eighth and tenth volumes of the Transactions of the Provincial Medical and Surgical Association, and the Reports of the Vaccination Section of the British Medical Association, contain many important facts bearing upon the identity of variola vaccinæ and human small-pox, and are well worth the careful study of every veterinary surgeon. But whilst these experiments seem so clearly to prove the identity of the variola of man with that of the cow, those of Chauveau point to an opposite conclusion. He performed numerous inoculations with small-pox lymph on the cow, which induced papular pustules, having no analogy with those of cow-pox. The lymph from those pustules implanted on man produced small-pox and not cow-pox; and it is stated in the Boston Medical Journal for 1860 that during that year Martin inoculated some variolous 
matter, taken from a pock upon the body of a man who had died of small-pox, into a cow's udder, and subsequently vaccinated some fifty persons with the matter derived from the cow. Most of those so inoculated were attacked, not with vaccina but with variola (small-pox), and three died. It is diflicult to reconcile such a wide difference in the results of experiments seemingly carefully performed, but I am not prepared to admit that the experiments of Chanveau and those of Martin have satisfactorily proved that those of others were wrong. In 1836 Dr. Basil Thiele of Kasan, in South Russia, inoculated some cows on the udder with the virus of human small-pox, the result being the production of vesicles bearing all the characteristics of the true vaccine vesicle. The lymph so produced from the variolation of the corvs continued to retain the specific properties of variola vaccinæ throughout seventy-five successive transmissions to the human body; and it is not stated that it had lost its specificity after so many transmissions.

In the face of such conclusive evidence, I think we are justified in concluding that in the experiments made by Chauveau, the virus, granting that it was properly selected, had not undergone that alteration during its transmission through the bodies of the inoculated animals which modifies and mitigates its virulence under ordinary circumstances, and that the virus selecied by Martin from a fatal case of small-pox was not proper for an experiment where human life was at stake.

Many experiments might be quoted (Ceely, Thiele, and others) to prove that great uncertainty and difficulty often attend the attempts to transfer the virus of variola from one animal to another; that when variolous disease affects the lower animals in a malignant form, it is capable of producing by inoculation a disease of similar severity in man, and that marked improvement sometimes takes place in the energy and in the quality of the virus by subsequent removes or inoculations in animals of the same kind, after the variolous virus had been successfully implanted in one of them; it seems to become less acrid, less virulent, and to acquire increased specific activity; capable of inducing more pronounced and perfect development of vesicles, with milder and less dangerous general symptoms.

Variola vaccinæ has been transmitted back to the cow from 
inan (retro-vaccination). By this process good human lymph loses some of its activity, for, aceording to Mr. Ceely, the phenomena in man, after vaecination with this retransmitted lymph, appears later, smaller vesicles are produced, but ultimately, after suecessive reinoculations on man, it regains its aetivity.

The disease vulgarly termed "grease" in the horse, believed by Jenner to be the origin of variola, has nothing to do with small-pox or cow-pox; but it is placed beyond a doubt that the horse is subjeet to a true equine pox, and that it is transmissible to the eow by inoeulation and eohabitation. It is stated that the equine pox oecurs as an epizootic when small-pox is epidemic. When small-pox was raging in Edinburgh in 1872, I saw one case of what I considered genuine equine pox. The animal (a mare) presented signs of fever, had a slight cough, loss of appetite, with eostive bowels for eight or ten days before any skin disease was observed. She was treated for a common cold, with fever; but at the end of the time mentioned small pimples were observed on varions parts of her body, more particularly about the shoulders and back. At first these pimples were very numerous, small, and pointed ; the great majority of them, however, withered, and a small seab fell off, leaving the skin bare of hair. About a dozen on each side of the shoulders aind upon the back increased in size, became vesicular, and afterwards pustular. These eruptions had very much of the eharacter of those seen in cow-pox, namely, pimples increasing in size from a point to that of a horsebean, beeoming vesicles in three or four days, and then pustules, depressed in their centres. In about ten days, or eighteen after the first manifestation of illness, dark, thiek, solicl scalss formed on the sites of the pustules. These scabs did not all fall off until fully a month had elapsed, and the hair was not completely restored in two months afterwards, when I lost sight of her. Jenner, Saccho, and others, have used equine lymph for the purpose of vaceination, and hmman small-pox has been transmitted through the horse to the cow, and so to the ehild in the form of cow-pox.

It has not been determined whether the small-pox poison originated in man, the eow, or the horse; whether man had the disease communieated to him from the lower animals, or whether horses and eows had it from man. The origin will most probably for ever remain a mystery, and we must be content with the 
knowledge that, through the keen penetration of Jenner, the disease directly communicated by vaccination from the lower to the higher animal has proved a blessing to millions of the human race.

There are many forms of eruption in the udder of the cow, which are sometimes confounded with those of variola; but those of variola have certain characteristics which distinguish them from all other, or so-called spurious, forms of the disease. The local symptoms of the true variola are heat, swelling, and tenderness of the teats for three or four days, followed by irregular pimply hardness of the skin, more particularly about the base of the teats. The pimples may sometimes be felt in five clays after communication; they assume a red hue when about the size of a pea, are very painful and hard, gradually increase in size, and in other three or four days attain that of a horse-bean, assuming a circular form on the udder, and an oblong on the teats. They rise in the centre, become more or less pointed (acuminated), containing at first a clear, and ultimately a turbid fluid. If the vesicles are broken, troublesome sores supervene, the discharge from which will communicate the disease to the milker, if he is not already protected by previous vaccination. The vesicles, if not broken, become depressed in their centres, and have an indurated margin, around the circumference of which the skin denotes active inflammation by a circle of redness, acquiring their maximum size about the tenth day, and are then pustular; and as the pustules dry, dark brown or black solid scabs or crusts form upon the surfaces. Some of these scabs may be seen semi-detached, others entirely so, exposing a raw surface, with a slight central slough. Tesicles, pustules, and scabs may be witnessed on the sane teat at one and the same time, indicating the formation of new crops of vesicles at different periods. The crusts, if left undisturbed, gradually become thicker, darker, and more compact until about the fourteenth day, and spontaneously separate about the end of three weeks, leaving shallow, smooth, oval, or circular pits of a pale rose colour, with some traces of surrounding induration.

The depression in the pocks is accounted for in various ways. Dr. Petzholdt thinks it is caused by the ducts of the cutaneous glands, which are ruptured as the pustules fill with pus and maturate, but which, in the early period of the eruption, bind 
down the cuticle to the cutaneous glands, thus producing the pit or umbilicus.

Dr. Gustav. Simon differs from the above opinion, and says that "variolous pustules are not always constituted alike. In many cases, where a central depression clearly existed, the epidermis was entirely raised from the subjacent cutis; and only at the spot corresponding with the umbilicus were both membranes united by a thin, whitish cord, which, as the microscope evidently showed, was a hair sac. The presence of a hair sac is not, however, essential to the production of an umbilicus, and a remarkable peeuliarity, first noticed by Rayer, characterises the variolous vesicle in those parts of the body where neither hair saes nor sebaceous follicles exist. Rayer found, on removing the epidermic cap of the vesicle, and wiping off the fluid collected beneath, that a little elevation existed in the centre of the denucled corium, whilst the circumference was visibly depresser below the level of the neighbouring healthy cutis. These elevations were found by Dr. Simon, who examined them microscopically, to consist of a file of papille of normal or nearly normal size and condition, whlle the depressed margin is paved with papille bent down or flattened. The explanation is simple. At the centre of the vesicle an organic connection (from some yet mexplained cause) exists between the cutis and cuticle, and at this point no fluid is effused; but around this centre, exudation of fluid ocenrs without imperliment, and tends to force the eutis and cuticle asunder."-(British and Foreign Medico-Chirurgical Teview, vol. iii.)

About the ninth day of the vaceine disease a purely vesicular eruption makes its appearance upon the udder. Within twentyfour hours the resicles contain a pellueid serous fluid. On the following day the fluid becomes turbid, the cuticle collapses or bursts, and a thin, brittle, flimsy crust forms, and speedily falls off. Successive crops continne to form and dessicate for three or four rreeks.

It is difficult to trace the origin of an outbreak of variola vaceinie in a herd of cattle. Outbreaks of it seem to follow similar inexplicable paths to those which characterise other contagious diseases. Cases spring up now and then which appear to be solitary, and cannot be traced to a cause. At other times it seems to be epizootic, and prevails at several farms at the same 
time. Unlike, however, the other contagious diseases of horned cattle, and unlike variola ovinæ, it is a disease which seldom or never proves fatal in this country. In some instances, the constitutional disturbance is rather severe, the mucous membranes being particularly prone to participate; the mouth becomes sore, and there is drivelling of saliva.

In hot climates the symptoms are succeeded by abdominal pains, profuse diarrhoea, rapid wasting of flesh, and sometimes death; but in temperate climes the general symptoms are slight climinution in the secretion of milk, with but little or no loss of appetite.

The contagium of variola vaccinæ is an extremely small form of micrococcus, usually called the Diplococcus varioles et vaccince (see Plate), in clear lymph, but in opaque, orange, and brown lymph there may be three or even four forms of cocci, the characteristic ones being in pairs or in quadruple arrangements, which latter many think the typical form. From the small size of the germs in vaccine and variolous lymph, Buist considers that they are more to be considered spores of micrococci than fully developed micrococci, and that this accounts for the less intense local effects after vaccination with clear lymph. Among the larger forms in opaqne lymph are to be found what look extremely like yeast cells (Torulce variolec), these being considered possible derivates from the micrococci. Some observers are of opinion that although the vaccine and variolous germs may not be actually special forms of the yeast organism, that at least both vaccination experiments with yeast and cultivation investigations seem to point out a similar origin.

When once the disease breaks out in a dairy, it is apt to spread to the whole herd, unless precautions be taken against its diffusion, by segregation of all affected animals and the employment of separate attendants, particular care being at all times taken that the milker of an affected cow shall not touch a healthy one. The milk should be drawn off with the tcat syphon, and, if the mammary gland becomes much inflamed, fomentations may be necessary, with other treatment recommended for " mammitis" (see Principles and Practice of Veterinary Surgery); but in all ordinary cases of the disease non-interference is to be strictly observed. 


\section{CHAPTER XXI.}

CONTAGIOUS DISEASES-continucd.

VARIOLA OVINAE (SHEEP-POX).

Synonyms.-Claveau or Clavelée (F.); Schafpocken, Schafpochenseuche, and Schafblattern (G.)

Definition.-A contagious and infectious eruptive disease, analogous to small-pox and cow-pox; runs a definite course, and occurs but once, as a rule, in the same animal. It is divided into two forms:-1st. A malignant, virulent, or confluent form; and $2 l$. A benign or discrete form. The malignant form never produces vesicles; the sheep lose their eyes; their wool falls off, the skin cracks in a zig-zag manner, and the nostrils become filled with a foetid discharge. In the benign form, genuine vesicles appear, which, after the scabs fall, leave pits in the skin, on which the wool never grows again. According to Professor Simonds the disease is not communicable to the cow or to children. Saccho, however, states that ovination is protective against small-pox.

\section{HISTORY.}

Sheep-pox is said by Mrr. Fleming to have appeared as an epizootic in England about A.D. 1277, but that it was well known in Britain more than two hundred years previons to 1275 , and was called the liot. The term variola had, however, been made use of in 569 (Animal Plagnes, 1875). But in more modern times sheep-pox was unknown in this country until 1847, when it broke out on a farm at Datchett, near Windsor, where it was introduced by fifty-six merino sheep, brought to this country in the ship "Trident," from Tönning, in Demmark.

Professor Simonds, in his treatise on Variola Ovinæ, says of 
these sheep-" When disembarked they appeared to be in health, and were sold by the salesman to whom, with other sheep, they were consigned. We have not succeeded in tracing the subsequent distribution of each separate lot of this cargo, but we are assured that many of the animals continue well. It is, therefore, evident that in this particular instance the malady was imported from Denmark; but this unfortunately did not prove to be a solitary case of its introduction.

"Within a day or two of the arrival of the 'Trident,' the 'Mountaineer' and the 'Princess Royal' came into port, each vessel having on board a number of merino sheep brought from Hamburg. . . . . Some of these sheep -507 brought by the 'Princess Royal '-were sold to butchers; others to farmers, as 'stock sheep.' The latter were bought in several quantities on 26th July by Mr. B. Weall, of Woodhall, Pinner ; Mr. Goodchild of Kingsbury; Mr. Pittman of the same place; and Mr. Choke of Barking. These purchased by the two last-named gentlemen are said to have continued in health ever since. Mr. Goodchild's sheep, however, showed symptoms of the disease very shortly after arriving at his farm, their illness being attributed to his having had them dipped or washed, for its true nature was not suspected. That portion of the cargo of the 'Princess Royal' which was purchased by Mr. Weall consisted of 80 merinos; and on the same day he bought 166 other sheep of the merino breed that had arrived by the 'Mountaineer.' These two lots were placed together, and subsequently, being sent to Pinner, were equally divided between Mr. Weall and his brother. In each moiety the disease showed itself, being first observed among the 80 sheep about ten days after their purchase, and from them it rapidly extended to others. Out of those belonging to Mr. B. Weall, 20 died in the acute stage of the malady, 27 more were sacrificed, and the residue was disposed of at a low price."

"We examined Mr. B. Weall's flock on the 7th of September, and found two of the sheep in the carliest stage of the malady; but most of them were recovering."-(Snroxds on Variola Ovince.)

The outbreak of 1847 committed great ravages in Middlesex, Surrey, Norfolk, Cambridgeshire, Suffolk, and Hampshire, and continued during 1847,1848, 1849, and 1850 ; the losses from 
it were also severe in 1852 , and more so in 1853 , at which time the practice of slaughtering sheep in or near London put an end to the disease until 1862, when it broke out at Allington, in.Wiltshire. This outbreak was supposed to be of spontaneous origin, as it could not be distinctly traced to foreign sheep. Mr. Gamgee visited the district at the time, and made many observations, from which he came to the conclusion that "the district turns out to be one not unfrequently visited by contagious disorders. . . . The district is traversed in all directions by driftways, so that drovers can pasture their sheep on the downs for days, and go from Bristol to London with the payment of a single toll. There are some notorious dealers who have no farm or down on which to keep their flocks. They pick up odd animals at a low price here and there, and drive over the downs, where they sleep, and move gently backwaras and forwards on the pretext of travelling, but in reality getting food for their flocks. Many instances have occurred of the spread of contagious disorders, such as scab, the foot-and-mouth disease, foot-rot, \&c., from these infected flocks passing over the downs along the Wans Dyke." The disease is not readily transmissible to other animals, but it appears that goats, pigs, hares, rabbits, and dogs are amenable to it.

These observations of Mr. Gamgee, along with the fact that sheep-pox is one of those diseases which never occur spontaneously in this country, are to my mind quite sufficient to account for the Wiltshire outbreak.

The early history of sheep-pox on the Continent of Europe, in some parts of which it is very prevalent, is very confused; it is certain, however, that it was introduced into Western Europe by diseased animals brought across the Russian frontier into Poland, Hungary, Prussia, Pomerania, \&c. Rammazini states that the malignant affection amongst cattle and sheep which prevailed in 1514,1599 , and 1691 was small-pox. From his description it is very probable that the disease of 1691, which chiefly attacked sheep, was in reality variola ovinæ, inasmuch as it was characterised by pustules similar in form, colour, and in the manner in which they went off. When they had died off after the suppuration, they left a black scar, like that which remains after the small-pox.

Professor Simonds instituted various experiments which prove 
that variola ovinæ is inoculable, contagious, and infeetious, and that its incubative stage varies from seven to thirteen days when communicated by inoculation, and from nine to twelve days in natural cases.

Like the poison of cattle plague, that of sheep-pox is both rolatile and fixed, existing in the blood, the tissues, secretions, and emanations, and may be carried from place to place in the clothes of attendants, in fodder, wool, skins, and particularly by dogs. Mr. Chas. Percivall informed Mr. Gamgee that he had seen a dog (Mr. Stephen Neate's) suffering from symptoms of disease identical with those of variola ovinæ, and there could be no doubt but that the dog contracted the disease from affected sheep (Domestic Animals); and it is pretty well demonstrated that a healthy flock is not secure from the disease at the distance of a quarter of a mile from affected sheep. Upon this head Continental writers are very clear, and they point out the danger of driving a healthy flock on the same road which shortly before has been travelled over by a diseased one. Chauveau has shown that the virus is a hundred times more infective, that the matter contains in an equal quantity thirty times the number of corpuscles, and is more active than that of vaccina. He has also shown that if vaccine matter be diluted with fifty times its weight of water, it propagates itself when inoculated with great uncertainty, while ovine matter may be diluted 1500 times before its virulence is appreciably affected.

The virus retains its vitality, when protected from the air, for a very considerable period, but is destroyed by the influence of air, heavy dews or rain, sunlight, a temperature of $122^{\circ} \mathrm{F}$. frost, and disinfectants, such as chlorine, permanganate of potash, and ozone, as well as by putrefaction, and if the wounds be suppurating, the matter from them will not infect.

\section{PATHOLOGY AND SYMPTONS.}

As already stated, the period of incubation varies from about the seventh to the thirteenth day, during which time there are no symptoms observable to a non-professional person. Like all specific fevers, however, there is an increase in the temperature of the animal body for some time prior to the outward manifestation of the disease. In thirty-six to forty-eight hours after the first manifestation of the disease, the temperature rises to 
$105^{\circ}$, or even as high as $107^{\circ}$ or $108^{\circ}$, but in the benign form it diminishes at the commencement of the eruption. In the malignant it is slower and later in diminishing, more irregular, and increases when suppuration occurs, becoming normal at the period of dessication. A careful observance of the temperature is of great importance, in order that affected sheep may be removed from the flock before any of the ordinary signs of the malady can be detected, and before the disease becomes infections. By carefully watching and examining their flocks, some farmers have been able to arrest the spread of the malady, and the course they have adopted has been that of segregating the diseased ones when the slightest rash made its appearance upon them. Mr. Charles Fielder of Sparsholt, near Winchester, says-" I employed two men to turn every sheep I had on my farm, and minutely inspect every one of them; and if they saw the slightest appearance of any rash, or a single pustule showed itself between the inside of the shoulder and the breast, where the skin of the sheep generally looks white and clean, and where it was sure to show itself first, I had it immediately taken away, putting the whole of the diseased together in one large field in the midule of my farm, at a distance from any road, as a protection to my neighbours. I followed the same course every morning, by having all my sheep turned and closely inspected, not looking to the trouble and expense, as I felt in my own mind that it was the only remedy I had to stop the infection; as I calculated, if I could only find out those sheep which had taken the disease and were breeding it, before it became infectious to others, I should be able to arrest its progress. Fortunately I was right in my calculations, for they daily decreased in numbers, although they still kept faltering for a fortnight or three weeks from the time I began tuming them (particularly in one flock where the Spanish sheep had been), before it, as I hoped, ceased altogether, as I have not found a diseased one for some time past."-(From the Farmers' Magazine, vol. xvi., page 524, quoted by Professor Simonds.) If separating the diseased from the healthy sheep after the first symptoms of the malady are discernible by an ordinary observer has so far been sufficient to arrest its spread, it can easily be understood that removal prior to the appearance of the rash will yet prove more satisfactory. Symptoms. - The first appreciable signs of the disease, after the 
termination of the incubative stage, are recognisable "by the existence of papulæ or nodules deeply imbedded in the dermis, having a florid red aspect. These show a preference for some parts of the integument, and are usually to be first detected on the inner side of the arms and thighs, and on the cheeks and lips, where the animal has a hairy and not a woolly covering. Other portions of the body are often simultaneously attacked, as the prepuce, labia, anms, and under surface of the tail-parts which are perfectly nude."-(Sinovds.)

In some cases there will be a diffused redness of the skin, in patches of various sizes, prior to the formation of the papulæ. Professor Simonds says that in inoculated cases this is so well marked that it constitutes a disease of the skin, analogous to roseola variolosa of the human subject, and establishes another similarity between these maladies.

During the papular stage the skin has a flea-bitten appearance, the eruption being at first in the form of small red points; these gradually enlarge, often uniting one with another. Professor Simonds considers that three days may be regarded as the period of papulation, and that it is generally longer in confluent than in the distinct form of the disease. The disappearance of the papulated eruption, the disease afterwards lying dormant in the system for three weeks and then breaking out, as described by Hutrel d'Arboval, was not witnessed by Professor Simonds.

Concomitant with the appearance of the rash, febrile symptoms are manifested, the animal becomes duil, with blood-shot eyes, is thirsty in all cases where the eruption is extensive, and the papulie confluent. Where the eruption is distinct the febrile symptoms are much milder. Continental writers state that the ontward signs of fever precede the eruption : the experience of Professor Simonds and others in this country does not confirm this, for in all cases seen by Professor Simonds the eruption preceded the constitutional disorder.

"The red papulæ gradually enlarge in size, then become elevated and transparent in their centres. The papula is now a vesicle containing a liquid at first transparent and then turbid. Many of the vesicles are very small, and if punctured yicld no fluid. The duration of the vesicular stage differs to some degree, and it has been observed that many of the papulie disappear willout vesicles being produced. The ovine vesicle is flat on its 
surface; and in this particular it forms a contrast both with the vaccine vesicle of the cow and the variolous of man. The serosity of the vesicles, first clear, becomes milky, turbid, less serous, and straw coloured, and ultimately by drying hardens into a crust, and is cast off with the epidermis."-(Simonds.)

Both Professor Simonds and Mr. Ceely are of opinion that the term pustule should not be applied to the eruption, except, as Mr. Simonds says, in the latter stages of extreme and protracted cases, where pus is in reality formed, and is succeeded by the ulcerative process.

"The period of desquamation or dessication depends on the extent of the original eruption, and also on its being distinct or confluent. It is likewise governed by the amount of inflammatory action which existed in the dermis, and is always protracted when suppuration has taken place."-(Simosds.)

The vesicles, scab, and crusts are liable to be injured in various ways, but more particularly by the sheep scratching themselves, and this interferes materially with the process of healing, and causes considerable irregularity in the duration of the malady. "In natural cases, even when mild," says Professor Simonds, " and when no cause retards their completion, a month, reckoning from the period at which the animal was first exposed to the contagion, usually passes before it is restored to healtl.

"The following summary of the gradations of the malady may De accepted as sufficiently accurate for practical purposes:-The first ten or eleven days are those of incubation; the twelfth and shirteenth of invasion; the fourteenth, fifteenth, and sixteenth of papulation; the seventeenth, eighteenth, and nineteenth of vesication; the twentieth, twenty-first, and twenty-sccond of suppuration; and the twenty-third to twenty-eighth of dessication and separation of the crusts."-(Sinonds on Variola Ovinoc.)

The same authority says that the constitutional symptoms, especially those which indicate the greatest danger, "are dejection, the head being held low; the infected animals separate from their fellows; mostly lie down in a corner of the field; the ears are pendent; the breathing quick and short; the eyelicls are swollen, and tears trickle down the face; the conjunctiva varies in shade from a bright scarlet to a modena red; a mucus discharge flows from the nostrils, and increases in viscosity as the disease advances, often becoming sanguineous in the 
latter stages; yellowish spots may likewise be seen scattered here and there on the pituitary membrane; the pulse early gives evidence of febrile excitement; at first it is quickened and somewhat increased in force, numbering about ninetyfive beats in a minute; later on it becomes tremulous and indistinct, even over the region of the heart; rumination is suspended, and all food refused; but the patients show a great disposition to take water, and also to lick earth, which, mingling with the mucus of the nose, assists in clogging the nasal openings, and renders the respiration more difficult. The alvine evacuations are but little changed in the majority of cases; in some, however, diarrhœa succeeds to a torpid condition of the bowels, and hastens the fatal termination; the temperature of the body is unequal, the feet and ears being genernlly cold, while the skin elsewhere is hot.

"These symptoms, more or less modified, are present from the commencement of the eruption, and seldom abate until the papular stage gives place to the vesicular, when the animals usually experience a relief. In all natural cases, the constitutional disturbance is great, and makes the probable termination of the attack donbtful, which is also rendered more uncertain when the confluent form of variola prevails; for the type of the disease, as elsewhere stated, governs to a considerable extent the amount of the fatality.

"Not only is the prognosis unfavourable in confluent variola, but it is equally so if the fever continues unabated, which is certain to be associated with an augmentation of the patient's sufferings. The breathing now becomes rapid and painful; the inspirations frequently, but more often the expirations, are accompanied with a moan ; frothy saliva is discharged from the mouth; the exhalations are fœtid; the wool separates from the skin on the application of the slightest force; ulcerations of the conjunctival membrane occasionally result; and sometimes the internal structures of the eye give evidence of disorganisation; the animal shrinks from the touch; the pulse gets more and more indistinct; great prostration of all the vital powers succeeds, and death closes the painful scene."

The per-centage of mortality arising from variola ovinæ varies considerably; very often half the sheep which are attacked succumb to the disease, and it is not unusual for nearly the whole 
flock to be swept away. Mr. Gamgee states that in one outbreak "the losses on 3811 sheep were 221 before inoculation had been practised, and the loss since has amounted to 537, or a total of 758 on 3811 , viz., very nearly $20(19.89)^{\mathbf{1}}$ per cent., whereas in 1310 not inoculated the loss amounted to 21 sheep, or 1.6 per cent."

It is evident that Mr. Gamgee intends the reader to understand that the small mortality in the 1310 was due to the precaution of scparating the diseased from the healthy animals during the earlier stage of the malady.

\section{POST MORTEM APPEARANCES.}

The post mortem appearances of small-pox are as follows:Body swollen, and exhaling a fotid odour; the eyes and nose closed with dry discharges; the mucous membranes of the mouth, nose, pharynx, cesophagus, larynx, bronchi, and rumen are covered with light copper-coloured vari or nodules, and occasionally small ulcers are seen on the epiglottis and other parts of the larynx. The lymphatic glands in various parts of the body are enlarged, and marked with red spots. The subcutaneous tissues are engorged with blood, and covered with solid red nodules, whilst purulent formations exist beneath the confuent papule. The areolar tissue of the face and extremities is often filled with effusion, and the skin itself is in some parts red, in others of a blackish hue, and the wool, if not already detached during life by the animal scratehing itself, easily comes off.

\section{PREVENTION AND TREATMENT.}

The prevention of the disease in this country depends upon the exclusion of animals from infected countries. The incubative stage being of such a duration, affected animals may easily pass the examination of the most careful inspector, no signs of illness being manifested during this stage.

If, however, variola should appear in a flock, the best way of dealing with it is that recommended by Mr. Fielder(see page 214), namely, the separation of all diseased sheep during the earliest

${ }^{1}$ In Mr. Gamgee's book it says very nearly 10 (19.89) per cent. ; the figure 10 is evidently a mistake. 
stage of the disease; and pastures, roads, and other places frequented by the diseased animals should be avoided until heavy rains have destroyed the contagium.

Inoculation or ovination is recommended by very high authorities. In this country, however, the experiment has not proved at all satisfactory. French veterinarians speak highly of ovination: thus, at the Alfort Veterinary School the mortality was only at the rate of 1 in 400 after ovination. Professor Simonds says our experiments are too limited to suggest correct conclusions, and they have shown a result so different, that, were we to found an opinion on the merits of ovination on them alone, it would not be in favour of the practice. The deaths have been at the rate of 20 per cent. Professor Gamgee condemns ovination entirely. Undoubtedly inoculation serves to increase the centres from which the contagium may extend in every direction, whereas segregation or slaughter at once diminishes the cause of the propagation of the disease, as well as the centres from whence it might spread.

Variola ovinæ, being a disease that runs a clefinite course, is not beneficially influenced by the action of medicines; on the contrary, the forcible introduction of drugs, or even of gruels, and other so-called nourishments, into the animal's stomach when it is incapable of digesting them, is calculated to do much harm. If the diarrhoea be excessive, chalk is to be mixed with the water which the sheep may drink; or if there be much fever, but no diarrhœa, chlorate, or nitrate of potash may be ordered in the same vehicle. It is essential, however, that the sheep be kept clean, have their nostrils sponged or syringed to prevent suffocation, have clean beds to lie upon, be well sheltered and kept warm, have plenty of fresh air, and, if they eat at all, be supplied with easily digested food.

On the Continent variola occurs among goats, pigs, dogs, and fowls.

Mr. Gamgee describes a form of chicken-pox in cattle, occurring on the Continent under the term varicella boum.-(See GungeE's Our Domestic Animals in Health and Discase, p. 256.)

VARIOLA EQUIN.E, OR HORSE-POX,

(Also called grease, constitutional grease, Soy's disease, and sore heels), is described by Dr. Fleming in his Sanitary Science 
and Police as an eruptive vesico-pustular malady, generally diffused in the different parts of the world inhabited by the horse. With the exception of the case mentioned at page 193, I have never witnessed anything approaching to variola in the horse.

It appears, however, that the eruption, preceded by a very slight, almost imperceptible, degree of fever, may appear on the skin at different parts, or over the whole body, on the nasal and buccal mucous membranes, and occasionally on the conjunctivæ.

The nasal eruption not only involves the Schneiderian membrane, but the nostrils and lips, and is apt to become confluent on the lips and the inferior parts of the limbs.

The contagium of the variola is transmissible from horse to horse, to the cow and to mankind, by contact and inoculation, but not by infection.

Variola equinæe appears to be a very mild and benignant disease; and as Dr. Fleming's work contains a full description, the reader is referred to it, as well as for descriptions of the goatpox (variola caprinæ), porcine pox (variola suillæ), variola caninæ, and the variola of fowls. Professor M'Eachran, of the Montreal Veterinary College, reports in the Veterinary Journal, August 1877, an outbreak of equine variola in Montreal in February and March of that year. 


\section{CHAPTER XXII. \\ CONTAGIOUS DISEASES-continued.}

\section{RABIES.}

Definition.-A disease originating in the canine, and, less frequently, in the feline race. During the progress of the malady a specific virus is developed in the saliva of the affected animal, which, being implanted through a wound, bruise, or thin epidermis without wound or abrasion, conveys the disease to other animals and to man. After an indefinite period of latency, it causes pain and stiffuess in the bitten part, excitement, feverishness, inability to swallow liquids, a tendency to vite, great prostration, and death. The poison is only recognised by its morbid effects in the animal economy.

\section{HISTORY,}

Rabies (Rabio, to rave), or, as it is sometimes erroneously called, so far as the lower animals are concerned, hydrophobia, is a disease which has been known from very early times.

Dr. George Fleming, in his work on Rabies and Hydrophobia, says-"Its great antiquity is undoubted. Plutarch asserts that, according to Athenodorus, it was first observed in mankind in the days of the Asclepiadre, the descendants of the god of medicine, Asculapius, by lis sons Podalirius and Machaon, who spread through Greece and Asia Minor, as an order of priests, prophets, and physicians, preserving the results of the medical experience acquired in the temples as a hereditary sect. They were the earliest physicians known to us, and it is not unlikely 
that they may have been the first to observe the madness of dogs transmitted to man." Dr. Bardsley, in his Memoir on Hydrophobia (Cyclopeedia of Practical Medicine, vol. ii.) says"We have already said that the disease was well known to Homer, and applied by him, with his usual critical exactness of similitnde, to the indiseriminate havoc with which Hector sweeps through the battlefield of his enemies."

Hippocrates alludes to hydrophobia in the faintest manner; but Democritus, who was contemporary with Hippocrates, according to Cælius Aurelianus, wrote upon the nature and treatment of the disease. Aristotle, in his De Historia Animalium says that the disease is communicable to all animals but man. Many other aneient and modern writers have described the fearful malady: amongst the latter may be mentioned Van Swieten, Sauvages, Cullen, Mead, Bardsley, Yonatt, \&e.

In 1271 wolves became affected with rabies in Franconia, and, contrary to their usual habits, they spared the herds and flocks and attacked human beings: upwards of thirty men fell victims to these attacks. In 1590 canine madness prevailed in Spain-(Blaine.) In 1590 epizootic rabies prevailed amongst the wolves in the provinee of Monthelliard. 'The further history of rabies may be obtained from Dr. Fleming's excellent treatise, to which the reader is referred.

Like other epizootic and contagions diseases, its prevalence is liable to many fluctuations, at times prevailing to a great extent, whilst for long periods the disease is almost unheard of. During the earlier portion of the present century rabies was very prevalent both in this country and on the continent of Europe, not only in dogs, but in foxes as well. Since then there has been an abatement in the frequency of the disease; but even up to the present, outbreaks are not very rare. In 1869 I witnessed the disease in a pack of hounds in the north: the nature of the disease was detected after one man had been bitten, and he fortunately resisted the action of the poison, although he took no precaution against its effects. The disease has again been prevalent in 1876-7-8 in the southern and lowland counties of England. 


\section{CAUSES.}

Opinions are divided as to the spontaneity of the origin of rabies. Some maintain that even in the dog and allied quadrupeds the malady never originates except by inoculation: whilst others hold that it originates spontaneously in the dog, even in this country.

After discussing the opinions of Blaine, Youatt, and Maynell, who affirm it owes its origin to a wound from a rabid creature, Fleming says- " There are few now-a-days who are not couvinced that it will occasionally appear in a spontaneous manner, and without any certain assignable cause. No doubt the transmission of the disease by inoculation furnishes by far the largest number of cases, and many of these, from the obscure manner in which the inoculation has been effected, appear to be due to other causes than that of a traumatic character; but, notwithstanding, the disease must have a commencement." However, he has changed his opinion, and now affirms that it originates from contagion only. In this view of the origin of the malady, facts and circumstances compel me to agree with Dr. Fleming; at the same time I am fully aware that the opinions of many observers are opposed to it. ${ }^{1}$ Professor Dick was of opinion that rabies always originated spontaneously-from atmospheric causes-a species of catarrh, which, by extension from the nasal mucuous membrane, through the cribriform plates of the ethmoid bone, involved the meninges of the brain, and produced the phrenzy, delirium, and the other train of nervous symptoms which characterise the disease. He also held the opinion that it was never caused by inoculation from the bite of a mad $\mathrm{dog}$; in fact that it was a non-contagious disease.(Veterinary Papers by Professor Dick.) This opinion of Professor Dick is still believed by a few, but fortunately very few, and the sooner it is dispelled the better, for nothing is more calculated to do mischief than the promulgation of what has been proved to be so erroneous. Rabies, once generated in the dog or cat, is transmissible by inoculation to every warmblooded creature.

1 It is but right that I should state that Dr. Fleming has changed his opinion, and is now strongly opposed to any but inoculation theory. 
The idea that hot weather is productive of rabies is also now dispelled. The following report, arranged by Professor St. Cyr, of the Lyons Veterinary School, is very instructive, showing that rabies is even more prevalent in the temperate months than in those of extreme heat and cold :-

\begin{tabular}{|c|c|c|c|c|c|c|}
\hline 1865 & & Rabid. & Suspected. & $\begin{array}{l}\text { Became Rabid } \\
\text { in Infirmary. }\end{array}$ & $\begin{array}{c}\text { Total } \\
\text { Admitted. }\end{array}$ & Total Rabid. \\
\hline January, . & • & 12 & 2 & 0 & 14 & 12 \\
\hline February, & . & ] 4 & 4 & 1 & 18 & 15 \\
\hline March, & . & 6 & 11 & 0 & 17 & 6 \\
\hline April, . & . & 14 & 20 & 1 & 34 & 15 \\
\hline May, . & - & 12 & 14 & 1 & 26 & 13 \\
\hline Tune, & . & 6 & 14 & 1 & 20 & 7 \\
\hline July, & . & 2 & 8 & 2 & 10 & 4 \\
\hline August, . & . & 7 & 3 & 2 & 10 & 9 \\
\hline September, & $\cdot$ & 1 & 3 & 0 & 4 & 1 \\
\hline October, . & - & 3 & 1 & 0 & 4 & 3 \\
\hline November, & - & 0 & 0 & 0 & 0 & 0 \\
\hline December, & $\cdot$ & 1 & 1 & 1 & 2 & 2 \\
\hline & & 78 & 81 & 9 & 159 & 87 \\
\hline
\end{tabular}

Sometimes the annual statistics show a predominance of cases in the summer months. But according to M. Bouley's observations there is no great difference in the seasons with regard to the disease. An analysis by M. Bouley of reports for the six years 1863-1868 gives-for the spring months, March, April, and May, eighty-nine cases; for the summer months, June, July, and August, seventy-four cases; for autumn, September, October, and November, sixty-four cases; and for the winter months, December, January, and February, seventy-five cases.

It was at one time maintained that rabies never became developed in the bitch, and it is now advocated that it is far less frequent in females than males. Professor Coleman stated in evidence in 1830 that on the occasion of rabies entering kennels, the mad dogs bit dogs but spared the bitches. This opinion of Coleman is still believed in, more particularly by medical writers. But there can be no doubt that bitches are as 
susceptible to the contagion by inoculation as dogs. This is well exemplified in what occurred a few years ago to a bitch pack of foxhounds belonging to Mr. Standish, South Shoreham, Southampton. The pack was nearly exterminated by rabies, through the disease having been introduced to the kennels by a hound which had strayed away for several days, and on its return was discovered to have been bitten."-(Veterinarian, vol. xliv.)

I think the reason for the supposition that fewer cases of rabies are seen in bitches than in dogs, is to be found in what seems to have escaped medical observers, namely, that there are fewer of them in the world: Indeed, the same explanation can be given on this point as on what appeared so strange to some during the prevalence of the cattle plague, that fewer bulls than cows were attacked by that malady.

\section{PATHOLOGY AND SYMPTOMS.}

The following circumstances in the pathology of rabies are worthy of notice, namely-First, That the period of latency after inoculation is very indefinite, and that it varies in different animals: Second, That inoculation does not always produce the disease, one-fourth of the inoculated animals generally escaping; and that the disease is not transmissible to man or other mammifers: and Third, That, notwithstanding the strongest evidence as to the microbic nature of the disease, the morphological character of the microbe is as yet undetermined. Cocci observed in the spinal cord of rabid dogs have been described by Babes (Les Bacterics, 1886), cultures from which were said by Babes to communicate the disease. Babes also describes a short bacillus. And again it is said that very fine bacteria have been isolated from the brain, cultures from which gave the disease. Researches are being still carried out which will in time doubtless determine the nature of the microbe. Virulence is said to be destroyed in thirty hours at a temperature $-20^{\circ}$, and in moist heat at $100^{\circ}$ in half an hour, and dessication in the air diminishes and even destroys the virulence in a few days. This fact has enabled Pasteur to modify his method 
of preparing his vaceine, which at first was transmitted from the $\operatorname{dog}$ to the monkey, in which animal the virulence became enfeebled after each transmission, until it became too attenuated to convey the disease, and was used as a preventive vaccine. Now, however, Pasteur inoculates a rabbit, in which the period of incubation is always short, through a trepan in the skull, having discovered that after successive inoculations from one rabbit to another virulenee becomes intensified, and the period of ineubation shortened, until it is redueed to six days. The virus then has attained its highest degree of virulence, and is called "virus fixe." The spinal cord of a rabit, having had this intensified rabies, having been suspended in a jar at a temperature of $73^{\circ} \mathrm{F}$. in an atmosphere kept dry by the presence of potash, gradually loses its virulence. The opinion that rabies could not be transmitted after an animal had been dead twentyfour hours, or after rigor mortis had been completely established, has been found to be erroneous; for it is now placed beyond doubt that virulence may be retained for a considerable time, provided putrefaction be prevented. The activity of the virus is also destroyed by various disinfectants, as such 1-1000 corrosive sublimate solution, 5 per cent. solution permanganate of potash, 50 per cent. alcohol; but 15 per eent. alcohol is said to preserve it for several days, and large doses are rendered innocnous by being rendered acid, alkaline, or putrefactive. The virus of rabies is contained in its most eoncentrated form in the saliva of the rabid animal; but it by no means follows that other parts of the diseased animal are free from its presence; indeed, direct experiments have determined that the virus is present in the blood, flesh, and other parts of the rabid animal. The virus of the rabid skunk, wolf, and hyæna appears to be more virulent and prompt than that of the dog.

It was at one time believed that the virus of rabies was innocnous when the bite was inflicted by any of the herbivora. Ubservation on accidental cases had led to this belief, and direct experiments seemed to confirm its correctness. Girard, Vatel, Huzard, Dupuy, Lafosse, and others failed to transmit the disease from the herbivora to the dog. Professor Coleman, Sir Astley 
Cooper, and others in this country believed that the disease was not communicated except by animals which "naturally employed their teeth as weapons of offence." Dr. Fleming mentions several instances where rabies was communicated by inoculation with virus ubtained from cows, sheep, and even domestic fowls, and argues "that the facility with which the disease can be transmitted by different species depends, besides the activity or degree of virulency of the infecting principle, upon the organisation, habits, or rather nature of the diseased. Flesh-eating or carnivorous animals, as is well known, generally attack other creatures with their teeth, which are well adapted for wounding and tearing; consequently they are the most successful in inoculating with the poison."

The virus having been introduced into the system, generally lies dormant for a very indefinite period of time, the shortest period in the dog being about seven days, and the longest 155 days, but in rare exceptions even longer than this; in man from three days to nine months, and even ten years in rare instances; in the horse, from fifteen days to three months, and even fifteen months; in the sheep, from fourteen days up to three months; and in the pig, from a few days-eight or nine-to as many weeks, or even months. The wound inflicted by the rabid animal generally heals rapidly. In $1862 \mathrm{M}$. Renault published the results of some experiments which had been conducted with the view of determining the period of incubation in the dog. Out of 131 dogs bitten by mad dogs, or inoculated with their saliva, 63 remained well at the end of four months; the disease being developed in the other 68 after intervals varying from five to 120 days. Thus-

In 25 dogs the disease set in betreen the 5th and 30 th day.

$\begin{array}{lll}\text { In } 31, & , & 30 \text { th and } 60 \text { th day. } \\ \text { In } 7 " & , & 60 \text { th and } 90 \text { th day. } \\ \text { In } 5 ", & 90 \text { th and } 120 \text { th day. }\end{array}$

Symptoms in the Dog.-There are no premonitory signs in the lower animals, at least none that can be detected-such as pain in the seat of the bite, melancholy and irritability, febrile disturbance, and stiffness about the neck and head. The dog, when the period of latency is passed, becomes restless, dull, watchful, withdraws from its companions, choosing solitude, shunning the light; hiding in corners, or below chairs or other 
furniture; being fidgety, lying down, then jumping up again in an excited, unnatural manner; it has a tendency to rove about, and if possible to escape from its room or kennel, and wander about the ccuntry. At first, the dog's power of recognising people about it does not seem lost; indeed there are moments when the usual faculties of the dog, its affection and liveliness, seem greater than usual. Along with these symptoms there is a desire to lick anything cold, to rest the nose on a cold object, and to pick up stones, bits of wood, straw, \&c. At this stage the tendency to bite is not observed, but as the disease advances the movements of the animal become unsteady; the eyes follow objects in a peculiar staring manner; sometimes the dog will stare at some imaginary object, then rush forward, and bite at anything that may chance to be in his way, or even at the air, as if he were catching flies; cushions, straw, and other objects by which dogs are ordinarily surrounded, are tossed about; the animal scratches the ground, snuftles as if on the scent; the appetite is lost, but the thirst is considerable, and the act of drinking is performed without much difficulty during the early stages of the disease. At a later stage, however, swallowingdeglutition-is performed with difficulty, or is not performed at all, owing to the spasmodic constriction of the throat; but even then the animal has no dread of water, does not shrink at the sight of fluids, but, on the contrary, will plunge its muzzle deeply into it, and endeavour to drink with great avidity. The coat is staring, the skin tight on the ribs, and the belly tucked up. The dog occasionally vomits, the ejected matter being tinged with blood; there is a brownish coating on the tongue; the nose and mouth are foul and offensive, and there is generally a flow of saliva from the mouth. A symptom is mentioned in connection with the disease in the human being, which is of doubtful presence in the lower animals, namely, the formation during the early period of the disease of lysses or eruption on each side of the tongue. If any eruption does occur, its presence is seldom detected.

The mad log, or indeed a rabid animal of any kind, brought into the presence of one of the canine species, exhibits great excitement, exasperation, and fury, with a desire to attack and lestroy what seems the object of its hatred. "The horse assaults it with its teeth and hoofs; the bull, cow, and ram with their horns; even the timid sheep, when rabid, becomes the assailant." 
--(Bouley.) The females of the canine species do not always lose their maternal affection; on the contrary, the young are attended to with great affection. In the course of a day or two after the first manifestation of the above symptoms, the characteristic signs become more marked; the desire to bite is greatly exaggerated; the pupils are dilated; the conjunctive recl and injected; the eyes alternately widely opened with fury, and then closed in a dull but fierce manner. The forehead becomes wrinkled, and the looks of the animal are terrifying and repulsive; the presence of a living object excites the rage of the sufferer, causing it to spring at and endeavour to bite it. Any shining object will bring on a paroxysm of rage and excitement, and water, if the light shines upon it, will do the same; but if placed in a dark place, or where light does not shine, the dog will endeavour to chink with avidity. Intermitting with the excitement are periods of great prostration, the exhausted animal lying down in the quietest spot it can find, insensible to all surrounding objects. All at once, however, it springs up, and becomes greatly agitated; the excitement, rage, and agitation being always much greater when the animal is surrounded by noises and objects; when away from these things, in a quiet place, the fits of rage are not so great, indeed sometimes scarcely observed.

The bark of the rabid dog is unnatural ; it is husky, spasmadic, and more of the nature of a howl. In some cases, the nervous symptoms are those characterising paralysis of the jaws, with inability to close the mouth; the lower jaw is dropped, the cavity of the mouth and its contents exposed, the tongue dry, and the buccal membrane of a brownish hue. This is the "dumb madness," thus called because the animal is unable to bark or' howl. Rabid dogs have a strange tendency to eat filth; they have been known to eat portions of dead dogs, and to swallow hair, coals, earth, excreta of all kinds, and these remaining in the stomach present a characteristic post mortem appearance. A mad dog, when loose, will travel an inmmense distance in a short time, generally rushing at everything that comes in its way, but preferring to attack other creatures than man, and finally endeavouring to return home, as if prompted by some instinct. The pulse is said not to be accelerated, nor are the respiratory movements much increased, except during the paroxysms; the bowels are constipated, and the urine is high-coloured; the 
gait is unsteady, the tail droops, the head is depressed, the nose protruded; the fits of rage become shorter, those of depression longer; scent, sight, and hearing are much impaired; insensibility to pain, which is generally present from the earliest stages, is more and more manifest; and finally paralysis, particularly of the hind limbs, supervenes, and if not destroyed, the dog dies, an emaciated, repulsive object, the desire to bite remaining to the last. In the dumb form, the ability to bite is lost, as well as the power to lap fluids. The exposed and protruded tongue is covered with a stringy, tenacious saliva; the desire to drink is even more intense than in the furious form, and attempts to lap and swallow become painful to witness. Squinting of one or both eyes has been observed; there is also abdominal pains in some instances, and an inflamed condition of the nasal cavities, larynx, and bronchial tubes. There is no tendency to harm and bite surrounding objects, and the dog dies from coma, exhaustion, and suffocation. Both forms of the disease terminate in death in from four to eight days. It is, however, reported that some chronic cases of rabies exist for an indefinite period.

With regard to the dread of water-hydrophobia-which has been looked upon by some as the diagnostic symptom of the disease, I may repeat that it is absent in the dog. The desire for water is always excessive; there is inability to swallow fluids, hence a dog will plung his nose deeply into water, lap it with avidity, but owing to the thirst being still unallayed, he will become greatly agitated, and is thus often thrown into a state of fury or of involuntary spasms.

Canses and symptoms in the Horse.-Tiabies in the horse occurs from the bite or contact of the saliva of a dog or cat, generally from that of the dog. The disease is manifested in a variety of ways. In some cases it commences by great apparent distress, with sudden perspirations over the body; unruliness, the horse stamping and pawing violently, finally becoming frantic, and destroying everything within its reach. In other cases, along with restlessness, there is manifested a desire to bite the seat of injury. In one case that came under my notice, where the horse had been bitten on the breast, three months before any symptoms of rabies were visible, the animal during the paroxysms continually bit the cicatrix, until at last the whole sternal region was denuded of its skin; there was also intolerance to light, great nervousness, the animal being easily startled, with fixity of the 
eyes, staring at some imaginary object, the pupil being the while dilated, and the ears moved backwards and forwards, as in a blind horse, when it hears some strange sounds. At one time, during the day and night upon which it was ill, it seemed to present some signs similar to those of laminitis, shifting its weight from one foot to another, and swinging its body backwards. At the same time we noticed convulsive twitchings of the superficial muscles, difficulty in swallowing, spasm of the throat, a hoarse cough, acceleration of the breathing, a quickened pulse, and a flow of saliva from the mouth. After a while it became very furious, fought violently, destroying everything within its reach. Gradually signs of paralysis of the posterior extremities became apparent, and at last it was unable to rise. It would, however, make violent efforts to do so, and during a rapid spring or jump one thigh was fractured. The efforts to rise were not discontinued even after this accident, and so violent did it become, and so strenuous were its efforts to get at something, that the other thigh was broken. It still continued to sit upon its haunches, and to fight with its fore feet, all the while tearing at its breast with its teeth. Seeing that both tibire were fractured, the owner consented to what had long before been urged upon him-to have the horse shot.

In the stallion and mare it is stated that the sexual desire is angmented; that the stallion has frequent erections, and neighs in a harsh tone, and the mare stands with her hind legs apart, showing signs of œestrum.

The remissions and paroxysms are less apparent in the horse than in the dog. In the latter, the animal seems for a time to be almost free from the disease, but in the horse the absence of anxiety, restlessness, and exhaustion is of much shorter duration, and the fits of violence more violent and prolonged, so much so, that it soon becomes prostrated, and dies in frightful convulsions upon the second, third, or fourth day.

Hydrophobia in man is generally characterised by a sensation of intense pain in the seat of the wound, the cicatrix beconning red and irritable, the pain shooting from the extremities (if the wound be situated upon them) to the trunk. It seems most probable that this sense of pain is felt in some instances by the horse, and is manifested by the persistent efforts to bite the seat of the wound, particularly, as in the case described, if it be within reach of the tecth. 
The symptoms of rabies in the cow, sheep, goat, \&c., are very similar to those in the horse; in all there is "loss or depravity of the appetite, prostration, great restlessness, increased excitability, muscular tremblings, a flow of saliva from the mouth, excitation of the sexual desire, especially in the bull, difficulty in swallowing, and manifestations of hallucinations as well as disagreeable sensations in the seat of the wound."-(Fleming.) And succeeding to these signs are those of great violence, paralysis of the hind extremities, emaciation, and finally coma and death.

Medical treatment, after rabies has established itself, is of no avail.

\section{PREVENTIVE TREATMENT.}

Complete excision of the bitten surface, as soon as possible after the infliction of the injury, is the best that can be adopted.

If the wound be superficial, the free application of the caustic potash (Potassa fusa) or of the nitrate of silver is sufficient; but if deep or much lacerated, the parts are to be carefully excised before the caustic is applied. Where this has been done in man seven out of ten escape, whilst if no such means are userl eight out of ten die.-(Bollisger.) It is very difficult in the lower animals, covered as they are with hair, to know whether the part bitten be confined to any particular region of the body, no matter how carefully the skin may be searched. It is therefore advisable to have the bitten animal shaved as quickly as possible, in order that all scratches be brought into view, and that they may be dressed with the caustic. In dogs preventive treatment should not be resorted to, but the bitten animal ought at once to be destroyed. It is very true that all dogs inoculated with the rabies poison do not become mad; the risk of their becoming so is, however, so great, and the mischief they might inflict is so grave and important in its nature, that the chance of their doing such mischief should never be allowed. Whilst the above precantions are considered necessary, it cannot be said that they are always effective in preventing the disease in bitten persons or animals. Pasteur, however, as is well known, had carried out his system of vaccination by attenuated virus to almost a universally successful conclusion, and he anticipated that, although the time was still 
distant when canine madness would be extinguished by vaccination, he hoped, pending that consummation, that he would be able to avert the consequences of a bite from a mad dog. He said-_" Thanks to the duration of incubation after a bite, I have every reason to believe that patients can be rendered insusceptible before the mortal malady has had time to declare itself."

M. Pasteur's anticipations were put to the test by a Commission appointed by the Minister of Public Instruction, consisting of MM. Bouley, President; Paul Bert, Bécland, Vulpian, Tisserand, Director of Agriculture, and Professor Villemin.

Forty dogs were submitted to the bites of rabid dogs. Twenty of these healthy dogs had been previously inoculated with attenuated virus, whilst the other twenty were not inoculated; the rabies showed itself exclusively among the non-inoculated dogs.

M. Pasteur was requested by the Commission to perform his proof experiments in a more expeditious and certain manner than by only submitting healthy dogs to the bites of rabid ones. He having found, first, that if nervous matter from the bulb of the brain of a rabid animal be inserted on the brain-by trephining-of a healthy dog; second, that when the virus was injected into the veins; and third, that when the dogs were repeatedly bitten about the head by rabid ones, a rapid and fatal rabies occurred within twenty days-therefore proceeded to carry out a series of experiments on protected and non-protected dogs, with the result that none of the protected animals had, up to 10th July 188t, shown any signs of the disease, whilst twelve of the unprotected ones, including two which were repeatedly bitten about the head, had succumbed to the disease.

The dogs still remaining healthy were to be watched by veterinary surgeons for a year, to see whether the inoculations hold good permanently or only temporarily. These experiments proved entirely successful, and, acting upon this conclusion, Pasteur continued to apply preventive inoculation to man, and with remarkable succéss.

The reprehensible practice of muzzling dogs in hot weather is fortunately dying away. Hot weather does not cause rabies; the muzzling of dogs might do so, and even if it did not cause the disease in question, it inflicts much misery upon the poor animals.

PATHOLOGICAL ANATOMY.

From the symptoms observed during life, the conclusions natu- 
rally suggests itself that the brain and its membranes are the seat of organic lesions. Indeed, the specific action of the poison appears to be exercised, particularly in the first instance, upon the medulla oblongata and the par vagum, the branches of which seem to lose their natural properties; hence the difficulty in swallowing, the depraved appetite, alteration of the voice-or its entire loss in the dumb form-as well as the convulsions of the respiratory muscles, are all due to derangement of this nerve; and as the nervous system of the animal becomes more and more deranged, complete paralysis of the respiratory muscles oceurs, and the animal dies from asphyxia.

The principal post mortem appearances are œdema or congestion, sometimes in patches, of the brain and spinal cord, particularly at the base and plexus choroides, effusion into the arachnoideal space, cerebral ventricles, and the cerebro-spinal substance, and softening of the membranes. On the lower surface of the medulla oblongata, at the origin of the seventh, eighth, and ninth pair of nerves, the membranes are generally highly injected, thickened, softened, and matted together. The liver, kidneys, spleen, and the muscular system are congested. The bladder is empty, and its mucous membrane covered with petechiæ. The lungs are greatly engorged with blood. The blood in the vessels is but imperfectly congulated, often black and tarry, sometimes bright and red, in appearance. The mucous membranes of the pharynx, œsophagus, stomach, and bowels are either greatly congested, with extravasation of blood on their surface, or diffusely inflamed. Patches of extravasation are particularly met with on the gastric mucous membrane, and account for the hæmorrhagic vomiting which is sometimes witnessed during the illness. The contents of the stomach are generally of a peculiar nature, consisting of hay, straw, stones-in fact, of a collection of the most incongruous materials, which, owing to depraved appetite, the animal has picked up during life. This appearance is of great value, as it proves most conclusively that the dog has died rabid.

The tongue is often wounded by the teeth; its papillæ congested; and the salivary glands enlarged and vascular. In "dumb madness" the congestions, more particularly those of the upper part of the respiratory and digestive tracks, are developed to a greater extent than in the other form of the disease. 


\section{CHAPTER XXIII.}

\section{CONTAGIOUS DISEASES-continued.}

\section{GLANDERS AND FARCY (EQUINA).}

Definition.-A malignant, contagious, and fatal disease, due to the introduction into the animal economy, or of generation within it, of a virus (said by Dr. Strück of Berlin to consist of an organism-the Bacillus mallei-about the same size as those of tuberculosis), which, infecting the whole system, shows specific effects more especially upon the Schneiderian mucous membrane, the lungs, and upon the lymphatic glands and ducts. Glanders, and its variety farcy, are capable of transmission to man, in whom the virus seems to increase in malignancy, ass, mule, sheep, goats, dogs, the feline species, and even to mice and rabbits. Cattle, pigs, and fowls resist the action of the contagium, even when inoculated.

\section{HISTORY.}

Glanders was described by Aristotle, ${ }^{\mathbf{1}}$ by Vegetius, ${ }^{2}$ and other early writers, under the terms morbus humidus, cymoira, capitis morbus, \&c.; and its variety farcy, as morbus farcimosus, vermis equi, vermis volaticus, farcina equi, \&c.

Glanders is a disease of temperate climates, rare in hot and very cold countries. The contagious nature of glanders was for a long time a disputed point; it is, however, now determined beyond question, the only difference of opinion being its capability of originating spontaneously or not; some veterinarians maintaining that it originates by contagion only, whilst others -and with whom I agrce-contend that it is capable of

1 Aristotle, De Hist. Anim., lib. viii. c. xxv.

2 Vegetii Renati Artis Veterin., lib. i. c. vii. 
spontaneous origin, and of propagation by contagion and infection.

Glanders may occur under at least four forms, namely, acute glanders, chronic glanders, acute farcy, called by some bud farcy, and chronic or button farey.

\section{CAUSES.}

It is now generally believed that equina originates from contagion only. I still have doubts upon this point, for it cannot be denied that many debilitating influences, such as old age, bad food, overwork, exhausting diseases, and general bad management; specific miasmatic or animal poisons, such as those generated in localities where large numbers of horses are congregated together, in camps, barracks, large eab or other establishments, even where the stables are well ventilated, lighted, drained, and the animals well attended to in every way, but more particularly where the stables are ill ventilated, badly drained, dark, and foul. Horses, when crowded on board ship, are very liable to this affection, and the Aralss in transporting their horses from Arabia to India always choose that part of the year when the passage is shortest, lest the accidents incident to a long voyage might oblige the hatches to be closed, and want of ventilation promote the development of glanders. I have also observed that glanders is developed in new stables, where the walls are not thoroughly dry, where in fact, in common language, they are said "to sweat"; and, finally, glanders occurs as a sequence to exhausting diseases, more especially if the animal be old, or of a bad constitution. These causes, and a generally vitiated condition of the animal system, may be said to predispose glanders-(1.) By causing the introduction into the blood of vitiated or decomposing material generated in the external surroundings of the animal: (2.) By inducing the formation of degenerated material within the animal system: (3.) By preventing the excretion of the degraded constituents normally generated within it by natural tissue changes, or excessively formed within it by various disordered functions, or introduced into it from without.

The most common forerunner of glanders, more particularly of that form of it known as farcy, is the disease commonly called 



\section{PLATE VIa.}
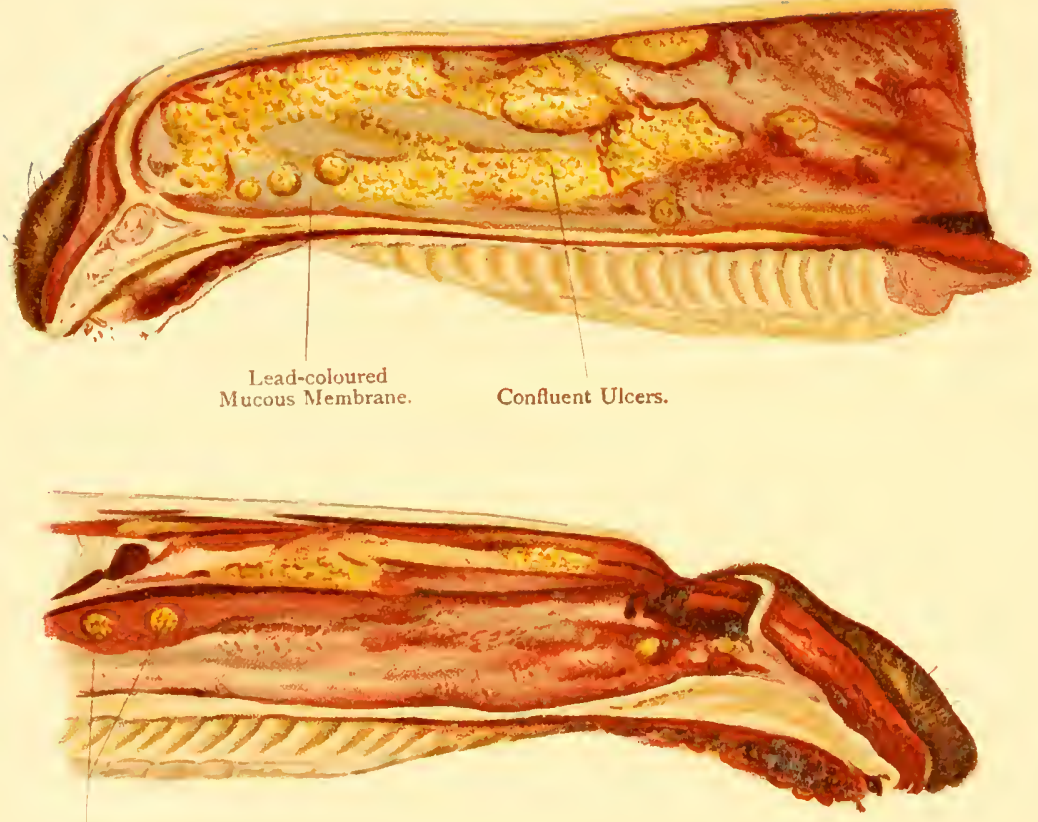

Uilcers.

GLANDERS AFFECTING NOSTRILS OF HORSE 
diabetes insipidus or polyuria. It cannot be said that in diabetes there is any obstruction to the excretion of degraded tissue; indeed, the reverse is the case, excretion of urine being enormously increased. If we look deeper into the matter we shall, however, see that the polyuria is associated with rapid tissue changes, rapid emaciation of the body being a most prominent symptom, with debility arising from degradation of tissue, and from the presence of the degraded materials within the eirculatory fluid. So apparent is this condition, that it has been truly said that diabetes, when arising from no cognizable cause, is often indicative of a general breaking-up of the constitution.

Although glanders and farcy are one and the same disease, differently manifested, farcy is apparently more frequently developed spontaneously than glanders. This circumstance: and the possibility of recovery from mild attacks of farcy, has led some to conclude that they are two separate disease; but direct experiment has proved that the virus is identical in both forms of the disease. The discharge from the nose of a glandered horse, when introduced into the systems of other horses, may in one produce glanders and in another farcy, whilst the pus from a farcy ulcer may produce in the inoculated animal glanders, farcy, or both; and the common termination of farey, if a horse affected by it be allowed to live a sufficient length of time, is glanders, and of glanders farey. Such an animal is described as being "both glandered and farcied."

\section{CONTAGION.}

Glanders, and its variety farcy, are highly contagious and infectious, and when once introduced into a stable are almost certain to spread amongst the horses there located.

As already stated, the contagium is found to be an aërobic hacillus discovered by Loffler and Schiitz, 1882, called the Bacillus mallei. These organisms are seen in the form of minute rods $1 \frac{1}{2 \pi 0}$ long, and from $\frac{1}{50800}$ to $\frac{1}{20} \overline{3} \overline{0}$ of an inch broad; they are rather thicker than the tuberele bacillus, and retain the methylene blue stain. They are found in the nodules in the lungs, liver, Schneiderian nembrane, and other involved 
structures, and are free or within the cells of the part. They are slightly motile, straight, or slightly curved, with rounded ends, and sometimes appearing as diplo-bacilli.

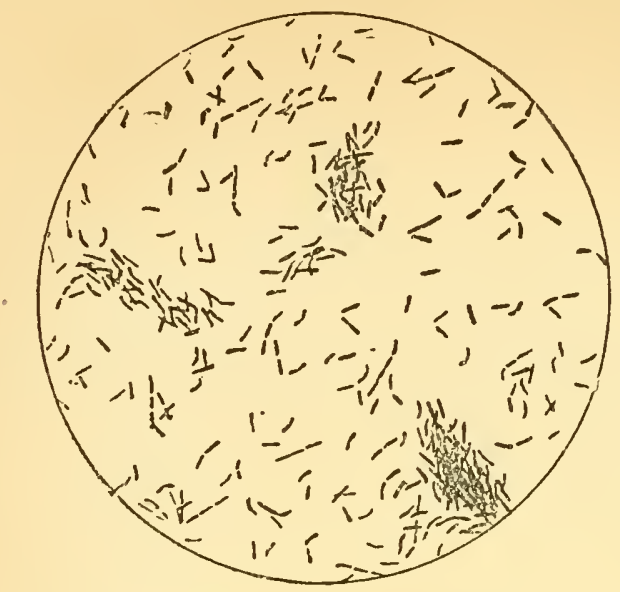

FIG. 7.-Glanders Bacilli.

They stain readily with aniline dyes, but do not retain their colour with Gram's method. The best stain is that of Schiitz: potash solution, 1 in 10,000, and concentrated alcoholic methylene blne, equal parts. Wash the section in a watchglass full of water, containing four drops acetic acid. Transfer for five minutes to 50 per. cent. solution of alchohol, fifteen minutes to absolute alcohol, clarify in clove oil, and mount in Canada balsam.

The specific poison can be introduced into the system either by the skin or mucous membranes. By inserting the virus under the slin with the point of a lancet, by rubbing the greasy heal of a horse, and by inoculating the mucons membrane of the nose, the disease has been produced.

With regari to the transmission of the contegium of glanders otherwise than by actual contact, opinion seems to be divided, some writers maintaining (and the experiments brought to bear upon their conclusions are formidable) that it is impossible to com- 



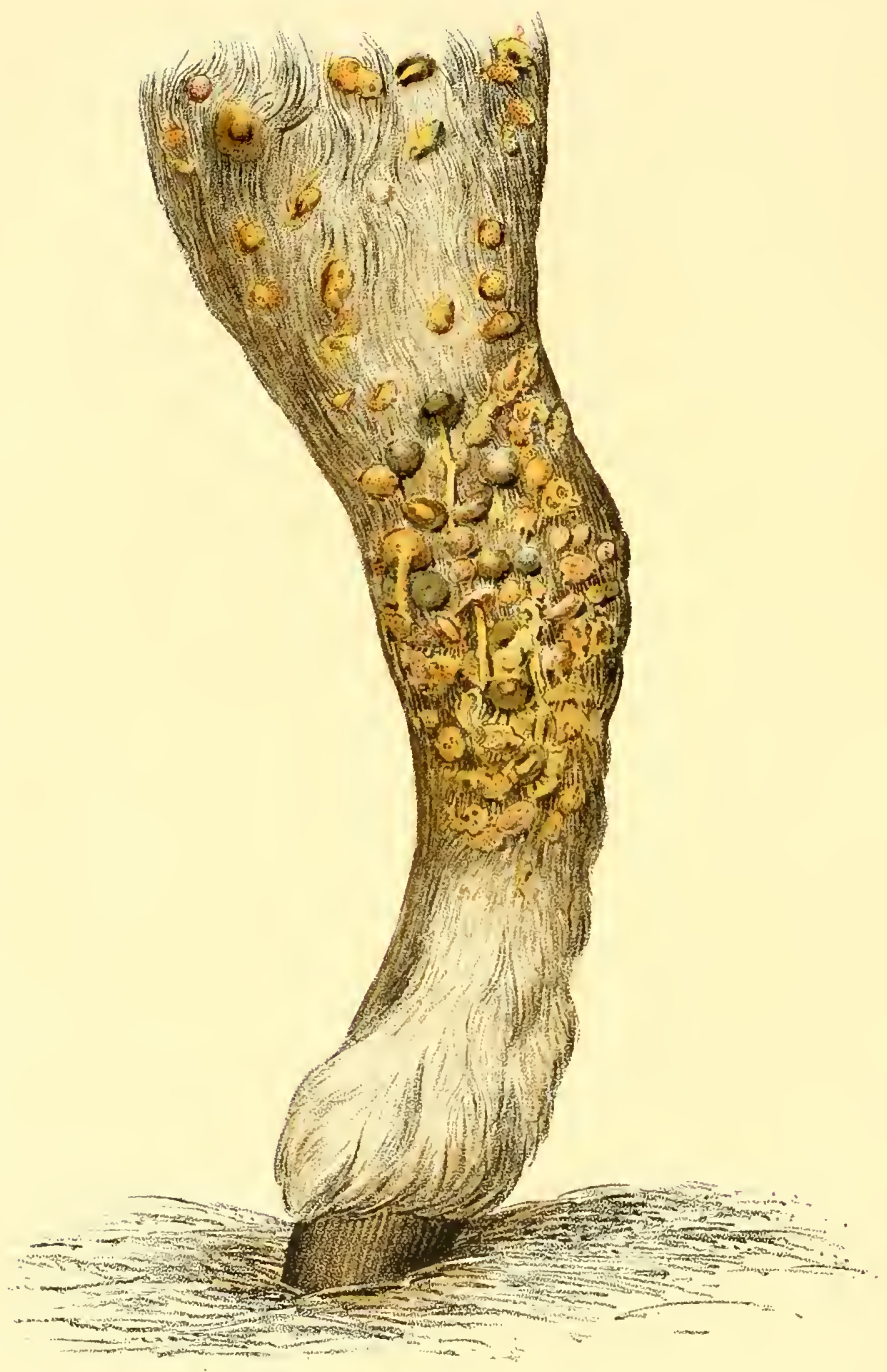

FARCY BUDS IN VARIOUS STAGES. 
municate it in any one of its forms by compelling healthy animals to inhale the expired air of those suffering from the disease, whilst others maintain that it is both contagious and infectious. The first opinion, if acted upon in every day practice, would, I opine, lead to disastrous results, and should not be entertained; for even if we were to grant that the virus is contained in the discharges from the ulcers, numerous experiments have proved that pus and other organised cells float in the atmosphere; the specific virus contained in these cells may thus be easily conveyed from one animal to another. Again, it must not be forgotten that in some instances glanders may exist without any external manifestations, i.e., without discharge from the nose, nor from farcy ulcers, but even in this form it is capable of propagation.

Farcied matter, made into balls, and introduced into the stomach of a horse, has caused glanders; and whichever way the virus has been introduced, once absorbed, it infects the whole blood, as has been proved by the experiment of the late Professor Coleman, who says- "I have produced the disease by first removing the healthy blood from an ass until the animal was nearly exhausted, and then transferring from a glandered horse blood from the carotid artery into the jugular vein of the ass. The disease in the ass was rapid and violent in degree; and from this animal, by inoculation, I produced both glanders and farcy." Professor Coleman also experimented on asses with virus obtained from man. He directed two asses to be inoculated with matter taken from the arm of a man labouring under the malarly, which resulted from a puncture received in dissecting a glandered horse, and both asses died of glanders. These experiments have becn repeated, and similar results obtained by Girard, Hering, and Leblanc.

Period of incubation.-Like all morbid poisons, that of glanders has its period of latency, which is, however, generally short. In the ass, the submaxillary glands become swollen and tender the second or third day after inoculation, and a discharge from the nostril occurs from the third to the sixth day. In some instances the incubation is much longer-from one to three or even six weeks; one case is mentioned in the Procès-verbal de $l$ E'cole de Lijons where the disease did not appear till the end of the third month after inoculation. The matter in this instance 
was obtained from a farcy ulcer, and the disease appeared at the precise point of the inoculating puncture.

\section{ACUTE GLANDERS.}

Symptoms.-The disease, preceded by increased temperature of the body, appears suddenly, ushered in by rigors, sometimes of a most persistent character. In one case which fell under my notice the rigors continned without intermission for three days and nights, at the end of which time a sanguineous discharge issued from both nostrils, the Schneiderian inucous membrane became violently inflamed and deeply ulcerated. More commonly, however, the rigors are not so persistent, but they are always more or less observable; the temperature is sometimes as high as $106^{\circ}$ or even $109^{\circ} \mathrm{F}$., the breathing is accelerated, the pulse feeble, rapid, and even dicrotonous, the leart's action palpitating and accompanied by metallic tinkling, the appetite fails, the pituitary membrane, at first of a dark copper colour, with patches of ecchymosis of a dark red hue, becomes purple, and the patches are rapidly converted into pitlike, ragged-edged ulcers, from which issues a copious sanious discharge. The submaxillary lymphatic glands enlarge. Other lymphatic glands inflame, enlarge, suppurate, burst, and expose raw, unhealthy-looking sores, from which issues a more or less ichorons and irritating purulent material. The eyes are weak, and a discharge issues from them; the nostrils are often swollen. The breathing is hurried, irregnlar, and impeded by the swelling of the nostrils, and by the glntinous discharge drying around them; abscesses speedily form along the course of the lymphatics of the face. The urine is pale, watery, and increased in quantity.

Acute slanders is rapidly fatal, and the post mortem examination reveals the following lesions:-The mueous membrane lining the nasal passages and facial sinuses is violently inflamed, and eovered with a numerous erop of pustules and uleers, from which a purulent or sanious fluid is freely discharged. In many instances, and where the disease has been very rapid, the inflammation is diffused over the whole surface of the mucous membrane, which then presents one uniform purple or purplish-black hue, its structure being exceedingly soft and friable from degene- 
ration, and it is easily detached from the bones. Now and then it will be found that the nasal bones, the septum nasi, the turbinated and ethmoidal bones, are in a state of necrosis, their surfaces bathed with a purulent discharge, and wholly separated from the mucous nembrane. The intermaxillary glands are congested and surrounded by a yellowish exudate, and the lymphatic ducts leading from them are thickened and congested.

The lungs in almost every case of glanders, acute and chronic, are more or less inflamed. Now and then the inflammation is diffuse, embracing perhaps the whole of one or a part of both lungs, whilst in others it is limited to some of the lobes, and presenting the appearance of a series of tumours and patches of congestion of different sizes, and in varying stages of development, scattered throughout the lung tissue. Many of these inflamed spots will be found to contain pus; hence they have been termed tubercles, and the nature of the disoase has, from this supposition, been laid down as tubercular; and so close is the relationship between tubercle and glanders, that the editor of the British and Foreign Medico-Chirurgieal Review says - "It is in glanders that Villemin thinks he has found the closest marks of analogy with tubercle, not only in its anatomy, but also in its symptoms and causation. He seems to have been conducted from the study of glanders direct to the inoculation of tubercle. The characteristic lesion of glanders is a small tubercle, which is strewn either in the mucous membrane of the nasal passages, or in the lungs, or, more rarely, in the liver and spleen. At first a greyish-white, firm granulation, composed of cells and nuclei apparently developed by hyperplasia of comnective tissue, it soon tends to soften centrally and form ulcers on the mucous membrane, cavities in the lungs. Like miliary tubercle, it occurs isolated or in clusters. Together with this little granulation, streaks and bands of fibrous tissue, as well patches of cheesy infiltration, are not infrequently met with in the lungs of glandered horses. It is interesting, too, that the same doults have been raised concerning the real nature of these 'infiltrations' in glanders as in tubercle. They are regarded by Villemin as one form of glanders, just as in man they are one form of tubercle As to which is the part primarily affected in glanders-the nasal membrane or the lungs-there is some difference of opinion; Virchow maintaining that the deposits in the lungs are always 
secondary and by metastasis from the nasal membrane; Phillipue and Bouley being convinced by repeated post mortem examinations that the primary lesions are always in the viscera, more particularly the lungs, and that the formations in the nasal membrane are invariably secondary. If, say they, a horse has the 'jetage' (discharge from the nose), it is already thoroughly glandered. It really matters very little which part of the body is first affected. In either case the analogy with a tubercular outbreak remains as strong as can be. The intestinal ulceration of tuberculosis-in which we see the counterpart of the nasal ulceration in glanders-is more often secondary to the pulmonary disease, but occasionally shows itself before any evidence of mischief can be detected in the lungs. Again, glandular enlargement of a severe and persistent kind constitutes an important part of glanders, as it does of tubercle. The mode of invasion is likewise identical in the two diseases; now acute, foudroyant, destroying life in a few days as by an overwhelming blood poison; now chronic, so as to last for years. Further, in the chronic form, the same recurrence of acute attacks complicating and adding to the chronic mischief is observed in glanders as in tuberculosis. To read a description of chronic glanders is, mutatis mutandis, to read an account of chronic phthisis. It is, therefore, not surprising that Dupuy goes so far as to say that glanders is a tubercular disease in the horse. In speaking of the supposed causes of tubercle, we purpose presently to follow out still further this remarkable thread of resemblance; but for the present it will suffice to say that glanders is transmissible by inoculation, and contagious from horse to horse, and that it is also unmistakeably communicable from horse to man. Call we hesitate to believe, says Villemin, that the parallel between tubercle and glanders must hear find its completion? To conclude, glanders and tubercle are so closely akin that they must be looked upon as nearly related species of the same genus." (Braithwaite's Retrospect of Medicine, vol. lviii.)

Whilst admitting the close resemblance between glanders and tuberculosis, and of the similarity of the microbes found in both diseases, we must hesitate to adnit their identity, for the broad fixct remains that inoculation with glanders produces glanders, whilst inoculation with tubercle is followed by the developmeut of tubercular nodules. 
The form of pneumonia seen in glanders is very characteristic, the inflamed part resembling an infiltration, with thrombi in the blood-vessels-gangrenous, emphysematous-of a greenish-black colour, and rapidly decomposing after death. The line of termination or demarcation between the healthy and inflamed lung tissue is often abrupt and very distinct. The tracheal and bronchial mucous membrane is more or less highly inflamed; in some instances covered with petechial spots or deeply ulcerated, and thickly covered with an unhealthy discharge, which exhales a gangrenous odour.

The changes which occur in the lymphatic glands in glanders are characteristic. The glands, irritated by the specific poison, become congested and enlarged; their cellular elements proliferate more or less rapidly, and are mixed with a citroncoloured exudate, which invades the surrounding connective tissue. In a few days the glands become dense and hard to the touch, more or less lumpy on their surface, and those in the submaxillary space fixed to the jaw by the now inflamed and indurated vessels which enter their deeper seated parts.

\section{CHRONIC GLANDERS.}

Symptoms.-In some instances the disease presents itself in such a mild form that the generál health is scarcely affected. There will be a discharge from one or both nostrils, generally from one nostril, and that very often the near (left) one. The submaxillary lymphatic glands are swollen and hard; the hardness and swelling are of a remitting nature, very often varying in size in a short period. For example, a horse may be left at night with scarcely any discoverable swelling, and found in the morning with a hard knot under the jaw, which is both easily seen and felt. The swelling may continue for several days, afterwards slowly disappear, and then reappear as rapidly as before. This condition may exist before any discharge issues from the nose, and a horse so affected is elegantly said to be "jugged." If the nostril of such a horse be examined, it will be found to be paler in colour than natural, or perhaps tawny, coppery, and sometimes of a dull leaden hue. The discharge of glanders presents a starchy or glue-like appearance, adheres to the nostrils, where it dries and accumulates, causing the nasal opening of the affected side to appear smaller or more contracted than in health. 
These appearances, in addition to a weak or debilitated condition of the eye of the affected side, may be all the symptoms present in a case of chronic glanders; indeed in some instances there may be nothing but the discharge from the nostril to lead the practitioner to suspect anything wrong with the animal, and the diagnosis is consequently very difficult, more particularly if the case is a solitary one; but where glanders is found to exist in a stud of horses, any suspicious symptom becomes significant. I have said nothing about the glanders-nleer, becanse in many instances of chronic glanders the ulcer is undiscoverable; indeed in some rare cases ulcers are never found either before or after death. For this reason Percivall limited the term chronic to that form in which no nlcers could be detected. He says, however, that they are always present in the frontal sinuses.

Before describing the more common form of glanders-namely, that in which ulceration is a characteristic sign, and called by Percivall "subacute glanders"-I shall call the reader's attention to what may be termed a spurious form of glanders; that is to say, a form of glanders where there is neither discharge from the nose, ulceration of the Schneiderian membrane, nor enlargement of the submaxillary glands; but in which all these may become apparent if the animal live long enongh. This form may be appropriately termed pulmonary glandcrs, and the symptoms are as follows:-The animal is languid, unthrifty, loses flesh, sweats on the slightest exertion; the visible mucous membranes are pale ; diarrhœa is easily induced, and there are occasional attacks of diabetes. There is a dry, dull, soft congh; the appetite is very capricious; the hair is easily removed from the mane and tail; the legs are sometimes very fine, sometimes more or less adematous. The affected animal may remain in this unsatisfactory condition for several weeks, or even months, and may die from marasmus and debility withont any other symptoms becoming apparent, or all the signs of glanders and farey may very rapidly develop themselves, and carry it off in a few days. If a case of this kind occur in the horse first attacked in a stud, or if in a horse where no others are kept, it is now possible to arrive at a satisfactory conclusion as to the nature of the malady by inoculating the animal with mallein, hereafter referred to. 
The post mortcm examination of a horse which has presented these symptoms prior to death will reveal the lungs studded with nodules, varying in size from a pin's head to a pigeon's egg, or even larger. Some of these nodules will be found to consist of an organised exudate-lymphoma-of a pearly grey appearance, and rather hard; others will contain pus enclosed in saes-vomicr; whilst others will be found in a degenerated condition, and presenting the appearance of rotten cheese, the degraded material consisting either of inspissated pus or a caseous metamorphosis of the exudate, in which the bacilli may be discovered by microscopical examination. In many instances the trachea is found studded with ulcers, and some of the deepseated lymphatic glands will be found enlarged, or in a suppurating condition. One remarkable pathological change which I have seldom failed to observe either in this or any but the most acute form of glanders, the splenic veins are filled with thrombi; the larger branches in particular being generally obliterated by hardened fibrinous coagula.

Glanders, as most commonly met with in this country, presents the following signs:-The horse is generally more or less off its feed, has a tendency to shiver on the slightest cold; its coat is rough and unhealthy, "lhas lost the bloom of health ;" it may or may not cough; the appetite is capricious; and perspiration is induced by slight exertion. There is a discharge of a starchy or gluey material from one or both nostrils; the discharge is often tinged with blood. In some instances recurrent hæmorrhage from the nostril is a premonitory sign of glanders. The mucous membranes are pale and unhealthy, and that covering the nasal chamber, from which the discharge issues, is studded orer with deep, pit-like ulcers. The ulcers are characteristic, being excavated, as if cut with a punch, but after a time they become ragged at their edges, irregnlar, enlarged in all directions, and confluent. The spaces between the ulcers are covered with hard yellowish pimples, which soon ulcerate. The eye of the affected side is weak, and Jooks smaller than its fellow, and an unhealthy discharge often issues from it over the face. The submaxillary lymphatics of the samc side enlarge and form a tumour-sometimes single, sometimes lobulatedwhich is more or less firmly adherent to the surrounding tissues. 
This tumour seldom suppurates; occasionally, however, I have seen it developed into an abscess, which, after having discharged a little unhealthy pus, has healed slowly, the tumour, however, continuing as large, or even larger than before the suppuration.

If the animal is allowed to live, these symptoms remit, and some of the ulcers may cicatrise, but they finally increase in intensity, and upon the application of any slight cause of disorder become rapidly developed into those of acute glanders. If the horse is well taken care of, it may do moderate work for a long time whilst affected with chronic glanders. In the course of time, however, symptoms of farcy appear; it then becomes a loathsome object, and if not destroyed, acute glanders sets in and carries it off in a very short time. ${ }^{1}$

Chronic glanders is more frequently seen than the acute form in the horse, but in the ass and mule the latter is more frequently met with.

In acute glanders the period of incubation is very short-from three to seven days; whilst in the chronic form the period is very uncertain, extending from a few days to several months.

\section{ACUTE FARCY.}

This form of the disease occurs either as the result of direct inoculation with the discharge of glanders or farcy; from infection through the medium of the air; as a sequel to some exhausting disease, particularly diabetes; from old age and other debilitating influences.

Symptoms.-The primary signs are those of fever, elevation of the animal heat to $106^{\circ}$ or even to $108^{\circ} \mathrm{F}$., rigors, loss of appetite, and swelling of the extremities.

The swellings of acute farcy, commonly confined to the extremities, manifest themselves by engorgement of a whole limb, resembling the swellings of acute 'lymphangitis or œedema, but presenting an uneven surface, increasing and decreasing suddenly, and attended with pain and lameness. When the engorgement of the areolar tissue diminishes, enlarged lymphatic glands and vessels will then be detected, forming buds and cords. The

1 For the differential symptoms of glanders and other diseases accompanied by a nasal discharge, see Principles and Practice of Veterinary Surgery, page 493. 
swellings, which are due to inflammation of the ducts and valves, point and burst, giving exit to a thin, purulent, yellowish material, which generally soon dries, and forms a yellow crust on the surface of the ulcer. These buds are generally found in groups, and away from the articulations.

In some instances farcy manifests itself by, first, the formation of a single painful swelling, which runs ou to suppuration, on the flexor tendons of a limb, below the hock or knee, and from which enlarged lymphatic vessels may after a time be traced. Other swellings form on various parts of the affected limb, with intervening enlarged lymphatic vessels, which ultimately burst and present ragged-edged, confluent, unhealthy-looking sores that discharge copiously.

In other instances, farcy is preceded by symptoms of rheumatism in some part of the body. Sometimes the muscles of the neck become acutely painful, and very often, but not always, swollen. The pain and swelling may disappear as suddenly as they came, and appear in some other part of the body, and this may occur repeatedly before any diagnostic signs of farcy manifest themselves; or the seat of pain may be the muscles of the chest, simulating pleurodynia or pleurisy, the animal groaning if made to move sharply or suddenly, and with a catch in his breathing diagnostic of thoracic pain. Again, one limb may suddenly become swollen and painful, causing much lameness. The tumefaction, heat, and lameness may recede, and attack the other limb; and such attacks may appear repeatedly and for several months, the animal's general health during the remissions being scarcely affected; but at length the disease assumes a more marked character, and unmistakeable symptoms of farcy appear, or glanders results, unpreceded by any true signs of farcy. In other instances, extreme lameness may appear in a limb without any traceable cause, and continue for days, or even weeks, without inducing any observable local change; generally, however, farcinous engorgement succeeds, and relieves excessive pain, and as a rule extreme pain is not of long duration. But it may be safely stated that equina is manifested in sucl a variety of ways as sometimes to mislead the most experienced. 


\section{CHRONIC FARCY.}

In chronic farcy the local symptoms generally precede any apparent febrile disturbance; but if the thermometer be employed for the examination of all horses in a stud where glanders exists, some elevation of the temperature of the body may be apparent in the infected prior to the development of any local symptoms.

These local symptoms consist of circumscribed inflammatory swellings, rumning in the direction of the principal vessels, which suppurate and burst, without much accompanying engorgement of the surrounding areolar tissue.

The circumseribed elevations or buls are connected together by corded lymphatic vessels, and wherever a valve is situated in a lymphatic duct, there a swelling will usually appear, and a bud will form. The buds are ranged in groups about the inner and outer aspects of the thigh, fore arm, flank, neck, and hend. From the circumstance that the enlarged cords and buds run in the same direction as the veins, the old farriers concluded that farcy was a disease of the veins; dissections, however, soon expelled this delusion, and the reason why the disease accompanies the vessels is explained by the fact that the lymphatics and blood-vessels run in company.

In some instances farcy is found confined to the cervical lymphatics. An examination of the neck along the course of the jugnlar vein will enable the veterinarian to detect the lymphatic duct swollen, hard, and presenting irregular knots along its course. Suppuration seldom occurs, but the animal sooner or later presents signs of glanders or of farcy in some other part of the boly.

Chronic farcy differs from the acute only in intensity and duration, and is the only form of equina which is at all amenable to treatment.

The Contagious Diseases (Animals) Act provides, however, for the destruction of glandered and farcied horses. I think that the provisions of the Act should be strictly carried out, and compensation paid from the imperial exchequer, as the disease, in. whatever form it appears, has hitherto remainerl incurable, and is always a source of danger both to human and 
animal life. It is stated, however, that cures have been effected by fourteen injections in forty days of mallein from a cat inoculated with glanders. Decroix and Bougome, two Algerian veterinary surgeons, are stated by Semner to have cured farcy by canterisation and extirpation of the farcy buds-an explodecl practice found to be useless in this country long ago. Brusaco, of Turin, and his pupils are stated to have cured 50 per cent. of glandered horses with carbolic acid, iodine, sublimate and sulphate of iron; whilst others claimed to have cured horses with tracheal injections of iodine and iodide of potassium; and in France they are satisfied with the curative effects of creosote in oil injected hypodermically. Semner again mentions that ox blood serum is useful in the cure of glanders. Pilarios states that he cured eight cases in this stage of the disease by repeated weak injections of mallein.

Glanders in man has been cured by frequent injections of mallein. Semner, however, prefers ox serum to mallein, and says that it has a more marked effect in destroying the virulence of the bacilli than mallein. Like Professor Macqueen, from whose observations (Central Veterinary Medical Society) I am now quoting - "To prevent misunderstanding, I ought to say that a glandered horse is said to be cured when it ceases to react to mallein. So much for the curability of glanders."(Veterinary Record, April 20th, 1895).

I entirely disapprove of the practice of working animals whilst affected with this malady, and would recommend the strictest surveillance by properly qualified inspectors.

\section{THE ANTISEPTIC PREVENTIVE TREATMENT OF EQUINA.}

When glanders appears in a stable of horses, in addition to the removal of all affected animals, and the inoculation with the mallein test of all in contact, it is most essential to attend carefully to the ventilation, drainage, food and water, and to the clean$\operatorname{sing}$ and disinfection of the stables, fittings, harness, and other appurtenances, the removal of contaminated wood-work, the painting of all fixtures, lime-washing, or even scraping and then lime-washing - the wash to contain a pint of crude carbolic aeid to every bucketful-and to the prevention of overerowding, exhaustive work, and all debilitating influences. It has been found serviceable to administer for several weeks to all horses in 
the same stud, with every meal, two ounces of the hyposulphite of soda, or two drachms of the chlorate of potash. American practitioners laud the hyposulphite in farcy, and say that the sores require no treatment except cleanliness.

- Tile Mallein Test.-Following the example of Koch, the discoverer of tuberculin, Helman of St. Petersburg successfully obtained the now recornised test for occult glanders, namely "mallein," which is a glycerine extract of cultures of the Bacillus mallei. This extract is sterilised by heat, and attenuated to ten times its weight with a two per cent. solution of carbolic acid. Thirty minims of this solution injected into a glandered horse causes an elevation of temperature of $2^{\circ}$ to $3^{\circ}$ in about nine to fifteen hours. There is also depression, increased rapidity of the pulse; a swelling at the seat of the inoculation, and of the nearest lymphatic glands, the lymphatic vessels surrounding the spot being also swollen and corded. Inoculations with mallein have no effect upon healthy horses.-(See Preparation of Tubereulin.)

\section{STOMATITIS PUSTULOSA CONTAGIOSA OF THE HORSE.}

The Veterinary Journal for November 1878 contains a report on this disease by Professors Eggreling and Ellenberger of the Berlin Veterinary College, translated from the Arehiv fuir Wissensehaftliche und Pratishe Thierbeillunde by G. A. B. I am indebted to Dr. Fleming for the woodcuts illustrating the eruptions characteristic of the disease.

They state that during the summer of 1876 several cases of a contagious disease appeared in and outside the clinic of the Berlin Veterinary College, presenting phenomena similar to those of variola, and chiefly affecting the mucous membrane and external integument.

Some cases outside the clinic had been declared by the inspecting veterinary surgeous to be suffering, or suspected of suffering, from glanders.

Most of the patients were from four to five years old, in good condition, and showing but slight indications of general illness; having on the whole a lively appearance, smooth and glossy coats, and eating bran mashes with good appetites; but whilst eating hay, large quantities of saliva flowed from the angles of the moutl. 
Some of the horses stood with depressed and stretched-out lieads; the temperature of the skin was warmer than natural, although the ears and extremities were cold. The pulse was 60 per minute, and full; the respirations normal. Even those showing the worst symptoms ate their food eagerly, but evinced pain during prehension, masticated slowly, swallowed with difficulty, and saliva was secreted in abundance, and a fine chlorophyl green discharge flowed from the nasal cavities; when water was taken large quantities of it was returned through the nose. The Schneiderian membrane was of a red rose colour; both the submaxillary glands were enlarged to about the size of a hen's egg-hard and knottybut unattached to the submaxillary bone or integument. The oral mucous membrane became intensely hot, red, and covered by a tenacious secretion. On passing the hand over it, small firm nodules, varying in size

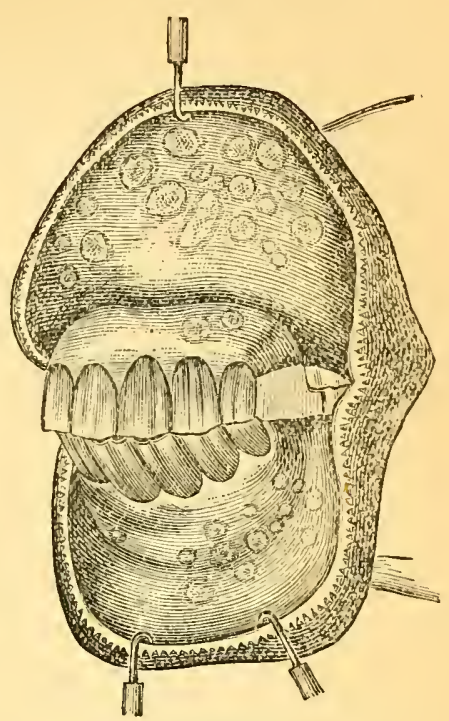

Fig. 8.-Ulceration of dental surface of lips. from a millet to a lentil seed, could be felt. These nodules rapidly increased in number and size, extending to the cheeks, tip of tongue, frcenum linguce, inferior surface of tongue and upper lip, and in one or two days later their summits presented a white appearance, or the epithelium was removed, and a small nlcerated surface was apparent, and in four or five days the ulcerations were numerous, the dental surface (see Fig. 8) of the lips, the tip of the tongue (see Fig. 9), frænum, and inferior surface of it, being

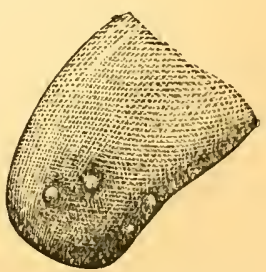

Fig. 9.-Ulceration of tip of tongue. specially affected.

About the sixth or eighth day the ulcerations began to heal. Four horses condemned as glandered, presented, in addition to the above phenomena, ulceration of the external integument, namely, on the upper lip, cheeks, and anterior extremities. 
The ulcerations of the skin in two of these cases were more numerous than in others observed before or since, and the prescapular glands were swollen in one case.

In one case the nodules appeared between the extermal nares and Schneiderian membrane; in another, on the skin of the lreast and fore extremities; in fact, on those parts on which saliva is most likely to be thrown, several nodules about the size of a pea, and two ulcers about the size of a threepenny piece were seen. On no other parts of the body could nodules or nlcers be found.

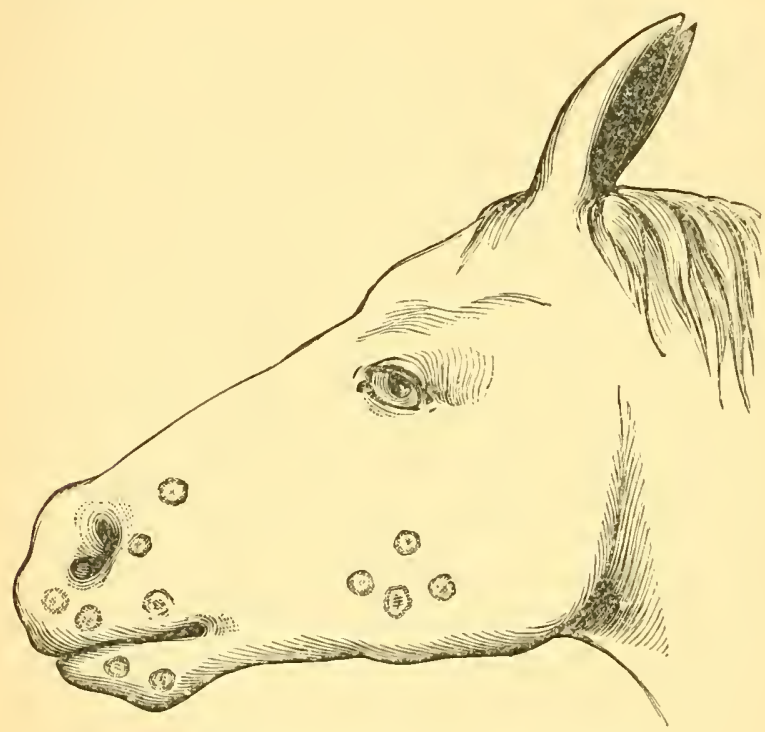

Fig, 10,-Ulceration of skin of lips and cheeks.

The disease runs a rapid course, the small nodules on the mucous membranes soon becoming prominent, presenting a smooth, round, and sharply defined surface, with a yellowishwhite summit, or the epithelium becomes removed, and they are transformed into round or slightly angular ulcers, varying in size from a threepenny piece to a shilling-they, in their turn, becoming encrusted, somewhat resembling pus scabs. Microscopically examined, they were seen to consist of pus corpuscles and epithelial cells, with red blood corpuscles. Fungi and micrococci were also present. The ulcers on the legs were generally larger than those on the mucosa. 
The healing process commenced in the ulcers about the sixth or eighth days, and all the cases under treatment resulted in the animals getting quite clear of the disease in from twelve to fourteen days.

Experiments as to the transmissibility of the disease by inoculation and infection were made on sixteen horses, two cattle, four sheep, one goat, three swine, two dors, one rabbit, and five men, with varying results. Four men and a number of horses took the disease by natural contagion, independently of experimental inoculation.

Below are given a few extracts from the diary kept during the course of the experiments.

$22 d$ May.-A seven months' old foal was successfully inoculated on the dental surface of the lip, the left ear, and the internal surface of the left fore leg; the resulting eruption was extensive and confluent, and the disease protracted, not having completely ceased until the twenty-first day.

$30 t h$ May.-A liealthy foal was placed with the above one, and another was inoculated on the vaginal mucous membrane. The inoculated one gave a negative result, but in the healthy foal the result was positive, the disease running its course, and terminating in eleven days.

31st May.-Another horse was placed with the last-mentioned foal. On the $2 \mathrm{~d}$ of June nodules were formed, the disease terminating on the 12 th. Others were inoculated with similar results, and the four foals which passed through all the stages of the disease before were again inoculated, but did not become infected a second time. One showed three small nodules, which disappeared without ulcerating in a few days.

24th May.-A calf was inoculated on the vulva. No symptoms appeared, and it was again inoculated on the 30th, after which the disease fully established itself, terminating in eight days. Others were inoculated with similar results.

23d May.-Sheep were inoculated with negative results. In pigs the result was also negative. In dogs, nodules appeared, but no ulceration. Rabbits were not affected.

29th May.-Mr. Häusel (student) inoculated himself. There was redness and unpleasant itching and swelling of the part, the formation of a pustule, over which a yellow-brownish crust formed, which fell off in fourteen days, leaving a deep cicatrix.

Other four gentlemen inoculated themselves without infection. 
A groom was infected, the disease running the course above described, he becoming quite convalescent in twelve days.

In contrasting this disease with that of glanders, the authors say-In a superficial examination the malady might be easily mistaken for that of glanders. In favour of this diagnosis we had its contagious character, the feebleness of the discharge from the nostrils, the swelling of the submaxillary and prescapular glands, accompanied with the formation of ulcers in the external integument and Schneiderian membrane (the last in one instance only). Close examination and observation, however, brought to light important differences between this disease and glanders. The most striking deviation was the appearance of the ulcer itself, which presented quite a different character to that of glanders. In these instances the ulcers were round, with sharply defined borders, varying in size from a threepenny piece to a shilling, the edges neither being serrated nor swollen as they are in glanders. The ulcerations were isolated, and independent of the course of the lymphatics. For instance, those on the lips and cheeks were irregularly distributed, and covered with a brownish-white scab projecting somewhat above the surrounding skin. When this was removed a white granular ulcerated surface presented itself. The ulcers were generally superficial, and rarely attained any depth, hardly ever reaching the subcutis. After the sixth day they readily healed.

The disease lasted in individual casos from twelve to fourteen days, and ran through a whole stable in about three weeks.

The discase in question differs then from glanders, more particularly in the character and distribution of the ulcers; also in the absence of lymphatic inflammation; in the quickness with which the ulcers healed; in the non-appearance of ulcers in the Schneiderian membrane; further, in the rapid course of the disease, its short incubative period, and the quickness with which it spread from one animal to another.

The virus seems to lose its virulence after passing through the system of several animals. From the scarcity of horses the experimentalists had to use cattle, when by degrees the lymph lost its infectious properties, until it finally ceased altogether. This characteristic separates the disease from variola, which it otherwise closely resembles. 


\title{
CHAPTER XXIV. \\ CONTAGIOUS DISEASES-continued.
}

\author{
MALADIE DU COÏT.
}

The name maladie du coït gives no further idea of the disease than that it results from the act of copulation. Other and various denominations, however, have been offered as expressing more definitely the character of this affection, some having been founded on its supposed analogy with the syphilis of the human being, and others upon the predominance of certain symptoms which have most particularly struck the observers.

Among these designations are the following:-Syphilis du cheval (syphilis of the horse); vérole; typhus vénérien; maladic pustuleuse et chancreuse maligne (frambœsia morbus pustulosus) (Erdley); épizootic chancreuse (Hering); maladie vénérienne du cheval; contagious eczema; paralytic disease of the horse; epizootic paralysis; epizootic paraplegia (Signol); donrine, \&c.

As all these terms and many others of a similar nature imply mistaken conceptions of the observers who so designated the disease, they have therefore been discarded, and the simple name maladie du coït, which (as I have said) is merely expressive of the fact that it is contracted during copulation, has been retained. It is difficult to give a correct defmition to this affection, the nature of which is not thoroughly understood, as may be seen from the following translation.

The nature of the disease is not plainly revealed by the symptoms and pathological changes. Some authors call it a nervous disease, and others rank it among the diseases of the generative apparatus.

It can scarcely be considered as vaginitis, nephritis, or angeitis. Though it is true that the symptoms and pathological anatomy show that these conditions exist, it must be remembered that they are consecutive, and that they do not form the base of the affection; also, that it is not linited to such organs as the vagina, the kidneys, the spinal cord, \&c. \&c.; for were it thus, according 
to the description of the disease, its nature would be seen to change too often.

It has been ranked in the order of inflammatory diseases, but it is quite different from them. In the inflammations, the circulation becomes accelerated, and the plasticity of the blood augments; while in this affection, on the contrary, the pulse is slow, the blood less plastic, there is a collapse of the vessels and non-turgescence; consequently no analogy exists between these two conditions. Not only is the blood less plastic, but greatly altered in its physical properties and constituent principles. It is black, no longer coagulates, assumes the consistence of gooseberry-jelly; in a word, has lost its vital and exciting properties, qualities indispensable to the free discharge of the nutritive functions. It presents characters identical with those of epidemic typhoid affections. Such at least is the opinion of the Commission at Tarbes.

The Germans, struck by the nervous symptoms which show themselves in the later stages of the disease, have considered it a nervous affection, and as it is nearly always accompanied by alterations of the lymphatic system, they have called it lymphatic-nervous-cachexia.

Others have looked upon it as a scrofulous, a nervous-phthisic, or scorbutic affection.

Its syphilitic nature has been admitted and rejected; admitted on account of the analogy of some local symptoms, and rejected on account of the non-transmissibility of syphilis from man to the horse by inoculation, and of the incfficacy of mercurial preparations in its treatment.-(M. Reynal, Traité de la Police Sanitaire.)

Thanhoffer says that the specific microbe is a micrococcus formd in the secretions of the vagina, in the semen, spinoneningeal fluid, the roots of the spinal (ischiatic) nerves, and in the blood. Hertwig says that it is transmissible by inoculation with the genital mucons secretions, the blood and other fluids appearing to be non-virulent, but further investigations into this subject are required.

\section{SIMPTOMS.}

As the disease manifests itself in two forms, benign and malignant, I will first detail the symptoms of the benign form in the female. 
Benign form, or Coital Exanthem.-The first symptoms of the benign form in the mare are often so slight that they do not attract the attention of the owner. They generally appear in from twenty-four hours to a fortnight after copulation. The animal strikes the ground several times in succession with its hind feet, whisks its tail, urinates frequently and in small quantities; in fact the symptoms are those presented by this animal at the period of cstrum. There is, however, this difference, that no matter how often the mare is covered, the sexual appetite does not appear to be satisfied.

The first local symptoms observable are a redness of the vaginal mucous membrane and the discharge of a muco-purulent fluid. At first this discharge is very slight; soon, however, the mucous membrane and the lips of the vulva become infiltrated, the discharge increases, at the same time becoming thick, viscid, and of a whitish-yellow colour, coagnlating around the vaginal orifice, and on the tail, perineum, or other external parts with which it may come in contact.

On the mucous membrane of the vagina may now be observed (though not invariably) a number of small pustules, about the size of a millet seed, changing into superficial ulcers, which heal rapidly and are replaced by others, that, like the first, are generally most abundant in the fossce navicularis, and on the clitoris.

According to M. Roll of Vienna (quoting from Maresch), during the prevalence of the maladie $d u$ coit in Bohemia, vessels of various sizes, containing a yellowish fluid, were observed on the mucous membrane of the vagina, instead of the pustules just mentioned. ${ }^{1}$

The general symptoms are œdematous swelling of the abdomen,

2 M. Rodloff says that the form designated benign by the authors is quite a distinct disease, and that the exanthematous condition, when found associated with what has been termed the malignant form, is accidental. Thus, what others call the benign form of the maladie du coitt, he calls "exantheme coïtale," observed in several species besides the horse, particularly cattle, and less frequently sheep and pigs, while to the malignant form he gives the name maladie du coit. The contagious microbe is located in the vesicles and pustules, and is transmissible from a mother to her suckling by the mouth, and from her legs by the postparturient secretions of the uterus, \&c. One attack does not give immunity even fur a few weeks. - (VerHeYes's Translation.) The distinctions between the two diseases are to my mind not sufficiently clear to warrant the division. 
perineum, and extremities, wasting, gencral weakness, and a slight engorgement of the submaxillary glands. The circulation, respiration, and even appetite remain unaltered in this form; the last may, however, become capricious. At this stage the symptoms commence gradually to diminish, and the disease usually disappears of itself in from a fortnight to two months. The only trace of it that can be seen four or five months afterwards is a slight thickening of the mucous membrane of the vagina, and that only in some cases.

It should, however, be remarked that this form may become aggravated and assume the malignant one.

In the stallion the benign form is difficult to detect. A considerable time may elapse before the manifestation of any symptom, and even then an intermittent odematous engorgement of the sheath may be the only one apparent.

The benign form in the stallion may become malignant as in the mare.-(Reynal.)

Malignant form (Dourine).-The local symptoms of this form in the mare are exactly the same at the onset as those of the benign form. Soon, however, they become more pronounced.

The lips of the vulva augment and diminish alternately; or one side only may be swollen, thus giving the organ a deformed appearance. Generally the swellings extend to the anus, and along the perineum to the mammary gland. The mucous membrane of the vagina presents numerous furrows, becomes still more infiltrated, and is studded with numerous patches of a blue or yellow colour, and on it may now be seen the pustules spoken of in the benign form.

The temperature of the vagina is elevated; the liquid that flows from it is more abundant, and also more irritating than in the preceding form, excoriating and denuding the skin of the thighs, \&c., over which it trickles.

On the external surface of the lips of the vulva, on the perineum, and on the inside of the thighs, lenticular pustules, which dry and form scabs, are sometimes seen. When these scabs fall they leave circular wounds, which heal rapidly.

The vulva and the mucous membrane of the vagina are now of a doughy consistence, and the latter presents a marbled appearance, the clitoris becomes swollen and indurated, and protrudes from the inferior commissure. The discharge is thick 
and purulent, of a reddish or greenish yellow colour, and exhales a very disagreeable odour.

The animal urinates often, and in small quantities, the evacuation being preceded, accompanied, or followed by a more abundant flow of the purulent discharge.

The ejection of the urine causes a great amount of irritation, and if the animal encounters any resisting obstacle behind her, she will rub herself violently against it.

Mares in foal generally abort about the third month. When they carry the full period, the foals to which they give birth are usually dead, or die in a few hours.

Stallion.-The manifestations of the symptoms in the stallion are exceedingly uncertain. Sometimes they remain latent for a very long period, and are only aroused by a repetition of the coït.

The sheath then becomes the seat of an cedematous swelling, extending in front to the umbilicus, and occasionally to the sternum, and behind to the scrotum, which may either retain its normal proportions or became slightly involved in the swelling.

According to M. Reynal, stallions have been observed to present no other symptoms than a swelling of the sheath for eight, ten, or even twelve months, and still they were affected by the disease.

In some cases the testicles, as far as external manipulation can decide, remain perfectly healthy; in others they become swollen, and the animal evinces great pain on the slightest pressure.

When the penis is protruded it may present no alteration, or it may (if destitute of pigment) have a reddish or marbled appearance. In some cases spots of various diameters, and of a deep violet colour, resembling ecchymosis, have been observed. When the organ is in a state of complete erection the glans penis acquires a volume so great that its return is accomplished with considerable difficulty. There is a hypersecretion of the urethral mucous membrane, which protrudes slightly from the meatus urinarius, attains a somewhat globular form, and a crimson or yellow colour.

Cicatrices have been observed on the penis, in greatest quantity near the glans, and the observers have thought it probable that they were the result of the healing of small super- 
ficial ulcers, caused by the destruction of vesicles which had formed on this organ.

After each evacuation of urine, effected after a number of painful efforts, and expelled in a small but continuous jet, the animal stamps violently, and whisks its tail, as if the passage of that fluid through the urethra had caused a considerable amount of pain.

In concluding the local symptoms, it may be remarked that paraphymosis is not an unfrequent result of the local swellings.

\section{GENERAL SYMPTONS.}

As these are the same in both sexes, it would be useless to consider them separately.

A more or less abundant discharge of a muco-purulent matter from the nostrils, and of tears from the eyes, sufficiently indicate that there is a general affection of the mucous membranes, of which those visible are highly injected.

The lymphatic ganglia, not only in the vicinity, but also those in the submaxillary space, are engorged.

The appetite is generally good, and the food thoroughly digested; nevertheless the animal gradually falls off in condition, and, to use a common expression, becomes hide-bound, with a dry and staring coat; the perspiration gives off a most offensive odour.

The sensibility of the lumbar region is so great that in some cases ordinary pressure will almost bring the sufferer to the ground. The presence of albumen has been observed in the urine.

General weakness soon manifests itself by the weight of the hind quarters being thrown alternately and incessantly from one posterior extremity to the other.

When the animal is caused to walk the gait is very uncertain, and the sudden flexion of the joints is sometimes so great as to cause it to fall. There is a considerable tumefaction around the larger joints, and especially around that of the haunch. This tumefaction is very painful, and causes the animal while at rest to lift its leg with a sudden jerk, after the manner in which a horse with stringhalt behaves wlen caused to move suddenly, but it places it more carefully on the ground again than does the latter. When both limbs are affected, this movement is manifested alternately. These symptoms of lameness are some- 
times intermittent, disappearing for a week or two, and then reappearing with a greater intensity.

After some time the appetite becomes exceedingly capricious, but is never entirely lost, the animal continuing in many cases to eat till within an hour of its death.

One or both hind extremities now become paralyzed, causing the sufferer to fall upon its litter, and the entire muscular system becomes atrophied, but more especially the hind quarters and legs.

The mucous membrane of the nose becomes of a yellow and the buccal membrane of a leaden colour; and there may be slight constipations.

Unless pneumonia or other complications of the respiratory organs set in, the respiration remains natural. M. Rodloff has often observed harses suffering from this disease become brokenwinded.

In the mare, one or other haif of the mammary gland sometimes becomes the seat of an inflammation, which usually terrninates in suppuration.

In the incubative stage of the disease, numerous cutaneous tumours form, which, unlike those of farcy, are entirely situated in the skin, and do not invade the subcutaneous tissues. They are of various sizes, but may be divided into three-a small, a medium, and a large size.

The small tumours, which appear suddenly, and may as suddenly disappear, have attained, when first seen, their full dimensions. This, however, is not absolutely without exception, as in some instances an increase of circumference has been observed. The middle and large-sized tumours commence by a circle, on which the hairs stare; the centre of the circle gradually fills in, and the tumour becomes fully developed within twenty-four hours. Their duration is variable, but may be said to be from four to nine days. The disappearance of the small tumours may be effected in a night, while that of the larger ones takes place more gradually; temperature and sensibility in the former are not above that of surrounding parts, while in the latter both are considerably exalted. Their disappearance is soon followed by the eruption of a fresh crop, which may be situated on the same or on different parts of the body.-(M. RodLoff. $)^{\mathbf{1}}$

1 Translated by M. Verheyen for the Recueil de Médecine Vétérinaire, 1855. 
The paraplegia, which almost invariably sets in before the termination of the disease, and which is described by some authors as its third stage, is occasionally accompanied by other paralysis, such as that of the lips.

Glanders and farcy are not unfrequent terminations.

\section{PROGRESS AND DURATION.}

The progress of the disease is slow; it does not terminate before three or four months at least, and it may extend over a much longer period. An apparent recovery should always be looked on with suspicion, as the affection is subject to intermissions. When the disease exists in a country, all stallions presenting persistent swellings of the sheath from unknown causes should be regarded as suspicious, and if in use as reproductors, their work in that sphere should be prohibited, for, although latent, the disease is none the less contagious.(M. REYNAL.)

Its duration is variable, extending from three to eight months, and even to three years.

A Ministerial Order in Prussia prohibits the removal or use, as reproductors, of affected stallions, and in the case of recovery they are not declared healthy until after the lapse of three years.

\section{ETIOLOGY.}

The original cause has been until now, and is likely to remain, in obscurity. A great many hypotheses, however, have been raised, and M. Lafosse, in his pathological treatise, gives a long dissertation on the effects produced by the crossing of various breeds, the change of climate necessitated for that purpose, the changeable condition of the atmosphere during the period at which the disease makes its appearance, which is always in spring, \&c. \&c. These, he says, are the predisposing causes, while copulation, too often repeated, by over-exciting the genital organs, and perverting the secretions of the parts which become primarily affected, is the exciting cause.

This theory of M. Lafosse is, however, open to grave objectious. If it be correct, why does not the disease appear every year and in every country in which the animals are exposed to the same causes? 
The supposition that the maladie $d u$ coït has been transmitted from man to the equine race, has not been rejected on account of the revulsion of feeling to which the thought of such a degradation gives rise, for the record of the debaucheries of Rome sufficiently show that sexual intercourse between man and the inferior animals has taken place. Even in this enlightened age the Arabs believe that by fornicating with the ass they can rid themselves of venereal diseases, and on this account some of the adherents of its syphilitic nature have founded the origin of this disease.

Its syphilitic nature, however, is rejected on better grounds.

The symptoms are not those of syphilis; the indurated chancres, or the ulcerations which invade the throat, lips, \&c., that characterise this disease in man, are not met with in the maladie du coït.

The inoculation of the syphilitic virus to the lower animals, as shown by numerous experimentalists, and more especially by the carefully conducted experiments of M. le Docteur M. A. Horand (Chirurgien en chef de l'Antiquaille à Lyons), and M. F. Peuch (Chef de service de clinique à l'école vétérinaire de Lyons), practised in 1868 and 1869, and published in 1870, is followed by entirely negative results. The mercurial treatment, so efficacious in syphilis, is not only entirely fruitless, but has been thought to hurry the death of the equine sufferer.

It would be useless to detail the hypotheses of the various authors, as they are all equally unsatisfactory.

The history of the disease, if minutely related, would occupy several pages. I will, however, limit myself to say that it was not known until the end of the last century, when it was first seen in Russia (in 1796), and that it has since invaded Africa, America, Egypt, and the majority of the European countries, No mention, however, has been made of it in Great Britain Spain, or Belgium.

\section{CONTAGION.}

The contagious properties of the disease have been denied by some, but the experiments of M. Lafosse are sufficient to convince the most incredulous. In 1852 that gentleman took fifteen healthy mares from a regiment stationed at Toulouse (where the disease had never been seen, and where it has never since 
reappeared), and presented them to affected stallions which had been brought for the purpose from the Dépôts de Tarbes, where the disease was then raging. Five of these mares became affected to a slight degree, the disease disappearing spontaneously. Five others became seriously affected, one of which only recovered; thus showing that five only entirely resisted, while four succumbed to the disease.

Other experiments could be related, but I think the above is quite sufficient to show that its transmission during the act of copulation is undeniable.

It is, however, just to observe that numerous experiments by inoculation were conducted without result, before the contagion was denied.

M. Hering, notwithstanding, succeeded in one instance in producing the disease by rubbing the mucous membrane of the vagina with the virulent matter.

It is worthy of remark that stallions offer more resistance to the contagion than mares.-(M. ReyraL.)

\section{POST MORTEM APPEARANCES.}

The post mortem appearances with regard to the genital organs do not coincide in different subjects. The lesions of the external organs and of the mucous membrane are, however, constant.

The sheath, when cut into, presents the appearance of a hard, homogeneous, yellow tissue, but on a closer examination the fibres of the areolar tissue may be distinguished, and between them the serosity which gives the appearance indicated.

The penis is partly protruded from the sheath, and slightly infiltrated towards its free extremity. On its external surface, though very rarely, small whitish elevations, formed by the infiltration of a small quantity of serum under the epithelium, have been observed. Small dried coagula of blood, which give the ecchymosed appearance mentioned in the symptoms, have also been enconntered; but the mucous membrane offers no other alterations than a slight protrusion, a thickening, and a yellowness in colour.

The mucous membrane of the vesiculæe seminales is said by some to offer a red or violet appearance, containing a yellowish thick matter of a purulent aspect; by others, the vesiculæ semi- 
nales, as well as Cowper's and the prostate glands, are said to be in a normal condition.

The testicles may either be healthy, atrophied, or hypertrophied. When hypertrophied a yellowish serosity is found in their interior. In the mare the mucous membrane of the uterus and vagina is thickened, ecchymosed, and of a reddish-brown colour. In the uterus a muco-purulent, yellowish-white, or a chocolate coloured fluid, analogous to that which flows from the vagina during the course of the disease, is found in greater or less quantity. It is said to have been so abundant in some cases as to have given the patient the appearance of being pretty far advanced in gestation.

The kidneys may be either in the normal condition or they may be hypertrophied. Their pelves contain a substance resembling the white of an egg, which has been found to be abundant in albumen. MI. Lafosse has found them to contain in exceptional cases a substance similar in appearance and consistence to honey.

The mucous membrane of the ureters and bladder in both sexes present a similar aspect to that of the womb in the female.

The muscles are friable, as are also the bones, especially the femur and ribs, of which the spongy tissue is generally impregnated with a dark-coloured blood, or with a yellow gelatinous matter.

The coxo-femoral and pubio-femoral ligaments are red, thickened, softened, and sometimes ruptured.

The synovia of the articulations is abundant, muddy, and presents the colour of blood.

The cartilages are yellow and soft, and have sometimes disappeared from the articular surfaces.

The fatty matter has everywhere disappeared.

The subglossal, sublumbar, and mesenteric ganglia, as also those in the vicinity of the urino-genital organs, are considerably enlarged, and of a yellow or reddish-brown colour. They are sometimes found to contain purulent matter. The French authors describe no other lesions in the cranial cavity than the superabundance of the subarachnoidean fluid, while the German authors affirm that the brain is in a degenerated state. They, however, agree that in some cases the spinal cord is softened, particularly in the lumbar region. It may, however, be re- 
marked that when the disease terminates in paraplegia, if this condition of the cord be not found, it is at least slightly congested.

Occasionally the volume of the sacro-lumbar plexus and sciatic nerves is augmented by a serous infiltration.

The digestive organs are healthy; in some instances, however, the liver and spleen are enlarged and softened.

The lungs are healthy (unless the disease has become complicated by pulmonary affections), but the mucous membrane of the bronchial tubes and larynx is slightly affected.

The sinuses of the head often contain a yellowish, oily matter, and the Schneiderian membrane is of a leaden colour, with red spots scattered over its surface.

The muscular tissue of the heart, like the rest of the muscles, is soft, friable, and of a pale yellow colour.

The blood is very much altered; it is fluid, defieient in fibrin, and, according to M. Lafosse, who has made microscopical examinations of it, the coloured corpuscles present a deformed appearance.

\section{TREATMENT.}

Antiphlogisties (including bleeding), emollients, tonics, stimulants, alteratives, \&c. \&c., liave all been employed in this disease; but with so little success, that until the appearance (in 1865) of M. Trelut's memoir it was believed incurable.

M. Trelut, after a careful study of the disorders produced in the organism by the maladie du coït, adopted an entirely different treatment, the efficacy of which is beyond a doubt.

Being struck by the fact that the blood of animals suffering from the disease was deficient in one of its most important elements, viz., the fibrin, he thought that if this constituent were renewed in the vital fluid the disease might be overcome.

He accordingly procured the necessary fibrin by stirring the blood of eattle when warm, thus separating the fibrin from the other constituents. The fibrin obtained in this manner was dried in an iron pot, in which a little butter had been melted to prevent carbonization; it was then divided into small particles and administered in the morning, fasting, in doses of sixty grammes (3xv.), either as an electuary, or added to half a quart of gruel as a drench. To stimulate digestion twenty or thirty grammes ( $\overline{\mathrm{v}}$. or 3 vii.ss.) of turpentine were administered every seeond 
morning with the drench. From 3i. to 3 ij.ss. of iron, reduced by liydrogen, alternated with from fifteen to thirty grains of the white oxide of arsenic, were also given, and food of the most substantial kind was allowed.

As the patients under the charge of M. Trelut increased, he found that a sufficient quantity of fibrin could not be obtained in the manner described. He then substituted for the pure fibrin of the blood of cattle, cooked horse flesh, very finely divided, and administered in the water which had served to cook it. When the bouillon was finished the flesh was mixed with honey and given as a electuary. Were it not important to utilise the soup, says M. Trelut, the flesh could always be given most conveniently in the latter manner.

The flesh was administered in doses of from 100 to 150 grammes.

When paralysis set in, cantharidine liniments, followed by mustard poultices, were applied to the abdomen; and as soon as an abundant effusion had been established, the pointed firing-iron was apphied, penetrating as deeply as possible, in order to fix the engrorgement. After the establishment of this engorgement the paralysis became gradually ameliorated, and in twenty-four hours, the animal, which was helplessly stretched upon its litter, was able to regain its feet without assistance.

The internal treatment requires to be continued at least fortyfive and at most 145 days, and on an average from two to three montlis.

By this treatment M. Trelut was successful in sixteen cases (in which the patients were seriously affected) out of seventeen.

The seventeenth was a mare which aborted, and the lesions of metro-peritonitis found at the autopsy would have been sufficient to account for her death.-(Memoire sur la Maladie dite du Coüt, par MI. Trelut, Recueil de Médicine Vétérinaire, Janvicr 1S65.)

Local treatment.-In stallions, during the first stage, or that in which the disease is confined to the genital organs, it was stated that good results had been obtained from castration; it is now proved that castration always aggravates the disease, and sometimes induces a fatal result.

In the mare, mucilaginous injections, followed, later on, by mild antisentics, astringents, and still later, if necessary, by the sul- 
phate of copper, sulphate of zinc, or nitrate of silver, \&c., have been recommended.

\section{COITAL EXANTHEMA OF HORNED CATTLE.}

It is stated in Friedberger and Fröhner's works on pathology and therapeutics of the domestic animals that this disease is more frequent in cattle than in horses simply on account of their greater number.

It is very contagious, as all the cows of a village may be infected by one bull, and it is said that it is communicated by friction of diseased against healthy cows, and by continual wagging of the tail. In oxen Numann has observed the eruption around the anus and sheath. Armbruster states that the contagion is conveyed by wooden drains-when animals lie down, their genitals coming in contact with these drains-when these are contaminated by diseased discharges. Schneiper says that the disease may affect the same subject several times at short intervals.

The symptoms are similar to those observed in the mare. There is intense pruritis, vaginitis with redness and tumefaction, muco-albuminous secretion; membrane covered with dark red spots, vesicles, and pustules of various sizes, which rapidly ulcerate, the discharge from which contaminates the perineal region and tail, drying up, forming crusts; micturition is difficult, and manipulation causes much pain. The appetite, secretion of milk, and other signs of general disturbance are frequently present. The duration of the disease seens to vary from eight to fifteen days, and more rarely from three to four weeks. Some cors abort. In the bull there is much tumefaction of the genitals, the penis studded with pimples, and there is a discharge of a yellowish purulent material from the urethra; phymosis is frequent; erection causes the ulcers to bleed; there is more or less constipation and loss of appetite.

Local antiseptic and astringent treatment is recommended. 


\section{CHAPTER X X V.}

CONTAGIOUS DISEASES-continued.

\section{CANINE DISTEMPER.}

Definition.-A febrile disease, due to the operation of a morbid poison, as the result of contagion and infection, and known in almost all parts of the world. The specific microbe has not been discovered, but it is known that the disease is transmissible by contagion and infection, and that, therefore, the virus is both volatile and fixed. It is most readily conveyed by the air. The virulence of the contagium contained in the discharges, the blood, \&c., is diminished if kept in a dry state for a time, but congelation and dessication seem to exert no power over it.

\section{PATIIOLOGY.}

The virus primarily indnces a febrile condition of the system, and specifically affects the mucous membranes of the nose and eyes. In some cases the poison destroys by shock, the animal dying, in a few hours, from collapse, without any true signs of distemper being developed. In other cases the action of the poison is concentrated upon the nervous system, causing epileptic fits, spasius of various muscles, and finally coma and death. Again, the liver seems to be the organ chiefly affected, and in other instances the intestinal canal or the bronchial mucous membrane is the seat of the local effects of the virus. Secondarily, the spinal cord or some of the spinal nerves become diseased, and, consequently, distemper often terminates in paralysis of the posterior extremities, or in chorea; the clonic spasm characteristic of chorea being generally more observable in the muscles of the neck and fore extremities. In consequence of the variety of forms which the malady assumes, it has been 
described as five different diseases, namely-(1.) The catarrlial : (2.) The respiratory or bronchial: (3.) The bilious: (4.) The intestinal: and (5.) The nervous. These are merely varieties of one and the same disease, and exemplify in a most remarkable manner the method in which the same virus or poison may act upon more parts or organs than one, as is the case with various medicinal and other agents.

The spasmodic condition of the body-the chorea-and the paralysis also illustrate how a morbid poison, after exhausting its powers upon one or more organs, may, after a space of time, affect other organs of the same body.

Distemper, like all contagious and infections diseases, has an uncertain but short period of latency. It, as a rule, affects the system only once; hence it is most prevalent in young dogs, and sometimes prevails as an epizootic.

Attempts have been made to discover the specific microbe of canine distemper. Some writers assert that it is a thin, short, bacillus found in the blood and discharges-(Semmer and Laosson)-small-sized corpuscles arranged in pairs, or four by four, like sarcina (Rabe) micrococci (Krajewiski). Mathis asserts that he has found a specific diplococcus in the discharges, and has obtained pure cultures, inoculations with which giving positive results, most of the young dogs died, having previously manifested the systems of the disease; those that lived obtained immunity.

Some writers have compared distemper to the typhoid fever of man. I have carefully dissected numerous fatal cases of distemper, and looked particularly for the specific lesion of typhoid, namely, congestion and tumefaction of Peyer's glands, but have failed to detect any growth in the gland cells of the intestines. In the intestinal form of the disease a generally congested condition, with even ulceration of the mucous nembrane, may be witnessed, but these ulcerations are very different to those observed in typhoid fever. Again, intestinal lesions are the specific distinctions of typhoid, whereas in distemper inflammation of the mucous membrane of the bowels is only seen in that form in which the virus seems to exert its influence on that part of the animal body, and it is only in a minority of instances that this effect is witnessed.

I can compare distemper to no human disease except measles, 
and the points of analogy are very great. In both diseases catarrhal symptoms are manifested; they are infectious diseases; they generally occur but once in a lifetime; they chiefly affect the young; in almost all cases of distemper there is some cutaneous eruption or rash, and desquamation of the cuticle; catarrhal ophthalmia, bronchial and pulmonary inflammation, and dysentery, are complications of both diseases, and, finally, convulsions sometimes occur both at the commencement and during the progress of measles and of distemper.

I am not aware, however, that measles is succeeded by either paralysis or chorea; nor do I mean it to be understood that distemper is communicable to man.

\section{SYMPTOMS.}

The primary symptoms are those of fever, associated with those of catarrh. The dog shivers, is dull, restless, with dry nose and injected eye. The appetite is partially lost; there is thirst and rapid loss of flesh and condition; the urine is high-coloured and scanty; the bowels are generally irregular, sometimes constipated, sometimes looser than natural; the freces are darkcoloured and foetid. In the course of a few days the catarrhal symptoms, which at first may have been limited to frequent sneezing, with a slight discharge from the nose and eyes, are fully confirmed. The nasal discharge is now more or less profuse; the eyes are weak, occasionally inflamed, and discharge tears and mucus. Very often the eyelids will be gummed together, and the animal thus rendered temporarily blind. Cough is present, at first dry and husky, afterwards moist. The breathing is sometimes much quickened, and the cough dry and painful, showing that the lung tissue and pleura are affected: the pulse may range from 120 to 150 beats per minute, and the temperature is elevated. In other cases the respiratory movements are but little affected, except when the bronchial tubes are filled with mucus, which is coughed up, and the breathing becomes much relieved. As the disease advances, debility rapidly increases, the dog being often at the end of the first week scarcely able to stand; the appetite becomes more and more impaired, and the digestive powers much debilitated. Food now partaken of or forced upon the animal is quickly 
ejected by vomiting, or passes through the intestinal canal in a foetid, ill-digested condition. At the end of about a fortnight these symptoms may abate in intensity, and the dog slowly regain its strength. Very commonly, however, complications occur which tend towards a fatal termination. The complications are as follows:-

1st. Pneumonia.-The breathing becomes laboured, rapid, and panting; the prostration of strength is very great; the dog is unconscious of surrounding objects; the pulse is frequent, feeble, and intermitting, and the feet, nose, and ears are icy cold. If the ear be applied to the chest the crepitating sounds of pneumonia will be detectecl.

2d. Jaundice.-This occurs from the presence of a blood poison, interfering with the normal metamorphosis of bile, from congestion of the liver, or most commonly from catarrhal inflammation of the mucous membrane of the biliary ducts; the swollen mucous membrane mechanically blocking up the tubes, and thus impeding the flow of bile--(See Liver Diseases.)

3d. Intestinal complieations. - Vomiting and purging are prominent symptoms. Sometimes there is true dysentery, the freces being tinged with blood; tympanitis and abdominal pain.

4th. Epileptie fits.-Spasmodic convulsions of varying intensity, coma and paralysis, more or less complete, occur as primary symptoms. These are to be separated from those signs of nervous alterations which occur as secondary affections in distemper. In the first case the brain and nervous symptoms are concomitant with or shortly succeed the attack; whilst in the latter, chorea, paralysis, or complete coma appear after the febrile condition has more or less abated, and when the dog seems in a fair way to recover.

Coninnctivitis is not at all an uncommon complication, and ulceration of the cornea, perhaps unpreceded by any inflammatory signs, may occur from mal-nutrition.

In other cases cellulitis or an erysipelatous inflammation of the extremities occurs, the inflammation sometimes suppurating in various parts: the suppuration, being of a diffuse or infiltrating character, causes much pain and rapid exhaustion.

In most instances some cutaneous eruptions are seen on the inner surfaces of the thighs and other parts, where the hair is thin and downy; the eruptions at first resemble flea-bites, but 
may become vesicular or even pustular; the skin is harsh, and much epithelium is thrown off, causing the hair to be filled with scales of scurf.

There are various conditions of the body which predispose the disease to attack the dog in the various ways above enumerated. The presence of worms in the intestinal canal may excite the intestinal form; previous high feeding and obesity the bilious; and the eccentric irritation of the nervous system caused by teething, worms, \&c. predispose the dog to the nervous form.

Distemper originates undoubtedly from contagion as well as from atmospheric infection, and usually rages as an epizootic. The majority of dogs in some districts suffer from the disease, whilst in other parts of the country it is scarcely ever heard of, unless brought there by a dog already diseased, and no class of dog is exempt. During the author's residence in Australia the disease was imported from Europe, and scarcely a dog in the colony of Victoria was unattacked. Nor were the wild dogs allowed to escape; hundreds of these were to be seen lying dead in the bush in various parts. It is also stated that it attacks the cat, wolf, hyæna, prairie dog, and monkey.

The contagium or virus, probably a microbe which may yet be attenuated and applied for the purpose of protective inoculation, will taint a kennel for a long time after the disease has disappeared; and it is always unsafe to introduce fresh dogs into such a kennel, if it cannot be proved they have passed through the disease, unless the walls, drains, fittings, \&c. are thoroughly cleansed and disinfected.

\section{TREATMENT.}

In the treatment of distemper it must always be remembered that the disease runs a certain but indefinite course, and that all the symptoms are but the result of the operation of a morbid material existing in the blood. If these facts be borne in mind, the practitioner is not likely to resort to those dangerous and exhausting remedies so generally recommended by writers on canine diseases. The administration of calomel, jalap, aloes, tartar emetic, digitalis, with the application of blisters or setons, is calculated at all times to do harm.

In the earlier stages, if the bowels are at all irregular, a small dose of castor oil is to be prescribed. The dose must vary in 
strength according to the size and age of the dog; from a teaspoonful for a small dog or young puppy, to an ounce for a wellgrown dog. This will remove any ill-digested or indigestible material from the intestinal canal.

After the laxative has operated, I have found from two to six grain doses of the hyposulphite of soda useful, modifying the symptoms most materially, and converting what has threatened to be a serious case into a mild attack. Care must always be taken not to administer any medicine in such large doses as to disorder the stomach in any way, or to cause vomiting, as it is of essential importance to keep up the animal strength by proper food, spontaneously partaken of. If there be much prostration of strength, a tea-spoonful of good sherry, or one drachm of spirits of nitrous ether, may be administered three or four times a day, in addition to the hyposulphite, with very great advantage.

This simple treatment, in addition to warmth, fresh air, a clean bed, and clothing for thin-haired dogs, with a plentiful supply of fresh cold water (or milk and water if the dog will take it) for the animal to drink, sponging of the nose and eyes not being forgotten, will often be successful. The food must be restricted in quantity, and of an easily digested nature. In my own practice I finct porridge and milk to be the best, provided the dog will take it; but if it be a pet dog, and used to pampering, it must have what food it will eat, and what it has been used to, in modified quantities. It may be laid down as a rule that the digestion of what the $\operatorname{dog}$ is fondest of will be more easily performed, provided that it is not allowed to eat too much. After the disease has continued for six or seven days, small doses-from one grain to three-of quinine may be administered, care being taken to discontinue it if it seem to disagree with the dog.

If there be much irritation of the stomach and vomiting, hydrocyanic acid, from two to four drops, Scheele's strength, will have a calmative effect on the gastric organs and allay the vomiting. Purging, if not excessive, should not be violently checked, but should the dog seem to lose strength from this cause, chalk may be first tried, and afterwards tincture of opium, from ten to twenty drops, three times a day.

The pulmonary complications are best relieved by the application of hot flannels to the sides, or hot fomentations may be 
substituted in smooth-haired dogs. In rough-haired ones, the hair saturated with the wet is a long time in drying, and the animal is apt to take cold. Nitrate of potash may be dissolved in the dog's drinking water, or given in solution in from six to twenty grain doses, as a febrifuge and diuretic; and the chloral hydrate may be given at night, particularly if the dog is sleepless. The nervous symptoms may depend upon reflex irritation. If from teething, the gums are to be lanced. If from the presence of worms, and this is a common cause, and tape-worm the parasite generally met with, from one scruple to one drachm of areca nut is to be administered. If the appetite is entirely lost, advantage must be taken of the thirst, and beef tea or milk allowed the dog to drink. If it will not drink spontaneously, nourishment must be forced upon it, in small quantities and oft repeated. A raw egg beaten up with a glass of sherry, carefully administered, will be of great service; in other cases, brandy and beef tea. I am, however, opposed, unless the prostration of strength threatens to prove rapidly fatal, to the forcing of large doses of stimulants, as they often cause much mischief, careful nursing and good attendance being much more beneficial than any mere medicinal remedy,

I have very little to say upon the treatment of the secondary nervous complications. They are due to a variety of pathological changes.

Paralysis sometimes results from obliteration or plugging of the spinal veins, which are found enlarged and pressing upon the cord: I have found this in several dissections of dogs which had died or had been destroyed whilst suffering from paralysisfrom atrophy of the cord, the nervous matter of which being in some instances replaced by a semi-fluid deposit, and from congestion and disintegration of its substance.

Some cases of paralysis after distemper, if kept long enough and well fed, will recover. As a rule, however, the loss of power remains, and the dog has ultimately to be destroyed. I have tried strychnia and other nervine tonics, but cannot say that they do much good. Iodide of potassium-given on the assumption that the paralysis resulted from the pressure of an exuclate on the cord-has been tried also by me, but with no very successful results. Blisters to the spine, setons, and even the application of the actual cautery, have also been tried in such 
cases. Such treatment inflicts much pain on the poor patient, but does not remove the disease or prolong life.

If the eyes are tender or inflamed they are to be bathed with a solution of boracic acid, and in order to relieve any pruritis and prevent scratching a five per cent. solution of cocaine should be applied every few hours; irritants, such as the nitrate of silver and sulphate of copper, should be avoided. Calomel, blown into the eye, may be tried in chronic opacity. 


\section{CHAP'TER XXVI.}

CONTAGIOUS DISEASES-continucd.

\section{ANTHRAX.}

Anthrax; charbon; gloss-anthrax; apoplexia splenetica; carbunculo contagiosa, \&c. (L.); charbon; chancre à la langue; mal de sang; sang de rate; typhomiè; fièvre putride, \&c. (F.); miltzbrand; miltzbrand-fieber; petechial typhus; pestfieber (Gr.); carbone; febbre carbonculara, \&c. (I.); apoplexy of the spleen; malignant sore throat; known in India as Loodiana disease, and in south Africa as horse sickness; in sheep as splenic apoplexy; in America, splenic fever, Texan fever, trembles, \&c.

The term charbon is applied by the French veterinarians for the reason that the regions of the body where the disease is localized are coloured black. Anthrax (a burning coal) is now adopted by most writers as a generic term, and applied to what is otherwise known as splenic ferer; but it throws no light on the nature of the disease, as others, septic and putrefactive in their nature, present a similar appearance of the blood.

Definition.-The disease consists in a special and primitive alteration in the blood, in which an organism termed the Bacillus anthracis is rapidly developed and propagated, and is more special to the herbivora and birds. Inoculation with the blood or tissue of animals which have died from it induces some one or other form of the disease,-in man, as a rule, malignant pustule. For this reason anthrax is looked upon and described as a truly contagious disease.

Anthrax appears at all seasons, but principally in the spring or during summer and autumn. It occurs either as a sporadic, enzootic, or epizootic disease, attacking animals of any age - the fat, vigorous, plethoric, as well as the lean, feeble, and languid. 
It is a remarkable fact that wounds, simple in themselves, in cattle subjected to the influence causing charbon, although not suffering from it, often become mortal.

\section{HISTORY.}

Anthrax has a very ancient history, and was known in Asia Minor at the period of the siege of Troy; but, leaving ancient history aside, it may be useful to mention that the seventeenth and eighteenth centuries were remarkable for the devastations committed by various epizootic outbreaks of anthrax. In 1617 it was prevalent and of such a fatal nature in the neighbourhood of Naples, that over 60,000 persons perished through partaking of the flesh of animals which had died of the disease. In 1731 it declared itself in several provinces of France, notably in Auvergne, Bourbonnais, and in Languedoc, where it was studied by Sauvages, and described by him under the term glossoanthrax.-(Nosologia Methodica, vol. ii. page 360.)

$1757,1763,1775,1779,1780$, and 1800 .-These years were signalized by a charbonous malady which extended nearly all over France, and affected all the domesticated animals. The disease was studied by Bourgelat, Chabert, Berdin, Huyard, Desplas, Detil, Gordine, Gilbert, and a great number of veterinarians. From 1800 to 1846 many outbreaks of charbonous disease were observed, generally in the hottest months. They were studied by Demoussy, Sansol, Pradal, D'Arboval, Mathieu, and others. During more recent periods, outbreaks have been studied by Roche-Lubin in Avignon; by M. Rey on the heights of the Alps; and in Eure et Loire by the Medical Society of the department; and by MM. Renault and Delafond, who were sent by the French Government, the one to Allier and Nièvre, and the other to Somme, to report on the disease. Within recent years the disease has been profoundly studied by Pasteur, Chauveau, and Koch, and others in France and Germany. In this country, however, little has been done for its elucidation; and though less frequent here than on the Continent, it is, however, quite as fatal in iț̣ character.

\section{ETIOLOGY.}

The influences which predispose to the development of anthrax are arranged by French veterinary writers under four 
principal heads, comprising respectively the influences of temperature; water spread over the surface of the earth, as in morasses and stagnant ditches; forage tainterl with decomposing animal and vegetable matters; and contagion.

Influence of temperature. - The hygrometric and thermometric conditions of the atmosphere, which always exercise a marked action on the organism, disposing it under certain circumstances to anthrax, are stated by several authors to be those characterised by humid and persistent fogs, coldness, and hunidity; a tempestnous atmosphere; alternations of burning heats and stormy rains.

It rages as an enzootic on the borders of rivers, and in low lands which have been inundated. In the months of July and August, signalized by excessive heat, charbon has been frequent. The years 1712, 1731, 1775, 1779, 1780, 1823, 1824, 1825, 1846, have furnished memorable examples. Under the influence of excessive and prolonged heat, the rivers, ponds, brooks, \&c. were dried up, the soil opened in crevasses, and the disease was developed to an enormous extent. In France it is stated that a very warm summer is never seen without charbonous diseases being prevalent; and it is concluded that a high temperature, especially if preceded by a damp or moist atmosphere, is very favourable to the development of charbon.

Influence of ponds, morasses, and stagnant vaters. - The history of the malady demonstrates that morasses are favourable to its development, as it is observed that it is frequent in countries where they occupy a large surface. It is also frequent in countries exposed to inundations, and where water stagnates on the surface of the soil; and where animals are made to stand in mosses and stagnant waters, the malady commits great ravages. This fact is remarkable, not only in France, but in other countries.

Observers who have closely watched these affections in this country, where it seldom appears in the horse, almost maninously conclude that in cattle and sheep they are due to dietetic errors; more particularly to suclden and violent changes in diet, whether that change be from a poor to a highly mutritsous, more particularly a nitrogenous diet; from dry and good food to watery unripe provender ; to damaged food of any kind; the influence of undrained lands; defective ventilation and drainage of stables; to food and water contaminated with the morbid 
products of animals which have died of anthrax. In one remarkable outbreak which came immediately under my notice, the disease appeared amongst sucking calves of the pure shorthorn breed, and which had never partaken of other food than what they obtained by sucking, the dams remaining healthy. Anthrax is also disseminated through the agency of flies, and Bollinger, who has observed that the disease is often most prevalent when flies are in the greatest abundance, has induced it in rabbits by inoculating them with flies caught on the carcases of animals dead from anthrax. The flies, however, resist the influence of the virus, although bacteridæ are found in them. Pasteur's assertion that the spores of anthrax are brought to the surface of the ground by earth-worms-contested by Koch-is now confirmed by Bollinger, who has found that five per cent. of the worms coming from an infected pasture-ground contained the spores of anthrax.

Dogs, cats, white mice, and Algerian sheep are said to have an immunity from the disease.-(Crookshank).

In opposition to the view of the spontaneous origin of anthrax, we have the observations of many eminent pathologists-and this view is now almost universally accepted-who maintain that the malady is dne to the propagation of a now well-known aërobic organism, the Bacillus anthracis, the history of.which is as follows

These organisms were first observed by Branell, and aftcrwards by Delafond and Gruby, in the blood of animals which had died of anthrax, as peculiar staff-shaped bodies, which Delafond designated bâtonnets, and which were believed to be products of putrefaction, and that anthrax was a septicamia or putrefaction of the blood. These batonnets were afterwards observed in 1850 by MM. Davaine and Rayer, and some time later Koch studied them, and found the aqueous humor of the ox's eye to be particularly snitable for their nutrition. With a drop of the

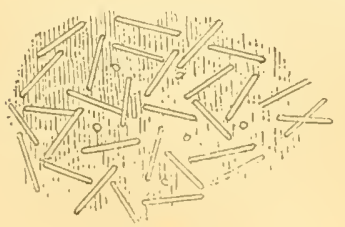

F.t. 11.-Transparent rods. aqueous humor he mixed the smallest speck of a liquid containing the rods, placed it under the microseope, warmed it suitably, and watched the subsequent action. During the first two hours hardly any change was noticeable, but at the end of that period the rods began to lengthen, and the action was so rapid 
that at the end of three or four hours they had attained from twenty to thirty times their original length, and at the end of a few additional hours had formed filaments in many eases a hundred times the length of the original rods; and further, it was seen that within the transparent rods little dots appeared; these became more and more distinct mntil the whole organism was studded with minute ovoid bodies like peas within their shell. After a time the integument fell to pieces, the place of
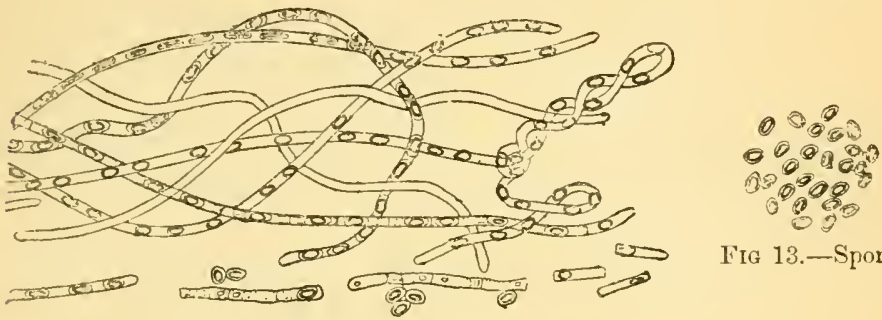

Fig 13.-Spores.

FrG. 12. - Spore-bearing filaments.

each rod being taken by a long row of seeds or spores. Koch concluded that these spores, as distinguished from the rods, constituted the contagium of the disease in its most deadly and persistent form.

By inoculating animals with the fresh blood of an animal suffering from splenic fever, he found that they invariably died within twenty to thirty hours after inoculation. By drying the infectious blood containing the rod-like organisms, in which, however, the spores were not developed, he found the eontagion to be fugitive, maintaining its power of infection for tive weeks at the furthest. He then dried the blood containing the fully developed spores, and exposed it to a variety of conditions. He permitted the dried blood to assume the form of dust, wetted this dust, allowed it to dry again, placed it for an indefinite period in the midst of putrefying matter, and subjected it to other tests. After keeping this spore-charged hlood, which had been treated in this fashion for four years, he inoculated a number of mice with it, and found its action as fatal as that of blood fresh drawn from the veins of an animal suffering from splenic fever, each spore in the millions con- 
tained in the diseased hlood being sufficient to produce the disease.

The bacilii are not always found in the blood of living animals suffering from the disease; indeed, they generally appear a few hours before death, which never takes place in less than twenty hours, and then only singly and in very sunall numbers. Their number, however, varies with the animal inoculated; in the guinea-pig they are numerous, sometimes exceeding the blood corpuscles; in the rabbit much smaller, and in the monse often absent altogether. If the disease has been induced by inoculation, they are present, though in variable numbers, in the inoculation carbuncle. Though the rods are not always found in the blood, the spores are said to be invariably present, and some assert that it is their product that destroys life.

The bacilli rods are straight or somewhat irregularly outlined, measuring from $\frac{1}{25000}$ to $\frac{1}{35000}$ of an inch in breadth, but they vary in length very considerably, those in the spleen being longer; the shortest rods being in length generally about twice the diameter of a human red corpuscle, the longer ones two or three times the length of the shorter; but when carefully examined, the latter will he seen in a process of clivision into

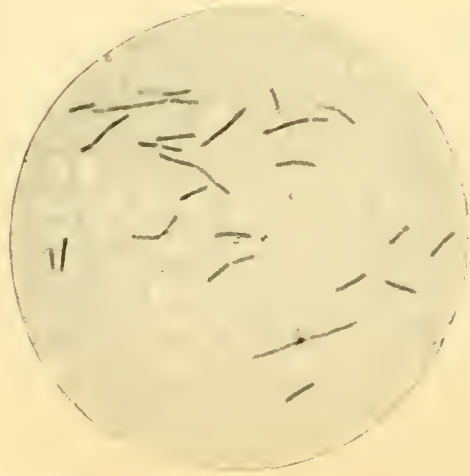

Fic. 14.-Anthrax bacilli and blood corpuscles. $\times 500$.

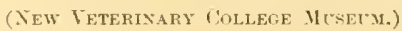

two or nore segments. They are lorvad at each ent, truncater? 
and slightly concave, and when united the concave ends enclose a lenticular space; they are non-motile, and their ends are not rounded but terminate sharply.

According to the observations of Koch, it appears that, whatever be the species of animal inoculated with anthrax blood, and no matter how many successive inoculations may be made, the bacilli multiply solely by fission, but only so long as the animal is alive; when dead a minute portion of its blood, placed in aqueous humor, and kept at a temperature of $35^{\circ}$ to $37^{\circ} \mathrm{C}$. $\left(95^{\circ}\right.$ to $98_{5}^{3 \circ} \mathrm{F}$.), the rods, as already stated, lengthen out very considerably. This process of lengthening of the rods into filaments is apparently effected by the temperature. In five hours a rod at a temperature of $32^{\circ}$ C. $\left(89 \cdot 6^{\circ}\right.$ F.) may have increased so as to be from eighty to one hundred times its original length, and in twenty-four hours the filament may be full of spores. If the temperature, however, be kept about $28^{\circ} \mathrm{C}$. $\left(82.5^{\circ} \mathrm{F}\right.$.), the spores may not appear till the thirty-sixth or fortieth hour. When the spores have once appeared, all the other changes go on at ordinary temperatures from $12^{\circ} \mathrm{C}$. $\left(53.6^{\circ} \mathrm{F}\right.$.) to $18^{\circ} \mathrm{C}$. $\left(64.4^{\circ} \mathrm{F}\right.$.), but not nearly so rapidly, even when the preparation is kept in the sun for a few hours daily, as when artificial heat is applied. On the other hand, a high temperature, $37^{\circ}$ to $40^{\circ} \mathrm{C}$. $\left(98 \cdot 6^{\circ}\right.$ to $104^{\circ} \mathrm{F}$.), at once checks all developmental changes.

The filaments differ in cultivated specimens very much in their arrangements. Sometimes they form a network-indeed a mycelium-made up of numerous, nearly parallel, unbranched threads, crossing each other at different levels; the threads are sometimes straight, but have generally a wavy outline. This condition may obtain throughont the whole preparation, but generally at some parts the filaments are extremely irregular and much convoluted.

Pasteur stated that the spores of bacilli remained toxic after boiling; and after being subjected to a pressure of twelve atmospheres of oxygen, Dr: Burdon Sanderson and Dr. Cossar Ewart tested the accuracy of this statement, and found that mice inoculated with the boiled and compressed solutions remained quite well.

The experiments of Bert, however, support to some extent 
the conclusions of Pasteur. In a series of experiments Bert submitted anthrax blood to the action of considerably compressed oxygen, and found the bacilli had disappeared, killed by the oxygen, and yet the blood retainect its virulence, for it killed rabbits, guinea-pigs, and dogs inoculated with it; and in another series of experiments Bert took anthrax blood containing bacilli, and added drop by drop of absolute alcohol to it, until a precipitate was formed, and which was dried in vacuum. This dried powder was injected under the skin, and it killed a rabbit, a guinea-pig, and even a dog.

If this alcoholized precipitate be dissolved in water and filtered, the filtrate is still virulent. If alcohol is again added to it, it forms a flocculent matter, which is deposited at the bottom of the vessel. Collected on a filter and dried, this precipitate is still toxic. It would therefore appear that the active or virulent element of anthrax resists absolute alcohol, and that it resists oxygen, and that it is precipitated by alcohol and soluble in water. It behaves itself something like diastase, except that, whatever may be its nature, it can reproduce itself to an indefinite extent; while it is asserted by some authorities that diastase cannot reproduce itself.

Putting aside the conclusions of Colin-that the bacilli are simple transformation of the blood-corpuscles - we are left between two sets of conclusions. Those of Koch and others point to splenic fever being due to a minute organism possessing wonderful powers of resistance and reproduction; Bert's to something independent of the presence or absence of animal or vegetable organisms-a ptomaine-which resist the action of compressed oxygen and absolute alcohol, and which would, he asserts, kill everything possessing life: this something he is of opinion is a substance analogous to diastase.

Blood containing bacilli, if dried in very thin layers, by being exposed to the air in a shady place, was found by Koch to lose its virulence and its power of developing elongated fibres after twelve to thirty hours. Thicker layers retained their powers for two or three weeks; and some still thicker for four or five weeks. After a longer time they were never capable of producing the disease.

Koch also found that if the bacilli were deprived of air they soon died. 
When rubbed up with the blood or aqueous or vitreous humor of an ox, and placed in a well-closed glass vessel, there quickly ensued an odour of putrefaction; the bacilli disappeared after twenty-four hours without the fibres enlarging, and lost their infective power. That their death was due to the absence of oxygen was shown by placing a drop of blood infected by the bacilli under the microscope. Examined by the micro-spectroscope it gave the bands of oxy-hrmoglobulin; the fibres in this drop increased four or five times in length in three hours, but after that time the oxygen was clearly used up, as the presence of the absorption band of reduced hæmoglobulin proved. From this moment the growth of the bacillar fibres ceased, although true putrefaction had not set in. .

When the spores and bacilli are separated from the blood by filtration, the blood is said to be rendered innocuous; and when pregnant animals become affected, or have been inoculated, the blood of the fortus does not become diseased, and other creatures can be inoculated with it and suffer no harm, the intervening membranes acting the part of a filter. The bacilli also disappear in liquids in the presence of carbonic acid, and the blood soon loses its specific property.

This proves that, to live and grow, the bacilli require to absorb oxygen and give out carbonic acid; hence they are what M. Pasteur terms "aërobic." If the fluid which contains them begins to putrefy they are destroyed, not only by being deprived of oxygen, but by being brought into contact with other organisms, such as the microbes of putrefaction, in the presence of which, and of all other low forms of organisms, they either do not develop at all, or develop with great difficulty. The organisms of putrefaction are not aërobic, and cease to move when brought in contact with oxygen; disappear, being transformed into refracting corpuscles, which in a suitable soil become motile, and multiply. with extreme rapidity in a putrefying fluid. If an animal be inoculated with it when in this condition, it does not die of anthrax, but of septicænia, the symptoms of which, when prodnced in guinea-pigs with the blood of a horse which had been dead of charbon twenty-four hours, and which contained the bacteria of putrefaction as well as some bacilli, and with the blood of a cow which had been dead forty-eight hours, and which contained a preponderating quantity of motile 
organisms, were violent inflammation of all the muscles of the abdomen and limbs, and here and there, especially on the ears, bullæ formed containing gas. The blood was diffluent, and on examining these animals immediately after death, M. Pasteur found that the muscles were filled with active vibrios of putrefaction, and in the peritoneal cavity they had undergone extraordinary development; one drop of this serosity taken from an animal still living affected another animal profoundly, while a drop of blood from the heart had no effect. The spores are very tenacious of life, resisting many germicides. The rods are destroyed by dry heat at a temperature of $212^{\circ} \mathrm{F}$., whilst the spores require to be exposed for three hours to a dry temperature of $283^{\circ} \mathrm{F}$. before they are killed. Some writers assert that the spores resist the action of boiling water, but Hamiltou says that they are killed after a few minutes' boiling: the rods, however, perish in a moist atmosphere at a temperature of about $140^{\circ} \mathrm{F}$.

Putrefaction and the action of carbonic acid gas, whilst destructive to the non-sporulated rods, have no effect upon the spores themselves; both spores and rods are, lowever, killed in ten minutes by corrosive sublimate-1 per 1000 solutionwithin twenty-four hours by a two per cent. solution of chlorine, bromine or iodine, and strong sunlight; but they seem to resist five per cent. solution after twenty days' exposure, twelve days phenic acid five per cent., and nineteen days teu per cent. solution of lysol. Iodoform seems to have no influence either on the spores or rods. Whilst the rods are destroyed by putrefaction, it is otherwise with the spores, which have a much greater degree of resistance, and it is due to this retention of virulence by the spores that anthrax continues in buildings, grass lands, dried fodder, and water. Grass and hay grown upon land where anthrax carcases have been buried months before have conveyed the disease, and the same may be stated of water, particularly spring water obtained from wells situated at a considerable distance, but below the graves of such animals.

Again, dilution of the fluid containing the bacilli with a moderate amount of water has no effect on its virulence, but a large quantity destroys it, and traces of carbolic acid prevent the development of the bacilli.

It has been stated that the bacilli destroy life-(1.) By acting 
as asphyxiants, depriving the blood of its oxygen; and (2.) By mechanically obstructing the blood-vessels. Against both these theories must be placed the fact that they are very few in number, indeed often absent altogether in the blood during life.

Anthrax is not transmitted by infection from one animal to another, for animals kept in the closest proximity to diseased ones, and placed under the most favourable conditions for infection through the air, do not become diseased.

Mice and rabbits seem capable of eating food containing bacilli with impunity, and flies can gorge themselves with the infected blood and suffer no harm ; but horses, cattle, pigs, dogs, cats, and ferrets succumb after partaking of food and water contaminated with the virus.

It is now generally admitted that animals are infected by spores contained in the food, which gain entrance into the circulation either through abrasions in the mucous membranes of the digestive track, or, as some assert, by the pulmonary mucous membrane; but this is doubtful. My own experience leads me to conclude that undecorticated cotton cake is the most fertile source of the disease of all the artificial foods, and it seems to acquire virulence after being kept for some time, particularly if neglected and allowed to become heated and mouldy. When freshly made, it seems to have no effect, and I have known several instances where cattle have eaten the same cake for weeks or even months before becoming affected, then all at once they have died off. I am of opinion that the spores- few in number-have been present in the cake from the first, and that they multiplied in the eake, which, as is well known, is imperfectly dried; thus, after repeated reproductions of the organisms, the cake has become sufficiently charged to induce the disease in its partakers. The decorticated cotton cake is differently prepared, being highly dried and submitted to great pressure; thus heating and fermentation are prevented, and any increase of the organisms rendered impossible.

When soil is contaminated with the blood or discharges of animals dead of anthrax, it is found that spore formation goes on actively in many moist and warm media, and it is stated that these spores may be conveyed by floods to surrounding pastures. It is also supposed that the organism may grow as a saprophyte 
on dead vegetable matter. Whether this be true or not, the vitality of the organism must be very tenacious, as it is well known that certain pastures are always dangerous to cattle, but more particularly after rain preceded by warm dry weather.

It is most remarkable that preventive inoculation has not succeeded in this country, although so successful on the Continent. Is it the fault of the operators, or what? A reference to experiments of Dr. Burdon Sanderson and Mr. Duguid (see below) ought to convince any one that the operation should be further tested, and that the Government should encourage all experiments calculated to increase the general welfare, instead of throwing obstacles in the way.

The local effects of inoculation of the skin with anthrax blood is as follows:-In twenty-four hours there is redness of the spot, with heat, swelling of the skin and subcutaneous tissue, extending from a third of an inch to an inch in depth.

The swelling increases in forty-eight hours to perhaps two inches, and on the third day, if the animal survive, to several inches; the heat and redness being most intense at the inoculated spot. The process extends in the connective tissue, particularly along the track of the lymphatics. In superficial inoculations bacilli can be seen in every instance in twentyfour hours, at a distance only of about one-fourth of an inch, but their after extension is not proportionate to the extent of the tumefaction, nor does the serum found in the swelling contain many of them until after forty-eight hours, when great quantities will be found in it; when the virus is iujected into the subcutaneous tissue death may occur without bacilli being found at the point of injection.

\section{INOCULATION FOR THE PREVENTION OF ANTHRAX.}

In 1878 it was discovered by Dr. Burdon Sanderson and Mr. Duguid that cattle might be inoculated with splenic fever from a guinea-pig, and though such inoculation caused the development of serious symptoms, the animals did not die; and in continuing these experiments it was found that cattle once so inoculated re. sisted the results of further inoculation,- - that in fact they could be thus rendered insusceptible to future attacks of splenic fever. Dr. Greenfield, in making a series of experiments with the view 
of obtaining a suitable virus for inoculation, found that the virus modified by transmission through the guinea-pig, and, cultivated nnder particular conditions, gradually lost its activity, and at last became practically inert; and it occurred to him that, by making use of this fact, a virus might be obtained so far modified as to be sufficient to ensure protection and yet not endanger the life of an animal inoculated with it, and this he found could be done with success.

The priority of this discovery is therefore claimed for England, but the merit of working out its details is undoubtedly due to Pasteur.

\section{VARIETIES OF ANTHRAX.}

The more recent investigators are disposed to confine the term anthrax to that form of disease characterised by black tumour, associated with the presence in the economy of the Bacillus anthracis; but as the term merely indicates a symptom -ramely, a black tumour, the charbon (coal-like) of the French -I intend to include under it all those diseases in which it is an expression or a symptom, contenting myself by pointing out in which of them the bacilli have been discovered, and which are proved to be contagious.

Chabert designates anthrax or charbonous diseases as foul affections of different natures, and external tumours terminating in mortification of the tissues, and divides them into three kinds, namely :-

1st. Charbonous or Anthrax Fever.-In this form there are no external manifestations, and it is rapidly fatal; the post morteru appearances being engorgement and congestion of the spleen, liver, mesentery, intestinal mucous membrane, the sublumbar tissues, lungs, and heart. In some cases death is preceded by roughness of the coat, dry, hot skin, excessive sensibility over the dorso-lumbar region, choking cough, glairy discharge from the nostrils, and a quick, strong pulse. Sometimes the animal may live long enough for level tumours to form under the skin; but very often the patients succumb without presenting the least symptom of the malady, or in a few hours after the first appearance of illness.

ANTHRAX IN THE IIORSE.

Anthrax in the horse rarely occurs in this country, but is 
prevalent in India, where it is said to attack the elephant as well as other animals.

Symptoms.-The animal may appear dull, walking with a heavy, feeble step, then falls prostrate in a state of somnolence; if it be standing, the head hangs down, resting on the manger or other solid body. It sometimes stands back in the stall, resting the body on the side, and finally becomes restive, stamps with the feet, looks to the sides and flanks, and shows other signs of colic.

If the disease comes on whilst the animal is at work, added to the above symptoms there is extreme lassitude, great weakness of the lumbar muscles and posterior extremities, with staggering gait. The skin has lost its suppleness, is hot, and slightly crepitates on the back, over the kidneys, and sides; the coat is rough and bristly in some parts; and there are partial or general tremblings of the muscles, and flow of saliva from the mouth. There are sweats, alteruating from hot to cold. At the base of the ears, and behind the elbows, the veins become augmented in volume. Above all, the lymphatic ganglia of the groin are swollen; and if the horse be entire, the testicles move rapidly up and down. Great excitement now sets in, the animal is irritable and timorous, and afterwards becomes unconscious of all around. The conjunctive are yellow or reddishyellow; and sometimes petechial spots are present on the visible uncous membranes.

The pulse is small and thready ; the beatings of the heart are, lowever, strong, and are accompanied by a metallic tinkle. The respiration is often irregular, and often associated with roaring.

These symptoms may insensibly disappear, or may be succeeded by a critical eruption. At other times, even after the animal has seemed to rally, aggravation of the symptoms takes place. The animal grinds its teeth, has violent colic, rolls about, carrying its head to its flanks; the muscles of the head and neck are agitated by convulsive movements; the eyes are hagrgard and wandering; the mucous membrane injected, and of a brown or red tinge; the heart beats with extreme violence, very irregularly, and accompanied by a strong metallic sound; the pulse is trembling or double, and very small; the respirations tumultuous and agitated; the nostrils chlated, and clots of yellow (almost sulphur coloured) serosity and blood escape 
by the nose; the month is filled with a mucous foam, the tongue is tumefied, and of a deep bluish-red colour (GLossoAnthrax); tears, sometimes tinged with blood, flow from the sunken and haggard eyes. The belly is sensitive when pressed upon, tympanitic, and the excremental matters are often liquid and mixed with blood clots; the rectum is often everted, appearing as a tumour, folded and livid; the temperature of the skin is lowered; the countenance is particularly anxious, the face shrunken. The muscular force now beeomes exhausted; the animal falls to the ground; convulsions come on, more particularly of the neck and extremities; and finally it suceumbs, after a few moments of calmness, which, succeeding a paroxysm, always precedes death. The disease may terminate in from six to forty-eight hours after the manifestation of the first symptoms. The ordinary time is from twelve to twenty-four hours, unless external eruption eliminates the morbid material from the body.

In the spring of this year, 18s4, a remarkable outbreak of charbonous fever, presenting the salient symptoms of Loodiana disease, occurred in a large stud of cart-horses under the care of Messrs. Leather, veterinary surgeons, Liverpool, and which had been, for some time previously, fed on an Indian pea (Pisum. sativum), called in Liverpool Indian mutters. MIUtur is the Hindustani word for the common pea (Pisum sativum), but that brought to Liverpool is different from the ordinary pea of this country, and resembles a lentil more than a pea. It is imported into Glasgow from India in large quantities, I believe, mostly as ballast, and has, I am told, not only been given to horses, but ground and mixed with various cakes for cattle, and with many fatal consequences.

However, in the outbreak at Liverpool, it appears that horses commenced to die very suddenly some time after the owners had commenced to use the mutters, and for several weeks after they had discontinued to use them they still died. The symptoms were roaring, hæmorrhage from the nose, great prostration, swelling of the throat, succeeded in many cases by sudden death. Other horses, however, lived a considerable period; but none recovered in which roaring had become pronounced.

I saw them in March, and found two dead on my arrival-one having only been dead a few hours; and from the blood of which I obtained the bacilli shown in the drawing. 
On examining the food everything was found to be of the best and cleanest quality; but the Indian mutters were very FIG. 14 A.

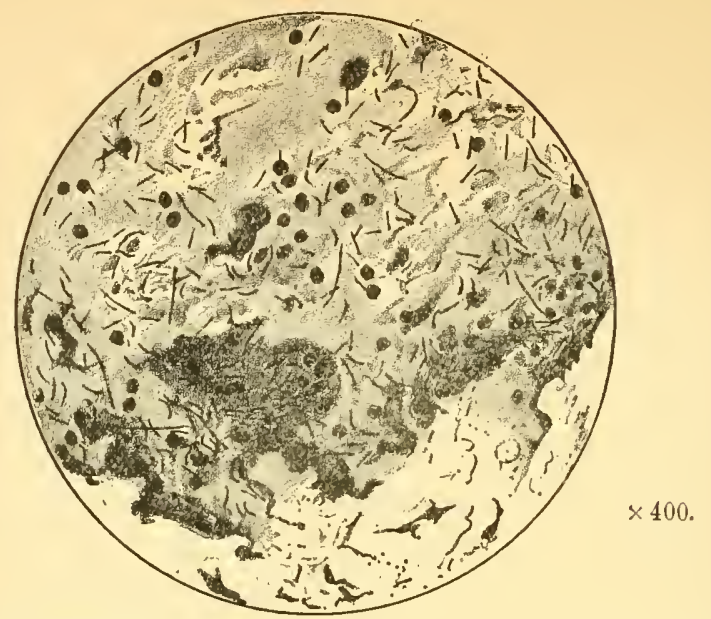

- Blood corpuscles.

Bacilli of various lengths, but about $\frac{1}{20 \frac{1}{4}}$ in. in diameter. These bacilli seem to differ from those of splenic fever, being rather smaller in diameter, and, so far as my observations go, multiply by fission only, not developing spores. This, however, requires confirmation.

dirty, dusty, and mixed with the excrement of rats; and from this dust bacilli identical with those found in the blood were cultivated; but in no instance did I succeed in obtaining bacilli from the interior of the grain. From this it may be inferred that the microbes were in the dirt surrounding the grain, but not in the mutters themselves, and that by proper washing and cleaning they might be a safe diet.

This, however, requires further investigation, as animals have died on the Continent presenting similar symptoms to those at Liverpool after being fed on the legumen "Lathyms sativus," a bitter legumen, but whether from a vegetable poison contained in the legumen or from bacillar growths has not yet been determined. ${ }^{1}$

In animals which have white skins, or where an eruption

${ }^{1}$ Further experience of the effects of lathyrus poisoning have led me to conclude that the hæmorrhagic engorgement of the throat and the epistaxis were accidental complications due to the tainted state of the food in these particular cises seen at Liverpool. The effects of feeding with lathyrus are now well known, and will be referred to in the chapters dealing with Dietetic Diseases. 
takes place in parts of the body void of hair or wool, red, brown, violet, or mulberry spots of bloody effusion are seen. These spots are independent of tumours and extravasations, and sometimes exist on the surface of the tumours. They are most commonly seen in the pig and sheep.

Ecchymoses are seen on the visible mucous membranes. When the fever progresses slowly these spots unite by confluence, and surround, notably in the pig and sheep, the whole body. Some of them become crepitous and emphysematous; others take the form of tumours, passing rapidly to the state of gangrene. In addition to these eruptions, there is often a soreness and swelling of the throat, infiltration of the upper end of the trachea, and a discharge of a lymph-like material from the nostrils, constituting what is termed gloss-anthrax, common in the pig which was fed upon anthrax flesh. It is also seen in the ox as well as the horse.

Charbonous tumours are generally of a black-brown colour. The phlyctenæe are filled with a brown liquid, which is very irritating; it sweats on the surface as drops of cold serosity. The tumours have little tendency to suppuration, and speedily become gangrenous. If these symptoms are added to those furnished by the mucous membranes, no difficulty need be felt in diagnosing charbonous tumours, phlegmonous and gangrenous.

Terminations. - When the tumours resist mortification, they terminate by delitescence, by suppuration, and by metastasis.

1st. Deliteseence.-The morbid products are effused, and constitute tumours, which are reabsorbed and expelled by the cxcretory organs. Examples of this kind of termination have been observed by veterinarians in Africa.

2d. Suppuration.- When the tumours progress in a slow manner, indurating gradually, suppuration may be brought abont by therapeutic and surgical means. Resolution is slowly induced, and it is not without pain that the necessary suppuration can be provoked. The animals remain poor and unthrifty, and often in the horse glanders and farcy conclucte this morbid state.

3i. Metastasis.-The amelioration of the symptoms which succeed the development of tumours is not often lasting. The products are reabsorbed and carried anew into the circulation. This unhappy crisis is amounced by the reappearance of all the symptoms proper to charbonous fever; their succession is so rapid that death may take place in from eight to ten hours. 


\section{SYMPTOMS OF ANTHRAX FEVER IN HORNED CATTLE, APOPLECTIC}

ANTHRAX, SPLENIC APOPLEXY, SPLENIC FEVER.

The symptoms in the ox are very analogous to those in the horse. The ox suddenly goes off its feed; rumination is suspended; there are rigors and tremblings; partial sweats bedew the body, which is alternately hot and cold. The dorso-lumbar region is excessively tender to pressure, and when it is the seat of the tumour, very acute pain is thus caused; the gait becomes staggering, and the animal rapidly exhausted. A recumbent posture is almost constantly maintained; the animal will now and then attempt to rise, but will rarely succeed in doing so. When standing, the back is arched, the legs stiff and rigid, but the standing posture is not long maintained. The animal looks towards its flank, falls into convulsions, and expels, without much effort, soft and bloody matter by the anus. The heart beats with violence against the thoracic walls; the pulse is small, rapid, irregular, intermittent, and sometimes double; the conjunctive red, injected, and reflect a blackish-red tint; the respiration is panting and plaintive; there is tympanitis of the abdomen; the tongue is bluish-red, and the mouth filled with mucus; blood escapes from the nose; the eyes are sunk in their orbits, and tears flow over the cheeks. The areolar tissue of the back and sides becomes crepitous to the tonch, and the animal dies during a convulsive exacerbation, or during the succeeding calmness. In some animals the excitement is so great that it is dangerous to go near them. The rapidity with which the symptoms succeed each other is variable, death taking place in the space of from a few minutes to twenty hours.

In cattle above two years old, particularly milch cows, the local lesions are often confined to great congestion of the spleen, and to a lesser extent of the liver and mucous membrane of the intestinal canal. In other, but rarer instances, the engorgement may be in the lungs, and should the animal survive for some days, decomposition of the extravasated blood is established, as expressed by foctor of the breath; the decomposed extravasated blood being absorbed into the circulation, causing death by septicxmia. I have also seen this condition in a sucking calf.

In true splenic apoplexy the spleen is often much enlarged, broken down in structure, and its capsule distended with a mass 
of tar-like blood. If one end of the organ be elevated, it will be seen that the blood will gravitate into the most dependent part, showing that the splenic tissue is disintegrated, and that the blood is more or less fluid.

Death from splenic fever is very suclden; in many instances an animal seen a few hours before apparently in good health is found dead, death having apparently occurred without a struggle. If, however, the disease is not so rapid in its course, it may be noticed that there are various alternations in the symptoms; in some instances an animal will be unwell for several days, suffering from a remittent fever; one day very ill, with rapid, feeble pulse, hurried and painful breathing, red and injected eyes, hot mouth, irregularity of the bowels, and redness of the urine. After continuing for some hours these symptoms may subside, and the animal commence to eat and ruminate. The febrile symptoms, however, often return, and in the end the sufferer too often succumbs.

In another form of anthrax without external tumours, the most prominent sign, in addition to the general disturbance, is the passage from the bowels of quantities of dark-coloured blood; and the disease is then denominated enterie or abdominal charbon, the post mortem characteristics being congestion of the intestinal mucous membrane, more particularly of the small intestines, which are covered with petechial spots, with incipient ulceration in their centres, extravasation of dark-coloured blood into the canal, and very often extravasations into the sullumbar areolar tissue; the fatty mass surrounding the kidneys being loaded with extravasated blood, in a disintegrated, brokendown, tarry, semi-fluid condition, or covered with petechiæ.

\section{SYMPTOMS IN SHEEP.}

In this country anthrax in sheep assumes the enteric form, but on the Continent of Europe splenic apoplexy seems to be the form by which they are usually attacked.

Braxy in sheep-an anthracoid disease-is a form of septicæmia, simulating anthrax in its post mortem appearance, but exhibiting no contagious properties and presenting no specific germ. Sce Septic Diseases. 
The other form of charbonous fever in the sheep, not commonly met with in this country, but which seems common on the Continent, is splenic apoplexy, the symptoms of which are similar to those witnessed in the ox. Indeed it may be men. tioned that the malady in all its forms may be accompanied by splenic congestion.

\section{ESSENTIAL CHARBON, OR THAT ARISING FROM INOCULATION- MalignaNt PUstule (PUSTUla maligNa) iN MaN.}

Definition.-Implanted on some uneovered part, the organism produces in the first instance a redness like the bite of a gnat, and afterwards a minute vesicle. A peculiar form of gangrenous inflammation is excited, which rapidly spreads from the point first affected to the neighbouring tissues. Hardening and blackening of the part is so extreme, and death of the tissue is so entire, that the part cracks when cut with a knife. No pain attends the ineisions; crops of secondary vesicles form round an erysipelatous-like areola, ehains of lymphatics become inflamed, the breath foetid, and death follows, amid all the indications of septie poisoning.--(Dr. Wr. Budd.)

Such is the disease in man, and its identity with charbon has been satisfaetorily proved by the fact that, when contracted by man, it has been conmunicated by inoeulation to the lower animals. Malignant pustule in man is coneurrent with charbon in cattle, \&c., and is a result of direct inoculation. Other cases oecur in which the exact vehicle of the poison cannot be identified, but these eases have all this significant peculiarity, that the disease is always seated on some part of the person which is labitually uncovered.-(AITKEN.)

Propagation.-The disease may be communieated to man in the following ways :-By direet inoculation, as in the case of butchers and others employed to skin the carcases of animals whieh have died of charbon, the poison finding access by means of the skin or hands or arms of the operators; by means of the skin or hair of animals dead of charbon; and there are many examples related by Dr. Budd which elearly prove that the virus, when once in a dried state, may retain its virulence for an indefinite period of time. Trousseau relates that in two factories for working up horse hair imported from Buenos Ayres, and in 
which only six or eight hands were employed, twenty persons died in the course of ten years from maliguant pustule. The disease may be communicated by eating the flesh of animals killed while affected with it, as also by using the milk and butter of affected cows.

\section{ANTHRAX IN THE PIG.}

The observations of Klein having proved that the very fatal disease amongst pigs known as anthracoid erysipelas, the blue sickness, pig typhoid, \&c., differs in many particulars from anthrax; the varieties witnessed in the pig are reduced to anthrax fever, gloss-anthrax, and anthrax with tumour. Anthrax fever is as rapidly fatal in the pig as in other animals, destroying life in a very short time, with but slight manifestation of sickness, killing by shock. In milder cases, however, there is loss of appetite, sudden prostration, sullen appearance, hanging ears, painful and haggard expression, vomition of a coffee-coloured fluid, continual convulsions, paralysis of the extremities, rapid alternations in the heat of the body, highly injected mucous membranes, and generally terminating in death.

Gloss-Anthrax-Malignant sore throat-Anthracoid anginaThis form of anthrax is most commonly seen in the pig when it has fed on the flesh of other animals which have died of the malady. It is rapidly fatal, the throat swelling enormously; the pharynx, larynx, tongue, \&c. becoming enormously swollen and gangrenous; an exhaustive diarrhœa, with great tenesmus and discharge of blood, often appearing prior to death.

I have known of several instances in which pigs have died in great numbers after having eaten of the flesh and offal of cattle which had died of quarter-ill and splenic apoplexy; and $\mathrm{Mr}$. Borthwick, V.S., Kirkliston, has told me an instance which occurred in 1872, in which twenty-five pigs died in two days after having eaten the flesh of a bullock which had died of splenic apoplexy.

\section{ANTHRAX IN POULTRY.}

Charbonous fever is amnounced in poultry by the following symptoms:-No appetite; feathers ruffled; walk difficult and staggering; foetid diarrhœa; great prostration; dragging of the wings; turgescence or blackness of the conjunctivæ; excessive 
sensibility of the extremities. The fowls squat, and do not look for a perch; the bill and comb become black; tumours, or red spots, which soon become black and gangrenous, form on the palms of the digits, and the animal dies in convulsions.

\section{PATHOLOGICAL ANATOMY OF ANTHRAX.}

Post mortem examinations disclose very manifest lesions, which explain the rapidity with which putrefaction has taken place in the tissues.

(1.) Exterior.-A short time after death the whole body is tumefied and disfigured from excessive formation of gas in the areolar tissue. The abdomen is considerably swollen from gas in the gastro-intestinal organs, and blood-clots escape from the nose and mouth; the rectum is reversed, and looks like a black tumour, from the centre of which gas and tainted liquids escape.

On different parts of the body, notally on the skin deprived of hair, red or mulberry spots, sometimes interspersed with yellow ones, are found.

After being opened, the body emits a very foetid odour.

(2.) Skin, cellular tissue.-In excising the skin crepitation is heard, which results from the disengagement of gas accumulated in the subcutaneous areolæ; black and liquid blood escapes from the parts cut with the knife.

On the tumours and engorgements the skin is found semidetached, and its internal surface presents spots of infiltration corresponding to those seen externally, and the tainted skins are at these spots without consistence, and depreciated in value.

The areolar tissue is the seat of blood and sero-albuminons infiltrations of yellow, red, or black colonr, which extend into the interstices of muscles and to deep-seated organs.

(3.) Nuscular tissue.- Yellow infiltrations on the surface, and to a less degree in the bed of the muscles, in the form of lines which put the fibrillæ in relief. The muscular system generally is impregnated with black blood, which communicates this colour to the whole frame.

The normal adhesions of the muscles to the bones, tendons, and aponeuroses are so relaxed that one may detach them without much effort. 
In the thickness of the muscular substance black spots are found from the escapement of blood. These morbid alterations are most marked in tumours and charbonous engorgements, constituting masses of serosity of a citron colour, which raise the skin, infiltrate the subcutaneous areolar tissue, and penetrate deeply into the organs of the body. Infected gas is disengaged from the surface of the divided tissues; here and there portions of the tissues are decomposed.

It is a remarkable and important peculiarity that in these vast engorgements no trace of inflammation can be found, no plastic material, no injections, no vascular arborizations, which characterise the inflammatory process.

M. Delafond has recognised the following microscopic particulars :-

(1.) That where tumours are situated the tissuc is penetrated by blood globules deprived in part of their colouring matter.

(2.) That the red colour of the tissue is due to the escape of the hæmatin from the red globules.

(3.) That the serosity of the infiltrations is fibrino-albuminous.

(4.) That this serosity is associated with a great number of very little globules, abnormally indented on their surfaces.

(5.) That the areolar plates, in which this serosity is deposited, are slightly opaque from coagulation of fibrin.

The morbic lesions of the solicls are evidently a consequence of the primitive alteration of the blood.

The large vessels-the aortæ, venæ cava, vena porta, the cavities of the heart-are filled with fluid blood reflecting a strong clark violet tint, presenting $n 0$ signs of coagulation. The walls of the vessels and of the heart possess a red colour, which resists washing, showing that the colouring matter has penetrated the tissues.

These alterations explain the formation of black spots, of effusions, of bloody extravasations, and of serous citron infiltrations on the surface and in the depths of the organs. At the same time the deficiency of fibrin renders the fluid incoagulable both during life and after death. The heart is flaccid, soft, and covered with bluish spots. The blood in both sides of the heart is black, liquid, and more abundant in the right than in the left side of that organ. When exposed to the atmosphere it retains its black colour, or is but very slightly reddened. Some deny that it changes colour at all. 
The pericardium is covered with ecchymosed spots. The pericardial fluid reflects a red tinge, more or less marked, according to the time which has elapsed between death and the autopsy.

(4.) Iymphatic system.-The ganglia of the cervical region, of the thoracic cavity, of the dorso-lumbar and inguinal regions, are always diseased. They are much increased in volume, ecchymosed, yellow or red, soft, impregnated with a great quantity of serosity, and a citron-coloured infiltration, and are easily pulpified by pressure between the fingers.

These characters are found in a degree more or less marked in the lymphatic ganglia of all the organs; on minute dissections being made, cords and little nodules are seen of a yellow colour, and of various forms and sizes, and the lymphatic vessels, especially those that have the tumours, are much distended.

(5.) Digestive apparatus. -- The peritoneum, epiploon, and mesenteries are irregularly covered by ecchymosed spots. The peritoncal cavity contains serosity of a deep colour, mixed with blood-clots. The large veins which follow the convolutions of the intestines have a bluish aspect, and are distended with very black blood. In the mesentery, and above all in the sublumbar region, charbonous tumours of various dimensions are found. They are very common in horses which have died of charbonous disease without external eruption.

The tumours are formed by a mass of very black blood of a syrupy and gelatinous consistence, and by an infiltration of serosity of a yellow colour. They are situated in the fatty masses that surround the kidneys, the pancreas, the posterior vena cava, the sublumbar ganglia, and between the folds of the mesentery, immediately above the convolutions of the intestines. The intestines present exteriorly a red colour, more or less marked, following the extravasation and escape of blood. The lesions found in the interior of the canal vary from a simple red discoloration to a dark congested state of the mucous membrane. When washed, the villi are seen congested and augmented in volume. In other cases blood is extravasated and fills the intestinal conduit, mixing with the alimentary matters. It rapidly decomposes, and emits a bad smell. Under the mucous membrane a yellow infiltration is found, extending into the sub-peritoneal areolar tissue. Peyer's patches do not 
present any characteristic alterations. In some cases the mucous membrane surrounding them has been seen to be ulcerated; and in animals of the bovine species the glands of Brunner have been seen to be augmented in volume.

(6.) Spleen.-This organ is the seat of very remarkable lesions, and in virtue of the general constancy of their character, they may be considered as the most conclusive expression of the existence of charbon. Its volume is often double, triple, or quadruple; it is larger, longer, and thicker, and its external surface has a livid blue or black colour. The enlargement is sometimes uniform, sometimes irregular, and is formed by a mass of blood distending the splenic capsule, which occasionally becomes ruptured, giving exit to thick, black blood.

When cut, black incoagulable blood escapes from the incision. On pressing and washing the tissues, the putrid matter is removed, and the fibres left are of a reddish-black colour.

(7.) Liver:-Augmented in volume, with the appearance of having been boiled. Its tissue is friable, easily cut, and from the cut surfaces great quantities of black blood escape.

(8.) Respiratory organs.-Some amount of serosity in the chest, of a muddy and slightly red colour. The costal and pulmonary pleuræe are covered with black spots. In the subserous areolar tissue there is a yeliow citron infiltration. which is continued into the interlobular areolar tissue. Gas is developed as in the subcutaneous areolar tissue, and brown and black spots are seen throughout the lung tissue and on the respiratory mucous membrane.

(9.) Nervous system.-The meninges of the brain and spinal cord are covered with black spots. The venous sinuses are filled with a very liquid blood, and in places yellow infiltrations are observed. The cerebral substance is ecchymosed, and clots of blood are seen on its surface.

The ganglia of the great sympathetic nerve are large, red, soft, and infiltrated.

(10.) Urinary apparatus.-Like the liver, the kidneys are augmented in volume. They are of a brownish colour, easily broken down, and the blood with which they are filled escapes when they are squeezed.

A consideration of the lesions found post mortem leads us to one conclusion-namely, that the alterations seen in the solids 
are due to a profound modification which has been established in the physical and chemical characters of the blood.

In the horse more particularly, the jelly-like yellow and serohæmorrhagic infiltrations are encountered nearly everywhere in the body where there is loose connective tissue, chiefly in the retro-pharyngeal and laryngeal tissues, along the course of the great blood-vessels of the neck, in the mediastinum, peritoneum, and about the kidneys. The corresponding lymphatic glands, especially the mesenteric, show sero-hæmorrhagic infiltrations, are considerably enlarged, and here and there in a state of incipient gangrene. The intestinal lesions in the horse are for the most part not sa diffusely spread; but still upon the mucous membrane, which is in a catarrhal state, odematous, and sprinkled with ecchymosis, there are found well pronounced carbuncles, which are the seat of more or less superficial sloughs. The intestinal contents are likewise often bloody and thinly fluid. The large glands, liver, and kidneys as a rule are swollen, the parenchyma cloudy, suceulent, and full of blood. In the blood, besides the presence of bacteria, the white corpuscles are found to be in considerable number. The red corpuscles for the most part are of lessened consistence, and manifest a tendency to cohere in little heaps. On microscopic examination of the carbuncles in the intestines, as well as elsewhere, and of the jelly-like hæmorrhagic effusion into the connective tissue, there are found in the capillaries, which are considerably diluted, besides a massing of white blood corpuscles (cellular oedema), numerous bacteria, and a finely granular mass, consisting partly of metamorphosed blood detritus, and partly bacterial germs. The thickness of the blood is due to its being deprived of water by the dropsical, jelly-like, and odematous effusions. The dark colour is caused by carbonic acid poisoning.-(BoLLINGER.)

\section{TREATMENT.}

When the symptoms of the disease, in any of its forms, are fully developed, but little good can be effected by medical or surgical interference. Some few cases may, however, be amenable to treatment.

The blood is fluid, dark, imperfectly coagulated, containing the constituents which unite to form fibrin in a diminished quantity, 
or in an altered or modified condition. How, then, is the viscidity of the blood to be restored? Direct experiments upon the blood removed from the body, and the treatment of hæmorrhagic diseases, have very satisfactorily shown that the chlorate of potash has a peculiar effect in this direction, and it is upon this substance that dependence must be placed. For young stock the following draught is to be administered:-

R. Pot. chlor. 3iii.

$$
\text { Aquæ, Oi. }
$$

M. and give three times a day.

For a full-grown animal an additional drachm may be given with safety; but if given in large doses intestinal irritation is apt to be induced.

On account of its well-known antiseptic properties, carbolic may prove very useful in the treatment of anthrax; indeed, I understand that Principal Veterinary Surgeon Collins has prescribed its internal administration with marked effect in India. It may be given largely diluted, two or three times a day, in doses proportionate to the species and size of the animal under treatment. M. Decroix, P.V.S., French Army, recommends the borate of soda, 100 grammes daily, dissolved in water, and given as a draught to the horse.

Purgatives generally do harm, and for the reason that there is some congestion of the intestinal mucous membrane.

During the course of treatment of charbon it is important that special regimen be used. The animal should be well nourished. Food easily digested and of good quality should be given. Tonic medicines, as preparations of iron, gentian, and decoctions of aromatic plants, may be used at the time of convalescence.

The prevention of charbonous fever is a matter of great importance. Some writers affirm that it is more commonly met with since the introduction of artificial manures, and that it is due to the presence of the nitrites conveyed from the soil to the blood in the water, herbage, turnips, \&c. I think that this view is not established, as the malady is met with where artificial manures are not at all used. If it were solely due to the nitrites or to any of the salts contained in artificial manures, its existence would have been unknown prior to the introduction of such manures. It is a fact, however, that when animals are poisoned with the nitrites, such as the nitrite of amyl, that the blood 
assumes a chocolate colour (Dr. A. Gangee); and it is also a fact that certain diseases are more prevalent on rich than on poor soils.

Preventive Treatment.-It is supposed by many veterinarians of experience that the introduction of setons is a very effectual preventive of charbon, and their operation is explained by the fact that in inflammations, artificially produced or otherwise, the coagulating properties of the blood are increased, as well as the phagocytes. The quantity of fibrin discoverable after the blood is removed from the body is appreciably increased.-(See Inflammation-Principles and Practice of Veterinary Surgery.) The safest and quickest method of creating an artificial inflanmation is by inserting a seton in the breast-the seton to be deeply inserted, and in order to increase its irritating properties it should be dressed with blistering ointment. If the disease has appeared amongst a herd before the veterinarian's attention is directed to any method of prevention, in addition to setons, the chlorate of potash is to he given, mixed with the animal's food or water for several days, the diet in the meantime being restricted, and all circumstances likely to promote the malady fully inquired into and removed.

It is also essential, not only as a means of prevention, but after the actual occurrence of the malady, that the excretory organs be kept performing their proper functions, and for this end gentle aperients and cliuretics should be employed. I have already pointed out the danger of administering lrastic cathartics, but there is no reason why the bowels should not be gently opened by laxatives, such as linseed or castor oil. If during the course of the malatly it is thought necessary to employ stimulants to rouse the flagging animal energies, the preparations of ammonia are to be aroided, as they cause the blood to loose its coagulating properties, both within and without the body; and, as already stated, an excess of anmonia already existing in the blood, alcoholic stimulants are to be given in preference. Acting upon this conclusion, I at one time administered acid preparations, and with some success, but a larger experience has taught me that the chlorate of potash is superior to all other medicines.

The flesh of animals which have died or have been killed whilst suffering from the disease should not be used as food, as it is apt to cause death, and the milk of affected cows has proved fatal to man. 
QUARTER-ILL, BLACK-LEG, OR CHABERT'S DISEASE.

Quarter-ill, black-leg, strike, symptoniatic anthrax, quarterevil, sarcophysema hæmostaticum bovis, infectious emphysema, are a few of the numerous names applied to a specific disease affecting cattle and sheep, and due to the presence of an organism, the Bacillus Chauvcei.

Quarter-ill was, until recently, supposed to be an external manifestation of splenic fever (anthrax), that in young cattle anthrax was always thus developed, whereas in older ones the disease was located in the spleen.

This disease is fortunately not nearly so common in Scotland as it is in England, France, and other countries on the Continent; but owing to better drainage of the land it is less common than formerly, even in England.

It occurs much more frequently in young than in old animals, as is well illustrated by the following record by M. Hess of 989 cases :-

\begin{tabular}{|c|c|c|c|c|}
\hline 439 & ", & " & 1 and 2 & years \\
\hline 83 & ," & ", & 2 and 3 & ", \\
\hline 65 & ", & ", & 3 and 4 & $"$ \\
\hline 10 & $"$ & ", & 4 and 5 & $"$ \\
\hline 18 & $"$ & $"$ & 5 and 6 & $"$ \\
\hline
\end{tabular}

Not only is it less frequent in old animals, but it is also less fatal to them. Animals affected under two years old almost invariably die, but animals over that age frequently recover: so much is this so, that many affirm all animals over three years of age to be free from clanger of contracting this disease.

But this conclusion, however, must not be implicitly believed in, as the following statements by Hess show there is even danger though the animals are beyond the stated agę.

Out of 36,000 animals, from six to twelve months old, 350 died of the disease ; of 13,000 animals, from one to three years old, 500 died; and of 135,000 , from three to six years of age, 120 ried; giving us the following per-centuges of total mortality-

Between 6 and 12 months, 972 per cent. died.

$\begin{array}{llll}\prime & 1 \text { and } 3 \text { years, } & 3.845 & \end{array}$


The disease is never, or hardly ever, seen in calves under six months old, unless they are fed on a diet which is not an exclusively milk one.

Quarter-ill, like many other diseases, has periods at which it is rife in the country, and other periods when it seems to be dormant.

It is most frequently seen when animals are changed from one pasturage to another, or from one condition of living to another, more particularly when the change is from poor feeding to rich pasturage. The disease is also most prevalent in lowlying pastures, and when there is rain and humid heat, though it may occur on any lands and in all kinds of weather.

Symptoms.-The symptoms of the disease are usually mostly as follows, but occasionally the initiatory ones do not indicate it very clearly, and may readily be taken for those of some other affection, and perhaps one which may be cured by treatment, and so consequently are apt sometimes to mislead even the expert:-Loss of appetite, dulness, listlessness, cessation of rumination, harshness and staring of the coat, elevation of temperature, rigors and local tremblings, coldness of the extremities, then lameness or stiffness when moved, arching of the back, and on examination of the skin a tumour is found forming under it in some part of the body. It may be on the head, neck, shoulders, dewlap, loins, genital organs, or mammary gland, but by far most frequently in either the shoulder or the loins.

M. Hess has observed that the tumour forms more often on the right side of the animal than on the left, but he can give no reasons why this should be so.

The tumour is found in regions which abound in muscular tissue, and where the connective tissue is loose, and seldom in the region of joints and tendons, and where the tissues are firm.

These tumours are ill-defined, and have no limiting membrane. Externally they may not appear of great size, but when carefully examined they are found to extend deeply into the subjacent tissues.

At first hot and painful to the touch, they rapidly become cold, insensitive, and dead in their centres, and when then handled are found to crepitate or erackle, due to the presence of evolved gases under the skin; their peripheries extend and penetrate into the surrounding parts until they attain enormous dimensions.

If incised, they discharge a dark-coloured and foetid, acid fluid, snceeded by a flow of frothy, citron-coloured serosity. 
As the disease progresses, the tumour or tumours enlarge, gases are evolved beneath the tissues, the animal evinces great distress, the breathing becomes greatly hurried, the temperature rises to a great height, the pulse beats at 120 to 130 per minute, feebly and intermittently; the expression of the face becomes haggard, tympanites (hoven) ensues, freces are passed involuntarily, the animal falls, becomes unable to rise, is attacked by fits, and either dies during one of these, or immediately after its cessation.

In the last stages the temperature falls below the normal, and decompusition sets in even before death.

There are, however, cases which do not exhibit the ordinary symptoms to commence with. In some the disease begins as colic, or some digestive derangement; and in others there may be lameness and stifliness, but no appearance of a tumour anywhere. On post mortem examination, the animal is found to be enormously swollen, due to the evolution of gases into the stomachs, intestines, and in the tissues under the skin. Bloody froth is seen issuing from the mouth, nostrils, and anus. On cutting into the carcase, gases of a sour odour escape, and are often accompanied by spurts of dark blood or yellow serosity.

The tumours are found to be black in their centres, and the muscles in their immediate neighbourhood to have the same colour. The further we proceed from the centre of the tumour the lighter the colour becomes, till, instead of being black, it is black and yellow streaked, then pinkish, and then surrounded by a citron-coloured portion.

In some cases the tumour is found in the muscles under the shoulder-in others in the diaphragm and in many varied situations, but these are rare compared to those found in the loins and withers.

Disposal of carcase.-It is the practice in some parts of England to slaughter cattle affected with the disease, to prepare them for human food by carefully removing the discoloured portions, and sending the rest of the carcase to market. We are opposed to this, and consider that this disease should be dealt with by the authorities, under the "Anthrax Order of the Contagious Diseases (Animals) Act," as the skinning and dressing the earcase is dangerous to human life.

The blood of an animal affected coagulates readily, and hence is quite different from that of anthrax (splenic fever). 
Cause of the Discase.-The cause of this disease is an anaërobic germ, which when in connective tissue and outside blood-vessels propagates itself with wonderful activity, and in so doing causes destruction of the tissues in its neighbourhood, the formation of the tumours, and ultimately death.

This germ is smaller than the true Anthrax bacillus, measures from $\frac{1}{5080}$ to $\frac{1}{\sqrt{2} 30}$ inch in length, and from $\frac{1}{50000}$ to $\frac{1}{250 \sigma 0}$ inch in breadth. It is found in the tumour, and the exuded fluids contained in and about it, in large numbers, is scarce in the blood itself; it varies in shape,-cylindrical when the contents are homogeneous, club-shaped when sporulated, but when the spore is central the bacillus becomes spindle-shaped, and often contains a clear spot or spore at one end, sometimes one at each end. It is motile, its movement being from side to side. Many of the rods contain spores, and have an irregular swollen appearance; they grow rapidly on glycerine, agar, and other cultivation fluids, liquefying gelatime, and evolving much gas, having an unpleasant sour odour. It is stated that the virulence is destroyed after the third cultivation, but returns on the addition of lactic acid.

If iodine solution be added, the germs stain a violet colour. In glycerine they grow readily and become motile.

They also stain with fuchsin, more particularly the spores contained in them.

When the virus is introduced into the system of a guinea-pig it becomes attenuated, and loses its great destructive powers.

Protective Inoculation.-It has recently been demonstrated that when the germis are introduced directly into the bloodstream, and not into the connective tissues, immunity to the disease is caused.

To obtain the germs for protective inoculation the following is the method recommended by Messis. Arloing, Cornevin, and Thomas, and others, and found to be extremely successful.

From an animal just dead of the disease take the blackest portion of the tumour, cut up into small pieces, mix with distilled water, then triturate in a mortar, squeeze through cloth, and filter through several folds of muslin which has been previonsly wetted with water, and of this fluid inject from five to ten drops into the jugular vein by means of a hypodermic syringe, taking the very greatest care that none of it escapes 
into the wound. If the operation be carefully performed, the animal will have immunity conferred upon it.

In many, for two or three days, there is dulness and disinclination to feed, but this soon passes off, leaving 110 ill effects.

But if the virus escapes into the wound, or if the animal has any bruises upon it, there we may expect to see the tumour of quarter-ill appearing, and death resulting.

Others again, instead of using the virus direct, inocnlate a guinea-pig subcutaneously, and cause the formation of a tumour containing germs, which are thus modified in their power, and after mixing with water, triturating, and passing through muslin, use it. Others again have used the dessicated virus prepared by drying in air portions of the deceased tissues. We, however, are of opinion that Cornevin, Arloing, and 'Thomas' method of using the virulent tissue mixed with water and filtered as above stated, is the best.

For general use, the virus contained in the fluid and muscles of the diseased parts are dried at a temperature of $89^{\circ}$ to $9 \tilde{3}^{\circ} \mathrm{F}$, the dried mass is then powdered, mixed with water and boiled. This is used as the first vaceine, and a second and stronger one is obtained by heating to $176^{\circ} \mathrm{F}$. only; others use cultures, but, as above stated, Arloing, Cornevin, and Thomas' is the most convenient method.

Many other methods of preventing this disease have been in use from ancient times, and seemingly with success; setoning, for instance, when performed on young animals not affected with the disease, but having been in contact with affected animals, almost invariably is said to protect the animal for the time being at least. We think that the setoning itself does not protect, but that the dietetic and hygienic alterations which accompany it materially tend to stop its progress, and the credit is thus given to the wrong agent. 


\section{CHAPTER XXVII.}

\section{CONTAGIOUS DISEASES-continued.}

\section{HOG CHOLERA-SWINE FEVER-PNEUMO-ENTERITIS.}

TrE term swine fever is applied in this country to a contagious and infectious disease of a very fatal nature. The pathology of infectious swine diseases has been profoundly studied on the Continent of Europe and in America, and it has been found that the pneumo-enteritis of Klein, typhoid fever of Budd, hog cholera, blue sickness, measles, erysipelas, and intestinal ferer are terms incorrectly applied to what was supposed to be one disease, but which has now been discovered to include three separate, although similar, pathological conditions; and the researches of Schutz, Löffler, Eggeling, Salmon, and others have brought to light that under these terms there are in reality three diseases, namely- $A$, Swine plague, also called rouget de porc, angina, petechial fever, \&c., characterised by septic gastro-enteritis, hæmorrhagic nephritis, with congestion of the spleen, inflammation of the heart, liver, and voluntary muscles, induced by a very small immobile bacillus, stainable by Gram and Weigert's preparations. $B$. Contagious or infectious pneumonia, pleuropneumonia, having a tendency to pulmonary gangrene and caseous products, and arising from an ovoid bacterium. C. Hog cholera, diphtheria, pneumo-enteritis, swine fever, \&c., characterised by an inflammation and ulceration of the glands, large intestines, enlargement of the mesenteric glands. The ulcerations of the glands of the large intestines are situated in the cæcum, immediately posterior to the ilio-cæcal valve, distinguished from swine plague by its slower development, its tendency to involve the pulmonary organs, and by differences in 
the specific microbes, which are said to be motile, oval bacteria, äerobic and anäerobic, unstainable by Gram's method, and but slightly coloured by Weigert's process. This is the disease usually found in Britain.

Definition.-Swine fever may be defined to be a highly contagious and infectious disease, having a period of incubation, after inoculation, of about five days, at the end of which period there is elevation of temperature to $10 t^{\circ}$ or $106^{\circ}$, succeeded by signs of general ill-health, and generally a rash on the skin.

When propagated by cohabitation, it appears, according to experiments performed by Dr. Lutton in America, that the dissease took about thirteen days to manifest itself in healthy pigs amongst which diseased ones had been introduced.

The disease is very common in Great Britain, Ireland, America, and various parts of the world.

It prevails as as an epizootic, and is the most fatal malady to which swine are liable; but pigs differ in their susceptibility to it.

Causes.-Like cattle plague, pleuro-pneumonia, \&c., swine fever appears to arise from contagion and infection only; no amount of bad management, filth, want of drainage, nor decomposing food being sufficient of themselves to induce it. The microbe is said to be a small oval motile bacterium, both aërobic and anaërobic, measuring $\frac{1}{2} \frac{1}{500}$ to $\frac{1}{1} \frac{1}{5} \overline{0} 0$ in length, and $\frac{7}{75000}$ of an inch in breadth. Klein, however, states that it is due to a small bacillus which in cultures assumed a long leptothrix-like filament which develops spores, which become free after the disintegration of the filamentous matrix ; and Detmer discovered a bacillus which he called Bacillus suis, and said it was the specific agent. The disease is transmissible by inoculation to the mouse and pigeon, and, according to Law (Cornell University), to the sheep and rat.

\section{PATHOLOGY AND SYMPTOMS.}

The symptoms are loss of appetite, general prostration, small and frequent pulse, hanging ears, sullen appearance, painful and haggard expression, watery eyes, conjunctivæ red and spotted, dirty secration about the eylids, generally preceded by a red 
blush and red spots on the ears, the abdomen, and internal aspects of the extremities.

The reddened spots are at first hot and painful to the touch, but afterwards become cold, humid, and insensibie even to the pricking of a pin. As the disease advances, tremblings and convulsions are manifested, the animal grinds its teeth, the flexor muscles of the limbs contract, and the animal stands upon its toes. These symptoms are succeeded by paralysis of the posterior extremities, or of the whole body, involuntary deficention and passage of high-coloured and even bloody urine. The bowels are at first generally torpid, but the freal matters may be soft and mixed with very black foetid blood, and thick tenacions mucus. Diarrhœa, however, often sets in; the defecation are then profuse and exhanstive; the breathing becomes catching and convulsive, a painful cough is present; the convulsions become more aggravated, and may continue to the end, or the animal becomes comatose, till death closes the scene.

In some cases the first observable symptoms remain stationary for a period rarying from twenty-four to forty-eight hours; then the surface of the body becomes burning hot, and very sensitive to the touch, notably at the sides and abdominal walls. If touched, the animal cries with pain, and to these signs are added tremblings, convulsions, grinding of the teeth, and tetanic contraction of the muscles; succeeled by rapid diminution of temperature. The conjunctivæ become brown; the eyes bleared; the tongue dirty, thick, and bluish; and the animal, extended on its litter, incapable of any regulated movements, succumbs in from twenty-four to forty-eight hours.

These symptoms are liable to various modifications, depending upon the intensity of the fever and the various localizations of the poison. In some cases, the virus seems to expend itself upon the serous membranes, inducing peritonitis or pleurisy; sometimes upon the mucous membrane, as expressed by bronchitis or broncho-pneumonic congestion and hæmorrhage, enteric congestion and ulceration, sometimes even to perforation or rupture of the bowel.

In many cases the animal is amaurotic, wanders to and fro, falls down, rolls and kicks, and seems to be in pain. Now and then it will rise from its bed and give a piercing cry, the whole body being involuntarily convulsed. 
The condition of the blood differs in a marked degree from that of anthrax, being fibrinous, red in colour, and as a rule contains neither bacilli nor the contagium of the clisease.

The experiments of Klein point to the conclusion that- " 1 st. The fresh blood of diseased animals does not, as a rule, contain the virus, as it fails to produce the disease when introduced into a healthy animal.

"Four animals were inoculated (at different times) with the fresh blood of diseasect animals. They remained healthy. When subsequently inoculated with virus-containing matter, they became smitten with the disease.

"In a fifth instance, however, fresh blood did produce infection. [And this same blood proved active after liaving been kept sealed up in a capillary tube for several weeks.] This blood was obtained from a very severe case with copious peritoneal exudation; in which were found peculiar, abnormally large, coarsely gramular cells; the same cells were also present in the blood; so that it appears probable that the blood became charged, by absorption during life, with matter from the peritoneal exurlation. This latter always contains the virus in an active state.

" 2. Experiments showing that fluid as well as solid lymph of the diseased peritoneum contains the virus in a very active state.

"Six successful inoculations with fluid peritoneal exudation.

"There is no difference of activity to be noticed between fresh exudation and one that had been kept sealed up in a capillary tube for several weeks.

"Solid lymph obtained from the peritoneal cavity of diseased animals, having been diried at a temperature of about $38^{\circ} \mathrm{C}$, proves very active.

"3. Experiments showing that parts of the diseased lung, ulcerated intestine, and also diseased spleen, contains the virus in an active state. Diseased parts of ling or intestine that were dried at a temperature of about $38^{\circ} \mathrm{C}$, retain their virulence unaltered.

"In all cases of pneumo-enteritis, the trachea as well as the bronchi have frothy blood-containingr mucous matter, possessed of infectious properties. It must be therefore supposed that the breath of a diseased animal is charged with the poison. On 
account of the diseased state of the intestine, also the dung is to be regarded as infectious.

" 4. Experiments showing that infection is produced by cohabitation with a diseased animal, or by keeping healthy animals in a place whence a diseased animal had been removed.

" 5. Several experiments were made to see whether feeding healthy animals on matter obtained from the diseased organs (intestinal ulcers especially) produces the disease. The experiment was always attended with success, if a lesion-abrasion existed in the mucous membrane of the mouth or pharynx; this was usually the case when the matter had to be introduced into the mouth while the animal was being held by assistants.

"There were, however, two cases which appear to prove that the disease cannot be produced by simple feeding.

"This was unfortunately at a time when I was not as yet acquainted with the fact that in many animals the disease is of so mild a form that it can harlly be recognised in the living animal. I have not made any post mortem examination of those two animals.

"But since then I have made two other experiments, in which the virus was brought directly into the stomach, by means of an india-rubber tube introduced per fauces and cesophagus. In both these instances the animals became diseased, and their intestines were most conspicuously affected.

"From the last three series of experiments, we may conclude that the principal way in which contagion of pneumo-enteritis is carried out, is through the instrumentality of the air and the food.

" 6 . This series comprises experiments to prove that the virus can be cultivated artificially-i.e., outside the body of an animal; in the case of splenic fever it has been successfully done by Dr. Koch.

"The experiments are seven in number:-( $a$.$) Two refer to$ cultivations commenced with fluid peritoneal exudation; (b.) In the five others the virus had been obtained by cultivation of dried lymph from the peritoneum of an animal suffering from the disease.

" (a.) The cultivation of the virus for the first two cases was carried out thus :-

" Fluid peritoneal exudation of a diseased animal had been 


\section{PLATE VI.}

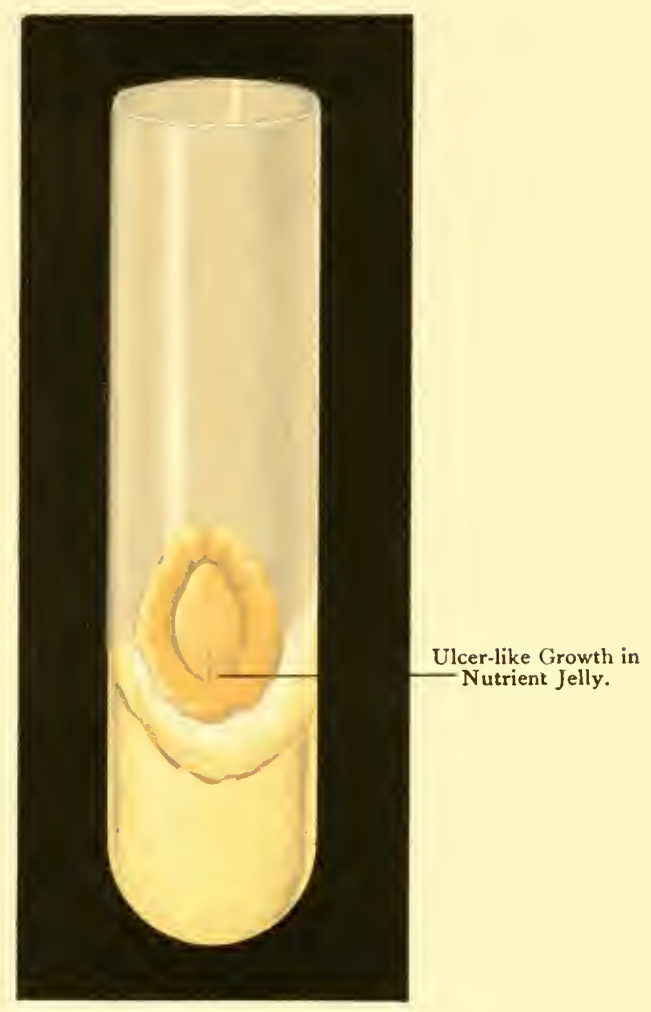

BACILLUS LUTEUS (SUIS). 
collerted and scaled up on November 6th, in a capillary glass tube. On the following day there was present a small clot due to coagulation. A minute speck of this clot was removed with the point of a clean needle, and with it was inoculated a drop of fresh aqueous humor of a healthy rabbit. This drop had been placed on a thin covering-glass, which, after the inoculation, was inverted over a small "cell," made by fixing a glass ring on an orclinary glass slide. The covering-glass was fastened on the glass ring by means of a thin layer of pure olive oil. The preparation was then liept in the incubator for twenty-four hours at a temperature of $32^{\circ}$ to $33^{\circ} \mathrm{C}$. After this time it was used to inoculate a new drop of aqueous humor in a similar manner to the one just described. We will call this the second generation.

"This new specimen was placed in the incubator, and kept there at a temperature of $32^{\circ}$ to $33^{\circ} \mathrm{C}$. for another twenty-four hours. In the same manner a third generation was started, by inoculating a fresh drop of aqueous humor. After having been kept in the incubator for several days, it was used to inoculate two animals at different times. Both animals became infected with the disease.

" $(b$.$) The other five experiments were carried out with virus$ cultivated from solid lymph of the peritoneum of a diseased animal. The lymph had been dried at $38^{\circ} \mathrm{C}$. (see series 2). A small particle of dried lymph is crushed into fine powder. With a granule of this, a drop of fresh aqueous humor is inoculated in the same manner as above described,-first generation.

"After having been kept in the incubator for two or three days at a temperature of $32^{\circ}$ to $33^{\circ} \mathrm{C}$, it is used to inoculate a second generation, care being taken to use a trace only of the fluid part, and not to come in direct contact with the original granule, which may be still discerned in the preparation.

"The specimen representing the second generation is kept in the incubator for a day or two. It is then used to inoculate a fresh preparation,- third generation. And, finally, this is used for establishing a fourth generation. After having been kept in the incubator, a part of it is used for inoculating two animals, the inoculation being carried out at different times.

"Both these animals became affected with the disease. Another portion of this fourth generation was used to start a 
fifth generation, then a sixth, a seventh, and an eighth generation. With this three animals were inoculated at different times, All three aninals became diseased in due time.

"In order to correctly interpret the results of this last (sixth) series of experiments, it is important to mention that inoculation with dried lymph, diluted far less than would correspond to the third generation in the last-named experiments, is followed by a negative result."

\section{MORBID AN.ITOMY.}

In the majority of animals, the skin about the perineum, groin, belly, and neck is swollen, and of a diffuse red or bluishred colour, and the ear lobes and the skin of the nose also red and swollen, whilst in some instances there are gangrenons patches of the superficial cutaneous structures. In many instances, however, this redness of the skin may be entirely absent, even after death; but the longer the animal lives, the superficial structures become, as a rule, the more swollen, and the dependent parts of the ears become deep red, puffy masses, from the surface of which the epidermis peels off.

The blood-vessels of the skin are more or less filled with blood or plugged with fibrin, and around the vessels lymphoid cells are discoverable; the suderiporous glands are greatly distended, and often filled with blood charged with large coarsely granular cells containing large clear vesicular muclei. The connective tissue of the corium contains fibrinous exudations and a yellow serosity.

The small intestines almost invariably, and the stomach more rarely, are congested and covered with spots of ecchymosis, both upon their mucous and peritoneal surfaces. The large intestines have always the most characteristic appearances. There are isolated or confluent, generally roundish, ulcerations at and around the ilio-creal valve, the rest of the mucous membrane being congested and studded with spots of ecchymosis. Klein says the whole large intestine down to the rectum contains ulcers; in the cxecum they are confluent, and measure several inches, extending transversely as well as longitudinally; while the whole remaining mucous membrane of the large intestine is much thickened, and in some parts the mucous tissue contains large accumulations of blood. The ulcers are of various 
aspects. The following forms may be seen-very minute, well defined, prominent yellowish-whitish specks of the size of a millet or hemp seed; then somewhat larger, more flattened, prominent, circular or oval, yellowish patches (with which in one case the whole mcuous membrane of the crecum seemed quite covered) of the size of a hemp seed, up to about oneeighth of an inch in diameter; next, flat, circular, or slightly oblong patches, situated on the crust of a fold of the mucous membrane, in size from one-eighth to one inch in diameter, generally black or grey (from bile pigment), except a very conspicuous, and, I may almost say, characteristic prominent rim, which is yellow. The ulcer generally shows a pale central or eccentric disc, around which the rest of the ulcer is arranged as concentric rings. Between these flat ulcers with concentric layers, and those uniform, yellowish-white, prominent patches and nodules, there are all intermediary forms. This is easily understood, if it is borne in mind that as the latter increase in size, the central part is transformed into that black or greyish mass. Besides these, there are very often formed on the surface of the mucosa minute whitish specks, just perceptible by the unaided eye. In one single instance have I seen ulcerations of the lower part of ileum. This case had exceedingly numerous ulcerations of the large intestines. The two uleers of the ileum were quite different from the ulcers generally found in the large intestine, for they were oblong, deep pits, surrounded by a thick, prominent wall of swollen mucous membrane, very much the same as ulcers of human typhoid. But under any circum-American pig slaughtered at Liverstances the microscope reveals pool, May 1879.

stanees the microscope reveals a $a$. Ilio-creal opening. marvellous difference between the $b, b, b$. Ulcers in various stages. two diseases; for in the pig these patches, whether nodules or ulcers, have absolutely nothing whatever to do with lymphatic 
follicles, whereas in human typhoid there is found in the first stage round, or generally oblong, prominent papulæ or patches of swollen lympl follicles, surrounded by swollen mucous membrane, after which stage the greater part of the swollen lymphatic patches dies, and is finally discharged as a slough leaving behind a pit-like excavation bordered by a well-defined fringe of nucous membrane, which is neither thickened nor indurated.

Next in importance to the intestinal lesions are those discovered in the lymphatic glands, which are congested, generally swollen, of a dark red colour, or infiltrated with blood, black, soft, and pulpy; those in connection with the intestinal canal are not only swollen, but infiltrated with a greyish-white opaquelooking matter.

The condition of the glands is sometimes very slight to the naked eye, there being merely swelling, and an apparent congestion ; but even here the microscope reveals bursting of bloodvessels in the cortical tissue, and hæmorrhage into the lymphatic follicles and sinuses of that part, amounting in severe cases to total destruction of the adenoid tissue by blood. In severe cases the medullary sinuses, and partly also the medullary lymphatic cylinders, become filled with extravasated blood. In so far as this condition is similar to what is found in anthrax. "This state of the lymphatic system is very characteristic, and, combined with the disease of the intestine, is of paramount importance to the diagnosis."-(KLEIN.)

Respiratory organs. - In a large number of cases, the tongue, month, fances, and pharynx are ulcerated, and sometimes gangrenous; the lymphatic ressels filled with micrococei; the lungs are generally congested in patches, with distinct mapping out of the lobes and lobules by cedema of the interlobular tissue, succeeded by hepatisation, at first red, subsequently opaque, or white specks or patches appear in the red substance, which, as they increase in size, become confluent. This is due to the fact that the bronchial tubes become gradually filled with a white, brittle, cheesy mass, progressing gradually from the finest ramifications on to the larger branches of the lobules and lobes. Finally the whole lobule is transformed into a discoloured, dry, hard, friable mass. The pleura of the corresponding parts is of course inflamed, being in some cases exceedingly thick, and 
covered with false membranes. In severer cases, the greater part of one lung and portions of the other may be thus changed, and on the external surface there may exist smaller or greater ulcerations. Except in very slight cases, there is generally a certain amount of pleural exudation; and in severer cases the pleura contains a considerable quantity of a thick, offensive, yellowish or discoloured exudation. In some severer cases the pericardium is also inflamed, containing a large quantity of exudation, and its walls being much thickened by false membranes. The same is also the case with the peritoneum, this being in some cases hyperæmic in parts, or even covered with solid lymph and pus.

The spleen and liver are, in severe cases, dark in colour, enlarged, and the kidneys are sometimes changed, being hyperæmic and covered with petechiæ, particularly in the pyramids and undemeath the capsule, which is easily stripped off.-(KLEIN.)

In the Privy Council Order dated 17th December 1878, which is termed also the "Typhoid Fever of Swine Order, 1878," it is laid down that typhoid fever of swine, otherwise called soldier disease or red disease, shall be deemed to be a disease (under the Contagious Diseases Animals Act, 1878) for the purposes of slaughter and compensation, notice of disease, Orders of Council, power of police, and power of entry; and, also (under the Animals Order, 1878), for the purposes of movement and exposure, movement of dung, burial or destruction of carcases, and general provisions, as also for cleansing and disinfection ; but this order has been very imperfectly carried ont.

Preventive inoculations have not been tested in this country, but Billings (Nebraska) claims that he has successfully induced immunity by inoculations with attenuated cultures, and Cornil and Chanternesse are said to have succeeded (Freidberger and Frohner) in attenuating the virus by subjecting the cultures to air and heat $93^{\circ} \mathrm{F}$. At the end of ninety days the virus no longer kills, but gives, immunity to the rablut and guinea-pig. This attenuation is transmissible to a series of cultures, and with these the following experiments were made. Four pigs were successively inoculated subcutaneously at eight days' interval, with a dose of two cubic centimetres of bouillon cultures, which were ninety, seventy-four, fifty-four, and eight days old, and finally with virulent virus. During the days following the inoculations the animals showed only a slight 
illness. Two months after the beginning of the experiments they received in their food one litre of virulent culture in bouillon. Two pigs which were not inoculated were subjected to the same treatment. Within ten days these latter died with characteristic intestinal lesions. The inoculated animals did not show any important morbid phenomenon for two months; after this lapse two dierl. The autopsy showed intestinal lesions, which were much developed, and had a chronic course, and very slight pulmonary lesions. Both of the other inoculated animals resisted.

These first experiments showed that the pig acquires immunity with more difficulty than the subjects of other species.

Swine plaguc, rouget de porc, angina, petechial or spotted fever, red disease, erysipelas, \&c., is common on the Continent of Europe, and is, according to Continental writers, a specific septicamia due to a fine bacillus resembling that of Koch's mouse septicemia. If inoculated in the mouse, the animal invariably dies; it is also often fatal in the rabbit, guinea-pig, birds, and the pig, but, according to Kitt, the field-mouse possesses immunity, as in septicxmia, to the action of the microbe, and he has also observed that its virulence is attenuated by once passing through the rabbit, and that after six days pigs may be inoculated with blood exudate collected at the point of inoculation, and thus obtain immunity. The microbe exists externally in different media, in damp earth, in water, and in plains and valleys with slow flowing strearns. Cornevin states that the horse, $\mathrm{ox}$ and sheep, and guinea-pig are immune to its action, as already stater ; it is a very fine cylindrical bacillus, measuring from $\frac{1}{25000}$ to $\frac{1}{12 \frac{1}{5} 00}$ in length by $\frac{1}{4200}$ in breadth; it is found in the blood, particularly in the capillaries in contact with their intima, in the white corpuscles, spleen, liver, kidneys, lymphatic glands, the bone marrow, exudates, freces, and urine; is non-motile, anaërobic, but grows in contact with air, and in a temperature as low as $45^{\circ} \mathrm{F}$. According to Kitt, it resists putrefaction. Schottelius says that it develops spores; Jut this is denied by Cornevin and Kitt, who base their objections on the non-virulence of dried virus.

This microbe, in reality the discovery of Löfler and Schutz, although ascribed to Pasteur and Thuillier, is destroyed by slow dessication in from fifty to eighty hours; by water at a tempera- 
ture about $83^{\circ} \mathrm{F}$. in twenty minutes, and in two minutes at that of $168^{\circ} \mathrm{F}$. Cold of about $20^{\circ} \mathrm{F}$. kills it in abont a fortnight, and salted meat within a month. It is, however, stated that it resists the action of saturated solutions of boracic acid, benzine, chloride of zinc, \&c., but is killed by chloride of lime, quicklime, \&c.

Symptons.-After a period of incubation of about three days, the disease is manifested by intense fever, constipation, red or purple patches upon the skin, particularly of the abdomen, lower parts of chest, ears, inner aspect of the thighs, \&c. There is often grinding of the teeth, muscular twitchings, vomiting, great weakness, paralysis of the hind quarters. The conjunctiva are dark red or reddish-brown in colour, and the patches on the skin become confluent, change from a clear red to dark red, and finally a bluish colour.

The constipation is succeeded by diarrhœa, the freces being often bloody and containing mucl mucus, and finally the breathing becomes much accelerated, the surface of the body bluish in colour. Death may occur in twenty-four hours or on the third or fourth day, but is seldom prolonged to over more than a week, and the per-centage of deaths is from 50 to 85 per. cent., or even higher.

A cure has heen attempted by the administration of emetics and calomel, but more attention is now paid to the arrest of the disease by segregation, disinfection, destruction of the carcases, and finally preventive inoculation has seemingly been of great value in some instances, whilst in others the benefits have been doubtful, and further researches will have to be made before its adoption can become miversal, The post mortem lesions indicate a more general septic condition; there is congestion of the spleen, liver, kidneys, and endocardium, with hæmorrhage into these organs and into the muscular structures. The spleen externally, however, is tense and resistent from distension of its capsule, but is soft internally and of a reddish-blue colour, and generally unassociated with hæmorrhagic infarctions or extravasations.

There is an intense congestion of the mucous membrane of the stomach and bowels, which is sometimes of a uniform dark red colonr, swollen, covered with superficial hæemorrhagic and diphtheritic patches; sometimes it has a marbled appearance. The solitary glands and l'eyer's patches are swollen, often covered with bloody mucus, surrounded by a red zone and very often ulcerated, as in the typhoid of man. 
Infectious pneumonia-swine plague of Ameriean wlitersis manifested by accelerated breathing, pulmonary gangrene, and caseous tumours, induced by an ovoid non-motile bacterium similar to that of the septicemia of the rabbil and the bacillus of chicken eholera, measuring about $\frac{1}{20} \frac{1}{00}$ of an inch in length and about $\frac{1}{5000}$ in breadth, proving fital, when inoculated, to the pig in from twenty-four to forty-eight hours. It induces an inflammatory cedema at the point of puneture, and proving fatal in sixty hours in a pig which had been given immunity against swine plague by inoculation. Aecording to Schutz, the contagium is very subtle and induces the disease by inbalation by the skin and digestive apparatus, is rapidly fatal, and is marked by redness, tmmefaction of the skin in the regions of the neck and legs; with congh, diffienlty of breathing, intense fever, and great depression.

The post mortem appearances are those of pueumonia, with several reddish grey hepatised spots, having yellowish neerosed spots in their centres of the size of a grain of sand, and associated with pleuritis and fibrinons pericarditis. The bronchial glands are enlarged, the liver, kidneys, spleen, and heart are softened and degenerate, but the lymphaties of the intestine are not usually altered; and in some ehronic eases of the lungs, lymphatie glands, tonsils, bones and tendons, caseons tumours, resembling those of tubereulosis-but not containing the tubercle bacillus-are diseovered.

The disease ean be inoculated into the rabbit, mouse, guineapig, fowls, and pigeons, but large doses are required to induee fatal results in the three latter. It is mostly transmitted from one pig to another by inlalation. 


\section{CHAPTER XXVIII. \\ CONTAGIOUS DISEASES-continued.}

\section{TUBERCULOSIS.}

Definition.-An infectious, infective, and inoculable disease induced by the action, particularly upon the hereditarily predisposed, of a bacillus discovered by Koch in 1882, and termed the Bacillus tubcrculosis, affecting many kinds of animals, and in consequence called by Lydttin the "universal panzootic."

Tuberculosis is most commonly seen in man, monkeys, and horned cattle, less frequently in the pig, horse, dog, and cat, and birds; amongst the latter, however, it sometimes assumes an epizootic form, attacking poultry, pigeons, pea and guinea fowls, turkeys; even small birds not being proof against its ravages. The smaller ruminants, sheep and goat, are very rarely attacked with tubereulosis; they may resist subcutaneous inoculation, but they are not proof against its intravenous inoculation. The direct introduction of the bacilli into the blood, and the repeated ingestion of tuberculous growths, induces the disease even in them. It is also found in the camel, giraffe, antelope, llama, gazelle, zebra, \&c., and it is now well known that the disease is transmissible from man to animals, and from animals to man.

The tubercular nodules vary in appearance, some being grey, like pearls, hence the term "pearl disease" in England, "perlsucht" in Germany; some being yellow, and some calcareous, these varying appearances indicating the age of the growths. They are non-vascular, and vary much in size, some being even smaller than a millet seed (miliary tubercle), very numerous, and invading one or many organs. Some larger ones are hard, greyish-red, pedunculated, or having broad bases, arranged in cluster's of various sizes, covering the lungs, thoracic walls, surface of diaphragm, the peritoneum, \&c.; or they are found in the form of great fluctuating masses filled with yellow pus, which is thick, grumous, full of calcareous grains, resembling mortar, or of an 
opaque yellowish matter infiltrating the tissues. These varying appearances gave rise to the opinion amongst Virchow and his followers that they were lesions of different diseases; and Villemin, who first discovered the inoculability of tuberculosis in 1865 , vainly tried to prove their identity by inoculations, but it remained for Koch to prove that they were all due to tubercular bacilli.

Examined microscopically, the nodules present giant cells with branched processes and small endothelioid cells, which are round or oval in shape, and outside a zone of lymphoid cells. The cells are surrounded by a more or less complete fibrous stroma. These tubercles are sometines scattered throughout the substance of the organs, having the form of distinctly rounded nodules, rather gelatinous in structure, and a pearly grey transparent appearance. In other cases it will be found that beneath the pleure, both visceral and parietal, there are large yelluw nodules, which give off numerous radiating branches. These are called "grapes," from the supposed similarity to that fruit, and between them are seen some red lines in which degeneration has not advanced to any extent. Each yellow patch is seen under a low power to consist of a number of follicles, each being degenerated-caseated-in its eentre, embracing the alveoli and their contents. There are no blood-vessels in the centre, but some vessels are seen with difficulty some distance from it, where the alveolar walls are slightly thickened. In order to differentiate the giant cells from those of an endothelial type and from the fibrous capsule, stain with picro-carmine, when the giant cells will present the yellow picric acid colour, and the others will be stained carmine; for the recognition of the bacilli other methods must be resorted to.

There is a difficulty in finding the bacilli in caseous tuhercles, particularly in eatlle, but they can often be demonstrated in material taken from the periphery of the nodule. If cavities containing pus are formed, such as are not uncommon in tuberculous horses, the bacilli can be detected very readily by the following process :-

A drop of water is placed on a perfectly clean cover-glass. Remove a small portion of the pus or caseating matter with the point of a neadie. then rub it with a drop of water already placel on the cuver glass. The cover-glass is then dried over 
a spirit lamp or Bunsen burner until the albumen is coagulated. A few drops of filtered carbol-fuschin solution is put on the cover-glass, and held by a pair of forceps over the flame until steam rises for five minutes. Wash in water, and decolorise by 25 per cent. sulphuric acid solution, and then wash in water. Counter stain with aqueous solution of methyl blue, then wash with water, dry over a flame, and mount in Canada balsam. The bacilli will present a bright red colour on a pale blue ground, and occur singly, in pairs, or aggregated in rosettes.

The bacilli vary in number in different lesions, and have no relation to the magnitude of the growths. Thus in a large nodule very few bacilli will be found after the most careful examination, whilst a smaller one may contain a large number. When the giant cells are numerous the bacilli are generally few in number, many of them having doubtless been ingested by the giant cells. They are easily detected in the expectorations of human consumptives, and in the thick purulent yellow discharge which is sometimes ejected from the mouths of cattle after long and violent fits of coughing, This material coughed into the mangers and upon partitions and walls of the cow-shed is, when dry, diffused through the air inspired by the healthy cattle, and thus becomes one of the great causes of the spread of the disease.

The transmissibility by inhalation, as well as that by ingestion and inoculation, has been proved experimentally. The animals experimented upon were compelled in a chamber to breathe, for several hours daily, air in which were fine particles of phthisical expectoration from persons with cavities in their lungs, mixed with water, and rendered into fine particles by a steam atomiser. Dogs alone were used, as they rarely suffer from tubercle. Eleven animals were experimented upon, and all were killed in a period varying from twenty-five to forty-five days, and, with one doubtful exception, presented well-developed miliary tubercles in both lungs, and in most of them tubercles were also found, but to a smaller extent, in the kidneys, and to a still smaller extent in the liver and spleen. Microscopical examination demonstrated the presence of the bacilli.

These experiments were conducted by Dr. Tappeinier of Meran, and a preliminary account of them led Dr. Max Schottelius to make similar ones, not only with the sputum of 


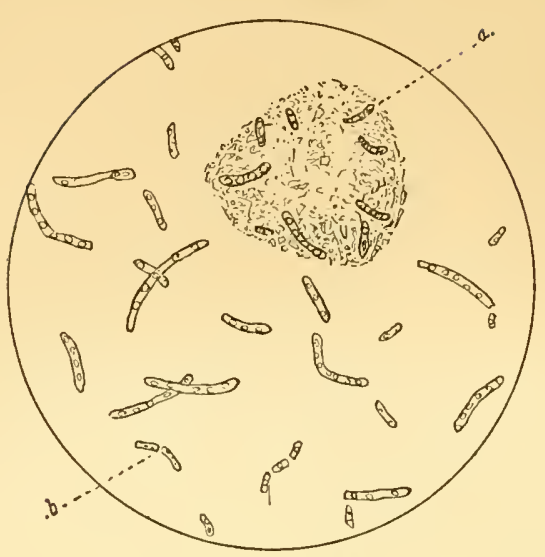

Fig. 15.-Tubercle bacillus.

$\times 1400$.

a. Giant cell invaded by bacilli.

b. Minute germs.

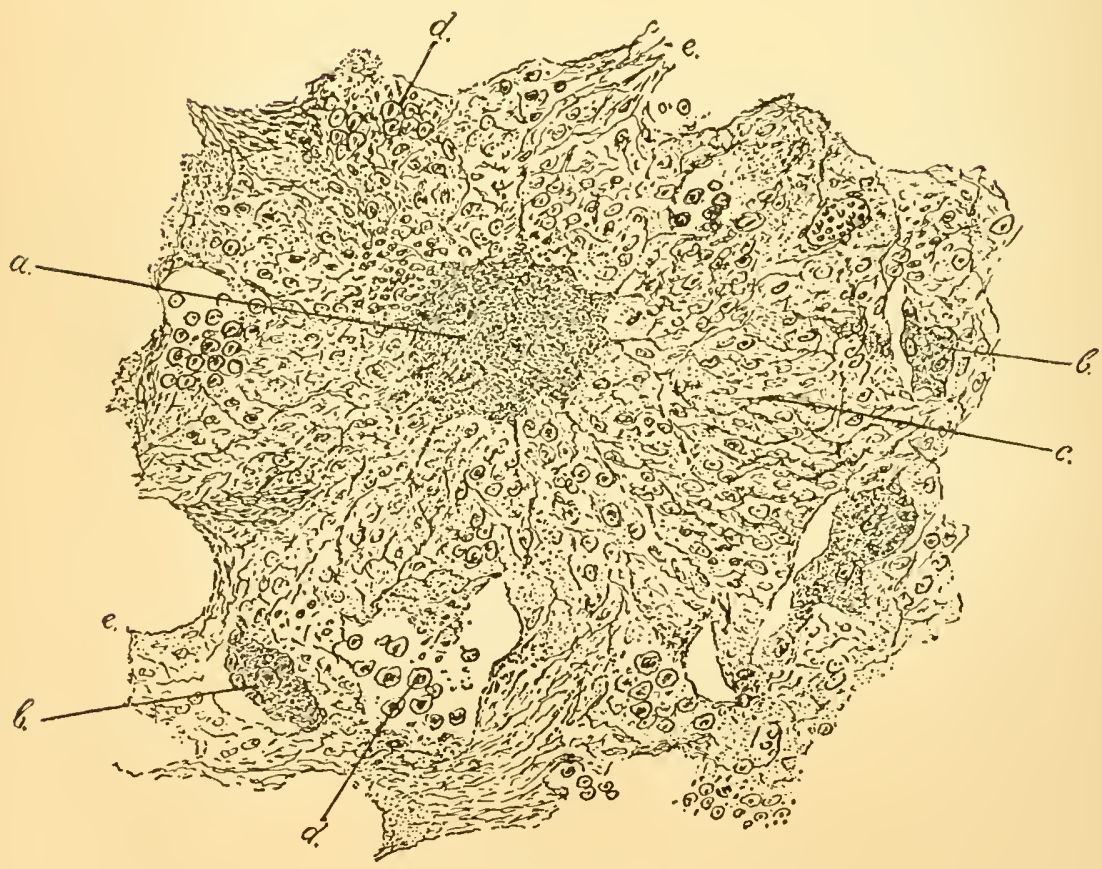

Fig. 16.-Tubercular nodule. $\quad \times 300$.

a. Caseated centre of nodule.

b. Giant cells.

c. Reticulum of nodule with epithelium-like cells.

d. Leucocytes, red cells, \&c.

e. Portions of adjacent alveoli. 
phthisical individuals, but also with that of persons suffering from simple bronchitis, and with pulverised cheese, brain, and cinnabar. The result was that miliary tubercles were found in the lung in all cases, and in equal quantity with both phthisical and bronchial sputum. Cheese produced a smaller quantity, pulverised brain still less, and the cimmabar least of all, merely a few whitish tubercles with pigmented centres, with an interstitial deposit of the substance, which had caused no inflammatory reaction; though the tubercles produced by non-specific matter closely resembled those of tuberculosis, they contained no bacilli, lience they cannot be classified as those of true tuberculosis.

Tuberculosis is described under three divisions, namely,-1st, Tuberculosis of the organs; $2 d$, of the serous membranes; and $3 d$, of the lymphatic glands; but generally these forms co-exist in the same subject, constituting the so-called "diffuse tuberculosis."

According to Nocard, it is shown that 40 per cent. of tuberculous animals are affected in both lungs and pleura, 20 to 25 per cent. in lungs alone, 15 to 20 per cent. in pleura and peritoneum, the remainder comprising either generalised lesions of all the organs, or lesions localised in the glands, the genital organs, mammi, tongue, osseous tissue, \&c., and that the lymphatic glands proceeding from the organs attacked are always more or less severely affected; and he makes this important statement, that in many cases when the animal is slaughtered quite at the conmencement of the disease tuberculons nodules are only found in the bronchial or mediastinal glands; and the pulmonary lesion which has served as the entrance gate for the contagion eludes the most careful research, or is only represented by a minute focus of disease much less important than the glandular alterations which it has preceded and caused.

Bollinger thinks it possible, or rather probable, that intestinal tuberculosis, consecutive to pulmonary phthisis, may be produced by the discharges (sputa) being swallowed and passing into the intestines, mesenteric glands, and liver, without there being generalisation through the blood-stream.

Symptoms in Cattle.-Tuberculosis is generally slowly developed, and its symptoms are often very obscure, whilst in a few instances its manifestation may he very rapid. In the latter case signs of ill-health appear, excited perhaps by parturition, a slight cold, indigestion with recurrent tympanites, or 
some other, perhaps trivial, cause; the illness continuing longer than usual, and the animal, being fat, is slaughtered to prevent loss, and the post mortem reveals the presence of tubercular tumours in various parts of the economy. In this case some of the tumours will be found in a softened, semi-fluid condition, the contents of which being absorbed, induce the continuance or aggravation of the otherwise trivial illness. Generalised tubereulosis only occurs when the blood-stream has become infeeted by the entrance of the bacilli. Nocard says:- "It often happens that apparently healthy animals slaughtered for human consumption are found to have several organs invaded by the disease, yet in such condition it camnot be said that the disease is generalised: these are successive localisations, and it is maintained that general tubereulosis only occurs when all the glands in the chain are destroyed, the lymph carries the bacilli into the thoracic duct, whence they are poured into the anterior cava, or, again, when a tuberenlar focus penetrates a vein of a certain size and pours into it the virulent material which it contains. Tuberculosis then assumes the character of a general disease, and all the vaseular tissues are virulent, and the tissues which are favourable to the growth of the baeilli, notably the liver and spleen and the marrow of the bones, become the seat of a number of specific granulations all of the same size and age, which constitute what is known in human medicine as gramular or acute miliary tubereulosis." Miliary tuberele in the spleen is said by Ostertag to be the surest sign of general tubereulosis. Although the animal may be fairly fat, the flesh usually is pale, watery, pitting under pressure, and prone to rapid decomposition.

In deep milkers, and in some highly bred cattle, the eachexia may seem to precede and accompany the tubereulosis, and generally the earliest signs are those of unthriftiness, and deterioration in the quality of the milk, which becomes thin and watery, a'though for some time it may keep up in quantity. If the eow be in calf, abortion is apt to occur ; if not pregnant, the condition called nymphomania is frequently present. The appetite is capricious; the mucous membranes pale; a congh of a dull character exists; the skin looks dull, the hair dirty; the animal does not lick itself, and, in the white parts, the skin is often observed to be yellow. Emaciation now proceeds more or less rapidly; the cough becomes troublesome, but there is seldom 
any expectoration or discharge from the lungs. The digestive organs are weak, the rumen prone to tympanites, and diarrhœa sets in, which soon renders the animal a mere bag of bones. Auscultation and percussion may find the lungs and contents of the thorax diseased or otherwise. There is generally some degree of pain and tenderness evinced by the animal when the sides are sharply struck or pressed upon, and very often a friction (pleural) sound is heard, and pressure upon the loins will cause the animal to cringe, groan, or otherwise evince pain.

As the disease advances the cough becomes more troublesome,--paroxysmal; louder than that of pleuro-pneumonia, and occasionally bronchinl discharges may issue from the mouth after a severe fit of coughing. As already stated, in this yellow, grumous, and viscid material the bacilli may be detected by microscopic examination. It, however, most frequently happens that the severest fit of coughing brings about no discharge, the material being swallowed. This also occurs in pulmonary glanders in the horse, in which abscesses may be found on post mortem examination filled with pus, freely communicating with the bronchi, from which during life no discharge has reached the nostrils, having been swallowed, as may be observed by watching the animal. It can be seen by standing at the left side that the act of swallowing is performed, and the course of the material followed by the eye during its course from the pharynx into the chest.

The animal rapidly becomes more or less hidebound, the hair dull, the expression dejected, the eyes watery and sunken, and the lids often covered with a scaly material, whilst a foetid discharge may issue from the nostrils. The respirations, as the disease advances, become greatly accelerated, short and jerky, each expiration being often associated with a moaning or grunting sound; the prostration is extreme, and the animal soon succumbs.

On percussing the walls of the chest the animal evinces some signs of pain, and a cough is often excited; there is decreased resonance and often signs of lung consolidations and of pleural change, whilst auscultation may reveal increased tubular sounds with diminished or absence of the vesicular murmurs, and often signs of consolidation. There may also be pronounced bronchial rales when the discharges have invaded the bronchi, and friction sounds when the pleural surfaces are roughened by 
the sub-pleual nodules and adhesions of the pleural surfaces. Some authors state that the external lymphatic glands are frequently enlarged: so far as my experience goes this is exceptional, and of no great diagnostic value. The temperature varies considerably in individual cases; in some very pronounced ones there is 110 great elevation, whilst in others this is extreme,$107^{\circ}$ or higher during the exacerbations, falling several degrees during the remissions, and these exacerbations and remissions are not infrequently met with in the pulmonary form.

As already stated, the calf is rarely tuberculons, and from the observations made at the abattoirs at Berlin and Copenhagen it was found that at Berlin the per-centage of tuberculous animals was 15.1 for oxen and cows, 1.55 for swine, 0.11 for calves, and 0.004 for sheep. At Copenhagen the per-centage was $17 \cdot 7$ for oxen and cows, $15 \cdot 3$ for swine, $0 \cdot 2$ for calves, and only 0.0003 for sheep. From its rarity in calves some writers have argued that tuberculosis is not congenital. This conclusion however, is against evidence, for cases are recorded where undoubtedly the calf has given evidence of the disease at or shortly after birth, where the lungs and various serous membranes-i.e., pleura and peritoneum-were covered with caseous and calcareous tumours, which must have been developer in utero. In two instances which came before me the arachnoid was covered with tubercles in an early stage of development, the calves dying from meningitis in an acute form. Infection of the foetus results from the penetration of the bacilli through the foetal membranes, or from their presence in the semen of the male or ovum of the female at the time of coition. We can understand this when we recognise that the ovaries of the female and the testes of the male are occisionally the seats of tuberculosis.

The liver, spleen, heart, mesenteric glands, and peritoneum may be invaded with many clusters of tubercles similar to those shown in figs. 15 and 16 , whilst not infrequently the ovaries and uterus are involved, causing continuous sexual excitement,nymphomania, - and sometimes discharge from the vagina, with non-impregnation after repeated serving: but should pregnancy occur, the animals generally abort, and according to Roloff this abortion is often the first sign of the infection of a herd.

Tubercle is also found invading the meninges of the brain and spinal cord, giving rise to fits of convulsions; to ptyalism and 
dysphagia and tympany when located in the pharyngeal glands; to purulent discharge when involving the penis of the male, uterus or vagina of the female; and colic, with alternating constipation and diarrheea, and flatulency after food, particularly if the liver be involved. The pericardium may be considerably affected without apparent symptoms, but after a time signs of eardiac irregularities beeome more and more apparent (see Heart Diseases), and sometimes the external lymphatic glands are enlarged; whilst in other eases an animal apparently quite healthy manifests symptoms of lanneness from no assignable cause, and this lameness is generally irremoveable, resisting all treatment, and after death the bones or joints present the usual characteristies of tuberenlar disease.

Tubereulosis, when affecting the mammary gland, is very frequently secondary to its development in other organs, but is supposed to be occasionally primary. Bang, Copenhagen, describes the condition as a diffuse, painless, hard swelling, involving one or more quarters of the gland, but generally the posterior ones. It differs from an ordinary inflammation of the mammary gland-mammitis or mastitis-in which the milk is curdled at commencement; but in the tubereular form the milk is at first normal, but at the end of a month it becomes watery, contains clots, and sometimes bacilli.

Bacilli are found with great difficulty; and Bollinger states that in fifty-five per cent. of examined cows where the milk was virulent the bacilli could only be found once in twenty cases. There is therefore great danger to human life when the nilk of tuberculous eows is made use of as food. No doubt innumerable cases of consumption are thus induced in the human being, particularly that form of tubereular disease tabes mesenterica, so common in young ehildren.

It must be partienlarly borne in mind by the veterinarian that, as a conservator of public health, he should at all times discountenance the consumption of the unboiled milk of tuberculous cattle not only by human beings, but by the lower animals, for it has been abundantly proved that the milk of a tuberenlous cow will, unless boiled, be dangerous to life; and when we reflect upon the fact that it is so largely consumed, partienlarly by infants and young children, we ean at least imagine the appalling consequences it may give rise to without being boiled.

The danger to human life from the ingestion of the milk of a 
tuberculous cow is greatly increased if the mammary gland be in a condition of inflammation, the seat of an abscess, or in a state of induration, as -in addition to the deficient nutritive value of all milk from tuberculous cows, which is blue in colour, thin in consistency, and in some cases contains cyanides-it will in such an instance be tainted with bacilli, and be a direct means of infecting the human being, particularly young and delicate infants. The boiling of milk and the thorough cooking of meat destroys with certainty the bacilli, and all suspected milk should be so treated. Let the veterinary surgeon, when called upon to give his opinion, consider the sacredness of his position, and-even if he chance to offend a client-do his duty towards his fellow-beings.

The majority of the veterinary profession are in favour of preventing the flesh of all tuberculous cattle-no matter what its condition and appearance may be-being used as human food; but I cannot support the wholesale condemnation of such flesh; and, whilst maintaining that such flesh, when properly dressed, and all tubercular growths with adherent flesh have been carefully removed, is fit for food, I must explain that if it present any appearance of being watery, pale, or otherwise unhealthy, I should not hesitate in at once expressing my conviction that it should not be used as food for man.

\section{TUBERCULOSIS OF THE HORSE.}

The horse is seldom affected with tuberculosis, and it is only seen in isolated cases, never attacking many animals in the same stable. An inquiry into the life history of tuberculous horses has enabled the author to discover that in several instances, where the history could be traced (the animals being still in the hands of the breeders), they had when foals been fed on cows' milk either after they had been taken from the mothers, or where the mare had been a bad mother or had died. In these cases the spleen and mesenteric glands were the seats of the lesions, which to a lesser extent involved the liver and bronchial glands.

As in cattle, the symptoms in the horse are generally very obscure, except in one particular, namely, that it is always associated with a more or less rapid emaciation. If the pulmonaryorgans are involved, there may be a cough, hurried respirations, particularly if excited by exercise or any other cause, and abnormal 
sounds may be heard in the chest on auscultation. If the contents of the abdomen are the seat of the disease, there may be frequent attaeks of eolic, with constipation or diarrhoca. Noeard has observed a very pronouneed diajetes insipiclus and irregular variations of temperature, but polyuria is not at all a constant symptom in my experience. Riemarkable to relate, all recorded instances in the horse have been isolated ones, though the animal may be one of a large stud. Is it possible that in the horse the virulence of the bacilli becomes destroyed, and the disease thus rendered non-infectious?

The tubereulin test may be applied to the horse as well as to eattle. In the majority of cases observed by the author, the spleen and mesenteric glands were the seat of the lesions; the liver, lungs, and bronchial glands being less frequently involved. In some instances the spleen has been enormously enlarged, and studded throughout its substance with grey translucent, yellow-cheesy tumours; whilst in the liver, lungs, and kidneys similar growths are seen, but are caseous in their eentres if truly tubercular. Those tumours found in these organs and consisting of white, round, firm sueculent tissue, invading the glomeruli of Malpighi and free from bacilli, are not to be considered tubereular; they are those of lympho-sarcoma, having some degree of organisation; whereas tubercle is incapable of a higher organisation than the grey nodule, is prone to rapid decay, retrogressive changes commeneing in the centre of the nodule, and, the caseous material being the product of the death of the cell elements, the surrounding growth is simply the hyperplasia of the normal tissue, due to the invasion of the bacilli; in fact, an effort to erect a barrier to the further inroad of the attacking bacilli.

\section{TUBERCULOSIS OF THE PIG}

Is much less frequently met with than in horned cattle, the average, according to statistics, varying from $1 \cdot 34$ to $1 \cdot 64$ per cent. in Saxony, a little more than 10 per eent. in Amsterdam, 04 at Roueu, .02 in the Duchy of Baden. In some districts it is never seen, and in Seotland it is so rare that at one time I was inclined to think the pig was immune. I have, however, observed it, and reported the results of the tuberculin test to the Royal College of Veterinary Surgeons; and in which one of the most prominent symptuns-not mentioned by authors-was an eruption of small 
tubercular nodules in the dermoid structures, invading the whole surface of the body.

Causes.-The ingestion of tubereular growths, or of tubercular milk. A tubereulous mother has, within my own knowledge, transmitted the disease to her offspring, and in some red Tamworth pigs of my own this was remarkably exemplified. A pregnant sow was sent to me from a celebrated breeder. In course of time she produced a litter, of which the majority dicl well, but shortly after the young pigs were weaned the sow beeame rapidly emaciated and was slaughtered; on post mortem she was found to have tuberculosis in an advanced stage, involving most of the organs.

One boar and one sow of this litter were kept, and after the sow became pregnant the boar was sold and not heard of again; the sow, a very large fine one, gave birth to eight or nine pigs, and shortly afterwards commenced to lose condition very rapidtly. An erythematous ermption appeared on the skin, and under the red spots hard nodules soon began to form; the young pigs throve for a short time, when some began to fall off rapidly and died tuberculous; the remaining apparently healthy ones were tested with tuberculin, and all reacted, and some time afterwards verified the value of the test, presenting signs of the eruption upon and nodules within the skin; rapid emaciation followed, and generalised tubereulosis was found in all. No cough was observed in these cases; the appetite remained fairly good for a time, it then became capricions, and sigus of indigestion became apparent, with constipation or diarrhoea. Pallor of mucous membranes, the tongue, oral cavity, and conjunctiva, as well as of the nose, was a prominent symptom in all the cases; the skin, in addition to the eruption, became very dirty, and the animals soon becane miserable objects, with sunken eyes and pendant belly.

The tuberculin test is the most reliable means of diagnosis, for in the absence of obvious symptoms of tuberculosis its detection becomes otherwise a matter of some difficulty.

Tuberculosis is seldom seen in the sheep or goat, but under certain conditions these animals are found not to be immune. Experimentally the disease has been transmitted, particularly by intravenous injection, which sets up an acute, generaliserl, miliary tuberculosis of a rapidly fatal type, and it is thought that prolonged cohabitation with tuberculous cattle gives the disease to both the sheep and the goat, although they, as a rule, 
resist subcutaneous and intraocular inoculation. The post nortem examination of these animals reveals invasion of the lungs, bronchial, mesenteric, and other glands, spleen, \&c., and when there is a discharge from the nose the bacilli can be found in abundance.

Care must be taken that actinomycosis, which is not a rare disease in the goat, be not mistaken for tuberculosis.

\section{TUBERCULOSIS OF THE DOG.}

Though by no means frequently affected, the dog is sometimes the subject of tuberculosis. In this animal the disease usually assumes the acute miliary or generalised form. A case recently occurred in the practice of the author. A fox terrier had been ill for three or four weeks and was bronght to the College. It died three days after admission. On post mortem examination nearly every organ was found to be affected with tubercular neoplasms. They appeared in great numbers on the surface of the pleura, peritoneum, and diaphragm. In the peritoneal covering of the bladder, in the substance of the lidney, and in the liver they showed as minute pearly points, while in the lung their confluence had formed nodes and excavations of various sizes. The heart was studded with nodules, a large one being placed on one of the cusps of the mitral valve. On the base of the heart and bordered by the pulmonary artery was an elongated tubercular abscess containing a couple of tablespoonfuls of pus. The spleen was apparently unaffected. The brain and testes were unfortunately not examined.

Cats are more frequently affected than dogs; indeed it is little suspected what a common cause of death consumption is in these animals. The discase in them also often appears as a generalised tuberculosis; sometimes, however, the abdominal viscera and membranes are affected to the exclusion of other parts. Then chains of tumefied glands can be scen along the course of the large intestines, and one or several large tubercular glands in the mesentery. They are extremely hard--cartilaginous in fact. In the liver, kidney, and spleen the tubercle follicles are as a rule quite small, and appear in sections as a round-celled infiltration, containing, especially in the liver, where they affect the portal space for the most part, beautiful examples of giant cells with their brightly shining zone of nuclei. When in this animal the lung is affected the formation of vomica is quite common. 
Tuberculosis of Birds (Tubcrculosis avium).--It is maintained by some and contradicted by other writers that the tuberculosis of birds differ in some essentials from that of mammals. Thus Rivolta, Strauss, Mafucii, and other experimentalists have failed to transmit the disease from mammals to birds, whilst Cadiot, Albert, Nocard, and others state that the inoculation is occasionally successful, and that the disease can be transmitted from one to another; and Nocard says: "It is true that the lesions observed in the guinea-pig as the result of the inoculation of Tuberculosis avium are widely different, nancely, large, red, and soft spleen, absence generally of tubercular nodules in the lungs and liver, but the latter as well as the spleen are crowded with tubercular follicles rich in bacilli, and a few transmissions from guinea-pig to guinea-pig are all that is necessary to enable these lesions to reproduce the type so well described by Villemin."

Lastly, the same writer says: "It must be remembered that the tubercle bacillus so resistant to all causes of destruction may, however, experience profound modifications by means of successive passages through the organs of divers species of animals. But if the modifications which it undergoes as the result of numerons transmissions through birds are profound enough to make the bacillus of Tubcrculosis avium a peculiar variety of the bacillus of Koch, they are not enough, in my opinion, to make these bacilli two distinct species."

The avian bacillus is longer than that of mammals, grows more rapidly and more readily on cultivation media, appearing as thick, moist, luxuriant spots, which preserve their vitality longer than those from man. They grow at a higher temperature $-109^{\circ} \mathrm{F}$ - - whilst those of the marmmal cease to grow at $104^{\circ} \mathrm{F}$.

'lo prevent the spread of avian tuberculosis it will be necessary to kill all the birds in the infected yard; the diseased ones being cremated or buried deeply in the gromnd, their bodies surrouided by a layer of some antiseptic, the healthy birds of course being disposed of for food as quickly as possible. All the wood-rork of the hen-houses should be destroyed, the walls scraped and whitewashed, the wash to contain carbolic acid, the excrement removed and burnt with the wood-work, and on no account should birds from an infected run be sold except for slanghter. 


\section{TREATMENT OF BOVINE TUBERCULOSIS.}

If the disease has passed beyond the very earliest stage, it is a waste of time and money to treat animals suffering from tubercular consumption. It is far better to slaughter and make the best of them; and in all cases it is better to make the animals fit for the butcher by ceasing to milk them, giving fattening food, such as oil-cake and good hay, avoiding grasses and roots, as they tend to produce indigestion, diarrhoea, and an acid condition of the digestive apparatus, and by administering cod liver oil in such quantities as the animal may digest and assimilate-say from six ounces to half a pint daily; if purgation is not induced, the latter quantity. The oil is best given mixed with lime water, and small doses of oil of turpentine may also be added with advantage, particularly if there be a tendency to indigestion, tympanites, or diarrhœea.

The following curative or preventive agents have been recommended,-iodine (Williams), mercuric chloride (Herroun), eucalyptus oil (M. Ball), salicylic acid (Griffiths), creasote (various), inhalation of turpentine, hydrofluoric acid, \&c. (various), but so far these have proved uncertain; paraffin has also been recommended, but the results do not warrant the continuance of any medicinal treatment.

I think the time has arrived when tubercular diseases ought to be included in the Contagious Diseases (Animals) Act, and that an endeavour should be made to prevent its increasing frequency, even if it be found impossible to diminish it, or stamp it out altogether.

\section{STRUMOUS ABSCESSES.}

In the Provisional Nomenclature of Diseases adopted by the Royal College of Physicians, London, tubercular diseases are considered under two heads, namely-(1.) Scrofula with tubercle, sometimes a concomitant of internal tuberculosis; and (2.) Scrofula without tubercle. This latter condition is witnessed in the sheep, in which the true tubercular nodule is rarely developed. This ruminant, however, is prone to suffer from strumous scrofulous abscesses in the submaxilliary, facial, and parotidean regions, sometimes commencing in the connective tissue about the jaws, neck, and face, and involving the lymphatic glands (strumous adenitis). 
These abscesses often appear, without any premonitory signs of ill health, as hard swellings, which sooner or later suppurate imperfectly, or burrow deeply in all directions; at first they are not painful, but often become very much so, and may multiply rapidly, appearing between the jaws, on the neck, about the eyes, lips, and nostrils, and occasionally upon other parts of the body, causing swelling, preventing the sheep from feeding, and rendering the breathing difficult, and snoring. In some instances swelling of the face and discharge from the nose are observed previous to the development of the tumours.

Young animals, particularly young fast-growing tups, if exposed to cold, are especially subject to this disease, but older animals of both sexes, as well as lambs, are not exempt.

If these abscesses are opened, the quantity of pus contained within them, not always commensurate with their size, is generally thick, and flows tardily, but afterwards becomes thin, and more or less ichorous; it is surrounded by a thick sac of low filurous material, like the walls of an old ulcer, and simuses are often found ruming from the cysts into the surrounding structures. Microscopically examined, the pus is found to consist of ordinary pus cells, rather shrivelled in appearance, but showing little or no tendency to caseation, and the tubercular bacilli are absent, but the pus may be loaded with the cocci of suppuration. In many instances the tumours undergo little or no change for two or three year's, the sheep seemingly suffering no inconvenience; but in other eases the animal loses flesh rapidly, the wool falls off in patches, symptoms of fever manifest theniselves, a cough is now and then heard, and the sufferer dies from exhaustion, anæmia, and sometimes dropsy. The flesh in this advaneed stage is pale and watery, but if an animal be slaughtered in the earlier stages it is fairly good.

The post mortem appearances are often quite local, inerely collections of pus in the various thick-waller abscesses observable before death; the pus will, however, be found to have burrowed from the alsseesses in various directions into the surrounding tissues. Now and then some degree of ulceration of the nasal mucous membrane may be detected, and abscesses found in the lungs, but these two latter conditions are by no means constant.

Strumous abseesses in sheep, or the conditions which lead to their formation, do not seem to be hereditary, as the stock 
of many tups affected remain quite free from the disease, provided they are not exposed to that cause-namely, coldwhich induces it in the progeny of those which have never been affected.

Treatment.-The cause or causes being removed, it will be necessary to open the abscesses as soon as pus can be detected in them, a matter of some difficulty, as their walls are very firm and unyielding; however, if by firm pressure with one hand, and manipulation of the tumour with the other, the pus can be felt fluctuating slightly, the operator need not hesitate, but make a bold incision, press out the semi-fluid matter, and dress the wound with some digestive, such as turpentine liniment. The parts are to be kept clean, dressed antiseptically daily; the wounds prevented from closing too quickly, and the animal fed liberally. A small quantity of sulphate of iron in the food often assists the recovery. A varying degree of swelling may exist for a time after the wounds have healed.

Abscesses about the throat in cattle are often supposed to be tubercular, when in reality they are due to the actinomyces. -(See Actinomycosis).

\section{PREPARATION OF TUBERCULIN.}

For diagnostic purposes this preparation of the products of the development of the bacillus in culture media has proved of incalculable value, experiments having been made by scientists in almost all parts of the world, and with the almost invariable result of inducing a reaction in diseased animals only, healthy ones, or those free from tuberculosis, remaining unaffected.

A pure culture of tubercle bacillus is inoculated into broth containing five per cent. of glycerine, and this is kept at a temperature of about $98^{\circ} \mathrm{F}$. in an inculator for six weeks. The broth, now teeming with bacilli and rich in their products, is sterilised by heating to $230^{\circ} \mathrm{F}$. in the steam steriliser. This kills the bacilli, while their products remain unaltered. It is now evaporated until one-tenth only of the original volume remains, and is filterect.

METHOD OF INOCULATION $\triangle$ ND EFFECTS OF TUBERCULIN.

Upon the discovery of tuberculosis in a herd, all animals in 
contact should be examined, the temperature of the animals to be operated upon should be taken and carefully noted for a day or two before the inoculations are made. The seat of operation should be the lower parts of the neck or behind the elbow. The part is to be well washed with a five per cent. solution of carbolic acid and the syringe disinfected with the same solution.

Taking up a fold of the skin, the needle is pushed into the loose subcutaneous tissue, which in these positions is abundant, and about 40 minims of the tuberculin injected. The temperature is to be taken ten, twelve, fifteen, eighteen, and twenty-one hours after the injection. The following are conclusions arrived at after very extensive trials, and published in the "Annales of the Pasteur Institute" :-

1. Tuberculin has a specific action on tuberculous bovine animals, which is expressed by a considerable rise in temperature.

2. The injection of a strong dose gives rise in tuberculous animals to an elevation of temperature of between 2 to 5 degrees Fahrenheit.

3. The same dose injected into non-tuberculous animals does not ordinarily produce any appreciable febrile reaction.

4. The febrile reaction appears most frequently between twelve and fifteen hours after the injection, sometimes at the ninth hour, very rarely after eighteen hours: it always lasts several hour's.

5. The duration and the intensity of the reaction are not related to the number and the gravity of the lesions; it seems even that the reaction may be more decisive in the case where, the lesion being very limited, the animal has conserved the appearances of health.

6. In the subjects gravely affected by tubercle, and especially in those which are fevered, the reaction may be little marked or absolutely wanting.

7. It is prudent to take the temperature of animals morning and evening for several days before the injcetion, because it may happen that from trifling and temporary affections, congestion and the like, there may be fluctuations of temperature productive of serious error. For these animals it is necessary to put off the injection. 
8. In certain tuberculous auimals, not fevered, the reaction consecutive to the injection of tuberculin does not reach more than $1.4^{\circ}$; still, as experience shows that in perfectly healthy animals the temperature may undergo variations of $1.4^{\circ}$ or more, only reactions above $2.4^{\circ}$ should be allowed to have any real diagnostic value. All animals in which the injection is followed by a rise of $1 \frac{1}{2}^{\circ}$ to $2 \frac{1}{2}^{\circ}$ should be considered as suspect, and ought to undergo a new injection after the lapse of about a month.

Crookshanks and Herroun have separated a ptomaine and an albumose from the crude glycerine extract of cultures, which, when ejected hypodermically into tubercular guinea-pigs, induces a rise of temperature, and its effect on tubercular glands in the cases associated with rise of temperature was to render them well defined, indurated, and painful. Other experiments have been made to determine the action of the amide group of organic substances upon the economy in health and in tuberculosis. In a report by Professor Samuel G. Dixon, M.D., and W. L. Zuill, M.D.D.V.S., Academy of Natural Science, Philadelphia, it is stated that, in endeavouring to discover the true nature of the active principle of tuberculin, a crystalline substance was produced that at once suggested the amide group,--allantoin, glycocin, tyrosin, kreatin and kreatinin, taurin, cystin, \&c. They determined to make use of kreatin, which was at hand, and injected a small quantity into tuberculous and healthy small animals, and afterwards by Zuill into cattle, with results resembling those obtained by tuberculin. Zuill states that the action of kreatin upon tubercular tissue is intensely energetic, causing its rapid necrosis, giving it the appearance of having undergone a cystic degeneration. Experiments with taurin also caused elevation of temperature.

Mr. George N. Kinnell, M.R.C.V.S., Pittsfield, Mass., reports, 1895 , that more definite results are obtained by using smaller doses of tuberculin. He says: "By a smaller dose I mean two-thirds of a minim; a large dose, the ordinary one from three to five minims-of Libbertz's Tuberculinum Kochii. The small doses will not cause reaction in the earlier stages, or where the disease is but slightly advauced, but will do so in 
advanced cases where a large dose has failed to cause elevation of temperature."

\section{SUGGESTIONS FOR SUPPRESSION OF TUBERCULOSIS.}

1st. For dealing with Non-Breeding Cattle.-When tuberculosis is suspected in an animal, it and all in-contact cattle should be subjected to the tuberculin test, and that all in which the reaction followed, if in good condition, be slaughtered, and the beef, if found fit after careful examination by a duly qualified veterinary inspector, be sold for human consumption. That the animal be valued prior to slaughter, and that the difference between the value of a milch cow and as beef be made $\eta p$ by the Government as in pleuro-pneumonia, or perhaps divided between Government and owner.

That all piners-wasters-reacting to the test, should be slaughtered and their carcases at once destroyed, no compensation being given, and that neglect to report such cases to the proper authority be considered an indictable offence.

That all animals in which the tuberculin reaction indicated that they were affected, but apparently showing no signs of ill-health, if not fit for slaughter, should be prepared for the butcher and slaughtered when fit, being in the meantime removed and isolated from those in which no reaction had taken place. That the healthy stock be again tested and the same rules applied.

2d. For dealing with Brceding Stock:-In dealing with breeding stock, I can do no better than quote from Nocard, who says: "All those engaged in agriculture, in breeding, rearing, feeding, or fattening, ought to carry out each for himself the prophylaxis of the disease. Each of them is directly interested in it. The methodical use of tuberculin, by denouncing the sick animals at the outset of the disease, permits one to isolate them, and to protect the sound animals from all danger of contamination.

As the young animals mostly escape the infection, breeding would not be seriously interfered with, and the vacant spaces would be filled in a few years. Of course, a farm once made healthy ought to be protected from re-infection. To effect this it would be sufficient to introduce into it no new aninals without having them previously tested with tuberculin. 
Professor Bang of Copenhagen has a similar scheme, and has, according to his last report, carried it to a successful issue. $\mathrm{He}$ proceeds as follows :-

The whole herd is tested with tuberculin, and thus divided into a healthy and a tuberculons section, which are separated from one another, and have separate attendants. The healthy section is tested every six months with tuberculin, and any animals which react are at once removed to the tuberculous section. Those animals of the tuberculous section which are obviously affected are got rid of ; but those which are apparently healthy are kept and used for breeding purposes as long as may be convenient, and as they will generally be fattened for slaughter before the disease is far advanced, the total condemnation of their carcases as butchers' meat will not, as a r'ule, be necessary. The calves of the tubercular section are removed to the healthy section immediately after birth, and are fed for the first day on colostrum, which has been heated to $65^{\circ}$ C.$149^{\circ}$ Fahr.-and subsequently on boiled milk, this boiled milk being from tuberculous cows.

At first these calves were kept in separate boxes, and only added to the sound section when they had snceessfully undergone the tuberculin test, but he now thinks that they may with safuty be removed to the sound section immediately after birth, and wait till the time of the general half-yearly testing with tuberculin.

In carrying out the ahove, I think the Govermment might safely devote an anmual sum of money to assist cattle-owners in suppressing so great and increasing an evil as tuberculosis; and, being of opinion that the schemes are tangible and reasonable, I would now suggest that tuberculosis be included in the statutory schedule of infectious diseases, and that compensation be paid to the owners of cattle affected with tuberculosis when such cattle are slaughtered in the public interest. 


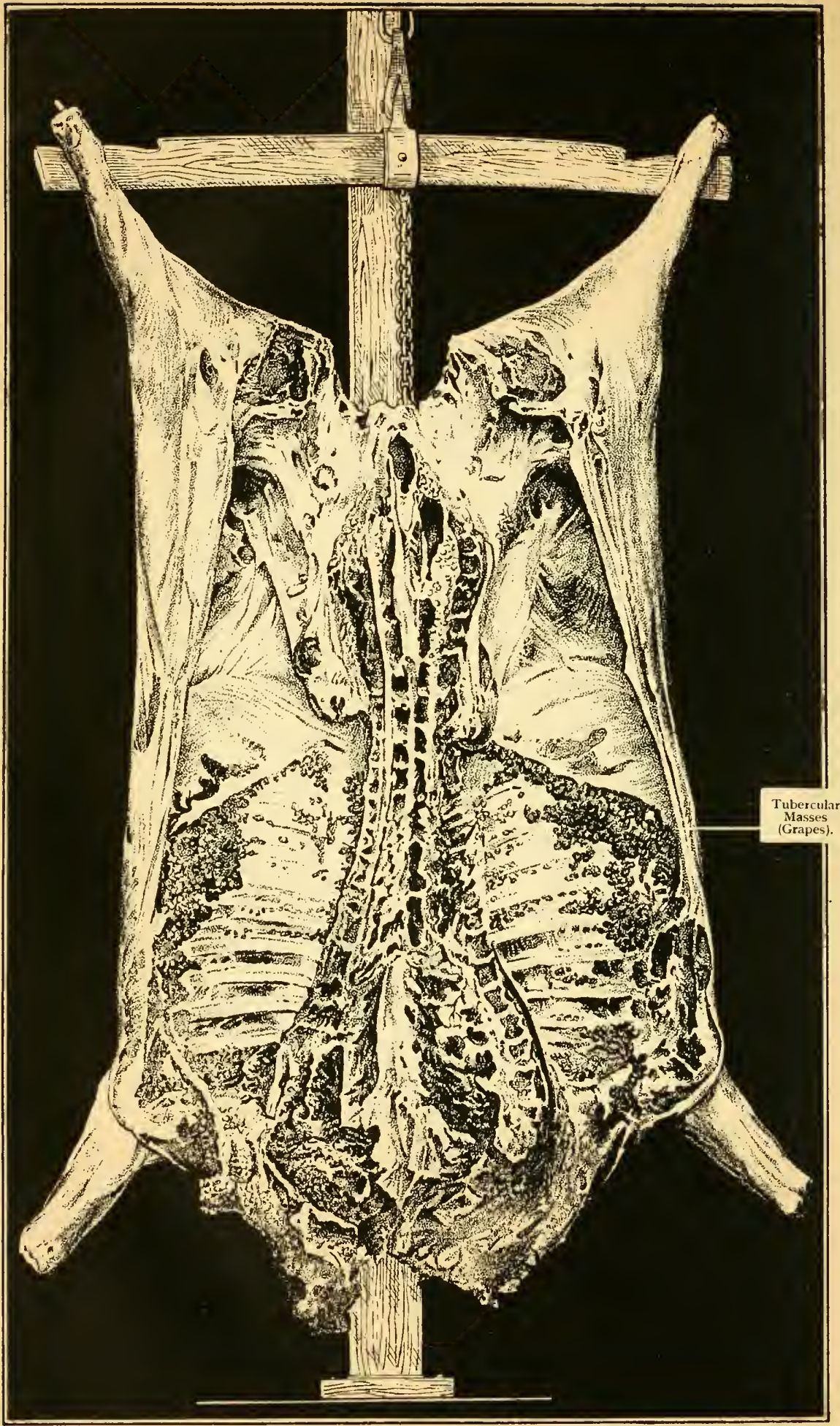

Fig. 17.-Tuberculosis Bovis. 


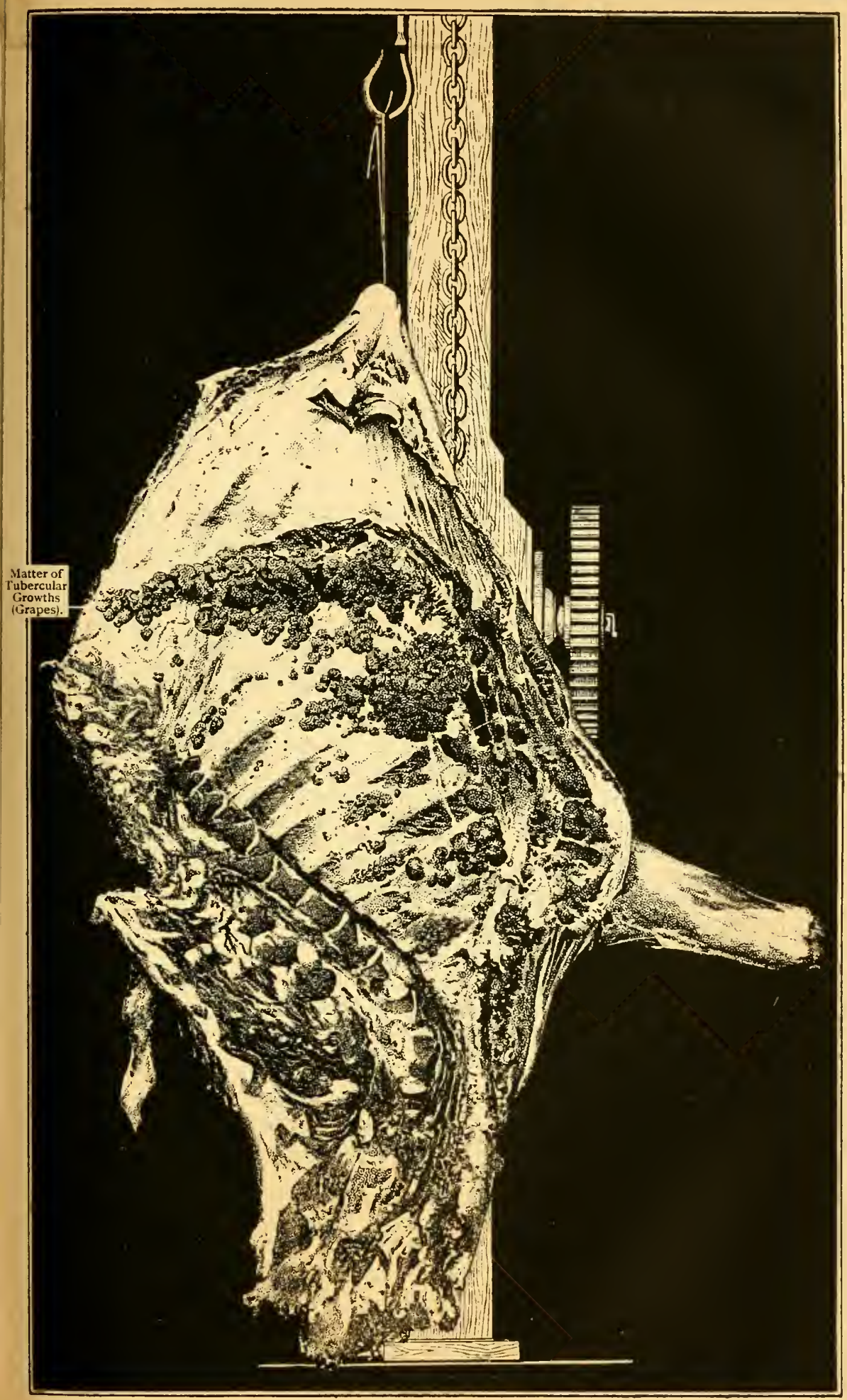




\section{( ' H A P'l E R X X I.}

ENZOOTIC AND EPIZOOTIC DISEASES.

\section{INFLUENZA.}

UNDER this term is inchuded three diseases, which every now and then prevail as epizootics in this and other countries, and which have been indiscriminately denominated fever of the horse, epizootis of the horse, nervous fever, putrid fever, infectious pnemmonia, typhus, \&c.

Falke, however, has reduced these to two conditions recognised under the term influenza,-namely, the red disease, for which he has reserved the term influenza, and the other form he has named typhus. Whilst giving due credit to Falle for endeavouring to abolish the mystification concerning influenza, I feel constrained to state that under the term I can recognise three different morbid conditions, namely-(1) Epizootic catarrhal fever: (2) Epizootic cellulitis or pink-eye; and (3) Epizootic pneumonia,-all being due to miasmatic or malarial-sapremic-organisms generally existent in large horse establishments, and liable to canse epizootic outbreaks under certain alterations of external surroundings, which seem to determine either the receptivity of animals subjected to their action, or a transformation of the vital properties of the microbes. For example, the disease may have been absent in a stable for a period of time, or until fresh animals are introduced. Then there is an outbreak of the malady amongst the freshly introduced animals, and from which it spreads indefinitely amongst those which have had immediate or mediate contact. Here I think we have an example of the action of a non-contagions facultative parasite becoming transformed during its passage through the animal body into a contagious parasitic microbe, and which at first is thus increased in virulence, but after a period of time the 
intensity of this virulence seems to become exhausted, and the microbe again is transformed to its original miasmatic nature, often to remain dormant until some cause is brought into operation by which its morbific effects are again reproduced.

\section{1st.-PANZOOTIC CATARRHAL FEVER OR INFLUENZA.}

An epizootic febrile disease attended with great prostration of strength, and with early inflammation of the nasal, laryngeal, and sometimes bronchial mucous membrane, complicated with irritability of the digestive nucous membrane. Occasionally the disease implicates the substance of the lungs, pleura, liver, the fibrous structures of the articulations, thecr of muscles and tendons, and the connective tissue of various parts of the body.

Synonyms.-Distemper; epiclenic catarrh ; epizootic catarrhal fever; (F.) courbuture, morfondure; (L.) febris catarrhalis, defluxio catarrhalis, \&c. The disease was first called influenza in Italy in the seventeenth century, because it was attributed to the influence of the stars.

Panzootic catarrhal fever or influenza has a very early history, but to trace this would be beyond the purpose of this work. In 1299 it appeared in Seville, and is referred to by the veterinarians Martin Arrendondo and Fernando Calvo, who derived their information from Laurentius Rusius. It killed more than one thonsand horses and seemed to be incurable.

"In 1648 an epizootic broke out amongst the horses of the French army in Gernany, and is described by Solleysell. It began by fever, great prostration, and tears running from the eyes, and there was an abundant discharge of a greenish colour from the nostrils."-(FLEMing.) In 1688 influenza was prevalent over the whole of Europe, affecting both men and horses. In 1699 Europe and America suffered from the disease, and again in 1732 it prevailed in both hemispheres. In 1767 it once more appeared in both hemispheres, and it is recorded that both horses and dogs were liable to its attacks. In 1776 , after a very severe winter and warm summer, with an earthquake in Wales, influenza spread over Europe, attacking horses and dogs first, and human beings after. Poultry died in great numbers of an epizootic with defluxion from the eyes. This epizootic was observed in Edinburgh in December, and in England at the commencement of January 
1788. Influenza was very severe in New York, and cansed great mortality amongst the horses of Maryland. During the present century the disease has raged with varying degrees of virulence in 1849-50, 1863-4; and the last outbreaks we have to record are-

Influenzc in Britcin in 1871-72.-This outbreak was mostly confined to the English metropolis; it was of a severe but not fatal type; in 1891-92 it was general all over Great Britain.

The American Horse Discase, 1872-73.-This disease broke out in Toronto, Canada, on October 1st, 1872. In nine days it had attacked nearly all the horses in the eity, and carriages could not be had for any price. On October 18 th it had reached Montreal, and was prevalent throughout Canada. On 14th October it had reached Buffalo; 17th, Rochester; 22d, Boston, New York, Brooklyn, and Jersey City ; 27th, Philadelphia ; 28th, Washington. It made its appearance in Nova Scotia on October 13 th.

Mr. James Law warmly advocates the contagious origin of this epizootic. Mr. Greene, M.R.C.V.S., St. John's, N.B., records the following inportant fact:- "I was always under the impression that influenza was both contagious and infectious till the late outbreak; since then I have altered my views with regard to the contagion and infection of that disease. One among several facts which I could mention will bear me ont in this question.

"During the month of July 1872 a horse had been put to grass on Partridge Island, in the Bay of Fundy. This island is three miles distant from this city. No other horse had been near the island from the date of his landing up to the time of the outbreak in St. John's, N.B., and on the 15th or 16 th of October, which was only two or three days after the first case was reported in this city, the horse on the island was affected with the most violent form of the epizootic.

"Would not the morbific matter have become diluted to such an extent (after travelling three miles) as to be inert?"(Veterinarian, April 1873.)

\section{ETIOLOGY.}

The microbe cause of influenza is not determined. The disease may occur spontaneously in various places, but its first source of origin cannot be indicated. It does not depend upon any known condition of the atmosphere, nor upon soil, seasons, or temperature. It prevails on every soil and geological for- 
mation, sometimes more so in low-lying districts than on the hills. It may appear at all times of the year; perhaps it is more commonly seen in autumn, winter, and spring than in summer. Very often, however, its worst form is seen during the hot summer months. Influenza has often appeared in foggy weather, but ontbreaks have occurred withont such a coincidence, and consequently no weight can be attached to this circunstance.

Its spread is not influenced by the wind: sometimes it moves against it.

"The outbreak of 1766 and the American one of 1872 succeeded to two very similar climatic conditions. The winters had been exceedingly severe and the summers unusually hot, and earthquakes had been frequent."-(LAw.)

Similar climatic conditions have, however, not been followed by an outbreak of influenza; and as in the case of fogs, mildews, \&c., the coincidence can only be looked upon as accidental.

Sudden changes of temperature appear to assist the development of the influenza poison, and exposure to cold predisposes the animal to the disease, but neither of these causes is sufficient of itself to produce it.

Ozone in undue quantity in the atmosphere has been supposed to be a cause of influenza. This is, however, highly improbable, as ozone, although an irritant to the mucous membrance of the nose when applied in large quantities, has no effect on the nervous system like the influenza poison.

Predisposing causes.-Animals crowded together in damp, illventilated, and otherwise unhealthy situations, are generally the first to suffer from influenza. In them it commits its greatest havoc. Young horses are more predisposed than those of maturer years; still, the old suffer severely, and are often carried away. Sex has no influence. Neglect of every description, as well as bad food and over-work, by debilitating, render animals subject to severe and early attacks of disease. But no amount of care will exempt them from it, as it appears in the stable of the nobleman as well as that of the poorest carter. The latter, however, experiences it in its greatest intensity, and at a much earlier period than the former.

\section{PATHOLOGY.}

The morbific agent absorbed into the blood gives rise to febrile disturbance and depression of the nervous centres. The 
period of latency, between the reception of the poison and the first manifestation of 'symptoms, is short but uncertain. To the febrile symptoms succeed those of the specific effect of the poison upon the murous membrane of the nose, eyes, throat, and respiratory track, as well as the intestinal mucous membrane and its ramifications in the biliary tubes. In some cases, the genito-urinal mucous membrane may participate in the inflammation, and in the majority there is more or less sympathetic or actual irritation of all the mucous membranes of the animal body.

In the majority of instances the fever precedes the catarrhal symptoms; the rigors, increased frequency of pulse, and elevation of temperature to $103^{\circ}$ or $104^{\circ}$ Fahrenheit, being observable prior to the appearance of any localization of the disorder. In other instances the irritation of the mucous membrane has been the first appreciable sign.

In uncomplicated cases the fever begins to abate in from three to five days, leaving the animal weak and prostrate.

It cannot be said that the fever terminates in a critical discharge, as in the human being. All that can be observed is that, coincident with the abatement of the fever, the secretions of the body become natural or slightly increased ; the critical sweat, diuresis, or diarrhœa so generally observed in the human being, are usually absent in the horse.

Complications.-The catarrhal form is the one to be regarded as the simple or uncomplicated; to this is added, in some instances, pulmonic, gastro-enteric, hepatic, and rheunatic complications, peri- or endo-carditis, the formation of thrombi, and nervous symptoms, as convulsive fits and coma.

Symptoms of the catarrhal form.-Actual rigor may or may not precede the other symptoms; very possibly the rigor is not observed. There is a dry, staring cont, the legs, ears, and nose are cold, with redness and dryness of the Schneiderian membrane, and an elevation of temperature to $103-4-5-7^{\circ} \mathrm{F}$. or higher. There is sneezing, a hacking cough, and shortly a defluxion, from one or both nostrils, of at first a thin, more or less acrid mucus. The eyes are lieavy, conjunctivæ injected and sometimes yellow, and tears flow over the face. The symptoms increase in intensity for two or even three days. The discharge from the nose becomes flaky and more profuse. In some cases the conjunctiva of one or both eyes is actually inflamed, as marked by opacity 
and imperfect vision or actual blindness, and there is partial or entire loss of appetite. The cough now becomes deep, sonorous, painful, and paroxysmal, convulsing the whole body, occasioning impatience, stamping of the feet, and great distress. The temperature of the surface of the body and extremities is very variable, sometimes high, sometimes low, or one leg hot, three legs cold, and vice versa. The pulse is feeble and easily compressed, numbering generally from sixty to eighty beats per minute. The mouth is hot and clammy, and the desire for water is intensified. The head is generally depressed. The animal is made to move with difficulty, and when compelled, he does so in an unsteady manner, swaying from side to side as if partially paralyzed. This muscular debility is much increased where there is great soreness of the throat and inability to swallow food or drink.

Soreness of the throat is indicated by "quidding of the food" (that is to say, the food is chewed and ejected from the mouth), or by its return through the nostrils. In the latter case an attempt to swallow excites a violent fit of coughing, and the food imprisoned behind the velum pendulum palati is forced into the nasal chambers. It there tinges the mucus discharges with its own colour; and when the horse is fed on grass the nasal discharge is thus turned green-a canse of great fear to some people, who at once conclude that the green discharge indicates something mysterious.

The conditions of the secretions.- In the earlier stages the fæces are dry, hard, pellety, and often covered with mucus; the urine high-coloured and scanty, sometimes tinged with bile, sometimes opaque, and sometimes of the consistence and appearance of linseed oil, containing albumen, or loaded with urea and hippuric acid.

In a period of time varying from three to five days the symptoms begin to abate in intensity. The discharge from the nose becomes thick, yellow, and profuse; the cough looser, moist, and not so paroxysmal; the pulse graclually falls in number and improves in tone; the action of the heart is less jerky; the appetite improves; the throat can now be handled without causing the animal to cough. Any swelling of the throat which might have been present gradually subsides, and strength is generally restored in from twelve to fifteen days. 
The leading character of the disease consists of an inflammation of the naso-pharyngeal mucous membrane; it also gives to the secretory organs a tendency to participate in the disease. Thus it is not at all uncommon for the submaxillary and parotid glands to become inflamed, swollen, and even to suppurate. When the critical stage has passed, the debility is generally very extreme, and the loss of flesh most marked. Indeed, it is a common observation that a horse in which the fever has abated and convalescence commenced, "looks as if the flesh has melted off his bones."

Owing to the debility of the circulation, dropsical swellings appear on the belly, legs, and chest. These, however, are not to be regarded in any way as serious, for as the animal regains its strength they will rapidly disappear.

In many instances, more especially in those which have been neglected or maltreated in the earlier stages, bronchitis is apt to supervene, and its accession is indicated by the congh becoming more paroxysmal, and the difficulty of breathing (dyspnœa) at first quite disproportionate to the other physical signs; the flanks heave, and the nostrils are widely dilated; in many instances the horse is said to fight for breath. The pulse, at first perhaps no quicker than the respiratory movements, which may be as frequent as 60 or 70 per minute, becomes very rapid-90, 100, or more beats per minute.

The auscultatory sounds are, roughness of the inspiratory murmur, best heard at the lower part of the trachea, and immediately behind the scapula, or the sibilant rale heard over the greater part of the chest. The first indicates bronchitis, involving the larger bronchi, the second inflammation of the smaller tubes.

The visible mucous membranes become livid, dark purple, or leaden in colour, the animal is semi-torpid from the action of non-oxidized blood on the brain and medulla oblongata. The discharge from the nose differs from that seen in the uncomplicated form, being scanty, and sometimes streaked with blood, whilst in other cases dark-coloured blood, extravasated from the engorged bronchial blood-vessels, will issue from the nostrils.

The pulse now falters, cold sweats appear on the body, and the animal sinks on the sixth, seventl, or eighth day.

The bronchitis or broncho-pneumonia of influenza is distinguished from other chest affections by the greater difficulty of breathing, by the respiratory movements being relatively more 
disturbed than the action of the heart, by the lividity, of the mucous membranes, and by the semi-comatose condition of the affected animal.

The pneumonic of influenza is characterised hy a soft, dull cough, by rapidity of the pulse, great coldness of the extremities and surface of the body, by crepitation on auscultation and dulness on percussion of the lower parts of the chest, the post mortem revealing desquamation and proliferation of the bronchial epithelium, the presence of catarrhal products in the tubes and vesicles, and by effusion of serosity, mostly confined to the lower portions of the lungs. In consequence of the asthenic nature of the inflammation there is a tendency to gangrene and disintegration of the lung tissue. This termination-a rare one it must be admitted-is expressed by foetor of the breath, sweats over the body, a faltering and excessively feeble pulse, rapid emaciation, looseness of the hair of the mane and tail, rapid sinking, and death.

Panzootic catarrhal fever is not very frequently complicated with plenritis, although, during some seasons, the latter disease rages as an epizootic.

Abdominal or cntcric complications. - In all catarrhal affections there is great irritability of the gastro-enteric mucous membrane, hence all teachers of experience warn their students to be careful in administering purgatives. Succeeding to the naso-laryngeal symptoms, those of abdominal pain will become manifest, the animal frequently looking at its flanks, alternately lying down and rising, kicking at its belly, and frequently passing small quantities of hardened freces, thickly coated and mixed with mucus. There is great debility, and some degree of torpor or somnolence; in some instances the splincter ani is relaxed, and the anus constantly open, the animal straining incessantly, and exposing the intestinal mucous membrane of a deep red colour. The conjunctivæ are yellow; the tongue is coated, dry, and shrunk; the animal is thirsty, and the urine is highcoloured and scanty. Great care must be taken in properly diagnosing this condition, for, if treated as ordinary colic, the result is almost sure to be fatal.

Towards the termination of influenza rhcumatic complications often appear; in some instances pain and stiffness in the joints appear early in the catarrhal form. Generally, however, rheuwuatism manifests itself towards the termination of the other symptoms. The animal becomes at first restless and uneasy, lifting its feet alternately from the ground; the joints emit a 
crackling sound when moved, then swell, after which the pain subsides. In other instances the rheumatic infiammation appears in some particular musele, or more frequently tendon, especially the great flexors of the feet. The swelling and inflammation are generally situated immediately below the knee or hock, involving the flexors-perforans and perforatus. The swelling is very great, hot, painful, hard, and causing severe lamenoss, which sometimes remains for a long time after the animal has regained its health in other respects.

Few horses suffer from more than one attack during one season; many cases relapse, but one attack in no degree protects against another at some future period.

Prognosis.-If properly treated influenza is rarely fatal; the old, bad constitutioned, and over-worked horses only succumbing.

\section{TREATMENT.}

The practitioner is always to bear in mind that influenza is a disease which runs a definite course, and that it is in noway cut short by any interference. The treatment must consequently be directed to relieve any distressing symptom, to allay irritability, and to support the strength.

Comfortable clothing to the body and extremities; food, consisting of warm mashes of bran, boiled oats, linseed, or barley, and an abundance of good cold water for the animal to drink, are always to be recommended. It is good practice to see that the water is so placed that the animal can get at it at its pleasure, for in many instances, if it is not thirsty, it will wash its mouth and cool its tongue, and feel grateful for the relief thus afforded. Inlaalations of warm water vapour, continued for an hour at a time, afford much relief, frequently mature the nasal discharges, and relieve the cough. The throat may be fomented with hot water and stimulated with an embrocation consisting of ammonia and oil, or at onee blistered with the eantharides ointment. Mustard applications are very commonly used. I must confess that I never use mustard, for it eauses very much distress, withont securing any marked abatement of the disease. If there be much prostration, doses of spirits of nitrous ether, or of the carbonate of ammonia, may be given in cold water two or three times a day; if the debility is not marked, nitrate of potash or sod. hyposulph. in the enteric form, dissolved in the animal's drink or mixed with its mash. Purgatives are 
always to be avoided, and any constipation which may be present in the earlier stages of the disease is best relieved by enemas of warm water. Should diarrhœa spontaneously occur it must not be checked, at least if not excessive. Any irregularity of the bowels should be overcome more by food than by medicines. If costive, a laxative diet is to be given; if the reverse, it may be advisable to give dry food.

If the appetite does not return in the course of the third or fourth day, milk should be substituted for water, and if the animal likes it, it should be allowed to drink of it abundantlysay three or four gallons per day. Skimmed milk suits better than unskimmed, as the latter is apt to induce some degree of diarrhea. If at any time the milk disagrees, and it will sometimes do so, it must be discontinued and gruel substituted. Neither milk nor gruel should be forced upon the animal by horning or bottling it down its throat in spite of its mute and often determined resistance; and it is a point worthy of remembrance that food thus forced is only calculated to disorder the digestive organs, and destroy all chance of a return of appetite. In the human being, dog, \&c., food thus forced would be vomited, the stomach would at once be able to express its rebellion, but in the horse vomition rarely occurs; and who knows what unpleasantness or even pain is inflicted by thus disordering the already delicate digestion, by the common, but to my mind barbarous, practice of forcing sundry quarts of gruel, beer, \&c. upon its unwilling stomach.

In mild attacks of influenza but little medical treatment is necessary; pure air, comfortable loose box, without draughts, well-drained and well-ventilated, with careful nursing and careful daily medical inspection, and the saline medicine above recommended, are all that is necessary. When convalescence has commenced, the restoration is materially assisted by a few doses of, first, vegetable, and, secondly, mineral tonics, good food, carefully regulated exercise, and careful grooming. If any cough remain, it may be necessary to apply a blister to the throat, and to administer such remedies as belladonna extract and camphor; and should the swelling of the limbs continue, the kidneys are to be stimulated by diuretics.

In some rare instances the local inflammation of the larynx is very great, and the tumefaction of the mucous membrane, vocal chords, \&c., interferes with the act of respiration. When this 
occurs the horse's nose is "poked out," that is to say, the face is elevated, and the respiratory passages placed as nearly in a straight line as possible; the eyes become prominent, the inspiratory movement is performed with great difficulty, and accompanied by a loud, roaring sound. No time should be lost in giving relief to an animal in this state, and if hot fomentations and steaming fail to give relief, tracheotomy must be performed.(See Principlcs and Practice of Veterinary Surgery, page 46.)

Influenza is also occasionally complicated with disorder of the liver, in which ease the mucous membranes are more or less tinged with yellow; the bowels are constipated, or irregularly relaxed and torpid, the fæces are of a dirty clay colour, and fœtid; the urine is high-coloured; the appetite is almost lost; and in some instances there are convulsive fits and comatose periods, which cause much alarm. Professor Dick was of opinion that these cerebral symptoms were due to the extension of the nasal inflammation to the meninges of the brain, through the cribriform plates of the ethmoicl. They are, however, due to the presence of biliary matters and products of degeneration of tissue in the blood, and are to be overcome by elimination through the natural channels. Some practitioners have termed this "bilious fever," but there is no necessity for any distinguishing term, as it is clue to tumefaction of the lining membrane of the bile ducts preventing the free flow from the liver into the duodenum of bile, which, accumulating in the liver, is absorbed into the circulation, giving the visible mucous membranes the characteristic yellow, jaundiced tinge.

I have never found it necessary to administer any liver stimnlant in this complication. All that is necessary is to keep the bowels regular by a mild aperient, such as a pint of linseed oil, and allow the animal such food as it will partake of which is of an easily digested and aperient nature. One condition which often prevails in this form requires modification, as it is apt to cause some degree of suffering, namely, an acid state of the stomach. The acidity of the stomach is manifested by grinding of the teeth, licking the walls, placing the tongue on cold objects, with drivelling of a clear saliva from the mouth. The bicarbonate of soda is very useful, giving speedy relief from this annoying symptom. The medicine is to be given two or three times a day, either dissolved in the drinking water, or as a draught out of a bottle. 
If there be abdominal complications, the colicky pains are to he relieved by warm fomentations to the belly, and small doses of opium, the bowels being regulated by oil. But even here 110 very decided and heroic steps are to be taken to relieve symptoms, it being always kept in view that they are the result of a cause which will not expend itself until a certain period, and that mere remedies, although perhaps affording relief at the time, are generally debilitating in their effects, and often tend to cause a fatal termination.

The sequelæ of influenza are, hydrops pericardii, hydrothorax, ulceration of the larynx, roaring, and sometimes glanders and farey.

\section{MORBID ANATONY.}

Uncomplicated influenza seldom proves fatal, except in the very old or otherwise debilitated animal; the most prominent lesions being great congestion of the mucous membrane of the respiratory track, extending even into the minute bronchi, some of which are filled with a muco-purulent discharge; the pulmonary lobules being consequently collapsed. The blood is dark, and is said to contain micrococci. The majority of fatal terminations are caused by the gravity of some local complication, such as pneumonia, bronchitis, \&c.

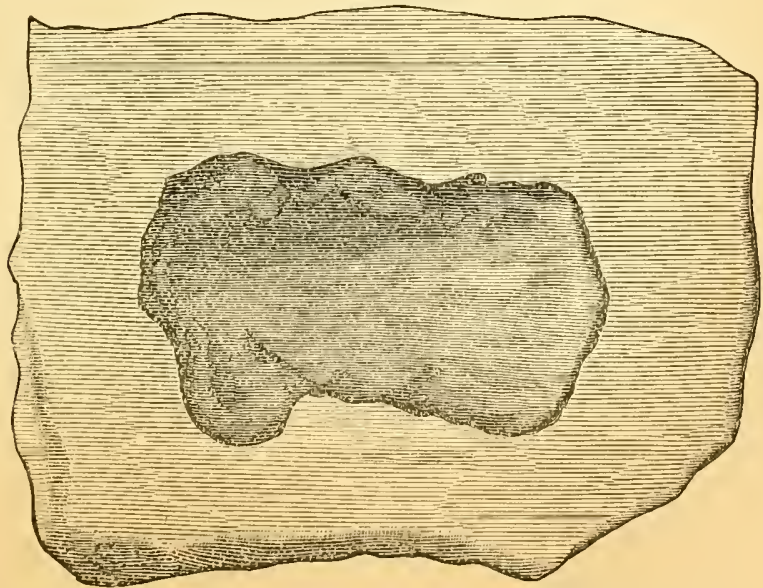

Fig. 19.-Congestive patch on mucous membrane of stomach. (Specimen sent by Mr. T. Taylor, V.S., Manchester.)

In Manchester it has been found that many dead animals present a dark patch of congestion, with incipient ulceration on the mucous membrane of the stomach, as shown above. 


\section{2l.-EPIZOOTIC CELLULITIS, OR PINK-EYE.}

The term pink-eye seems to be an importation from America -from one of the inconstant symptoms of the disease being redness of one or both eyes, and swelling of the eyelids.

Some suppose that it is a new disease, and that it first appeared in America in 1872 , the actual seat of its outbreak being Toronto, Canada, where it appeared on October 1st, 1872 ; in nine days it had attacked nearly all the horses in the city, and carriages could not be had at any price. On 18th October it had reached Montreal, and was prevalent throughout Canada. On Oetober 14th it had reached Buffalo; 17 th, Rochester ; 22d, Boston, New York, Brooklyn, and Jersey City; 27th, Philadelphia; 28th, Washington; and had been witnessed in Nova Scotia as early as October 13th.

It is characterised by great and sudden prostration of strengtl, elevation of temperature and other signs of fever, with pain in and swelling of the extremities; generally redness of the conjunctivæ, swelling of the eyelicls; and sometimes cranalgia; often colicky pains; congestion of the lungs, and in some rare instances an involvement of the brain and its membranes, expressed by phrenzy or coma.

The rate of mortality differs very widely,-_from 1 to 35 per cent.

In fatal cases death is dine to congestion and gangrene of the lungs, ante mortem clots in the heart and great vessels, enteritis, and extreme prostration of the system generally.

\section{CAUSES.-ETIOLOGY.}

Some writers maintain that it arises from contagion only, but there are many facts against this exclusive view. 1st. Its sudden and simultaneous oceurrence in various parts of a district or city. For example, a place may be entirely free from the disease one day, and upon the next outbreaks will occur in many and widely separated spots in that place. 2 $d$. Its occurrence in isolated places where contact with diseased animals is out of the question; for example, the first appearance of the disease in 1880 was at a farm in the Lothians at least one mile and a half from a railway station, and during the spring, when all the horses were kept at home plonghing. In a few days it was in Edinburgh and in many centres; but whilst I am convinced that it occurs as a malarial disease, I am equally 
convinced that it is highly contagious and infectious, and can be conveyed from diseased to healthy animals with great facility, Dièckerhoff says it may be readily communicated to healthy horses by the intravenous or subcutaneous injection of warm blood taken from a diseased animal ; and Mr. Archibald Robinson, F.R.C.V.S., Greenosk, says that it can be transmitted by the stallion which has had the disease months before to the mare by the act of coition.

A purely infectious disease like the pleuro-pnemmonia of the ox follows the great lines of commerce, and can be traced from place to place with a certain degree of exactitude, but a miasmatic disease, which may afterwards become infectious or contagious, cannot be followed from place to place. I am therefore forced to the conclusion that, like Roman and typhoid fevers in man, this disease, arising as it does from the entrance of a microbe into the animal system, is not due so much to that germ itself, but to certain properties which it has obtained from perhaps unknown conditions of the air; that the germ itself is constant in the surroundings of animals and under ordinary conditions quite innocnous, but let those conditions be altered, the properties of the germ become virulent and infectious.

There are several examples of the so-called spontaneous development of an afterwards contagious disease,-_tetanus being one of the most remarkable, and the same may be said of anthrax, which commonly originates without any contagion, and indeed, when developed in one animal, is only contagious by inoculation.

Now it appears that the infectious properties of this germ are generally developed during or immediately after a long continuance of wet weather. Several ontbreaks within my memory have been so ushered, but this is not miversal, as some writers state that it occurs in all weathers and in all elimates. This might be more correctly said of the catarrhal form than of the other.

The microbes (micrococci), which I have invariably discovered, as shown in the photo-micrographs, have a great tendency to arrange themselves in pairs. (See fig. 20.) They average about $\frac{1}{2+\frac{1}{000}}$ of an inch in size, grow freely in gelatine and agar-agar, and are easily stained with the aniline dyes. Further observations and experiments are necessary to confirm or contradict this discovery. 
The symptoms are as follows:-Some degree of lassitude; more or less failure of the appetite; the animal is inclined to be sluggish; his movements are chumsily performed, and he is inclined to "trip" with his toes; the eyes are heavy, eyelids

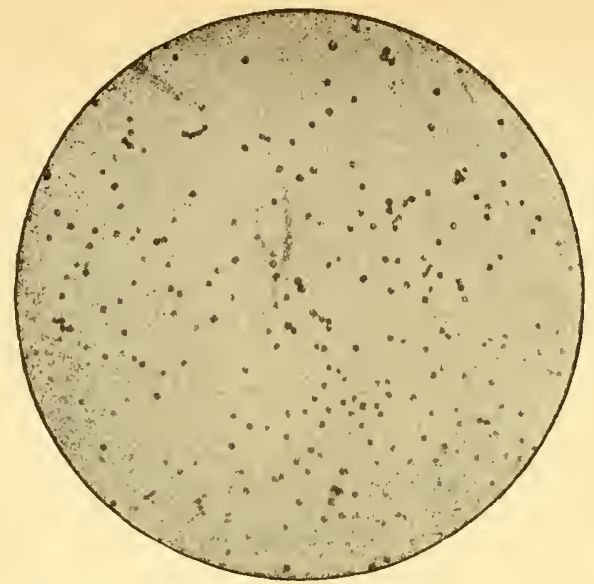

FIG. 20.-Microbes, mostly in pairs (Diplococci). $\quad \times 800$.

(New Veterinary College Museum.)

partly closed, in many cases some increased redness and some swelling of eyelids, with a discharge of tears over the face, and sometimes opacity of the cornea, and fibrinous clots in the aqueous humor of the eye.

If examined at this stage, the temperature may be from $102^{\circ}$ to $104^{\circ}$, or even $105^{\circ}$; the pulse from 60 to 70 per minute; respirations not particularly hurried; and if the horse be stopped from all work at this period the severity of the disease may increase, but very slightly, and recovery may be expected in the course of a week or nine days; but if the animal be kept at work, or, even if not working, in a badly ventilated or otherwise improper stable, the symptoms rapidly inerease in severity, the temperature rises to $106^{\circ}, 107^{\circ}$, or even as high as $108^{\circ}$, the respirations hurried, the pulse, increased in frequency to $80 \mathrm{or}$ even 110 beats per minute, is small and sometimes almost too indistinct to be felt by the most practised touch; the animal becomes excessively lame, sometimes immoveably so; or he is continually resting or pointing one foot and then the other, as if 
suffering agony in feet or limbs. In a short period of time the limbs begin to swell, more particularly about the lower joints, i.e., the hocks and knees downwards, and as the swellings become pronounced the pain disappears.

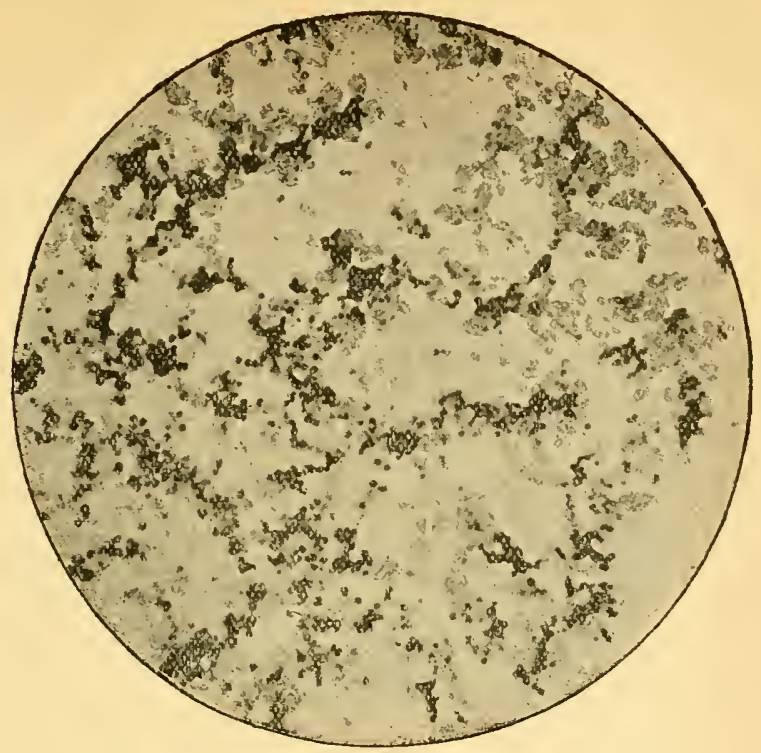

Fig. 21.-Microbes in groups. $\quad \times 800$.

(New Veterinary College Musecr.

As already stated, there is usually a cough and some degree of roughness of the throat and bronchial tubes, but in the last outbreak in Edinburgh these were conspicuous by their absence.

In some instances intestinal pain, expressed by restlessness, looking round at the flanks, scraping with the feet, and even rolling about, is present shortly after the first manifestation of illness; and in others-and these are the really dangerously fatal ones-well-marked signs of inflammation of the bowels are observable at an early stage, with intermittent action of the heart and total loss of appetite, or even extreme abhorrence of food, and occasionally of water.

The bowels are at first normal or slightly constipated; the freces covered with mucus (hence the terms syphus-mueosa and muco-enteritis), and their colour frequently indicates an absence of biliary secretion or the presence of altered bile; the rectum 
is often irritable, and the passage of fieces canses some degree of pain; on the third or fourth day the fieces become pultaceous and often of a brown colour, and later on diarrhoea may occur.

In other cases, including from the third to the sixth day of the disease, serious pneumonic symptoms are observed: there is an extensive exudation into the lung tissue-in fact, what is observable as taking place in the extremities, viz., exudation and swelling, is occurring in the lings, eausing the respiratory movements to become greatly accelerated, but if gangrene does not set in, indicated by foetor of the breath or discharge of dark-coloured blood from the nostrils, recovery may still be hoped for.

In other instances, even during convalescence, the pulse, hitherto moderately strong, and all other symptoms passing away, becomes rapidly feeble, and the animal suddenly dies, and on making a post mortem examination, firm clots or thrombi have been found in the heart, lungs, and blood-vessels, which have stopped the circulation and caused death.

'This termination has to be very carefully guarded against, as it may occur in the most promising cases. It is brought about as follows:-The disease is one which particularly attacks areolar tissue; and inflammation of this tissue, like that of the more firmly fibrous, causes the development of coagulating properties in the blood, and when-added to this coagulative tendencythe heart loses its tone, the blood coagulates in its cavities and blocks the circulation. In other instances horses have dieck wecks or even months aftcr they have passed through the $\mathrm{d} \cdot$ ase, and post mortem examinations have revealed plugging of the vcsscls of some important organ.

Another rare complication is witnessed by symptoms of irritation of the brain and its membranes, with effusion into the ventricles and sub-arachnoid space. As this complication manifests itself there will be at first delirium, the eyes staring, the pupils dilated, perhaps complete blindness, with unconscious restlessness, tendency to elevate the head, followed by paralysis commencing in the hind limbs and extending to the whole muscular system, with total blindness, unconsciousness, stertorous breathing, inability to swallow, and loss of voluntary movements: these conditions indicate a rapilly approaching fatal termination. To recapitulate, the symptoms may be briefly stated to be as follows :- 
Swelling of eyelids, often with diffuse redness of the conjunctivæ; congestion of the superficial vessels of the eyes, cloudiness of the cornea, and collection of yellowish fibrinous matter or blood-clots in the auterior chambers of the eyes. Sensitiveness to light, eyelids more or less closed, and discharge of tears; but in milder cases the eye symptoms may not be very distinct.

Pulse from 50 or 60 in mild to 100 or even 120 in severe cases.

Temperature from $103^{\circ}$ or $104^{\circ}$ in mild to $106^{\circ}, 107^{\circ}$, or even $10 S^{\circ}$ in severe cases. $108^{\circ}$ was observed in one case only in the College practice, and ended in recovery. During convalescence the temperature rapidly falls, and in many cases to below the normal-to $99^{\circ}$, and rises to normal-about $101^{\circ}$.

Gait awkward and slow, loss of tone in the muscles, particularly those of limbs, ears, and lips, and sometimes marked weakness in the hind quarters, and sometimes very great lameness.

The respirations from 15 to 20 in mild to 30 or 40 in grave cases; these, however, are subject to modifications, as in sorne horses treated at the College we observed the respiration even slower than natural at first, and increasing in frequency during convalescence. In some fatal cases the breath is foetid for some time before deatl, and respirations very rapid.

Mucous membrane of the month of a venous red colour; tongue dry ; frees at first dry, but on third or fourth day brown and poultice-like in consistence ; later on, diarrhœe in some cases.

Limbs slightly or considerably swollen; urine diminished, and sometimes albuminous, and in some cases of a chocolate colour, and containing broken-down blood-corpuscles.

\section{POST MORTEM APPEARANCES.}

Heart. - Congested; sometimes covered with mulberry coloured spots-petechix. Clots of blood in auricles, ventricles, and larger blood-vessels.

Blood.-Dark-coloured and viscid; white corpuscles increased in numbers, and many of the red ones broken down.

Lungs.-Generally congested; infiltrated with fluid, or filled with blood extravasation, which has undergone decomposition prior to death, and given rise to foetor of the breath. 
Liver - Kidneys.-Generally congested. Kidneys sometimes black.

Connective Tissue, particularly of the limbs, contains much fluid or semi-coagulated exudate.

Muscular System.-Generally pale and flaccid.

The Brain and its Membranes congested, and the eranial cavities containing a superabundant quantity of fuid in those cases where frenzy or coma have appeared during life.

Digestive System.-Mueous membrane of stomach sometimes congested; bowels inflamed, and oceasionally covered with petechial spots; contents generally fluid.

\section{MORTALITY.}

This seems to have varied during the 1890-91 outbreak in a most remarkable manner. Some practitioners who have replierl to a cireular issued by me, record a loss of only 1 to 2 per cent., whilst others return from 5 to 9 ; and it is reported that some have lost even a greater number. Our loss at the College was under 1 per cent., and at Piershill Barracks, out of a total of 59 treated by Mr. Phillips upon the principles of this College, there were no deaths.

\section{TREATMENT.}

The first and most important essential in the treatment of the disease is absolute rest as soon as the slightest indication of illness has been observed.

The animal, if possible, should be placed in a cool, wellventilated, but not cold, loose box, warmly but not too heavily clothed with sheet and hood; and when bandages do not eause irritation the legs should be bandaged. The diet should consist of bran mashes, and a moderate quantity of hay for the first few days, except where a horse has been used to and is fond of a linseed mash. In such a case one pound of well-boiled linseed should be mixed with the mash every night until the bowels are loosened, when, if there be any signs of diarrhœa, it should be discontinued, and dry eorn given. The mashes should be continued until the fever has subsided, and then gradually changed to the horse's ordinary diet. Cold water should be 
placed within the animal's reach, so that he can satisfy his, often inordinate, thirst night and day, and if greatly prostrate, the patient should be placed in slings. Medicinally, salines and other remedies recommended for the catarrhal form may be administered in accordance with the phase of the disease. If pain be prominent, the bromide of potassium has proved useful.

\section{3d.-Epizootic pneunonia, or pleuro-pneunonia of the HORSE-LOBAR-PNEUMONIA.}

Definition.-A croupous inflammation of the pleura and substance of the lungs, preceded and accompanied by a low typhoid or adynamic form of fever, whieh lasts from seven to fourteen days. It generally occurs but once in a season, but one attack does not render an animal exempt from a seeond or third. It is now considered an infectious disease, due to a micro-organism, which is asserted by some writer's to be the Diplococcus pneumonic of Frankel, identical with that found in the saliva of certain persons, and which produces septicemia in the rabbit. Others state that it is a streptoeoceus. Galtier and Violet describe two organisms-a streptocoecus and diplococeus-whilst Cadèac states there is only one microeoccus, often grouped in pairs, sometimes in chains. The microbes of Galtier and Violet do not stain with Gram, whilst all the others do. They are all facultative anaërobes.

For the last few years this form of epizootic has prevailed to a very great extent both in the north and south of Britain. Lrroneously called "influenza," it has seemingly and for some time taken the plaee of that affection, from which it differ's very materially. The true uneomplicated influenza is a disease of the mucous structures: this affects the serous covering and substance of the lungs.

In 1861-2, also in 1891 and 1894, this form of epizootic disease beeame very prevalent in the north of England, where it raged for many months, eommitting great havoe amongst horses of ali kinds, but particularly amongst those most exposed to the vicissitudes of the climate. It has prevailed more or less ever since, both in town and country, more particularly amongst young horses and those removed from pastures into stables. The know1?dge that nearly every newly purchased horse is liable to suffer from this or some other form of epizootic disease, deters many 
persons from keeping horses, and hence we find that many whose business requires that horses should be employed, prefer to let out their work to contractors and carriers. This repugnance to purchase and keep horses by men in business has also been increased by the great mortality which has generally resulted from this epizootic.

\section{SEMIOLOGY.}

The primary symptoms are very often more or less obscure. The animal is dull, dejected, off its food, performs ordinary labour with difficulty; lassitude, perspiration, and fatigue are easily induced. Examined carefully, the pulse will number from sixty up to eighty per minute-during some seasons eighty has been the common number-and the animal temperature will vary from $104^{\circ}$ to $106^{\circ}$. Sometimes there is a cough from the commencement; very often, however, there is no cough during the first three or four days. The extremities and ears are alternately hot and cold; the appetite is almost entirely lost; the alvine and urinary secretions are defective; the visible mucous membrane injected, and frequently of a rusty tinge; in some cases there is a rash in the mouth, a rusty or straw-colonred discharge from the nostrils. The animal does not lie down, Auscultation does not reveal much at first; in fact, for three or four days the animal may be said to be suffering from fever without local complications, but at the end of that period a hard, dry, painful cough is now and then heard; the thoracic walls are fixed; the breathing is abchominal; the elbows are turned out, and a line or hollow extends from the ensiform cartilage of the sternum to the anterior spine of the ileum, which denotes that the ribs are fixed, owing to pain within the chest. If the horse is now made to move suddenly, it emits a grunt or groan; in some instances it groans at each expiration, and if slightly rapped in the chest with the knuckles it will do so with pain; the breathing is catching and short, and auscultation will detect a friction sound.-(See Pleurisy.) Very commonly the right side only is affected, in some cases the left, and in rare instances both sides.

In the course of the next ensuing two days, the sounds of the chest indicate pleurisy, pleuro-pneumonia, or a complication of these with pericarditis. From the commencement of the 
manifestation of chest disease, the breathing becomes somewhat hurried, the pulse assuming a hardness of character which it did not possess at first; the number of its beats are not further increased; on the contrary, rapidity of the pulse may decrease as the exudation on the pleural surfaces advances, and it is not at all uncommon for one to witness an increased rapidity of the respiratory movements, gradual condensation of lung tissue, some effusion into the thoracic cavity, and a diminution in the number of the pulsations. If pericardiac complications are present the pulse is marked by a peculiar indistinctiveness and irregularity; the cardiac beat is generally loud and short; the impulse, however, is weak, and the arterial pulsations indistinct.

In some instances the whole body becomes stiff and sore, and the horse is disinclined to move, but stands with its nose extended forwards, nostrils dilated, and fore legs apart, presenting an appearance of distress, which is heightened by frequent looks at its sides, and by attempts to lie down, as if suffering from colicky pains. In such instances the respiratory movements are short, shallow, and much accelerated, numbering fifty, sixty, or even eighty or more per minute, whereas in the majority of cases they do not number more than thirty per minute, when auscultation may detect considerable exudation into the lung tissue.

An examination of the respiratory apparatus by percussion and auscultation-the only satisfactory method of diagnosing chest diseases-will enable the practitioner to detect the various changes as they occur.

1. For the first two or three days after attack both percussion and auscultation often give negative results; at the termination of that period percussion over the seat of the pleural inflammation will cause the animal to groan. Auscultation will detect a dry friction sound if the pleura be affected; absence of true respiratory murmur, and the presence of crepitations, large or small, if the lung-substance be involved; a combination of friction sound, and crepitations, if both pleura and lung tissue, and a "to-and-fro" rasping friction sound, with more or less palpitation, if the pericardium be involved.

These sinns indicate-1st. That the natural pleural secretion is arrested, and that the dry pleural surfaces rubbing on each 
other cause the friction sound ; 2 d. That engorgement of the lung tissue has taken place; and $3 d$. That the pericardial secretion is arrested, causing the "to-and-fro" sound at the heart.

2. In the course of from one to two days the above abnormal sounds disappear. Auscultation now reveals that in the part affected there is no sound at all, or that a tubular or hollow sound is heard. The absence of sound indicates either that the lung tissue has become impervious to air, or that a considerable effusion of serum has occurred into the thoracic cavity. If the former, the absence of sound becomes apparent by degrees only; that is to say, the respiratory sounds become gradually feeble as the consolidated part is approached, showing that at the border of the hepatized tissue the inflammatory process is less alvanced. There may be crepitations or tubal sounds, but when the absence of souncl is due to effusion of serum (hydrothorax) the sounds terminate abruptly at a certain distance from the floor of the thorax, the respiratory sounds being louder than natural above the line of termination, and in some instances the presence of fluid in the chest will be indicated by a "dripping sound," as of drops of water falling into a well. The tubal sounds indicate a less degree of consolidation; that air finds ingress into some of the bronchial tubes, but not into the more minute ones and air cells.

This condition may exist for three or more days, and then the crepitations reappear, showing that the exudate is breaking up and becoming gradually removed by absorption; the dull impervious part at the floor of the cavity becoming daily shallower, and the normal sounds slowly returning.

Percussion during the stages of consolidation and effusion produces a dull sound over the diseased part, and increased resonance over the healthy parts of the ehest. In all the unaffected portions of the lungs the respiratory murmur is increased.

The "to-and-fro" cardiac friction sound also disappears, owing to effusion into the pericardial sac, and reappears as the exudate is removed by absorption.

Very often after convalescence has been established the congh becomes more frequent; it, however, loses its painful liacking character, being now louder and stronger. In some instances, more especially if the exudate has been very abundant, a mal- 
condition of the system is induced by the abundance of effete materials in the circulation, removed by absorption from the transformed exudates. This has been compared by Professor Gamgee to the hectic fever of the human being. There is much emaciation, imperfect digestion, capriciousness of the appetite; the hair is easily removed from the mane and tail; irregularity in the surface temperature; and continuance of the increased temperature of the body, as indicated by the thermometer. If this condition of the system be not successfully combated, the respirations again become accelerated and the ribs fixed; there is flapping of the nostrils, which are greatly distended; the animal again discontinues to lie down. Anasarcous swellings may appear on the chest, abdomen, and the legs, denoting the presence of hydrothorax, to which the animal may eventually succumb in the course of an indefinite period-sometimes in two or three days only, sometimes in as many weeksor symptoms of farcy or glanders may appear.

\section{POST MORTEM APPEARANCES.}

In very acute cases, the appearances revealed by a post mortem examination are, intense redness of the pleural surfaces of one or both sides of the chest; the engorgement of the vessels embracing the visceral and parietal surfaces, and some degree of effusion and exudation of lymph. In some instances, the lungs show but little disease, whilst in others patches of inflammation are found throughout their substance. The pericardium and endocardium are generally more or less injected, and the death of the animal at this early stage generally results more from the cardiac complication than the pleural disease. It is but seldom that a case terminates so rapidly, unless the animal be kept at work after it has fallen ill.

If the horse live for two or three days after the pleural symptoms have become manifested, along with the injection of the vessels, an abundant quantity of serum will be effused into the pleural sac, floating in which, and loosely adherent to the pleural surfaces, bands of lymph will be found. These bands of lymph are loose and watery, and the serum is turbid or flocculent. If the horse has lain for some hours after death, and the post 
mortem is carefully performed. the effusion is clear at its upper and turbid at its lower part.

If the inflammatory process has been less rapid, the lymph is of a more consistent appearance, and forms over the inflamed parts floceuli of various sizes, or a distinct lining varying in thickness, and of a honey-combed appearance; but the lymph in the epizootic form does not possess the firmness, plasticity, and tendency to organization which is characteristic of that of the sporadic form of the disease. These bands of lymph are sometimes of very rapid formation, and they may even show some trace of organization in forty-eight hours after attack. 'This fact is of considerable importance legally, as it is often found that if a horse dies in a week or two after purchase, an attempt is made to compel the seller to refund the purchase-money on the ground that the animal was diseased prior to the date of sale. Old bands of lymph, and such as indicate previous disease, are firm, organized, of a glistening appearance, and unite the opposing surfaces more or less firmly together. New bands of lymph, on the contrary, are soft, watery, and the parts which they may attach together are easily separated.

In some instances, extravasations of blood give the lymph a red colour. This nust not be mistaken for vascularity, as the latter would denote organization and age.

Examined microscopically, the lymph exhibits the presence of numerous inflammatory corpuscles, oil globules, and illdeveloped fibres.

The exudate into the lung tissue is also wanting in plasticity, the lung cuts up soft and moist, a whitish fluid oozing from the cut surfaces.

In some cases, where the vital powers have been very low, or where they have become weakened by injudicious treatment, an abundant, turbid, foetid fluid has been thrown out, partaking much of the character of unhealthy pus; or abscesses form in the lungs, and in rare instances gangrene of the lungs has occurred.

The pulmonary complication is generally found at the inferior portions of the lung, and in many instances the exudate indicates that the process is wanting in the true character of plastic inflammation. 


\section{TREATMENT.}

From the foregoing observations, it will be understood that the local complications of this disease are preceded by fever, the pleural and pulmonary inflammation appearing after a few days have elapsed. The fever is of a typhoid or adynamic character, and the severity of the chest affection is generally in accordance with that of the premonitory fever. The fever, slight perhaps at first, is very often intensified by the suffering animal being kept at its ordinary work for some time after the appetite has failed. This inattention on the part of those in charge of horses is often a cause of great loss, inducing a fatal termination where recovery might reasonably have been expected. The febrile state and the local inflammation are increased by all debilitating influences, such as bad food, previous disease, natural delicacy of constitution, by the abstraction of blood, and very commonly by the administration of purgatives. Many horses are destroyed by the administration of aloes, a dose of which is often given when the horse is seen to be "off his feed." Superpurgation may not be induced, but the chest inflammation is much increased, and the animal often succumbs.

There is another practice in vogue, more particularly amongst horse-dealers, namely, that of giving a purgative (aloetic) ball to nearly every fresh horse they buy, and this is done without taking the health of the animal at the time into consideration at all. I am convinced that this is a most reprehensible practice, leading to grave pulmonary diseases and to death, more especially if an epizootic is prevalent.

The pathology of chest inflammation leads us to the conclusion that when once established it runs through certain stages, namely, congestion of the vessels, the outpouring of an effusion, and the formation of an exudate. The congestion, effusion, and exudation are determined by the intensity of the prececting fever. If the fever is slight, the local disease will be slight also; if, on the contrary, the fever is severe, the local inflammation will be proportionately grave. During some seasons the disease from its commencement is severe, and then the weakly succumb. But at other times this is not the case, and very few deaths result from the disease; but its character is always rendered more serious by irrational treatment. 
The duration of the fever cannot be cut short by any treatment, and attempts to do this are very often the cause of numerous deaths. Its severity, however, is amenable to modification by-

(1.) Complete rest whenever the slightest sign of illness is discoverable.

(2.) By housing the animal in a warm, dry, light, well-ventilated loose box.

(3.) By taking special precantions that no draught of cold wind hows upon it; for it must be remembered that cold, more especially cold wind, is the common cause of the disease, and that removal of the cause is the first step in the treatment of all diseases; and, again, that the severity of many affections is dependent on the "dose" of the cause. When this is heavy or long continued, the results will also be heavy and severe.

(4.) By clothing the animal and bandaging the legs, in fact by keeping up the equilibrium of the circulation; for if the vessels of the skin are prevented, by the operation of cold-acting upon and constringing them-from receiving a due supply of blood, internal congestions and inflammations are aggravated and often determined.

In addition to these rulus, the practitioner must remember that the disease, once established, is the effect of a cause which has been in operation for some time past; that the effects, whatever they may be, are the natural physiological responses of the animal body to the action of such cause or causes; and that all attempts to remove such effects or conditions by other than those processes which nature herself attempts, and generally accomplishes, can only result in disappointment and loss.

How, then, does the animal body rid itself of disease and its results? Briefly, in the one before us, the inflammation is the result of an irritant, acting for a certain time upon a certain tissue or organ. If the dose of the irritant is strong, the resulting inflammation will be strong also, and nothing will subdue that inflammation so long as the eause retains its strength and is allowed to operate. This inflammation is characterised by congestion, effusion, and exudation. When the cause is removed, or when its strength is exhausted, the congestion slowly disappear's, and the products of the inflammation, namely (1.) the effusion, is gradually taken up into the circulation and removed from the boly by the excretory organs; and (2.) 
the exudation or lymph, degenerated into a turbid fluid material -the pathological milk of Tirchow-is removed in the same nanner as the serous effusion, or is replaced by a fibrous structure, which binds the surface of the opposing parts together, and constitutes alluesion. When this adhesion is completed, the new structure becomes a part of the living body.

Seeing, then, that the various changes are natural results of the operation of a cause, the practitioner must be careful to avoid doing anything that is calculated to irritate or debilitate the animal body. At the same time he must be careful to keep the excretory organs in a natural condition, maintain the strength by appropriate diet, and allay morbid irritability by the administration and application of suitable remedies.

In the first place, the practitioner is to avoid irritating and debilitating his patient. In order to accomplish this, three methods of treatment now pursued by many practitioners must be abandoned, namely, bleeding, purging, and counter-irritation.

Blecding and purging, separately or conjointly, lower the animal powers, which are already too low, and prevent the physiological changes from taking place which are essential to the final removal of the disease. If they do not kill in a very short period of time, they cause an alteration in the inflammatory process, whereby large quantities of an aplastic material are formed, which, by blocking up the lung tissue, or filling the cavity of the chest, cause death by suffocation; and by their debilitating influence generally render the vital powers less able to resist even a mild attack of disease.

Counter-irritation.-This is the favourite method of treatment at the present time, and it is accomplished, or thought to be accomplished, by the application of mustard, cantharides, or other irritants to the skin of the sides and breast. It is applied on the principle that no two inflammations can exist in the body at the same time; that an artificial inflammation of the skin, excited by the irritant, removes or destroys that which is going on within the chest. Others say that it rouses the capillary circulation, removes congestion, \&c.

For many years I followed this method of treatment, and so plausible were the arguments in its favour, that I could scarcely bring myself to believe that it could do harm, although I saw that many horses died even when a blister had acted. I was at 
last induced to abandon it altogether, and the result has been most satisfactory.

Irritation of the sides and breast is injurious in all instances of chest disease in the horse, except in a condition presently to be described, and for the following reasons:-

1st. It eannot and does not remove the internal inflammation. Many post mortem examinations have convinced me of this. 1)uring the summer of 1873 I had a good opportunity of demonstrating this fact to my students. A horse newly bought by a lealer died from pleurisy, and its value was in dispute between buyer and seller. On viewing the carcase, both myself and students were struck with the violence of the inflammation of the skin upon the sides, induced by the repeated applications of irritants. Some of the students, having been pupils of gentlemen who advocated the "counter-irritation" theory, were under the firm belief that no internal inflammation could exist where the external was so very marked. The result, however, proved the fallacy of the hypothesis, for the pleura was intensely inflamed.

The internal inflammation being an effect, until it can be proved that an external irritant can remove the eause which has produced such effect, it is quite as reasonable to suppose that a blister on the palm will remove or prevent the effects of another on the back of the hand, as it is to think that a blister on the outside of the chest, no matter how soon applied, ean remove an irritant, or prevent it from affecting the internal structures.

$2 d$. Another argument which tells against blisters is the fact that their advocates differ as to the stage at which they ought to be applied. One party says they eannot be applied too soon, in order to counteract the internal inflammation, "by drawing the morbid action and the blood to the surface;" whilst another says that external irritants do harm whilst there is any fever present, and that they should always be applied after the pulse has fallen and when convalescence has eommenced, in order to stimulate the removal of the products of the inflammation. I, however, fail to see the necessity of doing this; for the removal of the exudates is a physiological process, best accomplished when uninterfered with, and counter-irrication at this period can only retard the recovery, or, by again setting up the febrile condition, bring about a fatal termination. 
There is, however, a very rare exception to these objections to external stimulants during the second stage of the disease, occurring in that condition where the horse neither gets better nor worse for several days - "lhangs fire," as it is commonly termed. In such instances a weak solution of mustard, by gently stimulating the skin, will often induce the beginning of convalescence, and act as a stimulant to the whole body. In no other condition are counter-irritants beneficial, and in this, care must be taken not to apply them too strong nor for too long a time. The sides should be quickly rubbed over with about two or three ounces of mustard mixed in a quart of warm water, and the parts covered over with paper or linen. If one application does no good, it is not advisable to apply another; but if the horse seems to improve for a time and then relapse, a second or even third application may be admissible.

$3 d$. The application of blisters causes pain and increases the fever. If applied to the sides they impede the respiratory movements by the pain they cause, and thus add to the distress and suffering of the animal. The cantharidine absorbed into the system causes irritation of the urinary organs, sometimes congestion of the kidney's, interferes with their excretory functions, and by the pain and disturbance thus induced adds materially to the febrile condition, and often causes the animal's death. Horses that recover from the disease naturally do so much more rapidly than those which have been blistered, and which are often a long time in overcoming the effects of the blister; with raw sides, they remain for weeks in their stables unfit for work. Sometimes, more especially if the blisters have been oft repeated, the animal becomes much emaciated, is unthrifty, the blistered skin will slough, and when it finally recovers the blemish remains for life. Finally, animals which die from the disease do so much easier than those which have been severely blistered. Indeed, the agony of death from disease bears no comparison to that from treatment.

Having now pretty strongly condemned what I consider to be wrong in principle and disastrous in practice, I must proceed to describe the treatment which has proved successful.

During the premonitory fever, in addition to housing, clothing, \&c., already described, the animal is to be allowed an abundant supply of cold water to drink, warm or cold brau mashes, which- 
ever it likes best, to eat, a boiled linseed mash every night, roots, such as carrots, turnips, or potatoes, with a handful or two of the best and sweetest of hay, or grass if in season. If the alternations of the temperature of the skin be very marked, two or three doses of spirits of nitrous ether are to be daily administered in warm water; and to excite the action of the lidneys, which is often in abeyance, the ether is to be supplemented by half-ounce doses of the nitrate of potash. Even when the fever is high and the symptoms acute my experience leads me to conclude that it is unwise to use depressants, such as aconite, antipyrine, \&e. If the bowels are costive, clysters of warm water are to be administered, and in rare instances, where the bowels are extraordinarily torpid, a dose of linsced oil. In the majority of cases, however, laxative food will effect all that is necessary, and it is better to avoid even the oil, except where the constipation continues, or where it causes measiness.

The chest is to be carefully examined at least once a day, and when convenient the animal temperature registered, as well as the pulse and number of respirations.

If at the onset of the pleural inflammation there be pain and distress, manifested by the horse looking to its sides and grunting, or attempting to lie down, opium or morphia subeutaneously administered is to be given to alleviate the pain, and in order to prevent its constipating effects, from eight to twelve ounces of oil are to be given. One or two doses will be sufficient, for when the dry condition of the first stage of the inflammation has passed away, pain generally disappears. If the symptoms are those of irritation rather than pain, bromide of potassium may be given in preference to the opium; and at any time during the progress of the disease, if irritability is much increased, the bromide will prove serviceable. In addition to the opium, warm, soothing-not hot, scalding-fomentations to the sides give great relief. These warm fomentations are to be applied for at least an hour two or three times a day in every severe case; and in all instances, whenever the breathing becomes catching or accelerated, they must be applied until relief is given. In many cases their effect in allaying pain and soothing the animal is most marked, the patient often giving a "sigh of relief" in a few minutes. The best metlod of applying them is either to wrap the horse's body in a thick blanket 
or horse sheet, and pour warm water upon it, placing a tub so as to catch the water as it falls from the sheet, or to wring cloths out of warm water and apply them to the sides. The latter method is the cleaner, as there is less water lost on the bedding, \&c.; but in the former the skin is not exposed during the fomentation, and is to be preferred. When the fomentation is concluded, the wet sheets are to be covered with a waterproof covering, or, if removed, the skin lightly rubbed with weak ammonia liniment, say one ounce of liquid ammonia to sixteen of cil, for the purpose of preventing the sensation of cold which is apt to follow warm applications.

As the local inflammation progresses the nitrate of potash is to be slightly increased. From one to two ounces may be given in the twenty-four hours, and it always acts best dissolved in the drinking water, as the horse can then sip it at its leisure. It lowers the animal heat, clcans the mouth, has some effect on the exudation, and stimulates the kidneys. When the diuresis becomes increased, the nitrate must be discontinued, and if the horse is progressing favourably, but little more is needed. In the course of a few days, if the appetite is not good, regetable, and afterwards mineral tonios, are to be given. In many cases, where the kidneys act very languidly a few doses of colchicum will prove of great service. I think the colchicum seeds are the best, made into a tincture, and given in doses containing one to two drachms, for two or three days, or until diuresis is induced. The colchicum, in virtue of its action in causing the elimination of the solid ingredients of the urine, removes from the economy much effete material, products of the metamorphosis of the inflammatory exudates.

Some veterinarians are very fond of giving the carbonate of ammonia as a stimulant in all typhoid diseases. If the kidneys are acting freely it is a very useful stimulant, but if the secretion of urine is diminished its administration only tends to load the system with ammonia-when it is already over-burdened with the products of tissue changes, all tending to be resolved to ammonia, carbonic acid, and water; but if administered in conjunction with the vegetable tonics in the later stages, it tends to stimulate the appetite, and restore strength. It should always be given in a ball, as solutions tend to irritate the mouth, and prevent the horse from eating. 
If the appetite is very much impaired, the horse must have an abundant allowance of milk to drink instead of water. Some horses are very fond of milk, and almost all will partake of it after tasting it a few times. If the debility is excessive, eggs beaten up with the milk will prove of great service. They supply those constituents which are required by the wasted tissues. If milk is refused, the animal must have gruel, linseed tea, or hay tea, if it will take them spontaneously, but nothing must be forced upon it.

of the termination of pleuro-pneumonia in hydrothorax I have only to say that since I have abandoned the heroic and counter-irritating treatment, hydrothorax has been almost unknown to me. Other terminations, such as rheumatism, roaring, thick wind, \&c., will be described under their proper heads. 


\section{CHAPTER XXX.}

\section{ENZOOTIC AND EPIZOOTIC DISEASES-continut.}

\section{MALIGNANT CATARRHAL FEVER OF THE OX.}

A malignant form of cáarrhal fever, sometimes, but erroneously, called glanders of the ox tribe, due to the operation of a morbid poison, which expends its specific effects upon the mucous membrane lining the sinuses of the head and nasal chambers; manifested by rigors, dulness, and debility, succeeded by the mucous membranes becoming of a bluish-red colour, the eyes closed, the eyelids swollen, and flowing of tears over the cheeks. There is a painful and frequent cough; the pulse is feeble; bowels costive at first, but diarrhœa soon succeeds. In the course of a few hours after the onset of the disease, a profuse discharge issues from the nostrils, mouth, and eyes; the sinuses of the face and head become filled with purulent matter, and in some instances the horns drop off.

The connection between the bone of the horn (flints) and the sinuses of the head is very intimate, for the bony process, the lorn core, which springs from the crest of the frontal bone is lollow, and this hollow is continuous with the frontal sinus. This hollow or sinus in the horn-process is very vascular, and its blood-vessels anastomose with those upon its periostal surface. Any influence, then, which causes inflammatory action in the sinuses, easily induces the same processes in those structures which are so intimately connected; and such we find it to be the case in malignant catarrhal fever,--inflammation is excited in the sinuses of the flint, which extends to the surface of the bones, and the consequent suppuration detaches the horny coverings.

The symptoms of this affection are not dissimilar to those 
of cattle plague, but it does not, like the latter, spread by contagion; indeed, it is a non-contagious disease, the result of exposure to cold. The discharge from the uostrils is more profuse than in the plagne, purulent or sanguino-purulent, and the horns fall off.

There may be appearanees on the month and in the visible mucous membranes very similar to those of rinderpest; and in some instances lameness and sloughing of the hoofs may lead one to suppose that it is an aggravated form of contagious eczena.

\section{TREATIENT.}

Temoval to warm, sheltered sheds; enemas, to relieve eonstipation ; stimulants, such as the carbonate or acetate of ammonia and the spirits of nitrous ether, with hot water vapour to the nostrils, constitute the treatment of the earlier stages. If the animal survive, these remedies may be sueceded by tonics, mineral acids, and good food. The disease is very fatal, and eauses death in from three to seven days. If the animal live over the latter period convalescence may be expeeted. Violent remedies, such as strong doses of salts, bleeding, calomel, \&e., always hasten the fatal termination. If aperients are imperatively called for, oil or treacle are preferable to the more drastie agents. 


\section{CHAPTER XXXI.}

ERUPTIVE OR PETECHIAL FEVERS,

USUALLY ASSOCIATED WITH EPIZOOTICS, ALTHOUGH THEY DO NOT THEMSELVES USUALLY ASSUME EPIZOOTIC CHARACTERS.

\section{I.-PURPURA HFMORRHAGICA, DIARRHA EMIA.}

This disease was arranged in the first edition of this work in the same group as the charbonous affections, which in several characteristics it simulates; but the life history of the morbid processes, the causes, the non-contagiousness, and the absence of organisins in the blood in this disease separate it from anthrax.

Fault has been found with the term "purpura hæmorrhagica," and Delafond has named the disease " diastashæmia." I think the term is more objectionable than purpura, as it merely implies a standing apart or a separation of blood; and were I to venture upon a new term, I would suggest "diarrhæmia"-Gr. día, through; " $\rho \epsilon \omega$, I flow; ai $\mu \alpha$, blood; or a disease characterised by breaking up of the blood, and ecchymosis.

Definition.-An eruptive non-contagious fever of an intermittent type, usually, but not uniformly, occurring as a sequel to another disease.

Pathology and symptoms.-The primary manifestations are often uncertain. In some instances the earliest symptom may be the expression of pain in one or more limbs, with but slight swellings, which, however, become more pronounced in a very short time. In other instances, the approach of the disease is shown by the appearance of a few petechial spots in the nostrils 
or papulæ on the skin, whilst in other cases the malacly is fully manifested in a very short period.

Similar to its analogue anthrax, purpura is a disease in which the blood is gravely altered, associated with extravasations, effusion of red coloured serum, preceded and accompanied by constitutional disturbance, languor, debility, sudden elevation of temperature to $104^{\circ}-106^{\circ} \mathrm{F}$, or even higher, the alternations of temperature being very great. The alternations in the following table are from the clinical report of a case which died at the College :-

\begin{tabular}{|c|c|c|c|c|c|c|c|c|}
\hline Date. & Hour. & Pulse. & $\begin{array}{c}\text { Temper- } \\
\text { ature. }\end{array}$ & \multicolumn{2}{|c|}{ Date. } & Hour. & Pulse. & $\begin{array}{c}\text { Temper- } \\
\text { ature. }\end{array}$ \\
\hline 1879 & & & & $187 !$ & & & & \\
\hline May 23. & $9 \cdot 20$ А. & 78 & $104 \cdot 2^{\circ}$ & June & 1. & 6 е.м. & 66 & $102 \cdot 2^{\circ}$ \\
\hline,$\quad$, & 6 P.s. & 84 & $106.4^{\circ}$ & , & 2. & 9 А.м. & 60 & $102 \cdot 1^{\circ}$ \\
\hline, 24. & 9 А.м. & 84 & $102 \cdot 3^{\circ}$ & ,, & & 6 р.м. & 66 & $102^{\circ}$ \\
\hline , & 6 р.м. & 72 & $102 \cdot 4^{\circ}$ & , , & 3. & 9 А.м. & 60 & $102^{\circ}$ \\
\hline , 25. & 9 A.M. & 60 & $101 \cdot 3^{\circ}$ & , , & ," & 6 P.M. & 66 & $101 \cdot 4^{\circ}$ \\
\hline & 6 р.мr. & 66 & $102 \cdot 2^{\circ}$ & & 4. & 9 А.м. & 66 & $101 \cdot 2^{\circ}$ \\
\hline , 26. & 9 А. М. & 60 & $102 \cdot 1^{\circ}$ & , & . & 6 P.м. & 72 & $103 \cdot 3^{\circ}$ \\
\hline & 6 P.M. & 66 & $104^{\circ} 1^{\circ}$ & , & 5. & 9 А.м. & 72 & $102^{\circ}$ \\
\hline,$\quad 27$. & 9 А. & 75 & $103 \cdot 1^{\circ}$ & , & , , & 6 Р.м. & 78 & $104^{\circ}$ \\
\hline & 6 Р.м. & 88 & $102 \cdot 3^{\circ}$ & & 6 & 9 А.м. & 78 & $104^{\circ}$ \\
\hline , 28. & 9 А.м. & 68 & $102^{\circ}$ & , & , & 6 р.м. & 88 & $105^{\circ}$ \\
\hline & 6 Р.м. & 78 & $103 \cdot 2^{\circ}$ & , & 7. & 9 A.M. & 96 & $105 \cdot 1^{\circ}$ \\
\hline , 29. & 9 А. М. & 68 & $102 \cdot 2^{\circ}$ & , & , , & 6 р.м. & 110 & $106^{\circ}$ \\
\hline , , & 6 р.м. & 72 & $102 \cdot 3^{\circ}$ & ," & 8. & 9 А.м. & 110 & $105.4^{\circ}$ \\
\hline , 30. & 9 A. & 80 & $104^{\circ}$ & , & ," & $10 \cdot 30$ A.3. & 108 & $105 \cdot 4^{\circ}$ \\
\hline & 6 P.M. & 86 & $103 \cdot 4^{\circ}$ & , & , & 1 P.s. & 108 & $106^{\circ}$ \\
\hline,$\quad 31$. & 9 А. М. & 75 & $102 \cdot 3^{\circ}$ & , & , & 3 P.M. & 86 & $105 \cdot 3^{\circ}$ \\
\hline , , , & 6 р.и. & 66 & $103 \cdot 1^{\circ}$ & & , & 4 р.M. & 72 & $104.4^{\circ}$ \\
\hline June 1. & 9 А.м. & 60 & $102^{\circ}$ & & , & 4.30 P.M. & & ath. \\
\hline
\end{tabular}

On the last day there was a copious discharge from nostrils and mouth. Horse kept on its legs till the last, and dropped down dead without a struggle.

The capillaries of the skin and mucous membranes are especially implicated in the congestions, and at all times there is a tendency to gangrene and decomposition of the extravasate, the products of which, being absorbed into the circulation, give rise to septicæmia and death. In some instances, thrombi form in the vessels of the parts more extensively and persistently swollen, and induce sudden death by embolism in the vessels of some vital organ, or a more protracted one by pyæmia. 
In other instances, effusion poured out into the lungs is converted into a fibrinous exudate, which tends rather to a retrograde metamorphosis than to organisation, forming a caseous or semicaseous mass, and interfering most materially not only with the integrity of the organ, but with the general health of the horse.

The more particular symptoms are petechial spots, of a dull mulberry or purple hue, on the visible mucous membranes. There is very often hæmorrhage from the mucous surfaces, the discharged blood being dark in colour and often foetid in odour. Extravasations also occur in the substance of several viscera, more particularly the lungs, spleen, and liver. The limbs, lips, and other depending parts of the body swell, the swelling arising from extravasation of blood into the areolar tissue, and from the transudation of serum, and feebly coagulable lymph. The swellings are characteristic: they are generally uniform, extending perhaps the whole length of a limb, and terminating superiorly very abruptly, as if a cord had been drawn around the part. They are painful, hot, hard, and in those portions where the skin is barely covered with hair, as the lips, nostrils, and inside of the thighs, shining; and if uncoloured, petechial spots are seen upon its surface, but where it is dark-coloured no spots are discernible. Vesicles of about the size of a pea are present upon the lower parts of the limbs, around the hock and fetlock joints; these burst and discharge an amber-coloured acrid serosity that scalds and excoriates the surface of the skin over which it flows. Cracks and fissures appear at the flexures of the limbs, from which an unhealthy amber or purple coloured discharge issues. Swellings appear about the sheath, abdomen, and breast; these, in the earlier stages often disappear from one part of the body and reappear in another. In many instance the face, lips, nostrils, and eyelids become fearfully swollen; the swelling being tense, uniform, and ending abruptly about the forehead. This swelling of the head is apt to cause a fatal termination by interfering with the respiratory function; the swollen nostrils diminishing the calibre of the nares, great dyspnœa is thus induced. By interfering with the movements of the tongue and jaws it also prevents the animal from feeding, and thus becomes an additional cause of debility. The skin over the swollen parts has a great tendency to slough, leaving large open and unhealthy looking sores, which 
discharge an unhealthy, fotid sanies. The pulse is feeble, often futtering, dicrotonous-double, and there is great prostration of the vital powers. The bowels are at first generally constipated, but purgation is easily excited. Sometimes the frees are mixed with blood, and abdominal pains are often present. In some instances there is soreness of the throat; but in purpura it is an accidental complication, and absent in the majority of cases. There is very often a hoarse or hollow cough, and a discharge of coffee-coloured fluid from the air passages. The petechial spots on the nose frequently slough, and leave a raw surface, from which issues an abundance of dark-coloured sanies, causing a snuffling sound in the nasal passages; and in some instances the tongue has been found swollen, with large, darkcoloured vesicles on its surface, containing much fotid material.

Some cases will commence to improve under proper treatment on the third or fourth day. Others take a much longer period, the fever being of a remittent character, aud at all times the animal is liable to exacerbations from very trivial canses.

It is with difficulty that the animal can be made to move, owing to the stiff and painful condition of the limbs; it will often stand rooted to one spot for hours or even days together.

The urine is generally ligh, often dark-coloured, and emits a strong odour of ammonia; it contains much solid matter, and if kept in a vessel very soon undergoes decomposition.

In many instances the enteric complications are very grave and important, and in every case there is a danger of sudden extravasation into the intestinal mucous membrane and alimentary canal, the extravasations being so excessive as to cause rapid death from internal hæmorrhage.

Causes.-In the great majority of instances purpura occurs as a sequel to some debilitating disease, more particularly catarrhal fever, and its origin can in most instances be traced to bad ventilation or drainage. When an animal suffering from catarhal fever is kept in a well-ventilated, well-drained stable, and otherwise properly cared for, purpura is scarcely ever seen; but if such an animal is housed in a badly drained, ill-lighted, and defectively ventilated stable, in fact, when it is compelled to inhale the products of decomposing urine, freces, and of its own breath for several days together, the blood becomes so empoisoned with effete products that it loses its integrity, accumulates in the 
capillaries and smaller veins of loose structures, more p.rticularly in the depending parts of the body, constituting those swellings which are so characteristic of purpura, and when withdrawn from the body it is only feebly coagulable. Again, I have witnessed the occurrence of the disease in loorses apparently recovered from influenza and strangles, placed to work or taken to exercise while still debilitated.

In rarer instances purpura may appear as a primary disease, traceable to defective drainage, ventilation, or bad food.

I have witnessed a few instances where death has occurred in the horse without the usual external manifestations; but where the post mortem examination revealed many of the characteristics of this blood disease, sometimes the animals have suffered from enteritis, sometimes they have sunk without manifesting any pain, have refused food, hung down their heads, the surface of the body being cold, they have become pulseless, and died in a few hours.

In one instance the animal suffered from several convulsive fits for three days, and died in one of them, the post mortem revealing darkness and fluidity of the blood, petechial spots on several internal organs, more particularly on the cerebro-spinal meninges. In this, as well as in the other cases, a careful examination of the tongue prior to death enabled me to detect the nature of the disease. This organ had a peculiar mulberry, purple, or claret colour, and that was the only symptom of blood alteration that could be detected, the Schneiderian memurane and conjunctivie being merely injected.

It may safely be concluded that the causes of purpura are of a septic nature, and are due to the absorption of products of decomposition extrinsic to the body; to the severity and rapidity of tissue change within the body, either owing to a previous disease or debilitating circumstances, and to their accumulation when naturally generated, owing to impairment of the excretory organs.

The acute symptoms are generally of a remittent type, ${ }^{1}$ and when the practitioner is consoling himself with the belief that the animal is improving, it often happens that at the next visit the symptoms have become much aggravated, and the patient is rapidly carried off, dying perhaps on the second, third, or fourth day. In many instances again the acute

1 This remittency rather points to the conclusion that purpura is not only due to the products of surrounding putrefaction, but to the presence of a inalarial or miasmatic non-contagious facultative microbe. 
symptoms subside, and the animal begins to feed, but rapilly becomes emaciated, with great muscular debility, and inability to rise after lying down; these symptoms being accompanied by, or independent of, extensive sloughings of the swollen parts. When sloughing takes place the skin and subcutaneous tissues assume a dry, leathery appearance, and separate tardily from the subjacent living structures; the discharge from the nose may continue, and the animal may die from repeated exacerbations, exhaustion, pyæmia, glanders or farcy, or from gangrene of the lungs, on the third, fourth, or even sixth week after attack. In some cases abscesses may be detected in various parts of the body, in the glands; or the lungs, liver, kidneys, \&c., may be infiltrated with purulent accumulations.

The post mortem appearances of those which have died during the early and acute stages of the disease are as follows :-

Darkness and fluidity of the blood; the subcutaneous tissues of the swollen parts filled with dark red or cinnamon coloured, feebly coagulable exudate. Dark spots will be found on the thecre of muscles, and in the muscular structures of various parts of the body. They do not penetrate deeply into the muscular tissues, but the stain gradually decreases as the muscle is cut into, and some trace of it may be found to extend perhaps an inch into the thicker muscles. The various serous membranes-the pleura, endocardium, pericardium, and cerebral meninges-will be covered with petechial spots, the mucous membranes stained black or blackish-green. This colour will not be uniform, but interspersed with petechial spots, and in some instances the membranes will be covered with a thick layer of imperfectly coagulated blood $;^{1}$ and, again, cinnamon-coloured, gelatinous coagula will be found on the heart, along with a serous effusion, filling the pericardial sac. The dark spots become black on exposure to the air. The blood has a violet tint, and is variously altered on exposure; in some instances it reddens, in others, like the spots, it becomes darker. Kept in a vial closely corked, it will retain its fluidity for a very long period. (Healthy blood, to which ammonia has been adeled, ean scarcely be distinguished from the blood of purpura.)

1 I have a beautiful drawing, given me by Mr. C. Stephenson, Newcastle, of a brain and spinal cord of a horse which died comatose from purpura, showing the arachnoidean cavity, cerebral and spinal, filled with extravasation. 
The mesenteric and other glands are generally congested, friable, and enlarged; the liver, spleen, and kidneys are variously altered,--sometimes merely congested, sometimes enlarged, softened, and containing a large quantity of dark-coloured fluid blood; the lungs dark, enlarged, pigmented, and more or less decomposed; the bronchi and trachea filled with dark-coloured extravasation.

Treatment.-It is useless attempting to treat this disease without first removing the animal from all offensive smells, bad drains, and bad ventilation. Pure air, light, warmth, and comfort are the first essentials. Secondly, it must be borne in mind that the great danger of a suddenly fatal termination is from extravasation into some internal organ, or even into the subcutaneous tissues. Some cases may die from the empoisoned condition of the blood, without the occurrence of any extravasation or even congestion, but these are rare compared with the first named.

For the purpose of overcoming this tendency to extrarasation, styptics, such as turpentine or ergot of rye, or astringents, as the tincture of the terchloride of iron, are sometimes successfully employed. If these are given in combination with an oleaginous purgative, any astringent effect which they might otherwise exercise on the alimentary canal is prevented. It must, however, be remembered that extravasations depend more upon the condition of the blood than upon the blood-vessels, and remedies which are calculated, either by their antiseptic or oxidizing properties, to alter the abnormally fluid condition of the blood, are better calculated to attain the object than those which merely act upon an effect of the disease. For this reason, the chlorate of potash has been prescribed, and with marked results. Whether this salt acts as a direct antidote to the septic poison or not, is a matter which I cannot explain. It is a fact that blood, when drawn from an animal suffering from purpura, or from one in a state of health, has its coagulating properties much increased when a small quantity of this salt is added to it. I have kept blood thus treated in a firmly coagulated condition for more than a year. The coagulation is so rapid and so firm that scarcely any serum is pressed out; and, what is most remarkable, there is but little contraction of the clot.

Acting upon my experience as to the effect of this salt in 
promoting coagulation, and at a time when physiologists believed that fibrin was a natural constituent of the blood, I commenced to treat purpura with the chlorate, for the purpose, as I then supposed, of increasing the fibrin in the blood, and thus prevent the occurrence of congestions and extravasations. The result was most satisfactory; the swellings rapidly diminished, and restoration to health ensued in the great majority of instances. One ounce in the twenty-four hours is quite sufficient after the first dose, which may be from one-half ounce to an ounce, given at once. If more than this be given, intestinal irritation is apt to be produced, and the disease thus complicated. In addition to the administration of the chlorate, it is necessary that the excretory organs perform their functions, in order that the morbid material and the products of tissue changes be eliminated from the system; for this purpose oleaginous aperients are to be administered. The latest method of treating purpura is by the intratracheal injection of a solution of iodine, and to the success of which I can bear testimony from actual experience; in fact, the effect has been most remarkable, improvement taking place very quickly in cases which threatened to terminate fatally.

I do not recommend those strong doses used by some practitioners. The solution I use is male as follows:-Iodine 4 grains, iodide of potash 8 grains, water 1 ounce. I have not fouml it necessary to use a larger quantity than this in the twenty-fonr hours; the whole may be injected once, or it may be divided into two injections daily.

Beyond this, I think medical interference is uncalled for; and it may be stated here that much advantage is derived from non-interference, both in this and many other ailments of the lower animals.

The convalescence is very often prolonged, great prostration remaining for a long time. The animal must therefore have a liberal supply of good food-oats, beans, hay, \&c.-and be carefully groomed and tended. The mineral tonics may be administered, and daily and carefully regulated exercise given.

External treatment.-If the head be much swollen, and there be a difficulty in breathing from tumefaction of the nostrils, continuous cold sponging must be ordered. The sponge may be dipped in cold water or in some weak astringent solutionvinegar and water, or the terchloride of iron tincture, largely diluted; if the cold sponging fails to reduce the swelling, warm 
may be substituted, particularly if the weather be very cold; but beyond doing this to the head, I am of opinion that all other interference does much mischief.

The swellings are but the result of the condition of the blood, and when the latter is restored to its normal standard the swellings will disappear. Indeed, an abatement of the swelling in one part of the body is often succeeded by tumefaction in some other, and perhaps more important structure or organ. The swellings are generally metastatic, and when they are confined to those parts of the body where they cannot endanger life, it is far better to leare them alone.

Puncturing the swellings is usually recommended and generally practised. In very severe cases of purpura admission of air in to the degraded tissues causes deep and sometimes intractable sloughings. Punctures are, therefore, inadmissible.

If there is dyspnoea from swelling of the throat or nostrils, tracheotomy must be performed, and it ought always to be done early, as it is essential that the lorse be enabled to breathe freely; in fact, it must not be forgotten that oxygen in abundance is required by the vitiated blood. The incision into the trachea should be antiseptically treated, as there is a great tendency to gangrene of the exposed structures; entrance of the gangrenous products into the bronchi, inducing septic pneumonia, thrombi in blood-vessels, pulmonary gangrene, and death.

\section{II.-SCARLATINA-SCARLET FEVER.}

A febrile disease, characterised by an eruption on the skin, petechial spots on the nose or under surface of the lips, soreness of the throat, and sometimes suppuration in various parts of the body, particularly in the submaxillary space.

Unlike the scarlatina which attacks the human being, it is a non-contagious disease, generally attacking but one or two horses in a large stud, amongst which some form of epizootic disease is at the time prevalent.

Dr. Copland, in his Mcdical Dictionary, article "Skin," says"First, That scarlatina was originally a disease of the horse, and that it formerly occurred, and has recently occurred, epidemically or as an epizootic among horses. Secondly, That it was communicated in comparatively modern times from loorses to man. Thirdly, That it may be, and has been, communicated also to the dog." If this be true, scarlatina of the present time inust be very different from what it has been in the past. I have seen numbers of horses suffering from it, but in no case did it ex- 
hibit any tendency to spread by contagion or infection; and I am of opinion that it is impossible to transmit it from the horse to any other animal.

Scarlatina is divided by medical writers into-(1.) Scarlatina simplex; (2.) Scarlatina anginosa ; (3.) Scarlatina maligna; and (4.) Scarlatina latens.

Of these the two first are observed in the horse, what is called the malignant form being identical with purpura; it is quite possible, however, for any of the forms to degenerate into purpura.

Scarlatina is usually associated with epizootic catarrh, and occurs in animals that have been for some days suffering from that disease; and the production of such an alteration in the blood as induces the scarlatina is due to defective ventilation or drainage of the stable in which the animal has been kept, or to over-crowding, by which the air becomes loaded with decomposing animal matters. Sometimes a weak constitution will convert a catarrh into scarlatina, and the severity of an epizootic disease may alter the blood, and give origin to scarlatina.

Semiology.-1. Scarlatina simplex.-On the third, fourth, or even as late as the sixth day after the commencement of epizootic catarrh, the animal is seen to be covered with blotches, upon the face, neck, body, and extremities. The blotches elevate the hair, but in many places there is scarcely any elevation of the skin, for if the hand be passed lightly over the apparent swellings, the skin is felt but little altered. In other parts of the body, particularly upon the inner aspect of the thighs, actual elevations of the skin in the form of rounded pimples can be both seen and felt. The nasal membrane will be covered with scarlet spots of variable size, and there will be a discharge from the nostril of at first a thin serous mucus, which afterwards becomes yellow or yellowish brown. The limbs are generally swollen, and the animal stiff in consequence. In some instances, no eruption is present, and the only evidence of scarlatina is found in the Schneiderian membrane, and perhaps the membrane of one nostril only will be covered with minute scarlet spots that escape the observation of all but the prastitioner.

Soreness of the throat is almost a constant symptom, and if it has preceded the rash, it will in all probability be more or less increased; but it by no means follows that soreness of the throat will appear concomitant with the rash. In a few days 
the rash and scarlet spots begin to disappear, and on the rash declining, some desquamation of the cuticle takes place, the skin being scurfy, and the coat dirty for some time afterwards.

2. Scarlatina anginosa.-The symptoms at first may be those of the simple form, which, instead of disappearing, may continue to increase in severity. The limbs rapidly swell, the swellings appearing in lumps or masses, large, hot, painful, and numerous. These swellings may also appear on the body and face; they are inconstant in their seat, aften disappearing from one place and appearing at another, whilst the intervening spaces may be covered by a rash similar to that of urticaria, and by blotches not elevated to the touch. The skin covering the blotches will often be found moist, and an amber-coloured serosity will ooze from it. The petechial spots on the membrane of the nose increase in size, and become more intense in colour, assuming a tinge of purple, more especially at their centres; in other cases they coalesce, forming one large block, which covers the whole nasal chamber.

The soreness of the throat now becomes very great, and is accompanied by a loud moist cough, and at each cough large quantities of a yellowish-red mucus are discharged through the nose and mouth. There is difficulty in swallowing, and in some cases in breathing, the inspiratory act being accompanied by a roaring noise, and by a snuflling nasal sound. The submaxillary lymphatic glands become enlarged, tender, and inflamed, and suppuration occurs after the other symptoms have begun to disappear.

In favourable cases the soreness of the throat will recede with the eruption about the fourth or fifth day, whilst in others it will remain for some days after the disappearance of the rash. In all cases that recover, however, both the exanthem and angina will have disappeared by the ninth or tenth day, leaving the animal weak, emaciated, with swollen limbs, and in a more or less unthrifty condition for some time longer.

The fever is usually proportioned to the severity of the sore throat, and very often, but not always, to the rash. In slight attacks, the fever, rash, and angina are of a mild character; but in the severer forms they are proportionately grave. The pulse, which is always of a weak or feeble character, varies from 60 or 70 in the mild, to 90 or even 100 in the severe forms; the respiratory 
movements are accelerated, and very rapid when congestion of the lungs is present; the urine is scanty, and of a thick yellow or brownish colour, smelling strongly of hippuric acid, and quickly becoming ammoniacal; the bowels are constipated, but diarrhcea is easily induced.

Mr. Haycock describes the malignant form. I am of opinion however, that what he thus describes is purpura, or a combination of purpura and scarlatina.

For a number of years I was of opinion that purpura and scarlatina were one and the same disease, but I have hacl occasion to alter this opinion, as typical cases of both diseases are not uncommon in Edinburgh. The distinguishing differences between the two diseases are to be found in the character of-1st. The petechiæ, which, in scarlatina, are composed of minute dots, forming a blotch by coalescence; the spots may be as large as in purpura, but each is composed of several smaller ones; they are scarlet in pure scarlatina, of a dark purple in purpura. $2 d$. The sore throat. This is never absent in scarlatina, and but rarely present, unless it is a symptom of previous disease, in purpura. $3 d$. The swellings. In scarlatina, these are at first in the form of lumps or masses, whereas in purpura they present an even surface, occupying the face, or a whole limb or limbs, and terminate abruptly above, as if a cord had been drawn tightly around the part. 4th. Scarlatina is often associated with swelling and perhaps suppuration of the glands, whilst in purpura this is not present; and finally, the latter disease is characterised by a tendency to sloughing in various parts of the body, and occasionally by gangrene of some extreme parts of the organism, such as the ears, which will present a shrivelled and blanched appearance, become dry, and slough, leaving a raw, unhealthy surface. The animal temperature may not distinguish the one disease from the other. If scarlatina be severe, the thermometer may register $103^{\circ}$, or even higher; in purpura this would indicate a mild attack.

Scarlatina is sometimes followed by inflammation of the joints, which is supposed to be rheumatic in character, and by suppuration in various parts of the body; but I am not aware that it is succeeded by renal dropsy, or associated with albuminuria, as in the human being. There is generally more or less œedema of the limbs, breast, and abdomen, but these are not 
necessarily due to any renal complication, as they are common sequelæ to most debilitating diseases in the horse.

Treatment.-In the milder forms of the disease it is sufficient to keep the animal in a warm, well-ventilated, light, loose box, to feed it on laxative food, and give small and repeated doses of the nitrate of potash, hyposulphite of soda, or chlorate of potash, in the food or water. The body must be elothed according to the weather, and the general comfort of the animal attended to.

In the graver forms, the animal must be carefully watched, more especially with regard to its breathing, for in some cases the glottis and other structures of the throat rapidly swell, the larynx becomes constricted, and the animal may die from suffocation. Whenever this is threatened, tracheotomy must be performed; and in all cases where the breathing is difficult, and accompanied by a loud roaring noise, this operation is to be resorted to, in order that the animal may obtain pure air to oxidize its already impure blood.

The throat is to be repeatedly bathed with hot water and enveloped in warm poultices, and the animal made to inhale the steam of hot water. The mouth is to be frequently washed out with salt and water, and if the coryza is excessive some of it may be applied to the nose. If the face and nares are swollen, they must be frequently bathed with cold water, and afterwards dressed with some astringent lotion, such as a solution of the tincture of terchloride of iron. If the bowels are very torpid, a gentle laxative may be administered, such as one pint of linseed oil, but active purgation must on no account be induced; the nitrate of potash may be given freely in the food or water, and abundance allowed of the latter, which must be cold and fresh. If signs of purpura supervene, the chlorate of potash must be substituted for the nitrate. If much depression is present, draughts of spirits of nitrous ether may be given, provided deglutition is not difficult; but if the act of swallowing causes pain and cough, nothing should be forced upon the animal, or suffocation may ensue. Indeed, where the throat is very sore, even the oil must be withheld, and the bowels relieved by enemas, or by the sulphate of magnesia dissolved in the horse's water. If he will drink this, it has a very good effect, but many horses will not drink at all if salts are dissolved in their water. After the first few days milk, or milk with eggs, is to be 
allowed in abundance, in order to support the animal strength. When the secretions are restored to their natural condition, and the soreness of the throat a little abated, a small ball containing carbonate of ammonia_the ball being well oiled-may be given two or three times a day with great benefit, and later on the mineral tonics, with bark or quinine. If soreness of the throat and cough remain after the subsiclence of the rash and fever, ulceration is to be suspected, and this can occasionally be detected by an examination with the oral speculum.-(See Principles and Practice of Veterinary Surgery, page 511.) It may be combated by a blister to the throat, or by direct application of the nitrate of silver solution to the part by means of a sponge tied to a piece of cane.

When suppuration has occurred, the abscess is to be opened, as the abscesses of scarlatina often become languid, and do not burst readily. ${ }^{1}$

Whenever the fever abates, and other signs of convalescence appear, food of the most nutritious quality must be allowed in moderate-not over-abundant-quantity.

Exercise should not be enforced until all febrile signs lave disappeared, and the animal has to some extent regained its strength; even then much exercise is to be strictly forbidden. I have seen the most severe and rapidly fatal purpura caused by exercising the animal too soon and too severely.

I have said nothing about the treatment of the external swellings of the limbs. I am of opinion they should not be interfered with, as they are but expressions of a condition of the blood which does not endanger the life of the animal, and will disappear spontaneously.

The post mortem appearances are similar to those of purpura, with the addition of inflammation and swelling of the throat.

1 The frequency of abscesses in scarlatina points to the conclusion that, in addition to its own peculiar miasma, one or more of the cocci of suppuration is present,-most probably the Staphylococcus pyogenes allus. 


\section{CHA P TER XXXII.}

\section{SEPTIC BLOOD DISEASES.}

Diseases due to ordinarily non-contagious facultative parasites, but which become infective by inoculation in suitable soils.

The most deadly microbes are Vibrio septicus of Pasteur, the pyogenic bacteria, especially the streptococci, and some are sufficiently viruleut to poison the most healthy; but generally the animal body whilst in a state of health is not affected by their presence, unless, indeed, they are very numerous.

Septic diseases are induced in two ways:- first, by the entrance of the microbes themselves into the general circulation (scpticcemia), in which case the symptoms and results are not proportionate to the dose,-i.e., fatal results may follow a very small dose; and sccond, by the entrance of microbic products-ptomaines only (sapramia) - in which case the symptoms are proportionate to the dose,-i.e., if large, the results may be immediately fatal, if small, it is soon excreted, and, having no reproductive powers, the effects soon pass off.

These microbes in their normal condition exist in external media, and only induce disease under circumstances which have rendered the animal body susceptible to the morbific effects of themselves or their ptomaines. For example, the body whilst in a state of health can generally resist the bacteria, unless very numerous; but when an organ or tissue injured-as in a wound -or diseased is exposed to the influence of such germs, they or their products overcome the resistance of the injured or diseased structures, penetrate the blood-stream, and give rise to grave disturbances, and even death. In proof of this I may refer to the experiments of Burdon Sanderson, who found that putrefactive fluids injected into the healthy peritoneal cavity of guineapigs produced no ill effects, but when the peritoneum had been irritated by an injection of some irritant, such as mustard and water, the virus penetrated the irritated membrane, inducing a general septicæmia, with rapidly fatal results. 
Putrefaction is a form of fermentation, and as the yeast plant in alcoholic fermentation splits up the elements of the sugar into those of alcohol by a process of digestion, so the various putrefactive microbes consume the constituents of materials in which they are locked, multiply rapilly when their food is abundant and suitable, alter its chemienl combinations, the products of such change being excreted, with or without fœtor, in the form of various chemical combinations, S.H. and N.H. and other organic bases, designated ptomaines, sone of which are injurious and some actually preservative. The writer's experience leads him to conclude that when the putrefactive materials are of vegetable origin, the products, subjected to sunlight and a plentiful supply of air, as in ponds, are generally imocuous, so far as the domesticated animals are concerned; but when they are of animal origin-cadaveric alkaloids, human ordure, decomposing animal fluids or solids, blood, milk, \&c. contaminating drinking water, the results are often disastrous.

Many of my readers have repeatedly seen eattle stancling in ponds cluring the hot summer weather for hours, polluting the water with their excreta, drinking freely of it, although in appearance it may resemble a green coloured gruel or porrilge more than water. Tet they take no harm, incleed seem to thrive on it. If such water be examined microseopically it will be found to be swarming with bacteria of various kinds. But if such water contain the products of the decomposition of animal fluids or solids, even when sunlight and air are plentiful, there will be a repulsive odour, and unless foreed, animals will not drink it; but when compelled for want of other supplies, the consequences are very prejudicial, the animals become emaciated, unthrifty, hidebound, often have diarrhœa, with irritation of the respiratory uncous membrane, cansing the animal to congh, and if the water be not ehanged death may result. The post mortem will reveal a general anxmic condition, with bronchopnemmonia, and perhaps some degree of congestion of the gastro-intestinal mucous membrane; as a rule, however, the lung lesions are in chronic cases more pronounced than those in the digestive tract. In some instances, and when the source of contanination is abundant, the animals may rapilly succumb to gastro-enteritis, witl colliquative and fotid diarrhcea, great prostration of strength, high fever-106- ${ }^{\circ} \mathrm{F}$. or more-and other 
signs of putrid intoxication, arising from the absorption of the microbes or of the cadaveric ptomaine.

These observations only apply to the putrefaction of vegetalle materials when occurring in ponds or ditches exposed to air and sunlight, for if the same materials are retained in leaky liquid manure tanks, and thus contaminate pump wells o1 other source of the supply of drinking waters, their effects are quite as disastrous as those emanating from animal sources. It is difficult to explain this fact, unless it be admitted that the alga and other vegetations of low origin, which grow abundantly in ponds exposed to sunshine and air, either destroy the septic microbes or produce an anti-ptomaine, which overcomes the virulence by breaking down the toxic materials into simpler forms-ammonia, \&c.

It is difficult to conceive a general septicemia without the actual presence of the microbes in the circulation, and undoubtedly, in many of the most fatal forms, the organisms are existent in the blood. As a rule, however, the blood does not seem to be a suitable medium for their development and growth; they soon disappear from it, being anaërobic, are destroyed by the oxygen, or are arrested in the capillaries of the liver, spleen, kidneys, \&c., and are there quickly destroyed; their virulence is thus overcome, and the disease is not transmitted from one animal to another.

In many cases, however, the germs remain external to the tissues, and act solely through their products, which gain entrance into the circulation and produce their virulent effects - sepsine poisoning.

In many instances septic microbes or their products gain entrance into the body from without-trammatic septicæmia, septic metritis, \&c. ; but there are other instances where they are formed within the body itself, and nicrobes, always existent in the intestines, may give rise to systemic and local disturbances of a grave or even fatal nature.

The intestinal mucous membrane-always in contact with decomposing materials - has the power, whilst in health, of excluding them from entrance into the circulation; but when that membrane is irritated in any way and by any cause, it not infrequently loses that power of exclusion, and the ptomaines, indol, skatol, gaseous products, \&c., instead of being climinated, 
are absorbed into the blood, inducing high fever, local inflammation, diarrhœa or dysentery-in the form called white scour in young animals - and even death.

I think the above in a great measure explains why inflammation of the feet is such a frequent concomitant of bowel affections, even a slight irritation of the mucosa being sufficient to induce it, such as that arising from an ordinary purgative administered whilst the animal is in perfect health; absorption of gaseons products, as in flatulent colic; of putrefactive products in septic metritis; and in broncho-pneumonia, when the catarrhal products have been retained in the bronchii, and there undergone putrefaction. Again, it may be stated that the sudden deaths, charactcristic of the disease termed braxy in sheep, are due more to the rapid evolution and absorption of noxious gases arising from the rapid fermentation of food eaten whilst in a partial state of decomposition, than to the tympanites by which braxy is generally recognised.

This form of poisoning has been named stercoræmia and intestinal septicemia, and is said to supervene upon a number of conditions, such as insufficiency of gastric juice to neutralise the majority of the germs which pass through the stomach, \&c.

In order to make this subject more easily comprehended, I have ventured upon the following arrangement:-(A) Sapriemia, where the products only are absorbed; and (B) where the microbes themselves, as well as their products, gain entrance into the circulation, and there increase and multiply. Under the first head may be included--

I. Traumatie Fever.-The mildest form of septicrmia. Causes. -Aseptic tissue necrosis, as in subcutaneous wounds, or in any effusion of blood, fibrin, or serum which does not undergo scpsis.

Symptoms.-Sudden e?evation of temperature-two to four degrees-as soon as shock has passed off", no other constitutional disturbance, the temperature falling in a few days, according to the extent of the absorption. If disturbance occurs, it is certain that some complication has arisen.

II. Malignant cedema (Koeh), gangrenous septicemia (Pasteur), is caused by the small sporogenous oedema bacilli called Vibrio septicus by Pasteur, which exist in large numbers, particulirrly in the upper layers of soil, and infection is easily produced by 
subentaneous connective tissue inoculation, but not by intravenous injection, or when applied to superficial scratches on the skin; the microbes, being anaërobic, are killed by the oxygen, and granulating wounds form an insuperable barrier. Chaureau says that a first infection confers immunity.

Some observers state that whilst the guinea-pig, goat, sheep, dog, horse, fowls, pigeons are susceptible to malignant œdema inoculation, cattle are absolntely refractory; but Kitt states that he has induced the disease in calves as well as in the other animals specified above; and further, that these bacilii are probably the cause of several diseases in cattle, such as septic parturition fever, surgical tumefactions, progressive inflammation of connective tissue, \&c.

In the cedema of the septic centre the bacillus is found in the forn of a rod with a spore at one end, or devoid of the spore, in which case it is of a slightly greater length; in serous membranes it reaches a considerable length, and becomes segmented into non-sporulated sections, and only invades the circulating blood towards the end of the disease or after death. They are little affected by antiseptics, but are killed by boiling for fifteen minutes and by a dry temperature of about $250^{\circ} \mathrm{F}$, and Coruevin states that by continuing the action of heat and antiseptics he has obtained a vaccine which confers a safe immunity on animals, and particularly the dog, against septicemia.

The bacillus is a rod measuring $4 \mathrm{~mm}$. in length by 1 mnı. in breadth, with or without a terminal spore, but, as already stated, it may be much longer in serosities; it is motile, having very active flexuous movements, quickly arrested by contact with oxygen, and slowly loses its virulence through the influences of putrefaction (two months, thus differing from the Bacillus Chauveau, upon which putrefaction has no effect). Chauveau and Arloing state that corrosive sublimate 1 to 500 does not kill it, and a three per cent. carbolic solution has no effect unless assisted by heat. Sulphurous acid, however, seems to have a rather powerful cffect upon it. It is preserved when dried even at $100^{\circ} \mathrm{F}$.

Symptoms.-Experimental inoculations in the connective tissues, particularly when protected from air, are followed by an celematous, doughy, sensitive, and crepitant swelling, extending in all directions, and there is intense reaction. The 
connective, adlipose, and muscular tissues in the neighbourhood become the seat of gelatinous infiltration containing a large quantity of very fœetid gases, and a reddish-yellow liquid teeming with the characteristic vibriones, absent in the blood during life, and only found in small numbers after death. The central part of the swelling soon lases its sensibility, and becomes moist, clammy, and cold-gangrenous; the circumferences of the swelling, however, are hot, tense, and extremely sensitive, the vibriones having evidently abandoned the centre, as only putrefactive germs can now be found therein, and invaded the surrounding tissues, where they continue to live at the expense of their vitality, and so on in a regular invasion until a general infection results, manifested by pulmonary oedema and congrestion of the intestinal mucous nembranes, the bacilli being found abundantly in the serous discharges, and but sparsely in the blood. The disease may follow surgical operations, particularly castration, when performed with unclean instruments.

III. Stercorcmia-Intestinal Toxcemia.-Absorption of bacterial products from any tissue or a mucous membrane, whether abraded or not-(a.) when the products are those of pyogenic bacteria, or (b.) those of ordinary putrefaction, e.g., Protcus $L$. colicommunis.

Symptoms. - These vary in propartion to the dose. (a.) The temperature rises several degrees, and remains so till absorption ceases, from the products becoming arrested in the course of natural drainage, such as free suppuration, free incisions, and irrigation, when arising from a wound. Constitutional disturbance well marked. (b.) When the dose is large, rapid collapse may follow.

(c.) Surgical shock is simply a sapremia, in which death occurs in one to two days after severe symptoms, especially those seen after abdominal operations, castration, \&c., owing doubtless to the enormous rapidity with which the peritoneum alusorlis the toxic products.

IV. Hectic.-A chronic form of sapriemia seen typically in long-continued " mixed infections," in chronic tuberculosis, where there is a constant absorption of small quantities of virus.

Symptoms.-Irregular, with a tendency to periodicity of elevations and remissions, progressive emaciation, loss of appetite, and death from exhaustion. 


\section{POST-PARTURIENT SAPREMIA AND SEPTICAMIA,}

Retention of the fotal membranes, even for an hour, in the mare is apt to be succeeded by a most acute and fatal form of septic infection manifested by the symptoms of acute metritis (which see). The cow, however, seems to resist the rapid invasion of the organisms and their products, and manifestations of the septic invasion are not observable in the majority of cases for some time after parturition.

The fœetal membranes are naturally expelled in a few hours, sometimes immediately after the birth of the offspring; and in a few days afterwards another discharge appears, known amongst cattle people as the "second cleansing,"-lochial discharge, consisting of mucus tinged with streaks of blood, and the débris or cast-off products of the uterine mucous membrane. Occasionally, however, this second discharge is retained, and undergoes microbic changes, the ptomaines of which are absorbed-and it seems to be a true sapræmia-into the circulation, giving rise to the following symptoms.

From the third to the sixth week after calving the cow begins to lose condition, although her appetite at first is not diminished; but gradually rumination is suspended, and the appetite entirely fails; a peculiar odour is now noticed to emanate from lier; the milk falls off in quantity, and has the disagreeable smell; it has also a disagreeable flavour, and is bluish in colour. Bowels are constipated, and the fæees are of a dark colour, and covered with mueus, the vaginal and uterine secretions suspended, and the vulva has a dried-up and contracted appearance.

The urine is high coloured, often albuminous, and has the same smell as the other secretions; the pulse is not much affected in the earlier stages, but if the disease advances it becomes soft, and sometimes dicrotonous; the temperature varies from $103^{\circ}$ to $105^{\circ} \mathrm{F}$.

In the more advanced stages there is grinding of the teeth, which may end in delirium and death. Mr. John Bell, of Carlisle, who has had great experience in the treatment of this disease, prescribes the following:- "Saline aperients combined with calomel at commencement; a good large poultice of flour and mustard to the loins; and when the brain symptoms set in, a dose or two of chloral hydrate, which has invariably the desired 
effect. Recovery is hastened by the administration of tonics. It is essential to diet the animal upon sloppy food for the first few days, when the early symptoms are observed, or otherwise the treatment is of no avail."

I think the success which seems to have attended Mr. Bell's experience would be enhanced if the vagina and uterus-provided the tube of the syringe could be introduced through the os without force-were washed out at least twice a day, particularly at the commencement of the disease, with watery solutions of boracic acid, Condy's fluid, weak solutions of corrosive sublinate, or other well-known antiseptics; and, to counteract the odour of the intestinal discharges, the hyposulphite of soda might be administered with advantage.

\section{TRUE SEPTICAMIA.}

Multiplication of the Microbes in the body.-The symptoms here do not depend upon the strength of the dose, the entrance of the minutest quantity being sufficient to lead to a fatal result. It is seen in braxy, septic metritis, \&c., and also after the suppuration of a thrombus in phlebitıs, when the septic emboli are carried into the blood stream, lodge in the lungs and other organs of the body, according to the seat of the initial lesion, and as a sequel to strangles and to suffocative catarrh-acute bronchitis-when the catarrhal products are retained in the bronchii until they become decomposed, and thus imprison the blood stream. See Bronchitis.

The symptoms are not in proportion to the dose, as in sapræmia. There is sudden rise of temperature, prostration, weak pulse, and death in a few days or hours from shock or coma; there are small liemorrhages into serous sacs, with diarrhcea, pericarditis, endocarditis, pleurisy, \&c.--See Septic Metritis. Death is often preceded by subnormal temperature. Acute pyæmia resembles septicœmia, indeed it might be described as a secondary septicremia, owing to the microbes in the emboli being set free in the circulation, these multiplying and producing further quantities of septic ptomaines. Chronic pyæmia resembles hectic sapræmia.

\section{BRAYY.}

Septicamia Gangrenosa.-Braxy.-Striking of Blood, \&.c.-This is a well-known disease of sheep, particularly of young one-year- 
old sheep-hoggs-prevailing during the autuinn and winter months, and is known in Scotland under two different terms, namely, "dry braxy" and "wet braxy," the latter form being characterised by more or less effusion of a reddish coloured serosity into the peritoneal cavity. This latter condition is to be looked upon as an endeavour to excrete the morbid material from the circulation, and may be compared to the compensatory pleurisy and hydrothorax witnessed in septic broncho-pneumonia subsequent to parturition, or as a result of the ingestion of food containing ptomaines of animal origin.

The Symptoms.-Unfortunately in the majority of cases a sheep left quite well at night is found dead in the morning, and the shepherd judges of the nature of the disease by the swollen and discoloured appearance of the carcase. But if seen some time prior to the fatal result the first symptom seems to be manifested by a short step, then the animal stands apart from its companions with the head depressed and back arched. Tympanites rapidly follows, the stomach becoming enormously swollen and distended with gases, and is resonant on percussion The other signs are those of colicky pains, lying down and rising alternately, or standing apart with the lead and ears hanging down, the eyes dull, and the back arched; prostration and swelling of the belly rapidly increase, the sheep then goes down, and, although struggling, is unable to rise, when death closes the scene.

The post mortem appearances are analogous to those seen in cattle, namely, a great tendency to rapid decomposition of the blood and tissues; the blood-vessels are full of dark semi-fluicl blood; bloody froth issues from the nose, mouth, and sometimesanus; the flesh has a dark red appearance; petechial spots are visible upon the serous membranes and in the subcutaneous tissues; the abomasum and duodenum are highly congested, and covered with large dark-coloured spots of ecchymosis; the alimentary matters are mixed with blood, and the mucous membrane generally infiltrated in its substance, and coated on its surface with a layer of semi-coagulated, dark-coloured blood. The peritoneal cavity is generally filled with serum, and the bowels distended with foetid gas.

The pathology of braxy has yet to be determined, but it seems to be one of the forms of mycotic yastro-enteritis arising from 
the ingestion of grasses in a semi-putrefactive condition, as it is only commonly seen after the grasses have been more or less destroyed by frost, and is most common in frosty rimy weather, or when a hoar frost has covered the ground. When grasses in this half-frozen and withered state are eaten, the stomach, debilitated by the coldness or low temperature of its contents, is to some extent paralysed, and consequently disabled from performing its function. As its contents become warm, fermentation rapidly takes place, ptomaines and noxious gases are freely evolved, which, gaining entrance into the circulation, set up a fatal septic or putrid intoxication. In order to prevent this fermentation, I would suggest that, where it is possible, the sheep should be fed on hay in the early mornings, in order to moderate the keenness of the appetite, and thus ensure a slower and more perfect mastication; and also, by clevating the temperature of the cold food, prevent lowering the temperature and vitality and function of the digestive organs. When sheep are folded on turnips, "sickness" might be prevented if this were done.

Treatment.-Unfortunately there is seldom any opportunity of testing the efficacy of any remedy, but should there be an opportunity, the hyposulphite of soda might be administered, say in half-ounce doses, dissolved in half a pint of water, for a wellgrown hogg or sheep. This remedy counteracts putrefaction, and has a slightly aperient effect on the bowels; or carbolic acid or any of the other disinfectants are also serviceable, and to prevent death from suffocation the rumen should be punctured, and the gases allowed to escape.

The flesh of sheep which have died, or been slaughtered whilst on the point of death, is generally salted and driedmade into mutton ham-and eaten with impunity in many parts of Scotland, and it can be truly said that it is at least equal, if not superior, to venison, and any one who is fond of high game would thoroughly enjoy a nicely brandered cut of it.

Septicæmia and pyæmia in young animals are known under the terms pyæmic omphalitis, navel-ill, pyæmic arthritis, jointill, erysipelatons disease, also called black spaud in Scotland. Omphalitis and arthritis will be found described in my Veterinary Surgcry.

Even a more marked form of malignant œedema or gan- 
grenous septicemia prevails and is known in Scotland by the term black spaud, a gangrenous form of disease seen in lambs under a week old, and characterised by the skin and subcutaneous tissues surrounding and extending from the umbilicus, becoming slightly swollen and assuming a dark purple colour. The discoloration and swelling are sometimes in large patches, covering the lower parts of the abdomen, breast, and sides, but more frequently they consist of one large diffuse patch, embracing the regions mentioned, and extending to one or both shoulders, hence the term black spaud (shoulder).

This disease sometimes commits great havoc, the author having known as many as ten score lambs being carried away at one sheep farm in a few days.

Etinlogy and Pathology.-If during the first few days of the lamb's life, or before the umbiliens has completely closed, and a hard eschar formed, the unbilical cord be brought into contact with decomposing materials, both the germs and the products of the septic process may gain entrance into the umbilinal canal, and there, acting upon its contents, set up the putrefactive process, which, extending into the surrounding tissues, induces locally congestion and thrombosis of the blood-vessels, characterised by diffuse mulberry coloured patches, with or without suppuration of the cord, and generally great and increasing prostration, and a rapidly fatal collapse, death occurring in a few hours after attack.

On many farms the sheep slaughtered for food are skinned in the lambing sheds, the blood and offal allowed to remain on the ground, the skins thrown across the beams of the roof to dry; and the skins of others which have died of disease or accident are also brought there, and allowed to remain there, very often until the next clipping season.

From the foregoing observations it is evident that infection of the umbilical cord by the products of putrid decompositions which cover the floor of lambing grounds, particularly lambing sheds, is the starting-point of the infection.

Can it be wondered that lambs dropped in such an atmosphere fail to escape the contanination?

Careful observers, whilst agreeing that permanent lambing sheds, contaminated as they necessarily must be by decomposing materials, are the great cause of both black spaud and joint-ill, state that both of these forms of septicrmia are 
more prevalent during cold east winds; and they reason as follows:-1st. That during cold weather the ewe does not cleanse so freely and quickly-indeed in some instances, owing to the debility induced by the cold, expulsion of the foetal nembranes does not occur; that the retained membranes putrefy, giving rise to foetid discharges and emanations: $2 d$. That in order to receive warmth the lamb clings more closely to the mother, and is consequently more exposed to become infected by the septic matters. Many who have experienced losses from this disease have now dispensed with permanent lambing sheds, and lamb their ewes in an open but well sheltered spot, or in temporary removeable sheds.

Treatment. - The disease is not amenable to medical or surgical treatment, therefore its prevention should be carefully studied.

All lambing sheds should be abolished; but if this is impossible, they should be thoroughly cleaned, lime-washed, and disinfected before the commencement of the season, all skins and decomposing materials being first removed. The bedding shonld be dry, frequently removed and replaced; all blood, foetal membranes, \&c. swept away at the end of each day, and before putrefaction has commenced, or else the place will soon become tainted. If the disease has made its appearance, the ewes should be lambed somewhere else. If in the absence of sheds the lambing take place in the open air, the ground should be clean and protected from cold, particularly east winds, as these are acknowledged to predispose to the disease, and some antiseptic dressing, such as carbolised oil or sublimate solution, applied to the umbiliens.-(See Joint-Ill, Veterinary Surgcry.)

Septic diseases may become contagious amongst animals under certain conditions, as in metritis of the ewe. Here the initial or spontaneons attack spreads amongst lanbing ewes, but has no effect upon the males and barren females.

\section{SEPTIC PNEUMONIA OF CALVES AND LAMBS.}

This disease has prevailed to a considerable extent in various parts of England, Scotland, and Ireland during the last few years, committing great havoc amongst calves a few weeks or 
months old, killing them very quickly. Poels, who las studied this disease, states that it is due to the presence of a facultative bacterium which is found in the soil of certain farms, and the disease is thus rendered persistent on such infected lands. It is an ovoid bacterium with rounded ends, with a clear centre, measuring from $\frac{1}{50} \overline{0} \overline{0}$ to $\frac{1}{16000}$ of an inch in length, and $\frac{1}{5000}$ in breadth, staining with aniline colours, but not by Gram's or Weigert's. The same, or a similar bacterium, is found in the broncho-pneumonia of American cattle. The same microbe is said to be found in the diarrhoea of calves- "white scour"and calves fed with small doses of bouillon cultures, about $1 \frac{1}{2}$ drachms, become diseased, and die in one to two days. The same germ is found in the contents of the intestines of healthy calves, but destitute of pathogenic properties, and it is supposed by Jensen that it only becomes virulent under certain abnormal conditions, perhaps attributable to the diet, and that this virulence becomes increased by subsequent passage from calf to calf.

The symptoms are those of pneumonia, with or without diarıhœa.

There is a cough, with discharge from the nostrils, and the catarrhal products contain the microbes, which are thus spread around with the expectoration and freces. Anyhow, it is admitted that the disease is transmitted from one animal to another, and Poels says that he has found the microbes in all the organs; and he is supported by Jensen, who has found them in the blood, spleen, liver, lungs, kidneys, heart, and in the mucous membrane of the intestinal canal.

The disease is occasionally very fatal amongst sheep, particularly lambs and others under one year old (hoggs), and Galtier states that it is identical with pneumo-enteritis of the pig, and that he has transmitted it from the pig to the sheep, guinea-pig, rabbit, and from these latter he has retransmitted to the pig and sheep. He has also transmitted it to the dog, goat, calf, donkey, and poultry, and to a goat in an advanced state of pregnancy, inducing abortion. The foetus when examined was found to have in the thoracic and abdominal cavities a serosanguinous exudate, containing the microbes, and reproducing the disease by inoculation.

The post mortem appearances are those of intense pulmonary congestion, with or withont pleurisy. In some cases a gelatinous 
exudate is found on the pleural surfaces, whilst in others they have simply presented a more or less deeply congested appearance. On cutting into the lung structure, minute yellowishwhite spots are to be seen, and if the lung be pressed, a mucopurulent discharge will be seen to issue from the inflamed bronchi, and if this discharge be examined microscopically it will be seen to contain the microbes more or less abundantly.

The pericardium, heart, liver, kidneys, and digestive tract will also often be found nore or less inflamed.

Treatment.-Assuming that the usually harmless microbe becomes virulent under abnormal conditions of the body of its host, and in order to limit the spread of the disease, separation of the sick from the healthy should at once be resorted to, and the surroundings cleansed and disinfected. Medicinally but little can be done beyond endeavouring to destroy the virulence of the microbe by the administration of carbolic acid, liyposulphite of soda, or remedies having a similar effect, as the condition of the animal might justify. 


\section{H A P TER X X X III.}

\section{BLOOD DISEASES.}

\section{ANAMIA-SURRA.}

A FATAL form of anæmia has been observed on the Continent of Europe, in India, and Burmah. Some observers state that it is due to a fine bacillus in the blood, and that it is infectious; whilst Evans, Burke, Steel, and Lingard, who have studied it in India, where it is well-known under the name of SURR, have discovered that it is due to the presence of hæmatozoa-the Trypanosoma-in the blood of the affected animals,-horses, mules, camels, elephants, donkeys. The hæmatozoa are also found in rats and bandicoots, and in honour of its discoverer, Evans, it is now called Hcematomonas Evanse.

Evans, when he first discovered the parasite, thought it was a spirillum, but further examination convinced him that it belonged to the animal kingdom, presenting when fresh and active an apparently round body, tapering in front to form a neck, and terminating in a blunt head, and posteriorly a tapering tail, extending from which is a long slender lash. $\mathrm{He}$ also states that at the head end there appeared in one or two cases a circlet of pseudopods, and as the body slowly died in serum it gave the appearance of flattening out. After watching carefully all the forms and movements of the parasite, he concluded that on each side of the body there existed two pin-like papillæ, one near the neck and the other close to where the tail began; but he only observed them in a very few instances, and supposed they were of the nature of psendopods.

The parasite is very active in its movements, with an undulatory eel-like motion, moving generally with head foremost, but occasionally in the direction of the tail lash, when, as Captain Appleton, A.V.D., describes it, tugging at and worrying a red corpuscle : they thus attack and disintegrate the red corpuscles. 
Sometimes two will unite and swim off as one body. Evans thought that they joined heads and tails; others thought that they fastened with their tails in opposite directions.

The disease is not contagious or infectious in the ordinary sense, but can be communicated by subeutaneous inoculation, and by the introduction of blood containing the parasites into the stomachs of healthy animals.

Steel cliffered from Evans as to the exact classification of the parasite, maintaining that it resembled the spirillum of relapsing fever in man. Notwithstanding this error, it must be admitted that he did good work when the disease broke out in British Burmah in 1855, confirming its communicability by inoculation of blood containing the parasites to the dog, horse, mule, \&e., that the disease was relapsing in its nature, that the parasite appeared as the temperature rose, and disappeared during the non-febrile periods; and he concluded as follows:- "That relapsing fever of mules is invariably a fatal disorder, characterised by the periodical occurrence of attacks of high fever, during which a special organism closely resembling the spirillum of relapsing fever in man is found in the blood. This organism is one-sixth of the size of a blood corpusele in width, and three to six times in length, \&e. All observers conclude that the parasites are not always present in the blood of the diseased inimal, but come and go in successive broods."

Seeing that Evans and Steel differed in opinion as to the exact classification of the surra organism, Evans referred the natter to the late Dr. Timothy Lewis, who concluded that it resembled, but was not exactly like, that which he had discovered in the blood of rats, now called Trichomonas Lcwisi. The matter was further referred to Crookshank.-(" Journal of Royal Microscopical Society," part 6.- "Flagellated Protozoa in the blood of diseased and apparently healthy animals.") He says, page 916 :- "In the face of these conflicting opinions, Dr. Evans was good enough to place in my hands for investigation some preparations of the organism in the blood as well as material from the lungs and intestines of a camel that had succumbed to the disease.

"On examining a stained preparation with a power of 200 diameters, a number of the parasites could be distinguished in the field of the microscope, and with a $1 / 12$ and $1 / 18$ O.I. (oil 
immersion) the individual characters were clearly brought out. These were quite sufficient at once to dispel the idea of its being a spirillum. It was obvious that it was a more highly organiser micro-parasite, presenting very peculiar and distinctive structural appearances.

"The first glenee at the parasites recalled the appearance of nematode hæmatozoa, as if, indeed, they might be embryonic Filcrice; but wheu I had carefully studied several specimens, and had further undergone the searching examination entailed by the accurate focussing necessary to obtain a number of sharply defined photo-micrographs, I came to the following conclusions:

"The somewhat tapering central portion or body of the parasite is continuous at one end with the whip-like lash, and at the other end terminates in an acutely pointed stiff filament or spine-like process. Here and there, possibly from injury or want of development, the spine-like process appears to be blunted or absent. By carefully focussing on the upper edge of the central portion, I discovered the existence, much more markedly in some of the parasites than in others, of a longitudinal membrane, with either straight or undulating margin. The membrane is attached along the body, arising from the base of the riwid filament, and becomes directly continuous at the opposite end with the flagellum. In some cases the edge only is deeply stained, giving the appearance of a thread continuous with the flagellum, so that one might be easily led to overlook the membrane, and imagine that the flagellum arose from the opposite end of the body, at the base of the spine-like process.

"Close to the base of the spine-like process, a clear urstained spot is in many parasites easily distinguished, and at the opposite end there is in some the appearance of the deeply stained protoplasmic contents having contracted within the faintly stained membranous investment. Where the longitudinal membrane has a wavy outline the undulations are much more marked in some cases than in others. Here and there the wavy outline appears first on the one side of the central portion and then on the other, but there never is any wary outline on both sides of the same part of the body ; and this was explained hy careful examination, which showed that in dying the somewhat ribbon-like parasite had become doubled on itself. The discovery of this undulating membrane at once suggested to my 
mind an explanation of the lateral psendopodia described by Evans.

"If we imagine that we are looking down upon the parasite, with the edge of the membrane towards us, one can conceive that the rapid undulations, first on one side and then on the other, might give an image upon the retina which could be construed as due to protrusion of lateral psendopodia.

"I may add that I could not discover in the stained preparations any trace of the circlet of pseudopods, and I think the undulating membrane may account for this appearance also."

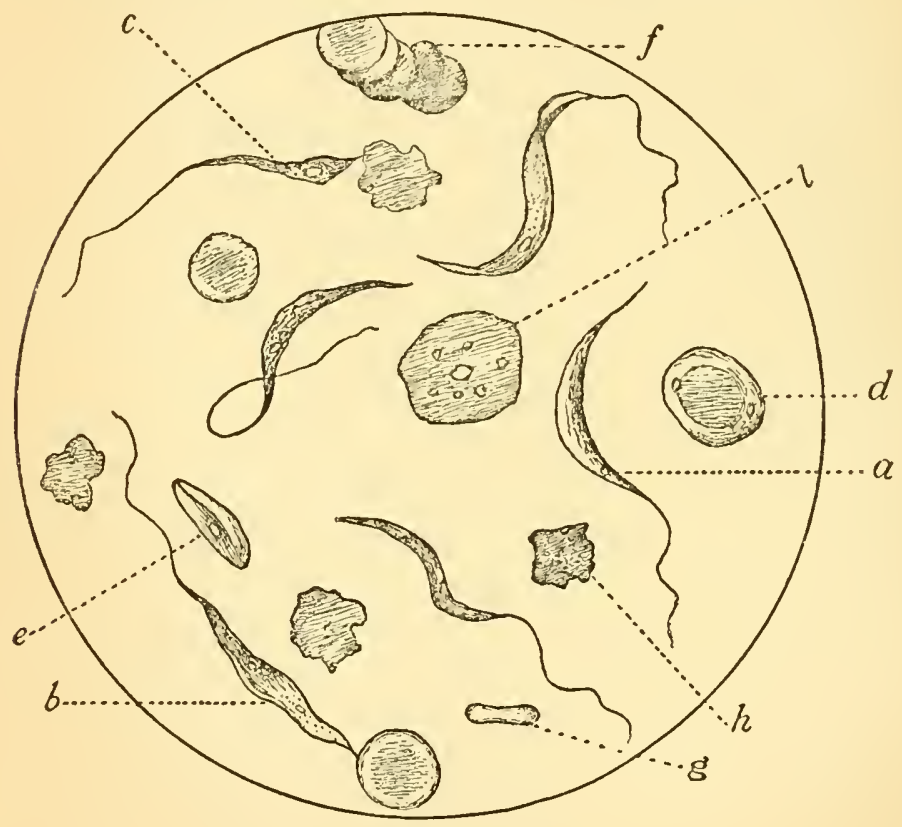

Fig. 22.-Monads in Rat's Blood.

a. A monad in free motion among the cells.

b. Another, attached to a red corpuscle.

c. Angular form.

d. Encysted form.

e. The same seen edgeways.

$f$ and $g$. Normal red blood corpuscles.

h. Crenated red blood corpuscle.

i. White blood corpuscle. 
Owing to the somewhat curved and twisted shape of the parasite, and the curling of the flagellum, in the stained preparations it was difficult to make exact measurements, but I was able to ascertain that the average width, according to whether the membrane was visible or not, varied from 1 to $2 \mathrm{~nm}$., and the length of the body from 20 to $30 \mathrm{~mm}$. The flagellum was about the same length as the body.

Similar if not identical parasites are found in the blood of rats, bandicoot, mudfish, carp, and apparently inducing no inconvenience to their hosts. I cannot, however, agree with Crookshank that the parasites in surra are only associated with the disease, the impoverished blood affording a suitable nidus for their development, while the contaminated water nay be the common source of the organism and the disease.

It was discovered by Lingard that one attack did not render an animal immune to a second, and the following experinient is interesting as bearing on this point. Twelve months after an Australian horse had been cured of surra by the administration of arsenic, iodide of arsenic, and mercury, this horse was inoculated with a minute drop of surra blood-a mere trace of soiled blood being smeared over a scratch on the muzzlein order to ascertain whether any immunity had been given by the previous attack. The latent period of the second attack occupied seven days. The progress of the disease was marked as usual by paroxysms of fever and intermissions, the former occupying a much more prolonged period-twenty-two daysand the latter being shorter than usual-only two days.

Death took place on the fifty-third day of the disease, clearly proving that one attack of surra is unable to protect against a second attack.-(Lingard, Report on Surra to the InspectorGeneral, Civil Veterinary Department, Simla, 1895.)

Symptoms of Surra.-Outbreaks of this disease occur in India and Burmah chiefly after the rainy season. The more prominent symptoms observed at first are a capricious appetite, dulness, and slight fever, which after a few days' rest frequently pass off without medical treatment. When a relapse occurs, which is alnost invariable, there is an increase of fever, the mucous membranes are yellow in colour, and petechize become apparent, also local or general urticaria. There is generally some oedema of the limbs, also beneath the belly, chest, and sheath. The 
urine becomes much altered, viscid, of a dark yellow or orangegreen colour, frequently containing albumen and blood. The animal becomes emaciated, and great thirst is invariably present. There are occasionally catarrhal symptoms. The lungs may be more or less congested, and breath fotid. There are periods of remission, marked by improved health, which is merely transitory, as repeated attacks of inereasing intensity finally end the animal's liie. This disease, according to the condition and age of the animal attacked, may last from a few days to several months before death oceurs. It is very fatal. The disease, until quite recently, has not been amenable to any line of medical treatment. Professor Lingard, however, has been experimenting with the arsenic treatment, with some amount of success in patients possessed of vigorous constitutions.

The investigations as to the origin of this deadly parasite, the cause of surra, point to the probability that it is to be found in the food and water. The miero-orgnnism (apparently identical with that of surra) found in the blood of 30 to 40 per cent. of rats experimented upon, surgests the necessity of preventive measures being particularly direeted towards ensuring the cleanliness and purity of all kinds of food grain, more partieularly when it has lain in any quantity for some length of time, and subjected to the risk of eontamination with the excreta of the rat speeies. The purity of the drinking water also demands attention. As to medicine, it is obvious that in this, as in all other diseases due to micro-organic invasion of the blood fluid, the effects are as yet unreliable.

TIIE TSETSE FLY DISEASE, OR NAGANA, IN ZULULAND.

Surgeon-Major David Bruce, A.M.S., has published his investigations into the causes and nature of this clisease-December $189-$ - and seems to have satisfactorily proved that it is identical with surra; the hæenatozoon being probably conveyed to horses, cattle, donkeys, and dogs by the tsetse fly, and characterised, like surra, by a rapid diminution of the red corpuseles-from $5 ! 1$ millions to $2 \frac{1}{2}$ millions per cubie millimetre.

Dr. Bruce records several experiments, the value of which will be gained by reading the following "Postscript." 
" On account, then, of the lack of absolute proof furnished by the last experiments, I set myself on $22 \mathrm{~d}$ November to try to infect susceptible animals with the disease by being bitten by tsetse flies brought frequently to the top of the Ubombo from the low country and straightway placed on the animal.

"The greatest care was taken that the flies were caught on a perfectly healthy animal, as to have allowed them to puncture one already affected by the disease would naturally vitiate an experiment.

"The time which elapsed between catching the flies and placing them on the animal under experiment varied from four to seven hours.

"The following experiment gives the dates, the number of flies used, and the result :-

\section{"Exp. 225. Horse in good condition.}

"Nov. 22. Brought up ten tsetse flies from the low country ancl placed them on this horse.

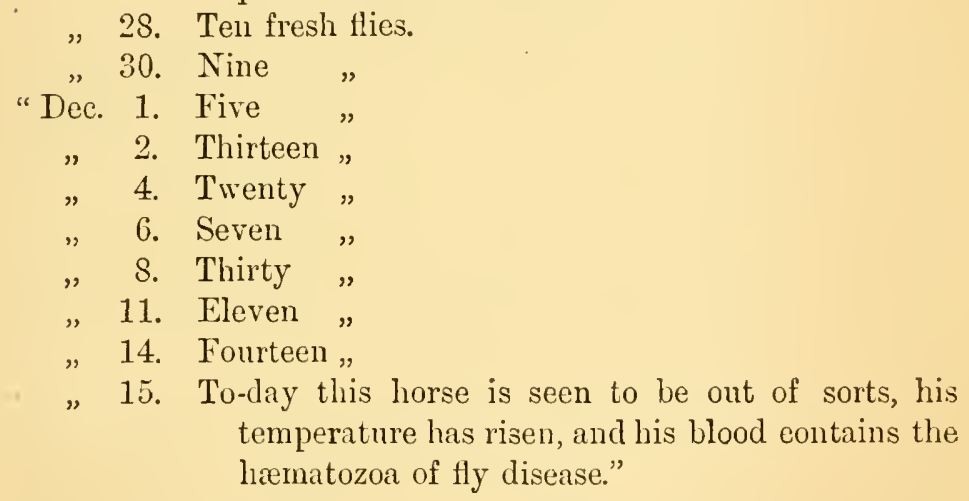

The temperature of this horse was $99^{\circ}$ on 19 th November, and after fluctuating a good deal between $99^{\circ}$ and $102^{\circ}$, rose to over $102^{\circ}$ on 15 th December, when the hæmatozoa were discovered in his blood, and was over $104^{\circ}$ on 17 th December.

Dr. Bruce adds-" From this case, then, and from the evidence I have already brought forward, I consider myself justified in believing that the tsetse fly in a state of nature does convey the disease to susceptible animals, and the question now arises, from what source does the fly obtain the living virus." 


\section{CHAPTER XXXIV.}

BLOOD DISEASES.

\section{IXODIC ANAMIA-TEXAS FEVER, \&O.}

LATER studies of this disease have brought to light that it assumes two forms: an acute fatal type, as seen in America in the hot summer months, and a mild, rather prolonged, usually nonfatal form, recognised by an examination of the blood, which must reveal the micro-parasite in the red corpuscles and plasma. This form is seen in the autumn when the heat of summer has passed away, also during October and November, and, rarely, in the first week of December. It is stated that the difference between the acute and mild type is accounted for by the fact that during a stage of its life-the small stage-the Texas fever parasite circulates in the blood in a condition differing from that observed in acute cases; that in the latter it rapidly destroys the blood corpuscles, giving lise to hæmoglobinuria,red water,-whereas in the mild form the destruction of the corpuseles is much more prolonged, and not associated with hremoglobin in the urine.

It has also been observed that in certain cases when an animal has recovered from the acute attack, and the number of red corpuscles has nearly reached the normal number, they again diminish, and many of them contain the parasite in its small stage. The reason for the recurrence of this disease is not clearly defined,-whether these second attacks are merely relapses or reiufections. As already stated, the symptoms of the mild form are indefinite, and may be confused with a variety of mild diseases, but the ACUTE FOIN is manifested suddenly in the hot summer months, and simultaneously in all animals of a herd which have been exposed to the same infection together.

The fever, characterised by a temperature of from $105^{\circ}$ to $108^{\circ} \mathrm{F}$., precedes outward symptoms for several days, the animals 
apparently quite well. The pulse and respiration then become accelerated, and the urine is now observed to have the appearance of blood, varying in colour,-reddish, claret, or blackish red water (hæmoglobinuria) in the most acutely fatal cases. Out of forty-six fatal cases (Burean of Animal Industry, Washington, 1893), hæmoglobin was found in the urine in the bladcler of thirty-three after death. It is uncertain whether this condition of the urine is present in all cases of the acute form, the opinion of the reporters to the American Bureau being that it "depends upon the rapidity with which the red corpuscles are infected and destroyed. A slow destruction may allow other organs to excrete the debris and thus forestal the discharge of hæmoglobin in the urine." But if free from hæmoglobin, the urine at the height of the fever is found to contain a small quantity of albumen, to have a specific gravity of 1030-40, may be strongly alkaline and effervescent when treated with acids; but as the disease advances its specific gravity will fall to $1010-12$, its reaction becoming neutral or slightly acid. It sometimes contains small numbers of red corpuscles, which may be derived from small hæmorrhages in the pelves of the kidneys, regularly observed post mortem.

The bowels are, as a rule, constipated during the high fever, but as the fever subsides the freces become softer and tinged with bile. After a few days' illness the debility becomes very great, and whilst the animal is standing, trembling of the lind quarters and limbs are prominent features in many cases: the blood is then found to have become very thin, pale, and watery, due to the destruction of the red corpuscles. In the earlicr stages, if freshly drawn blood be allowed to stand, the serum will be found to present a very dark red colour, indicating the presence of much colouring matter in solution. Later on, this colour may not be present, but thinness of the blood, owing to destruction of the red corpuscles, seems to be the "most essential character of Texas fever."

The duration of the disease varies, proving fatal or disappearing in a few days. Recovery, however, is associated with great debility; degenerations may occur in organs, and frequently there are relapses. Some animals never regain their health; in other's, recovery takes place after weeks or months. High temperature rarely lasts longer than eight to ten days; it is then followed by a period of normal or subnormal temperature, 
the falling of the temperature marking the cessation of the destruction of the corpuscles, and the disappearance of the parasite from the blood. The mortality varies greatly. A midsummer outbreak, when acute in its nature, is the most fatal, and from this time there may be all gradations towards the mild, non-fatal form of the disease.

Etiology.-Several observers have reported the discovery of various forms of bacteria in this disease. In 1883 Salmon described a diplococens obtained from spleen cultures. Detmers found bacilli and micrococei in the liver, but none in the blood; and in 1888 Billings claimed the discovery of what he terms the "true germ," an ovoid bacterium, staining at the ends, similar to that of swine plague. All these contentions, however, lave now been apparently terminated by the discovery of a parasite in the blood corpuscles, conveyed from Sonthern to Northern cattle by eattle ticks (Boophilus bovis). At the experimental station of the Bureau, near Washington, it was found by experiments in 1889 that the disease ean be produced by ticks artificially hatehed in the laboratory.

The parasites thus conreyed are found in certain proportions of the red eorpuscles in the form of rounded or somewhat ovoid or pyriform bodies, isolated or in pairs, in the acute form of the disease, whilst in the mild form it appears that from 5 to 50 per cent. of red corpuscles are found to contain cocens-like bodies, sometimes on the border, and outside the corpuscles; they are from $\frac{\pi}{8} \frac{1}{000}$ to $\frac{1}{50} \frac{1}{8} 00$ of an inch in diancter, sometimes dividing into two; they, as well as the larger pyriform bodies seen in the acute form, stain with aqueous solutions of aniline dyes, and with hemotoxylin. Both the large and small forms are considered to be different stages of the same parasitic protozoa (Pyosoma trigeminum, Dr. Theobald Smith). The smaller ones are found in cattle exposed to the disease late in the season, or cluring a second attack or relapse after passing throngh an acute attack, and in milder cases during or previous to the season (hot) of the acute disease.-(See Fig. 23.)

Post-mortem Appearances.-Congestion of the vessels and occrsional patches of extravasation in the subcutaneous tissues, blood sometimes thin and watery, lungs normal or discoloured by congestive patches, heart congested; petechial spots on peri- and endocardium, spleen congested, its capsule streaked from distension of its ressels. The weight of the 
organ varies, according to the stage of the disease in which the animal dies, the more rapid the fatal termination the larger the spleen. Generally it is from two to four times its natural weight, distended, firm to the touch, its capsule attenuated, the pulp dark brown, glistening, and homogeneous. The markings of the Malpighian bodies and of the trabeculæ have disappeared.

The liver is gravely altered, enlarged, sometimes of a dark

FIG. 23.

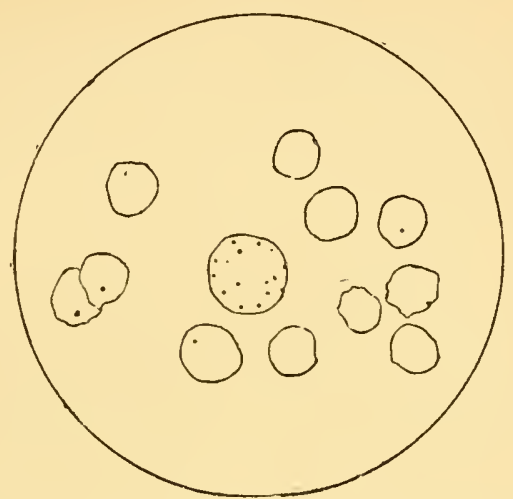

Micro-Parasites seen as minute points, observed in the mild form of the disease.

colour from blood congestion, or light coloured from extreme bile engorgement; gall bladder full of thick grumous bile; when incised the cut surface presents a uniformly brownish-yellow colour, or else a mottled appearance, also seen on the surface. There is occlusion of the biliary canaliculi and ducts, and a more or less extensive fatty degeneration of the liver cells, and a tendency to necrosis of the inner zone of the lobules.

The kidncys vary in colour according to the severity and stage of the disease when death occurs. In the carly stages, when the bladder contains port-wine coloured urine, they are enlarged and of a uniform dark brownish-red colour, and the vessels are uniformly distended with red corpuscles. The secreting structures are not scemingly altered. In cases which die after the acute stage and fever have passed away, they are paler than natural and quite flabby, having pigmentary deposits in the convoluted tubules, and their epithelium so loaded with a reddish-yellow pigment that they are easily traceable in their windings by their colour. In those cases where the capillaries are filled with red 
corpuseles, the microbes are usually found in all the corpuseles. Extravasations of blood are found in the pelves, and in most cases the bladder is found to contain urine mixed with hæmoglobin, whilst ecchymosed spots are seen on the inner surface of the mucosa.

The digestive apparatus, with the exception of the abomasum. which is frequently congested, generally presents no definite alterations. The pylorie portion of the abomasum is found to contain deep ragged excavations with hæmorrhagic bases. The constancy of these ulcers, probably the result of vascular occlusion, is considered by Moreau Morris to be more certain indieation of Texas fever than any other lesions commonly present.

The intestinal lesions are congestions and pigmentation, with an increased flow of bile.

Prevention and Treatment.-If the disease is suspected, the herd should be examined thoroughly for ticks, and the animals' temperature taken. The combination of ticks and fever, or the presence of the former in a locality where they do not naturally exist, may be considered a sure sign of the imminence of Texas fever. If the ticks are found they should be carefully removed and the cattle at once transferred to uninfected grounds. The examination for ticks should be done repeatedly, and all ticks destroyed. These measures may not prevent all attacks, nor cut short the disease after it lias once shown itself, but the reporters are satisfied that fewer animals will sucemb. A single infection is sufficient to cause severe and prolonged disease, as shown by the experimental injection of blood; but the mortality seems to be lower than in natural exposures, where the infection is intensified with every additional tick.

No special line of medical treatment las as yet been tried. Quinine and its preparations are proposed as possibly valuable, and methylene blue, recommended for malaria, is referred to as worthy of trial.

Inoculation with the blood of Southern cattle, apparently healthy, and with that of diseased Northern cattle, transmits the disease, and if this is done after the hot summer weather has passed, the induced disease may be of a mild character; but it appears that immunity is not assured, as it is reported by Dr. Dinwiddie that of vaccinated and non-vaceinated cattle sent to Texas $66 \frac{2}{3}$ per cent. of the vaceinated and $88 \frac{2}{3}$ of the nonvaccinated died. 
Had there been a well-marked immunity conferred by inoculation, it would have been possible to have improved the method of procedure; and perhaps carefully prepared blood serum, freed from the organism, might have been of great value. One remarkable instance is mentioned in the report, namely, that the Texas fever parasite was found in the blood of a North Carolina animal three years after it had left the permanent infected territory.

Similar diseases, manifested by coloured urine, are reported to prevail in the marshy regions of Roumania. These were investigated by Babes in 1888, and disseminated largely by draught oxen: in the Cape of Good Hope also conveyed from place to place by yoke oxen. By keeping communication with the territory north of the colony, these cattle, like those of the Southern States, seem not to infect others directly, but they infect ground over which they have passed. It also prevails in Australia, and is due to the same cause, ticks being numerous in that country.

Infected pastures have been found to be harmless to sheep, and it is thought that other domesticated animals may graze unharmed in such pasture.

The discovery that the disease is conveyed by ticks explains what has litherto been very mysterious, namely, that Northern cattle become affected by the disease when pastured on lands previously grazed by Southern cattle apparently in perfect health. This fact leads to the conclusion that Southern cattle, recovered, perhaps, from previous attacks, have acquired immunity, though covered with ticks.

Although it is clearly demonstrated that Southern cattle are dangerous when they bear the cattle tick, and that this tick conveys the micro-parasite from Southern to healthy Northern cattle; but transmission from Northern cattle is very rare.

Northern cattle have ticks on them, but only those which survive the disease or die after a prolonged attack ripen the tick on their bodies. Those which die of an acute attack in a short time after infection have only immature ticks on them. If the fever has occurred early enough in the season to permit a second generation of ticks to appear before the cold weather arrives, another outbreak during the same season may occur. Usually the first outbreak occurs in August, and the second, to be looked for late in September or early in October, is so mild as to pass unobserved. If, however, the first outbreak occur 
in July, the second may appear in September, and perhaps be of greater virulence.

When the disease is induced by inoculation, experiments have proved that it has no contagious properties,-in fact, that it is not transmitted by cohabitation, but by the intermediation of the cattle tick. But, as already stated, it seems to be concluded that preventive inoculation has so far been a failure, for the effect produced in the body of an animal by the destruction of the red corpuscles equal in amount to all those circulating in the body at any given time should make much more impression than any method of inoculation is likely to do. Not being, so far as at present known, a ptomaine-produeing organism, like bacilli, \&c., this is as might be expected. Yet such an attack not only does not always prevent a second attack, but may not prevent death during a second attack. Aside from the diffieulties attending the production of immunity, under any circumstances the difficulties of preparing a "vaceine" according to the method hitherto practised are at present insurmountable. The microorganism cannot be cultivated. The infection of Northern cattle might be prevented by careful destruction of the tieks upon Southern eattle before their removal to Northern teritories, or by prohibiting their migration during hot weather. These suggestions are not, however, made by the reporters, but they attach great importance to the protection of animals taken south into permanently infected pastures. They state that it is probable that if ealves be taken they may, withont treatment of any kind, survive the infeetion upon southern pastures, and become gradually insusceptible. But in case of animals more than twelve to eighteen months old the first attack might be fatal ; and, perhaps, the simplest way of dealing with older eattle is to endeavour to induce immunity by exposing them to infection at some specified time in autumn. In the latitude of Washington it was found that the most convenient time is the middle of September. In more northerly latitudes the exposure should be correspondingly earlier. Cattle exposed in this way invariably take the fever, but the mortality is very small. Such animals may die of a second attack during the succeeding summer, but a mild exposure during the following autumn may furnish a sufficient protection.

Another method of inducing the disease is the injeetion of blood from infected cattle, which generally induces-if practised 
after the hot summer weather-mild attacks of the disease. The blood of apparently healthy Southern cattle will serve the same purpose.

Exposure to ticks during the cool autumn months is also recommended, being simpler, requiring no operation, the ticks being easily procurable from the permanently infected Southern territory.

In concluding their observations upon these methods of protection, the reporter's say that their statements concerning the possible uses of mild infections as a means of subsequent protection must be regarded as mere suggestions, which may or may not prove of practical utility on a large scale.

The Tick - Ixodes bovis (Riley). - This parasite was first described by Professor C. V. Riley in 1868 as a reddish, coriaceous, flattened species, with hody oblong-oval, contracted behind the middle, and with two longitudinal impressions above this contraction and three below it, more especially visible in the dried specimens. Head short and broad, not spined behind, with two deep round pits. Palpi and beak together unusually short, the palpi being slender. Labium short and broad, densely spined beneath. Mandibles smooth above, with terminal hooks. Thoracie shield distinet, one-third longer than wide, smooth and polished; coxie not spined. Length of body $0 \cdot 15$ of an inch; wilth 0.09 of an inch. Further observations have made it clear that the above description probably applies to the adult male only, the colour of the females being olive brown on the back and slate-coloured on the belly. Dr. Cooper Curtice has shown that in the earlier or larval stage the young tick has three pairs of legs; after another week's sojourn on a calf (kept in an artificially heated stable, the season being advanced-November 15 th) it was ready to moult. The emerging nymphal stage was provided with an additional pair of legs; after another week on the calf the tick was ready to moult again, and became sexually mature. It is thus shown that in this particular species of tick there are two periods of moulting before the parasite becomes mature. Curtice likewise created for it a new genns-Boophilus. It is classified by Dr. George Marx-(Journ. Comp. Mcdicine and Veterinury Archives, July 1891 and January 1892)-as follows : -Class, Arachnida-Order, Acari; Sub-order, Cynor-Cynohesta; Family, Rhipistornicke; Genus, Boophilus; Species, bovis. A full-grown tick is said to lay on an average about 2100 
eggs in from eight to fifteen days; the ticks which take the longer time lay the larger number of eggs. In hatching these eggs in glass dishes, with damp soil or leaves, the dishes being kept close to prevent the escape of the young brood, it was found that the period of incubation varied according to the surrounding temperature. When the weather was very hot, the hatching wis completed in from fifteen to eighteen days. When the season is more alvanced, or the temperature lowered, the period of hatching is prolonged to twenty-five, thirty, or even forty days.

Conclusions.-(1.) Texas cattle fever is a disease of the blood, characterised by a destruction of red corpuscles. The symptoms are partly due to the anæmia produced, partly to the large amount of débris in the blood, which is excreted with difficulty, and which causes derangement of the organs occupied with its removal.

(2.) The destruction of the red corpuscles is due to a microorganism or micro-parasite which lives within them. It belongs to the protozoa, and passes through several distinct phases in the blood.

(3.) Cattle from the permanently infected territory, though otherwise healthy, carry the micro-parasite of Texas fever in their blood.

(4.) Texas fever may be produced in susceptible cattle by the direct inoculation of blood containing the micro-parasite.

(5.) Texas fever in nature is transmitted from cattle which come from the permanently infected territory to cattle outside of this territory by the cattle tick (Boophilus bovis).

(6.) The infection is carried by the progeny of the ticks which mature on infected cattle, and is inoculated by them directly into the blood of susceptible cattle.

(7.) Sick natives may be a source of infection (when ticks are present).

(8.) Texas fever is more fatal to adult than to young cattle.

(9.) Two mild attacks or one severe may preveni a subsequent fatal attack.

(10.) Sheep, rabbits, guinea-pigs, and pigeons are insusceptible to direct inoculation. (Other animals have not been tested.)

(11.) In the diagnosis of Texas fever in the living animal the blood should always be examined microscopically, if possible.

The regulations of the American Department of Agriculture provide for the prevention of the introduction of the disease into 
non-infected territories by the complete isolation of all cattle from infected territories between March 1st and December 1st of each year; on the proper disinfection of the litter and manure from such cattle during transportation. Furthermore, such cattle can only be transported into minfected territories for immediate slaughter during the prescribed periods.

There are further directions in the American Report, from which the foregoing has been compiled, without the scope of this work. The author may, however, state that the Report, covering 126 pages of closely printed matter, in addition to nine pages of illustrations, is a most valuable one, conducted with great care by Theobald Smith, Ph.B., M.D., and F. L. Kilborne, B.Agr., B.V.S., under the direction of Dr. D. E. Salmon, Chief of the Bureau of Animal Industry, Washington, 1893.

\section{IXODIC AN EMIA IN JAMAICA.}

In July last, 1896, I was commissioned to go to Jamaica to study a fatal disease which had prevailed amongst the cattle of that island for the last few years; and, after careful consideration and two months' experience, during which period I examined diseased cattle in all parts of the island, I arrived at the conclusion that I had to deal with Texas fever, of which the following is a short description.

Cattle enfeebled from any cause seem to form a more favourable habitat to the ticks, and are more predisposed to the disease than the healthy and strong; but the healthy and strong have no immunity, as they are frequently attacked, and succumb. I have therefore arrived at the conclusion, after carefully weighing all the facts and circumstances which have been brought before me, both directly and indirectly, that the disease is due to the attacks of ticks, and (with the exception of the Mysore cattle) that no cattle have immunity. Some are but slightly invarled by the ticks; some, owing to strength of constitution or other causes, seem to throw off the effects with but little appreciable suffering or lamage, whilst in others the attack is acute, and terminates fatally in a few days after the first manifestation of the disease; and I re-assert that, after carefully weighing the evidence, I have arrived at the conclusion that the disease is, as presented to me, a modified form of Texas fever, now prevailing in other parts of the world besides Jamaica, and that it is carried from place to place by ticks. 
The disease in itself cannot be called contagious or infectious; there is, therefore, no necessity for burning the carcases of dead animals.

Animals suffering from the disease, if perfectly freed from ticks, do not transmit the disease, no matter how severe the attack may be; but if invaded by the parasite, the pastures on which they graze are first contaminated by the mature ticks, which drop from cattle, and in alout seven days lay their eggs in the grass; the eggs hatch in about other twenty days-sometimes a longer period-and the young ticks are at once ready to crawl on to the cattle. They then become again encapsuled and emerge from the second shell in fourteen or fifteen (lays.-(See p. 423). If these figures be added together, it will be found that the shortest possible time, after tick-infected cattle are turned out into a field, in which the disease may appear is about forty days; but the period of first attacks may be much longer than this, as all the eggs are not laid upon the same day: there is therefore a daily hatching for consecutive days. (Own observations: Texan ticks placed in bottle on 5 th September commenced to lay on 17th.)

It is rather unfortunate that during the earlier part of our visit the animals examined, both prior to and after death, were suffering from the disease in its chronic form, and in which many of the marked symptoms and post mortem conclitions of acute 'Texas fever were absent; but during the latter portion of the visit more decidedly marked signs of that disease were observed.

One characteristic sign of acute Texan fever (as described by the American writers) - red water or blood-coloured urine, hæmoglobinuria-was alssent. They state that " the one sign regarded as peculiar and pathognomonic (characteristic) in this disease is the discharge of urine having the colour of blood. This colour is not dne to a discharge of blood from the kidneys and subsequent breaking up of the red corpuscles, but to a filtration of the colouring matter of broken-down red corpuscles (hæmoglobin) already in solution in the circulation, into the urine in the excretory structures of the kidneys." This statement is perhaps too obscure to be easily understood. It means that the red blood-cells are broken up in the blood-vessels, and that the colouring watter thus set free tinges the fluid portion (which is naturally colourless), and which, when excreted by the kidneys, presents a red appearance. Now this sign, considered 
so important by the American authors, and stated by them to be present in 33 out of 46 cases, did not present itself except in one doubtful case. The animal (a cow) was suffering from a very prolonged but not severe attack, and had latterly passed red water. I was of opinion, however, that this tinge was due to some injury to or breaking down of the urinary aplaratus, as the colour was dne to actual (coagulating) blood, and not to the colour of broken-down blood-cells. The information obtained from all who were acquainted with the disease, that red water is not a symptom, wonld have cansed me much surprise had I not known that in Australia red water is only observed after the animal has been travelled. (One of the constituents of the blood absent in healthy urine, viz., albumen, was found in all the specimiens of urine examined.) Other signs of Texan fever, such as enlargement of the spleen and of the liver, serosanguinous or red watery condition of the fat, \&c. abont the kidneys, were absent. The spleen had in no instance shown the enlargement described by the American writers, but the liver in some three cases was slightly enlarged, and presented a pale yellow mottled colour, dne to bile congestion and fatty degeneration. When cut into, the tissue presenter a mottled appearance throughout, and was bloodless, whilst the larger blood-vessels discharged a very dark-coloured and thick bloorl. Put whilst this characteristic of acute Texan fever was present in these few cases, the liver itself did not present the three to five pounds enlargement over that of health, as stated to be the case in that disease; in fact, we dicl not find one liver to be appreciably increased in weight, but the bile was generally increased in quantity and mixed with mucus. The appearance of the heart also differed from that described in Texas fever, the petechial spots passing along the intra-rentricular groove and near the base, extravasation, \&c. being absent, but the heart itself was found to be pale and undergoing a retrograde change, as seen in the Texas disease after the subsidence of the fever.

Temperature.-In most cases there was more or less elevation of temperature. In one case it was as low as $101.4^{\circ} \mathrm{F}$; this was in a very emaciated and debilitated calf. As a rule, however, the temperature ranged from $103.5^{\circ}$ to $106.8^{\circ} \mathrm{F}$.

The apperercence of the Blood and-Tissues.-Animals that were grazed upon morasses and boggry grounds were upon post 
mortem examination found to have flukes (Distoma) in the liver, and in some cases in the lungs, but, except in one case, the liver flukes were few in numbers.

In districts in which there are no rivers, and where the drinking water is obtained from ponds supplied by the rains, the Strongylus contortus was found in varying numbers; but we concluded that, though they might aggravate the conditions, they were not the cause of the disease, as in the majority of the post mortem examinations neither flukes nor strongyles were present. In two cases the lesions of broncho-pneumonia-as seen in American and Canadian cattle-were found, and these were supposed to be tubercular. No tuberculosis was met with during the whole investigation.

Where the cattle obtained water from wells and rumning streams, the post mortem appearances were as follows:-The organs and tissues did not to the naked eye materially deviate from those seen in health. There was bloodlessness (anæmia), characterised by pallor of the organs and some degree of degradation of their structures. The blood, however, was thin, watery, pale-in some instances scarcely tingeing the fingers of the observer-whilst microscopically it presented those characteristies reported upon by my son, Dr. Williams.

The symptoms during life were-1st. great depression, the animal presenting signs of languor and debility, the appetite was indifferent, and rumination irregular and infrequent; there was emaciation, the loss of flesh being often extreme; the ears drooping, and the visible mucous membranes, i.e., those of the nose, month, eyes, \&c., exceedingly pale. In one uncomplicated case only dicl we observe yellowness of these membranes. There was sometimes diarrhoea, but we did not witness one case in which there was constipation. The pulse in all cases was exceedingly small and weak, ranging from 65 to 120 beats per minute. About 85 beats, however, or abont twice the natural number, seemed to be a medium. The urine was pale in colour generally, and in all instances when tested was found to contain a small amount of albumen. The breathing, except in calves, or when the animal was distmbed, was about 20 movements per minute, or about one-third more than the natural number. There was no cough nor a discharge from the nose, or other signs of disease of the lungs, except in the cases alrealy cited. The cyes had a sunken appearance, and the ears drooping, and 
the animals had a staggering gait, particularly if hurried or excitel. In several instances they fell to the ground when moved sharply or turned round suddenly; in one instance the animal fell down dead, so great was the general prostration and weakness of the heart's action.

The skin about the shoulders, lower portion of the neck, and other parts easily rubbed by the animal, was generally denuded of hair, and in many instances presented a rough scaly appearance. This symptom has been described as one preceding the advent of the tick, but such is not the case, as it was found upon close examination that the skin was covered with ticks underneath the undisturbed hair of the host. I therefore conclude that this symptom is a sequence to and not a precursor of the clisease, as supposed. Some penkeepers call this " mange," and seem to think that it has no connection with the disease. On close examination it was found that there were not only fully matured parasites present, but many in an embryonic stage,- that is to say, covered by a shell of thin membrane; upon breaking which, two young ticks, namely, a male and a female, were usually found in close contact, the male being of a brownish colour and having four pair of longish legs, the female larger, also having four pair of whitish legs, and paler in colour than the male.

The surface of the body was generally more or less covered with ticks, which presented at least three varieties, viz.- 1 st. the large blue cow tick, also called the dog tick-Ixodes ricinus-the first tick known on this island, and not supposed to be injurious ; 2d. the silver shield tick-Ixodes scapulatus-which clings much more tenacionsly to its host, and is difficult to remove. This tick has four maxillary palpi, which, when close together, resemble a spear, but separate from each other at their points when the skin is pierced. These two kinds of ticks, as well as the grass lice, or the young ticks of both kinds, are supposed to be non-injurious to cattle, but I cannot subscribe to this, as they must do harm, and at least assist in the induction of the anrmia or bloodlessness which follows. The third form-the one stated to have been unknown by the majority of penkeepers and others until pointed out to them-was bronght before my notice about the third day of our investigation, having been found shortly before upon a lot of cattle badly attacked by the wasting disease. Upon examining this tick I arrived at the con- 
clusion that it was very similar to, or identical with, specimens of the Texan cattle tick, described as follows by Professor C. V. Riley. -(Journal of Medicine and Veterinary Archives, 1891 and 1892.) The life history of this tick, referred to at page 423 , is somewhat as follows:- "Adult females kept confined in bottles lay their eggs. These eggs are placed in covered glass dishes containing a little soil and kept in a warm place. After a period of three or four weeks the young ticks appeared; these were placed on a calf kept in an artificially warmed place, the weather being cold. The earliest or larval stage, as it emerged from the egg, had three pairs of legs. After one week's sojourn on the calf it was ready to moult. The emerging nymphal stage was provided with an adclitional pair of legs, and after another week's sojourn on the calf the tick was ready to monlt a second time, and become sexually mature." I may state that I have found the parasite both before and after the second moult, and can confirm the statement that during the larval stage it has only three pairs of legs, and four after its first and second moult. Experiments have proved that each mature female confined in a bottle remains quiet for several days - from two to four and a half, according to the American observers, but a longer period, from eight to twelve days, in one case under my notice; then a few ergs will be observed on the mouth and surrounding part. The number of eggs and the period of oviposition vary when kept in a temperature of $60^{\circ}$ to $78^{\circ} \mathrm{F}$; the laying was observed to continne from eight to fifteen days, and that those ticks which took the longest time laid the largest number of eggs. Each full-grown female will average 2100 eggs, which appear as dark red brownish masses of oval bodies.

As all the evidence brought before me pointed out that this tick was unknown to the majority of the cattle-owners and their servants, I made application to the Colonial Secretary, the Honourable Fred Evans, to obtain specimens for me through "The Bureau of Animal Industry, Washington, U.S.," of ticks found on Texan cattle; and in compliance with that request, the acting secretary of that department sent some specimens, which were immediately forwarded to me by the Colonial Secretary. These specimens, collected in Manhatta, Ks., correspond in every particular with the Ixoules bovis, also called Boophilus bovis, \&c., found on the cattle here.

Looking upon the tick, and, without confining myself to the 
"foreigner" alone, as the cause of the disease-which it indnces first of all by introducing some virulent secretion into the blood of its host, which causes fever, alteration in certain organs in acute cases, the development of bodies - which may be living or otherwise-into and around the blood corpuscles, diminish the quantity and deteriorate the quality of the blood, and finally induce death by robbing its victim of its life-sustaining fluid-I am of opinion that every effort must be made to destroy the tick upon the ground and upon the bodies of its hosts. The female tick, fully or partly charged with ova, leaves her host and deposits the eggs on the surrounding vegetation and upon the ground, which there remain until they are hatched.

During my investigations into a tick disease in Scotland, it was found that old and withered grasses and decaying vegetable matter of all kinds formed a cover for the ticks, and that they preferred to deposit their eggs where there was an abundant cover, and thus secure their non-destruction during the cold of winter. Although protection against low temperature is not necessary in Jamaica, there is plenty of evidence to show that when decaying and withered vegetation is allowed to remain on the ground there the ticks will be found, and I have been informed, over and over again, that during some parts of the year eattle will not face guinea-grass; that when placed in such pastures they will show signs of excitement, jump over the fences, and thus abandon even the most luxuriant pasture. Now this proves that the ticks are there in abundance, and no wonder, when it is considered that guinea-grass, said to be eaten down, leaves a withered stubble often two or three feet long, which forms a cover for the tick to deposit its ova upon, where they hatch, and are ready for the first cattle they may come in contact with.

I therefore think that when cattle are removed from guineagrass the stublble should be destroyed by burning.

I am told that, when guinea-grass is burnt standing upon the ground, the destruction is imperfect, and that the heat simply. favours the hatching of the young tieks, the ova or eggs being under the stones, \&e. If this be the case, the stubble should be eut down, collected into heaps, and burnt, the ashes remaining being spread over the land, and thus return something back into the soil. If it be proved by analysis that the soil is deficient in some essential ingredient, this shonld now be applied, as fertility. 
of the ground not only insures better crops, but renders that ground unfavourable to ticks and other parasites, as proved in Scotland by liming and otherwise improving tick-infested pastures. In addition to the destruction of all guinea-grass stubble, decaying and dead vegetations of all description should, when possible, be burnt, and the ashes spread over the land. During my travels in Jamaica I have witnessed many thousand tons of all kinds of vegetable matter allowed to remain on the ground, harbouring vermin and preventing the growth of useful materials; whereas, by collecting such refuse into heaps and burning, not only would the harbour for vermin be destroyed, but the ashes so obtained would to some extent, by improving the land, compensate for the trouble and ontlay.

With regard to the destruction of the Ticks themsclves.-A united effort must be made to remove the cause of the disease, by diminishing, or, if possible, wholly destroying the ticks; and for the purpose of killing them on their host I have, after due consideration as to the cost, and knowing full well that the profit on an $£ 8$ to $£ 10$ four-year bullock will not allow much expenditure of money, to recommend the following treatment; but, if money be not spent and an earnest and united effort be not marle, I cannot speak as to the future consequences.

For the destruction of the ticks on the cattle I recommend the following as the cheapest and most reliable dressing:--to every 4 gallons of linseed oil add 1 gallon of tar and $1 \mathrm{lb}$. of resin. The tar and resin to be melted with a little of the oil before being added to the loulk, and applied to all parts of the tick-infested skin; if one dressing the not sufficient, a second should be applied in a few days. All large ticks might be removed by being picked off and carefully destroyed, not thrown upon the ground and stamped upon, but burnt or smothered in tar. Oil destroys the tick by suffocating it, and any oil will do this, but boiled linseed oil is a cheap oil, and being a "drying oil," will remain longer on the surface of the body than a nondrying oil. Jeyes' Fluid and Sheep Wash have proved of service in destroying the ticks, but the mixture of linseed oil, \&c. will answer the same purpose and be more permanent in its effects.

In America and Australia cattle dipping is now resorted to, and where the cost can be afforded it should be done in Jamaica, for after all it must be confessed that to see the cattle attendants dress any animal is but a sorry sight. If the dressing is not 
thorough, it is money thrown away; every crevice fold, and depression should be thoronghly dressed, the cavities of the mouth, \&c. examined, the ticks removed, and the parts dressed; the eyelids should also be carefully examined, and the ticks gently pulled off.

Every effort should be made to conserve and increase "tickdestroyers," such as the Black Tick Birds, of which I saw two kinds, one a long-billed and the other a short-billed bird. I look upon these birds as the greatest friends to the cattle owners. I have had much amusement in watching these birds, as there seems to be an understanding between them and the cattle, whereby they are assisted and encouraged to destroy the ticks.

The domestic fowl is also very valuable as a tick destroyer, and for this purpose it could economically be kept in increased numbers. The long-legged varieties have a great advantage over the shorter legged ones, as they can reach much higher and thus remove more ticks, which usually do not favour, at least to any great extent, the lower parts of the limbs. Tick-destroying birds, such as the starling and perhaps the song-thrush, might be imported.

To a stranger visiting the island the scarcity of birds is a striking feature. I have been told that it is due to the mongoose, which has not only diminished the number of wild birds and domestic fowls, but other tick-destroying creatures, such as the ground lizard. Now this destruction of the natural tickdestroyers should, as far as possible, be prevented,-1st, by legal protection, $2 d$, by encouraging the slaughter of the mongoose, a small reward being offered for every head brought to officers appointed for the purpose of receiving them.

For further information see my Report to His Excellency the Governor of Jamaica, Veterinary Journal, December 1896.

\section{MORBID ANATOMY AND MICROSCOPIC APPEARANCES.}

BY T. A. WILLIAMS, M. B., C.M.

As Principal Williams, owing to his constant travel about Jamaica, inspecting and examining healthy and diseased herds, las not himself har time for microscopic and bacteriological examination of the specimens we took from live and dead cattle, this duty devolved upon me. The following is a summary of my observations.

As the enumeration in his Report of the many affections to 
be considered shows, our attention was prominently directed towards the microscopic characters of the blood:-

A. In the first place, great anæmia was almost always present. The difficulties in making exact observations with the hæmatocytometer in open pens were considerable; and as we had also to make other blood and visceral preparations-which to be of any value must be done with rapidity-the red corpuscles of the blood were enumerated in only a few instances. The smallest number found was 700,000 per cubic millimetre. This number indicates a most profound anæmia, being more than seven times less than the normal number.

The hæmoglobin of the blood was also found greatly diminished, but as far as my limited observations went, not to so great an extent as the number of red cells.

B. The features of the red cells were greatly altered in the more grave cases characteristic of changes in all pernicious anæmias. Their measurement (normally $7 \cdot 5$ micro.) varied from 3 to 9, 10, or even 12 micro. Their shape, instead of being perfectly discoid, was in many instances irregular, with prolongations in all directions. They were often curved on themselves, and were sometimes without pigment. In some I observed a central area about 4 micro. in diameter, which did not stain with basic or acid aniline dyes, surrounrled by a more transparent zone about 2 micro. wide, staining faintly with basic aniline dyes, but becoming decolorised by a weak solution of acetic acil.

c. The white corpuscles did not appear to be essentially modified, and no detailed study of them was made.

D. The blood also contained in several cases one or more of the following abnormal bodies:-

(1) In the first acute case observed, that seen in Trelawny, I found a few bodies shaped like a short banana, averaging 4.6 micro. long and about 2 micro. broad, very highly refractile, and not staining deeply with basic aniline dyes. They were seen only in the red cells, not free in the plasma.

(2) Numerous irregnlarly rounded bodies abont 1.5 micro. in cliam., within, generally near, the margin of and be- 
tween the red cells. They were much more numerous in the blood exuding from the cut organs, especially the liver, spleen, and, above all, the kidney. They stained more deeply than the former by Lœfler's method, but were decolorised completely by Gram's method.

(3) Faint bodies, in some foci of a pale brick-red tinge, never observed within but only between the cells.

The purposes of our inquiry are sufficiently served by the fact that the bodies I have observed are identical in essential respects with some of the forms described as being found in the blood by those who studied that fluid in the disease known as Texas fever.

Any slight differences which may exist between what I and others have seen are only to be expected in view of the modifications which the disease must necessarily exhibit under such various conditions in different countries. The study of this condition is yet in its infancy, and one has only to think of the very striking homology between this disease and malarial fever in man, to realise how many differences must necessarily be found in so many forms of the same disease.

The minute examination of organs not apparently abnormal to the naked eye shows the following:-

(1) The liver :-

(a) The liver cells were, in some cases, the more acute in a state of clondy swelling, their nuclei being almost all obscured. A fatty degeneration of the cells was presented in the more chronic cases, and in many a slight amount of abnormal pigment appeared within the cells.

(b) The bile capillaries in two cases were full of a dark yellow pigment, appearing as beautiful radii from the central vein of the hepatic lobule.

(c) The blood capillaries were visible only here and there, and did not present any abnormality either in their walls or contents. I have not yet observed any abnormal contents in the capillary blood of the organs.

(2) The chief abnormality in the spleen was the presence of an unusual number of masses of granular siennacoloured pigment within the large cells of the splenic pulp and the endothelioid cells of the stroma. Also a 
large number of minute isolated granules of similar appearance extend throughout the spleen substance. This is probably broken-down blood pigment, and is an invariable accompaniment of any disease in which such extensive destruction of red blood-cells takes place as in this. The amount of pigment varies with the rapidity of destruction of blood-cells, and if very great (which only occurs in very acnte cases) it is excreted by the kidney, giving rise to the appearance of the urine which produces the striking symptom of red water. In no instance lave we observed such a degree of blood change. In none of the cases has there been even sufficient to canse the thickness of bile which is one of the characteristic changes in the severe forms of this disease.

(3) The kidneys showed extensive cloudy swelling in their cells, their outlines being quite indistinct, while in a case which had been ailing for weeks the cells appeared quite normal.

(4) The other organs present, with the exception of anæmia, no morbid pathological change.

\section{BIOLOGY OF THE ORGANISM.}

The organism appeared to be, as in Texas fever, either the protozoon Pyosoma bigeminum of Theobald Smith, or a form closely allied to it. If any difference exists, it is chiefly in the thickness of the flagella, which here appears to be greater. Nearly all the stages of development, as described by American authors, and figured in "The Johns Hopkins Hospital Reports for 1896," have been seen in my specimens.

The American authors seem to be uncertain of the order of the developmental stages, and I confess to be also somewhat uncertain upon this point. Further observations of perfectly fresh blood in the fields (a matter of great difficulty in a hot climate) may lead to a correct conception of all the developmental changes.

In my own specimens there appear to be at least nine stages which show natural segmental changes, as seen in the photomicrographs. 'The illustrations Nos. $25,25 \mathrm{~A}, 25 \mathrm{~B}$, and $25 \mathrm{c}$, show what appears to be by far the most common form in the ox. 
IXOIDI AN.EMIA-TEXAS FEVER, ¿N.

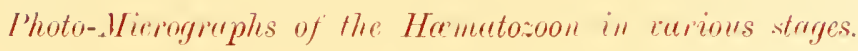

The subjects of the following Photo-Micrographs have heen worked ont and photographer by my colleague Dr. Hunter, from specimens prepared in Jamaira by my son Thomas A. Williams, Al. B., C.M.

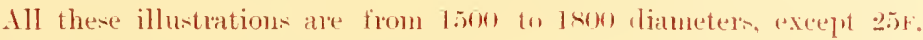
2.51 , and 2.5.5, which are 2100 , lianters. 



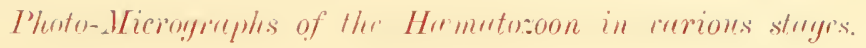

Fig. 24.

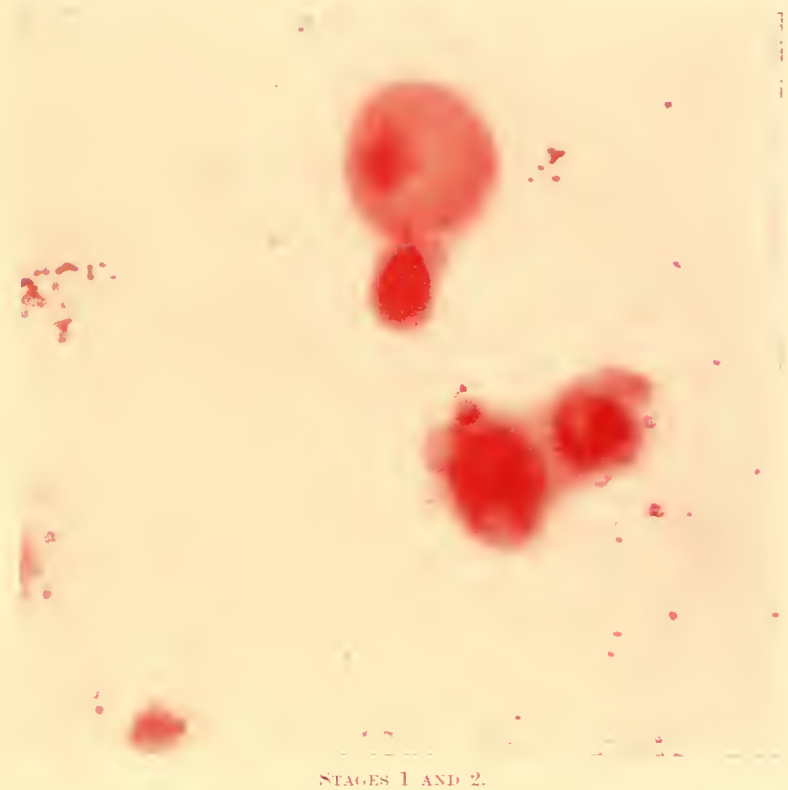

Bloou of Blue or Cow Tick. slowing l)lood corpuseles and plusmolit of various sizes. chiefly in the ylasma ; average size alont $i \mu$. in clianeter; the smaller bollies are the more highly refractive.

Fili. 25 ,

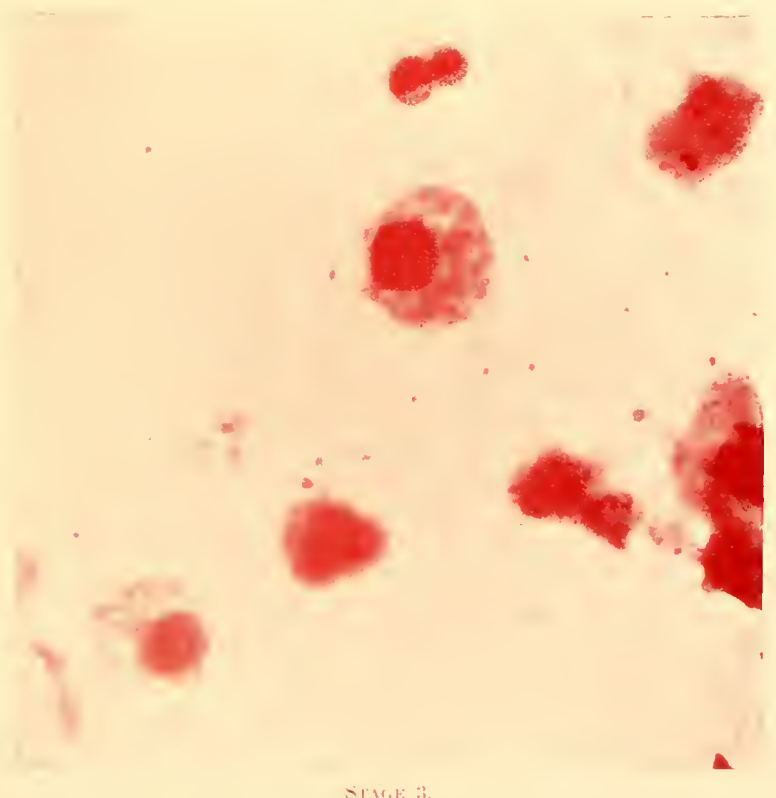

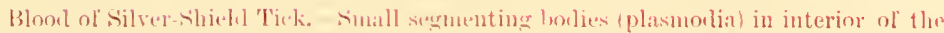
band corpuscles. This maty be calles the thimb stage of tevelopment. 



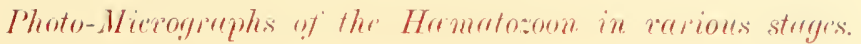

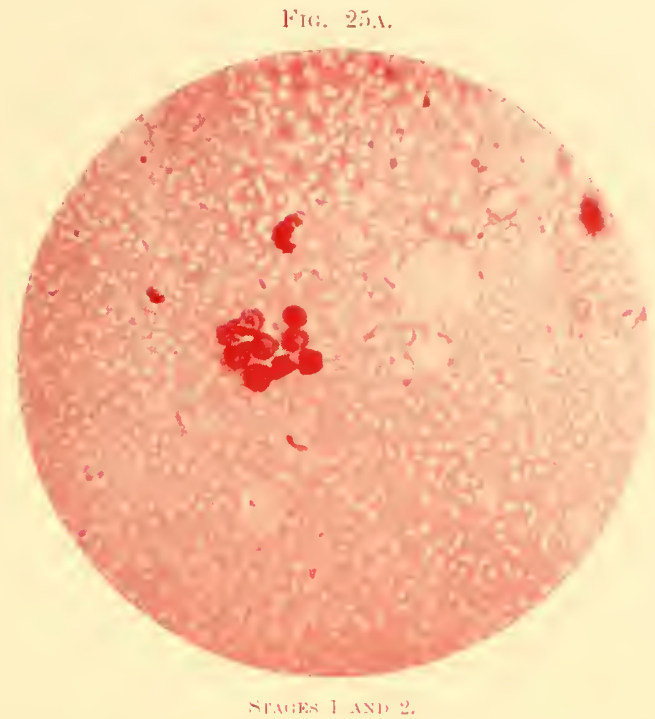

Blood of 'lexas 'Tick. showing platsua rowlent with snall highly refractive lonlies:

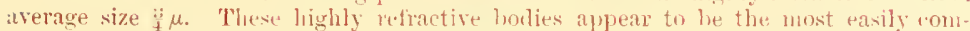
mmuivated form of the organisun. It will he observerl that there " "ocens-like "borlies are much moe numeton: in this than in the two previous illustrations (Figs, 2t and 25) : a cluster of spherioal segmentime boties, consilerably pigmenterl, and coccilioilal in appearance, is seen near the centre; the libot corpuseles appear to bave unclergone. but slight changes.

\section{Fili, 25 is.}

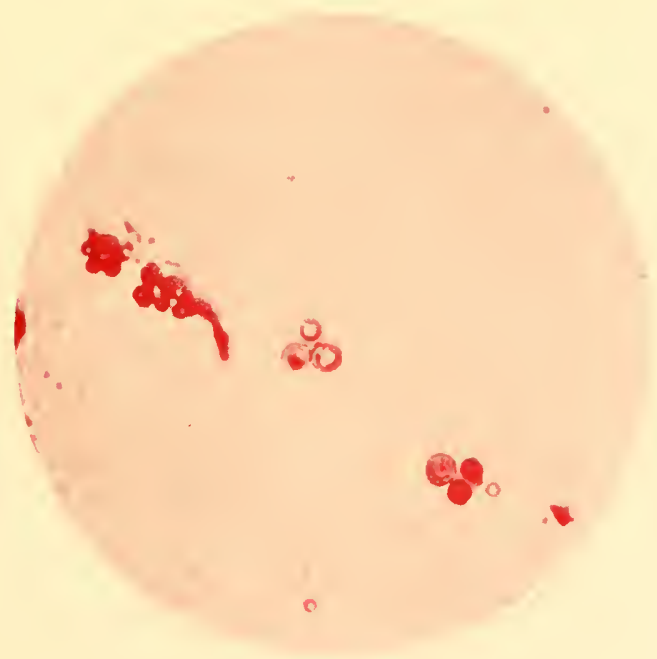

STA:FE 1, 2, AN1 4

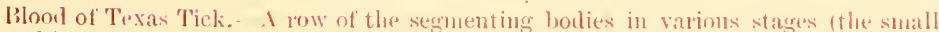

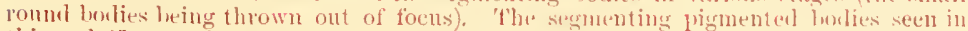

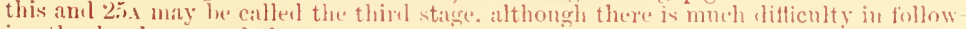
ing the levelopmental changes. 



\section{Photo-Micrographs of the Hematosoon in verious stuges.}

FIG. $25 \mathrm{c}$.

STA(k 3, same as sepll in Tick Fig. 25.

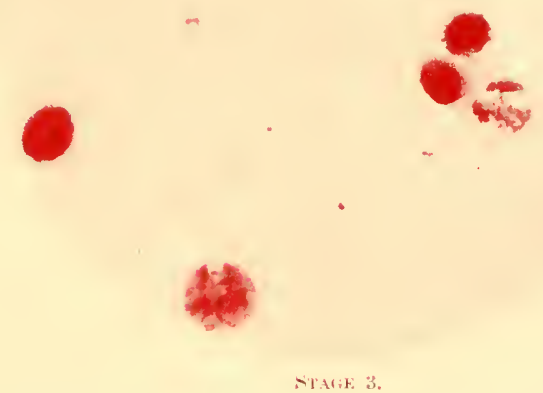

Blood of $\mathrm{Ox}$, showing small segutenting bodies(plasmorlia) in interior of the red blowt corpuscles. These stain with great difficulty, amul are here representerl by the colourlesw (white) portions. The resl corpnseles are shrunk to about half their normal size.

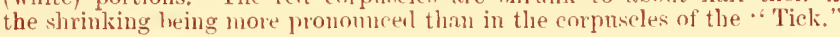

FIG. $25 \mathrm{~N}$.

\section{8}

S'TAFiE 4

Blood of $\mathrm{Ox}$, showing enlargenent of free segmenting holles, the previons stages of which are seen in Figs. 2.5. : and 25in. The boulies are seen here perfectly splurriral, with distinct and sunofl outlines, and show, alparrntly, uo internal structure. They arr not, however, vacuous, hut contain transparent "mloplasm, which is less refractive than the small spheres noticel in the first four illustrations. They vary in size from I $\mu$. to $1 \frac{1}{2} \mu_{0}$ in cliameter. 

IXOUIC ANAMIA-TEXAS FEVEL, ETC.

Photo-Mirrographs of the Hometosurn in rarious stregrs.

FIG. 25E.

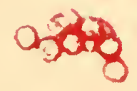

STAR: i,

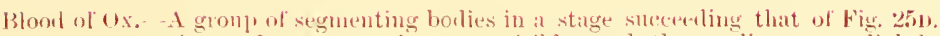
Ueve traces of internal segunentation are visible. amol the ontlines are cliglitly untcherl.

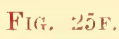

Silutiti.

Bhool of (x) A nore alvances stage of segmentation, where the organism is

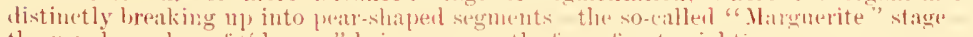
the usual mumber of " leaves" being aplatently from live to cight: 



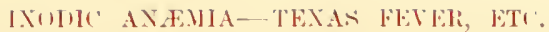

Photo-Mireagraphs of the Hematusoon in rerious striges.

FIt; 25 t.

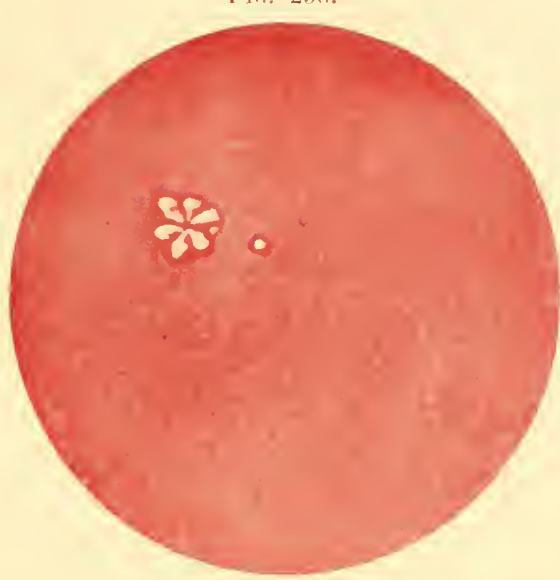

S'IAT.E: $i$

bloml of Ux.-From seat of fresh hite of 'Texis 'T'ick. with " dak hack-gromm illumination." showing a vely perfect "Marguerite" of six leaves, and al sumll refinetive splure. The blood cells are faintly seen, witl contained plasmolia.

Fl(: 25H.

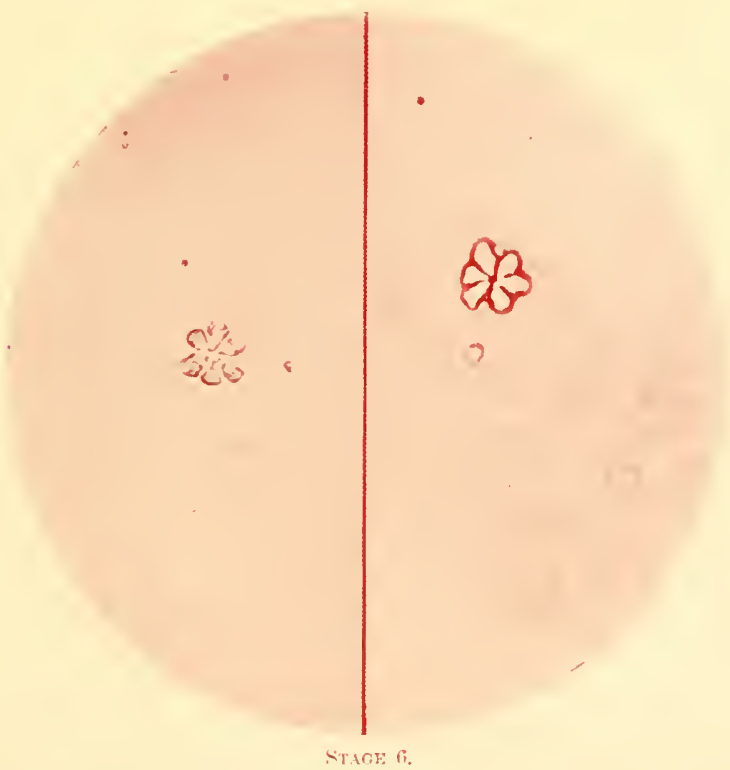

f'wo other viows of Fig. 2.x: under diflerent illummation amb foens: the altered hood corjuseles heing hetter sern than in son. 

IXIDIC ANEMLA-TEXAS FFTEL, ETS.

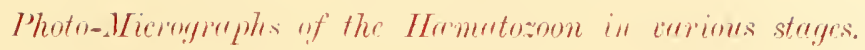

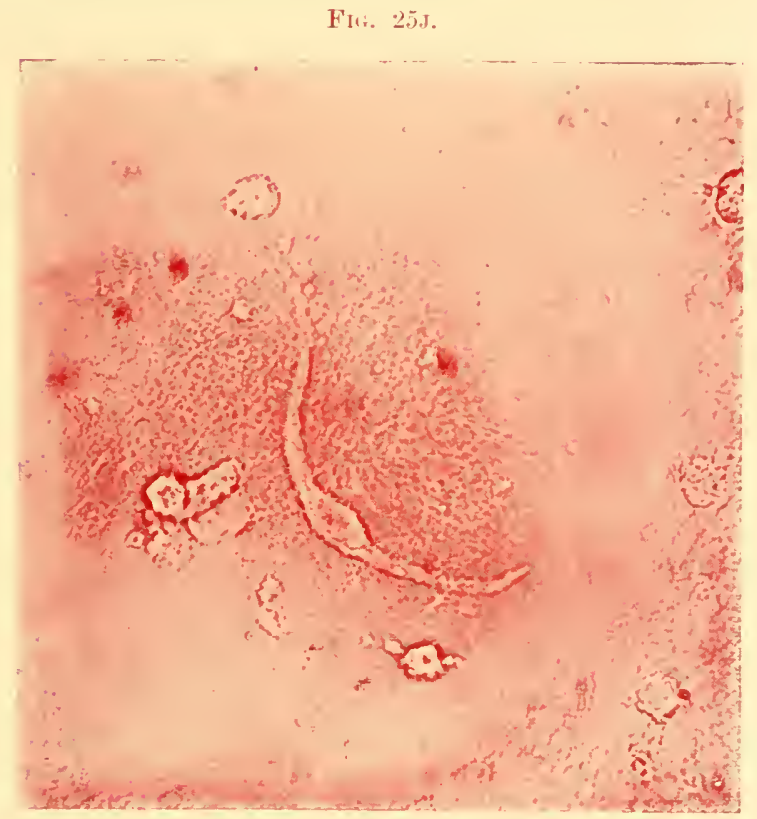

STACE 9.

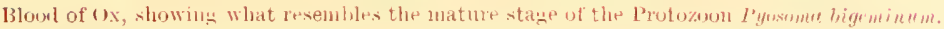
This Hayellate stage must be rarely reached in eattle, fin there are rery tow sesen in the specimens; but they may probubly be more numproms in actite cases, mue of which were seth hy ute. The plate shows varions stages of the disintegration of the nunal, which seems r.stremely liable to break up alter removal firm the lorly. (Imlend this Hagellate stage

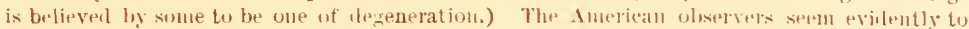

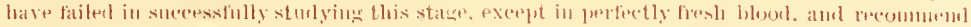

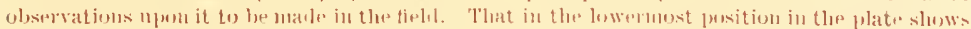

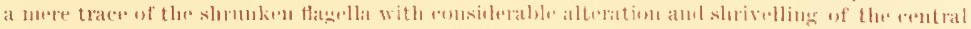

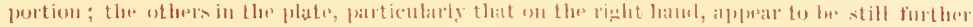

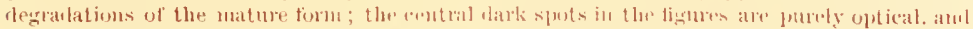

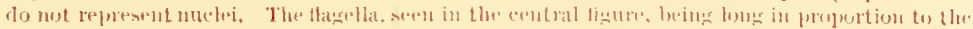

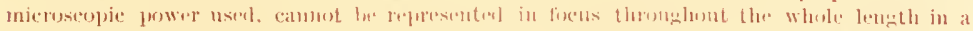
photograpls, as they ale wot all on sme plane. It will le ditlicult to establish the irlentity of

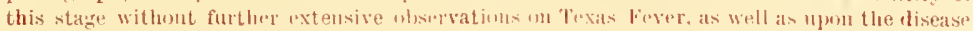
surra, secu in the hurse in Inulia. (sine sinru.) 



\section{Summary.}

(a.) That the disease as witnessed by me is a chrouic form of Texan fever conveyed from place to place, and transmitted from one animal to another through the intervention of the tick.

(b.) The infection is conveyed by the progeny of ticks which have matured on infected cattle, and is inoculated by them directly into the blood of susceptible cattle.

(c.) It is stated that two mild attacks, or one severe one, will probably prevent a subsequent fatal attack.

(d.) That animals under one year old, though not immune, are not so frequently attacked by the slower form of the disease, and that if such be nursed over one or more mild attacks, they may probably obtain immunity. Ticks being the cause of the disease, there should-

(1.) Be a united effort to destroy them on the cattle by external dressings, and on the ground and pastures by burning.

“Dipping ponds," as now used in America and Australia, should, where possible, be preferred to the applications by hand. Plans of the most recent and most effective ponds or baths should be obtained.

(2.) To prevent the further introduction of ticks into the country by an effective system of quarantine.

(3.) After destroying the ticks on the cattle, the "animal strength" should be maintained by keeping them in a quiet sheltered place and feeding well, without causing them to travel for their food.

(4.) To avoid drastic medicines. If the cattle drink pond water, it will be safe to conclude that the disease may be complicated and aggravated by the Strongylus contortus in the fourth stomach, for the destruction of which a dose of sulphate of copper should be administered, and, if necessary, repeated two or three times; but when cattle obtain their water out of running streams, or from clean tanks, the copper is not necessary. If the animal be feeble, the following may be administered to act as a tonic and as a food, viz.:Sulphate of iron, nitrate of potash, and common salt (chloride of sodium), of each one drachm in a pint of pimento tea, once a day for a week or so. 


\section{CHAPTER XXXV.}

\section{SPORADIC DISEASES.}

\section{RHEUMATISM.}

ACUTE RHEUMATISM, OR RHEUMATIC FEVER.

Definition.-An inflammation of the fibrous structures of the joints, tendons, ligaments, thecæ of muscles, or of the heart and closed cavities, due to a specific condition of the blood, and accompanied by fever, stiffness, and lameness. The inflammation is metastatic or erratic in its character, disappearing from one part of the body to reappear in another, without any appreciable cause. The inflamed parts are generally swollen and hard, but in some instances no swelling can be detected.

Characteristic of rheumatic inflammation both in man and the lower animals are-(1.) The rarity of suppuration. This process may and does sometimes occur, especially in horned cattle, from inflammation of the superficial coverings of the diseased tissues, cansed by external bruising, or the application of powerful external irritants. (2.) The occurrence of cardiac complications. (3.) A natural predisposition or diathesis, which predisposes to the malady without any ostensible cause. The form of the disorder in this instance is of a less acute nature, but may become acute by the addition of any trivial cause of disease.

Etiology.-The application of cold; damp; or it may result from other diseases, as epizootic diseases, contagious eczema, or from a natural predisposition without apparent cause.

Semiology.-The symptoms of rheumatic fever are as follows: - Sudden lameness, with or without swelling of some particular articulation, such as the stifle, hock, or fetlock joints; the flexor tendons, immediately below the knee or hock, in the sesamoidean bursa, the thecæ of the muscles of the loins and quarters, or of those of the thoracic walls, constituting pleurodynia. The lameness may be preceded by some febrile disturbance or a malaisé condition, expressed by yawning, dulness, or dejection. 
The lameness often disappears from one part of the body and suddenly reappears in another. Very often the lameness is symmetrical, that is to say, it will be due to inflammation of the same joints in both legs, say in two stifle or in two hock joints. The fever is acute and sthenic when it is not preceded by some epizootic disease; the pulse is hard and unyielding; the mouth hot and dry. The urinary secretions are impaired and altered; the urine, which in health, when tested with litmus paper, gives an alkaline reaction in the herbivora, is neutral, or more or less acid, and if microscopically examined is found to be loaded with hippurates of soda and ammonia, and hippuric acid.

There is generally some degree of costiveness, and if blood be drawn, the coagulum will be firm and large; indeed, in no disease is there such a rapid and sensible increase of fibrin in the blood as in acute rheumatism-in some cases as much as 10 parts in 1000 of blood have been found. The swellings of the affected parts quickly assume a hardness due to exudation, but, as already stated, suppuration rarely occurs. The elevation of temperature is sometimes very great- $104^{\circ}, 105^{\circ}$, or $106^{\circ} \mathrm{F}$; when over $105^{\circ} \mathrm{F}$. it is always indicative of great danger. I have known it as high as $109^{\circ} \mathrm{F}$. in a cow.

\section{CHRONIC RHEUMATISM.}

The symptoms of this form are mere modifications of those of the former, except that fever may be entirely absent. It is, however, much more persistent, less metastatic, and leads to alterations of structure, consisting of ulceration of articular cartilage, eburnation of the bones, and the formation of osteophytes, much more commonly than the acute, an attack of which may leave the animal quite well at its termination. Sometimes, however, the acute degenerates into the chronic, and an animal subject to the chronic is very often attacked by the acute form. In chronic rheumatism not only are the white fibrous structures altered, but the bones in various parts of the body may become subject to various pathological changes; tumours or bony excrescences form on the bones of the pelvis, spinal column, and in the fringes of the synovial membranes; distortions also occur. I have seen the neck twisted by large bony tumours on the cervical vertebræ, arising from this cause; also enlargement and ancliylosis of the joints, ulceration of articular cartilages, particularly of the navicular bursa, eburnation of the exposed bony extremities, and 
ossification of the walls of the heart. The soft structures above and below the affected joints in horned cattle sometimes swell, suppurate, and continue to discharge pus for a lengthened period, the animal rapidly wasting, and finally becoming worthless.

Both in the acute and chronic forms the pericardium, endocardium, and cardiac valves may become inflamed. This complication, however, is of much more frequent occurrence during an acute attack. The pulse becomes jerky and wiry, the heart's action short, sharp, and angry, the cardiac impulse is often awanting, the pulse intermittent, and a to-and-fro sound accompanies the heart's movements.

\section{PATHOLOGY.}

What the true nature of the so-called rheumatic poison really is, pathologists are not agreed upon, some asserting that it is lactic acid, whilst others are not satisfied on this point. It is certain, however, that the urine both in man and in the lower animals assumes an acidity which is quite unnatural; but the acid found in it is not the lactic, but the uric and its salts in man, and the hippuric and its salts in the herbivora. The suggestion that rheumatism is due to the presence of an excess of lactic acid originated with Dr. Prout. The lactic acid originates by the conversion of the starch of the food into lactic acid, which then combines with oxygen to form carbonic acid and water; and anything which interferes with this change may lead to the accumulation of the acid in the system. Dr. Garrod, however, found the serum of the blood distinctly alkaline in thirty-five cases of rheumatism in the human being.

In every case of rheumatism met with by myself, and where an examination of the serum has been made, it has been found alkaline. The only experiments which go at all to establish the connection of lactic acid with rhennatism are those of Dr. W. B. Richardson, who injected lactic acid into the peritoneal cavity of dogs, and found that it caused death, not by inducing peritonitis, but peri and endocarditis, with thickening of the cardiac valves; the post mortem examination showing a red thickened state of the mitral valve, with fibrous bead-like deposits on its surface; the endocardium lining the left ventricle intensely inflamed, resembling bright red velvet, and the aortic valve of a deep red colour, and its borders thickened 
and everted; the pericardium red, injected, and covered with lymph.

Dr. Fuller believes the poison of rhemmatism to be identical with some natural excretion of the skin. The researches of Dr. Parkes lead him to conclude that the disease is due to some substance rich in sulphur; whilst other writers and observers look upon rheumatism as simply an inflammation of the fibrous and serous membranes, the predisposition to which arises from deficiency of healthy tone in these textures, rendering them liable to be inflamed by the influence of a variable temperature. The character of rheumatism, more especially its metastatic tendency, and the symmetrical development of its symptoms, tend to show that an alteration in the blood exists; the only constant alteration is, however, the great increase of fibrin. We are thus led to the conclusion that, whatever the morbid material may be, it is one formed within the economy, and due either to a faulty secondary digestion and assimilation, or to an abnormal metamorphosis of tissue and the retention of the products of such metamorphosis within the animal body. In man copious perspiration is a characteristic sign of rheumatism, and it is to be noticed that this perspiration has an acid or sour smell. In the lower animals perspiration may be copious, but sourness of smell is not appreciable.

In rheumatic endocarditis in man, the left side of the heart is only affected, as a general rule; and it has been inferred by Dr. Richardson that the chemical change whereby the morbid matter of acute rheumatism is produced, is completed in the pulmonary circulation; that when the blood in the pulmonary capillaries is exposed to the air, the acid quality of the poison is produced; that the poison thus formed is carried by the arterial circulation to be disposed of by decomposition or elimination, or both; and that it does not return as an acid by the veins, but simply as a product which admits of retransformation in the pulmonic circuit into the acid state.

TREATMENT.

In the Horse. - A gentle laxative may be given with advantage, and there is no such objection to an aloetic purge as in some of the diseases which have been enumerated. If the pain be excessive, opium may be combined with the purgative, and 
repeated once or twice a day. To prevent its constipating action on the bowels, the opium may be combined with calomel, but calomel should not be given until the bowels are "set" after the purgative. If the pain is not excessive, aconite and the nitrate of potash are more suitable than opium, and in all cases the nitrate of potash is to be freely administered in order to alter the character of the bloorl, to act upon the excretory organs, and to influence the vascular system. I am of opinion that the nitrate has a more jowerful influence on the disease, and is less debilitating than the bicarbonate of potash or the liquor potassæ. In combination with the nitrate, colchicum has a favourable effect, and in cases where debility is not great, it should bè substituted for the aconite about the third or fourth day. If the sufferer be strong, young, and in good condition, a moderate bleeding is usually prescribed; as a rule, however, the conditions do not warrant the abstraction of blood. Indeed, it is now generally agreed that although bleeding, by relieving congestion, may give temporary relief, it enhances future suffering and danger, by increasing the irritability of the heart, and consequently predisposing it to rheumatic inflammation.

Local treatment.-If the pain in the inflamed parts be very great, it may be advisable to endeavour to relieve it by warm fomentations, and the application of a liniment, containing some of the extract of bellarlonna, opium, or aconite; but valuable time should not be wasted if these afford no relief, and blisters are to be freely applied to or near the inflamed parts. The treatment by blistering is called that of elimination, and those who advocate it most strongly use it only. According to Dr. Herbert Davies, physician to the London Hospital, the action of blisters, by promoting a serous discharge from the blistered surface, affords a ready means of exit to the rheumatic virus.

The success of this treatment depends upon the amount of vesication produced by the blister; and in oraer to promote the free flow of serum, the blistered parts are to be subsequently covered by linseed meal poultices. According to Dr. Davies, the blister treatment causes a speedy diminution in the frequency of the pulse, rapid subsidence of the joint affection, and lessens the liability to cardiac inflammation. Within twenty-four hours after the removal of the blister, the urine is stated to become alkaline in reaction. 
Dr. Davies does not apply the blister upon, but near to the inflamed part. I can speak with great confidence of the benefit of treatment by blistering in the lower animals; the blisters are, however, quite as effectual when applied to the inflamed parts.

In the Ox.-The same external treatment is applicable as in the horse ; the nitrate of potash may, however, be given in larger doses, and the bowels acted upon by the sulphate of magnesia or soda instead of aloes. Cows will often, when suffering from rheumatic fever, refuse to rise from the recumbent posture. In such cases repeated change of bedding is absolutely necessary; great cleanliness, dryness, and comfort must be secured, and the inflamed joints wrapped in dry flannel, in order to prevent bruising and the occurrence of the suppurative process.

With regard to cardiac complications, I think the cow is not quite so liable to suffer as the horse: this is, however, a matter of opinion merely.

If suppuration occur in or near to an articulation, it is best at all times to put the ox or cow out of its sufferings, as recovery seldom or never takes place.

The diet in all cases, if the animal be not already debilitated by previous disease or other cause, nust at first be light and easy of digestion, as mashes and hay, or green food, if in season; as the fever subsides, stronger food may be cautiously allowed; and if depression be a marked symptom, cinchona and ammonia are to be prescribed in suitable doses.

Continental writers describe articular rheumatism as a febrile infectious disease, most frequently affecting horned eattle, and often due to abortion or non-delivery; and they consider that the absorption of septic products of the uterus is the sole cause of articular rheumatism. I cannot agree with this view, and the writers have evidently confounded rheumatism with septic conditions sometimes witnessed immediately subsequent to parturition, abortion, or co-existent with retention of fœtal membranes or of a dead foetus:

The treatment of rheumatism, according to Continental writers, is a very complicated affair, consisting of irritating and stimulating frictions to the affected regions, the induction of diaphoresis, massage; the administration of salicylate of soda, salol, niphthol, antipyrine, laxatives, tartar enetic, tartar colchicum, pilocarpine, \&c. The "reader" can have his choice; the "anthor" has tried a few of the remedies and found them inoperative, unless for harm. 


\section{CHA PTER XXXVI. \\ SPORADIC DISEASES-continued.}

\section{DIETETIC DISEASES.}

In order that the health of the animal body may be duly maintained, it is necessary that the various kinds of food should be sufficient in quantity and undeteriorated in quality. It is also necessary that the solid food contain at least four classes of constituents, each of which plays an important part in supplying the various wants of the economy, either as to supplying materials for tissue formation or for force. These constituents are-(1.) Nitrogen, nitrogenous or proteid food, which seems most fitted to nourish muscular and other albuminoid tissues; on this account the substance containing it has been called plastic or nitrogenous food. Substances defective in nitrogen, and having an cxcess of carbon or hydrogen, are called (2.) carbohydrates or starchy foods, consisting of elements which easily break up into carbonic acid and water, supply materials for combustion, and thus assist in maintaining animal heat and other energies required by the system. (3.) Fats, or hydrocarbons, like the carbohydrates, have no nitrogen, require a great supply of oxygen, and evolve a large amount of animal heat. Food must also contain (4.) saline materials, in order that the solid structures of the body be built and maintained in health; the saline constituents also aid in the processes of assimilation and elimination, of carrying new materials into the system and old materials out of it. If these constituents are absent, or are present in undue proportions, health cannot be maintained, and common experience has taught us that all animals are kept in the best health when fed on a mixture of foods.

Although chemical analyses have enabled us to know the quantities of nitrogen, carbon, \&c. contained in the food, it does not follow (as General Fitzwygram justly remarks) " that the food which possesses these constituents in the greatest abundance 
will produce the most beneficial results. Digestibility, readiness of assimilation, absence of unduly heating properties, and many other qualities, are needed in order to make a substance possessing the necessary ingredients available as food. Chemistry is a valuable but not an infallible guide, and its indications require to be tempered by the test of experience."

Professor Dick said that a horse may be kept without work, but taking a little exercise, in fair condition on 12 lbs. of hay and 5 lbs. of oats per day; but if a good amount of work is to be got out of it, the horse should have 14 lbs. hay, 12 lbs. oats, and 2 lbs, beans. In this diet of the horse at rest there are $29 \cdot 2$ ounces of flesh-formers, and 150 ounces of heat-givers and fat-makers; and in the work diet there are 251 ounces of heat-givers and $59 \cdot 1$ ounces of nitrogenous constituents.

It is not my intention to enter into a lengthy discussion on the above subject. My experience leads me to conclude that the scale laid down by Professor Dick is too limited; that it is necessary to give to a horse at rest several pounds more corn per day in order to keep it in good health. Every-day experience teaches us that no horse can be kept in health without exercise, and no horse taking sufficient exercise to keep it in health can maintain its vigour and flesh on 5 lbs. of oats per day.

I am also of opinion that all horses at work should have a mixture of oats, beans, and hay; say $14 \mathrm{lbs}$. oats, and $2 \mathrm{lbs}$. to 4 lbs. of beans per day. I find this to be the cheapest and best of food. It must be given either whole or crushed, dry, and it is advantageous to mix it with chopped hay. The quantity, however, must vary with the size of the animal.

The corn and hay must also be of good quality, sweet, free from mildew, well harvested, old, and dry.

For further information on the subject of feeding, the reader is referred to Sellar and Stephen's Physiology at the Farm; General Sir F. Fitzwygram's Horses and Stables; Low, Playfair, Voelcker, and others.

Effects of over-feeding.-Too much non-nitrogenous food favours che development of fatuess and obesity. This is well seen in pet logs and cats fed on cream, sugar, and tit-bits of various linuls. These animals die before half their days are over from latty infiltration and legeneration of the heart, liver, and other oryans. Lions, tigers, and other carnivora liept in menageries 
also suffer and die from fatty degenerations, and old carriage and other favourite horses die from the same cause, when pampered and fed with carbonaceous food.

Accumulations of the flesh-forming or nitrogenous elements in the blood, and their non-elimination, cause the development of many blood diseases, as has been already, and will be further shown. For example, over-feeding on linseed cake induces a septic condition of the blood. Analyses of oil-cake have shown that it contains over 22 per cent. of nitrogenous materials, whilst dried wheat contains only 12.47 per cent. of albuminous compounds. Again, decorticated cotton cake contains a much larger per-centage of flesh-forming matters than even linseed eake, and the proportion of oil is higher than in the best linseed cake. The following are the analyses of the two cakes:-

\section{DECORTICATED COTTON CAKE.}

Average analysis of seven Samples. (VOELCKER.)

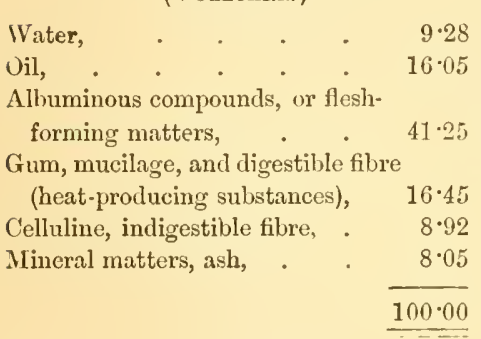

\section{OIL-CAKE.}

(Professor Johnston.)

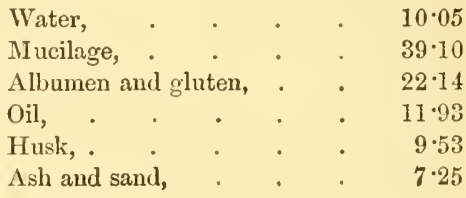

$10 \cdot 05$ $39 \cdot 10$ $22 \cdot 14$ $1 \cdot 93$ $7 \cdot 25$

The nitrogen of the food is not all assimilated in the system, and a large portion passes away with the excreta: on this accomnt the dung produced by cake-fed stock is particularly valuable. Should anything occur to interfere with the functions of the excretory organs, the nitrogenous compounds accumulate in the system, and there set up a variety of diseases more or less grave and important. It will thus be seen that great care and discrimination are to be exercised in feeding stock upon highly nutritious food; and provided the feeder has an eye more to the inaintenance of the health of his stock than to the richness of his manure, he will bear this in mind.

Whilst over-feeding leads to the development of disease, deficiency of food leads to no less grave results; and this 
deficiency may relate both to quantity and quality. "A deficiency of food," says Dr. Letheby, "especially of the nitrogenous part, quickly leads to the breaking up of the animal frame. Plague, pestilence, and famine are associated with each other in the public mind, and the records of every country show how closely they are related." It is stated that in cases of very gradual starvation an urgent feeling of hunger is not a prominent symptom, and even when it exists at first, it usually soon diminishes, and is succeeded by a feeling of exhaustion and faintness, and even a loathing of food, if abstinence has been long protracted. -(R. B. Holland.)

Whilst it is essential to health that food should be sufficient, but not over-abundant in quantity, and that its quality should be so regulated as to supply all the wants of the economy, it must be confessed that the lower animals will live for a very long period on very common fare, provided it be sufficient in quantity, and they are not exposed to extreme cold; but to have health, energy, and condition, both food and water must be good in quality.

A dietetic disease may be defined to be a morbid condition of the body, brought about by food or water deteriorated in quality, iusufficient or over-abundant in quantity, or containing some ingredient directly poisonous or injurious to the animal economy. 


\section{CHAPTER XXXVII.}

\section{SPORADIC DISEASES-continued.}

\section{DIETETIC DISEASES-continued.}

\section{DIABETES INSIPIDUS, POLYURIA, HYDRURIA- AZOTURIA-OXALURIA-RED WATER-ASTHMA, BROKEN IVIND, \&c.}

\section{DIABETES INSIPIDUS, POLYURIA, HYDRURIA.}

A DISEASE characterised by great thirst, excessive discharge of urine, rapid emaciation, languor, and debility. In the majority of instances it is caused by deteriorated food, but in some cases it seems to be due to some constitutional cause, produced through derangement of the assimilative functions, either in the digestive canal, the solid organs, or the blood. When induced by no traceable cause, and where change of diet has no effect in checking it, the disease is generally premonitory to farcy or glanders, and is symptomatic of a breaking up of the tissues of the body.

Etiology.-Diabetes is induced by two kinds of causes, namely, intrinsic and extrinsic. The intrinsic causes are those originating in defective assimilation, or rapid tissue metamorphosis, owing to the actual presence of the glanders poison, or to a condition of the system tending to its development; the polyuria being induced by the action upon the kidneys of those constituents, products of tissue change, which are naturally eliminated by them. In some instances diabetes results from indigestion, disappearing when the digestive apparatus is restored to its normal condition. It also accompanies other diseases where the digestive process is defective.

The extrinsic causes are to be found in the food which the animal consumes; I am not aware that it is ever induced by the water it drinks. Dark-coloured, highly-heated hay generally 
causes much thirst and diuresis, probably containing some ingredient which acts as a stimulant to the kidneys, but it does not induce diabetes so readily as hay that is musty, oats or beaus which have been musty or damp, even if kiln-dried, and bran having a peculiar greasy odour, more especially foreign bran.

Diabetes sometimes occurs as an enzootic disease-not due to any atmospheric influence, but following a bad harvest, and caused by damaged fook. All writers agree in condemning kilndried oats. I am of opinion that these, if of fair quality when put on the kiln, are not so hurtful as is generally supposed, and that they are much more likely to cause disease if given when damp or musty.

In a stud of horses where glanders prevails, the slightest error in feeding the apparently healthy animals will often bring on profuse diuresis. In one stud under my care, a supper of warm boiled food was sure to be succeeded by several animals being attacked by inordinate thirst and diuresis. Habitual feeding on boiled food is a very common cause, and although animals so fed may look moderately sleek, and lay on fat, they are incapable of performing the same amount of work as others fed on similar food given uncooked.

Semiology and Pathology.-The diagnostic signs are excessive thirst and profuse urination: in addition to these there is failing of the appetite. Some teachers say the appetite is increased; I have never known this in the horse, but in the dog there is often a craving for flesh. The visible mucous membranes are pale or sometimes rusty yellow; the skin is harsh and the coat unhealthy looking; the animal is debilitated and rapidly loses flesh. The pulse is often slower than natural, sometimes quicker, and always atonic. The mouth has a sour odour; the horse prefers unclean food and water, and is fond of licking the walls and manger. The urine is of a very pale colour, sometimes as clear as clean water, and has a specific gravity little higher than distilled water, the specific gravity of which is 100 , whilst that of diabetic urine is from 100.2 to $100 \cdot 3$, and that of healthy urine in the horse ranges from $100 \cdot 30$ to 10050. According to Lassaigne, the diabetic urine of the horse contains-water 98.0 ; urea, benzoate of soda, acetate of potash, acetate of lime, chloride of sodium, and free acetic acid, 1.5; mucus and sulphate of lime, 0.5 ; and differs from healthy urino 
in (1.) being more watery; (2.) containing acetic acid, and in being free from earthy carbonates.

The diabetes mellitus differs from the insipidus, from the circumstance that the urine contains sugar. I am not aware that this form is ever seen in the herbivora; but I have repeatedly met with it in dogs, and in almost every instance the creatures had been fed for a length of time on boiled liver. MI. U. Leblanc reports a case in a dog and another in a monkey.(See GamaeE's Domestic Animals.)

Thcrapeutics.-Investigate carefully the quality and quantity of the food, which in all cases, whether it be apparently good or indifferent, ought to be changed. If the horse is fed on oats and hay, try a change to beans and a fresh sample of liay; if fed mostly on beans, change to oats. The bran and other fodcker must also be changed, as any of these articles may contain some undetectable material which operates unfavourably on the economy, some constituent which acts as a constant irritant upon the body and kidneys. It is advisable to give an aperient, and to restrict the diet to hay and mashes for some days. Much relief will be afforded to the animal by giving the bicarbonate of soda in the food or water. I prefer this to chalk, a remedy held in well-deserved esteem.

When the aperient has operated, iodine is to be administered, commencing with two-drachm doses twice a day, and diminishing the dose as the thirst disappears.

Iodine was first prescribed for diabetes by Professor Dick, who discovered, whilst experimenting with it in the treatment of glanders, that it had the effect of allaying the sense of thirst in a most remarkable manner, and ever since then it has been held to possess specific powers in this affection. There is no doubt whatever as to its therapentic value, for not only does the thirst diminish, but the appetite improves, and all the other symptoms disappear as soon as the system is charged with the iodine.

It is usual to mix clay with the horse's drinking water; if soda bicarbonate is prescribed, there is no need to give dirty when clean water can be obtained, and it is cruel to restrict the water in quantity to any great extent. The horse must rest, and be carefully attended to for several days.

In the dog the disease is fatal. I hiave tried various remedies, such as feeding on milk or on flesh entirely; creosote, opium, 
astringents, the bromide of potassium, as well as the iodine treatment, and have seen no permanent benefit from any of them. Both iodine, or iodide of potassium, and the bromide moderate the symptoms for a time, but usually the benefit lias been of short duration-a cough has come on, rapid emaciation, decay, and death.

Post mortem appearances.-Both in horses and dogs, pallor of the organs and tissues of the body generally; in dogs, enlargement of the liver and caseous tumours in the lungs-the latter being probably accidental complications.

\section{AZOTLTIA.}

Under the term of azoturia, or nitrogenous mine, I intend to describe a disease to which attention was drawn in this country by Haycock, who named it, at the suggestion of Mr. James Moore, V.S., London, "Hysteria." In Gamgee's Domestic Animais, the same malady is described as "enzootic hrematuria of horses "- " schwaze harwindi" of the Germans, who state that it occurs in horses, and very rarely in mares. Mr. Haycock, on the contrary, says it occurs in mares only, and for this reason has called it hysteria. I have, however, seen it both in mares and horses, and have satisfied myself that in no instance is there blood in the urine, but that the dark coffee colour is due to the presence of urea and colouring matter.

The symptoms are well described by Haycock, who says"In all cases of this nature which I have treated the disease commenced very suddenly. They [the subjects thereof] began to exhibit an unusual degree of restlessness, to perspire profusely, which symptoms or states were speedily succeeded by a disposition to lie down; by great sluggishness, loss of motor power in the hind limbs; violent spasm of the large muscles of the loins and lind quarters (the gluteal muscles were excessively cramped), and the shoulders; the pulse in two of them rose from sixty to eighty beats per minute, and the respirations were greatly increased; they made several efforts to rise upon their feet, but from the total loss of all motor power in the hind limbs, they were unable to do so; two out of the three every now and then strained violently, and ejected, per vaginam, excessive quantities of coffee-coloured urine, which consisted principally 
of blood; the perspiration was excessive also. In two cases the more violent symptoms subsided for a time; they became cool in the skin, and partook of food and water, but were totally unable to rise. This improvement did not prove of any long duration; they commenced struggling again with renewed violence with the fore limbs, and continued to do so until they died." Gamgee gives the symptoms of the disease as it occurs in Germany as follows:- "Stiff gait, weakness of the hind quarters, frequent pulse, redness of the visible mucous memlranes, anxious expression of countenance, and sweating; a remarkable swelling of great firmness occurs over the loins and hips, and there is a copious discharge of urine of a very dark red or brown colour. Great difficulty of breathing ensues, tetanic symptoms supervene, and death."

The two descriptions agree in most particulars; both contain the diagnostic symptoms of the malady, namely, the tonic spasm of the gluteal, lumbar, and some scapular museles, and the profuse discharge of dark-coloured urine.

Mr. Haycock was of opinion that the disease had some connection with the period of cestrum, that it was the result of undue excitability, and le compares it with hysteria as described by Copland in his Medical Dictionary. Mr. Gamgee offers no hypothesis as to the nature of the malady. Professor Dick describes it as sprain of the psor muscles.

At first I was inclined to think, with Haycock, that it was a disease of the mare, but further experience led me to conclude differently, for $I$ have as frequently seen it in the gelding as in the mare; and examinations of the urine, both chemical and microscopic, have convinced me that there is no blood passed by the kidneys, but immense quantities of urea and a less notable quantity of hippuric acid.

'The pathology of the disease is, in my opinion, a hypernitrogenous condition of the blood and system generally, due to over-feeding and want of exercise; the excessive secretion of urine and excretion of urea being physiological results due to the presence of effete products-metamorphoses of nitrogenous food-in the blood. The history of every case that I have met with, and of all those recorded by Haycock, points to this. He says-- "The first case which I treated, the animal had rested about a week, at the end of which time it was put 
into harness upon a very hot day, and driven slowly for a distance of five miles, and was seized on the road. The second case also occurred after the mare had rested a week or a littie more, when it was taken out of the stable on a very sultry afternoon, and galloped severely, and shortly afterwards was seized. The subject of the third case rested from Saturday morning to Monday morning, at which time it was put to its usual labour, and driven slowly for about a mile, and was seized ere it had got to the end of the journey, short as it was." The above corroborates my experience of every case of this malady in a most remarkable manner. Varying periods of rest were succeeded by an attack on the first journey, the animals always leaving their stables in higher spirits than usual, and giving rise to the remark, "He never looked better than when he first turned out; in fact we could scarcely hold him, he was so spirited." I never met with a case that was attacked in the stable prior to some amount of exercise. It seems necessary that some degree of muscular exertion be performed, and the only way in which I can account for this is, that the blood before exercise contains a superabundant quantity of albumen unappropriated by the tissues, and that the exercise, by increasing the rapidity of the circulation and of the respiratory movements, induces a rapid oxidation of such superabundant albumen, whereby it is transformed into urea, hippuric acid, \&c., with which the blood becomes over-loaded, and the kidneys stimulated to excrete what is proving deleterious. Albumen is occasionally present in the urine, but this is by no means constant; its presence, however, points to an aggravated form of the disease, and is often prognostic of a fatal termination.

The presence of such effete material in the circulation prorokes tonic spasms of the muscles, loss of motor power in the posterior, and sometines, but rarely, in the anterior extremities, tetanic convulsions, and, finally, death, by extreme muscular prostration, simulating motor paralysis. In every fatal case that I have observed, the spasms and convulsions have been succeeded by extreme muscular debility, the muscles contracting but feebly on the application of a stimulus, the heart and diaphragm partaking of this prostration, and the animal dying from asthenia and apnoea. In other cases the animal has overcome the primary and violent symptoms, but has remained partly paralyzed in one hind extremity. 
Post mortem examination reveals the blood dark-coloured, having an ammoniacal smell, and semi-fluid; congestion of the lungs ; elots of dark blood on both sides of the heart; the bladder filled with dark or coffee-coloured urine, and sometimes softening of the liver and kidneys.

Congestion of the sheaths of the great gluteal nerves lias been present in some cases, in which the spinal cord at the lumbar region has been found red, congested, and softened, but in others these appearances have been absent, the spasm and loss of power being due to the effect of the altered blood on the muscular tissue.

Examination of the urine.-The urine is to he examined as soon as possible after it has been obtained from the animal, as it quickly becomes ammoniaeal. In every case its specific gravity is much increased-1.185 or higher. It is highest during the first few hours after attack, as it often becomes lighter in colour, even in those cases which succumb after the first day or two. Boiled, the urine generally gives no reaction, exeept that ammoniacal gas is emitted. Tested with nitrie acid, it occasionally beeomes almost solirl, but the solidity is rarely due to coagulation of albumen, but to the precipitation of erystals of nitrate of urea in great abundance. These erystals appear as mica-like scales of a brown colour, and fall to the hottom of the glass. At first the addition of the acid canses much effervescence, the urine being strongly alkaline, containing ammonia carbonate. When the effervescence has eeased the urea is rapidly precipitated, and the liquid loses much of its dark colour. Microscopically examined with a quarter-inch objective, it presents the appearanees seen in fig. 26 .

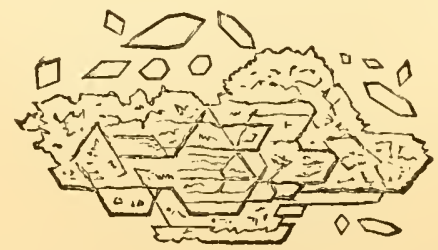

Fig. 26.-Crystals of Nitrate of Urea.

In some samples of urine analysed by Ivison Macadam sugar has been detected.

If the serum of the blood is earefully prepared, the erystais of nitrate of urea can be detected in it by the following process :- 
Take a given quantity of serum, and percipitate the albumen by boiling, filter and evaporate the liquid to dryness over a water bath. Treat the residue with alcohol, which dissolves urea readily, evaporate the alcoholic extract to dryness, and add a little water, so as to make a syrupy mass, which should be plunged into a freezing mixture, and a few drops of pure nitric acid added to it, when crystals of nitrate of urea will soon be found in it.

There is no doubt that this malady attacks mares more particularly during the period of estrum. This can be accounted for in two ways, independently of any association with hysteria, namely-(1.) Because mares whilst in this state are very often kept off work for a few days; and (2.) They are in a highly excitable nervous condition, and more apt to suffer from spasmodic diseases, the causes of which may be very trivial.

In the spring of $1870 \mathrm{I}$ was called to see a horse in a dying state from this disease. I found that four had already died from it. They were farm horses, which in consequence of the frost had not been worked or exercised for some time. As long as they were kept in the stable they seemed all right, and immediately on the frost giving way they were put to work. They were all attacked, and succumbed in a few days; some of them dying early in the disease, others living for some days; and, what was very remarkable in those which struggled longest, the muscles lost all power of contractility, and in the one I saw there was no rigor mortis after death.

Treatment.-The therapentics of this disease may be summed up in a few words, namely, - - keep the various excretory organs acting freely, in order to assist nature in expelling the degraded products from the circulation. For this purpose a cathartic or oleaginous aperient onght to be administered without delay. The kidneys generally act freely enough, and need no stimulus; but should they cease to perform their functions, diuretics, and more particularly colchicum, are to be used. The animal should always be placed in a large, well-bedded, dry, loose box, with plenty of short straw or chaff around it, which is to be removed as soon as it becomes damp or wet. If unable to rise, it is to be turned from side to side two or three times a day, or more frequently if it becomes uneasy. Encmas are to be administered until purgation commences, and plenty of diluents allowed. Generally the 
horse is thirsty, and will drink cold water freely and to manifest advantage.

There is no necessity to aggravate the disease by either stimulants or sedatives. They always do harm during the first stages of the malady. If however, towards the third day the animal seems depressed, moderate doses of spirits of nitrous ether may be given two or three times daily; and about this time attempts should be made to get the horse on its lergs. If unable to to so without assistance, the slings should be placed under it, and it is to be gradually placed on its feet. At first it will be much disinclined to stand, and will be apt to fall from muscular weakness; but if the limbs are supported and smartly hand rubbed for some minutes, they will regain much of their lost power, the horse will begin to "feel himself," as is commonly said, and will often commence to feed and do well. It should be kept in the slings for a, few days, but taken out occasionally for a little exercise; and when allowed to lie down for the first time, it should be carefully watched, as it is apt to knock about very much of unable to rise with ease.

During the first few days the diet is to be of the lightesi description and restricted in quantity, but when convalescence has commenced, it must be moderately nutritive and easy of digestion, as much muscular debility exists in the digestive apparatus as well as in the voluntary muscles. At this stage a few doses of nux vomica may be given with advantage.

I have never seen any benefit accrue from external applications to the loins or back. On the contrary, fomentations, frictions, stimulants, or blisters increase the irritability of the animal, cause it to struggle when it otherwise would remain quiet, and do much harm.

If the pathology of the disease be borne in mind, the practitioner will at once see that the symptoms will cease as the morbid material in the blood is eliminated, and that the first and great aim of treatment is to effect this, after which the muscular debility must command his attention; and if there are indications of failure of the heart's action in two or three days after attack, stimulants are to be administered with freedom.

Any of the ordinary stimulants will answer the purpose, but perhaps we slıould exclude ammonia, as the blood-poisoning products are too similar to that medicament. 


\section{OXALULIIA.}

A condition of the system manifested by dulness, capricions appetite, loss of flesh, debility, stiffness about the loins, a branlike scurf on the surface of the boly-pityriasis-"hide-bound," and the presence of the oxalates of lime in the urine.

This disease is most commonly seen in hunters or other horses whose work is irregular, and which have to undergo long periods of fasting, as during a long and severe day with the hounds, the result of such long fasting being a weakened or debilitatecl condition of the digestive organs, whereby the products of the primary digestion are imperfectly formed, and unfitted for assimilation and consumption in the processes of nutrition and calorification.

The disease is also induced by food rich in saccharine matters, as carrots, turnips, or other roots, more especially if the digestion is deranged. Oxalates may also appear in the urine during recovery from other diseases, more especially during a protracted recovery from an enzootic or inflammatory affection.

Oxalic acid is a product of imperfect combustion or oxidation of the amyloidal and nitrogenous matters of the body. It may also result from an imperfect condition of those matters primarily; a perverted acid condition of the digestive organs, impediment to the respiratory function, or from a superabundance of food, more especially if combined with insufficient exercise. Various vegetables contain oxalic acid-the sorrelsbut I am not aware that these are partaken of by the horse, or that they are ever the cause of this disease. The pathological importance of a deposit of the oxalates has been considered doubtful by writers on human medicine. Some writers affirm that they result from chemical changes in the urine whilst in the renal passages, and that their occusional presence is no indication of disease. In our patients, however, their continual presence generally attends a form of indigestion in which an acid condition of the alimentary track is a constant accompaniment.

Semiology.-Loss of flesh; capricious appetite; a dry, immoveable, scurfy condition of the skin; the horse is said to be hide-bound and scurfy; a desire to lick the walls, and frequent urination of a palish amber-coloured urine, the act of micturition being often accompanied and followed by signs of uneasi- 
ness, such as whisking of the tail, or even attempts to lie down, or kicking at the belly with the hind feet. The mouth has sometimes an acid or sonr smell, the tongne is covered with a whitish fur, and there is generally a degree of constipation or irregular action of the bowels. An animal thus affected is stiff in the loins, and is easily fatigned.

Etiology.-The canses are to be found in irregularity of feeding, want of sufficient exercise, irregular exercise and work, such as fatigning journeys with long intervals of rest; improper food; or it may originate in weakened digestion from an mnknown canse.

Conclition of the urine.- The mine at each emission is scanty; of a pale amber colour; clear, like pale sherry, and is neutral or acid in its reaction. If examined immediately with the microscope, crystals of the oxalates will most probably be detected. In some instances, however, the crystals cannot be detected with the microscope for a day or two after the urine has been passed, the oxalic acid heing present in its free state, and afterwards combining with the lime and forming at least two distinctive forms of crystals:-1st. and most common, Octahedra ( 1 ) 2 . Dumb-bell (b) (the dumb-bell is very rare in the horse). -See fig. 27.
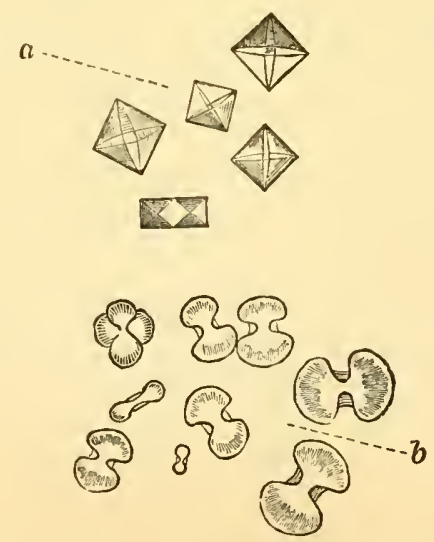

FIG. 27.-Crystals of Oxalate of Lime.
(a.) Octahedral form.
(b.) Dumb-bell form.

These crystals are soluble in nitric acid withont effervescence, are insoluble in water, and are unaltered by boiling in acetic acid or liquor potassie. 
Beneke, a German pathologist, has studied the question of the origin of oxalic acid in the human frame, and has arrived at the following conclusions:-

"1. Oxaluria, a condition which accompanies the lighter or severer forms of illness, has its proximate cause in an impeded metamorphosis-that is, an insufficient activity of that stage of oxidization which changes oxalic acid into carbonic acid.

"2. Oxalic acid has, if not its sole, its chief source in the nitrogenous constituents of the blood and food: everything, therefore, which retards the metamorphosis of these constituents occasions oxaluria.

" 3 . Such a retardation of the metamorphosis of the azotizer constituents of the blood may be cletermined by the following causes:-

"(a.) Abuse of azotized articles of food (direct retardation).

"(b.) Abuse of saccharine and starchy articles of food (indirect retardation).

“(c.) Insufficiency of the red corpuscles, and (cventually) diminished oxidation.

"(d.) Insufficient enjoyment of pure, fresh, ventilated air.

"(e.) Organic lesion, which may in any way impede respiration and the circulation of the blood.

" $(f$.) Conditions of the nervous system which bear a character of depression, whether these arise primarily from mental derangement or from pathological states of the blood.

"4. Excess of alkaline bases in the blood, which, as numerous observations tend to show, plays an important part among the etiological conditions of oxaluria; and it is not improbable that an increased production of lactic and butyric acids in the digestive canal, consequent thereupon, impedes the development of the red corpuscles, and thereby generates that chlorotic state which so often occasions and accompanies oxaluria.

“ 5. Catarrhal conditions of the intestinal mucous membrane, in case they are accompanied by oxaluria, have at most only a common source. They may determine oxaluria by causing deranged digestion, but cannot be considered as its proximate cause."

Oxalic acid differs from carbonic acid in possessing an atom less of oxygen and one less of carbon, and we cannot be sur- 
prised at its oceurrence in the urine, when we remember how sliglit a deviation in the oxidation of tissue may ause its formation.

Treatment.-In the first instance, it is necessary to alter the deranged condition of the digestive apparatus, and for this purpose a purgative is often rendered imperative. When the bowels have been operated upon, the condition of the system (the oxalic acid diathesis) is to be overcome, and the digestion improved by mineral acid tonies, the nitro-muriatie acid in preference to others, given in a decoction of gentian or other vegetable stomachic bitter. The diet must be carefully regnlated, and all roots and food containing much sugar withdrawn. 'The quality of the drinking water nust also be looked to, and if it contains an excess of lime, it must at once be changed, for if the oxalic acid is to be eliminated from the system it must be removed in a soluble form, and every remedy will fail if an excess of lime is present in the circulation. For the same reason alkalies generally are not to be administered, as they are transformed into oxalates, more or less insoluble, in the body.

Bearing in mind that non-oxidation is a source of oxalic acil, the practitioner will order regular exercise, pure air, and good grooming.

The irritation manifested in the passage of the urine is due to the direct irritation of the oxalic acid and oxalates on the numcous membrane of the minary passages, and the frequency of the act of micturition is due to the same source. If not very harassing to the animal, no special treatment is required for ts removal, for as the urine regains its natural character the irritation passes away. Should it be really a source of much annoyance, enemas containing belladonna or opium may be used.

In some cases of oxaluria, when the mineral acids have failed, the disease has rapidly disappeared when acid phosphate of soda has been substituted.

RED WATER, IIFMO-ALBUMINURIA (SIMONDS); BLACK WATER, bloody URine, HeMaturia (GAMGEE); MUIR-ILL, ETC.

A disease of the bovine tribe characterised by the emission of red-chocolate or black urine, containing albumen and the 
colouring matter of the blood in a broken down or disintegrated condition. The disease is associated with great prostration, febrile excitement, palpitation of the heart, a double, dicrotonous, or trembling pulse, pallor of the mucous membranes, and diarrhœa, succeeded by obstinate constipation of the bowels.

The disease is divided into two linds, namely, parturient and non-parturient red water. The pathology of both is the same, and a description of one will serve for both, except indeed that the parturient form oceurs in from eight to fourteen days after parturition.

Etiology.-The immediate cause of the disease is to be found in the nature of the food. In the puerperal form the cause is generally traced to the turnips the cows are fed upon. Mr. Cuming of Ellon says that "turnips contain about 10 per cent. of saccharine matter, and 1 or $1 \frac{1}{2}$ per cent. of vegetable albumen. A liberal allowance of turnips increases the quantity of milk, the sugar of that milk being directly supplied by the turnips; but the cow cannot make milk out of sugar alone, and the other elements of secretion, namely, the albuminoid casein, not being duly afforded, the tendency is to draw upon the blood, and through that fluid upon the bedy at large, for what is deficient." This theory can only hold good in reference to the puerperal form of the disease. Red water, however, prevails among calves, stirks, oxen, and bulls, and extensive experience has shown that impoverished pastures, heathy moors, and woody districts are subject to have the cattle grazing upon them affected with the disease in an enzootic form, during some seasons.

On the Continent the disease has been recognised as connected with damp lands and wet seasons. It occurs in bad weather, and when food is scanty and of inferior quality. It is seen always on pasture lands, and never in stall-fed animals, except in Britain, where it occurs amongst cows fed on turnips grown on damp land.

All authorities are agreed that the disease is induced by food containing an excess of water and diminished proportions of flesh and fat forming matters. I think Mr. Cuming is wrong when he states that a disproportion of sugar produces it. Turnips only produce it when they are given over-abundantly, and 
without a due admixture of other food. I am not aware that locust beans were ever known to cause it, and the quantity of saccharine matter in them is very great indeed. Again, it is very satisfactorily proved that when turnips are grown on welldrained and properly manured land, they do not cause the disease, provided the season in which they are grown has not been unusually wet, and if they are used in moderation; but when given indiscriminately, and withont due admixture with other food, such turuips are almost sure to induce the malady. Indeed, it may very properly be concluded that red water is due to insufficient feeding. The food may be sufficiently abundant in quantity, but it is defective in quality, the result of such deficiency being the breaking up of the blood and tissues, whereby they are excreted or expelled from the body as so much effete material.

Puthology.-Examinations of the urine in this disease have led to no very definite results. Professor Simonds named the disease hremo-albuminuria, from the fact that the urine always contained albumen.

In the Vetcrinarian for June 1840 an analysis is reported by Mr. Hughes, a student. The analysis was made in the previous Jalluary, and is as follows :-

Relative proportion of the different Substances in an Imperial Pint of Uine.

Albumen and other animal matter,

Urea, with fatty colouring matter,

Sulphate of soda, .

$112 \cdot 50$

Phospliate of soda,

$22 \cdot 50$

$10 \cdot 00$

Muriate of soda, .

$20 \cdot 00$

Iuriate of lime, .

$25 \cdot 00$

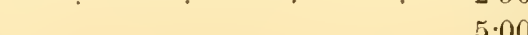

Lactate or acetate of solla, . . . 10.00

Silica, phosphate of magnesia, with lactite or acetate

of lime or magnesia, . . . . $10 \cdot 00$ Lass, 
Mr. Cuming of Ellon throws some doubt upon the accuracy of the above analysis, and gives analyses of his own and of Mr. Deuchars, V.S., his assistant, which differ from it to some extent. Being dissatisfied with any reported analysis, I procured some specimens of urine, and found that it may be satisfactorily concluded that albumen is always abundantly present, and that the hrematin of the blood, escaped from the corpuscles to a great extent, gives colour to the urine, which may be of a dullish red or a brownish-black appearance, the difference in the colour being due to the varying quantity of hrematin, and to a chemical alteration of its iron, arising from the presence of sulphide of hydrogen and ammonia. The following analysis, made by Dr. Macadam, will show the chemical constitution of a sample of urine, selected as on average one from several which were sent to me from various parts of England, Scotland, and Ireland :-

28 th March 1874.

Analysis of Samples of "Red Water" received from Prineipal Williams, of the New Veterinary College, Edinburgh.

Specific gravity at $60^{\circ}$ F. 1041 (Water $=1000$ ).

In 100 parts by weight.

Organic Matter-

Urea, . . . . 1.26

Albumen, . . . . . 1.82

Extractive matter, . . . . 1.28

$4 \cdot 36$

Saline Matter-

Chloride of sodium, . . . 152

Oxide of iron, . . . . 0.05

Phosphates, . . . . 0.68

Other saline matter, . . . 0.74

Wutcr, . . . . $\begin{array}{r}2.99 \\ 92 \cdot 65 \\ \hline 100 \cdot 00 \\ \hline\end{array}$

It may be mentioned that every specimen emitted a more or less powerful odour of rotten turnips, and was strongly alkaline in its reaction. Microscopically examined, crystals, as shown iu 
the figure, of eholesterine, hippurates, phosphates, and epithelial scales were found more or less abundantly.

Fig. 28.

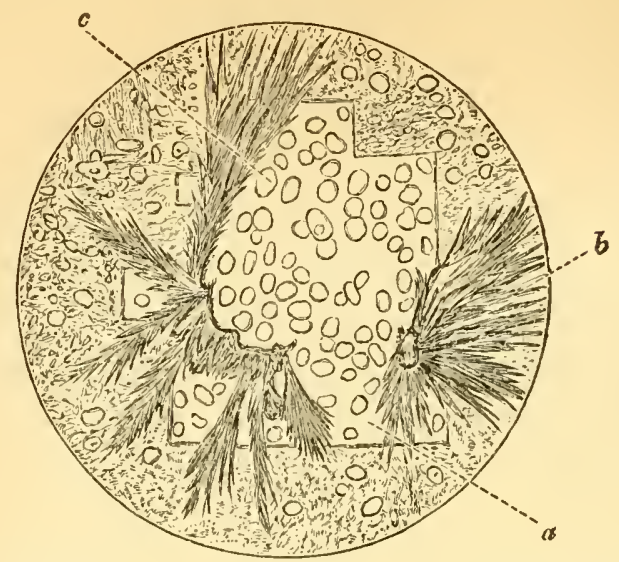

$\begin{array}{lll}\text { (a.) Cholesterine. (b.) Phosphates. } & \text { (c.) Mucous corpuscles. } \\ \text { (450 diam.) } & \end{array}$

From examinations of samples of urine, and from the history of eases lindly furnished by numerous veterinarians, as well as from what has more immediately come under my notice, I am induced to conclude that the disease originates in an impoverished condition of the blood, arising from want of proper food; that the albumen of the blood is thus degraded in quality, and as such is unfit to be appropriated for the nourishment of the tissues, and is consequently excreted by the kidneys and expelled from the body; that the blood globules are in a broken-rlown or disintegrated condition, arising from solution of their outer layers or cell walls, when their coloured interiorhematin-escapes, which, mixing with the serum of the bloor, and being eliminated by the kidneys, gives the characteristic tinge to the urinary secretion. This mal-condition of the blood is succeeded by diseases of various organs; most notably congestion of the liver; irritation of the intestinal mucous nembrane, as expressed by diarrhoa; paralysis of the intestinal canal, giving origin to an apparent constipation, and in some instances to severe cerebral disturbance, manifested by delirium and convulsions. The irregularity of the heart's action, as weil as the feebleness and peculiar trembling or thrilling of the pulse, 
are aiso expressive of a mal-condition of the blood, and characteristic of the anæmic state.

The post mortem appearances indicate a condition of anæmia. The subcutaneous tissues are white; the blood-vessels and cavities of the heart are empty, leading one to suppose that the animal had been bled to death; ecchymoses are seen on the serous membranes, and on the lower surfaces of the kidneys; the uterus in the parturient form contains some dirty coloured mucus, and sometimes presents spots of so-called inflammation. In no case are there indications of nephritis, the kidneys, on the contrary, being blanched, and the uriniferous tubes enlarged, evidently dilated with a viscid fluid. The rectum generally contains fæces covered with a dark-coloured mucus; in nearly all cases the liver is darker than is natural; the gall bladder distended sometimes with a dark viscid, sometimes a thin yellow bile. The contents of the lacteals and thoracic duct are occasionally found to be of a dark red or brown colour; but the most constant pathological conditions, in addition to the anæmia, are softening and engorgement of the liver, and fulness of the gall biadder.

Semiology.-In the parturient form, the disease is developed in from eight to fourteen days after parturition, with general derangement, diarrhœe, and loss of milk. In all cases, when examined particularly, the pulse indicates considerable constitutronal disturbance, being $80,90,100$, or upwards, having a peculiarly full beat, thrilling or double ; the artery relaxed, and easily yields to the onward wave of blood; the back is arched, and in some cases there is considerable straining. Constipation succeeds the diarrhœa, and the fæces passed are dark in colour. Palpitation of the heart is apt to occur, whilst the pulse becomes feebler and feebler. Mr. Steel of Biggar says that the vagina has, in the parturient form, a contracted or puckered-up appearance; that the milk drawn from the cow prior to the attack is particularly disposed to froth in the pail, and sometimes has a red sediment at the bottom after standing.

Treatment.-The preventive treatment.-Seeing that the disease is one arising from insufficient nourishment-- " a disease of the poor man's cow," as expressed by one writer-is a simple matter. About the parturient period, great care is to be taken that the animal's food be of a proper quality, in proper propor2 II 
tions as to all its flesh-forming, heat-giving, and saline constituents. If the malady be manifested in other than parturient animals, its source of origin will generally be found to exist in the pastures, which are on undrained, impoverished, or naturally inferior soils, in rank, unnutritious food, and in defective feeding of all kinds. If the causes be removed-the animals properly fed-it will be found that the further manifestation of the disease will be arrested.

The medicinal and hygienic treatment of the disease is very varied and often unsatisfactory. Its pathology, however, points to a rational method, namely, that the great end in view is the restoration of the blood to its physiological condition by the administration of albumen, which can be easily effected by giving eggs and milk; and several of the veterinarians who sent me samples of urine, have, on my recommendation, pursued this line of treatment with very successful results. In addition to the highly albuminous food, stimulants, and the chlorate of potash, from its well-known action on the blood, may be given with benefit. As to the administration of purgatives, I am of opinion that the more drastic ones should be avoided, and that the diarrhœa, which is a very constant symptom at the commencement of the disease, should be encouraged by mild olcaginous aperients, as it is the means by which the unsuitable food contained in the alimentary canal is expelled from the body. If there be any appetite, it is clear that food of a nutritious but easily digestible nature must be allowed in moderation,

Treatment by styptics.- Some veterinarians, looking upon the malady as hæmaturia, administer styptics and astringents, for the purpose of restraining the bloody discharge. This view of its pathology is incorrect, and consequently the treatment which is based upon it need not be discussed.

\section{ASTHMA-BROKEN WIND.}

Asthma, like diabetes, might with propriety be classified amongst constitutional diseases, for now and then it arises from no cognizable cause. I have, however, deemed it advisable to classify it with the dietetic diseases, as in the great majority of instances it originates from errors in feeding.

Definition.-A non-inflammatory disease, characterised by. 
difficult and peculiar breathing; the inspiratory movement is performed with ease, the expiratory by two apparent efforts. The difficulty in breathing is constant, but is liable to remissions and severe exacerbations. A peculiar cough, called "the loroken-winded cough," is a constant symptom; indigestion and flatulence aggravate the dyspnœa.

Etiology.-The causes of broken wind, as laid down by writers, are very numerous and complicated. Youatt says that emphysema of the lungs is present in almost every case that he has examined. Haycock ascribed the disease to hepatization and induration of a portion of one or both lungs, chronic disease of the digestive organs, or of the nerves of the respiratory apparatus, rupture of the diaphragm, and inflammation of a low subacute character of the mucous membrane of the bronchial tubes and minute air cells. Gibson attributed it to enlargement of the contents of the chest. Dr. Lower supposed that it arose from rupture of the diaphragmatic nerve. The Professors of the Veterinary College at Lyons (1826), after performing a variety of experiments, were led to conclude that the dyspnca arose from a reversed situation of the diaphragm, caused by nervous derangement. M. Godine, Jun., professor at Alfort, considered it as a natural defect in the normal and relative proportions of the right and left side of the heart. M. Demussy, in a Memoir to the Royal Society of Agriculture of Paris (1823), affirms that by direct observation he discovered that those districts of France where hay or other dry food is most used, are subject in a peculiar degree to broken wind amoing their horses.

Coleman said it originated in mechanical rupture of the air cells. Blaine supposed it arose from emphysema of the lungs, due to the formation of gases within the lung tissue.

'To give my own opinion, I have no hesitation in asserting that broken wind is generally due to improper food, more particularly to bad, musty, or coarse hay, containing a large quantity of woody fibre, from being allowed to become too ripe lefore being cut, and to a superabundant allowance of hay of any kind, with a deficient supply of corn.

Mr. Anderson, V.S., Glasgow, has very carefully investigated the causes of broken wind, and has been led to conclude that in most instances it is due to habitual over-loading of the stomach with coarse, indigestible food; in some cases the stomach has 
been found much enlarged, and containing masses of dry food adherent to its walls.

In some districts the disease prevails, or used to prevail, to a great extent. In some parts of Wales I have very frequently noticed many broken-winded horses; and upon inquiry, have discovered that their fodder has been of the worst possible description. I quite agree with Professor Gamgee that broken wind is at first a purely nervous affection, dependent on the condition of the digestive system, and in which the pneumogastric nerve is especially involved, and that the organic lesions are the effects of the nervous disorder. Some horses are peculiarly prone to suffer from broken wind, where no fanlt can be detected in the method of feeding. Such animals are constitutionally predisposed to indigestion, tympany, and irregularity of the bowels; they are generally round, shallow-chested horses, but are not bad thrivers before they become aflected in the wind.

Pathology.-The smaller air tubes are surrounded by involuntary muscular tissnes; in broken wind, owing to the irritation of the par vagum, the function of this muscle is interfered with. In the early stages, and during the paroxysms of broken wind, it is spasmodically contracted; in the later stages most probaluly it is paralyzed; in either condition there is arrest of respiration. The irritation arises from the action of indigestible food upon the cardiac branches of the vagus, and is reflected to the pulmonary branches of the same nerves. Recent experiments by Bert have convinced him that the lungs contract under the influence of the vagus, branches of which, it will be remembered, supply both the lungs and stomach, as well as other organs. The action of the bronchial muscle surrounding the tubes is brought into play during the expiratory movement, whereby the lungs are assisted in expelling the tidal air. The same thing happens in the lungs of a horse suffering from broken wind as in the lungs of a human being suffering from asthma. Owing to nervous irritation, there is arrest of respiration, and this can occur either in expiration or in inspiration; the arrest during expiration being the more easily induced, indeed "in some animals it is impossible to obtain arrest during inspiration."-(PAul Bert.) The arrest takes place in the act (inspiratory or expiratory) which happens to be going on at 
the instant of application of an irritant to the nerve; " the lung being, as I take it, seized and fixed by spasm, and immobility of the bronchial muscles."-(Dr. J. C. Thorowgood.)

In the horse the arrest is always during the expiratory act, which, in consequence, hecomes prolonged, difficult, and calls for a double contraction of the abdominal muscles to force the air out of the distended and now immobile bronchial tubes. I have noticed that very round-chesterl horses sometimes become broken-winded without apparent cause, and I conclude that difficult expiration may occur in them from limited thoracic contraction. When the conformation of the chest is round, respiratory movements, at least thoracic movements, are very limited in extent; for when the chest is naturally round, its conformation is but little altered during the respiratory movements. An animal so formed is incapable of taking a deep inspiration, and as incapable of performing a strong expiration; the lungs of such contain much residual air, the thoracic walls are more resonant on percussion, and in them probably arrest of expiration is most easily induced. Every horseman knows that round-chested horses, although apt to put on fat and look well, are not so capable of performing the same severe and fast work as deepchested animals; in fact that they are " not so good in their wind."

The abnormal condition of the bronchial tubes and air vesicles is at first due to purely nerrous derangement, but this is very rapidly succeeded by organic or structural change. Inflation of the air cells to excess prevents the free circulation of the capillary blood-vessels, and thus the nutrition of the bronchial muscle and mucous membrane is weakened; degeneration succeeds, and the lungs become more and more emphysematous; the air cells undergo dilatation, their parietes become stretched and disorganized, and air now infiltrates into the meshes of the connective tissue of the lungs. In this manner both vesicular and interlobular emphysema may be induced. Various other changes may occur in the thoracic organs, the most constant being hypertrophy and dilatation of the right side of the heart. By bearing these facts in view, much of what seems irreconcilable may be understood, and what have been looked upon as causes will be seen to be but mere effects.

The morbid anatomy of broken winil differs according to its duration at the time of the animal's death, which is generally due 
to another discase or to an accident. If death occurs soon after asthma has been manifested, no morbid change may be detected. Professor Dick said he had examined many cases where no disease could be discovered. In other cases the stomach has been impacted with food. This impaction of the stomach will only be observable in an animal which has died suddenly, or has been killed, as in one suffering from another disease the appetite would be impaired, and the horse would not eat for a time prior to its death.

If the disease has continued for a period of time, the following lesions may be detected:-Emphysema of the lungs, chronic thickening of the bronchial tubes, dilatation of the air vesicles, with anæmia, degeneration, and rupture of their walls, pallor of the pulmonary parenchyma, and eccentric hypertrophy of the right side of the heart, or the whole cardiac substance may be variously altered in structure.

Symptoms.-The inspiratory movement is performed with ease, but the expiratory by two apparent efforts, at the conclusion of which the miscles relax, and the flanks fall with peculiar force. There is a singular cough, which seems to be "ejaculated with a sort of grunt through the upper part of the trachea, perhaps from a sympathetic connestion with the parts below."-(BLAINE.) Auscultation will detect the respiratory murmur weakened or absent; there is a loul, sonorous, sibilant wheeze, heard particularly loud towards the posterior part of the chest. Rhonchus and sibilus of every tone and variety may be heard all over the chest. There is staguation of air in the lungs, and the sounds may change both in character and site, owing to the varying clonic contractions and relaxations of the bronchial muscle. A rubbing sound is heard, if rupture of air cells has occurred, denoting the presence of air in the lung tissue. On percussion, the resonance of the thoracie walls is increaserl, showing that the lungs are distended with air; the chest itself seems rounder than is natural, and the thoracic movements are very linited, whilst the abdominal ones are violently increased during a paroxysm. The symptoms are much more severe after the animal has been fed, and after the spasmodic fit has passed away, the breathing may be tolerably easy until it is fed again. The digestive organs are weak and easily disordered; flatulence is a prominent symptom; and the animal is often harsh and dry in its coat, pot-bellied, and unthrifty. 
Treatment.-Gireat attention to diet. The food and water should be carefully regulated as to quantity, and be of the best quality. Feeding on bran mashes, containing a few ounces of linseed oil with lime water, has proved serviceable in the practice of Mr. Anderson of Glasgow. A purgative should occasionally be administered, and the alimentary track always kept in proper order.

The old farriers used to make a supplementary anus for the horse by passing a red-hot iron into the rectum from immediately below the tail, through which a leaden ring was inserted, to keep it patent. There is no need to resort to this barbarous and insane method; but they supplied a reason for doing it, namely, that the artificial anus allowed the flatus to escape from the lowel easily, and that it did away with the disagreeable sound which accompanies that act when it is naturally performed.

The symptoms of broken wind may be palliated by all methods which improve the digestion, and by remedies that are calculated to give them tone; hence arsenic, alkalies, bitters, more especially nux vomica, and the various tonics are serviceable. All mere remedies, however, must be of secondary importance to hygiene and dietetics.

"Horse-coupers" resort to various methods for relieving the breathing of broken-winded horses. These persons know well enough that the animal breathes moderately well when the stomach is empty; they therefore take good care to keep it short both of food and water, and give it a sharp trot to unload the bowels. Shot, lard, gunpowder, opium, and other remedies are then poured down its unoffending throat, and most of these remedies seem to exercise a sedative or "stilling" effect, and the unwary purchaser only knows too late how cleverly he himself has been "sold."

There is one consolation, if not for the buyer, at least for the public generally, and that is, broken wind, like many other diseases the results of ignorance, is fast becoming a thing of the past.

LEAD POISONING-LEAD PALSY-PLUMBISM.

Definition.-A series of morbid phenomena induced by the absorption of the salts of lead contained in solution in the drinking water or in the food. 
Lead poisoning is a disease generally confined to those districts of the country where lead smelting is carried on. It may, however, occur accidentally in any part where animals have access to lead paint, or splinters of leaden bullets, which are often scattered about in quantities near rifle targets. It also occurs in cattle pastured in fields manured with town ashes, which often contain scrapings of paint-pots, waste paint, \&c.

In whatever form the lead gains access to the stomach, it must be rendered soluble before it is absorbed into the circulation. Lead paint, leaden bullets, and other insoluble forms of lead, are acted upon by the acid secretion of the true digestive stomach, and are thus rendered soluble and fit for absorption into the animal economy. The portions of insoluble lead found in the rumen, \&c. of horned cattle are to be looked upon as indicating that lead is present, and that some of it is absorbed into the system, rather than as actual causes of any symptoms present prior to death. Lead in its metallic state appears to be devoid of medicinal or poisonous action. Four ounces of shot were given to a dog at the Veterinary School of Lyons without effect. The metal may sometimes, however, be converted within the body into an oxide or active salt.-(FinLay Dur.) Shot is used by low horse-dealers to relieve the symptoms of broken wind, and doubtless some of the lead is dissolved by the gastric acids, and acts as a sedative upon the gastric nerves.

Plumbism, or lead poisoning, results from the absorption of lead into the circulation. It is thus diffused through the whole body, and exerts its toxic action upon all the organs and tissues of the animal frame, but it does not affect all parts alike, but accumulates in some more than in others.

Dr. George Wilson (see Monthly Journal of Medical Science, May 1852), found that the spleen yielded lead most abundantly; next to the spleen the liver yielded most; then the lungs, afterwards the kidneys, then the heart, and the intestines least. Lead has also been found in the brain and in the muscles; and the blue line observed in the gums of animals and men poisoned with lead, is supposed by Toures to be due to the lead in combination with the tartar of the teeth.

Lead having once entered the body, leaves it very slowly, and may be detected in it months after an animal has ceased to receive lead with its food or water. It enters into combination 
with the tissues of the body, and is retained in the system as an. insoluble compound, and only leaves the body on the breaking up or disintegration, medicinally or otherwise, of such insoluble compound.

The symptoms of slow lead poisoning in the lower animals are, capriciousness of the appetite, loss of flesh, convulsive fits, a blue line seen on the gums where teeth are present, the gums in the interdental spaces showing no trace of this colour. Colic is sometimes absent, and constipation is not so constant a symptom as in man; indeed I have repeatedly seen a diarrhœic condition of the bowels with leaden-colomred and fotid stools. In the horse, roaring is considered, in Wales, the first symptom of lead poisoning, and every roarer in the lead districts about Holywell and Flint is said to have had lead (plwom). Mr. Shenton of Bakewell, in a letter to Mr. Finlay Dun, thus describes the symptoms of lead poisoning in eleven horses which came under his observation in 1852:-- "There was a rough, staring coat, a tucked-up appearance of the abdomen, and a slightly accelerated pulse; in fact, symptoms of febrile excitement, which usually passed away in about a week. About this time large quantities of grey-coloured matter were discharged from the nostrils, and saliva from the mouth; but at no time was there enlargement of the submaxillary, lymphatic, or salivary glands. Neither was there constipation of the howels, which appears to be nearly always present in cases of lead poisoning in man. Fits and partial paralysis came on at intervals; and when the animals got down they often struggled for a long time ineffectually to get up again. The breathing up till this period was pretty tranquil, but now became so difficult and laboured that the patient appeared in danger of suffocation. The pulse was in no case above sixty or seventy; and I ascribed the difficulty of respiration to a paralyzed state of the respiratory apparatus. The animals did not live more than two or three days after these symptoms appeared.

"The post mortem appearances varied but little. The lungs and tracliea were inflamed; the lungs engorged with large quantities of black blood; the trachea and bronchi filled with frothy spume. In all cases but two the villous part of the stomach presented isolated patches of increased vascular action; and in ail cases the intestines, especially the large ones, wore infiamed. 
The blind pouch of the cæcum was nearly gangrenous. There was nothing remarkable about the liver, spleen, or kidneys, except that they were of a singularly blue appearance. The brain and spinal cord were not examined."-(FInLAY Dus's Materia Medica.)

Lead palsy in man is characterised in its later stages by the blue line around the teeth, ulceration of the mouth, colic, and 'pileptic fits, preceded, however, by palsy of the upper extremities. The extensor muscles of the hands and fingers become first paralyzed, so that when the arms are stretched out the hands hang down by their own weight, causing what is termed "vrist-drop." This paralysis is succeeded by atrophy of the affected muscles.

In the diagnosis of lead palsy in man the electric current plays an important part, for it is found that when the palsy is induced hy lead the excitability of the muscles is much diminished, whilst it is normal in ordinary paralysis. "Therefore, when the museles of a paralytic limb move well under the influence of the olectric current, we may fairly conclude that there is no lead in the system."-(Dr. Alimaxs on Paralysis, Neuralgia, \&e.)

Mr. Herapath reports in the Chemist for 1855 (see F. Dun's Materia Medica) some interesting cases of lead poisoning, which followed the erection of smelting furnaces on the Mendip Hills in 1853. The inquiry appeared to commence half a mile from the chimmey, and to extend half a mile further. Oxide, carbonate, and sulpliate of lead were found on the herbage, hedges, and hay. On the live stock "the effects of the metal were, a stunted growth, a leamness, shortness of breathing, paralysis of the extremities, particularly the hinder ones, the flexor muscles of the fore legs were affected, so that they stood upon their toes, swelling of the knees, but no constipation or colic as in the human species; in a few months death followed. In the young the symptoms were more conspicuous and the mortality yreater. Lambs were yeaned paralytic. When three weeks old they could not stand, although they marle great efforts to do so. In attempting to feed them from a bottle they were nearly suffocated from paralysis of the glottis. Twenty-one died early out of twenty-three. Colts also died; and those that lived could not be trotted 150 yards without distressed breathing. Pigs confined to the sty were not injured, but if allowed to roam were soon affected. The milk of cows and sheep was reduced in quality and quantity, 
and cheese made from the former had less fat in it. I foumc in the milk of both minute traces of lead. The dead subjects showed the mucons surfaces to be paler than natural. The lungs had large portions of a dark red colour, with circumscribed edges, not like ordinary inflammation, but evidently surcharged with fluid. There was a blue line seen in the gum of the lowel' jaw, which Dr. Taylor said in court was not caused by lead poison, as it dicl not occur, as in the human subject, on the upper edge of the gum, but where the gums first come into contact with the teeth, about three-sixteenths of an inch below the top edge. I therefore lissected out this line, which was about three-quarters of an inch in length, and the thickness of sewing cotton; and by aid of carbonate of soda and the blowpipe reluced a spangle of lead from it, quite visible to the jury without the aid of the microscope."

I am not aware that there are any cases on record of animals poisoned by water conveyed in leaden pipes; such a thing is not, however, impossible, and where other sources of lead are ron-apparent, it will be advisable to search into this.

Treatment.-In the rapidly developed form of lead poisoning from the accidental ingestion of lead paint, bullet spray, \&c., the treatment must be directed to prevent the solution of the lead in the stomach and intestinal canal, by administering such agents as enter into combination with lead, forming insoluble combinations. For this purpose the bowels are to be acted upon ly a solution of sulphate of magnesia, to which sulphur and sulphuric acid have been added. By this means the lead in the intestines is converted into an insoluble sulphate and sulphicle. If pain be present, opium or hyoscyamus is to be added to the purgative. The acid is to be continued for some time, as it is only by keeping the digestive apparatus charged with it that the solution of the lead by the gastric juices can be prevented, for the thin crust of sulphate, which probably forms on the surface of the portions of lead, may be thrown of, and the metal, or its oxide or carbonate, as the case may be, again exposed to the action of the gastric juices.

In addition to the purgative and acid, diluents are to be freely administered, and purgation actively liept up for some days. It is also necessary to keep up a copious discharge of urine, witl: the view of eliminating the absorbed lead from the body. 
When the bowels are thornughly acted upon, and if the sulphuric acid does not seem to agree with the animal, sulphur and vegetable tonies may be substituted. The sulphur is partially transformed into sulphuretted hydrogen, which combines with the lead, and forms an insoluble sulphide.

In the slower form of lead poisoning, and where the lead has been slowly accumulating in the system, it will be well to eonsicler the chances of success before attempting a cure; and in none but the mildest eases should this be attempted, as the termination will prove neither ereditable to the practitioner nor profitable to the owner.

In all cases a small dose of the sulphate of magnesia is to be administered, and when this has operated, the elimination of the poison is to be attempted by the iodide of potassium.

The priruiple upon which the iodide of potassium acts has been pointed out by Melseus, who assumes that the learl is retained in the body as an insoluble componnd with the tissnes. The iodide, after its absorption into the lood, combines with the lead, and forms with it a new and soluble salt. The poison is thus liberated from its union with the injured part, dissolved out from the damaged fibre, and once more set afloat in the circulation, and along with the remedy cast out with the urine. Great eaution is at first necessary in using this remedy, as at the moment when the compounds, fixed in the body, become dissolved or transformed, the phenomena of acute poisoning may occur. The dose must therefore be small at first, and either increased or diminished, as the patient seems to bear it.

Galvanism is also recommended for lead poisoning in man; and, if necessary, may be tried in the case of a valuable liorse or cow.

It will be apparent that removal of animals from the souree whence the lead is obtained must be the first step in the treatment.

The soluble salts of lead, such as the acetate, when given in poisonous doses, cause nausea, quick, small, hard pulse, colic, stiffness of the limbs, paralysis of the optic nerves, insensibility, and death; and on post mortem examination the gastric mucous membrane is found reddened from eongestion, or grey, and having a macerated appearance, owing to the chemical action of the salt. 


\section{PARAFFIN OIL POISONING.}

I have met with one instance where several head of cattle on a farm were slowly poisoned by drinking from a stream into which the refuse from paraffin works was discharged.

The symptoms were, gradual loss of flesh, and a persistent diarrhoa, and it was only on a post mortem examination being made that the cause of death was discovered. The appearamces were as follows:-

Anæmia, wasting of the muscles of the body generally; absence of fat, and other signs of a prolonged mal-nutrition; the characteristic lesions were discovered on opening the abdominal cavity. The intestines had a greyish-black appearance throughout, and were covered with very dark grey or blackish spots of a dull appearance. These spots were found to consist of the various intestinal glands, surcharged with the pigmentary matters of crude paraffin. The glands of the mesentery were more or less enlarged, and presented the same dark appearance; the lacteals were of the same colour, and were found to contain paraffin; the liver presented nothing very unusual, but the kidneys, more especially on their inferior surfaces, were covered with the dark spots.

These animals, at least those which I had the opportunity of seeing, had been removed from the field, through which the polluted stream flowed, some months prior; they had continued to eat moderately well, but everything they took seemed to pass through the intestinal canal as if the power of absorption hail been entirely lost; and when the condition of the glands and lacteals was examined, this could be accounted for; indeed it may be said that the animals had died from slow starvation, arising from impermeability of the chyle vessels, due to their being blucked up by crude paraffin. Although so long a time had elapsed since the paraflin had obtained access into the body, the characteristic smell was retained; and even many months after they were removed from the body, and preserved in spirits, the odour was still recognisable.

\section{RHODODENDRON POISONING.}

Several cases of poisoning by the Rhododendron hydridum having been observed by my late student, Mr. Gun, Veterinary 
Surgeon, Beauly, he kindly wrote me the following particulars, which are well worthy of attention:-

"On Sth April last I was asked to attend a lot of fourteen eattle, one and two-year-olrls, and on examination the following symptoms were noticeable:-staggering, swaying from side to side, partial paralysis of hind extremities; some of the animals would press their heads to the wall and strike out with their fore limbs, is if endeavouring to get forward, then vomit a green ropy spume. One, a yearling stirk, was unable to get up, the hind extremities being completely paralyzed; eyes staring and fixed in their sockets, pupils not dilated, nucous membranes very pale, great straining, passing small quantities of hard, dark foetid fieces covered with nucous. The symptoms varied very much, as some of the animals, especially the two-year-olds, would lie down during the spasms of pain, and kiek the belly with the hind legs. Vomition was a marked symptom, and came on at intervals, when the animal would groan with pain, romit, then a spasmodic cough came on, which lasted for a few minutes. When administering medicine, if the animal's head was much raised above the level of the body, the creature would instantly fall as if struck with an axe. On examining the substance vomited, it was full of the flower buds, leaves, and young shoots of the rhododendron in a semi-masticated state. An inspection of the park grazed by the cattle was made, when about three cart-loads of the prunings of rhododendron were found thrown over the fence by the gardener; the prunings having been recently, as was seen, eaten of by the cattle.

"Treatment.-Linseed oil, with potass carbonate, and small doses of tincture opii in the eases suffering from colic was in every instance successful. The yearling stirk affected with paralysis was treated as above, with the addition of the iod. potass., which in a few days had the desired effect.

"P.S.-The first symptoms of illness were noticed fifteen hours after the eattle lad eaten part of the prunings."

\section{YEW POISONING.}

Considerable difference of opinion seems to exist as to the poisonous or non-poisonous properties of the leaves of the yew tree; some maintaining they are not poisonous except in a 
partly dried or decayed state, whilst others state that eattle can eat them with impunity, either dry or green.

Numerous instances are, however, recorderl which prove that the yew is poisonous to eattle, sheep, pheasants, \&c.; and my own experience enables me to agree with this opinion, namely, that the yew, English and Irish, is poisonous, both in its green and its dry state.

Professor Tuson (Veterinarian, January 1877) says, "The whole subject of poisoning by yew is so important, that I have undertaken to investigate it, in the hope of being enabled to determine the nature of the toxic principle of the plant, as well as to explain away the many eontradictory statements that have from time to time been made relative to its fatal effects."

It is to be hoped that Professor Tuson will be able to perform his promise upon. a matter so very important. By doing so he will confer a benefit on agriculturists and the veterinary profession.

The symptoms are rarely noticed, as the toxic action is very rapidly fatal, destroying life without there being any very deeided manifestation of symptoms beyon? loss of appetite, great prostration, feeble pulse, coldness of the surface of the body, and rapid sinking of the vital powers.

The post mortem appearances are equally unsatisfactcry, and but little can be deteeted beyond the presence of the leaves and twigs in the stomach.

In investigating into the eause of deatl of one lot of eattle poisoned, several of which were found dead, it was discovered that some elippings from yew trees had been thrown into the park the previous evening, and in those which had died first the poison was found in the greatest abundanee; others of the cattle lingered for several hours, and in these the leaves were less in quantity.

From what I observed in making the examination, it would appear that it is not essential that the yew leaves should pass beyond the rumen to exert their toxic properties, as in some of the eases no leaves were found beyond this viscus, whilst in others a few were found in the reticulum; in those that lived the longest some of the leaves had reached the omasum. No doubt some of the juice expressed during mastication would become absorbed and eause death. 
Treatment.-The indications for treatment are the removal of the toxic vegetable as quickly as possible; and, provided the cause has been discovered by the history of the occurrence, or the post mortem examination of the animal or animals already dead, and seeing that vomition is induced with so much difficulty, I see no objection to the performance of rumenotomy, in order to remove all the contents of the rumen; but before resorting to such an operation, the practitioner should endeavour to overeome the effects of the poison by large and repeated doses of stimulants, such as ammonia and alcohol, friction to the skin, and warm clothing. Should this succed, the operation may not be necessary. At the same time, it must be remembered that, even if the first effects be thus obviated, the animal will continue in danger until the poison has been expelled. The process of rumination re-established will again subject the plant to the action of the saliva; its remaining toxic properties being thus set free may induce a fatal collapse, while perhaps all has been thought secure. Purgatives will be essential, and ought to be administered without delay.

\section{MILK SICKNLSS.}

A form of disease in which the milk and flesh become poisonous, and which, when partaken of by man or animals, induce a violent and even fatal disease.

It appears, as reported by Professor Law of Cornell University, that the disease has been observed by Professor Kerr every summer in the mountains of the Blue Ridge, which runs down to the western portion of North Carolina; among the hills farther south by Dr. Salmon; by Drs. Phillips and Schmidt on the upper water of Scioto, Ohio; and by Mr. Beardsley in Illinois, and adjacent States.

Etiology.-Mr. Law says_ "There is no uniform geological condition in the different unhealthy districts to account for their dangerous tendencies. Dr. Phillips says-' It is found in localities among the Green River hills in Kentucky; it occurs in some localities along the Wabash River in Indiana, as well as here and there in our own heavily timbered uplands in north-western Ohio.' And yet a constant condition of its preralence is the unreclaimed state of the land on which it appears. In the Carolinas and Georgia it is found only on the highest 
mountains; and in Kentueky, Ohio, Indiana, and Illinois, only on the lieavily timbered lands or marshy river bottoms in their virgin condition. But the removal of the primeval forest, the drying of marshy spots, and the general cultivation of the soil, is the signal for its disappearance; and thus Dr. Phillips again remarks- In many localities where it formerly prevailed to a dangerous extent within the memory of the present generation, as in Green and Madison counties, in the State of Ohio, it has now, by reason of the cultivation of the soil, been entirely exterminated. Indeed, the disease may be said to be rapidly disappearing before our extending civilisation.'

"The recent microscopic observations of Dr. Phillips have done more to elneidate the cause of milk sickness than all the wild lypotheses and crude observations of a century before. He has found vegetable organisms in the blood; and as these will explain fully all the peculiarities of the disease, they may be provisionally accepted as the probable disease germs. He says'In the month of June last, I had under my care a typical case of milk sickncss. I made a eareful microscopical examination of the urine every day; and upon the fourth day after the case came under my care, I drew a few drachms of blood, and immediately placed it under the microscope at the bedside, while yet warm and liquid. I found it contained a great number of living, moving spiral bacteria, similar in their general appearance to those spiral bacteria described by Professor Lebert as abounding in the blood of relapsing fever patients. I also found in the urine of that patient those same spiral bacteria, and, co-existing with them, the sphero-bacteria, in segments of two to six or eight. I had opportunity to examine the blood of but one patient; the urine of several was obtained and examined, furnished from patients under the care of one of my professional neighbours-all showing the presence of both these described forms of baeteria. I sent specimens of the blood and urine to a professional friend, having a state reputation as a microscopist and pathologist, and had the pleasure of receiving, within a few days, a full endorsement of my observations.' 1

"This will sufficiently aceount for the fact that particular districts, fields, woods, waters, or plants are at times found to be

1 It ought to be stated that Dr. Schmidt denies the existence of bacteria in the blood of his cases. 
infecting; while the same plants or waters, containing identical chemical elements, are at other times or places immocuous. It wili further explain why only a certain proportion of the animals or men exposed to the morbid influence succumb to it; while the remainder, whose systems or vital fluids are in a condition chemically incompatible with the propagation of the bacteria, escape. It is quite in keeping with the fact that the disease is produced in widely different conditions of climate, vegetation, soil, geological formation, and management; and that no combination of these is able to produce it, unless the disease germ has been introduced. It explains, too, the permanent preservation of the virus in cheese and other comparatively stable products of the sick. It is equally in keeping with the following circumstances, relating to what may be considered predisposing causes of the disease:-

"The excess of effete matters in the blood evidently favours its development. Thus, overwork, severe exertion-even for a limited time-want of sleep (as in the case of the members of a family watching with a sick relation), a fit of constipation, or a slight attack of malarial fever, all strongly conduce to the development of the malady. 'It is curious to note that children often suffer less than adults, probably because their emunctories are usually more free, and they are less exposed to exhausting and depressing conditions. In infected cattle, the symptoms are easily developed or aggravated by subjecting them to fatigue; and in mill-sick districts, it is a common practice to test the soundness of stock by giving a good run before purchasing.

"Another very remarkable fact is, that milch cows rarely show the disease, though conveying it to other animals through their milk. Dr. Phillips says-' It is not unusual, where persons suffer from this disease, to find, at the same time, calves using the same cow's milk sick with 'trembles;' while the cows are to all appearance in perfect health. The dry cattle are much more frequently attacked with 'trembles' than cows which are secreting milk in abundance.' To the same purpose Dr. Schmidt remarks - Cows giving milk do not show the disease at all, but their ealves have it all the time as long as they suck the milk; dogs fed on milk get it.' Dr. Phillips says - ' A calf gets it from the cow in the milk, - the calf dies; and the pigs which eat the carcase get the disease and die; the $\log _{\mathrm{g}}$ which eats the pig, and 
the buzzard which eats the dog, all in due time contract the same disease.' Dr. Schmidt and others speak in similar terms.

"This transmission of the disease through a long series of successive victims, which has been shown in "scores of careful observations,' demonstrates the important fact of the specific nature of the poison and its multiplication within the system; while any simple mineral or vegetable poison in similar circunstances would soon have been rendered inert by dilution.

"The presumption is that all races of domestic animals are susceptible, and may contract the disease, either from the milk, flesh, or other products of the sick, or directly from ingestion of the poison in food and water. Phillips even snggests the air as a possible medinm of infection, but adduces no facts in support of the opinion.

"Symptoms.-In milch cows these may be so slight that they are entirely overlooked, unless the animal is excited. The outlet for the poison through the mammary secretion appears to protect these, in a great measure, from the graver phenomena. When violently excited, however, and especially when driven fast for some miles, they are seized with a rigor and all the more dangerous symptoms of the disease. The breath, too, has at all times that fœetid, sweet, mawkish smell which characterises the disease in all animals.

"In steers, and other dry cattle and shcep, the condition is characteristic, even in decubitus: a position which is maintained by the animal as much as possible, evidently because of the great nervous prostration and extreme muscular weakness. The lhead resting on the ground, the swcetness of the breath, the blood-shot eyes, and the staring coat, are marked features, though appetite and rumination may be preserved. The worst cases lie prone, with head extended on the ground, and show an utter indifference to all sense of fear or danger; and the wildest of them whine under the prostrating influences of the disease, and allow themselves to be handled in any way withont the slightest effort at resistance or escape. The nervous debility and torpor are not confined to the voluntary muscles, but all the organic functions are largely impaired, the bowels in particular are obstinately constipated, and when death ensues it is by asthenia.

"The milder cases, those even that present no appreciable 
symptoms when at rest, are seized with trembling when made to undergo the slightest exertion, assume a very haggard appearance, drag their limbs slowly and stiffly, and after a drive of four or five miles will often suddenly drop down dead.

"The prostration is even more marked in sheep, and in very many cases they seem to be quite unequal to the task of rising.

"In vomiting animals, emesis is a prominent symptom. Calves tremble while sucking, and often stop suddenly, eject the contents of their stomachs, fall to the ground, and perish. In pigs and dogs, vomiting and constipation are prominent symptoms; swine burrow under their litter, and are roused with difficulty, and dogs get 'the slows,' and are unable to follow their master. The result of exertion is the same in all.

" Convalescence in this disease is as peculiar as any other feature in its clinical history; and to this peculiarity I would invite especial attention, inasmuch as these facts are important in the study of its special pathology. Convalescence is rarely marked by a critical evacuation of any kind. I have no recollection, in any case, of a critical perspiration to mark the initiation of convalescence. A moist skin during the active stage of this disease, in my opinion, never occurs. Diuresis, or diarrhoea, as a rule, does not occur as a critical discharge. It is true a resumption of these functions occurs, but not in quantities to be regarded as critical. I have occasionally seen diarrhœa occur as a sequel, with typhoid symptoms; but I have always regarded it as a result of injuries inflicted by the injudicious use of drastic purgatives, or as an evidence of uramic poisoning from retained excreta. Ordinarily, convalescence is marked by a cessation of the characteristic symptoms, and a gradual resumption of the impaired and suspended functions,-only this and nothing more. There is a popular belief that a patient once suffering from this disease never fully recovers; and that, upon exposure to heat and fatigne, he is liable to a relapse. This, it is true, does occur occasionally, but not as a rule. On the contrary, I could point to scores of cases where recovery has been perfect and complete.'-(Dr. Philuips.)

"Mr. Beardsley also testifies to the permanency of the effects, and liability to relapse in many cases.

"Lesions in Cattle.-Beside the presence of fungi in the blood, and the tendency to capillary stasis in different organs, the 
fullowing have been noticed:- 'The stomach and intestines are inflamed (?); the mucous coat is measurably destroyed; the entire coats are in some cases gangrenous; they have a dark or black appearance, which is not due to a mere congestion; the coats are not firm, though they are not easily broken down. In the ventriculus, or paunch, and indeed throughout the whole alimentary canal, is found a substance conglomerated and resembling cemented sawdust, in the shape of balls, somewhat elongated, and perfectly adherent to the coats of the digestive tubes.'-(Dr. Schindt.)"-Veterinary Journal, Sept. 1877.

CAKE POISONING-CASTOR OIL SEED (RICINUS COMNUNIS) AND CROTON SEED (CROTON TIGLIUM) POISONING.

It is well known that both castor oil seed and croton seed are very poisonous, even a less quantity than one per cent., when present in the food - usually feeding cakes - causing serious illness, and even death. Mr. J. W. Leather, in The Analyst, July 1892, says: "It requires but a very small quantity of these seeds-probably three or four seeds or less per lb.-to cause serious illness or death to stock." Mr. Leather was kind enough to communicate his method of detecting this adulteration of feeding cakes to me in a letter, June 12, 1892, at which time I was engaged in investigating several outbreaks of a disease of a serious and fatal nature occurring amongst cattle fed on a particular linseed cake. His method I found to answer every purpose, and I have since repeatedly tested it. Boil about a pound of the material with one to two per cent. of hydrochloric acid for one hour. Decant the upper liquid from the sediment several times; boil the residue with dilute alkali for another hour, then wash by decantation until the washing water is colourless. Treat the residue with half a pound to a pound of bleaching powder (chloride of lime), and add sufficient water (a quart to three pints) to keep the temperature from rising. This is frequently stirred and then left to stand for several hours, although the bleaching action is generally complete in about four hours. It is then repeatedly washed by decantation, and all the lime removed. This process renders the testa of the seeds generally met with in cattle foods sufficiently transparent to be recognised by the microscope,-in fact bleached, soms being completely decolorised; whereas in the case of the croton 
and castor oil seeds the outer integument is washed away, and the principal part of the testa remains black and opaque, and at the edges some indication of the structure can be seen.

Subjected to the action of sodium hypochlorite, even for several days, the castor and croton seeds retain their colour, whilst those of linseed, cotton, locust bean, \&c. are readily bleached. Free chlorine gas has a more powerful action, and will gradually bleach both croton and castor seeds, but it requires more time, and even at the end of three or four days their colour is not entirely removed.

After passing through this bleaching process the castor oil seed testa has a much smootlier and shiny exterior than the croton seed, and this is an important point in their distinction, as microscopically the structure of each is very similar, being built up of closely packed bundles of fibres of the same thickness; but on the outermost edge of a transverse section of the croton testa there is thickening of each bundle of fibres, absent in a section of castor oil seel.

Experimentally Mr. Leather has added the testa of castor oil seed, weighing $0: 223$ gramme to $1 \mathrm{lb}$. cotton cake, subjected it to the bleaching process, and has recovered 0.200 gramme; and out of 0.074 gramme croton seed testa mixed with $1 \mathrm{lb}$. cotton cake recovered by picking out 0.060 gramme. In performing these experiments it was found that a mouldy croton bean became bleached after the lapse of a day. When microscopically examined, this bean was found to have been penetrated through and through with a fungus. It may be mentioned that the lilium of cotton seed does not bleach so readily as the other portions, but there is no difficulty in distinguishing it, for the pieces are round, have no sharp edges, and are marked by alternating rings of grey and black on the surface. If the material to be acted upon is roughly handled, the testa is broken into small fragments, and may thus be overlooked.

Differences in the appcarance of the outer integnments of the Castor and Croton Seed.-Castor Oil Seed before bleaching.-The seeds are oval, and bluntly rounded at one end, while at the other they come to a point, where there is an aril, easily broken off. The testa or outer covering is smooth and glazy, grey in colour, with very characteristic longitudinal striations of darkbrowr, yellow-brown, or black. 
Croton seeds are oval ard triangular or imperfectly quadrangular in shape; but, as in the case of castor seeds, are only seen in the ground state, in cakes. The testa or outer seed coat is cinnamon brown in colour, and when scraped shows a blackish appearance, and underneath the brittle testa there is an inner pale-coloured part which surrounds the yellow endosperm.

Symptoms.-Both croton and castor oil seeds have similar effects upon the animal economy. In a short but variable time, depending upon the extent of the adulteration, the animals fed upon such cakes prezent symptoms of great prostration, uneasiness, and excessive diarrhœa, the watery alvine evacuations containing clots of blood and large quantities of mucus. In calves stomatitis is a prominent symptom, the mouth being covered with thick layers of epithelium, which readily peel off, exposing the mucous membrane red and congested in appearance; there is much flow of saliva, and champing of the lips, as seen in foot and mouth disease.

The post-mortem appearances are those of gastro-enteritis.

Treatment.-Demulcents, such as starch, gruel, linseed tea, with opium and chalk, to allay irritation, and some antiseptic, such as carbolic acid or small doses of soda hyposulphite, to arrest stercoral putrefaction, with stimulants to overcome the great depression and prevent a fatal collapse. Eggs, milk, and wher fluid nutrients may also be necessary in order to overcome the feebleness of the heart's action.

Powerful astringents should not be administered in the earlier stages, as they, by cliecking the outpouring of fluid from the vessels, prevent the removal of the poison and increase the congestion.

\section{LATHYRIASIS.}

A disease induced in the horse by feeding on a leguminous seed called the Lathyrus sativa, - the vetchling. Professor Stewart Macdougall, of the New Veterinary College, has given an interesting account of this plant in the Veterinary Journal, January $189 \tilde{\jmath}$.

He says the natural order Leguminosæ includes a very large number of plants, having among them diverse properties, some being mutritious, others purgative and astringent, and still others poisonous. The latlyyrus belongs to the sub-order Papilionacex, 
which also contains lupins, whin, broom-clover, vetches, haricot bean, scarlet runner, peas, gram, lentil, \&c.

There are several varieties of Lathyrus sativa, showing slight differences in colour of flower, in size and colour of seeds, in colour of foliage (darker or lighter), and in strength of growth; but they have one characteristic in common, namely, in being wedged, angular (triangular), or hatchet shaped. They differ in colour in various countries where they are grown. According to Harz, quoted by Macdougall, the variations are as follows:-

\begin{tabular}{|c|c|c|c|c|}
\hline Locality. & & Colour. & $\begin{array}{c}\text { Largest } \\
\text { Diameter. }\end{array}$ & $\begin{array}{l}\text { Weight of } \\
100 \text { seeds in } \\
\text { Grammes. }\end{array}$ \\
\hline $\begin{array}{l}\text { Carinthia, . } \\
\text { Carinthia, . } \\
\text { Austria, . } \\
\text { Sicily, . } \\
\text { Bohemia, . } \\
\text { Verona,. . } \\
\text { Greece, . . } \\
\text { Sicily, d . } \\
\text { East India, } \\
\text { East India, }\end{array}$ & - & $\begin{array}{l}\text { Greenish yellow, . } \\
\text { Reddish yellow, } \\
\text { Yellowish green, } \\
\text { Pea-coloured, spotted, } \\
\text { Reddish pea-coloured, } \\
\text { Pea-coloured, } \\
\text { Partly faint pea, partly brown, } \\
\text { Pale pea, } \\
\text { Dark niarbled on brownish } \\
\text { ground, } \\
\text { Deep reddish brown, black } \\
\text { spotted, . }\end{array}$ & $\begin{array}{r}10 \cdot 1 \\
10 \cdot 3 \\
9 \cdot 5 \\
12 \cdot 0 \\
8 \cdot 3 \\
15 \cdot 0 \\
7 \cdot 6 \\
14 \cdot 7 \\
7 \cdot 1 \\
7 \cdot 5\end{array}$ & $\begin{array}{l}20 \cdot 871 \\
25 \cdot 830 \\
23 \cdot 756 \\
41 \cdot 423 \\
16 \cdot 637 \\
56 \cdot 545 \\
18 \cdot 438 \\
48 \cdot 991 \\
13 \cdot 619 \\
16 \cdot 527\end{array}$ \\
\hline
\end{tabular}

The nutrient ratio is $1: 175$, the characteristic being richness in nitrogenous constituents. The seed contains $8 \pm$ pel $^{\circ}$ cent. of dry substance, yielding 25 per cent. proteid, 1.9 per cent. fat, 54.5 per cent. non-nitrogenous extract, 4.1 per cent. fibre, and 2.9 per cent. ash. For further information upon the botanical and other characters of the plant the reader is again referred to Professor Macdougall's article.

So far as my knowledge goes, only two kinds of the seeds have been concerned in the production of the disease, namely, a small dark-coloured seed, known as Indian Mutters, brought from India mostly as ballast to such ports as Liverpool and Glasgow, and a larger white variety, popularly called the dog-tooth vetch or dog-tooth pea, and sometimes sold under the name of Russian peas.

The terms Matar, Muther, Mutter are used in India for 
peas generally. Whether the seeds used are of the smaller dark kind or of the larger white kind, they have been equally injurious.

Professor Macdougall says that he found the seeds in poultry food in several of the Edinburgh grain-dealers' shops, added as as an adulteration. Some of the dealers did not trouble about the presence of the lathyrus seeds, whilst others objected to them, not on the ground that, so far as they knew, the seeds were injurious, but that they were an inferior quality of pea.

Dr. Voeleker states that he has discovered several cases where feeding cakes have been adulterated with lathyrus, and that injurious effects followed their use.

The effect on man using the seeds as daily articles of food is a sudden and inenrable paralysis of the lower limbs, and there are records of epidemics of paralysis following their use, both in Asia and Europe.

Symptoms in the Horse.-The animals, seemingly in a perfect state of health whilst at rest, on being put to work or even exercised, particularly if the weather be cold, are seized with roaring and great difficulty of breathing, some immediately dying from asphyxia unless tracheotomy be performed, others gradually overeoming the dyspnoa and remaining apparently well whilst kept in repose.

The following is the description of the symptoms given by the manager of the Bristol Tramway Company,-case at law reported.

123 horses out of a stud of 800 fell ill owing to feeding on mutters. "The horses suffered from what was styled, for want of a better name, 'an epidemic of falling.' They would fall suddenly, without any accountable reason. $\mathrm{Cab}$ and carriage shafts were broken claily, as also were the ear poles." And again, "A horse being exereised was taken suddenly ill; it roared, its flanks heaved, its month was kept wide open, the nostrils were distended (dilated), and the tongue hung out and became livid; it stared and staggered, and threatened every moment to fall down strangulated and suffocated, and during this paroxysm, which lasted several minutes, the perspiration ran freely off every part of the horse."

Principal M'Call, who was eonsulted in the Bristol ease, having seen much of the disease in Glasgow, and who advised 
tracheotomy, with the best results, says that mere excitement is sufficient to induce a paroxysm.

In $188+$ the Messrs. Leather, veterinary surgeons, Liverpool, discovered the disease in a stud of 74 cart horses, out of which 35 became ill, 19 died, 2 were killed as useless, and 14 recovered. They had 3 or 4 lbs. of mutters mixed with their ordinary food per day, which consisted of 20 lbs. of grain, for three months. Professor M'Call states that 2 lbs. per day could be continued for six weeks or so before symptoms of the attack become apparent; and it is remarkable that upon some of the horses in these studs, as well as in others that have come under my observation, the poisonous qualities of the seeds seem to lave no ill effects.

In Liverpool, Glasgow, and Bristol it appears that the dark seeds only were used, but there are several reports from Bedlington, - $\mathrm{a}$ number of pit ponies; Eastwood, 20 or 30 horses; Newcastle, 12 or 15 ; near Sheffield, reported by Mr. Alson, V.S.; 12 horses at Hamden Colliery, near Birmingham,-where the disease was induced by the large light-coloured seed.

Both in man and the horse the lathyrus is a cumulative poison, the symptoms showing themselves the sooner the greater the proportion of seeds contained in the food. It appears from the evidence of many observers that boiling renders the seeds innocuous. Thus the late Mr. Thomas Greaves told me that he knew that they had been used regularly for years after being boiled withont any bad effect; and Professor M'Call mentions that in the neighbourhood of Glasgrow 100 bolls of the seeds lay unused for some time, until they were by his orders boiled, steamed into a pulp, and given in quantities of $1 \frac{1}{2}$ lbs. per night per horse, without any bad effect. In the case of human beings Watt says "there is a certain capriciousness" in the effects of the poison on different individuals; and, adopting the view that the poisonous effects are due to a volatile alkaloid, he suggests that persons eating thin cakes made from the sativus grain do not suffer, as all parts of the cake being exposed to a high temperature, the poison is eliminated, whereas in food preparations exposed only to moderate temperature sufficient of the poison remains to act injuriously on those partaking of such food.

Professor Macdongall states that the seeds cause paralysis and spasm in pigs, and that when fattened on the meal they lost the use of their limbs, but became very fat whilst lying on the 
ground. Cattle seem to resist this poisonous affect, but it has been stated that the seeds are injurious to milch cows, and that sheep fed on small quantities receive no harm. Pigeons are said to lose the power of flight. Geese eat them with impunity. Poultry do not eat them readily.

The true nature of the poison is not satisfactorily determined, lut may be a volatile alkaloid. Astier claims to have isolated an alkaloid. Further experience has convinced me that the organism found in an extravasate form around the throat of one of the Liverpool horses was an accidental complication, as extravasation was not witnessed in any except the Liverpocl horses. The mutters in that instance were very dirty, and fouled with rodent excrement, from which a microbe was cultivated homologous in appearance with that found in the extravasate.

The North of England Institute of Mining Engineers, at a meeting held at Newcastle-on-'Tyne, December 14, 1895, formulated the opinion "that a reasonable quantity of these peas is eaten with impunity by pit horses in that district." The "reasonable quantity" referred to turns out to be, according to the statement of Mr. Hunting, a quarter of a pound per day, and from what is known I have no doubt but this is a correct statement. If this could be correctly carried out, doubtless it is probable that no bad effects would follow. To my mind, however, there is a risk of imperfect mixing, which would result in a greater share of the seeds being eaten by one horse than by another.

At the same meeting Mr. Clement Stephenson stated that Mr. W. Hunter, M.R.C.V.S., Newcastle-upon-Tyne, had discovered that all the affected horses, upon which tracheotomy had been performed, had recovered. Mr. Hunter, however, stated that they had not entirely recovered, but had greatly improved in their breathing, and worked daily without distress, although the tubes had been removed some months ago.

In order to prove the extreme danger of feeding upon full quantities of the lathyrus, I annex a letter from Mr. Green, M.R.C.V.S., Indley, who a short time ago had several cases under treatmuent, and in which the proprietor of the horses obtained damages from the corn dealer.-(See Report of the Dudley cases in Veterinary Periodicals.) 
Mr. Green writes as follows :-

"To Professor Williams.

“ Dudley Veterinary Infirmary,

“ January 9, 1896.

"Dear Sir-In reply to yours, I may say, in the Dudley case of poisoning of horses with the Lathyrus sativus, the cab proprietor bought twenty-five bags as split peas, and commenced using them at once, giving the full feed, and I have no hesitation in saying they had from eight to nine pounds a day for seven days with bran and chaff. On the ninth day the horses began to stop in their work, stale frequently, show slight abdominal pains, fall on their knees when standing in the stable or on the cab-stand, and about the twelfth day the roaring appeared. The corn was suspected, and discontinued on the tenth day. Nothing relieved them but tracheotomy. None of the horses have thoroughly recovered, and in my opinion they never will.-Yours faithfully,

A. Green."

\section{LUPINOSIS.}

A disease said to be due to the ingestion of plants belonging to the genus Lupinus of the Natural Order Leguminose.

The name lupin is said to be derived from lupus, a wolf, because the plants "devour all the fertility of the soil," but the derivation is doubtful. None of the lupins, numerous though the species are, and very common in our garlens, are natives of Britain, their chief home being the countries in the neighbourhood of the Mediterranean Sea and the temperate regions of North and South America. The members of the genus are shrubs or sub-shrubs, and can be recognised by their digitate leaves. One of the best known is the species with white flowers - Lupinus albus-grown in Southern Europe, either for fodder. or as a manure for the purpose of ploughing the crop in. The Greeks and Romans, in spite of the bitter taste of the seeds, acquired a liking for them, and used them as an article of diet. Cattle, too, are known to have been fed on them after the bitter taste had been removed by steeping and boiling. The yellow lupin (Lupinus luteus) has sometimes been recommended as a forage plant in places where a poor, light, and sandy soil would grow nothing else. In spite of its vitterness, which made it unpalatable to stock generally, sheep are said not to refuse it, and to thrive on it. One authority remarks that cases of poisoning 
have been known to follow its use, but were not understood Others speak of lupin seed meal as excellent for young calves.

A bitter prineiple, probably alkaloidal, seems to be characteristic of the various species. We have certain knowledge in the case of two at any rate, viz., Lupinus albus and Lupinus angustifolius. From the seeds of these an alkaloid has been isolated, called lupanine. Bitter to the taste, it is said not to be hurtful to man, but experimentally to be poisonous to frogs.

The Root Tubercles of Leguminose.-The Leguminosæ are well termed "nitrogen collectors," for they collect quantities of nitrogen, and this withont making the soil poorer, but, on the eontrary, leaving it richer in nitrogen at the end of their growth. Experimentally it is known that in the absence of nitrogen in the soil they can flourish.

The Leguminosæ, then, possess some means of supplying themselves with nitrogen, means denied to the generality of plants, which are dependent almost entirely for their nitrogen on the nitrates in the soil. The Leguminosæ, almost alone among plants, are able to make use of free nitrogen of the air or soil, and this power is associated with the presence of nodules or swellings in their roots. These swellings vary in size in the different species. Examination by the mieroscope of a transverse section through such a swelling shows a multiplication in number of the cells of the ground tissue of the root. In these cells there can be seen, in addition to the protoplasm, a number of bacteria. These bacteria pass into a form to which the name "bacteroid" has been given. These bacteroids expose a large surface to the air, and make use of the free nitrogen preparatory to its being combined into such forms as the plants can absorb and use. We have here a useful partnership between a lower plant and a higher, between bacteria on the one hand and the leguminous plant-bean, elover, lupin, \&c.-on the other. The bacteria are first of all nourished by the plant, and later the contents of the bacteroids are absorbed by the plant.

If a soil be sterilised and the germs thus killed, no tubereles will be formed until the soil be in some way "microbe seeded."

If this disease were due to a ptomaine or toxine generated by the action of the bacilli upon the eonstituents of the plant, the fuet that lupinosis is only induced in animals grazed on poor pastures could be easily understood, but conditions identical with those 
observed in lupinosis are seen in this comntry, and the disease prevails as an epizootic in certain parts of North America, particularly in two counties of Nova Scotia and Antigonish, where it is called "The Pictou Disease;" and M'Eachran of Montreal, who investigated it in 1880 , came to the conclusion that it was not due to the ragwort, the plant supposed to be the eause by the inhabitants, but to the defiviency of nutritive materials in the animals' food,-a conclusion supported by my experience-see Cirrhosis of the Liver-and also by the observations of Continental farmer's, who believe it due to the exhaustion of the soil, weakened for want of proper eultivation; and it is remarkable to observe that lupin when first grown upon poor land may induce the disease, but is innoenous from land in which it has been grown for a number of years.

Again, in Nova Scotia the farmers are also fishermen, and allow their farms to beeome deteriorated for want of proper cultivation.

There is nothing said in the report of the "I'icton Cattle Disease" as to whether lupins are grown in the particular districts where the disease prevails, but they are plentiful in America. I am therefore in the dark on this point, and am forced to suppose that it is probable that a toxine may be formed in other leguminous plants when grown in poor soils.

The disease is said to attack the horse, ox, goat, sheep, and deer; inoculable in the $\log$, but the rabbit seems to have inmunity.

The disease seems to be most prevalent anongst sheep in Prussia, Silesia, Hanover, North Germany, committing great havoc; and in the bulletin of the Royal College of Economy of Prussia, February 1880 , it is stated that out of 240,000 sheep in one district of Pomerania, 14,000 and 13,000 died in one year. In the Nova Seotia report there is no mention of sheep.

The disease seems to appear in two forms, viz., an acute form, characterised by acute hepatitis, the liver soft and friable, and in some instances enlarged by fatty infiltration, the fat supposed to be transported from the subcutaneous adipose tissue to the liver; this is followed, if the animal live, by an acute hepatie atrophy.

Dr. Wyatt Johnston, Montreal, says - "The condition of the liver in the early stages is a very peculiar one. There is a very 
great secretion of bile; the gall bladder is very much distended with bile, sometimes containing over a quart. Notwithstanding this, there is an excess of bile passed in the stools, which are dark, and contain large quantities of bile.

"In the early stages the liver shows marked signs of parenchymatous degeneration, or cloudy swelling; later on a fatty change in the liver cells is very common.

"In the later stages of the disease,- - that is to say, in animals which have been ill two or three months, the liver alwaysshows a marked condition of cirrhosis, and the liver cells have disappeared, and are replaced by delicate fibrous tissue.

There is a peculiar condition of the submucosa of the fourth stomach, which is distended with a clear viscid fluid, so that the folds are swollen out in round dropsical masses, the distention being due to the fluid. In some cases, when the cattle have been ill for over a month, a number of ulcers are seen in the stomach. In the sheep the changes are similar to the above; the alterations of the liver consist mainly in chronic interstitial hepatitis, with hypertrophy of the connective tissue (see Cirrhosis of the Liver) congestion of the portal system, followed by ascites, serous tumefaction of the spleen, and of the gastrointestinal mucous membrane."

Symptoms.-In the Picton disease, the symptoms, as describel by M[Eachran, are loss of condition, with diarrhœea at an early stage, and the milk acquires a bitter taste and unpleasant odour. This change in the milk is often the earliest symptom; there is a soft pulse; a very prominent bulging of the eyes; a roughness of the coat. In about a week the abdomen begins to swell owing to the development of ascites, and if the animal is killed in from one to three weeks from the commencement of the symptoms, the ascites is abuudant, the fluid amounting to several bucketsful. With this ascites there is a disappearance of the fat about the kidneys, so that the adipose tissues become filled with a clear fluid, which apparently replaces the fat. In some cases the temperature semains normal, although the pulse is increased in rapidity.

As described by Continental writers, the symptoms in sheep, are loss of appetite and elevation of temperature, which falls considerably before death: this elevation is sometines observed on the day following the ingestion of toxic lupin. Symptoms of 
jaundice generally, but not always, appear on the second, third, fifth, or sixth day, and may come on suddenly, or slowly and gradually, being first seen in the eye; there is also weakness and stupefaction, stiffness of gait, the recumbent posture being generally maintained; in some cases great excitement, grinding of the teeth, spasmodic agitation of the jaws, and in some instances true trismus from the third to the twenty-second day after the attack. Constipation may be an early symptom, the freces coated with yellowish or pitchy-brown mucus mixed with blood. Diarrhoa follows. Chronic lupinosis, said to be induced by a prolonged use of moderately toxic plants, assumes the form of anrmia without the symptoms of jaundice.

In the horse, induced by lupin seeds in the oats or litter, the symptoms are those of gastro-enteritis, cercbral depression, grinding of the teeth, head carried low, with uncertain and staggering gait, elevation of temperature - $106^{\circ} \mathrm{F}$. in some casesaccelerated respiration, more or less marked jaundice, constipation, the freces being covered with mucus and having a fotid odour, diminished secretion but frequent emission of small quantities of highly coloured urine. These symptoms continue for about a week, and recovery is complete in about sixteen to twenty-one days. Directions are given for the treatment of lupinosis, which must be mainly preventive, there being no specific antidote. When impossible to exclude the lupin, it is recommended that it should be mixed with good food and given preferably to old animals; that the toxic fodler should be put in small heaps and left exposed to the action of rain, which washes the poison from the superficial layers, or to macerate the toxic lupins for forty-eight hours in a solution of soda-1 in 100 -renewing the liquid from time to time, or to subject the lupins to the action of steam for at least two hours under the pressure of at least two atmospheres.

When the disease has manifested itself, it is recommended that alkaline liquids should be avoided, as they dissolve the toxine and increase the danger, and that some acid should be added to the water in order to render the lupin and toxine insoluble, and the poison removed from the intestinal canal by oleaginous purgatives.

I have endeavoured to condense much of what has been written on lupinosis for the benefit of students particularly, but 
I have very grave doubts as to disease being due to the lupins, for not only have we similar conditions in the Pictou disease, but in this country acute and chronic diseases of the liver are frequently met with, particularly in sheep-see Liver Diseasesand on the Continent. Hautner has seen identical conditions in sheep fed on malted potatoes, and several observers state the same conditions are induced by inundated pastures and by pea, bean, and vetch stubble, \&c. 


\section{CHAPTER XXXVIII. \\ SPORADIC DISEASES-continued.}

LOCAL DISEASES.

LOCAL diseases are those which have their primary seat and origin in any one tissue, function, or organ. They are sporadic diseases, and are generally, but not always, associated with constitutional disturbance, which, however, depends upon, and is secondary to, the original local affection.

\section{(A.) DISEASES OF THE NERVOUS SYSTEM.}

\section{EPILEPSY - CHOREA.}

Compared with the nervous diseases of man, those which affect the lower animals are few and insignificant. This can be accounted for in a variety of ways, but more particularly by the absence of the higher mental functions in the lower animals, and "in the way of life," from their non-participation in the excitements, pleasures, hopes, and fears, as well as in the indulgences of the human race.

There are certainly some instances of what might be considered aberrations of intellect or morbid fancies in the lower animals, inore particularly in the dog, by which the docile become ferocious, the good-tempered extremely irritable, the intelligent stupid or listless.

Animals also vary in degree of mental power: some are sharp, intelligent, easily taught, possessed of good memories, and are capable of manifesting a great degree of affection to those around them; while others are dull, hard to teach, have bad memories, and seem to live for themselves only. Notwith- 
standing these varieties in the temperament and intelligence of animals, the purely mental-psychological-diseases are absent in our patients.

\section{EPILEPSY,}

A peculiar nervous state, difficult to define, but in which complete loss of consciousness prevails for a short time, associated with spasmodic contractions of the muscles, succeeded by debility, and sometimes by a desire to sleep. Epilepsy occurs amongst all animals, but is most commonly seen in young dogs.

The pathological condition of the brain which gives rise to epileptic fits is not yet determined; indeed, in fifteen out of twenty cases in which the brains of epileptic human patients have been examined, the structure of the brain has been found quite healthy. Occasionally, however, the brain and its membranes are actually diseased; thus the membranes may be found thickened, inflamed, or even ossified. In one horse, subject to epileptic fits, an absccss in the white matter of each hemisphere was found by me; in another, tumours in the choroid plexus; but in some instances it is due to some condition of the blood, as in those fits which are associated with catarrhal fever. In young dogs epileptic fits are associated with dentition, and with wornis in the intestinal canal or stomach.

Dr. Brown Sequard's researches into the origin of epilepsy are quoted at length by Mr. Gamgee in his Domestic Animals in Health and Disease, page 458. They are very interesting, but the tendency at present is to consider epilepsy certainly as a brain disease, but as one in which no definite pathological condition can in all cases be determined; and that the loss of consciousness, associated with excessive mobility, leads observers to regard those parts of the brain in the immediate vicinity of the cella turoica and basilar portion of the occipital region-for example, the central ganglia or medulla oblongata-as parts where, in future, morbid anatomy may yet discover a lesion.

Symptoms.-An animal in apparent health is seen to stagger and stare-the dog cries out at first, but is afterwards quite dumb-and then to fall into more or less violent convulsions, the whole system being argitated. The urine and frees are sometimes passed involuntarily, the eyelids are closed, and if they are opened and the eyes examined, they will be seen to be in- 
jected, sometimes convulsively agitated, at other times in a condition of strabismus (squinting), and sometimes fixed. During the fit the pulse is frequent, hard, sometimes intermittent, at other times scarcely perceptible; the respiration is stertorous, and sweats bedew the body.

I have carefully studied epilepsy, and have concluded that the condition termed vertigo or megrims is separate and distinct from what may be designated epilepsy, which is a disease, functional or otherwise, arising from extrinsic or intrinsic nervous irritation; whereas megrims is a term applied to all diseases which are associated with periods of insensibility or convulsions. I therefore limit the term epilepsy to a nervous affection characterised by convulsions, and which may appear either in the stable, kennel, or outside, at exercise or rest; whilst vertigo is applicable to a condition of syncope arising from disease of the leart or pericardium, and which occurs whilst the animal is at work or exercise. The epileptic subject, especially the horse, may be occasionally recognised whilst the animal is perfectly quiet, by a shaking of the head, and a working backwards and forwards of the ears, also by an occasional vacant, upward stare. This shaking of the head must not be confounderl with that caused by ill fitting bridles and head-stalls. Some horses are peculiarly square on the upper portion of the sides of the neck, and it will often be found that the borders of the neck are chafed by the head-gear. This should be carefully looked to before an opinion is given as to the soundness or unsomndness of a horse that shakes its head.

Treatment of Epilepsy.--If the fits are due to eccentric or peripheral causes-for example, to teething, worms in the stomach or intestinal canal, or indigestion, and this is not an infrequent cause, particularly in cattle-the first thing to be done is to remove these sources of irritation, by lancing the gums, extraction of offending teeth, and, by the use of vermifuges and purgatives, to expel wor'ms or other intestinal irritants.

If the fits are due to disease of the nervous centres, all causes which may induce excitement or congestion of them are to be avoided; the food must be light and digestible, and the intestinal track cleared by means of purgatives, whilst the congestion, or a tendency to it, of the medulla oblongata and brain is best prevented or overcome by extract of belladonna. In the dog, 
intrinsic epilepsy has sometimes given way to the salts of silver, zinc, or iron.

\section{CIIOREA.}

Definition.-A serious of automatic muscular movements, rarely seen except in the dog; clonic spasms, sometimes affecting the muscles of the face only, but generally involving those of the head, neck, and fore extremities; due ta alterations in the spinal cord, or to disease of the facial nerve when affecting the face only.

The pathological conditions which give origin to the peculiar twitching or convulsive action of the muscles, characteristic of chorea or St. Vitus' dance, are not exactly determined, many pathologists who have investigated cases of the disease having failed to detect any definite morbid appearances. Consequently it has been regarded as an entirely functional disease, or as a disease due to an altered condition of the blood, the precise nature of such alteration not being known, and alsa as a symptom of some diseases of the heart.

In the dog, it may with good reason be said to be first due to an altered condition of the blood, generally brought about by, or giving origin to, the disease called distenuper, of which it is so often a distinguishing feature; whilst in the horse, spasmodic contractions of the thoracic and cervical muscles, of the nature of chorea, are often associated with cardiac diseases of a rhemmatic type.

Symptoms in the Dog.-The convulsions may be confined to one fore leg or shoulder, of which there is a peculiar jerking action, like a series of pulsations. At other times both fore legs are affected, when there is a nodding or depressing of the liead and neck. In many cases it is restricted to a constant jerking of the head and lower jaw, with marked twitchings of the superior cervical muscles; the muscles of the eyelids, and sometimes the muscles of the eye itself, are also affected. The convulsions do not cease whilst the animal is lying down, but they generally, although not uniformly, cease during sleep. In some cases the dog will remain otherwise healthy for an indefinite period; but if the choren attaclis it whilst in a debilitated state, the expenditure of muscular action, and the general restlessness, slowly undermine the remaining strength; the dog becomes emaciated, subject to fits, and finally paralysis and death supervene- 
The post mortem appearances are very indefinite. If succeeding a recent attack of "distemper," redness and softening of the spinal cord, meningeal dropsy, and in some cases spots of congestion on the medulla, optic thalami, and corpora striata, are discoverable; whilst in cases which have become chronic no lesions have been discoverable after death. Most probably, however, some change has taken place in the composition of the nervous system, which has not been detected; and this supposition is supported by the evidence that in acute or recent cases morbid changes are usually detectable. In some cases the disease disappears spontaneously, more especially if mild, ant the patient be moderately strong.

In the horse chorea may usually be regarded as a concomitant of other diseases, and will be mentioned in its proper place.

Treatment in the Dog:-Attention to the condition of the bowels, removal of all sources of irritation by mild purgatives, generous diet, and tonics.

In human medicine, Dr. Walshe recommends the extract of cannabis indica as the most efficacious remerly, in doses of one-fomth of a grain thrice daily. In other cases much benefit has accrued from the cold bath. In my own practice I have found that camphor and spirits of nitrous ether are sometimes very useful, allaying the restlessness and diminishing the convulsions. At the same time the animal strength has been improved by good food and sulphate of iron. I find that the best diet for $\log$ is milk and oatmeal porridge in the morning, and an allowance of flesh, according to size, in the evening. Bromicle of potassium or chloral hydrate, oxide of zinc, or arsenious acid, have been reconmended.

The foregoing diseases not being essentially due to inflammation, congestion, or organic alteration of any particular part of the cerebro-spinal system, I have placed them under the general head of "Diseases of the Nervous System." Having done this, I now purpose giving a detailed description of those nervous diseases to which a locality can, with some certainty, be ascribed. 


\title{
CHAPTER XXXIX.
}

\section{SPOIADIC DISEASES-continued.}

\author{
LOCAL DISEASES-continued.
}

\section{(B.) CEPHALIC DISEASES.}

INFLAMMATION OF THE BRAIN SUBSTANCE-CEREBRITIS.

THIS is a very rare affection, and its occurrence can only be proved by post mortem examinations. Mr. Gamgee says that it is impossible in practice to distinguish between inflammation of the brain-cerebritis, and inflammation of its coverings-meningitis. In this he is so far correct; but pure cerebritis does occur, if we are to look upon the formation of abscesses in the white substance of the cerebrum as evidences of inflammation.

I have seen two cases which presented this appearance post mortem, and another, that had all the symptoms of cerebral abscess, and recovered, but was peculiar in its motions and action, had epileptic fits, was unable to back, turned with difficulty, and moved its limbs automatically as long as it lived.

The three cases mentioned were suffering, or were recovering from strangles, the advent of the brain symptoms succeeding to the formation of the abscesses in the intermaxillary space. In the two that died, the symptoms were, great drowsiness, slowness of the pulse, inability to masticate food, although a desire to eat still remained, staggering gait, dragging of the feet, and subsequently coma, with stertorous breathing, amaurosis, paralysis, embracing the muscles of the head, trunk, and extremities, rapid sinking, and death. In one case the voluntary muscles were quite flaccid, and were incapable of any movement; whilst in the other some contractile power still remained, and now and then feeble struggling would take place. It was able to move 
the limbs. but very slightly, and possessed a little sensibility when pricked with a pin. In both cases the sphincters were relaxed, and urine flowed involuntarily.

In the third case-the one which lived - the symptoms were those of incomplete paralysis of the whole muscular system; there was drooping of the lips, semi-closure of the eyelids, staggering gait, automatic muscular movements, and when the horse was moved, muscular action continled for some time. When its head was pressed against a solid object, such as the walls of the stable, it would continue to move its limbs, and press its head and body forward with great force; indeed there seemed to be a tendency to go straight forward. The desire for food was pretty good, and although mastication and deglutition were performed with difficulty, it was able to take a fair amount of support, consisting of mill, eggs, gruel, \&c., in addition to ordinary food. It continued in this condition for about fourteen days, when it burst through the box door during the night of 31st December 1862, and was found prostrate, and covered with snow, in my yard in Bradford, when the groom went into the yard on the morning of 1st January; it was immediately dragged into its box, and covered over with clothing and straw. It lay prostrate, steaming with perspiration, thus induced, for three days, during which time it scarcely moved. As the bowels were constipated, a full cathartic was given, which responcled on the second day; the urine flowed involuntarily, doing away with the necessity of using the catheter. On the third morning some degree of conscionsness returned, and it was now alole to drink water-small quantities of which had been carefully given it out of a bottle-and in the evening it rose to its feet. It now gradually recovered, but ever afterwards showed some degree of aberration of mental powers- "intellect," if the term is properand was always ealled the "cranky horse" by the men about it. I conclude that this was a case of small abscess in the cerebral substance, from the fact that it was at the time affected with a pyogenic disease-strangles-and from the similarity of the symptoms to those seen in the cases in which I had the opportunity of making post mortem examinations; and I account for the partial recovery on the supposition that the pus had either broken down and was absorbed, or had become inspissated, and the medullary tissue of the brain accommodated to its presence. 
The differences between cerebral and meningeal diseases are arranged as follows by Aitken, and as, to some extent, they may prove useful in diagnosis, I take the liberty of quoting them:-

\section{CEREBRAL DISEASE.}

1. From the outset, or from a very early stage of development, there is loss of some one or more of the proper nervous functions, such as paralysis, anæsthesia, losis of memory.

2. Cerebral disease is not conmonly attended by high-marked exaggeration of function, such as furious delirium, convulsions, intense hyperæsthesia, or tenderness.

3. Little vascular excitement attends cerebral disease, nor is there irequently any highly marked general disturbance.

4. Paralysis and anæsthesia, losses of volition, ideation, perception, and the like, characterise cerebral disease.

\section{MENINGEAL DISEASE.}

1. It is not till some time after the detection of signs of disease that diminution or loss of nervous function takes place.

2. The subsequent diminution or loss of nervous function which suoceeds the prolonged existence of "head symptoms" is generally precedod in cases of meningeal disease by extremely severe excitement or exaggeration of functions, such as pain, tenderness, furious delirium, or oonvulsions.

3. In meningeal affections there is usually much local vascular excitement, with general disturbance.

4. Spasms, or convulsions, pain, and delirium are the general features of meningeal disease.

In all the three cases of cerebral abscess mentioned, there was the absence of the above-mentioned indieations of meningeal disease; and indeed the post mortem of the two fatal ones proved this, and even the automatic movements of the limbs of the third ease clearly proved that volition was to a great extent destroyed.

In each of the brains examined, the abscesses were symmetrical, situated in the anterior lobes of each hemisphere, and were seen, after the removal of the dura mater, as two buiging prominences, measuring about an inch in diameter, which, when opened, gave exit to a thick, whitish pus, of good consistence and free from smell. The walls of the abscesses consisted of the 
medullary substance, covered here and there in one case with small red points; whilst in the other the surrounding substance was of the natural colour.

\section{TREATMENT.}

But little can be suggested in the way of remedial treatment. If the anirnal lives sufficiently long, the liquor puris may be absorbed, and the shrivelled pus cells remain, as in the case recorded. But little assistance can be given to this, beyond keeping the bowels open by an occasional cathartic, to act as a derivative, and attending to the diet of the animal; the food in all instances must be nutritious but non-stimulating. Care must also be taken that the animal does not injure itself by falling; and to prevent injury to the head, some soft substance, such as a sack stuffed with straw, should be placed in front of the patient, in order that it may rest its forehead upon it. 


\section{CHAPTER XL.}

\section{SPORADIC DISEASES-continued.}

\section{LOCAL DISEASES-continued.}

(C.) ENCEPHALITIS, INFLAMIIATION OF THE BRAIN AND MIEMBRANES - CEREBRO - MENINGITIS, PHRENITIS, MAD STAGGERS, PHRENZY, CONA, SLEEPY STAGGERS, STOMACH STAGGERS, ABDOMINAL VEPTIGO, \&.

Definition.-A morbid condition of the brain and its membranes, attended with congestion of the vessels, effusion into the ventricles and subarachnoid space, with exudation of lymph between the convolutions, and arachnoid and pia mater.

Percivall, Plaine, Dick, and others describe two forms of staggers-the one arising from inflammation of the encephalon, and the other from engorgement of the stomach, respectively denominated "mad" and "stomach staggers;" and we are led to conclude that in the latter disease the brain is only sympathetically affected, or is disturbed by reflex irritation. Mr. Gamgee says, "I regard phrenitis as almost an unknown disease." If by this Mr. Gamgee means the phrenzy or mad staggers of our forefathers, experience compels me to agree with him; but if he intends to convey the idea that encephalitis, or inflammation of the brain and its membranes, occurs as a traumatic disease only, I am bound, by the sane experience, to differ from him, as experience amongst the living, and examinations of the clead subject, have convinced me that the conditions named "mall," when not caused by violence, traumatically, or by tumours, \&c., and "stomach staggers," are symptomatic of but one and the same malady, namely, inflammation of the brain and its meninges, and that the varying degrees of violence, convulsions, frenzy, 
stupor, coma, immobility, or paralysis, depend upon the seat of the congestion or inflammation at the time such symptoms are manifest. For example, the coma and loss of function become apparent when the brain proper is congested, or when pressed upon by effusion; the convulsions, tetanic spasms, and frenzy when the pressure is not so great, and when, in all probability, the membranes are the seat of the congestion or irritation; and paralysis of a part of the body, generaily the posterior extremities, when there is inflammation of the spinal cord and its membranes.

There are many pathological conditions of the brain which give rise to synuptoms of frenzy, such as tumours, some noncornizable morbid poisons, uræmic poisoning, rabies, the irritation of melanotic deposits, \&c. ; but inflammation gives rise to loss of function, and instead of the exalted condition, so graphically described by Percivall, we have in true cerebritis stupor, coma, and general paralysis.

Mr. Gamgee describes a form of encephalitis in lorned cattle arising from alcoholism. The cases are reported by Mr. George Dundas in the Edinburgh Veterinary Review. "The disease is due to a practice, prevalent in some parts of Scotland, of giving "burnt ale" to cows, in the neighbourhood of distilleries. The ale is given by steeping straw in it; and the animals will also drink it freely. They often sleep soundly after such a beverage, and sometimes symptoms of intoxication are manifest. The symptoms are as follows:-The head is turned singularly to the side, and is slightly elevated. The pupils are widely dilated, and the eyes have a remarkably wild appearance. On approaching the animals, they wink rapidly, and tremble. There is marked heat of head, horns, and ears. When pressed with the finger in the axilla they fall instantly, and when pulled by the lead they incline to turn over. The pulse is about seventy or eighty per minute."

"After death, all the organs are found healthy except the nervous centres, and both brain and its membranes are found congested. This congestion often extends into the spinal canal, and the pia mater over both the brain and cord is the seat of red spots. The redness is either ramified, or is obviously due to blood extravasation. Clots of blood have been found in the lateral ventricles, and around the spinal mareow in the cervical 
regions. There is evidently softening of the brain substance as a direct result of this condition."-(GAMGeE's Domestic Animals n Health and Disease.) I have never witnessed this effect of alcohol on cattle, although I an familiar with the fact that the "dreg" from distilleries is largely used for feeding purposes, and that it is an excellent adjunct to other foods.

The most common form of congestion of the brain and its membranes, both in the horse and horned cattle, is that called stomach staggers, sleepy staggrers, or grass staggers-a disease which sometimes rages as an enzootic.

\section{STMPTOMS.}

The animal at first appears dull, listless, falls asleep whilst standing, or drowsily nods its head; eats slowly and at intervals; the breathing is generally slower than is natural, sometimes slightly accelerated, but it is always of a somewhat snoring description. The pulse is also slower than natural, from twentysix to thirty, full and rolling. The animal walls with a straggling gait, staggers, and seems as if about to fall. If suddenly disturbed whilst in the somnolent condition, it looks around excitedly, shivers violently, and seems affrighted; but soon becomes calm again, and may remain so for a short period, especially if kept in a dark, quiet place. It now and then thrusts its head against the rack or wall of the stable; moves the limbs automatically; rears, hangs back, and breaks the halter, or gets its fore feet, into the manger, and elevates the nose high up into the rack. The eyes, mouth, and rectum are injected, and of a yellow tinge. In some cases, the yellowness of the mucous membranes is a very prominent symptom. As the disease advances, the extremities become alternately hot and cold; sweats bedew the body; there will be twitchings or elonic spasms of the superficial muscles of the neck, breast, and hind quarters. Violent convulsions will now cccur; the whole body becoming stiff and rigid, and the respiratory movements extremely difficult. During these spasmodic-tonic-attacks, the tail will be elevated, the membrana nictitans drawn over the eye, as if the animal were suffering from tetanus, and the pulse frequent, hard, and wiry, the eyes fixed and amaurotic, the mouth clammy, and the urine may be ejected by a convulsive 
effort. All at once the tonic contractions subside, and there will be great nuscular debility; the legs bend, the animal totters, sometimes falls, and when down will fight convulsively, and for a time be unable to rise. By slow degrees consciousness to some extent returns; the animal may then regain its feet, and will perhaps commence to feed; then fall asleep, with its mouth full of food, or look about in a wild, staring, vacant. manner, or ramble unconsciously about the box, striking its head against everything that may come in the way, the eyes amanrotic, and the animal quite blind.

Sometimes there is flaccidity or paralysis of the muscles supplied by the cranial nerves, the lips are pendulous, and the tongue hangs out of the mouth. At other times there will be tonic spasm of the facial and masticatory muscles, and some degree of trismus present.

The disease may attack the animal whilst at grass in the field. It is then observed to ramble about in an unconscious, drowsy manner, until it meets with some solid object, against which it fixes its head, and then moves its limbs continuously.

In cases which have been noticed at the commencement of the attack, some degree of rigor has always been observed premonitory to the development of other symptoms.

The above symptoms are subject to some variations. In some animals the comatose and paralytio conditions are the most prominent throughout, whilst in others delirium, convulsions, and spasmodic contractions alternate with the stupor. In other cases, again, the spinal system seems most affected when paralysis, without loss of consciousness, or with but a slight degree of drowsiness, is present.

There appears to be a general diminution of the various secretions; the costiveness is obstinate; the urine is secreted in small quantities, and is particularly high in colour. The function of the liver is also suspended, and the whole system becomes tiuged with the non-excreted biliary colouring matter -biliverdine.

As the disease advances the coma is more profound, or the fits of excitement and frenzy more frequent, and of shorter duration, leaving the animal mure and more enfeebled; the pulse is now small and quick, and the breathing stertorous and difficult; profuse sweats bathe the body, the sphincters relax, the 
animal is unable to stand, and dies fighting convulsively, or in a state of profound coma.

\section{ETIOLOGY.}

It is very generally held by writers upon veterinary medicine that the malady arises from an over-loaded and impacted condition of the stomach, that the cerebral symptoms are purely reflex or sympathetic, and that no actual disease of the brain or its meninges is present. These assertions are not supported by the results of investigation into the morbid anatomy of the malady, further than that the stomach has been generally found filled with food. Now, if engorgement of the stomach were the cause of the train of symptoms seen in this malady, then coma, delirium, or paralysis would be general in the majority of cases of engorgement, seen in the routine of general practice, but this is not the case. Numerous instances of engorgement, impaction, even to rupture of the stomach, constantly come under the notice of the practitioner, but signs of any brain uffection scarcely ever occur. We must then look to something more than mere impaction as the cause, and I think this will be found in the nature of the food.

In Scotland it has been called "grass staggers," from the fact that it occurs when the animal is fed on green food; but ordinary green food does not induce it. I have very carefully noted every case which has fallen under my care for some years, and I find that grass, more particularly rye-grass, when it has commenced to ripen, or when it has been cut, and allowed to heat and ferment before being used, is a fruitful source of this disease. I find that my observations are borne out by those of Professor Dick and others, and that not only horses and cattle are liable to the disease from this cause, but sheep, anc especially lambs.

Mr. Brydon, V.S., Traquair, in a letter to Professor Dick, says that lambs are often destroyed by eating the tops of rye-grass; and that he has found the tops of rye-grass two or three inches in length in their stomachs, causing infiammation.

Professor Dick again says_ " From what has been stated it will appear that, when rye-grass begins to ripen, a change should be made in the food by placing the animals on other pasture. The grass should be cut before it has quite ripened, as it will be found in that state innocuous." He then refers to a statement made 
by White of Exeter, in White's Farriery, that the disease occurred in one farm in South Wales, from hay made the previous year (1800), and concludes that "it seems more than probable the hay had been over-ripe. when made, and that the process of withering had not destroyed the irritating or noxions, or perhaps narcotic, quality of the over-ripe grasses."-(Veterinary Paper's by Professor Dick.)

I quite agree with Professor Dick that the disease is due to some peculiar narcotic principle, that is developed in the grass at this time, or which may be developed by the process of heating and fermentation, when cut at an earlier stage of its growth; for every-day experience proves to us that food, even rye-grass included, has no effect in producing symptoms of cerebral disturbance, when used in its ordinary condition, and that it is only when in a transition stage, as it were, between grass and hay, that it seems to possess toxic qualities.

After careful observation I have arrived at the conclusion that the seat of this disease is in the brain, the spinal cord, and their meninges; that, owing to the quality of the food, a degree of narcotism is first produced, speedily succeeded by congestion and other changes to be described in the morbid anatomy, and that owing to this derangement of the great nervous centres, paralysis of the digestive apparatus is the result, and the stomach becomes sometimes engorged, from the fact that the animal continues to feed, when the digestive as well as other functions are in abeyance.

This disease seems to have first attracted notice in 1787 , the summer of which was hot and dry. It raged in the sonth-west of England and Wales in 1800 and 1819, the summers of which were also hot and dry, prevailing most commonly amongst horses at grass in low, wet pastures, where the grass was rank. It was supposed to arise from their eating ragwort or staggerwort-Scnecio Jacobce-a plant supposed to contain a poisonous principle, or some other poisonous herb; but of this there is no absolute proof. We have, however, sufficient evidence to prove that it originates when animals eat rye-grass in the condition described. Mr. Gamgee says the disease may arise from gastric derangement, brought about by eating wheat, or even oats and bran, in large quantities. My experience convinces me that this is not the case, and that mere over-loading is more apt to pro- 
duce rupture, tympanites, inflammation of the bowels or feet, or intestinal apoplexy.

All kinds of damp fodder soon become covered with rusts or moulds. Amongst the former, the Puccinia graminis uredo, or red rust, has induced disease in rabbits, characterised by gastric irritation, vertigo, and convulsions (Frank). There are also black moulds-microphytes-closely related to yeast plants, indeed said to be a less advanced stage of the yeasts, and these are now proved to cause the formation of ptomaines, having a deleterious effect upon cattle, manifested by symptoms of depression, abdominal pains, constipation, succeeded by a fotid and sometimes bloody diarrhœa, bellowing and groaning, some degree of tympanites, and sometimes paralysis of the tongue and pharyngeal muscles, with continned movements of the jaws, salivation, and cough. These latter symptoms are more particularly due to black rust-Tilletia caries. These moulds and rusts are not uncommon on ill-dried feeding cakes, and are undoubtedly a source of much mischief.

\section{MORBID ANATOMY.}

From various dissections, very carefully made, it is found that impaction of the stomach is but an occasional complication. In many cases some degree of congestion of the villous coat has been present, and this appearance led Blaine to conchude that the disease was a specific form of gastritis; but this is not an invariable lesion, and is most probably as much induced by the action of medicinal agents as by food. The stomach may be quite empty; sometimes it contains some amount of food, and at other times it is impacted; there is always a congested condition of the lungs, as is the case in death by coma. The brain and its membranes are invariably congested; the former, after removal, seems to be in a swollen condition; the dura mater seems stretched, and the convolutions appear broader and flatter than natural, as if they had been pressed against the cranial walls. The vessels of the pia mater are injected and tortuous, and that membrane itself is easily laccrable, and may be stripped from the surface of the convolutions without tearing the cortical substance, which of itself looks darker than natural. On cutting into the brain both grey and white matters are 
studded here and there with red points. The plexus choroides is large, highly injected, sometimes covered with a thin film of exudation, and the lateral ventricles are filled with fluid.

In the spinal form of the disease, namely, that characterised by paralysis of the hind extremities, the congestion and effusion are generally limited to the spinal cord and its membranes in the dorso-lumbar region; the arachnoid space is filled with serosity of a reddish colour; the pia mater is congested, and the cord itself in a red, softened condition.

Prognosis.-In the horse the probabilities of recovery are much greater than in oxen and sheep, in which it is very fatal. The recovery in the horse, however, has been slow and protracted, some degree of paralysis in many cases remaining for several weeks after the acute symptoms liave passed off. Recovery may be expected when the symptoms do not become much aggravated during the first day or two, when the delirium is not excessive, and when the coma is not continuous and profound. In the spinal form the animal may fall and be unable to rise, very early in the disease; this is but of little moment, provided the animal does not struggle much, and work itself into a state of fever and exhaustion. In the cerebral form, inability to retain the standing posture is always a bad sign, more especially if the delirium be continuous. The age of the animal must also be taken into consideration; the young and strong may recover, the old and debilitated succumb.

\section{TREATMENT.}

In the early stages, when the pulse is full, and has tone, bleeding has a beneficial effect, by directly unloading the vessels of the brain. Indeed, I have witnessed most marked results from this, the almost unconscious, amaurotic animal regaining its sight and consciousness in a short time. Four, five, six, or even eight quarts can be taken from the jugular with advantage. If, on the contrary, the pulse be fceble, accelerated, as in the later stages of the disease, bleeding only hastens the animal's death. A smart cathartic is always to be administered, and no danger need be apprehended from superpurgation. The purge may be composed of from six to eight drachms of aloes, and a 
drachm of calomel for the horse; twenty ounces of sulphate of magnesia, and fifteen croton beans for a full-sized ox or cow; after which the animal is to be kept as quiet as possible; all food must be removed; plenty of water allowed to drink; an enema now and then administered; and the head bathed with cold water. The attendant is to do all this quietly and calmly; there must be no shouting, or any noise to disturb the animal, nor is it to be eontinually dosed with medicine. Time must be allowed for the purgative to act, and in some cases there is no response for two or three days; but if the symptoms abate, no alarm need be felt, as the bowels are always slow to act when in a paralyzed condition. Should the symptoms inerease in severity, belladonna might be tried, in two-drachm doses of the extract, or half-grain of atropine injected subcutaneously.

I think this form of constipation offers a fair field for the chloride of barium, about ten grains of which might be intravenously administered to induce rapid action of the bowels.

When the acute symptoms have passed off, it may be necessary to apply blisters to the loins, or at the back of the poll; but during the earlier stages, more especially if the horse is restless, nothing should be done to irritate and annoy it, or it may work itself into a state of excitement which speedily terminates in death. In addition to blisters, I have found the iodicle of potassium, given twice a day, to be exceedingly useful in promoting the absorption of any exudate or effusion; and should the bowels remain torpid, and an imperfect paralysis continue, nux vomica may be added with advantage. It may be here remarked that the arrestation of the urinary, biliary, and intestinal secretions are results of cerebral disturbance, and that when this is removed the secretions regain their natural condition. Purgatives are useful as removers of the cause, which is food containing deleterious principles, and as derivatives. If the pulse be feeble, small doses of stimulants-ammonia-may be administered. I am, however, no advocate for large doses of stimulants, as they tend to excite the animal and malie it restless, when quietude is of the greatest importance. 


\section{CHAPTER XLI.}

\section{SPORADIC DISEASES-continued.}

LOCAL DISEASES-continued.

\section{(D.) CEREBRAL APOPLEXY.}

Definition.- " A disease essentially characterised by the sudden loss, more or less complete, of volition, perception, sensation, and motion, depending on sudden pressure upon the brain (the tissue of which may be morbid), originating within the cranium."(Aitken.)

Apoplexy is of two kinds:-1st. That arising from degeneration of the cerebral vessels, with rupture of them, and extravasation of blood upon or within the substance of the brain; and $2 d$. That in which congestion of the cerebral vessels -not of themselves necessarily diseased-is the primary condition; this, when excessive, resulting in rupture and extravasatioll.

\section{SEMIOLOGY}

Apoplexy from the rupture of degenerated blood-vessels.-There may be some premonitory symptoms, such as staggering and partial paralysis, but generally the animal falls suddenly without warning. After falling it may lie prostrate, in a state of unconsciousness, without the power of voluntary motion, perfectly insensible to surrounding objects, and dead to all ordinary feeling, with its eyes wide open and presenting a ghastly stare, the pupils dilated and insensitive to the light-amaurotic. The breathing is stertorous, the pulse small, rapid, and thready, the surface of the body cold, or bedewed with a cold sweat, the limbs flaccid, the mouth open, and filled with frothy saliva, and in some severe cases the sphincters are relaxed. In other 
instances the animal may still retain the power of muscular movements; but they are irregular, and intermixed with spasmodic contractions. Whilst down, it fights convulsively, presses the back of its head violently against the wall or other solid body. Some degree of opisthotonos is present, the back is arched downwards, and the hind legs extended backwards. The eyes move about convulsively, or there may be persistent strabismus of one or both of them; the pupils may be dilated or contracted, alternately contracted and dilated, or they may be natural, or one may be contracted, and the other natural or even dilated. The respiratory movements are sometimes spasmodic; now and then there may be a stertorous sound; at other times sighing, and sometimes expressions of great pain, as if the animal had been sharply wounded with a eutting instrument.

These symptoms may alternate with intervals of quietude, when the animal will fall into the comatose condition. The pulse may then fall below its natural standard, and the respiratory movements may become slower than natural, with heavy, deep inspirations. The various secretions are, in all cases, suspended, and the animal gradually sinks from increasing brain pressure, or suddenly from renewed extravasation.

The symptoms of apoplexy present some degree of variety in their character, and in the course of experience one cannot but remark that conditions which were laid down by our teachers as being invariably present, are sometimes altogether absent, or are present in a very modified degree. For example, it is generally taught that when a patient is apoplectic and comatose, the pupils are widely dilated, and the eyes amaurotic; but this is not always the case, neither is contraction of the pupils an invariable sign of brain disease. In many cases the condition of the eyes is quite normal. Again, the loss of consciousness varies very much; in one case we have complete insensibility, in another a degree of hyperæsthesia to touch and sound, as expressed by convulsions and tremors, when the animal is touched or spoken to. In one case, although a large clot, extending from the superior surfaces of the crura to the base of the brain was discovered post mortem, it was observed that when left alone the horse would lie more or less quietly; immediately it was disturbed it would fight convulsively, the breathing would become spasmodic, and it would dash its head with 
violence upon the ground; when pricked with a pin it showed extreme agony.

Siertorous breathing.-This symptom, like the other, is not invariably present, indeed it is often absent in undoubted apoplexy. At all times it indicates severe lesion, and that the pons varolii or medulla oblongata are implicated.

\section{MORBID ANATONY.}

Apoplexy from degeneration is seen in old horses, in which the post mortem examination reveals the cerebral arteries in an atheromatous condition. The first attack generally proves fatal, but I have seen two cases of relapsing apoplexy, in which distinct post mortem traces of former extravasation were found.

The clots are either superficial or deep seated. Superficial clots are generally supposed to be clue to extermal violence. This is, however, not always the case. Extravasations are found in the lateral ventricles, from a rent in the corpus striatum, or from rupture of some of the vessels of the choroid plexus, upon the surface of the cerebrum, and at the base of the brain; in one instance there was, in addition to a tumour, extravasation from degeneration and rupture of the vessels of the falx cerebelli.

The extravasations are occasionally circumscribed, but most commonly diffused, and spread over a more or less extensive surface, extending from the lateral to the fourth ventricle, and sometimes surrounding the crura cerebri.

In rare instances the symptoms may take some days before they become fully developed. This is due to very slight but increasing hæmorrhage. In the majority of cases the attack is sudden, and the loss of power almost instantaneous. Congestion of the brain-as in the last described disease-occasionally terminates in apoplexy, or rupture of the cerebral vessels and extravasation of blood; but congestive cerebral apoplexy is a rare disease in the horse, that which is usually met with being due to degeneration of the vessels. 


\section{CHAPTER XLII.}

\section{SPORADIC DISEASES-continued.}

\section{LOCAL DISEASES-continued.}

\section{(E.) APOPLEXY FRON CONGESTION.}

This form of apoplexy, except as a sequel to encephalitis, is very rare in the horse, but is of frequent occurrence in cows, and is variously termed parturient apoplexy, milk fever, dropping after calving.

Definition.-A parturient disease, characterised by suppression of the lacteal secretion, congestion of the brain, and apoplexy.

\section{ETIOLOGY.}

Various opinions are held upon the causes and origin of parturient apoplexy, some writers maintaining that it is a blood disease, whilst others state that conformation or anatomical peculiarity is sufficient to account for its occurrence in the cow, and its absence in the mare. The late Mr. Barlow, in a most profound and remarkable paper, traced its origin to derangement of the great sympathetic nerve, with consequent arrest of secretion, resulting in general congestion, which became localized in the brain and spinal cord; whilst other observers express an opinion that the disease results from derangement of the digestive apparatus, and that it is a peculiar form of indigestion.

Without entering into a critical examination of these various hypotheses, I may venture to state that there is no evidence of the primary presence of a blood poison. Mr. Gamgee ventures upon the assertion "that notwithstanding the sporadic nature of parturient apoplexy, in cattle it is marked by the development of a poison capable of inducing a similar disease in other animals, 
of affecting the liuman frame, and hence of rendering the flesh of animals affected by it unfit for human food."-(Domestic Animals.) If this assertion were correct, the number of the human race would ere this have been much reduced, for it is a well-known fact that the flesh of cows slaughtered whilst suffering from parturient apoplexy is a common article of diet, and that no bad consequences result from it, provided the animal has been slaughtered early, before the system has been empoisoned by the excessive doses of medicines which are so generally prescribed in this malady, and antecedent to a general vitiation of the animal solids and fluids by the accumulation of effete materials.

The anatomical theory is upset by the fact that the disease is inseparable from domestication and stimulating food; peculiar to deep milkers, and scarcely ever succeeding difficult parturition, which most assuredly would be the case if shortness of neck, excessive-natural-vascularity of the brain and its membranes were predisposing causes.

The other theories, namely, that which traces the origin of the disease to derangement of the function of the ganglionic or sympathetic nerve, and that ascribing it to indigestion, may conjointly, I think, be looked upon as offering at least an explanation.

Parturient apoplexy rarely occurs prior to the third period of parturition, but I have seen it, in two instances, succeed the second calving. Cows of all breeds are subject to it, provided they are deep milkers. It seldom attacks cows in which the lactiferous system is not highly developed, no matter of what breed they be. Age, and the profitable property of giving an abundant quantity of milk, are acknowledged and well-known predisposing causes; so much so, that dairymen very often decline to buy a deep milker immediately prior to a third or subsequent parturition. This tendency to the malady is promoted and intensified by warm weather and stimulating food, which is generally allowed in great abundance. All cows, but more particularly those which are profitable milkers, ought to be well fed; but it should be remembered that prior to the act of parturition, deep-milking cows, which are dry or nearly so, rapidly become plethoric, and that this state of body, at the time when the calf is born, is one which very commonly excites 
this fatal malady; hence, in feeding an animal of this kind, great care should be taken that the food be not over-abundant, too highly nitrogenous, or too watery. A previous attack of parturient apoplexy also predisposes to a second, and generally fatal attack of the malady.

The exciting causes may be looked for in the act of parturition itself, mistake in the dietary, and in the season of the year.

In rare instances, parturient apoplexy occurs during or immediately prior to the birth of the calf; but almost invariably the first symptoms are not manifested before the lapse of some hours-from twenty to twenty-four hours is a very critical period-or even two or three days after parturition.

When symptoms of the malady are not manifested for some time after the birth of the calf, it will generally be found that the secretion of the mammary gland has been in an average, or even abundant quantity, that the appetite has been good, and rumination naturally performed. Premonitory to other symptoms, it is generally seen that the secretion of milk is suspended.

The arrest of the lactiferous secretion is doubtless due to some disturbance of the organic system of nerves, but how this arises it is difficult to determine, unless we take into consideration that the great sympathetic is developed to a greater extent in deep milkers than in other cattle, and that in consequence it is more susceptible, and more prone to derangement from trivial causes, especially at this critical period.

In the natural or healthy condition, the blood required for the support of the fotus in utero is diverted after the parturition into the mammary gland, for the purpose of supplying material for the formation of milk. One portion of the ganglionic system is thus brought into increased action, whilst that which during the pregnancy had been developed in the uterus, being no longer required, falls into a condition of at least temporary atrophy.

If we bear in mind that the blood required to support the fœtus prior to its birth is diverted, as it were, in the healthy state to the mammary gland, when the mother is delivered, in order to supply material for the secretion of milk; and if, in addition to the arrest of the lactiferous secretion, the varions excretory organs cease to perform their functions, it can be easily understood how the whole vascular system becomes preternaturally engorged or congested. Such then is the condition 
of the system in this affection, not only prior to the occurrence of the local cerebral congestion, but throughout its course, and after the animal's death, when the vessels are found engorged and the tissues loaded with effusion.

I think that the particular congestion of the brain and its meninges is determined by the state of mental excitement which is always present at this period, and this argument is borne out by the fact that the removal of the offspring from its mother is a fruitful exciting cause of the so-called milk fever. That the lower animals are eapable of expressing strong maternal affection is patent to all observers. Who has failed to witness the restlessness, loss of appetite, and unhappiness of many a cow deprived of its ealf? and this is a fruitful source of derangement of health.

To exemplify this, a case of general paralysis in a she-goat came under my notice some years ago, which I will here relate. The goat's kids, four weeks old, were taken away from it by the owner, who, on returning in about an hour's time, found the poor animal completely paralyzed, moaning piteously, and evidently in a state of great distress. It remained in this condition for several days, when the owner brought it to the New Veterinary College for my opinion. When I saw it, it was quite conscions, but powerless, lying at full length upon its side. I liept it for several days, but it gradually sank and died. Now in this case there was no cause of disease but mental distress, and if this be the case with the goat, most assuredly mental distress may be a canse of derangement in the cow. In addition to the removal of the calf from the mother, it is a common practice to make sudden and violent changes in the animal's diet. For the food to which it has been used, thin bran slops, gruel, \&c. are substituted: this of itself would be sufficient to induce indigestion and serious constitntional disturbance.

That middle-aged animals are more prone to the malady is explained by the facts that the lactiferous powers are greater in them than in the young, and that the functions of the ganglionic nerves are more active, and consequently are sooner deranged.

Atmospheric conditions have also to some extent an influence in predisposing to the malady. During warm spring weather it is much more prevalent than in cold seasons: from this cause many newly calved cows are simultaneously attacked, leading some to suppose the disease to be enzootic. 


\section{SEMIOLOGY.}

In many instances the symptoms appear suddenly, and with great violence, the disease running its course, and terminating fatally in a short period, but most commonly there are some premonitory signs. The secretion of milk is stopped, the cow hangs its head, ceases to feed, and paddles with its hind feet. By-and-by the breathing becomes hard and rapid; it sways from side to side; the hind legs double at the fetlocks, and at last it falls. The eyes are blood-shot, in some cases wild and staring; the eye-lids twitch spasmodically, and an abundant flow of tears runs over the cheeks. The ears, horns, and forehead are now intensely hot; the animal lies either in a state of perfect stupor or coma, or dashes itself violently about; the head is thrown from side to side, and there is danger of the horns being knocked off.

In some cases the nose rests firmly upon the ground, and if the head is lifted up, it will fall back like some lifeless body; at other times the head is brought back to the side, where it remains firmly pressed against the shoulder or neck. There is often a disposition to lie upon the side, in which case the neck is stretched out, and the limbs extended, the eyes glassy, and the mouth open; tympanites soon sets in, and the cow will die in a very short time if its position be not altered. Very early in the disease the power of vision is lost, the eyes being amaurotic ; the power of swallowing is also lost, or very imperfectly retained. The cow may remain in this condition for several hours, provided it is made to lie, by packing, in the natural position on the stermum, evincing but few signs of life, except the act of breathing, which is generally stertorous; now and then there is emitted a gurghing sound of gas and fluid regurgitated from the rumen.

The pulse, which at first is generally full, and more or less accelerated, becomes small, quick, and almost imperceptible; the breathing is more and more difficult; in some cases convulsions set in, whilst in others death occurs from a gradual sinking of the vital powers; the sphincters are paralyzed, the mouth open, and the breathing partially oral, with puffing of the cheeks at every expiratory act.

In some instances the symptoms of delirium are very violent; 
the animal struggles violently whilst down; works itself on to its side in spite of all precantions; dashes its head wildly about; bellows, groans, and strains violently; very shortly the abdomen becomes tympanitic, and the breathing more laboured and difficult; there is eructation of foetid gases from the rumen, and death rapidly supervenes.

In other cases conscionsness in some degree returns, the eyes become natural, the cow pricks its ears as if it once more could hear sounds, the secretion of milk returns, the body cools, and seems as if convalescence were in reality established. These flattering signs are, however, too often succeeded by a return of the worst symptoms, and it very frequently succumbs.

In some instances the bowels may be relaxed in the earlier stages; and this will greatly depend upon the previous treatment of the animal. This is, however, very soon succeeded by nonexcretion both of fæecs and urine. Constipation, or rather nonpassage of the fæces, is always a marked sign, not that there is any actual dryness or costiveness, but that the muscular walls of the intestines are in a paralyzed condition, the peristaltic action being lost. The urine, generally pale in colour, and free from albumen, scantily secreted by the kidneys, is retained in the bladder, which has also lost its power of contractility.

\section{PROGNOSIS.}

I consider it a good sign, if, after the animal has fallen, it is able to rise, although it fall again, and become perfectly comatose. This conclusion is a result of experience, but I can offer no explanation of it. If in the course of thirty to forty hours consciousness gradually returns, and the animal makes attempts, but not too violent, to rise, the bowels commence to act, the secretion of milk, and a desire for food return, a successful termination, at least of the apoplexy, may reasonably be expected. If, on the contrary, the sphincters relax, the coma becomes more and more profound, or the delirium continue, and if, in addition to these, the dropping of the jaw and oral breathing, which are always indicative of grave lesions, become manifest, a fatal issue is to be expected. 


\section{POST MORTEM APPEARANCES.}

The vessels of the body generally are filled with dark-coloured blood, the tissues are moist; the uterus is generally large and congested: this may, however, be unassociated with the disease, as it is the natural condition of the womb so soon after parturition. Extravasations of blood are found at the base of the brain, on the medulla, and cervical portion of the spinal cord. In some cases, however, actual rupture of the vessels has not occurred; great congestion of the vessels and the red pointspuncta vasculosa-have been very marked when the brain has been cut with the knife, and in others no marked congestions have been seen, having in all probability disappeared post mortem, or perhaps inmediately previous to the animal's death -congestion being generally an ephemeral condition; but in all instances there is an accumulation of fluid in the ventricles, and the brain substance itself is loaded with serosity.

The digestive organs, kidneys, liver, heart, present no special signs; the serous membranes are sometimes covered with petechial spots, the lungs are engorged as in all cases of death from paralysis of the respiratory apparatus. Thinking that perhaps the symptoms of intoxication seen in the disease were due to uræmic poisoning, I have very carefully examined the kidneys, but have never been able to detect any special lesions which support that view. It is quite possible, however, that urea is superabundantly present in the circulation, but I do not think that it has any special connection with the origin of parturient apoplexy. Further observations are, however, required before this matter is satisfactorily solved. I have never detected albumen in the urine.

\section{TPEATMENT.}

In the earlier stages, whether the animal is standing, or lying prostrate and in a state of coma, if the pulse be not excessively weak, and the heart's action almost fluttering, recourse must be had to venesection. Slow and deep breathing, with a tendency to stertor, add greatly to the necessity of immediate bleeding. The beneficial action of the withdrawal of blood is shown by the pulse becoming fuller, stronger, and better in tone. The 
opening into the jugular is to be a large one, in order that the blood may flow freely to relieve the congestion, to check, if possible, a further extravasation of blood or effusion of serum, and to divert its active flow into the head; but it must not be carried out so as to debilitate the leart's action. When the pulse becomes fuller and stronger, the bleeding is to be stopped; from three to five quarts will generally be sufficient.

The bleeding is for the purpose of removing pressure from the brain, and although the pulse may indicate stimulants rather than depletion, it will be found that as the blood flows the tone of the pulse will improve, for the weakness of the pulsation, the want of impulse, and debility of the heart's action, are results of brain pressure. If, however, the surface of the body be cold, the animal tympanitic, the heart's action fluttering, and the pulse almost undetectable, bleeding is calculated to do more harm than good, as the heart's action would now be further impaired, and the amount of arterial blood sent to the brain diminished; for it must be remembered that the cerebral congestion is now less due to an over-abundant supply of arterial blood than to pressure upon it by venous or capillary engorgement; that, in fact, the brain-engorged as it may appear-is in an anæmic condition in regard to its arterial supply; and when this is the case, paralysis of the heart is to be prevented, if possible, by the use of stimulants. It is the practice of many veterinarians, and of its success I can speak with confidence, to bleed to relieve congestion, and administer a stimulant to increase the heart's action; to enable the heart, in fact, to send arterial blood into the brain by overcoming the resistance of the congestive venous pressure.

If the cow is already down when first seen by the practitioner, his first care must be to see that it is made to lie as near the natural position-on the sternum--as possible, and this he will do by having it packed up at the side by bundles of straw, or, what is better, sacks filled with straw, firmly wedged under the quarter and shoulder, the head at the same time being properly propped by the same means; and care being at all times taken that the cow is prevented from injuring its head by striking it against hard bodies. When this is done, venesection may be had recourse to.

In all cases, whether the pulse be small or full, a strong 
cathartic should be administered, consisting of sulphate of magnesia, with croton, ginger, or other aromatic, care being taken that the animal is not clroked, as the act of deglutition is often performed with difficulty. I always try if the animal can swallow a little water from a bottle before administering any medicine. If the patient shows any difficulty in doing this, the medicine is to be given with the stomach pump. I am of opinion that the cathartic should not be repeated, having seen the repeated administration of even simple fluids cause tympanites and death. Cold water is to be applied to the head; the spine along its whole course stimulated with strong ammonia liniment, and the animal warmly, but not heavily, clothed. Every two or three hours it is to be turned and packed on the other side of the body; the mammary gland stripped and hand rubbed, and should there be signs of exhaustion or failure of the heart's action, a stimulant is to be administered; the best way of doing this is to give ammonia carbonate in a ball, which, after being oiled, is to be pushed by the hand as far as possible into the pharynx, when it will slowly gravitate into the rumen. If the tympanites is not relieved by the anmonia, the rumen is to be punctured by the trocar, and the cannula left in. Enemas of warm water are to be repeatedly injected into the rectum, the urine withdrawn, either by the finger introduced into the bladder, by the female catheter, or by introducing the nozzle of the ordinary injection syringe.

Some practitioners recommend nux vonica to be given every few hours. There is no objection to a single dose of this being given along with the cathartic, but its repeated adninistration can only do harm; the paralysis depends upon engorgement, and spinal or nervine stimulants in the acute stages of the disease are contra-indicated.

The pouring or pumping of large quantities of fluids, consisting of sundry quarts or even gallons of water, with treacle, ginger, \&c., for the supposed purpose of causing purgation, is also to be condemned; the fluid absorbed, whilst the excretory organs are not performing their functions, only adds to the general plethoric condition.

To prove the injurious consequences of this practice, as well as that arising from feeding the animal upon thin sloppy food 
after parturition in order to prevent this disease, I will quote a parallel from human pathology.

Niemeyer says, under the liead Hypertrophy of the IIeart"Such patients must beware of immoderate eating and drinking, in order to avoid the plethora which, although but transient, always follows the free use of food and drink. How often does the long-threatening apoplexy set in in the midst of the plethora which has developed after a long and hearty meal? In this connection, I may mention an act of folly which I have often seen practised by tavern-keepers and itinerant winedealers. The latter suppose that by a free use of water they can counteract the pernicious influences to which they expose themselves, although it is evident that the plethora arising after a full meal would only be increased by an immoderate addition of fluid."

When consciousness has returned, and the animal seems in a fair way of recovery, no medicines should be administered; but if at any time there are signs of exhaustion or sickness, small doses of spirits of nitrous ether and water may be administered, provicled the animal is conscious and can swallow freely.

In some instances the apprtite is inordinate immediately after consciousness has returned; the animal will greedily devour any lay or straw that may be about it. This must be prevented by muzzling it at once, or a fatal attack of indigestion with tympanites will be the invariable result. Small quantities of water or thin gruel may, however, be allowed, and all food given very sparingly until rumination has been fully reestablished.

There are two sequelæe which often annoy the practitioner when he has congratulated himself upon a successful case, namely, congestion of the lungs, and paralysis of one or both hind extremities.

Congestion of the lungs appears about the third or fourth day. The cow, in the meantime, having perhaps been on its feet repeatedly, giving milk freely, \&c., suddenly begins to breathe heavily and quickly, the pulse becomes oppressed and rapid, and the animal quickly sinks. In some instances the lung complication is due to inflammation of the bronchial tubes, excited by the incautious administration of fluids during 
the comatose stage; such fluids having passed into the trachea and lungs, and causing inflammation, which is manifested whilst the animal is otherwise recovering. An abundant secretion of mucus, with cough and mucous rales, indicate this, but undoubtedly congestion of the pulmonary system arises frequently, and independently of the irritation of foreign agents in the bronchial tubes, and is due to the mal-condition of the blood, resulting from retention within it of effete materials, and from debility of the heart's action. It is best treated with stimulants and hot fomentations to the sides. It is, however, very fatal.

The other sequel, namely, paralysis of the hind extremities, very often baffles all attempts to overcome it, and is due to inflammation and red softening of the cord in the lumbar region. If the cow is not up about the second day after consciousness has returned and after it has commenced to improve, paralysis may be expected to be present. It is very true that some cows, out of sheer obstinacy, will refuse to rise upon their feet, although the practitioner knows well enough they can do so if they like.

Many a cow will do this until it sees some liateful object, such as a $\operatorname{dog}$ - and it is a common practice to introduce a dog into the byre-when her ladyship very soon takes to her feet. This is a harmless, and sometimes a very effectual practice. At other times, believing that paralysis is present, and is to be overcome by severe counter irritation, the veterinary surgeon has applied the actual cautery to the lumbar region. The touch of the hot iron has been enough; with a loud bellow and a dash, it has jumped to its feet, and, if not fastened, has made good use of its hitherto supposed paralyzed legs.

When paralysis is actually present, and if the animal is in good condition, it is better to make it into beef, as too often the use of the limbs is never restored, more especially if it is a heavy, fat cow.

The treatment consists in the application of the actual cautery to the loins; blisters, purgatives, succeeded by iodide of potassium and diuretics, to excite the absorption of any exudation which may press upon the cord, and finally nux vomica and sulphate of iron. In some instances one leg ouly is paralyzed, and the animal is able to rise with a little assistance. Many 
cows, however, resist any attempts to help them, and will struggle up if left alone; drag the paralyzed limb, "knuckle over" at the fetlock for a time, and finally recover.

I may state, in conclusion, that parturient apoplexy is a recurring disease, and that it is not safe to allow a cow to calve after it has once been down; and if the owner consults his own interests, he will milk it as long as possible, and then prepare it for the butcher.

I have heard of several cases of post-parturient paralysis in which dislocation of a hip-joint has been discovered post mortem. 


\section{CHAPTER XLIII.}

SPORADIC DISEASES-continued.

LOCAL DISEASES-continued.

(\$.) ADVENTITIOUS SUBSTANCES IN THE BRAIN AND CRANIAL CAVITY-CAUSING, GENERALLY, SYMPTOMS OF CONDITIONS VARIOUSLY TERMED SLEEPY STAGGERS, IMMOBLITY, COMA, \&c. \&c.

TUMOURS.

Tumours are generally found in the choroid plexus. They are very commonly met with in both plexuses, sometimes larger in one than in the other; very often quite symmetrical in size and position. They grow slowly, and scarcely ever affect the health of the animal, or give any indication of their presence, until they have attained a size varying from a pigeon's to a hen's egg. They consist of a caseous material, mixed with a calcareous matter; they are in fact composed of an exudation which has undergone the caseous and calcareous degeneration. These calcareous particles are called brain sand-psammoma-and are never found post mortem presenting signs of recent origin.

I have found these psammomatous tumours in horses of various ages, but most commonly in old horses.

When they have attained the size above mentioned, they, in some instances, give rise to severe convulsive fits, a staggering gait and inability to perform work, whilst in others their presence is not indicated by any symptoms prior to a final stroke, simulating apoplexy, to which the animal rapidly suceumbs. In those instances, when they occasion disturbance prior to the fatal attack, the symptoms vary somewhat in character; in one animal there will be unconsciousness, with elevation of the head and fore part of the body; in another-and this is rather a 
frequent symptom-there will be a tendency to keep the head in a depressed position. In one case this depression of the head was very marked, but this did not happen until the animal-a mare-was worked or exercised; when kept quiet it appeared in the best of health in every way, but if exercised, no matter how slowly, depression of the head-the nose being almost brought to the ground-would always oceur. This animal had, shortly before presenting these signs, recovered from an attack of farcy, affecting the cervical and facial absorbents. When I saw it I expressed an opinion that there was a tumour in or upon the brain, due to or connected with the previous farcy. I may mention that the cervical lymphatics were still enlarged, but showed no signs of recent inflammation. The probable age of these ventricular tumours is sometimes a question of importance as connected with the soundness of a horse prior to purchase; and it is well to bear this in mind, as an animal may die from an attack of cephalic disease, and upon a post mortem examination being made, these tumours may be found in the lateral ventricles. The clinical history of these points out that they are of slow growth; that they do not cause any disturbance until they have attained a certain size: they do not, at least in all the instances that I have met with or heard of, give rise to disturbance, increasing in severity as they slowly grow; but all at once, when they have attained the size of a small egg, signs of a severe cerebral disease occur, which ultimately proves fatal. Examination of the brain and tumours reveals-1st. The lateral ventricles nearly or completely filled by the growths; $2 d$. The tumours themselves firmly encapsuled in the folds of the choroid plexus; $3 d$. Absence of congestion, injection of the vessels, and other signs of recent inflammation; and $4 t h$. A caseous and calcareous change of the interior of the tumours.

Thickening of the dura mater:- Thickening and induration of the dura mater consequent upon a slow chronic inflammation is of very rare occurrence. I have one case on record, however, in which the dura mater attained a thickness varying from one inch at the base to several inches at the anterior part of the cranium, causing absorption of the descending (orbital) plates of the frontal bones and wings of the ethmoid, and filling the frontal sinuses. The horse in which this was discovered had. presented signs of brain disease, coma, immobility, partial 
paralysis, amaurosis, and paralysis of the muscles of mastication, for a considerable period before its death. The post mortem examination revealed that the bones above mentioned had been absorbed, the frontal sinuses filled with a white mass resembling brain, but which proved to be thickening of the dura mater; the brain compressed, very solid in consistence; the iateral ventricles almost obliterated by approximation of their sides and roof, and the brain generally anæmic. There was no previous history to this case. It is very probable, however, that this condition was a result of an injury, such as a blow upon the head, causing perhaps partial detachment of the dura mater without fracture of the bones, and consequent chronic inflammation.

In another case there was enlargement of the lachrymal gland causing absorption of the bones of the orbit, and death by pressure on the brain. The cranial bones as well as the brain are now in my possession, and were obtained from an old horse, which had presented no signs of disease, except a slight difficulty in masticating its food for a few days prior to death, and at first was supposed to be another specimen of induration of the dura mater : but further examination proved it to be due to enlargement of the lachrymal gland. In addition to penetrating the cranium, the tumour partly filled the frontal sinus on the same side, causing partial absorption of the orbital process of the frontal bone; but the most remarkable circumstance in connection with the progress of the case was the fact that at no tirne was there any bulging or undue prominence of the eye ; a condition that one would have supposed would have been a more likely one to occur than absorption of the osseons walls of the orbit.

The tumour measured several inches, was lobulated in character, and microscopically was found to consist of numerous cells imbedded in a few very delicate fibres. The tumour presented no signs of retrograde change, and partook of the character of lymphadenoma in many particulars.

TUBERCULAR DEPOSITS IN THE MENINGES OF THE BRAIN.

Tubercular meningitis - a disease not rarely met with in the liuman being, more especially in the young-is a very rare affection in the lower animals. I have seen it in calves about three inonths. 
The symptoms were those of nervous derangement, partial paralysis, squinting, contraction of the pupils, fits of convulsions succeeded by coma, loss of motor power, amaurosis, and death. In some cases there will be indigestion, purging, the freces being white, and consisting of the milk the animal was fed upon, loss of flesh, hardness of the belly, and emaciation. In others the digestion may be good and the body fairly nourished.

In addition to small tubercular deposits, scattered here and there on the membranes-caseous and calcareous, scrofulous tumours were found in the peritoneum, in the mesenteric glands, and in the pleura. Very probably the condition termed hydrocephalus, or dropsy of the brain, is due to the development of tubercle during fotal life.

\section{EXOSTOSES}

Are met with in horned cattle. These consist of enamel-like growths of a globular nature, sometimes convoluted, and attached by a pedicle. They dip, along with the dura mater, into the convolutions of the brain, and seem to be due to ossification of the dura mater.

Mr. Gamgee reports a tumour having the appearances of an ossified brain, which is in the Milan Museum; and states that these growths sometimes attain the size of an ox's brain, without inducing any apparent disorder until the animal's sudden death.

I have seen in the horse tumours having a resemblance to dentine invading the temporal bones, both externally and internally; and these, like the bony tumours of cattle, have induced no marked symptoms during life.

\section{HYPERTROPHY AND ATROPIIY OF THE BRAIN}

Are conditions unknown in the lower animals. In my work on the Prineiples and Practice of Veterinary Surgery, page 18:, a case is described of apparent hypertrophy of the brain; but in reality the apparent enlargement was due to development of fibrous tissue in the brain, which of itself was not enlarged. In the case of thickening of the dura mater mentioned at page 504 , the brain was atrophied by compression owing to the thickening of the dura mater. 


\section{MELANOSIS OF THE BRAIN AND MENINGES.}

Small, black, melanotic nodules, varying in size from a pea to that of a bean, were found in the brain and on its meninges in an aged grey stallion which had suffered for a number of years from "stringhalt." Not only were they found in the cranial cavity, but along the course of the spinal cord, being particularly numerous in the lumbar region.

\section{ENLARGENIENT OF TIE PINEAL BODY.}

This body is sometimes found enlarged, and sometimes converted into a cyst, containing a whitish viscid fluid. The pituitary body may also be found in a similar condition. These appearances are met with in old subjects on the dissecting table. There are no appearances which indicate their presence during life.

Parasites in the Brain.-(See Parasitic Discases.)

\section{SOFTENING OF THE BRAIN.}

I have already stated that softening of the brain occurs as a sequel of epizootic cellulitis, and that it is due to plugging of the cerebral arterial branches.

All the conditions mentioned in this chapter may give rise to what is termed immobilité by French veterinarians-coma somnolentum by writers on human medicine, and sleepy staggers ly old writers on farriery; a manifestation or expression of brain disease characterised by general dulness, a tendency to fall asleep when allowed to stand quiet for a while, tripping or falling, staggering gait, slow pulse, and deep respiration. An animal suffering from this will often fall asleep with its mouth full of food. It is, however, easily aroused, and if this be done suddenly, will manifest fright, trembling, and a disposition to delirium.

For the relief of these symptorns an occasional purgative ought to be administered, the animal carefully fed, and worked gently, so long as it remains serviceable, after which it should be humanely destroyed, as it is useless to endeavour to cure what is organically changed. Hence all irritating materials in the form of blisters, setons, \&c., should be avoided. 


\section{CHA P TER X L IV. \\ SPORADIC DISEASES-continued. \\ LOCAL DISEASES-continued. \\ (G.) DISEASES OF THE SPINAL CORD AND ITS MENIBRANES.}

INTLAmmation of the spinal cord and its meninges is described as two separate diseases by liuman pathologists, and is termed myelitis, when attacking the substance of the cord; rachidian arachnitis and spinal meningitis, when involving the membranes only. I think that in the lower animals the distinction is unnecessary. I shall therefore describe the discase under the generic term.

\section{SPINITIS.}

Idiopathic inflammation of the spinal cord and its membranes presents itself in the horse in two forms, namely, acute and chronic.

The symptoms of acnte idiopathic spinitis are not always alike. In some instances the paralysis, which results in every case, may be manifested suddenly, and with little or no previous premonitory signs of ill health, the animal losing all power of movement over all parts posterior to the seat of inflammation; whilst in others, symptoms of cramp or spasm, great restlessness, and a high degree of fever may for some time precede the paralysis.

The following case will illustrate this form of attack:-A grey cart gelding, six years old, in previous good condition, and at regular work, was observed to perspire profusely, to breathe quickly, and to express signs of great restlessness; the hind feet were alternately lifted from the ground with great 
violence, the great muscles of the thighs were violently cramped, and such was the degree of pain that it became almost unmanageable. It was led home and placed in a loose box, where it lay or almost fell down. For a while it seemed relieved, but after a time commenced to struggle violently, and at last managed to regain the standing posture. The cramp of the limbs returned; after an interval it again lay down, and when first seen by me it was struggling violently, breathing heavily, and groaning with pain; the pulse about ninety, and full; mucous membranes injected, and the body bedewed with sweat. It again struggled to its feet, passed some rather high-coloured urine; remained quiescent for a short period, and then the great struggle again commenced. It was placed in slings, into which it threw its weight, and gave a sigh of relief; after an interval of about fifteen minutes it commenced to struggle violently, its hind legs were dreadfully cramped, and we were obliged to lower it down on to its bedding, when it again seemed to receive relief. It was bled and a purgative with belladonna administered, the loins and back were fomented witl hot water, mustard applied, and afterwards covered with a sheep-skin. In a while, however, it commenced to struggle afresh, and we were compelled to raise it by pulleys and slings. For two days and two nights it thus remained, now and then free from pain, then in dreadful agony; it seemed that all movement hurt it dreadfully, every jerk and start causing it to groan and to jump as it were in agony. At first the muscular convulsions were confined to the posterior extremities, but the fore limbs became after a time affecter. The poor horse seemed to dread moving its limbs, but a sudden start would ensue, and all its limbs would move convulsively and with great force. We were compelled alternately to sling and let it down, for in no posture could it obtain ease for more than a few minutes at a time. On the third morning the spasms left it, the bowels responcled to the purgative, the respirations became slower, but the pulse was feeble, thready, and greatly accelerated, beating about 130 per minute. It could now stand for some hours, but if made to move it did so with great difficulty, dragged its hind limbs, staggered, and was inclined to knuckle at the fetlocks; the off fore limb was also partially paralyzed, and was moved with difficulty. When last seen by me it was standing with its head over 
the box door, and looking pretty cheerful, had drank some gruel and eaten a little hay, and I was in hopes it might rally; but shortly afterwards it fell completely paralyzed, lost both motor power and sensitiveness; there was no struggling now, it lay passively on its side, looked quite conscious, and died without a struggle. A post mortem examination revealed the spinal cord and meninges highly congested, and the subarachnoid space filled with serosity. The dorsal, lumbar, gluteal, and the muscles of the thighs, as well as the serrati magni, levatores humeri, triceps extensor brachii, and the other muscles which had been violently cramped during life, presented a dark, congested appearance.

The spinal congestion was most marked in the dorso-lumbar region, and both roots of the spinal nerves, and the nerves themselves for some distance, presented the same appearance. The arachnoid, pia mater, and dura mater were intensely red; the substance of the eord looked reddish, and on cutting through it black blood exuded abundantly from its centre.

The other form of spinitis is that manifested by sudden loss of power, withont previous irregular muscular action, and may be appropriately termed inflammatory paralysis; for, in addition to the paralysis, there is fever, constipation of the bowels, diminished secretion of urine, and other signs of constitutional disturbance. This form is apt to be confounded with fracture of the vertebræ, more especially of the lumbar vertebre, in which generally the paralysis is complete immediately after an accident. Whereas, when one of the dorsal vertebre is fractured, displacement may not immediately occur, displacement and paralysis occurring perhaps in a few hours after the accident.

The history of the case will have to be taken into consideration in arriving at a diagnosis. Broken back generally suceeeds a traceable accident, acute spinitis without any appreciable cause.

The first form of spinal inflammation is apt to be confounded with azoturia. The dark colour of the urine which characterises that disease is absent in spinal inflammation, and the spasm, which in azoturia is mostly confined to the gluteals, affects various muscles, and is of a more clonic or alternating kind. 
CHRONIC SPINITIS.

This again presents itself in two forms, namely, that associated with tonic spasm of the voluntary muscles, and that associated with paralysis of particular muscles.

Chronic spinitis associated with exalted muscular contractility is in some districts termed the "cords," from the fact that the superficial muscles have a prominent or corded appearance.

The symptoms are at first obscure. There is some degree of stiffness about the spine, the animal turns with difficulty, and often groans when made to do so suddenly. Some particular muscle is now seen to stand out from its fellows, and present a permanent rigidity and hardness. Very often the muscles of the shoulders or the levatores humeri and cervical muscles are first affected; but gradually the whole body becomes more or less stiff, the limbs are flexed with difficulty, the nose elevated, and the animal seems as if affected with chronic tetanus; the facial muscles, however, remain normal, and the animal continues to feed well. After the lapse of some weeks, the lind limbs become feeble; there is knuckling over at the fetlocks, crossing of the feet, staggering gait; the animal seldom lies down, and rises with great difficulty, and eventually becomes paralyzed, and has to be destroyed.

In the other form the first symptoms are, staggering gait, weakness of the hind legs, crossing of the feet, and a gradually increasing loss of motor power, until finally the animal becomes powerless, unable to rise from the recumbent posture, and has to be destroyed. In none of these cases-acute or chronic-do we find that the sphincters lose their power of contractility, nor is common sensibility lost. Indeed, in the acute form, I have often thought there was hyperesthesia, and that the animal dreaded the touch of its attendant. These conditions indicate that the meninges are the principal seat of the inflammation.

The post mortem. - The spinal cord is red, injected, and softened(red softening), the meninges thickened, the arachnoid covered with an exudate, which in some places joins its opposed surfaces, or is filled by a semi-purulent or reddish serosity. In one case, which died during very cold weather, the fluid, being frozen, after death presented a very beantiful appearance.

Idiopathic spinitis differs from that arising from external 
injuries or disease of the bones, in which the dura mater is inflamed on its adherent surface; the areolar tissue uniting it to the spinal canal is loaded with venous blood, or sometimes broken down, and the dura matter separated from its attachments.

The causes of idiopathic spinal inflammation are very obscure. Very probably it is often due to rheumatic inflammation localized in the membranes primarily, the cord itself becoming secondarily aflected. In Principles and Practice of Vcterinary Surgery, page 248, I have pointed out that hereditary tendency is a cause of spinal affections.

\section{TREATIFAT.}

The acute forms are to be treated antiphlogistically; blood may be withdrawn and purgatives administered; depletives must not, however, be pushed too far, as acute spinal diseases have a lowering or debilitating effect upon the system. The purgative may be combined with belladonna, which will to some extent relieve pain and spasm, as well as overcome the congestion of the spinal vessels. Hot fomentations to the back and loins are indicated in the earlier stages, and blisters, or even setons, should there be but a partial recovery. It must be remembered that a continuance of the symptoms, after the acute stage has passed off, depends upon some degree of effusion or exudation, and that means must be taken to promote absorption. On this account iodide of potassium with diuretics is inclicated. Ergot of rye (Sccale cornutum) in combination with the iodide of potassium is well spoken of by physicians, and may be tried in the lower animals.

In all cases great care must be taken that the urinary bladder be regularly emptied; and if micturition is not naturally and easily performed, the catheter must be used at least thrice a day. If the animal is recumbent, this should never be neglected. The attendants must also be directed to turn the patient every now and then, see that enemas are regularly administered, and that the bed is kept clean and dry. 


\section{CHAPTER XLV. \\ SPORADIC DISEASES-continued.}

LOCAL DISEASES-continued.

(H.) PARAlysis.

PALSY or paralysis may be seen in various forms rather as a symptom of a lesion than as a disease itself, the term palsy or paralysis being commonly restricted to that form where motion is lost, while the term ancesthesia implies a palsy of the nerves of sensation.

In order to understand the various lesions which may cause paralytic symptoms, it is advisable that a glance at the physiology of the spinal cord should now be undertaken.

1. The spinal nerves have two roots; and Sir Charles Bell proved that the anterior (inferior in the lower animals) conduct the power of motion, whilst the posterior (superior) are devoted to sensation only. At one time it was thought that if the corresponding columns of the cord were cut or injured, loss of sensation only would result when the superior columns were cut, and loss of motion if the inferior were cut across; but such is not actually the case, for it has been demonstrated that if the posterior columns are cut across, the result is not numbness and insensibility, but hyperæsthesia and loss of co-ordinating power in the parts posterior to the section, with some local pain, due not to any sensitiveness in the columns themselves, but to the cut having traversed through the posterior roots of the nerves. The same results are obtained if the restiform bodies and small superior pyramids are divided, and as these parts are connected with the cerebellum, and the superior columns of the cord, the deduction is, that the channel which 
conducts the action of the cerebellum upon the body is composed of the restiform bodies and small posterior pyramids in the anterior part, and of the superior columns of the cord in the posterior part of its course.

Seetion of the inferior columns, if made immediately behind the medulla oblongata, is not followed by any very obvious loss of paralysis or loss of sensation, unless the cut be extended to the lateral column, when loss of motion is the result, as if the columns were divided behind this point. It is therefore apparent that in the anterior part of their course the inferior columns have not an intimate connection with the corresponding roots of the spinal nerves. Posterior to this, however, they lave a close connection with the inferior roots of the spinal nerves and with voluntary motion. They have also some conneetion with sensibility, as a certain degree of numbness is produced by injuries which give rise to loss of motion.

Inferior pyramids.-Section of one of these pyramids in any part of its course is followed by immediate loss of voluntary movements in the muscles below the cut on the opposite side of the body; but sensation is not affected in any appreciable manner.

Lateral columns. - In the cervical region, for a short distance behind the point at which the inferior pyramids of the medulla oblongata decussate, the lateral columus of the spinal cord are directly concerned in conveying the stimulus of the will to the muscles on the same side of the body, for when they are cut across paralysis of the muscles of the same side occurs.

In the lower part of the neck, and regions posterior to this, the same results are not seen; for when they are divided in these regions, some trifling paralysis ensues, with some degree of anæsthesia and loss of co-ordination.

Olizary bodies.-When one of these is divided, a persistent spasm of many muscles of the same side is the result.

Grey substance of the cord.-Dr. Brown Sequard says that the grey substance of the cord is an important conductor of sensory and mutor impressions. Paralysis, without loss of sensation on the same side of the body, are the results of cutting across one lateral half of the grey substance of the cord. Anæsthesia on both sides of the body, paralysis of neither side, are the strange results of making a longitudinal section midway between tha two lateral halves. 
The superior half of the white matter may be divided at one point, and the inferior half at another a little anteriorly, so that all the white fibres shall be divided transversely by the one cut or the other, without any material continuity of the cord or clamage to the grey matter; and when this has been done, irritation of the sensory nerves connected with the parts below the section excites the sensation of pain as strongly as ever. Hence it follows that the impulses which excite pain reach the brain through the grey matter, and so long as a small portion of the grey matter remains intact, these sensations are transmitted.

If one-half of the cord be eut through transversely down to its very middle, so as to interrupt all continuity of both white and grey matter, irritation of the skin of the same side will give rise to as much pain as if the cord were not cut, but all voluntary power will be lost in the museles of that side below the section. It thus follows that the channels which convey sensory impulses must cross over from the side of the cord which they enter to the opposite side, and that the motor influences sent down from the brain must travel along that side of the eord by which they pass out.

There is increased temperature and sensibility on the side in which sensation is preserved, and diminished temperature on the side in which sensation is lost, especially if the section is made near the medulla oblongata.

It would seem that the injury acts upon the vasa-motor nerves contained in the cord, as well as upon the motor and sensory nerves (it may be here stated that the vasa-motor fibres do not arise from the sympathetic ganglia, but simply pass through them on their way from the spinal cord to the upper dorsal region), eausing paralysis of the vasa-motor nerves on the side in which there is increased temperature and sensibility, and irritation of the vasa-motor nerves on the side in which there is diminished temperature and anæsthesia; for the experiments of Dr. Brown Sequard, Claude Bernard, and others have proved that when the cervical sympathetic is paralyzed by dividing it, a state of congestion, of which the most conspicuous signs are a blood-shot state of the conjunctiva and lining membrane of the ear and nostril, with a contracted pupil, and increased temperature, is at once set up on the same side of the head, and also 
that when the end of the divided nerve is irritated, the immediate result is dilatation of the pupil, with an immediate cooling and blanching of the parts which were blood-shot and warm.

Injuries to the cervical portions of the spinal cord are followed by a remarkable elevation of the animal heat. In one case of injury in a man where there was a forcible separation of the fifth and sixth cervical vertebrae, with extravasation of blood into the theca vertebralis and laceration of the lower part of the spinal cord, although the breathing was performed by the diaphragm only and very imperfectly, the pulse weak and countenance livid, the thermometer indicated $111^{\circ}$ F.-(Iuxley, Brown Sequard, Claude Bernaid, Dr. Ratcliffe.)

Such, then, are a few facts which serve as indications of the seat of spinal lesions.

In all cases of clisease or injury to the cord the paralysis oecurs in the parts to which all the nerves originating posteriorly to the seat of the lesion are distributed. If the injury or disease be in the sacrum the tail alone is paralyzed; if in the lumbar region the hind legs are paralyzed; and if the disease or injury be very great the sphincters of the anus and bladder may be paralyzed. If the injury be higher up, in addition to loss of voluntary power in the hind limbs, the abdominal muscles will be paralyzed, and the expiratory movements performed with difficulty.

If the disease be low down in the cervical region, the muscles of the fore limbs, as well as those of respiration, will be paralyzed, and both expiratory and inspiratory movements will be difficult; and if the injury be above the middle of the neck, or anterior to the fourth pair of spinal nerves, death will at once result from paralysis of the diaphragm, the serrati magni, sealeni, intercostales, and other muscles of inspiration.

Paralysis as seen in the lower animals may be divided into1st. Paraplegia; 2d. Hemiplegia; and $3 d$. Paralysis originating in injury to a motor nerve, as paralysis of the lips, described at page 496 of Principles and Practice of Veterinary Surgery.

\section{PARAPLEGIA,}

Or Paralysis affecting a portion of the body transversely.-Of this kind three forms are met with in veterinary practice, namely 
-1st. Centric, or that arising from disease of the spinal cord or its membranes. Symptoms simulating paralysis may occur from occlusion of the iliac arteries.-(See Veterinary Surgery.) $2 d$. Eceentric or reflex paraplegia; and $3 d$. Paralysis due to blood poisoning.

Of centric paraplegia but little need he said; it is symptomatic of conditions of the spinal cord and its membranes already deseribed, or it may result from injuries to the cord arising from violenee, fractures, \&c.

\section{REFLEX PARAPLEGIA,}

In horses, cattle, and dogs, spinal paralysis is met with in which no disease of the eord or its meninges can be detecter post mortem in those which die, and which, in many cases, is quite ephemeral, passing away when its cause is removed.

The causes of reflex paralysis as seen in the horse are those originating in indigestion, constipation of the bowels, and, in the mare, uterine irritation.

Paralysis arising from indigestion is oceasionally witnessed during attacks of colic, the loss of power coming on rather slowly; the animal is at first seen to show weakness of one hind limb, with knuckling over at the fetlock, and difficulty of moving it, then the opposite limb may become affected; and if the loss of motor power be great, the animal falls upon its haunches, and is unable to rise. If it be a docile animal and will lie quietly, the paralysis may disappear in a few hours, or leave only such traces as weakness of the limbs and staggering gait, which may take some days to pass away.

In mares, during the period of ostrum, I have repeatedly seen eases of paraplegia, generally preceded by some irregular muscular aetion; persistent tonic spasm of the muscles of the lind quarters, continual kicking with the hind feet, with great injection of the vulva, succeeded by loss of motor power, and even death.-(See Hystcria.) In dogs, paralysis, even affeeting all parts of the body except the heal, is not an infrecuent result of the irritation of teething, indulgenee in rich food, and want of exercise. In horned cattle paraplegia is not an infrequent symptom of indigestion, arising from impaction of the rumen, und fiom post-parturient nterine irritation. I have alrealy men- 
tioned a case of general paralysis succeded by congestion of the brain and death in a goat, arising from grief at the loss of her kids ; this may justly be called emotional paralysis.

The differences existing between reflex paralysis and that from disease of the cord is found in the circumstance that recovery is not at all an infrequent result, and that when death occurs either from the gravity of the primary disease, restlessness of the animal, or other circumstances, no spinal post morten lesions are discoverable.

It is supposed by Dr. Brown Sequard that reflex paraplegia is not due to spinal congestion, but to a condition diametrieally opposed. He believes that a state of irritation, commeneing eccentrically, is propagated along the vasa-motor nerves, of which the result is, primarily, contraction of blood-vessels in, and, secondarily, exclusion of the due amount of blood from, one or more of the three parts following-the spinal cord, the nerves proceeding to or coming from the cord, the inuscles, and that the proper activity of the nervous tissue is starved into paralysis from want of blood. This view is founded by Dr. Brown Sequard upon the fact that a state of irritation in the vasa-motor nerves may proceed from a distant point, and prochee contraction of the ressels, and upon the fact that traces of organic disease are wanting after death in many cases of reflex paraplegia.

The following are among the causes of reflex paraplegia in the limman being:- Irritation of the urethra; inflammation of the bladder; diseases of the prostate and kidneys; enteritis; the presence of worms in the intestines; dysentery; diphtheria ; diseases of the lungs and plemra the irritation of teething; irritation of the cutaneous nerves generally following cold and wet; and diseases of the knee joint.

Four different conditions of the muscles are observed in paralysis in the human being, some of which are also observable in the lower animals:-(1.) A condition little different from that of health, but less firm, less excitable by the galvanic stimulus, when the paralyzing lesion is not of an irritative kind. (2.) Complete relaxation of the muscles, characterised by imperfect nourishment and rapid wasting-so rapid that in a few days the size of a limb experiences a marked diminution. Such museles scarcely, if at all, respond to the galvanic stimulus. (3.) Contraction of the muscles, with rigidity and wasting (the 
flexors being always more rigid than the extensors), a condition which is due to chronic shortening of the muscles themselves, and generally associated with muscular atrophy. (4.) Nutrition not impaired, constant firmmess and rigidity, incomplete paralysis, increased susceptibility to galvanic stimulus.

In the treatment of reflex paraplegia the removal of the eccentric cause must always be the primary object of the practitioner. In the horse, if there be colicky pains and constipation, opium and purgatives, sncceeded by belladonna, and an alkaline carbonate; the soda biearbonate in all cases arising from indigestion. If congestion of the cord is suspected, belladonna or the ergot of rye are indicated. Generally, however, it is found that in the lower animals reflex paralysis is ephemeral, and passes away when its cause is removed.

In the conv the stomach and bowels are to be unloaded by strong catliarties. Sulphate of magnesia, with croton, if there be no actual inflammation of the stomachs; oleaginous purgatives, with opium, belladonna, or hyoscyamus, if inflammation is suspected.

In the doy reflex paralysis is often accompanied by great irritation of the stomach, vomition, and intestinal pains. Castor oil and hydrocyanic acid are the most useful agents in affording relief. If the stomach be too irritable to retain the oil, the hydrocyanic acid is to be given alone, or in combination with carbonate of soda, until undue irritability is allayed, then the aperient is to be administered. Iodide of potassium and the soda earbonate agree better, and are more useful in the dog than all other remedies after the aperient lias operated. If the disease be dne to decayed teeth or the irritation of teething, relief must be afforded by extraction of the teeth or lancing of the gums. In all animals cathartics, enemas, fomentations to the loins, hot baths for the dog, and emptying of the bladder, where possible, are not to be lost sight of. If paralysis continue for a time, nux vomica and ferruginous tonics may be prescribed advantageously, and the loins may be blistered, and galvanism applied.

\section{HI:MIPLEGIA.}

Hemiplegia is that form of paralysis in which one lateral half of the body is affected. It is a very rare form of clisease iu the lower animals. I have, however, seen it in the horse, 
and Mr. Mahony, V.S., Templemore, in a letter to me, December $1 S 7 S$, describes a well-marked case. I have also seen it in the cow and in the dog.

M. Girard, Jun., has left a case on record, quoted by Mr: Percivall, which bears out many points in the physiology of the cord already referred to. He says- "The sensibility of the left -the affected-side proved extremely acute. The lips and alie of the nose were drawn to the right side, the contrary to that to which the head and neck turned. The occlusion of the nostrils was such that the air made a blowing noise in its passage through them. The left ear was palsied, and the tongue slightly distorted. The lips and nostrils retained their sensibility, though in a diminished degree to what it was on the unaffected side. When oats were laid before the horse, it seized them with the right side of the mouth, the left remaining motionless. It experienced a great difficulty in mastication, and succeeled only in swallowing a part of its food, the remainder staying behind, lodged between the cheek and molar teeth. It could manage to pick up its oats from a plain surface, and when presented to it in a trough, it plunged its muzzle into the middle of them, opening wide its month. It could drink but slowly, and with difficulty, and only by thrusting its mouth deeply into the water. The nostril of the affected side perceived scents. It could walk, lut could hardly sustain itself after but a short exercise. If attempted to be turned to the left side, this instability became still more manifest. Pressure upon the vertebral column from the head to the tail seemed to give great pain. The respiration, although sonorons, was regular. On the fourth day, the animal, unable any longer to stand, sank down, and after several turns and ineffectual struggles to rise, rolled over and lay upon its right side. Its bowels were relieved by manual operation, its bladder with the catheter; though even after this it passed its dung, but conld not posture itself to pass its urine. Its pulse, like respirntion, remained undisturbed. It died on the seventh day."

In the case of the cow the attack was of an acute kind. The animal was grazing in a field with a lot of others, and was left quite well at milking time in the morning. The field being some distance from the house, it was not seen again before evening. It was then fomd prostrate on the ground, lying upon its left side, and was mable to rise. 
When I saw it I observed the following symptoms:-The left ear was pendulous, left eyelid drooping and closed, the eye squinted outwards. The left lip hung down, the angle of the mouth was lower than on the opposite side, the muscles were loose and flaccid, and the lips drawn to the right side. The tongue protruded, and when put into the mouth was drawn to the right side. The neck was twisted and the head drawn to the left side. Deglutition was imperfect, but the breathing was not appreciably affected. The superficial blood-vessels of the right side were engorged with blood, and stood out prominently all over the trunk and neck. The same side was warm, whilst the opposite side was cold, and the hair was "pen-feathered."

In endeavouring to place the animal on a hurdle for the purpose of removing it to a shed, it was observed that it rolled over from the left to the right side, on which side only could it be made to lie. The bowels were constipated, and the belly tympanitic, the sphincter ani was rather relaxed, the vulva flaccid, and ragina protruding. The animal was quite conscious, but inclined to somnolency. It was bled, a purgative administered, and the back was fomented and dressed with strong ammonia liniment. On the following morning it was able to rise, but both the legs of the right side remained partially paralyzed for some weeks. The affection of the face, however, passed away during the first night, and it was able to partake of food on the following morning.

This was a case of hemiplegia due to some ephemeral disease of one side-the left-of the brain; and from the fact of the outward squinting, amaurosis, and drooping of the upper eyelid, the conclusion is, the functions of the third nerve being interfered with, that the brain disease-ephemeral as it wasinvolved the posterior part of the left crus cerebri. It must be remembered that, in all cases of disease affecting one side of the brain, the paralysis is on the opposite side of the neck and trunk, but on the same side of the face. It was remarkable however, in this case, that although the loss of motor power was complete, sensibility remained intact.

Paralysis from blood poisoning is witnessed in azoturia, lead poisoning, canine distemper, and grass staggers. In the human being, as well as in horses, cattle, and birds, a form of paralysis arises from the use of the vetch known as the Lathyn'us sations. 
-(See Lathyriasis, page 4S\%.) The eanine distemper poison leads in many instances to softening of the cord, in which its substance is brokeu down and softened almost to a pulp. In recent cases the softened cord is red or yellowish-red in appearance, due to some degree of aecompanying congestion. In the more ehronic eases, or those which have lived for a longer period, the red appearance is absent. White softening may, however, occur quite independently of the red or inflammatory form, and is due to cedema or effusion of serum. I have repeatedly seen not only softening but apoplexy of the cord occur as a sequel to eanine distemper, and in which the neurilemma of the roots of the nerves were filled by a elear fluid material, and quite devoid of true nervous matter. In softening, the grey matter of the cord is always more affeeted than the white. I have never seen the converse condition of the cord, namely, hypertrophy, nor induration of its substance. The treatment is the same as that for paraplegia, and in the case of a valuable animal galvanism should be tried for a prolonged period. Young sheep grazed on newly limerl land are often affeeted with spinal paralysis.

\section{LOUPING-ILL, OR TREMRLING.-(Ixodic Toxcmire. $)^{1}$}

LoupING-ILL.-The term "louping-ill," supposed to be deriver? from hloupa, an old Scanclinavian term for "a staggering gait," as applied to the disease, is general in some parts of Scotland, whilst in others it is known by the term "trembling." It seems to prevail more in the Silurian lill districts of Scotland, and extends from the Hebrides to the sonthern extremity of Dumfriesshire, being equally prevalent in the district of Langholm as in the isle of Skye. There is this difference, however, namely, in the southern parts it rarely occurs to any extent but cluring the early summer months, eommencing about the middle of April, and disappearing before the end of June; whereas in Skye there are two annual outbreaks,-one in early summer, and another in antumn, commencing at the end of August, and terminating in a month or six weeks.

It is stated that it affects, but to a slight extent, cattle, horses, and pigs. I have seen well-marked cases in horned eattle, but never in horses and swine.

Symptoms.-The symptoms, as observed during my investigations in 1881 and 1882 , were as follows:-

${ }^{1}$ Condensed from Reports to the Highland and Agricultural Society of Scotland, 1882-97. 
No. 1.-A three-year-old ewe was seized on the 18th May, just before giving birth to a dead lamb. When seen on the 19th she was standing with head erect and brilliant eye, the upper muscles of the neck spasmodically contracted, eausing the nose to be protruded as in tetanus. When made to move, she did so very stiflly and automatically, the limbs being lifted very ligh, aurl the feet planted on the ground in a peculiar jerky manner, like a horse with stringhalt. There were tonic or persistent spasms of the muscles of the loins, back, and neck, and clonic or intermitting and irregular spasms of the other muscles, particularly those of the limbs; the breathing was very jerky, quick, and irregular, being from fifty to sixty per minute when quiet; pulse 102 , and temperature $105.5^{\circ} \mathrm{F}$.

On being exeited the ewe fell prostrate on the belly and chest, and lay with head and neck extended; on being raised, walked away trembling violently, the trembling being due to alternate contraction and relaxation of the muscles.

On examining the skin three kinds of ticks were found, but they were not very numerons.

She was killed, and on making a post mortem examination of this ewe, nothing very special was discovered; the flesh was pale, and the carcase thin, but there seemed to be no abnormality of the cord or spinal canal, beyond a slight inerease of the spinal fluic. There was no jelly-like exudate on the cord, nor any congestion of the cord, or of the membranes.

No. 2.-Another ewe was recovering. She had trembled for a day, then became paralyzed, and went down and lay three days, but was then up and walking about, but in an unsteady semi-paralyzed manner.

No. 3.-Ewe hogg; had been ill four days; was not observed to tremble, but found down, paralyzed. Was in good condition; in fact almost fat. Lay quietly on the right side; eyes open, no dilatation of the pupils, nor any squint. When suddenly approached, or otherwise disturbed, was very frightened, and struggled violently. When hind leg was lifted up and allowed to fall there was no attempt at motion, but when the fore leg was lifted in the same way there was a to-and-fro motion before reaching the ground.

When raised, and held up by hand, it could move its fore limbs pretty freely, and could stand and bear weight upon them; 
lut the hind ones remained extended backwards, eompletely paralyzed, and the muscles of the hind quarter were clonically convulsed.

Sensibility was dininished in the hind limbs, but there seemed to be some degree of hyperasthresia in the fore ones, as these moved very rapidly and violently when pricked. The sensibility of the trunk seemed to be normal.

The temperature was $105.5^{\circ} \mathrm{F}$., pulse 106 , and respirations, when not disturbed, 30, but when excited the breathing became very rapid, jerky, and convulsive. The post mortem examination revealed nothing very partienlar in the spinal canal, brain, nor in any other organ. The flesh was not at all pale or ansmie, as in the majority of the cases examined, and fat was abundant and very healthy looking.

During 1881 abont three score of sheep and lambs were examined after death, and about one-third of these were considered to have had "louping-ill," whilst others were found to have had-(1.) "Joint-ill" (smppmrative arthritis); (2.) Anamia, from pure starvation; (3.) Impaction of the stomach with wool in young lambs; (4.) Abscesses in various parts.

In one lamb, sent in from Dumfriesshire in 1882, there was a downward squint in both eyes even when in repose, but in others there was no squinting unless under excitement, when, as in the first case described, there was often a convulsive motion in both eyes.

We were informed that many sheep seemingly in good health often made a jump and fell down, some dead, some eompletely paralyzed, when suldenly frightened or approached by a dog, and that there were very often no other indications of the disease.

Causes.-These are ascribed to a variety of external circumstances, such as soil, pasture, geological formation, altitude, weather, and the influence of ticks.

I. Influcnce of pasture, geological formation, and altitude.The late Mr. Brotherston, Kelso, who made an extensive examination of the geological formation of lomping-ill districts, came to the conclusion that it is found on very different soils, geological formations, altitudes,-close to the sea, as in skye, and on soils and formations so far apart as the Lower Silurian, the Upper Silurian, the Old Red Sandstone, through the Carboniferons to the Basalt of the Tertiary period, and that it existed equally 
upon all these, provided old grasses were allowed to remain on the ground. (By old grasses is meant the growths of the last and previous years.)

Mr. Brotherston was the first to promulgate the opinion that the canse of the disease was contained in the withered grasses, as these grasses were extensively covered with a variety of micro-fungi; and that these fungi acted upon the animal body in a manner similar to that induced by ergot; that, in short, "louping-ill" was a species of ergotic intoxication or nervons excitement. This was a very reasonable conclusion, for, as before stated, the disease was not found unless portions of the ground were more or less thickly covered by grasses of last year's growth, all of which were covered with micro-fungi.

It was important to set this question at rest, and on 9th May 1882 four one-year-old sheep were obtained from a district in which lomping-ill was mnknown, and were fed at the College up to the 15th day of June, thirty-six days in all, upon these withered grasses, carefully collected by Mr. Brotherston in the Hawick district. After that, six ewes and lambs were added to the number, and fed upon the same grasses, and remained quite free from disease. Later on, the grasses were mixed with ergots, but no effect was induced beyond a slight loss of appetite and some degree of purging.

By introducing a small portion of fungus-covered grass into a cultivation fluid, long thread-like filaments conld be grown, but these differed from what is to be hereafter mentioned, and the above experiment almost conclusively proved that neither the grasses nor the fungi then seen were capable of directly inducing the disease.

Doubtless animals fed mpon such poor food, particularly if compelled to range over the hills in search of it, are rendered feeble and incapable of resisting the influence of disease-exciting canses, and in consequence the mortality is thus rendered much greater than where the pastures are richer and more nutritious.

II. The influenee of the weather.-The minority of those acquainted with the disease hold the opinion that a sudden change of weather of any kind, whether from good to bad or from bad to good, is sufficient of itself to induce the disease; whilst others maintain that it appears only during the prevalence of cold east winds. 
These opinions are entitler to some consileration, as it is an actual fact that the mortality is very much greater after a sudden change, particularly-and in every instance that came under observation during the investigation-a change from warm to cold and inclement weather.

But neither change of weather nor exposure to its inclemency are of themselves sufficient to induce the disease, nor does the latter in any of its phases simulate an ailment brought about by elimatic influences; and the only conclusion that can be deduced from the above premises is, that inclement weather, by its debilitating influences, predisposes to disease, and canses many animals to suceumb to what otherwise might have passed off without very grave consequences.

One flockmaster observed that he believed the great majority of the sheep in an infected district had the disease once in their lives in greater or less severity during the scason; that if the food were good and the weather favourable, but few beeame seriously ill, and fewer died; but if the weather became suddenly cold or wet, the disease was able to make a deeper impression upon the animal body, and many then succumbed; and further, if the disease were due to the weather, why was it confined to certain districts, whilst in others equally cold and exposed it was entirely unknown?

Being satisfied that the grasses, geological formations, and weather were insufficient to cause the disease, one other proballe somrce of it remained to be considered, nanely-

III. The influence of ticlis (Irodes).-The belief is general amongst all acquainted with louping-ill that it is closely allied to ticks; the majority of farmers, indeed, believing that the parasites, by fixing themselves in the skin and sucking the blood, cause such extensive irritation, pain, and weakness, that the animals perish from these causes, and that the convulsive spasms and paralysis are merely symptoms of irritation and debility. A very brief acquaintance with the disease, however, compelled the anthor to conclude that, no matter how elosely allied the tick and the disease were, irritation and loss of blood had little or no connection with the disease under consideration; for in many instances but few of the parasites were found on some of the diseased sheep, whilst many healthy ones were swarming with them, and seemingly feeling but little dis- 
comfort. It was, however, noticed that when they were very numerous on lambs, such lambs presented the disease in an aggravated form, as if the dose of the disease-inducing material had been a strong one. The belief in the influence of the tick, or its close commection with the disease, being so general, it was necessary to consiler this point very carefully, with the view of determining if any relationship existed between the parasite and the disease. The result of observations on this point was confirmatory of the popular idea; for it was found-1st. That where louping-ill existed there the tick was sure to be : 2 $九$. That where the parasites were absent there was no louping-ill; and it is a remarkable fact that upon ground having a westerly or souther'n aspect ticks and louping-ill prevail, whilst on arljoining grounds with easterly or northerly aspects, upon which the grasses are short, thus providing no cover for the ticks, the disease is rare: $: d$. That the appearance of the parasites on the sheep in April and early summer was concomitaut with the annual ontbreak of the disease: $4 t h$. That in Skye, where it is

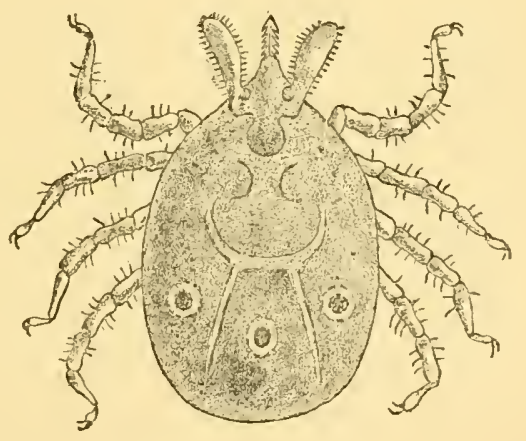

FIG, 29.-The Tick.

said the disease appears both in spring and autumn, the tick being also there in autumn as well as in spring.

Ticks are occasionally found on land free from louping-ill. From this it must be concluded that the parasites are the conveyers or inoculators of a virus. By improvement of the land by ploughing and liming, by artificially feeding the sheep, and destroying or otherwise disposing of old grasses - the natural 
covers of the tick, as will be shown hereafter-louping-ill has been entirely eradicated.

The tick is a true blood-sucking parasite, belonging to the family Ixorlicle, the class Archnide, and order Acarince. The mouth of the tick is provided with a serrated beak or rostrum, which enables it to pierce the skin, and retain its hold very firmly, and almost without effort, as the barbs or serrations point lackwarts.

The species are very numerous, liut the best known are the Ixodes ricinus, dog-tick, the Ixodes reduvius and Ixodes plumbens, sheep and cattle tick. Those found during the investigation were recognised by Mr. Moore, British Museum, as Ixodes rieinus, crinaceus, and marginatus.

During this investigation we found ticks upon every variety of soil, and at different altitndes-close to the sea level in Skye, and at a height of 2000 feet in the inland districts; in fact they will be found as high as sheep will go, proviled always old grasses are abumlant to afford cover, as they are thus protected from injury, and are left at comparative rest during the periods they are not obtaining blood from the animals which they attack. Prompted by humger, they quit their hidingplaces, and attack any animal that may come in their way, by plunging their beaks or sucking proboscides deeply into the skin, particularly about the face, root of ears, lower parts of breast, between the thighs, in fact into those parts of the skin covered more with fine hair than wool.

Thus fixed, they will hang for days together sucking the blood, until their bodies become distended to eight or ten times their original size; they then (quit their hold, fall to the gromud, and lay their ova, which remain dormant until next spring, and, shortly after they are hatched, they most probably attack the sheep, and after they once bite remain firmly fixed in the skin.

It was a noticeable fact that when dead or dying sheep were examined the ticks were found alrealy dead, or in a dying condition, in almost every instance of louping-ill.

Rabbits, ferrets, stoats, weasels, and more rarely hares, are attacked by ticks, but there are no instances recorded of these animals becoming infected by louping-ill. This is probably due to these hosts proving unsuitable habitats for the development of the organism inducing "louping-ill," but horses are 
said to present symptoms of the disease during the loupingill season.

When they first leave the ground to attack animals they are very small, sometimes no larger than a pin's head, and when very numerous make the part quite black by their numbers, and they adhere so closely that scraping them off will tear up the skin.

Being satisfied that the parasites had a close connection with the disease (the proofs that they were co-existent upon certain lands, and simultaneous in their appearance, being overwhelming), but failing to see that they caused grave disease and death by inducing irritation and loss of blood to any great extent, it was necessary to discover if any organism existed in sheep dead from louping-ill, and if so, to trace its source of origin, indeed to demonstrate if such an organism was common to the tick and to animals affected with louping-ill. This was a matter of some difficulty, as in the majority of the animals examined after death no really characteristic lesions could be detected.

In many, all parts of the loody presented the appearances of health, death having been evidently due to intense nervous irritation.

In some, of course, it will be understood that there were signs of other diseases, but these in reality had no connection with the animal's death, and we are now referring to louping-ill pure and simple. In some instances there is a jelly-like formation within the spinal canal, sometimes extending in a more or less uniform layer from one end of the canal to the other, but rarely extending within the cranium. It is sometimes in patches here and there, particularly embracing the roots of the spinal nerves; generally there is a large patch in the lumbar region, then smaller ones scattered along the dorsal region; often a large patch at or about the junction of the cervical and dorsal, and very frequently a large and much redider patch immediately posterior to the foramen magnum, or entrance into the brain cavity. This jelly is seen both in lambs and in aged sheep in great perfection. It was first seen in sheep by me at Langholnı on 20th May 18S1, in a four-yearold ewe. I have also seen it in oxen, as well as in sheep and lambs. It is, however, not constant, even in cases that 
have been unwell for several days, whilst in others that have been down three or four days it is abundant. I make particular reference to this jelly, as some to whom I have shown it have asserted that it has no connection with the disease, that in fact it is a myxomatous condition of the fat surrounding the spinal corl, - a conlition, they say, not inconsistent with health in young animals; and that what I considered mycelia, the development of which within the boly being supposed to be an impossibility, were nothing more than capillaries, and the spores blood corpuscles.

Figs. 30 and 31. -Microscopic Vieiws of Gelatinous Mitter frour Spinal Canal containing the Organisy in its most advayced stage.

FIG. 30.

(Drawing by Dr. JAS. IIUnTER, 1882.

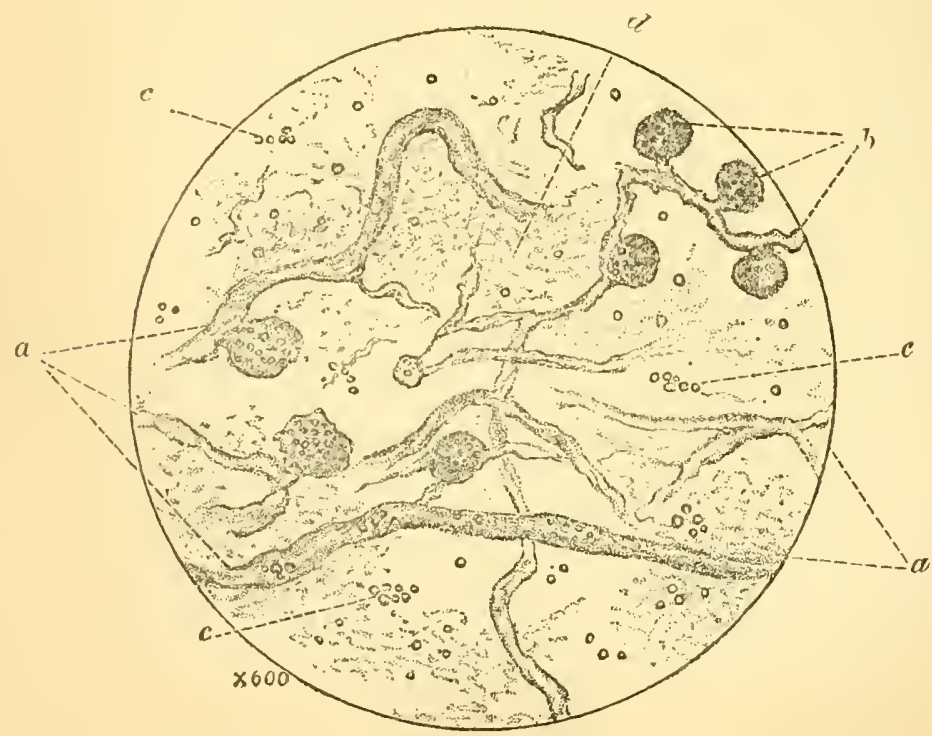

a. Spore-bearing filaments. c c. Free spores. b. Peculiar globular masses of spores. d. Micrococei or spores.

This jelly is a slimy tenacious substance, of a straw colour, and, as seen by the nakeil eye, lying external to the dura mater, and when examined microscopically is found to contain an organism, presenting the appearances seen in the illustrations.

Figures 30 and 31 have reference to the appearance seen 
in fresh specimens of this jelly under powers of 600 or 700 diameters, when the most characteristic structures are those to which I have applied the term mycelia, and which, as stated ahove, were considered to be capillaries by some savants to whom I had shown the specimens. At $\alpha$ in figures 30 and 31 are seen these filaments in various forms; they appear to consist of an extremely delicate limiting membrane (appearing better defined in filaments of medium size than in the larger ones) of highly refractive semi-fluid contents, and, at intervals, masses of spores of a still higher refractive power than the fluid in which they lie.

FIG. 31.

Another view of Fig. 30.

(Drawing by Dr. JAS. Hunter, 1SS2.)

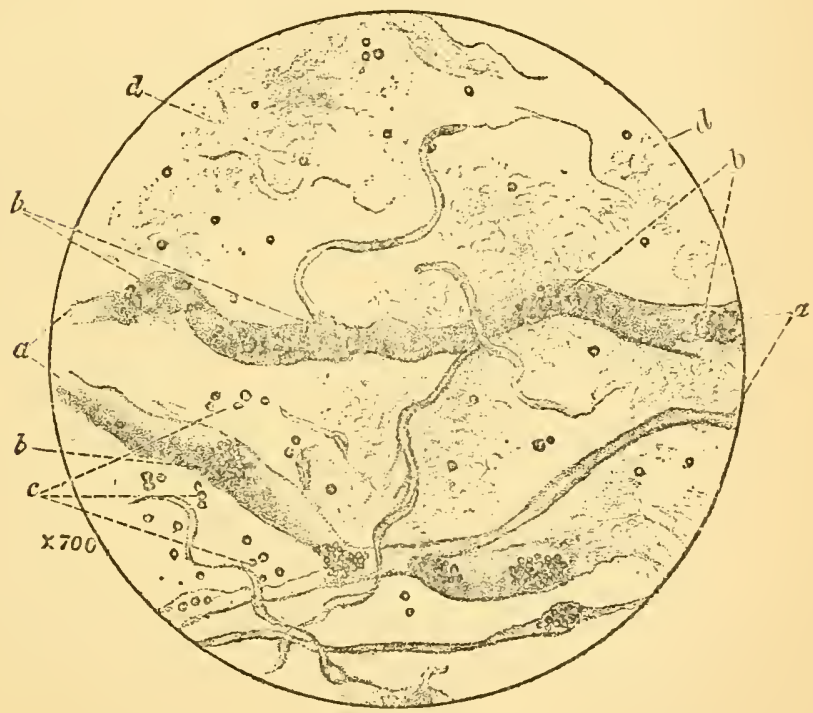

$a$ a. Filaments. $\quad b b$. The spore masses in the filaments.

$c c$. Free spores. $\quad d$. Micrococci or spores.

These spore masses are seen at $b$ in both figures, and at $c$ there are a few free spores ontside the filaments. Figure 31 shows the appearance I have most commonly observed, figure 30 represents a less common appearance, where they resemble berries upon shor't stalks.

As arguments against these mycelia or filaments being capillaries, I would point to their peculiarity of branching, the 
minute size of many of these branches, their behaviour on staining; and further, that the contents, instead of being shrivelled blood-corpuseles, differ very much from such bodies in their uniformity of size, smoothness of outline, and high refractive power; but, finally, the appearance of identical filaments in fluids from cultivation of the blood and spinal fluids of sheep affected with this disease sets aside completely any possibility of their being capillaries.

The fresh spinal subarachnoid fluid obtained from spines in which the jelly or muxa is absent, when dried on a glass slide and stained with methylaniline, is seen to contain a considerable number of bodies, like those marked $a$ in the illustrations, and some epithelial scales, but no motile or rod-like bacteria or bacilli. When a minute drop of this clear and fresh spinal fluid is cultivated in mutton broth, even at the ordinary temperature of a room, it gives rise to the following series of changes:-first, in about eighteen hours the fluid is seen to be more or less turbicl, and if examined microscopically under a good light, and with power over 600 diameters, it will be seen to be crowded with extremely minute organisms of a cylindrical or elub shape, with slightly romcled encls, these cylindrical rods being either free or attached in chains. Some of the rows are single, some double, and a few are triple or even quadruple.

The single free rorls are motile, and stain readily with methylaniline blue or violet, and when so stained are easily observed.

Figure 32, drawn from a specimen procured from a cnltivation in mutton broth of spinal fluid from an affecter sheep, shows these rods free and in several states of development, as well as masses of zoogloa with bacilli embedded therein.

A fow hours later, longer chains are developed, and rod-like londies having active motion are very abundant, whilst at other points distinct mycelia or spore-bearing tubes identical with those seen in the glutinous matter from the cord are visible, and masses of zoogloea with micrococci are seen scattered throughout the field,-figures 33 and $34, c \epsilon$.

To gain an irlea of the individual character of these rods, and also of their attachment in single or multiple chains, I refer the reader to figures 34 and 35 .

A free rod, as at $d$, figures $3 t$ and 3.5 , presents a some- 
what cylindrical outline, slightly rounded at the extremities, with a bright line (due to refraction) rumning down the centre, and slightly swollen out at either end. (The appearance of this line is less given by the drawing than are the other characters.) The middle part of each rod is a little constricted, but much less so than Bactcrium termo; its length is about midway between that of Bacterium termo and of Bacillum anthracis, and the rounding of its ends is also intermediate between these two, but more nearly approaching the characters of the latter.

FIG. 32.

Cultivation frou Spinal Fluid.

(Drawing by Dr. Jas. Hunter, 1852.)

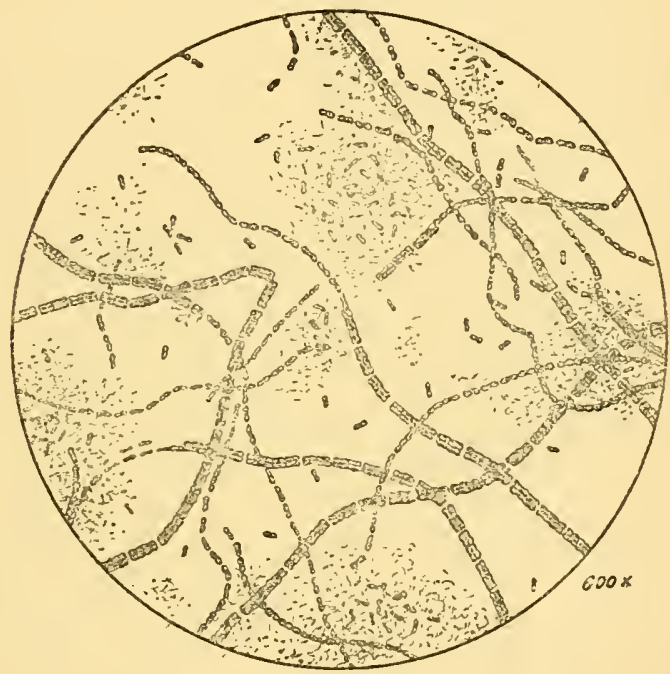

The character of the chains or rows of rods may be seen in figs. 32 to 35 , the former under 600 diameters, showing the general appearance of single and donble rows, the latter of double rows moler a ligher power (1200) and in various stages of detachment.

These chains present yery much the appearance secn in many low forms of regetalle life, only differing in point of size. Figure 32 shows that in the double rows, so long as the rods are in lateral contact, elear division lines mark the chains transversely at intervals corresponding to the lengths of two rods, and that also less clistinct cross lines are visible midway between these, marking off each bundle, as it were, into four. The single 
chains often present here and there an occasional filament in position alongside (see figures 34 and 35). In all of the figures are to be seen masses of zooglœe with embedded bacteria, as well as many free bacteria in the intervals. In figure 35 many of these seem to have been breaking up into débris (these are shown in fainter lines); incleed it was noticed that by allowing putrefaction to take place the appearance of the Bacterium termo was always rapidly followed by this result, and finally by the total disappearance of the bacillum under consideration. Perhaps more interesting than the appearance of the rods in all the cultivation fluids, whether from the spinal fluid or from the blood, is the appearance of these filamentous tubes marked $\varepsilon$ in figures 33 and 34 , as proving the identity of the organisms seen in the jelly from the spinal canal, and those developed from cultivation of fluids from the affected animals, as well from the bodies of sheep ticks, as will be seen from what follows.

An examination of the blood alone gave no very satisfactory result. Here and there, however, spore-like bodies were seen: these, however, were neither numerous nor constant; but by inoculating mutton broth with a minute quantity of fresh blood a result was obtained, but not in all instances, exactly identical with that resulting from the cultivation of the spinal fluid. In the course of from twenty-four to thirty-six hours the cultivation fluid was seen to be more or less turbid, and on the microscopic examination of a stained specimen free bacilli were seen in great abundance; a few were also seen in rows. On the third or fourth day mycelia, with spores in their centre or covering their walls, became visible, as well as masses of zoogloea with numerous embedded bacilli and micrococci, as seen in figure 33 .

The microscopic appearances of the jelly-like substance found in the spinal canal, and the development of the same mycelinm, and spores with motile bacilli from the blood, \&c., although not as yet clearing up entirely the life history of these organisms, showed that a new discovery had been made, and that the disease in question was due to the irritation of the nervous system through the spinal cord, in the fluids of which this organism seems to find a suitable nidus for complete development.

The experimental feeding of the sheep on fungoid grasses having given no result (p. 553), the anthor had but one resource left by which he hoped to determine whether the tick conveyed 
the disease to the sheep or not, and for this purpose ticks from diseased sheep were introduced into the cultivation ressels containing the mutton broth, and the results were most conclusive. In a few days the fluid became turbid, and was found

Fig. 33.

\section{Blood Cultivation from Diseased Sheep.}

(Drawing by. Dr. JAS. HUNTER, 1582.)

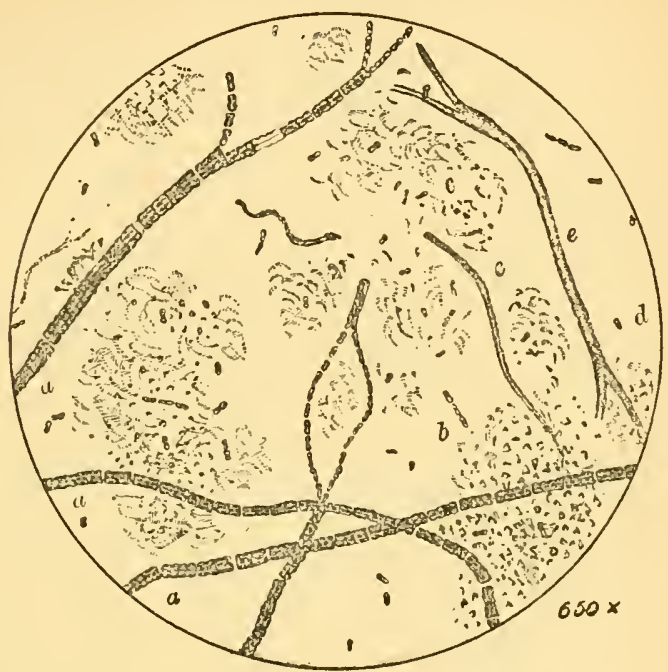

a. Multiple rows of rods or bacterioid segments (pathogenic mould).

b. A few rods in a single row.

c. Mass of zoogløa with micrococci.

d. A single rod.

c. Mycelium with spores.

to contain numerous bacilli or rod-like bacteria, in no respect distinguishable from those got in the fluids from the diseased sheep; and further, that the peculiar filaments with spores in their interior were also seen (figure $34, e$ ).

The strawish-coloured mould which soon covered the ticks, when stained, dried, clarified with oil of cloves, and mounted in balsam, presented the appearances seen in figures 34 and 35 .

Now this proved nothing beyond the fact that the organism was in the tick; but whether the tick received it from the sheep, or the sheep from the tick, was a point yet to be determined, and for this purpose ticks were obtained from the grass and perfectly healthy sheep, and treated in the same way. The con- 
clusion arrived at was that the organism was communicated by the tick from louping-ill districts to the sheep, for the same organism was found to be developed, but not quite so quickly, in the cultivation as in that containing the tick from sheep suffering from louping-ill.

Fig. 34.

Cultivations from Blood of Tick.

(Drawing by Dr. JAS. HUNTER, 1882.)

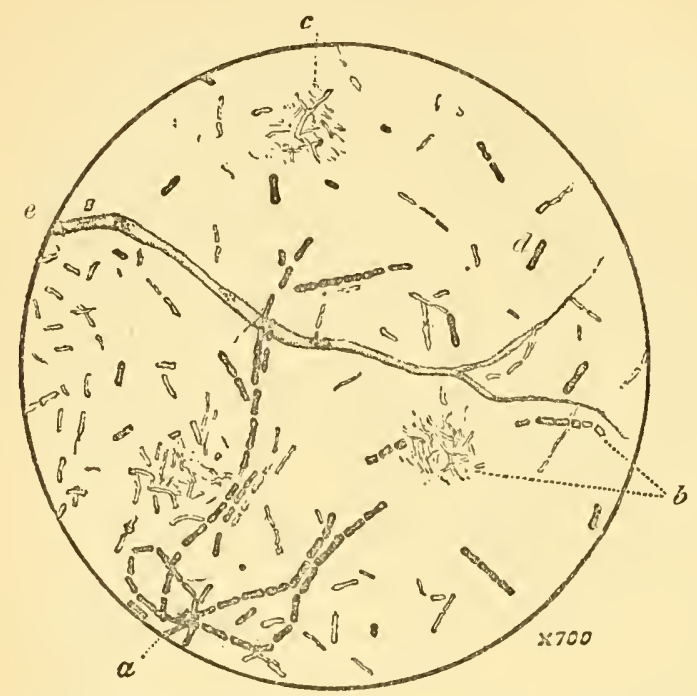

1. Single rows of bacterioid segments with traces of a double row remaining.

b. A few rods seemingly imperfect.

c. Zoogloea. d. A perfect and entire rod. e. Mycelium.

This last experiment, repeated over and over again, proved the fact that the ticks experimented with contained the spores of the organism, and were capable of infecting sheep; and that the reason that all sheep were not infected was simply the fact that many sheep were able to resist the invasion of the organism, or suffered so slightly that they were not observed to be ill.

Many sheep even badly affected recover from louping-ill, and it is fair to conclude that the organism has but a transient effect upon many others; that in fact, as is the case with other diseases, many are able to throw it off, and are most probably by this inoculation rendered proof in many or even the majority of instances against any further infection. This conclusion is 
borne out by a circumstance well known to flockmasters and shepherds, namely, that sheep reared in districts free from louping-ill are much more liable to take the disease, when subjected to its cause, than those reared where the disease and ticks prevail.

FIG. 35 .

Cultivations from Blood of Tick.

(Drawing by Dr. Jas. Hunter, 1Ss2.)

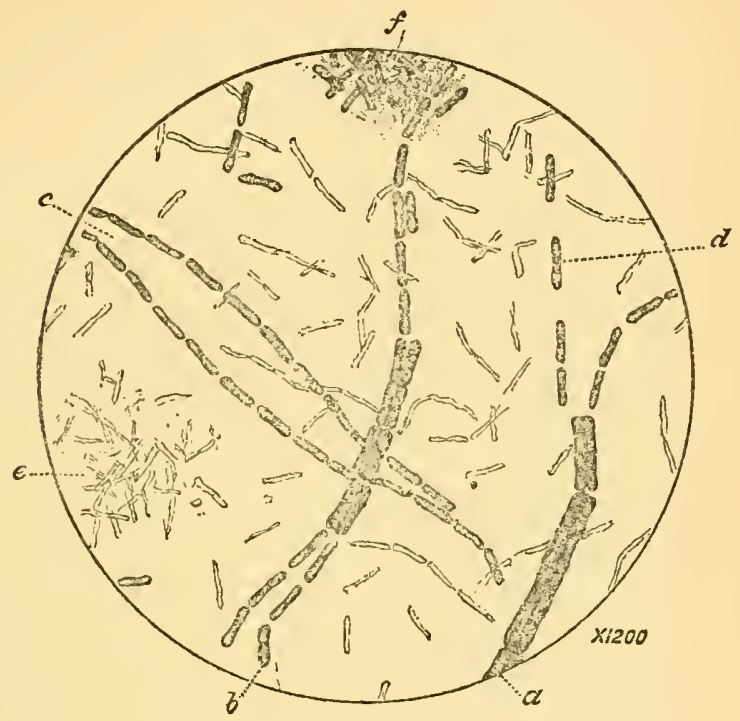

a. Double row splitting into two single rows, which separate longitudinally.

b. Double row in another stage of division.

c. Two single rows lying nearly parallel,

d. A single rod.

e. Zoogloa with disintegrating segments. $f$. Zooglœa with free segments.

It will be noticed that the mycelia or filaments and bacilli or rods differ in size, and to some this seems antagonistic to the view that it is a specific organism.

It must, however, be remembered that these different appearances are due to the varying ages of the growth, and that the organism seems only to arrive at maturity in the spinal canal, where, in the myxa sometimes there found, it may be seen in its highest development, and it is not denied that the organism may be multiple.

Dipping the sheep with any of the recognised sheep-dips will also destroy the ticks, but this procedure cannot be very con- 
veniently effected at and about the lambing season, and if lambs were dipped, great care would have to be exercised that the dip be not too strong, or else the remedy might prove worse than the disease.

The great question, however, is, can ticks be destroyed and the disease prevented? and I think I am in a position to answer in the affirmative.

It must be borne in mind that, as already stated, ticks are only found where the last and previous year's grasses are rank and afford cover for the parasites, and the remerly for the prevention of the disease is to eat down if possible, cut, burn, or otherwise remove, during the autumn all the long grasses of the previous summer's growth.

Putting aside the problem which is yet unsolved, namely, the possibility of the louping-ill organism having to pass one of its stages of existence as a micro-fungus upon some grass (such as the Nardus stricta, which is found in great abundance on the poor soil in such localities) before entering the bolly of the tick, or whether it is hereditary, as it were, in the borly of some ticks, leaving the pasture bare, and subsidising it with hay or corn, recommends itself to the careful consideration of all interested in sheep, for it is a fact placed beyond all doubt, that by rendering the pastures bare the covers and hatching-places of the ticks are thus destroyed. In confirmation of this conclusion, I take the liberty of giving the experience of an Ettrick sheepfarmer, Mr. Nichol, Crosslee and Newborongh on Ettrick, who was visited by Mr. Brotherston and myself on 24th June $18 S 2$.

Mr. Nichol stated that he entered the farm of Newborough twelve years ago, and that the louping-ill was then very bad. Between Whitsunday term (May 26th) and August he lost ten per cent. of his lambs-thirty-two out of sixteen score-besides several ewes. He thought the farm was not worth having. The parks were all in rough grass. Next year he bought some cattle, and far more the following year; ate, cut down, and even burned the old grasses; drained and limed the parks, using six tons of lime per acre to the light and eight tons to the heavy land. He continued to eat, cut, and burn down the grasses as bare as he could, and both the "lomping-ill" and the ticks became scarcer and scarcer. The third spring after he entered the farm it was very cold and sleety up to and after the "cutting" time. Ticks were very much scarcer than they had 
been before, and louping-ill was very much diminished with him, whilst on other farms it was, as might have been expected from the state of the weather, very bad. He thought, but wrongly, as he has now found out, that the wet, coarse weather was the cause of the scarcity of the ticks.

In six years both louping-ill and tick had both almost entirely disappeared-an odd one now and then-and he has had scarcely any since.

He has kept extra stock, both of cattle and sheep, during summer. The number of cattle kept has increased from forty to one hundred and twenty, which were kept during the summer of 1881. The cattle are sold about October, but about thirty head are kept on the hills during winter, part of them being tied at night. Last year Mr. Nichol stated, "Yon may believe the pastures were eaten to the bone." During our visit, however, we found that the grasses were more luxuriant and of much better quality than on any neighbouring land.

Mr. Nichol very quaintly remarked, "Those who can afford to let their sheep die, do so, but I cannot afford this, so give corn."

One farmer whom I know very well congratulated himself that his heavy loss had taken away all his weakly sheep, and he had now a good strong stock to breed from, stock that would stand anything, even starration.

One moment's rational consideration of this matter would open the eyes of even the most prejudiced, and convince them that starvation never leals to strength, but to the converse condition, and that a strong healthy stock, although able to live through a hard winter without any food but what they can scrape up, are rendered weak and debilitated; and that the mortality amongst the ewes is enormous during the lambing season, that many dead lambs are brought forth, and that where both mother and offspring survive, the latter often dies from starvation, the ewe being unable to afford it its natural sustenance. It must also be borne in mind that exhanstion of soil, micro-fungi on grasses, ticks, poverty, and louping-ill are coexistent.

In conclusion, I would earnestly recommend all who may read these observations to carefully consider the suggestions laid down, to eat up or destroy the old grass, which, when left on the ground, becomes a source of mischief not only in 
itself, but by affording a cover and harbour for parasites, which I think have been proved to be the source of enormous losses.

With regard to the ked, Melophagus ovis, found upon all sheep, and having no connection with the disease under consideration, it was discovered that those removed from sheep suffering from louping-ill contained the germs of the organism, and that it could be cultivated as readily as that of the tick itself, whereas, when it was removed from sheep healthy and not upon louping-ill land, no results were obtained, although several experiments were made. The fact that ticks induce more diseases than louping-ill is now fully recognised (see Texas Fever).

1894 ,

\section{Further Investigations, 1894-6.}

\section{Louping-Ill Inoculations at the College.}

May 2. Sheep inoculated at 3.25 P.M. with cultivation from sheep killed at Brown's farm, Dalmally, whilst suffering from louping-ill. Temperature $103^{\circ} \mathrm{F}$.

, 3. Temperature $102^{\circ} \mathrm{F}$.

, 4. Partial paralysis of the fore limbs; convulsive twitchings or "trembling "; foaming at the mouth. Temperature $102^{\circ} \mathrm{F}$.

, 5. Complete paralysis of the fore limbs; partial paralysis of the hind limbs ; convulsive movements, \&c. increased. Temperature $104^{\circ} \mathrm{F}$.

"6. Sheep died, 8 A.M. Post-mortem revealed louping-ill lesions.

On the same day, May 2, from ten to fifteen living ticks were placed on each of the remaining sheep, six in number. These were examined on the following morning, when it was found that the ticks had almost entirely disappeared; they were reexamined daily until June 2, and, giving no evidence of infection, all the ticks having evidently dropped off without becoming adherent to the skin of the sheep, the following experiments were made:-

\section{Inoculations with Cultivations from Ticks.}

.Tune 2. Sheep No. 1 inoculated, 2.30 P.r. Temperature $104_{5}^{\circ} \mathrm{F}$.

,3. Temperature $103^{\circ} \mathrm{F}$.

, 4. Temperature $104^{\circ} \mathrm{F}$.

, 5. Temperature $104^{\circ} \mathrm{F}$.

"6. Temperature $104^{\circ} \mathrm{F}$. Results negative.

, 9. Sheep No. 2 inoculated, 2 P.м. Temperature $104_{0}^{\circ} \mathrm{F}$.

, 10. Temperature $105^{\circ} \mathrm{F}$. 
June 11. Temperature $105^{\circ} \mathrm{F}$.

,12. Temperature $103^{\circ} \mathrm{F}$. Results negative.

"16. Sheep No. 3 inoculated, 1 р.м. Temperature $103^{\circ} \mathrm{F}$.

", 17. Temperature $103^{\circ} \mathrm{F}$.

, 18. Temperature $102 \cdot 4^{\circ} \mathrm{F}$.

, 19. Temperature $102 \cdot 6^{\circ} \mathrm{F}$.

,20. Temperature $102^{\circ} \mathrm{F}$. Results negative.

", 16. Sheep No. 4 inoculated with tick cultivation in egg albumen, 1 P.M. Teinperature $103 \cdot 4^{\circ} \mathrm{F}$.

,17. Temperature $103^{\circ} \mathrm{F}$.

,18. Temperature $103^{\circ} \mathrm{F}$.

,, 19. Temperature $103 \cdot 2^{\circ} \mathrm{F}$.

, 20 . Temperature $104^{\circ} \mathrm{F}$. Results negative.

,, 20. Tup No. 5 inoculated, 4.30 Р. . Temperature $103^{\circ} \mathrm{F}$.

,, 21. Temperature $105^{\circ} \mathrm{F}$.

, 22 . Temperature $103^{\circ} \mathbf{F}$.

, 23. Temperature $103^{\circ} \mathbf{F}$.

, 24. Temperature $103 \cdot 6^{\circ} \mathrm{F}$.

,25. Temperature $103^{\circ} \mathrm{F}$. Results negative.

,29. Sheep No. 6 inoculated in vein. Temperature $104^{\circ} \mathrm{F}$.

, 30. Temperature $104^{\circ} \mathrm{F}$.

July 1. Temperature $103 \cdot 8^{\circ} \mathrm{F}$. Results negative.

June 29. Sheep No. 1 reinoculated in back (subcutaneously). Temperature $103^{\circ} \mathrm{F}$.

, 30. Temperature $103 \cdot 8^{\circ} \mathrm{F}$.

July 1. Temperature $103^{\circ}$ F. Results negative.

The attempt to induce the disease by placing the ticks upon healthy sheep, and by inoculations with the organism cultivated from ticks, having proved unsatisfactory, and the season being too far advanced for further experiments, I determined to adopt another line of procedure during the season 1895. I may here state that all the experiments with living ticks had hitherto given negative results: ticks, except small ones and those which had not yet become adherent to the sheep, conld not be induced to cling to a fresh host; no matter how many fairly or fully grown ticks were placed on a sheep, they would disappear in a few hours.

I then determined to send sheep during the following year to tick-infested districts; to muzzle these sheep in order to prevent them from grazing; to feed and water them indoors with food sent from the College; and to allow them to remain in the infested district until infested with the ticks, then have them returned to the College for examination and further study.

"April 21, 1896.-We visited Mr. Hamilton, Leithen Hall, and an adjoining farm, occupied and owned by Mr. Carruthers. 
He removed there from the south of Scotland (the Hawick district) about fifty years ago, and placed a large number of sheep from that part on his new farm. The mortality was very great, quite 30 per cent. He redueed this percentage by limiting the introduction of fresh sheep, and devoting his energies towards the inerease and retention of sheep home bred. The loss was thus reduced to 10 per cent; and when he became the owner of the land he limed and drained to such an extent that at this date a case of louping-ill is very rare. He is a firm believer in 'no ticks, no louping-ill.' He frequently witnessed in the old days bullocks affected with the disease. He still washes his sheep with Bigges' Dip in solution, and lays down the rule to be good to the land, and louping-ill will disappear wherever it has been seen.

"At four A.M. on the morning of the 22d April we aceompanied the shepherd to the hill, and found some 600 ewes and their lambs all in good health. About thirty six hours previously the shepherd had found a hogg dead that for two days previonsly showed very pronounced symptoms of louping-ill. He had, after skimning it, preserved the carease for our examination in a cavern on the hill. We found it to be cool and quite fresh, with the head cut off. This we regretted, but he stated that a large quantity of fluid had escaper from the cranial cavity on section. At nine A.M. we (having had the carease and head earried to the farm-house) made an examination of all the internal organs, also of the brain and spinal cord. The jelly-like exudate was present in great abundance, particnlarly in the lumbar portion of the spinal canal; the spinal cord was inflamed in patches along its whole length, but the jellylike exudate was confined to the lumbar region.

"The test sheep having arrived-they having been driven from the station with a mouth-piece on each, which effectually prevented them from feeding - the muzzles being removed, they were fed with oats and hay and watered, also rested for a few hours. The mouth-pieces were then re-applied, and about one o'clock they were turned on to a field where ticks were known to exist. About ten A.M. the following day they were returned to the outhouse to be fed and watered. A few minute ticks were seen about the inner parts of the fore legs. At one P.M. they were again turned out with their mouths securely covererl. Every day until Tuesday, the 28th, when the sheep were re- 
turned to the College, this system was carried out. One of the ticked sheep died on 10th May and another on 12th." 1

On May 13 ten sheep, out of eleven obtained from Mr. Skirving, were sent from Edlinburgh to Leithen Hall, as I supposed the ticks would now be more numerous. They safely arrived, were muzzled in the usual way, and remained there until the 21st, when they returned apparently healthy.

The student, Mr. Morgan Williams, who accompanied these sheep, wrote to me as follows:- "The sheep were all muzzled, and examined for seven days, but owing to the early season this year very few ticks were found on the farms, neither were there any sheep affected with louping-ill during the above period. Out of the ten sheep two only were found with ticks on, and, as far as could be seen, there were only three ticks on each. Nothing abnormal was detecter on these from the time they came under my charge until they were brought back to the College. Mr. Hamilton informed me that fewer of their sheep were infested this season with ticks, and consequently fewer were affected with louping-ill; that they were all clipped this season, as recommended by Professor Williams. He also informed me that a top-dressing of salt, 10 cwt. to the acre, gratuitously supplied by Mr. Thompson, V.S., Aspatria, was applied to one park, which had been previously badly infested with ticks; but since the application of the salt the sheep cannot be kept away from the dressed park. But Mr. Hamilton covild not inform me whether this dressing had made any difference as regards the ticks, because, as above stated, the sheep were lipped, and they did not find any on the sheep after the application (dip), irrespective of the part of the farm the sheep were grazed on."

On their return to the College, 21st May, these ten sheep were carefully examined, but no ticks could be found upon any of them. They were carefully watched, and their temperatures taken daily until 27th May, when four, including the one retained at the College; were inoculated with cultivation material from the spinal canal of the ticked sheep which had died on the 12 th. The cultivation material was filled with the welldeveloped organism. One of these sheep clied on May 29, having presented very acute symptoms, which we supposed were due to some septic blood-poisoning; but on making a post-mortem examination, the condition of the spinal cord and

1 Report by Mr. R. Moir, M.R.C.V.S., who kindly took charge of the sheep. 
brain left no doubt in our minds as to the true nature of the disease. Although there was no jelly-like exudate in the spinal canal, the cord itself was inflamed in patches, and the organism could be detected microscopically and cultivated in the usual way. On 1st June a seeond inoculated sheep died, and on 5th June another died. Thus three out of four inoculated on $27 \mathrm{th}$ May died. The fourth remained bealthy.

On May 5 I visited Mr. James R. W. Wallace's farm of Auchenbrack, near Thornhill, and there met Professor Wallace, who takes a keen interest in this question. We found several sheep and lambs affected-some dying, some recovering, and some dead; but Mr. Wallace thought the mortality had not been as great as usual. The symptoms during life and the post-mortem conditions revealed were undoubtedly those of louping-ill. No experiments beyond tube cultivations were made, but it was arranged-Mr. Wallace generously offering to perform the experiment free of expense-to buy about a score of sheep and place them muzzled on that part of the farm where trembling and ticks committed the greatest havoc. The sheep, nineteen blackfaced hoggs, were bought on May 13, and fourteen of them were muzzled and turned out during the day, and described by Mr. Wallace as follows: "13th May.-Will turn out fourteen of the ewe hoggs on the hill on which there is trembling, with the muzzles on, and take them in again in the morning, and driving them to the fields free from trembling to feed during the day. 26th May.-The sheep have been a week on, and I only see one tick each on three of them. The season being so early, I think the time is now past, as fewer of my own sheep have been affected this year; but I will go on with the hoggs this week, and then turn them on sound land free of trembling for a while, till we see if anything turns up." On 1st June one of the ewe hoggs died, and was sent to the College, where a post-mortem examination was made, but no trace of louping-ill could be detected. On 6th July Mr. Wallace wrote again as follows: "The blackfaced ewe hoggs I had muzzled and put to the hill to test for trembling have all done well since they were tumed out. It was rather late for this early season, so a better result might be got another year. There were not so many ticks and less trembling with me this season than usual, but some places were worse. I did not think the hogg that died, died of trembling, but I thought it better to send you the carcase, as it was one of the lot." 
Photo-Wicroyraphs of the Oryanism, 1s97.

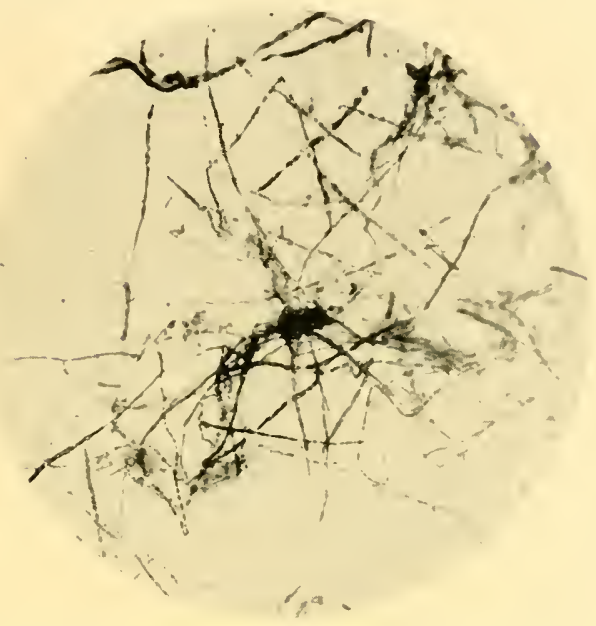

Fig. 35.1. - Filamentous stage of the organism in spinal canal of sheep dead from louping-ill, May $1886 . \times 500$.

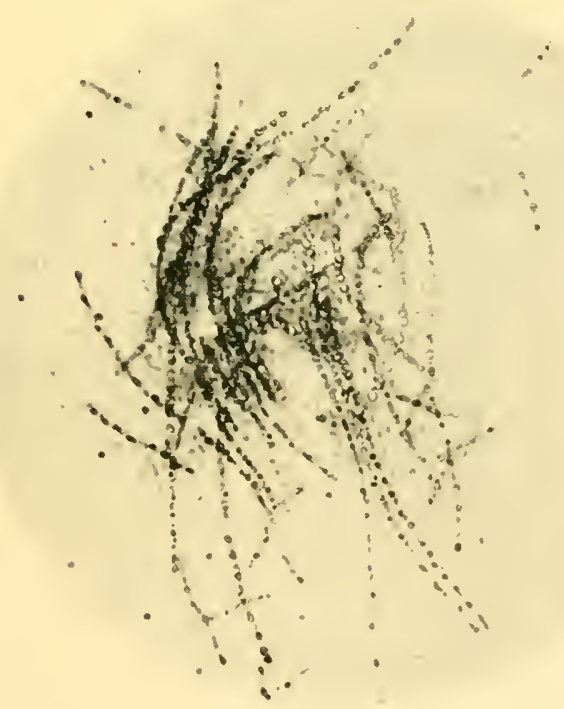

FIG. 35 k. - Filaments breaking up into spores. ('ultivation from blood of sheep inoculated with material shown in Fig. 35. $\times 1500$. 
Photo-1heroyraphes of the Oryanism, 1897.

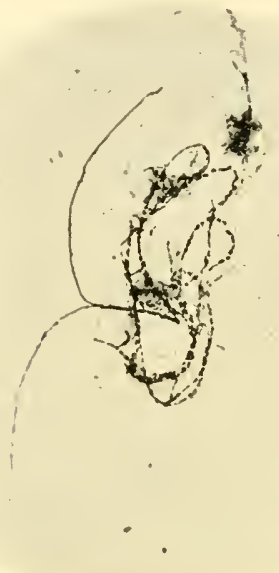

Fiti. 35r. - Cultivation from tick, tenth day. $\times 500$.

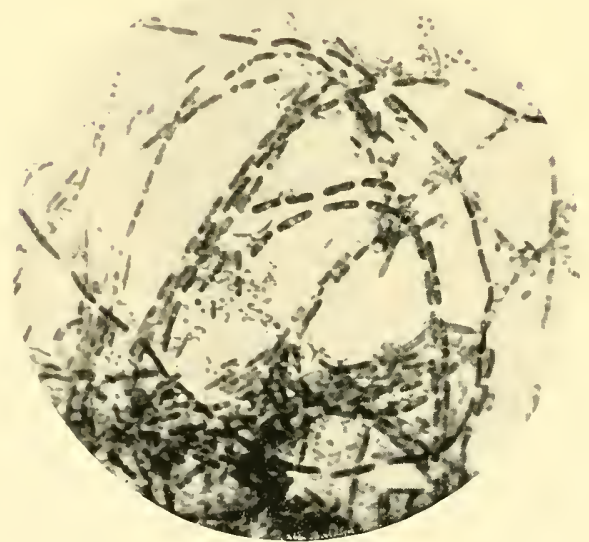

Fig. 350.-Portion of Fig. 35c under a higher power. $\quad \times 1500$. 
Photo-Microgiaphs of the Organism, 1897.

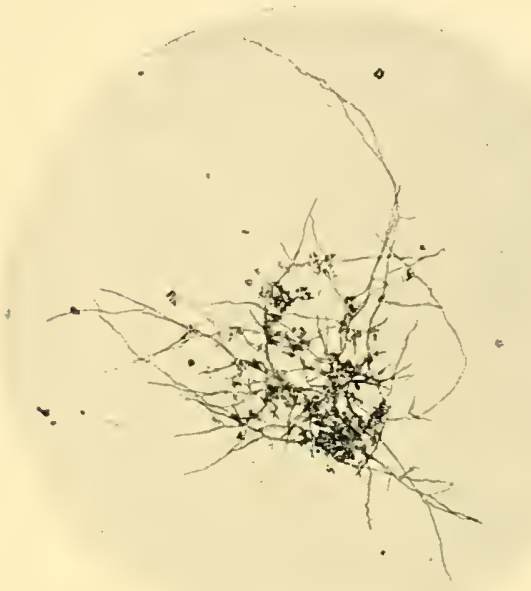

Fig. 35E. - Jelly from spinal canal of Twiglees lamb, $1882 . \times 500$.

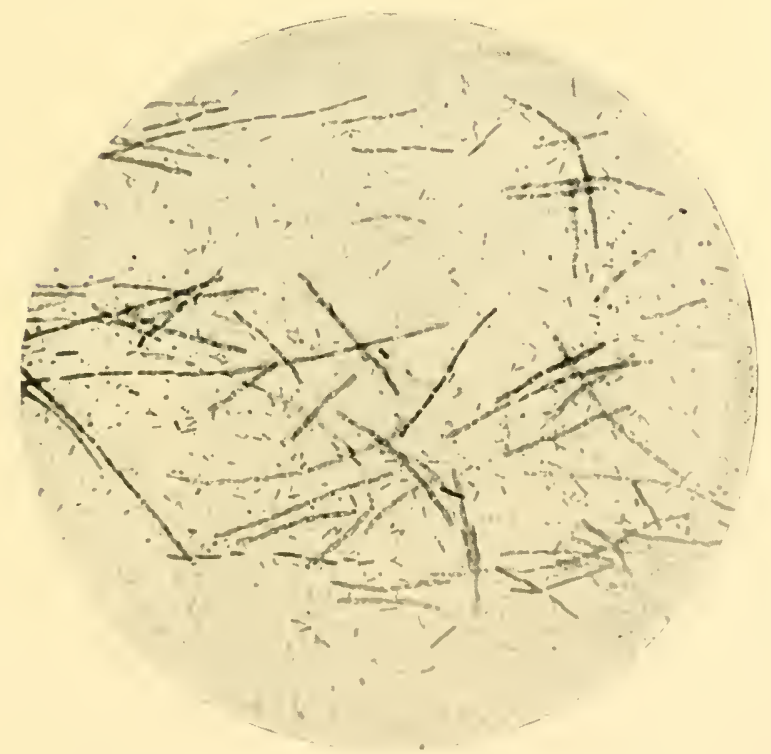

FIG. 35F.-Cultivation from cardiac blood of sheep which dierl after being inoculated. June $5,1896 . \times 1500$. 


\section{LOUPING-ILL.}

Photo Mirroyraphs of the Oryanism, $1 \times 97$.

FI *. 35k, - Cultivation from spinal canal of ticked sheep, died at Collegre. May $10,1896 . \times 1500$.

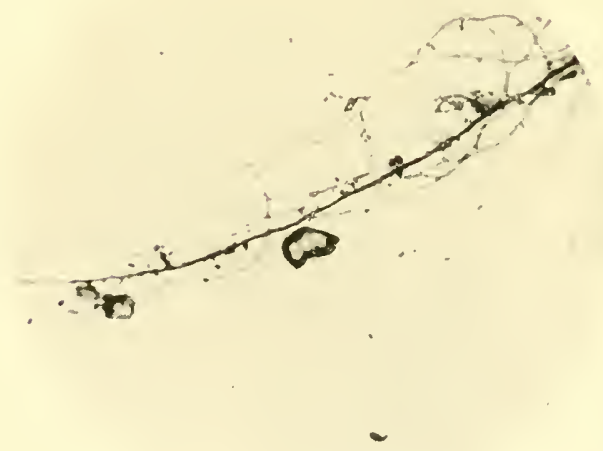

FIG. $35 \mathrm{H}$. Cultivation of spinal fluid from sheep dead from louping-ill, 1896. $\times 500$ 
Photo-Mierouraphs of the Oryanism, 1s!97.

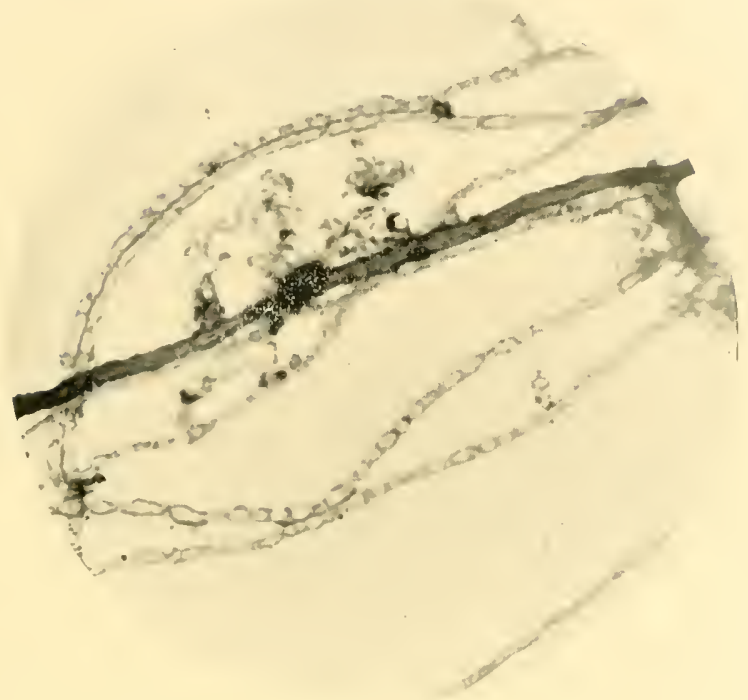

FIg. 35r. - Portim of Fig. 35k under a higher power. $\times 1500$.

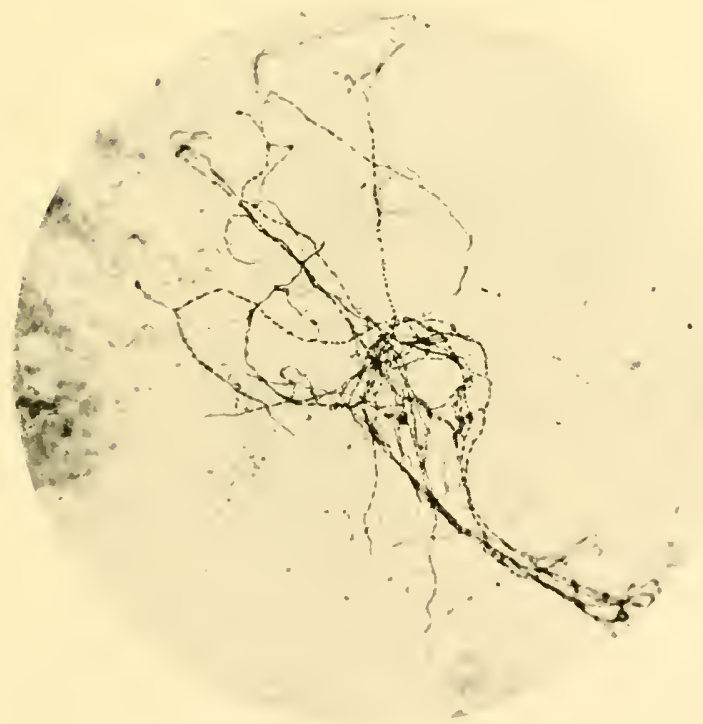

Fu. 35k.-Cultivation from cardiac blood, Auchenlsack lamb (agar-agar ten (lays), May 1s9t;. $\times 500$. 

By comparing the photo-micrographs with the microscopic drawings, both by Dr. Hunter, published in my article on Louping-Ill in $1882,{ }^{1}$ it will be observed that the similarity, if not identity, is maintained throughout. From the long and exhaustive investigation (in which I have been assisted by my son, W. O. Williams, and Dr. James Hunter, and to whom my thanks are due) of this disease, I an now more convinced than ever that the organism or organisms are most probably developed in one stage as minute moulds on old grasses-in this condition, innocuous to the higher animal, until it has passed through the tick; that in the body of the tick it attains properties which are virulent when subsequently communicated to the sheep by the tick.

It will be observed that in the louping-ill organism there are met with, during its complete life history, those involving series of transitions assumed by G. Zopf and other bacteriologists to be more or less presented during the development of many micro-organisms. Leaving it, as most have done, to be decided whether or not the forms seen in such cases as the present are the exact connterparts of saprophytes, typical micrococci, bacteria, or bacilli, there is no doubt that such variations are seen during the growth of the louping-ill organism. But further, when it has been found that the inoculation of healthy animals by certain of these forms has led to the appearance of the others in great constancy under varied conditions, the identity of a series of forms can no longer be disputed. It must, however, be confessed that as yet the true relationship and significance of the individual forms have not been so fully made out as could be wished from a purely biological point of view; but the pathogenic action peculiar to the organism has, by all the usual modes of research, been placed beyond cavil. This is not to be wondered at, when it is remembered that several well-known micro-organisms have stages in their development that aré by no means fully understood. Apparently the simple unsegmented saprophytic filaments are generally met with in the spinal myxcedema of the affected animal, whiie the ligher forms of spore filaments, fully segmented and free rods, closely resembling the typical bacillus, were seen, so far as the present observations go, mostly in culti-

1 See Transactions of the Highland and Agricultural Society. 
vations outside the aninal body. They, however, presented themselves with great constancy in cultivations of the spinal fluid and myxodema-jelly-like material-under every possible variation, produced by change of media, place, and manner of experimenting. It must therefore be surmised that they are either various stages in the life history of the same organism, or that, as in some other diseases, multiple organisms are present. As an organism certainly more nearly allied to the moulds than to any pathogenic type previously described, it is interesting as being, so far as I am aware, the first known instance of what appears to be a mould, in one stage at least, proving capable of producing pathogenic effects upon the higher animals, and it is also interesting to note that this pathogenic property is probably acquired after passage though the body of the tick, a state of matters now found to exist in other diseases.

When this disease was first investigated by me, the influence of the tick in inducing the so-called Texas fever, and other similar sources of inoculation, had not been investigated; but now it is proved beyond all doubt that the tick disease, as well as other diseases communicated by insects, is now known in various parts of the world.

One remarkable circumstance might, in conclusion, be added, viz., that whilst in this comntry the tick is but seldom injurious to other animals than sheep, in other parts of the world its pathogenic effects are mostly confined to horned cattle, although it attacks human beings, sheep, horses, mules, \&c.

\section{NEURITIS-NEURALGIA.}

Inflammation of the Nerves-Neuritis.-When one considers the very delicate organisation of nervous tissue, and the extreme sensibility of the sensory nerves, a most striking fact presents itself,--namely, the rarity or almost total absence of inflammation of the nerves. The nervous substance contained within the cranium, as well as that constituting the spinal cord, is subject to inflammatory changes; but it may truly be said that, except as a result of blood alteration-as in azoturia, where the sciatic nerves, and traumatic tetanus, where the nerves connected with the wound and their neurilemme are often found 
inflamed-neuritis is almost unknown, or, if it does exist, has not as yet been correctly diagnoser.

Neuralgia, or pain in the course of a nerve, and recurring in some few cases at stated intervals, whilst in others the recurrence is very uncertain, is also a disease which is rarely or never met with in the lower animals. I certainly once heard of a case which presented some signs of having neuralgia of the face, but whether it was that or pruritis the practitioner under whose care the horse was placed could not tell. The symptoms were persistent rubbing of the face against any solid object for a certain period each clay, with shaking of the head and great restlessness.

The principles of treatment applicable to neuralgic diseases will entirely depend upon the cause. If a nerre leading from a wound be inflamed, then fomentations and emollients must be applied; whilst belladonna should be rubbed along the course of the nerve, or, if the pain be excessive, morphia may be injected subcutaneously into the tissues of the painful part.

Should it be considered that neuralgic pains arise from a malor clebilitated condition of the animal generally, then measures must be resorted to which are calculated to restore the body to its normal condition, and in many cases aperients, followed by nervine tonics, such as arsenic, nux vomica, iron, or, where it is deemed necessary to soothe pain, belladonna, stramonium, yellow jessamine, or the bromides, may be administered for a short period. If pain on the course of a nerve threaten to become chronic, a smart blister will be imperatively called for. 


\section{CH A P TER X L V I. \\ SPORADIC DISEASES-continucd.}

LOCAL DISEASES-continued.

\section{(I.) DISEASES OF THE RESPIRATORY ORGANS.}

BEFORE entering upon the consideration of the particular diseases of the respiratory organs, it will be necessary to point out certain modes of physical examination by which the diagnosis of these diseases is accurately traced.

1. Auscultation.-In its technical sense this term indicates the act of listening to the sounds of the interior by means of the ear applied to the surface of the body.

Auscultation may be practised dircctly by applying the ear to the part; or indircetly through the medium of an instrument called a stethoscope. Generally direct or immediate answers every purpose; and in veterinary practice, at least, indirect or mediate auscultation is seldom practised. Auscultation, discovered by Laennec, was introduced into veterinary practice by Delafond and Leblanc.

Many of the sounds characteristic of abnormal conditions in the human being are not heard in the lower animals; hence auscultation, as well as percussion, is less satisfactory in the hands of veterinary surgeons than in those of the physician. There are various reasons for this, and not the least of them is the fact that the thoracic walls of man are much more thinly covered with muscular tissue than those of the lower animals. Again, a large portion of the thoracic cavity in our patients is covered by the scapula, which form insuperable barriers to examination. These, in aduition to the movements of the panniculus camosus, the horizontal position of the body, oblique arrangement of the diaphragm, the pressure of the digestive 
organs, and very often the restlessness of the patient, render this method of examination much less satisfactory than it otherwise would be; but, notwithstanding all these drawbacks, auscultation is of immense advantage, and by it we are enabled to arrive at conclusions that would otherwise be impossible.

To become familiar with the knowledge to be acquired by auscultation much time and labour must be devoted to it, first on healthy, and afterwards on unhealthy animals; in fact the ear must be trained by long experience.

The stethoscope can be bought at any instrument maker's, and the simpler it is the better. In applying it to the chest, its funnel-shaped end is to be accurately and firmly applied to the surface, and the opposite end in perfect apposition to the ear.

When immediate auscultation is practised, care is to be taken that the ear be accurately applied to the skin, or if the state of the surface does not admit the ear, a single fold of a thin cloth only should be allowed to intervene, as the rubbing of two folds or surfaces may create a sound and puzzle the examiner.

2. Percussion.-This process consists in striking upon the surface with the view of eliciting sounds, by the nature of which an opinion may be formed of the conditions of the parts beneath. Like auscultation, percussion is either immediate or mediatc. The former was employed by Avenbrugger, and the latter invented by M. Piorry, who gave the name of "pleximeter" to the intervening body.

In immediate percussion, the ends of the fingers are brought together and supported by the thumb, and the parts are struck perpendicularly to the surface, or the parts may be rapped with the knuckles of the closed hand, the force of the blows being regulated by the depths of the parts to be examined, and the size and delicacy of the animal. In the cat or dog gentle blows with the tip of the middle finger are generally sufficient.

In mediate percussion, the pleximeter is generally a flat, oval, or circular piece of ivory or gutta percha, or the left index finger of the operator, which is certainly the most convenient and best intervening body which can be had.

M. Poirson of Paris recommends that percussion should be performed by means of a common sewing-thimble placed on the middle or fore finger, so as to include a small portion of air between the end of the finger and that of the thimble. The intensity of sound elicited is said to be thus greatly increased. 
Some operators substitute a small hammer for the fingers, the head being made of wood, ivory, or metal, with its percussing surface covered by a softish and somewhat elastic substance, as felt, caoutchouc or gutta-percha. The more satisfactory method, and the best and simplest, is immediate percussion by the tips of the fingers or knuckles; the part percussed to be unclothed, and struck perpendicularly to its surface, otherwise the character of the sound will sometimes be confusing.

3. Palpation.-Touch is useful in the diagnosis not only of chest diseases but of many other ailments. By this method we detect tenderness, heat, coldness, fluctuations, tumours, and a variety of conditions indicative of disease.

If an animal be affected with pleurisy, firm pressure in the intercostal spaces will cause wincing, grunting, or even groaning.

4. Mensuration is recommended by the French veterinarians. It has, however, found little or no favour in this country, as it is considered that an amount of disease sufficient to alter the relative size of the sides of the chest is otherwise determined than by measurement. In the cat, $\log$, and cow, the intercostal spaces are expanded and dilated in hydrothorax of one side, if of long standing; and in some cases of chronic pneumonia in cattle I have confirmed the observations of Delafond, that one side of the chest is sometimes palpably larger than the opposite side.

Mensuration as applied to the chest, says Mr. Gamgee, " consists in the application of a cord or tape to the similar parts of the two sides of the chest, in order to ascertain whether any difference exists in their relative prominence. For the larger animals a tape of three or four feet long, and for the smaller, one of a foot and a half to two feet, may be employed. One end is placed immediately behind the withers, and the line carried downward to the middle of the sternum, which part is marked by a knot; a second measurement is mate from the withers to the eighth rib, or the commencement of the cartilages of the false ribs, and similarly marked; a third measurement is made from the lower end of the third rib to the commencement of the cartilages of the false ribs; lastly, the tape may be earried from the posterior border of the shoulder along the middle region of the chest as far as the last rib. The same measurement may then be made on the opposite side of the thorax, care being taken that they be always made at the same stage of the respiratory act, as otherwise false 
results will be obtained. It is well, indeed, to measure the different parts after both inspiration and expiration, that any difference in the expansion of the two sides of the thorax may be ascertained."

5. Inspection.--Some symptoms are observed by inspection only-the condition of the visible membranes, the attitudes and movements of the body, the expression of the countenance, the character of the respirations, the degree of fulness, or the converse, of the several parts. In human medicine the state of the larynx can be thus detected by an instrument called a laryngoscope, but owing to the length of the oral cavity, the dimensions of the velum pendulum palati, and the position of the larynx itself, the laryngoscope has not yet been made available for veterinary purposes.

6. "Succussion consists in grasping the thorax between both hands, and shaking it quickly so as to elicit sound. It is only useful when gaseous and liquid matters coexist in the pleura; in such cases, however, a splashing or churning sound may be heard. It will strike every one that this measure is only applicable to the smallest class of animals."-(GamaEe's Domestic Animals, page 537.) I have never practised this method of examination, nor do I recommend it, and here describe it in Mr. Gamgee's own words.

\section{RESPIRATORY SOUNDS.}

The respiratory sounds, normal and abnormal, detectable by auscultation, are divided into four classes, namely, Nasal, Laryngeal, Tracheal, and Thoracic.

\section{NASAL SOUNDS.}

In the normal condition a soft, to-and-fro, blowing noise, of equal intensity on both sides, is heard when the ear is apprnached to the nostrils. This sound is increased with exercise, and then bears a resemblance to the sound of a large bellows. There is no sound detectable throngh the parietes of the nose nor the sinuses of the head except after exereise, when a slight snore may be detected throngh the first and a mumur in the latter.

In some horses the nasal sound is abnomally increased with 
exercise, and can be heard at a considerable distance from the animal, eausing the horse to be termed a "high blower." This sound is generally much greater when the animal is gently trotted or eantered than in the gallop, and is londer in the expiratory than in the inspiratory act. The increase of sound arises from flapping or crackling of the nostrils, and has no connection with any disease. "It arises," says General Sir Frederick Fitzwygram, "from powerful museular development in the part. If a horse so formed be pushed to its speed and continued for some time, it will be seen that it can intermit the noise at its will; and when it really becomes distressed at its pace it will have something else to do than flap about its nostrils, and the sound will then cease altogether."-(Horses and Stables.) I have repeatedly observed that some horses will emit this sound when they are first put into training after resting for a while, but when they are bronght into condition the sound entirely ceases, and I have thought that the sound was due to flaccidity of the dilatores nares muscles. In other horses, again, high blowing is due to a natural narrowness of the nostrils and nasal passages. It never seems to interfere with the horse's usefulness, and, consequently, is not to be considered an unsomudness.

Snoring is diagnostic of polypus, thickening of the Sclmeiderian membrane, or some other obstruction to the passage of air throngh the nasal chambers.

A snuffling sound indicates some disease, accompanied by discharge from the nose, as purpura, glanders, eatarrh, \&c.

Whistling may be due to a tumefied condition of the Schneiderian membrane. This is, howerer, rare, and may be mistaken for a sound arising in the larynx or even in the lungs, which sometimes so retains its foree within the nasal chambers as to lead one to think it originates there. Auscultation of the several parts will, however, enable the practitioner to determine where the sound originates.

Sneezing or snorting indicates an irritable condition of the Schneiderian membrane, and is present in many cases in the early stages of catarrh. Sometimes I have seen it very persistent after the subsidence of the catarrhal symptoms, or existing independently of any apparent disease, coming on in paroxysms when the animal has been at work. One fit of sneezing has 
followed another, causing the animal a deal of distress. Steaming the head has succeeded in allaying it in some instances, whilst in others it has been necessary to apply a blister to the face.

Epistaxis, or bleeding from the nose, occurs as a symptom of various diseases, such as glanders, purpura, malignant catarrhal fever. It may also be inauced by injuries, rupture of the small nasal blood-vessels during severe exertion, sneezing, or it may arise from the presence of a polypus. When arising spontaneously or from injury, it must be arrested by the application of cold astringents or by plugging the nostril with cotton wool or tow. Percussion applied to the nasal region and simuses of the head yield in the young horse but an indistinct resonance; the sound increases with age as the sinuses undergo change. The presence of pus, tumours, or coagula deadens the sound. If there be an extensive accumulation of pus, or a large tumour in the simuses, the sound is completely deadened, the bone is painful to the touch, and often bulging in appearance.

\section{AUSCULTATION OF THE LARYNX-LARYNGEAL SOUNDS IN HEALTH} AND DISEASE.

In health there is a faint to-and-fro respiratory sound. In disease the laryngeal sounds are varied and important; they consist of grunting, whistling, coughing, roaring, and trumpeting. Laryngeal sounds, with the exception of "grunting," constitute unsoundness. If they be of a temporary nature, and due to irritation or tumefaction of the mucons membrane, pressure of abscesses or tumours of a removeable nature, the unsoundness may pass away; but if of a permanent character, no matter how tritling they be, they distinctly indicate unsoundness, not only interfering with the usefulness of the horse for the time being, but generally having a tendency to increase in intensity with age, and often causing a horse to become unserviceable. I do not mean to say that a whistler or roarer is not fit for work, but I assert that the infirmity is a drawback, and an aninal so affected is worth less money in the market.

1. Grunting.-If a horse, when struck at or suddenly moved, cmits, during expiration, a grunting sound, it is called a "grunter." Such a sound may or may not have any connection with disease of the larynx. A horse will grunt with pain when 
suffering from pleurisy, pleurodynia, and other diseases. Some horses habitually grunt when struck at or moved sudienly. A great number of cart-horses are so affected, and big horses of all breeds are very apt to be grunters, whilst they may be quite sound in their wind. Horses with heavy jaws and ill-set necks often emit this sound; and again any horse may do it if it has been fed for a time with bulky food. The sound is always to be regarded with suspicion, and the animal further tried for its wind, as it generally accompanies roaring or whistling. If the grunter, however, stands the tests used to deteet roaring, without making any noise in its breathing, it may be considered sumel Grunting is often symptomatic of oceult glanders.

2. Whistling.-This sound is of two kinds, a soft or moist and a dry or hard whistle.

Soft whistling is due to acute laryngitis, when a considerable increase of mucus has taken place, and when the mucous membrane is temporarily swollen. When due to the presence of mucus it partakes more of a wheezing sound, and is generally diminished when the animal coughs. The sound arising from tumefaction of the laryngeal mueous membrane is greater during the inspiratory than the expiratory act. It is very similar in character to the dry sound to be described; but in addition to the sound, symptoms of catarih, fever, or acute irritation of the larynx are present. This sound indieates a temporary unsoundness, and in many instances it is unsafe to pronounce a decided opinion for several days or even weeks after the subsiclence of other symptoms. In such instances the mueous membrane has undergone some degree of thickening; there is relaxation of the vocal eords, a want of tone in the larynx generally, that require some time to overeome. It is impossible to pass an animal of this kind as perfect, and it would be unwise to give a decided opinion until all abnormal sound has disappeared.

Dry whistling.-This sound has been generally looked upon as a modification of roaring. $\mathbf{i}$ an, however, of opinion that whistling and roaring are due to different pathological conditions of the larynx, and that they may exist indeperidently of each other; that roaring does not always terminate in whistling, nor whistling in roaring.

Whistling, like roaring, is a sound emitted more particularly during inspiration, and is due to a diminution of the calibre of the larynx, or sometimes trachea, owing to a permanent thicken- 
ing of the mucous membrane, distortion of the neck by tight reining, the presence of an immoveable tumour in the trachea, or by any cause which diminishes the area of the passage of the air to and from the lungs. Whistling, although loudest during the inspiratory movement, is by no means always absent during the expiratory act; careful auscultation is, however, necessary to detect it. If entirely absent during expiration. the seat of the lesion causing it is to be looked for in the larynx or upper part of the windpipe. Whistling, like roaring is often traceable to hereditary taint, and is an unsoundness.

3. Roaring.-This symptom of disease consists in a loud unnatural sound emitted during the inspiratory act. Roaring is sometimes symptomatic of acute laryngitis, and then only indicates a condition of temporary unsoundness.

The cause of roaring is, however, generally found to be due in the majority of cases to atrophy and fatty degeneration of the muscles of the larynx. The origin of the paralysis is involved in some mystery; but it is generally accepted by veterinarians that, inasmuch as it is generally confined to the left laryngeal muscles, the explanation is to be found in the fact that the recurrent or ascending laryngeal nerve on the left side leaves the pneumogastric further back than on the right, and winds round the posterior aorta; whereas the right is given off opposite the first rib, winding round the dorsal artery, and consequently the left nerve is more apt to be implicated in any disease of the chest than the right. This explanation is unsatisfactory on many accounts. 1st. Roaring is not a common sequel to pulmonary disease : $2 d$. It is not an accompaniment of thoracic disease, which would most certainly be the case if the nerve were implicated in an inflammatory affection : $3 d$. Many roarers whose history has been known from their birth have never suffered from any chest affection, nor indeed from any disease beyond a common cold, and sometimes not even from that slight ailment: 4th. Mares and ponies are less often affected with roaring than large geldings and stallions, but are quite as susceptible to chest diseases; and lastly, dissections have failed to discover any change in the nerve trunk itself, although the animals dissected have been confirmed roarers for years previous to their death. 
It is very true that division or ligature of either of the recurrents will be succeeded by roating, and tumours involving the recurrents may cause the same sympton; and Dr. Warburton Begbie says that "no more interesting variety of local paralysis exists than that which is dne to the interference with the recurrent or motor laryngeal nerve, produced by an aneurism of the arch of the aorta, or by a cancerous mediastinal tumour. Well-marked atrophy of the muscles of one side of the larynx las, under such circumstances, been found." But in the form of disease involving the laryngeal muscles, which eommonly causes roaring, there is no change in the nerve itself, nor can the loss of motor power be traced to pressure upon the nerve trunk by any tumour, aneurism, or adventitious substanee.

The disease of the muscles is, however, essentially nervous in its origin, and may, I think, be classified as a form of wastung palsy-paralysis atrophica-originating in the laryngeal muscles themselves. Wasting palsy is defined by Dr. William Roberts, in Reynolds' Systcm of Mcdicine, to be " an atrophie degeneration of certain groups of muscles, indepenclent of any antecedent loss of mobility, or of any metallie poisoning."

I have already stated that the atrophic change is trsually seen on the left side of the larynx. It, however, by no means follows that the muscles of the right side are entirely free from disease; indeed, in many instances, they distinctly partake of the atrophic change, though to a less extent.

Why the change is greater on the left than on the right side, is one of those things for which no more satisfactory explanation can be given, than why the ulcers of glanders are oftener seen in the left than in the right nostril.

Roaring is generally gradually developed. At first, the sound may be intermitting, and days or even weeks may elapse during which the animal may make no noise, although put to severe exertion, as if the muscles had, at the time the sound was emitted, been debilitated from some ephemeral disturbance of nutrition. As the loss of muscular substance progresses, there is a corresponding and permanent loss of power, and what at first was intermitting is now a permanent infirmity. This intermission of the sound is not, however, the ordinary method by which the disease manifests itself. More commonly the noise or roar, slight at first, gradually, but often very slowly, increases in 
intensity, thus marking or indicating the progress of the muscular change, and inability to dilate the laryngeal opening.

Causcs.-Putting aside all exciting causes of alteration of the air passages, to be hereafter mentioned as causes of roaring, I think that laryngeal muscular atrophy is due to hereditary predisposition; and it is a well-known fact that certain breeds, the produce of certain sires, are nearly all roarers. I have repeatedly observed this, not only in racers, but in other breeds of horses. Indeed, I know one breed of Clydesdales which are nearly all roarers, both mares and horses. Horses and geldings are, however, more liable to become roarers than mares, which seldom, except where the hereditary taint is very strong, become roarers. Small ponies are scarcely ever affected, although they are subject to colds, laryngitis, and pulmonary diseases.

A horse predisposed to become a roarer generally suffers from laryngeal irritation from trivial causes, and usually the infirmity appears after repeated attacks of cold and sore throat.

Other causes of Roaring.-Concluding that the majority only of cases of roaring are due to the condition described, it is necessary to mention that the sound may arise from other and incidental conditions which, although not so frequent, are quite as important.

Roaring may be due to disease of the nose, nasal polypi, depression of the nasal bones from previous fracture (see Principles and Practice of Voterinary Surgery), osseous tumours in the nostrils; closing of one nasal chamber by false membrane or disease of the bones; tumours on the posterior nares, called "bellones" by horse-coupers, falling into the glottidean opening, causing intermitting roaring; constriction of the trachea ; tumours in the thoracic cavity; distension of the guttural ponches; disease of the pharyngeal and parotidean lymphatic and salivary glands; tight reining; fractures of the tracheal rings, or any cause of distortion of the larynx.

In addition to the sound emitted during inspiration, the roarer generally has a cough which is diagnostic, being a loud, harsh, dry sound, half roar, half cough; and the generality of roarers are also grunters. It will also be found that the sensibility of the larynx is diminished in confirmed roarers, and that consequently it is difficult to make them cough in the ordinary way by pressing the larynx. In testing a loorse for its wind, it is usually the 
practice with some to place it against a wall, and threaten it with the whip; if it grunts, it is further tested; if not, it is merely made to congh by pressing the larynx, and if the cough has a healthy sound the animal is generally passed sound.

This plan is not always satisfactory, and the better way is to have the animal galloped, or if a cart-horse, to move a heavy load some little distance, when, if it be a roarer, it is sure to make a noise.

Treatment of Roaring.-If the sound can be traced to any removeable cause, the practitioner knows what to do, but the removal of the causes of the form of roaring generally met with is, however, a matter of great importance. Many experiments have been tried; in some rare instances the application of blisters, or the actual cantery, to the skin of the laryngeal region, has succeeded, not only in arresting the progress of the atrophic change in the muscles, but in materially improving their strength and tone; but in order that this may prove effectual, the animal is to be treated in the very earliest stages of the disease. In addition to "firing," I have only to suggest that the clilorate of potash is worthy of trial, as it is found to lave some power in arresting fatty degeneration.

In very bad roaring the sound may be modified by pads attached to the bridle and fitted over the false nostril. These pads regulate the quantity of air taken in at such inspiration; for it is observable that when a roarer is pushed in its work or paces, that the nostrils dilate greatly, thus admitting a large volume of air, which by its weight and pressure causes a further falling in of the arytenoid cartilage and an increased constriction of the laryngeal opening. If this method be inefficient, tracheotomy is to be performed, and the tube kept in the trachea for the remainder of life. Excision of the left arytenoid cartilage (presuming that the left muscles are atrophied) has been suggested by Guinther of Hanover, and has, I believe, been more or less successfully carried ont. The operation is to be performed by making an incision through the skin into the superior part of the trachea, the collapsed cartilage seized by forceps or tenacula, and excised by means of a pair of scissors.

It will be apparent that a bad roarer, no matter what the means may be by which the sound is modified, is only useful for slow work; and lastly, I liave always observed that confirmed 
roarers become bad thrivers, and often succumb to trivial diseases.

Roaring being due to a progressive disease, it is necessary that the veterinarian should reject as unsound every animal which makes the slightest roaring or whistling sound in its breathing.

\section{COUGH AS INDICATIVE OF DISEASE.}

Cough is the sound produced in the larynx by the violent expulsion of air from the lungs, and is symptomatic of various diseases. Cough is divided into dry and moist.

Dry cough is divided into short, hacking, hollow, brokenwinded, and spasmodic. The dry cough is symptomatic of irritation and clryness of the respiratory mucous nembrane. In the early stages of laryngitis it is loud and long, becoming afterwards rasping and then moist. In chronic disease of the larynx it is loud, roaring, and often bollow. In the early stages of bronchitis it has a hollow metallic sound; it afterwards becomes moist, and is more or less painful throughout the diseasc. In pneumonia the cough is short, seems as if proceeding from a solid organ, and is accompanied in the later stages by a rusty, tenacious expectoration.

The cough of pleurisy is dry throughout, is painful, hacking, sometimes as if cut in two, the animal being seemingly afraid to complete the act by one effort.

The broken-windcd cough is at first spasmodic, becoming, as the disease advances, feeble, short, and single, the animal being unable to relieve itself by the action of the chest and lungs; hence the suppressed cough becomes diagnostic.

The hollow cough, a sepulchral sound, varying in intensity, indicative of clironic disease, and on this account is termed a "chronic cough."

The moist coughs indicate an inflamed and humid condition of the respiratory mucous membrane.

There are various other kinds of coughs associated with diseases of the heart, digestive organs, and the process of dentition; these are valuable as aids to diagnosis, when studied in connection with other symptoms. They are mostly dry coughs.

Horses with narrow, shallow chests, weak loins, and long legs are very generally predisposed to cough from very trivial causes. 


\section{TRACHEAI SOUNDS.}

Except at its entrance into the chest, the trachea yields no sound. At this point, however, a blowing sound-tracheo-bronchial respiration-can be heard, more prolonged during the expiratory act. In disease this may be increased. When the bronchi contain much mucus or other fluid, a moist rattlemucous rale-may be heard, accompanied by a wheezing, gurgling, or spumous or frothy sound.

\section{TIIORACIC SOUNDS.}

The thoracic sounds are divided into pulmonary, pleural, and cardiac. The pulmonary sounds are of two kinds, namely, the vesicular and the bronehial sounds.

1. Vesicular, also called the respiratory murmur, is heard during both inspiration and expiration. During the inspiratory act it is a soft diffused murmur of a gentle breezy character: slightly harsher and more hollow during expiration, and not above one-fourth the length of that during inspiration. It is caused by the entrance and expulsion of air to and from the terminal portions of the bronchi and air sacs. The intensity of this sound is increased with exercise or other causes of quickened respiratory movenents, as fever; is louder in chests thinly clothed with flesh; stronger in the young than in adults, hence, when strong, it is called "puerile"; it is also louder when the stomach is empty. Puerile respiration depends upon the smaller size of the air vesicles, and the greater elasticity of the lung tissue; in the very old the murmur is scarcely preceptible, and is called "senile." Senile respiration may also result from slow breathing, or any cause which obstructs the entrance of air into the lungs. If either modification is present to the same extent in the same region of both sides of the chest, they indicate no other condition than the natural ones above indicated; but if the murmur is puerile on one side and senile or absent on the other, the first indicates a compensating action of that lung, supplementary to diminished action in the other. The vesicular murmur is best leard in the superior portion of the lower third of the chest, from behind the elbow and shoulder to about the ninth rib, whence it diminishes in force, 
and is altogether lost over the sixteenth. In the inferior part of the chest it climinishes at the seventh, and is lost at the tenth rib. On the left side it is mixed in the lower and anterior regions, immediately behind the shoulder, with the sounds of the heart.

2. The Bronchial or Tubal sound resembles the blowing of air quickly through a tube; it is higher in pitch than the vesicular murmur, and more rapidly evolved. It is nearly as prolonged during inspiration as expiration, with a distinct interval between the two. This sound is distinct over both the middle and upper thirds of the chest; lotidest immediately behind the scapula and caput magnum, or the nearer we can approach the ear to the lifurcation of the trachea and larger bronchial tubes; diminishing in intensity to the twelfth rib, where it is superseded by the vesicular, and entirely lost at about the seventeenth rib. It also diminishes in intensity as it approaches the upper part of the lower third of the chest, where it becomes lost in the vesicular sound. The tubal sound becomes diagnostic of disease when it is heard in the inferior portions of the chest.

In the ox, the bronchial sound is heard much lower down in the right side, owing to the large tube which passes to the anterior lobe of the right lung.

The true thoracic sounds are very often complicated with abdominal rumblings or gurglings, due to the movement of food, fluids, and gases within the alimentary canal ; these are loudest in the posterior part of the chest, are irregular in their occurrence, and bear no relationship to the respiratory movements. A little practice will soon enable the student to distinguish these from the respiratory sound.

Percussion.-The sound obtained by percussing the healthy chest is loudest over those parts where the bronchial sounds are best heard, except indeed in those parts most thickly clad with muscular tissue. On the left side the resonance is very clear immediately belrind the shoulder to the twelfth or thirteenth rib, where it gradually diminishes. If the parts are here struck forcibly, the intestinal resonance may be induced.

Mr. Percivall has pointed out what may be easily verified in practice, that the sound along the right superior region grows londer from the posterior border of the shoulder to the last rib, whilst on the left it gradually diminishes on the same line. 
This incrense of sound on the right side is due to a resonance from the arch of the colon. If the blows are light, this sound is not brought out, and when heavy, it is more of a tympanitic character, resembling that of an emphysematous lung. Along the middle region louder sounds are elicited than either above or below, more particularly between the fiftl, sixth, seventh, and eighth ribs, from whence it diminishes to the fifteenth, and then becomes tympanitic or abdominal on the left, and dull on the right side, owing to the opposition of the liver. In the inferior part of the chest the sound is weak, but clear, from the fifth to the eighth rib on the right side, where it becomes dull, responding to the liver; whilst on the left side the resonance is almost absent over the fifth, sixth, and seventh ribs, opposite to the lieart; it becomes clearer over the eighth, behind which it loses its intensity, and is lost at about the thirteenth rib.

\section{MORBID SOUNDS.}

The morbid sounds indicative of diseases of the respiratory apparatus elicited by auscultation may be divided into bronchial, pulmonary, and pleural.

BRONCIIIAL SOUNDS.

These are of two kinds, namely, 1 st. Dry; and $2 d$. Moist.

1. Dry sounds are subdivided into large and small, or rhonchus and sibilus.

(a.) Rhonchus, a hoarse, sonorous murmur, sometimes of a humming, cooing, or snoring character, compared to the bass note of a violin or cooing of a pigeon, especially marked during expiration, but coexistent with both movements, and due to a narrowing of some part of the larger bronchial tubes. It may be heard at the front of the chest very plainly, as well as behind the shoulder. It is sometimes cansed by portions of viscid mucus adhering to and obstructing the larger tubes, acting as vibrating tongues as the air passes by them. If the aclherent mucus be removed by coughing, the sound may disappear for a time, and then reappear; when not removed by coughing, it may be due to tumefaction of the bronchial mucous membrane; and 
if permanent in a given spot, it may arise from the pressure of a tumour or other cause which may flatten an air-tube. Phonchus essentially belongs to the larger tubes, denoting partial narrowing; is a dry sound, and, if uncomplicated, indicates a condition of no great danger. It is also called vibration, sonorous rhonchus, and sonorous rale, and is generally associated with bronchitis.

(b.) Sibitus, Sibilant Rule, Sitilant Rhonchus.-A high-pitched whistling, hissing, clicking, wheezing sound of variable intensity and duration, coexistent with both respiratory movements, but more especially marked during inspiration, arising from tumefaction or accumulation of viscid mucus in the small bronchial tubes. I once heard a dairynan call this a "chaining sound," from its resemblance to rustling of an iron chain. It is associated with bronchitis situated in the smaller tubes, and is best heard over those regions where the vesicular murmur is most audible in health. It indicates greater danger than rhonchus, and if present over a large surface of both sides, a condition of great gravity.

2. Moist sounds.-The dry, bronchial sounds are succeeded in bronchitis by moist ones, termed rales, rattles, or bubbling sounds.

(a.) Mucous Rhonchus or Rale.-The bursting of bubbles of some size, unequal and varying in number, modified by coughing and expectoration, coexisting with both respiratory movements. It is due to the bubbling of air through liquid-mucus, blood, or pus-in bronchial tubes the size of a crow quill, heard in those regions where the tubal sound is most apparent in health. Succeeding dry rlonchus, it indicates the moist stage of bronchitis.

(b.) Small bubbling Rhonchus or Ruttle, subcrepitant or submucons Rules or Rihonchi, succeeding sibilus, coexistent with loth movements, but loudest during inspiration, and due to the bubbling of air through a more or less viscid fluid in the minute bronchial tubes at their peripheral distribution; heard in bronchitis affecting the smaller tubes, and during the resolution of puemonia.

(c.) Gurgling Rattles, cavernons Rhonchi or Rales.-The bursting of bubbles, obriously of a large size, with a hollow, gurgling sound, or a metallic sound if the bubbles be small, coexisting with both respiratory morements, is associated with romicre or 
excavations from tubercle, dilatation of bronchi, pus in pleura, with a bronchial fistula and deliquescence of gangrenous lung structure.

\section{PULIIONARY SOUNDS.}

1. Crepitations.-Crepitant rale or rhonchus; compared to the solind produced by rubbing slowly and firmly between the finger and thumb a lock of one's hair near the ear, or to the crackling of salt when scattered over hot coals. It is heard during inspiration only, and indicates the primary stage of pneumonia. "Probably due to the sudden expansion of clelicate tissue, altered in its physical properties by the inflammatory state, and which probably undergoes minute ruptures."--(AITKEN.) This sound is best heard in the lower third of the chest, replacing the vesicular mummur. After continuing for a short period it may disappear, and the vesicular murmur return, indicating the resolution of the inflammation. Usually, however, the crepitation becomes fainter and fainter, and is substituted by-

2. Tubal or Bronchial sound.-The tubal or bronchial sound, when heard over the inferior portions of the thorax, indicates some degree of consolidation or hepatization of the lung tissue. It shows, in fact, that the minnter ramifications of the bronchi and air vesicles have become impervious to air, by the pressure of an exudate within and upon their walls, and that the larger tubes of the part are still perrious. In health, both vesicular and tubal sounds are emitted by all parts of the lung tissue, but can be detected by auscultation in certain parts only. For example, the tubal sound is so loud in the upper and anterior parts of the chest, as to mask or hicle the vesicular murmur to a great extent; and, conversely, the vesicular murmur being londest at the inferior portions, masks or renders inaudible the tubal or bronchial sound. When, however, those portions of the lungs, namely, the minute tubes and air vesicles, are rendered impervious-the larger sized tubes still remaining pervious-it naturally follows that the sound emitted by air passing in and out of the larger tubes now becomes audible.

In many instances, however, the tubal sound continues for a short time only, and is succeeded by-

3. Absence of sound.-When this occurs it indicates that the exudation is excessive in quantity, that the larger as well as the 
smaller tubes have been rendered impervious, or that effusion has taken place into the cavity of the thorax.

Consolidation of the lung tissue, and the presence of fluid in the thoracic cavity, can be recognised the one from the other(1.) By the previous character of the sounds; and (2.) By the manner in which the absence is marked. In hydrothorax the termination of the area of absence of sound is sharply defined superiorly; above the "water line" the pulmonary sounds are generally exaggerated. In consoliclation the area of absenee of sound has no sharply defined limit; at the lower parts of the chest, and for some inches upwards, there may be no sound at all ; then faint tubal sounds are heard, which increase in intensity as the ear is moved upwards along the thoracic walls. Now and then a blowing sound is heard in cousolidation of the lungs, confined as it were to the superficies of the lung, indicating hepatization of the deeper-seated portions of the organ, leaving the subpleural parts still pervious. My experience leads me to the conclusion that this superficial blowing sound is a bad symptom, indicating a creeping pneumonic inflammation, having a tendency to a fatal issue.

4. Secondary crepitations.-Consolidation, as above described, is succeeded by the breaking up and absorption of the exudate. This process is marked by the advent of erepitations of a bubbling nature, slowly evolved, few in number, dissimilar and irregular in occurrence, more audible during inspiration than during expiration ; they mark the resolution of pneumonia, and are suceeded by the natural sounds more or less modified. Such are, then, the sounds of pneumonia. They are subject to modifications, and to be variously mixed one with the other during the various phases of the disease.

\section{PLEURAL SOUNDS.}

1. Friction sound.-A grazing, rubbing, grating, creaking, irregularly jerking, superficial noise, heard more particularly in inspiration, or in both respiratory acts. It results from the rubbing together of the two opposed surfaces of the pleura, chiefly heard at the lower part of the ehest, where the pulmonary organs lave the greatest freedom, and is indicative of dryness of the serous surfaces, or any cause of roughness upon them. This sound is succeeded, when hydrothorax occurs, by- 
2. Absence of sound in the inferior part of the thorax, reaching to a certain line, above which the respiratory sounds are heard.

As the fluid accumulates, the absence of sound ascends. In some cases of hydrothorax one or two other sounds are heard, namely, metallic tinkling, and a gurgling or splashing sound. These sounds are only heard when gas or air and fluid coexist in the pleural cavity. The metallic tinkles may oceur from drops of fluid, imprisoned in false membranes, falling from the ronf" of the pleural cavity into the liquid beneath, or may result from the bursting of bubbles of air or gas on the surface of the liquid.

In the horse the pleural sounds are less distinct than in the ox and dog.

The sounds termed bronchophony, pectoriloquy, xgophony, and amphoric resonance, proluced by modifications of the voice during articulation, do not apply to any morbid sounds heard in the lower animals.

\section{CARDIAC SOUNDS.}

If the ear or stethoscope be applied to the lower part of the shoulder or arm, or if the left (near) fore leg be extended, and the humerus pulled forward as far as possible, the sounds of the leart can be made out.

The sounds of the heart in health are two in number: first, a longish, dull sound, then a sharp sound, snceeded by a pause, and then the recurrence of the sounds in a regnlar and uniform manner, provided the animal is not excited nor in any way disturbed. The first sound coincides with the hardening of the ventricles, the complete closure of the auriculo-ventricular valves, and the opening of the arterial orifices; is cansed principally by the sudden distension of the ventricles, and is of the same nature as the noise made by all muscles in contracting against a resistance.

The second sound is undonbtedly due to the sudden closure of the sigmoid (semilumar) valves at the conclusion of the ventricular contration. This has been proved by experiments on living animals, the sound being destroyed by hooking back the semilumar valves.

The impulse of the heart may be felt by placing the hand directly upon the left side of the chest, immediately behind the elbow. It is very distinet in flat-sided and lean animals, less 
distinct, or even entirely absent, in round-chested and fat ones. Diminished impulse, when not due to the above cause, indicates feebleness of the heart's action from disease, as fatty degeneration of its muscular tissue; hydrops pericardii, when the apex may be prevented by the effusion from coming into contact with the thoracic wall; or it may arise from weakness of the system generally, attenuation of the cardiac walls, and dilatation of the cavities. Emphysema of the lungs may also diminish the impulse, the enlarged lung overlapping the heart; and adhesions of the pericardium to the pleuræ of opposite sides may bind down the heart so that the impulse will not be felt.

The impulse of the heart is temporarily increased by any cause of excitement, fear, exercise, fever, or pain. When excessive it is called palpitation.

Increased impulse, when not traced to the above-named causes, or when it is easily induced, may depend upon organic disease of the heart. It is stronger than natural in hypertrophy of the cardiac walls, and particularly so if such hypertrophy is associated with dilatation. It may, in such cases, be slow, gradual, and donble, and this kind of impulse is due to no other condition of the heart; indeed it is one of those few symptoms which throw light upon the condition of parts within, for, notwithstanding much observation carefully recorded, we are bound to confess that, unless cardiac diseases be aggravated, we are unable, either by the character of the sounds or impulse, to diagnose them with that certainty arrived at by those who practise on the human patient.

\section{MORBID SOUNDS.}

1. A to-and-fro friction murmur, synchronous with the heart's movements, indicates pericarditis or pericardial effusion.

2. A bellows murmur with the first sound indicates mitral insufficiency; stricture of aortic orifice; disease of the aortic valves, or deposits on the ventricular surface of the mitral valves; or it may depend upon an altered condition of the blood itself, as in anæmia, in which case it resembles a churning sound, heard also in the large veins and arteries. These anæmic murmurs vary with the condition of the blood. Sometimes there is a continuous hum heard at the base of the jugnlars, and to which the French have applied the term "bruii de diable." 
3. A bellows murmur with the second sound indicates aortic insufficiency; roughened auricular surface of the mitral valves, or mitral obstruction. The most common sound heard in the horse is a murmur masking or hiding the second sound, and indicating semilunar insufficiency. When the murmur is double, occupying the periods of both cardiac sounds, it indicates mitral obstruction and insufficiency.

The sounds of the heart may be seemingly intensified by consolidation of the lungs and by hydrothorax, and diminished by emphysema of the lungs. In some instances of consolidation the sounds may be more distinctly heard on the right than on the left side: this indicates hepatization of the right lung, with compensating dilatation of the tubes of the left one.

Metallic tinkling, coincident with the cardiac impulse, is sometimes associated with the booming sounds of endocarditis.

\section{PERCUSSION IN DISEASE}

Supplementary to auscultation, percussion is a valuable aid to diagnosis, more especially of pleural and pulmonary diseases.

1. Increased resonance indicates dilatation of the bronclial tubes and air vesicles; if partial or confined to one side only, and if associated with dulness of the opposite side, increased resonance may merely indicate a compensatory respiratory effort. In such a case the respiratory murmur will also be increased. Augmented resonance is, however, generally associated with decrease or absence of the true respiratory sounds, and accompanied with wheezing, creaking, crackling crepitations, indicative of pulmonary emphysema, as in broken wind, rupture of the air cells, and chronic bronchitis.

2. Diminished resonance indicates consolidation of the lungs, hydrothorax, pleural exudations, tubercles, congestion of the lungs, pulmonary apoplexy, \&c. When confined to the inferior parts of the chest, replaced abruptly superiorly by increased resonance, it is diagnostic of hydrothorax. When the dulness gradually disappears, it is indicative of hepatization of the lung tissue, being greatest where the consolidation is most complete.

It is stated that enlargement of the heart is indicated by an increased area of cardiac dulness; for my own part, I have never been able to make this out. 


\section{CHAPTER XLVII.}

SPORADIC DISEASES-continued.

LOCAL DISEASES-continued.

(1.) DISEASES OF THE RESPIRATORY ORGANS-continued.

CATARRH AND LARYNGITTIS.

CATARRI.

Synonyms.-Common cold, coryza, a defluxion or rumning at the nose, and is witnessed in the horse, ox, sheep, and other animals.

Symptoms.-Catarrh is indicated by sneezing, runuing from the eyes, redness and dryness of the Schneiderian membrane, succeeded by a discharge, at first thin and colourless, which soon, however, becomes turbid, yellowish-white, and profuse. It is associated with a varying degree of fever, dulness, and debility.

Causes.-Alternations of temperature, hot, ill-ventilated stables, exposure to wet and cold. A strong predisposition to cold exists during the process of changing the coat. Young animals, more especially if newly brought into warm stables, are pre-eminently liable to suffer. In some instances it is complicated with laryngitis, and in all cases, if the animal be neglected, the catarrhal inflammation is apt to spread from the nose over the whole surface of the respiratory mucous membrane.

Treatment.-This is a very simple matter if adopted in time, and consists in placing the animal in a well-ventilated loose box, clothing the body if the weather be cold, feeding upon bran mashes, boiled linseed, and a small quantity of good hay for a few days.

In the early stages the nasal irritation will be much relieved 
by making the animal inhale steam, and this is best done by holding its head over a bucketful of hot water, and stirring the water with a wisp of hay. A few doses of nitrate of potash are beneficial in the early stages as a febrifuge and diuretic; if the supervening debility be extreme, tonics and good food are to be prescribed. Some writers recommend that a purgative should be given: this is a practice which cannot be too strongly condemned, as in all catarrhal affections there is a tendency to the spread of the irritation, and a purgative may cause a fatal inflammation of the mucous membrane of the digestive organs; but, provided that the effect of such treatment is not immediately fatal, a simple disorder is rendered complicated and serious, and, should the animal ultimately recover, the convalescence is much more prolonged and difficult. Any undue constipation of the bowels is best combated by laxative food, enemas of warm water, and perhaps eight or ten ounces of linseed oil. If the cough be troublesome the throat may be stimulated or lightly blistered. For the results of catarrh, see Principles and Practice of Veterinary Surgery, page 517, et seq.

\section{LARYNGITIS.}

Acute Laryngitis.-An inflammation of the lining membrane of the larynx, indicated by difficult breathing, discharge from the nose, and febrile disturbance.

Pathology and Symptoms.-Laryngitis is not an uncommon nor yet an unimportant disorder, sometimes killing quickly, and at all times a dangerous disease. The gravity of acute laryngitis depends upon the nature, character, and extent of the inflammation. In acute inflammation, embracing the epiglottis and rima glottidis, there is such a rapid and extensive effusion into the submucous tissue, and formation of mucus upon the free surface of the mucous membrane, as to canse almost a total obliteration of the glottal opening, and the death of the animal from suffocation. This rapid effusion into the submucous tissue and swelling of the membranes constitute what is termed " odema glottidis," and it is to this rapid effusion that the danger to life is due. The respirations become suddenly difficult, the inspiration being particularly prolonged, and attended with a peculiar harsh sound, succeeded 
by a short expiratory movement, sometimes, but very rarely, attended by a hoarseness. The animal's nose is protruded, the superior respiratory passages being thus made to approach as near a straight line as possible; the eyes prominent, conjunctivæ red and highly injected, with abundant flow of tears. There is a peculiarly anxious and distressed expression of the face, the alæ of the nostrils are dilated, the nasal cliambers are reddened, there is a hoarse rasping cough, sweats bedew the body, the legs and ears are cold, the latter often drooping; the animal mauifests its distress by frequently stamping with its feet-the fore ones particularly. The slightest excitement aggravates all these symptoms; the pulse, which may at first be hard and full, soon becomes rapid and indistinet-fulness generally remaining; the visible mucous membranes now assume a livid appearance from non-oxidation of the blood; prostration of strength becomes extreme; the animal staggers, finally falls, and dies after a few struggles. The above is a description of by no means a common, but of an aggravated form. of acute laryngitis. In many eases the symptoms are much less severe, but they partake of the general character of those above described. There is generally a discharge from the nose, even in the early stages, and the act of deglutition is performed with great difficulty. In some instanees this is due to the inflammation extending to the pharynx; in others, however, there seems to be no real pharyngeal complication, for if tracheotomy be performed, the difficulty in swallowing is immediately removed. It appears to me that the difficulty in swallowing is due to the momentary pressure of any liquid or solid upon the inflamed epiglottis, causing inereased interruption to the ingress of air. Restore the freedom of breathing by the operation of tracheotomy, and the passage of fluids or solids from the fauces into the pharynx no longer prevents the free ingress of air, and hence the swallowing again becomes easy. In those eases, however, in which the diffieulty in swallowing persists, the pharynx is inflamed, and the foor is returned into the nose, tinging the mucus with its own colour.

The causes are the same as those of eommon cold.

Treatment.-In an aggravated ease this must be prompt. Inhalation of steam, and hot fomentations to the throat, may be tried for a short time, but if the distress is not speedily relieved, 
tracheotomy is to be resorted to. In the milder eases, inhalations, fomentations to the throat, succeeded by blisters, with febrifuges ; light, soft diet, warm clothing, comfort, and pure air, constitute the necessary treatment.

It may be observed that when deglutition is diffieult, all medicines should be given in the animal's food or water, the latter being abundantly supplied, as enforcement may eause violent fits of coughing, and even suffocation. Belladonna sometimes has a good effect in the earlier stages; it is best given as the extract, placed between the teeth.

This disease is always sueceeded by great prostration of strength; and in order to prevent this as much as possible, milk-in conjunction with eggs beaten up, or boiled hard and powdered-should be allowed the animal to drink, alternately with water, gruel, or linseed tea. But none of these should upon any consideration be foreed upon it by horning or bottling, for, as I have already stated, this is a dangerous practice, and one ealculated not only to excite violent fits of coughing in all diseases of the throat, but indigestion, and disorder of the digestive apparatus in all other ailments, and thus destroy what little appetite the patient might possess.

Sequelc.-Thickening of the mucons membrane, ulceration of the rima glottidis, atrophy of laryngeal muscles, and follicular growths upon the laryngeal entranee.

Thickening of the mucous membrane is best removed by a course of iodide of potassium and blisters; ulceration of the rima glottidis, by solution of nitrate of silver applied to the part by a sponge fastened to a rod. The follieular growths have been removed by the applieation of solution of corrosive sublimate-forty grains to the ounce of water; and to prevent the progressive atrophy of the muscles, I would recommend a trial of the chlorate of potash.

Chronic Laryngitis.-(See Roaring.) 


\section{CHAPTER XLVIII. \\ SPORADIC DISEASES-continued.}

LOCAL DISEASES-continued.

(I.) DISEASES OF THE RESPIRATORY ORGANS-continued.

CROUP-CYNANCHE TRACHEALIS : DIPHTHERIA.

Croup.

Youxg cattle, varying from a few weeks to a few months old, are subject, when kept on low, damp pastures, more especially in meadows near rivers, and during the fall of the year, to a form of inflammation of the throat, characterised by the formation of a fibrinous exuclate or false membrane of a greyish-white colour, sometimes brown or yellow, extending orer the larynx, trachea, and sometimes fauces, or the bronchial mucous membrane.

I have never seen this disease in any but young calves, and in one district only, namely, on the borders of the River Alun, near Mold, Wales. Fowls are subject to croup, and Mr. James Law, in the Edinburgh Veterinary Review, vol. iii., page 216, hints that the carnivora are subject to it.

Croup differs from ordinary catarrhal laryngitis in a most remarkable manner. In laryngitis there is an increased formation of mucus, which is discharged as quickly as it is formed. In croup, an exudative process attends the inflammation in the larynx and trachea, which induces the formation of false membranes, varying in thickness and consistency, some of which being several lines in thickness and very opaque, whilst others are so thin that the mucous membrane is easily seen through them. Some are so firm in consistence that they can be detached for a considerable length without tearing, whilst others are almost diffluent. In colour they vary from a dirty greyish- 
white to a yellow or yellowish-brown. If an animal be examined post mortem, after the disease has existed for four or five days, the windpipe and larynx will be lined for a considerable distance, in some places partially only; in other parts the entire circumference will be embraced by the false membrane, forming a complete hollow tube or cylinder.

Some writers maintain that croupous inflammation is a more intense form of inflammatory action than that witnessed in ordinary (catarrhal) inflammation of nucous membranes. Dr. Copland, however, states that subacute or slight inflammatory action may be inferred as having existed in connection with an increased proportion of fibro-albuminous matter in the blood, whenever we find the croupal productions in the air passages. Neither of these views seems to account for the peculiarity of croupous inflammation, and I do not think that it is due to any specific cause. Indeed, direct experiment has shown that severe irritants induce the formation of false membrane on mucous surfaces; and we must rest content with the conclusion that in young animals growth is rapid, and that the new formation is so rapid in growth, and so plastic in consistence, as to remain more or less firmly adherent to the structure from which it is developed.

The causes, as already indicated, are damp and cold, particularly exposure to heavy dews at night.

Symptoms.-These generally commence with a hoarse cough, discharge of frothy saliva from the mouth, and of mucus from the nose; the animal is unthrifty, has some difficulty in swallowing; sometimes swellings appear in the parotid and submaxillary regions, succeeded by difficulty in breathing, the inspiratory act being accompanied by a crowing noise, and by spasm of the laryngeal muscles, causing violent paroxysms. In other cases the spasmodic affection of the larynx and difficult breathing may occur without any premonitory warning. The pulse, hard and quick at the commencement, becomes feeble and indistinct as the clisease advances, the fits of coughing more and more troublesome and violent; paroxysms constantly occur, particularly if the animal be subjected to any sudden excitement. In the course of two or three days flakes of the non-vascular and unorganised false membrane are coughed up; the expectoration becomes more profuse; being loosened and detached by 
a fluid poured from the mucous follicles, until finally separated and cast out; and if the case progresses favourably the false membrane is not again formed, the suppurative process terminating the inflammatory action.

If the stethascope be applied to the larynx and trachea, a peculiar trembling may be detected in places where false menbrane exists.

Treatment.-When the symptoms are very alarming, the breathing difficult, and the noise loud, tracheotomy should be immediately performed; indeed it is essential that air be admitted early in every severe case, for should the operation be delayed, the blood becomes so overloaded with carbonic acid and effete materials, that the animal succumbs to a condition of blood poisoning.

The head is to be steamed for several hours consecutively, and the hot water used for that purpose may contain carbolic acid or iodine. Nitrate of silver in solution may be directly applied to the diseased mucous membrane, as directed in a former page. The solution commonly in use contains half a drachm to the ounce of distilled water.

The medicinal treatment will greatly depend upon the condition of the animal, but little good need be expected from any very heroic remedies. In the early stages the nitrate or chlorate of potash are to be given in small doses, or the hyposulphite of soda in the animal's drinking water. If there be much prostration, spirits of nitrous ether, with camphor, may be prescribed, and in all cases a gentle oleaginous purge may be ordered if the bowels are costive. It must not be forgotten that the patient must be kept in a warm, dry shed, and have the bocly clothed if the weather be cold.

\section{DIPITIIERLA.}

Diphtheria may be described as a specific blood disease, associated with sore throat of great severity, attended with extreme prostration, and characterised by exudation of false membrane upon and pulpification of the mucous membrane of the throat and sometimes that of the nose.

In the Veterinary Journal for August 1875, Mr. W. Robertson, Kelso, reports some facts in connection with the occurrence of diphtheria in $\operatorname{dogs}$ and horses. and places on record the causes, 
spread, and subsidences in two particular outbreaks-one amongst lorses, the other amongst dogs.

Mr. Robertson restricts the term " diphtheria" to "that specific sore throat ordinarily regarded as contagious, accompanied with much systemic disturbance, and tending to laryngeal croup."

In both the outbreaks the fatality was great, all the horsesfive in number-dying; in the case of the dogs, there were three or four recoveries from between thirty and forty seizures.

As seen in the horses the seizure was in every instance sudden; the animals-farm horses in good working condition, of different ages-apparently in the enjoyment of the fullest amount of health and vigour one day, were on the following found unaccountably ill; two, indeed, were only noticed unwell in the evening, having worked all day, and fed with their usual zest, hoth in the morning and at mid-day. Attention was first directed to the animals from their inability or disinclination to drink when offered water; some pushed their noses into the trough or pail, and seemed attempting to swallow, but at once desisted, or the water returned by the nose. The breathing was at the same time noticed to be rather faster than natural, and the nose slightly pushed forward; occasional muscular tremors at this time showed themselves, and the animals were rather restless or uneasy. There was little or no cough, and when it did exist, it seemed merely to result from attempts made to swallow. The glands of the throat were slightly swollen from the first, but certainly did not increase much during the period the animals lived. The temperature rose rapidly, and continued high until shortly before death; while the pulse was accelerated about one-half, but was considerably less in volume and force. In one case there were well-marked symptoms of abdominal pain from the outset of the disease, with no abdominal organic lesion observable after death to accomnt for these. In this instance there was also very noisy breathing; this, however, was accounted for at the post mortem examination, which showed more extensive involvement of the larynx and trachea than any of the other's. None of these animals lived over the fifth day, and two of them died within forty-eight hours.

I have never witnessed a disease similar to that described by Mr. Robertson in the horse ; but during the winter 1878-9 I had the opportunity of seeing several dogs, the property of various 
owners, that had this fatal form of sore throat, and which presented signs very nearly approaching those of diphtheria as described by physicians.

The symptoms were those of great prostration and languor; flow of tears from the eyes; a very sunken and pinched appearance of the face; the power of swallowing completely lost; imability to close the mouth, which in all cases was persistently open, the muscles of mastication, as well as those of deglutition, having lost their function either from paralysis or inflammation. Indeed, the first symptom observed was a dropping of the lower jaw, and on this account it was supposed by the owners the disease was dumb madness; a more or less copious discharge of a viscid ropy saliva from the mouth, dirty yellowish-red tongue, the neck stretched and rigid, the glands slightly swollen, œdematous, and painful to the touch. Diarrhœa was present in all, and the posterior extremities were paralyzed.

The mucous membrane of the mouth and fauces was of a dark red colour, swollen, tense, and glistening from extraneous infiltration, but no ulcers were observable.

Three of the dogs died in from twenty-four to forty hours after they were first observed, but the fourth, a staghound, lived for four days, the symptoms in it having been much more gradually developed. In none of the dogs were convulsions or coma present; they all remained conscious to the last, evidently dying from diarrhœa and exhaustion.

No history could be obtained with any of the dogs. One was a lady's pet dog, and was daintily fed; another was a brewer's yard dog, always on the chain; so it was impossible, in these two cases at least, that the disease could have been induced by any tainted-infected-food, even if diphtheria had prevailed amongst the inhabitants of Edinburgh. Such, however, was not the case, diphtheria being entirely absent at that time.

The disease did not seem contagious or infectious, there being several dogs at the College at the time; some of these, owned by students, being confined in the boxes in which the diseased ones lad died for the purpose of testing its contagiousness. One dog was inoculated, the results being negative.

Mr. Robertson traces the outbreaks which he witnessed to unsatisfactory sanitary conditions of the stable and kennels. These being drained and better ventrlated the disease disappeared. 
Post mortcm appearances.-The post mortcm appearances in four different cases examined at the College in 1879 were pretty much alike. A frothy nucus escaped from the nose and mouth, and the joints remained quite flaccid for three or four days.

On eutting through the skin, the larger veins were found to be engorged with extremely black fluid blood; and on pricking an artery, blood of the same colour and consistency exuded from it. The mucous membrane of the mouth, and all other mucous cavities, was very much congested, and covered by a frothy mucus; that of the fauces and tonsilar cavities being extremely so in two cases, having commenced to undergo granular degeneration; in a third, a false membrane had formed over the glottis.

All the cavities of the heart were filled with black fluid blood, and only here was there any appearance of its coagulating, and the pericardial sac contained a quantity of fluid.

The surface of the lungs looked unnaturally red, with here and there small dark spots; and on cutting into its tissue, the same dark fluid blood exuded from it.

The stomachs, as in rabies, in two eases were filled with straw, stones, and other rubbish; but in the other two no such materials were found there.

The kidneys were also engorged with black blood.

Some interesting experiments are published in the Veterinary Journal for August 1875 as to the transmissibility of diphtheria, which the reader may profitably consult.

Treatment.-The treatment indicated by the symptoms is that calculated to destroy a virus, and for this purpose antiseptics, such as carbolic acid, might be worthy of trial. If the power of swallowing be completely lost, subcutaneous injections might be sried, and the throat repeatedly dressed with it or a solution of permanganate of potash.

Experimental inoculations with the diphtheric products of man have hitherto failed to induce the disease in animals, but it has been discovered that the serum of horses inoculated with diphtheric products furnishes an antitoxin which not only gives immunity, but has a powerful curative action when the disease is actually established in the human being; if applied in the early stages, the mortality is reduced as much as two-thirds. 


\section{CHAPTER XLIX. \\ SPORADIC DISEASES-continued.}

LOCAL DISEASES-continued.

\section{(I.) DISEASES OF THE RESPIRATORY ORGANS-continued.}

\section{INFLAMMATION OF THE RESPIRATORY ORGANS- BROX'CHITIS.}

Division.-This disease may, according to its seat, be arranged under four heads, namely, "tracheo-bronchitis," where the lower part of the trachea and larger tubes are the main seat of the inflammation; "bronchitis proper," where the medium-sized bronchi are the chief seats of the disease; "capillary bronchitis," where the smaller bronchi are chiefly implicated; and catarrhal, lobular, or broncho-pneumonia, where the smallest bronchi and alveolar walls are involved in the inflammatory process. For simplicity of description I shall retain the generic term bronchitis, dividing it into acute and chronic.

The character of the inflammation, whatever part of the respiratory tract may be affected, is what is understood as catarrhal-that is, an inflammation in which, instead of an exudation rich in fibrin, there is a fluid secretion containing a large quantity of mucus and cellular elements. In this particular it differs most essentially from inflammation of the lungs, originating in the parenchyma and from pleuro-pneumonia, in which the pleural surface as well as the lung structure is involved. The exudate in these is termed "croupous" or fibrinous.

Causes.-Bronchitis, wherever its seat, is generally dne to exposure to cold; it may supervene on an attack of ordinary catarrh, particularly if the animal be neglected, exposed to wet and cold, or kept in ill-ventilated stables. It may also arise without any premonitory catarrhal symptoms in both horses and cattle during voyages by sea, particularly if the weather be rough and stormy, and the animals battened down. During 1877 the author had the opportunity of seeing bronchitis assuming the form of suffocative catarrh, and which proved fatal to many, amongst foreign horses imported at Leith. An instructive fuct 
in connection with these cases was that it appeared only after rough and stormy passages; when the weather was fine no eases were observed. It also arises from the absorption of septic matters, as seen in post-parturient septic broncho-pneumonia, particularly when the fotal membranes are retained, and in the so-called corn-stalk disease in American cattle described by Billings (see p. 177), and as a result of drinking water tainted with the putrefactive products of animal matters. A similar condition is deseribed by I'oels, Holland, and I'rofessor E. Lienaux, Brussels, as septic pleuro-pneumonia in calves, and in which they have demonstrated a mobile ovoid microbe $.001 \mathrm{~mm}$. to .0015 long by $.0005 \mathrm{~mm}$. in breadth, identical in appearance to the one observed by Nocard, Billings, and myself (see Plate V1 ${ }^{1}$ ).

Bronehitis, like laryngitis, may be caused by the inhalation of irritant matters, and by the accidental entrance of foreign materials, as medieines or food, into the bronchial tubes. Inflammation of the bronehial tubes arising from the latter cause usually occurs in horned cattle, often as a sequel to parturient apoplexy, in which affection the power of deglutition is in a great measure lost, and where the sensibility of the glottis is, during the eomatose stage, greatly diminished or entirely absent. In such cases fluid medicines incautiously administered enter the trachea and bronchi, and these may cause immediate death by suffoeation, or if not immediately fatal, induce a severe and perhaps fatal inflammation.

Again, during the state of coma, semi-fluid ingesta are apt to flow into the mouth through the flaccid osophagus, particularly if the cow lies with its head and anterior extremities lower than the posterior ones. In parturient apoplexy there is also very often during the earlier stages some extent of antiperistaltic action of the oesophagus, with eruetations of gases from the rumen; along with such gases semi-fluid ingesta gain entrance into the fauces, and owing to the paralyzed state of the glottis fall into the larynx and trachea.

Catarrh or bronchitis, from other than mechanieal eauses, may, particularly in eattle, if the accompanying cough be long and powerful, eause some degree of vomition. The food thus vomited, or in other words coughed up, sometimes gains entrance into the trachea, and causes a fatal issue.

Along with Mr. Borthwick, Kirkliston, I saw cases of this in a herd of Irish cattle brought to Seotland, which were suffering from bronchitis and gastric irritation from neglect and exposure. 
Four of the herd became much worse than the rest, one died, and the other three were slaughtered. In all of them the bronchial tubes were filled with ingesta, ejected into the fauces during violent fits of coughing. Again, in several specimens of the lungs of American cattle slanghtered at Liverpool, supposed to be affected with pleuro-pneumonia, food was found in the bronchi. Is it not possible that during a rough voyage cattle may suffer to some extent from sea-sickness, and even vomition, and that the vomited matters may gain access into the trachea and bronchi? In others of the condemned American cattle the irritation was associated with the presence of filaria in the bronchi. Both the ingesta and the parasites were present only in a minority of the diseased lungs examined, and could therefore be only looked upon as accidental concomitants.

Food sometimes gains access into the trachea in the course of dissolution, or even after death, particularly if the rumen be rather full of moist food; it will then be found in the greatest abundance in the trachea and larger bronchi, whereas in those instances in which it has been in the tubes for some time before death, the food will often have disappeared from the larger into the smaller tubes and air cells.

I have witnessed one case of fatal bronchitis in the horse, due to the entrance of vomited ingesta into the bronchi. Some days prior to its death fifteen minims of Fleming's tincture of aconite had been administered; this brought on attempts at vomition and great distress. The animal's respiration continued very highly accelerated after the effects of the aconite had passed off, and continued until the animal died. A post mortem. examination revealed the fact that romition had occurred, and that the small quantity of food thus expelled liad entered the larynx, and gained access to the bronchi.

\section{ACUTE BRONCHITIS.}

Symptoms.-Bronchitis consists of congestion of the bronchial tissues, associated at first with dryness, narrowing, and rigidity, and subsequently moisture, dilatation, and relaxation of the tubes.

Owing to these changes, the vibrating sounds caused by the passage of air through the inflamed bronchi undergo variations, which indicate pretty clearly the dry ur moist condition of the parts, or, as some term it, the dry or moist catarrh. 
As the symptoms are developed, the cough becomes hoarse, ringing, loud, and paroxysmal; the respirations are in some instances greatly accelerated, indeed out of all proportion to the pulse. For example, the pulse may be seventy or eighty per minute, and the respirations as numerous, or even more so: this indicates bronchitis affecting the smaller tubes and alveolar walls-catarrhal pneumonia-collapse of a more or less extensive area of lung structure, or even occlusion of non-inflamed bronchi and air vesicles by the gravitation into them of the catarrhal Huid, as shown in the woodcut.

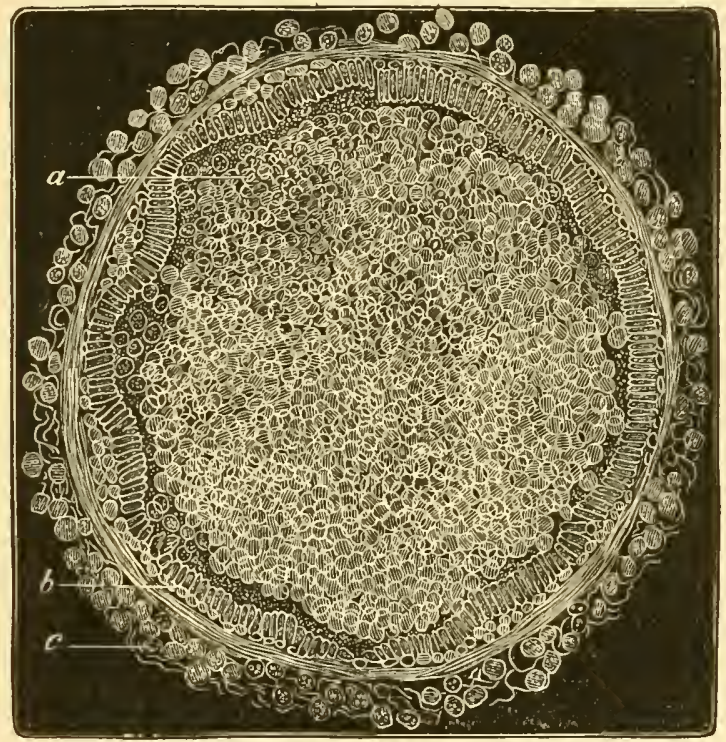

FIG. 36. - Small healthy bronchus in acute bronchitis, occluded by a plug of catarrhal secretion. -350 diam. $a$, Catarrhal plug ; $b$, Epithelium lining bronchus; $c$, Surrounding adventitious coat infiltrated with cells. -(From American ox condemned at Liverpool for pleuro-pneumonia.)

Bronchitis of the larger tubes is naturally less dangerous than the other two, and only proves fatal by inducing the two abovementioned conditions, namely, collapse and occlusion of a more or less extensive breathing surface.

Amongst the foreign horses above alluded to, it was noticed, where the discharge of muco-purulent matter was most profuse, although some of the animals seemed to recover from the febrile, 
disturbance and accelerated breatling of the acute stage, that they succumbed in from fourteen to thirty days afterwards from gangrene of the collapsed lungs, or putrefaction of the fluid incarcerated in the bronchi and air cells; both of these conditions being expressed by foetor of the breath, exhaustive diarrhœa, metastatic inflammations of the articulations and feet, complete loss of appetite, rapid emaciation, fluttering pulse; at first great elevation of temperature- $106^{\circ} \mathrm{F}$. or more; partial sweats upon the body, gasping respiration, some abdominal pain, and other signs of general septicæmia.

In no case of pure bronchitis is the breathing painful, but short and quick, the thoracic as well as the abdominal muscles being brought into full play; this distinguishes it from the breathing characteristic of pleurisy, in which the ribs are more or less fixed and the respirations abdominal. In ordinary cases of bronchitis the animal is dull, listless, sometimes semi-comatose; hangs its head; is generally thirsty; ropy saliva fills the mouth, which is hot and moist. The visible mucous membranes are injected, and present a varying degree of lividity, due to nonoxidation of the blood. The animal stands in a corner or moves listlessly about. If in a lox, and the door be open, it stands with its head to the open air, from which it evidently obtains relief. The bowels are generally somewhat constipated, the freces covered with mucus, but they easily respond to purgatives, showing that the alimentary mucous membrane participates in the irritation. The urine is high-coloured, scanty, and if examined will be found to contain urea, mucus, and colouring matter in excess, and the chlorides in diminished quantities.

As already stated, bronclitis of the larger tubes is not ordinarily a fatal disease, but when affecting the smaller bronchi and alveoli, particularly if associated with a profuse discharge of a yellowish coloured, more or less tenacions fluid, which occludes the smaller bronchi and air cells, it is the most fatal chest disease that the author is acquainted with. This tendency to gravitation of the catarrhal fluid is explained by the fact that the columnar and ciliated epithelium are shed in the earlier stage of the attack, and take no part whatever in the after changes which ensue. It is never seen again till the signs of acute inflammation, such as distension of the vessels and cedema of the basement membrane, have passed off. Subsequently it is gradually reproduced. 
The muco-purulent material thus incarcerated is driven or impacted by the ramrod-like action of the inspirated air into the periphery of the smaller tubes and vesicles, and there constitutes those masses which may undergo putrefaction in the horse, causing septicimia, as already explained, and caseous masses, mistaken for tubercle in the ox.

The physical signs of bronchitis are as follows:-Percussion returns a more or less resonant sound, but auscultation will enable the practitioner to detect the nature and extent of the bronchial inflammation. Fihonchus, confined to the upper and middle third of the chest, with true respiratory murmur over the lower part, will indicate inflammation of the larger and middle sized bronchial tubes, and a condition of comparatively little danger. Sibilus, heard at the lower parts, indicates a condition of much greater danger, and that the disease involves the smaller tubes and air vesicles. Inspiration is generally shortened, expiration prolonged, and more distinctly accompanied by the abnormal sounds. These sounds are succeeded at a later stage by moist bubbles, rattlês, or rales-mucous rales. At first the discharge expelled by coughing is thick, tenacious, and gelatinous, or watery and scant. The lower animals do not, however, expectorate in the true sense of the word; some discharge issues from the nose, but the greater part of what is coughed up falls into the fauces, and is swallowed. As the disease advances, however, a profuse discharge issues from the nostrils, and the inflammation gradually subsides. The cough becomes less hoarse, more vigorous, and even more frequent than at first; but it gradually disappears, the discharge becomes again thinner, clearer, and eventually ceases.

In some instances all sounds disappear from a certain part of the lungs. This is due to occlusion of the tubes and vesicles by the catarrhal secretion, or to more or less collapse of the vesicular tissue, dependent on obstruction to the passage of air during inspiration by glutinous or inspissated mucus. This collapse is often confined to individual lobules, which are thus condensed, leavy, indurated, and of a dark colour, and may ultimately become hepatized, atrophied, or even emphysematous. 


\section{PATHOLOGY AND MORBID ANATOMY.}

Inflammation of the bronchial tubes, like that affecting other mucous membranes, is attended with changes in their epithelium, the secretion of the glands, and in the surrounding tissues.

It is rare to meet with a fatal case of tronchitis during its earlier stages, and but for the accidental slaughter in Liverpool of the American cattle already referred to, it would have been difficult to have given the details of the morbid anatomy.

The appearance of the lung in the earlier stage of bronchitis, with collapse, that is to say, when it is observed prior to the commencement of secondary changes or phenomena, is as follows:-There are patches over its surface that have fallen below the level of surrounding parts; sometimes these depressions measure an eighth of an inch in depth; they are of a bluish-purple colour, and variable in size. The parts around them are of a light pink hue, and are either healthy or in a more or less emphysematous condition.

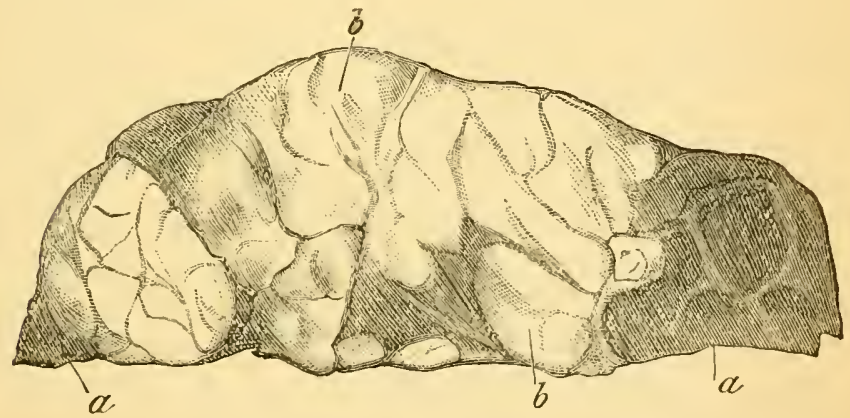

FIG. 37.-Portion of lung from American ox slaughtered at Liverpool, and showing bronchitis in the very earliest stages ( $a$ a, collapsed lobules) from obstruction of tubes. The elevations $(b b$, non-collapsed lobules) are slightly emphysematous.

The depressions consist of certain lobules in a state of collapse arising from occlusion of their bronchial tubes by pus or other material. The collapsed portions are bhish-purple in colour; non-crepitant, and depressed, resembling foetal lungs, sinking slowly in water.

Collapse of the lung tissue-atelectasis-indnces more or less 
congestion and subsequent inflammation; consequently it is found that broncho-pneumonia often succeeds bronchitis, due to the absence of the expansion and contraction of the air vesicles which normally aid the pulmonary circulation, and to arrestment of the blood-flow owing to imperfect æration. This congestion is soon succeeded by effusion of serum, and the bluish-purple collapsed portions become darker in colour and less resistant in consistence. They, however, retain some degree of elasticity, for, if not too rudely pulled out, they do not tear as in pleuro-pneumonia; if cut into and exposed to the atmosphere for a few minutes, the bluish-purple colour becomes bright scarlet. It is important to bear in mind that the pneumonic process which supervenes in bronchitis is principally confined to those portions of the lungs in which collapse has taken place. Sometimes the collapse is isolated, invading but small portions of the lungs: this condition is not rarely witnessed in parasitic bronchial disease. These limited collapsed portions vary in size, are rather wedge-shaped, and have their apices towards the obstructed bronchus. The lung tissue surrounding them may be more or less congested, or it may be emphysematous, but no juice is exuded from them when cut into, as in acute pleuro-pneumonia.

Professor Gairdner was, I believe, the first to show that condensation of the vesicular substance occurs as a result of mucous or other obstruction in the air-tubes leading to the condensed portion. It is at first sight difficult to understand how incomplete obstructions of the bronchi-and these obtain much more frequently than absolutely complete occlusion-cause collapse. One would suppose that some quantity of air would gain access into the vesicles, but such is apparently not the case; and it seems that the air gradually finds its way out by the edges of the obstructing substance. The expiratory force, so long as there is air in the vesicles, constantly tends to dislodge the obstructing body by pushing it towards the wider (proximal) end of the tube, whilst the inspiratory drives it inwards towards the narrower tubes, which it effectually occludes. The entrance of air is thus more or less effectually opposed, and its exit permitted, so that ultimately the vesicles beyond become completely emptied; in fact the plug acts as a valve, allowing the air to pass in one direction, but opposing its passage in the other. Where the 
obstruction is complete from the commencement, the air is absorbed.

It had been supposed by Laennec that the emphysema, or, more correctly, the over-clistension with air of the parts surrouncling the collapsed lobules, was due to what he thought a fact, that the act of inspiration was more powerful than that of expiration, so that though air could be drawn through the obstruction, it could not be breathed out. In consequence, it accumulated in the ultimate pulmonary vesicles, became expanded by heat, and so acted mechanically as a dilator. Dr. Gairdner, however, pointed out that expiration is a much more powerful act than inspiration, and that there is never any difficulty in causing expulsion of air, provided always there be no obstruction in the tubes. Emphysema, then, does not occur in the vesicles connected with obstructed tubes, but in those which are adjacent. When the lungs are free from disease the column of air presses equally in all the tubes and vesicles; but when one portion connected with any obstruction is collapsed, then the adjacent parts are over expanded, so as to occupy the space previously filled by the former.

At a later stage the contents of the obstructed bronchi are pushed by the weight of the descending or inspired atmosphere into the most minute bronchi, alveoli, and air vesicles, always from the centre towards the periphery, and appear as minute white points beneath the pleural surface. They are well shown in the figure.

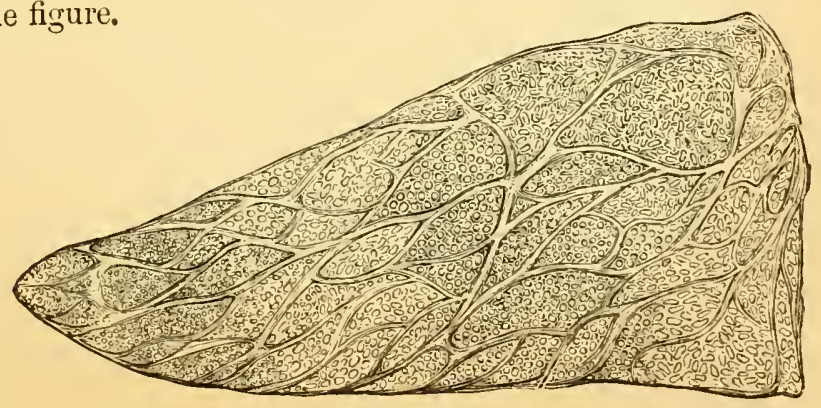

FIG. 38.-Pleural aspect of pulmonary lobe from American ox slaughtered at Liverpool ; alveoli filled with muco-purulent matter; pleural surface intact. The microscopic examination revealed broncho-pneumonia in some of the alveoli (see fig. 40); whilst others showed no traces of inflammation (see fig. 36), but were merely filled with the inhaled bronchial secretions. 
On cutting into the lungs, it will be found that the large and small tubes, and sometimes the trachea, contain an amount of fluid. This condition, as well as the collapse, is limited in the majority of instances to the small or anterior lobes of the lungs, and rarely, except by extension, affects the large lobes, not only in ordinary but in mechanical bronchitis. This fact is of importance, as pleuro-pneumonia contagiosa, with which the disease under consideration has been confounded, generally commences in the larger lobes, either in their centres or towards their posterior edges.

The fluid contained in the tubes is thick, and has a yellow colour; in the trachea it is more or less frothy; and is abundant in the smaller bronchi. as shown in the figure.

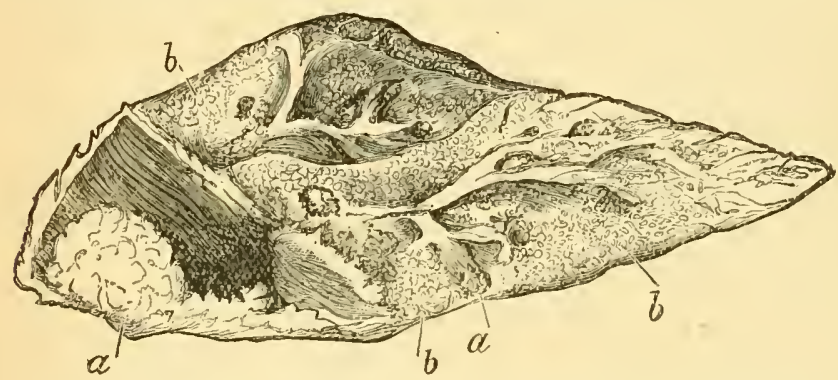

Frr. 39.-Section of portion of lung; the external aspect is shown in fig. 38. The larger $(a)$ and smaller bronchi and air vesicles $(b)$ filled with purulent matter.

If the lungs in this condition be squeezed, little pellets of yellow matter are pressed out: sometimes these pellets are too small to be seen by the naked eye, and require the aid of a magnifying glass. If the bronchitis be associated witin catarrhal pneumonia, elevated patches will be apparent on the cut surface, having a greyish-red colour. They are soft to the touch, and if squeezed, the same muco-purulent matter exudes from them, or from a small bronchus which may happen to communicate with the particular group of vesicles implicated.

Dr. Hamilton, in his series of papers on bronchitis published in the Practitioner for 1879 , states it is a matter of difficulty in man to get at the first change which ensues in the bronchi in acute catarrh. He has, however, been able to verify his observations by an examination along with myself of the lungs of American cattle slaughtered in the earlier stages of bronchitis; 
in fact before any external signs of disease were manifested. He says - " On careful comparison, however, of many cases, we feel assured that the first deviation visible is a relaxation and distension of the abundant plexus of blood-vessels ramifying in the inner fibrous coat, immediately beneath the basement membrane - that is to say, of the branches of the bronchial artery. They become engorged with blood, so that on transverse section they appear like little cavities distended with blood corpuscles. In a few hours afterwards the basement membrane ${ }^{1}$ becomes much more apparent than it usually is, and at the same time more clear and homogeneous, while the surface is thrown into many folds. These changes in the basement membrane are apparently due to its becoming odematous, serous fluid being infiltrated into it from the underlying plexus of distended vessels; and we shall see that, as the acute irritation continues, this adematous state of the basement membrane becomes more and more a well-marked feature. The next change, so far as we have been able to calculate, occurs in from twenty to thirty

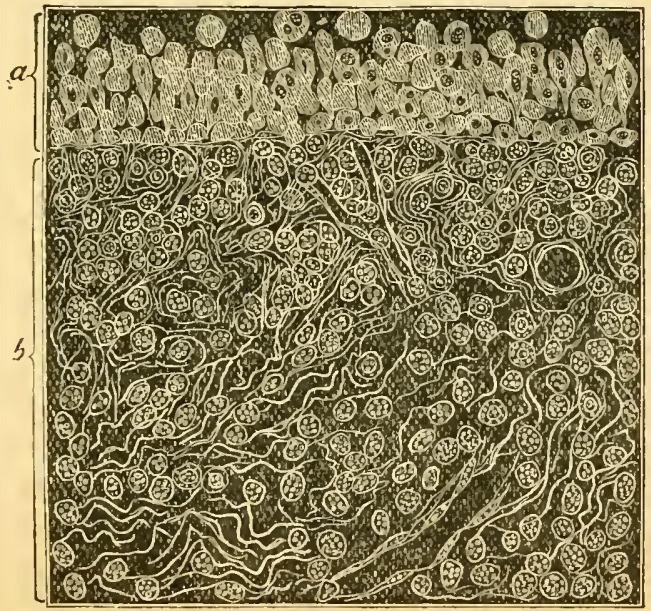

Fig. 40.-Bronclius (medium sized) in acute bronchitis.--(American ox slanghtered at Liverpool.)

(a) Deep layer of epithelium, germinating and throwin off catarrhal cells.

(b) Inner fibrous coat, infiltrated with inflammatory cells. (480 diam.)

The columnar epithelium shed.

1 The basement membrane is not so apparent in the lower animals as in man. 
hours after the primary distension of the vessels, and consists in the loosening and desquamation of the columnar epithelium at the foci of greatest congestion.

"The columnar epithelium is thus shed at a very early stage of the attack, and takes no part whatever in the after changes which ensue. It is never seen again until the other signs of acute inflammation, such as the distension of the vessels, and œdema of the basement membrane, have passed off. Subsequently we shall see that it is gradually reproduced. The cause of this desquamation of the columnar epithelium seems to be the œdema of the basement membrane loosening its underlying attachments, very much in the same way as the vesicles which form in an acute inflammatory affection of the skin loosen the attachments of the superficial layer of epidermis. The removal of this protective covering from the mucous membrane naturally leaves the latter in an exposed condition, and no doubt the feeling of rawness experienced in acute catarrh of the bronchi is due to the cold air acting upon an over-stimulated and exposed mucous membrane. And, further, it can easily be understood that, where this desquamation takes place to an inordinately great extent, the loss of the ciliary action of the columnar cells will seriously interfere with expectoration, and tend to cause the catarrhal products to gravitate downwards towards the smaller bronchi and air vesicles. This description essentially coincides with what Socoloff found experimentally in animals (Virchow's Archiv, vol. lxviii, p. 611), in which he induced an artificial bronchitis by the injection of irritants, such as potassic bichromate, into the air passages. He states that one of the first changes which ensued was the desquamation of the columnar cells, and that they took no part in the catarrhal inflammatory process." This early shedding of the columnar cells, and their non-reproduction until after the subsidence of the inflammatory process, is a fact of real importance, as it goes a long way to explain the occurrence of those caseous tumours mistaken for tubercle, and so often confounded with that growth.

The pneumonic process, which may supervene either by extension of the inflammatory process from the tubes to the alveoli, or the irritation of inhaled inflammatory products subsequent to collapse, is, in the earlier stage, commonly limited to scatterer groups of air vescicles, hence the term lobular which is applied 
to it. It causes the portions affected to appear as scattered, illdefined nodules of consolidation, irregular in size, and passing insensibly into the surrounding tissue, which is variously altered by collapse, emphysema, and congestion. These nodules are of a reddish-grey colour, faintly granular or smooth, slightly elevated, and soft in consistence. As they increase in size they may become confluent; and in a more advanced stage they become paler, drier, firmer, and to some extent resemble ordinary grey hepatization.

Microscopically examined, they are seen to consist of cellular elements accumulated in the alveoli.

The disease may, as already remarked, terminate fatally by the absorption of the putrescent catarrhal products, by gangrene of the collapsed lungs, or by sudden effusion of fluid into the bronchi, constituting what is termed suffocative catarrh. If a fatal termination does not ensue, the contents of the alveoli undergo degeneration, and are gradually removed, by discharge, or by absorption; or, by coalescence, form caseous masses, which may become encapsuled, undergo the calcareous change, and thus become innocuous; or may induce a diathesis favourable to the actual development of tubercle in the ox, and to symptoms

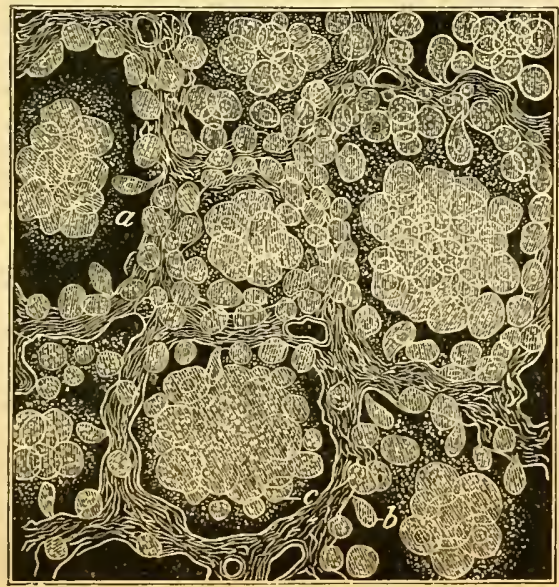

FIg. 41.-Acute catarrhal pneumonia (American ox).Section through several air vesicles. Shows the alveolar cavities filled with large granular catarrhal cells $(c)$. (b) Catarrhal cells sprouting from the alveolar wall. (a) Coagulated mucus in which the catarrhal cells lie.-(480 diam.)

simulating phthisis pulmonalis in the horse-that is to say, an 
accumulation of catarrhal products, epithelial and other cells, within the pulmonary alveoli; cellular infiltration and thickening of the walls of the alveoli and bronchi; incrense in the interlobular comective tissue, with, in some instances, the occurrence of fibrinous masses, intermixed with leucocytes in the alveoli, as demonstrated by Zenker of Dresden, but without the occurrence of tubercular tumours (grapes) in the serous membranes and parenchyma of organs.

In all cases of bronchitis the bronchial glands undergo some change. In the earlier stages they are increased in size, contain the products of the bronchitis conveyed by the lymph tract; become more or less friable in consistence; and in more advanced bronclial inflammation distended with catarrhal elements; botl glands and contents undergoing the cascous metamorphosis, the products of which may either liquefy or become infiltrated with calcareous matter.

\section{TREATMENT.}

Venesection is to be avoided; indeed bronchitis is characterised by depression and debility from its earliest stages. In the very commencement of the disease the irritation is generally modified by a moderate dose of opium. In the horse, ox, and sheep remedies termed expectorants are quite useless, having no effect. In the dog, however, they are said to act. They are antimonial wine, ipecacuanha, hyoseyamus, \&c.; but I do not think that they are of much service, and are not to be compared to chloral hychate in from two to four grain doses. The great principle of treatment, however, in all animals after the very earliest stage of the disease has passed off-when, and then only, it may be possible to cut short the attack by a full dose of opium - is to assist in promoting the natural course and termination of the inflammation. It has already been pointed out that in the earlier stages the bronchi are dry, and that subsequently they become moist; and as the moist stage becomes, as it were, matured, the irritation disappears. Such, then, is the natural course, and the practitioner is to assist in promoting this by causing the animal to inlale steam, medicated or simple, the medicated steam being made by adding camphor, creosote, or carbolic acid to the hot water. When the discharge is profuse, 
indicating the involvement of a large area of tubes, and a condition of real danger, the inhalation of steam is of the utmost importance, as such inhalation modifies the viscidity of the catarrhal fluid, and facilitates its discharge from the bronchi. And in order to prevent as much as possible the putrefaction of the catarrhal product, such steam should be medicated with carbolic acid. In addition to this the sides are to be bathed with hot water, and rubbed over with oil or a weak liniment to keep off the sensation of cold. If the bowels are costive, enemas are to be administered, but on no account are aloetic purgatives to be given; for obstinate constipation-a very rare complication - a moderate close of oil may be giten. It is far better, however, to keep the alimentary canal in proper order by enticing the animal to eat laxative food, such as linseed mashes, carrots, or grass, if in season. Some practitioners recommend that sulphate of magnesia be given in four-ounce doses daily in the horse's water until the bowels respond. For my own part I have found that horses generally refuse to drink such water, although they may suffer much from thirst; and I generally content myself with ordering half-ounce doses of nitrate of potash twice daily in the water or mash. Good nursing, warm clothing, pure air, and a good stable or loose box, are essentials which are not to be forgotten.

In the course of bronchitis, it will frequently be observed that symptoms simulating those of diabetes insipidus become developed; the animal becomes very thirsty and urinates profusely. These need not cause any alarm; they are due to the absorption and elimination of effete materials and various salts - chloride of sodium particularly - which have been retained, probably in the inflamed part, during the active stage of the discase. If the patient be freely supplied with water, and enticed to partake of good food, the diabetic symptoms pass off, and the animal will speedily become convalescent.

Should debility remain, with cough and irritation, hyoscyamus and tonics are to be administered; and if the case threatens to become chronic, a blister may be applied to the throat and breast, and the iodide of potassium prescribed.

Chronic bronchitis in the horse may cause what is termed thick wind, chronic cough, and eventually emphysema from rupture of the rigid and altered air vesicles and small bronchi. 
Metastatic or embolic broncho-pneumonia results as a sequel to septic metritis, absorption of products from septic wounds, or from impure drinking water or tainted food.

\section{CURONIC BRONCHITIS,}

As indicated by a lond, metallic cough, emaciation and debility, is not an infrequent disease, particularly in horned cattle, and is due to thickening of the bronchial and alveolar walls, and to caseous masses. It is best treated by hydrocyanic acid, in doses varying from $\eta \mathrm{xx}$. to $\Pi \mathrm{lx}$., Scheele's strength, in combination with nitrate of potash and bicarbonate of soda, twice per day, a strong blister to the breast, and careful housing and nursing. A morbid change, termed bronchiectasis or dilatation of the bronchial tubes, arising from a chronic interstitial pneumonia, induced by acute croupous pneumonia, broncho-pneumonia, pleurisy, and the inhalation of solid irritating particles, is witnessed in the human being. It is characterised by thickening of the interlobular septa and alveolar walls; and when the fibrosis is extensive, the lung is diminished in size, the tissue is smooth, dense, being in parts almost cartilaginous in consistence, and irregularly mottled with black pigment. The alveolar structure of the lung is in most parts destroyed, and on section the dilated bronchi are seen as numerous large openings scattered over its surface. The dilated bronchi frequently become the seat of secondary inflammatory processes, which inay lead to ulceration, and ultimately to extensive excavations of the indurated tissue; but there is a complete absence of caseous changes. The pleura is almost invariably thickened and adherent, the new formation in the earlier stages usually contains new blood-vessels, but later the tissue contracts, and the vessels become destroyed; the contraction of the false membrane may also induce deformity of the chest, and twist the neck to one side. I have seen a case similar to the above description in a dog in which the lungs contained quantities of sand. This dog had been several years in Africa hunting with its master. Before death it was observed that the right side of the chest was depressed, and this was found to be due to carnification and contraction of the adherent lung and connecting new formation. 


\section{CHAPTER L。 \\ SPORADIC DISEASES-continued.}

LOCAL DISEASES-continued.

\section{(I.) DISEASES OF THE RESPIRATORY ORGANS-continued.}

\section{CHRONIC EMIPHYSEMA OF THE LUNGS.}

Cinronic Empirsena of the lungs is of two kinds, namely-(1.) $V$ esicular, due to enlargement and dilatation of the air cells, with gradual effacement of the blood-vessels distributed over their walls. There is anæmia of the affected parts, a tendency to dilatation of the right cardiac ventricle, and a disposition to anasarca of the limbs. The dilated cells vary in size, and when very large it is probable that several vesicles are dilated into one cavity by rupture of the vesicular walls and partitions. These dilatations may be clearly seen through the pulmonary pleura, as they protrude from the surface of the lungs; they are pale, sometimes quite white, the tissue drier than natural. Vesicular emphysema is seen in chronic broken wind, and is due, as already explained, to degeneration of the bronchial tissues. It is, however, a result of bronchitis, and several theories are put forward to explain the origin of emphysema. Dr. Elliotson considers that a want of due expansion of the lungs is the most common cause. He says- "Whatever prevents any one part of the lungs from expanding when the thorax expands, whether it be a material obstruction of the bronchial ramifications or a compression of them, or whatever else, it will occasion those parts which remain dilatable to keep dilated in a corresponding increased degree, in order to fill up the vacuum which the expansion of the chest occasions. Wher we inspire we dilate the chest, and the air rushes down the trachea, and the lungs follow 
the dilated portions. If there be any part of the lung that will not, dilate, then other parts are over-cilated to fill up the vacuum, and in that way those parts which we distend are overdistended, in order to compensate for the want of distension in other parts; and when once over-distended they are often unable to recover themselves, just as is the case in other parts of the body-the urinary bladder for example.

2. Interlobular Emplyysema, or accumulation of air or gases in the meshes of the comnective lung tissue, may arise from rupture of the walls of the air vesicles. This result of rupture is, however, an uncommon occurrence, for generally, when the cells are ruptured, they break one into another, and form permanent enlargements, with rigid walls, by coalescence; but acute emphysema is, however, seen in various diseases, especially of horned cattle, from rupture of air cells or from the evolution of gases, which infiltrate and distend the pulmonary areolar tissue. It may be induced artificially by the injection of the bicarbonate of soda into the veins. It is also seen in an animal which has been destroyed by "blowing," i.e., by forcing air into the jugulars.

Snarry, of York, in a private letter to me, records sereral cases of subcutaneous emphysema in cattle, the synptoms of which were as follows:-

The breathing is short, catchy, the air expelled by a double effort, and often associated with a grunting or moaning expiratory sound. In most cases the head is drooping, eyes duli, and the ears flaccid; whilst in others the animal manifests great anxiety, and even some amount of dread or terror, as if each respiratory movement were a source of pain, and possibly there is actual pain, caused by the tissues being dissected by each ingress of air; indeed it may be said that when the patient has completed one respiratory movement its seems to dread the pain of the next. There is always some stiffness of gait, particularly when turned round, and in many instances a subentaneous emphysematous swelling makes its appearance, sometimes on the first day, but more frequently on the second, third, or fourth day, or even later, after the manifestation of illness. This emphysematous swelling may be first noticed on one or both sides of the ehest, the loins--one or both loins ; but it gradually, sometimes quickly, extends from its original seat to neck, head, 
back, sides,-in fact all over the body. The crepitations may not be very pronounced at first, probably from the fact that the emphysema is deep-seated, and mechanically pushing the superimposed tissue in the outward direction; but in a very short time the crackling sounds emitted on pressing the parts leave no doubt as to its true nature. The bowels are generally constipated, and some recover when free action of the bowels has been established. Some of Mr. Snarry's cases occurred prior, others subsequent, to parturition. In none of the cases was there any great elevation of temperature, $103^{\circ} \mathrm{F}$. being the highest.

\section{TREATMENT.}

No permanent benefit can be expected from any treatment. Much relief may, however, be afforded by careful dieting, keeping the bowels regular, and when the paroxysms of dyspncen are very great, by the administration of sulphuric ether, chloral hydrate, or the bromide of ammonium. A horse thick in the wind, or suffering from chronic cough, is an unsound animal. 


\section{CHAPTER LI.}

\section{SPORADIC DISEASES-continued.}

\section{LOCAL DISEASES-continued.}

\section{(I.) DISEASES OF THE RESPIRATORY ORGANS-continued.}

\section{CONGESTION OF THE LUNGS-PULMONARY APOPLEXY-MECHANICAL ENGORGEILNT.}

APART from inflammation of the lungs, which will be described immediately, the lower animals, particularly the horse, are apt to suffer from congestion of the true pulmonary blood-vessels, i.e., the branches of the pulmonary artery. This congestive condition is not only seen during the progress of many diseases, such as laminitis, traumatic arthritis, enteritis, pneumonia, heart affections, and various prostrating epizootics ; but often originates in the horse during severe exertion, more especially in the hunting-field when the animal is not "in condition," or is taxed beyond its strength. As a question of jurisprudence, the occurrence and results of congestive pneumonia, or, more correctly, pulmonary apoplexy, is a matter of some importance, for many instances have occurred, and will doubtless again occur, of horses dying from this affection within a few days after purchase, and the seller has been more than once mulcted in damages to the full value of the animal.

In order to bring a horse into "condition" for severe exertion, it is necessary that it be gradually trained. First of all, it is for a time walked for one or two hours daily, in order that the locomotor muscles be gradually brought into tone for stronger and severer work; then it is trotted, and afterwards galloped and sweated. Useless fat is thus removed, and the muscles of locomotion, as well as those of respiration, are brought into a condition and tone which enable them to perform the severest and fastest exertion; the tone and power of the heart are also 
increased and elevated, whereby it is enabled to pump an increased supply of blood to all parts of the body, in order to maintain their strength and integrity during the time they are so greatly taxed. In fact, it may be said that the horse's respiratory organs - wind-circulation, and muscular action, are elevated into a high state of functional perfection by careful training, and a horse in this condition will perform during a long run with the hounds or on the race-course, without danger to his health and life. But if a horse that is not thus trained-not in condition, no matter how good its health might be-is suddenly put to severe and prolonged exertion, when neither its muscular, pulmonary, nor circulatory systems are fit to undergo the fatigue consequent thereon, it will be seen that, first of all, the breathing becomes frequent and distressed; the heart beats tumultuously, but with little impulsive force; the voluntary muscles, consequent upon want of tone and exhaustion, obey the will imperfectly. It goes "all abroad," as the horseman says. The breathing becomes more and more distressed, and at last it falls, and perhaps dies from actual suffocation, consequent upon the pulmonary vessels being overloaded with non-oxidized blood; and after death the lungs are found gorged with blood, black in colour, and prone to rapid decomposition, giving origin to the expression "black as your hat, and rotten as a pear," and to the idea that the animal had suffered from some chronic disease. In giving an opinion on a case of this kind, the veterinarian must bear in mind that the blackness, tendency to putrescence, or even deliquescent condition of the lnng tissue, are results of acuteness of attack, and not of any previous disease; and it may always be accepted that mere engorgements and blackness, without the formation of an exudate, are positive evidence that the disease is not of long standing.

Another cause of congestion of the lungs is actual want of air in badly ventilated stables, the congestion liere arising from the stop-cock action of the pulmonary capillaries already described -(see Death by Suffocation) - which contract, and prevent, as it were, as much as possible the passage of impure blood into the left side of the heart and systemic circulation. Pulmonary apoplexy is also termed hæmorrhagic infarction, and, as explained by Dr. Yeo, is "universally admitted to depend on a local impediment to the circulation, such as an embolus impacted 
in an artery. There being no arterial anastomosis in the lung, such a plug has a very marked effect. The embolus cuts off the normal supply of blood from the part, and the pressure in the arterial branches beyond the stoppage falls to zero. The blood, however, can still find its way through the capillaries into the branches at the distal side of the plug. The branches of the occluded artery are thus reduced to the condition of occluded veins, and as they have none but capillary connections, they may be said to form blind ends to the adjacent arteries. The blood then trickles into these arterial branches and fills them, but no outward flow can take place, therefore they become intensely engorged with stagnant blood. Under these circumstances, the inner coat of the vessel is deprived of its nutrition, for which the constant renewal of the blood is required. This starvation of the minute vessels renders them unfit for their function; they lose their power of retaining the blood, which escapes into the neighbouring textures, forming the dense black consolidation now known as hæmorrhagic impaction."

\section{SYMPTOMS.}

The symptoms of pulmonary apoplexy are very distressing. The animal stands with outstretched legs, and seems to fight for breath; the nostrils will be seen opening and closing quickly, the flanks to heave rapidly; the eyes are blood-shot and wild, or sunken and dull; there is a tremor all over the body; the legs and ears are deathly cold, and cold sweats bathe the body: the pulse is small and indistinct ("the oppressed pulse of pnenmonia" of the old writers), perhaps beating from 100 to 150 per minute in extreme cases; the heart's action tumultuous, but without strength.

In some cases there will be some discharge of frothy blood from the nose ; in others hæmoptysis is absent, and if the jugular or other vein is opened, the blood trickles from it, black, thick, and scanty. Death occurs by apnœa. ${ }^{1}$

1 The term apnœa is used by physiologists to indicate a condition of breathlessness induced by excess of oxygen (Handbook for the Physiological Laboratory, by Sanilerson and others, page 318 ), and by pathologists that of suffocation or asphy ria 


\section{TREATMENT.}

In the first place, it is necessary to have the horse so placed that it can obtain as much pure air as possible. It is therefore to be tied with its head to the box or stable door, the body is to be smartly rubbed, not knocked, with wisps of hay or straw, and afterwards warmly clothed. When the surface of the body and extremities are very cold, I have found it to be a good practice to place the feet in hot water and bathe the legs for several minutes, then rub them over with a stimulating embrocation, and bandage them in thick flannel as high as possible. If proper bandages cannot be obtained, hay or straw ropes are to be substituted. The medical treatment must be that which is calculated to equalise and balance the circulation, and for these purposes stimulants are to be recommended, such as the ethers, spirits, wine, or even ale when notling else can be obtained. I have also found that the tincture of arnica, in one or two ounce doses, acts very satisfactorily; it seems to stimulate the cutaneous circulation, and on this account is well calculated to restore the equilibrium of the circulation. If the congestion does not give way to the above treatment, bleeding is to be resorted to, in order to relieve the pressure on the pulmonary vessels, and the engorgement of the great veins and right side of the heart; from four to six quarts of blood may be withdrawn with safety, but it is better not to repeat it. I am of opinion that it is a cruel practice, and one calculated to do harm, to apply mustard or other powerful irritant to the sides. Horses, when thus treated, begin to be excited in a few minutes after the irritant has been applied, paw, move about, lie down, and perhaps roll in pain; the breathing becomes more accelerated, and loss of strength rapidly follows. I hold it to be imperative that the veterinarian should enjoin perfect quietude and repose, and if anything is to be applied to the sides or breast, let it be warm water, in order to soothe and calm the distressed sufferer.

After the symptoms of congestion have passed away, it is not improbable but that they may be succeeded by those of inflammation of the lungs. Bearing in mind the probability of this sequel, the treatment of the animal for some days must be of the most careful description; the diet must be light, sparing, and easy of digestion; the water must be supplied abundantly, 
and even in the earliest stage, if the animal be thirsty, water is to be freely allowed; doses of nitrate of potash given in the water or mash; the air of the stable must be abundant and pure - but the horse is not to be exposed to draughts-and the body kept warm by clothing.

\section{POST MORTEM APPEARANCES.}

The post mortem appearances are, congestion of the pulmonary vessels, with rupture of some of them, and extravasation of blood into the parenchyma, constituting pulmonary apoplexy, whilst others are plugged by emboli. The lungs resemble the spleen, and the term splenification has been applied to this condition. When cut into, the lungs present a deep, dark, purple colour, the vessels are filled with dark blood of a tarry consistence, whilst here and there, interspersed thronghout the lung substance, därker points are seen, indicating where extravasation has occurred. Although much heavier and more condensed than natural, the lung tissue will generally float in water; thus differing from the condition of hepatization, which is present when death has occurred from pneumonia. The right side of the heart and great veins are filled with blood of a dark appearance and tarry consistence. The left side of the heart also contains some quantity of blood of the same appearance and consistence.

It may be stated that hypostatic congestion may occur post mortem or during the death struggle. This always occurs in the most depending part of the lungs, is to be distinguished by its situation and the antecedent symptoms, the animals having died from other diseases, and is not to be confounded with any diseased condition of the lungs. 


\section{CHAPTER L II. \\ SPORADIC DISEASES-continued.}

LOCAL DISEASES-continued.

(I.) DISEASES OF THE RESPIRATORY ORGANS-continued.

\section{LNFLAMMATION OF TIIE LUNGS-LOBAR (SPORADIC) PNEUMONIA.}

Synonyms and Varieties.-Peripneumonia; peripneumonia vera (as opposed to peripneumonia notha, or capillary bronchitis); fcbris pneumonica; acute pneumonia; chronic pneumonia; lobar pneumonia ; interlobular pneumonia (an affection of the interlobular tissue); primary pneumonia, secondary pneumonia (signifying differences in origin); specific or glanderous pneumonia. I retain the terms sporadic and lobar, as being indicative of the origin as well as of the seat of the disease.

\section{PATHOLOGY.}

Congestion or engorgement of the lungs has been already described as a result of arrested pulmonary circulation arising from over-exertion, debility of the heart's action, embolism, or to any other circumstance preventing the due arterialization of the venous blood, and causing hæmorrhage from or stasis in the terminal branches of the pulmonary artery.

Inflammation of the lungs is a disease in which all the textures of the pulmonary substances are more or less involved; and this distinction may be made between congestive and inflammatory pneumonia, that, in the first the lesion lies primarily in the pulmonary vessels, and, in the second, in tissues supplied by the branches of the nutrient vessels of the lungs, namely, the bronchial arteries, the pulmonary branches becoming subsequently involved, and having no inconsiderable share in 
furnishing the exudative materials, which characterise the condition of hepatization or consolidation that occurs in pneumonia.

The various changes which occur in the course of pneumonia are-1st. Arterial injection, characterised by a brighter colour, and dryness of the pulmonary tissue, a condition indicated by harsh respiration and crepitating sounds. $2 d$. The arterial injection is succeeded by a condition of engorgement, congestion of the pulmonary vessels, and incipient œdema of the lung. The substance of the lung is engorged with blood and bloody serum; externally it is of a dark red colour, and crepitates but slightly under pressure; it also pits under the finger, and is more easily torn than healthy lung; is heavier than natural, inelastic; its cells are filled with liquid, mixed with air. On being cut a large quantity of bloody serosity escapes from the cut surface, which is frothy in the earlier stages from admixture with air. This, along. with the commencing consolictation, proves that the effusion has been poured into the bronchial tubes and air vesicles as well as into the pulmonary tissue. During this stage the lung tissue will still float in water, although it is heavier and less crepitant than natural.

If a very thin section be made, and examined microscopically, the capillaries will be found filled with blood; the air vesicles enlarged and granular, with incipient division of their nuclei; and exudation corpuscles, mingled with red globules, which have escaped from the vessels and are seen in the alveoli.

3d. Red Hepatization.-If the inflammation continue, other changes occur in the lung substance. The lung is red externally and internally; is solid, sinks in water, and no longer crepitates under pressure ; it, however, tears easily and breaks down under pressure; and on this account AnIral objected to the term hepatization, and termed the second stage of pneumonia red softening-ramollissement rouge. The colour is less livid than in the earlier stage, being a dull reddish-brown, becoming brighter on exposure to the air. This reddish-brown colour is never very uniform, and in some animals diversity of colour is very marked: thus in the ox the inflamed lungs present such a variety of colour as to cause the term "marbled" to be applied to it. This is due to the very distinctly lobulated anatomical character of the ox's lungs, and the consecutive nature of the lobar pneumonia. Dark red spots indicate the 
earlier or second stage and the grey spots the third or later stage of the disease, which is called-

4th. Grey Hepatization, termed by Andral ramollissement gris, or grey softening. In the horse, perhaps the term grey softening may not be inappropriate, but in the ox solidification expresses the condition much more accurately. The cut surface of the part which has run on to this stage presents a grey tint, the redness of the preceding stage liaving passed away, and the granular character is less distinct. In the horse, dog, \&c. the tissue has very often lost its firmness, and has become soft and pulpy, and an abundant dirty-looking puriform material oozes from the cut surfaces. In the ox the grey portions are generally smooth, glistening, and firm. In some instances the smooth, glistening appearance is observed in the inflamed lungs of horses, but the firmness is rarely so great as in the lnngs of oxen, in which the tissues of consolidated parts retain their marbled appearance, and acquire a resisting character, or are transformed into a more or less firm cheesy material. In some instances, however, the grey exudate, even in the ox, is broken down into a more or less pulpy material.

If a portion of hepatized lung be torn, and the torn surface examined with a magnifying glass, the tissue will appear to be composed of a crowd of small red granulations, lying close to each other, air vesicles clogged up, thickened, and made red by the inflammation. In the catarrhal form of pneumonia commonly seen in the horse, the exudative products are mostly accumulated in the interior of the air vesicles, but in the exndative form (croupous) seen in the ox, and in cases of pleuropneumonia in the horse, the exuded materials not only fill the air vesicles but the interstices of the pulmonary connective tissue.

Suppuration of the lung is a more advanced state of grey liepatization; the lnng is softer, yellower, and more pulpy, but the condition is not materially distinct from the others, as pus cells are present in the advanced stages of pnenmonia, and the distinction between softening, grey hepatization, and suppuration is more one of terms than of reality. Diffuse suppuration of the lungs is not rarely witnessed in newly calved cows; exposed to wet and cold, it runs a rapid course, terminating fatally in three or four days, the lungs being engorged with puriform materials. It is a 
remarkable thing that suppuration of the lungs very rarely runs on to the formation of abscesses when the inflammation is not due to a specific cause, such as glanders or pyæmia. I have certainly seen abscesses in the lungs of both oxen and horses, but the event is a very rare one, and Sir Thomas Watson endeavours to account for the rarity of pulmonary abscesses in a very ingenious manner. He says-" "When I was speaking of inflammation in general, I pointed out to you the remarkable influence which the presence of air in contact with the inflamed part has in accelerating or determining the event of suppuration. In a recent cut through the skin the admission or the exclusion of the air to the cut surface will make all the difference between the adhesive and the suppurative inflammation; and so in other cases which I then mentioned, and will not now trouble you by repeating. Now it seems to me that the same principle obtains in inflammation of the lung. First, there is an effusion of serum and blood, then of lymph and blood; but the air, passing into the surrounding sounder tissue, and penetrating for a time even the inflamed portion itself, causes the suppurative process to supersede the adhesive; and so no wall of circumvallation is formed by the coagulable lymph, as is the case in areolar tissue when not accessible by the air."-(WATson's Lectures, page 81.)

Gangrene is more generally a result of congestion than of inflammation of the lungs, but its occasional occurrence in pneumonia is indisputable. Sometimes it occupies a large portion of the lung, and is not circumscribed, sometimes it is more limited. The affected parts are dark, dirty olive or greenishbrown in colour; fœtid in odour; moist, wet, and diffluent in consistence. The occurrence of mortification has been ascribed to thrombosis occurring in the branches of the pulmonary artery -(Huss, Carswell) - and to the destructive effect of the inflammatory process destroying the vitality of the tissue, or to an arrest of the circulation by the excessive accumulation of its products in the interior of the air cells.

Pneumonia may be double (bilateral) or single (unilateral), that is to say, it may affect one or both lungs; double pneumonia is, however, very uncommon. I have for many years carefully noted the site of pneumonia, and have found that the inflammation is much more commonly situated in the right than in the left lung, both in horses and in cattle, in epizootic, sporadic, 
and contagious pneumonia; that, generally speaking, the inflanmation commences in the inferior portions of the lungs; that it invades the tissue from below, upwards; that in all cases the bronchi are more or less involved in the inflammatory process; and that when the pneumonia is superficial, the pleura participates in the inflammatory process, losing its normal colour and translucence, becoming opaque, and covered with a layer of fibrinous material; that the exudation, whether it be into the parenchyma or on the surface, is fibrinous. When the disease originates deeply within the lung substance, the pleurisy may be and occasionally is absent.

When the pulmonary inflammation terminates in resolution. or a gradual return of the lung to its normal condition-and this termination is the most frequent one in ordinary pneumonia, provided the animal be properly treated-the exudates liquefy, undergo fatty degeneration and disintegration, and become so altered that they can be removed by absorption, and as the softened matters become absorbed, the circulation is gradually restored, and the lung slowly attains its normal character.

In glanderous pneumonia, the exuded materials are transformed into an ichorous, infecting, puriform fluid, and in pleuropneumonia-bovina contagiosa they undergo a caseous necrotic change; the inflammatory process meanwhile continuing in other portions of the lungs until a large part of them becomes consolidated, impervious to air, and the animal dies from suffocation and allemia.

The various stages of pneumonia are manifested by certain sounds detectable by the ear when applied to the sides of the chest. In the first stage, and previous to the occurrence of much engorgement, a crackling sound is heard, mingling with the vesicular murmur, over the inflamed part; the sound is of the sinallest and finest kind of crepitation, similar to that emitted by salt when thrown on hot coals. As the inflammation aclvances the crepitations become more and more pronounced, until they entirely supersede the vesicular murmur. These crepitations do not remain long; they are superseded either by a return of the vesicular murmur, indicating the resolution of the inflammation by a tubular sound, or an entire absence of sound.-(See Auscultation.) 


\section{ETIOLOGY.}

The causes of sporadic pneumonia are similar to those of bronchitis, laryngitis, \&c., namely, exposure to cold and wet, sudden chills, and housing in very cold, draughty stables. Horses kept in ill-ventilated stables are undoubtedly rendered susceptible to many diseases, and to pneumonia amongst the rest, but they will bear impure air even better than cold draughts blowing directly upon them. I have repeatedly observed that the slightest cold contracted by a horse kept in a draughty stable has almost invariably been succeeded by pneumonia, and that if the animal were not removed to a more comfortable situation the disease tended to a fatal termination. It has already been stated that inflammation may succeed the congestive condition induced by severe exertion; it may also be induced by irritating gases; smoke of burning hay and straw; foreign bodies entering the lungs from the bronchi, examples of which are not uncommon in cows, as sequelæ to parturient apoplexy, where medicine finds its way into the trachea and bronchial tubes, and in horses choked by irritating and powerful remedies, such as ammonia.

Direct injuries to the lung through wounds in the thoracic walls are not always succeeded by much inflanmation, the lungs appearing to lave remarkable powers of recovery from direct injury, provided it be not crushed, or that foreign bodies or extravasated blood are not forced into the wound in its tissue.

Pneumonia may also be induced by any material altering the composition of the blood, epizootic influences, purpura, anthrax; accidental products accumulating in the blood; mechanical or solid materials (thrombi) formed elsewhere, and conveyed to the lungs by the blood, as in phlebitis; infecting influeuces of materials conveyed to the lungs, as in pyæmia or glanders. It may be the result of passive congestion arising from disease of the heart or weakness of the circulation, induced by exhausting diseases or old age; or it may be the localization of a specific disease, as in pleuro-pneumonia contagiosa.

\section{SYMPTOMS.}

The symptoms of pneumonia, namely, the greatly accelerated respiratory movements, oppressed pulse, and other signs of excitement described by Youatt, Gamgee, and others, are not by 
any means diagnostic of pulmonary inflammation. In many cases the breathing in the earlier stages is not difficult, and one is often surprised upon auscultating the chest to find that consolidation has proceeded to a great extent without any very distinct symptoms having been presented.

In the earlier stages, the symptoms are acceleration of the pulse; in very many instances it will average eighty beats per minute; hotness of the mouth, and elevation of the temperature to $103^{\circ}, 104^{\circ}$ or even $106^{\circ}$; there is a dry, dull cough, coldness of the extremities, and some degree of rigor. In some instances the disease is ushered in by a severe rigor, succeeded by a hot stage; the mucous membranes are red and injected, the conjunctivæ presenting sometimes a rusty yellow tinge; the animal does not lie down, and if loose wanders occasionally about in a dull, depressed manner, now and then eating a mouthful of food. It is stated that the animal stands with its limbs outstretched and head protruded. I have, however, failed to observe this, except in very acute and congestive cases, until the disease has made considerable progress. There is some degree of constipation, but the frecs are mixed with flakes of mucus; the bowels are irritable, and do not tolerate purcatives. If complicated with pleurisy, the breathing is more distressed, abdominal, and painful, but in pure pneumonia there is an absence of any very painful symptoms. I have carefully noted the breathing in many cases of pneumonia, and found that the respiratory movements may not be above twenty per minute for several days after attack-a difference so slight from the normal number, that one is apt to overlook it altogether. The observations of Dr. W. Gairdner, that " the dyspnœa of pure pneumonia is a mere acceleration of the respiration, without any of the heaving or straining inspiration observed in bronchitis, or in cases when the two diseases are combined," hold good both in horses and cattle'; but congestive paroxysms are not at all infrequent during the progress of the malady, during which the breathing is not only greatly accelerated, but also laborious and distressed. As the disease advances, the respiratory movements become somewhat accelerated, until the period of crisis, when usually the breathing becomes much quickened. As a clinical fact, it may be stated that the fever continues for some days-five, eight, or sometimes longer-and that it then gradually subsides, the pulse falling 
several beats per minute, the month becoming cool, the elevation of the temperature gradually subsiding, the appetite returning; the secretion of urine, which during the febrile period had been scanty, of a high specific gravity, loaded with urea, and deficient in chlorides, is now abundant. Whilst these symptoms of returuing health are becoming apparent, it will be found that the respiratory movements are increased in rapidity, and auscultation will reveal the fact that consolidation of the lung increases during the subsidence of the febrile symptoms.

This had led some pathologists to conclude that all pneumonias are specific fevers terminating by an exudation into the lung tissue, in the same manner as variolous fever terminates in an eruption on the skin. I cannot agree with this view, inasmuch as the pneumonia is a concomitant condition from the commencement, and I look upon the exudative process as affording relief to the febrile symptoms, in the same manner as an ordinary swelling gives relief in such diseases as lymphangitis and ordinary inflammation of areolar tissue.

It will be seen that pneumonia is dangerous during two stages: first, during the early fever, which may destroy life by its intensity; and, secondly, during the period of lung hepatization, which may prove fatal by so altering the lung tissue as to produce suffocation.

These, then, are the two periods during which the practitioner has to exercise his skill and care. In some instances death may occur subsequent to the consolidative stage from rapid deliquescence of the inflammatory products, extensive suppuration, or contamination of the blood by degraded exudates absorbed into the circulation.

In human medicine, the absence of the chlorides in the urine during the earlier stages, and their return in increased quantities in the convalescent stage, is looked upon as of great importance. This is a matter which requires further observations in veterinary practice. In most instances, a discharge of a rusty-tinged more or less viscid material from the nose is observed in the horse. This is sometimes streaked with blood; it is not, however, a constant symptom, as in all probability much of what might be expectorated by the human being is swallowed by the lower animals. The character of the discharge is significant; in bronchitis and catarrhal pneumonia it is more or less 
purulent, and if excessive, indicates a condition of great danger. In croupous pneumonia it is amber coloured, viscid, but not abundant.

It is agreed upon by all observers that a horse will not lie down during the intensity of any chest disease. This is not a diagnostic sign of any particular affection of the horse, as it will persistently stand while suffering from many other maladies.

The ox will lie on the sternum during all the stages of pulmonary diseases.

When the breathing is very rapid, the horse will stand with the ellows turned outwards, and the toes turned inwards. If great prostration succeeds, the position of the limbs is altered. It will then stretch its feet apart; the elbows will be turned inwards, and it will balance the trunk upon the extremities.

The pulse of pneumonia is very variable; sometimes full, with a degree of hardness; sometimes full and soft; at other times it is small, irregular, intermittent, or double-the latter condition marking a congestive condition of the pulmonary vessels and right side of the leart and veins, as well as an anæmic state of the arterial system.

In ordinary pneumonia, running its course to a favourable termination, the period of consolidation, which is indicated by absence of sound, or a small degree of tubular breathing, is succeeded by the advent of secondary and larger crepitations, which increase in number and intensity for two or three days, then, gradually diminishing, are superseded by the normal respiratory sounds.

To sum up, it may be stated that the diagnostic signs are those revealed by auscultation and percussion. First of all, small crepitations, indicating injection of the nutrient arteries and dryness of the lung tissue; secondly, tubular breathing, or absence of sound, indicative of engorgement and consolidation; and thirdly, the reappearance of the crepitations, now of a larger character, pointing out that the exudate is undergoing metamorphosis, and becoming absorbed.

The secondary crepitations are sometimes of a bubbling character, and if associated with sunken eyes, a wrinkled expression, and feebleness of the pulse, indicate a condition of great gravity, either of extensive suppuration or of moist gangrene. Both these conditions may be associated with fœtor of the breath, the latter particularly being accompanied by a horrible odour. 
During all the stages of pneumonia, increased dulness is elicited by percussion.

Inflammation of both lungs, or double pneumonia, must be regarded as a source of very serious danger from the great impediment it presents to the breathing, and deaths from it are much more numerous than from the single form. It is, however, very rarely met with.

Pneumonia is sometimes latent, and may remain undiscovered perhaps until after the animal's death. In all cases of ill-defined maladies, it is necessary that the chest be thoroughly examined, when disease of a very grave nature may occasionally be detected, although the animal has never presented any very evident signs of serious lung disease.

During the prevalence of epizootics, pneumonia is apt to assume a marked adynamic or typhoid form, attended with an early breaking down of the pulmonary tissue. This is, however, rare, and the most common form of chest disease met with is a combination of pneumonia with pleurisy, in which the symptoms of both diseases are inseparably blended.

Acute pneumonia, especially if associated with bronchitis, is sometimes complicated with laminitis or inflammation of the feet.-(See Principles and Practice of Veterinary Surgery.) Acute inflammation may terminate in a chronic form of pneumonia, in which the inflammatory products undergo fibrous or caseous degeneration, keeping up a continued degree of irritation, unfitting the animal for work, and gradually destroying life by inducing anæmia, glanders, or hydrothorax. The symptoms of non-contagious pneumonia and pleuro-pneumonia in the ox are much more acute than those of pleuro-pneumonia contagiosa.

Hamoptysis, or bleeding from the lungs, is not generally associated with pneumonia, but may be witnessed in congestion, accidental rupture of vessels, glanders, purpura, and in a passive form in some epizootic disease or influenza; in which case I have seen the blood of a dark colour, and not frothy as in ordinary hæmoptysis.

\section{TREATMENT.}

Bleeding.-The abstraction of blood was considered the sheetanchor in pneumonia. Percivall says- "I will take it for granted 
that pneumony, either in its congestive or inflammatory form, has set in; which being the case, it becomes the imperative duty of the practitioner, without any regard whatever as to the state of the pulse or the condition of his patient, to abstract blood the moment he is called in. Generally speaking, a large orifice in the jugular vein is to be preferred to a small one; in cases of imminent danger it is absolutely indispensable. The quantity of blood to be abstracted must be as great as the patient will bear; and our sure guide in this is the effect which the efflux of blood has upon the pulse at the jaw. While the blood is flowing, keep your fingers applied upon the submaxillary artery; so long as you feel that pulsating, so long may the stream of blood be continued; but the instant the vessel collapses under the pressure of the fingers, and pulsation is no more preceptible, let the bloodcan be removed and the vein pinned up. . . . The quantity of blood we shall be able to draw on the first occasion will vary in different subjects and under modified circumstances; it may amount to a couple of gallons, it may not exceed a couple of quarts. . . A second blood-letting is often borne better than a first. When the quantity taken in the first instance has has been but small and inadequate, if we will only wait a few hours until reaction appears to have taken place, we shall commonly be able fully to accomplish our object. Six, twelve, or twenty-four hours after the first full blood-letting, guided by the exigencies of the case, principally by the state of the breathing and pulse, it may become necessary to repeat the bleeding, regulating the quantity, as before, by the perception of the pulse at the jaw. I have found it requisite to bleed thrice during the first eighteen hours."-(Percivall's Hippopathology.) This was the belief and doctrine not only of Percivall, Youatt, and others, but of Professor Dick, who taught and maintained its correctness up to the time of his death. A glance at the pathology of the disease, no less than actual statistics, will enable any unprejudiced observer at once to see the fallacy of the doctrine; and veterinary surgeons, with but few exceptions, have been long convinced that bleeding most materially increases the mortality of the disease. The experience of the medical profession has led them also to arrive at a similar opinion, and Dr. Wilson Fox, in Reynolds' System of Medicine, sums up the conclusions upon this head as follows:- 
"(1.) That indiscriminate bleeding immensely increases the mortality of the disease.

"(2.) That it is especially fatal in old people and young children, in patients of exhausted constitutions, and in those suffering from chronic diseases, and particularly in Bright's disease.

"(3.) That it is absolutely unnecessary in the majority of cases of young adults and also young children.

"(4.) That in the vast majority of eases it has no infinence whatever either in cutting short the disease, or in lessening its duration or diminishing the pyrexia, but that occasionally these results appear to follow its use when practised early.

" (5.) That in the majority of cases it hinders the critical fall of temperature and delays convalescence.

" (6.) That in the majority of cases, as shown especially by Dr. Beunett's and Dietl's data, recovery is equally, if not more rapid, when it is not practised as when it is resorted to.

" (7.) That in a few eases a moderate venesection may be necessary in the early stages to avert immediate danger of death from asphyxia."

The above conclusions have reference to moderate bleeding only, repeated bleedings being condemned by Dr. Fox as " a system whose impropriety it is scarcely needful to discuss further."

Bleeding, then, can only be safely practised in the very earliest stages of a few exceptional cases, in which symptoms of dyspnœa are very urgent; and even then it must not be pushed so as to debilitate, for more horses die from the prostration of strength at later periods than from the occasional suffocative effects of the earlier and congestive stages.

Bleeding in some cases, no matter when it is practised, seems to afford a relief to the breathing, but this effect is only temporary, and disappears in a very short time, the rapidity of the respiratory movements in pneumonia being dependent on oedema or consolidation, conditions upon which the withdrawal of blood can have but little or no effect.

Convinced of the inutility and danger of venesection, many veterinarians, undoubtedly influenced by the teaching of Dr. Tod, fell into the other extreme, and treated pneumonia by large and repeated doses of stimulants. What possible good effect 
this kind of treatment has upon an ordinary case of pneumonia is beyond my comprehension; it can only add to the irritation of the inflamed part, and increase the amount of exudation, if pursued in the earlier stages. I have seen it extensively tried, and must confess the results have been most disastrous. In the later stages, during the deliquescence and absorption of the exudate, if the pulse be small, or in any degree presenting the double or dicrotonous character, when the system is depressed by the obnoxious effects of large quantities of effete materials in the blood, moderate doses of stimulants are both necessary and beneficial. The practitioner should, however, wait until the consolidative stage has to some extent disappeared, and secondary crepitations established. Four or six ounces of whisky or brandy may then be given two or three times a day; not every hour, as recommended by some practitioners. The effect of the first doses must be carefully noted ; if they stimulate the appetite, they are to be repeated; but if they cause distress in the breathing, or any signs of exacerbation, they are to be discontinued.

During the pyrexia from seven to ten drops of Fleming's tincture of aconite may be given with advantage. It appears to exercise a benefieial influence, allaying irritation and lowering the temperature both in horses and cattle, and is to be preferred to opium, belladonna, or any other sedative. In the dog, the liydrate of chloral is to be preferred to aconite. The bromicle of potassium or ammonium also exercise a calmative effect, and may be given to all animals in suitable but not large doses.

Calomel, opium, digitalis, tartar emetic, seem to have no effect in curtailing the duration of the malady. Opium is only admissible when the pneumonia is associated with pleuritic pain.

Purgatives are inadmissible in the horse on account of the sympathetic and irritalle condition of the alimentary mucous membrane.

If constipation be extreme, enemas, or a small close of oil, are to be preferred to the more active cathartics. Aloes is contraindicated, and should never be given. In horned cattle a saline cathartic may be given in the early stages, for the purpose of unloading the stomachs and alimentary canal, and thus give greater freedom to the respiratory movements.

Nitrate of potash given dissolved in the animal's drinking 
water seems to diminish the amount of exudation, and modify the fibrinous condition of the blood. It has also a cooling or detergent effect upon the system generally, modifying the pyrexia, and, by acting upon the kidneys, assists in the excretion of effete materials from the blood. In some instances, particularly when the kidneys remain torpid, its dinretic effect is increased by a few doses of colchicum. But the administration of the nitre or the colchicum should not be persevered in too long, as they both tend to debilitate, and whenever free diuresis is established, they should be discontinued. In some instances, particularly if the patient be debilitated or out of condition, spirits of nitrous ether may be substituted for the potash salt.

Blisters.-For many years I liave held that the application of the so-called counter-irritants, whether they be cantharidine blisters, mustard, turpentine, or any other powerful irritant, is not only useless but dangerous in the earlier stages, and uncalled for when convalescence is progressing favourably.

In the earlier stages, they add to the distress, prevent the freedom of the respiratory movements, and increase both the fever and fibrinous condition of the blood; and large cantharides blisters, the cantharidine of which is absorbed into the blood, induce a condition of blood poisoning characterised by albuminuria and great prostration, and by irritating the urinary passages increase both the distress and the fever. I am glad to find that this conclusion-a conclusion, I may state, which has met with much opposition-is endorsed by physicians. Dr. Wilson Fox says-" Blisters, in the earlier stages of pneumonia, are to be considered as both useless and as greatly inereasing the distress of the patient. When resolution is progressing favourably, they also appear to be quite unnecessary. In a few cases when resolution is delayed, or when there is evidence of a small amount of pleuritic effusion, they may, I believe, in adults be occasionally employed with apparent advantage. In children they are almost invariably inapplicable. Warm fomentations or poultices to the sides often give great relief to the pain. I liave by no means satisfied myself that any advantage accrues during the acuter stages from any more irritant applications, whether mustard or turpentine, though in cases of threatening collapse, or when dyspnoa is severe, they have occasionally appeared to afford relief."-(See ReyroLDs' System of Medieine, vol. iii., p. 701.) 
In the horse the application of either mustard or turpentine causes very great distress, a high state of excitement, an increase of the febrile disturbance, and often tends to hasten a fatal termination. For these reasons the indiscriminate employment of such remedies is highly condemnable. Warm fomentations to the sides, however, afford relief; and they should be persistently smployed in all cases marked with rapidity or distressful breathing.

When the extremities remain very cold, the return of warmth and. circulation will be much assisted by frictions, or the application of a mild embracation and bandages; and, to sum up, it may be stated that the successful treatment of pneumonia consists in the following essentials:-1st. Placing the patient in a wellventilated loose box, where the air is pure, abundant, but not too cold. The necessity of pureness of air will be understood by reference to the observations of Sir Thos. Watson quoted at page 559. If the air be impure, suppuration of the lungs to an extreme extent will be apt to be established, as impure air contains organic germs in great abundance, and these, gaining access to the inflamed part, increase the tendency to the formation of pus. In the winter months, especially if the weather be cold, I have noticed that coldness of the air increases the pulmonary inflammation, and that after a very cold night an animal has been in a state of approaching collapse in the morning. To prevent the occurrence of the mortality from the above cause, veterinary establishments ought to be furnished with loose boxes, where the air can be artificially warmed to a temperature of at least $70^{\circ} \mathrm{F} .2 d$. The surface of the body, extremities, and head are to be kept warm by suitable, but not too heavy, clothing; frictions, and the application of non-irritating stimulants to the extremities, being useful in cases where they are deathly cold. 3d. The horse is to be allowed as much cold water as it will drink; but it is not advisable to allow it to drink a large quantity at once. If it be permitted free access to water whenever it pleases, by placing it in its box or stall, it will generally partake of it in sufficient but not over-abundant quantity. $4 t h$. The febrile condition must be treated according to the intensity of the symptoms. If the fever be high, nitrate or chlorate of potash are to be administered, but if, on the contrary, the fever be of an adynamic type, carbonate of ammonia 
with camphor may be given in the form of a ball, in addition to the potash salt. 5 th. If the bowels be constipated they are to be carefully regulated by a moderate dose of oil, and by enemas. 6th. During resolution of the inflammation care must be taken that nothing be done to check the action of the excretory organs. Should moderate diarrhca or increased diuresis come on, they are on no account to be checked, as they are merely critical discharges, whereby the effete materials in the blood are eliminated from the economy. $7 t h$. In the earlier stages the food is to be simple, laxative, cooling, and nutritious. Bran and boiled linseed mashes, a moderate allowance of good hay, roots if in winter, or grass if in summer. During the later stages strong food, as oats, beans, with grass or roots, are to be allowed in moderation; and should the appetite be bad, stomachic stimulants, as spirits of nitrous ether, with gentian or nux vomica, are useful adjuncts; but should the patient refuse to feed, milk and eggs are to be freely supplied. This it will drink if stinted in its water; it should never be forced upon it by horning, nor should gruels, hay tea, large doses of ale, porter, \&c., be forced upon it.

I may state, in conclusion, that in all cases where depression and debility are prominent symptoms, with a small, feeble, rapid pulse, stimulants should be cautiously used, and if after a few doses they appear to do no good, they are to be discontinued, and the case left without much meddling or interference; and that bleeding is but rarely called for, and only in cases where the dyspncea is great, and the danger to life imminent. Even then it is not to be pushed to the extreme recommended by some writers and teachers.

It must be borne in mind, as already pointed out in the chapter on Degenerations, that pneumonia, pleurisy, bronchitis, and other diseases, even of a mild type, are apt to terminate fatally in overfed and pampered animals, such as those kept for show purposes, no matter how discreetly they are treated; for in such animals the vital organs-heart, liver, kidneys, \&c.-will be found in such a state of degeneration as to prevent the performance of their functions under the altered conditions of induced disease. So long as the animals are not subjected to any trivial cause of disease, function seems to be performed; but whenever the system is suljected to any trial, such as a chill, fatigue, parturition, \&c., the powers of the altered organs are wholly or partially lost, and death is the result. 
CHAPTER LIII.

SPORADIC DISEASES-continued.

LOCAL DISEASES-continued.

(I.) DISEASES OF THE RESPIRATORY ORGANS-continued.

\section{PLEURISY-PLEURITIS.}

Inflammation, partial or general, of the serous membrane that lines the cavity and covers the viscera of the thorax, atteuded with effusion of serum, exudation of lymph, or, rarely, the formation of pus.

Pleuritis, or a combination of it with pneumonia, is the most common form of chest inflammation met with during the prevalence of the easterly winds of spring and early summer, and has been fully described at page 346 .

In the lower animals, exposure to cold is the most common cause of pleurisy. It has followed clipping when an animal has been exposed to cold, and Duvieusart states that he has seen three hundred cases of pleurisy in a flock of sheep shorn in February, thirty of which died.-(GaMgeE's Domestic Animals.) It will sound strange to veterinary surgeons to be told that cold is now believed to have no effect in causing pleurisy in the human being : such, however, is the belief.

Pleuritis in the horse often partakes of a rheumatic character; the inflammation being induced by the same cause-coldand assuming a similar metastatic type and character, changing its seat from the pleura, sometimes to the pericardium, endocardium, or to the ligamentous or tendinous structures of the extremities. In more than one instance navicular disease has succeeded an attack of pleurisy, and I have one specimen in my possession showing ossification of the heart 
caries and eburnation of the navicular bone, as results of acute navicular arthritis succeeding an attack of rheumatic pleurisy.

At the outset the inflammatory phenomena commence in the subpleural tissue, the blood-vessels of which become congested. At first the congestion presents an irregularly spotted appearance; the spots, however, multiply rapidly, and the red colour becomes diffuse by their confluence.

In other instances the pleural surface presents a streaky appearance, whilst in others the whole of the pleura, pulmonalis and costalis, presents a bright red congested appearance over its surface. The secretion of the pleural surfaces is at first suspended, and if the ear be applied to the side of this stage a dry friction sound will be heard.

The dryness of the membrane is very shortly succeeded by effusion of serum, and the formation of an exudate which has a tendency to become organized into a false membrane, causing the adhesion of the two pleural surfaces.

It is important that the time in which these false membranes may be formed be clearly ascertained. The experiments of Andral throw some light upon this subject, which is important to the veterinarian as a question of jurisprudence.

Andral made experiments upon the pleura of rabbits by injecting acetic acid into them.

He sometimes found at the end of nineteen hours soft, thin, false membranes, traversed by numerous anastomosing red lines.

In other rabbits, placed under circumstances which appear to be exactly similar, no such result had taken place at the end of a much longer period; and the pleura contained only a serous or puriform liquid, mixed with unorganized flakes of lymph.

Experiments performed by myself upon healthy subjects (horses) in 1874 proved that a false membrane, presenting some degree of organization, may be found in twenty-four hours after the injection of an irritant into the pleural sac, and that the puriform appearance mentioned by Andral is an evidence of an inflammation of longer standing, arising from degeneration of the inflammatory products.

It may be briefly stated that all cases of pleuritis terminate either in the exudation of lymph, which may liquefy and be re-absorbed, or in the formation of a permanent false membrane. But the most ordinary result is effusion of serum, constituting a limited hydrothorax, even to invasion of the 
walls of the thorax, a natural result of a diffuse and intense inflammation; and that in pleuro-pnenmonia contagiosa of the ox there is often œdema of the thoracic walls without actual hydrothorax.

Pleurisy may be either double or single; generally it is single, and confined to the right side.

Pleurisy is sometimes, but very rarely, caused by direct violence to the thoracic walls. Pleurisy is often secondary to other diseases, such as septic broncho-pnenmonia-the transit or corn-stalk disease of American cattle-and of many forms of blood poisoning.

\section{SYMPTONS.}

The disease at the outset is characterised by some degree of rigor, very often a mere chill, manifested by a staring coat, and coldness of the surface of the body. This is, however, succeeded by signs of pain, often mistaken for colic, during which the horse paws, and perhaps lies down and rolls; it eventually becomes stiff and sore, and if suddenly approached, or if rapped upon the affected side, will groan. The acts of respiration are performed rapidly and incompletely, the ribs are fixed, the respiration is mostly abdominal, and a hollow line extends along the inferior border of the false ribs, from the steruum across the flank to the anterior spine of the ilium. A dry, short, painful cough supervenes; the pulse is hard and quick. If the side be closely examined, the muscles covering the affected part will be noticed to tremble or quiver; this lasts but a short time only, and is succeeded by diminished motion. These signs, as well as the pain upon pressure, indicate that the intercostal muscles participate in the inflammation, and that pleurisy is generally associated with pleurodynia, first described by Haycock.

When the pleurodynia, or inflammation of the muscles, is very iutense the animal moves in a very rigid manner, steps slowly and very short; is greatly dejected, the back is arched, the skin exhibits great tenderness when subjected to pressure. I may state that I have seen some cases that were so stiff and sore as to fall when compelled to move.

Hydrothorax.-When an animal dies of pleurisy, especially if it has survived the first stage, a considerable amount of effusion of serum will be found, constituting dropsy of the chest, or hydrothorax. In some instances a large quantity may be found in a day or two, and in some rare cases without any perceptible 
disease of the pleura. The symptoms of hydrothorax are short, quick, laboured respiration; the pulse small, quick, soft, often intermitting; auscultation reveals absence of sound in the inferior part of the chest, or a sound resembling that of drops of water falling into a well, as has been already explained. The hydrothorax in the horse generally invades both sides of the cavity of the thorax, for a communication exists owing to the loose diaphanous or web-like structure of the mediastinum, as pointed out by Rigot, Delafond, and Bouley. This natural communication is in some instances obliterated by exudation of lymph on the mediastinal surface, in which the hydrothorax will be confined to the one side. In other instances the serous effusion, confined in sacs of adventitious products, will be confined to certain circumscribed parts of the chest only. The liquid effused is composed of serum mixed with flakes of lymph, and is generally more or less clear upon the surface, turbid in the lower parts from admixture witl particles of exudative materials; it is sometimes, however, tinged with blood. If the clear liquid be placed in a glass and left at rest, it generally separates into clot and serum, proving that it contains the constitnents of fibrin, which coagulate on exposure to the atmosphere.

In forty-three cases observed by M. St. Cyr, the effusion from the first to the seventh day presented a port wine colour in nine cases, a sero-sanguineous appearance in six, muddy or greyish in three. From the eighth to the fifteenth day the port wine colour was observed in two, the sero-sanguineous in three, muddy greyish in four, and limpid in six. From the sixteenth to the thirtieth day the colour was limpid in five cases, and after the thirtieth it was also limpid in three cases. M. St. Cyr draws the conclusion that the liquid begins to clear up towards the end of the second week, and that it is clear after the twentyfifth or thirtieth day, and that this is the epoch from the acute to the chronic state of pleurisy.

English veterinarians generally maintain that the advent of hydrothorax is rnanifested by an apparent improvement of the pleuritic symptoms. They state that the pulse falls, the breathing becomes easier, \&c. This is so far true, but is not diagnostic, as the abatement of the symptoms indicates the subsidence of the pain and fever, and that the dry condition of the pleura has passed on to that of increased effusion, its natural sequence. 
Now, if the effusion continue or remain unabsorbed, or is poured out in greater quantities than can be taken up by absorption, it naturally follows that the condition of hydrothorax will be the result; if, on the contrary, the effusion be moderate in quantity, or if absorbed, it as naturally follows that the improvement of the symptoms is but the forerunner of convalescence.

In addition to increasing difficulty of breathing, a difficulty sometimes so great as to call into operation the action of muscles other than those of respiration, extending even to the caudal ones, causing an upward and downward motion of the tail similar to that of a pump handle, the other characteristic signs are dropsical swellings, commencing generally at the sternum, extending along the floor of the abdomen, and finally invading the areolar tissue of the extremities; flapping of the nostrils, protrusion of the head, and increased roundness or bulging of the ribs, and the hair of the mane and tail is easily pulled out. In some instances of hydrothorax the legs remain unnaturally fine, even to the fatal termination.

It may be mentioned that the appetite is capricious, and that the symptoms generally are subject to variations-the diagnostic ones being those detected by percussion and auscultation.

\section{TREATMENT.}

The treatment of pleurisy in its acute stage differs but little from that of pneumonia, unless, indeed, the pleuritic pain be acute, then opium is essentially necessary. In the very earliest stage of the inflammation, when the pain is usually most acute, a dose of tincture of opium, in combination with a pint of linseed oil, has usually a very decided anodyne effect, particularly if its soothing effect be assisted by warm fomentations to the affected side. The opium may be repeated if the pain continues; usually, however, the acute pain subsides in a few hours; and, provided the pulse remain hard, aconite may take the place of the opium. If the pulse be soft and showing little irritation, but little treatment, beyond warm fomentations to the sides, clothing the animal warmly, allowing it to drink freely of cold water, in which an ounce of potash nitrate is daily dissolved, and supplying it with nutritious, easily digestible food. is necessary. The fomentations to the sides are 
not, however, to be neglected, so long as the breathing remains at all laborious; experience having taught me that they not only soothe the irritation and pain, but that they most materially promote convalescence, and diminish the tendency to hydrothorax and death, but they should not be applied for more than half an hour at a time; their too long-continued application often becomes annoying to the animal and causes restlessness.

Bleeding, although often indicated by the hardness of the pulse, seldom does any good; it certainly has no effect on the duration of the disease, even in cases where its primary effects have seemed to afford relief to the pain and respiratory embarrassment; and in the majority of instances where it has been indiscriminatcly performed, it has delayed the convalescence, increased the tendency to hydrothorax, and increased the rate of mortality.

Blistcrs.-Of the application of the so-called counter-irritants, I can only repeat what I have taught, that with rare exceptions they are injurious. Veterinarians, however, generally maintain otherwise; and in support of their arguments appeal to the practice of physicians, who, with rare exceptions, until lately practised this method of treatment. Within the last few years opinion has greatly changed; and in the latest book on medicine, namely, "Iieynolds", Dr. Austm, article Plcurisy, page 942, vol. iii., says- "'The treatment by so-called 'counter-irritation,' as pursued by many physicians, is no less repugnant to me than is that by mercury or bleeding. Let me make two admissions :In the first place, the mere application of a mild mustard plaster, or, still better, of a hot poultice or epithem, undoubtedly may give some ease, perhaps even arrest incipient inflammation; and the use of 'small flying' blisters, in the limited attacks of pleurisy, which are so common in phthisis, muloubtedly appears to give relief in many cases. But the use of large blisters, especially if kept open, appears to me both riseless and often prejudicial." I need not here repent what must be already selfevident, that blisters, especially those extensive ones ordinarily applied, only inerease the pain, the embarrassment of breathing, the general febrile condition, and the tendency to excessive efiusion and exudation. There are instances, however, in which the application of a moderate mustard liniment proves beneficial; these are the cases in which the disease makes but little progress towards recovery, in which the symptoms remain without much alteration for better or worse for some days. Tlis con- 
dition is eharacterised by languor, prostration of strength, a pulse standing about eighty, with seanty secretion of high-coloured urine, a continued elevation of the bodily temperature, capriciousness of the appetite, and a respiratory embarrassment.

From the tenth to the fourteenth day after attack, the above symptoms being present, the application of the mustard liniment, and succeeding warm fomentations to the sides, promotes the absorption of exuded materials, stimulates the systern generally, and inereases the excretion of effete materials from the econony, evidenced by diuresis, improvement of pulse and respiratory movement, and a rapidity of convalescence, which otherwise could not have been established. In thus applying the cutaneous stimulants care must be taken that their effects do not exceed that of stimulation or gentle irritation; if the application be sufficiently strong to induce soreness of the sides, it is ealculated to do harm, not only by increasing the pain, but also by exaggerating the adynamic or typhoid condition. In no instance where resolution is progressing satisfactorily should this treatment be applied.

The colchieum, as recommended for pneumonia, may advantageously be administered in combination with iodide of potassium, or the vegetable or mineral tonics, as the ease may be, when absorption and excretion of the inflammatory products are not progressing satisfaetorily. When debility and anæmia are associated symptoms, I have found the salts of iron, more especially the tincture of the terchloride, to have a marked effect in promoting the absorption of the inflammatory products, fluid and solid. I have a far higher opinion of the effects of iron salts than of iodine or iodide of potassium. The practitioner is, however, at liberty to combine the iodine with the iron.

Paracentesis Thoracis.-In all cases where effusion is excessive, no time should be lost in giving relief to the symptoms of distress by the operation of tapping. I do not mean to advocate early tapping in cases of effusion, being strongly of opinion that absorption of the fluid is calculated to save more lives than its evaeuation; but when danger to life is imminent from its excessive quantity, the operation should never be delayed until prostration and debility are associated with the dyspnœa. The most successful method of performing paracentesis thoracis is by 
means of an instrument, consisting of a trocar and cannula, guarded by a tap, by which a small quantity of the fluid is withdrawn, the exact nature of which can be identified. If fluid be present it can be withdrawn, an elastic pipe being attached to the tap, and the serum allowed to flow into water. Should the fluid be purulent or too thick to flow through the cannula, a suction syringe is to be applied, the force of which is to be sufficiently strong to cause its evacuation.

Dr. Bowditch's rules for performing this operation are as follows:- "Find the inferior limit of the sound lung behind, and tap two inches higher than this on the pleuritic side, at a point in a line let fall perpendicularly from the angle of the scapula. Push in the intercostal space here with the point of the finger, and plunge the trocar quickly in at the depressed part; be sure to puncture rapidly, and to a sufficient depth, as you may be balked by the false membranes occluding the cannula.

"It will sometimes happen that with the greatest care and trouble we are unable to get a flow of fluid at the part where we first puncture; it is then our duty to try elsewhere, for our failing may be owing to unusual thickness of the false membranes in the lowest inch or two of the pleural cavity. We thereupon repeat the puncture a little higher up, and further towards the axillary line, and here we perhaps find fluid; at any rate no harm has been done by the two punctures.

"The circumstances under which paracentesis ought to be performed for pleurisy are the following:-

" 1 st. In all cases of pleurisy, at whatever date, where the fluid is so copious as to fill one pleura, and begins to compress the lung of the other side; for in all such cases there is the possibility of sudden and fatal orthopncea.

" 2 d. In all cases of double pleurisy, when the total fluid may lie said to oceupy a space equal to half the united dimensions of the two pleural cavities.

" $3 d$. In all cases where, the effusion being large, there have been one or more fits of orthopncea.

" $4 t h$. In all cases where the contained fluid can be suspected to be pus, an exploratory puncture must be made; if purulent, the fluid must be let out.

" 5 th. In all cases where a pleuritic effusion occupying as much as half of one pleural cavity lias existed so long as one month, and shows no sign of progressive absorption." 
In our patients the puncture can be made in the fifth or sixth intercostal space, and as near to the anterior margin of the rib as possible. It is usual to divide the skin with the lancet, and to stretch it before introducing the trocar, so that, when the fluid is withdrawn, the external wound is not directly opposed to that in the tissues. By this method admission of air into the cavity of the thorax is preventecl.

Physicians are agreed that it is not necessary to extract the whole of the fluid, and that the removal of just so much as may be necessary to relieve substantially the mechanical distress will, in most cases, give the necessary spur to the natural process of absorption, by means of which the rest of the fluid will be taken up. Should the cannula become blocked up by fibrinous coagula, and the escape of the fluid thus prevented, a whalebone probe must be introduced.

After the operation the animal's strength must be maintained by good food and stimulating tonics, such as nux vomica, ferrous sulphate in combination with hydro-muriatic acid. If the animal does not feed, it should be supplied with milk and eggs instead of its drinking water for a day or so; but, for reasons already given, all such food must be partaken by, not forced on, the animal. 


\section{CHAPTER LIV. \\ SPORADIC DISEASES-continued.}

LOCAL DISEASES-continued.

\section{(K.) DISEASES OF TIIE HEART AND ITS MEMBRANES.}

THE weight of the heart of the horse varies according to the animal's weight and size-from 8 to $20 \mathrm{lbs}$; the average, however, is about 10 lbs. In the ox, sheep, and pig the weight of the heart is to that of the weight of the body as 1 in 220, and in the $\operatorname{dog}$ as 1 in 90. Anything much above this average is indicative of hypertrophy.

Heart diseases, althongh rare in the lower animals compared with their frequency in man, and much more difficult of diagnosis, are neither infrequent nor unimportant.

For the convenience of description they may be arranged under two heads, namely-1st. Diseases of the heart and valves; $2 d$. Diseases of the cardiac membranes.

1st. Diseases of the heart are divided into functional and organic.

(a.) Functional derangement of the heart, characterised by palpitations, irregularity, or intermittence of the pulse, may arise from debility, indigestion, blood poisoning, as in purpura, hemoalbuninuria, epizootic catarrhal fever, \&c.

Palpitation of the heart is often due to dyspepsia, disappearing with the indigestion and reappearing with another attack, both in the horse and dog. It also arises from nervousness, and if a nervous animal be approached rapidly and roughly, the beatings of the heart are often distinctly heard; and if the pulse be felt, it may be irregular and intermitting. If one were immediately to form a diagnosis that these results were due to heart disease, a few minutes' further observation would point out the error.

Palpitations and irregularities are symptomatic of other disorders, when they occur occasionally only, and when other sigus 
of heart disease are absent; if persistent, they indicate, separately or conjointly, some organic lesion of the heart or its menibranes. liepeated examinations of the patients are necessary before forming a conclusion.

(b.) Organic Discases.-Carditis, or inflammation of the substance of the heart, is always circumscribed; a general or diffused inflammation, which would be immediately fatal in consequence of destruction of function, is fortunately never witnessed. Partial or circumscribed carditis, unless it be due to injuries, and associated with exocardial effusion and exudation, presents no symptoms during life, and is only discoverable post mortem by the presence of a small abscess or circumscribed deposits of lymph; but although the cardiac walls are not subject to inflammation, they undergo varions alterations inimical to the wellbeing of the animal, and may be described as follows.

1. Hypertrophy.-This may be of three kinds:-

(1.) Simple hypertrophy, where the walls are merely thickened without alteration of the cavity.

(2.) Eeccntric hypertrophy, where the walls are thickened and the cavities enlarged.

(3.) Concentric hypertrophy, where the walls are thickened and the cavities diminished.

These conditions are manifested by a more or less persistent palpitation or increased cardiac force, the cardiac sounds being often heard upon both sides of the chest. They are due to some obstruction to the circulation consequent upon chronic pulmonary disease, constriction of some of the large vessels, and are best combated by a moderate diet, freedom from excitement, and, if depending upon broken wind or any impediment to the pulmonary circulation, by small doses of arsenic.

The most common organic change which occurs in the hearts of horses and dogs is that due to atrophy and fatty degeneration. This change is generally met with in aged subjects, and in those which have been pampered and irregularly exercised. It is also a result of pericarditis, and as a sequel to epizootic diseases in which the pericardium has been involved. It is also incluced by blood affections, as purpura, azoturia, and scarlatina.

During life the progress of atrophy and degeneration is manifested by a more or less slowly increasing debility of the circulation. exemplified by diminished cardiac impulse, irregu- 
larity of the pulse, and a tendency to cedema of the extremities, with difficulty or inability on the part of the animal to perform ordinary labour.

I have repeatedly watehed the progress of this disease, and have found that when the animal is near death there has been capriciousness of the appetite, extreme muscular debility, a peculiar rusty-red appearance of the visible mucous membranes, and a want of correspondence between the pulse and the contraction of the heart. The earliac sounds have been often loud, amounting to palpitation, but the impulse has been feeble, and the pulse weak and irregular, arising from the fact that the feeble cardiac impulse is not transmitted.

In hypertrophy of the heart, more partieularly that of the left ventricle, when the walls are increased in thickness, and the eardiac contractions proportionately strong, the blood is propelled into the arteries with increased force, and the pulse is strong and hard, so long as the circulation is unimpeded by aortic obstruction; but when the heart is atrophied, or its walls attenuated, and its cavities enlarged, the pulse will be of the opposite character-soft, weak, and irregular. If the hypertrophied heart's action be intermitting, the strong pulse will be intermitting also; but in atrophy the pulse will be intermitting and irregular, even providing that the beatings of the heart be regular in their succession.

The post mortem examinations reveal the heart apparently enlarged, but the enlargement is due to dilatation of its eavities and attenuation of its walls. The muscular structure of the whole organ presents a pale or fawn-coloured appearance, is soft to the tonch; and when examined mieroscopically, the most noticeable change is the absence of the transverse strix of the muscular fibres, with here and there true fatty degeneration. In the dog, atropliy of the muscular fibres is generally due to fatty infiltration, which, pressing upon the muscular fibres, cause their removal by ahsorption. But in the horse the ehange is more commonly found in the sarcous elements themselves, by which they first lose their truly muscular eharaeteristics and their power of contractility, and finally become converted into a fatty inaterial.

I cannot recommend any treatment ealculated to arrest the progress of this ehange in the horse, but would suggest that the chlorate of potash (which has the power of arresting some 
degenerations) might be administered. For the dog, mild purgatives, regular exercise, and light food constitute the best treatment, for, as already pointed out, the atrophy is generally simple, except in old dogs-depending upon the pressure of the fatty infiltration.

\section{PERICARDITIS.}

Inflammation of the pericardium is often associated with epizootic diseases. In the ox it frequently arises traumatically.

When not arising from injury, its causes are similar to those of pulmonary diseases and rheumatism, with which it is often associated. There are but few instances of epizootic pleuro-pneumonia in the horse but are complicated with inflammation of the pericardium; indeed, during some seasons, epizootic diseases partake of the rheumatic type, and involve not only the pleura, pericardium, and endocardium, but also the fibrous structures of other parts of the body.

Symptoms.-In addition to general signs of fever, which is often of a severe type, there is a peculiar irritability and hardness of the pulse, indicative of irritability of the heart; the pulse is a short, angry beat, and the heart's action is often irregular; sometimes bounding and violent, at other times feeble and fluttering. There is coldness of the extremities, and acceleration of the respiratory movements.

Leblanc, who is followed by Percivall, Gamgee, and others, says that a marked contrast between the violent heart-beats and smallness of the pulse are characteristic signs of endocarditis, and that they are very important in distinguishing endocarditis from pericarditis. I cannot confirm this conclusion, having repeatedly witnessed these symptoms in pericarditis unassociated with disease of the endocardium. Generally, however, both membranes are implicated in the inflammation, and it is a most difficult matter, in the lower animals at least, to distinguish between the two affections.

Associated with this inflammation, spasms or cramps of the superficial muscles aie often witnessed. Most frequently the cramps are confined to the muscles of the pectoral region and neck; but occasionally those of the posterior extremities are also more or less violently affected. The physical sign of pericarditis is a to-and-fro friction sound, resembling a rasping murnur, 
syuchronous with the cardiac movements. This sign is subject to variety in tone and degree, depending upon the nature and extent of the exudation or effusion. If much fluid be present, the sound becomes lost, being replaced by a dull, churning noise; as the effusion diminishes by absorption, the to-and-fro friction sound returns, unless, indeed, the pericardium has become generally adherent to the heart's surface. If this somnd be associated with a deep-seated blowing or bellows murmur, which sometimes begins to be distinguished when the other ceases, or, in the words of Watson, appears to supervene upon it or to take its place, the combination of sounds indicates an affection of both the external and internal membranes-pericarditis and endocarditis. Dr. Stokes says, "that in respect of Inorbid anatomy, cases of pericarditis may be arranged into three classes. In the first class are to be placed those in which there is a slight though general effusion of coagnlable lymph. In the second, those in which there is smperadded the secretion of serum in abundance, causing distension of the pericardial sac. In the third class are to be placed those cases in which signs of muscular excitement, if not of myocarditis, are added to the preceding conditions.

"As the disease advances from the first to the last of these forms, there is progressive increase in the violence of the inflammation, denoted in the second form by the occurrence of excessive serous effusion, and in the last by the altered and impaired condition of the heart itself. Death tends to occur by syncope, induced by paralysis of the left ventricle. The muscular substance of the heart is paralyzed, being of a dirty brown or yellow colour, flabby, and easily torn-a condition which speedily leads to passive dilatation of the heart, general cachexia, and dropsy."

Post mortem examinations, as conducted in the dissecting rooms, go to prove that pericarditis, as evidenced by alterations of structure, is not such a common form of disease as enclocarditis. In the human being it is stated that about thirty-three per cent. of cases examined post mortem, varying from the ages of eighteen to thirty-nine, and about seventy-one per cent. from ages between forty-eight and eighty, show white spots in the heart. These white spots are supposed by some pathologists to be due to previous pericarditis, and by others to attrition from rubbing 
of the part against the pericardium applied to the sternum, induced by dilatation of the heart or impeded action of the lungs. These white spots are seldom met with in the pericardium of the horse, whilst they are not uncommon on the endocardial surface.

Traumatic Pericarditis.-In ruminants, particularly cattle, foreign bodies often find their way into the pericardium, wounding both it and the heart. Whilst the carditis so induced is circumscribed, and merely surrounds the point of puncture, the pericardial inflammation and exudation involve the whole surfaces of the membrane.

Cattle are exceedingly fond of chewing and swallowing all sorts of substances; for example, nothing seems to give greater pleasure to a cow than to have an old boat or other piece of leather in its mouth, and this it will chew at with evident gratification. An old brush is also a dainty morsel, and I have seen as a consequence of this that the pericardium has been pierced by the brush nail. Many kinds of sharp-pointed materials have been found in the pericarclium of cows. I have seen hair-pins, horse-nails, needles, table-knife, iron wire, \&c. \&.c.; and, as a matter of fact, the smarter the maids are in a dairy the greater the danger of this disease amongst the cattle, for smart dairymaids use numerous hair-pins, some of which become lost amongst the food and are swallowed by the cows.

In some instances the foreign body is gradually forced from the chest into the thoracic walls, as proved by the following three cases related to me by Mr. Malcolm Walker, V.S., Alexandria, Dumbartonshire, in a letter dated August 1888 :-

"The first was an Ayrshire milch cow, said to be off her feed for a few days. On examining her, I found the pulse weak and irregular, the rumen impacted and flatulent. I ordered a tonic ball to be given three times a day, the ball containing ginger $\bar{j}$ i., carb. ammonia 3 ii., made up with treacle. In two days she seemed better; she had been ruminating occasionally, the pulse still weak, irregular, and an anxious look about her. I saw her again in two days, when she appeared much worse; the nose poked out, small, weak, irregular pulse; laborious breathing, dropsical swelling below the sternum; the sounds of tho 
heart almost inaudible. I then diagnosed it traumatic disease of the heart, ordered tonics, stimulants, and good nourishment. The swelling on the ehest increased to a considerable size. I punctured it in several places, and it gradually diminished ; the appetite slowly returned, and the cow seemed to improve every day and get all right again for a few weeks; but she still had the anxious look, and a grunt when she moved about. I was called to see her again in about four weeks after the first attack; the owner said she was very lame in the off fore leg. I had her taken out of the byre, which was dark, and found her lame, caused by a swelling on the off side of the chest, immediately above the sternum and close to the point of the elbow. This swelling being hard, and very painful to the tonch, I suspected the presence of some foreign body. I made a free incision inito it and introduced my finger, and felt the sharp point of a needle. I enlarged the wound so as to admit my finger and thumb, then I got hold of a very large darningneedle and took it out; it was the largest needle of the sort I ever saw -it was 5 inches long. I treated the wound with fomentations and earbolic dressings for about two weeks, then I had it thoroughly washed and examined, and found a small dark-coloured cord, which came away with a gentle pull. As long as this cord remained in the wound there was a very offensive smell from it, but very soon after it was removed the wound healed up and the cow did well.

"The second case was in a mileh cow, that had been treated four weeks by another practitioner for indigestion. I at once suspected some chest disease, from the grunt she had, and her thin, emaciated appearance. I went to the left side of the chest to auscultate, when my cheek came in contact with a very painful nodule, about the size of an egg. I examined it carefully, and felt something hard in its centre. I cut down on this hard spot, and came on the point of a needle, which I removed with the forceps, it being over the average size of darning-needles too ( $4 \frac{1}{2}$ inches). The grunt immediately left her, and the appetite improved every day. I dressed the wound the same as in the first case, and removed the cord in three weeks. The wound healed quickly, and the cow is now as well as ever she was.

"The third case came under my notice on the 1st of this month (August). This eow had been treated by another practitioner for some days (for what I don't know). The owner told me she hacl been off her feeding and wasting away for some time. I observed a swelling on the left side of the ehest, above the sternum, and close to the point of the elbow. This swelling was also very painful to the touch; it was about the size of my two hands. After manipulating it earefully, I could feel the hard nucleus that I felt in the others. I cut down on it, and got hold of a darning-needle of a smaller size, and 
half of the eye broken off. I ordered the wound to be treated with fomentations and dressed with carbolic liniment. I have not seen the cow since, but the owner writes me stating that she appears all right, and feeding well. I expect to see her about the end of this week to remove the cord, as in the others.

"I may mention that all these wounds, after the needles were removed up to the time the cord or slough was taken away, had a very offensive smell."

Traumatic pericarditis is generally manifested very insidiously, diagnostic symptoms being often absent for long periods after the foreign body has been swallowed, no symptoms being present, so long as it remains in the digestive cavity, nor during its course to the heart, until it materially interferes with the functions of that organ. I have repeatedly observed instances where cows have presented no other sign of disease than an occasional attack of flatulence or indigestion, and have been sleek and well-doing, until the act of parturition, when they have rapidly succumbed, and the post mortem has revealed the pericardium immensely thickened, adherent at its surfaces, and the exudate in a condition of organisation, sometimes of a consistence resembling cartilage; the adhesions between the reticulum, diaphragm, and mediastinum and the walls of the canal being so organised as to leave no doubt of the lengthened period since the injury had been inflicted; the act of parturition, more especially the contractions of the abdominal muscles, having evidently altered the position of the hitherto quiescent foreigu body, and thus excited a fresh attack of inflammation. More commonly, however, the symptoms of the lesion have become gradually diagnostic; at first symptomatic of indigestion, with capriciousness of the appetite, flatulence, and eructation of gases, and gradual emaciation. After a while, the pulse becomes exceedingly small; the jugular veins are distended; there is also a well-marked jugular thrill or pulse, extending even as high as the bifurcation of those veins, associated sometimes with palpitation of the heart. To these succeed œdema of the intermaxillary areolar tissue, gradually extending down the neck to the dew-lap; in some instances clonic spasms of the superficial, particularly the cervical muscles. Continental writers state that the diagnostic symptoms of traumatic from simple pericarditis 
are a loud gurgling cardiac sound and eructation of gases. The gurgling sound is supposed to arise from the greater consistency of the pericardial effusion, and its admixture with various gases. The eructations are supposed to arise from the communication between the pericardium and stomach, allowing the gas formed in the former to pass into the latter, and thence into the mouth. If these latter symptoms were constant, their importance and value would be beyond doubt. I have, however, seen cases in which neither eructations, gurglings, nor splashing were detectable, and in 1857 I reported two cases in the $V$ eterinarian, in which absence of all cardiac sounds was mentioned, this absence of sound being ascribed by me at the time to the thickness and plasticity of the exudate rendering all sounds inaudible.

In some instances there is a painful congh and disturbance of the respiratory movements; in other cases cough is absent, and the respiratory movements unaffected, or even slower than natural. The post mortem examination tends to prove that the pericardial exudations are of varying ages, and that the animal has suffered from repeated attacks of pericarditis. This conclusion is arrived at by the fact that two or three, or even four layers of exudation, more or less separable one from the other, are found; the older exudate presenting the appearance of a corneous change, being in consistence similar to cartilage, and not easily cut with a knife; then another layer of exudation of apparently more recent origin; then another layer or two of more recent origin still, all being more or less tinged with the chemically altered metai. In some instances the pericardial surfaces are intimately adherent one to another; in others they are here and there connected loosely by bands of lympl, separated from each other by a turbid fluid, whilst in others the exudate is prevented from becoming adherent by the interposition of fluid. It is, however, more or less vascular, and gradually assumes the appearance of a villous membrane.

Unless the foreign body can be removed as in Mr. Walker's cases, it is obvious that no treatment can be suggested for this form of pericarditis, and it is advisable to make the best of the animal before emaciation renders it worthless. For the prevention of this, attendants on cattle should be enjoined to see that the food be free from any of the above-named foreigu bodies. When cattle are attended by females the danger is greater from the pins, \&c. worn in the dress becoming mixed with the food. 
The treatment of idiopathic pericarditis must be directed to allay pain and undue irritability. For this end aconite is recommended; if pain be great, repeated doses of opium are to be administered; the bowels are to be kept regular by moderate doses of oil, and the absorption of the effusion promoted by diuretics. It was supposed at one time that calomel had the power of causing the removal of the exudate, and it was consequently largely employed; but its administration is now generally condemned, and Sir Thomas Watson confesses, in the following remarkable words, that the hope which he once cherished that the inflammation could be controlled by the constitutional influence of mercury has faded away. He says-" Pericarditis has been known, not seldom, to spring up while the patient was already under mercurial salivation. I am obliged therefore to recant the advice which I was formerly in the habit of giving in respect of mercury as a remedy for acute pericardial inflammation." If debility be present, the weakened heart must be supported and invigorated by moderate doses of stimulants in combination with opium. Bleeding, except to relieve urgent symptoms in the earlier stages of the disease, had better be withheld, as there is a strong tendency to an early diminution of the cardiac energy. Blisters are not called for in the earlier stages of the disease, but their application may, in some rare instances, be necessary to promote the absorption of the effusion. Tonics, more especially the salts of iron, prove useful in promoting the absorption of the effused fluids, and are to be given alternately or in combination with diuretics or the iodicle of potassium. In the rheumatic form colchicum is indicated. Digitalis, so highly recommended by some authors, appears to me to act injuriously; it destroys the appetite, is uncertain in its action on the heart, and, if persevered in, its toxic, cumulative effects are apt to cause serious derangement.

To sum up, it may be stated that warm fomentations to the the side, warm clothing, bandages to the legs, with careful administration of remedies calculated to relieve such urgent symptoms as may arise during the progress of the disease, and allowing the animal a plentiful but not over-abundant supply of easily digestible nutritious food, are the general principles of safe treatment. If there be danger of death from hydrops pericardii manifested by orthopnœea, obstruction to the venous 
circulation, and a serious interference with the heart's action, paracentesis is to be performed; a small trocar being used, which is to be introduced carefully at the side of the sternum, between the cartilages of the fifth and sixth ribs.

\section{ENDOCARDITIS.}

As a sequel to articular rheumatism, endocarditis is a more frequent form of disease than the last described one. It is an inflammation of the membrane lining the cavities of the heart, and presents symptoms similar to those of pericarditis; the difference being that in its purity the blowing sound-the "bellows murmur" already referred to-takes the place of the to-and-fro friction sound. The marked venous pulse, and the want of correspondence between the pulse and the cardiac impulse, laid down as diagnostic of this disease by Leblanc and Gamgee, are seen in other cardiac affections, and are of no diagnostic value. I am, however, of opinion that the clonic spasms of the superficial muscles, already mentioned, along with hurried breathing and a tendency to syncope, if the head be suddenly elevated, or the animal in any way disturbed, are more marked in this than in any other cardiac affection. It is very true that in cardiac degeneration there is a tendency to syncope from debility of the circulation. This condition, however, differs from acute endocarditis by the absence of febrile disturbance. Endocarditis is much more fatal than any other acute cardiac affection, for the reasons that it is often associated with a mal-condition of the blood; that it leads to valvular alterations, and to a deposition of fibrinous coagula on the valves, which destroy life by interfering with the circulation of the blood, or, carried away by the blood to other parts of the body, by obliterating the capillaries of other organs, leading to softening, abscesses, or sudden death, or undergoing degradation by poisoning the circulatory fluid.

Morbid Anatomy.-The first effect of inflammation of endocardium is seen in the form of red spots, streaks, and patches. 'The redness is always most intense in the neighbourhood of the valves, which in some instances lose their integrity, and become ruptured or detached from their tendinous cords. Supervening upon this, lymph is exuded both into the substance of the 
membrane and upon its free surface. That upon the free surface is often washed away by the current, but, generally speaking, it is found between the folds and upon the free surfaces, constituting warty excrescences upon the valves.

In a specimen now before me, obtained from a cow which had three months prior to death suffered from rheumatic arthritis, there are no less than five-anci-twenty of these excrescences, more or less organised, attached to the surfaces of the tricuspid valve. They vary in size from a pin head up to a large nut; some of them being in the auricle and some in the ventricle, attached not only to the valves but to the chordre tendiner, carnæ columnæ, and musculi pectinati, whilst the spaces between the tendinous cords are filled with coagulated blood. In the left side of the heart there are also traces of deposition within the mitral valve, having the shape of irregular white spots. It will be thus seen that there is not only a deposition upon the free surfaces, but also an exudation in the membrane itself. In some instances the valves become ulcerated, and even the cardiac walls perforated, establishing a communication between the two cavities.

The growths of endocarditis differ from those clots or coagula which form during or after the agony of death, and they must not be confounded with the corpora aurantii, which are generally more or less enlarged in aged subjects. The coagula, which form during or after death, are not adherent to the parietes, are soft and easily removed.

In the treatment of endocarditis particular care must be taken to pursue no treatment calculated to lower the heart's action, as debility of the circulation greatly favours the tendency to coagulation of the blood in the heart, and the consequent formation of these coagula. For the same reason, remedies which have the power of modifying the coagnlation of the blood, such as the nitrate of potash, or, when debility is present, the bicarbonate of ammonia, are to be prescribed; and for the reason that inflammations, artificial and natural, increase the fibrinous condition of the blood and its tendency to coagulation, blisters, setons, and all other remedies which constitute the so-called counter-irritant treatment, are to be avoided. The treatment recommended for pericarditis is applicable for endocarditis, with this exception, that those sedatives which diminish the cardiac energy are to be 
carefully and cautiously administered. They are certainly useful, more particularly aconite, in relieving disquietude and irritability; but they should be given in small doses only, and for the reason above stated, that all remedies calculated to lower the action of the heart promote the tendency to fibrinous coagulation.

The results of endocarditis are various diseases or alterations in the form and structure of the valves, leading to ulceration, perforation, pulmonary apoplexy, and ultimately to the death of the animal.

\section{DISEASES OF THE VALVES.}

For various reasons, such as the distance of the heart from the thick and muscular thoracic walls, valvular diseases are exceedingly difficult of diagnosis. They are generally due to a change of structure, caused by endocarditis, mechanical rupture, or morbid growths. The result is imperfect valvular action, indicated by difficulty of breathing when the animal is subjected to exercise, the venous or jugular pulse, the so-called vertigo or megrims, sometimes a tendency to cedema of the limbs, and modifications of the cardiac sounds. These modifications are as follows:- $\Lambda$ bellows murmur with the first sound indicates mitral disease or insufficiency; a bellows murmur with the second sound indicates aortic insufficiency. Beyond this my experience does not enable me to describe more definitely any train of symptoms which are of diagnostic value. Indeed, I have repeatedly found the cardiac sounds modified, a distinct venous pulse, and irregularity of the heart's action in various conditions unassociated with valvular diseases. For example, the jugular pulse, which would lead one to conclude that the regurgitations were due to tricuspid incompetency, is generally present in various alterations of the cardiac walls, and in pericarditis, whether it results idiopathically or traumatically. Practically it may be stated that an animal suffering chronically from the above symptoms is only fit for the slowest kind of work.

Ulcerative and warty endocarditis are said by Ziegler to differ nnly in the degree of their intensity. In the one there is a growth of white fibrous and elastic tissue in the valvular reduplications, which is sometimes absorbed; at other times the valves become thickened, form abnormal adhesions, and 
contract the urifices. Fibrinous exudates form on the surfaces of the valves, and these, when carried away, are conveyed to the lungs, if on the right side, or to the brain, kidneys, \&c. when on the left side, inducing embolism. Warty excrescences on the va!res, observed in cases of swine fever, have been supposed to be the result of or associated with that disease; but according to Mr. Cope (Report of Board of Agriculture, 1894), out of 3065 hearts taken from suspected cases of swine fever, 1039 were found to have that disease, and out of this number 270 showed disease of the valves, but of these only 1.07 were associated with swine fever. This shows that disease of the valves in the pig is induced by other causes than as well as swine fever.

It appears that the slightest wound on the surface of the valves is followed by a fatal enclocarditis, said to be induced by micro-organisms, which are harmless in the normal circulatory apparatus. The Staphylococcus aureus, Streptococcus pyogencs, and the cocci of septicrmia have induced similar conditions.

\section{ANGINA PECTORIS.}

The condition termed angina pectoris, or breast-pang, a disease characterised by agonizing pain and distress in the human being, undescribed, so far as I am aware, in the lower animals, is a form of disease which I believe sometimes affects the horse. This disease, in man, is defined as a pain or spasm referable to the lower part of the sternum, extending to the left scapula and root of the neck, and manifesting itself in paroxysms of great severity. I feel confident that in one horse $I$ have seen this condition in a well-defined form. The subject in question was an aged cart-horse brought for my examination in the year 1873. For twelve months previous the horse had done but little work, owing to the fact that when he was excited by work or exercise he manifested the most exquisite agony in the near (left) fore limb, the muscles of which, more particularly the pectorals and those of the neck, became violently convulsed, the limb itself being alternately rigidly fixed by muscular contraction and powerlessly paralyzed, so that the animal was quite unable to use it, and if forced to move he painfully dragged the limb, or sometimes fell to the ground. Whilst at rest the paroxysms seldom occurred, 
but I noticed that there was a continual spasmodic twitching of the above-mentioned muscles, as well as a dread on the part of the animal when suddenly approached. On examining the heart and circulation there was a distinct jugular pulse, great irregularity of the heart's action, a loud cooing or blowing sound and strong impulse, indicative of hypertrophy, and such a want of correspondence between the cardiac energy and feeble pulse as to lead me to the conclusion that the horse was suffering from valvular incompetency or impediment, to overcome which hypertrophy of the cardiac walls had become established.

Angina pectoris is supposed by physicians to be due to dilatation of the cardiac cavities, degeneration of the walls, some condition of the aortic valves which permits the regurgitation of blood into the heart, or some sudden impediment to the coronary circulation. The condition of the horse in question pointed to the conclusion that the canse was due to aortic impediment. Unfortunately I lost sight of the animal, and I am unable to confirm this view by any post mortem examination. The latest theories upon the cause of this painful malady are as follows:-

(1.) Neuralgic affection, commencing for the most part in pneumogastric nerve, and spreading in different directions.

(2.) That it is due to such an acceleration of the movements of the blood, by exercise or otherwise, that it arrives at the heart faster than it can be transmitted onwards, and accumulates in its cavities so as painfully to distend them.

(3.) Ossification of the coronary arteries, which, by failing to supply sufficient blood to the cardiac walls, so impairs their strength that the heart is incapable of contracting upon the increased quantity of blood within its cavities, brought about by exertion or excitement.

\section{CYANOSIS.}

The blue disease described by some veterinary authors, and depending upon non-closure of the foramen ovale, is a condition which is only met with, and that very rarely, in very young animals. It is due to admixture of the venous with the arterial blood in the left cavities of the heart, owing to the foramen of communication existing in fotal life remaining pervious after birth. It is manifested by blueness of the visible mucous 
membranes, difficulty in breathing, and coldness of the surface. Animals so affected live but a short time.

\section{ECTOPIA CORDIS.}

Misplacement of the Heart.-The most common form of cardiac misplacement is that in which the heart is situated outside the chest. I have specinuens in my possession in which both ventricles and auricles are outside the chest, and in which the heart communicates with the interior of the body through large foramina in the steruum. 


\section{CHAPTER LV.}

\section{SPORADIC DISEASES-continued.}

LOCAL DISEASES-continued.

\section{(L.) AFFECTIONS OF THE DIAPHRAGM.}

SPASM OF THE DIAPIRAGM,

Often confounded with palpitation of the heart, is generally caused by over-exertion, such as a fast run in the hunting-field; it is also sometimes seen in tetanus. Its most prominent symptom is a convulsive motion or jerking of the whole body, accompanied by a dull, thumping noise, unconnected with the pulsation of the heart, emanating posterior to that organ in the region of the diaphragm. In some instances the impulse of the heart is barely perceptible; the pulse is small and weak, and there is great difficulty in breathing. The spasmodic movements of the diaphragm are not synchronous with the pulse: this, along with the fact that the sound proceeds from parts posterior to the heart, at once points out the difference between this affection and cardiac palpitation.

Treatment.-Generally speaking, a good diffusible stimulant, with warm clothing and quietude, is all that is necessary. Should the symptoms continue, opium is to be administered, and if at any time the dyspnoa be great, and the animal in langer of dying from apnoa, it may be necessary to abstract a moderate quantity of biood in order to relieve undue pulmonary congestion.

\section{RUPTURE OF THE DIAPHRAGM.}

Many cases of this lesion are reported by veterinarians. I am afraid, however, that in most of them the rupture has been post 
mortem, arising from the pressure of the intestines, distended with gases evolved after death. It is stated that the rupture, when occurring during life, is always found in the tendinous portion of the muscle, and that that occurring post mortem is in the fleshy part of it. In nearly every subject brought for dissection, if kept unopened until the abdomen has become much distended, the diaphragm is found ruptured. I am not acquainted with any symptoms diagnostic of this lesion, except those of abdominal pain, distressed breathing, and general disturbance. Mr. Robinson of Greenock tells me, however, of a case in which for two days prior to death there was general uneasiness, pawing with the fore feet, and, what might be looked upon as diagnostic, a dark patch of perspiration was seen to be constantly present on the skin opposite the diaphragm on one side. On making a post mortem a knuckle of intestine was found in the cavity of the thorax, forced through a round aperture in the diaphragm, opposite the patch of perspiration. Beyond this, I can gather no reliable information from the writings of veterinary authors which would justify me in laying down any rules for its recognition. If I were to do so, what I might state would be calculated more to mislead than to enlighten.

Should the lesion be found post mortem in the tendinous portion, or if at any time the gap be stained with extravasated blood, the probabilities are that it occurred during life. 


\section{CHA P TER L V I.}

\section{SPORADIC DISEASES-continued.}

\section{LOCAL DISEASES-continued.}

\section{(M.) DISEASES OF THE DIGESTIVE ORGANS.}

Wiтh very rare exceptions, diseases of the digestive apparatus are results of errors in feeding. Some of them have been already discussed under the head of Dietetic Diseases, and need not again be referred to. I may here, however, briefly observe that horses are best kept in health and working condition when fed upon an admixture of food requiring thorough mastication, and that horned cattle are best kept in health when, in addition to the more nutritious aliments, they are freely supplied with food requiring remastication, such as hay, grass, or straw.

Observations on the diseases of digestive organs point to the conclusion that in the horse the intestines are more liable to suffer from disease than the stomach; whilst in the ox and sheep the reverse is the case; and in the dog, consequent upon its power of vomition, the stomach is more rarely disordered than one would be led to expect from the nature of its food, \&c.

In the horse the stomach is a simple organ, small in comparison to the size of the animal, and in contrast with the volume of the intestines. It is but slightly called into action during the digestive process, and provided the food be properly masticated and incorporated with the salivary secretions, it is arrested for a short time only in the stomach, but is passed onward into the intestinal canal, where the process of digestion is completed. On this account the intestines are more liable to disease. It is also a remarkable fact that easily digested food, if given over-abundantly, is apt to derange the small intestines, whereas food containing much woody fibre, such as over-ripe hay, more particularly rye-grass, coarse straw, \&c., accumulates in the large intestines, and there causes derangement, inflammation, and even paralysis of the intestinal muscular tissue. It is also a fact worthy of notice, that if food be given artificially prepared by boiling or steaming, it is retained in the stomach itself, and if given over- 
abundantly, causes distension, inflammation, paralysis, and even rupture. This is accounted for by the circumstance that food thus imperfectly prepared for digestion is retained or imprisoned by the action of the pyloric structures, and thus distends the stomach by its bulk, or by gases evolved by the process of fermentation, which is apt to ensue. By bearing these facts in remembrance, the practitioner will to some extent be able to arrive at a correct idea of the seat of gastric or intestinal disease.

The food of the horse contains an abundant quantity of starchy materials, and the process by which these are rendered soluble commences in the nouth, not only by their admixture with the salivary secretions, but by a chemical change, through which the non-soluble starch is converted into dextrine and grape sugar, and made fit for the action of the intestinal, biliary, and gastric secretions, and for absorption by the vessels of the gastric and intestinal walls. For the purpose of performing this process the liorse is provided with twenty-four mill-stones, in the form of molar teeth, which have the power of crushing and triturating the hardest food, and of an extensive system of salivary organs, which secrete-most actively during the process of mastication -a fluid which most effectively blends with, and chemically changes, the food thus triturated. On this account we find that, when horses are sufficiently but not over-abundantly fed with dry food of a proper quality, the stomach rarely suffers from disease. An error in the diet, however, or a sudden change from one kind of food to another, not only deranges the stomach, but the intestinal canal as well.

In the ox and sheep, the large and complicated stomach not only digests, but also prepares the food for digestion. For example, ruminating animals eat and swallow the coarsest food very rapidly, and they are provided with a large receptacle for its retention, in which it undergoes maceration and reduction to smaller particles by a slow churning movement in the rumen and reticulum, which facilitates its trituration during the process of rumination and after-solution by the digestive fluids.

Without entering further into a physiological consideration of the process of digestion, it will be seen that the stomach of the ox much more actively participates in the process of digestion than that of the horse, and that it is thus rendered more liable to disorder in its first and second, as well as its third and fourth compartments, than the simple. single stomach of the horse. 


\section{CHAPTER L VII.}

\section{SPORADIC DISEASES-continued.}

\section{LOCAL DISEASES-continued.}

\section{(N.) DISEASES OF THE STOMACH.}

\section{INDIGESTION WITHOUT ENGORGEMENT.}

From various causes, such as improper food, the process of dentition, diseases of the teeth causing imperfect mastication, ravenous feeding, the presence of other diseases, debility of the stomach itself resulting from some constitutional predisposition, or from food given at uncertain and rare intervals, a condition of indigestion is induced in the horse. In young animals the same is induced by draughts of cold milk; removal from the dam at too early an age, or, what is commonly the case in some districts, compelling the dam to work shortly after the birth of the offspring, and allowing it to suckle at rare intervals and when the dam is heated.

Symptoms.-In the horse the symptoms are, loss of appetite, or depravity and capriciousness of it, manifested by the animal eating at irregular intervals, or having a desire to eat filthbulimia-with sourness of the mouth, and usually increased thirst; the animal soon becomes hide-bound, has a dry, scurfy skin, there is irregularity of the bowels, and frequent escape of flatus by the anus. If caused by imperfectly masticated food, such as whole oats or coarse hay, these may be found in the fæces. In addition to the above diagnostic symptoms, there may be a dry cough, or irregularity of the pulse, which may be slower or faster than natural; colicky pains may also be present in some cases, occurring more particularly in an hour or two after the animal has partaken of food; whilst in others, fits of giddiness-megrims-and even paralysis occur, the latter condition being not seldom seen in cattle, and very often in dogs.

In the young the above symptoms are more commonly associated with diarrhoa than in the older animal, in which constipation is generally present. The freces often resemble the colour of the food: for example, if the horse be fed on 
dark-coloured hay or clover, the freces will be dark-colonred also; if, on the contrary, it be fed on oats, the freces will be light in colour; and in the young animal, when fed on milk, it will often resemble it both in colour and consistence, mixed, however, with large masses of curdled milk, and often very foetid. I have often noticed that when indigestion is induced by clover, the urine is very dark in colour, and deposits a thick, almost brick-coloured sediment. This condition of urine, however, need not cause any apprehension, as it is often seen in the slover-fed animal without any disease being present. The urinary deposit mostly consists of carbonate of lime, the tinge being due to the colouring matter of the food. Indigestion, however, is a fertile source of deposits in the urine, which result from imperfect nutrition of the tissues, or a chemical change in the constituents of the blood-plasma, due to the products being imperfectly prepared, or containing some material unfit for healthy nutrition, as already referred to in the former parts of this work. ${ }^{\mathrm{I}}$

Treatment.-Carefully inquire into the cause, and remove it. If due to the process of dentition, the presence of unshed crowns of the temporary teeth irritating and wounding the mouth, or to any irregularity of the dental apparatus, these must be attended to according to the directions laid down under their several heads. In all instances where such causes are not in operation, even when the cause cannot be traced to the food, it will be necessary to make some alteration in the diet, and to examine the various alimentary matters in order to detect the offending one if possible.

If diarrhœa be not excessive, and the animal thereby much debilitated, it will be advisable to give a mild aperient or a moderate cathartic. To the young animal a dose of castor or linseed oil; to the older, a moderate dose of aloes, combined with a vegetable bitter, ginger, or gentian. After the laxative has operated, I have found that the bicarbonate of soda, with

1 Continental writers conclude that the diarrhœea or white scour of young animals is an infectious disease, developed from the first to the third day after birth, less frequent after the fourth day, and that the milk has nothing to do with its causation. I cannot subscribe to this opinion. The disease, as observed in this country, is due to irregular or unnatural feeding; and by unnatural I mean forcing young animals to swallow their milk without sucking it. Diarrhœa and dysentery are, however, observed in the young when infected with the septic diseases already described. 
gentian, or, where the stomach seems much debilitated, nux vomica, to have a most beneficial effect, not only improving the appetite, and removing the acidity-pyrosis or "heartburn"which is usually present-manifested by a tendency to lick the walls or other cold or alkaline material-but strengthening and improving the gastric apparatus.

It may be here stated that the mineral tonics, particularly the salts of iron, are inadmissible, and generally do harm in the earlier stages of gastric debility, or indigestion, often destroying what little appetite remains, and becoming combined with sulphuretted liydrogen in the intestines, tinging the frecs a black colour-a certain sign in all instances that the iron is not digested or absorbed into the circulation, where alone it can be beneficial, and that it is doing harm. It is very true that even in health large doses of iron salts tinge the fæces, but moderate doses have not this effect to any great extent. In some cases the alkaline-bitter treatment fails in having the desired effect. When this occurs the mineral acids, particularly the nitro-muriatic, may prove beneficial. In foals and calves pepsine can be administered, as in all probability the indigestion is due to imperfect secretion of the gastric glands; even in the older animal this is often presumably the case, and more especialiy when the disorder occurs without apparent cause, the same remedy will prove beneficial. It is almost needless to observe that the dieting of the animal is to be carefully conducted, and that pure air, moderate exercise, and good grooming are essentials to good digestion. Occurring in the winter, if the animal be thickly clothed with hair, clipping will act almost magically, restoring the digestion and appetite, which may have been long impaired, notwithstanding remedies, in the course of a few hours. In the cow, chronic indigestion, as exemplified by recurring tympanitis and other symptoms similar to those observed in the horse, and when not occurring from recognisable external causes or other diseases, is often due to the presence of some foreign body in the rumen or reticulum, removeable in some instances by the operation of rumenotomy, hereafter to be described. In all chronic indigestions of the cow this operation is recommendable. I have repeatedly performed it successfully, even where no foreign body has been found, the mere removal of the longretained food having been sufficient to restore the organ to its 
healthy condition. In young calves, indigestion, associated with convulsive fits, is sometimes due to hair-balls; these after a time become gradually disintegrated by the movements of the stomach, and the symptoms may slowly disappear. In rare instances hair-balls are found in fully grown cattle, and, as in calves, result from the auimals licking each other. In calves the symptoms of urgency are often relieved by stimulants, such as the carbonate of ammonia or turpentine; should the indigestion, however, remain for a considerable period, recourse must be had to the operation already referred to. It may be here mentioned that common salt given in the food promotes digestion in all animals. In the dog indigestion is manifested by frequent retchings or vomitings and fotor of the breath, and is best treated by a brisk purgative, antacids, and a restricted diet.

INDIGESTION WITH ENGORGEMENT-IMPACTION OF THE STOMACHPLENALVIA - GASTRIC TYMPANETES-HOVEN.

Distension of the stomach may arise from repletion with solid food, or from the evolution of gases arising from solids or liquids contained within it undergoing the process of fermentation, or disengaged from the gastric walls when the stomach is empty, as occurring in conditions of great prostration.

The causes in the Horse.-Impaction of the stomach results from the ingestion of food too abundant in quantity, or greedily swallowed and imperfectly masticated. In those parts of the country where the cooking of food for horses is a common custom, it is found that deaths from diseases and lesions of the digestive apparatus are very common. From the reasons already hinted at, namely, that it is necessary for the food to undergo not only the process of trituration by the teeth, but that it requires to be chemically altered by combination with the saliva, it will be understood that food prepared in any other way, as cooking by boiling and steaming, is unfitted to be acted upon by the stomach, and is consequently retained within it-the animal meanwhile continuing to eat-until its walls become distended, paralyzed, or even ruptured.

Some kinds of food, nutritious in themselves, and theoretically calculated to be proper for the horse, are found practically to be highly dangrerous. Wheat, for example, which is highly 
nutritious, containing, according to Sir H. Davy, 955 parts of nutritious matter in 1000, is found to be improper, deranging the stomach, causing purgation, laminitis, and death. Barley again has a similar effect, and for these reasons is found to be an improper article of diet.

When, however, from various circumstances, such as damaged crops, it becomes compulsory to cook the food, it is necessary that it be given with the greatest caution, in small quantities, and at intervals. Bran again, so useful an article when combined with other foods, or as an occasional mash, if given in large quantities, is retained undigested, and induces a condition of repletion which often proves fatal. Some kinds of hay, musty or otherwise damaged, or too ripe previous to being cut, barley and foreign straw, are also common causes of impaction; whilst green foods, particularly when animals are first put upon them, or if given too abundantly, not only induce engorgement, but also undergo formentation in the stomach, and thus induce tympanitis.

In horned cattle and sheep the same conditions are produced, most commonly by damp grasses, turnip-tops, maltcums, clovers, \&c.; indeed it may be stated that various foods, if given overabundantly, will cause tympanitis, but none so speedily as green clover.

In horned cattle, tympanites-hoven or blown-arising from retention of food in the rumen, frequently accompanies other diseases. It is wonderful how great a quantity of food is found in the rumen of an animal which has died from a disease which has existed for several days or even weeks. In many instances, where an animal suffering from pleuro-pneumonia has not partaken of any solid food for a period extending perhaps over a fortnight, it has been found that the rumen contains several bucketfuls of alimentary matters. The rumination having been suspended, there has been no true digestion, and the food partaken of prior to the occurrence of the illness has lain, as it were, in a mass in the inert and paralyzed rumen, having a tendency to undergo fermentation, and thus induce tympanitis.

An additional cause of retention in the rumen is found in disease of the salivary glands. Fluorens asserted that from the period of feeding to that of rumination there is a constant abundant secretion of saliva, which is constantly swallowed; if 
this be stopped, the contents of the rumen become hard and unfit for regurgitation. In this way rumination is suspended, and tympanitis induced. These observations have been confirmed by Colin, who also found that if the parotid ducts were opened, and the secretion thus prevented from flowing into the mouth, rumination became suspended.

The act of vomition, rarely performed by the horse, occurring only as a symptom of a grave lesion or disease, might be easily performed by ruminants; in fact the regurgitation of the food during the act of rumination indicates this facility, but vomition itself seldom occurs.

It is not my purpose to enter into a physiological discussion upon this matter, but merely to state that I am of opinion that vomition is rendered difficult in the horse by folds of mucous membrane at the cardiac orifice, and that, if from any cause, such as inordinate distension or rupture of the stomach itself, flaccidity, dilatation, or paralysis of the lower end of the cesophagus - the rugæ become unfolded, and the cardiac orifice opened-that vomition can and does occur. The valve of Gurlt, which is described by some authors as a spiral valve at the cardiac opening of the stomach, has no existence.

The true source of the rarity of the act in ruminants is, I think, satisfactorily pointed out by Mr. J. S. Gamgee, and is due to the fact that these animals are nauseated with great difficulty. I have, however, witnessed very forcible vomition in cattle suffering from indigestion, as well as the passive return of food into the mouth, which occurs during the profound coma of parturient apoplexy. Indeed, upon more than one occasion such ingesta have found their way into the trachea and bronchial tubes, and have caused a fatal pneumonia in two or three days after recovery from the parturient disease.

The dog, pig, and cat are easily nauseated by various remedies, and vomit with great facility.

Symptoms in the Ox and Sheep.-Tympanites is diagnosed by a swelling on the left side, which may appear during the time the animal is feeding, or shortly after; the breathing is difficult and laborious, becoming more so as the gas is generated and as the swelling increases. The oppression of the breathing is manifested by the general appearance of the animal; there is expansion of the nostrils, moaning during the expiratory movement, 
eructations, dribuling of saliva from the mouth, and some degree of uneasiness; rumination is suspended; the bowels soon become constipated, and if the tympanites is extreme, there will be a prominence and wildness of the eye which is characteristic of obstruction to the entrance of air into the lungs.

The moan or grunt, which is heard not only in tympanites, but in various forms of indigestion, even when unaccompanied by distension, is similar to that of pleuro-pneumonia; and on this account indigestion has been mistaken for pleuro-pneumonia, and credit claimed for curing the latter disease when it had no existence. The moan of indigestion is rather more prolonged, and resembles a groan more than the grunt of pleuro-pneumonia. The gases evolved have been found to be composed of carburetted hydrogen, sulphuretted hydrogen, carbonic acid, and in some cases carbonic oxide. Unless relieved, the animal will die, either from pressure of the distended stomach upon the diaphragm, causing suffocation, or from the absorption of noxious gases into the circulation. In some instances, tympanites is chronic, and, as already stated, may depend upon the presence of foreign bodies in the rumen.

Treatment.-In very urgent casees, the most effectual treatment is that of puncturing the rumen with a trocar, and allowing the gases to escape through the cannula. The operation is to be performed on the most prominent part of the swelling, and at equal distances from the spine of the ilium, last rib, and transverse processes of the lumbar vertebræ. When the symptoms are not very urgent, tympanites can be relieved by stimulants and stomachics, such as carbonate of ammonia, turpentine, alcoholic preparations, or the vegetable spices, particularly if given in warm ale. After the symptoms of urgency have been removed, cathartics are to be prescribed, such as salts, with croton and aromatics, care being taken that the animal be kept upon a restricted diet for some days after recovery. When the tympanitis becomes chronic, stomachic stimulants, particularly nux vomica, are to be prescribed; but if, along with the attention to dicting, they fail to give relief, it may be necessary to open the rumen, when the cause may be detected and removed.

IMPACTION OF THE RUMEN WITH SOLID MATTERS.

The symptoms are similar to the above, with the exception 
that the swelling, resonant when tapped with the fingers in tympanites, is dough-like and pits on pressure. In some instances I have noticed that the pitting remains for a considerable period after the pressure is removed, indicating that the coats of the rumen have lost their muscular tonicity, and that its morement is in abeyance, or entirely lost. I have also noticed that in sucl cases medicinal remedies do harm until the viscus has been to some extent emptied, and that the best method of doing this is to perform rumenotony before the powers of life become exhausterl.

The best method of performing this operation is as follows :After securing the animal by the nose, with its right side to the wall, plunge a sharp bistoury into the rumen, commencing the puncture midway between the last rib and the spine of the ilium, and from four to five inches from the points of the transverse processes of the lumbar vertebræ, cut downwards until the wound is large enough to admit the hand.

Some practitioners recommend that a towel or handherchief be introduced into the wound, in order to prevent the food falling into the peritoneal cavity. I find it much better to place a suture at the lower part of the incision through the lips of the double wound. When this is done, the contents are to be removed by the hand; the parts are then to be thoroughly cleaned; the incision in the stomach to be first stitched up, its edges being turned inwards, so as to get the peritoneal coat into apposition. The best material for the sutures is small catgut or fiddle-string. The external wound may then be closed with a stronger suture of strong waxed twine, over which a stiff pitch plaster it to be applied. Cathartics are to be administered, succeeded by vegetable bitters, more especially nux vomica, and the animal is to be carefully fed. In less urgent cases, where the rumen still retains some tone and power of movement, removal of the impaction is to be effected by cathartics and stimulants, the best cathartic for this purpose being an admixture of croton, aloes, and sulphate of magnesia, succeeded by a plentiful supply of fluids, treacle, and an occasional dose of ginger or ammonia. To restore tone to the rumen when debilitated, common salt dissolved in cold water has been recommended. This can do no harm, and failing other remedies, may be given in tympanitis and impaction. 


\section{IMPACTION OF THE THIRD STOMACH.}

Variously termed fardel-bound, vertigo, maw-bound. This is a very popular disease, some writers ascribing every case of constipation to impaction of the omasum, basing their conclusions upon the fact that the contents of this viscus are in a dry and hard condition when examined after death. But seeing that this is its natural state, and that, when animals have died from what appeared to be obstinate constipation, its contents liave been found moister than natural, I have arrived at the conclusion that what is supposed to be impaction of the third stomach is in reality an inflammation of the mucous membrane of the true stomach-abomasitis-or true gastric inflammation. I do not mean, however, to state that the third compartinent does not participate in the disorders of the others; but, on the contrary, that disease commencing in the rumen, reticulum, or abomasum, soon involves the third compartment. I shall not, therefore, separate the description of this disease from abomasitis.

In the gastritis of ruminants a highiy disturbed condition of the nervous system is a listinguishing symptom, evidenced either by a high state of delirium, coma, or convulsive fits, indicative of disturbance of the brain proper, or by paralysis of the posterior extremities, when the area of the disturbance is limited to the posterior parts of the spinal cord. Paralysis, convulsions, and coma are also frequently seen in dogs when suffering from gastric affections, and the same may be said, but in a less legree, of the horse.

In addition to the above symptoms, the gastritis of ruminants is characterised by more or less diarrhœa, soon succeeded by an apparent obstinate constipation, which, however, is not due to an obstruction by impacted food, but to cessation of the peristaltic action of the intestines, the contents of the stomachs being found generally more or less fluid after death. In many instances the animal strains violently, and passes both blood and mucus, showing that the inflammation has extended into the intestinal canal, and it is said that a hard swelling may be detected on the right side, arising from distension of the third stomach. In many cases, however, general swelling of the abdomen, tympanitis, supervenes early in the discase, and greatly adds to the animal's suffering. 
Outbreaks of gastritis prevail in some parts of Scotland during the spring and early summer, and is known by the tern "grass staggers;" and Mr. Clark of Coupar-Angus, in a paper read before the Scottish Metropolitan Veterinary Association, July 4th, 1894, has given an able deseription of this disease after an experience of twenty years in a district where the disease prevails to a remarkable extent. He states that animals of all ages and breeds become attacked when placed in fields favourable to the development of the disease, such fields being generally poor land or good land poorly treated, and which have been sown with too large a portion of rye-grass and too small a quantity of permanent grasses. When the quantity of rye-grass has been diminished, and that of the permanent ones increased, the disease has disappeared from land known to be favourable to its development.

The disease appears in about a month after the cattle have been turned out, and to disappear upon the advent of the white elover, which occurs from the 10 th to $1 \bar{t}$ th of June in $\mathrm{Mr}$. Clark's district.

The disease is dangerous on first year's grass, less so on the second, and rarely, if ever, on a third year's crop; but it has been known not to attack on the first, but during the second year's crop.

Artificial feeding seems to diminish and modify attacks, but prevention ean only be effectual by the climinution of the quantity of rye-grass sown, increase of the natural ones, and better treatment of the land.

The premonitory symptoms are obscure: the animal loiters about, feeding occasionally, and when lying down there is a flapping or restless movement of the ears and intermittent tremors at the elbow and flank, and any excitement, even at this stage, such as driving from the field, is apt to induce loss of vision. The next symptom is purging, the freces being black and watery, with entire loss of appetite, failure of milk, grinding of the tceth, accelerated pulse, cold extremities, blindness, but the sense of hearing seems to be very acute. There is no elevation of temperature.

In three days the erisis is reached, when the animal may become intensely excited, bellows fiercely, presses the head against the valls, and has violent tier.ors; and, if unfastened, 
scrambles up against the walls, and gives every evidence that it is suffering pain of the most formidable character.

The post mortem reveals acute gastro-enteritis, involving the abomasum and small intestines, and in the rapidly fatal cases the contents of the omasum are in a soft and normal condition, - thus doing away with the idea that the disease is due to impaction of the maniplies (omasum); but if the disease has been more prolonged before death or slaughter, it will then be found that the food is hardened and the walls of the viscus covered with small red or congested spots, "which," Mrr. Clark states, and I agree with him, "has given rise to the erroneous opinion that the animal has suffered from obstinate constipation. Such, I feel certain, is not the case, the hardened or dry con. dition being the result and not the cause of the disease." $\mathrm{Mr}$. Clark thinks that the disense is induced by an irritant, being also more or less narcotic in character, causing malassimilation of food, and as a result non-nutrition of blood, accompanied by inflammation of the true stomach and intestines, this being the only constant morbid condition found on post mortem inspection.

The treatment recommended as the most successful is linseed oil as a purgative, Epsom salts being highly objectionable, and calculated to reduce the chanees of recovery. A large percentage of cases are fatal, and bleeding has proved injurions. When brain symptoms set in, benefit can he obtained by the application of cold water to the head.

When the animal is in good condition Mr. Clark advises slaughter, and mentions that some farmers are under the necessity of renting grass parks-permanent pasture-to tide over the dangerous period of May and June.

Professor Dick said he was successful in treating this disease by repeated bleeding, even to faintness, large doses of purgative medicines given in large quantities of gruel, cold water to the head, \&c. He, however, considered the disease to be mere impaction. At the present time no one would think of pursuing such an irrational course in cases of acute gastritis; and having the knowledge that the constipation results from loss of function rather than impaction, we will do well to recommend a course of treatment calculated more to modify that inflammation thar to overcome the seeming constipation. For 
this purpose sedatives, such as aconite or belladonna, with antacids-bicarbonate soda or potash-and one or at most two moderate doses of an oleaginous aperient, with an abundant supply of fluids for the animal to drink, fomentations to the abdomen, and enemas, are to be prescribed. This treatment is much more calculated to save life than the indiscriminate use of powerful cathartics and stimulants. I speak advisedly, having witnessed many animals destroyed by the administration of repeated doses of cathartics in order to overcome constipation in this affection, the parties prescribing not being perhaps aware that an inflamed part loses its function, and that before the function and the peristaltic movement of the bowels can be restored the subsidence of the inflammation is essential, and that such inflammation is much more likely to be increased than diminished when the inflamed tissue is irritated by drastic cathartics or other remedies, which are supposed to rouse up the action of the bowels. If passage of the frees is not restored in the course of twenty-four hours after the administration of the aperient, it does not follow that it is necessary to repeat it ; time must always be allowed in all inflammatory diseases for the inflammation to subside, and for the weakened or debilitated parts gradually to resume their normal functions. The late Professor Strangeways was very successful in the treatment of this affection. At the commencement he gave a dose of oil, with sedatives, and after the febrile symptoms had to some degree subsided, from eight to twelve ounces of sulphate of magnesia, fifteen grains of quinine, and a few drops of sulphuric acid, ample time being allowed before any additional cathartic was given. It was very seldom necessary to repeat the medicine, as the bowels became gradually restored to their natural healthy condition.

Impaction of the stomach in the dog and cat are naturally orercome by romition, which also occurs sometimes in the pig, - but should this not oceur, an emetic is to be administered.

The dog suffers from catarrhal inflammation of the stomachgastrom hoea-induced by improper food, or occasionally by enzootic influences, in which there is a high degree of fever, hot nose, blood-shot eyes, and quick pulse, abdominal pair, constipation, and frequent or almost constant attempts to vomit; a dense mucus tinged with bile being sometimes thrown up. The most 
sliccessful treatment consists in allaying the gastric irritation by small doses of hydrocyanic acid and antacids, and when this is effected, gently moving the bowels by a small dose of castor oil. Enemas may, however, be beneficially employed in the earlier stages, and if the abdomen be tender or swollen, fomentations or a warm bath. If the strength seem to fail, the pulse small, \&c., stimulants are to be cautiously given. As a rule, however, they do more harm than good, and if the first dose is not succeeded by visible improvement they are to be discontinued.

\section{SYMPTOMS OF GASTRIC IMPACTION IN THE HORSE.}

Pawing with the fore feet, especially the near one, eructations of gas, sometimes attempts at vomition, with occasional discharge of saliva from the mouth, some degree of fulness of the abdomen, colicky pains, tremors of the superficial muscles, particularly those in the region of the left shoulder, with partial sweats upon the body, are more particularly the symptoms of this disease.

True gastritis, except from the action of dircet irritants, such as poisons, is but seldom seen in the horse; its stomach may be distended even to rupture, without any marks of inflammation being discoverable after deatl.

When inflammation of the stomach is induced by arsenious acid, the symptoms are great pain with uneasiness, the animal alternately getting up and lying down, tympanitic abdomen, fxces mixed with mucus, the saliva foetid, and its secretion increased, mouth hot, extremities cold, nausea, purging, and great prostration of strength with delirium. When the irritation is caused by the bichloride of mercury, there is, in addition to the above symptoms, a profuse discharge of saliva from the mouth. The antidotes for arsenical poisons are the hydrated sesquoxide of iron, chalk, albumen, or magnesia, and for the mercury salt, white of eggs, the symptoms of irritation being combated by opium, oleaginous purgatives, and demulcents.

\section{TREATMENT OF IMPACTION OF TIE STOMACH IN THE HORSE.}

Aloetic purgatives; if combined with tympanitis, oil, turpentine, or ammonia; enemas, fomentations to the abdomen; care at all times being taken that the animal be prevented from 
throwing itself suddenly down and causing a fatal lesion, namely-

\section{RUPTURE OF THE STONACH.}

The symptoms are, sudden tremors, particularly of the fore extremities; in many cases extension of the near fore limb, profuse perspiration, great prostration of strength; the animal breathes heavily, staggers in its walk, looks round to the flanks, and is generally seized with symptoms of vomiting, during which the head is suddenly depressed, the nose bronght down to the sternum by spasmodic contractions of the inferior cervical muscles, and in many cases there occurs an actual expulsion of food from both the mouth and nostrils.

The value of vomition as a diagnostic symptom of rupture of the stomach is certainly very great. Occurring in rupture of the stomach, it is said to be due to the muscular coat having first given way, thus allowing the mucous membrane to protrude, and the rugæ of the cardiac orifice to become unfolded. This conclusion is true in part only, for vomition will occur in rupture of the colon or other intestine, and in dilatation of the cesophagus. It must, however, be admitted that the act of vomition is much more complete in rupture of the stomach and dilatation of the cardiac orifice than it is in any intestinal lesion. The differential symptoms of rupture of the stomach, which occurs in the great curvature towards its pyloric portion, and a paralyzed condition of the cardiac orifice, are chiefly those manifested by the general condition of the animal. In rupture, prostration and rapid sinking of the animal powers are very extreme, the pulse feeble and fluttering, and death soon closes the scene; whilst in the other condition, though sweats bedew the body, and the animal exhiljits extreme agony, the vital powers still remain tolerably strong; the pulse, though frequent, still retains some fulness, and the surface of the body and extremities are never deatllly cold as in rupture.

Abdominal pain, as ordinarily manifested by rolling, striking violently at the belly, \&c., is not a constant symptom of rupture. In some cases the animal will stand immoveable, breathing heavily, being seemingly afraid to perform any movement which may increase its anguish; in other instances, the ordinary symptoms of abrlominal pain, combined even with delirium, are present. 
I have observed that all medicinal remedies incrcase the severity of the symptoms. This is undoubtedly due to their escaping into the peritoneal cavity. Mr. Percivall asks the questionsCan vomiting take place after rupture? Will the real stomach retain any power of ejection? Would ejection of the coutents upwards be produced by the abdominal muscles and disphragm without the aid of the stomach? And replies, "I should very much doubt it. I should rather feel inclined to the opinion that the act of vomiting should be taken as a proof of the entireness of the stomach. At all events," he says, "we may lave rupture happen without vomiting; and, consequently, we must cease to regard that symptom as pathognomonic, though we may justlo consider it, in company with others, as one throwing much ligity upon the nature of the case. Onr guides, in the absence of any one infallible patlognomonic sign, must be-the history of the case, the subject of it, the circumstances attending it, the inflated or enlarged condition of the abdomen, the symptoms of colic or gripes ceasing and beconing succeeded by cold sweats and tremors, the pulse, from being quick and small and thready, growing still more frequent, and at length running down and hecoming altogether imperceptible; the countenance denoting gloom and despondency of the heaviest character, with or without vomiting."

This graphic description contains some errors. For example, the enlargement of the abdomen is not generally associated with ripture; on the contrary, the belly in some instances is smaller than natural, the abdominal muscles being rigid and tense from tonic spasm, and the condition of rupture is undoubtedly associated with vomition, for it not unfrequently happens that the animal dies immediately after that act.

In addition to impaction from over-feeding, the stomachs of old horses particularly become ruptured from degeneration of their walls. When this occurs the gastric walls are exceedingly thin, and atrophied for some distance around the breach; and if examined microscopically the tissues are found to have lost their histological character, being reduced to a granular débris or an oily material.

There is no treatment for this lesion; and if the veterinary surgeon is satisfied that it has occurred, he will do well to order the animal to be put out of its misery. 


\section{CHAPTER LVIII.}

\section{SPORADIC DISEASES-continued.}

LOCAL DISEASES-continued.

\section{(O.) DISEASES OF THE BOWELS:}

\section{CONSTIPATION.}

THE bowels of some horses are naturally torpid. Constipation, however, may be looked upon more as a symptom than as a disease in itself. So long as the animal remains in health there is no necessity to employ active remedies for the removal of constipation, and all that is necessary is to give an occasional bran or linseed mash. Should the condition be caused by the nature of the food, such food must be changed, and one of a more laxative description substituted.

In many diseases constipation results from debility of the bowels, and is to be overcome by remedies, the action of which on the healthy borly may be considered astringent in virtue of their tonic effects, such as the salts of iron, cinchona bark, gentian, nux vomica, and other tonics and bitters.

Constipation may also arise from paralysis of some portion of the intestines, and if large and repeated doses of cathartics be administered, a fatal termination may be looked for, either from their toxic effects upon the system generally, or their direct irritation on the intestinal canal. It is, therefore, advisable to act cautiously in all cases of constipation, to allow plenty of time for the paralyzed bowel to regain its tone, to rouse it by stimulants and nervine tonics, and to administer enemas, which may contain turpentine. One symptom of paralysis of the bowels is diagnostic, namely, the absence of intestinal murmurs. Another may be mentioned of not infrequent occurrence, 
especially if the paralysis be in the large intestines, namely, a dilated, dry, and non-contractile condition of the rectum, which feels, when the hand is introduced, as a large cavity with passive walls.

\section{COLIC.}

Colic is of two kinds, namely, 1st. Spasmodic; and 2d. Flatulent. Spasmodic Colic.-A spasmodic contraction of the muscular coats of the intestines, which may run on to inflammation, due to improper food, sudden changes of diet, exhaustion from overwork, particularly if associated with long fasting, and to other circumstances, trivial in themselves, and quite insufficient as causes if uncombined with other disturbing influences. For example, a drink of cold water is often supposed to cause colic. Now water, no matter how abundantly it might be drank, as is witnessed in diabetes, does not cause colic; but if an animal be exhausted by a long journey, or bathed in profuse perspiration, cold water may then cause disturbance and abdominal pain.

Subcutaneous injections of chloride of barium, 12 to 15 grains, or of physostigma, 2 grains, are exceedingly serviceable in noninflammatory constipation, but exceedingly dangerous where the condition is associated with signs of inflammation.

Colicky pains are also symptomatic of intestinal concretions, parasites, introsuseeption, mesenteric abscesses, and of diseases of other organs, such as the pleura, kidneys, liver, \&c. On this account colic has been divided by some authors into true and false; the true including the colicky pains arising from all intestinal diseases involving structural change, and the false those from other causes.

Amongst other causes of colicky pains may be enumerated mesenteric abscesses, succeeding strangles or other suppurative disease, ulcers in the stomach, the irritation of numerous parasites, cancer, and chronic inflammation and thickening of the intestinal walls.

I have seen two cases of chronic induration of the duodenum and pylorus. The symptoms in both were as follows:Capricious appetite, slight colicky pains succeeding in about two hours after a meal, a peculiar staring appearance of the eye, hanging back in the stall to the full length of the collar shank, a peculiar frightened look, irregularity of the heart's action, the 
pulse sometimes slower, sometimes faster than natural, but always irregular; gradual emaciation and death. The post mortem appearances, a thickening of the submucous areolar tissue, which presents a whitish and fibrous character, and being united most intimately with the mueous and muscular coats, which were both pale, thickened, and eontained much translucent material. The whole mass was firm, and resisted the knife.

Symptoms.-When colic is truly intestinal the symptoms are, sudden pain, pawing, kicking at the belly, looking round at the flanks, lying down, rolling, struggling in a variety of ways, or lying outstretched; then suddenly rising, shaking the body, and remaining for a short period free from pain. After a short interval, however, the symptoms return, sometimes in an aggravated, occasionally in a modified form, and this occurs again and again, until the animal is either relieved or dies from enteritis, pain, and exhaustion. During the paroxysms of pain the breathing is accelerated, sighing, or panting, the pulse is observed to rise in frequency, and to become more or less full and hard; during the intervals of ease it may fall to its normal condition. At the commencement of the attack there is generally a frequent evacuation of small quantities of fæces, which are sometimes hard, sometimes soft; the urine is passed in small quantities, or there are frequent but ineflectual attempts to micturate; and if an examination be made per rectum, the bladder will very often be found full and distended, the urine being retained by a firm eontraction of its neck. In some instances, more especially if the animal has been fed on moist grasses, potatoes, or unripe eorn, there is diarrhœa and eseape of much foetid flatus.

The seat of the spasm is sometimes in the small, often in the large intestines; the symptoms during life, however, do not enable us to ascertain this with certainty. I have, however, observed that pawing with the fore feet, frequently looking round to the side, with very aente and sudden pains, point to the small intestines as being the seat of the spasm. In some instances there has been violent agony, a tendency to rear, and to kiek savagely with one hind foot, when the disease has been confined to the small intestines, whilst a disposition to back or press the hind quarters against a wall or other solid object is almost a constant symptom of impaction of the colon.

Flatulent colic, whether oceuring primarily or subsequent 
to an attack of spasmodic, is a condition from which much more serious results are to be apprehended than the spasmodic.

Its causes are-weakness of digestion, but most commonly food which easily undergoes fermentation, such as raw potatoes, green clover, a mixture of maltcums and brewer's grains, wheat, and boiled food.

In this form of colic the expression of pain, though not so acute, is much more constant than in the spasmodic form; the abdomen is more or less tensely swollen, and resonant upon percussion; the pulse soon becomes rapid and feeble, the breathing difficult and mostly thoracic, the extremities become cold, there is more or less delirium, the animal reels to and fro; twitching of the muscles, retraction of the lips, and if relief be not afforded, death ensues either from asplyxia, blood poisoning from absorption of gases, or rupture of some portion of the intestines. When the animal lies down or rolls it is observed that it performs these acts much more carefully than when suffering from spasm.

Tympanites may arise independently of any cognizable extrinsic cause. Occurring during the progress of another disease, it is always to be looked upon as indicative of a very grave condition, that the animal powers are so exhausted as to be bordering upon dissolution, and becoming amenable to chemical laws. Tympanites also occurs in obstructions of the intestinal canal from calculi, tumours, or other mechanical causes, and generally indicates the approach of death.

Treatment of Spasmodic Colic.-Slight attacks are often permanently relieved by tincture of opium, with spirit of nitrous ether, administered as a draught with water, or with eight to ten ounces of linseed oil. If relief be not afforded, say in half an hour, and if the colic be not associated with inflammatory symptoms, an attempt must be made to remove the source of irritation by means of cathartics or aperients. The late Professor John Gamgee recommended as the sole treatment the administration of a ball containing from eight to ten drachms of aloes, according to the size of the horses, if fed upon dry food; but if the food were of a succulent nature smaller doses were to be given, with enemas of warm water. He condemned the use of anodynes and stimulants. Other practitioners prefer to give from twelve to twenty ounces of linseed oil, combined 
with a stimulant such as carbonate of ammonia, spirit of nitrous ether, or with oil of turpentine; others prefer tincture of opium; others belladonna or tincture of aconite, Indian hemp, chloral hydrate, and other fancied anolyne or stimulating drugs. For my own part, I now prefer linseed oil with tincture of opium and spirit of nitrous ether, followed, if necessary, by sodium hyposulphite, one or two ounces dissolved in water, at intervals of three or four hours, with an occasional enema of warm water. All eases of bowel affections should be examined per anum at the commencement of the attack, as there is a possibility of detecting and removing a cause lodged in the rectum. The enema tube should consist of guttapercha piping at least four feet long, with a rounded nozzle, and introduced by gentle-not forcible-pressure as far as possible into the bowel. If the urinary bladder be distended, pressure from the rectum with the palm of the hand will often assist the act of urination; if this be insufficient, relief must be given by means of the catheter.

In many parts of the country colic is looked upon as a disorder of the urinary organs by non-professional people, and from the fact that the first sign of recovery is often the act of urination, countenance is given to this opinion. There is doubtless a spasmodic contraction of the constrictor vesicæ in the majority of colic cases; and relaxation of this spasm is a concomitant of that of the intestines; hence rapid recovery generally takes place after the act of micturition.

When the contents of the bowels are found by rectal examination to be hard, an attempt should be made by manipulation or kneading with the hand in the rectum to break down the impacting masses, and thus assist the bowels to resume the peristaltic action.

In conclusion, it may be stated that if the attack be a very slight one, a single dose of the opiate will often give permanent relief. There is one thing that I should warn the practitioner about, and that is, no certain dependence can be placed upon this, and that in all cases where the animal cannot be watched for some time afterwards the aperient is not to be omitted; and upon no account is a horse attacked late at night, and thus relieved, to be left without the aperient being administered, for it has often happened that when all are asleep in bed the pains have returned, and the horse in the morning found dying or dead. 
Treatment of Flatulent Colic.-In addition to the administration of an aperient for the removal of the cause, it becomes essentially necessary that the symptoms of urgeney be promptly relieved. Carbolic acid or the hyposulphite of sola to some extent averts fermentation and the further formation of gases, and thus prove very useful. If, however, the tympanites be severe, or seem to increase under the prescribed treatment, the colon should be immediately punctured with a Toop trochar and cannula, to enable the gases to escape, and thus give immediate and permanent relief. The method of procedure is as follows :- Select the most prominent part of the swelling, which is generally about midway between the last rib and anterior spine of the ilium, upon the off-right-side; asepticise the spot, then make an incision with a small bistoury, and introduce the point of the trochar rather obliquely upwards, in order to allow the escape by drainage of any fluid which may be afterwards exuded, and thus prevent the formation of an abscess; when the bowel is punctured, the stilette is withdrawn, when the gas will be freely expelled; and in order to prevent further fermentation it will be necessary to inject, by means of a Toop syringe, through the cannula, about three-quarters of an ounce of pure earbolic acid, dissolved in twenty ounces of warm water, into the intestine. I feel confident that we have saved many horses in the College practice since adopting this treatment.

If the tympany is not entirely removed by the escape of the gases, the operation may be performed on the opposite flank with great benefit. There is no danger in the operation : in one case it was performed thirteen times, giving relief of several hours' duration after each puncture, in a case of tympany associated with a large calculus in the colon. The horse lived several days, and on making an examination of the seats of puncture only three or four small reddish spots were seen, with no surrounding inflammation.

\section{IMPACTION OF THE COLON.}

As already stated, animals over-abundantly fed, or kept upon food containing much woody fibre, are liable to suffer from the accumulation of such matters in the colon and cacum. The diagnostic characters of this, in addition to symptoms of general abdominal pain, are a tendency to push backwards, to press the 
tail against any solid object, to resist by violent straining the introduction of the hand and enemas into the rectum. In some cases the hard and impacted mass may be felt by the hand introduced into the rectum, and there is also some enlargement or distension of the abdomen. The propriety of administering cathartics by the month for the relief of this condition is a question of very great importance, for it has often happened that rupture of the colon las occurred in the course of some hours-generally about twenty-after the administration of an aloetic ball, the condition of the intestinal canal, when examined post mortem, having been found as follows:-The stomach and small intestines more or less emptied of alimentary matters, or containing large quantities of fluid; the large intestines distended with a mass of more or less hardened material, or partly hard and partly soft; the mucous membrane sometimes highly congested, sometimes pallid, but ruptured, and the contents more or less escaped, as in ruptured stomach, into the peritoneal cavity. I have repeatedly witnessed this, and the question has arisen in my mind whether the rupture has not been induced by the contents of the small intestines having been forced into the already over-burdened large ones by the action of the purgative. Concluding that this was the case, I have injected aloetic solutions into the rectum, and endeavoured to excite the commencement of purgation in the large intestines. In some instances this has suceeeded, whilst in others it has failed; the failure has been due to the expulsion of the medicine by the animal. I have found it the best method to dissolve two ounces of aloes in a pint of hot water, and to administer it with a syringe having a long flexible tube, at a temperature of about $90^{\circ} \mathrm{F}$. If this be retained for an hour or two, it generally has some effect, but if immediately expelled, it ought to be repeated. I am well aware that writers on materia medica will say that the specific action of aloes is limited to the large intestines and rectum, no matter how it gains entrance into the economy, and that this result will be obtained by its administration by the mouth. My experience leads me to the conclusion that, although it may irritate the posterior bowel during its exit from the body, its primary effect is to stimulate the peristaltic action and glandular secretion of that part of the intestines with which it first conies in contact.

Piupture of the colon from impaction, or when resulting from 
degeneration of its muscular eoat, is manifested by symptoms similar to those of rupture of the stomach. If oceurring from impaction, it will be found that the passage of enemas, which had previously been difficult, owing to the violent straining or resistance of the animal, becomes suddenly easy; the hand introduced into the rectum meets with little or no resistance, the intestine itself being dilated and paralyzed.

\section{DISEASES OF THE RECTUM.}

The condition of degeneration observable in the stomach and colon is sometimes met with in the reetum, eausing a gradually decreasing contractile power of the bowel, in eonsequence of which the freal matters accumulate to an inordinate extent, and are only expelled by violent straining and contraction of the abdominal muscles. In such instances, it is observed that the freces of the horse, which in its normal condition is divided into globular pellets, is diseharged from the body as a huge mass, resembling a large German sausage, and as thick as a man's leg. I have observed this condition, which may be denominated paralysis of the rectun, as a result of injury of the spine or sacro-lumbar nerves, and independently of injury in old horses habitually kept upon coarse, indigestible food. When resulting from injury, it has sometimes disappeared, either spontaneously, as the lesion which eaused it beeame repaired, or by the assistance of nervine tonics, more particularly nux vomica, and blisters to the sacro-lumbar region. In many instances, it has been necessary to empty the bowel by the hand four or five times daily, and to administer enemas to prevent pain and colicky symptoms. Rupture of the reetum has sometimes oceurred from this eause.

Rupture of the rectum has also oecurred within my experience from the formation of an aneurism of the hremorrhoidal arteries, the pressure of the aneurismal tumour having caused atrophy, not only of the muscular and peritoneal, but also of the mueons coat of the intestine, and rupture of these being caused by some sudden effort; hrmorrhage has occurred both into the intestinal canal and peritoneal cavity. Rupture has also been witnessed as a result of a tumour, and as an accident during parturition.

Aceidental rupture of the rectum, posterior to its peritoneal 
lining, is not necessarily fatal; but if anterior to the termination of the peritoneum, the escape of freal matters and of blood into the peritoneal cavity will cause death. In one case, where the rectum was pierced by the foot of the foal during parturition, causing a recto-vesical fistula, I was enabled to bring the lips of the wound together by metallic suture, and induce their ultimate union, the mare eventually doing well.

Inversion of the rectum is caused by violent straining during parturition, or attempts to expel fæcal matters; as a result of paralysis; in the coma and spinal paralysis witnessed in parturient apoplexy in the cow; and in pigs it is a common occurrence owing to constipation. In clogs it results from the impaction of bones or other hard bodies in the bowel, causing violent straining. Its reduction in all animals must be preceded by thoroughly emptying the bowel of all sources of irritation. When this is effected, the bowel, thoroughly washed and oiled, is to be carefully returned into its proper position, and retained there by a suture across the anus, or a rope or truss properly adjusted (West's clamp), and the sense of pain and irritation soothed by opium. For several days the bowel is to be carefully emptied by the hand, or enemas, and the animal fed upon a restricted diet. Purgatives are to be avoided; the bowel may, however, be lubricated with oleaginous enemas.

In recurring inversion, which commonly happens in the pig, excision of the protruded intestine may be successfully performed; and in all cases where it is found impossible to return the bowel, the engorged mucous membrane is to be carefully dissected from the subadjacent structures, and a reduction thus effected. It now and then happens that the sphincter of the anus closes firmly, forming a constricted neck, preventing the return of blood to such an extent that the protruded intestine speedily becomes gangrenous. If the sufferings of the aninal be not very great, it is generally advisable to allow a short time to elapse before removing the sphacelated mass, in order that new adhesions may form; but if the animal suffers severely, it is advisable to insert sutures for the purpose of causing union between the gut and anal opening, and at once remove the whole protruded mass with a sharp bistoury, the subsequent pain and straining being alleviated by a full dose of opium. When several feet of the intestine are protruded, as sometimes happens after parturition, it becomes an impossibility to returu 
it. The intestine is generally lacerated from being trodden upon, and humanity renders it necessary that the animal should be put out of its sufferings.

\section{HAMORRIIOIDS OR PILES.}

With the exception of the dog, the domestic animals rarely suffer from piles, which consist at first of congestion of the mucous membrane at the verge of the anus, and subsequently of dilatation of the hæmorrhoidal veins, constituting small tumours. These sometimes protrude outside the anus, and bleed frequently. The diagnostic symptoms of piles are switching of the tail, and a tendency to rub it against the wall; pain during the act of defecation, and the freces being tinged with bloorl. The dog sits on its liaunches and pulls itself along in that position. The examination of the anus will reveal the presence of vascular tumours, and of much congestion and swelling of the mucous membrane and skin.

Treatment.-The cause of hremorrhoids depends upon some obstruction to the portal circulation, constipation, and frequently on retention of hardened fieces in the rectum; the treatment must therefore be directed to the removal of these by manipulation, gentle laxatives, and a restricted diet. The uneasiness may be modified by fomentations, enemas, and the application of some mild astringent. Astringent ointments are also useful, more particularly an ointment consisting of equal parts of oak galls and hog's lard; the benzoate of zinc ointment is also a useful application.

\section{IMIPERFORATE ANUS.}

A congenital malformation met with in all the domesticated animals, particularly in the pig. It gives rise to symptoms of colic, and generally proves fatal shortly after birth. In some instances the anal opening is well former, but the rectum at a short distance from it forms a "cul de sac," which is not continuous with the alimentary canal. In other instances the anal opening is permanently closed by skin, the bowel terminating in a pouch underneath it. When this condition is met with, relief is possible by puncturing the intestine, thus forming an artificial anus. 


\section{CHA P TER LIX.}

\section{SPORADIC DISEASES-continued.}

LOCAL DISEASES-continued.

(O.) DISEASES OF THE BOWELS-continued.

\section{INFLAMMATORY DISEASES OF THE INTESTINES- ENTERITIS.}

ENTERITIS, or inflammation of the bowels, may safely be stated to be the most rapidly fatal inflammatory disease to which the horse is liable, destroying life in the course of a few hours. Indeed, it is very doubtful whether the disease recognised as enteritis by veterinarians is a true inflammation at all, as its course and progress, rapid termination, and post mortcm appearances tend to confirm the opinion that it partakes more of the nature of apoplexy than of inflammation. It is very true that impaction, constipation, introsusception, the presence of calculi, or the action of irritant poisons, may cause great congestion and inflammation of the intestinal membranes; but the disease to be described originates sui generis, and very often without the occurrence of an immediate and recognisable cause of direct irritation. This fact has led some practitioners of very great experience, amongst whom may be mentioned the late Mr. Lawson of Manchester, to arrive at the conclusion that enteritis never originates in colic, spasmodic or flatulent. Many writers assert that enteritis is situated in the small intestines, more particularly in the ileum and jejunum. My experience, however, leads me to the conclusion that it is more commonly situated in the cxcum and colon than in any other part of the intestinal canal, but no portion of the tube is exempt. The only recognisable causes are, over fatigue, cold 
from exposure, or from washing with very cold water whilst the animal is heated, and thereafter inadequately clothed.

\section{PATHOLOGY.}

Various writers state that the inflammation is situated in the muscular coat. This is evidently a mistake, as the congestion is of the greatest severity in the mucous membrane, the redness of which is of a deep venous colour, approaching to blackness, in patches of various extent, and associated in many cases with extravasation of blood into the canal. Of course, when such extreme congestion of the mucous membrane exists, all the coats are more or less implicated, but the primary and gravest condition is limited to the mucous membrane.

The submucous tissue is generally much thickened; there is loss of cohesion, the mucous membrane being easily stripped from its attachments; some effusion of serum into the intestinal canal, causing purging in cattle and dogs; but in the horse purging seldom or never occurs, though the contents of the bowels may be found fluid after death. This seeming constipation results from paralysis or loss of function, and is marked liy complete retention of the frecal matters. In some rare instances enteritis may terminate favourably in the horse; but in the great majority of cases mortification results, or the animal dies from the debilitating effects of liæmorrhage into the intestinal canal, or from prostration of the nervous system induced by pain. Gangrene may result in eight or ten hours, the animal rapidly succumbing. In some instances, however, death may not result for several days. In one instance a horse lived for a period of five days, the bowel being found sphacelated, the process of ulceration having commenced at the edges of the gangrenous patch. It is very rarely that ulceration occurs in the horse, but it may be witnessed both in cattle and dogs.

\section{SYMPTOMS.}

The first noticeable signs are those of abdominal pain; generally, however, they are preceded by some degree of constitutional disturbance, rigors, accelerated breathing, repeated evacuations of small quantities of frees, and general depression; 
the mucous membranes soon become deeply congested, the mouth dry, the tongue contracted, and now and then of a brownish colour, the appetite of course being lost; the pulse is liard, wiry, and quick; the belly is tender upon pressure; the abdominal muscles more or less contracted; and if tympanites be absent the belly may seem tucked up and smaller than natural. Byand-by the symptoms of dulness and depression give place to those of excitement and pain; the horse stamps the ground with the feet, strikes at the belly, lies down, but much more carefully than in spasmodic colic, or makes feints to do so; it may roll upon its back; turns its eyes anxiously towards the flanks, pants, blows, and sweats with pain. There are no sharp paroxysms of pain with intervals of ease as in colic, but the pain is constant, distressful, and agonizing; in some cases so much so, that the sufferer seems afraid to express it, except by a most anxious expression, which is a characteristic and diagnostic symptom. Now and then the animal will stand persistently with the head in a corner, and paw the ground for hours together with one or both fore feet alternately. The pulse is hard, wiry, and quick, often rangiug from $S 0$ to 120 beats per minute; and as the clisease advances, it becomes thready and imperceptible; the animal sighs, or even groans with pain; the perspirntion runs off the body; the skin is never dry, at one time hot, at another cold; the countenance becomes haggard, the eyes expressive of delirium, pupils dilated. The horse may throw itself about in a most dangerous manner, or walk round its box incessantly; then it will stand, balance itself, its legs give way, when it may fall and die after a few convulsive struggles, or suddenly all symptoms of pain may subside; it will then stand quiet, and even drink or endeavour to feed; its breathing becoming more or less tranquillized; but the haggard expression of the face still remains; the pulse continues thready and imperceptible; cold sweats bedew the body; the belly becomes tympanitic; gangrene has now set in; it trembles incessantly; the legs and ears are deathly cold; the mouth cold; the breath rold, and even foetid; the lips drop pendulous; the eyes become more amaurotic, and after a varying interval death closes the scene, the bowels remaining inactive to the last. If, however, in three or four hours from the commencement of attack there be some abatement of the symptoms; if the surface of the body 
become dry, if there be a passage of flatus or fieces, the pulse becoming frller and softer, and the characteristic anxiety leave the expression, a favourable termination may be anticipated. This, however, is rarely the case.

\section{TREATMENT.}

Concluding that the constipation which is so prominent a symptom in enteritis is due to obstruction from alimentary natters, veterinarians generally administer powerful eathartics. It has, however, been shown quite conclusively that it is always dangerous to propel fiecal matters through an inflaned portion of bowel; and that in most cases the effort is useless, so far as exciting peristaltic action in the inflamed portion is concerned. Purgatives, however, stimulate and excite the muscular and excretory action of the healthy intestines anterior to the inflamed part; the result of this is, that the inflamed part becomes more and more distended, inflammation is increased, the bloodvessels become ruptured; it becomes softened, and its vitality is ultimately destroyed. It is therefore a fact that the inflamed part, the function of which is lost, consequent upon the inflammation, affords an imperliment, and that purgatives, as a rule, have no true purgative effect-that is to say, they do not cause the discharge of fiecal matters by the anus.

Two great principles are therefore recognised, namely-first, to relieve pain, and, second, to arrest as far as possible all movement of the intestines; and for these purposes opium is to be administered in large doses. For the horse, one, two, or even four drachms of the powder may be administered, succeeded by smaller quantities at short intervals, or by the subcutaneous injection of morphia (the solution of the meconate of morphia being recommended. The first subcutaneous injeetion should contain, in addition to 5 to 8 grains of morphia, a half grain of atropia; but the atropia should not be repeated for at least twenty-four hours, whilst the morphia may be administered every few hours, according to the severity of the symptoms. In addition, hot fomentations to the abdomen are useful; and they should be continuonsly applied for at least an hour at a time. Enemas of warm water may also be gently alministered: they are not, however, to be repeated too often, and if at any time they increase the pain, they should be discontinued. 
If, after the abatement of the active symptoms, the bowels remain torpid (as they generally do), the practitioner is by no means to attempt the removal of this torpidity by the administration of cathartics or aperients of any kind; for if the animal is to recover, it must be dependent upon the restoration of function and tone to the inflamed bowel; and it is well known that for this end perfect quietude of the inflamed part must be maintained.

The advisability of bleeding in enteritis will depend entirely upon the condition of the animal. If the pulse be moderately full, if depression be absent, an alstraction of blood commensurate with the strength of the vital powers will be followed generally by abatement of the symptoms, and if performed early is beneficial. Should the appetite return, great care must be taken that the food be of the simplest and of the most easily digestible kind, such as scalcled bran and boiled linseed given in moderate quantities; the eating of dry food being prevented by a muzzle. Care must also be taken, when the functions of the bowels are restored, that no undue accumulation of fæcal matter's be allowed to remain in the rectum. The question of administering stimulants during the acute stage will also greatly depend upon the condition of the animal; they usually do more harm than good, but if tympanites be present, one or two doses may be tried. If they give relief they may be continued, but if, on the contrary, they aggravate the pain or seem to have no effect, they are to be discontinued. It must not be lost sight of that antiseptics, such as carbolic acid or hyphosulphite of soda, in proper doses are important factors in counteracting putrefaction of the contents of inflamed bowels, and the rapid gangrene of the inflamed part, as well as a septic condition of the blood. If, however, they fail to reduce the tympany, relief by puncture must be immediately given (see Treatment of Flatulent Colic). 


\section{CHAPTER LX.}

\section{SPORADIC DISEASES-continucd.}

LOCAL DISEASES-continued.

(O.) DISEASES OF THE BOWELS-continued.

\section{INTUSSUSCEPTION AND VOLVULUS.}

Intussusception (from intus, within, and suscipio, to receive), and volvulus (from volvo, to roll $\mathrm{up}$ ), a twisted bowel. Under these names various forms of entanglement of the intestines, giving rise to abdominal pain, enteritis, and death, have been described.

\section{INTUSSUSCEPTION.}

By intussusception is meant the prolapse or slipping of a portion of intestine into the cavity of that immediately posterior to it. In consequence of this the natural course of the intestines is interrupted by a kind of knet, consisting of three successive portions of bowel; the immediate effect of which is obstruction to the passage of the intestinal contents, and to the return of blood from the imprisoned portions of intestine involved, along with which some portion of the mesentery must be included.

Both the small and large intestines are subject to it in all the domesticated animals. When, as is generally the case, it occurs in the large intestine, the cecum caput coli is that usually inrolved, and instances have occurred, both in horses and cattle, of recovery after sloughing of the invaginated cxecum. Within my own experience, a cow treated by me, after seven days of obstinate obstruction of the bowels, expelled the gangrenous portion, measuring several inches in diameter, and the animal recovered. 
Mr. Aitken, V.S.; Dalkeith, has a case on record of a cow which recovered after five days of illness, the gangrenous portion being expelled with the fæces. Before recovery from intussusception can occur, it is necessary that the opposed peritoneal surfaces become adherent, and the imprisoned portion separated by ulceration.

Invagination of the small intestines, owing to the severity of the inflammation, is necessarily fatal.

Symptoms.-In the cow already mentioned, the symptoms were those of enteritis and obstinate constipation. The treatment consisted in the administration of opium. In Mr. Aitken's case drastic cathartics were administered from the commencement. The symptoms in the horse, as related by the late Mr. John Field, are as follows :- " Pain; restlessness, in some cases approaching to madness, unrestrainable; wandering about; rolling on the back; sweating, in some cases profuse; crouching; sitting on the hind quarters, almost diagnostic; anxious countenance; frequent feeble pulse; belly at first of natural size, subsequently fuller, in some cases distended, dependent upon the locality of the intussusception; membranes, in advanced stage, turgid, injected ; mouth moist and clean, or furred and offensive ; respiration accelerated; continued restlessness; rearing with fore legs into manger, and standing upon that point d'appui, looking back from side to side; extremities cold; pain absent, tranquil; sighing or snorting; death. The sighing may exist in some cases and not in others, and in some retching and vomiting."

I am, however, of opinion that there is no diagnostic symptom of volvulus, intussusception, calculus, or strangulation of the intestines in the horse; that the above symptoms are common to all, characteristic of none. In the dog and pig, however, vomiting of stercorous matters is generally witnessed; but even in these animals this symptom may be induced by any cause of obstruction. Stones accidentally swallowed, pieces of bone arrested in the small intestines, are frequent sources of obstruction in the dog.

Treatment.-It has been proposed to cut down upon and mechanically remove the source of mischief. I think, however, that the operation would be as bad as the lesion. If intussusception be due to contraction of one portion of intestine, and the slipping in of that contracted portion into the healthy portion 
behind, remedies calculated to relieve spasm may prevent its occurrence, but cannot overcome it when once established.

\section{VOLVULUS, OR TWISTED BOWEL,}

May occur in either the small or large intestines, and, like strangulation of the intestines by pedunculated tumours, or from their entrance into the inguinal canal or the foramen of Winslow, are but rarely witnessed, even in the horse, and their exact diagnosis is attended with difficulty. It is true that some practitioners assert that when a horse resists the introduction of the hand into the rectum by straining, that it is indicative of volvulus of the colon; but if, on the contrary, the rectum be found passively distended-hollow-that it indicates impaction and paralytic loss of function of that bowel. I have seen many cases in my time, and can safely say that these diagnoses are unsupported by extended observation. Many theories have been advanced to explain the etiology of volvulus. A case published in the Veterinary Journal, January 1897, by Mr. Locke, M.R.C.V.S., Manchester, was likely induced by several falls while a high-spirited horse was taxed beyond his strength in trying to pull a heavy load out of a deep place. Rolling whilst suffering from colic has been perhaps rightly blamed, and aneurisms of the mesenteric artery, by causing anæmia of a portion of the bowel, has also been ascribed as a canse.

The diagnosis is extremely difficult. There is continuous pain, - mild perhaps at first when the large colon is twisted, severe from the first when the small bowel is involved; borborygma is absent, and no fæces passed after the very earliest stage; on rectal examination there may be a difficulty, if the backward pressure is great, in introducing the hand into the rectum.

Jelkman states that there is no difficulty in diagnosing volvulus per rectum, and even of effacing it by manipulating through the bowel. Copions enemata of oil and warm water, administered through a long tube, and the administration of anodynes-morphia or chloral hydrate-with attempts to reduce the volvulus by manipulation, by rolling the animal, and, as a last resort, laparatomy might be performed. As a rule, however, the progress of the congestive change is so rapid that there is little chance of saving life. Anæsthesia will render reduction a much easier matter, as well as give the aninal at least temporary relief. 


\section{CHAPTER LXI.}

\section{SPORADIC DISEASES-continucd.}

\section{LOCAL DISEASES-continued.}

\section{(O.) DISEASES OF THE BOWELS-continued.}

\section{INTESTINAL CONCRETIONS.}

THE common seat of the various concretions is found to be in the large intestines, where they sometimes attain a large size, and as much as twenty-five pounds or more in weight. Occasionally, but very rarely, they have been found in the stomach weighing four or five pounds.

They were studied by the late Professor Morton, and classified under three heads, namely, 1st. Phosphatic calculi ; $2 d$. Oat-hai calculi; and $3 d$. Mixed calculi.

1st. Phosphatic Calculi.-These are hard, smooth, and polished on their external surface, bearing much resemblance to a common pebble. If a section be made of a phosphatic calculus, it will be found to consist of concentric layers arranged around a nucleus, generally consisting of a piece of iron or stone. If several of them, or any other form of calculus, be formed together, they are generally flattened upon their sides, or concave on one side and convex on the other, the convexity of one stone fitting into the concavity of another, and so on. This form of calculus is now much more rarely met with than formerly. This arises from the circumstance that the food of animals is much better prepared and cleaner than it used to be, and containing, therefore, fewer accidental materials or foreign bodies, which formed the nuclei.

Their chemical composition, according to Girardin, is as follows :- 


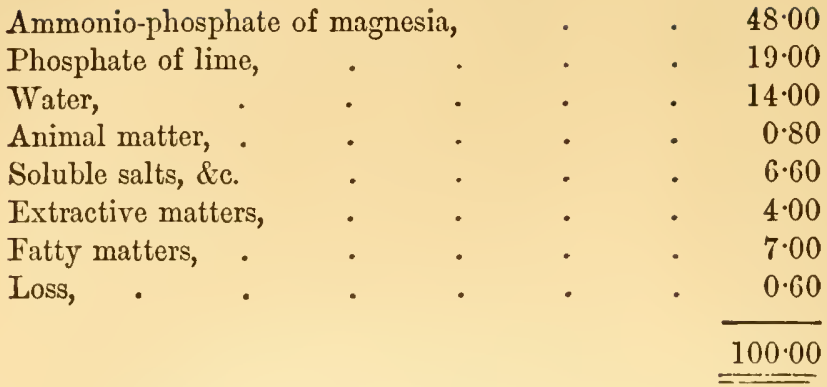

From the above analysis it would be seen that the calculi contained some animal matters; in fact the earthy materials are glued together by mucus, \&c.

Their occurrence is accounted for as follows by Professor Morton:- "In the cereal plants, certain of the phosphates are met with, and in considerable quantities. It is then to this food that we are to look for their origin, coupled with a morbid state of the digestive functions, by which it does not undergo the necessary change, probably from the gastric juice not being sufficiently powerful to dissolve these phosphates. A foreign body being accidentally taken into the stomach, which may be a nail, or anything else, as a piece of stone, serves as a common centre, the phosphates arrange themselves in their turn, and in doing so they blunt that which by its sharpness would wound the lining membrane of the organ. If from its magnitude the calculus is nnable to pass through the pylorus, the stomach becomes its residence. They will give rise to colicky pains, depravity of appetite, perhaps to wind-sucking or crib-biting, and in the end will invariably, by accumulation destroy the life of the horse.

$2 d$. Oat-hair calculi, generally found in the cæcum or colon, consist almost entirely of the beard of oats, barley, or other grain. They sometimes attain a large size, but are light in comparison with the first-named. They, however, assume the same shape, and are occasionally mixed with phosphatic salts.

$3 d$. The mixed calculi are composed of phosphatic salts, fæcal matters, oat-hair, or any indigestible matter which may accumulate in the intestine.

In addition to these, food may adhere to the mucous membiane, constituting what is termed a stercoral concretion, con- 
sisting very often of straw-knots, \&c., firmly glued together. This will sometimes be found perforated through its centre, so as to admit the passage of fæces.

The presence of these concretions at some time or another constitutes a fatal obstruction. Many symptoms are laid down as being diagnostic of calculus, such as sitting on the haunches, lying upon the back, \&c. I am not, however, aware that there is any diagnostic sign, beyond those of violent abdominal pain and obstruction, by which they may be discovered during life; and they can only be guessed at by recurrent attacks of colic and constipation. In one case which fell under my notice there were no symptoms of abdominal pain, but merely constipation, which nothing could overcome. For two days after the animal had been observed to be off its food the pulse remained natural; on the third day, however, the abdomen became tympanitic, the pulse rose rapidly, and the animal died; and the post mortem revealed the presence of a phosphatic calculus, about ten pounds in weight, firmly lodged in the single colon. In one case examination per rectum enabled me to feel and remove a calculus from the rectum, the animal being immediately relieved. This shows the necessity of such an examination in all cases of abdominal pain.

Cases are recorded of polypi or other tumours in the stomach and in the intestines. Gastric polypi finally plug up the pyloric orifice, and give rise to fatal distension.

To prove the fallacy of forcing the action of the bowels in cases of mere constipation, and to show how long a horse may live without passing any fæces, I may mention that I had a horse under my care that had no action of the bowels whatever for twenty-five days, yet it made a good recovery. It was noticed that after a purgative had been administered the pulse becarne greatly accelerated, mucous membranes injected, and that it manifested signs of pain, which continued for several hours, in fact until the medicine was excreted by other organs. Except when suffering from the effects of medicine it ate a moderate quantity of food. Another case lived eighteen days, passing no fæces, and showing no pain except when irritated by purgatives; towards the end of that day it showed pain, and died from calculus in the single colon. Upon this point the following letter from a late student, Mr. Martin, now in London, is instructive :- 
"London, 6th June 1879.

"Mr DEAR Sir-In reply to your note you kindly sent me of 29 th ult. in reference to the case of stoppage of the bowels of a horse I had under my care, I have now to inform you that he died on Monday evening, Nay 26, it being the thirtieth day that no fæces had passed from him. Within six hours of his death he started blowing, suffering a deal of pain, abdomen distended very much. Next morning, on post mortem examination, I found the bowels gorged, and a calculus firmly fixed in the single colon, weighing $2 \frac{1}{2}$ pounds, 5 inches in diameter. All the other parts were perfectly healthy. It appeared to me that the bowels becoming so full, caused him to die sooner than he would have done had less solid food been given him, although the result would have been the same." 


\title{
CHA PTER LXII.
}

\section{SPORADIC DISEASES-continued.}

\author{
LOCAL DISEASES-continued. \\ (0.) DISEASES OF THE BOWELS-continued.
}

DIARRHEA.

THIS term is applied to all cases of simple purging in which the fæces are loose, liquid, and frequently discharged without any coexistent inflammation. Diarrhœa may be a spontaneous effort to discharge from the intestines something which is obnoxious to them or to the system generally. It is also induced by a variety of canses in all animals, such as indigestible food; sudden changes of diet, particularly from a dry to a moist one; medicinal substances; parasites; derangement of the liver; or copious draughts of water when the animal is heated.

Some animals are particularly prone to diarrhœe from trivial causes; narrow-loined, flat-sided, and loosely coupled horsesthat is to say, horses in which the space between the ilium and last rib is large-and those which are of a nervous temperament, are apt to purge without apparent cause. They are vulgarly called "washy" horses. Such horses will start upon a journey in the best of apparent health, but before they have proceeded any great distance, will commence to purge more or less freely. They are hard to keep in condition, and require the best of food. If, however, put to slow work they will sometimes do well enough.

\section{SYMPTOMS.}

The symptoms of diarrhœa are purging, the fæcal matters being semi-fluid, of a dirty-brown colour, without offensive odour, 
or clay-coloured and fotid. If the condition continues long, the animal looses flesh, the appetite fails, and in some cases ascites, or even farcy and glanders, may supervene.

\section{TREATMENT.}

When the purging arises from the presence of some offending matter in the intestinal canal, its expulsion must be aidecl-and this applies to all our patients-by a moderate dose of castor or linseed oil, and the diet must be changed. Purging in the dog is often induced by a too exclusively farinaceous diet, and it is impossible to arrest it without an entire change of food. If the purging arises from no apparent cause, or if the bowels do not regain their normal condition after the action of the aperient has subsided, it will be necessary to administer calmatives, such as opium or mild astringents, such as chalk; proceeding, however, very cautiously, as too sudden a check may induce complications of a grave character. Boiled starch or flour gruel may be allowed the animal to drink; the food must be of the sweetest and besi kind, and given in moderate quantities.

If there be much fœtor the sulphite or hyposulphite of soda may be very advantageously given dissolved in the drink, or mixed with the food. If the animal be depressed and weak, moderate and repeated doses of the spirits of nitrous ether will afford relief, and promote the restoration of the bowels to their natural condition. Should this treatment prove futile, the more powerful astringents, such as catechu or kino, or, what has proved more successful with me, the oil of turpentine and opium beaten up with eggs, are to be administered. Some practitioners recommend the mineral acils, such as the aromatic sulphuric acid, these remedies having a tonic as well as an astringent action upon the bowels. Warm clothing and perfect quietude are necessary. 


\section{CHAPTER L X III.}

\section{SPORADIC DISEASES-continued.}

LOCAL DISEASES-continued.

(O.) DISEASES OF THE BOWELS-continued.

\section{SUPERPURGATION.}

Although a form of diarrhœa, from its gravity, and the important legal questions it may give rise to, superpurgation deserves a separate consideration. It is well described by the late Mr. Haycock. He says- "Superpurgation, or over-excitement of the intestines from the undue action of purgative medicine, is a condition of the bowels which the veterinary surgeon is frequently called upon to treat. The symptoms associated with the action of a purgative upon the bowels are quickened rate of the pulse, attencled with a partial but temporary loss of its force; the breathing also is a little hurried. This is the most conspicuous when purging is about to commence, and the animal is nauseated. If the purging, however, does not go on to an undue extent, these symptoms soon subside, the pulse becomes normal, and the nausea succeeded by a desire on the part of the animal for food; but should the animal be of weak constitution, or be taken out of the stable and ridden or driven a considerable distance while the purging continues, or taken out too soon after what is called the 'setting' of the physic, or if the purgative dose be too powerful in the first instance, or if two or more of these circumstances or causes act in association, the purging will in all probability becone excessive, and the life of the animal may be placed in danger. Purging may continue for a long time; but so long as the patient is lept quiet in the stable, so long as the appetite remains good, and the pulse maintains its regular, slow, and round beat, little or no danger need be apprehended; 
but if the appetite fail, if the pulse becomes thready, and the patient weak, it behoves the owner to act with promptness." "The following symptoms," says that excellent observer John Field, "indicate the violent and too long-continued action of purgatives, and invariably portend a fatal termination:-Staring glassy eyes; frequent, indistinet, feeble, and sometimes threadlike pulse; purging offensive matters, with or without distension of abdomen, or distended abdomen without evacuations; offensive mouth; tongue pallid or whitish with fur, and pasty; smell quite peculiar; respiration tranquil, but it becomes laborious when the belly becomes enormously distended; extremities warm; the horse usually stands still, sometimes paws or wanders abont, and but rarely lies down."

Superpurgation does not always depend upon the strength of the dose. In some instances as little as four drachms of aloes have been succeeded by fatal consequences. Again, horses in an obese condition, and those suffering from slight colds, are easily acted upon by purgative medicines, and are apt to sink from superpurgation. A full dose of aloes, from six to eight drachms, operating quickly, is seldom succeeded in healthy animals by any evil consequences; the same quantity, however, if divided into two or more doses, lias a much more depressing effect, and is apt to be followed by serious consequences. In the first instance, the quantity, by its strength, insures its own expulsion; whilst in the second, the aloes is absorbed into the circulation, excites a toxic effect upon the system generally, and reduces the horse to such a state of debility that its succumbs to the purgative influence. The explanation of the tendency to superpurgation in the horse is to be found in the fact that its bowels are extremely vascular in comparison with those of other animals, and that the effect of the purgative acting upon sc vascular a surface is grave and serious. I have ahready pointed out that many young horses, when first brought into the stables are rendered susceptible to various diseases by the debilitating influences of indiscriminate purging, and that such a method of treatment is uncalled for and irrational. In addition to the symptoms described by Messrs. Haycock and Field, I have observed that those of laminitis are induced by purgatives, and that when they occur they indicate a condition of great gravity.

The post mortem appearances are those of congestion of the 
intestinal mucous membrane generally, concentrated in many cases in that of the cæcum caput coli; a thick, tarry appearance of the blood, and extreme blackness, congestion, or apoplexy of the lungs, the blood being, as it were, deprived of its watery elements, altered in its composition, rendered too viscid to circullate through the pulmonary capillaries, and so altered chemically as to be rendered unfit for perfect oxidation.

In order to prevent the occurrence of superpurgation after the administration of an aloetic, or, more particularly, a mercurial and aloetic purgative, it is necessary that the practitioner should order the animal to be fed on an easily digestible diet, such as warm bran mashes; that the quantity of water should be restricted, and that the chill be taken off it, for nothing is so apt to induce inordinate intestinal action as large quantities of cold water whilst the animal is in physic. It is also necessary that no green food, roots, or other articles of diet, containing much water, and laxative in themselves, should be allowed at this period. If a purgative does not seem to take effect in from twenty to twenty-four hours after its administration, moderate walking exercise is to be prescribed, for it is a fact that the longer a purgative is retained in the body, the greater the danger from its superaction; if, however, purging has actually commenced, exercise, by increasing it, is apt to cause harm. It is therefore necessary to keep the horse quiet until the physic has " set."

\section{TREATMENT.}

So long as the horse remains moderately lively, the pulse but slightly accelerated, the countenance natural, and so long as some appetite remains, it is unnecessary to take any active measures to restrain the purging, which is the natural and physiological response of the intestines to the action of the cathartic; it is therefore irrational and dangerous to check it, and all that is requisite is to allow the horse to partake of remulcent drinks, such as thin flour gruel, if it will do so spontaneously, at the same time kecping it perfectly still, warmly clad, and taking care that it does not drink too freely of anything whatever. Should there be any colicky pains, moderate doses of opium are to be administered. Care, however, must be taken that the purging be not checked even by these means too 
suddenly; as the consequence of this would be congestion of the intestinal mucous membrane, denoted by tympanites, great prostration, cessation of the purging, and the other symptoms described by Mr. Field, finally terminating in death.

If the purgation continue, an endeavour should be made to overcome it gradually but not too quickly. For this purpose I know of nothing better than tincture of opium, chalk, and flour gruel. Two ounces of the tincture are to be given, with the same quantity of prepared chalk, mixed with a quart of flour gruel, every three or four hours until the purging is checked, hot applications being in the meanwhile applied to the abdomen, great care being taken that the animal has no access to cold water or other fluid, as its thirst is great and it is apt to drink inordinately; but it is essential that it should have small quantities of flour gruel or other emollient drink, not only to allay the painful and feverish thirst, but to keep the blood in a proper fluid condition to circulate through the minute pulmonary capillaries. If the prostration be very great, stimulants, as wine or brandy, are to be tried; if they seem to act beneficially, they are to be continued, but not otherwise.

As a medico-legal question, the occurrence of superpurgation after the administration of a simple and moderate cathartic by the veterinarian is one of great importance. In no case where due caution has been taken, where the dose has not been more than the necessities of the case required, and where the above mode of after treatment has been enjoined, should the veterinarian be held responsible.

Superpurgation is typical of inflammatory diarrhœa, no matter what its cause may be, and as the symptoms and treatment are identical, there is no necessity for further description. It may, however, be mentioned that a form of diarrhœa, even more fatal in its consequences, accompanied by a greater degree of prostration, and terminating fatally in a shorter period of time than that induced by medicinal cathartics, is brought about by an over-abundant feeding upon raw potatoes. Again, the same condition is induced by the ingestion of food containing sand, small pebbles, or other materials incapable of being digested. There is no cause, however, so rapidly fatal as raw potatoes, the toxic properties peculiar to the natural order "solanacece" being seemingly exerted upon the system gene- 
rally, in addition to the irritating effects of the tuber itself upen the intestinal canal.

The treatment of inflammatory or acute diarrhœea when arising from these causes becomes a matter of extreme delicacy. For, in the first place, the retention of the irritating material in the intestinal canal is a source of danger in itself; and, secondly, the extreme purgation which it induces is no less a cause of fatal termination. It therefore follows that the practitioner, on the one hand, must take care not to check the efforts of nature to expel the offending materials from the body too suddenly by the administration of powerful astringent remedies; and, on the other hand, that he should endeavour to modify the violent purgative and irritating effects by the administration of demulcents, calmatives, such as opium, and antacids, such as the bicarbonate of soda; and to support the animal's strength by diffusible stimulants, such as the ethers, wine, and alcoholic stimulants, in moderate and oft-repeated quantities; fomentations to the abdomen, and other means calculated to allay irritation and febrile disturbance, being by no means neglected, and to modify fermentation the hyposulphite of soda might be advantageously administered. 


\section{CHAPTER LXIV.}

\section{SPORADIC DISEASES-continued.}

\section{LOCAL DISEASES-continued. \\ (O.) DISEASES OF THE BOWELS-continued.}

\section{DYSEN'TERY.}

WhiLsT superpurgation and acute diarrhœea most commonly attack the horse, chronic diarrhœa and dysentery are much more commonly seen in cattle, dogs, \&c. Dysentery, sometimes termed "colitis," or "bloody flux," consists of an inflammation having a tendency to terminate in ulceration of the mucous membrane and glandular structures of the large and sometimes the small intestines. Dysentery is observed both in an acute and chronic form; the chronic in horned cattle being often dependent upon the scrofulous diathesis, with tubercular deposit and ulceration of the intestinal glands.

\section{CAUSES.}

Except as a concomitant of other diseases, such as rinderpest, or when induced by direct irritants, acute dysentery is a rare form of disease. It may, however, be induced both in horses and cattle by bad food or putrid water; some kinds of pastures, especially those situated upon moorlands and in shady places; on this account it was called "moor-ill" and "wood-evil" by the old writers. Occasionally it is seen as a complication of "red water " and "parturient peritonitis."

In the dog it is witnessed as the effect of cold and damp, and in the intestinal form of distemper.

\section{SYMPTOMS.}

In the ox the acute form is attended witl shivering fits, variable temperature of the body, arching of the back, and increased sensibility or tenderness of the loins; a furry tongue, and clammy mouth. The animal grunts, yawns, grinds its 
teeth, and at short intervals discharges a quantity of thin excrementitious material, mixed with pellets of hardened fæecs and blood. There is much straining, and irritation of the rectum and anus, which appear sore and red; some abdominal pain, evinced by arching of the back, whisking and extension of the tail, associated with tympanitis, great dulness, thirst, rapid emaciation, and an aphthous condition of the mouth.

In the chronic form there is great emaciation, a verminous condition of the skin, looseness of the teeth, and anasarcous swellings of the intermaxillary space; the fæces become deeply tingred with blood, contain much mucus, and after a time an admixture of fotid purulent matter. Ulceration about the anus ultimately appears; the freces are discharged involuntarily; the eyes soon become dim and sunk into the orbit, and the animal dies.

Both in diarrhœa and in dysentery of a chronic nature the freces contain gaseous materials, which cause the appearance of little air bubbles upon their surface when discharged from the body. The urine of an animal suffering from dysentery is of a high colour, increased specific gravity, containing an inordinate quantity of urea, proving the rapidity of the tissue changes occurring within the economy.

\section{TREATMENT.}

Both in the acute and chronic form much benefit is to be expected from the administration of a mild oleaginous aperient, succeeded by opium and antacids. Should these not succeed, styptics, as the oil of turpentine, or astringents, as tannic acid, sulphate of copper, terchloride of iron, or alun; and to overcome the foctor of the freces, hyposulphite of soda, or other deodorizers, may be given with advantage. When the disease is essentially chronic, associated with much emaciation and a tubercular diathesis, cod liver oil in such doses as the animal will tolerate up to four ounces, given twice a day, mixed with four or five eggs, will prove advantageous. It must be remembered, however, that all treatment nust be subservient to careful dieting; cake, good hay, and other articles easy of digestion and nutritive, being at all times recommendable. For the dog the same agents, in doses proportionate to the size of the animal, along with proper diet, such as rice-milk or flour porridge, are to be prescribed. 


\section{CHAPTER LXV.}

\section{SPORADIC DISEASES-continued.}

\section{LOCAL DISEASES-continued.}

\section{(O.) DISEASES OF THE BOWELS-continued.}

\section{ASCITES, OR DROPSY OF THE ABDOMEN.}

Peritonitis, or acute inflammation of the coats of the abdominal cavity, having been already described in Principles and Practice of Veterinary Surgery, I need only refer to it here as a condition which may lead to an alteration of the peritoneal structure by the inflammation becoming chronic, and to a collection of fluid in the cavity, constituting ascites, which may be defined to be a collection of fluid in the peritoneal sac-the nature of such fluid being sometimes simple, consisting of a pale straw-coloured serum, sometimes more complex, the serosity being mixed with flakes of lymph, and inclosed in cysts or cavities by the adhesions of false membranes.

\section{CAUSES.}

Direct disease of the peritoneum is a very rare cause of ascites in all the domesticated animals except the ox, in which it is found frequently, as a result of tubercular growths- " grapes"studded over the surface of the membrane.

Ascites results most frequently from diseases of the liver, the heart, or the portal blood-vessels; as a consequence of cardiac debility in some exhausting diseases; and from the ingestion of improper food, as when animals are kept during winter upon poor, ill-drained, or moorland pasturc. It may also arise from anæmia and deficiency of albumen in the bloos, whether induced by parasites, as in the "rot" in sheep, or independently 
of such invasion from any cause inducing deficiency of albumen in the blood. It also occurs in some forms of septicæmiabraxy-and is looked upon as a condition of "crisis," or an endeavour to excrete the morbid material from the circulation. In sheep and lambs a sanguineous form of ascites is not rarely met with, variously termed red water, water braxy, and "maladie rouge," or diarrhæmia, by the French. In this latter condition, not only is there a transudation of the fluid of the blood, but also of the colouring material (see Purpura), the globules being sometimes dissolved, sometimes entire, and more or less altered in appearance in the serosity. Ascites is also witnessed as a complication in hydrothorax and dropsy of the pericardium.

In the dog, dropsy of the belly is generally caused by disease of the liver, induced by over-feeding and want of exercise.

SYMPTOMS.

These are denoted by enlargement of the belly, sheath, and sometimes the legs, the enlargement being due to effusion, as indicated by fluctuation, and a dull sound on percussion. As the fluid increases, the breathing becomes thoracic; and in the sheep and ox odema of the submaxillary areolar tissue and inferior cervical region, with emaciation, feeble pulse, irregularity of the bowels, looseness of the hair and wool, and other symptoms common to exhausting diseases, supervene.

\section{TREATMENT.}

When arising from a removeable cause-as improper foodan improvement in the health and a disappearance of the collected serosity may be effected by generous diet, occasional aperients, and by tonics, more particularly the salts of iron. When associated with deficiency of albumen in the blood, it may be useful to introduce albumen into the economy by administering ergs to those in which the appetite is absent, and to those which still eat, by feeding on oil-cake and other nutritious diets, the effect of common salt as an aid to healthy digestion not being lost sight of. When ascites is due to organic diseases of the liver, heart, or other organ, treatment is of no use, except to palliate the symptoms, and in all cases it is better to put the animal ont of its suffering. Paracentesis abdominis, or tapping, gives temporary, lut seldom permanent relief. 


\section{CHA P IER LXVI.}

\section{SPORADIC DISEASES-continucd.}

\section{LOCAL DISEASES - continued.}

\section{(P.) DISEASES OF THE LIVER.}

Diseases of the liver are rare in the domestic animals, with the exception of sheep, which are destroyed sometimes in great numbers by various diseases of the liver, induced by exclusive feeding upon turnips, particularly Swedish turnips, causing a fatty or degenerative condition of the organ; by stimulating food, long continued, such as the various calies, inducing congestions and softening, and by the invasions of the "distoma" or fluke worms, giving rise to the disease termed "the rot," or of "strongyles," which appear as small bladders throughout the organ, and cause anæmia and death. But neither the horse, ox, nor dog is exempt from hepatic affections.

\section{CONGESTION OF THE LIVER.}

Under this head three forms are included, namely-1st. Passive congestion of the hepatic and portal veins, arising from anything which interferes with the circulation of the blood, such as disease of the lieart. In my paper on Traumatic Pericarditis, published in the Veterinarian in 1857, I pointed out the coexistence of this condition of the liver. Its occurrence is explained as follows:-In heart diseases there is stagnation of blood in the vena cavæ and hepatic veins; in time, as explained by Frerichs, this is propagated to the portal vein, and to the organs from which it takes its origin; the liver then becomes turgid with blood, and the congestion extends to all the veins of the digestive system. In the same manner consolidation of 
the lungs, by arresting the circulation of the blood, causes congestion of the liver and enteric veins, ann is one cause of the diarrhœe which is so often an accompaniment.

The second form of congestion is that termed "active," in which the arterial capillaries are mostly involved. This form is induced by food of a stimulating nature, given over-abundantly, particularly during hot weather, or when the animal is not receiving sufficient exercise. It has been already pointed out that animals rapidly got up for sale frequently suffer from a diseased condition of the liver. Dr. William Budd points out how congestions of the liver occur so commonly in the human being; the same reasons are applicable to some extent to the lower animals, particularly as hepatic congestions are generally met with in over-fed, slowly worked, pampered horses, such as those belonging to brewers, or people whose business requires animals for show as well as for labour. He says-" Amid the continual excesses at table of persons in the upper and middle classes of society, an immense variety of noxions matters find their way into the portal blood that should never be present in it, and the mischief which this is calculated to produce is enhanced by indolent or sedentary habits. The consequence often is that the liver becomes habitually gorged. The same or even worse effects result in the lower classes of our larger towns from the inordinate consumption of gin and porter." Compared to its frequency in man, active congestion in the lower animals is exceedingly rare. The late Professor Coleman was of opinion that this rarity was due to the simplicity of the liver in the horse-having no gall bladder-compared to its complicated structure in man. This view is evidently erroneous, as the livers of other domesticated animals are furnished with gall bladders, and it cannot be said that they are more liable than the horse to hepatic congestions, except from errors in feeding, to which they are perhaps more frequently exposed. Hutrel d'Arboval takes another view, and describes the exemption from disease to be due to the little areolar tissue entering into the composition of the horse's liver; this view is evidently as erroneous as that of Coleman.

Symptoms.-In hepatic congestions, as proved by post mortem examinations of animals which have died from "ramollissement," or softening, with perhaps rupture of both liver and its capsule, 
traces of repeated attacks of congestion, indicated by varions differences in colour and in the consistence of the hepatic textures, are not unfrequently present without there having been any manifestation of symptoms during life, beyond occasional loss of appetite, and perhaps a little dulness. Now and then, however, the following symptoms are observable:-Abdominal pain, the animal looking to the right side; yellowness of the mucous membrane; high brownish colour of the urine; constipation of the bowels; the fieces sometimes of a clay colour and fotid, with a sour, acid, or even offensive condition of the mouth; grinding of the teeth; a desire to eat earthy substances, or lick the walls; and in very rare instances pain, manifested by lameness in the off (right) shoulder, with varying degrees of febrile disturbance.

Treatment.-Bearing in mind that the engorgement may become so excessive as to cause rupture of both gland and capsule, practitioners must abstract such a quantity of blood as the character and nature of the pulse will warrant; so long as the artery is round, the pulsation distinct-no matter how severe the seeming dejection and debility may be-a free abstraction of blood will be succeeded by amelioration of the symptoms, as they are but consequences of the state of hyperæmia, the removal of which being of paramount importance.

With regard to medicine, cathartics, followed by neutral salts, as the sulphate of magnesia, cause a rlain from the portal system, and thus relieve the congestion. In the dog, elaterium has a special effect, by causing watery stools. A recurrence of the congestion is to be prevented by a restricted diet and regular exercise.

The third form of congestion is that due to engorgement with bile, arising from obstruction, parasites in the ducts, or inflammation of their mucous membrane, by which their calibre becomes diminished, and the flow of bile consequently arrested. This condition is associated with epizootic diseases, and is termed by some veterinarians "bilious influenza," and is characterised by yellowness of the visible mucous membranes and high coloured urine, with the symptoms of the epizootic from which the animals suffer.

The treatment of this form calls for no special comment, except that the administration of the so-called liver stimulants, as 
calomel, is contra-indicated, for the reason that the secretory powers of the gland are not interfered with, but that it is incapable of discharging the secreted bile, owing to the tumidity or swollen condition of the lining membrane of the small bile ducts; salines and a non-stimulating diet are therefore indicated.

\section{HEPATITIS, OR INFLAMMATION OF THE LIVER.}

Hepatitis, or inflammation of the liver, is one of the rarest diseases affecting our domesticated animals, the majority of cases diagnosed as such being probably due to congestion. It sometimes, lowever, occurs, and cases are recorded where the post mortem examination has revealed the presence of abscesses, transformed exudates, and even ossification of the products of inflammation.

The inflammation may have its seat in Glisson's capsule, when it is called "perihepatitis," and examinations of old horses slaughtered for dissection point out the fact that this form of inflammation-the formation of false membranes, and the adhesion of the liver to the diaphragm-is of no rare occurrence, unaccompanied, however, with any symptoms during life which point to its presence. Even in the human being, perihepatitis is rarely accompanied by serious derangement, unless the inflammation extends to the portal or hepatic veins, or causes obstruction of the larger bile ducts-events of rare occurrence. -(Frerichs.) In all probability this inflammation of Glisson's capsule accompanies the pleurisies to which the horse is so particularly liable.

Hepatitis, or inflammation of the glandular structure, may occur in a circumscribed or in a diffused form; the circumscribed leading on to suppuration-as in cases mentioned by the late Mr. John Field and others - or to the formation of patches of fibrous tissue, which appear as firm, light-coloured spots scattered throughout the crgan; whilst the diffused form induces rapid degeneration of the glandular structure, with softening and atrophy of the organ, or an indurated condition of it.

In the primary stage of the acute diffused form, patches of hyperæmia occur, the peripheral portions of the lobules being filled with a more or less fluid exudate. The viscus is swollen in proportion to the number and size of the inflammatory patches; 
the parenchyma is loosened and lacerable. As the inflammation advances, the red colour fades, and is replaced by a brownish or greyish-red tint in some parts, and yellowish-red or pale yellow in others. This condition, as well indeed as nearly all morbid conditions of the liver, is often accompanied by a similar state of the kidneys, and sometimes of the spleen.

Causes.-The causes are similar to those of congestion. "In hot countries hepatitis assumes an epizootic form, especially about the end of summer. It is almost always connected with inflammation of the other abdominal organs. After death the liver is found congested, of a greyish-red colour, and weighing from forty to fifty pounds. In addition to ordinary symptoms, there is irritation of the skin. Lessona deseribes such an epizootic as having occurred in Italy in 1827."-(GAMGEE.)

Symptoms.-The animal is dull, inactive, has a heavy head, lustreless eye, and loathes its food. Mr. Percivall says, "the horse seems as if it was suffering some inward pain; but it is clearly not of an acute kind. It has not lain down during the past night; its dung-balls are small and dark-coloured; its urinary discharges seanty; and there is manifestly a strong fever arising in the system. The fever runs on, and commonly on the second or third day after its onset turns out to be what farriers call "the yellows'-known by them to be so from the remarkable circumstance of the mouth and eyes assuming that colour. The inner surfaces of the lips and cheeks, the tongue, the conjunctivæ, and in some cases the transparent cornea and iris as well, turn yellow, manifesting the diffusion of bile over the body; and the same is further demonstrated by the deep golden dye of the serum of the blood. I have also observed yellow matters floating about in the aqueous humor. The dung-balls are deeply imbued with bile, and in some cases enveloped in a viscid, bilious, nucous matter as well ; their colour is that of a reddishbrown, leaving when rubbed upon white paper much the same stain as opium would. If any urine be caught, it will be found to be thick, to exhibit the same bilious tinge, and to deposit, on standing, a copious lateritious sediment. The horse will probably lie down quiet, and yet not appear easy, but from time to time turn a dolorous look at its side, and soon after raise itself up again; and if the right side be pressed against it will flinch, or bite, or otherwise express tenderness there." In 
addition to the above symptoms, lameness in the off shoulder has been observed. My own experience leads me to conclude that this is a rare symptom, and is more particularly symptomatic of the existence of abscesses or of foreign bodies in the gland. In one case a thorn has been discovered; in other two, which canie under my immediate observation, a stocking needle was found in one, whilst in the other a calcified exudate, surrounded by purulent material, was detected after death. In the latter case, the symptoms of liver disease, which had continued for many months, became associaterl with those of chronic tetanus, and, finally, with ascites. I think it right to mention that in another animal which had died suddenly, in addition to a calcareous concretion, rupture of the liver was found, the animal having manifested no symptoms of disease during life.

Treatment.-In the earlier stages of acute hepatitis, purgatives and sedatives, more particularly aconite, or, if the symptoms be very acute, nauseants, as digitalis or veratrum. In human practice, ipecacuanha stands in high repute. It is given in large doses for the purpose of causing nausea, profuse diaphoresis, and frequent bilious motions. Its value in the treatment of the liver diseases of horses and cattle has been proved to be very great, and it is almost invaluable for the dog and other carnivora. It will be clearly understood that calomel and other so-called liver stimulants are inadmissible in this condition; if, however, the liver remain sluggish after the subsidence of the febrile symptoms, one or two doses may be given in combination with stomachics.

\section{CHRONIC HEPATITIS.}

This form of disease may occur as a sequel to or independent of acute hepatitis.

It tends to create various changes in the substance of the liver, whereby it either becomes enlarged and softened in structure, or hardened, indurated, and diminished in bulk.

Induration of the liver is described under a variety of names -cirrhosis, interstitial hepatitis, hob-nailed or gin-drinker's liver, nutmeg liver, and chronic atrophy; and the nature of the changes which occur in the liver substance are explained in three ways :-

(1.) Dr. Goodeve, who takes the view of Dr. Budd, says that 
"when it commences with inflammation or congestion, the course which leads to atrophy is as follows:-Fibrinous exudation takes place; this occupies the portal canals, and extends even into their minute ramifications, so that the very lobules may be separated by the exudation. Livers in this early stage are much enlarged, are firm and tough-sometimes very tough-the external surface, perhaps, merely uneven, with commencing granulations, and the capsule more or less thickened and opaque. On section there is found considerable vascularity, an amorphous, albuminous exudation, tailed or spindle-shaped cells and fibrocellular tissue separating the lobules. In more advanced stages the fibrous tissue is more decidedly developed. Subsequent to the formation of new fibrous tissue, contraction follows, with constriction of the vessels lying in the course of the new tissue, impediment to the circulation in the small branches of the portal veins, starvation and wasting of the tissue by them."-(See Reynolds' System of Medicine, vol. iii. page 345.)

(2.) According to this view it is supposed that the fibrous tissue is hypertrophied and condensed, rather by a degenerative action than by one which can be termed inflammatory. Dr. Handfield Jones says - " The change seems to be of a similar kind to that which produces cartilaginoid induration of the capsule of the spleen, stiffening of the valves of the heart, and contraction of its orifices, which can scarcely be regarded as an inflammatory origin. We are confirmed in this view by having often observed various minor degrees of condensation and thickening of the Glissonian sheaths, in cases where there was no trace of inflammatory action, as well as by a circumstance which has hitherto been quite unexplained-that is, that the spleen, albeit exposed to the backward pressure of the blood, retarded in the splenic vein, does not become distended in the way that one would expect, but is often, on the contrary, small and soft. In such spleens we have often observed very many of the nuclei throwing out fibres, which is certainly not the natural metamorphosis; and hence it seems not improbable that in this way, owing to increase of fibrous tissue in its substance, the parenchyma of the spleen is less distensible than usual, and has a contrary tendency to shrink and collapse."

(3.) Cirrhosis is attributable to degeneration of the secreting tissue, independently of inflammation, arising from an unsuitable 
pabulum passing through the liver, causing a smaller demand for and diminished afflux of portal blood, wasting and absorption of the lobular structure, leaving masses of the connective tissue, which waste less rapidly than the secreting ones; this change being similar to atrophy, as it occurs in muscular tissue, and due to similar causes, namely, imperfection in the quality and diminution in the quantity of the blood.

The cases to be immediately described support this latter view, and that in all probability the atrophic change may also be partly due to the liver not being called upon to perform its function, owing to the animals having been kept in a state of semi-starvation. The cases referred to are as follows:- In the month of January 1873 Mr. James C. Dixon, veterinary surgeon, Rothbury, Northumberland, forwarded a sample of urine from a mare, requesting my opinion as to the nature of her disease. After duly examining the urine, I expressed an opinion that the animal was suffering from some affection of the liver, and wrote for a description of the symptoms. Mr. Dixon replied that he saw the mare on the 7 th December 1872 ; it was then dull, hanging its head, and off its feed; the breathing slightly accelerated; the pulse eighty, small and feeble, but there was no cough; the breath was rather foetid; the bowels regular. In the course of a few days the pulse was down to forty-four, and it was much improved, the bowels regular; but at this time Mr. Dixon ceased visiting it. Shortly afterwards he was told that it drank too much water, and passed large quantities of urine. An examination of the food showed that both the hay and corn were very mouldy and bad. By this time it had lost much flesh, and was "awfully tucked up" in the flanks. The eyes were quite yellow, the fæces light coloured, glazed, and having a very oftensive smell; the urine of a deep amber tint, and passed in great quantities. It continued alternately better and worse for some time, but losing much flesh. Mr. Dixon says, "I never saw an animal lose condition so fast in my life, except cattle when in the advanced stages of red water. After death," Mr. Dixon says, "the liver seemed dyed with bile. I have seen grocers have coarse paper the colour of this liver." A portion of this liver was sent to me, and found to be identical in appearance with the indurated liver of the human being. Externally the organ was more or less roughened in 
some places, firm to the touch, and broke down under the finger less readily than natural; in fact was firm, dry, tough, and fibrons, and on section the lobules were in parts completely replaced by a white fibrons tissue, and in others surrounded by an increased quantity of the same structure, giving to the cut surface a mottled granular appearance similar to that presented in the interior of a nutmeg.
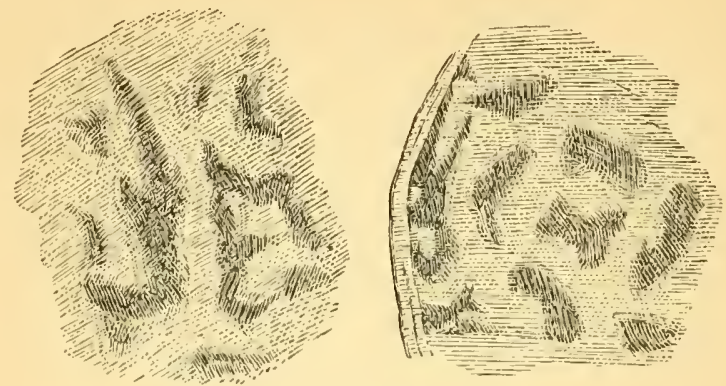

FIG. 42.-Cirrhosis of the Liver.

The white parts consist of white fibrous tissue surrounding the atrophied gland structures, slightly maguified.

The kidneys of the same animal were in a stage of degeneration.

Two other animals died on the same farm, namely, a mare and a six months' foal, between the time mentioned and the 25th of March, from liver and kidney disease. The symptoms and appearance of the mare are deseribed by Mr. Dixon as follows:"The owner says that for ten days or so before it left off feeding it was very dull, and took a deal of driving. It left off feeding on a Thursday, and I was sent for on the following Saturday, when it presented the following appearance and symptoms:-Standing with head depressed; dull and oppressed; eye heavy, and eyelids partially closed; resting from one lind foot to the other now and again; ears, legs, and body of a natural lieat; the pulse small and weak; the breatling slightly increased (the pulse and breathing very like what we find in influenza); fotid breath; bowels easy and natural; the urine rather high colonred. In a few days its pulse came down to sixty, and continued to do so gradually for ten days until it came down to forty-four, at the same time increasing in strength. I did not see it again for ten days or so, and in the course of 
eight days it got decidedly worse, and was now very thirsty and passing urine very often; pulse agitated, and fifty-six; fearfully tucked up in the abdomen; lost condition terribly; anxious for water; bowels now confined; frees light in colour and covered with mucus; eye quite yellow; membrane in the space between the last incisor and first molar of the upper jaw yellowish in tinge; breathing increased; evinced pain on pressure on the false ribs on right side up and down, anteriorly and posteriorly. Next day pulse down to forty-four; anxiety for water and frequent staling gone; and continued thus for about ten days; eating well; was more lively; the breathing more gentle; the bowels kept regular. By the end of this time it fell off again, and shortly alter it improved, and was better upon the whole; but the urine was now passed in a small stream, and instead of being amber-coloured was of a reddish-brown appearance; evinced no uneasiness when voiding it; continued to eat in a fair way for a week or so. By this time its eye was not so yellow; it left off feeding, and its pulse was now of a very different character-sixty, rather full artery, and rather wiry; it gradually sank, and died quickly. It never coughed during the time it was ill, and it lay on both sides. The eye at first was not yellow, but to me appeared blanched."

Mr. Dixon also sent me the symptoms presented by the foal. He says- "I was called to the foal on the 13th of March (but I saw this foal in December, when it was a nice, thriving, promising animal), but about eight days before I was called to it I was passing the farm and looked in. The foal was in a box. I went in and handled it, and I was perfectly struck by its appearance-just shaggy hair, skin and bone; the hair of a dirty dry colour, in fact quite darkened to a mousy brown, while before it was a lightish brown; pot-bellied. I examined the hay in the rack, took out a handful of dry, musty, black stuff, more fit to litter pigs; so I was called on the day mentioned. It seemed inclined to hang down its head, or oftener stood with it in the manger, resting on the under jaw ; fore legs apart; pulse eighty, with some degree of power in it; eye yellow; the pot-belly gone, and rather tucked up; pain on right side (like the rest). Next day comatose, pushing its head in corner of box, cold legs and ears, and dead next morning. Great debility and weakness were present from the first. I 
may add that the foal had never refused eating until the day I was called to it; it ate none after that, and it was not confined in the bowels."

It appears from the history of these cases that cirrhosis of the liver was due to a gradual starvation, and that the atrophy of the hepatic secreting structures was part of the general emaciation-atrophy-of the whole body, which was so rapidly going on, consequent on want of proper food.

Cirrhosis, consisting as it does of an organic change in the gland, tends to a fatal termination, but the rapidity of its course and progress may in some instances be modified by the removal of its cause.

It may be observed that the same condition of the liver has been discovered to prevail as an epizootic in Novia Scotia, under the term of the Pictou disease, and on the Continent of Europe as lupinosis; and the concurrence of evidence seems to point out that it is due more to the want of proper pabulum to be acted upon by the liver, than upon any toxic material; and I understand Mr. D. Hutcheon, the Chief Veterinary Surgeon to the Cape Colony, says that similar conditions of the liver are common in the colony, as a consequence of periodical periods of semi-starvation-when the grasses are scarce-succeeded by sudden repletion during the rapid growth of the said grasses during the rainy seasons.

SOFTENING-RAMOLLISSEMENT-RUPTURE OF THE LIVER.

Softening and enlargement of the liver is due probably more to repeated attacks of congestion or engorgement than to a truly inflammatory change.

Softening of the liver, very often without there having been any indications during life, terminates in sudden death from rupture of the gland and its capsule. In some instances, however, there are certain symptoms of disease of the organ, such as pallidity or yellowness of the visible mucous membranes, occasional attacks of what appears to be colic, nausea, and irregularity of the bowels.

These attacks of apparent illness usually subside in a short time, leaving the animal seemingly in perfect health. They are, however, apt to recur, the patient finally rapidly sinking 
from what at first appeared a slight attack of colic, or from some sudden or violent exertion. Death does not always immediately occur from rupture, as it would appear that the liver may become repeatedly ruptured without the occurrence of a fatal hæmorrhage. So long as Glisson's capsule remains intact, the hæmorrhages are not fatal; and even rupture of the capsule itself, when limited in extent, is not always followed by immediate death. In a case mentioned by Mr. Siddal (l'ercivall, vol. ii.), a horse had been ill, and subject to frequent fainting fits for upwards of three weeks before it died, which appeared afterwards to have been caused by small ruptures of the peritoneal coverings of the liver in different places, from all which it rallied, not sinking until the grand rupture had happened. I have met with cases which, remaining dull and off their feed after an attack of colic, have suddenly sunk; and post mortem examination has revealed the existence of several small ruptures, and the final one, of larger size, from which several gallons of blood have escaped into the peritoneal cavity. When small ruptures occur, the hæmorrhage becomes arrested by coagulation of the escaping blood; this may become encysted by the formation of a fibrous membrane round it, the extravasated blood becoming shrunk by absorption of its fluid parts, collections of fine crystals of hæmatin being left.

The symptoms of approaching death are, fainting fits; violent colicky pains; the animal breathing heavily, looking round to its right side; there is rapidly increasing pallor of the mucous membrane; coldness of the mouth, and of the body generally; dilatation of the pupils; the animal propping itself on its limbs, or by the side of the stall; and a running down pulse. If these symptoms be preceded by yellowness of the mucous membrane, by tinged urine, or clay-coloured freces, they are diagnostic that the hæmorrhage proceeds from rupture of the liver.

Causes.-The causes which induce softening and disorganization of the liver are identical with those inducing congestion, namely, stimulating food, or want of sufficient exercise; and animals which die from this lesion are generally fat and sleek in the coat.

It is stated that the liver may become ruptured by kicks or blows and severe exertion. This seems to me, however, very improbable, without there being a previous disorganization of the organ.

Post mortem appearances.-On cutting into the abdomen a 
large quantity of a semi-coagulated dark-coloured blood will be found in the peritoneal sac; the tissues of the body are blanched, and generally intermixed with much fat. With regard to the liver itself, it is found to present two pathological conditions: first, highly eongested, enlarged in every direction, its vessels filled with dark semi-fluid blood, and its tissues with much serosity.

Now and then various parts of it present a peculiar mottled appearance, the centre of the lobules being of a dark red colour, whilst their peripheral portion is of a yellowish-white; this condition constituting what is generally termed the "true nutmeg liver." Sometimes the organ has acquired the enormous weight of sixty pounds or more.

In the second form, we find that the liver is not so greatly enlarged as in the first, its capsule presents a peculiar slatecoloured tint, and the whole organ may be of the same hue, or stained deeply yellow. Both the gland substance and the capsule are friable, easily reduced to a pulp if touched with the finger, and having more the consistence and appearance of moist, bluish, or yellowish clay than of an organized structure.

Whilst horned cattle, and particularly sheep, are liable to suffer from disorganization of the liver, they seem to escape fatal termination by rupture. Sheep, however, die in great numbers from liver disease about the period of parturition; sometimes without presenting any symptoms of liver disease, or of any disease whatever; sometimes anremic, and occasionally with well-marked symptoms of jaundice.

It is impossible to account for these varieties in the symptoms when the pathological conditions of the organ are identical; and we can only accept them as facts which further researches may throw light upon.

Treatment.-This can be only prophylactic. An inquiry into the causes will show that the errors which lead to the gradual disorganization of this organ are those of improper feeding. In the horse and ox, food highly charged with nutritious elements, and mixed, or but slightly mixed, with that of a coarser nature. In sheep, as already pointed out, food containing saccharine, and but little nitrogenous materials, as when they are fed exclusively upon turnips.

It is therefore necessary, in order to prevent these various alterations of structure, that the proper dieting of animals should 
always be attended to; that when sheep are put upon turnips, nitrogenous food should be allowed them throughout the whole turnip season in some shape or other, as hay, straw, or small quantities of cake, or bean meal.

\section{JAUNDICE-ICTERUS, OR THE YELLOWS.}

Although spoken of as a disease, jaundice is in reality but a symptom of many affections in which the tissues of the body are dyed yellow. Although a result of many organic diseases of the liver, it sometimes occurs when that organ is healthy, and is not rarely absent when it is in a state of disorganization. It may be produced artificially by ligature of the common duct, which proves that obstruction of the flow of bile into the intestine can cause the disease. It may also occur when there is no such obstruction. And to account for these varieties of causes various theories have been alvanced:-First, that the bile is formed in the blood, and is merely removed by the liver, and that jaundice is a consequence of non-separation of the bile; second, that in some diseases the hrmatin of the blood is changed into bilepigment, with disintegration of a large number of blood corpuscles, thus assigning a blood origin to the colouring matter of jaundice; third, that it arises from abnormal diffusion of bile, proceeding from some alteration in the circulation of the blood in the liver, or else to defective metamorphosis or impaired consumption of bile in the blood. It is now, however, generally admitted that some of the ingredients of the bile are generated in the liver-namely, the bile acids, glycocholic and taurocholic acids found in the bile combined with soda bases; whilst others -the bile pigment, biliverdine, bilirubin, or cholepyrrhin and cholesterine-exist in the blood, and are merely separated by the liver in a manner similar to the separation of the urea from the blood by the kidneys. It is now admitted that jaundice may arise from two distinct canses:-1st. Suppression or nonelimination; and 2d. From reabsorption of bile.

The two forms are distinguished by the presence of the biliary acids in the urine when it arises from reabsorption, and their absence when due to suppressed secretion. The test is as follows:-To a couple of drachms of the suspected urine add a small fragment of loaf sugar, and afterwards puur slowly 
into the test tube about a drachm of strong sulphuric acid. This should be done so as not to mix the two liquids. If biliary acids be present there will be observed, at the line of contact of the acids and urine, after standing for a few minutes, a deep purple hue. This result may be taken as a sure indication that the jaundice is due to obstructed bile ducts. On the other hand, the absence of this phenomenon, and the occurrence of merely a brown instead of a purple tint, although in the earlier stages of jaundice equally indicative of suppression, is no indication of the cause of the suppression, which must be gleaned from other circumstances.-(HARLEY on Jaundice.) Non-secretion of bile may arise from a variety of causes:-1st. Innervation; 2d. Disordered hepatic circulation; $3 d$. Absence of secreting structure, as in atrophy, the invasion of tubercle, and the degenerations.

Jaundice from reabsorption is arranged as follows:-1st. Obstruction by foreign bodies within the bile duct; $2 d$. Obstruction by inflammatory tumefaction of the duodenum or of the lining membrane of the duct, with exudation into its interior; $3 d$. Obstruction by stricture or obliteration of the dnct; $4 t / \mathrm{t}$ Obstruetion by tumours closing the orifice of the duct or growing in its interior; $5 t h$. Obstruction by pressure on the duct from without; $6 t h$. Obstruction by parasites.

Looking upon jaundice, which is manifested by yellowness of the mucous membranes, as symptomatic of disease, it will be necessary, before the practitioner prescribes any particular treatment, that he shonld examine the urine in order that he may determine whether it arises from any cause obstructing the flow of bile into the intestinal canal, or from a disordered condition of the liver itself, consequent upon which true bile is not secreted.

Treatment.-In jaundice from suppression, if the cause of the suppression be congestion or inflammation of the liver, the treatment recommended for those conditions is to be pursued. If, on the contrary, those conditions be absent, liver stimulants, as calomel with aloes, may be administered. In the dog, podopholyn, with hyoscyamus; or benzoic acid, from 10 to 15 grains, divided into three doses, daily.

As repeated cathartics are not admissible in the lower animals, benefit is often derived from taraxacum, nitro-muriatic acid, and 
in some cases ox gall. It must, however, be understood that no permanent benefit may be expected if the jaundice be due to cirrhosis or the degenerations. The animal will, however, continue to perform moderate work, provided it be properly and carefully dieted.

In jaundice from reabsorption, the cause of obstruction must be inquired into. If it be due to catarrhal inflammation of the mucous membrane, purgatives, more especially in the horse, are contra-indicated, the constipation of the bowels being more safely removed by mild aperients, such as four-ounce doses of the sulphate of magnesia, or half a pint of linseed oil administered daily until the freces become pultaceous. The debilitated state of the liver-which sometimes remains after acute attack, and in which the organ performs its functions irregularly, sometimes secreting inordinately, at other times scarcely secreting at all; the inordinate secretion being characterised by bilious purging, some portions of the fæces being coffee-coloured, tinged with altered bile; and the non-secreting condition by clay-coloured freces with fotor-is to be nvercome by tonics, more especially iron, cinchona bark, or quinine, and a carefully regulated diet. Should the animal indicate, by licking the walls or grinding its teeth, that the stomach and bowels are in an acid condition, great relief will be afforded by the antacids, lime water, or the carbonates of soda or magnesia.

It may be observed that the cause of obstruction will, if long continued, induce such organic changes in the liver itself as to destroy its secretory function; hence we find in such diseases as the "rot" in sheep that the biliary acids are not present in the urine towards the later stages.

In dogs suffering from jaundice and ascites, elaterium, by inducing watery stools, often gives temporary relief, and prolongs the life of the animal.

Necrottc Abscesses are rather frequently found in the liver particularly in the livers of young cattle. They rarely give any indication of their presence during life, the animals dying apparently from some mysterious cause. They are either isolated or, rarely, in groups, and are often supposed to be tubercular, but upon microscopic examination the tubercle bacilli are found to be absent, and there is no appearance of successive growths, as in tuberculosis. Each abscess is sur$3 \mathrm{~L}$ 
rounded by a capsule or zone of condensed hepatic tissue of a dark brown colour, surrounding a mass of brokell-down fibrinous material having a yellowish or sometimes brownish-white appearance.

In my opinion these supposed abscesses are broken down thrombi or emboli, consequent upon a mild attack of omphalitis during early life, remaining dormant for an indefinite period, but from some cause or other becoming liquefied, and thus infect the blood stream, inducing death by septicæmia.

Gall Stones are very rarely found in the lower animals, and there are no symptoms indicative of their presence during life, beyond those which may be induced by any disease of the liver itself. It may, however, be mentioned that in horned cattle which have died from any exhausting disease, with prolonged absence of appetite, the gall bladder is generally distended with an inspissated bile, in which much sediment may be found adhering to the mucous membrane, and that in sheep deposits sre found when the gall ducts have been dilated by "flukes." 


\section{CHAPTER LXVII. \\ SPORADIC DISEASES-continued.}

LOCAL DISEASES-continued.

(Q.) DISEASES OF THE SPLEEN AND PANCREAS.

Diseases of the Spleen.-Various organic changes, as atrophy, hypertrophy, thrombosis, tubercle, cancer, the presence of hydatids, lymphadenoma, and ossification are found post mortem; but there are no symptoms during life which indicate their presence. The late Mr. Walter Lewis, of Crewe, mentions that in two cases of diseased spleen there was a tendency to run backwards in the stall and elsewhere. I have already stated that I have witnessed this symptom in disease of the duodenum (see ante, page 680.) I am informed that in America the horse suffers from an intermittent fever in which the spleen becomes enlarged. It is stated that enlargement of the spleen may be detected per rectum. I doubt, however, its possibility, unless, indeed, the spleen be of an enormous size. In one case which came under my own notice, lymphadenoma of the spleen (shown in the frontispiece of the first edition of this work) was guessed at by the absence of signs of disease of any other organ, slight increase of the white globules in the blood, pallidity of the mucous membrane, a stiffness of the back, and a gradual wasting of flesh. In another case lymphadenoma of the spleen was diagnosed from the history of a case which had a lymphadenomatous tumour in the parotidean region. This animal had latterly fallen off its appetite, and was subject to slight recurring colicking pains, but there were no other symptoms of internal disease. I removed the tumour from the neck, but after finding out its histological character, gave an unfavourable prognosis, being of opinion that another of the same nature was in existence, 
most probably in the spleen, giving rise to the colicky symptoms. The animal died a few days afterwards, and a hard lymphomatous tumour twenty-five pounds weight was found in the spleen, involving walls of stomach, duodenum, \&c.

I carefully examined the blood of this animal, and found there was scarcely, if any, increase in the white corpuscles (leukæmia), a condition sometimes associated with hyper-development of lymphatic tissue.

I have met with several cases in which the lymphatic glands in various parts of the body, but more particularly in the cervical and subcapular regions, have been enlarged, with development of lymphatic tumours in the spleen and liver.

The same kind of growth is also found associated with farcy, and in the case from which the illustration was obtained the liver weighed thirty-five pounds, being studded throughout with the white tumours shown in the figure.

FIG. 43 .

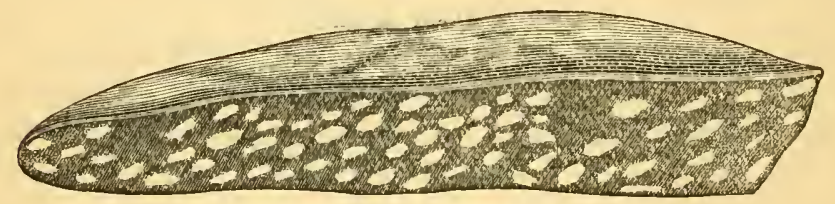

The development of lymphadenomatous growths, although found associated with farcy, is not to be considered as due to the presence of the glanderous poison, as they frequently occur when there is not the slightest suspicion of malignant disease.

Lymphadenoma, for a long time confounded with tubercle, differs very materially from that product; in fact, whereas tubercle rapidly undergoes degeneration, lymphoma has little or no tendency to retrograde. Microscopically lymphoma consists of a delicate network of fibres, within the meshes of which are contained numerous cells-lymph corpuscles. In the early stage of the growth the number of cells is very great, and many of the cells may contain two or even more nuclei, but in a more advanced stage the proportion of cells is smaller, and the reticulum forms most part of the growth.

The physical characters, according to Green, vary according to the rapidity of the growth. The rapidly growing forms, in 
which the cellular elements are numerous, are of a greyish colour and soft brain-like consistence, much resembling encephaloid cancer. These often attain an enormous size, and infiltrate the neighbouring structures. They have been called by Virchow lympho-sarcoma. Those which are more slowly developed, and in which the reticulum constitutes the greater portion of the growth, are much harder in consistence, sometimes being almost cartilaginous.

I have never witnessed the lympho-sarcoma of Virchow. In the case from which the figure was drawn, the tumours, although in the aggregate weighing so heavy, were individually no larger than a thrush's egg, and were moderately firm in consistence.

The origin of these tumours is a matter of obscurity. I have seen them in the well and in the ill fed animal.

The above remarks will apply to diseases of the pancreas. It must be, however, held in remembrance that the pancreatic juice las the property of converting the fatty matters of the food into an emulsion. The presence, then, of fat or oil in the fæces of the dog, or of any other animal of which fat is an ingredient in its food, points to some diseased condition of the pancreas. 


\section{CHAI'TER LXVIII. \\ SPORADIC DISEASES-continued.}

LOCAL DISEASES-continued.

\section{(R.) DISEASES OF THE KIDNEYS.}

Owing to the fact that the lower animals are free from mental emotions, the cares and troubles of the world, and that they do not indulge in alcoholic drinks, the kidneys are in a great measure exempt from those diseases which so often destroy human life.

Diseases, however, do occur with which the veterinarian has to cope. Physiologically, the kidneys excrete from the body those materials resulting from metamorphosis of tissue which would, if retained, act injuriously upon the system generally; and the secreted material-the urine-differing as it does in various animals, contains certain constituents, some of them identical, some dissimilar. The presence of these constituents in normal or abnormal quantities, or their absence, indicate to the investigator various morbid conditions of the kidneys, as well as many changes which occur in the body during disease. The urine is naturally alkaline in the herbivora, and acid in flesheating animals. It consists of a large amount of water; a nitrogenous substance called urea; an acid-hippuric in that of herbivora, and uric in the carnivora; colouring matter called uro-hæmatin ; inorganic salts; organic substances of an ill-defined nature called extractive matters.

The density of healthy urine of the horse appears to range from 1030 to 1050 ; that of the ox has a specific gravity ranging from 1032 to 1040 ; that of pigs 1010 to 1012 ; and of the goat 1008 or 1009.-(Von Bibra.) The amount of water in the urine varies much, according to the quantities of fluids that have 
been taken into the system, and the condition of the intestinal canal, skin, and the surrounding atmosphere.

The amount of urine daily excreted by healthy horses varies, according to the observations of Colin, from 44.033 to $61 \cdot 646$ English pints; and the amount of water in 1000 parts of urine, according to the analyses of Von Bibra, Boussingault, and others, from 880 to 930 parts; whilst in that of the ox, Von Bibra found 912.01 and 923.11 ; in that of the pig from 980 to 990.

Urea is the most important product of tissue change; it has the same chemical composition as the carbonate of ammonia, to which it is readily converted when heated a little above the temperature of boiling water, or when kept in contact with decaying animal matter. When pure, it crystallizes from a watery solution as a white, semi-transparent, crystalline body with a bitter taste. The crystals are quadratic prisms, with rectangular terminal planes; it readily combines with nitric acid, forming the nitrate of urea, which speedily crystallizes; and the addition of nitric acid to urine is the method of detecting its presence in abnormal quantities. The crystals of the nitrate of urea, when rapidly formed, are flat, shining, rhomboidal plates (see fig. 26, p. 454); when slowly crystallized, fine prisms. Nitrate of urea is soluble in water and alcohol, but only sparingly so when they contain an excess of nitric acid; it is insoluble in ether.

It combines also with oxalic acid, forming the oxalate of urea (Ur. $\mathrm{C}_{2} \mathrm{O}_{3} \mathrm{H} \mathrm{O}$ ), which crystallizes in prisms and quadrilateral tubes. Urea forms insoluble compounds with the nitrate or protoxide of mercury.

Although found in the urine, urea is not formed in the kidneys, but is excreted by them from the blood; and, as already pointed out in former chapters, it results not only from metamorphosis of tissue, but also from excess of food, as well as from the decomposition of uric and hippuric acid. Its formation from uric acid has been experimentally proved by Neubauer, who on giving rabbits from 31 to $46 \frac{1}{2}$ grains of uric acid with their food, the quantity of urea excreted in the twenty-four lours was augmented from 32.5 to $65 \cdot 1$ grains. Uric acid, when acted upon by permanganate of potash, is transformed into several substances, one of which is urea. 
The elimination of urea is also influenced by water. The more water an animal drinks the more urea will it excrete. Common salt also produces an augmentation in the excretion of urea.-(Böcker, Bischoff.) Certain foods diminish the daily excretion of urea, such as sugar, starch, fat; in fact, says Dr. George Harley, "my idea is that all nitrogenized foods augment, and that all non-nitrogenized foods diminish, the amount of urea eliminated by the kidneys."

Increased elimination of urea occurs in all febrile diseases, and is indicative of abnormal metamorphosis of tissue; and its amount bears a close relationship to the exaltation of temperature and intensity of fever. Retention of urea is a very unfavourable circumstance, and is symptomatic of intense congestion of the kidneys, exudation and hæmorrhage into the uriniferous tubes, and desquamation of their epithelium, with destruction of their secreting cells.

Uric acid, $\mathrm{C}_{10} \mathrm{H}_{4} \mathrm{~N}_{4} \mathrm{O}_{6}$, is a white crystalline, feebly acid, tasteless, organic substance, insoluble in ether or alcohol, and but sparingly so in water: 2000 parts of hot and 11,000 parts of cold water are required to dissolve one part of uric acid. It is soluble in strong sulphuric acid, and is transformed by dry distillation into urea, cyanic acid, hydrocyanic acid, carbonate of ammonia, and an oily coal. It unites with bases such as potash, soda, or ammonia, forming with them crystalline urates. The urine of herbivorous animals contains little, if any, uric acid; but it is found in the solid urines of serpents, birds, and insects, and in the liquid urines of omnivora and carnivora, in combination with potash, soda, and ammonia.

The pathology of uric acid is of little consequence to the veterinarian. It is derived from the same source as urea, and whatever accelerates oxidation increases the amount of urea and diminishes the uric acid, and whatever diminishes oxidation decreases the urea and increases the uric acid in the urine, thus proving that urea is a more completely oxidized product than the uric acid. In the dog and other carnivora uric acid calculi have sometimes been found.

Hippuric acid $\left(\mathrm{C}_{18} \mathrm{H}_{9} \mathrm{~N} \mathrm{O}_{6}\right)$ is a beautiful white crystalline, slightly acid body, constantly found in the urine of all but the purely carnivorous animal. It is soluble in 400 parts of cold water, and its solution is sufficiently acid to redden litmus 
paper; it is also soluble in ether and alcohol. Its quantity in the urine varies greatly. When the quantity of urea is small that of hippuric acid is almost always large, and vice versa. According to Von Bibra and Boussingault it varies from 5 to 15 parts in 1000 of the urine of healthy horses; and it has been stated by some chemists that the urine of horses which are subjected to very hard work contains no hippuric acid, but much benzoic acid instead. "The last part of the statement," says Mr. Gamgee, "seems doubtful, though it would appear that hard work checks the excretion of hippuric acid, and increases the quantity of urea excreted." The observations of Maack oppose this conclusion. He found that horses kept standing in the stable passed very little hippuric acid, but large quantities of urea, and Dr. G. Harley made similar observations regarding London stall-fed cows. They did not, however, examine the urine after these animals had been exercised; their experiments are therefore incomplete. Hippuric acid is increased in rheumatism, red water, and in diseases of the respiratory organs; in fact, in all conditions in which the blood is imperfectly aërated, either from obstruction to free respiration or sudden arrestment of the cutaneous functions, as well as from an imperfect condition of the blood itself, rendering it incapable of oxidation.

Extractive matters, as creatine, creatinine, lactic acid, and benzoic acid, which is transformed into hippuric acid in the body-for it has been found that when benzoic acid is given to an animal an almost equal amount of hippuric acid is eliminated by the kidneys - are found in the urine. Sometimes urine contains a large quantity of mucus derived from the pelves of the kidneys, ureters, bladder, or urethra, indicating some degree of irritation of the mucous membranes. After parturition, as might be expected, the urine generally contains much mucus, derived from the mucous membrane of the vagina.

Saline matters, consisting of various materials from the food and tissue, as the chlorides, phosphates, sulphates, and carbonates, are present in healthy urine, whilst other salts, originating in mal-nutrition of tissues, or from defective secretion of other organs, such as the oxalates, are sometimes met with. For the method of determining the presence of these, the reader is referred to Harley on the Urine, Roberts on Urinary and Renal Diseases, Beale, and others. The most common inorganic deposit which 
is found in the urine of the horse is the carbonate of lime, which appears as a chalky sediment if the urine be kept in a vessel for some hours.

In the ox and sheep, when fed upon turnips and linseed cake, the urine generally becomes highly charged with the phosphates, which are very often spontaneously precipitated upon the mucous membrane of the bladder, urethra, and the long hairs surrounding the prepuce. I have some specimens in my possession of the ammonio-magnesian phosphate in the form of tubes deposited upon the hairs surrounding the preputial opening. The occurrence of phosphatic deposits in the lower animals results from the food upon which they are kept being highly charged with phosphatic salts. We consequently find that when animals are fed upon turnips and other foods grown upon land highly manured with dissolved bones and other phosphatic preparations, that the urine becomes highly charged with these materials, and that they are apt to be deposited in the form of gravel upon the mucous membrane of the urinary passages, more especially upon the occurrence of any cause of irritation, such as a common cold or other catarrhal affections. They sometimes induce obstruction to the flow of urine if situated in the urethra, or a suppression of its secretion, either by extension of the irritation primarily excited in the urethra or bladder to the kidneys, or when deposited in the pelves of the kidneys by an inflammation of the glands. In order to overcome the further deposition of this salt a change of diet is essential, and to dissolve what is actually deposited, nitric acid in fifteen-drop doses, largely diluted, may be administered three times a day.

Pus or blood are occasionally met with in the urine, and indicate irritation or inflammation of the urethra, bladder, or kidneys. When from the urethra, bladder, or ureters, the extravasated blood has no definite form, but if from the kidneys, blood and lymph are moulded into the shape of the uriniferous tubes.

Albumen is recognised by its property of coagulating upon the application of heat and of nitric acid. If the urine be alkaline the albumen may not coagulate upon boiling, but if a few drops of nitric acid be added it is immediately precipitated. Sometimes, if the urine be boiled, a precipitate may be formed, consisting of the phosphates; these are dissolved by nitric acid. 
Again, if the urine contain much urea, a precipitate is formed when nitric acid is added; this is, however, dissolved if the liquid be boiled, and the determining test for albumen is that it is neither dissolved by heat nor nitric acid. The presence of albumen in the urine is diagnostic of congestion, inflammation or degeneration of the kidneys, or that condition of the system already described under "Red Water." Albumen is also present in the urine in some cases of indigestion, and indicates that the albumen of the serum is in a condition which renders it unfit for the nutrition of tissues.

The occurrence of oxalic acid in the urine has already been referred to.-(See Oxaluria.)

\section{THE URINE.}

A study of the following table will at once give the reader an idea of the condition of the urine in health.

URINE OF BULLOCK IN HEALTH.

\begin{tabular}{|c|c|c|c|c|c|c|}
\hline & & & $\begin{array}{l}\text { Quantities in } \\
\text { twenty-four } \\
\text { hours. }\end{array}$ & $\begin{array}{l}\text { Specific } \\
\text { Gravity. }\end{array}$ & $\begin{array}{l}\text { Degree of } \\
\text { alkaline re- } \\
\text { action. }\end{array}$ & $\begin{array}{c}\text { Grains of Urea } \\
\text { in twenty-four } \\
\text { hours. }\end{array}$ \\
\hline & & & PTS. OZ. & & & \\
\hline 1st day, & & & $\begin{array}{ll}4 \quad 7 \\
4\end{array}$ & 1050 & $\begin{array}{l}149 \cdot 01 \\
188 \cdot 46\end{array}$ & $\begin{array}{r}930 \cdot 40 \\
1094 \cdot 90\end{array}$ \\
\hline $\begin{array}{l}2 \mathrm{~d}, " \\
3 \mathrm{~d}\end{array}$ & : & : & $\begin{array}{ll}4 & 15 \\
4 & 17\end{array}$ & $\begin{array}{l}1049 \\
1045\end{array}$ & $\begin{array}{l}188 \cdot 46 \\
179 \cdot 70\end{array}$ & $\begin{array}{l}1094 \cdot 90 \\
1156 \cdot 36\end{array}$ \\
\hline 4th, , & . & . & 515 & 1047 & $144 \cdot 63$ & $1290 \cdot 33$ \\
\hline 5th ," & & . & 106 & 1051 & $207 \cdot 74$ & $1485 \cdot 12$ \\
\hline 6 th,$"$ & . & . & 410 & 1053 & $162 \cdot 15$ & $1021 \cdot 62$ \\
\hline 7th , & . & . & 43 & 1055 & $197 \cdot 22$ & 108783 \\
\hline 8th, , & . & . & $\begin{array}{ll}4 & 17\end{array}$ & 1048 & $226 \cdot 15$ & $1220 \cdot 10$ \\
\hline 9th , & & 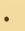 & $511 \frac{1}{2}$ & 1048 & 195.98 & $1186^{\circ} 46$ \\
\hline 10th ", & & - & $5 \quad 12 \frac{1}{3}$ & 1048 & $203 \cdot 36$ & $1221 \cdot 80$ \\
\hline 11th ," & . & . & 510 & 1052 & $220 \cdot 89$ & $1248 \cdot 66$ \\
\hline 12 th $"$ & . & . & 47 & 1053 & $194 \cdot 60$ & $1208 \cdot 68$ \\
\hline 13th ", & - & . & 30 & 1058 & $192 \cdot 84$ & $1305 \cdot 00$ \\
\hline 14th ,", & - & . & 48 & 1056 & 178.82 & $1018 \cdot 22$ \\
\hline
\end{tabular}




\section{SPORADIC DISEASES-continued.}

LOCAL DISEASES-continued.

\section{(R.) DISEASES OF THE KIDNEYS-continued.}

\section{INFLAMMATION OF THE KIDNEYS-NEPHRITIS.}

A RARE disease in the lower animals, but in human medicine it is described under three heads, namely-Suppurative, Interstitial, and Tubal. Except from the irritation of a calculus, suppurative nephritis-pyelitis-is a very rare form of disease in the lower animals. I have, however, met with a few cases where it has arisen independently of such source of irritation. In one case (a cow) it was associated with inflammation of the urino-genital mucous membranes, consequent upon difficult parturition; indeed it seemed to be due more to the absorption of infective materials from the inflamed mucous membranes, which discharged a foetid, purulent material very profusely, than to an extension of the inflammation. The next case was that of a foal two days old, which had died from acute suppurative arthritis. A post mortem examination revealed not only the presence of pus in the tissues surrounding the articulations, but sundry purulent points in the kidneys, the pus in which being more or less inspissated or caseous.

I look upon this case as important, as to some extent it supports the view that " joint disease" occurring in young animals is not always a primary disease, but secondary to the formation of pus in other parts of the organism. Generally, however. nephritis embraces all the structures of the kidneys, often commencing in the mucous membrane of the uriniferous tubes, afterwards involving the parenchyma, and terminating in resolu- 
tion, or in desquamation of the lining of the epithelium and degeneration of the secreting structures, sometimes in suppuration, and in very rare instances gangrene.

Causes.-Nephritis is said to occur from injuries, such as blows and strains. My experience leads me to think that this idea is exaggerated, and that it occurs, and that but rarely, from the internal administration of irritant diuretics, as turpentine, the resins, or cantharides, the absorption of cantharidine from large blisters, the irritating effects of croton oil when given as a purgative, and still more rarely from the effects of stimulating food, or of the long-continued effect of cold, such as cold water dropping upon the animal's back, directly applied to the loins, Many ailments and injuries are mistaken, I think, for nephritis, more especially if they are characterised by a straddling or stiff gait.

Symptoms.-There is considerable fever and colicky pains; indeed the malady closely simulates colic with fever. There is a hard, frequent pulse; increased thirst; short, rapid breathing; hot, clammy mouth, and constipation of the bowels. There may be some stiffness of the loins and disinclination to move, but it by no means follows that stiffness, a straddling gait, tenderness of the loins, arched back, are constant symptoms; indeed, I am led to conclude that they are present only in the minority of cases, and that the only signs by which the true nature of the disease can be determined are a scanty secretion or total suppression of urine, desire to micturate frequently, the animal stretching itself in vain attempts, passing perhaps but a few drops of a concentrated, highly coloured, and irritating secretion, which, if carefully examined microscopically, will be found mixed with sanguineous or fibrinous casts of the uriniferous tubes, blood globules, epithelium, and even pus cells. If tested by heat or nitric acid, a more or less abundant precipitate of albumen will be thrown down. In those instances where there is total suppression of urine, the application of the determining test is impossible. The true, nature of the disease may, however, be arrived at, from the fact that when fever, with colicky pains, is present, in addition to emptiuess of the urinary bladder, frequent attempts to urinate, but no urine being discharged, that the non-secretion is due to inflammation of the kidneys, which are unable to perform their functions in consequence of the inflam- 
matory process. Cases now and then occur in which the only symptoms present are suppression of urine, with fever; but in other instances there are signs of pain manifested by frequent lying down, rolling, sighing, \&c.; whilst in others the seat of pain is pointed at by the animal turning round and endeavouring to bite or scratch its loins. In one instance this symptom was very marked, and called forth the observation from the attendant that the horse wanted to bite its near hind leg, which was elevated and brought forward as if for that purpose; the animal, however, seemed to desire to bite its back, but was unable to do so. Should the suppression-ischuria-be prolonged, other symptoms, namely, those of blood poisoninguræmia--arise. The retention of urea in the blood does not affect all animals alike. In the dog, insensibility and coma soon accur; but in the horse the effect seems to resemble a moderate alcoholic intoxication; the eye becomes brilliant, there is partial unconsciousness, but no loss of motor power. In one case, where total suppression continued for five days, no signs of coma or even somnolence were observed; but the animal became incapable of directing its movements, which were automatic, and from right to left, in which direction it continually moved round the box, and no force could compel it to move in the contrary direction. As the system becomes loaded with urea, the freces and secretion of the skin emit a strong uriniferous or even ammoniacal odour; the breath becomes fœtid; the bowels constipated and tympanitic, with frequent vomition in the dog. In the case above mentioned, it was observed that the near (left) side was covered by a continual sweat, which became more and more uriniferous as the disease advanced; the skin of the opposite side was dry, hard, the coat staring, and on the fourth day the near hind limb was partially paralyzed; and the horse was frightened if it were suddenly touched or approached. In the stallion, retraction of the testicle on the affected side may be present.

Post mortem appearances.-In the acute form of general nephritis one or both kidneys may be involved; generally one is more especially affected, being enlarged, of a dark colour, and streaked with patches of congestion. It is easily lacerable, and. if cut with the knife, a deep-coloured fluid flows from the cut surface, which, if examined microscopically, will be found to 
contain pus cells in abundance; there is exudation into the tubes and desquamation of the epithelium, constituting "epithelial casts." Pyelitis, however, gives rise to the formation of abscesses, which are principally confined to the cortex of the kidney. These vary in size from a pin-head to a pigeon's egg, or even larger. Abscesses are multiple and surrounded by congested tissue, and sometimes by submucous hæmorrhage; this was specially observable in the cow referred to. They are easily seen through the capsule of the kidney as many yellowish white spots.

Interstitial nephritis consists of an inflammation of the interlobuar connective tissue, similar to cirrhosis of the liver, and leads to contraction of the affected kidney.

Treatment.-This must be according to the ordinary principles of the treatment of inflammation. If the pulse be strong, a full bleeding will be of much service, not only allaying the febrile disturbance, but, by acting as an evacuant, removing much effete material from the economy. The intestinal canal is to be freely acted upon by cathartics, for direct experiment has shown that the urea, \&c. which accumulate in the blood are by this means eliminated from the body, and the symptoms of uræmic poisoning delayed. For the horse, aloes; the ox, the sulphates of magnesia or soda; the dog, calomel and jalap, with enemas of warm water, warm fomentations or poultices to the loins, succeeded by mild mustard applications.

If the suppression continue for several days, or if at any time uræmic intoxication is apparent, it becomes necessary to excite the secretion of urine, and the best and safest method of doing this is by the application of digitalis to the skin in the form of a decoction, repeatedly applied as a fomentation to the loins, or as a poultice. It must, however, be discontinued immediately after the kidneys have commenced to react. I can speak with great confidence of this remedy if thus applied.

If pain be a prominent symptom it must be relieved by opium.

It is scarcely necessary for me to say anything against the application of cantharidine blisters, as the practitioner is aware that they have a most irritating effect upon the urinary organs.

As sequelæ to nephritis, atrophy and degeneration of the gland may result. If the inflammation be confined to one, it is 
found that the other kidney generally becomes hypertrophied, having a double function to perform. In the case mentioned, where the suppression continued for five days, the left kidney was found to be, when examined some years afterwards, a mere flabby bag, its substance destroyed, and the ureter impervious; the right kidney, on the other hand, was much enlarged, and almost lealthy. This condition of atrophy of one, and hypertrophy of the other, was diagnosed at the time it was attended, for the enlarged organ was easily detectable by the hand introduced into the rectum. Both Percivall and Gamgee quote a case of suppuration of the kidney, described by D'Arboval, occurring in a mare which had fallen into a hole.

\section{ALBUMINURIA.}

The occurrence of albumen may be due to various causes unconnected with disease of the kidneys, as in "red water" in cattle, some forms of indigestion in the horse, as well as from cerebro-spinal irritation. The application of large cantharidine blisters may also be followed by albuminous urine. According to some this is due to the toxic effects of cantharidine upon the blood, inducing a condition of that fluid simulating that resulting from the action of morbid poisons. According to others it is a result of the direct irritating effects upon the kidneys of the absorbed cantharidine. This latter view seems to me to be the more correct one, for not only does it irritate the kidneys, but the urinary passages generally, as manifested by frequent and difficult urination-strangury-which is best treated by opium, demulcents, as linseed tea, and bicarbonate of soda.

Persistent albuminuria arises from that degenerated condition of the kidney termed by medical writers "Bright's disease." It is, however, very rare in the lower animals. It is clescribed by veterinary authors under the term of albuminous nephritis and granular degeneration of the kidneys.

The urine in this disease is permanently albuminous, and if examined microscopically will be found to contain a number of thread-like cylinders, which are in fact slender fibrinous coagula monlded to the shape, and discharged from the urinary tubes of the kidneys. They are generally studded with minute epithelial cells, which have been detached from the surface of the urini- 
ferous tubes, and in some instances they are mixed with oil globules, which denote not only the presence of desquamative nephritis, but of fatty degeneration also.

The anatomical characters of the kidneys in this disease are of two kinds, and are spoken of as the large white and the small red kidney. If a longitudinal section of the large kidney be made, its cortical portion is seen to be much increased; the organ is soft in consistence, smooth upon its surface and upon its investing membrane; whilst the small kidney is hard and red, rough upon its surface, and its investing membrane firmly adherent.

Symptoms.-In addition to some stiffness of gait, there is continued desire on the part of the animal to stretch out in the stall, and in this position to continue, as described by Mr. Percivall, "with its fore legs extended under the manger, and its hind ones backwards, unless disturbed, all day long, not for the purpose of staling, but apparently because that posture seemed an easy or a comfortable one to it." The diagnostic signs are present in the urine itself, which is albuminous, containing fibrinous easts of the uriniferous tubes, epithelial cells, or perhaps oil globules, whilst in quantity it may be very materially diminished or increased. I had the opportunity of watching the progress of a case for a number of years, and of making a post mortem examination after the animal's death. The general health seemed but little affected. There was some shortness of breath, the animal never lying down, and some stiffness of gait; the urine varied in colour and density, sometimes dark, sometimes pale, but always albuminous and copious in quantity. The horse became paralyzed, and was destroyed. Examination of the kidneys showed that they were red and small, with numerous cysts, containing a thin transparent fluid in their interior. I was unable to account for the copious secretion of urine, but I find the same thing occurs in man; and that in the large white kidney the secretion is scanty, has a higher specific gravity, and contains clear, fibrinous, wax-like, and sometimes oily casts, with occasionally a little blood, and is frothy; whilst in the small red kidney the urine is copious as to quantity, and in the advanced stages pale, of a very low specific gravity, and contains granular casts of the uriniferous tubes.

Treatment.-When resulting from degenerative changes in the $3 \mathrm{C}$ 
glands, treatment of albuminous urine can only be palliative, relieving the kidneys as much as possible from the labour of elimination, by keeping the bowels in a relaxed condition by proper food, the skin warm, avoiding exposure to cold, and preventing anæmia by mineral tonics or the mineral acids, and putting the animal to such labour as it is capable of performing. When arising from other than disease of the kidney, albuminous urine, which is but a symptom, will disappear as the causes of such diseases are removed.

\section{FLOATING KIDNEY.}

I have met with one case wliere the right kidney in a cat was situated subcutaneously between the two last ribs. Supposed to be a tumour which had arisen in consequence of a bite from a dog, it was removed by me early in 1879; and, what is most interesting, the cat was none the worse of the operation, and is alive at the present time and as well as ever.

\section{H EMATURIA-RENAL CALCULI.}

The occurrence of blood in the urine is due to a variety of circumstances, as acute congestion, degenerations already described, cancers, melanosis, and to the presence of calculi.

Renal calculi in the horse are composed of the carbonate of lime, and their presence is discoverable by the condition of the urine, which is charged with earthy materials, and by intermitting discharges of blood, occasional colicky pains, more especially after the animal has been severely worked or exercised. They are, however, exceedingly rare, and are best treated by the administration of hydrochloric acid, by the avoidance of food and water rich in saline matters, and by keeping the digestive and secretory functions well regulated by diet, careful grooming and exercise when admissible, and the avoidance of liard, calcareous water. In some instances the calculi become impacted in the ureters, inducing a more or less rapid degeneration of the lidney and suppuration within its substance, causing extreme agony and death. If the ureter becomes much dilated, the dilatation may be discovered by an examination per rectum, in the form of a fluctuating swelling upon either side of the pelvis. 


\section{CHAPTER LXX. \\ SPORADIC DISEASES-continued.}

LOCAL DISEASES-continued.

(S.) CYSTITIS, OR INFLAMMATION OF THE BLADDER.

EXCEPT as a result of the irritating effects of cantharidine or croton oil absorbed from a blister, or administered internally, I have never seen this affection in any of the lower animals, and am disposed to coincide with many veterinarians, that it never occurs except from the above causes.

Symptoms.-These are manifested primarily by excitement, followed by prostration of strength, the animal appearing to suffer much pain and distress; the urine is passed frequently, and with difficulty and pain; the freces are covered with mucus and mixed with blood. When induced by cantharides or croton administered by the mouth, the inflammation extends throughout the whole alimentary canal, and there is redness of the buccal mucous membrane, with difficulty of swallowing, and attempts at vornition in horses and cattle, the genito-urinary organs being also much affected; but if the above irritants be absorbed from a blistered surface, their effect is concentrated upon the urinogenital organs, causing in most animals strangury and apparent sexual excitement, and if these symptoms are not soon allayed, death will result in two or three days.

Treatment.-If from a blister, wash and cleanse the blistered part; internally, hyoscyamus or opium, bicarbonate of soda, demulcents, such as linseed tea, mucilaginous drinks, milk, and white of eggs will be found beneficial.

\section{RETENTION OF URINE.}

An inability, total or partial, of expelling by natural effort the urine contained in the bladder. It is caused by spasm of the 
neck of the bladder; so induced, it is often a complication of colic; paralysis of the bladder. Retention is a common complication in parturient apoplexy and in paraplegia; enlargement of the prostate in aged males; cystic or urethral calculi; cancer of the penis; any cause of obstruction at the urethral opening; prolapsus of the uterus or vagina, and excessive accumulations of freces in the rectum, and of dirt in the sheath. Inability to urinate may also result independently of the above causes and of any disease of the bladder or urethra, when an animal is unable or unwilling to rise upon its feet, as in azoturia, laminitis, or paralysis.

The symptoms are frequent and ineffectual attempts to urinate; if standing, the animal will stretch itself out, strain violently, and groan with pain, discharging but a few drops of urine, or none at all. Examination per rectum will enable the practitioner to feel the distended bladder with the hand, and this distension of the bladder is the diagnostic symptom. Such an examination will also often enable him to discover the cause; if from enlarged prostates, these will be felt as oval bodies immediately within the pelvis pressing upon the urethra. I have one case recorded where the animal had suffered, according to my informant, for five weeks from what appeared to be incontinence of urine-there being a continual dropping of urine niglit and day -but which proved, upon examination, to be a case of retention from the pressure of large prostates. The bladder was enormously distended, and the continual dropping of urine was the mere overflow; upon the catheter being introduced relief was immediately given by the withdrawal of a large quantity of urine. It was necessary to introduce the catheter two or three times a day for about a week, when the enlarged prostates, under the influence of iodine internally administered, and applied as an ointment to the perinæum, diminished in size, and permanent relief was afforded.

Paralysis of the bladder, if not caused by cerebro-spinal or spinal derangement, may be induced by retention of urine, as when an animal is compelled to perform a long journey without the opportunity of relieving itself. In this case the muscular fibres of the bladder, unnaturally stretched by the pressure of the contained urine, lose their tonicity, and become unable to contract upon their contents. Whatever be the cause of 
the retention, relief must be afforded by the introduction of the catheter, and it is highly important, in all cases where animals retain the recumbent posture, to examine the condition of the bladder, and afford the necessary relief by the introduction of the catheter. Sometimes, however, the evacuation may be effected by firm but not violent pressure upon the bladder with the open hand introduced into the rectum, taking care that the mucous membrane of the rectum be not injured by the finger nails or by too violent pressure. In introducing the catheter in the cow, care must be taken not to injure the thin, delicate, membranous valve which guards the urethral opening; and in order to introduce the catheter without causing injury, the valve is to be lifted upwards by the finger, and the point of the catheter carefully introduced beneath it. If retention be due to the accumulation of dirt within the sheath of the penis, it may not be necessary to introduce the catheter, but to wash the parts thoroughly with soap and warm water; a little carbolic acid in the latter will render this operation much less offensive to the nostrils by destroying the fotid smell.

\section{INCONTINENCE OF URINE.}

This is the reverse of retention, being a continual flow of uriue. It arises from a variety of causes, namely, paralysis of the sphincter vesicæ, the muscular power of the walls of the bladder remaining intact; calculi; and from pervious urachus shortly after birth.

For the treatment of the two latter causes see Principles and Practice of Vcterinary Surgery; and for that arising from paralysis cathartics may be necessary, succeeded by nux vomica and cantharides, with injections of cold water into the rectum.

"Incontimence is said to occur in pigs after eating of polygonum, hydropiper, and lapathifolium."-(GAMGEE.)

The following terms are applied to the checked discharges of urine:-suppression-ischuria; painful discharge of little urine -dysuria; and the passage of urine in drops-strangury. 


\section{CHAPTER LXXI.}

\section{SPORADIC DISEASES-continued.}

LOCAL DISEASES-continued.

\section{(T.) HYSTERIA.}

Concurrent with the period of cestrum in the female, the following symptoms-clenching of the jaws, grinding the teeth, difficulty of swallowing, some degree of trismus, squinting of the eyes, tonic spasms, alteruating with those of a clonic kind, continual kicking in an irregular uncontrolled manner with one or both hind feet, stamping of the fore feet, and other signs of nervous excitement, have been observed in the mare and female ass.

In one case that I saw, the two fore shoes and one hind shoe had been thrown off by the violent kicking and stamping. Attempts were made to remove the remaining one, which was loose; they were, however, unavailable, as the slightest touch caused a most violent kicking. The animal would fall, the whole trunk and neck being perfectly rigid, whilst the limbs continually moved in a violent and spasmodic manner. The power of volition seemed to be completely lost. The gluteal muscles were exceedingly hard and prominent. The rapid opening and closing of the vulva and its highly injected mucous membrane indicated the hyperasthæsia of the genital organs. Urine was passed abundantly, at first pale and watery, but afterwards highly coloured, and loaded with solid matters.

In another case the symptoms were rather different. The animal was prostrate, rigid, with its eyes turned upwards, as if in a trance. They were both bled, and an endeavour was made to nauseate them with aloes, and to allay the nervous irritability with opium. One recovered, the other died. For the prevention of this condition I can only suggest that the sexual desire be gratified if possible, whenever such a state of excitement short of the above presents itself. 


\section{CHA PTER LXXII.}

\section{SPORADIC DISEASES-continued.}

LOCAL DISEASES-continued.

\section{(U.) DISEASES CONCURRENT WITH OR IMMEDIATELY SUCCEEDING PARTURITION.}

ACCIDENTAL conditions connected with parturition, requiring the aid of a surgeon, will be found fully described in the Principles and Practice of Veterinary Surgery, as it is my intention to confine my present observations to those of a more purely medical character.

Under the common term "milk fever," at least three separate diseases are generally described, namely, parturient fever, parturient apoplexy (already described), and acute metritis.

\section{PARTURIENT FEVER.}

Dcfinition.-A benign form of fever, seen in the cow, mare, \&c., occurring about the second or third day after parturition, and generally terminating in recovery in from twenty-four to forty-eight hours.

The symptoms are those characteristic of general febrile disturbance; the pulse generally full and strong, the breathing accelerated, the visible mucous membranes injected, the secretion of milk suspended, the mammary gland hard and slightly erythematous, bowels constipated. The cow may assume the recumbent posture, but the power of maintaining the standing one is not lost, nor are there any signs of unconsciousness or of cerebral disturbance. Unlike parturient apoplexy, it attacks animals of all ages, but it is most commonly met with after the first parturition, and is induced by cold, or some dietetic error. 
and seems to be connected with that condition of exciternent associated with the secretion of milk.

Treatment.-The treatment of this affection is very simple, a mild oleaginous purgative, combined with a dose of the spirits of nitrous ether, warm clothing, und a restricted diet being all that is required.

ACUTE METRITIS, OR INFLAMMATION OF THE WOMB.

A very fatal form of inflammation, occurring in a few hours or within two or three days after parturition.

Whilst parturient apoplexy and parturient paralysis are generally met with in the cow, acute metritis, or, more correctly, metro-peritonitis, attacks the cow, mare, ewe, the bitch, and the sow.

Pathology.-A diffuse inflammation, primarily situated in the mucous membrane of the uterus, soon, however, extending to the uterine veins, giving rise to the formation of clots or thrombi, and extending to the peritoneum and intestines. The inflammation is characterised by its tendency to spread rapidly over a large surface, and by the rapid formation of an abundant quantity of a dark chocolate-coloured fluid exudation, which stains the tissues of the organs involved, and is poured out upon the free surface of the uterine mucous membrane, from whence it is discharged per vaginam, and which, by its acridity, causes much irritation and straining-tenesmus. Examined microscopically, it is found to consist of the débris of disintegrated blood globules, pus, and blood corpuscles, and an abundant quantity of granular material slightly intermixed with shreds of imperfect lymph floating in a reddish-coloured serosity.

This affection may be looked upon as being due to pyæmic or ichoræmic poisoning, the whole mass of blood becoming altered in its character, dark, feebly coagulable, with the appearance of secondary spots of inflammation and ecchymosis in the lungs, the brain, and other organs.

The occurrence of the disease is due to over-fatigue, as from over-driving immediately prior to the act of parturition, wounds inflicted upon the uterus or vagina during difficult delivery, the retention of the fotal membranes, which, rapidly decomposing, infect the blood by absorption of the putrescent products. It 
may also be induced by obesity, more particularly in the bitch, exposure to cold, or any other debilitating influence.

The disease may occur within a few hours after parturition, or its appearance may be protracted to the third or fourth day. and the chances of a favourable termination are in accordance with the lateness of the attack. If occurring within the shorter period, it is almost invariably fatal.

Symptoms.-Restlessness, paddling of the hind feet, loss of appetite, almost a total suppression of milk, the few drops that might be squeezed from the mammary gland being of a bluish tint, thin, and watery, sometimes of a reddish or even chocolate colour, containing granular matter, more or less curdled in appearance, and exhaling a peculiar odour. The vulva is small, contracted ; the vaginal mucous membrane sometimes of a dark purple hue, or a deep red colour, with petechial spots and purple streaks. There is violent straining, discharge of a coffee-coloured, fotid liquid, and, as the disease advances, an exhaustive fœid diarrhœa. The prostration of strength is extreme; the pulse is feeble, threacly, and very rapid; the heart's action tumultuous; the belly tympanitic; the surface of the body cold. So long as sufficient strength remains, the animal will frequently lie down and rise again; will kick the belly, and show other signs of abdominal pain; and in the mare these symptoms are sometimes complicated with those of inflammation of the feet. As the debility increases, the animal will be unable to maintain the standing posture, but whilst recumbent, will still manifest symptoms of abdominal pain by rolling from side to side, looking round at the flank, and by violent attempts to regain its feet; the pulse becomes feebler and feebler; the breathing hurried and thoracic; at last the eye becomes amaurotic, and complete unconsciousnes sets in; the animal dying in from twelve hours to perhaps two days after attack.

Whilst practising in Mold, I had the opportunity of seeing this disease extensively. Mold at that time was the terminal station of the railway, and calving cows were driven in from long distances in Wales for the purpose of being conveyed to the English markets. They would sometimes calve on the road, and were of necessity driven onwards with the rest of the herd, and too often succumbed to this fatal malady.

Treatment.--Bleeding, purgatives, and depressants of all kinds 
hasten the fatal termination, and the only hope of recovery must be based upon attempts to restore the diminished vital powers by the administration of alcoholic stimulants, the removal of the uterine and abdominal pain by opium and hot fomentations to the loins and abdominal walls, and to destroy the septic properties of the contents of the uterus, and soothe its irritated and inflamed mucous membrane by injections of warm water containing opium and antiseptics, such as "Condy's Fluid," hyposulphite of soda, or carbolic acid largely diluted.

Amongst ewes, the disease is known by the term "inflammation," and great success has been obtained in its treatment by the application to the inflamed uterus of carbolic acid one part, olive oil ten parts. The same treatment is applicable to other animals.

Contagions Mammitis.-A disease rarely met with in Britain, but said to prevail in other comntries, and stated by Nocard and Mollereau to be due to a rounded or ovoid micrococcus $1.25 \mu$. in length by $1 \mu$. in thickness, and forming long straight or sinuous chains, sometimes bilobed in way of division, and found in the milk and the walls of the excretory ducts. It is aërobic and anaërobic, and its growth in cultures is arrested by weak solutions of boracic acid, and by a three per cent. solution of carbolic acid, and it is recommended that the milkers of cows thus affected should wash their hands previous to milking with this carbolic solution.

Experimental inoculations with pure cultures into the teat have reproduced the disease in the cow and goat. Even on the first day the milk from the inoculated udders was swarming with micrococci, had an acid reaction and a curdled appearance, and finally the udder became inflamed. A fatal form of gangrenous mammitis is seen in milch ewes-said by Nocard to be due to a microccocus $0 \cdot 24 \mu$. in liameter, associated in groups of four or more, but never in chains, stainable by Gram's method, anaërobic, coagulating the milk and turning it sour. Curdling of milk, as well as other changes, viz., putrefaction, viscous milk, blue milk, red milk, and yellow milk, are all due to various microbes, and should be counteracted by cleanliness and the application of antiseptics, such as boracic acid. 


\section{CHAPTER LXXIII.}

\section{PARASITIC DISEASES.}

REMARKS ON CLASSIFICATION-NEMATODA-TREMATODA-CESTODA - ACANTHOCEPHALA-DIPTERATRACHEARIA-TABLES OF ENTOZOA.

\section{CLASSIFICATION OF PARASITES.}

Parasites are distinguished as Endoparasites when living in the interior of their hosts, and as Ectoparasites when external. With the Ectoparasites we have at present nothing to do, but will confine our attention entirely to the Endoparasites. Among the most important endoparasitic animals or Entozoa are those spoken of collectively as "worms," including not only round. worms resembling the earth-worms (Lumbricus terrestris), and hence popularly spoken of as tumbricoids, but also worms resembling a band or a tape (tape-worms), or a leaf (flukes), as well as the thorny-headed worms (Echinorhynchus). The round-worms and thorn-headed worms form together the class Nemathelminthes, while the flukes and the tape-worms constitute the class Platyhelminthes. These two classes form the parasitic division of Cuvier's sub-kingdom Verues.

These classes are further broken up into natural orders; thus the class Nemathelminthes includes the two orders Nematoda and Acanthocephala, and the class Platyhelminthes the two orders Trematoda or flukes, and Cestoda or tape-worms.

These natural orders are further subdivided into families, genera, and species. Then, again, it must not be forgotten that there are other kinds of internal parasites, commonly designaterl "bots." These creatures are not usually classed with the entozor, or helminths proper, because they are merely the larval stages 
of growth of various species of gadfly. These flies are generally included in the genus Estrus of entomologists, the Estrido forming a rather numerous family of the class INSECTA, and belonging to the order called Diptera. As the attention of the profession is often called to these singnlar creatures, it will be desirable to give a brief account of their development and habits.

There is yet another series of intermal parasites, long ago called Pentastomes, from the notion that they were furnished with five months. These worms are also occasionally brought under the notice of the practitioner of veterinary medicine; and in one remarkable instance recorded by the late Professor Dick, three of these entozoa cansed the death of a valuable sporting dog. These Pentastomes, in fact, are allied to the true ticks, and therefore must be grouped along with all those spider-like creatures, which, in common with the spiders themselves, breathe by means of peculiar air-vessels termed tracheæ. In other words, the Pentastomes belong to the order Trachearia, forming a division of the class Araciridia.

It thus appears that, without taking into consideration certain minute parasitic organisms belonging to the lowermost class of animals, and misnamed "cattle plague bodies," we have to deal with no less than six well-marked orders of invertebrate animals, one or more species of each of these orders being liable to play the part of parasite within the body of some one or other of our various domesticated animals.

To such of the parasites as have an especial interest for the profession, and which are included in one or other of the six orders, namely, Nematoda, Trematoda, Cestoda, Acanthocephala, Diptera, and Trachearia, the reader's attention is now invited.

Classification of Entozoa.

Sub-Kingdom Vermes (Worms).

Cl. I. Platyhelminthes (Flat-worms).

(N. O. 1.) Cestoda.

(Fam. a.) Taniadce (Tape-worms).

Gen. Tania.

(Fam. b.) Bothriocephalidae (Pit-headed worms). Gen. Bothriocephalus. 
(N. O. 2.) Trematoda (Flukes).

Gen. Distoma.

Amphistoma.

Cl. II Nemathelminthes (Round-worms).

(N. O. 1.) Nematoda (Round-worms proper).

(Group a.) Polymyaria.

Gen. Ascaris.

Eustrongylus.

Filaria (including Spiroptera).

(Group b.) Meromyaria.

Gen. Oxyuris.

(Group c.) Holomyaria.

Strontgylus (including Dochmius).

Gen. Trichina.

Trichocephalus.

(N. O. 2.) Acanthocephala (Thorn-headed worms).

Gen. Echinortiynchus.

Sub-Kingdom Arthropoda.

Cl. I. Insecta.

N. O. Dipter $a$.

Fam. Estriace.

Gen. Estrus; the larvæ of some species are Entozoa.

CI. II. Arachnida.

N. O. Trachearia.

Gen. Pentastoma.

\section{NEMATODA.}

This order is treated of first because it includes the parasites emphatically called "worms." When a horse is said to have worms, we know that in nine cases out of ten the animal is passing lumbricoids of large size (Ascaris megalocephala), or it is troubled with maw-worms (Oxyuris curvula). In like manner, when a dog is said to have worms, it is either meant that the animal is passing or throwing up round-worms (Ascaris marginata), or that it is parting with the falsely so-called maw-worms, which, after all, are neither more nor less than the free and independent segments or proglotticles of some species of tape-worm. Worms 
in cattle and sheep are not often spoken of as such, and little attention is paid to those of the pig. With the cat, however. it is far otherwise, for we have known veterinarians whose assistance has been eagerly sought in view of ridding this donestic pet of its internal enemies, and especially of the nematode entozoon called Ascaris mystax. In addition to the above, there are other species belonging to the nematode order, to which a larger interest is, or ought to be, attached by the professional man. To this series belong the little flesh-worm (Trichina spiralis); the thread-worm which occasions the "lamb disease" (Strongylus filaria); the very similar nematode that produces husk or hoose in cattle (Strongylus micrurus); and the worm which gives rise to aneurisms in the horse and ass (Strongylus armatus). No member of the veterinary profession should be totally unacquainted with the natural history of these important species of parasite.

Except as regards the Trichina spiralis, it cannot be affirmed that we have an entire knowledge of the course of development undergone by any of the above-mentioned nematodes. It is true that, with more or less deviation from a common plan, all of them must pass through similar stages of growth, from the time of their first formation in the egg up to the period of sexual maturity. All, moreover, must in some way or other conform to a known law of their existence, which involves at least one change of residence before they can acquire the adult condition within the body of the last or ultimate bearer. Herein lies the difficulty in tracing out the development of most of the species; nevertheless, from the facts already made known by experimental research, it is not too much to hope that we shall hereafter become possessed of a knowledge of all the nore important phenomena connected with the development of the nematode worms.

The Trichina spiralis, as ordinarily known, is a small sexually immature nematode, usually found lodged within capsules or cysts, and occupying the muscles of some animal, such as the pig, or of man limself. When the little worm is removed from the cyst its entire length will be found not to exceed the $\frac{1}{25}$ th of an inch. In its full-grown or sexually mature state it is still a very minute worm; the males measuring only the ${ }_{1}^{\frac{1}{8}}$ th of an inch, whilst the females, which are more than as large again, reach up to about $\frac{1}{8}$ th of an inch. 
The professional importance of the trichina more directly concerns the medical man than the veterinarian; nevertheless, since the human disorder termed trichiniasis takes its origin from the consumption of animal food, especially pork, it is clearly the duty of the latter to understand the nature of the malady thus provoked, and to acquaint himself with the phenomena of the parasite's development. The experiments of various helminthologists, and especially those of Davaine, have distinctly proved that when small animals, such as rats, rabbits, and cats, are largely infected, they, like man himself, readily succumb to the disease. In the case of larger animals, a very great amount of infection is necessary to give rise to any external symptoms. So complete, indeed, does this immunity appear to be, that a pig experimented on at the Royal Veterinary College showed no sign of the disorder, although from subsequent post mortem evidences it was calculated that its flesh contained sixteen millions of living worms.

Until very lately we flattered ourselves that in England and Scotland there was no such thing as trichina existing in our home-reared porkers; but so far as the former division of the country is concerned, this immunity can no longer be said to exist. Not only have several English fed pigs been found to harbour spiral flesh-worms, but in the year 1871, as recorded by Dr. Dickenson, an outbreak of trichiniasis occurred in a farmer's family in Cumberland, this attack resulting from the consumption of pork reared by themselves. As Mr. Gamgee has well remarked, "If pigs are permitted to swallow the germs of hurnan parasites, as in Ireland and in many British piggeries, we must expect hams, bacon, and pork sausages to be charged with the embryonic forms of human entozoa." Very much more, of course, might be said on this subject in relation to questions of hygiene; but our object in these pages is merely to show the necessity of a general acquaintance with the subject.

The symptoms, whether occurring in man or animals, are generally believed to be due to the wounds and consequent irritation set up by the worms during their wanderings in the tissues of the host; but whilst this is true as a canse of the phenomena occurring in the second stage of the disease, it is obvious that the earliest symptoms, often accompanied with diarrhcea, are due to intestinal irritation alone. Some authors 
have, indeed, contended that there is no such thing as traumatic injury caused by the wandering parasites, but our best experimentalists in helminthology are one and all in favour of the view here advocated.

Whilst many admirable memoirs have been written on the structure and development of trichina (the literature of the subject being of very great extent), we have on the whole satisfied ourselves that the clearly enunciated statements and conclusions of Leuckart are worthy of every conficlence. The following is a brief résumé of his conclusions, given almost in his own words :-

1. Trichina spiralis is the juvenile state of a little round-worm.

2. The mature Trichina inhabits the intestinal canal of numerous warm-blooded animals, especially mammals.

3. The intestinal Trichince attain sexual maturity on the second day after their introduction into the stomach.

4. The eggs are developed within the parent worm into minute filaria-like embryos, which are born free from the sixth day onwards.

5. The new-born young soon commence wandering, penetrating the intestinal walls, and passing directly through the abdominal cavity into the muscles of the host.

6. The directions in which they proceed are in the course of the intermuscular connective tissues, the majority of the embryos resting in the muscles of the abdomen and thorax.

7. The embryos penetrate the separate muscular bundles, and at the expiration of fourteen days they will have acquired the size and organization of the spiral flesh-worm.

8. Soon after the intrusion of the parasite the infesterl muscular fibre loses its original structure; and after a while the spot occupied by the rolled-up entozoa becomes spindle-shaped, within which the well-known lemonshaped cysts are formed.

9. The further development of the muscle trichinæ is altogether independent of the formation of these cysts, the walls of which become hardened by calcareous deposition, and thus, moreover, males and females are already distinguishable in the larval state.

10. The immigration of the young parasites in large numbers 
produces very serious or even fatal consequences; and thus it happens, that in proportion to the quantity of imported parasites the symptoms resulting will be either severe, dangerous, or even fatal.

It has been stated by some writers that even pigs occasionally display symptoms of trichiniasis, the signs of the disease in the animal being loss of appetite, quiescence, aversion to all kinds of movement, and even partial paralysis of the limbs. In the human subject it is well known that the symptoms are much more severe, many of the patients enduring the most intolerable agony, until at length death mercifully comes to put an end to their sufferings.

Here it will not be out of place to mention that cats are liable to be affected with another disease very closely resembling trichiniasis, which may appropriately be called olulaniasis. The disorder is occasioned by a minute trichina-like nematode termed by Leuckart Olulanus tricuspis. It gains access to the lungs in the larval state, producing death by suffocation.

Our knowledge of the history of the development of the larger round-worms is very incomplete; nevertheless the causes of the prevalence of these worms in particular localities and during certain seasons are not far to seek. It is clear that their final stage of growth is accomplished with great rapidity, otherwise we should not meet with lumbricoids in pigs and puppies scarcely three weeks old. Large round-worms have also been found in very young colts. The ordinary lumbricoids of the horse, of the pig, and of man, so very closely resemble each other, that by some they are regarded as mere varieties of one species (Ascaris megalocephala, A. suilla, and A. lumbricoides). Whether they are so or not is of little practical moment, for it seems quite certain that a perfect knowledge of the earlier stages of development of any one of them would furnish a clue as to what obtains in the others. It is probable also that the lumbricoids of the dog and cat undergo similar clianges.

The eggs of the common round-worm have been kept alive by Davaine for more than five years; and various observers have watched their development in fresh water up to the stage of imperfectly developed embryos, and have kept them alive in this condition for three months. 
Dr. Davaine administered some of his five-year-old embryos to rats, and had the satisfaction of finding a few of their eggs in the frees, with their embryos still living, and striving to get out of the shells. He administered others to a cow, also introducing some into the stomachs of dogs in sinall linen-covered flasks. As a general result, it may be said that the embryos escaped from their shells; but the contents of those eggs in which the process of yolk-segmentation had not arrived at the stage of embryonal formation remained undigested. So far back as the year 1853 Verloren reared embryos in the eggs of the dog's round-worm within a period of fifteen days in distilled water. Dr. Cobbolıl has also reared the embryos of this species (Ascaris marginata) in fresh water, and has kept them alive for a period of seventeen months. At the expiration of this period, and during the warm weather, some of them escaped from their shells.

Accorling to Davaine, the eggs of many nematodes will readily retain their vitality though long exposed to dryness, but their contents will not go on developing during this period of exposure. In the case of Ascaris tetraptera of the mouse, however, embryonal formation goes on in spite of the absence of external moisture. He has noticed the same thing in the oxyurides of rodents. Dryness does not even destroy the eggs of Ascaris lumbrieoides and Trichocephalus dispar. It would seem, in short, that the eggs of nematodes, which normally take up their resilence in cats, $\operatorname{dogs}$, and carnivorous aminals which reside in arid regions, will develop embryos in oro without a trace of moisture. Davaine is of opinion that it is not necessary that nematode embryos should pass through the body of any intermerliary bearer; and he believes that they are often directly transferred to the stomach of their appropriate hosts whilst adhering in the condition of an impalpable dust to the coats of their bearers, whence they are detached by the animals themselves when licking the fur. With the eggs of Ascaris megalocephala Dr. Cobbolit has performed several experiments, having reared the embryos in simple fresh water, and found them capable of escaping from their shells during warm weather. He also succeeded in rearing these larve in pond mud, noticing at the same time that after their exclusion they grew more or less rapidly up to a certain point, after which they appeared to stop, as if waiting transference to some host for the further accom- 
plishment of their larval growth. The addition of horse-dung to the soft wet mud in one case, and of cow-dung in another, neither appeared to advance nor retard the process of embryonal growth so long as the embryos remained in their shells. On the other hand, when the embryos were reared in simple horsedung, purposely kept moist, they attained a higher degree of organization than did those which were reared in water and wet mud. Having watched hundreds of these larvæ under varying conditions, Cobbold came to the conclusion that after their escape from the egg, their growth, strength, and activity are favoured, if they lappen to have gained access to fluid media containing impurities. Ditch or muddy pond water would appear to be eminently favourable to the development of the escaped larvæ up to a certain stage of growth.

That warmth is eminently favourable to the development of all kinds of parasites is a well-established truth; and in the case of most nematodes it appears to be absolutely essential to the formation and hatching of the embryos. Take the case of Oryuris, for example. As Leuckart observes (Die Menschlichen Parasiten, Bd. ii. s. 326), "One only needs to expose the eggs of the human thread-worm to the action of the sun's rays in a moistened paper envelope, when in five or six hours the tadpoleshaped embryos become slender elongated worms, which are not unlike the sexually mature oxyurides in form, exhibiting rather lively movements under the influence of the warmtl." The power of warmth is thus very obvious in the case of oxyuris, since without a certain degree of temperature the earliest embryonal change cannot be accomplished. As in the oxyuris of man, these early changes are sometimes accomplished whilst the eggrs, discharged from the maternal worm, still lie in the fæces or rectum of the bearer; so also it is probable that similar changes occur in the eggs of Oxyuris curvula whilst they still remain in the rectum of the horse. According to Leuckart, the escape of the embryos of the human oxyuris ordinarily takes place when the eggs are swallowed by a new human bearer; but from the observations of Heller, it is also quite certain that any person may infest himself by swallowing the eggs which have come from oxyurides dwelling in his own person. In either case the escape of the embryos from the egg is brought about by the action of the gastric juice acting upon the egg-shell. The 
further changes resulting in the formation of the perfectly mature oxyuris are accomplished within the alimentary canal of the bearer. Here we have cases in which the adult sexual form of the parasite lays eggs in the alimentary canal of its host. Favoured by the warmth and moisture of the alimentary canal, the embryo reaches a certain stage of development while still enclosed within its egg-shell. If these eggs, expelled with the frees, reach a new host, the embryo is hatched, and develops into a sexual adult. This is the mode of development in Trichocephalus affinis, and almost certainly in Oxyuris vermicularis of man, Oxyuris currula of the horse, and Ascaris lumbricoides.

Thus Dr. Heller found, post mortem, young round-worms, of the species Ascaris lumbricoides, in the intestines of an imbecile. There were eighteen specimens, the largest of which had only acquired the length of about half an inch, whilst the smallest gave a long diameter of only 275 millimetres, or let us say roughly the ninth part of an inch. Thus Heller, in part at least, bridged over the gap which had formerly existed between the size of the embryo at the time of expulsion from the egg and the large sized ones which alone had been formerly observed in the alimentary canal, and in this case we are almost certain that the eggs are hatched in the alimentary canal, where the embryos attain sexual maturity.

The mode of development is usually slightly different in Ascaris and Strongylus, where the eggs have a thin shell, and the embryo enjoys for a time a non-parasitic existence in water or mud. Here it grows in size, but develops no sexual organs. Sometimes it accidentally attaches itself as a parasite to a freshwater mollusc, but it undergoes no change there. Eventually, gaining access to a proper host, it reaches sexual maturity, and the cycle of its life is repeated. This is the mode of development of Dochmius trigonocephalus of the dog, of Dochmius duodenalis of man, and of various species of Ascaris, c.g., Ascaris acuminata of the frog. In the case of some of the strongyles, there can be no doubt that the earlier larval transformations are undergone after the eggs have been expelled and lodged within soft soil or mud. Leuckart has proved this in the case of Strongylus hypostomus, whose rhabditiform young cast their 
first skin in about three weeks, at which time, as happens with many other larval nematodes, they part with their tails.

The case is still more complicated when the nematode requires two hosts to complete its metamorphosis. In some cases it is the egg which is taken into the first or intermediate host, in other cases it is the larva. We have a good example of a parasite requiring two hosts in the case of the Spiroptera obtusce of the monse. The eggs pass out of the alimentary canal of the monse, and are eaten by the meal-worm. The eggs are hatched, and the larva, after living in the meal-worm for about five weeks, forms round itself a capsule of connective tissue. If meal-worms containing these encysted larvæ are eaten by mice, the larve leave their capsules and become the sexually mature Spiroptera obtusa of the mouse. The Filaria sanguinis hominis, or Filaria Bancrofti, is another good example of a parasite which requires two hosts. The sexually mature worm is found in the human tissues, and is viviparous, producing numerous larve, which make their way into the blood. The blood is sucked by mosquitos, and thus the larvæ get into the mosquito, where they increase in size, and undergo various changes. When the mosquito dies the larvæ pass into the water and live for a time a free life. Ultimately they gain access to the intestines with the water, and, boring into the tissues, produce again the larvæ on attaining sexual maturity in their human bearer.

A third example of a nematode requiring two hosts is the Trichina spiralis. The sexually mature worm lives in the alimentary canal, and is viviparous, producing numerous larvæ, which make their way out of the alimentary caual into the muscles, where they encyst. When flesh containing the sexually immature larvæ is eaten, the cystic stage comes to a close, and sexual maturity is attained in the alimentary canal, where the larvæ are again produced.

The eminent Russian traveller Feltschenko observed the primary larval changes to take place in the young of the guineaworm during their sojourn in the alimentary canal of cyclopes, these entomostracous crustaceans being destined to play the part of intermediary bearers. In like manner, there can be little doubt that the young of the strongyles, which occasion husk and lamb disease, undergo their primary changes of de- 
velopment either within soft mud alone, or within the bodies of small slugs and other minute denizens of herbage, or possibly within the bodies of larval insects and minute entomostracans, inhabiting ponds, ditches, and running streams. It thus seems that whilst some nematodes can accomplish their developmental processes without any lengthened sojourn outside their final bearer, others, on the contrary, require particular, varied, and prolonged conditions which shall enable them to undergo certain preliminary changes altogether exterior to and apart from the bodies of their ultimate hosts. In short, as Leuckart points out, we have two distinct groups of strongyles: those which lead a free life in their larval state, undergoing a certain grade of development in mud and water; and those which pass through certain larval changes of growth within the bodies of insects and other intermediary bearers.

So much for the natural history of the nematoda, or order of thread-worms and round-worms, on which much more might be said, were we not limited to a general view of all the groups of internal parasites. As it is, the reader will not fail to perceive that, speaking generally, it is now clearly understood how cattle and sheep and other animals obtain one frequent form of lung disease. It is almost needless to add that the labours of helminthologists have thus contributed largely towards the formation of rational principles on which to base successfully both a radical and prophylactic method of treatment.

\section{PLATYHELMINTHES.}

The flukes and tape-worms which belong to this class have flattened bodies, hence the name Platyhelminthes or flat-worms. They are hermaphrodite, and are usually furnished with organs of attachment, such as suckers and hooks. Usually there is an alternation of generations, i.e., the young one is not like its parent, but must pass through various metamorphoses in order to reach the adult form.

\section{TREMATODA.}

This order comprises the flukes; and it is of great interest to the veterinarian, inasmuch as he is occasionally consulted in 
reference to the well-known disease in sheep termed rot. This disorder is unquestionably due to the presence of the common liver fluke (Distoma hepaticum), a parasite that is seldom more than an inch in length. Other animals than sheep are liable to be infested by it, but, except in the case of cattle, hares, and rabbits, it only very rarely occasions severe disease. In about a score of instances this entozoon has been detected in the human body. It sometimes also infests the horse.

For many years past investigations have been conducted with the special purpose of ascertaining the manner in which cattle and sheep infest themselves with this parasite; and although we have not succeeded in tracing out all the stages of growth of the common fluke itself, researches amongst the trematodes generally have enabled observers to arrive at conclusions of the highest practical importance. Intelligent cattle-breeders and agriculturists have all along observed that the rot was particularly virulent after long-continued wet weather, and more especially so when there had been a succession of such seasons; and further, that the flocks grazing in low pastures and marshy districts were much more liable to invasion than those which pastured on higher and drier grounds; but, what is most interesting, they also observed that an exception occurred in the case of those sheep feeding in the extensive salt water marshes bordering our eastern shores. It was probably this latter circumstance which suggested the common and useful practice of mixing salt with the food of sheep and cattle, both as a preventive and curative agent. At all events, as will appear in the sequel, the intelligible explanation of the good effected by this simple practice, is intimately associated with a correct understanding of the mode of development of the parasite in question.

The symptoms, treatment, and pathological appearances connected with rot are not here discussed, the present chapter being limited to the natural history of the entozoa. Even within this limitation the subject is too large to be treated of exhaustively and in detail; but for all practical purposes it is probably sufficient to follow the plan we have adopted in the case of trichina, namely, to offer a series of conclusious, such as appear to be well established by the independent researches of various helminthologists. In this connection it is 
only necessary to add that the labours of Steenstrup, Leuckart, Van Beneden, Pagenstecher, Moulinie, Davaine, Filippi, and Simonds have played a most conspicuous part. It may also be observed that, notwithstanding many assurances to the contrary, the present state of our knowledge does not justify our going beyond the data and conclusions here given. The statement made by one naturalist, to the effect that the larvæ of the common fluke reside in the mollusc called Succinea putris, has not received confirmation. That the larvæ are not limited to a single species of molluscan bearer is, to say the least, extremely probable. All things considered, perhaps the following may, in the present state of the science, be regarded as a fair exposition of the genetic relations and phenomena exhibited by the common liver fluke.

1. The liver fluke, in its sexually mature state (Distoma hepaticum) gives rise to the disease commonly called rot; this affection being also locally termed coathe (Dorsetshire, Devon), iles (Cornwall) and bane (Somersetshire). In France it is known as the Cachexie aqueuse, and more popularly as pourriture. In Germany the epidemic disease is called egelseuche, and in a more limited sense either die Füule or die Leberkrankheit.

2. The rot is especially prevalent during the spring of the year, at which time the fluke itself, and innumerable multitudes of the free eggs, are constantly escaping from the alimentary canal of the bearer. The germs are thus ordinarily transferred to open pasture-grounds, along with the fæces of the bearer.

3. As it has been shown by dissections that the liver of a single sheep may harbour several hundred flukes, and as also a single adult fluke is capable of throwing off several thousand eggs, it is certain that any rot-affected flock is capable of distributing millions of fluke germs in the egg condition.

4. Such flukes as have accidentally escaped their host per anum do not exhibit powers of locomotion sufficient to enable them to undertake migrations. Their slight movements, however, subserve the purpose of concealing 
them in the grass where they have fallen, and also, probably, aid in the further expulsion of egçs, which latter can only pass from the oviduct in single file, one at a time.

5. After the death of the escaped flukes, the further dispersion of the eggs is facilitated by the subsequent decomposition of the parent worm, and also by its disintegration, occasioned by the attacks of insects. It has been calculated that the uterus of a full-grown fluke may contain upwards of forty thousand eggrs.

6. By the agency of winds, rains, insects, the feet of cattle, dogs, rabbits, and other animals, as well as by man himself, the freed ova are dispersed and carried to considerable distances; and thus it is that a considerable proportion of them ultimately find their way into ponds, ditches, canals, pools of all kinds, lakes and running streams.

7. The eggs at the time of their expulsion exhibit the alrealy segmented yolk in a state of fine division. The ego contents continue to develop outside the parent's body; the granular matrix finally becoming transformed into a ciliated embryo, which, when set free, follows the habit of infusorial animalcules in general by swimming rapidly in the water. The escape of the embryo is effected at the anterior pole of the egg-shell, which is furnished with a lid that opens in consequence of the action of prolonged immersion, aided by the vigorous movements of the contained embryo.

8. The ciliated, free, swimming embryo of the common fluke, at the time of its birth, exhibits the figure of an inverted cone, its anterior extremity, which is broad and somewhat flattened, supporting a central proboscis-like papilla. A small pigment spot, placed dorsally, and having the form of a cross, is supposed to be a rudimentary organ of vision. After the lapse of a few days the cilia fall off, the embryos then assuming the character of planaria-like creeping larvæ. 
9. Notwithstanding its abridged locomotive powers, the nonciliated larva sconer or later gains access to the body of an intermediary bearer, within whose tissues it becomes transformed into a kind of sac or sporocyst. In this condition the larva is capable of developing other larva in its interior by a process of budding. The sporocysts vary in character, and when highly organized are called rcdice; they are often also called nurses; the latter term being generally applicable to all forms of trematode larvæ which reproduce by internal budding.

10. The progeny of the more highly organized "nurses" (sporocysts or redix) are furnished with tails, in which characteristic stage of growth they constitute the wellknown cercario or higher trematode larvæ. In this stage they migrate from their intermediary molluscan hosts, and pass into the water to lead for a time an independent existence.

11. There is every reason to believe that before the cercarice succeed in gaining access to their final or definitive hosts, they re-enter the bodies of molluses or aquatic insects. $^{1}$ This they accomplish by means of a boring apparatus; and, having previously cast off their tails, they encyst themselves beneath the surface of the skin. In this new situation they acquire a still higher degree of organization, thus realising the so-called tailless or pupa stage.

12. The pupæ or encysted cercarice are at length passively transferred, along with its fodder or its drink, into the digestive organs of the ultimate host; and it is thought that the cysts serve the purpose of a protective covering until the larvæ have passed into the true or digestive stomach, in which organ the action of the gastric juice, by dissolving the sac, liberates the pupce.

${ }^{1}$ From the investigations of $\mathrm{Mr}$. Thomas, it is assumed that the intermediate host of the liver fluke is not the slug as supposed, but a small aquatic animal, which has hitherto been overlooked, or that the roots of grasses play the part of hosts to the larval forms of distomates.-(Vetcrinarian, April 1882.) 
From the stomach the tailless larvæ succeed in entering the common liver duct and its branches, in which situation they rapidly acquire all those internal organs which characterise the adult flukes. In this way the life cycle is completed.

From the researches of Van Beneden, and especially also of Pagenstecher, it would further appear that the degree of multiplication of the larvæ and the extent of their organization are largely affected by varying states of the season, in association with other co-ordinating external conditions. This conclusion is important practically. For example, the highly organized germ-sacs or sporocysts (redice) are capable, under favourable climatal states, of developing not only the ordinary tailed cercariæ in their interior, but also new germ-sacs? It would seem, further, that there is no recognisable limit either to the variety or to the extent of larval fluke development. No wonder, therefore, that an accidental concurrence of favourable conditions, such as happens in particular seasons, should be followed by the disastrous outbreaks recognised as epidemics of rot. As we cannot regulate the character of the seasons, so neither can we prevent the occurrence of many epizootics. Helminthological science, however, does enable us to lessen the amount of disease, by affording an adequate insight into the nature of the causes concerned in its production. We may, indeed, yet be able to ascertain with precision what intermediary bearers are immemediately concerned in the harbouring of the cercarean or pupal representatives of the common fluke; and should these guests be found to reside in the body of some one particular and readily accessible mollusc, it is obvious that the collection and destruction of the intermediary bearer in question would operate to limit, if it did not, in course of time, altogether serve to eradicate the disease.

Guided by indications of the order just mentioned, Dr. Rowe, of Mount Battery, Goulburn District, Victoria, has proposed a somewhat rough and ready method of stamping out the rot, which disease, by the way, appears to be far more constant as an epizootic in Australia than it is with ourselves. In brief, the plan suggested was to burn the whole of the grass where 
rot-affected sheep have been pasturing, to destroy the diseaser animals themselves, and, after a time, to restock with sound animals.

There can be little doubt that this method would prove effective for a while; but since, to insure a permanent result, a frightful sacrifice would have to be made in the first instance, it is to be feared that the experiment could not be undertaken without severe and rather unconstitutional legal enactments. Moreover, supposing the colony were rendered entirely free from rot, the original exciting cause, which we are told brought the malady into the country somewhere about the year 1855, might again reintroduce the disorder. On this suljert, however, the reader will do well to consult the notice of Dr. Rowe's observations, as recorded in the Veterinarian for February 1873. In perusing the communication in question, it should be borne in mind that the observations proceed from the pen of an observant and extensive stock-owner.

\section{CESTODA.}

This natural order of flat-worms has acquired its name from the circumstance that most species in the adult condition resemble a tape or band, hence also the common name tapeworm. Sometimes, however, the resemblance to a tape fails altogether, as is oonspicuously the case with the little tape-worm of the dog (Tonia echinococcus). The absence of a mouth and of an alimentary canal are very characteristic of the order, so that in the tape-worm we have an animal feeding by the process of osmosis.

If an adult tape-worm be examined we find that it consists of segments. The anterior segment is differently constructed from all the rest, and is variously designated as the head, nurse, or scolcx. Behind the anterior segment or head follows a chain of seginents for reproductive purposes, the gcnerative scgments or proglottides. These generative segments are produced by a process of budding from the head. In the same worm we find them at all stages of development, unripe, half-ripe, or completely ripe segments, the youngest nearest the head. It is quite important to observe that the head produces all the rest of the worm by a process of budding, so that if all the body be 
removed and the head loft behind, the worm will be again reproduced.

The head or scolex, in addition to its power of continually budding off chains of segments, is provided with organs by means of which it fastens itself to the wall of the alimentary canal. These organs of attachment in the Bothriocephalus take the form of two longitudinal grooves, hence the name, while in the Tania the sides of the head are provided with four sucking discs. In addition to the four suckers, some tape-worms (Tonice armatc) have circles of hooks anterior to the suckers, each hook formed in a little pocket. These hooks have sharp projecting points, which serve as anchors for the tape-worm. Their presence or absence, their size, form, and number, are often very characteristic, and their examination is often facilitated by the use of caustic potash, in which the hard chitinous material of which they are composed is insoluble.

Following the head come the youngest segments, as yet narrow and short, and not plainly distinguishable from one another, often spoken of as the ncck. Proceeding backwards from the neck, the segments become broader and longer, and more distinct from one another. The hindmost segments have reached the full size, are capable of separating from the others, and can even have an independent existence for a time. Ultimately these detached segments wander out of the body, or are expelled with the frees, when they die and decay; but the ripe eggs provided with their hard shells retain their vitality, and are ready, under favourable conditions, to undergo a series of metamorphoses, whose final term is the adult tape-worm.

Each segment is provided with a complete set of male and female reproductive organs, so that, if we talke into account the fact that the segments can detach and lead for a time an independent existence, we may regard each segment or proglottis as a complete hermaphrodite individual. In the youngest segments the sexual organs are not yet developed, but in the half-ripe segments the male and female organs are distinguishable, whereas in the completely ripe segments the uterus loaded with its eggs is the conspicuous part. The form of the uterus of the ripe segment is very characteristic, and may be readily observed by pressing the ripe segments between two slips of glass, and examining with the naked eye or a pocket lens. For example, in 
the Bothriocephalus the uterus has the form of a rosctte, whereas in the Tania it is usually tree-like, and by counting the number of uterine branches we are often able to determine the species. At the same time the sexual openings can be observed-(1.) On the margin, and alternately right and left, i.e., on the left margin of one segment, on the right margin of the next, on the left margin of the third, and so on, c.g., Twnia comurus, Tonia serrata, Tania marginata, and most Tcenia ; (2.) Two marginal openings for each segment, one on the right margin and one on the left (only in Tania cucumerina and Tonia expansa); (3.) Openings not marginal, but in the middle of the ventral surface, e.g. Bothriocephalus.

If the eggs from the uterus of a ripe segment of a Tania be examined, they appear to the naked eye as coloured points, and under the microscope as round or oval bodies. The shell is thick, and is seen to consist of little prisms cemented together. In the interior of the shell the embryo is visible as a little solid body provided with six hooks. The egrgs of the Bothriocephalus present quite a different appearance.

These eggs are hatched when they gain access to the alimentary canal of a suitable host, which is rarely the same as the host of the adult Tcenia. Stimulated by the warmth and acted upon by the gastric juice of the alimentary canal, the shell is more or less dissolved, and the six-hooked cmbryo or Proscolex becomes free. Helped by its hooks, the embryo bores its way through the wall of the alimentary tract, and is carried along with the blood, or otherwise, to some place suited for its further development, it may be the connective tissue, muscles, liver, lungs, or even the brain, as is the case with the Conurus ccrebralis in the brain of sheep. Having reached a suitable resting-place, the solid embryo drops its hooks, and, acting as a foreign body, causes local exudations and new formations of granular matter and connective tissue. Thus the surrounding tissues form nutritive material and a connective tissue capsule for the solid embryo. The embryo, fed at the expense of the surrounding tissues, rapidly grows in size, loses its solid character, and becomes a hollow sac filled with fluid. In this condition the creature is spoken of as a cystic or bladder-worm, or more commonly as a measle or hydatid, and this cystic stage is quite comparable with the sporocyst of a trematode-worm. The cystic- 
worm, still enclosed in its capsule, undergoes further important changes. At one or more points in its wall a process of budding takes place, each bud growing inwards in the form of a hollow pocket, in the interior of which the suckers, or suckers and hooks, are developed. Fach bud has now the form of an invaginated tape-worm head, and when evaginated is quite like that of the adult tape-worm, differing mainly from it in being hollow.

In other cases the cystic-worm undergoes more complex changes, the buds developing not into tape-worm heads, but into secondary cysts, while the buds on the secondary cysts become the tape-worm heads, as is the case in Echinococcus cysts.

These different kinds of cystic-worms, which are merely the larval stages of tape-worms, have received different names.

(1.) Cysticercus-cyst filled with serum, and provided with only one head.

(2.) Cysticcrcoid-cyst withont serum, only one head.

(3.) Conurns-cyst filled with serum, and provided with many heads.

(4.) Echinococcus, primary cyst, gives rise to secondary cysts filled with serum, each secondary cyst producing numerous heads.

(5.) Acephalocyst, without heads.

Thus the tape-worm eggr, when hatched, gives rise to a sixhooked embryo, the six-hooked embryo changing into a cysticworm, with one or more tape-worm heads, and that is the end of one part of its development.

The cystic-worm having developed its heads, can remain for a length of time without undergoing further change, cases being known in which human beings have carried an Echinococcus cyst for over thirty years. But if flesh, lung, liver, or brain containing cystic-rorms be eaten by certain animals, then a new course of development begins. The head of the young worm comes out of the cyst, which is digested by the gastric juice, the head being protected from the action of the juice by the carbonate of lime with which it is loaded. The head is now free, attaches itself to the intestinal wall, and begins rapidly to form a chain of segments, which develop their sexual organs and their eggs, and thlis we have now the cystic-worm changed into an adult I'onia, 
with its head and segments. The ripe segments containing the eggs detach and get out of the body, and now the eggs are again ready to commence anew their life cycle.

The development of the Bothriocephalus differs in several respects from that of the Tania. The egr-shell is provided with a lid. In its interior the embryo is formed, the outer layer of cells forming the ciliated coat, and the central mass of cells the six-hooked embryo. The ciliated embryo comes out of the shell by the opercular aperture, and by means of its cilia swims about in the water. Experiments have shown that the embryos of Bothrioccplalus latus can develop into a sexually mature worm without passing through a cystic stage. The eggs were taken and kept for six months in fresh water till the embryos were formed. A very young pup was supplied with milk containing these eggs and the ciliated embryos. On the 10th July the pup was fed with the eggrs, and on the 25th August it passed a Bothriocephalus latus $42 \frac{1}{2} \mathrm{~cm}$. long, - that is, about 17 inches. On the 9th September it was killed, and three Bothrioccphali were found in its intestines.

Interesting as are the phenomena connected with the origin and development of the various tape-worms found infesting our domesticated animals, it is not necessary for the practitioner of veterinary medicine to acquaint himself with the natural history details of all the forms which are apt to come under his notice; nevertheless he will find it useful to have a general knowledge concerning some of them. Thus, he should know that no less than six different kinds of tape-worm infest the dog. Of these, he should be further aware that one (Tania serrata) is acquired by swallowing the larvæ (Cysticercus pisiformis) which reside in the bodies of rabbits and hares; that another (Tcnia marginata) is obtained by devouring the larvæ (Cysticercus tenuicollis) which reside in the viscern of the sheep and pig; that another. (Tcenia conurus) is obtained by ingesting the larvæ (Conurus cercbralis) which infest the brains of sheep and other animals; that another (Tania echinococcus) is developed when the dog swallows the lnvre (Lchinococcus vctcrinorum) found in the viscera of various animals, especially the pig; and lastly, that yet another (Tcenia cucumerina) results from swallowing the dog louse (Trichodcctes latus), which harbours its representative cysticercus or measie. 
To describe minutely all the changes through which these and other tape-worms allied to them pass, would require a separate treatise. We are concerned chiefly to present a general view of the subject; and, perhaps, in further illustrating the phenomena of tape-worm life, we cannot do better than select that particular species which, in its larval state, gives rise to the familiar disease, variously termed vertigo, gid, staggers, and sturdy.

Practical veterinarians have repeatedly asked in what manner sheep become affected with gid. For them it is not sufficient to be told that the sheep obtains the worms from the dog on the one hand, and that the dog in its turn obtains tapeworm from the sheep. There are, as we have seen, several tapeworms liable to reside in the dog, and only one of these cestodes is concerned in the matter at issue, and that is the Tocnia conurus.

If the head of a yearling affected with gid be opened, one or several hydatids will be found in the brain. These hydatids must not be confounded with the common and often much larger hydatids found in the viscera of various animals, nor with the slender-necked hydatids liable to infest the abdomen of the sheep. The brain-hydatids in question are coenuri, being readily recognised by their polycephalous character; that is to say, they are furnished with numerous processes termed heads. The fullgrown gid hydatid is always lodged within a sheath or cyst, and when removed, whether by operation or post mortem, invariably displays these heads at the surface. To the naked eye, indeed, the heads may merely exhibit the appearance of minute whitish granulations, especially if they happen to be inverted and retracted within the walls of the hydatid. The cœnurus otherwise presents the general appearance of an ordinary bladderworm, containing in its interior a clear, amber-coloured, watery fluid.

On suljecting the so-called heads to microscopic examination, they will be found to display a double crown of minute hooks in front, besides four sucking discs, such as are commonly seen on the head of an ordinary tape-worm. A single large cœnurus may support several hundreds of these heads; each head in reality representing a young tape-worm. This relationship has been proved by experiment. If, for example, a fresh cœnurus be given to a dog, each of the hundred or more heads becomes con$3 \mathrm{E}$ 
verted into a tape-worm, having characters quite distinct from all the other tape-worms that are liable to infest the dog. About eleven weeks would be necessary for the tape-worm to assume its perfect or sexually mature condition. Thus, in one experiment, where only five days were allowed to elapse before the canine bearer was killed, the heads were found alive, and separated from the hydatid, but displaying no trace of any body. In a second experiment, where three weeks elapsed, the young and sexually immature tape-worms had only attained the length of one inch and a half. In a third experiment, where the interval extended to two months, the tape-worms had acquired a length of eighteen inches; but the eggs, even then, were not perfectly developed.

A period of three months being somewhat more than sufficient for the maturation of the gid tape-worm, the perfect ova will by this time be found escaping from the dog along with its freces. These eggs, in common with those of other tape-worms, display six-hooked embryos in their interior. Wherever the infested dog wanders and passes excrement per anum, there will it be privileged to distribute the ova. The eggs, if left to themselves, would do no harm; but by various agencies they are further distributed over the pastures where yearlings and sheep are grazing. Millions of tape-worm germs are thus annually seattered far and wide. In clue course the ova are swallowed by grazing animals. When the ova have arrived within the true digestive stomach, the gastric juice dissolves the shells, and the minute six-hooked embryos forthwith make their escape. They speedily set about migrating on their own account; and having, by means of the hooks in question, bored their way into the blood-vessels, they are carried to and fro in the current of the circulation. By virtue of some selective capacity they seem to know when they have arrived within the vessels of the brain, in which organ, after escaping the vessels, they bore their way to the final resting-place. Here, by a process of transformation, they part with their hooks, and gradually acquire the bladderworm state, in which condition they vary in size from a pin's head to that of a large walnut. To attain the perfect polycephalous state, they require a period of about ten weeks, and thus the whole eycle of development is accomplished within something like five months, 
It must be allowed that the process of development above recorded is one of the most astonishing of all the biological phenomena with which the naturalist is acquainted. Not merely are the necessary changes of host remarkable, but the characteristics marking each phase in the life-history of the entozoon itself are still more noteworthy. At one time of its career the creature is a mere bladder-worm, at another an elongated tape-worm, at another a minute six-hooked embryo, and finally, once more, a bladder-worm. But this is not all; inasmuch as its development, in one very important particular, differs from the process undergone by the ordinary beef and pork tape-worms (Tonice mediocancllata and Tonia solium). Thus, whilst the egg of the common tape-worm is only capable of developing onward into a single sexually mature tape-worm, the solitary egg of the Toniia conurus, as we have seen, becomes transformed into a multitude of tape-worms. In this respect, it is true, our parasite is, in some sense, eclipsed by another tape-worm that is resident in the dog (Tonia echinococcus); but, with this exception, we know of nothing comparable to it within the limits of cestode reproduction.

Amongst the other tape-worms of general interest to the professional man are those which are derived from the consumption of pork and beef. The measles of pork (Cysticercus ccllulosus) are transformed into the Tounia solium, whilst those of beef (Cysticcrcus boris) are transformed into the Tonia mcdiocancllata. These tape-worms, as such, are only known to infest the human body. The tape-worms of cattle and sheep, as well as those of the horse, probably all belong to that group of tape-worms whose larve are normally resident in the bodies of insects and other non-vertebrated animals. This explains why their separate developmental histories have not hitherto been fully made ont by helminthologists.

Those who desire further details on this head should consult the standard treatises of Leuckart and Kïchenmeister, and also Cobbold's manual of the parasites of our domesticated animals, in which the medical and sanitary importance of the beef tape-worm is dwelt upon at cousiderable length. Lastly, we desire to call particular attention to the fact that cysticerci or measles have been found in mutton. These small cystic worms are undoubtedly the representatives of a distinct species 
of tape-worm that probably resides in the human bearer. Some, account of the parasite (Cysticercus ovis) is given in the supplement to Cobboid's larger treatise on Entozoa (p. 30), the selfsame entozoon being much more recently and fully described by Dr. Maddox in the Monthly Microscopical Journal for June 1873. We now know, therefore, that mutton and beef, as well as pork, may become measled; these three kinds of measles being perfectly distinct from each other, and all severally derivable from different species of tape-worm.

\section{ACANTHOCEPHALA.}

As already stated, this order of helminths is represented by a parasite which occasionally takes up its residence in the intestines of the pig. It is known as the large thorn-headed worm, or Echinorhynchus gigas. The male commonly measures three or four inches in length; whilst the female often exceeds fifteen inches, examples having been recorded beyond two feet. The head is furnished with an armed proboscis, by means of which the worm anchors itself securcly within the mucous membrane of the small intestines. This parasite is tolerably abundant in France and Germany, but very little is known of it in England. Professor Verrill, writing for the "Report of the Connecticut Board of Agriculture," speaks of this parasite as " the commonest and most injurious intestinal worm found in swine. These parasites," he adds, "not unfrequently perforate the walls of the intestine, and stray into other parts of the viscera, producing serious disease. Sometimes the intestine of a hog is found perforated by so many of these holes that it cannot be used in the manufacture of sausages. In severe cases the hogs are weak in the loins, and have the membranes in the corners of the eyes swollen, watery, and lighter coloured than usual. The excrement is hardened and highly coloured, and the animal often keeps up a continual squealing and grunting, especially in the morning. Such hogs are generally cross and morose, biting and snarling at their companions, but usually too weak to defend themselves if attacked in return, and easily thrown down. Finally, the weakness increases until the poor creatures are unable to walk about or to stand." 
Aithough the development of the Echinorhynchus of the hog has never been fully traced, there can be little doubt that its mode of development is precisely similar to that known to occur in other members of the genus. Dr. Guido Wagener has furnished us with some admirable illustrations of the eggs and embryos of various species (Sieb. and Köll., Zcitsch. vol. ix.), but it remained for Leuckart to explain that these creatures during growth exhibit the characteristic phenomena of alternate generation (Gött. Nachrichten, 1862). His experimental investigations were chiefly made with $E$. proteus and $E$. filicollis. Professor Leuckart caused some fresh water crustaceans (Gammari) to swallow the eggs of these small thorn-headed worms, and he had the satisfaction of observing that in a few days the embryos quitted their egg-shells and passed into the bodies of the unsuspecting intermediary bearers. After a series of further changes (which Leuckart regarded as comparable to those of a true alternate generation, and not simply metamorphotic), the young parasites rapidly increase in size; the original skin of the embryo being cast off " as soon as the echinorhynchus occupies the whole interior of the embryo." The young parasites acquire sexual organs whilst still lodged within the intermediary bearers, so that, within about a week after they are transferred to the intestinal canal of their proper and ultimate piscine host, their development into the adult state is completed. What ordinarily takes place in the case of these echinorhynchi of the fish must more or less appertain to the echinorhynchi of the hog. Swine, as we all know, are not very particular as to what they eat or drink, consequently they have abundant opportunities of swallowing insects, gammari, entomostracous crustaceans, or other minute creatures which are destined to harbour the larve of acanthocephalous parasites.

According to Schneider the eggs of the Echinorhynchus gigas are discharged in the fæces of the pig, which harbours the sexually mature adult. The eggs are devoured by maggots, and, reaching the stomach, are hatched. The embryos, which are provided with spines, bore their way into the body cavity of the maggot where they develop a young Echinorhynchus in their interior. The maggots are in turn devoured by the pig, in which the Echinorhynchus again reaches sexual maturity and produces ova. 


\section{DIPTERA.}

As remarked at the commencement of this chapter, some of the flies are apt to prove troublesome as internal parasites. With those dipterois or two-winged insects which, as external parasites, occasion suffering to animals; we have here little or nothing to do; but since some of these forms of insect life play the double part of attacking their victims from within as well as from without, it is desirable to speak of such of them as fairly, in one phase of their life, come under the general class of internal parasites.

In this relation there is a particular gromp of insects that distinguishes itself above all the others. This is the so-called bot-producing family, comprising various forms of gadflies (Estrido).

As enemies of the horse, ox, and sheep, the gadflies have acquired notoriety from the earliest times. Thus they originally obtained their family title from the ancient Greeks, who called

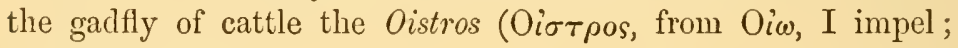
Latin (Estrus).

When any person became unduly excited they said he had a fit of the Oistros. One can readily see the force of this expression after noticing how ontrageously excited and furious a herd of cattle becomes when attacked by gadflies. As, however, it is not with the gadflies, viewed in the light of external enemies or parasites, that we have here to deal, we may dismiss this part of the subject by observing that, in the case of cattle, the gadflies have the ingenuity to select as their victims young beasts from two to three years old. The hide of an older beast is more difficult to pierce.

Since different species of gadfly attack different animals, and several kinds of fly, in the larval state, infest one and the same animal, it is desirable to speak of the forms belonging to our various domestic animals separately. In the first place we will consider those of the horse.

The common gadfly of the horse (Estrus equi) attacks the animal whilst grazing late in the summer; its object being not to derive sustenance, but to deposit its eggs on the coat; and this it accomplishes by means of a glutinous material causing the ova to adhere to the hairs. The parts of the animal selected 
are chiefly those of the shoulder, base of the neck, and inner part of the fore legs, especially about the knees; for in these situations the horse will have no difficulty in reaching the ova with its tongue. When from any cause the animal licks those parts of the coat where the eggs have been placed, the moisture of the tongue, aided by warmth, hatches the ova, and in something less than three weeks from the time of the deposition of the eggs, the larve thus make their escape. As maggots, they are next transferred to the mouth, and ultimately to the stomach of the equine bearer along with food and drink. Of comrse a great many larvæ perish cluring this passive mode of immigration; some being dropped from the mouth, and others being crushed in the fodder during mastication. It has been calculated that out of the many hundreds of eggs deposited on a single horse, scarcely one out of fifty of their contained larve arrive within the stomach. Notwithstanding this waste, we are all of us familiar with the circumstance that the interior of an animal's stomach may become completely covered with the larvæ in the condition of "bots." Whether ferw or many, they are retained in this singular abode chiefly by means of two large cephalic hooks, which are inserted into the mucous membrane.

As soon as the bots have attained their perfect growth, as such, they voluntarily loosen their hold, and allow themselves to be carried along the alimentary canal until at length they make their escape with the fæces. It is said that during their passage through the intestinal canal they not unfrequently reattach themselves to the mucons membrane, thereby occasioning severe intestinal irritation. When thus lodged in the neighbourhood of the anus they seriously inconvenience the animal. In all cases, however, they sooner or later fall to the ground. When once transferred to the soil they bury themselves beneath the surface in order to undergo the change whereby they are transformed from the bot state into the pupa condition. At length, having remained in the soil for a period of six or seven weeks, they finally emerge from their pupal envelope or cocoon in the active life-phase of the imago or perfect dipterous insect. It thus appears that these creatures in the form of bots ordinarily pass about eight months of their lifetime in the digestive organs of the horse. 
That bots are capable of giving rise to severe disease in the horse there cannot be any reasonable doubt, but it is not often that the disorder is correctly diagnosed, since it is only by the passage of the larvæ, or by their adherence to the verge of the anus, that the practitioner can be made aware of their presence. We are not called upon to dwell on this fact of the subject in the present chapter, but may remark in passing that Mr. J. S. Woods, V.S., has published in the Veterinarian a case of tetanus in a mare associated with the larvæ of Estrus equi, and Mr. J. T. Brewer, V.S., has also given a case in the same journal, where the duodenum of a horse was perforated by bots.

Several other species of CEstrus victimise the horse; one of the most formidable of these being the $E$. hamorrhoidalis. This fly is especially annoying in the initiatory stages of the attack, because, unlike the common species, it selects the lips and nostrils as the principal locality for the lodgment of the ova. According to Bracey Clark the mere sight of the insect produces extreme agitation, the horses wildly galloping to and fro in their usually vain endeavours to evade these winged tormentors.

The common bot-fly of the ox (Eistrus bovis) passes through transformations similar to those undergone by the gadflies of the horse. It differs, however, in one important particular; for, in place of acquiring its larval condition as a bot within the stomach, it takes up its residence for that purpose beneath the animal's hide. In this situation its presence gives rise to the formation of small tumours, termed warbles. The facts, in short, are as follows:- Selecting, as before remarked, young beasts in good condition, the fly lights on the back on either side of the spine. The animal darts away in alarm, often bellowing furiously and frightening its companions. The whole herd forthwith rush about in a frantic manner; and it is said that the mere buzzing of the insects is sufficient to render yoked animals quite unmanageable. In a short time the insect succeeds in perforating the skin by means of an ovipositor, one agg being deposited in each opening. After a time the egg is hatched, and the young during growth produces sufficient inflammation to lead to the formation of the well-known warbles. Within the tumour the bot is placed with its head downwards, its tail being applied to a small external opening in the warble, in order that it may receive sufficient air for the purposes of respiration. 
When the bots are mature they make their escape, and fall to the ground, burying themselves in the turf, or hiding underneath stones. During the process of metamorphosis the skin of the bot becomes transformed into a cocoon, and in course of time the pupa or chrysalis stage is completed. In this state it remains as a grub for a month or six weeks, at the expiration of which period the lid of the cocoon comes off, and the perfect insect or imago is set free.

The common bot-fly of the sheep (Estrus ovis) neither chooses the stomach nor the back of its bearer as a place of residence during its acquisition of the larval condition, termed the bot. This insect may be regarded as a worse tormentor than either of its common congeners above mentioned. It attacks the nostrils of the sheep, and the distress thus occasioned is so great that the poor animals, in order to avoid the flies, will often bury their nostrils in the dusty hollows of cart-ruts, further protecting their heads with the fore feet. The members of any flock thus attacked will also collect together in groups, and jostle against one another with their heads downwards, so as to avoid the flies as much as possible. When struck by the fly they stamp the ground violently, and exhibit other signs of distress, sometimes amounting to agony. According to Mr. Riley, as quoted by Verrill, the young larva is itself deposited at the margin of the sheep's nostrils, having quitted the egg whilst yet within the oviduct of the parent insect. Be this as it may, the young larræ having once gained access to the nasal passages, have no difficulty in retaining their hold, at the same time that they cause fresh distress to the inhappy bearer. Within the cranial sinuses they firmly anchor themselves by means of a pair of cephalic hooks, and in this situation they remain until they have perfected this stage of their larval development. Considering the situation of these creatures, there need be no astonishment at the fact that their presence sometimes gives rise to terrible sufferings on the part of the sheep; the afflicted animals occasionally perishing under the inflammatory action thus set up. Stock-owners and farmers term this disease grub in the head, and it is often asserted by them that the grubs gain access to the substance of the brain itself. They are perfectly sure they have seen maggots in the brain. and no arguments of the veterinarian, derived from a study of the osteology of the 
sheep's head, will serve to convince them that they are in error. Without dwelling upon this point, we have further to observe that the perfected bots usually pass from the nostrils to the ground by the same way that they entered, and thence-forward, having penetrated the soil, they accomplish their subsequent metamorphosis in a manner very similar to that of their congeners. The pupal state is acquired in about two days, but they remain concealed in the soil for a period of six or eight weeks. At the expiration of this period the lid of the cocoon is raised, and the insect prisoner makes its escape in the usual manner.

In perusing the above remarks, it will be noticed that, if $\mathrm{Mr}$. Riley's statements are to be accepted as correct, the gadfly of the sheep reproduces viviparously. This is a point of considerable interest, since, so far as we are aware, all the other gadflies bring forth their young in the egg condition. According to Verrill, who quotes from the First Annual Report on the Noxious Insects of Missouri (given in the Connecticut publication already cited) for 1868, Mr. Riley states that Mr. Cockrill had removed upwards of three hundred living larvæ from the body of a single gadfly. Soon after the flies have effected their escape from the cocoon, they set about operations for the continuance of the species; and as they are neither furnished with a mouth nor other means of taking in nourishment, it is obvious that the pleasures they enjoy during the winged state must be exceedingly short-lived.

\section{TRACHEARIA.}

As with the dipterous insects, so with the members of this large order or division of arachnidans. Whilst many species are externally parasitic, only a very snall number can in any true sense be called entozoa. One species, however, of the present group is not only internally parasitic in the larval state, but also in the full-grown or sexually mature condition; consequently it has even more right to be regarded as an entozoon than any of the gadflies. The parasite in question is the Pentastome tenioides. In the adult state, this worm occujpies the nasal and frontal sinuses of the dog, sheep, and horse; and in one of its larval stages it is found either encysted or free in the viscera 
of various animals, especially ruminants, as well as in man himself. The larva, which is commonly described as the Pentastoma denticulatum, usually measures about one-fifth of an inch in length ; but the adult males are three-quarters of an inch long, whilst the females occasionally measure as much as four inches from head to tail.

The history of the development of these curious parasites is somewhat remarkable and of great practical interest. The female discharges her eggs whilst within the nose of the dog. From the nasal passages the eggs are constantly discharged by sneezing and otherwise. They are thus scattered by the canine host in all directions, and by the drying of the slime they adhere very readily to vegetable and other matter. Afterwards, on being transferred to the stomach of ruminating and other animals, their embryonic contents are set free by the dissolution of the shells. The embryos, which are furnished with two pairs of claws, then bore their way into the liver and other viscera. They next become encysted and change their skins. After a time, the larvæ are set free, but their wanderings are ordinarily of no avail, unless portions of the infested animal are brought in contact with the nose of the ultimate bearer. In the case of the dog, this commonly happens when the animal is engaged in devouring portions of fresh viscera carelessly flung to it. The larvæ are thus brought in contact with the dog's nose, and then, by means of the hooks and spines with which they are armed, the young pentastomes readily adhere to the nose, and in a short while crawl up the nasal passages, where they rapidly acquire sexual maturity. It is not so clear how they make their way into the nasal cavities of the sheep and horse; but in all probability some larvæ escape from the bodies of their intermediary bearers into open pastures, and thence into the nasal organs whilst these animals are grazing. Dogs that frequent knackeries and slaughter-houses are particularly liable to become infested by the adult parasite. Such larvæ as do not succeed in escaping their cysts perish by calcareous degeneration.

To the veterinarian these facts of development are chiefly important as explaining how dogs contract the parasite; and although instances of suffering from these entozoa may not be common, there is every reason to believe that examples of the kind are not unfrequently overlooked. It is certain that dogs 
sometimes suffer severely from worms in the nose, the parasites giving rise to nasal catarrh, accompanied by fotid discharges. There is one remarkable case on record, in which death was occasioned by pentastomes. This is the instance already mentioned as having been made public by Professor Dick in the pages of the Vetcrinarian for 1840 . The communication is most interesting and instructive. Three of these parasites having wandered into the fauces and trachea of the dog, its death resulted, partly from spasm of the laryngeal muscles, and partly from inflammation of the left lung, accompanied by excessive bronchial secretion. In short, the animal was süfocated.

\section{ASCARIDES AND LUMBRICI.}

The Ascaris megalocephala of the horse and Ascaris suilla of the hog are considered by some helminthologists to be identical with the Ascaris lumbricoides of man. Dr. Cobbold and others take exception to this conclusion, and consider that they are distinct speeies.

These worms, best known amongst veterinarians as the lumbrici, resemble the common earth-worm in size and shape; the males are shorter than the females, which sometimes measure from twelve to sixteen inches in length. They are found in the small intestines; sometimes, but rarely, in the stomach. If few in number, they occasion no inconvenience to the bearer; but if numerous, and particularly if they infest the stomach, they, like bots, may cause colic, indigestion, unthriftiness, and emaciation. After the death of the bearer, several of them are generally found matted together, and coiled up in the form of a ball, leading one to conclude that they have thus caused an obstruction during life. Numerous observations in the dissecting room enable me to state that they assume this form after the death of the host; that they congregate together, interlace one with the other, very shortly after the animal which they infest has died.

In the dog, round-worms-Ascaris marginata-especially if they enter the stomach, cause convulsive fits, vomiting, and sometimes death.

Oxyuris curvula, or better known to veterinarians as ascarides, 
are small white worms-needle or whip worms-which com monly infest the rectum, and very often the colon, being very abundant in the flexures of that intestine. They escape by the anus, and cause irritation, manifested by the horse rubbing its tail against any hard substance, such as the walls of its stable, or whisking it about in an irritable manner. If the anus and perineum be examined, small masses of a yellowish-white looking substance will be found adhering to the skin: these are the eggs of the worms discharged from the body.

Treatment.-It is a difficult matter to destroy these parasites. Sometimes an aloetic cathartic will cause the expulsion of numerous lumbrici from the horse, but the remedy is uncertain. Oil of turpentine stands in high repute, and doubtless is more anthelmintic than any other in the horse, but it cannot always be depended upon. Aconite, in some instances, will cause the expulsion of the parasites, as is seen when it is employed in the treatment of inflammatory diseases. When associated with emaciation and debility, the salts of iron prove of much service; they not only have the effect of destroying the worms, but, by causing an improvement in the general health of the animal which they infest, render it an unfit habitat for the parasites, for it is a well-known fact that when the condition of the animal's body is weakly, it is more liable to be infested by parasites of various kinds.

In the dog, emetics will sometimes cause the expulsion of the Ascaris marginata when in the stomach; if these fail, santonine, in from three to five or eight grain doses, according to the size of the animal, or the etherial extract of the male shield fern, is to be administered every second or third day, taking care to watch their action upon the patient, as santonine will sometimes cause straining and other signs of irritation.

The oxyuris may sometimes be destroyed in great numbers by enemas, consisting of decoctions of quassia, gentian, or even wormwood. These enemata are to be frequently repeated, and their action may be aided by purgatives. The introduction of a small piece of mercurial ointment into the rectum is a very common practice in some parts of the country, and it seems to answer very well, not only preventing the migration of the parasites, but actually destroying them. 
There are many other remedies recommended by helminthologists, many of which are very serviceable, whilst others are only calculated to do harm. I need only refer to the insane practice of administering ground glass and other mechanical irritants, in order to warn the reader against what is absurd.

Other nematode worms infesting the lower animals, and found in the blood-vessels and eyes, are briefly referred to in my work on Veterinary Surgery, to which the reader is referred. 


\section{CHAPTER LXXIV.}

\section{PARASITIC DISEASES-continued.}

\section{DISEASES CAUSED BY NEMATODA OR ROUND-WORMS.}

\section{PARASITIC DISEASE OF THE LUNGS.}

Is the calf and lamb, bronchial irritation, arising from the presence of nematode parasites, termed strongyles, is of frequent occurrence, and is variously termed The Husk, Hoosc, Phthisis pulmonalis verminalis, and Parasitic bronchitis.

In the lamb, the parasite, termed Strongylus filaria, is from one to two and a half inches long; the female is white, larger than the male, which is of a yellowish-white colour, and its body is of uniform size, but tapered at both ends. The head is short, stumpy, rather angular, but not tuberculated as in other strongyles. Extending from the mouth is a short oesophagus, entering the stomach, from which a straight intestine is continued nearly to the extreme end of the tail. The tail of the female is pointed; the oviducts, filled with eggs and live young, extend into the vulva, which is situated close to the anus.

The anatomical situation of the parasites in the lungs of lambs and sheep is not always the same. In lambs they are found not only in the bronchial tubes, but also in the lung-substance, whilst in sheep they are generally encysted in the parenchyma of the lungs, giving them the appearance of being filled with small tubercular deposits; indeed, the disease was for a long time looked upon as a true tubercular affection. I am of opinion that within the last few years the disease is more commonly met with, and during the winter and spring of 1873-1874 I had more opportunities of witnessing it than in any former years.

The lungs of sheep which have been the hosts of these parasites are thickly covered with numerous small nodules, varying 
in size from a pin-head to a hemp-seed, or even larger, and resembling small vesicles or bisters. Some are filled with a clear fluid; others contain a soft material, consisting of granular matter; whilst others are hard and gritty, -and all contain minute worms coiled upon themselves.

The varying degrees of hardness and consistence of the nodules mark their age; the vesicle seems to be their earliest development; the soft, solid condition a more mature condition; and the gritty state shows that the wall has undergone calcification, and that the worm has been lodged in the lung tissue for a considerable period.

The presence of these parasites in the lung does not always cause irritation or inconvenience to the host. The lungs of sheep killed in the primest condition are found loaded with them. Occasionally, however, they induce debility, anæmia, and cause death, more particularly in lambing ewes, at or about the period of parturition. Many ewes died from this cause during the lambing season 1874.

In lambs, however, the parasites find their way through the softer lung structure into the bronchial tubes, and there give rise to irritation, and to the symptoms of the "lamb diseasc."

It is supposed that the parasite is developed in the lamb only, and that those found encysted in the lungs of sheep have been long imprisoned as it were by a boundary line of plastic infammation, which finally becomes calcified, and offers an impassable barrier to the movement of the strongyle.

Whilst admitting the greater frequency of the affection in lambs than in sheep of a more mature age, I cannot subscribe to the above conclusion, as the results of examinations of the lungs of four or even five year old ewes have shown conclusively that many cysts are not in a state of calcification-a condition that they certainly would be in, if the parasitic invasion had occurred when they were lambs. The manner by which parasites gain access to the lungs has been a matter of controversy. Ur. Edward Crisp, in an essay on this disease, for which a prize of $£ 30$ was awarded by the Bath and West of England Agricultural Society, accounts for their presence in the lungs by direct passage into the trachea from the mouth, where they have been forced from the stumach during the act of rumination. Mr. Dickinson, M.R.C.V.S., Boston, Lincolnshire, Professor Armatage, 
as well as Professor Gamgee, oppose this view. Mr. Dickinson says - "The idea entertained of a direct passage of these to the lungs appears to me paradoxical and in nowise probable. Their migration, I am inclined to believe, is a work of time; and hence, as I have frequently observed, the parasites abound in the alimentary canal in large numbers, give rise to aggravated symptoms which terminate fatally when no worms or their eggs are to be detected by the naked eye, at least in the lungs or bronchi." Professor Armatage informs me he has also observed this in many instances. Mr. Armatage says- "The presence of Strongylus filaria in the lungs of lambs and sheep, I think, cannot be explained by any theory which describes-no matter with what minute exactness and elaborate detail - the unnatural and, I might add, almost impossible mode of their passing direct through the windpipe to these organs. We all know, as possessing some slight knowledge of physiology, how a hair, a breadcrumb, drop of water, \&c., will irritate the glottis or entrance to the windpipe, and give rise to the most painful and convulsive coughing. These are, however, objects of an inanimate character, and irritate by mere presence. How much greater, then, would be the effects of a live worm or worms insinuating themselves on the delicate structures, and especially when the natural barbs or hooklets, as described by Professor Simonds, are put in operation. The supposition, I think, suffers much under the great probability that violent coughing taking place on the entrance of a worm or worms would entirely expel them. If they are expectorated in large numbers from the recesses of the bronchial tubes, they will most assuredly be compelled to evacuate much more rapidly at their entrance to the windpipe." The same gentleman further says- "We must not overlook the important fact that young lambs are principally affected. In them the tissues are more easily pierced, and their passage from the lung tissue to the bronchial tube readily effected. In older animals they remain enveloped within a matrix of cretified substance and metamorphosed lung tissue, in order, as it were, to guard against their effects. If their passage to the lungs were always more direct, the opposite would be the case, and our old animals would die as rapidly as the young ones." Professor Gangee says- "The migration from the mouth or alimentary canal to the lungs certainly requires a more complete explanation than has hitherto becn given." 
There is no doult in my mind but that the ova and young parasites taken up with the food, in the first place gain access from the alimentary canal into the circulation, and are conveyed into the lung-substance, where they are deposited, the parasites when mature piercing the tissues and entering the bronchial tubes, and there cause the irritation symptomatic of the disease, whilst those remaining encysted in the lung cause little or no inconvenience (see fig. 42). It is very true that in many instances the parasites are found fully matured in the digestive canal, and doubtless the conclusions of Dr. Crisp are due to this fact. We can, however, easily understand that the heat and moisture of the stomach are quite sufficient to cause the ova to hatch and some of the embryos to mature in the intestinal canal, whilst other embryos pierce the intestinal wall and are taken into the circulation, and inature only when they have been deposited in their proper habitat; and a few may become fully devcloped in the blood. I have on two occasions seen the parasite in the cavities of the heart and in the blood-vessels; and I think this fact conclusively points out the correctness of this view of their migration.

The tenacity of life in the young strongyle is very great. Ercolani found that they showed signs of life on being moistenerl after drying for thirty days, and at other times after having been immersed in spirits of wine at $30^{\circ}$, or in a solution of alum and corrosive sublimate.-(GAMGEE.)

The number of embryo worms in the lungs of one sheep is very great; if to these we add those hatched in the digestive canal, we can easily understand, when these are severally discharged from the infected animal, how a pasture may become infected with parasites and ova to such an extent as to infect a whole flock.

Dr. Crisp says the disease is due to over-stocking, and especially to the feeding of lambs off a second crop of clover after the first crop has been consumed by sheep. The mere feeding of lambs with the second crop of clover, after the first has been consumed by sheep, would not of itself be sufficient to cause an outbreak of the lamb disease; but when we consider that a very large number of sheep are infested with the parasites, from which they seem to suffer no harm, we can easily understand how, by the expulsion of some of these from the affected a pasture may become fouled by the parasites and their ova. 
I can offer no explanation as to the source from whence the Strongylus filaric is originally derived, and can only say with Dr. Cobbold, that, "with the exception of the Trichina spiralis, I am not aware that we have a thorough knowledge of the entire life history of any of the nematode species which infest the human body; nor, indeed, can I call to mind any round-worm infesting the lower animals, whose life phases, migrations, and peculiarities of structure during development are satisfactorily known."

We know, however, that the disease prevails particularly in low, damp situations; on lands subjected to be flooded by the overflowing of rivers, or after heavy rains occurring during early autumn or the latter part of summer.

Symptoms.-These are subject to some modification, depending upon the seat of the parasites. In the majority of cases, they are found in the lungs, in others in the lungs and digestive organs, whilst in others again they are found in the stomach and bowels only-the lungs being entirely free from them. When in the intestinal canal the symptoms are those of dysentery, with fœtid stools; there is much tenesmus or straining, and occasionally clots of foetid blood are discharged per rectum. The lodgment of the parasites in the pulmonary tissue and bronchial tubes cause, in the lamb, irritation and inflammation, indicated by cough, rubbing the nose on the ground, and accelerated respiratory movements, whilst in the more mature animal-the sheep-the presence of the parasite may induce no diagnostic signs, even in those which succumb, and it is only after death that the cause of the emaciation and anæemia, which may have been present during life, is discovered. In many cases the abdominal symptoms are accompanied by depraved appetite, intense thirst, and even colicky pains.

When the parasites are in both the lungs and bowels, there will be a combination of pulmonary and enteric irritation, ancl very rapid sinking. If the expectoration and alvine discharges be carefully examined some of the parasites are sometimes discoverable.

Treatment.-This naturally divides itself into preventive and curative.

To prevent the disease lambs require to be fed on fresh pastures; second and third year crops are to be specially avoided, if they have been previously grazed by sheep. If the 
seasons are damp, the flocks are to be pastured on the hill sides, or on dry pastures; and if the grass be scarce, it is to be supplemented by artificial food.

To cure the disease, or rather to destroy its cause-the parasites-inhalations of chlorine gas have been recommended. In using this agent great care must be taken that it be sufficiently diluted with air, so as not to destroy the patient as well as the parasite, for I have heard of individuals who have applied it sufficiently strong to destroy all their patients. It is therefore better to compel the animals to inhale it from the chloride of lime, to which sulphuric acid has been added, than to manufacture it in the ordinary way, namely, by the admixture of common salt, peroxide of manganese, and sulphuric acid. Should the animals be thought too weakly to stand the chlorine gas, sulphurous acid may be substituted, and this is so cheap and so much safer than the chlorine that I much prefer it. It is made by burning sulphur, which, combining with oxygen during the combustion, gives off fumes of sulphurous acid. Of course it will be understood that when animals are made to inhale either of these gases, they are to be confined in some building. When the parasites are in the intestines, several doses of turpentine are to be administered. The debility from which the animals suffer is best combated by stimulating food, as the cakes, to which the sulphate of iron-from ten to twenty grains for each lambhas been added. Rock salt should also be allowed the animals to lick, or a small quantity of common salt added to the food.

Hoose in Calves. - This disease very closely resembles that in lambs; and is caused by a parasite termed the Strongylus micrurus (Mellis), which gains access to the pulmonary tissue and bronchial tubes through the circulation, the ova being absorbed from the digestive canal. This parasite is very tenacious of life, and will be seen to be quite lively in the pulmonary organs several days after the death of its host. It is one of the armed strongyli, has a filiform body, and a mouth with three papillæ.

This disease prevails in low-lying districts, on land near rivers, more especially after heavy floods, and is mostly seen in the months of August, September, and even October, in calves under one year old, and very rarely in those rising two years old. ${ }^{1}$

1 I found this parasite in the lungs of several full-grown American oxen during the spring of 1879 . 
Sometimes the worms are very numerous in the trachea and bronchial tubes, and not unfrequently one finds them congregated together, after the animal's death, in a perfect ball, effectually obstructing the windpipe.

I have watched the movements of the embryos of this parasite under the microscope, and have seen them burst, through the walls in which they have been enclosed, and make their escape.

This parasite has also been found in the air passages of the horse and ass. A similar affection has been observed in the pig, the parasite being called Strongylus suis. In poultry the disease termed the "gapes" is caused by a parasite called Sclerostoma syngamus.

The symptoms in the Calf.-The seat of the irritation is indicated by a bronchial cough, "husk or hoose," loss of flesh, a varying degree of constitutional disturbance, and death by suffocation if the sufferer be not relieved. If any mucus be coughed up and examined the parasites may be discovered. Bronchial irritation occurring in calves during summer or autumn should always be looked upon with suspicion, and its source thoroughly inquired into. If any calves be already dead, a careful post mortem examination ought to be made, in order that the pathological condition of the lungs should be determined. If none are dead, the mucus fram the nose should be examined, when in all probability some of the parasites will be discovered.

Treatment.-The calves are to be warmly housed if the nights be cold; the affected animals are upon all occasions to be removed from the healthy; not that the disease is contagions in itself, but that the parasites or their ova, discharged from the sick, are apt to gain access into the bodies of the healthy, and for the same reason the healthy should be removed to fresh pasture and to dry situations, as the fields upon which the disease has prevailed will, for a time at least, be tainted by the parasites and ova.

Inhalations of chlorine or sulphurous acid are recommended. If this be carefully done, the sufferers may be kept surrounded by either of these for about fifteen minutes each day, until the disease disappears; two or three inhalations are generally sufficient. If inhalation be objected to, turpentine may be administered in gruel daily, or, what has succeeded well with me, from ten to twenty minim doses of Scheele's hydrocyanic acid, with 
carbonate of soda, and some bitter stomachic, as gentian or chamomile, twice per day. The acid seems not only to have the effect of destroying the parasites, but also of allaying the bronchial irritation in a very short space of time.

The "gapes" in fowls, due to a parasite in the air passages, the Sclerostoma syngamus, and indicated by gaping, gasping for Jreath, sneezing, and frequent attempts at swallowing, is best treated as recommended by Professor Cobbold and others.

"First. When the worm has taken up its abode in the trachea of fowls and other domesticated birds, the simplest plan consists, as Dr. Wiesenthal long ago pointed out, in stripping a feather from the tube to near the narrow end of the shaft, leaving only a few uninjured webs at the tip. The bird being secured, the webbed extremity of the feather is introduced into the windpipe. It is then twisted round a few times and withdrawn, when it will usually happen that several of the worms are found attached. In some instances this plan entirely succeeds. But it is not altogether satisfactory, as it occasionally fails to dislodge all the occupants.

"Secondly. The above method is rendered more effectual when the feather is previously steeped in some medicated solution which will destroy the worms. Mr. Bartlett, superintendent of the Zoological Society's Gardens, employs for this purpose salt, or a weak infusion of tobacco; and he informs me that the simple application of turpentine to the throat externally is sufficient to kill the worms. To this plan, however, there is the objection that, unless much care be taken, the bird itself may be injuriously affected by the drugs employed.

"Thirdly. The mode of treatment recommended by $\mathrm{Mr}$. Montagu appears worthy of mention, as it proved successful in his hands, although the infested birds were old partridges. One of his birds had died from suffocation; but he telis us that change of food and change of place, together with the infusion of rue and garlic instead of plain water to drink, and chiefly hemp-seed, independent of the green vegetables which the grass plot of the menagerie afforded, recovered the others in a very short time.'

"Fourthly. The plan I have here adopted, by way of experiment, of opening the trachea and removing the worms at once. This method is evidently only necessary when the disease has 
advanced so far that immediate suffocation becomes inevitable; or it may be resorted to when other methods have failed. In the most far-gone cases, instant relief will follow this operation, since the trachea may with certainty be cleared of all obstructions.

"Lastly. The most essential thing to be observed, in view of putting a check upon the future prevalence of the disease, is the total destruction of the parasites after their removal."

Intra-tracheal injections for hoose in calves.-Recent experiments have demonstrated that drugs may be injected into the trachea with impunity, and that hoose may be most advantageously treated with remedies administered by this method. Turpentine, prussic acid, carbolic acid, and creosote may be used in this way. The turpentine and creosote should be mixed with either oil, or an emulsion of oil, water, and alkali, as in the following:-B Ol. tereb., 3ij. ; ol. olivæ, 3j.; potassæ carb., gr. xx. ; aque,, $5 \mathrm{j}$. Inject this quantity daily for three days. The injections are given with the hypodermic syringe. Make a small incision through the skin at the site for tracheotomy, pass the needle of the syringe into the trachea between two of the rings, and inject.

Corrosive sublimate, one of the most powerful parasiticides known, might be tried in the form of intra-tracheal injectiongr. $\frac{1}{6}$ to gr. $\frac{1}{4}$ in 3iij. or 3iv. of water.

\section{PARASITIC GaStRic CATARRH.-(Gastrorhoea Parasitica.)}

A disease of the fourth stomach of the sheep, caused by the presence of the nematode worm called the Strongylus contortus.

The following is a short history of an outbreak of disease in sheep induced by this parasite, and to which my attention was called by Mr. Connochie, V.S., Selkirk, in March of this year, 1884.

It appears that the owner of the sheep in question had placed his lambs, after weaning time, upon a very luxuriant pasture, but from the nature of the autumn of 1883 , which was very wet, the grass became rank and the soil damp, notwithstanding it had been previously well drained both on the surface and underground.

After thriving well for some time, they began to lose Hesh very rapidly, and gave other indications of disease; they were then removed to another pasture of good clean grass, and shortly afterwards placed on turnips, oats, and Indian corn. This change seemed to have the desired effect, as they commenced to thrive and lay on flesh; but in the month of February 18s4, when nearly iat and fit for the butcher, the now hoggets suddenly 
presented signs of disease. Their appetite at first became capricious; they then ceased eating their food altogether, but would devour earth or any rotten or decaying vegetable matter. From this time other and more serions symptoms soon developed themselves, such as great restlessness, lying down and suddenly rising again, pawing with the feet and showing signs of abdominal pain. The bowels now became very irritable, and from being constipated became very loose, the evacuations being like muddy water. At this stage a most unnatural desire for water was evinced, the sheep roaming about in search of it, and when driven away from it, immediately returning to it. Along with this insatiable thirst, there was also an almost constant desire to urinate, the urine passed being as clear as water. The cough now became hacking, but intermittent, each fit of coughing continuing until the animal succeeded in ejecting a quantity of frothy mucus, which relieved it for a time only, as the fits soon returned and became more frequent; during the intervals a flow of watery saliva continued to drivel from the mouth, accompanied by a constant grinding of the teeth, and a continual twitching of the nostrils and upper lips.

During the development of the above symptoms the hoggs had most rapidly lost flesh, the adipose and muscular tissues being rapidly atrophied; the abdomen becoming greatly distended, causing the animal to present a most miserable appearance. All the above symptoms increased gradually and steadily, until death closed the scene.

After trying various remedies without success, Mr. Connochie advised the owner, Mr. Elliot, Hollybush, Galashiels, to call me into consultation.

Before seeing the sheep in consultation with Mr. Connochie, one carcase was sent to the College for examination.

In the lungs of this sheep the Strongylus filaria was found, but not in great numbers; the parasites were also found in the bloodvessels of the lungs, causing embolism; and in the fourth stomach the Strongylus contortus was found in great numbers. After this examination I visited Hollybush, and saw several sheep in an advanced stage of the disease, and, in addition to the above symptoms, furnished by Mr. Connochie, found that the majority presented aggravated symptoms of cedema of the head, affecting the nostrils and interfering with the breathing, and involving the intermaxillary space and inferior cervical region as low down as the breast. 



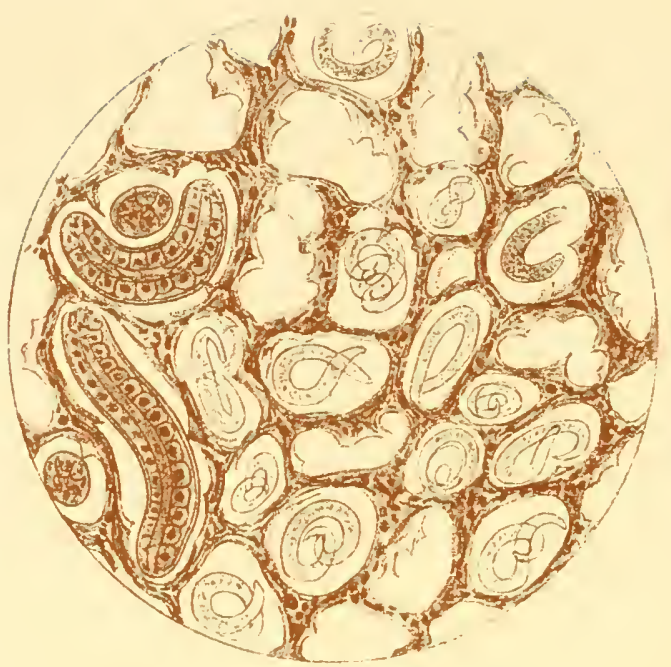

Strongylus Filaria, embryonic stages, in alveoli of Lungs. $(\times 200$.

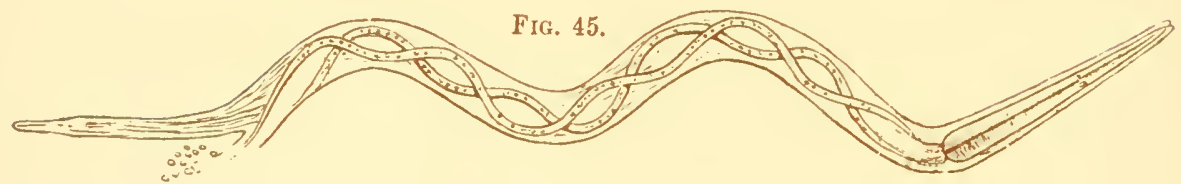

Convoluted Uterus.

Strongylus Contortus.
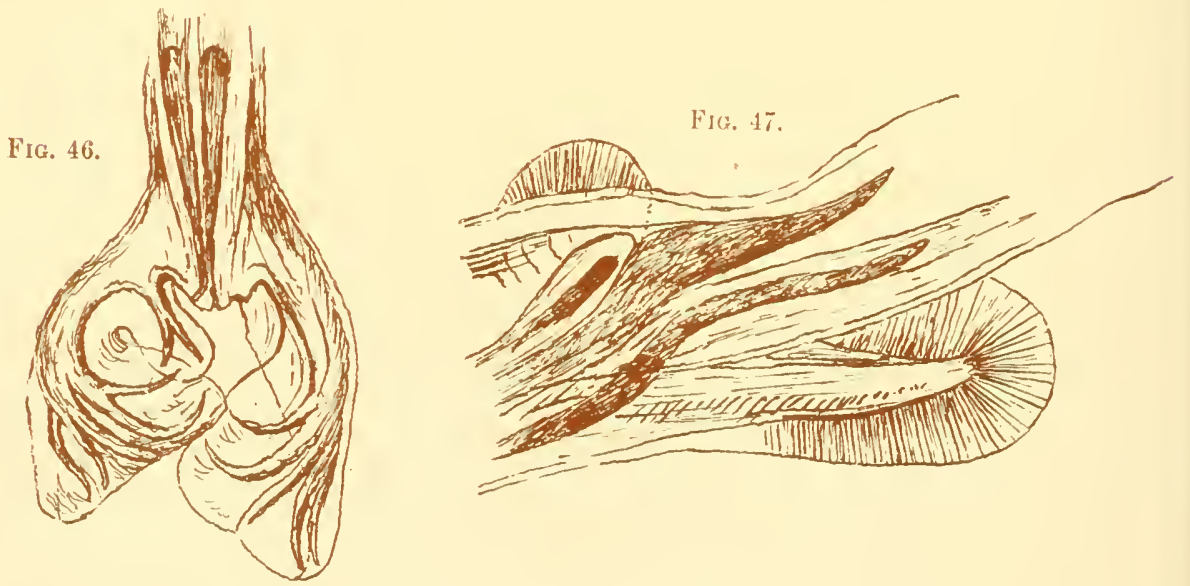

Strongylus Contortus.-Bursa of Male. Strongylus Contortus.-Genital papilla of Female. 
On making a post mortem examination of one sheep killed for the purpose it was found that the Strongylus flaria was absent, but that the true stomach (abomasum) contained a quantity of a dirty looking fluid, in which the Strongylus contortus could be seen wriggling in great numbers.

The parasites were so small as to be seen in the fluid with difficulty by the naked eye, but by gently moving the walls of the viscus, the whole fluid would seem to be one seething, fermenting mass of wriggling, moving worms; whilst the body of the animal was very anrmic, the muscular tissue greatly wasted and very pallid, the fat fairly abundant, but presenting the very white appearance seen in liver-rot and other anæmic diseases.

The Strongylus contortus has been studied in Germany by Gerlach particularly, and the following is a brief resumé of his conclusions.

The Strongylus contortus occurs in the fourth stomach of the sheep and goat, and when present in large numbers causes clisease (Magenwürmerseuche). At the same tine, as was first noticed by Gerlach, the Strongylus filaria is usually present in the lungs. In the summer the Strongylus filaria is most abundant; in autumn the Strongylus filaria and Strongylus contortus are almost equally numerous; while in winter the Strongylus contortus prevails, and the Strongylus filaria has almost disappeared. Hence the stomach disease caused by Strongylus contortus breaks out in winter and spring in those lambs which have survived the lung disease of summer and autumn. ${ }^{1}$

These facts agree with what was observed at Hollybush, namely,-1st. The appearance of disease early in the autumn amongst the sheep. This would be lue to the lung disease cansed by Strongylus filaria. 2 2 . The recovery of many of the sheep affected when the diet was changed, i.e., when removed to a clean pasture, and $3 d$. The appearance of the disease later on in the season, in February 1884, and resulting from the Strongylus contortus, confirmed by the post mortem examinations, in one of which only a few Strongyli filarioe were found.

From this connection between these worms, Gerlach was led to make the following experiments:-Eggs of Strongylus filaria were given to healthy goats and lambs, and, four months after the feeding, Strongylus contortus was found to be present. From these experiments Gerlach concluded that Strongylus contortus

I The Strongylus contortus is prevalent in Jamaica in districts where animals are confined to "pond water," and is injurious to eattle and destructive to sheep and goats. 
could develop from the eggs of the Strongylus filaria, but opinions differ upon this point.

The name Strongylus contortus occurs first in the writings of Rudolphi, and the species was described from specimens found in the month of November in the fourth stomach of a lamb. Fabricius also found them in the intestine.

Description.-The body is cylindrical, red or white, and tapers very gradually towards the anterior extremity, where is the mouth (fig. 45), which is unprovicled with papillæ or other appendages, and leads into the muscular pharynx, rather more than $1 \mathrm{~mm}$. in length, in a worm $2.5 \mathrm{~mm}$. long.

The male is smaller than the female, and is from 10 to $16 \mathrm{~mm}$. in length, and $0.2 \mathrm{~mm}$. in diameter.

The terminal bursa consists of two elongated ovoid lobes, each of which is supported by five ribs, and attached to the left lobe is a smaller one, supported by two ribs. There are two spicules about $0.5 \mathrm{~mm}$. in length, each one tapering to a fine point, which is slightly curved ontwards. Under a high power each point is seen to be tipped by a small knob (fig. 38).

The female is from $18.30 \mathrm{~mm}$. in length, and $0.3 \mathrm{~mm}$. in diameter. The body tapers very suddenly to an extremely fine point, and about $3 \mathrm{~mm}$. in front of this is the genital opening, furnished with two papille, of which the left is much larger than the right (fig. 47). The uterus is convoluted, hence the name Contortus.

Symptoms.-The symptoms of the stomach cliscase caused by the Strongylus contortus are, according to Gerlach, not sufficiently marked to allow of a certain diagnosis. The animals are languid and dejected, and tend to become lean and anæmic; the excrement is thin and brown, mixed with slime, or at times with blood. At last cachexia occurs, and death ensues from starvation (Erschöpfung). On post mortem examination the fourth stomach is found with hundreds of the Strongylus contortus covering its surface.

Treatment.-Good food is recommended, e.g., roasted malt, seeds, lupine hay or lupine seeds, and the following medicines :Chabert's oil, 1 part.

Oil of turpentine, 3 parts.

The dose being one tea-spoonful per day.

Kamala has been recommended, and the picrate of potash is highly spoken of by Zurn. 
Rabe used picrate of potash in the small dose of $0 \cdot 12$ grms., or about 2 grains per day for three days, and was successful in his treatment. The picrate of potash was given in thick linseed mucilage.

The doses of picrate of potash as given by Zurn are-

For a lamb 0.30 grms. or about 5 grains.

For a sheep $1 \cdot 25, \quad$ or about 20 grains.

Divided into two doses per day.

It is now believed that the ova of these parasites, passed from the body of their host, retained their vitality in damp places only, and that where nice bites of green grass, such as that growing on the sides of open drains and damp spots in pastures otherwise dry, are sources of danger, the parasitic ova becoming ingested with such grasses. The prevention of the disease by the destruction of the worms should be attempted by first digging up, where possible, all such green grasses, and inverting the sods. 2d. By a liberal application of salt to the land, and, where possible, an allowance of it to the sheep.

During wet seasons the above precautions are impossible, and all that can be clone is to remove to the driest pastures, give corn where possible, and an allowance of common salt in the food.

\section{STRONGYLUS TETRACANTHUS.}

In March 1873, an Iceland pony rising two years old, which had been under my care for some weeks, died from what appeared to be an exhausting disease, and, as stated in a letter to Dr. Cobbold, there were no very marked signs of the presence of parasites during life. The appetite was good; there was no cough ; and we were led to suppose that there were worms by the absence of disease more than anything else. There was gradual emaciation, but no diarrhœa, and at no time were the frecs fotid. As bearing upon the discovery of this parasite, I may state that Dr. Knox, who wrote in the Edinburgh Heclieal and Surgical Journal for 1836, says-" On two occasions my friend Mr. Dick found parts of the large intestines of a horse presenting the following appearance: the gut being simply laid open so as to exhibit the mucous membrane, a number of dark spots are observed, evidently exterior to the inner membraue." And, further on, speaking of the worms, he adds: "they liave no cyst, and vary from the tenth of an inch to a length 
of at least seven-tenths. They have a digestive tube divided into numerous compartments or sacculated; a mouth unarmed."

Professor Dick's own statements, as communicated by Dr. Knox, are particularly interesting. He says - "I have found these worms in several horses, and at different stages of their growth, from the size of a pin-point to that of an inch and a half in length, and in two instances in the blood-vessels.

"They seem almost always to produce a bad form of diarrhœa, and seem to depend on the food or situation in which the animal has been previously kept. The horses I have found affected with these have always been running out previous to their becoming ill."

During the spring of 1874 I harl again the opportunity of studying the disease caused by this parasite, or rather parasites, for I am of opinion that there were at least two kinds present in the subjects examined by me, some being embedded in the intestinal walls and others in the intestinal canal, the latter being a reddish-looking worm, very similar to a small earthworm, from two to three inches in length. Those embecked in the intestinal walls, as well as the appearance of the intestines, are delineated in the following woodeuts.

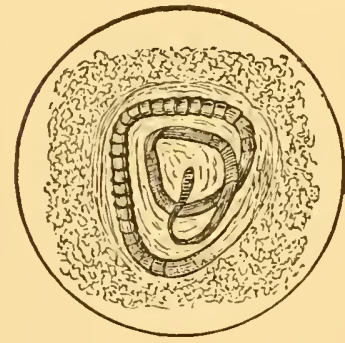

Fig. 48 .

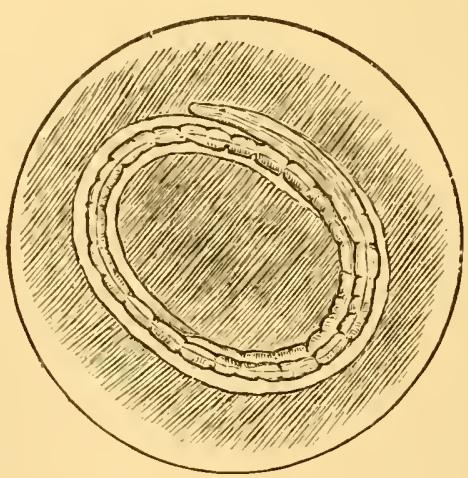

FIG, 49 .

Microscopic appearance of Strongylus tetracanthus embedded in mucous membrane of Iceland pony, described in the text. These worms were of a bright red colour. (About 100 diam.) 


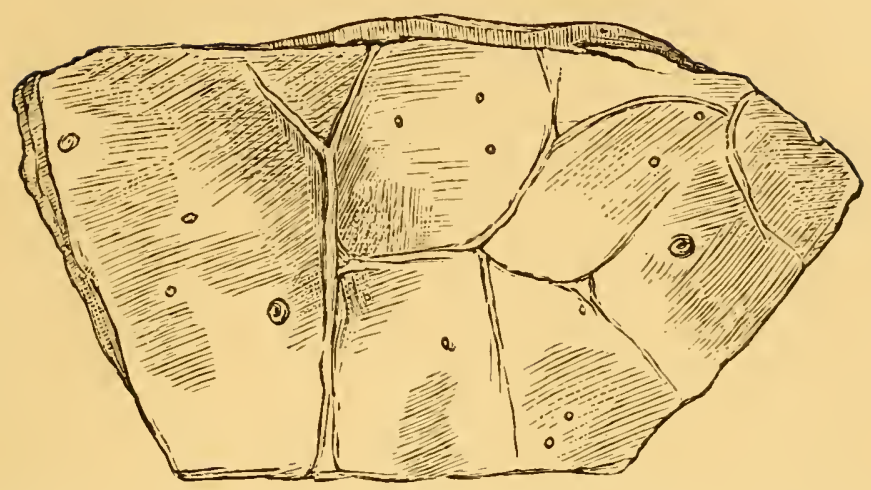

FiG. 50.-Appearance of mucous membrane of colon of Iceland pony, natural size.

I may state that all the ponies affected were rising two years old, and that several had died before my opinion was sought. They had been imported from Iceland during the summer and autumn of 1873 , and thriven tolerably well until late in the winter. They were at pasture during the whole winter and spring, being allowed hay in addition to what they could graze. There were many older animals amongst them, the lot being a large one, all of which were quite free from the parasites.

The question naturally arises, Are these parasites peculiar to Iceland? and at the first glance, my own experience would compel me to answer in the affirmative; but the observations of others at once point to the conclusion that other than Icelandic nonies are subject to the invasion, and Dr. Cobbold has arranged the following list of those who have written upon the subject:-

"Dick:-_Worms at different stages of growth;' in MS. to Dr. Knox, 1836.

"Knox.-'Animals similar to Trichina;' in Edin. Med. and Surg. Jou'n. (loc. cit.)

"Diesing._'Nematoideum equi caballi ;' Syst. Helm., vol. ii. 1851.

"Littler._' Extremely small ascarides;' in a letter to Mr Varnell; The Veterinarian, 1864, p. 202.

"Varnell.-'Entozoa in various stages of growth;' The Veterinarian, ut supra, p. 265.

'Williams._-'Entozoa from the intestinal walls;' in MS. to the author, Narch 13, 1873.

"Colboll.-Trichonema arcuata, a new species of nematode (with description as below). 
"Body spiudle-shaped, more narrowed posteriorly than in front; head truncate, with circular mouth, cup-shaped buccal cavity, and muscular œesophagus; tail of the male drawn out to a long fine point, that of the female being abruptly pointed; reproductive papilla of the female situated at the lower part of the upper third of the body.

"Size.-Males, $\frac{1}{10}$ to $\frac{1}{8}$ of an inch in length, by $\frac{1}{1 \frac{1}{30}}$ to $\frac{1}{120}$ in breadth; the females averaging $\frac{4}{10}$ of an inch in length by $\frac{1}{40}$ in lireadth."

The opinion that this was a new species of nematode was found to be a mistake, as even Cobbold had referred to it in 1873, and Küchenmeister in 1857.

Symptoms.--In the pony under observation in 1873 diarrhoea was absent throughout. Mr. Justus Littler, who was then a student with me, was able, however, to see some points of resemblance in the symptoms to those described by his father in the letter to Mr. Varnell, above mentioned. In the lot observed in 1874, diarrhoea of a foetid character, along with a more or less rapid ennaciation, was present in all the cases, and 1 conchnde that, in conjunction with the ages of the affected animals, diarrhoe and emaciation may be considered as diagnostic symptoms. Four of the $\mathbf{1 8 7 4}$ lot were so reduced as to be unable to stand; they were recumbent, very quiet, with pallid mucons membranes; the appetite was, however, good. The question again arises, Was the diarrhcea due to the presence of the larger worms in the intestinal canal? and one is led to conclude that, from the fact that none were discovered in the first case, this symptom arose from this cause, and that so long as the parasites were passive in the intestinal walls they did not cause superaction of the bowels, but as they became matured and burst through the tissue surrounding them, they irritated the intestines and caused purgation. In addition to the dark spots on the mucous nembrane of the colon, delineated in the woodcut, the intestinal glands were, in all the cases examined, more or less enlarged, many of them containing pus and what appeared to be the débris of the parasites. In every instance the dark spots, which in reality indicated the seat of the worms, were confined to the colon. An experiment was made with the flesh of the first pony upon some fowls, and, as stated by me in my letter to Dr. Cobbold, two of them died, with the livers and other viscera filled with parasitic ova and embryos. 
Mr. Cawthron of Hadlow has found these parasites enveloped in cysts of vegetable débris.

Treatment.-In the cases treated in 1874 we were successful in every instance by administering two-drachm doses of the oil of turpentine night and morning, along with eggs and milk well beaten up. The eggs and milk were administered, not only for the reason that they form a good vehicle for turpentine, but to afford nourishment and support to the debilitated animals.

This is a very interesting subject, requiring further investigation, and it may turn out that the larger worms are a more mature form of the smaller specimens, or that they are a distinct form altogether.

\section{TRICHINOSIS.}

The Trichinc spiralis is a minute round-worm, measuring about $\frac{1}{35}$ of an inch in length, originally found in the muscular tissue of pigs, eels, cats, dogs, badgers, hedgehogs, pigeons, poultry, moles, crows, vultures, \&c., and transmissible by ingestion of trichinosed flesh to other animals, and to man; but, according to some observers, carnivorous birds are exempt from the invasion of the parasite. The anterior extremity of the worm is rather pointed, its posterior thick and rounded; has immature sexual organs, and lies coiled up in an oval cyst. The cyst, which measures about $\frac{1}{\frac{1}{4}}$ of an inch in length, appears to be no essential part of the worm, but forms around it after it has taken up its location; the walls of the cyst are laminated, transparent, and thick, generally studded externally with calcareous matter.

The trichina cysts occupy the striated muscular tissue, and in some specimens it has been estimated that each cubic inch of the striped muscles of an infected hog may contain from thirteen to thirty-five thousand worms. The cysts appear in the muscles as minute white grains, visible to the naked eye, and with their long diameter corresponding to the direction of the muscular fibres. In a cat experimented upon by Leuckart each ounce of muscle was calculated to contain 325,000 trichinr; and Dr. Cobbold estimates that a man of medium bulk may easily hirbour 20,000,000. Dr. Bellfield and Mr. Atwood, at Chicago, fed a rat, weighing two ounces, with infected pork in small quantities every two or three days for six weeks. No impairment in the health of the rat resulted; it was then killed, and 
its body was found swarming with living trichinæ; the observers estimated there were no less than 100,000 in the whole carcase. They also found that rats may be fed occasionally with small numbers of trichinæ without in any way disturbing the health; and the inference drawn from these experiments has been, that any animal or man may take live trichinæ in small numbers occasionally without injury. These observers further believe that many more human beings than have been hitherto supposed are infected with trichinæ; indeed that the majority of us are carrying these worms in our muscles. So sure have they felt of this fact, that one of them ate twelve living trichina on 20 th November 1878 , and after more than three weeks had elapsed-when the ease was reported-not the slightest effect had been experienced. These gentlemen also discovered that small portions of sulphurous acid dissolved in the brine in which hams are pickled will kill the trichinæ. The per-centage of acid was not then fully determined, but the amount is so small that it is no detriment to the meat for commercial purposes.

This worm was first discovered by Professor Owen in 1835 in a piece of a man's muscle, which presented a peculiarly speckled appearance. These specks were found by Professor Owen-as had been previously shown in similar instances by Tiedman and Mr. Hilton-to consist of minute encysted entozoa, which he named Trichina spiralis owing to its hair-like and spirally coiled form; but until 1860 it was only known as a pathological curiosity. At that date, however, Zenker of Dresden showed that, however harmless the encysted parasite might be, the gravest symptoms, and even death itself, might be caused after its reception into the bowels, during the process of its reproduction which then ensued, and during that of the migration thence of the young trichinæ into the voluntary museles.

It is not yet determined how long the larval trichinæ retain their vitality, but there is no doubt that they may live encysted in the muscular tissue for many years, and retain life after the death and putrefaction of their host. They do, however, perish in situ sooner or later, and usually undergo calcareous change.

When the trichinæ capsules are swallowed, they are dissolved by the gastric juice, and the contained parasites set free; they then rapidly undergo clevelopment, and attain sexual maturity, 
the female acquiring a length of $\frac{1}{8}$ inch, the male $\frac{1}{15}$. The males are filled with sperm-corpuscles, and the females densely stocked with ova, which are hatched within the uterus, from whence the living embryos escape into the intestinal canal of the host, and at once commence active migration. They first attach themselves to the intestinal mucous membrane, eat through the intestinal walls, and find their way to the small vessels and lymphatics of the bowels, from which they are conveyed by the blood stream to all parts of the body. They have been found during this period in almost every part of the organism; in the intestinal walls; in the abdominal cavity, in the mesentery and mesenteric glands, in the comnective tissue, and, in an as yet unencapsuled condition, in the muscular tissue itself.

The immature trichinæ taken into the stomach become mature on the second day; on the sixth and following days, up to the end of the second or even third week, the embryos are born, and commence operations, and probably reach their destination in a week or two, and by the end of a month or a little more have come to the conclusion of their labours. Although the trichinæ fix their abode in the striped muscular tissue, they are rarely if ever found in that of the heart, but are often specially abundant in the muscles of the larynx.

The experiments of Dr. Bellfield and Mr. Atwood support the conclusions of previous observers, that the manifestations of disease in animals experimentally infected vary according to the number of trichinæ introduced; if these have been few in number, no ill consequences have resulted, but when more numerous, the disease has been of an aggravated or even fatal character.

Symptoms. - The symptoms of trichinosis in man, as concisely described in Tanner's Practice of Mcdicine, are as follows:"'The symptoms of trichiniasis vary in degree, being mild or severe according as only a few or many of the worms have been swallowed, as well as in proportion to the number of the progeny and the extent of their migrations. Thus, Dr. Althaus remarks that in the epidemic of Burg, near Magdeburg, a woman who had eaten a quantity of raw pork with bread, fell ill and died; her child, who had sucked a spoon used by the mother, suffered slightly and recovered.-According to the accounts given by most authors, the earliest symptoms are loss of appetite and 3 G 
general malaise; to which succeed nausea and retchiıg, prostration, diarrhœa, a sense of thorough indisposition, and a painful stiffness about the neck and arms and legs. This pain is due to the immigration of the young trichinæ into the muscles; and it is accompanied with high fever, and an œedematous swelling about the eyelids and face. The pulse is frequent, and there are copious offensive perspirations ; but although the temperature of the body is raised it does not reach the same height as in typhus and typhoid fever. For some days the stiffness of the limbs continues to increase; while all the muscles seem to be painful and swollen and very sensitive to the touch. The movements of the intercostal muscles in respiration are attended with suffering, so that repose is impossible; while there will be tronblesome hiccup if the diaphragm be invaded, with hoarseness and loss of voice where the laryngeal muscles get inhabited. Neuralgia of a very severe description, in the cœliac and mesenteric plexuses, has likewise been present in certain cases. When a large quantity of trichinous meat has been eaten, so that the immigration of the trichinæ into the muscles is great, the patient may lie almost paralyzed in a state of great exhaustion. The facial œedema generally lasts about a week, its disappearance being followed by swelling of the feet and legs, and ultimately of the trunk. There is no effusion, however, into any of the cavities; nor does the urine become albuminous, although it is always lessened in quantity and may be loaded with urates. About the beginning of the fourth week the patient is in a pitiable condition. The pulse and respirations are very frequent, the tongue is red and dry, the pain is severe, the sweating is profuse, the mouth can scarcely be opened, no sleep can be obtained, and there is great anxiety or delirium; death not unfrequently occurring with all the symptoms of profound exhaustion. Such complications as pneumonia, peritonitis, and pleurisy with effusion, are not uncommon. In favourable cases, however, the pain and swelling and diarrhœa abate; the oppression of the chest passes off; sleep is obtained; a desire for nourishing food is evinced; the power of the limbs is regained; and there is only left great anæmia, with a falling off of the hair, \&c. The parasites have taken up their abode in the muscles, and have fortunately become encysted."

It appears that the symptoms are much more severe in the 
human being than in the lower animals; and Dr. Cobbold mentions that a pig experimented upon at the London Veterinary College, and in which it was calculated that about sixteen millions of encysted trichinæ had been developed, manifested no symptoms of suffering; other pigs, however, showed much general disturbance and suffering, arising from the irritation of the worms in the intestines, and during their passage into the muscular tissues. The irritation of the alimentary canal, which lessens towards the end of the first week after pigs have swallowed trichinæ, is denoted by loss of appetite, vomiting, colic, tympanitis, and diarrhœa; dulness, arching of the back whilst standing; and pigs incline to lie down and hide themselves in their litter in this as in many other diseases. These symptoms, with the exception of the diarrhœa, generally disappear in from six to eight days. When the trichinæ have been numerous, the membraneous passage of a great number of them have, in the experience of Lenckart, induced in some a fatal peritonitis; and in others, a form of enteritis, with ejection of false membrane.

"The muscular symptoms appear towards the termination of the second or during the third week after the ingestion of the trichinosed flesh; while the intestinal phenomena become less marked, and the fever increases in intensity. At this stage the patient is often lying; in walking, it carries its back raised and the limbs stiff, and manifests signs of pain; mastication, opening the mouth, and swallowing are difficult; the voice is harsh, husky, and weak; the respirations laboured and loud; cutaneous œedema appears in different places, together with the intense pruritis already alluded to ; the conjunctival membrane is most frequently infected, and emaciation rapidly sets in. If the number of migratory trichinæ is very great, death may be the result, though this is somewhat rare in the pig. Usually the different symptoms disappear gradually, and it is only in very debilitated animals that convalescence is slow. The presence of trichinæ in the muscles does not appear to exert any subsequent influence on the development or fattening of the animals which harbour these strange creatures; on the contrary, it has been stated that they fatten more readily than before they were infested."-(Fleming's Sanitary Science and Police.) The determining test, however, is the discovery of the parasites in the intestinal discharges, or in fragments of muscular tissue extracted by a harpoon. 
The vitality of the trichinæ is not destroyed unless the meat in which they are contained is exposed to the influence of the heat of boiling water for a sufficient time to insure that every particle of it has been acted upon by that degree of heat. The mere toasting of ham or bacon is insufficient to destroy the worms, and smoked ham and German sausages are, unless well cooked, sources of danger. Many remedies have been suggested for the destruction of the trichinæ in the treatment of the disease, more particularly picric acid, picro-nitrate of potash and benzole, carbolic acid, sulpho-carbolate of soda, \&c., but none of these have been proved to have any effect; indeed, in trichinous pork of a pig killed with picric acid, the worms were found alive by W. Müller of Homburg. 


\section{CHAPTER LXXV.}

PARASITIC DISEASES-continucd.

\section{TREMATODA.}

\section{ROT IN SHEEP.}

Rot is a disease of low lands, marshy grounds, and wet seasons, and observers are agreed that the flooding of pastures suffices to taint them for a season, owing to the dissemination of the Distomata; for it is pretty satisfactorily proved that the ova of the fluke pass out through the intestines of the sheep, and fall on the pastures. The ova hatch in moist places, and become transformed into ciliated embryos, which, when set free, swim rapidly in the water, and thus spread over pastures in wet seasons.

Remarkable outbreaks of this disease have occurred in England. Professor Simonds, in his essay, records the outbreaks of $1809,1816,1824,1830,1853$, and 1860. "In the outbreak of 1853-54 many thousands of sheep were swept away, and not only in undrained districts, but also in others of a more healthy character. But since 1830, however, no outbreak can at all be compared to the one of the autumn of 1860. Speaking in general terms, it may be affirmed that all the western and southern counties of England, together with several of the eastern and midland, suffered to a ruinous extent. As in former years so in this, the attacks of the disease were due to an excess and long continuance of wet weather. 1860 will long be remembered by agriculturists, not only as producing the rot among sheep, but likewise for its baneful effects on the root crops, as also on the hay and corn harvests." 1872 again was a wet year, and rot was very prevalent, appearing on land that was considered sound during ordinary seasons. 
In conjunction with the Distomata in the liver, it is not an unusual thing to find other parasites infesting the bodies of sheep. Strongyles in the abomasum, intestines, and lungs, cysticerci, \&c.-indeed symptoms similar to those of rot-are sometimes present independent of the fluke, and this circumstance has led some erroneously to conclude that the debilitated condition of the sheep renders it a favourable habitat lor the parasite; that, in fact, the mal-condition induced by damp food is the cause of the disease and not the effect, hence it is termed watery cachexin, cachexia aquosa, by some writers.

It is important that this disease be distinguished in its earliest stages, and in order to enable the sheep-farmer to do this, the following rules are given by Mr. Beattie in the Transactions of the Ilighland and Agricultural Society of Scotland, vol. iii., 1807 :-

"The first thing to be observed is in the spring, when they are dropping their lambs. A sound ewe in good order drops a lamb covered with a thick and yellow slime, which the ewe licks off it, and the rule is, the sounder and the higher condition the ewe is in, the darker and thicker will be the slime; but when they observe a ewe drop a lamb covered with thin watery bubbles, and very white, they note her down as unsound.

"About the month of September, when they intend to dispose of their draught ewes, they put all their sheep into a fold, and draw them by the hand; that is, they catch them all, viz., the ewes they design to sell any of, and clapping their hand upon the small of the back, they rub the flesh backwards and forwards betwixt their fingers and thumb and the ends of the short ribs. If the flesh is solid and firm, they consider her as sound; if they find it soft and flabby, and if, when they rub it against the short ribs, it ripples, as we term it - that is, a sort of crackling is perceived-as if there was water or blubber in it, they are certain she is unsound. This is the most certain of all symptoms, but is not to be discerned with any degree of certainty but by an experienced hand; for although, as I have here related it, it seems a very simple affair, and easily acquired, yet it is well known that many shepherds, who have followed sheep all their lives, never arrive at anything like certainty in judging by the hand, whilst men of superior skill will seldom be mistaken, and will draw by no other rule. Yet still it must be acknow- 
ledged that the seeds of this disease will sometimes lie so occult as to baffle all skill, and that no man can, with absolute certainty, draw a flock tainted with the rot. There is another method, to which men of inferior skill resort, which is more easily acquired. They take the sheep's head between their hands, and press down the eyelids; they thereby make the sheep turn its eyeball, so that they get a view of the vessels in which the eyeball rolls. If these are thin, red, and free of matter, they consider the sheep as sound; but if they are thick, of a dead white colour, and seem as if there was some white matter in them, they are confident she is rotten. This is a pretty general rule, and easily discerned; but I think it is not so certain as when they are judged by the back; for in firm heathy lands the eye of a sheep is far redcler than it is in sheep upon grassy lands; and in some boggy lands, the eye is never very red, be the sheep ever so sound, so that there you cannot so well judge by the eye; but when you see the eye of a sheep a good deal whiter and thicker, and more matter in it (I mean the vessels in which the eyeball rolls) than the run of the flock amongst which it feeds, you have reason to suspect it is not sound."

In some instances the progress of the rot is very rapid, but usually it is slow and insidious. At first the affected animals appear to thrive very fast, but inactivity and dulness supervene; the mucous membranes become pallid, the flesh wastes, the general surface of the skin loses its ruddy colour, becomes dry and devoid of that oily condition which is natural to the fleece of the sheep. As the disease progresses, the flanks become hollow, the back rigid, weak and tencler about the loins, as evinced by wincing when this part is pressed by the hand, and the spine sticks out prominently; the fleece drops off in patches, the belly enlarges, the eyes become yellow, and dropsical swellings appear in different parts of the body, particularly around the throat. There is often much thirst, depravity of the appetite, diarrhœa, general stupor, the pulse is weak, the heart's action tumultuous, and anæmic murmurs are heard. As demonstrated by Delafond and others, the blood is deficient in albumen, thin, watery, and on this account the serum transudes through the walls of the vessels, collects in the loose areolar tissue of the depending parts of the body and in the cavities, constituting the condition of dropsy which is seen in rot. 
Professor Simonds states that a dry scaly state of the skin on the inner parts of the thighs, particularly where it is uncovered with either wool or hair, is early recognised, and that an examination of the eye will materially assist in determining the question of disease. "If the lids are everted, the membrana nictitans being pressed forward, it will be found that in the early stages of the malady, and especially if the animal has been excited by being driven a short distance, the vessels of the conjunctiva are tinged with a pale or yellowish-coloured blood, and that the whole part has a moist or watery appearance. Later on the same vessels are blanched, and scarcely to be recognised, excepting perhaps one or two, which present a similar watery condition, or are turgid with dark-coloured blood." In some cases these symptoms are complicated by others, induced by strongyles in the air passages and alimentary canal.

\section{PATHOLOGICAL ANATOMY.}

The tissues of the body are generally wasted, flabby, pale or yellow, and watery; there is an absence of the firmness and colour of healthy mutton. The peritoneal cavity contains a more or less abundant quantity of serum, which may be of a clear straw colour, or more or less yellow, in which occasionally fragments of lymph and false membranes are floating; the digestive organs are remarkably blanched; the liver is hard, scirrhous, irregularly knotted on its surface and margins, and sometimes united by false membranes to the surrounding organs. In colour it is either a dirty chocolate brown, deeper in some parts than others, or has a yellowish tint, intermixed with pale yellow spots. Flukes are found in the bile ducts, which are filled with a dark thick secretion; on further examination the ducts are found sacculated at various points, the distended portions generally containing many flukes massed together. The canal walls are much thickened in some places, and coated with calcareous matter on their internal surface. Professor Simonds mentions a case where a concretion was found as large as a hen's egg, which, when broken up, was found to contain about a dozen dead flukes. He also states that "the coats of the ductus hepaticus, as also of the ductus communis choledicus, are not unfrequently so thick as to be upwards of ten times their normal 
substance, and likewise as hard as to approach the nature of cartilage."

Respecting the number of the flukes, the greatest variation exists. Dr Cobbold says- "The presence of a few flukes is totally insufficient to cause death; consequently, when a sheep clies from rot or is liilled at a time when the disease has seriously impoverished the animal, then we are sure to find the organ occupied by many dozen, many score, or even several hundred flukes. Thus from a single liver Bidlow obtained 800, Leuwenhoeck about 900, and Dupuy upwards of 1000 specimens. Even the occurrence of large numbers only destroys the animal by slow degrees, and possibly without producing much physical suffering, excepting perhaps in the later stages. Associated with the above described appearances, one not unfrequently finds a few flukes in the intestinal canal, whilst a still more interesting pathological feature is seen in the fact that the bile contained in the liver ducts is loaded with flukes' eggs. In some cases there cannot be less than tens or even hundreds of thousands. Not a few may also be found in the intestinal canal, and in the excreta about to be voided." And lastly, says the same author, "it need scarcely be added that it is by no means unfrequent to find one or even several other kinds of entozoa coexisting with the fasciola in the same sheep, the most common form being that of the larval echinococcus."

\section{TREATMENT.}

All observers agree that the growth and development of the fluke is impossible on dry land, and that the disease is unheard of on dry pastures except during wet seasons, and as the "rot," once established, is difficult to overcome, it behoves the flockmaster to pay every attention to its prevention. For this purpose it is necessary that unsound sheep pastures should, after being drained, for a time at least, be applied to other purposes.

During moist seasons, and when there is a fear of the disease appearing on sound pastures, the natural food should be supplemented by cakes, corn, beans, or other nutritious diet, in addition to common salt and the sulphate of iron.

As to the beneficial effect of common salt, all writers are agreed that it prevents the development of the parasites, for it 
is tolerably certain, as mentioned by Dr. Cobbold, that the larvæ of the Fasciola hepatica exists only in the bodies of fresh-water snails and small aquatic animalcules. Seeing this is the case, and that salt marshes are exempt from the invasion of the fasciola, would not the application of common salt to the land be worthy of a trial.

For sheep that are actually affected but little can be done, at least in the way of a permanent cure. If the disease be slight, its progress may be retarded, and the animals made fit for the butcher, by removing them to a dry pasture, supplying them liberally with food of a highly nutritious nature, and administering salines and tonics. 


\section{CHAPTER L I X VI.}

\section{PARASITIC DISEASES-continued.}

CESTODA.

Trie most important disease of stock caused by this order of parasites is that variously termed

\section{STURDY, TURNSICK, OR GID.}

This is a species of vertigo induced by the Conurus cerebralis, which is the hydatid or larval form of the tape-worm, called the Tonia conurus. Many conclusive experiments have been made in this and other countries, recorded in works on helminthology, which proves that the mature Tonia conurus infests the intestines of the dog, from which perfect segments are expelled. These segments, falling on the pastures where sheep are feeding, are swallowed along with the herbage, and, as explained by Dr. Cobbold, the six-hooked embryos, escaped from the ova contained in the segments, commence their wandering in the tissues of the higher animal, and, by virtue of their selective capacity, escape from the blood-vessels of the brain, and bore their way to their final resting-place in that organ. Even when the brain is reached, it by no means follows that every embryo attains its full growth. Indeed, it is only usual for one to flourish, sometimes two of equal size, but seldom more. It has, however, been observed that when one hydatid cyst has been removed, that another has developed in a different part of the brain. This proves that the brain serves as a proper habitat to one or at most to two hydatids at a time.

This disease attacks cattle as well as sheep, but in this country it seldom attacks any but sheep, its victims being lambs under 
one year old, sheep above two years old being rarely affected. It prevails to a great extent on unenclosed lands, and for the reason that shepherds have to be assisted by dogs, whilst on enclosed pastures, and where sheep are unattended by dogs, the disease is scarcely known.

Symptoms.-The usual form of sturdy is that due to the location of a hydatid in one of the cerebral hemispleres. The sheep so affected, when caused to move, turns from right to left, or left to right, as the case may be; but when the parasite is lodged between the hemispheres, the animal steps high, and goes forward in a straight line; the head is then carried upwards, and there may be a varying degree of amaurosis in one or both eyes. Sometimes the animal becomes both blind and deaf, and is unable to follow its companions. When the hydatid is lodged in the cerebellum, the animal's movements are performed without control. The head is elevated; the limbs are moved with difficulty and automatically; one or two steps are taken forward, when the animal starts with a bound, but immediately falls, and is unable to rise for a time.

When the animal is first affected the symptoms are generally severe, from the congestion and irritation; as the contents of the skull adapt themselves to the hydatid, the brain symptoms subside more or less; but as the parasite grows, the symptoms become more severe, until paralysis is induced, and the animal can no longer stand.

As many tumours and hydatids of different species are found in the brain which do not induce symptoms of sturdy, Davaine believes that the nervous substance is irritated by the heads of the parasite, which protrude from the bladder, and by means of their hooks and sucking dises penetrate the brain substance nearly two lines in depth. As the cœnurus grows, the animal becomes more and more emaciated, and death occurs, unless the sheep be relieved naturally or artificially. The natural method is by the bones of the skull becoming absorbed, the skin accidentally broken, and evacuation of the hydatid. This is, however, very rare.

The bones of the skull, however, are generally softened, and if in about three weeks after the first manifestation of the symptoms the head be pressed with the thumb, a remarkable degree of softening will be found at one part of it, as if the skull 
were wanting in that particular spot. Into this softened spot a trocar and cannula are introduced, and after the trocar is withdrawn-leaving in the cannula-a syringe is applied to the latter, and the bladder extracted.

"The prevention of the hydatid disease," says a writer in the Agricultural Gazctte, "must begin at its source, and the first step is to expel the tape-worm from the intestines of the dog or dogs which are employed in the farm. It may be objected that this measure will be applied too late to remedy the mischief if the existence of the tape-worm is not suspected until some of the flock become affected with "gid;" but in fact the existence of tape-worm should always be suspected, and dogs should be treated periodically with areca nut as a measure of precaution. A dose of half a drachm to two drachms of the grated nut may be given now and then with safety, even when the tape-worm segments are observed, and if the remedy is not followed by the expulsion of a worm, the animal may be deemed secure. A second important means of prevention is to prevent the infection of dogs by destroying bladder worms immediately on their removal, instead of throwing them either to the dogs or in places where the animals can easily discover them. If this plan were universally adopted cystic entozoa would soon be diminished in number, and one source of loss to the stock-owner be averted. Sheep suffer from the entrance of embryos, which are expelled from the intestines of the dogs. Dogs become infested with the tape-worm in consequence of introduction into their digestive organs of the larval forms of the parasites, which exist in the brain of the sheep, being given to them, or placed within their reach. This interchange can be prevented by the exercise of a little common care.

"Sheep which are pastured on common lands, or fields through which a right of way exists, are exposed to risks of various kinds from which animals in isolated positions are exempt, and under such circumstances it is impossible to apply any effectual measures of prevention. In the case of "gid" some amount of danger is incurred from wild animals; foxes, for instance, may harbour the Tonia conurus, and a few sheep may here and there become infested with bladder worms from eating the eggs expelled from the intestines of those animals, but these causes of infection are occasional and remote, and do not materially affect 
the main question. Of the method of prevention, if the Tcenia conurus were to be expelled from every dog in the country, and effectually destroyed, the disease " gid," among sheep, would be very rare indeed.

"In reference to the treatment of the disease we have nothing to urge. Puncture or removal of the cyst in many cases alleviates the distress which the animal suffers, but permanent cure is rare, and, knowing this, the farmer wisely consigns the animal to the butcher. He should, however, always bargain for the return of the head, which contains the parasite, in order that he may burn, bury, or otherwise destray it, and thus render thousands of embryos inert."

The various other diseases caused by tæniæ, in different stages of development, and other parasites, almost too numerous to mention, are not intended to be described in the present work; helminthology being a specialty upon which many works have been written. In connection with this I may refer to one small work, namely, The Internal Parasites of our Domesticated Animals, by Dr. Cobbold, as being the only book which deals exclusively with the entozoa of interest to the veterinarian. Under the able direction of Dr. Tommasi, an Italian edition of this manual has been published at Florence.

I may, however, state that I have found areca nut, with the etherial extract of the male shield fern, to be the most certain remedy for the expulsion of tape-worms, a tabular arrangement of which is given on the following page.

The drawings of the parasites have been mainly taken from Zurn, also from Heller, Krabbe, Ieuckart, Cobbold, Kuchenmeister, and others. 


\section{Tabular Arrangement of Entozoa.}

\section{N. O. Nematoda.}

Genus Ascaris.

(a.) A. megaloceplata of horse and ass; found in the small intestine, oceasionally passing from the duodenum to the gall ducts.

(7.) 'A. lumbricoides of man, pig, cattle; found in small intestine. The Ascaris of the pig is sometimes reckoned as a different species, viz., $A$. suilla.

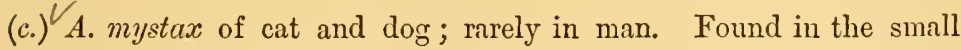
intestine. The Asccris of the dog is sometimes reekoned as a different speeies, viz, $A$. murginata.

\section{Genus Eustrongylus.}

(a.) E. gigas of dog, horse, eattle, and very rarely man; found in the kidneys, bladder, and areolar tissue, beneath the peritoneum; sometimes free in the peritoneal cavity; also in the heart of the dog.

\section{Genus Filaria.}

Species (a.) F. lachrymalis of horse and ox; found in the lachrymal duets.

99

(b.) F. papillosa of the horse, ox, and ass; found in the globe of the eye, said to be in the anterior chamber, but usually in a cyst within the cornea, also in the peritoneal and thoracic eavities, in the diaphragm and abdominal muscles, and in the araehnoid membrane of the brain.

" (c.) F. immitis of the dog; found in heart, pulmonary arteries, and blood, henee spoken of as Hematozoa.

(d.) F. trispinulosa of the $\operatorname{dog}$; found by Gescheidt in the capsule of the crystalline lens.

Genus Spiroptera (Filaria of some authors).

Species (a.) S. megastoma of the horse; found in tumours usually at the cardiác end, more rarely at the pyloric end of stomach; when these tumours are pressed pus and bundles of the worms are obtained. S. microstomu of the stomach of the horse is considered as a large variety of S. megastoma.

" (b.) S. sanguinolinta of the dog and wolf; found in tumours of the cesophagus and stomach. 
Species (e.) S. strongylina of the pig, \&c.; found in the stomach.

$n$

(d.) S. scutata resophagea bovis of ox, also described in the horse ; found in the mucous membrane of cesophagus. Worms supposed to be the same are found in tongue of pig and œsophagus of sheep.

(e.) S. hamulosa of the common fowl.

$"$

(f.) S. cincinnata (Onchocerca reticulata) of horse; found in the foot ligaments wound round the elastic fibres.

Larval forms of nematodes are found in tumours in the sub-mucous layers of the wall of the alimentary canal.

\section{Genus Oxynu'is.}

Species $(a .)^{2} O$. curvula of the horse and ass; found very frequently in the cæeum and colon.

$"$

(b.) O. vermicularis of man; found in the large intestine and rectum.

Genus Dochmius (Strongylus of some authors).

Species (a.) D. hypostomus of the deep, goat, and other ruminants; found in the intestine.

" (b.) D. tubceformis of the cat; found in the dnodenum.

" (c.) D. trigonoceplaalus of the $\log$; found in the stomach and small intestine. A rariety is declared to exist in the right side of the heart.

" (d.) D. cernuns of shcep ; found in small and large intestine.

" (e.) D. duodenalis (Anchylostoma duodenale) of man; found in duodenum.

\section{Genus Strongylus.}

Species (a.) S. armatus of horse; sexually mature adults found in crecum and colon, seldom in the duodenum, in the pancreas and tunica vaginalis of testis; the larval forms in aneurisms in the intestinal arteries, \&c.; the eggs come out with the excrements and develop free living larval forms in water; the free living larvæ get into alimentary canal with the water, and thence into the blood-vessels, where they cause aneurisms; becoming sexually mature they leave the aneurisms, and bore their way into the alimentary canal, where they copulate.

(b.) S. tetracanthus of horse; found in small intestine and cæcum; the larval forms are free under the epithelium or enclosed in capsules in the mucous membrane of 
the large intestine, where they attain sexual maturity, and then leave the mucous membrane and enter the alimentary canal.

Species (c.) S. dentatus of pig; found in large intestine.

" (d.) S. syngamus, from the trachea and bronchial tubes of fowls.

" (e.) S. radiatus of the ox, and several other ruminants. From the small intestine and colon.

$" \quad(f$.$) S. venulosus of the goat; found in the small intestine.$

$" \quad(g$.$) S. micrurus of cattle, horse, and ass; found in aneu-$ risms of the arteries of the cow, and in the trachea and bronchial tubes. In the tough, yellow mucus of the air passages and bronchi they are to be found in countless numbers.

Species (h.) S. filaria of sheep and goat, camel, \&c. ; found in the trachea, bronchial tubes, and lung-parenchyma. The ripe worms are coughed up and die. From the bodies of the dead females the embryos come forth. When they get into water they develop further, and live as free ner todes (or possibly as parasites on an intermediate host). These gain access to the stomach of the sheep with the water, wander into the lungs, and encyst in the parenchyma. When mature they leave the parenchyma for the bronchi. The forms described as Nematoideum ovis pulmonale are considered as embryos of S. filaria.

(i.) S. paradoxus of the pig; found in the trachea and bronchial tubes.

(k.) S. filicollis of the sheep; met with in the small intestine.

(l.) S. ventricosus of cattle; found in small intestine.

(m.) S. inflatus of cattle, rare; found in colon.

(n.) S. contortus of sheep and goat; found in the abomasum, usually associated with S. filaria.

Stephanurus dentatus of pig; found in or about the kidneys. The kidney-worm of the Americans; Leuck. art's Sclerostoma pinguicola.

\section{Genus Trichina.}

Species (a.) T. spiralis; sexually mature adults in alimentary canal producing living young, which bore their way into the muscles and encyst, forming the immature larval T. spiralis or Muscle Trichina; found in man, pig, ox, rabbit, rat, \&c. 


\section{Genus Trichocephalus.}

Species (a.) T. dispar of man.

"(b.) T. affinis of sheep and goat; rare in cattle; found in cæcum.

" (c.) T. depressiusculus of the dog; found in the cæcum.

" (r.) T. crenatus of the pig and wild boar; found in the large intestine.

\section{N. O. Trematoda.}

Genus Distoma (Fasciola of some authors).

(1.) Distoma hepaticum.-Leaf-like form, with a small sucker round the month, and a little behind this a ventral sucker, also small. Habitat, gall ducts and gall-bladder (usually only in spring) of sheep, cattle, goat, and pig. Rarely in horse, ass, cat, and very rare in man.

(2.) Distoma lanceolatum.-Lance-like form, with a tolerably large ventral sucker. Habitat, gall ducts and gall bladder of sheep, cattle, goat, pig. Wandering specimens also in blood-vessels and heart.

(3.) Distoma campanulatum, according to Ercolani, occurs in the liver of the dog.

(4.) Distoma conjunctum, found in the bile ducts of Indian dogs.

(5.) Distoma species have been found in muscles of pig, more particularly in the diaphragm.

\section{Genus Amphistoma.}

(1.) Amphistoma conicum, cone-shaped, thick posteriorly, with a large sucker at the posterior end. Habitat, paunch of cattle.

(2.) Amphistoma truncatum of cat.

\section{Genus Hemistoma-Holostoma.}

$\checkmark$ (1.) Hemistoma alatum of dog, wolf, and fox; found in small intestine.

(2.) Hemistoma cordatum of cat.

\section{Genus Gastrodiscus.}

(1.) Gastrodiscus polymastos, a dise-shaped trematode found by Dr. Sonsino in Egyptian horses. 
N. O. Cestoda.

(Fam. 1.) Taniadae.

\begin{tabular}{|c|c|c|c|}
\hline MATORE WORMS. & Habitat. & $\begin{array}{l}\text { Larval, Crstic, or } \\
\text { Hrdatid Stage. }\end{array}$ & Coymon Habitat. \\
\hline 1. Tænia m & Intestines of man, & 8 & \\
\hline 2. Trenia solium, & Do. of do. & Cysticercus cellulosæ, & Do. of pigs. \\
\hline 3. Tænia serrata, · & of dog, & Cysticercus pisiformis, & $\begin{array}{l}\text { Entrails of hares aucl } \\
\text { rabbits. }\end{array}$ \\
\hline 4. Tænia cucumerina, . & of do. & $\begin{array}{l}\text { Cysticercus cucumeri- } \\
\text { nus. }\end{array}$ & $\begin{array}{l}\text { Body of dog-Iouse-- } \\
\text { Trichodectes latus. }\end{array}$ \\
\hline 6. Tænia cœnurus, . . & of do. & Cœenurus cerebralis, . & Brain of herbivora. \\
\hline $\begin{array}{l}\text { 6. Tænia echinococcus or } \\
\text { Hydatid tapeworm. }\end{array}$ & Do. & $\begin{array}{l}\text { Echinococcus veterino- } \\
\text { rum. }\end{array}$ & $\begin{array}{l}\text { So-called bladder worms, } \\
\text { in liver, heart, lungs, } \\
\text { bones, \&c. }\end{array}$ \\
\hline 7. Tænia marginata, & $\begin{array}{l}\text { Do. of do., } \\
\text { wolf, \&c. }\end{array}$ & $\begin{array}{l}\text { Cysticercus tenuicollis, } \\
\text { or slender-necked hy- } \\
\text { datid. }\end{array}$ & $\begin{array}{l}\text { Liver, walls of bile ducts, } \\
\text { mesentery, pleur, } \\
\text { pericardium, diaph- } \\
\text { ragm, \&c., of sheep } \\
\text { and pigs. }\end{array}$ \\
\hline 8. Tænia crassicolis, & of cat, & Cysticercus fasciolaris, & Liver of rat and mouse. \\
\hline
\end{tabular}

\section{Tcenia whose Cystic forms are unknown.}

1. Tænia nana of man.

2. Tænia expansa of ox, sheep, gazelle, chamois, \&c.

3. Tænia denticulata of ox.-France and Germany.

4. Tænia plicata of small intestines and stomach of horse.

5. Tænia mamillana; large intestines of horse.

6. Tænia perfoliata. Cæcum, and sometimes small intestines of horse.

7. Tænia elliptica of cat. Said by Van Beneden to be same as T. cucrimerina of $\log$.

8. Tænia infundibuliformis,

9. Tænia proglotina,

10. Tænia crassula,

11. Tænia malleus,

12. Tænia lanceolata,

13. Tænia setigera,

14. Tænia sinuosa,

15. Tænia fasciata,

Common fowl, water fowl, pigeon, and other domestic birds.

(Fam. 2). Bothriocephalidee or Pitheaded Tape-Worms.

Bothriocephalus latus. Intestines of dog.

Bothriocephalus cordatus.

Bothriocephalus fuscus.

Bothriocephalus reticulatus.

Bothriocephalus dubius. 




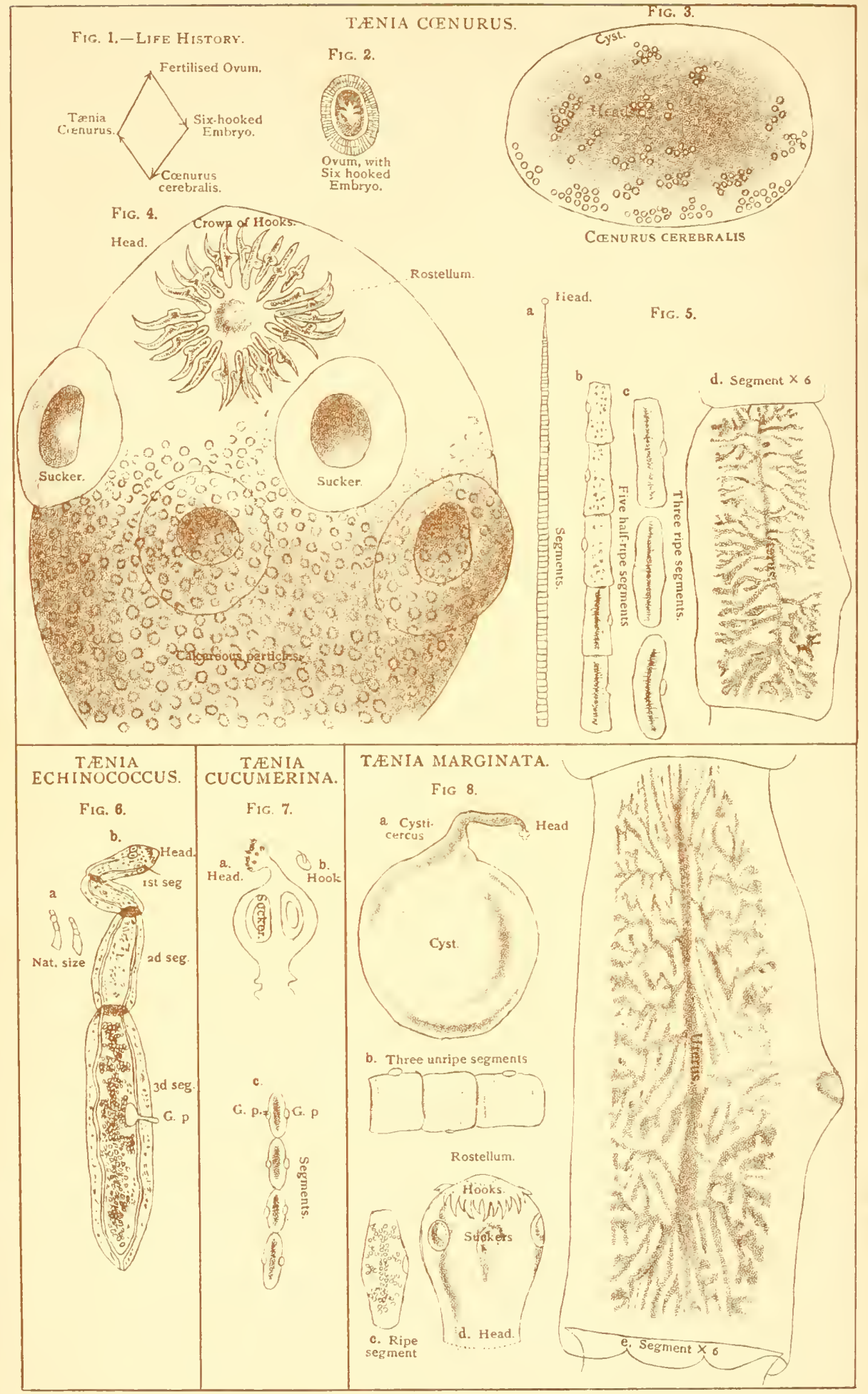


Tenia Cemurus.

\section{PLATE VII.}

Fig. 1.-Life History. Ovum, six-hooked embryo, Canurus cerebralis (sheep), Tonia ccenurus (dog).

Fig. 2.-Six-hooked embryo, surrounded by shell of ovum, highly magnified. Notice shape of ovum; the shell consists of layers, each layer composed of little prisms cemented together.

Fia. 3.-The non-sexual cystic stage, Coenurus cerebralis. Notice the numerous heads or scolices with which the cyst is provided; hence the name coenurus.

FIG. 4.--Head or scolex magnified 350 diameters. Notice rostellum and the crown of hooks, twenty-seven in number (usually twenty-eight), arranged in two rings. Four oval suckers, two being seen through the head. The calcareous particles are very numerous, and are often mistaken for ova.

FIG. 5.-Tcenia ccenurus: a shows the young anterior part of the worm (natural size). A complete worm may have as many as 220 segments. $b$ and $c$ show the segments as they ripen. Observe how the uterus gains in conspicuousness as the segments ripen. Notice also the genital pore on the margin of each segment, and their alteration in form as they become older. $d$. Segment magnified to show the uterus.

\section{Tenia Echinococcus.}

Fig. 6. -The sexually mature tænia- $a$. natural size; $b$. magnified. Notice the small size of the worm, the three segments, and the head provided with hooks. G. p., genital pore.

\section{Temia Cucumerina.}

FIG. 7.-Head and segments of the sexually mature tænia.

a. Notice the protrusible rostellum of the head. Only two of the four suckers are seen (highly magnified).

$b$. Hook attached to the middle of a disc.

c. Four segments (natural size). Notice the rounded ends, giving the segment the appearance of a cucumber; hence the name cucumerina. Also that each segment is provided with a pair of genital pores (G. p.)

\section{Tenia Mirginata.}

Fig. 8. - a. The Cysticercus temicollis. Notice the round cyst, the head evaginated, and the long thin neck; hence the name tenuicollis. $b$, c. Notice the alteration in the form of the segments. $d$. Head with suckers and hooks. e. Segment magnified, showing uterus. 
Tania Serrata.

\section{PLATE VIII.}

FIG 1.- $a$. Six-hooked embryo, surrounded by egg-shell.

b. Cysticereus, with head evaginated, called pisiformis from its pea-like form (nat. size).

c. Part of the cysticereus magnified head invaginated, provided with suckers and hooks. Numerous calcareous particles are seen.

d. Notice the saw-like margin of the segments, hence the name serrata.

e. Shows how the old segments alter in form.

$f$. Segment magnified to show the uterus.

Thenia Solium.

FIG. 2.- $a$. Cysticereus with head protruding.

b. Head magnified, showing the suckers and hooks.

c. Ripe segment, magnified 6 diameters

Thenia Perfoliata.

FIG. 3.-Observe the short but broad segments lying together, like the leaves of a book; hence the name perfoliata. Notice the tapering posterior end, showing that it is a young worm.

Tania Expansa.

FIG. 4.--Observe that each segment is provided with two genital pores like $T$. cucumerina, but that the segments have quite a different form.

Tania Crasicicollis.

FIG. 5.--Segment magnified.

Bothriocephalus Lates.

Fig. 6.-Orum highly magnified. Note the shape and observe the shell provided with a lid for the escape of the eiliated embryo.

FiG. 7.-The ciliated embryo, with a six-hooked embryo formed in its interior.

Fig. 8. -The six-hooked embryo casting off the ciliated coat.

FIG. 9.-The head. Observe $(b$. $)$ one long groove or pit in the side of the head; the second groove not shown; $(c$.$) a section$ of the head showing both grooves.

Fig. 10. Segment magnified to show the rosette-like uterus.

FIg. 11.-Shows the ventral genital pore. In a tape-worm the genital pore is margimal. 


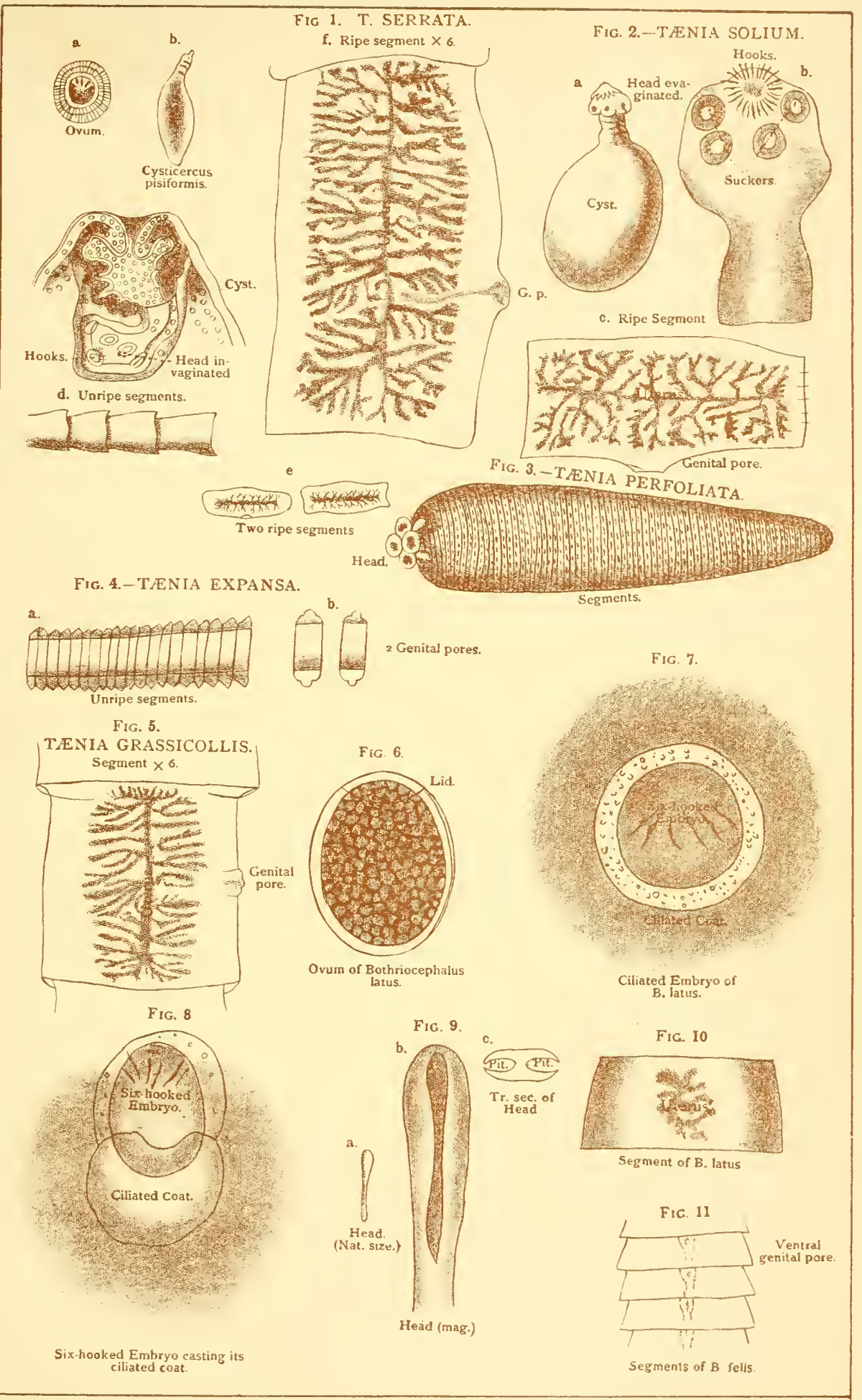


PLATE IX.

Fig. 1.

Head. ASCARIS MEGALOCEPHALA.

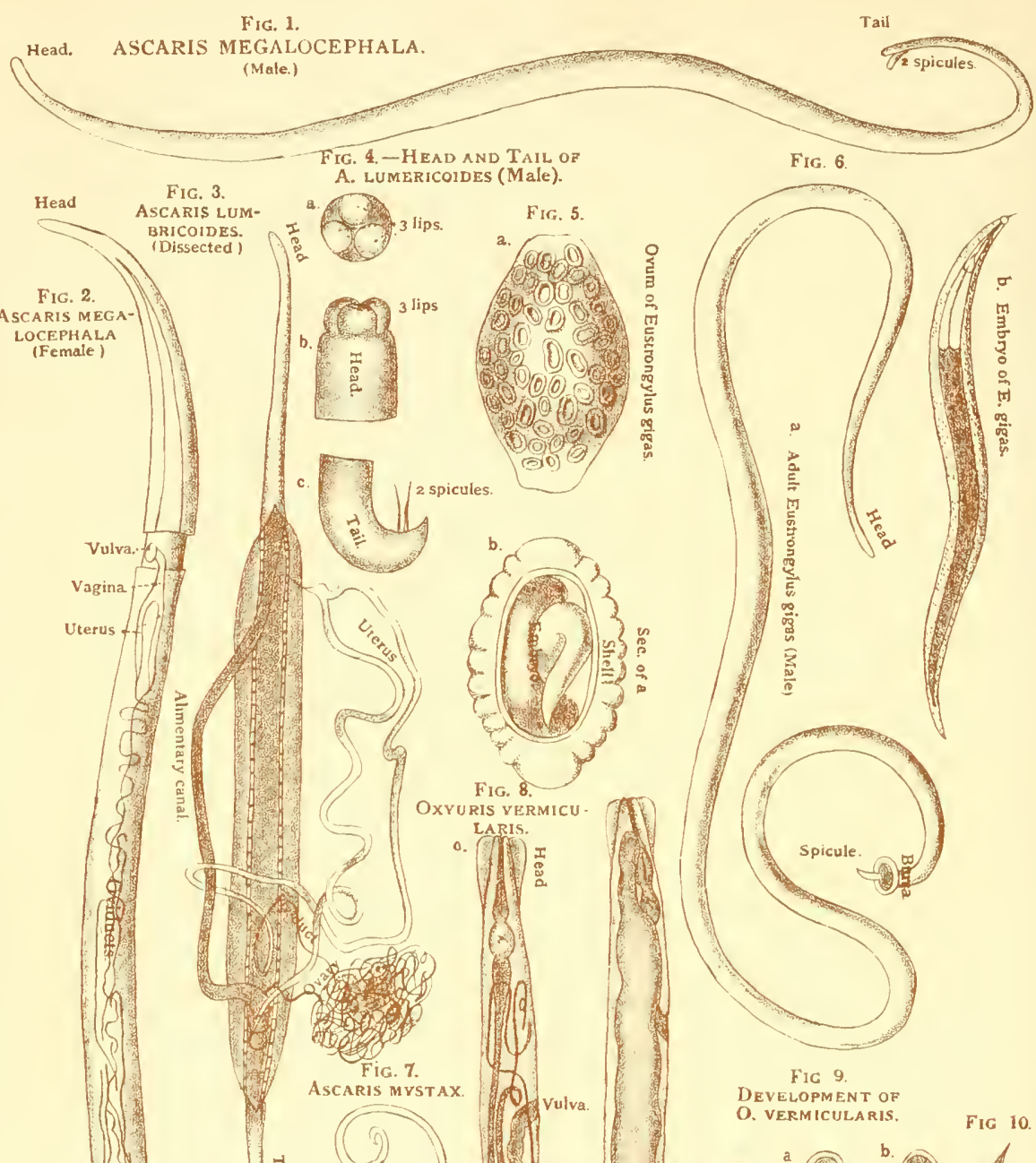

FIC 9.

DeVElopment OF

O. VERMICULARIS,

FIG 10
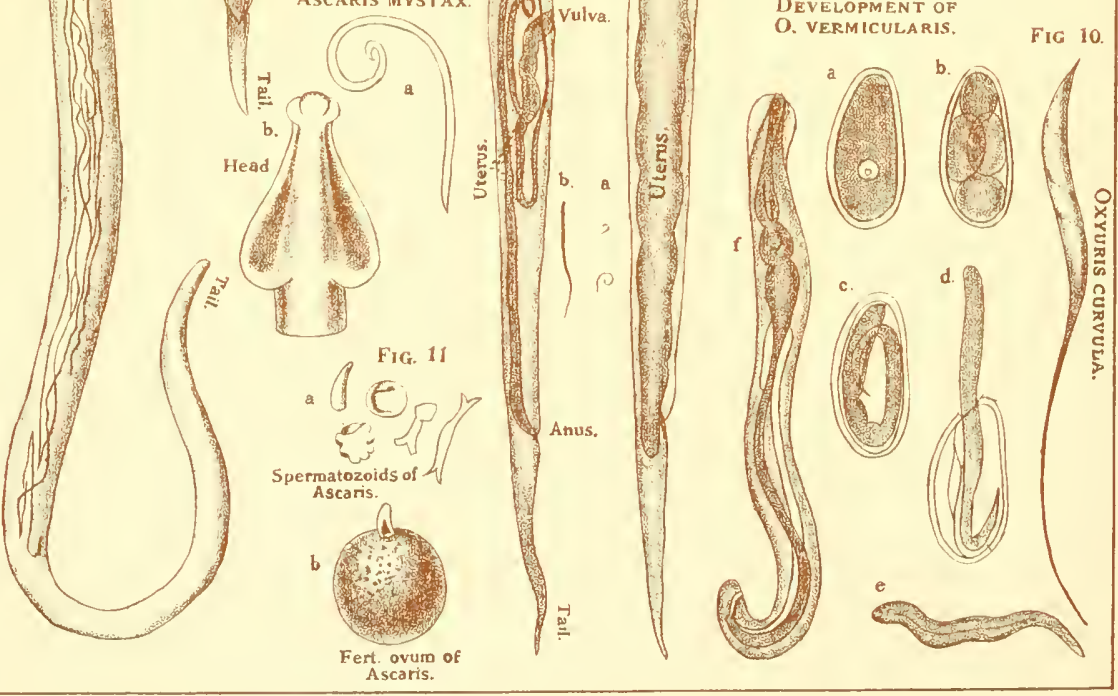


\section{PLATE IX.}

Ascaris Megalocephala.

Figs. 1, 2.-Male and female worms (nat. size).

Ascaris Lumbricoides.

FIG. 3.-Dissection of a female, with the parts laid out.

Fig. 4.- $a, b$. Head; c. Tail with spicules.

\section{Eustrongyuus Gigas.}

FIG. 5.-The orum; $a$ shows the form of the shell and the markings on its surface; $b$. is a section of the ovum, showing the thick shell with the embryo eoiled up in the interior.

Fig. 6.-a. Male worm (nat. size); $b$. Embryo worm.

Ascaris Mrstax.

FIG. 7.- a. Natural size ; $b$. Heart-shaped head (mag.)

OxyURis Vermicularis.

FIG. S.-a. Male (nat. size).

b. Female (nat. size).

c. Female (mag.) showing the internal organs.

d. Female (mag.) loaded with eggs, so that now the conspicuous part is the swollen uterus.

FIG. 9.-a. Ovum (mag.) ; b. Division has commenced; c. Embryo forming; $d$. Embryo emerging from the egg shell: $e$. Embryo free from the egg ; $f$. Further advanced.

Oxyuris Corvula.-Fig. 10.

Fertilisation of Ascailis.

Fig. 11.-a. Amœboid spermatozoids.

b. Impregnation of the ovues. 


\section{PLATE X.}

Strongylus Tetracanthus, formerly described as Trichonema Arcuata.

Fig. 1.-Larva (slightly mag.) from mucous membrane of an Iceland pony. The worm was of a uniform rose colour.

Fig. 2.-Head (mag. 80 diameters).

Parasites of Grouse Disease.

FIG. 3.-The parasites embedded in the mucous membrane of the cæcum (mag. 10 diameters).

Strongyli from Bronchial Tissue of a Three-year-old Sheep.

FIG. 4.-Magnified 80 diameters.

Strongylus Filaria.

Fig. 5.-From lung tissue of a sheep (mag. 80 diameters).

Parasite from Liver of a Domestic Fowl.

Fig. 6.-The fowl had been fed on the flesh of an Iceland pony infested with Strongylus tetracanthus. The whole of the abdominal viscera of the fowl were covered with small tubercular-looking ubjects containing worms, embedded in a tough, almost cartilaginous substance (mag. 80 diameters). 
FIG. 3.

FiG. 1.
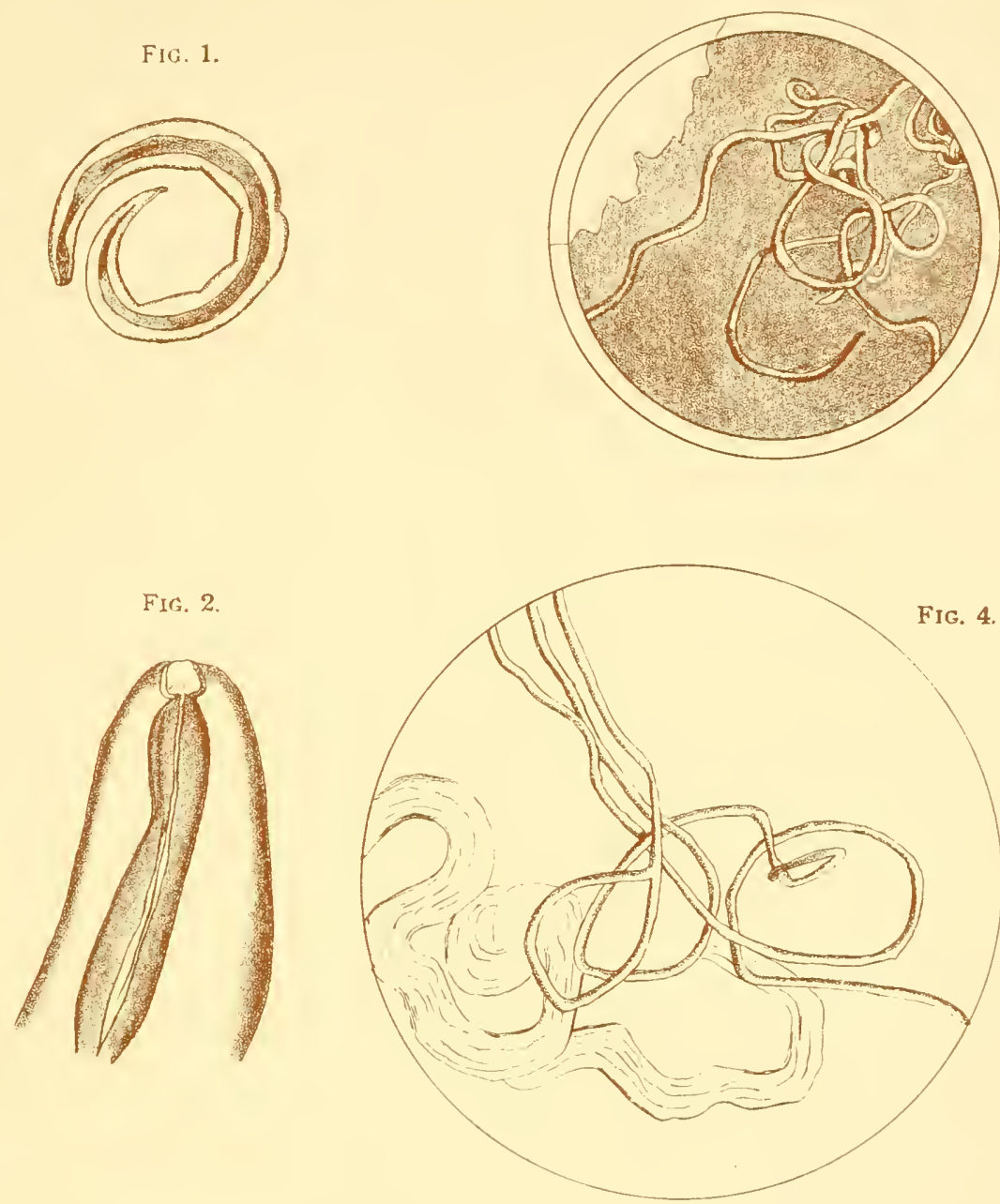

FIG. 5.

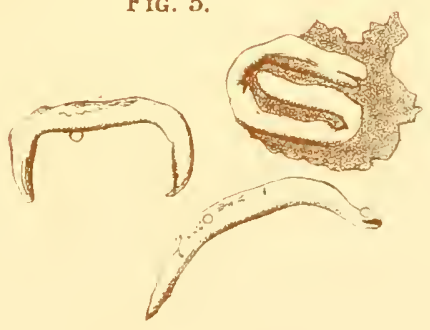

Fig. 6.

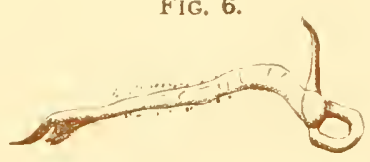




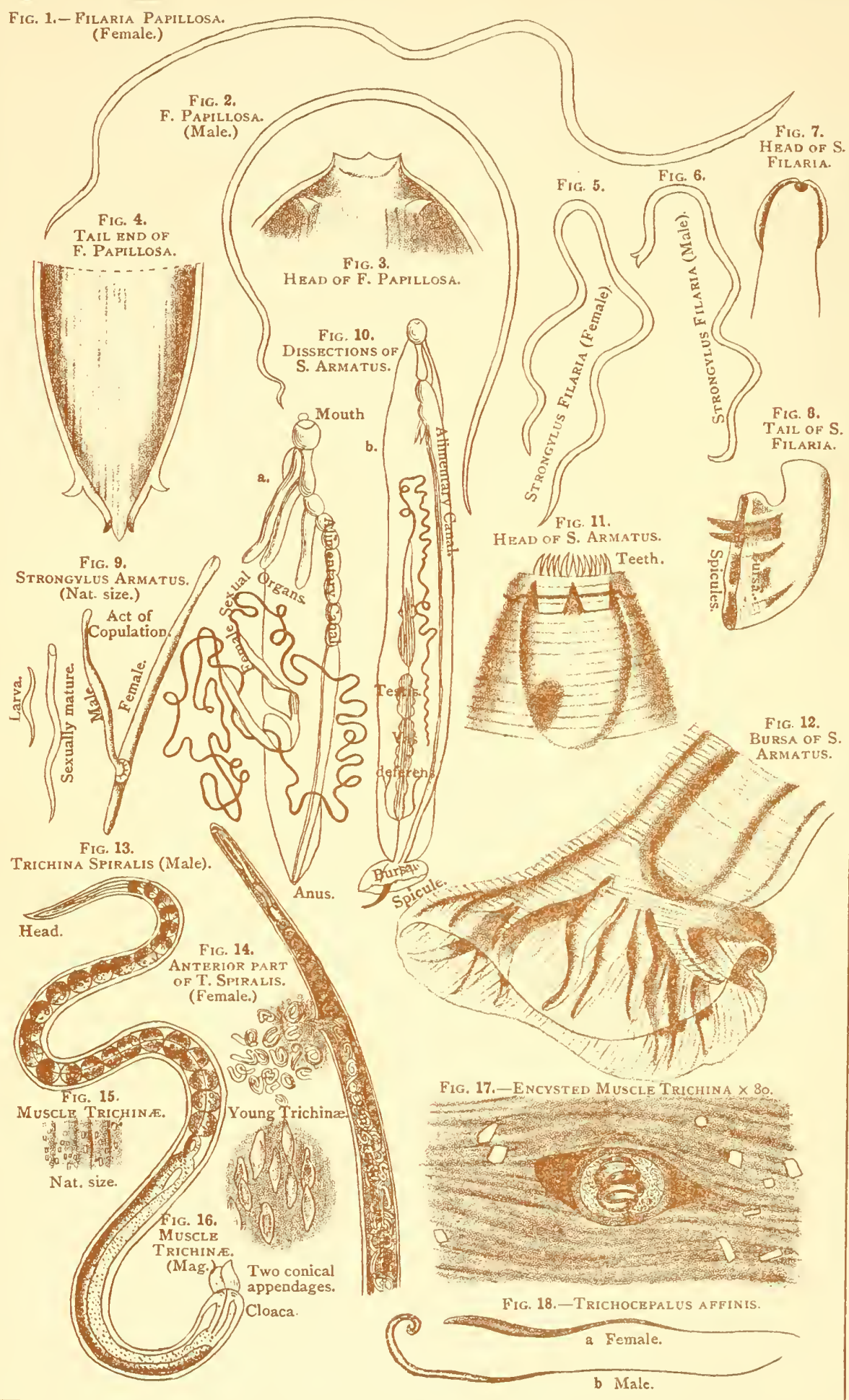




\section{PLATE XI.}

\section{Filaria PapiliLosa.}

Fig. 1.-Female (nat. size).

Fig. 2.-Male (nat. size). Notice the spirally wound tail.

FiG. 3.-Head (mag.)

FIG. 4.-Tail (mag.)

\section{Stroxgylus Filaria. (See Fig. 4, Plate IV.)}

FiG. 5.-Female (nat. size).

Fig. 6.-Male (nat. size).

FIG. 7.-Head (mag.) Observe that the head is round, and provided with a round naked mouth.

FIG. 8.-Tail of male (mag.) Observe the bursa with the two spicules projecting from it.

\section{Strongylus Armatus.}

Fig. 9.-The larva and sexually mature worm from the aneurism of a horse. The act of copulation is also shown; the small male is attached to the larger female by its bursa.

FIG. 10.- Shows the anatomy of the mature male and female.

Fig. 11.-The head. Observe the ring of teeth round the circular mouth.

Fia. 12.-The bursa. Notice the arrangement of the ribs.

Trichina Spiralis.

FIG. 13.-MIale worm from the alimentary canal of a man (mag.) Observe the pointed hoad and the round tail end provided with a pair of conical appendages for purposes of copulation.

Fig. 14.-Part of a female from the alimentary canal (mag.) Observe the young worms, some emerging from the sexual aperture, and others still in the interior.

Fia. 15.-Encysted trichinæ in muscle (nat. size).

FIG. 16.-The same (slightly magnified).

Fig. 17.-Muscle trichina. Observe the spirally coiled worm in the interior of the cyst.

\section{Trichocephalus Affinis.}

FIG. 18.-Observe the long, thin head end of the worm, hence the name Trichocephalus. 


\section{PLATE XII}

Life History of a Trematode.

FIG. 1.-Fertilised ovum, ciliated embryo, sporocyst, redia, cercaria (developed as an internal bud in the interior of the sporocyst or redia), encysted form, fluke.

Ovum of Distoma Hepaticum (mag.)

Fig. 2.-Observe the shell opening by a lid, and the embryo eseaping through the opening.

Cifiatev Eubryo of Distoma Hepaticum.

Fig. 3.-Observe the cilia, the cross-like eye spot, and the large touch papilla projecting from the broal anterior end.

Sporocyst (mag.)

Fig. 4.-Observe the ciliated coat cast off, the internal buds developing into cercarix, and the alimentary canal without an anus. A highly organised sporocyst is called a redia.

Tailed Cercaria.-Fig. 5.

Amphistomum Conicum.

FIG. 6.-Observe the conical form, the anterior and posterior sucker, the alimentary canal without an anus, and the much ramified water vascular system.

Organisation of Distomum Lanceolatum.

FIG. 7.-Observe the lanceolate form, oral sucker (O. S.), ventral sucker (V. S.), the bifureating alimentary canal (A. C.), the testes (T.), uterus and female organs, and the water vascular system (W. V. S.).

Distonum Hepaticum.

Fig. S. Natural size. Near the anterior end the ventral sucker is shown.

\section{DIPTERA.}

Gastrophilus Equi.

Fig. 9.-Male and female (nat. size).

Fig. 10.-The larva or bot.

FIG. 11.-The pupa from which the fly has emerged.

FIG. 12.-Part of the stomach of a horse, with larvæ (bots) living in its interior.

\section{TRACHEARLA.}

Pentastomum Temioldes.

Fig. 13.--Internal organs.

Pentastonum Denticulatum. - Fig. 14. 
PLATEXII.

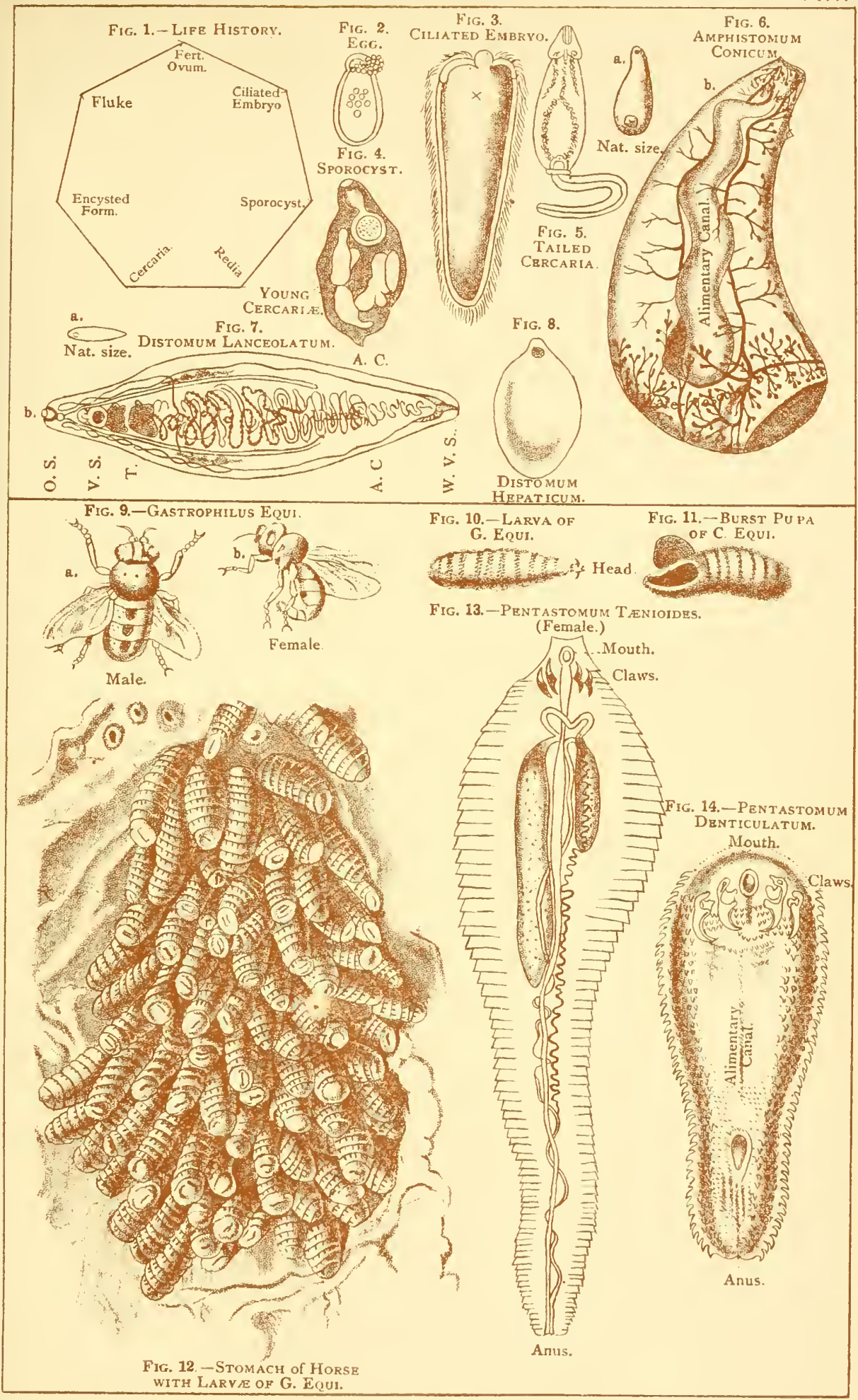





\section{CHAPTER TAXVII.}

\section{B A CT E R I A.}

BACTERIA, a division of the smallest aud simplest of plants -rounded, ovoid, or spiral in shape-are micellular and devoid of chlorophyll. They consist of protoplasm enclosed in a membrane, having a great affinity for certain stains, and are, in common with vegetable matter's, not destroyed by potash, ammonia, or weak acids, and have the power of reproduction, some also of movement. They are divided into four great classes, each of which is recognised by the shape and size of its individuals, and also sometimes by their method of growth.

I. Spherobcteriens, very small, rounded or ovoid in shape, and may be either micrococci or monads. If the latter, which are believed to be spores, they may be traced growing into tubules; if micrococci, the globular form remains.

II. Microbacteriens, large rod-shaped, and may be bacteria or bacilli. If bacteria, they remain short; if bacilli, they may grow into lengthened filaments, having rounded cells - spores. These rods vary in shape; some are swollen in the middle, some pisiform, swollen in the middle and attenuated at the ends; others constricted in the middle, as Bacteriens termo; and some enlarged at one end-club-like. They are again divided into three groups- $(a$.) coloured bacteria, found in ovine milk, \&c.; (b.) zymotic; and (c.) pathogenic bacteria.

\section{Desmobacteriens.}

\section{Spirobacteriens.}

Bacteria have been carefully measured, and the results are as follows :-

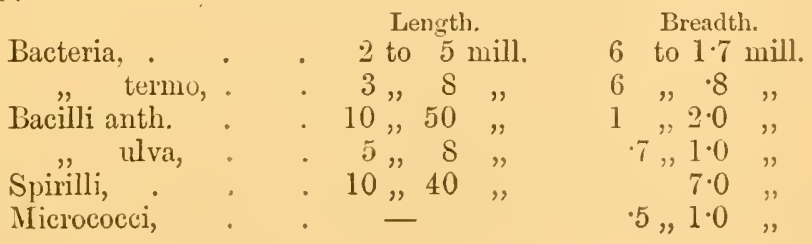




\section{PLATE XII. - BACTERLA, or SCHIZOMYCETES.}

(Gr. schisis, a splitting.)

(Fig. 8 is after Ewart, the rest after Dodel-Port, based on Dr. Koch's photographs.)

Bacteria are those organisms which produce the change in organic bodies known as Putrefaction. Hay Bacteria, developed in an infusion of hay, may be profitably examined first. Take some fresh hay, pour hot water upon it, and allow to stand. In the course of a day or two the liquid becomes turbid, due to the presence of Bacteria, and latterly it has the smell of decaying organic matter. If a drop of this liquid be examined under the highest power of the microscope, it will be found to contain Bacteria of simple form.

Figures X 3000, exoept Fig. 8.

Fig. 1. Micrococci (Gr. mikros, little; kokkos, a berry) are simply small, round, or oval cells, occurring free, or in chain-like rows, or united into a gelatinous mass. They are remarkable for the bright colouring matters with which they are tinged-red, blue, \&c.

Micrococcus prodigiosus-the blood-red Micrococcus-is a spherical form, appearing as blood-red, slimy drops on stale potatoes, bread, damp wafers, and the like. From its sudden appearance (often arising in the course of a single night) it has often been superstitiously regarded as an evil omen, as stories of "bleeding bread" or "bleeding wafers" testify. The colouring matter is insoluble in water, but may be extracted by alcohol or ether.

FIG. 2. A chain of Microçocci found in putrefying blood.

This chain has probably originated from the repeated division of a single individual. The single cell lengthens as it grows, then forms a sort of figure of 8 preliminary to division, and this repeated again and again would give rise to the chain.

FIG. 3. A gelatinous film or Zooglœa.

This film or scum forms on the surface of putrefying fluids, and consists of a number of Micrococci embedded in rows in a gelatinous material. This arrangement in rows has probably been produced, as in Fig. 2, by repeated division, as some are found in that condition.

FIG. 4. Bacteria (Gr. bakterion, a staff), or Cylindrical Forms-the two red blood-corpuscles are merely represented to show relative size.

These forms are the first found in the body after death. They are short or long rods, multiplying by transverse division.

FIG. 5. Rods from putrefying vegetable matter, with a vibratile cilium at each end, by means of which they wriggle about

FIG. 6. Spirochoete (Gr. chaite, hair), or Relapsing Fever Bacteria, occurring in the blood of fever patients.

The spiral filaments are flexible and exhibit wave-like movements, which is often revealed by the motion imparted to the bloodcorpuscles in the neighbourhood.

FIG. 7. Spirillum-to be found in puddles in summer where there is decaying vegetable matter.

They form inflexible spiral filaments, of one or several turns, and have a vibratile cilium at each end.

FIG. 8. Development of Spirillum- $-a$ to $i$.

(a.) Zoogloea-stage-motionless forms embedded in gelatinous material.

(b.) Vibrio-stage-bow-shaped forms passing into spiral forms.

(c.) Filamentous-stage-the last elongated.

(d.) Filamentous-stage-further developed forms, in which the filament is long and motionless.

(e.) Filamentous-stage-Spore-producing filameut.

(f.) Filamentous-stage-ripe and motile filament.

(g.) Filamentous-stage-filament breaking up.

(h.) Spores which encyst and divide to form sporules.

(i.) Spores germinating-little comma-shaped bodies which repro duce the original Spirillum.

Life History Diagram.-The stages are here given through which Spirillur passes in order to complete the cycle of its life. 

I N D E X. 



\section{N D E X.}

Abdominal Breathing, . . $\quad 41$

Abdominal complications of Influenza, 353

Abdominal Vertigo,

Abnormal Heat, Determination of, Abomasitis,

Treatment of, .

Abscesses, Strumous,

, $\quad$," $\quad$ Semiology, .

Acanthocephala,".

Treatment.

Acephalocyst,

Acid, Benzoic,

,, Hippuric, .

, Lactic,

, Uric, .

Active Symptoms,

Acute Bronchitis,

Acute Metritis,

$\begin{array}{lll}, & \text { ", } & \text { Pathology of, } \\ \text { ", } & \text { Symptoms of, }\end{array}$

Acute Rheumatism,

Adventitious Substances in the Brain and Cranial Carity, .

Affections of the Diaphragm,

Albumen in Urine, of the Blood,

Albuminoid Degeneration,

Albuminuria,

Ale, Burnt, Effect on Cows,

Alfort, Experiments at,

Aloes cansing Superpurgation.

, in Bronchitis,

American Horse Epizootic,

Ammonia, Carbonate of, in Azoturia.

Amyloid Degeneration,

Degeneration, Case of,

", Degeneration, Charactci of Tissues affected, substance, Reactions of,

Analysis of Blood, of Urine in Red Water,

Anatomy, Importance of, Anæmia-Surra (see Blood Diseases), $40 ?$
," Symptoms of, - . 82

Treatment of,

Anænic Blood, Character of,

Analysis of Morbid Material,

Anasarca, a Symptom of Anæmia,

Angina Pectoris,

Animal Heat, Production of,

Anthrax (see Contagious Diseases),
338

338

339

788

783

745

744

745

744

760

761

761

438

531

670

746
Anthrax, Inoculation for Prercution of 288

Antiseptic Prevention of Equina, 249

Anus, Imperforate, . . . 695

A pnoea, . . . . . 116

,, Symptoms of, . . 117

Apoplectic Anthrax (see Contagions

Diseases), . . . . 294

Apoplexy, Cerebral, . . 516

from Congestion (see Sporadic Diseases),

from Congestion, Causes of, 519

., $\quad$ Tost mortem of, 525

, Sequelæe of, $\quad 528$

,. Synlptoms of, 523

Treatment of, 525

from Ruptured Vessels, $\quad 516$

Parturient, . 519

Pulmonary, . . 621

Arachnida, . . . 761

Arrangement, Tabular, of Entozoa, 764

Arrest of Nutrition, . . . 56

Arterial injection of the Lungs, . $\quad 630$

,, movements, . . . 29

Ascarides, . . . . . 796

Ascaris Marginata, . . . 765

, Megalocephala, . . 765

" Lumbricoides, . . . 769

"Suilla, . . . $\quad 769$

"Acuminata, . . 772

" Tetraptera, : $\quad . \quad 770$

Mystax, . . 766

Ascites, . . . . 720

,, Causes of, . . . 720

,. Sanguineons, . . . 721

, Symptoms and Treatment, 721

Asthenia, . . . . 116

Asthma, . . . . . 466

," Causes of, . . . 467

" MLrbid Anatomy of, . 469

". Relief of, . . . 471

". Symptoms of, . . . 470

"Treatment of, . . 471

A tony of Walls of Vessels causing

Congestion, . . . . 96

Atrophy and Degeneration of Tissue, $\quad 52$ ,, caused by some Drugs, . $\quad 54$

". Canses of, . . . 53

", compared with Degcneration. 57

, Definition of, . . . 52

," Numerical, . . . . 52

"Simple, . . . 52

A trophied Tissnes, Changes in. . 54

Auscultation in Plemrisy. . . $\quad 367$ 
Auscultation in Respiratory Discases, PAGE ", of the Larynx, . 579 Azoturia, . . 4 451 , Post mortem of, . . 454 , Symptoms of, . . 451 ,, Treatment of, . . 455 " Urine in, . . . 454

Bacilli Anthracis, . . . 282

Bacteria, Classification of, . . 843

$\begin{array}{ll}\text {, } & \text { Desmobacteriens, : } \\ \text { Microbacteriens, : } & \text { Spherobacteriens, : } \\ \text {, } & \text { Spirobacteriens, } \\ \text { ", } & \text { Length and Breadth of, } \\ \text {, } & \text { Effects of Heat on, }\end{array}$

of certain Chemicals 0112

Bacon-like Degeneration, . . 62

Beale, Dr., on Bioplasm, . . $\quad 54$

Beef Measles, on Rinderpest Poison, 142

Beneke on Oxalic Acid, : : $\quad 459$

Bennett on Oxidation of Tissues, . 44

Benzoic Acid, . . . . 745

Bichat, Experiments of, . 118

Bioplasm, Dr. Beale on, . . 54

Blackleg, . . 305

Black Spand (see Septic Blood Diseases), . . . . 405

Black Water, . . . . 460

Bladdcr, Inflammation of, . $\quad . \quad 755$ "Worm, . . . 782

Bleeding, Effect of, on Fibrin, . $\quad 74$

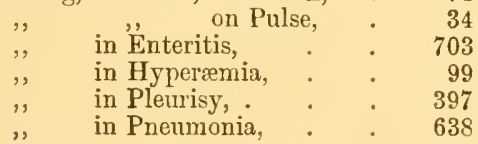

Blood-

Congestion causing Gangrene, . $\quad 99$

Treatment of, . . 100

Contagion and Contagious Diseases-

Origin, . . . . 129

Koch on, . . . . 129

Facultative Parasites, . . 130

Defective Excretion, . . 88

Diseases-Classification, . . 126

Hyperæmia, Definition and Causes, 94

Collateral, . . . . 94

Mechanical, . . . . 96

From diminished Cardiac Power, 96

From Gravitation, . . 97

From altered Conditions of Blood, 97

From Defective Secretion, - 98

Lipæmia, . . . . 102

Mellitæmia, . . . $\quad 93$

Rules for treating Diseases dependent on Blood loaded with Effete Materials, . . 93

Septicæmia-Progressive Gangrene, 105 Artificial Induetion of, . 105 Modes of Death, . . . 113
BLoon-continued.

PAGE

Death beginning at Heart, . 114

,, ," at Lungs, . 116

," , at Brain, . 121

.' " ". with the Blood, 121

Septicremic "Virus, Experiments with, . 106

Transformation of Chyle and of the

Tissues, . $\quad . \quad$. $\quad 89$

Blood, Changes in the, . $\quad 71$

," Healthy, . . : 71

$\begin{array}{ll}\text { Healthy, } & 71 \\ \text { Analysis of, : } \quad: \quad & 71\end{array}$

Constituents vary, . . 72

Water in, . . . 72

Experiments of Zimmerman, 72

Albumen of, . . . 73

Fibrin, . . . 73

Excess of (Hyperplasma, Hyperinosis), . . 74

Effect of Bleeding on, . 74

Deficiency of (Hypoplasma, Hypinosis), . 75

Want of coagulating power, 75

Substances influencing, $\quad \mathbf{7 5}$

Partial suffocation from Fire, its influence on, . 76

Splenic Apoplexy, . 76

Modern theory - Globulin and Fibrinogen, . 76

Huxley on Coagulation, 77

Coagnilation of the, of different animals, . . 78

Effect of Chlorate of Potash, 81

Crassamentum indicative of state of the System, .

Anemia, Hypœnia, Oligæmia,

Symptoms of, . . 82

ANASARCA, - . 84

Treatment, . $\quad$. 85

Evil of forcing Food, - 84

Clipping Horses, . $\quad 86$

Character of Anæmic Blood, 86

Plethora, . . . 87

Sir Thomas Watson on, 87

Treatment, . . 87

Changes from Defective ExCRETION, $\quad \cdot \quad 89$

Experiments of MM. Prevost and Dumas, . $\quad . \quad 89$

Influence of Urea, . $\quad 89$

Transformation of Chyle, AND OF THE Tissues,

Source of Urea, . . $\quad 90$

Process of Nutrition, - $\quad 90$

Kinds of Food, . . $\quad 91$

Quantity of Food, $\quad 92$

Nitrogenous Food, $\quad 92$

Uræmic affections and principles of Treatment, . 93

Changes in the, in Rinderpest, $139-140$

Death beginning with the, 123 
Blood, Changes IN THE-continued. , in Urine,

, Pressure increased, causing $\mathrm{Hy}$ -

Blood Diseases perremia

AveMIA-Surra,

Symptoms of,

Ixodic Axrmia (see Texas Fever),

TEXAs Fever-Fornis of,

Aeute Form, Symptoms of,

Appearance of Blood and Tissues in,

Conclusions,

Destruction of Ticks,

Etiology,

Habitat of Ticks, .

Hæmoglobinuria in,

In Jamaica, .

Morbid Anatomy and Nicroscopie Appearances,

Post mortem appearances,

Prevention and Treatment,

Symptoms,

Temperature in,

The Tick, History of,

The Tick, Life History of 423,430

Tsetse Fly Disease or Nagana in Zululand,

Temperature in,

Dr. Bruce on,

Bloody Urine (see Sporadic Diseases),

Botliriocephalus, .

Bots in the Horse,

Oxand Sh- 761

Bouley, Professor, Experiments of, in

Pleuro-Pneumonia,

Bowels, Diseases of (see Sporadic

Diseases),

Brain, Adventitious Substances in,

, and its Membranes, Inflammation of the,

,, and Meninges, Melanosis of,

"Hypertrophy and Atrophy of,

, Death beginning at the,

.. Exostoses in,

,. Inflammation of,

, Softening of,

Tumours in, .

Thickening of Dura Mater,

Tubercular Deposits in Meninges of, 533

Brauell, Dr., on Rinderpest,

Braxy (see Septic Blood Diseases),

Breathing, Quickened,

, Abdominal,

,, in Broken Wind,

, Irregular,

, Stertorous, .

, Thoracic,

'Brodie, Sir Benjamin, Experiments of 41

Broken Wind (see Sporadic Diseases), 466 Congh of,

Bronchial Sounds, Dry and Moist, 588, 589
PAGE

605

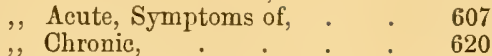

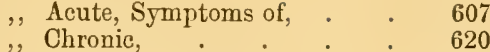

,, in Foreign Horses, . . 606

,, of Influenza, . . . 352

, Pathology and Morbid Anatomy of, 611

"Parasitic, . . 769

,, Signs of, . . . . $\quad$. 610

,, Treatment of, . . . 618

Broncho-Pneumonia (Corn-Stalk

Disease), . . . 177

Brown, Prof,, on Milk in Eczema, 190

Brown's, Professor, Thermometer, 46

Cake Poisoning (see Sporadic Diseases), 485

Calcareous Degeneration, . . 60

419 " , , Causes of, 60

$425 \quad, \quad, \quad$ Conditions of, 60

433 Calculi (see Sporadion, Calcification, 61

418 , Mixed, . . . 708

420 , Oat-hair, . . . . 708

428 " Phosphatic, . . . 707

427 , Renal, . . . . 754

423 Calorific Functions, Symptoms afforded by,

Canine Distemper (see Contagious

Diseases), . . . . 269

415 Cannabis Indica in Chorea, . . 502

415 Cantharides in Nephritis, . . 751

Caseation, . $\quad . \quad$. 59

Castor Oil Seed Poisoning (sce Sporadic

Diseases), . . . . 485

Catarrh-Symptoms and Treatment of, . . . . 595

184 Cattle Plague (see Contagious Diseases), 137

Cerebral Apoplexy, . . . 516

707 ,, Morbid Anatomy of, . . 518

531 , Semiology of, . . $\quad \mathbf{5 1 6}$

Cerebritis (sce Sporadic Diseases), 503

532 ,, and Meningitis, differences be-

535 tween, . . . . 505

534 ,. Treatment, . . . . 506

121 Cerebro-Meningitis, • . . 507

534 Chabert's Disease (see Contagious

532 Diseases), . . . . 305

535 Chorea (see Sporadic Diseases), . 501

$533 \quad$, Symptoms of, . . . 501

532 ,, Treatment of, . . . 502

145 Coma, Post mortem appearances of, 502

402 Congestion of Lungs, . . . 624

40 Contagion and Contagious Diseases-

Origin of, $\quad \cdot \quad$. $\quad 129$

Koch's Postulates on, : $\quad 129$

Pathogenic Microbes in, . 130

Classification of Microbes, . 132

Pleomorphism, . . . 134

Pcriod of Incubation, . . 135

Anthrax(Charbon)-Definition of, 277

History, . . . 278

Influence of Temperature, - $\quad 279$ 
Contagion an Contagious Diseasescontinued.

Influence of Ponds, Morasses, and Stagnant Water,

Contagion, Forage,
Bacillus Anthracis,
Essential CHArbox,
Definition and Propagation of,
In Pig,
Gloss-Anthrax, :
In Poultry,
Pathological Anatomy of,
Treatment,
Inoculation for prevention of,
Varieties of,
In Horse, Symptoms of.
Gloss-Anthrax,
Charbonous Tumours, :
Terminations,
In Horned Cattle, Symptoms of,
In Sheep, Symptoms of,

BLACK-LEG, • • • • 305

Canine Distemper-Definition of, 269

Pathology, . . . . 269

Symptonis, . . . . 271

Complications, . . . 272

Treatment, . . . 273

Cattle Plague-Definition of, 137

Pathology and Symptoms of, 138

Post mortem appearances of, . 147

Skins of Animals affected with, 151

History of, . . . . 152

Treatment of, . . . 157

Eczema Contagiosa-

Definition of, . . . 188

Pathology and Symptoms, . 188

Treatment, . 194

Character of Milk in. . 190

GuaNoEns ANn Farey (EqUixa) -

Definition and History of,

Causes of

Polyuria forerunner of,

Contagion,

Period of Incubation,

Acute Form, Symptoms, and Post mortem appearances,

Chronic Form, Symptoms and Post mortem appearances,

Acute Farey, Symptoms of,

Chronie Farey,

Antisertic Prevention of Thinina,

Lquina, 249

Mallein Test

$23 \check{3}$

236

236

237

239

240

243

246

248

Stomatitis Pustule Conto

InfeCtious Amortion-

Causes of,

Nocard on, in Cow and Mare,

Carbolic Aeid in jrevention of,

malanie mu Cö̈t,

Nature of,
Contaginn and Contagious Dineanes-continued.

Benign Form, or Coïtal Exanthema, Symptoms of, . $\quad 256$

Malignant Form, . . . 258

General Symptons, . . 260

Progress and Duration, . $\quad 262$

Etiology, . . . . 262

Contagion, . . . . 263

Post mortem appearances, . $\quad 264$

Treatment, . . . 266

Coïtal Exanthema of Holned Cattle, . . . . 268

Plevio-Pxeunonia Contariosa, 160

Definition of, . . 160

Pathology and Symptonis, . 160

Premonitory Symptoms of, . $\quad 161$

Palpable or obvious Symptoms, 162

Post mortem appearances, . 164

Dr. Yeo on, . . 167

Caluse of, . . . . . $\quad 171$

Prevention of, . . . $\quad 172$

Disinfection, . . $\quad 172$

Inoculation, . . . . 172

Conclusions of Belgian Commission, . 174

Pathosenic Microbe of, : 176

Compared with Bronchitis, . $\quad 177$

QUARTER-ILL, . . . . 305

Symptoms of, . . . 306

Post mortem appearances of, . $\quad 307$

Causes of, . . . 308

Protective Inoculation, . 308

RABIES-Definition and History of, . 221

Causes of, : : $\quad 223$

Pathology and Symptoms, . 225

Causes and Symptoms in Horse, 230

Preventive Treatment, . . 232

Pathological Anatomy, . 233

Post mortem appearances, . $\quad 234$

RINDERPEST IN SHEEP, · . 159

Results of Inoculation, . . $\quad 159$

SPLENIC A POPLEXY, . $\quad 294$

Sptuenic Fever, . • • 294

Swine Plague-Definition of, $\quad 310$

Pathology and Symptoms, . 311

Experiments of Klein, . . 313

Morbid Anatomy, . . 316

Symptoms, . . . . 321

Tumerculosis-Definition of, . 323

Appearanee of Tubercular Nodules, . . . 323

Experiments of Dr. Tappeinier, 325

Divisions of, . . . 327

Symptoms in Cattle, . $\quad . \quad 327$

Of Mammary Gland, . . $\quad 331$

In Horse, . . . . 332

In Pig, . . . $\quad 333$

In Sheep and Goat. : . 334

In Dog, . . . 335

In Birds, . . . . 336 
Contagion ann Contaglots Diseasescontinued.

Treatment of Bovine, . . $\quad 337$

Strumous Abscesses, . . $\quad 337$

Tunerculis-Preparation of, 339

Method of Inoculation and effects of,

Suggrestions for suppression of,

rariola Vaceisa (Com-Pox), .

Synonyms, Definition, Pathology, and Symptoms,

Trinsmission from

Man to Cattle, 203

Equine Pox, . . . 206, 219

Prevention and Treatment, . 209

VARIOLA Orina, . . . 210

Synollyms, Definition, aud History, 210

Pathology and Symptoms, . 213

Professor Gamgee's Statistics,

Post mortem appearances,

Prevention and Treatment,

Inoculation (Ovination),

Varicella Boum,

Variola Equina,

Cough, Dry,

Moist,

Varieties of, .

Croton Seed Poisoning (sce Sporadic

Diseases),

218

218

$21 \mathrm{~s}$

219

219

219

42

Croup (see Sporadie Diseases), $\quad$. $\quad 599$

Symptoms of, . . . 600

Treatment of, . . . 601

Cyanosis, . . . . 668

Cynanche Trachealis (see Croup), . $\quad 599$

Cystitis, . . . . 755

Degeneration, Colloid, . . . 62

Mucoid, . . . 62

Pigmentary, . . . $\quad 62$

Amyloid, . . . . 62

Lardaceous, . . . . 62

Amyloid, Characters of, $\quad . \quad 63$

Diabetes Insipidus (see Sporadie Diseases),

Diaphragm-Spasm of, Rupture of, .

449

670

670

381

Diarrhæmia,

Diarrhœa-Symptoms of, Treatment of,

Digestion, Secondary, .

Digestive Orgaus, Diseases of,

Diphtheria (see Sporadie Diseases), Post mortem appearances of, .

Treatment of,

Diseases, Functional, Structural, .

Diseases of Borvels,

Digestive Organs,

Heart aud its Membranes,

Tídneys,

Respiratory Organs.

Spinal Cord aud liembranes,

708

672

654

742

595

536
Diseases of Spleen and Pancreas, .

PAGE

739

", Stomach, coneurrent with or immedi-

ately succecding Parturition,

758

721

$7 \cdot 21$

718

719

Dyspnoa,

Ectopia Cordis,

Eczema Contagiosa (see Contagions

Diseases),

Emphysema, Chronic, of Lungs, . Vesicular,

Interlobular,

Treatment of,

669

188

621

621

622

623

Encephalitis (sce Sporadic Diseases), 507 Symptoms of, . . . 509

Etiology of, . . . 511

Morbid Anatomy of, . $\quad 513$

Treatment of, . . $\quad 514$

Endocarditis-Morbid Anatomy, . 664

Enzootic and Epizootic Diseases-

Epizootic Cellulitis or Pixi-Eye-

Causes-Etiology,

358

Post mortem appearances, . 363

Nortality, : . . . $\quad 364$

Treatment, . . . . 364

Epizootic Pneumonia-Definition, 365

Semiology, . . . . 366

Post mortem appearances, $\quad 369$

Treatment, . . . 371

INFLUENZA-Morbid Conditions of, 346

Panzootic Catarrhal Fever-

Symptoms of, . . 347

Etiology, . . . . 348

Predisposing Causes, . $\quad 349$

Pathology, . . . . 349

Complications, . . $\quad$. 350

Prognosis, . . . . 354

Treatment, . . . . 354

Morbid Anatomy,. . . 357

Maligrant and Catarihal

FEver IN OX-Symptoms, $\quad 379$

Treatment, . . 3S0

Prnk-Ere (see Epizootic Cellulitis), 358

Pleuro-Pneumonia (see Epizootic Pueumonia),

Epilepsy-Symptoms of, . . 499

Treatment, . . 500

Epizootic Cellulitis (see Enzootic

Diseases), . . . . 358

Epizootic Pneumonia (see Euzootic

Diseases),

365 per Petechial Fevers-

Diairillmia (sce Purpura Hæmorrhagica),

Purpula Hemorrhagica-

Definition,
Pathology and Symptoms, 
Eruptive or Petechial Fevers-contd.

Causes,

Post mortem appearances in acute cases,

External Treatment, . . 388

Scallatina, . . . . 389

Divisions of, . $\quad 390$

Semiology-Scarlatina Simplex, 390

Treatment, Scarlatina Anginosa,

Post mortem appearauces, .

Scarlet Feter (see Scarlatina), Exudations, Soft,

", Consolidated, .

, Specific,

Farcy,

" Acute, Symptoms,
" Clironic,
"Treatment, .

Fardel-liound,

Fattening Horses for sale,

Fatty Blood, Lipæmia,

," Degeneration,

\begin{tabular}{|c|c|c|}
\hline , & Causes of, & \\
\hline$"$, & Laryngeal M & Iuscles \\
\hline ," & $\begin{array}{l}\text { leading to C } \\
\text { tion, } \\
\text { of Mnscle, }\end{array}$ & Casca- \\
\hline Embolism, & . . & . \\
\hline Infiltration, & & . \\
\hline ," & Causes of, & from \\
\hline , & $\begin{array}{l}\text { Degeneratio } \\
\text { from fattening }\end{array}$ & n, \\
\hline
\end{tabular}

Feeble Pulse,

Legal importance of, 740

Fibrin altered by partial Suffocation,

Effect of Bleeding on,

,. Excess of, in the Blood,

," Huxley on,

,2 Process of formation of,

Fibrinogen, .

Moderu Researches on,

Filaria Bancrofti,

, Strongylus,

," Sanguinis hominis,

Fitzwygram, General, on Food.

Flat-Worms,

Flatulent Colic,

Fleming, Dr., on Rabies, .

Flukes,

Food, Constituents of, .

, Kinds of,

", Nitrogenons, bad in excess,

" Quantity of,

Foot-and-Mouth Disease,

Forcing Food, Evil of,
Formation of Thrombi, $\quad 98$

Fowl Cholera Microbe, Cultivation of, 135

Foul appearance of Mouth, . . 27

Frequency of the Pulse, , . 33

Functional Diseases, . . . 50

Gadfly, . . . . . 760

Gall Stones, . . 708

Galvanism in Lead Poisoning, . 462

Gamgee, Professor, on Inoculation in Rinderpest, . . . . 166

Gamgce's, Professor, Statistics in Variola Ovina, . . . 221

Gangrene of the Lung, . . 605 from Congestion, . . $\quad 97$ 
Treatment of,

Chronic,

, in Pleuro-Pneumonia, 165

," Red, . . 630

Hide-Bound a Symptom of Indigestion, 28

Histology,

Hog Cholera (see Contagious Diseases), 310

Hoose,

In Calves, : . : .

Horse, Pleuro-Pneumonia of,

Hoven,

Husk,

Huxley on Coagulation of the Blood.

Hyaline Substances,

Hydræmia, .

Hydrothorax from Pleurisy,

in Pleurisy,

Hygiene, or Prophylaxis,

Hyperæmia (or Congestion),

Bleeding in,

Collateral,

Definition and Causes,

Escape of Blood Globules in,

From increased Blood Pressure,

From relaxation of Vessels, .

Influence of the Nerves in, .

Mechanical,

Results of, . . 98

Treatment of, : : 100

Venous, Causes of, . $\quad$. 99

From diminished Cardiac power, 96

From Gravitation,

From altered conditions of the Blood,

From Defective Secretions, .

Hyperinosis,

Hyperplasma,

Hypertrophy,

Canses of,

Handfield Jones on

Leading to Tumours.

Of the Heart,

Hypinosis, .

Hypoplasma,

Hysteria,

Icterus,

Idiopathic Symptoms, .

Impaction of the Omasum, of the Rumen with Solid

Natters,

of the Stomach,

of the Stomach in Carnivora, 685 of the Stomach, Symptoms

in the Horse,

of the Stomach, Treatment

in the Horse,

of Third Stomach,

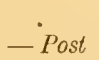

804

365

677

799

47

82

647

647

94

100

94

94

94

95

96

98
Inpaction of Stomach in Jog and Cat, 685

$\begin{array}{cc}\text { Horse, } & 686^{\circ} \\ \text { Symptoms of, } 686\end{array}$

Treatment of, 686

Imperfect Ventilation, . . 20

Imperforate Anus, . . . 698

Inanition, Death by, . . . 116

Incontinence of Urine, . . $\quad 757$

Increased Nutrition, . . . 69

Indigestion with Engorgement (see Sporadic Diseases), . . 677

Causes in Horse, . . . 677

Causes in Horned Cattle, . 678

Symptoms in Ox and Sheep, . $\quad 679$

Treatment of, 680

Indigestion withont Engorgement, 674

Induration of the Liver, . . $\quad 727$

Infectious Abortion (see Contagious

Diseases),

Infectious Pneumonia (see Swine

Plague), . . . . . 322

Infiltration, Fatty, . . . 65

Inflammation and Hyperæmia confounded, . . 98 of the Bladder, . $\quad 755$ of the Bowels, . $\quad 699$ of the Brain, . . 503 of the Kidneys, . 748 of the Liver, . . 725 of the Lungs, . $\quad 624$

Bleeding in, 634

Causes of, 634

Counter-ir-

ritation in, 642

Symptoms

of, . 634

Treatment of, . 638

of the Womb, . 760

Inflammatoly Diarrhœa, . . 711

Influ Diseases of the Intestines, 699

of Age in predisposing to

Disease,

Influenza (see Enzootic Diseases), $\quad 345$

Inoculation of Plenro-Pneumonia, 172

in Rinderpest, Professor

Gamgee on, . . 157

Mr. Rutherford on, . $\quad 175$

a sucecssful Preventive, $1: 7$

in Variola Ovina, . 219

Insecta, . . . . 763

Inspection of Chest, . . . $\quad 576$

Interlobular Emphysema, . . 621

lntermitting Pulse, . . . $\quad 36$

Intestinal Concretions, . . 707

Intestines, Inflammatory Diseases of, 699

Intratracheal Injections in Hoose, $\quad 807$

Introduction, . . . . 1

Intussusception, . . . . 704

Iodine in Diabetes, . . . 450 
Ischuria,

lxodic Anæmia (sce Blood Diseases),

Jaundice,

Treatment of

Jones, Handfield, on Hypertrophy,

Jugular Vein, Obliteration of,

Kied, Sheep-Melophagus Ovis,

Kiidueys, Diseases of, ?

Kidney, Floating.

Kinds of Food,

Metamor 1 hoses,

Congh, .

Koch, Experiments of, with septicemia,

Lactic Acid,

Lamb Disease,

Lardaceous Degeneration,

Laryugeal Museles subject to Fatty Degeneration, ;, Sounds,

Laryngitis (sce Sporadic Diseases),

Symutoms of,

Treatment of,

Sequelie of,

Chronic (see Roaring),

Larynx, Auscultation of the,

Lathyriasis (sce Sromadic Diseases),

Lead Poisoning (sie Sporadic Dis.

eases),
Legal importauce of Fatty Infiltration, $\quad 67$

Lime in the Water in Oxaluria, . 458

Lipæmia, or Fatty Blood,

Liver, Affections of (see Sporadic Dis. cases),

Congestion of,

Symitoms of,

Treatment of,

Cirrhosis of, .

Discases of, .

Gall Stones in,

Induration of,

Inflummation of

Necrotic Abscesses in

limpiture of,

$$
\begin{gathered}
\text {-Canses and post mor- } \\
\text { tem aplearances, } \\
\text { Treatment of, . }
\end{gathered}
$$

Softening of,

Lobar Pnemmonia (see Enzootic Diseases),

Local or General Symutoms,

Lomping-Ill or Trembling-Ixodic

Toxemia (sce Sporadic Diseases),

Lower Animals, Vomition in.

Lumbrici,

Lumbricoides,

Lungs, Affections of (see Sporadic Diseases),

Lumgs, Congestion of the,
PAGE

757

416

735

736

70
Lungs, Death beginning at,

Gangrene of, . . $\quad 632$

Hepratization of, . . . 630

Inflammation of, . . . 629

Snppuration of, . . . . 631

Parasitic Diseases of, . . 799

Post mortem appearances of, . 628

Pathology of, . . . 629

Etiology of, . . 634

Sympitoms of, . . 634

Treatment of, . . . 638

Lupinosis (see Sporadie Diseases), 492

Lymphadenoma of the Spleen, . $\quad 739$

Mad Staggers, . . . $\quad . \quad 507$

Maladie du Coït (sec: Contagious Diseases), 255

Malignant and Catarrhal Fever in $\mathrm{Ox}$ (see Enzootic Diseases), . . $\quad 379$

Mammitis, Contagions, . . $\quad 762$

Meehanieal Engorgement, . . 624

Nellitenia, . . 93

Metritis, . . . 760

Symptoms and Treatment, . 761

Milk Sickness (sre Sprorarlic Discases), 480

Morbid Elementary Products, . 47

Morloid Materials, Classification of, 47

Morlid Phenomena, . . 23

Mucous Membranes, Redness of, . $\quad 26$

Tesication of, . . $\quad 26$

Petechial Sjots, . $\quad . \quad 26$

Lividity of, . . . . $\quad 26$

Various Colomrs, . . . 27

Nurir-Ill (see Sporadic Discases), . 460

Naegelli on Bacteria, . . . 135

Nasal Somnds, . . . . 577

Necremia, . . . 123

Necrobiosis, . . 53

Negative Symptoms, . . . 35

Nematoda, . $\quad 765$

Diseases cansed l,y, . . $\quad 799$

Nematodes. $\quad 831$

Nenutoid Worms-Genus Oxymis, $\$ 32$

, , ",$\quad$ Ascaris, 831

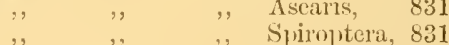

", ", ", "Trichina, 833

," , ". Tricoce-

") phalns, 834

., , , , Filaria, 83I

., , , ", Dochmius, 832

,, , , , Strongylus, 832

, . . , Eustrongylis, . 831

Nephritis (see Sporadic Diseases), . $\quad 748$ l'ost mortem and Treatment, $\quad 750$ Sequelie of, . . . 751 Symitoms of, . : $\quad 749$

Nervous System, Diseases of, . 498

Nenralgia, . . . . . 5736 Nemitis, . . . . $573 \pi$ 
New Formations from Hypertrophy,

Nitrate of Urca,

Nitrogenous Food,

Non-Spontaneity of Contagious Diseases discussed,

Nosology,

Numerical Atrophy,

Nutrition, Arrest of,

$$
\begin{aligned}
& \text {, Increased, : : : } \\
& \text { ", of Tissue, } \\
& \text { ", Process of, . }
\end{aligned}
$$

Oat-Hair and Mixed Calenli,

Obliteration of Jugular Veiu,

Oljjective Symptoms,

Estridæ,

Estrus Bovis,

,Equi,

", Hremorrhoidalis, Ovis,

Oil-Cakes, Analysis of,

Oligæmia,

Olulanns Tricuspis,

Omasum, Impaction of,

Opaque Vaccine Lymph, Variolous,

Organic Diseases,

Origin of Contagions Diseases, ", of Epizootics, Theories of, .

Osteoclasty,

Over-Feeding, Effects of,

Ovination,

Ox, Pleuro-Pnemmonia of the,

Oxalate of Urea,

Oxalic Aeid, Bencke on,

Oxaluria (sec Sporadic Diseases), . Canses and Symptoms, .

Lime in Drinking-Water,

Treatment of,

Urine in

Oxidation of Tissues,

Oxyuris Curvula, ,, Vermicularis, .

Palpation of Chest,

Panzootic Catarrhal Fever (see Enzootic Diseases),

Pancreas, Diseases of,

Paracentesis Thoracis,

Paraffin Oil Poisoning (sce Sporadic Diseases),

Paralysis (see Sporadic Diseases), . ,, Constipation from,

$$
\text { , General Sympitoms, }
$$

Paraplegia,

$$
\text { Reflex, . }
$$

Treatment of,

Frontispiece

Frontispiece

50

129

346

19

445

219

160

714

459

457

457.

460

460

458

44

765

772

576

473

739

651

477

541

689

544

544

545

547

Parasitic Diseases-

Remarks on Classification of Parasites, . . .

Classification of Entozoa, .

Nimatodu, or Found Worms, Trichinia Spiralis, .

765

766,815
PARASITIC DISEAsEs-contineed.

Ascarirles Megalocephala Suilla, and Lumbricoides, Davaine,

Strongyli,

Trematode, or Flat-Worms-

Flukes,

Rot from Fasciola Hepatica, .

,, History of,

", Symptoms of,

"Pathological Anatomy of,

, Treatment of,

, in Australia,

Cestoda, or Tape and Cystic (Bladder) Worms,

Proglottides and Strobila, :

Tape-Worms of the Dog, .

Coenurus Cerebralis cansing Vertigo or Gid, .

Cysticereus Bovis,

Cysticercus Cellulosus (Measles of Pork), . . . . 787

Acanthocephala, . . . 788

Echinorrhyuchus Gigas, 788

Dipterc, . . . 790

Estridre (Gadflies), Esstrus Equi, 790

Bots in the Horse, . . 791

Bots in the Ox and Sheep, . 792

Warbles, . . 792

Trachearia, Pentastoma Tæuioides and P. Denticulatum,

Diseascs causcd by Nematoda, . $\quad 799$

Parasitic Diseases of Lungs or Husk, and Symonyms, . 799

Strongylus Filaria, . $\quad$ • 829

Symptoms and Treatment, . 803

Chlorine and Sulphurous Acid, 804

Hoose in Calves, . . . 804

Strongylus Micrurus, . • 804

Symptoms and Treatment, . 805

Treatment of, with Intratracheal Injections, . . . 807

Gapes in Fowls, . . . 806

Selerostoma Syngamus, . 806

Parasitic Gustric Catarih, . 807

Definition, . . . 807

Strongylus Contortus, . . 807

Description of S. Contortus, . $\quad 810$

Symptoms, . . 807

Treatment, . . . 810

Worm found in Ieeland Ponies, (Strongylus Tetracantluus), 811

Description, . . . 814

Symptoms, . . . . 814

Treatment, . . . . 815

Trichinosis, . . . . 815

Trichina Spiralis, : $\quad 815$

Experiments of Dr. Bellfield and

Mr. Atwood, Chicago, . $\quad 815$

Trichina Spiralis in Main, . . 816

, $\quad$," $\quad$ Tymptonis of, 
Parasitiu Diseases-continucd. Ascaridcs (Lumbrici),

PAGE

Treatinent,

Diseases caused by Trematoda, .

Rot in Sheep,

Distomata,

History,

Symptoms,

Pathological Anatomy,

Treatment,

Discases caused by Cestoda,

Sturdy, Gid, Turnsick, induced by Cœnurus Cerebralis,

Symptoms,

Trocar,

Treatment of Tape-Worm,

Tabular Arrangement of the Cestoda,

Parasites, Remarks on Classification,

Parasitie Bronchitis,

" Disease of Lungs,

"Gastric Catarrh,

Plenalvia,

Plethora,

Pleural Sounds

Pleural Sounds, . $\quad 591$

821 Pleurisy (see Sporadic Diseases), . 645

821 Plemrisy, Auscultation in, . . 646

Pleuritis

$\begin{array}{ll}\text {,. Bleeding in, } \quad \text {. } & 650 \\ \text {,. } & 650\end{array}$

," Hydrothorax, . 647

, Paracentesis Thoracis in, 651

") Pereussion in, . . 648

", Post mortem of, . . 645

". Symptoms of, : : 647

", Treatment of, . . 649

835 Pleuro-Pueumonia Contagiosa (see

Parkin, Dr., on Origin of Rinderpest,

Parturient Apoplexy, ,, Fever,

Passive Symptoms,

Pasteur on Anthrax, \&c

Pathogeny,

Pathognomonic Signs,

Pathognostic Signs,

Pathology,

Essentials to the Study of, :

General and Special,

Pentastomes,

Pentastoma Denticulatum,

Tænioides,

Percussion, in Disease,

, in Pleurisy,

576

163

594

648

657

Synptoms of,

Traumatic,

Treatment of,

Perversion of Secretory Functions,

Petechice of Scarlatina and Purpura,

Plienomena, Morbid,

Phosplate, Excess of, in Urine, Phosphatie Calculi,

Phrenitis (Phrenzy)

Phthisis Pulnonalis Verminalis, .

Physiology, Importance of,

Pig M.easles, of the Spinal Cord,

Pigmentary Degeneration,

Piles, .

"Cause and Treatment,

Pineal Body, Enlargement of,

Tumours of the,
Pink-Eye (see Enzootic Diseases),

657

659

663

43

391
763

799

\section{Contagions Diseases)}

Pleximeter, .

Plumbism (sce Sporadic Diseases),

Pneumo-Enteritis (see Contagions Diseases),

Pneumonia, .

Poisoning by Parattin Oil

602

375

477

477

478

Polyuria, Yew,

449

236

Positive Symptoms, . . . 25

Potatoes, Raw, Effects of, . . 716

Precursory Symptoms, . . . 24

Predisposing Causes, . . . 5

Premonitory Symptoms, . . . 24

Prevention of Death from Apnoea, $\quad 118$

of Pleuro-Pneumonia, . 172

Prevost and Dumas, MM., Experi-

ments of,

89

Process of Nutrition, . . . 90

Products, Mlorbid Elementary, . 47

Production of Animal Heat,. . 44

Proglottides, . . . . 780

Prognosis,

4

$\begin{array}{lr}\text { Forming of a, } & 25 \\ \text { in Congestive Apoplexy, } & 498\end{array}$

in Eneephalitis, ? 514

in Influenza, . . 354

Prognostic Symptoms, . . . 24

Progressive Gangrenc, : . $\quad 105$

Prophylaxis, . . . 108, 128

Proscolex, . . . . . 782

Prostate Gland, Enlargement of, . $\quad 756$

Proximate Canses, . . . 4

Pulmonary Apoplexy, . . . 624

,, Sounds, . . . $\quad 590$

Pulsations, Mntral Relations of, . $\quad 35$

Pulse, The, . . . . . 29

,, Single, . . . . 30

"Double, $\quad 39$

, Feeble, . . . 37

764, Frequent, . . . 33 
Pulse, Gaseons, - • $\quad \begin{array}{r}\text { PAGE } \\ \quad-\quad 37\end{array}$

, Hard,

, Infrequeut,

, Intermittent,

, Jerking,

, Large and Simall,

Quick,

Rate of, in various Aninals,

Slow,

Snall,

Soft,

Strength of,

Symptoms afforded by the,

Weakness of,

Varieties of,

Venous,

Volume of,

Puncturing the Colon, .

Pupre Distoma,

Purpura Hrmorrhagica (see Eruptive Fevers),

Causes of,

"Petecliæ of,

Post mortem of, .

,. Symptoms of,

,, Treatment of,

Pus in Urine,

Quantity of Food,

Quarter-Ill (see Contagious Diseases), 305

Quick Pulse,

Quickened Breathing, .

Rabies (see Contagious Diseases), .

Ralc, Cavernous, .

," IIncous,

Ramollissement, .

Rate of Pulse in various Animals,

Reactions of Amyloid Substance, .

Rectum, Inversion of the,

Redìt, Rupture of the

Red Water (see Sporadic Diseases),

Redness of Visible Mucous Membranes, 26

Reflex Paraplegia,

Renal Calculi.

Respiration, Difficulty of,

Respiratory Functions, Symptoms,

$$
, \text { Organs, Diseases of, . }
$$

Results of Mechanical Congestion,

Retention of Urine, . .

Reynolds' Classification of Discases,

Rheumatic Complications of Inflnenza, Fever,

Rheumatism, Acute,

$\begin{array}{lll}, & \text { Chronic, . } & 439 \\ , & \text { Symptomis of, . } & 438 \\ & \text { Treatment of, in the } \\ & \text { Horse, . } \\ \text { Treatment of, in the Ox, } & 441\end{array}$

Rhododendron Poisoning,
PAGE

", Cavernons, . . $\quad 589$

" Nucous, . . . $\quad 589$

", Snall Bubbling, . . 589

Rigor, Symptoms of, . . . 29

Rinderpest, . . . . $\quad 159$

,$\quad$ in Sheep, : . . $\quad 159$

,, Inoculation in, . . 157

Roaring, . . . . . 581

" Causes of, . . . 581

," Treatment of, . . . 584

Rot, . . . . 775

,, from Trematoda, . . . 821

,, in Anstralia, . . . $\quad 779$

,, Symptoms of, . . . 822

"Treatment of, $\quad . \quad \cdot \quad \cdot \quad \cdot \quad 825$

Round-Worms, . . . . 765

Rumen, Impaction with Solids of the, 680

Rumenotomy, . . . . 681

Rupture of the Diaphragm, . . $\quad 670$

", of the Liver, . . . 732

, of the Stomach, . . $\quad 687$

Rye-Grass a canse of Encephalitis, $\quad 511$

Saline Matters of Urine, . . 745

Sanderson, Dr., on Rinderpest, . 144

Sapræmia (see Septic Blood Diseases), 398

Saprophytes, . . . . 130

Sangnineous Ascites, . . $\quad$. 720

Scarlatina (sce Eruptive Fevers), . $\quad 389$

Scarlet Fever (see Eruptive Fevers), $\quad 389$

Schizomycetes, . . . . 132

Sclerostoma Syngamus, . . $\quad 805$

Scolex, . . . . . 780

Scurfy Skin, Symptoms of, . . 28

Seat of Diseases, . . . . 50

Secondary Digestion, . . . 44

". Symptoms, . • . 24

Secretion, Defective, a Cause of Congestion, . $\quad$. 98

,, Symptoms afforded by, 42

", Perversion of, . . 43

Semiology, Colliquative, · $\quad 43$

Sequelæ of Congestive Apoplexy, . $\quad 528$

,, of Influenza, . . . 357

,. of Laryngitis, . . . $\quad 598$

Sepsin, Poisoning by, . . . 107

How induced, . . . 395

BuAck Spatn-
Etiology and Pathology, $\quad 405$

Treatment, . . : : 406

Braxy - Symptoms, : $\quad 402$

Post mortem appearanees, . 403

Pathology, . . . . 403

Treatment, . $\quad . \quad$. 404

Post-Pantulient Sapremia and SePticenil, . . . 401

SAIREMIA, · . . $\quad 398$

Tranmatic Fever Sympitums, . 398

Malignant (Edenia Symptoms, 399 
SEPTIC BLuUn, DIsEAsEs-cuntinued.

Stercorremia Symptoms,

Hectic Symptoms, . . 400

Septic Pneumonia of Calves and LAMBS,

Symptoms,

Post mortem aplearances,

Treatment,

Septicemia Gangrenosa (see Braxy),

Striking of Bluon (see Braxy),

TuUE SEPTI'enila, .

VARIOLA VACCINA-

Synonyms and Definition,

Pathology and Symptoms,

VAIIOLA OVINAE-

Synonyms and Definition,

History of,

Pathology and Sympitoms,

Simonds on, .

Gamgee on, .

Post mortem appearances,

Prevention and Treatment,

VAIIOLA EqUina,

VARICELLA BUIM,

Septic Pneumonia (see Sertic Biood

Diseases),

Septicremia (see Septic Blood Diseases), 402

Septicremia Gangrenosa (see Septic

Blood Diseases),

Septicremia, Post-Parturient (see Septic

Blood Diseases),

Septicrmic Vims, Experiments with 105

Sheep-Pox, . . . 210

Sheep, Rinderpest in, . . . 159

Shivering a Sympton of Indigestion, 29

Shock, Death from, . . . 115

Sililus, . . . . 589

Signs of Disease, . . . . 23

Simple Atrophy, . . . . 52

Six-hooked Embryo, . . $\quad 782$

Skin, Scurfy, a Symutom of Indigestion,

Sleepy Staggers, .

Small Pulse,

Snoring,

Soft Pulse,

Softening of Caseons Matter, of the lirain,

Solanacere, Toxic effects of the,

Sounds, Absence of Plenral, .

Alusence of Pulmonary,

", Bronehial Dry,

,

, Moist,

Cardiac, .

oist,

Friction,

Laryngeal,

Morbid Bronchial,

Nasal,

Cardiac,

on Percussing Cliest, Plemal,
Somuls, Puluonary, PAiE

590

590

577

586

586

587

586

90

690

51

51

4

Sphygmograph, : $\quad: \quad 29$

Spinal Cord and Nembranes, Diseases of, $536^{\circ}$

Spinitis (see Sporadic Diseases), . $\quad 536$

,, Post mortem appearances of, 538

, Clironic,

" Cleatment, : 540

Spiroptera Ohtnsa, . . . 773

Spleen, Diseases of, . . . 739

,, and Pancreas, Diseases of, 739

, Lymphadenoma of the, . 739

Splenic Aproulexy (see Contagions

Diseases), . . . • 294

Splenie Fever (see Contagions Uiseases), 294

Spplenic Aproplexy, . . . 294

Sporadic Diseases

Rinematism, . . . . 438

Acnte or Rhemmatie Fever, . 438

Definition, Etiology, Semioloug, 4:38

Chronic Rhemmatisnu, . . 439

Pathology, . . . . 440

Treatment in the Horse, . 441

Local Treatinent, . . 442

Treatment in the $\mathrm{Ox}$, . $\quad 443$

DiETETIC DISEASES, . . . 444

Constitnents of Food, . . ' 444

General Fitzwygram on Fooù, 4.14

Eflects of Over-feeding, . 445

Analysis of Oil-cakes, . . $456^{\circ}$

Definition of Dietetic Disease, 447

Diaibetes Instrines, Polynria, 448

Definition and Etiology, . 448

Semiology and Pathology, . 449

Diabetes Mellitus, ? . 450

Treatment, . . 450

Iodine, . . . . . 450

Fatal in the Dos, . . . 450

Post mortem appearances, . 451

Asthma, Broken Wini, . 466

Definition and Etiology, . 467

Pathology, . . . . 468

Morbid Anatomy, . . $\quad 469$

Symptoms, . . . . 470

Treatment, . . . . 471

Modes of rehieving Broken Wind, 471

Ren WATER, HeNu-A LBUNINURIA,

Black Water, Hemattria,

Mitil-Ill, $\quad$. . . 460

Definition and Etiology, . 461

Pathology, . . . . 462

Analysis of Urine, 462 
SPORADIC DisEases-continued.

Post mortem appearances,

Symptoms,

Treatment,

AzoT' IRI $\Lambda$,

Definition and Symptoms,

Post mortem appearanees,

Examination of the Urine,

Treatment,

Oxaluisa,

Definition,

Semiology and Etiology,

Condition of the Urine,

Beneke on Oxalic Acid,

Treatnient,

Examination of Drinking Water,

Rhodonendron Poisonisg, .

Treatment,

Yew Puisonisg, . . .

Post mortem,

Treatment, .

Milk Sickisess,

Etiology,

Symptoms,

Cake Poisonixg, Castur Oil Seed and Cruton Seed Puisoning

Adulteration, Cause of,

Method of Detection,

Differences in appearance of

Castor aud Croton Sceds,

Symptoms,

Post mortem appearances,

IAATHYRIASIS,

Definition of,

Varieties of Lathyrus sative, .

Nutrient Properties in, .

Professor Maedongall on,

Symptoms in the Horse,

Lupixosis,

Origin of

Varieties of Lipuins,

Symptoms, .

Lean Polsonisg, Lean Palsi, Plunisism,

Definition and Canses, .

Symutons,

Post mortem appearances,

Finlay Dun on,

Treatment,

Galvanisn, .

Paraffix Oll Polsonici,

LOCAL DISEASES,

Definition,

Dismasta

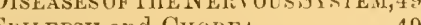

EPILEPSY and CHores $A, \quad \cdot \quad 498$

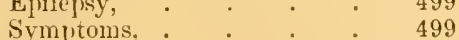

Treatment, . . . . 500

Chorea-Definition, Symp,toms, 501

l'ost mortem of, . . . 502

Treatment,
PAGE

Sporane Diseases-continued.

PAfE

Cepilalic Diseases,

INFLAMMATION OF TIIE BRAIN Substaxce, or Cerebritis,

Post mortem of,

Compared with Meningitis, . $\quad 505$

Treatment, . . . . 506

ENCEPHAlitis, . . . 507

Definition, . . . 507

Effect of Burnt Ale on Cows, $\quad 508$

Symptoms, . . . 509

Etiology, . . . . 511

Morbid Anatomy, . . . 513

Prognosis, . . . . 514

Treatment, . . . . 511

Apoplexy, Cerebral, . • 516

Definition, . . . 516

Morbid Anatomy, . . . 518

Symptoms, . . . . 516

APOPLEXY FROM CONGESTIONParturient Apol'lexy or MILK FEYER, . • • 519

Definition and Etiology, . $\quad 519$

Symptoms, . . . $\quad 519$

Prognosis, . . . . 524

Post mortem of, . . $\quad 525$

Treatment, . . . . 525

Sequelæ, . . . . 528

Advextitious Substaxces in

Bran axd Craxial Cavity, causing various Symptoms, 531

Tumours, . . . 531

Thickening of the Dura Mater, $\quad 532$

Tubercular Dep̧osits in Meuinges, 533

Exostoses, . . . 534

Hypertrophy and Atrophy, - 534

Mlelanosis, . . 535

Tumou's of the Pineal Body, 535

Softening of the Brain, . . 535

Diseases of SPINAL Culid AND ITS MEMbRANEs. • • 536

SplNitis, . : $\quad 536$

Chronie Spinitis, : : : 539

Post mortem of, . $\quad$. $\quad$. 539

Treatment, - . $\quad$. 540

Paralysis, . . . . 541

Physiology of the Spinal Cord, 541

PARARLEGA, . . . 544

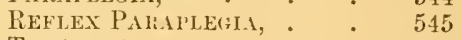

Trentment, . . . . 547

HeMIILEGIL, : $\quad \cdot \quad \cdot \quad 577$

Cases of, . . . $\quad 548$

Lovpreste-Ih, or Themblisg

(Irodic Toxcemiu), . . 550

Origin of, . $\quad . \quad 5 \quad 550$

Symptoms, . . . . $\quad 550$

Causes, . . . $\quad 552$

Influenee of Pasture, \&c. . $\quad 552$

Influence of Weather, . . $\quad 553$

Influence of Ticks, . . 554

Cultivation of Tick Organism, 557

Further Investigations into Causes of, . . . . 568 
Sroradic Diseases-contimed.

Inoculation with Cultivation from

Tick,

Experiments in Inoculation, .

568

570

Photo-Micrographs of Organisn, 572

Neuritis, Nevilalgia, . $573 a$

Diseases of the Resplitatory Organs, Diarnosis-Ausculta

ties Stetloscope, tion, Stetloscope,

Pereussion,

574

Plexinieter,

Palpation, $\quad 576$

Mlensuration, . . $\quad 576$

Inspection, . . . . 577

Succussion, . . . . 577

Respiratory Sounds, . . 577

Nasal Sounds, . . . 577

Epistaxis, . . . . 579

Anscultation of the Larynx, . $\quad 579$

Laryngeal Sounds, . . $\quad 579$

Grunting, . . . 579

Whistling, . . . . 580

Roaring, . . . . 581

Causes of Roaring, . . $\quad 583$

Treatment of, . $\quad 584$

Cough, . . . . 555

Kinds of, . . . . 585

Tracheal Sounds, . . . $\quad 586$

Thoracic Sounds, . . . 586

Vesicnlar Sounds, . . . 586

Bronchial or Tubal sounds, . $\quad 587$

Sounds on Perenssion, . . 557

Morbid Sonnds, . . 588

Bronchial Sounds, Dry and Moist, 588

Rhonchus, . . . 588

Sibilits, * . 589

Moist Sounds, . . . 589

Mucous Rhonchus or Rale, . $5 \$ 9$

Small Bubbling Rhonchus, Cavernous Rhonchi,

Pulmonary Sounds, . $\quad 590$

Clepitations, 590

Tubal or Bronchial Sounds. . $\quad 590$

Alisence of Somd, . . 590

Secondary Crepitations, . $\quad 591$

Plemal Sounds, . . 591

Friction Sonnds, . . . 591

Absence of Sound, . . 592

Cardiac Sounds, . . . 592

Morbid Sounds, $\quad 598$

Percussion in Disease, . . 594

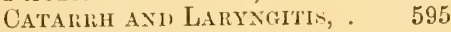

Synonymis, Symptoms, Causes, and Treatment of Catarrh,

Laryngitis, Pathology and Symptoms,

Treatment, 597

Sequelie, . . 598

Croup, Cranunue Trachealis, 599

Definition, . . . . 599

Symptoms, . . . . 600

Treatment, : $\quad$. 601
SPORADI: DISEASES-continued.

PAGE

Diphtheria,

" Mr. Robertson on, 602

" Cases of, . . 603

", Post mortem appear-

ances, . . 604

, Treatment, . 604

BRoNCHITIs, . . . 605

Division, . . . . 605

Causes, . . . 605

Symptoms, . . . 607

Pathology and Morbid Anatomy, 611

Treatment, . . 618

Chronic Form, . . 620

EMPHYSEMA OF THE LUNG, Clironic and Vesicular, . . . 621

Interlobular Emphysema, $\quad 622$

Treatment, . . . . 623

Congestion of the Lungs, Pulmonary Apoplexy, . . 624

Causes,. . . 625

Symptoms, . . . . . $\quad 626$

Treatment, . . . . 627

Post mortem of, . . . 628

FFlaMMATION OF THE LUNGS, Lobali (Sporadic) Pxeunoxia, 629

Synonyms, Varieties, and Patho$\log y$,

Arterial Injection, 630

Red Hepatization, . . 630

Grey Hepatization, . . 631

Suppuration, . . . . 631

Gangrene, . . . . 632

Etiologry, . . . 634

Symptoms, . . . . 634

Hæmoptysis, . . . 638

Treatment, . . . . 638

Bleeding, . . . . 638

Blisters, . . 642

Plemrisy and Pleuritis, . $\quad 645$

Definition, . . . 645

Symptoms, . . . 647

Hydrothorax, . . 647

Treatment, . . . . 649

Paracentesis Thoracis, . . 651

Diseases of the Heant ani) i's

MLMBRANEs, . . . 654

Functional Derangement, . 651

Organic Diseases, . . . 655

Hypertrojhy, . . . 655

Pelicaridits, . . . 657

Synptoms, . . . . 657

Trammatic form, . . . 659

Treatment, . . . . 663

Exuocarnitris, . . . 664

Morbid Anatomy and Treatment, 664

DisEases OF THE VALVES, . 666

Angina Pectomis, . $\quad 667$

Cyanosis, . . . 668

Ectopia Cordis, . . . 669

AFFECTIOSS OF THE DIAJHALGM, 670

Spasm and Treatment, . . 670

Rupture of, . . . . 670 
Sporanic Diseases-continued.

Diseases of the Digestive Organs,

Comparison of Organs in different Animals,

Diseases of the Stomach, .

Indigestion, .

Causes and Symptoms, .

Treatment, · •

Indigestion with Engorgement,

Synonyms and Causes in Horse,

Canses in Horned Cattle,

Vomition in the Lower Animals,

Symptoms in the Ox and Sheep,

Treatment,

Impaction of the Rumen with Solid Matter's,

Rumenotomy,

Impaction of the Third Stomach,

Abomasitis,

Treatment,

Impaction in the Dog and Cat,

Gastrorrhoea in the Dog,

Symptoms of Gastric Impaction in the Hor'se,

Treatment of Gastric Impaction in the Horse,

Rupture of the Stomach,

Symptonus,

Vomition,

Causes, .

Diseases of THE Bowels,

Constipation, Causes and Treatment,

Colic,

Spasmodie Colic,

Symptoms of,

Treatment of,

Flatulent Colic

Symptoms, .

Treatment,

Puncturing the Colon, .

Impaction of the Colon,

Rupture of the Colon,

Rupture of the Rectum,

Inversion of the Rectum, . 697

Hæmorrhoids or Piles, . . 698

Cause and Treatment, . . 698

Imperforate Anus,

Inflammatory Diseases-Enteritis, 699

Canses,

Pathology and Symptoms,

Treatment,

702

Bleeding,

Intussusception,

704

Definition,

704

Symptons and Treatment, . 705

Volvulus,

706

Intestinal Coneretions, . . $\quad 707$

Calculi,

707

Diarrhcea

Definition and Symptons,

Treatment,
Spopadic Diseases-continued.

PAGE

Superpurgation, . . . 713

Causes of, . . . . 714

From Aloes, . . . . 714

Prevention after a Cathartic, $\quad 715$

Treatment, . . . . 715

Inflammatory Diarrhœa, . $\quad 716$

Raw Potatoes producing, . 716

Dysentery, . . . . 718

Definition and Causes, . . 718

Symptoms and Treatment, . 718

Aseites or Dropsy of the Abdomeu, 720

Causes, . . . 720

Symptoms and Treatment, . $\quad 721$

DISEASES OF THE LIVER, . 722

Congestion, . . . . 722

Symptoms, . . . . 723

Treatment, . . . . 724

Elaterium in the $\mathrm{Dog}$, . $\quad 724$

Hepatitis or Inflammation of the

Liver,
Causes and Symptoms,

Treatment, . . . 727

Chronic Hepatitis, . . 727

Induration (Cirrhosis), . . 727

Cases of Cirrhosis, . . $\quad 729$

Softening, Remollissement, Rupture of the Liver, . . 732

Causes and Post mortem of, . 733

Treatment, . . • . 734

Jaundice, Ieterus or the Yellows, 735

Causes, . . . . 735

Treatment, . . . . 736

Necrotic Abscesses, . . 737

Gall Stones, . . . . 738

Diseases of the Spleen AND Paxcreas, . . 739

Lymphadenoma of the Spleen, 739

DIsEases OF THE KIDNEYS, . 742

Urine in the Lower Animals, 742

Urea, . • . . . 743

Oxalate of Urea, . . . 743

Uric Acid, . . . $\quad 744$

Hippuric Acid, . . . 744

Extractive Matters, . $\quad 745$

Saline Matters, · . . 745

Exeess of Phosphates. . . $\quad 746$

Pus and Blood, . . . $\quad 746$

Albumen, . . . $\quad 746$

Analysis of Urine in Health, 747

Nephritis-Inflammation of the Kidneys, . . $\mathbf{7 4 8}$

Definition and Causes, . . $\quad 749$

Symptoms, . . . . 749

Post mortem, . . . 750

Treatment, . . . . 751

Cantharides, . . . 751

Sequelæ, . . . . 751

Albuminuria-Calıses, . . $\quad 752$

Symptoms, . . . . 753

Treatnient, . . . . 753

Floating Kidney, . • $\quad 754$

Hienaturia-Renal Caleuli, . 754 
Sporadic Diseases-contimued.

Cystitis-Inflammation of the Bladder,

Symptoms and Treatment,

Retention of Urine,

Enlarged Prostates,

Incontinence of Urine, .

Hysteria,

Parturient Fever

Acute Metritis, or Inflanmation of the Womb,

Definition of

Pathology,

Symptoms,

Treatment,

Contagious Mammitis,

Sporocyst,

Statical Symptoms,

Stertorons Breathing, .

Stethoseope,

Stomach, Diseases of, .

$\begin{array}{ll}\text { " } & \text { Causes of, } \\ \text { Inpaction of the, } & \text { Rupture of the, } \\ \text { " } & \text { Staggers, } \\ \text { " Symptoms of Rupture of, }\end{array}$

Stomatitis Pustulosa Contagiosa in

Horse,

Strancrurs,

Strength and Weakness of the Pulse,

Striking of Blood (see Sentic Blood

Diseases),

Strongyli,

Strongylus Filaria

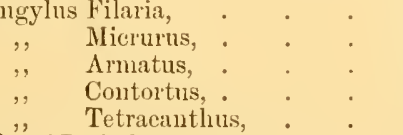

Study of Pathologr, Essentials to the,

Sturdy,

, Symptoms of,

"Trocar in

Snccinea Putris,

Suceussion of Chest, . . . 577

Sulphurous Acid as an Anti-Parasitic, 804

Superpurgation,

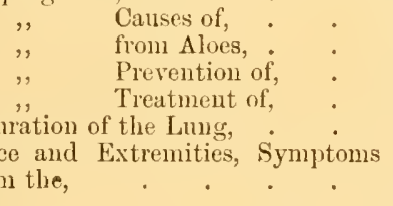

Suppuration of the Lmin, . . 631

Surface and Extremities, Symptoms

from the,

Surra,

Swine Plague (sce Con tagions Diseases), 310

Sympathetic Symptoms, . • 24

Symptomatology

Symptoms and Signs of Disease-

Symptoms,

Definition,

Lucal or Gencral.

Idioprathic,
PAGE

755

755

756

757

758

759

760

760

760

761

761

762

778

25

41

576

674

674

680

687

507

687

687

$2: 0$

757

59

403

772

799

804

832

807

811

827

828

829

776

713

714

714

715

715

631

4

23

24
Symptoms and Signs of Disease-contd.

Sympatletic or Secondary, . 24

Premonitory or Precursory, . $\quad 24$

Commemorative, . . . 24

Diagnostic, Prognostic, Therapeutic, 24

Objective, . . . . 24

Active or Dynamical, . . $\quad 25$

Passive or Statical, . . . 25

Positive, . . . . 25

Negative, . $\quad$. $\quad . \quad 25$

Diagnosis, Art of, ․ $\quad 25$

Pathognomonic or Pathognostic, 25

Example of Diagnosis, . . $\quad 25$

Forming of Prognosis, . . $\quad 25$

General, . . . 26

Visible Mucous Membranes, . $\quad 26$

Surface and Extrenities, . 28

Pulse, . $\quad 29$

Erequency of, . . . 33

Quick, . . . . 33

Slow, . . . . . . 35

Infrequent, . . . . 35

Volume of, . . . . $\quad 36$

Feeble, Small, IIard, . . $\quad 37$

Soft, . . . . . 38

Double, . $\quad . \quad$. 39

Strength and Weakness, Canses of, 39

Venous, . . . 39

Reniratory Finctions, . 40

Quickened lireathing, . . 40

Difficulty of Respiration, (Dyspnœea), 40

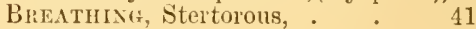

Abdominal, . . . . 41

Thoracic, . . . . . 41

Irregular, . . . . 41

Cough, Varieties of, . . 42

Secretions, . . . . 42

Perversion of, . . . 43

Calonific Functions, . . 43

Abnormal Heat, . . . 45

Syueope, Kinds of, . . . 114

Synomyms of Catarth, . . $\quad 595$

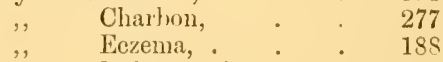

," Inflammation of the Lungs, · 629

Influenza, . . 316

Maladie du Coït, . 255

Plenalvia, . . 677

Pleuro-Pnenmonia, . 160

Rinderyest, . . 137

Tabular Arrangement of Entozoa, 831

Tape-Worms, . . . . 780

, Arrangement of Species of,

835

of the Dog, : . $\quad 784$

Treatment of, . 829

æriæ,

" Conurns, . . . 835

," Crassicolis, . . . 835

,. Cucumerina, . . . $83 .$,

,2 Echinococens, . . \$35 
Tenia, PAGE

Tænia, Expansa,

, Marginata,

", Mediocanellata,

", Serrata,

, Solium,

Temperature of Surface and Extremities,

Animal, in Health,

Thermometer, Rise" in Disease,

" Animal, in Health,

Therapeutic Symptoms,

Therapentics,

Texas Fever-Ixodic Toxremia (see Blood Diseases),

in Jamaica (see Blood Diseases), Thoraeic Breathing,

Thorter-Ill, Hydatid in Cerebellum,

Thrombi, Formation of,

Ticks, Ixodes,

Tissue, Atrophy of,

Tissues, Degeneration of

$\begin{array}{ll}\text { "Nutrition of, } & \text { Oxidation of, } \\ \text {," Transformation of the, Effect }\end{array}$

Tonic Spasms, on Blood,

Tracheal Sounds,

Trachearia,

Tracheotomy in Scarlatima,

Trammatic Pericarditis,

Trematoda, . causing Rot in Sheep, .

Trembling, or Louping-Ill,

Trichina Spiralis,

Trichonema Arcuata, Strongylus

Tetracanthus,

Trichinosis,

Triehiniasis,

Trichocephalus Affinis,

Dispar,

Tricks of Horse-Corper's,

Troear in klatulent Colic,

Tsetse Fly Disease (see 13lood Diseases), 414

Tubal Sounds,

Tuhereular, Cheesy matter not,

Deposits in Brain,

766,815

811

815

767

772

770

784

471

694

587

60

533

Tuberculin (see Contagions Diseases), 339

Tuberculosis (see Contagious Diseases), 323

Tumours, Caseous,

", from Hypertrolyy,

") of Pineal Body,

Tympanites, Causes of, in Horned Cattle, .

in $\mathrm{Ox}$ and Sheep, Treatment of,

Symptoms in the Ox and

Turusick, Sheep,

Urea, Composition and Source of,

, Demonstration of Nitrate of,
Urea, Influence of, in excess, . $\quad 89$

743

743

89

744

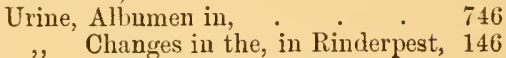

" Excess of Phosphates in, . 746

, Extraetive Matters of, . . 745

". in Azoturia, . . . . 454

", in the Lower Animals, . . 742

, in Oxaluria, . . . 457

, Ineontinenee of, . . . 757

, Pus or Blood in, . . . 746

", Retention of, . . . 755

," Saline Matters of, . . 745

Use of Thermometer, . . . 44

Variation in Constituents of Blood, $\quad 72$

Varicella Boum (see Contagions Diseases), • . . . 219

Varieties of Congh, . . . 42

,, of Pulse, . . . 43

Variola Equine (see Contagious

Diseases), . . . . 210

Variola Ovinæ (see Contagious Diseases), 219

Variola Vaccine (see Contagious

Diseases),

202

Venous Pulse, . $\quad . \quad$. $\quad . \quad 39$

Ventilation, Imperfect. • . 20

Vermes, . . . . 763

Vertigo, . . . 500,682

, from Worms, . . . 500

Vesication of Visible Mucous Membranes, 26

Vesicular Sounds, • . . 586

Veterinary Medicine and Surgery, Distinction,

Visible Mucous Membranes, Appearance of, . . . . 26

Vital Processes eonstituting Disease, 49

Volume of the Pulse, . . . 36

Volvulus, . . . . . 706

Vomition in the Horse, . . 687

,, in the Lower Animals, . 679

Warbles, . . 792

Wastiny Diseases, Death from, . 116

Water Braxy, . . . . 721

,, in the Blood, . . . 72

Watson, Sir Thomas, on Plethora, $\quad 87$

Waxy Degeneration, . . . 62

Whip-Worms, . . . . 797

Whistling, . . . . . 580

Woorara Poison, Experiments with, 122

Yellows, The, . . . . 735

Yellow-colomred Mucons Membranes, 26

826 Yew Poisoning (see Sporarlic Diseases), 479

743 Zimmerman, Dr., Experiments of, it

454 Zoo-Pathology, . . . . 4 




\section{$x^{2}-$

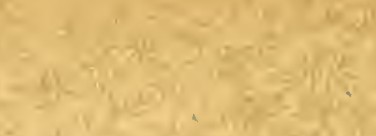

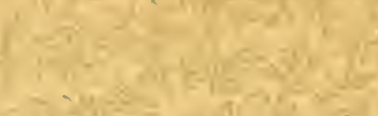

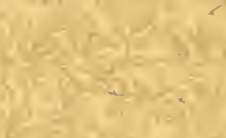

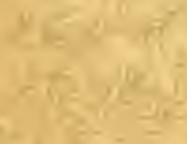

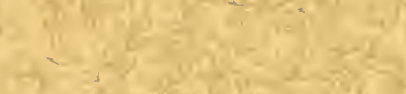

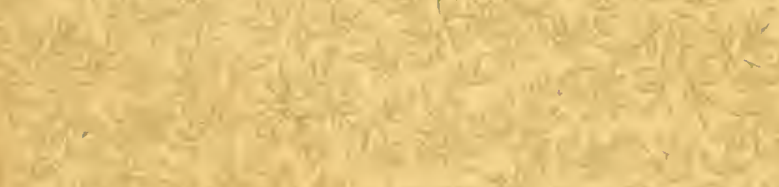

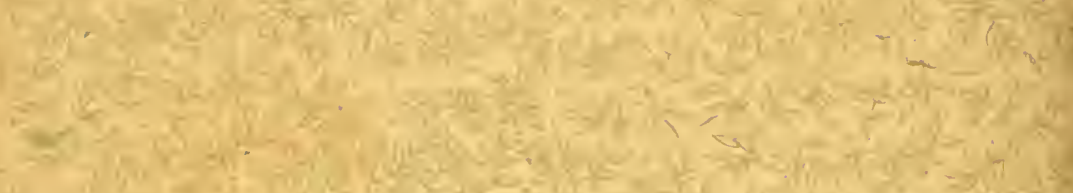

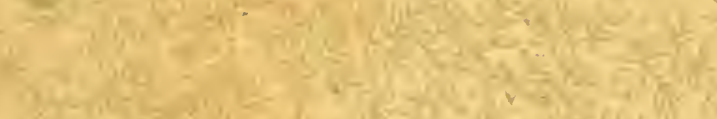

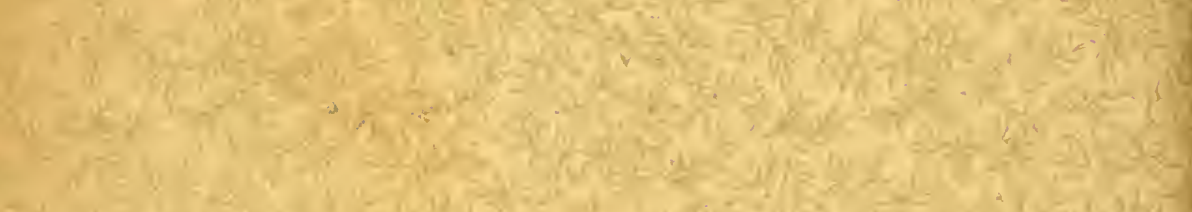

-
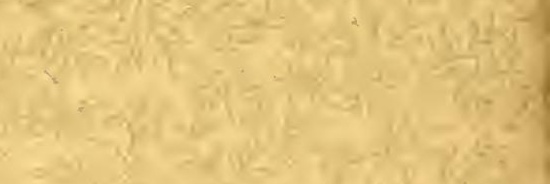

Webster Family Litary of Vatefhary Medicine

Cunmines Sehool of Votemary Modicine at

Tutis University

200 Westboro Ŗọad

North Grafton, MA 01536 


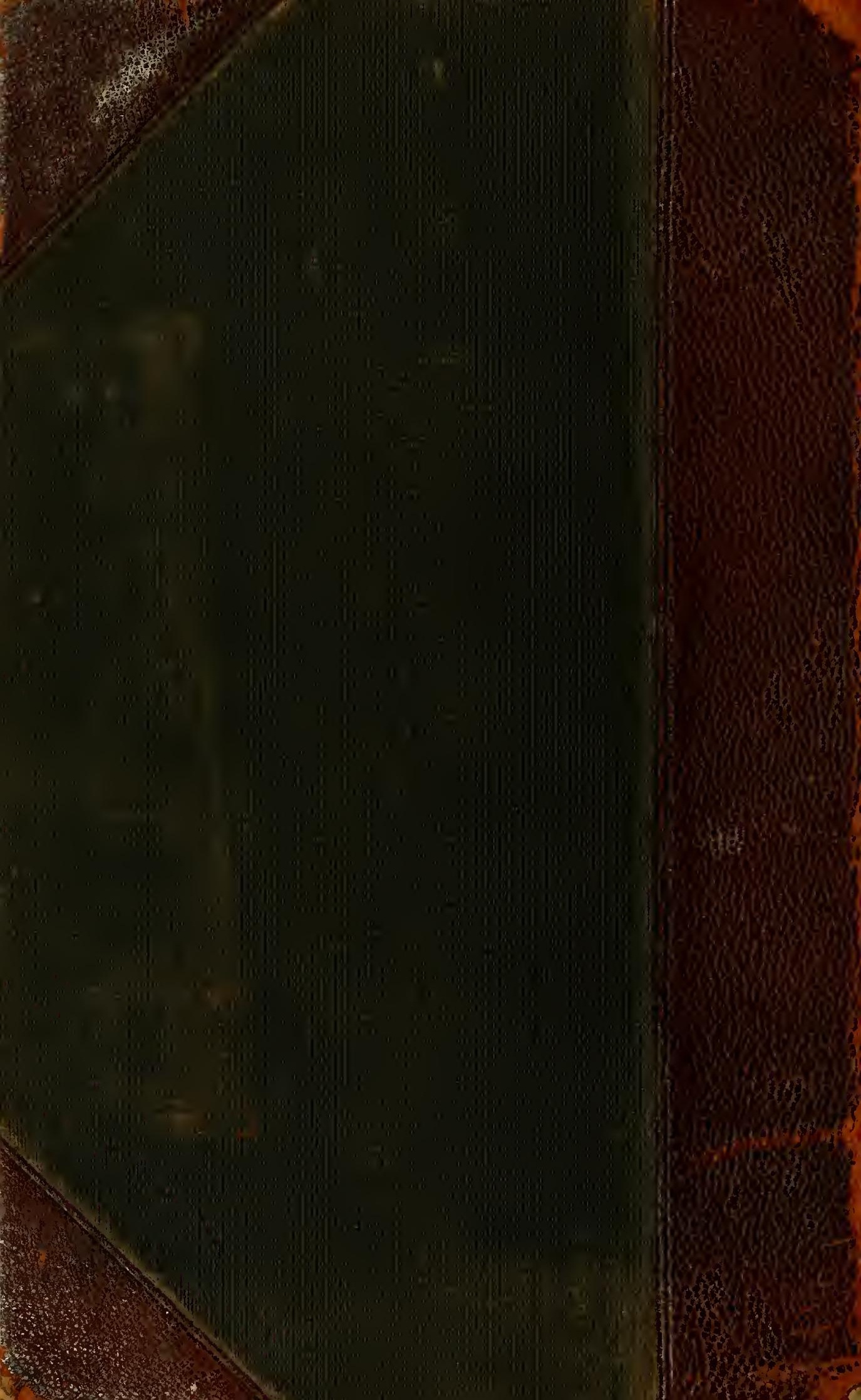

